
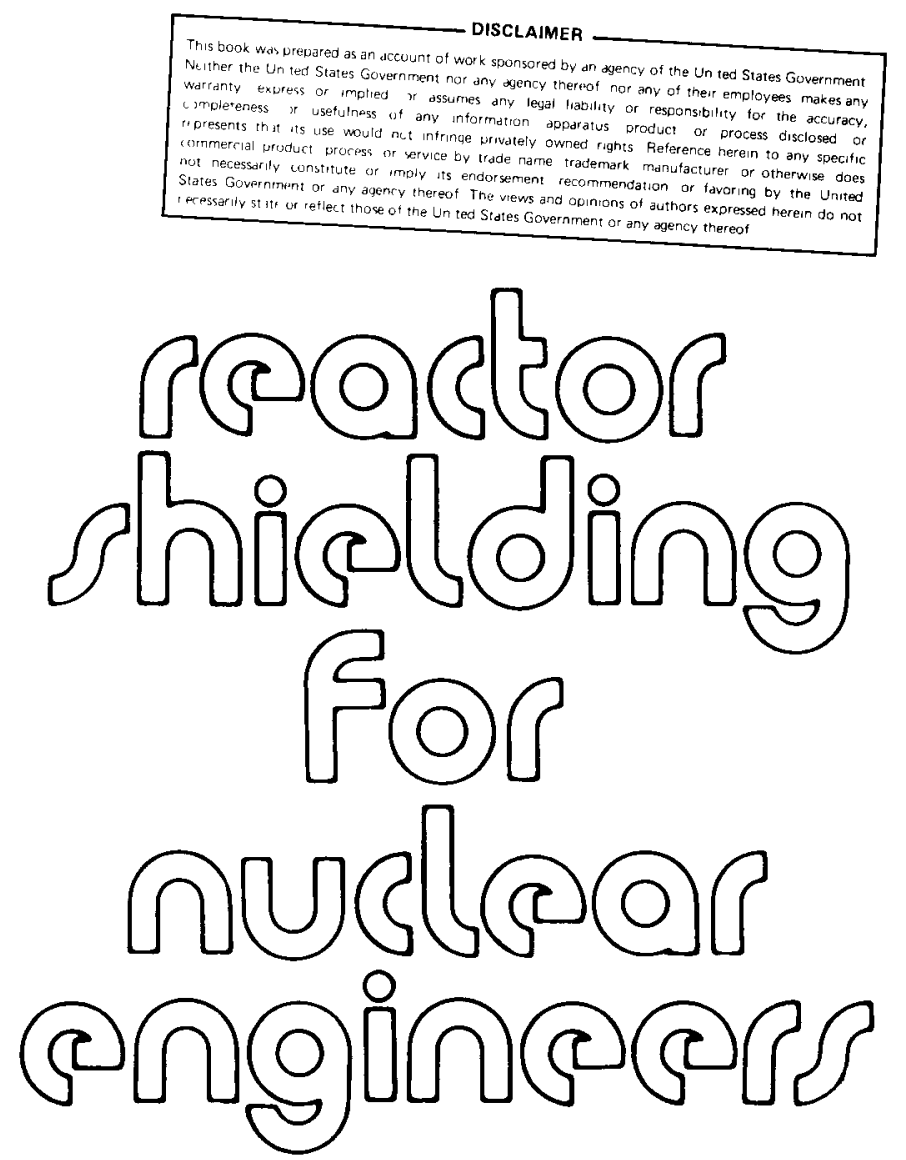

M.M.SCHAEFFER, Editor

President, Radiation Research Associates, Inc.

Prepared for

Division of Reactor Development and Technology U.S. Atomic Energy Commission

Reprinted by the Technical Information Center, U. S. Department of Energy 
Available as TID-25951 for $\$ 28.00$ from National Technical Information Service U.S. Department of Commerce Springfield, Virginia 22161

International Standard Book Number 0-87079-004-8 Library of Congress Catalog Card Number 73-600001 Distribution Category UC-80

May 1973; latest printing, September 1981 


\section{DISCLAIMER}

This report was prepared as an account of work sponsored by an agency of the United States Government. Neither the United States Government nor any agency Thereof, nor any of their employees, makes any warranty, express or implied, or assumes any legal liability or responsibility for the accuracy, completeness, or usefulness of any information, apparatus, product, or process disclosed, or represents that its use would not infringe privately owned rights. Reference herein to any specific commercial product, process, or service by trade name, trademark, manufacturer, or otherwise does not necessarily constitute or imply its endorsement, recommendation, or favoring by the United States Government or any agency thereof. The views and opinions of authors expressed herein do not necessarily state or reflect those of the United States Government or any agency thereof. 


\section{DISCLAIMER}

Portions of this document may be illegible in electronic image products. Images are produced from the best available original document. 


\section{Preface}

Shielding technology has matured considerably in the last decade, and shield physics must routinely be translated into shield design. Since the publication in 1959 of Fundamental Aspects of Reactor Shielding, by Herbert Goldstein, new generations of computers have become available to exploit techniques heretofore considered too costly, and new measurement techniques have been devised. The energy and angular distributions of neutrons and gamma rays can be followed, both in theory and in practice, throughout their transport histories. Such powerful tools have brought correspondingly large dividends to the shielding community.

These advances and their underlying fundamentals are recorded in this volume, which is intended as a text for a two-semester course in reactor shielding directed at an advanced undergraduate or graduate level. The reader is assumed to have some familiarity with calculus through partial differential equations and with nuclear physics through particle interaction theory, although pertinent aspects of the latter are reviewed in Chap. 3. The material is arranged to cover fundamental transport considerations in the first semester; portions of Chap. 4 could be reserved for the second semester. The second semester could then consist of special topics, such as Monte Carlo techniques, albedos, ducts, shield-analysis projects, seminars on experimental shielding, and shield design. Instructors will doubtless follow plans of their own choosing. Chapters 2 through 6 have problems appended, with solutions given at the back of the book. Metric units have been used exclusively. Citations of classified literature have been avoided, and technical reports have been referenced only where no journal articles could be given.

Although titled Reactor Shielding, this text should be applicable in related areas where neutron and gamma-ray attenuation are important, as in nuclear 
weapons shielding and in isotope source applications. The study of space radiation and high-energy-accelerator shielding, although closely related to the present subject, has been considered outside the scope of this book.

Dr. Samuel Glasstone originally conceived the idea for this text; he concluded that the book was needed and originally proposed to prepare it. In the preliminary planning of the project, the U.S. Atomic Energy Commission asked me to collaborate with Dr. Glasstone. Notwithstanding many plans and discussions for this collaboration, Dr. Glasstone had to relinquish his role in order to carry out a number of other projects. It is a pleasure to acknowledge his efforts in the planning of this book and his useful critiques of early drafts. I sincerely regret that our proposed association could not be continued.

For their assistance in the preparation of this manuscript, I am greatly indebted to many people in a number of ways. First, no book on shielding could be readied for publication at this time without acknowledgment of the pervasive influence of one man, the late E. P. Blizard. Not the least of his many contributions to the development of the technology was his encouragement of the efforts of others, including my own effort in preparing this manuscript.

The many services and suggestions provided by the staff of the Radiation Shielding Information Center, Oak Ridge National Laboratory, were extremely helpful, particularly in scanning the current literature. It is a distinct pleasure to acknowledge many useful discussions with others at ORNL: Lorraine Abbott, Clyde Claiborne, Charles Clifford, Paul Stevens, and Dave Trubey, each of whom supplied references and data in addition to contributions cited elsewhere. My colleagues Mike Wells and Bob French have also contributed in this way and in their forbearance.

I owe thanks for reviews and comments on various portions of the manuscript to Arthur Chilton and his students at the University of Illinois, Don Dudziak of Los Alamos Scientific Laboratory, Charles Eisenhauer of National Bureau of Standards, Cliff Horton of Rolls Royce, Ltd., Richard Faw of Kansas State University, Norman Francis, David Mesh, and their associates of General Electric Knolls Atomic Power Laboratory, Gene Hungerford of Purdue University, John Lamarsh of New York University, Fred Maienschein of Oak Ridge National Laboratory, Ed Profio of University of California at Santa Barbara, and Leigh Secrest of Texas Christian University. I am particularly indebted to Lew Spencer of National Bureau of Standards for his detailed review of the complete manuscript and 
for his many useful suggestions. Most of these reviewers provided recommendations based on teaching experience in shielding.

The guidance and counsel of John Inglima during the planning stages and of Robert Pigeon during the manuscript drafting, both of the U.S. Atomic Energy Commission, is gratefully acknowledged. For technical editing I am grateful to Jean Smith and Marian Fox, also of the U.S. Atomic Energy Commission, and, for typing a difficult manuscript, to Monsita Quave of Radiation Research Associates, Inc. I am especially grateful to Ceil Schaeffer for relieving me of many burdensome proofing tasks and, most of all, for her understanding and encouragement.

N. M. Schaeffer

May 1973

\section{Note to Reader}

The occasion of the 1981 reprinting gives me the opportunity to comment on the currency of this text, now eight years after initial publication. Several observations should be made: (1) The methodology of shield analysis as described here continues in general use. Many computer programs are now available which are successors to those mentioned in the text; the newer versions tend to be more-efficient or more-convenient versions of the forebears that were originally described. (2) Many of the tabulated data originally included for the reader's convenience have been superseded by newer results. We urge the user to check for later references with the Radiation Shielding Information Center, Oak Ridge National Laboratory, if the accuracy of his results is important or if he is in doubt about the latest available computer programs. (3) The shield designs reviewed in Chap. 10 are obviously dated but should retain some classroom value for discussion of the principal design considerations.

The general acceptance and use of this text has been particularly gratifying, and I thank the numerous teachers and students who have offered suggestions and corrections, which have been incorporated where possible.

N. M. Schaeffer

August 1981 


\section{Contributors}

H. C. Claiborne

Oak Ridge National Laboratory, Oak Ridge, Tennessee

S. T. Friedman

Consultant, Los Angeles, California

C. W. Garrett

Radiation Research Associates, Inc., Fort Worth, Texas

(Now with National Academy of Engineering, Washington, D. C.)

L. G. Mooney

Radiation Research Associates, Inc., Fort Worth, Texas

N. M. Schaeffer

Radiation Research Associates, Inc., Fort Worth, Texas

W. E. Selph

Radiation Research Associates, Inc., Fort Worth, Texas

(Now with Intelcom Radiation Technology, San Diego, California)

P. N. Stevens

Oak Ridge National Laboratory, Oak Ridge, Tennessee, and University of Tennessee, Knoxville, Tennessee

D. K. Trubey

Oak Ridge National Laboratory, Oak Ridge, Tennessee 


\section{Contents}

PREFACE ............................ . iii

CONTRIBUTORS ........................ vi

1 HISTORICAL BACKGROUND .... . . . . . . . . . . . . . 1

2 SOURCES OF RADIATION . . . . . . . . . . . . . . . . . . . . 11

2.1 Gamma-Ray and Neutron Sources . . . . . . . . . . . . . . . . 12

2.1.1 Gamma-Ray Sources . . . . . . . . . . . . . . . . . . . . 12

2.1.2 Neutron Sources . . . . . . . . . . . . . . . . . . . . . 17

2.2 Basic Mathematical and Physical Concepts . . . . . . . . . . . . . 19

2.2.1 Differential Distributions . . . . . . . . . . . . . . . . . . . 19

2.2.2 Average and Most-Probable Values . . . . . . . . . . . . . . . . 24

2.2 .3 Solid Angle . . . . . . . . . . . . . . . . . . . . . . . 24

2.2.4 Measures of Radiation Intensity . . . . . . . . . . . . . . . . 26

2.3 Spatial and Directional Characteristics . . . . . . . . . . . . . . 39

2.3.1 Spatial Distributions . . . . . . . . . . . . . . . . . . . 39

2.3.2 Directional Distributions . . . . . . . . . . . . . . . . . . . 42

2.4 Energy Distributions . . . . . . . . . . . . . . . . . . . . . 48

2.4.1 Energy Distributions of Gamma-Ray Sources . . . . . . . . . . . . 49

2.4.2 Neutron Spectra from Fission . . . . . . . . . . . . . . . . . 54

2.4.3 Effect of Medium on Spectra . . . . . . . . . . . . . . . . . 57

References .......................59

Exercises . . . . . . . . . . . . . . . . . . . 60

3 INTERACTIONS OF RADIATION WITH MATTER . . . . . . . . . . . 63

3.1 Cross Sections . . . . . . . . . . . . . . . . . . . . . . . . 63

3.1.1 Microscopic Cross Section . . . . . . . . . . . . . . . . . . 64

3.1.2 Macroscopic Cross Section . . . . . . . . . . . . . . . . . . 65

3.1.3 Radiation Reaction Rates . . . . . . . . . . . . . . . . . 66

3.2 Radiation Interactions . . . . . . . . . . . . . . . . . . 67

3.2.1 Photon Interactions . . . . . . . . . . . . . . . . . . . . . 68

3.2.2 Neutron Reactions . . . . . . . . . . . . . . . . . . . . 83

3.3 Responses to Radiation . . . . . . . . . . . . . . . . . . . . 94

3.3.1 Absorbed Dose . . . . . . . . . . . . . . . . . . . . . . 95

3.3.2 First-Collision Dose and Kerma . . . . . . . . . . . . . . . . . . 99

3.3.3 Exposure . . . . . . . . . . . . . . . . . . . . . . . . 103

3.3.4 RBE Dose; Dose Equivalent . . . . . . . . . . . . . . . . . 105 


\section{CONTENTS}

3.3.5 Maximum Absorbed Dose; Maximum Dose Equivalent . . . . . . . . . 108

3.3.6 Multicollision Dose $\quad . \quad$. . . . . . . . . . . . . . . . . . . 112

References . . . . . . . . . . . . . . . . . . . . . . 113

Exercises . . . . . . . . . . . . . . . . . . . . . . . . . . 114

4 RADIATION TRANSPORT . . . . . . . . . . . . . . . . . . . . . 119

4.1 Fundamental Considerations . . . . . . . . . . . . . . . . . . 120

4.2 The Boltzmann Transport Equation . . . . . . . . . . . . . . . 123

4.3 Spherical Harmonics Method . . . . . . . . . . . . . . . . . . 129

4.4 Discrete-Ordinates $S_{n}$ Method . . . . . . . . . . . . . . . . . . 132

4.4.1 Transport Equation and Phase-Space Geometry . . . . . . . . . . . 134

4.4.2 Derivation of Finite-Difference Equation . . . . . . . . . . . . . 136

4.4.3 Numerical Solution of the Discrete-Ordinates Equation . . . . . . . . 144

4.4.4 Advantages and Disadvantages . . . . . . . . . . . . . . . . . 148

4.5 Moments Method . . . . . . . . . . . . . . . . . . . . . . 149

4.6 Application of Diffusion Theory . . . . . . . . . . . . . . . . . 160

4.7 Invariant Imbedding Method . . . . . . . . . . . . . . . . . . 163

4.8 Kernel Technique . . . . . . . . . . . . . . . . . . . . . . 168

4.8.1 Gamma-Ray Calculations . . . . . . . . . . . . . . . . . . 169

4.8.2 Neutron Techniques . . . . . . . . . . . . . . . . . . . 178

4.9 Combination Removal-Diffusion Methods . . . . . . . . . . . . . 188

4.9.1 The Spinney Method . . . . . . . . . . . . . . . . . . . . 190

4.9.2 Variations of the Spinney Method . . . . . . . . . . . . . . . 192

4.9.3 Differences in Current Methods . . . . . . . . . . . . . . . . 199

References . . . . . . . . . . . . . . . . . . . . . . . . . 201

Exercises . . . . . . . . . . . . . . . . . . . . . . . . . . 204

5 MONTE CARLO METHODS FOR RADIATION TRANSPORT ․ . . . . . 207

5.1 Sampling from Probability Distribution Functions . . . . . . . . . . 209

5.2 The Evaluation of Integrals . . . . . . . . . . . . . . . . . . . 216

5.3 Source Parameters . . . . . . . . . . . . . . . . . . . . . . 218

5.3.1 Selection from an Energy Distribution . . . . . . . . . . . . . . 218

5.3.2 Selection of Spatial Point of the Source Particle . . . . . . . . . . . . 219

5.3.3 Selection of Initial Direction of Source Particle . . . . . . . . . . . 221

5.3.4 Source-Biasing Parameters . . . . . . . . . . . . . . . . . 223

5.4 Path Length . . . . . . . . . . . . . . . . . . . . . . . . . 225

5.5 Collision Parameters . . . . . . . . . . . . . . . . . . . . . 234

5.6 Particle Parameters After Collision . . . . . . . . . . . . . . . . 236

5.6.1 Neutron Elastic Scattering . . . . . . . . . . . . . . . . . . 236

5.6.2 Neutron Inelastic Scattering . . . . . . . . . . . . . . . . . . 238

5.6.3 Compton Scattering . . . . . . . . . . . . . . . . . . . . . . . . .

5.6.4 Particle Absorptions . . . . . . . . . . . . . . . . . . . . . 240

5.6.5 Calculation of Emergent-Direction Cosines _ . . . . . . . . . . . . 241

5.7 Particle Scoring . . . . . . . . . . . . . . . . . . . . . . . 242

5.8 Statistical Variance . . . . . . . . . . . . . . . . . . . . . . 247

5.9 Demonstration Monte Carlo Program . . . . . . . . . . . . . . . 251

5.10 Programming Suggestions . . . . . . . . . . . . . . . . . . . 254 
References ....................... . 257

Exercises . . . . . . . . . . . . . . . . . . 258

6 SHIELD ATTENUATION CALCULATIONS . . . . . . . . . . . . . . 261

6.1 Analysis of the Source . . . . . . . . . . . . . . . . . . . 261

6.2 Direct Solutions . . . . . . . . . . . . . . . . . . . . . 263

6.3 Application of Parametric Data . . . . . . . . . . . . . . . . . 264

6.3.1 Moments-Method Differential Energy Spectra . . . . . . . . . . . . 265

6.3.2 Monte Carlo . . . . . . . . . . . . . . . . . . . . . . . 270

6.3.3 Measured Data . . . . . . . . . . . . . . . . . . . . . . 274

6.3.4 Fitted-Parameter Data . . . . . . . . . . . . . . . . . . . 277

6.4 Simplified Solutions . . . . . . . . . . . . . . . . . . . . . 283

6.4.1 Applications of Gamma-Ray Buildup Factors . . . . . . . . . . . . 284

6.4.2 Applications of Neutron-Removal-Theory Kernels . . . . . . . . . . 286

6.4.3 Other Point-Kernel Applications . . . . . . . . . . . . . . . . 288

6.4.4 Methods for Estimating Low-Energy Neutron-Flux Density . . . . . . . 298

6.5 Application of Kernel Technique to Calculations of

Secondary Gamma-Ray Dose . . . . . . . . . . . . . . . . . . 301

6.5.1 Calculation for Slab Shield . . . . . . . . . . . . . . . . . . 304

6.5.2 Calculation for Semi-Infinite Shield . . . . . . . . . . . . . . 308

References ......................... 310

Exercises . . . . . . . . . . . . . . . . . . . 311

7 ALBEDOS, DUCTS, AND VOIDS . . . . . . . . . . . . . . . . . 313

7.1 Introduction to Albedos . . . . . . . . . . . . . . . . . . . . 313

7.2 Definitions . . . . . . . . . . . . . . . . . . . . . 315

7.2.1 Differential-Dose Albedos . . . . . . . . . . . . . . . . . . . . 316

7.2.2 Total-Dose Albedos . . . . . . . . . . . . . . . . . . . . . 317

7.2.3 Other Albedos . . . . . . . . . . . . . . . . . . . . . . 318

7.3 Neutron Albedos . . . . . . . . . . . . . . . . . . . . . . . 318

7.3.1 Fast-Neutron Albedos . . . . . . . . . . . . . . . . . . . . 319

7.3.2 Intermediate-Neutron Albedos . . . . . . . . . . . . . . . . . . 331

7.3.3 Thermal-Neutron Albedos . . . . . . . . . . . . . . . . . . . . . 332

7.4 Gamma-Ray Albedos . . . . . . . . . . . . . . . . . . . . 341

7.5 Secondary-Gamma-Ray Albedos . . . . . . . . . . . . . . . . . 350

7.6 Applications of Albedos . . . . . . . . . . . . . . . . . . . . 355

7.7 Ducts . . . . . . . . . . . . . . . . . . . . . . . . . 356

7.8 Line-of-Sight Component . . . . . . . . . . . . . . . . . . . 357

7.8.1 Rectangular Ducts . . . . . . . . . . . . . . . . . . . . . 360

7.8.2 Rectangular Slots . . . . . . . . . . . . . . . . . . . . . 363

7.8.3 Cylindrical Ducts . . . . . . . . . . . . . . . . . . . . . 365

7.8.4 Cylindrical Annulus . . . . . . . . . . . . . . . . . . . . 366

7.9 Wall-Penetration Component . . . . . . . . . . . . . . . . . . 367

7.9.1 Application to Cylindrical Ducts . . . . . . . . . . . . . . . . 370

7.9.2 Application to Partially Penetrating Cylindrical Ducts . . . . . . . . . 372

7.9.3 Comparison with Experiment . . . . . . . . . . . . . . . . . 374

7.10 Wall-Scattered Component . . . . . . . . . . . . . . . . 375

7.10.1 Analog Monte Carlo Calculations . . . . . . . . . . . . . . . 376 
7.10.2 Albedo Methods . . . . . . . . . . . . . . . . . . . . 380

7.10.3 Additional Experimental Investigations . . . . . . . . . . . . . 398

7.11 Voids . . . . . . . . . . . . . . . . . . . . . . . . . . 404

7.11 .1 Single Voids . . . . . . . . . . . . . . . . . . . . . . 404

7.11.2 Small Random Voids . . . . . . . . . . . . . . . . . . . . 412

References . . . . . . . . . . . . . . . . . . . . . . . . . 414

8 SHIELD HEATING, AIR TRANSPORT, SHIELD MATERIALS,

AND SHIELD OPTIMIZATION . . . . . . . . . . . . . . . . . . . 419

8.1 Shield Heating . . . . . . . . . . . . . . . . . . . . . . . . 419

8.1.1 Gamma-Ray Heating . . . . . . . . . . . . . . . . . . . . 420

8.1.2 Neutron Heating . . . . . . . . . . . . . . . . . . . . . . 426

8.1.3 Charged-Particle Heating . . . . . . . . . . . . . . . . . . . 429

8.2 Air Transport . . . . . . . . . . . . . . . . . . . . . . . . 430

8.2.1 Infinite Air Medium . . . . . . . . . . . . . . . . . . . . 432

8.2.2 Air-Over-Ground Calculations . . . . . . . . . . . . . . . . . 437

8.2.3 Air-Ground Interface Effects . . . . . . . . . . . . . . . . . . . . . 439

8.3 Shield Materials . . . . . . . . . . . . . . . . . . . . . . . 443

8.3.1 Considerations in Materials Selection . . . . . . . . . . . . . . . 445

8.3.2 Shield Materials for Stationary Reactor Systems . . . . . . . . . . . 447

8.3.3 Shield Materials for Mobile Reactor Systems . . . . . . . . . . . . 450

8.3.4 Comparison of Attenuation Properties . . . . . . . . . . . . . . 456

8.4 Shield Optimization . . . . . . . . . . . . . . . . . . . . . . 462

References . . . . . . . . . . . . . . . . . . . . . . 465

9 EXPERIMENTAL SHIELDING . . . . . . . . . . . . . . . . . . . 471

9.1 Detectors for Shielding Experiments . . . . . . . . . . . . . . . 472

9.1.1 Active Neutron Detectors . . . . . . . . . . . . . . . . . . 472

9.1.2 Passive Neutron Detectors . . . . . . . . . . . . . . . . . . 474

9.1.3 Active Gamma-Ray Detectors . . . . . . . . . . . . . . . . . 475

9.1.4 Passive Gamma-Ray Detectors . . . . . . . . . . . . . . . . . 476

9.1.5 Interpretations of Detector Output . . . . . . . . . . . . . . . . 476

9.2 Shield-Material Measurements . . . . . . . . . . . . . . . . . . 477

9.2.1 Reactors . . . . . . . . . . . . . . . . . . . . . . . . 477

9.2.2 Accelerators . . . . . . . . . . . . . . . . . . . . . . . 493

9.2.3 Fixed Sources . . . . . . . . . . . . . . . . . . . . . . 503

9.3 Phenomenological Measurements . . . . . . . . . . . . . . . . 507

9.3.1 Air-Transport and Air-Ground Interface Experiments . . . . . . . . . 507

9.3.2 Duct Penetration . . . . . . . . . . . . . . . . . . . . . . 514

References . . . . . . . . . . . . . . . . . . . . 516

10 SHIELD DESIGN . . . . . . . . . . . . . . . . . . . . . . . . 519

10.1 Iterations in the Shield Design . . . . . . . . . . . . . . . . . 520

10.1.1 Preliminary Conceptual Design . . . . . . . . . . . . . . . 521

10.1.2 Detailed Conceptual Design . . . . . . . . . . . . . . . . . 522

10.1.3 Final Engineering Design . . . . . . . . . . . . . . . . . . 524

10.2 Fast Breeder: Enrico Fermi . . . . . . . . . . . . . . . . . . 527

10.2.1 The Reactor Plant . . . . . . . . . . . . . . . . . . . . 528

10.2.2 Shield Design Criteria $\quad . \quad$. . . . . . . . . . . . . . . 532 
10.2.3 Reactor-Shield Systems . . . . . . . . . . . . . . . . . . 534

10.2.4 Shreld Costs . . . . . . . . . . . . . . . . . . . . . . . . . 548

10.2.5 Calculational Techniques . . . . . . . . . . . . . . . . . 548

10.2.6 Comparison of Measurements and Calculations . . . . . . . . . . 553

10.3 Fast Breeder: Dounreay Fast Reactor . . . . . . . . . . . . . . 559

10.3.1 Calculational Model for Bulk Shield . . . . . . . . . . . . . . 562

10.3.2 Measurements . . . . . . . . . . . . . . . . 563

10.3.3 Effect of Streaming . . . . . . . . . . . . . . . . . . 565

10.4 Heavy Water, Natural Uranium: Agesta . . . . . . . . . . . . . 566

10.4.1 Description of Reactor and Shield . . . . . . . . . . . . . . 566

10.4.2 Calculational Model . . . . . . . . . . . . . . . . 567

10.4.3 Measurements . . . . . . . . . . . . . . 570

10.5 Boiling Water: Pathfinder . . . . . . . . . . . . . . . . . . 574

10.5.1 Calculations . . . . . . . . . . . . . . . . 578

10.5.2 Survey Measurements . . . . . . . . . . . . . . . . . 579

10.6 Ship Propulsion: N.S. Savannah . . . . . . . . . . . . . . . . 579

10.6.1 Description of Ship, Reactor, and Main Shielding . . . . . . . . $\quad 580$

10.6.2 Shielding for Refueling and Control Rod Maintenance . . . . . . . 581

10.6.3 Shield Design Criteria . . . . . . . . . . . . . . . . . . 583

10.6.4 Lead-Polyethylene Shield Construction . . . . . . . . . . . 585

10.6.5 Attenuation Calculations . . . . . . . . . . . . . . . . . 587

10.6.6 Measurements . . . . . . . . . . . . . . . . . . . . 591

10.6.7 Comparison of Measurements and Calculations . . . . . . . . . 592

10.7 Space Power: SNAP-10A Flight Test . . . . . . . . . . . . . . 595

10.7.1 Shield Analysis . . . . . . . . . . . . . . . . . . . . 595

10.7.2 Flight Test Results . . . . . . . . . . . . . . . . . . 598

References . . . . . . . . . . . . . . . . . . . . . . . 602

Appendix A: GAMMA RAYS FROM INELASTIC NEUTRON SCATTERING

AND FISSION . . . . . . . . . . . . . . . . . . . . . 605

Appendix B: NEUTRON FLUENCE-TO-KERMA CONVERSION

FACTORS FOR STANDARD-MAN MODEL . . . . . . . . . 611

Appendix C: CYLINDRICAL TISSUE PHANTOM . . . . . . . . . . . . 616

Appendix D: COORDINATE SYSTEMS, VECTOR OPERATIONS,

AND LEGENDRE POLYNOMIALS . . . . . . . . . . . . . 617

D.1 Coordinate Systems . . . . . . . . . . . . . . . . 617

D.2 Coordinate-System Transformation . . . . . . . . 617

D.3 Vector Operators and Functions . . . . . . . . . . . . 619

D.4 Dirac Delta Function . . . . . . . . . . . . . . 621

D.5 Legendre Polynomials . . . . . . . . . . . . . 622

D.6 Associated Legendre Polynomials . . . . . . . . . . . 623

D.7 Associated Spherical-Harmonic Functions . . . . . . . . 625

Appendix E: EXPOSURE BUILDUP FACTORS . . . . . . . . . . . . . 626

Appendix F: COEFFICIENTS FOR GAMMA-RAY BUILDUP FACTORS ～. . 628

Appendix G: GRAPHS AND FORMULAS OF EXPONENTIAL

INTEGRAL FUNCTIONS . . . . . . . . . . . . . . . . . 642 
Appendix H: TABLES OF ATTENUATION FUNCTIONS FOR FINITE SLAB GEOMETRY ． . . . . . . . . . . . . 653

Appendix I: RANDOM-NUMBER GENERATORS . . . . . . . . . . . . 663

I.1 Properties of Random-Number Generators (RNG's) . . . . . 664

I.2 Recursion Equations for RNG's . . . . . . . . . . . . 665

I.3 Testing RNG's . . . . . . . . . . . . . . . . . . . 668

I.3.1 Global Tests . . . . . . . . . . . . . . . . . . . 669

I.3.2 Equidistribution . . . . . . . . . . . . . . . . 669

1.3.3 Independence . . . . . . . . . . . . . . . . . . 670

I.4 Pathological Numbers . . . . . . . . . . . . . . . . 671

1.5 Useful RNG's . . . . . . . . . . . . . . . . . . . 672

Appendix J: DEMONSTRATION MONTE CARLO PROGRAM $\quad$. . . . . . . 674

Appendix K: MOMENTS-METHOD RESULTS FOR FISSION-NEUTRON PENETRATION IN Be, C, $\mathrm{CH}, \mathrm{CH}_{2}, \mathrm{H}$, AND $\mathrm{H}_{2} \mathrm{O}$. . . . . . . . 687

Appendix L: GAMMA-RAY DIFFERENTIAL ENERGY SPECTRA FOR WATER AND LEAD $\quad$. . . . . . . . . . . . . . . . 692

Appendix M: GRAPHS FOR NEUTRON ATTENUATION

CALCUlATIONS . . . . . . . . . . . . . . . . 700

Appendix N: GRAPHS OF THE $\psi$ FUNCTION ． . . . . . . . . . . . . 717

Appendix O: CONSTANTS FOR EMPIRICAL EXPRESSIONS

OF ALBEDO DATA $\ldots . . . . . . . . . . .726$

Appendix P: RADIATION PENETRATION OF CYLINDRICAL DUCTS • 734

SOLUTIONS TO EXERCISES . . . . . . . . . . . . . . . . . . . . 737

AUTHOR INDEX . . . . . . . . . . . . . . . . . . . . . 771

SUBJECT INDEX . . . . . . . . . . . . . . . . . . . . 775 


\section{Historical Background}

Early reactor shields were largely a matter of educated guesses. The complex of phenomena that had to be considered for an accurate shield analysis was an imposing obstacle. Microscopic-particle interaction processes were reasonably well understood, but their relative importance depended on largely unknown physical parameters called cross sections. Bulk attenuation properties of materials for two principal radiations of interest, neutrons and gamma rays, were also unknown. Even for an empirical approach, there was no opportunity under the wartime pressures of the Manhattan Project to launch a systematic investigation of the attenuation properties of materials. It was obvious that hydrogenous materials were needed for neutrons and dense materials for gamma rays. It was also evident that simple exponential attenuation based on the total cross section was a thoroughly inadequate concept for determining layer thicknesses. The shield of concrete and paraffinized wood for the Argonne National Laboratory graphite pile in 1943 was adequate for gamma rays and was overdesigned for neutrons. The $\mathrm{X}-10$ reactor at Oak Ridge National Laboratory (ORNL) included a 2.1-m concrete shield, of which the central $1.5 \mathrm{~m}$ contained a special mixture incorporating the mineral haydite. The large water-of-crystallization content of haydite made it appear especially useful for neutron attenuation. This shield was also overdesigned for neutrons and about adequate for gamma rays, although streaming problems were evident for both radiations around access holes in the shield. $\dagger$

The special requirement for a thin shield for the Hanford reactor was dictated in 1944 by the maximum length of aluminum tubing that could be

tHistorical material for this chapter has been drawn from H. Goldstein, Everitt Pinnel Blizard, 1916-1966, Nuclear Science and Engineering, 27: 145 (1967), the dedication of a special issue prepared as a memorial to E. P. Blizard. Additional information was graciously provided by Mrs. L. S. Abbott from the archives of the Neutron Physics Division, Oak Ridge National Laboratory. Mr. C. C. Horton of Rolls Royce, Ltd., has kindly provided reminiscences of British developments. 
drawn. E. Fermi and W. Zinn had made some provisional attenuation measurements in Chicago in 1943. H. Newson and L. Slotin made some gold-foil measurements for masonite and iron slabs in the core hole at the rear of the X-10 pile in 1944. A young engineer, C. E. Clifford, was assigned to help them. The Hanford reactor shield was built of iron slabs sandwiched between masonite layers. Although initially a good neutron attenuator, the masonite suffered severe radiation damage and decomposed. The CP-3 (Chicago Pile-3, 1944) shield was composed of ordinary concrete; although thicker than necessary, it performed satisfactorily.

The early reactor projects clearly demonstrated that the design of a shield for neutrons and gamma rays that was optimal, efficient, or economical required answers to a great many questions. In 1946 the Navy initiated an intensive study program for a nuclear-powered submarine, and the Air Force, a similar study for a nuclear-powered aircraft. Space and weight limitations for these nuclear applications added more impetus to the open questions in shielding. In the spring of 1947, E. P. Blizard, then a Navy physicist assigned by Capt. H. Rickover to ORNL, was directed to start a program of shielding measurements. He proposed a program of neutron and gamma-ray attenuation measurements through several types of concrete placed in the rear core hole (a $60-\mathrm{cm}$ square aperture) of the $\mathrm{X}-10$ reactor. C. E. Clifford of the laboratory staff was assigned to work with him because of his experience with measurements for the Hanford shield in 1944. Slabs of material were placed in the aperture, and detectors were positioned within and beyond the slabs. This effort marked the first organized research program in reactor shielding. A spiral-duct mock-up placed in the hole demonstrated that properly designed passages could penetrate the shield without transmitting excessive radiation. These studies also led to the recognition that the production of secondary gamma rays by neutron interactions in the shield was clearly a significant design consideration.

By 1948 shielding studies supporting various reactor projects were in progress at Hanford, Knolls Atomic Power Laboratory, Bettis Atomic Power Laboratory, and Massachusetts Institute of Technology (MIT). As additional results of measurements in the $\mathrm{X}-10$ core hole were made, Blizard became convinced that too much radiation streamed around the test samples for accurate measurements and a better facility was needed. He concluded that a fission plate-a thin disk of enriched uranium covering the core holewould provide a local source of fission neutrons and would be more accessible for attenuation measurements. Clifford suggested that a tank of water be placed adjacent to the fission plate so that materials and 
instruments could be submerged, which would greatly reduce the radiation background. These two ideas culminated in the Lid Tank Shielding Facility, which began operating in 1949 .

In the United Kingdom shielding research efforts were started in 1948 and were geared to the British philosophy of reactor development: large gas-cooled reactors for plutonium production to be followed by development of these systems for electricity generation. The research reactor BEPO had just been completed; it had a $15-\mathrm{cm}$ iron thermal shield followed by a bulk shield of barytes concrete and had a layout similar to the Oak Ridge $\mathrm{X}-10$ reactor. The Windscale reactors were under construction in 1948 and included a thermal shield similar to BEPO but Portland concrete was used rather than barytes. Early design calculations were made by B. T. Price, D. J. Littler, and F. W. Fenning.

A shielding group was set up under C. C. Horton as part of Fenning's reactor physics group at Harwell to investigate shielding problems connected with large concrete shields, heating effects, and radiation streaming in the large ducts that are integral to gas-cooled systems. In these systems heat generation in the first $30 \mathrm{~cm}$ or so of the shields was recognized to be an important problem, and Horton, later with K. Spinney, developed some models to predict the distribution of heat generation by neutrons and gamma rays. Horton, J. R. Harrison, and D. Halliday of the Harwell group also initiated a program of duct-streaming measurements in 1952 at the BEPO facility.

During an intensive working session at ORNL in shielding in the summer of 1949 with interested participants from a number of installations (one of many organized by Blizard), T. A. Welton of MIT developed the concept of the removal cross section for treating neutron attenuation in heavy materials mixed with hydrogenous materials. Recognizing the importance of the removal concept, Blizard initiated a new series of measurements in the Lid Tank to verify applicability and to obtain removal cross sections for many materials. The removal-cross-section concept quickly came into widespread use and became the principal method of treating neutron attenuation. Two decades later it is still regarded as a useful, valid technique for many applications. So great were the demands on the Lid Tank that a second fission-plate facility was constructed on the reactor at Brookhaven National Laboratory, and a program of additional removal-cross-section measurements was carried out under the direction of R. Shamberger (Chap. 4).

Blizard proposed an additional test apparatus for complete $4 \pi$ shields since they could not be tested in the Lid Tank. Tests for the mock-up for the 
Materials Testing Reactor (MTR) indicated that this type of reactor would make a useful source for shield tests. Construction was authorized, and the Bulk Shielding Reactor (BSR) was completed in 1950. The facility was so versatile that it became the pattern for swimming-pool research reactors around the world. The BSR group included L. Meem, F. Maienschein, and R. Peelle. Numerous basic and applied results were forthcoming on materials, shield mock-ups, and a definitive measurement of the fission gamma-ray spectrum (Chap. 2).

The British workers also realized the need for a special facility; they required data to support the design of large shields for power reactors. A group under the direction of Fenning was set up to design and build this reactor. Horton was responsible for the physics and general layout of the facility. The reactor (LIDO) was completed in 1956. Unlike the Oak Ridge facility, the entire pool was constructed above the ground to allow access to three caves in the shield wall, in which substantial dry mock-ups could be placed. The reactor could be traversed through the pool to provide a source for these mock-ups, and an important design criterion for the pool layout was that construction of a mock-up in one cave could be carried out while experiments were continuing in another.

Aircraft shielding required measurements away from the ground; thus Blizard and Clifford conceived the idea in 1952 of a facility in which a reactor might be suspended at a sufficient height to eliminate the effects of ground scattering. They planned an arrangement of four towers in a rectangle with cable hoists for elevating a BSR-type reactor and crew compartment $60 \mathrm{~m}$ above ground. The Oak Ridge Tower Shielding Facility began operation under Clifford's direction in 1954, and it proved versatile in applications far beyond the ill-fated nuclear aircraft program (Chap. 9).

Although destined for cancellation in 1961, the aircraft nuclear propulsion (ANP) program produced a number of other useful shielding efforts. The Nuclear Aerospace Research Facility at Convair, Fort Worth, Tex., included two reactors: the Ground Test Reactor (GTR), a copy of the BSR, and the Aircraft Shield Test Reactor (ASTR). In 1954 B. Leonard and N. Schaeffer proposed a program of ground and flight studies with these reactors to resolve the major shielding uncertainties affecting airframe design. The GTR was operated in a small water tank suspended from a crane at a height of $30 \mathrm{~m}$ to obtain an early measurement of ground scattering. It was also placed in a mock-up consisting of the empty fuselage of a retired aircraft (the XB-36) with a shielded cylinder representing a crew compartment. From these measurements and concurrent air-transport results at the 
Oak Ridge towers, the large contribution of secondary gamma rays produced by neutron radiative capture in air was first observed in 1955 . The importance of these secondary gamma rays was a surprise to both groups; previous estimates of the probability for gamma-ray production by neutron capture in nitrogen had been too low, and these measurements were the first to reveal the discrepancy. The ASTR was carried in the aft bomb bay of a specially modified B-36 in a series of test flights from 1955 to 1957 at altitudes from sea level to $11 \mathrm{~km}$. The program provided data on radiation transfer by air and aircraft structure from reactor to shielded crew compartment. The program culminated with a joint effort at ORNL in which the ASTR and the crew compartment were suspended at the towers in the same relative positions as when installed in the B-36 (Chap. 8).

The decade from 1951 to 1961 is the period when shield technology came into its own. The major facilities were all in operation from 1954 onward, and large shielding groups at General Electric in Cincinnati, Ohio, Pratt and Whitney in Hartford, Conn., Convair in Fort Worth, Tex., and Lockheed in Marietta, Ga., were participating in the ANP program. The submarine effort was concentrated at the Westinghouse Bettis Laboratory near Pittsburgh, Pa., and the General Electric Knolls Atomic Power Laboratory in Schenectady, N. Y. The Oak Ridge group was extremely busy supporting both efforts. These groups contributed to the technology by developing design methods, by measuring attenuation through shield materials (including mock-ups of various shield designs), and by devising new experimental and analytical approaches. The demise of the ANP program and the successes of the nuclear submarine are well known. The U.S.S. Nautilus sailed on nuclear power for the first time in January 1955. This date is to be compared with 1954,1956 , and 1957, the years in which nuclear-fueled electric plants first went on line in Russia, Great Britain, and the United States, respectively.

The nuclear-applications programs gave impetus to the development of shield-analysis methods as well as to large-scale experimental programs. By the early 1950 s an intensive program in radiation physics was under way at the National Bureau of Standards (NBS) under the direction of U. Fano. G. W. Grodstein published a definitive set of X-ray attenuation coefficients, and L. V. Spencer's method-of-moments solution of the Boltzmann transport equation was first described. Shortly afterward a group at Nuclear Development Associates, Inc., under the direction of $\mathrm{H}$. Goldstein joined with Spencer and Fano in an intensive program of moments-method calculations, which culminated in 1954 with publication of the Goldstein 
and Wilkins report on gamma-ray buildup factors. R. Aronson, J. Certaine, M. Kalos, and P. Mittelman, with Goldstein, applied the method to neutrons. Fano, Spencer, and M. J. Berger published a definitive exposition of gamma-ray penetration in 1959 , which included a summary of the moments method as well as other techniques (Chap. 4).

Work on neutron attenuation in the United Kingdom followed a somewhat different path from the efforts in the United States. Horton and J. D. Jones devised the removal-diffusion method, the first results of which were reported at the second Atoms for Peace conference at Geneva in 1958. Since 1956, A. Avery and J. Butler have further developed these techniques at Harwell (Chap. 4).

Although $\mathrm{H}$. Kahn of Rand Corporation published two papers on the application of Monte Carlo techniques to shielding in 1950, in which he identified virtually all the principal concepts, widespread use of the technique and its subsequent development had to await the improvement of the digital computer. Prominent among early contributors in delineating the techniques and concepts were E. Cashwell and C. Everett of Los Alamos Scientific Laboratory (LASL) and G. Goertzel and M. Kalos of Nuclear Development Associates, Inc. Kalos and F. Clark of ORNL reported on the theory of importance sampling and finite variance estimators. At NBS, E. Hayward and J. Hubbell reported photon albedo calculations in 1953; M. Berger and J. Doggett extended their results in 1955. The first successful Monte Carlo applications in air scattering were reported in 1957-1958 by Berger, C. Zerby of ORNL, and M. Wells of Convair. The completion of the O5R system of Monte Carlo programs by R. Coveyou at ORNL in 1958 must be regarded as a significant advance in shield technology. The O5R system required a great deal of its users, but it was extremely flexible and widely used. At the Geneva (Atoms for Peace) conference in 1964, Blizard and Mittelman reported on eight major Monte Carlo programs in use in the United States. The MORSE Monte Carlo code of E. Straker, P. Stevens, D. Irving, and V. Cain was completed in 1969; it has produced results in excellent agreement with analy tic solutions (Chap. 5).

Monte Carlo has been regarded as one of the sophisticated techniques, but the workhorse method of shield design has been the point-kernel approach. Blizard, J. Miller, D. Trubey, and G. Chapman of ORNL and J. MacDonald, W. Edwards, and J. Moteff of General Electric (GE) made notable contributions to the use of removal cross sections. K. Shure of Westinghouse Electric Corporation developed an analysis technique for metal-hydrogenous shields based on a combination of point kernel and a 
numerical method using spherical harmonics called a $P_{1}$ multigroup (later a $P_{3}$ ) solution of the one-dimensional transport equation. In the development and application of gamma-ray buildup factors to kernel techniques, the work of J. Taylor of Westinghouse, M. Capo of GE, M. Berger and J. Hubbell of NBS, R. French of Convair, M. Grotenhuis of Argonne, F. Clark and D. Trubey of ORNL, and A. Chilton of the University of Illinois should be listed as principals in devising empirical representations of the data and simplified schemes for its application (Chaps. 4, 6, and 8).

From the outset many investigators in shield analysis sought manageable numerical techniques for achieving analytical solutions of the Boltzmann equation. Of all the efforts in this direction, such as the method of moments, spherical harmonics, numerical integration, and invariant imbedding, perhaps the most significant in terms of present usage is the discrete-ordinates method. B. Carlson of LASL had developed a discrete-ordinate method for reactivity calculations in 1955 which became known as $S_{n}$ and which has been successfully applied to a variety of transport problems. F. Mynatt and W. Engle of ORNL developed ANISN in 1965, which incorporated improved differencing and convergence techniques and made the method more suitable for shielding applications. A two-dimensional version of ANISN called DOT was described a year later by F. Mynatt, F. Muckenthaler, and P. Stevens (Chap. 4).

The $S_{n}$ programs, although not without problems in some geometries, have been used with a great deal of success in obtaining detailed radiation distributions in complicated two-dimensional geometries. Several laboratories have recently studied the utility and applicability of coupling Monte Carlo and discrete-ordinate calculational links. Thus the latter is used for those portions of a geometry reducible to two dimensions and the former where the description requires three dimensions.

A more complete historical survey would include the developments and researchers in nuclear instrumentation for shielding. As shield analysis has been paced by the development of the digital computer, so shielding experimentation has been gaited to innovations in particle detectors and fast electronics. A survey of neutron and gamma-ray detectors is given in Chap. 9.

In the foregoing chronology we have been limited to an outline of United States shielding research with only brief insertions of corresponding British activities. Significant and occasionally large shielding efforts have also been maintained elsewhere, notably, Belgium, Canada, France, Italy, Japan, the Netherlands, Norway, Russia, Sweden, and West Germany. These 
programs principally support national power reactor developments, although the literature also contains many reports of maritime and space reactor shielding studies from Europe and Asia.

We have used some terms that will be meaningless to the uninitiated. However, the chapter references will aid the curious in locating the appropriate explanations; the objective here has been to trace the early developments and to introduce some of the literature.

The newcomer will find the following earlier books on this subject to be useful references: The first handbook in reactor shielding was published in 1956 and was edited by T. Rockwell, ${ }^{1}$ who had been in the original shielding group at Oak Ridge. B. Price, C. Horton, and K. Spinney ${ }^{2}$ of the British group active in reactor shielding wrote the first text to appear (in 1957) on the subject. The text by Goldstein ${ }^{3}$ was published in report form in 1957 and appeared in hard cover in 1959. The Shielding volume of the Reactor Handbook, edited by Blizard and Abbott, ${ }^{4}$ was published in 1962. T. Jaeger ${ }^{5}$ wrote a text on Principles of Radiation Protection Engineering, which was published in 1960 in German and translated by L. Dresner of ORNL for publication in English in 1965. From the standpoint of dissemination of shielding information, probably the most important event was not a publication date but the founding in 1962 of the Radiation Shielding Information Center (RSIC) at Oak Ridge. Originally organized by K. Penny, D. Trubey, and B. Maskewitz, RSIC has performed a remarkable job of serving the needs of the shielding community. The specialized needs of civil defense have lead to a separate technology of fallout shielding, which is available in a 1962 monograph by Spencer ${ }^{6}$ and a 1966 collection edited by Kimel.?

From 1966 to 1970, ORNL published Chaps. 2, 3, 4, and 5 of the Weapons Radiation Shielding Handbook, edited by Abbott, Claiborne, and Clifford. ${ }^{8}$ Authors for this handbook contributed revised material from the earlier publication to the present text. Recently the Engineering Compendium on Radiation Shielding, ${ }^{9}$ R. G. Jaeger, editor-in-chief, was published, Vol. I in 1968, Vol. III in 1970, and Vol. II in press. This compendium is sponsored by the International Atomic Energy Agency, Vienna, and is an excellent source for the international shielding literature.

The extensive Russian shielding literature deserves further mention here since it is referenced in only a few instances elsewhere in this work. A guide to the Soviet literature was published by J. Lewin, J. Gurney, and D. Trubey ${ }^{10}$ for RSIC in 1968. A recent computer scan of Russian entries in the RSIC bibliography produced over 200 entries. Most of these 
articles are available in English translation 1 to 2 years after their original Russian publication. Current reports will be found in Soviet Atomic Energy (Atomnaya Energiya). ${ }^{11}$ Collections that are useful reports of current progress are found in a series entitled Problems in the Physics of Reactor Shielding; Vol. 4 is the most recent volume available in English. ${ }^{12}$

\section{REFERENCES}

1. T. Rockwell III (Ed.), Reactor Shielding Design Manual, USAEC Report TID-7004, 1956.

2. B. T. Price, C. C. Horton, and K. T. Spinney, Radiation Shielding, The Macmillan Co., New York, 1957.

3. H. Goldstein, Fundamental Aspects of Reactor Shielding, Addison-Wesley Publishing Company, Inc., Reading, Mass., 1959.

4. E. P. Blizard and L. S. Abbott (Eds.), Reactor Handbook, 2nd. ed., Vol. 3, Part B, Shielding, Interscience Publishers, a division of John Wiley \& Sons, Inc., New York, 1962.

5. T. Jaeger, Principles of Radiation Protection Engineering (English translation by L. Dresner), McGraw-Hill Book Company, Inc., New York, 1965.

6. L. V. Spencer, Structure Shielding Against Fallout Radiation from Nuclear Weapons, National Bureau of Standards, Monograph 42, Superintendent of Documents, U. S. Government Printing Office, Washington, D. C., 1962.

7. W. R. Kimel (Ed.), Radiation Shielding Analysis and Design Principles as Applied to Nuclear Defense Planning, Report TR-40, Office of Civil Defense and Kansas State University, 1966. See also C. H. Koontz (Ed.), Shelter Design and Analysis, Vol. 1, Fallout Radiation Shielding, Report TR-20, Office of Civil Defense, Revised 1970.

8. L. S. Abbott, H. C. Claiborne, and C. E. Clifford (Eds.), Weapons Radiation Shielding Handbook, USAEC Report DASA-1892, Oak Ridge National Laboratory, 1970.

9. R. G. Jaeger (Editor-in-Chief), Engineering Compendium on Radiation Shielding, Springer-Verlag, New York, Vol. I, 1968; Vol. III, 1970; Vol. II, in press.

10. J. Lewin, J. Gurney, and D. K. Trubey, A Survey of Recent Soviet Radiation Shielding Work, USAEC Report ORNL-RSIC-23, Oak Ridge National Laboratory, 1968.

11. Soviet Atomic Energy, Consultants Bureau, New York, monthly.

12. D. Broder, A. Veselkin, Yu. Yegorov, A. Suvorov, and S. Tsypin (Eds.), Problems in the Physics of Reactor Shielding, Report JPRS-51083, Joint Publication Research Service, 1970. 
$$
\text { . }
$$ 


\section{Sources of Radiation}

Shield analysis is usually subdivided into three or four phases; separate calculations are performed for the radiation components that are significant at each phase. Occasionally two or more of the phases may be combined, but each represents a distinct consideration in a shield analysis. The first phase involves defining the source in sufficient detail to provide the parameters for input to the succeeding phases. The second phase involves calculating the intensity and distribution of the radiation that penetrates the shield about the source; it is the principal phase in the analysis of a reactor shield. The third phase consists in determining the intensity and distribution of radiation traveling from the shield to the receiver. "Receiver" describes the points or region for which the radiation intensity is desired, e.g., a radiation detector (real or hypothetical), a human for whom biological exposure is desired, or equipment for which the exposure is desired to assess possible radiation damage. This third phase typically involves a calculation of radiation interactions in air. A fourth phase may be defined if the receiver has a separate shield, e.g., for some applications of nuclear propulsion it is advantageous to divide the shielding and use separate shields at the reactor (the source) and the crew (the receiver).

Thus the first task in a shield analysis or a shield design is to define and characterize the radiation sources. This chapter contains a classification of the various types of sources encountered in reactor shielding and a brief description of each in a form convenient for shield analyses; the characteristics important to the shield designer are emphasized.

Discussions of the nuclear and atomic processes that give rise to the emission of radiation are given in any nuclear physics text. Also, the shield designer is primarily concerned with the characteristics of the emitted radiation, so only a cursory description of the physics of radiation production is given here. In addition, some mathematical and fundamental 
radiation physics concepts are reviewed which are necessary tor the development of the material in this and succeeding chapters.

Subsequent chapters are devoted to the other phases of shield analysis and to topics relevant to their application.

\subsection{GAMMA-RAY AND NEUTRON SOURCES}

The primary concern in reactor shield design is the specification of suitable barriers around sources of neutrons and gamma rays to limit the radiation exposure to biological systems or equipment that must function in proximity to these sources. Both types of radiation are sufficiently penetrating to be difficult to attenuate; yet they are sufficiently interacting to be damaging to tissue and other materials.

Several other types of radiation arise from the fission event or from the interaction of fission neutrons with nuclei. These include charged particles and neutrinos.

Neutrinos, which possess no charge, mass, or magnetic moment, cannot interact with matter except through the very weak, purely nuclear forces. Thus, despite the fact that they carry away $5 \%$ of the power of a reactor, they do not pose a shielding problem because they are incapable of causing damage.

Conversely, charged particles are of little concern because they are so highly interacting that relatively small amounts of material provide an adequate barrier. The absorption of energy associated with charged particles may, however, be an important consideration in the thermal design of a system.

The sources of radiations of primary interest in a reactor, neutrons and gamma rays, are discussed in the following sections.

\subsubsection{Gamma-Ray Sources}

A variety of sources contribute to the gamma radiation produced by a fission reactor. The relative importance of these sources to the total gamma-ray intensity depends primarily on the reactor design; within a given reactor the importance of the components may vary with position in the reactor and with reactor operating history. For example, at a point near the reactor core, the prompt fission gamma rays may be most important during reactor operation, the fission-product gamma rays may predominate for the first few hours after shutdown, and subsequently the gamma rays from activated materials in the vicinity may be more important. 
In power reactors the most intense gamma-ray component penetrating the shield is frequently that from neutron interactions in the thermal shield, pressure vessel, or biological shield. However, in special situations any of the sources discussed in the following paragraphs may be of importance, and each must be considered as a potentially significant source by the shield designer.

(a) Fission Gamma Rays. The discussion on gamma rays from fission and fission products is limited to those from ${ }^{235} \mathrm{U}$. Spectral distributions and energy partitions are not known as well for other fissionable materials; similarity to ${ }^{235} \mathrm{U}$ is frequently assumed, but some differences have been observed. Strictly speaking, gamma-ray energy released in fission is divided into four time ranges, the first and last contributing more than $90 \%$ of the total energy released as gamma rays. These time $(t)$ ranges are:

Prompt, $t \leqslant 0.05 \mu \mathrm{sec}(7.25 \mathrm{MeV})$.

Short-life, $0.05<t \leqslant 1.0 \mu \mathrm{sec}(0.43 \mathrm{MeV})$.

Intermediate-life, $1.0 \mu \mathrm{sec}<t \leqslant 1.0 \mathrm{sec}(0.55 \mathrm{MeV})$.

Delayed, $t>1.0 \mathrm{sec}(6.65 \mathrm{MeV})$.

The values in parentheses are for the gamma energy released per ${ }^{235} \mathrm{U}$ fission and were taken from an evaluation by Holden, Mendelson, and Dudley ${ }^{1}$ except the value for prompt fission, which is quoted from a recent note by Peelle and Maienschein. ${ }^{2}$ Prompt fission gamma rays have energies from $10 \mathrm{keV}$ to $10 \mathrm{MeV}$. An average of $8.1 \pm 0.3$ photons are released per fission, and these photons carry off $7.25 \pm 0.26 \mathrm{MeV}$ per fission event. Prompt fission gamma rays can contribute a significant amount to the total gamma-ray field at points near the reactor core during reactor operation and should be included in the core-shield analysis. The energy distribution of prompt gamma rays is discussed in Sec. 2.4.1.

The short-life interval is similar to the prompt in energy distribution and accounts for $5.9 \%(0.43 \mathrm{MeV})$ of the prompt gamma-ray energy release.

The intermediate-life interval is also usually assumed to have the same energy distribution as the prompt interval with an energy release of about $0.55 \mathrm{MeV}$.

(b) Fission-Product-Decay Gamma Rays. The two middleweight nuclei resulting from a fission event are called fission fragments or fission products. Because of an excess of neutrons, most of approximately 80 possible initial fission-product isotopes are radioactive, initially decaying by beta emission. The beta decay is followed by gamma emission whenever the beta decay 
results in an excited state of the daughter nucleus. In a few special cases, only gamma radiation is emitted.

The vast majority of daughter products of the initial fission fragments are also radioactive, decaying with the emission of beta particles and gamma rays. Having, on the average, about three stages of radioactive decay before they become stable, fission products form a complex mixture of gamma-ray emitters with half-lives ranging from less than a second to millions of years. Of the approximately $21.5 \mathrm{MeV}$ per fission emitted by fission products, the greatest fraction is carried by beta particles and neutrinos. About $6.65 \mathrm{MeV}$ per ${ }^{235} \mathrm{U}$ fission is emitted by the fission product as delayed gamma rays; over three-quarters of this energy is released with $10^{3} \mathrm{sec}$ following fission.

Fission-product activity will not dictate the thickness of the shield surrounding an operating reactor. There are a few shield-design situations, however, where protection from fission-product activity is the primary criterion. Included in this category are primary loops in circulating-fuel reactors and enclosures for the shipment or dismantling of used fuel rods or assemblies. Knowledge of the dose rates from fission products is essential to the formulation of procedures to be followed in any maintenance involving compromise of the primary shield.

Delayed gamma rays can be classified into two groups, depending on the time of their emission following fission. Early fission-product gamma rays are those emitted within a few minutes after fission; they contribute to the total core source during the operation of a reactor. They can also be of prime importance in regions external to the core during the operation of a circulating-fuel reactor. Late fission-product gamma rays (those which are emitted several minutes or longer after fission) are not of much importance during reactor operation, but they can be a very significant source following reactor shutdown.

Because most of the fission-product energy is contained in the early fission products, fission-product intensity reaches a state of quasiequilibrium in a reactor core that has operated at steady state for only a few hours. Thus the early decay gammas are sometimes lumped with the prompt fission gamma rays and are considered a part of the prompt source. Energy spectra for both the early and late fission products are discussed in Sec. 2.4.1.

(c) Capture Gamma Rays. Radiative capture of neutrons by nuclei at thermal and epithermal energies produces secondary gamma rays, commonly called capture gamma rays. They are emitted promptly after neutron capture 
to relieve an unstable energy situation generated within the compound nucleus.

The total energy available for gamma rays from capture is the sum of the kinetic energy of the incident neutron and its binding energy in the compound nucleus. Since the probability of capture decreases rapidly with increasing kinetic energy, capture reactions generally are of importance only for neutrons with kinetic energies below $25 \mathrm{keV}$. Typically, neutron binding energies are in the region of 6 to $8 \mathrm{MeV}$, although they can range from 2.2 to about $11 \mathrm{MeV}$. Thus binding energy is the greatest component of the total gamma energy emitted.

Although this energy may be carried off by only one photon, it most often is shared by two or more photons as the compound nucleus decays through several excited states. The energy distribution of capture gamma rays can range from sharp discrete line spectra to almost continuous spectra produced by cascading decay; the capture interaction process is further discussed in Chap. 3.

Since investigators studying capture spectra are primarily interested in nuclear processes, many times only discrete spectral lines with significant intensities are reported. In using such data, we must be careful to ascertain whether or not the entire neutron binding energy is contained in the reported gamma spectrum. Where it is not (differences can be as much as $30 \%$ ), adjustments should be made by increasing the number of photons emitted to account for the total amount since this energy is usually an important contributor to the radiation that penetrates the shield.

Capture gamma rays are a significant source and occasionally constitute the most important consideration in shield design because of their high energy and the fact that they are generated throughout the shield.

(d) Inelastic-Scattering Gamma Rays. In neutron inelastic scattering, part of the energy of the incident neutron is carried off by the scattered neutron and part is absorbed by the target nucleus. The latter is left in an excited state and subsequently decays by gamma-ray emission. An alternative process that may be involved is the formation of a neutron-heavy compound nucleus by union of the target nucleus and the incident neutron. The extra neutron is then emitted, and the target nucleus is left in an excited state. The time between neutron interaction and gamma-ray emission is negligible $\left(10^{-14} \mathrm{sec}\right)$.

As with capture gamma rays, the excess energy may be carried off by one or more photons. However, gamma rays from this source are generally 
less penetrating than those from neutron capture; their energies range from a fraction of $1 \mathrm{MeV}$ to several $\mathrm{MeV}$. Since the energy of the incident neutron must exceed the energy of the first excited level of the target nucleus, inelastic scattering becomes more important as the neutron energy increases. In general, neutrons must be in the $\mathrm{MeV}$ region to generate significant gamma rays by this process.

(e) Reaction-Product Gamma Rays. The reaction-product source results from a process resembling that of inelastic scattering except that some particle other than a neutron is ejected from the nucleus. The nucleus is left unstable and emits a gamma ray. An example is the ${ }^{10} \mathrm{~B}(n, \alpha)^{7} \mathrm{Li}$ interaction, which is accompanied by the emission of a gamma ray of approximately $0.5 \mathrm{MeV}$. Sources of this type are significant only in materials containing isotopes which have a reasonable probability of undergoing a particular reaction and which are located in strong neutron fields. In reactors boron-containing materials generally are the only materials that fit these criteria.

(f) Activation-Product Gamma Rays. Capture and inelastic-scattering gamma rays are emitted simultaneously with the neutron-target nucleus interaction. However, the nucleus formed by a neutron interaction may be radioactive and decay with a half-life that can range from seconds to years, emitting photons and other radiations in the process. These activationproduct gamma rays may be of importance to the shield analysis and are of particular concern after reactor shutdown. They may also be emitted in significant quantities from materials which have been exposed to the high neutron flux of the core and which are subsequently circulated in regions external to the reactor shield. Irradiated samples and reactor coolants both fit this description.

For example, the ${ }^{16} \mathrm{O}(n, p){ }^{16} \mathrm{~N}$ reaction produced by fast-neutron activation of water emits gamma rays with energies of 6.1 and $7.1 \mathrm{MeV}$. The half-life of ${ }^{16} \mathrm{~N}$ is $7.13 \mathrm{sec}$, short enough to produce high activities in irradiated-water-coolant streams. Liquid-metal fuels and coolants must also be considered as a source of activation-product gamma rays. In sodium mixtures, ${ }^{23} \mathrm{Na}(n, \gamma){ }^{24} \mathrm{Na}$ produces 1.38 - and $2.76 \mathrm{-MeV}$ photons with a half-life of $14.8 \mathrm{hr}$.

(g) Annihilation Radiation. A few activated materials decay by the emission of positrons, which are annihilated by subsequent combination with electrons. Because of the relatively short distance that positrons travel before annihilation, the process can be considered to occur at the time of 
decay and at the site of the activated nucleus. Two $0.511-\mathrm{MeV}$ photons are emitted from each positron-electron reaction. High-energy gamma rays also can react in a converse process called pair production (discussed in Chap. 3 ) to produce electron-positron pairs. These positrons also are annihilated near their source in an identical manner and contribute further to the source of $0.511-\mathrm{MeV}$ gamma rays.

(h) Bremsstrahlung. The acceleration and deceleration of electrons in the atomic electric field produces electromagnetic radiation called bremsstrahlung. The process is identical to that occurring in the X-ray tube and is an important consideration only where high-energy beta particles (or accelerated electrons) interact with materials of high atomic number. An example is found in the use of lithium as a coolant. Neutron absorption in ${ }^{7} \mathrm{Li}$ produces ${ }^{8} \mathrm{Li}$. The latter undergoes decay to ${ }^{8} \mathrm{Be}$ by emission of beta particles with energies as high as $13 \mathrm{MeV}$. Bremsstrahlung produced by these high-energy electrons as they slow down in piping or containment materials requires evaluation as a gamma-ray source.

\subsubsection{Neutron Sources}

By far the greatest neutron source in an operating reactor is that created by the fission process itself, in which, along with the prompt gamma rays, free neutrons are released as part of the fission event. However, other reactions can also produce neutron sources of importance to the shield designer, and all sources discussed in this section should be considered in a shield analysis.

(a) Fission Neutrons. Approximately 2.5 neutrons are emitted per fission event in ${ }^{235} \mathrm{U}$ by thermal neutrons (more in other cases), and they carry away a total energy of approximately $5 \mathrm{MeV}$. Although energies can range from the $\mathrm{eV}$ region to beyond $18 \mathrm{MeV}$, the average energy of $a^{235} \mathrm{U}$ fission neutron is about $2 \mathrm{MeV}$, and an upper limit is often taken to be $14 \mathrm{MeV}$. In fact, less than $1 \%$ of the total energy of fission neutrons is shared by neutrons whose energies exceed $10 \mathrm{MeV}$. However, these high-energy neutrons are very penetrating, and in some cases they can be of overriding importance. Prompt fission-neutron spectra are discussed more fully in Sec. 2.4.2.

For shielding purposes, fission neutrons may be assumed to be evolved simultaneously with the fission event. The very small fraction $(<1 \%)$ with delayed emissions requires consideration as a separate source only in the case 
of a circulating-fuel reactor where the fuel loop extends beyond the core shield.

(b) Activation Neutrons. Under certain circumstances the decay of a radioactive nucleus can be followed by the emission of a neutron. This occurs when the energy of excitation of the daughter nucleus is in excess of the binding energy of the last neutron in the nucleus. In fact, when such an energy imbalance exists, neutron emission is the preferred mode of release. An example is the beta decay of ${ }^{17} \mathrm{~N}$ with a 4.14 -sec half-life, which leaves an ${ }^{17} \mathrm{O}$ nucleus with more than enough excitation energy to eject a neutron. Neutrons from this source have a most probable energy around $1.0 \mathrm{MeV}$. Nitrogen-17 is formed by the ${ }^{16} \mathrm{O}(n, p){ }^{17} \mathrm{~N}$ reaction and can be important in fast-neutron bombardment of water.

(c) Photoneutrons. A photon whose energy is greater than the neutron binding energy of a nucleus can impart enough energy to the nucleus to cause neutron emission. The photon energy required to make such a reaction possible exceeds $7 \mathrm{MeV}$ for all but a few nuclei, and the probability for the photoneutron reaction is quite low until photon energies above $10 \mathrm{MeV}$ are reached. Thus photoneutrons do not contribute a significant source component in the vast majority of reactor shielding problems. The few nuclei whose neutron binding energies are low enough to create a possible problem in reactor shielding include ${ }^{2} \mathrm{D},{ }^{9} \mathrm{Be},{ }^{13} \mathrm{C}$, and ${ }^{6} \mathrm{Li}$. The threshold photon energies for these isotopes are 2.23, 1.67, 4.9, and $5.3 \mathrm{MeV}$, respectively. Since all four occur in moderator materials, these exceptions are occasionally important.

(d) Particle-Reaction Neutrons. The interaction of alpha particles with nuclei of lithium, beryllium, oxygen, boron, and fluorine produces neutrons. Thus these elements are often combined with alpha-active isotopes, such as polonium or plutonium, to form neutron sources for use in experimentation or reactor start-up. Neutrons from this source may be important to shielding and safety during assembly or in the pre-start-up environment of reactors containing beryllium in the fuel-element material. Similarly, neutrons from $(\alpha, n)$ reactions in oxygen may be dominant in oxide fuel elements.

The energy distribution of these neutrons is broad since the neutron kinetic energy depends on its angle of emission relative to the direction of the incident alpha particle as well as on the kinetic energy of the alpha particle. In polonium-beryllium and plutonium-beryllium sources, for example, emergent-neutron energies range from $<1$ to $>10 \mathrm{MeV}$. 
Neutrons may be generated by other charged-particle interactions, but they have not been important in reactor designs to date. Incident neutrons with energies above $10 \mathrm{MeV}$ can excite a compound nucleus sufficiently to emit two or more neutrons. Such reactions are rarely of importance in reactor shield design.

\subsection{BASIC MATHEMATICAL AND PHYSICAL CONCEPTS}

The physical properties of radiation sources and fields are discussed in the remaining sections of this chapter and in subsequent chapters. The manner in which these properties are characterized requires an understanding of certain basic concepts, including differential and integral distributions, normalization and averaging techniques, solid-angle concepts, and definitions of flux density and current density. This section contains a review of these necessary basic considerations.

\subsubsection{Differential Distributions}

Most properties that characterize radiation fields and sources are functions of one or, more often, several independent variables. Mathematical functions may be used to describe the dependence of such a property on its variables; functions of this type are called differential distributions. Errors in the treatment of these distribution functions are among the more common mistakes in shielding practice. Because of the need for precision in these concepts, a careful explanation of the methods of treating such distributions is in order.

Consider, for example, a radiation source nonuniformly distributed in a three-dimensional slab whose emission rate varies with both time and one spatial dimension. Such a source can be described by a function of four variables, $P(x, t ; \Delta V, \Delta t)$. Its values give the number of source particles emitted in time interval $\Delta t$ centered about time $t$ from a spatial volume element of size $\Delta V$ centered at point $x$ in the slab. The dependent variables $\Delta V$ and $\Delta t$ are functions of the independent variables $x$ and $t$, respectively. Such a function is sufficient to characterize completely the spatial and temporal properties of the source.

The concept can be generalized to any number of variables. The general form can be written as

$$
P(x, y, z, \ldots ; \Delta x, \Delta y, \Delta z, \ldots)=D(x, y, z, \ldots) \Delta x \Delta y \Delta z \ldots
$$


The function $D(x, y, z, \ldots)$ is called a differential distribution function, $\uparrow$ and its values give the number of events per unit measure of each of the independent varıables, $x, y, z, \ldots$, involved.

In the radiation-source example, the differential distribution

$$
D(x, t)=\frac{P(x, t, \Delta V, \Delta t)}{\Delta V \Delta t}
$$

gives the number of source emissions that occur per unit volume and per unit time in volume element $\Delta V$ about $x$ and in time interval $\Delta t$ about $t$. It has units of $\mathrm{l}^{-3} \mathrm{t}^{-1}$, for example, particles per cubic centımeter per second.

Differential distributions are often used to describe energy spectra of sources and fields. For example, if $D(E)$ is a differential distribution describing the continuous energy spectrum of a photon field, the values of $D(E)$ give the number of photons per unit energy interval about $E$, and $D(E) \Delta E$ is the number of photons whose energies are in the energy interval $\Delta E$ containing energy $C$

Although differential distributions are useful in conjunction with finite intervals (1.e., $\Delta V, \Delta t, \Delta L$ ), which need not be infinitesimals, they are frequently applied to differentials. Thus $D(x, y, z, \ldots) d x d y d z$ specifies, for a distribution of events, the amount that lies in the differential volume element $d x d y d z \quad$ containing the point $(x, y, z, \quad$.$) . The integral of a$ differential distribution function over the entire domain of all variables gives $N$, the total number of events or quantity contained in the distribution,

$$
N=\int_{x} \int_{y} . D(x, y, \ldots) d x d y \ldots
$$

In our two examples, $\int_{\mathrm{x}} \int_{\mathrm{t}} D(x, t) d t d x$ is the total number of particles emitted from the radiation source and $\int_{\mathrm{E}} D(L) d E$ is the total number of photons of all energies contained in the energy distribution. Differential distributions may be displayed in graphical form, as shown in Fig. 2.1.

It is important to recognize that the value of $D(x)+$ at a selected value of $x$, say $x_{1}$, does not give the number of events occurring at point $r_{1}$. Rather, $D\left(x_{1}\right)$ is the number of events occurring in a unit interval of $x$ that contains the point $x_{1}$. Thus, although the unit selected to measure the independent

tMathematicians reserve the term distribution function for integral distributions $\left[1 \mathrm{e}, H_{1}(x)\right.$ and $F_{2}(x)$, Eqs 224 and 225 ] however, our use of the term here is consistent with common usage in the radiation shrelding field

†The discussion that follows is easily extended to many independent variables 


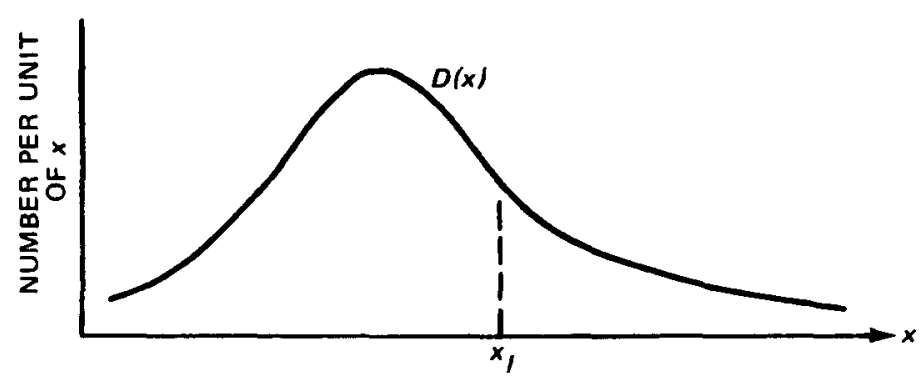

Fig. 2.1-Differential distribution.

variable $x$ is arbitrary (i.e., if $x$ is a length, centimeters, feet, yards, etc., can be used), it is essential that this unit be specified in order for the distribution function and its plot to have meaning.

Distributions are sometimes expressed in other forms. The cumulative distribution function for a differential distribution of one variable is defined by

$$
F_{1}(x)=\int_{-\infty}^{x} D\left(x^{\prime}\right) d x^{\prime}
$$

where $F_{1}(x)$ gives the amount of the distribution that lies below $x$. $f$ Alternatively, the function

$$
\Gamma_{2}(x)=\int_{x}^{\infty} D\left(x^{\prime}\right) d x^{\prime}
$$

gives the amount of the distribution above $x$. It is sometimes called the survivorship function.

Often, distributions are normalized to the total distribution, an operation that is valuable in comparing different distributions of the same variables:

$$
\begin{gathered}
f(x)=\frac{D(x)}{N}=\frac{D(x)}{\int_{-\infty}^{\infty} D(x) d x} \\
f_{1}(x)=\frac{F_{1}(x)}{N}=\frac{\int_{-\infty}^{x} D\left(x^{\prime}\right) d x^{\prime}}{\int_{-\infty}^{\infty} D(x) d x}
\end{gathered}
$$

†In Eqs. 2.2-4 and 2.2.5, - and $\infty$ can be replaced by any $a, b$ such that $a<x<b$; this merely alters the range of the distribution. 


$$
f_{2}(x)=\frac{F_{2}(x)}{N}=\frac{\int_{x}^{\infty} D\left(x^{\prime}\right) d x^{\prime}}{\int_{-\infty}^{\infty} D(x) d x}
$$

where the function $f(x)$ is the fraction of the distribution per unit interval about $x, f_{1}(x)$ is the fraction of the distribution lying below $x$, and $f_{2}(x)$ is the fraction lying above $x$. Note that $f(x), f_{1}(x)$, and $f_{2}(x)$ are constrained to the interval $[0,1]$, and $f_{1}(x)+f_{2}(x)=1$. If $x_{b}$ and $x_{u}$ denote the lower and upper bounds of the distribution,

$$
\begin{aligned}
& f_{1}\left(x_{b}\right)=f_{2}\left(x_{u}\right)=0 \\
& f_{1}\left(x_{u}\right)=f_{2}\left(x_{b}\right)=1
\end{aligned}
$$

and

$$
\int_{x_{b}}^{x_{u}} f(x) d x=1
$$

Differential distribution functions can be used to obtain the portion of the distribution or the number of events that occur over a specified region. In the photon-field energy-spectrum example,

$$
\int_{E_{1}}^{E_{2}} D(E) d E
$$

gives the number of photons with energies between $E_{1}$ and $E_{2}$. Such integral distributions may be plotted in histogram form (Fig. 2.2). The ordinate $D\left(\Delta x_{i}\right)$ is given by

$$
D\left(\Delta x_{i}\right)=\int_{x_{i}}^{x_{i+1}} D(x) d x
$$

and gives the total number of events within $\Delta x_{i}$, where $\Delta x_{i}=x_{i+1}-x_{i}$. Observe that the shape of a histogram depends on the selection of the increments, $\Delta x_{i}$. The distribution function that is plotted in Fig. 2.2 with equal increments could give a histogram of the form shown in Fig. 2.3 if different increment sizes were chosen. However, if a differential histogram is constructed in which

$$
D^{\prime}\left(\Delta x_{i}\right)=\frac{1}{\Delta x_{i}} \int_{x_{i}}^{x_{i+1}} D(x) d x
$$




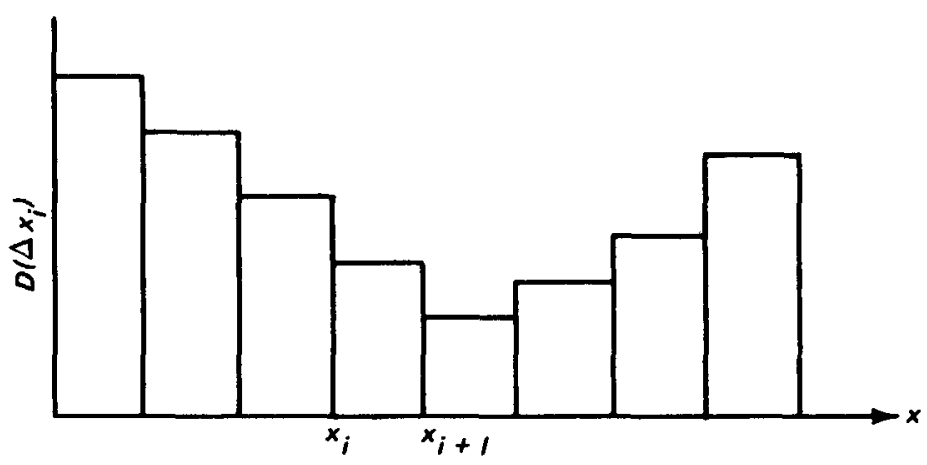

Fig. 2.2-Histogram for constant intervals.

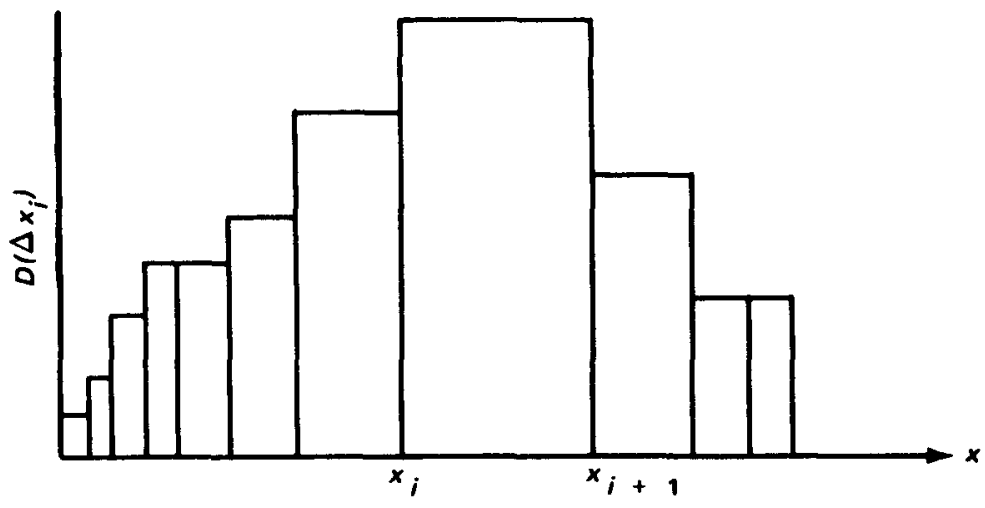

Fig. 2.3-Histogram for variable intervals.

the shape of the histogram always approximates the distribution function $D(x)$, and the approximation improves as the number of intervals is increased. Note that

$$
N=\sum_{i} D\left(\Delta x_{i}\right)=\sum_{i} D^{\prime}\left(\Delta x_{i}\right) \Delta x_{i}
$$

If a differential distribution function has more than one independent variable, new differential distributions are obtained when an integration is performed over some, but not all, of the variables. Consider the following differential distribution function, which can be derived for the radiationsource example:

$$
D^{\prime}(t)=\int_{\text {all space }} D(x, t) d V
$$


Most of the time, differential data presented in shielding analyses have been integrated (either mathematically or through the inherent characteristics of an experimental device) over one or more of the variables involved. Such data are commonly denoted singly differentiated, doubly differentiated, etc., depending on the number of independent variables that remain after integration of the fundamental multidimensional distribution.

\subsubsection{Average and Most-Probable Values}

Several parameters derived from differential distributions are of value in characterizing the distribution. One is the integral value $N$ (Eq. 2.2-3), which gives the total number of events in the distribution and is often used for normalizing purposes.

The average value of an independent variable within a distribution is obtained by computing the integral

$$
\begin{aligned}
\bar{x} & =\frac{1}{N} \int_{x} \int_{y} \ldots x \cdot D(x, y, \ldots) d x d y \ldots \\
& =\int_{x} \int_{y} \ldots x \cdot f(x, y, \ldots) d x d y \ldots
\end{aligned}
$$

which is called the first moment of the differential distribution.

The most-probable value of an independent variable of a differential distribution is its value where the distribution function has its maximum value. Note that the most-probable value may not be unique; for example, if $D(x)$ is constant over all $x$, every value of $x$ is a most-probable value.

At times, higher moments of differential distributions are useful (e.g., in the computation of standard deviations and variances). The $n$th moment about the variable $x$ is defined by

$$
\bar{x}^{(n)}=\frac{1}{N} \int_{x} \int_{y} \ldots x^{n} \cdot D(x, y \ldots) d x d y \ldots
$$

Examples of calculations of average and most-probable values are given in the exercises.

\subsubsection{Solid Angle}

Directional characteristics are essential to a complete description of radiation fields and sources. Since the concept of solid angle is used in specifying directional properties, some elementary definitions are reviewed. 
The solid angle subtended at a point by a surface is the projection of the surface on a sphere of unit radius surrounding the point. Consider the solid angle subtended at the point $D$ by $K_{1}$ in Fig. 2.4. (We could consider $K_{2}$ or a surface of any other shape.) The area of the projection of $K_{1}$ on the sphere of unit radius is a solid angle. A unit of solid angle is called a steradian. Since the area of a sphere is $4 \pi r^{2}$ and that of a unit sphere is $4 \pi$, there are $4 \pi$

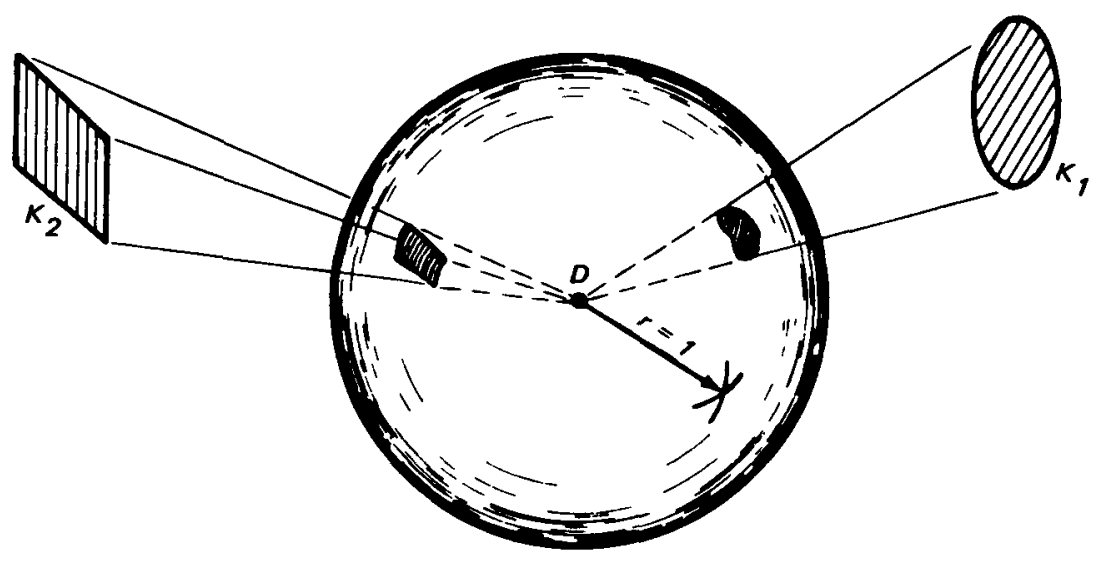

Fig. 2.4-Solid angle.

steradians about a point. If the unit vector normal to surface $K_{\mathbf{1}}$ is $\mathbf{n}$ and the unit vector along $r$ through $K_{1}$ is $\Omega$, then the solid angle is the scalar product $\mathbf{n} \cdot \Omega\left(K_{1} / r^{2}\right)$, $\dagger$ when $r$ is the length of the radius vector to $K_{1}$.

Radiation-source and -field angle distributions are defined by differential functions of the form $D(\Omega)$, where $D(\Omega)$ gives the distribution per unit solid angle along the direction of vector $\Omega$. In polar coordinates, where $\theta$ measures polar and $\phi$ measures azimuthal angles, $\$$ the differential area on a unit sphere is given by

$$
d A=\sin \theta d \theta d \phi
$$

and is numerically equal to the differential solid angle, $d \Omega$, subtended by $d A$. Thus, if $D(\Omega)$ is the differential angle distribution of a point radiation

†Strictly speahing, this formula is accurate only when $K_{1} \ll r^{2}$.

$\ddagger$ Poldr and azımuthal angles are defined in Sec 2.3.2., where directional characteristics of radiation sources are discussed. 
source located at the center of the sphere, $D(\Omega) \sin \theta d \theta d \phi$ particles are emitted which pass through $d A$, and

$$
\int_{\theta_{1}}^{\theta_{2}} \int_{\phi_{1}}^{\phi_{2}} D(\Omega) \sin \theta d \theta d \phi
$$

gives the number of source particles having directions bounded by polar angles $\theta_{1}$ and $\theta_{2}$ and by azimuthal angles $\phi_{1}$ and $\phi_{2}$. Note that in the preceding discussion, the angles $\theta$ and $\phi$ define the directional vector, $\Omega$, which has a unit magnitude.

\subsubsection{Measures of Radiation Intensity}

Essential to a discussion of intensity is the concept of a receiver, or a detector, a mathematical concept used in defining the necessary units and describing the fields. As will be seen, theoretical detectors take the form of points, surfaces, and volumes, depending on the quantity being measured. Measures of radiation intensity commonly used in shield analysis include particle density, flux density, current density, absorbed dose rate, kerma rate, and exposure rate. The first three are used to characterize a radiation field and are defined in this section. The last three are used to characterize radiation interactions with matter and are discussed in Chap. 3. The time integral of the last three quantities over an operating cycle or exposure duration provides a basis for estimating the damaging effect of the radiation involved.

The quantities discussed in this section are obtained by taking a limit of measured radiation-particle densities $t$ in a finite volume or passing through a finite area as the volume or area approaches zero in size. This is a very special limiting process that requires careful definition to avoid a contradiction between the mathematical concept of a differential and the physical reality of the random nature of particle tracks. In moving to such a conceptual limit, we can reach a point where the detector size is so small that for further reductions in volume the probability of finding a particle within it also approaches zero, and thus the limit of the particle density is zero. In the special limiting process implied in the definitions that follow, this is not

tThe terms particle and density are used in a general sense. Particle refers both to particles having rest mass and to photons. Density refers to a differential function of one or more variables, i.e., particles $/ \mathrm{cm}^{2}$, particles $/ \mathrm{cm}^{3}, \mathrm{MeV} \mathrm{cm}^{-3} \mathrm{sec}^{-1}$, etc. 
allowed to occur. Rather, in setting the limit, we stop shrinking the detector volume or area as soon as a further reduction in size would not change the value of the density quantity being measured. At the same time, we must keep the detecting volume large enough to contain a statistically significant number of particles or particle interactions. In radiation fields with very steep gradients, this special limiting process raises difficulties because the two conflicting requirements on detector size may be mutually exclusive. In such cases we introduce an averaging process to overcome the conceptual problem.

A complete description of a radiation field includes the number of particles of a given type and energy which at a given time exist at a given position and travel in a given direction, all particle types, energies, positions, and directions being considered at all times. Such complete descriptions are seldom required, however, except in the most sophisticated particletransport calculations, and a radiation field cannot be measured in such detail. Therefore particle populations are usually described in terms of a lesser number of parameters.

Instead of the indefinite number of variables used in the previous discussion on distributions in general $(x, y, z, \ldots)$, we now limit the discussion to the seven dimensions needed to define the kinematics of a particle. These are the three spatial coordinates: the particle kinetic energy (velocity is used alternatively), two angles defining direction, and time. The three spatial coordinates can be specified as the radius vector, $r$, and the two direction angles reduced to a unit direction vector, $\Omega$. These variables define phase space, which is indicated functionally as $f(\mathbf{r}, E, \Omega, t)$.

The following discussion of particle, flux, and current densities is adapted from a recent report by Stevens and Claiborne. ${ }^{3}$ They have followed the recommendations of the International Commission on Radiation Units and Measurements, ${ }^{4,5}$ which this text has also attempted to follow.

(a) Particle Densities. Knowledge of the particle density over all phase space is equivalent to a complete solution of a particle-transport problem and comprises more information than is available from most calculational schemes now in use. When given in seven-dimensional phase space, particle density is defined by

$$
n(\mathbf{r}, E, \Omega, t) d E d \Omega
$$


the number of particles per unit volume at space point $r$ and time $t$ having energies in $d E$ about energy $E$ and directions in $d \Omega$ about the unit direction vector $\Omega$.

The particle density so defined is doubly differential, in energy and in direction, and less detailed forms will often suffice. For example, we may use the steady-state $f$ particle-density differential in energy only, commonly called the differential particle density and defined by

$$
n(\mathbf{r}, E) d E
$$

the number of particles per unit volume at space point $\mathbf{r}$ having energies in $d E$ about $E$.

$$
n(\mathbf{r}, E) d E=\int_{4 \pi} n(\mathbf{r}, E, \Omega) d \Omega d E
$$

Or one may use the steady-state total particle density, defined as the number of particles per unit volume at space point $r$ and given by

$$
n(\mathbf{r})=\int_{4 \pi} \int_{0}^{\infty} n(\mathbf{r}, E, \Omega) d E d \Omega=\int_{0}^{\infty} n(\mathbf{r}, E) d E
$$

(b) Flux Densities. Even though the concept of particle density is basically simple and has a unique interpretation, experience has shown that the flux density, ${ }^{4}$ or, as it is commonly called, the flux, $\$$ serves better as the dependent variable in solutions of the transport equation (discussed in Chap. 4). The flux density is related to the particle density through the particle's speed, and, when described in terms of seven-dimensional phase space, the flux density is given by

$$
\Phi(\mathbf{r}, E, \Omega, t)=v n(\mathbf{r}, E, \Omega, t)
$$

where $v$ is the particle's speed and corresponds to the energy $E$. (The speed is the scalar magnitude of the particle's velocity vector, v.)

tThe steady state, or time independent, condition is denoted in phase space notation by dropping the time sy mbol $t$, seven-dimensional phase space becomes six dimensional and in phase space notation is given by $(r, E, \Omega)$

$\ddagger$ Although this quantity is truly a density and the International Commission on Radiation Units and Measurements ${ }^{4}$ recommends the use of the term flux density, the simpler term flux is ingrained in shielding terminology and is used extensively elsewhere. We have used flux density exclusively in this text. 
The flux density defined in the preceding paragraph is doubly differential and is usually referred to as the angular flux density. Greater insight into the use of the angular flux density as the dependent variable in mathematical descriptions of particle transport is provided by its interpretation either as the track lengths traversed per unit volume and time or as the flow of particles per unit area and time.

Flux density is a measure of a radiation field in terms of its potential for interaction with the material through which it is passing and is measured with volume detectors. In the theory of radiation interactions, nuclear forces (or coulomb forces) are additive; i.e., no shadowing of nuclei or electrons by others occurs. This implies that the probability of a radiation particle interacting with matter is directly proportional to the number of nuclei (or electrons) in whose vicinity it passes. This number, in turn, is proportional to the distance traveled by the radiation particle. Thus the total interaction probability of a radiation field with matter is proportional to the sum of the distances, or total track length, traveled by all the radiation particles traversing the medium, and the interaction rate is proportional to the total track length generated per unit of time.

The track length interpretation of angular flux density follows from the observation that the speed of an individual particle can be considered as its scalar track length per unit time. The product of particle density and speed is then the sum of the track lengths traced by all the particles within a unit volume per unit time, $\dagger$ in which case the definition of the angular flux density would be

$$
\Phi(\mathbf{r}, E, \Omega, t) d E d \Omega
$$

the total track lengths traversed per unit volume and time at space point $\mathbf{r}$ and time $t$ by particles having energies in $d E$ about energy $E$ and directions in $d \Omega$ about $\Omega$.

The interpretation of the angular flux density as a flow of particles per unit area and time is closely related to the concept of angular current (to be discussed in the next section). It will be shown in the discussion on current that the angular flux density is identical to the magnitude of the current vector $\mathbf{J}$ and thus can be interpreted as

†Note that the per-unit-time units of the flux density are associated with the particle's speed, which is a function only of the energy. However, the time dependence of the flux density is a consequence of the time behavior of the particle density, which does not have time units even though the time symbol, $t$, is included in the phase-space notation to denote a dependence on time. 


$$
\Phi(\mathbf{r}, E, \Omega, t) d E d \Omega
$$

the flow per unit area and time at space point $\mathrm{r}$ and time $t$ of particles having energies in $d E$ about $E$ and directions in $d \Omega$ about $\Omega$.

When the transport and the deposition of the particle kinetic energy are of interest, the energy-flux-density differential in energy and angle is often used. This quantity, called the angular energy-flux density, is defined by

$$
I(\mathbf{r}, E, \Omega, t) d E d \Omega
$$

the energy flow per unit area and time at space point $\mathrm{r}$ and time $t$ due to particles having energies in $d E$ about $E$ and directions in $d \Omega$ about $\Omega$, and is related to the angular flux density by

$$
I(\mathbf{r}, E, \Omega, t)=E \Phi(\mathbf{r}, E, \Omega, t)
$$

Many calculational models employ less detailed descriptions of the flux density; for example, they may use a description in which steady state is assumed and the angular dependencies are removed by the appropriate solid-angle integration. This reduces the description to four-dimensional phase space and results in a dependent variable representing the particleflux-density differential in energy. Commonly known as the differential flux density, this quantity is given by

$$
\Phi(\mathbf{r}, E)=\int_{4 \pi} \Phi(\mathbf{r}, E, \Omega) d \Omega
$$

Like the angular flux density, the differential flux density can be interpreted in terms of track length per unit volume and time or in terms of the number of particles that enter a unit sphere per unit time. In the latter case, the solid-angle integration can be regarded as a summing of particles that enter a sphere of unit cross section regardless of their directions of motion. The sphere is, in effect, generated by the rotation of a circular unit area during the integration over a $4 \pi$ solid angle (see Fig. 2.5). In this context the definition for the differential flux density can be restated as

$$
\Phi(\mathbf{r}, E) d E
$$

number of particles having energies in $d E$ about $E$ which enter a sphere of unit cross section per unit time at space point $r$. 


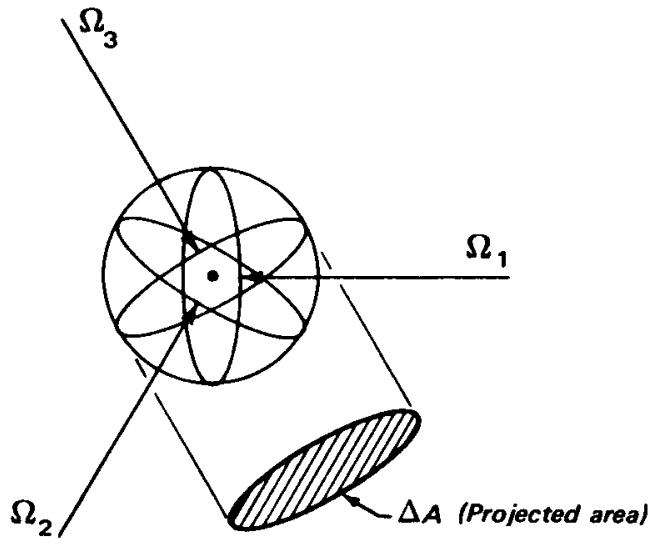

Fig. 2.5-Incremental sphere concept of flux density.

Although this definition of the differential flux density is descriptive, it is not exact. The mathematically rigorous definition is

$$
\Phi(\mathbf{r}, E) d E \equiv \lim _{\Delta A \rightarrow 0} \frac{N(E) d E}{\Delta A}
$$

which implies the limit process $\Delta A \rightarrow 0$, with $N(E) d E$ denoting the number of particles having energies in $d E$ about $E$ which enter an incremental sphere of cross section $\Delta A$ per unit time.

The concept of the incremental sphere is the best way to visualize the energy-flux-density differential in energy. Referred to as the differential energy-flux density, this quantity may be defined by

$$
I(\mathbf{r}, E) d E
$$

the energy flow per unit time into a sphere of unit cross section at space point $\mathbf{r}$ due to particles having energies in $d E$ about $E$. The differential energy-flux density is given by

$$
I(\mathbf{r}, E) d E=\int_{4 \pi} I(\mathbf{r}, E, \Omega) d \Omega d E=E \Phi(\mathbf{r}, E) d E
$$

Other quantities used are the total flux density, the total energy-flux density, the group flux density, and the group angular flux density. The total flux density, defined alternatively as the total particle track length per unit volume and time at space point $r$ or as the number of particles that enter a 
sphere of unit cross section per unit time at space point $\mathbf{r}$, is obtained by integrating the differential flux over all energies:

$$
\Phi(\mathbf{r})=\int_{0}^{\infty} \Phi(\mathbf{r}, E) d E
$$

Similarly, the total energy-flux density, defined as the total energy flow per unit time into a sphere of unit cross section at space point $r$, is obtained by an integration of the differential energy-flux density over all energies:

$$
I(\mathbf{r})=\int_{0}^{\infty} I(\mathbf{r}, E) d E
$$

The total flux density has only limited application to practical shielding problems because of the strongly energy-dependent nature of the particle behavior. A more useful approach is to divide the total energy range into $\mathrm{L}$ energy intervals, called energy groups, $\uparrow$

$$
\Delta E_{G} \equiv E_{g}-E_{g+1} \quad G=1,2, \ldots, \mathrm{L}
$$

and to define the group flux density as the integral of the differential flux density over the corresponding energy group,

$$
\Phi_{G}(\mathbf{r}) \equiv \int_{E_{g^{+1}}}^{E_{g}} \Phi(\mathbf{r}, E) d E
$$

with the constraint that

$$
\Phi(\mathbf{r})=\sum_{L} \Phi_{G}(\mathbf{r})
$$

The group angular flux density (group flux density differential in angle) has a similar definition and is obtained by integrating the angular flux density over a specific energy group:

$$
\Phi_{G}(\mathbf{r}, \Omega) \equiv \int_{E_{g+1}}^{E_{g}} \Phi(\mathbf{r}, E, \Omega) d E
$$

tThe subscripts $g$ and $g+1$ refer to the upper and lower limits, respectively, of the $G$ th energy group, and $G=1$ corresponds to the highest energy group. An alternate convention would associate $G=1$ with the lowest encrgy group; the subscripts $g+1$ and $g$ would then correspond to the upper and lower energy limits. $I$, is the number of groups. 
For problems that involve directional symmetry, the group angular flux density can be rewritten in terms of a new angular variable $\mu=\Omega \cdot r /|\mathbf{r}|$, the direction cosine:

$$
\Phi_{G}(\mathbf{r}, \mu) d \mu=\Phi_{G}(\mathbf{r}, \Omega) d \Omega
$$

The group angular flux density can then be defined as the total particle track length per unit volume and time at space point $\mathbf{r}$ of particles with energies within energy group $\Delta E_{G}$ and directions defined by direction cosines that lic in $d \mu$ about $\mu$.

In neutron physics, another variable used to characterize a neutron speed is lethargy. The lethargy, $u$, of a particle with energy $E$ is given by

$$
u=\ln \frac{E_{0}}{E}
$$

where $E_{0}$ is an upper limit of $E$, often taken to be $10 \mathrm{McV}$ in fission reactors. Appropriately, lethargy increases as energy decreases and

$$
\Phi(\mathbf{r}, u)=L \Phi(\mathbf{r}, E)
$$

where $\Phi(\mathbf{r}, u)$ is the differential flux density per unit lethargy at point $\mathbf{r}$.

A useful measure of total exposure to a flux density for applications involving energy deposition is the integral quantity called fluence. Fluence is defined by the International Commission on Radiation Units and Measurements $^{4.5}$ as the quotient of $\Delta N$ divided by $\Delta A$, where $\Delta N$ is the number of particles that enter a sphere of cross-sectional area $\Delta A$ and the $\Delta$ 's imply the special limiting process described at the beginning of Sec. 2.2.4. This definition is equivalent to regarding fluence as a time-integrated flux density over some specified time interval. As such, the fluence can be written

$$
I=\int_{t_{1}}^{t_{2}=t_{1}+\Delta t} \Phi(t) d t
$$

where $\Delta t$ corresponds to some specified time interval and $\Phi(t)$ can be any one of the several kinds of flux density described in the preceding text. For example, the energy fluence is

$$
F(\mathbf{r})=\int_{t_{1}}^{t_{2}=t_{1}+\Delta t} I(\mathbf{r}, t) d t
$$


(c) Current Densities. The characteristic property of the current variable is its close relation to the convective (leakage) effects in the theoretical description of particle transport. The most general form of the current variable is differential both in energy (or speed) and in angle. Called the angular current density, or, more frequently, the angular current, this quantity is symbolized by $\mathbf{J}(\mathbf{r}, 1, \Omega, t)$ and is defined as the directed flow per unit area (normal to the $\Omega$ direction) and time at the space point $r$ and time $t$ of particles having speeds in $d v$ about $v$ and directions in $d \Omega$ about $\Omega$.

The relation between current and particle density can be established by considering that (1) the product of $\mathbf{v}$ and the particle density can be regarded as a vector sum of the individual codirectional velocity vectors $(\mathbf{v})$, yielding the resultant vector $\mathbf{J}=n \mathbf{v}$ or (2) that the $(v \times d t \times d A \times N)$ particles contained within the volume element shown in Fig. 2.6 will all exit through

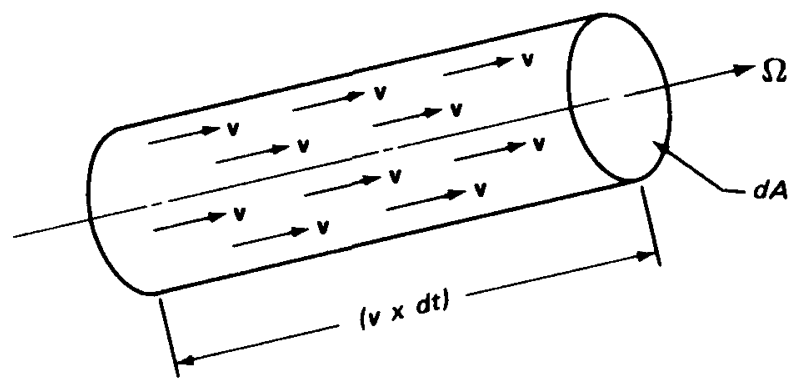

Fig. 2.6-Particle flow concept of current. The particles contained within the volume element will all exit through the differential area $d A$ within $d t$ if $\mathbf{v}=\Omega$.

the differential area $d A$ within differential time $d t$ if $v=v \Omega$. These models can be expressed mathematically in terms of the angular current as

$$
\mathrm{J}(\mathbf{r}, v, \Omega, t) d v d \Omega=\Omega v n(\mathbf{r}, v, \Omega, t) d v d \Omega
$$

and, since the particle's kinetic energy is a function of its speed, Eq. 2.2-36 can be rewritten

$$
\mathrm{J}(\mathbf{r}, E, \Omega, t) d E d \Omega=\Omega v n(\mathbf{r}, E, \Omega, t) d E d \Omega
$$

where $\mathbf{J}(\mathbf{r}, E, \Omega, t) d E=\mathbf{J}(\mathbf{r}, v, \Omega, t) d v$ and $n(\mathbf{r}, E, \Omega, t) d E=n(\mathbf{r}, v, \Omega, t) d v$. Then, when $v n(\mathbf{r}, E, \Omega, t)$ is identified as the angular flux density, the relation 
between the angular flux density and the angular current noted in Sec. (b), above, is obtained:

$$
\mathbf{J}(\mathbf{r}, E, \Omega, t) d E d \Omega=\Omega \Phi(\mathbf{r}, E, \Omega, t) d E d \Omega
$$

Other less detailed forms of the angular current are the group angular current, $\mathbf{J}_{G}(\mathbf{r}, \Omega)$, and the total angular current, $\mathbf{J}(\mathbf{r}, \Omega)$, which are obtained by in tegrations over an energy group and all energies, respectively:

$$
\begin{gathered}
\mathrm{J}_{G}(\mathbf{r}, \Omega) \equiv \int_{E_{g+1}}^{E_{g}} \mathrm{~J}(\mathbf{r}, E, \Omega) d E=\Omega \Phi_{G}(\mathbf{r}, \Omega) \\
\mathrm{J}(\mathbf{r}, \Omega) \equiv \int_{0}^{\infty} \mathrm{J}(\mathbf{r}, E, \Omega) d E=\Omega \Phi(\mathbf{r}, \Omega)
\end{gathered}
$$

It is apparent that the angular current variables all have essentially the same simple relation with the corresponding angular flux density because the energy integrations are performed directly on the flux density. For example, in the case of the group angular current,

$$
\begin{aligned}
\mathbf{J}_{G}(\mathbf{r}, \Omega) & =\int_{E_{g+1}}^{E_{g}} \Omega \Phi(\mathbf{r}, E, \Omega) d E \\
& =\Omega \int_{E_{g+1}}^{E_{g}} \Phi(\mathbf{r}, E, \Omega) d E=\Omega \Phi_{G}(\mathbf{r}, \Omega)
\end{aligned}
$$

The integral of the angular current over all directions ( $4 \pi$ solid angle) constitutes a vector summation, and the resultant vector is regarded as the net current, often called simply current.

The net current differential in energy only, referred to as the differential net current, is defined as the net flow per unit area and time at space point $\mathbf{r}$ and time $t$ of particles having energies in $d E$ about $E$, where the unit area is normal to the direction of the resultant vector $\mathrm{J}(\mathbf{r}, E, t)$, or $\mathrm{J}(\mathbf{r}, E, t) d E$,

$$
\begin{aligned}
\mathbf{J}(\mathbf{r}, E, t) d E & =\int_{4 \pi} \mathbf{J}(\mathbf{r}, E, \Omega, t) d \Omega d E \\
& =\int_{4 \pi} \Omega \Phi(\mathbf{r}, E, \Omega, t) d \Omega d E
\end{aligned}
$$

which is the angular flux-weighted vector summation of the unit vectors $\Omega$ over a $4 \pi$ solid angle. The group net current, $\mathrm{J}_{G}(\mathbf{r})$, and total net current, $J(r)$, are $4 \pi$ solid-angle integrations of the group angular current and total angular current, respectively: 


$$
\begin{gathered}
\mathrm{J}_{G}(\mathbf{r})=\int_{4 \pi} \mathrm{J}_{G}(\mathbf{r}, \Omega) d \Omega=\int_{4 \pi} \Omega \Phi_{G}(\mathbf{r}, \Omega) d \Omega \\
\mathrm{J}(\mathbf{r})=\int_{4 \pi} \mathrm{J}(\mathbf{r}, \Omega) d \Omega=\int_{4 \pi} \Omega \Phi(\mathbf{r}, \Omega) d \Omega
\end{gathered}
$$

The flow of $\Omega$-directed particles across an arbitrarily oriented differential area is a necessary concept in the description of the directed flow of particles in terms of a specific coordinate system and can be related to the angular current, $\mathbf{J}(\Omega)$, $\dagger$ by consideration of Fig 2.7 , where the direction vector, $\mathbf{n}$, is

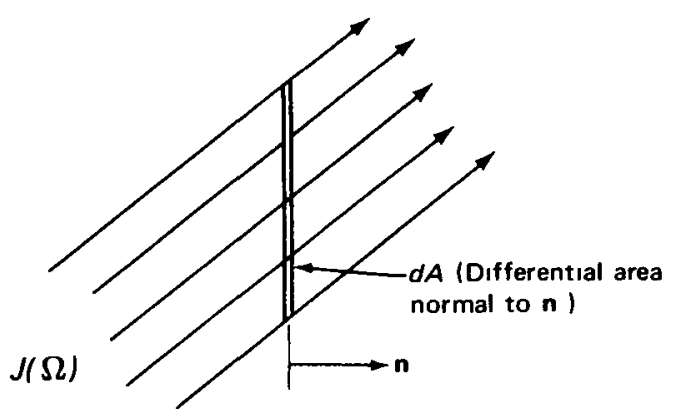

Fig 27-Schematic diagram of partıcle flow across an arbitrarily oriented surface

normal to the differential area The number of $\Omega$-directed particles crossing the differential area $d A$ per unit time is equal to $\mathrm{J}(\Omega) \cdot(\mathbf{n} d A)$. A scalar current, $J_{n}(\Omega)$, that describes the flow of the $\Omega$ directed particles per unit area normal to the direction $\mathbf{n}$ is defined as

$$
J_{n}(\Omega) d A \equiv \mathrm{J}(\Omega) \cdot(\mathbf{n} d A)
$$

It follows that

$$
J_{n}(\Omega)=\mathrm{n} \cdot \mathrm{J}(\Omega)=\Omega \cdot n \Phi(\Omega)=\cos \theta \Phi(\Omega)
$$

where $\mathbf{n}=$ the unit vector corresponding to an arbitrary direction

$n=$ a coordinate-identifying subscript, for example, $n \equiv x$ when $\mathbf{n} \equiv \mathbf{i}$ $J_{n}(\Omega)=$ the flow of $\Omega$-directed particles per unit area (normal to the direction $\mathbf{n}$ ) and tıme

\footnotetext{
fIn this further discussion of current the notation $\mathrm{J}(\Omega)$ will be used to denote any of the angular currents and $\mathrm{J}$ the corresponding net current for example $\mathrm{J}(\boldsymbol{\Omega})$ may represent $\mathrm{J}(\mathbf{r}, E, \Omega, t) \mathrm{J}_{G}(\mathbf{r}, \boldsymbol{\Omega}, t)$, or $\mathrm{J}(\mathbf{r} \Omega)$ and $\mathrm{J}$ ma y $\mathrm{rcprcsent} \mathrm{J}(\mathrm{r}, \boldsymbol{F}) \mathrm{J}_{G}(\mathbf{r})$ or $\mathrm{J}(\mathrm{r})$
} 
$\Phi(\Omega)=$ the angular flux variable corresponding to the angular current $\mathrm{J}(\Omega)$

Note that $J_{n}(\Omega)$ is a scalar quantity but is uniquely related to the coordinate system through the direction unit vector $\mathbf{n}$. The corresponding vector current is given by

$$
\mathrm{J}_{n}(\Omega)=\mathrm{n} J_{n}(\Omega)=\mathrm{nJ}(\Omega) \cdot \mathrm{n}
$$

where the vector $\mathrm{J}_{n}(\Omega)$ is the component of the vector $\mathrm{J}(\Omega)$ with respect to the $\mathbf{n}$ direction and $J_{n}(\Omega)$ is the projection of the vector $J(\Omega)$ on the $\mathbf{n}$ axis and also the magnitude of the component $\mathbf{J}_{n}(\Omega)$.

The three components of $J(\Omega)$ in cartesian coordinates are given by

$$
\begin{aligned}
& \mathrm{J}_{x}(\Omega)=\mathrm{i} J_{x}(\Omega)=\mathrm{i} \Phi(\Omega)(\mathrm{i} \cdot \Omega) \\
& \mathrm{J}_{y}(\Omega)=\mathrm{j} J_{y}(\Omega)=\mathrm{j} \Phi(\Omega)(\mathrm{j} \cdot \Omega) \\
& \mathrm{J}_{z}(\Omega)=\mathrm{k} J_{z}(\Omega)=\mathrm{k} \Phi(\Omega)(\mathrm{k} \cdot \Omega)
\end{aligned}
$$

and $J(\Omega)$ is equal to the vector sum of the three components:

$$
\begin{aligned}
\mathrm{J}(\Omega) & =\mathrm{J}_{x}(\Omega)+\mathrm{J}_{y}(\Omega)+\mathrm{J}_{z}(\Omega) \\
& =\mathrm{i} J_{x}(\Omega)+\mathrm{j} J_{y}(\Omega)+\mathrm{k} J_{z}(\Omega)
\end{aligned}
$$

It is important to recognize that, although they have the same units, the scalar quantity flux density is not equivalent to the vector quantity current density. The latter includes directional properties of the radiation field but the former does not. Current density should be considered as a measure of the passage of particles through a surface (a density per unit area), and flux density should be considered as a measure of track length in a volume (a density per unit volume). Only in rare instances are the two numerically equal at a point in a radiation field. The following examples illustrate this point.

A plane source $S_{1}$ (Fig. 2.8) emits monodirectional and monoenergetic particles at the rate of $10^{10}$ particles $\mathrm{cm}^{-2} \mathrm{sec}^{-1}$ in a direction normal to the surface. Let us compute the current density and flux density:

(a) At a point in a plane $A$ whose normal $\mathbf{n}_{A}$ is parallel to that of $S_{1}$.

(b) At a point in a plane $B$ whose normal $\mathbf{n}_{B}$ is at an angle $\theta$ with respect to $\mathbf{n}_{A}$. 


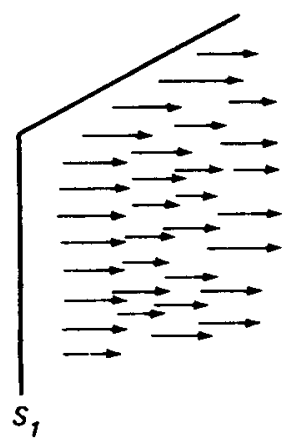

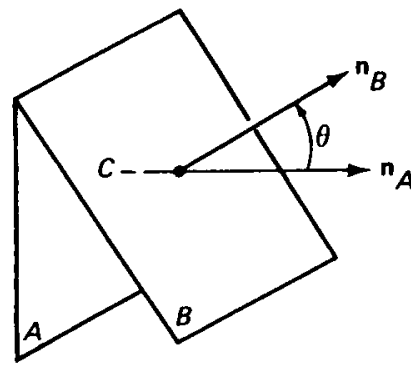

Fig. 2.8-Plane sources.

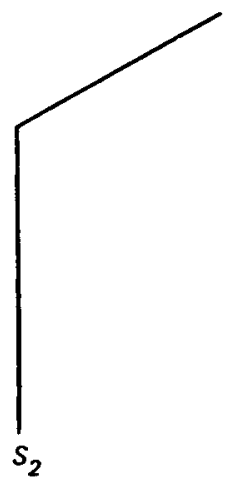

We proceed as follows:

(a) The flow of particles per unit time and area in the positive direction through plane $A$ is $10^{10}$ particles $\mathrm{cm}^{-2} \mathrm{sec}^{-1}$. The flow rate in the opposite direction is zero. Therefore the net current density, $\mathbf{J}$, is in the direction $\mathbf{n}_{A}$ and is $10^{10}$ particles $\mathrm{cm}^{-2} \mathrm{sec}^{-1}$. To obtain the flux density, $\Phi$, consider a unit cube ( 1 by 1 by $1 \mathrm{~cm}$ ) whose center lies in $A$ with two faces parallel to $A ; 10^{10}$ particles $/ \mathrm{sec}$ enter the cube, and each generates a track length of $1 \mathrm{~cm}$ in crossing the cube. Thus the total track length generated in the cube per unit time is $10^{10} \mathrm{~cm} / \mathrm{sec}$, and, since the cube volume is $1 \mathrm{~cm}^{3}$, the flux density is $10^{10}$ $\mathrm{cm} / \mathrm{sec} \div 1 \mathrm{~cm}^{3}=10^{10}$ particles $\mathrm{cm}^{-2} \mathrm{sec}^{-1}$.

(b) The net flow rate through a unit area of plane $B$ is $\cos \theta$ times that through a unit area of plane $A$; i.e., the component of $\mathrm{J}$ in the direction $\mathbf{n}_{B}$ is $\mathbf{J} \cdot \mathbf{n}_{B}$, and the current density in the direction of $\mathbf{n}_{B}$ is $\cos \theta \times 10^{10}$ particles $\mathrm{cm}^{-2} \mathrm{sec}^{-1}$. The flux density is not a function of the direction $\mathrm{n}_{B}$ and is $10^{10}$ particles $\mathrm{cm}^{-2} \mathrm{sec}^{-1}$.

Suppose now that we add a second plane source, $S_{2}$, also shown in Fig. 2.8, which emits monodirectional particles with the same energy but in a direction opposite to that of $S_{1}$ at a rate of $6 \times 10^{9}$ particles $\mathrm{cm}^{-2} \mathrm{sec}^{-1}$. We compute the current density in the direction $\mathbf{n}_{A}$ and the flux density at a point in plane $A$ when both sources are emitting particles simultaneously. The particle flow rate is $6 \times 10^{9}$ particles $\mathrm{cm}^{-2} \mathrm{sec}^{-1}$ in the negative direction and $10^{10}$ particles $\mathrm{cm}^{-2} \mathrm{sec}^{-1}$ in the positive direction. Thus the net current density is $4 \times 10^{9}$ particles $\mathrm{cm}^{-2} \mathrm{sec}^{-1}$. The track length contribution from $S_{2}$ is $6 \times 10^{9} \mathrm{~cm} \mathrm{sec}^{-1} \mathrm{~cm}^{-3}$. The flux density is thus $1.6 \times 10^{10}$ particles $\mathrm{cm}^{-2} \mathrm{sec}^{-1}$. 


\subsection{SPATIAL AND DIRECTIONAL CHARACTERISTICS}

A number of parameters are needed to characterize a source. It may be distributed in space and emit a variety of radiation types, its intensity may vary with time, and the radiation it emits may be distributed in both energy and direction of emission. Spatial and directional properties of typical radiation sources are discussed in this section; important energy and time distributions are considered in the following section.

\subsubsection{Spatial Distributions}

Radiation sources are classificd into four spatial categorics, point, line, surface, and volume, depending on the number of dimensions they encompass. Often, however, a source of two or more dimensions is considered as a configuration of sources of lower dimension. For example, a volume source may be viewed as an aggregate of point sources distributed throughout the volume.

The spatial variables describing sources are independent of each other and of other source parameters. Thus sources of any number of dimensions and any spatial distribution within those dimensions can have independent distributions in radiation type, direction of emission, energy, and time. Direction, energy, and time distributions may vary from point to point in the spatial distribution.

(a) Point Source. A source emitting radiation from a single point in space may be considered the fundamental source configuration since all other sources can be constructed from numbers of such point sources. Total point-source strengths arc measured in units of particles or MeV per second. When point sources have distributions in direction, energy, and time, differential distributions characterizing such dependencies have typical units of particles $\sec ^{-1} \mathrm{MeV}^{-1}$ steradian $^{-1}$. Differential point sources are of great value in developing analytic functions of radiation-source and -field properties. For example, at point $\mathbf{r}$ let $S(\mathbf{r})$ be the emissions $\mathrm{cm}^{-3} \mathrm{sec}^{-1}$ of a volume-distributed source. A differential point source is then given by $S(\mathbf{r})$ $d V$ particles/sec. If $f\left(\mathbf{r} ; \mathbf{r}^{\prime}\right)$ is a function relating a radiation-field property at $\mathbf{r}^{\prime}$ to a point source of unit strength at $\mathbf{r}$, the field property generated at $\mathbf{r}^{\prime}$ by the entire volume source is given by

$$
F\left(\mathbf{r}^{\prime}\right)=\iint_{\substack{\text { source } \\ \text { volume }}} f\left(\mathbf{r} ; \mathbf{r}^{\prime}\right) S(\mathbf{r}) d V
$$


In fact, functions relating two spatial positions, such as $f$ above, play an important role in shield analysis. They are called point kernels and are discussed more fully in Chap. 6 .

A source need not be infinitesimal in size to be classified as a point source. When a radiation field at a detector point is being evaluated, a point-source approximation may be used to represent a volume or area source if the source dimensions are small compared to the source-detector distance. In making such an approximation for volume-distributed source configurations, we must be careful to consider radiation interactions that occur within the source volume (self-shielding), and adjustments should be made if required.

(b) Line Source. A source with emission confined to a line is a line source; total line-source strengths have typical units of particles or $\mathrm{MeV}$ per second per unit length of the source. Like all sources, line sources can have differential energy, angle, and time distributions that may vary along their length. Typical units of differential distribution describing such line sources

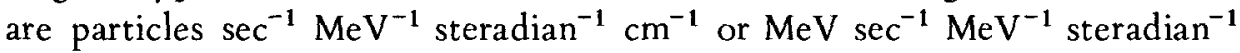
$\mathrm{cm}^{-1}$. Examples of approximate line sources encountered in reactor shielding problems include pipes carrying radioactive material and long, thin fuel pins.

(c) Surface Source. A surface source is one in which radiation emanates from a plane or other two-dimensional surface. The units of source strength are particles or $\mathrm{MeV}$ per unit time per unit source area (i.e., particles $\mathrm{sec}^{-1}$ $\mathrm{cm}^{-2}$ ). Since surface source strengths have the same units as flux and current density, care must be taken not to confuse the three quantities. Surface-source strength is a scalar, specified in terms of a particle emission rate per unit area of source surface. The need for such care is illustrated in Sec. 2.3.2, where it is shown that a differential surface-source strength function, although sharing the same units, may not be the same as the differential function defining the flux density generated by that surface source.

Surfaces that can be described mathematically are of high value in specifying surface sources since many times functions describing such surfaces admit to analytic integration and are convenient in hand and computer numerical computations. Also, volume sources are often characterized by the radiation passing through their exterior surfaces. Such a representation often allows the shielding analysis to be divided into two phases: (1) determination of the radiation transport within the source, resulting in the definition of an equivalent surface source, and (2) analysis of 
the radiation transport external to the source. In such an approach the calculations involved in phase (1) need not be repeated for shield-design parameter studies, which are a part of phase (2). Reactor-core and pressure-vessel surfaces are examples of often-used equivalent surface sources.

(d) Volume Source. A radiation source distributed throughout a closed surface constitutes a volume source. Typical volume sources encountered
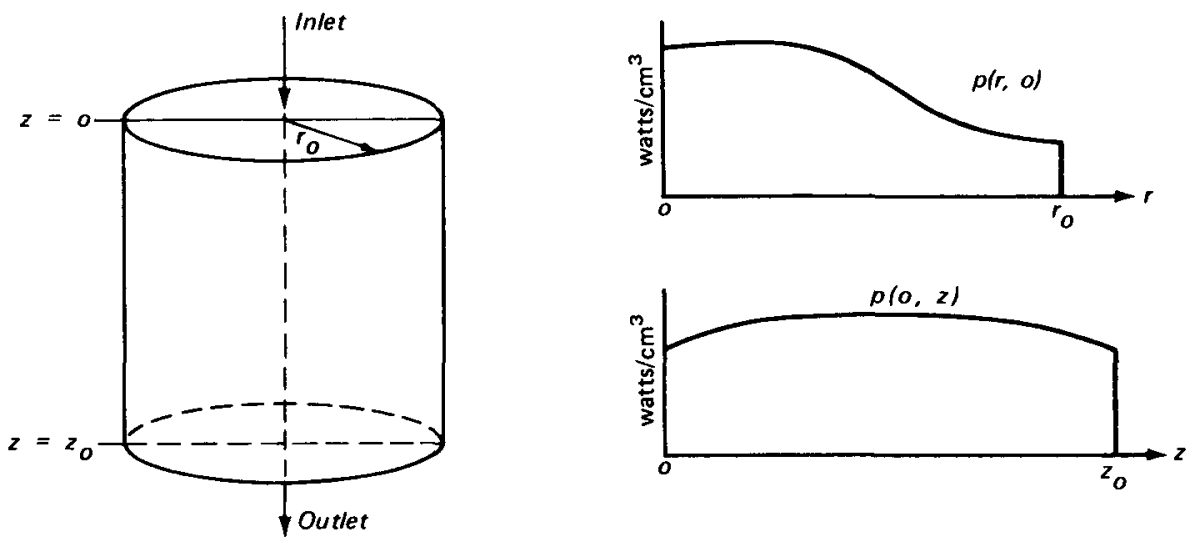

Fig. 2.9-Cylindrical volume source.

include cylinders, cubes, slabs, spheres, and other regular geometries in which total source strengths are expressed in particles or energy per unit volume per second.

As an example of a common volume source, consider a cylindrical reactor core whose power distribution is symmetrical about its axis and whose radial and axial power distributions are separable $\nmid$ (see Fig. 2.9). Let $p(r, 0)$ and $p(0, z)$ be the differential power distributions (watts $/ \mathrm{cm}^{3}$ ) along the radius at $z=0$ and along the reactor-core axis, respectively. If $K$ particles/watt-sec are emitted in the fission process, the differential particle source density, $S(r, z)$, at $(r, z)$ is given by

$$
S(r, z)=\frac{K}{p(0,0)} p(r, 0) p(0, z) \text { particles } \mathrm{cm}^{-3} \sec ^{-1}
$$

HIf a function $p(r, z)$ can be written $p_{1}(r) p_{2}(z)$, the variables are separable. 
and the total reactor source is

$$
S=\int_{r=0}^{r_{0}} \int_{z=0}^{z_{0}} S(r, z) 2 \pi \mathrm{r} \mathrm{dz} \mathrm{dr} \text { particles } / \mathrm{sec}
$$

In more complex systems, power-distribution functions describing volume sources may not be separable. In such cases, if analytic expressions are unavailable or unwieldy, a power map can be constructed, and integrations can be performed numerically.

\subsubsection{Directional Distributions}

The directions of travel of radiation particles are another important characteristic of radiation fields and sources. As suggested in Sec. 2.2.3 on solid angle, particle directions are usually defined relative to the origin of a conveniently chosen coordinate system. Once such a coordinate system is established, a direction is specified by a vector, $\Omega$, parallel to the direction of travel and having unit magnitude.

Frequently, a radiation field or source is symmetrical in direction about one or more lines or planes in space, and it is usually convenient to choose the coordinate system such that one or more axes lie in the plane or line of symmetry. In such cases directions can then be specified by a single angle. For example, Fig. 2.10 shows that two angles, $\theta$ and $\phi$, are required to specify direction without symmetry. The angle $\theta$ is called the polar angle and the angle $\phi$ the azimuthal angle in such a system. If a field is symmetrical about a line, aligning the $z$-axis with that line enables one to specify direction by use of the polar angle alone, and the field is said to have azimuthal symmetry.

Although the direction of $\Omega$ is always taken to be parallel with the direction of travel of the radiation particles, there is no universal convention that specifies whether $\Omega$ points toward or opposite to that direction of travel. Thus care must be taken to specify accurately which is the case when direction distributions are defined. For example, when the directional properties of a point source are given, the coordinate system is usually chosen to have its origin at the point, and $\Omega$ points in the direction of travel of the emitted particles. However, when directional properties of a field at a point detector are given, the origin is again situated at the point, and thus the direction of $\Omega$ is opposite to the direction of travel of the particles arriving at the detector point.

As with the other properties of fields and sources, directional properties are specified by differential distribution functions. Direction functions are 
sometimes separable from other distribution functions describing the source or field, in which case, for example, the differential flux density, $\Phi$, may be written

$$
\Phi(\mathbf{r}, \Omega, E, t)=F_{1}(\mathbf{r}, E, t) F_{2}(\Omega)
$$

and direction attributes can be examined independently of other parameters. In cases where the directional dependence is not separable and analytic manipulation of the total differential function is not possible, multidimensional maps may be constructed and numerical manipulations performed to obtain directional characteristics.

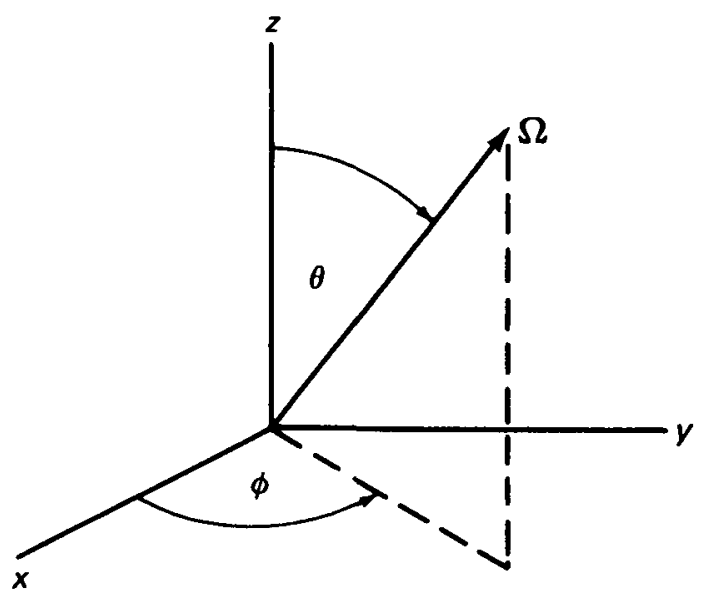

Fig. 2.10 -Direction vector $\Omega$.

The following sections discuss some of the more common direction distributions encountered in shielding problems. The exercises at the end of this chapter provide additional insights into the definition and manipulation of direction distribution functions.

(a) Isotropic Distributions. An isotropic direction distribution is one in which all directions of travel are equally likely. Since there are $4 \pi$ steradians of solid angle surrounding a point, the normalized isotropic differential distribution function is a constant function, $1 / 4 \pi$ per steradian, independent of angle or direction.

Many nuclear reactions that cause the emission of radiations are considered to be isotropic in nature. Thus the neutrons and gamma rays emitted from a fissioning nucleus, fission products, activated nuclei, and 
electron-positron annihilations may be assumed to be isotropic. However, certain reactions, most notably those which involve a scattering process, are not isotropic, and assuming so can lead to significant errors (see Chap. 3).

Point, surface, and volume-distributed sources can be isotropic. When analyzing isotropic surface sources, we must be careful to ascertain if the quoted source strength applies to one or to both sides of its surface. Suppose

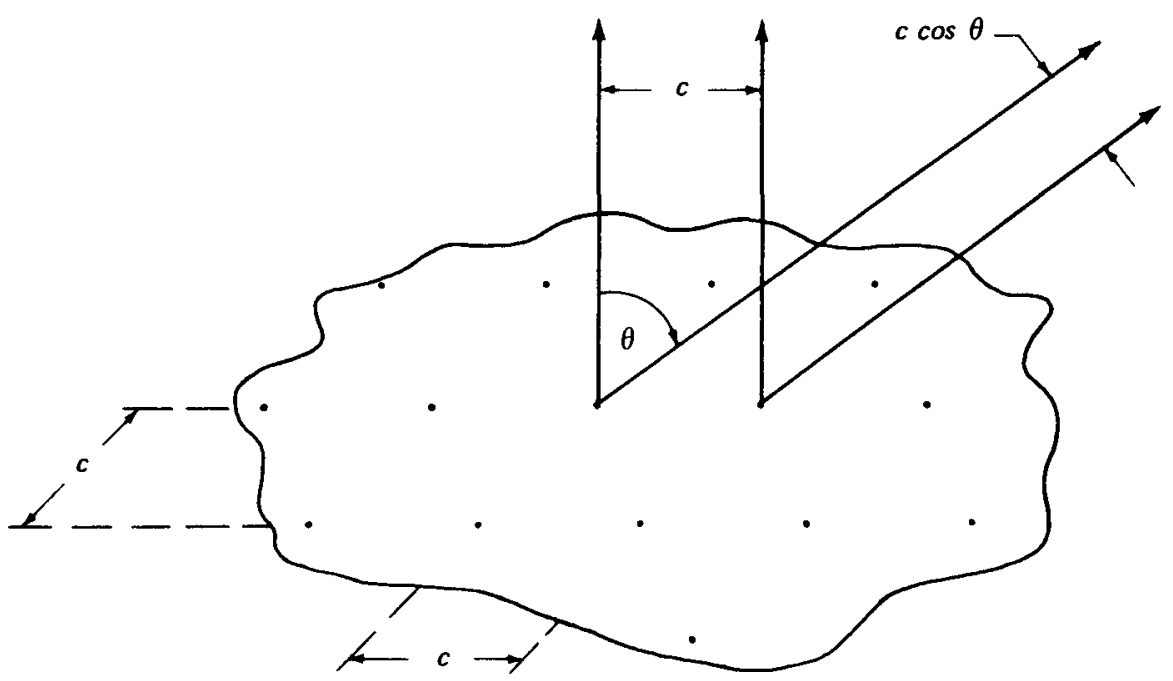

Fig. 2.11-Point isotropic sources in a plane.

we are given a plane source that isotropically emits $S_{a}$ particles $\sec ^{-1} \mathrm{~cm}^{-2}$. In some texts, $S_{a}$ applies only to the $2 \pi$ space above the plane; in others, $S_{a}$ applies to $4 \pi$ space. The two interpretations of $S_{a}$ differ by a factor of 2 . We will choose the latter alternative and assume, unless stated otherwise, that $4 \pi$ emission is intended.

Isotropic point sources distributed in a finite volume do not necessarily generate equivalent isotropic surface sources on the boundaries of the volume since particle interactions within the source medium may change the directional distribution of the emerging particles. Also, isotropic surface sources do not generate isotropic flux densities at detectors removed from the source plane, as shown by the following example.

Consider a plane isotropic surface source approximated by equally spaced isotropic point sources spaced $c$ centimeters apart, as shown in Fig. 2.11.

As the polar angle $\theta$ is increased, tracks of particles from the surface move closer together and consequently have a flux density that is higher by a 
factor of $1 / \cos \theta$ than the particles emitted normal to the surface. The angular distribution of the flux density above such an isotropic surface source is given by $\dagger$

$$
\Phi(\theta)=\frac{1}{4 \pi} \frac{S}{\mathrm{c}^{2} \cos \theta} \text { particles } \sec ^{-1} \text { steradian }^{-1} \mathrm{~cm}^{-2}
$$

where $S / c^{2}$ is the total surface source strength (in particles $\mathrm{sec}^{-1} \mathrm{~cm}^{-2}$ ).

(b) Cosine Distribution. Radiation emerging from the surface of a volume-distributed source often depends on the cosine of the angle between the normal to the surface and the direction of emergence. In many cases the dependence closely approximates or is exactly a cosine distribution of that

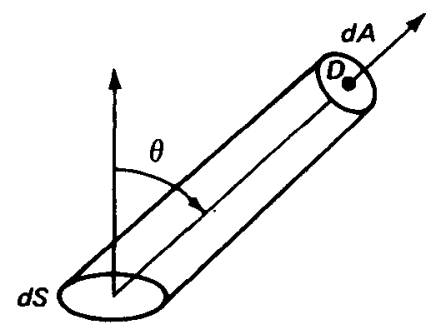

Fig. 2.12-Cosine distribution.

anglc. For such a source, if $S_{a}$ particles $\mathrm{sec}^{-1} \mathrm{~cm}^{-2}$ is the source strength, the differential source angle distribution function [demonstrated in Sec. 2.3.2(d)] is $(1 / 2 \pi) S_{a} \cos \theta$ particles $\sec ^{-1}$ steradian ${ }^{-1} \mathrm{~cm}^{-2}$ emitted along a direction inclined at an angle $\theta$ to the normal (Fig. 2.12). As before, the tracks in direction $\theta$ are closer together than those which are normal to the surface $(\theta=0)$, and we must divide by $\cos \theta$ to obtain the flux density. The flux density at $r$ is then

$$
\frac{S_{a}}{2 \pi r^{2}} \text { particles } \mathrm{cm}^{-2} \sec ^{-1} \operatorname{steradian}^{-1}
$$

(c) Other Distributions. Various other analytical expressions have been derived to fit observed or expected angular emittance patterns. Surface-angle functions of higher powers of $\cos \theta$ have been used to approximate the

†Proof is given in Problem 2.6. 
leakage characteristics of a volume-distributed self-absorbing source whose activity per unit volume increases with depth into the source. If the differential angle surface-source function of such a source is proportional to $\cos ^{n} \theta$, the differential flux density above the surface is proportional to $\cos ^{n-1} \theta$. However, in some cases the complexity of the leakage pattern may prohibit use of an analytical approximation, and numerical techniques must be employed.

In the treatment of radiation reflection from a medium, the intensity varies with the azimuthal angle $\phi$ between the incident and reflected directions as well as with the polar angle $\phi$. In such cases the angular distribution will be a function of the variables $\theta$ and $\phi$, as shown in Fig. 2.13.

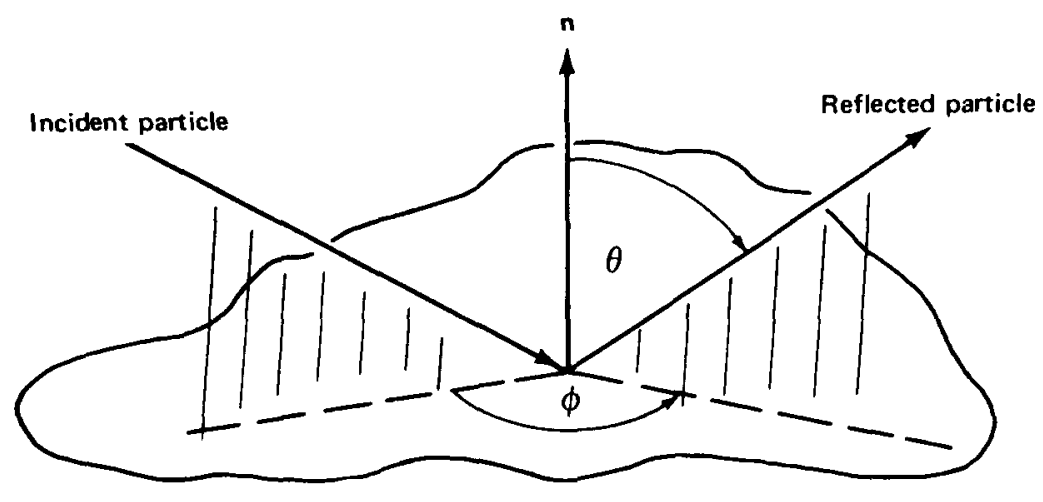

Fig. 2.13-Reflection angles.

The intensity in a given direction is still reported per steradian (or other designated increment), but both angles are required to designate the directional properties of the scattered radiation from a beam incident at a specified angle.

(d) Normalization and Monodirectional Approximation. When an analytic expression has been used to represent the variation of emittance rate with angle, the expression may be normalized to the total source strength, $S_{0}$, by the relation

$$
S_{0}=\int_{\Omega} K G(\Omega) d \Omega
$$

in which $G(\Omega)$ is the functional variation of the source with angle and $K$ is a normalization constant. Thus, for a cosine distribution from a plane surface source, $G(\Omega)=\cos \theta$, and 


$$
S_{0}=\int_{\phi=0}^{2 \pi} \int_{\theta=0}^{\pi / 2} K \cos \theta(\sin \theta d \theta d \phi)
$$

$S_{0}=2 \pi K$ particles per unit time per unit area, and $K=S_{0} / 2 \pi$. The source differential angular distribution function is then given by

$$
\begin{aligned}
S(\theta) & =K G(\Omega) \\
& \left.=S_{0} \frac{\cos \theta}{2 \pi} \text { particles (unit time) }\right)^{-1} \operatorname{steradian}^{-1} \text { (unit area) }
\end{aligned}
$$

Occasionally other powers of the cosine are useful in describing an angular variation. For $G(\Omega)=\cos ^{n} \theta$ (where $n$ is an integer), the normalization is

$$
S_{0}=2 \int_{0}^{2 \pi} \int_{0}^{\pi / 2} K \cos ^{n} \theta \sin \theta d \phi d \theta
$$

and

$$
\begin{aligned}
K & =\frac{(n+1) S_{0}}{4 \pi} \\
S(\theta) & =\frac{(n+1) S_{0}}{4 \pi} \cos ^{n} \theta
\end{aligned}
$$

In a like manner we can show for an isotropic distribution from a plane surface source emitting $S_{0}$ particles per unit time and area that $G(\Omega)=1$, $K=S_{0} / 4 \pi$, and

$$
\left.\left.S(\theta)=\frac{S_{0}}{4 \pi} \text { particles (unit time) }\right)^{-1} \operatorname{steradian}^{-1} \text { (unit area) }\right)^{-1}
$$

For a calculation of the transport of radiation with variable angular distribution, it is sometimes convenient to assume monodirectional sources with representative directions. The results are combined and normalized to the incident distribution. Such an approximation assumes that the differential distribution shown in Fig. 2.14(a) may be approximated by the monodirectional beams shown in Fig. 2.14(b). In normalizing these two sources, we must assign representative solid-angle increments $\left(\Delta \Omega_{i}\right)$ to each of the discrete sources and weight the monodirectional intensity by use of the relation

$$
N\left(\Omega_{i}\right)=\int_{\Delta \Omega_{i}} K G(\Omega) d \Omega
$$




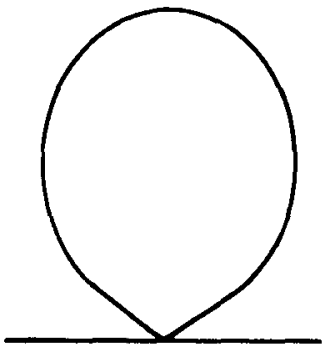

(a)

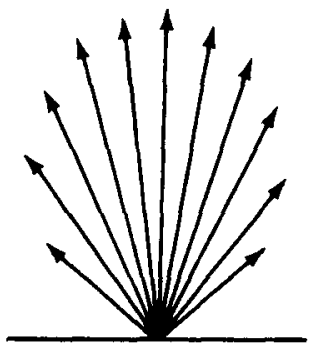

(b)

Fig. 2.14-Angular distribution represented by array of monodirectional sources.

where $N\left(\Omega_{i}\right)$ is the monodirectional source strength of the $i$ th source pointing in direction $\Omega_{i}$. The total source strength is given by

$$
S=\sum_{i=1}^{n} N\left(\Omega_{i}\right)=\int_{\text {all } \Omega} K G(\Omega) d \Omega
$$

where $n$ is the number of representative monodirectional sources used.

\subsection{ENERGY DISTRIBUTIONS}

Monoenergetic radiation fields are rarely encountered. A few monoenergetic gamma-ray sources are available in the form of separated isotopes with no daughter products, and monoenergetic neutrons are obtained from charged-particle reactions in accelerators. Even though only one radiation energy is emitted by these sources, interaction with environmental materials or within the source itself will lead to the presence of radiation of various degraded energies in the radiation field.

Some isotope sources emit two or three discrete energies; other sources, such as fission, provide a continuum of evolved radiation energies within certain energy ranges. In analytic treatments an energy-distributed radiation field is often approximated as a series of monoenergetic sources. All the radiation within an energy increment is assumed to have the same energy, which may be chosen as the midpoint of the increment, the average energy within the increment, or some weighted average based on a particular interaction probability. This is analogous to approximations used in the other distributions, such as the use of a fixed direction to represent an angular increment, a point source to approximate a source-volume increment, etc. Methods that are used to select the representative energy of 
an energy increment are discussed in Sec. 2.2.2. In particular, the average energy is often used, in which case the integral of Eq. 2.2-16 is carried out over the range of the energy increment.

For many purposes an average energy will not suffice, and the actual energy distribution must be carried through the analysis from source to detector. The term spectrum is used interchangeably with energy distribution, following the practice of the spectroscopist.

\subsubsection{Energy Distributions of Gamma-Ray Sources}

A few relevant characteristics of the spectra of gamma-ray sources encountered in reactors were included in the discussion of the various source types (Sec. 2.1.1). The two most important sources are the prompt fission reactions and fission products; their energy spectra are discussed in the following two sections. Capture and inelastic neutron-scattering gamma-ray spectral data are given in Appendix A.

(a) Prompt Fission Gamma-Ray Spectra. The spectrum of gamma rays given off simultaneously with the fission of ${ }^{235} \mathrm{U}$ has been rather extensively studied. The measurements of Peelle and Maienschein ${ }^{2}$ are the most accurate published data; they contain uncertainties of at most $15 \%$, and, in most energy regions, the uncertainty is less. Figure 2.15 presents the differential energy distribution (photons fission ${ }^{-1} \mathrm{MeV}^{-1}$ ) measured by Peelle and Maienschein. ${ }^{6}$ Kirkbride ${ }^{7}$ found that the spectra for ${ }^{233} \mathrm{U}$ and ${ }^{234} \mathrm{Pu}$ were not significantly different from that for ${ }^{235} \mathrm{U}$. Even ${ }^{252} \mathrm{Cf}$, which fissions spontaneously, exhibits a prompt gamma-ray spectrum very similar to that of ${ }^{235} \mathrm{U}$ (Ref. 8). The spectrum of Fig. 2.15 may be approximated by the segmented fit:

$$
\Gamma(E)= \begin{cases}6.6 & 0.1<E<0.6 \mathrm{MeV} \\ 20.2 \exp (-1.78 E) & 0.6<E<1.5 \mathrm{MeV} \\ 7.2 \exp (-1.09 E) & 1.5<E<10.5 \mathrm{MeV}\end{cases}
$$

The equation agrees with the experimental spectrum plus uncertainty to within $10 \%$ from 0.6 to $7.5 \mathrm{MeV}$ except at 1.2 and $\sim 5.0 \mathrm{MeV}$, where it deviates $\sim 16 \%$. The constant yield agrees with experiment from 0.1 to $0.6 \mathrm{MeV}$ to within $\sim 20 \%$ except at $0.26 \mathrm{MeV}$. An approximation for the energy region from 0.01 to $0.6 \mathrm{MeV}$, which may be adequate for shielding 


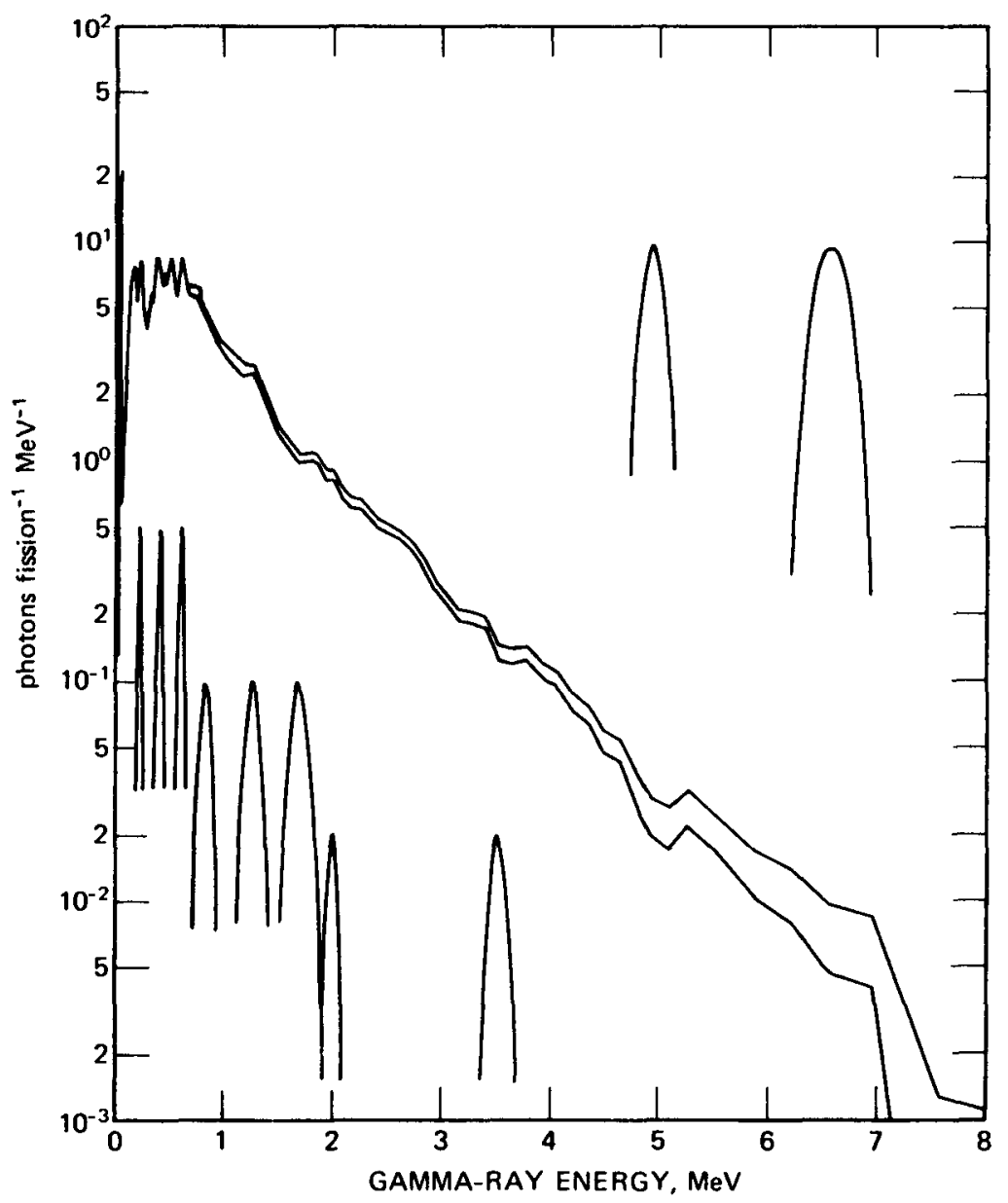

Fig. 2.15-The energy spectrum of gamma rays emitted within $69 \mathrm{nsec}$ after fission of ${ }^{235} \mathrm{U}$ by thermal neutrons. The two lines, which represent the random (largely propagated from counting statistics) $2 / 3$ confidence limits on the spectrum, are drawn as straight lines between adjacent mean window energies. The nearly Gaussian shapes shown at the lower left and upper right indicate the energy resolution (From Peelle and Maienschein. ${ }^{6}$ )

calculations, is the emission of 3.75 photons/fission of average energy $0.324 \mathrm{MeV}$.

Although the approximation of Eq. 2.4-1 is useful, for most applications accuracy demands the use of a numerical representation of the spectrum rather than these less-precise analytical functions. Photon yields per fission in fine and broad energy groups are given in Appendix A. 


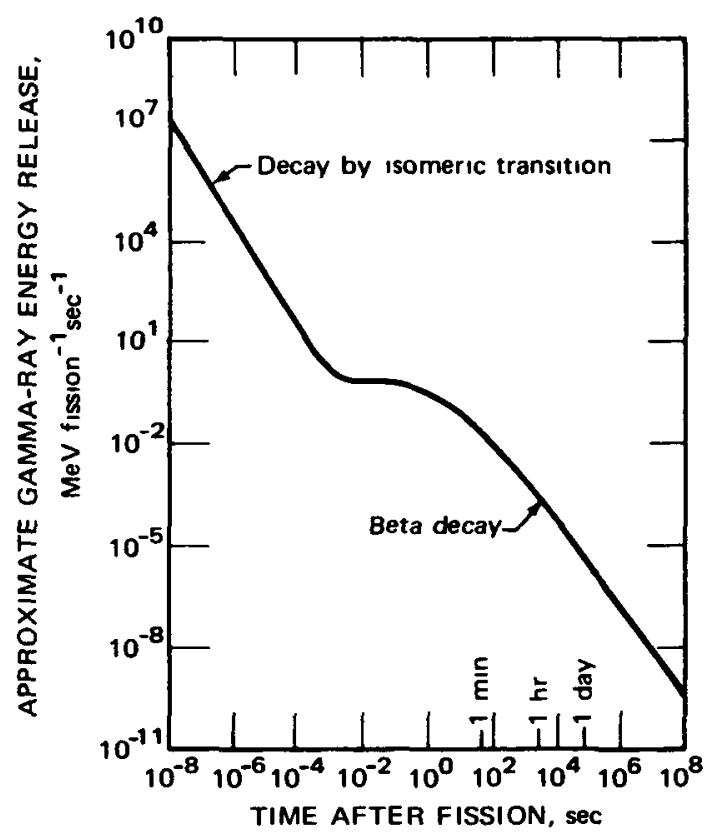

Fig 2 16-Gamma ray energy release from fission of ${ }^{235} \mathrm{U}$ as a function of time after fission The so called prompt fission gamma rays are emitted at times much shorter than those shown on the graph (From F C Marenschein, Engineering Compendium on Radiation Shielding, Vol 1, p 76, Sprınger Verlag, New York, 1968)

(b) Fission-Product Gamma Rays. Analysis of fission-product decay is often complicated by the time history of fission-product generation. Data on the decay of products of simultaneous fissions are of interest in the analysis of nuclear-weapon fallout or of a short reactor power burst. Of more general interest in reactor shielding is the decay of fission products built up during sustained operation or over complex operating cycles.

Two types of events contribute gamma rays to fission-product decay. Between $10^{-8}$ and $10^{-3}$ sec after fission of ${ }^{235} \mathrm{U}$, decay of isomers in excited states to the ground states contributes most of the decay gamma rays. After about $10^{-1} \mathrm{sec}$, beta decays of the unstable nucle1 contribute most of the decay gammas. Figure 2.16 illustrates the relationship of these processes.

In an operating reactor the primary importance of the gamma rays from early decays is their contribution to the steady-state environment. Decay gamma rays reach saturation very soon after start-up. Gamma rays from these short-lived isotopes constitute the majority of all fission-product gamma rays; about $75 \%$ are released within $10^{3} \mathrm{sec}$ after fission. If the 
fission-product gammas are integrated over time, the magnitude and shape of the energy distribution is close to that for prompt fission gammas. Maienschein ${ }^{9}$ gives

$$
N(E)=7.4 e^{-1.10 E} \text { photons fission }{ }^{-1} \mathrm{MeV}^{-1}
$$

as a simple, though approximate, expression for the fission-product spectrum. Equation 2.4-2 corresponds to an estimated total fission-product gamma-ray energy release of $6.8 \pm 1.0 \mathrm{MeV} /$ fission, which is composed of $5.9 \pm 0.7 \mathrm{MeV} /$ fission for $1 \mathrm{sec}<t<10^{8} \mathrm{sec}$ and $E>0.28 \mathrm{MeV}$; $0.6 \pm 0.6 \mathrm{MeV} /$ fission, $E<0.28 \mathrm{MeV}$; and $0.3 \pm 0.2 \mathrm{MeV} /$ fission for $t<1 \mathrm{sec}$. As noted in Sec. 2.1.1, this component is often combined with the prompt fission gamma rays.

Measurements of the gamma radiation evolved by decay of the fission products between 0.2 and $45 \mathrm{sec}$ after fission were made by Engle and Fisher. ${ }^{10}$ Listed in Table 2.1 are the values of the total radiation integrated over energy and over the time period from 0.2 to $45 \mathrm{sec}$ for four fissionable isotopes. Comparison of these values with the totals from the long-lived fission products shows that a significant fraction of the fission-product energy is evolved within the first $\mathbf{4 5} \mathrm{sec}$ following fission.

Table 2.1-RESULTS OF DELAYED GAMMA SPECTRA INTEGRATED OVER ENERGY AND TIME FROM 0.20 TO 45.0 SEC AFTER FISSION ${ }^{10}$

\begin{tabular}{lccc}
\hline Isotope & Photons/fission & MeV/fission & MeV/photon \\
\hline${ }^{233} \mathrm{U}$ & 2.02 & 1.97 & 0.975 \\
${ }^{235} \mathrm{U}$ & 3.31 & 3.18 & 0.961 \\
${ }^{238} \mathrm{U}$ & 5.50 & 5.08 & 0.924 \\
${ }^{239} \mathrm{Pu}$ & 3.26 & 2.86 & 0.877 \\
\hline
\end{tabular}

The energy spectra for five time intervals are shown in Fig. 2.17 for fission of ${ }^{235} \mathrm{U}$. Engle and Fisher ${ }^{10}$ give similar data for other fissionable isotopes, ${ }^{238} \mathrm{U},{ }^{233} \mathrm{U},{ }^{232} \mathrm{U}$, and ${ }^{239} \mathrm{Pu}$. It is worth noting that the spectra for these isotopes vary considerably from that of ${ }^{235} \mathrm{U}$ ( $1 / 2$ to 2 times) in this early time interval.

The long-lived (late) fission products are unimportant during operation of the reactor, but they represent a significant source of radiation after shutdown, particularly for a reactor core with many hours of operating history. From a knowledge of the direct yield and half-life of each fission 


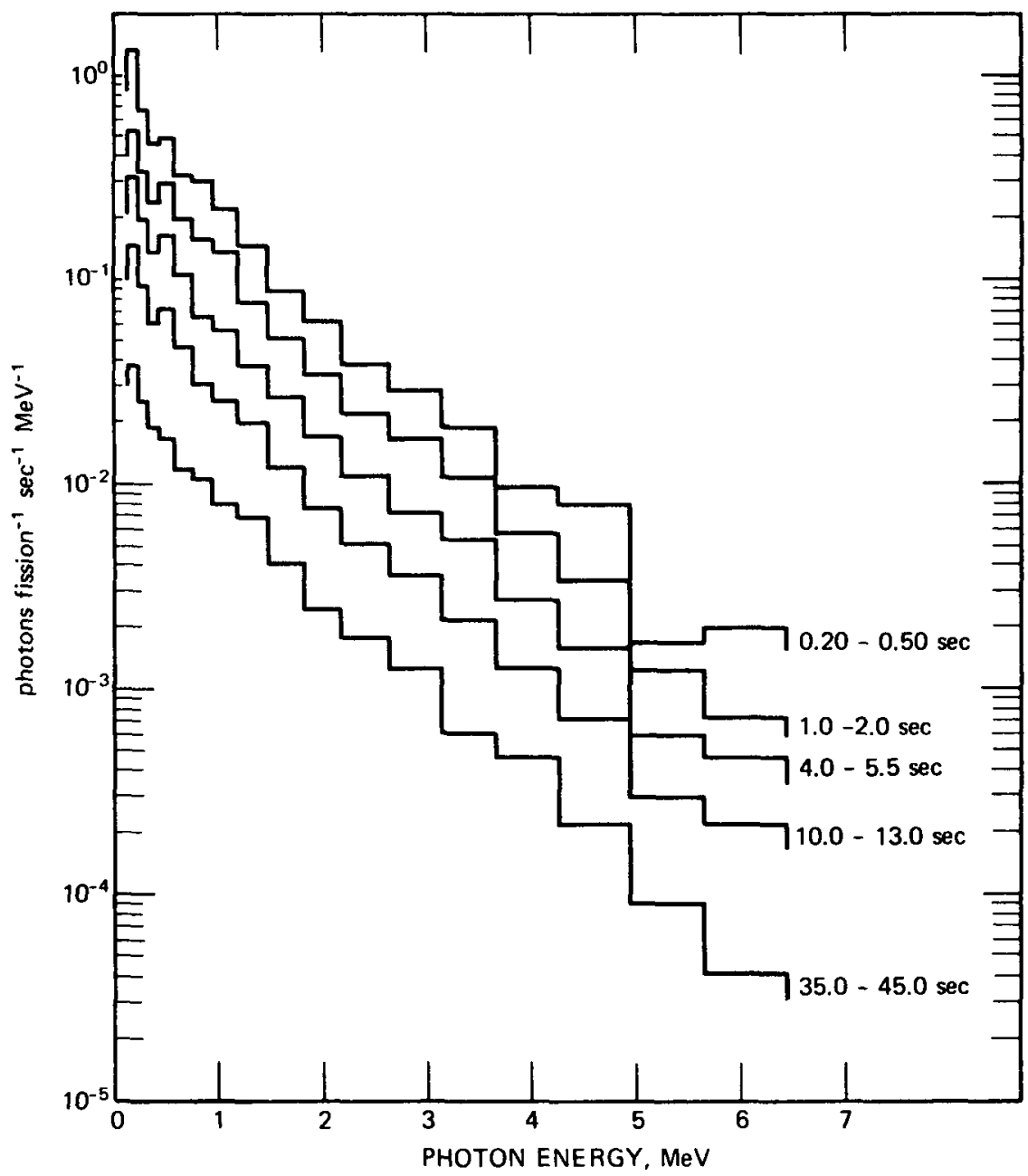

Fig. 2.17_-Photon spectra of gammas for different time intervals following ${ }^{235} \mathrm{U}$ fission. (From Engle and Fisher. ${ }^{10}$ )

fragment and the intensity and energy of each emitted gamma ray, along with similar data for each daughter isotope in each decay chain, it is possible to calculate the energy and time distributions of fission-product gamma rays as a.function of reactor operating time. In this manner the important gamma emitters in each time and energy interval can be identified, and more importantly a source term for the shutdown reactor core may be obtained for any operating history. Although the task is onerous and exacting, a number of investigators have tackled it with results that compare increas- 
ingly better with measurements as the number of identified decay chains increases. Perhaps the most authoritative work was published by Perkins and King ${ }^{11}$ and later updated by Perkins. ${ }^{12}$ The results of the later work are given in Appendix A. For instantaneous fission and 1, 10, 100, and $1000 \mathrm{hr}$ of reactor operation, these data give disintegration rate, beta-ray energy release, and total gamma-ray energy release and further subdivide the gamma rays into seven energy intervals. Unfortunately, the uppermost energy interval contains everything above $2.6 \mathrm{MeV}$. Results calculated by Scoles ${ }^{13}$ avoid this shortcoming since his energy intervals are $1 \mathrm{MeV}$ wide, except the topmost, which contains only one line at $5.4 \mathrm{MeV}$. These results were used considerably for some years since they were uniquely suited to shielding problems for times to $10^{6} \mathrm{hr}$ of reactor operation and to $10^{4} \mathrm{hr}$ after shutdown. Perhaps a reader of this text will update this work.

For approximate calculations the total fission-product decay rate is given within $20 \%$ from $10 \mathrm{~min}$ to 30 days after fission by Goldstein ${ }^{14}$

$$
\Gamma_{T}(t)=1.5 t^{-1.2} \mathrm{MeV} \text { fission }{ }^{-1} \sec ^{-1}
$$

where $t$ is the time (in seconds) after fission.

\subsubsection{Neutron Spectra from Fission}

The energy distribution of neutrons from fission is obviously one of the principal inputs in the preparation of the source term for a reactor. The energy range of importance in shielding for thermal fission in ${ }^{235} \mathrm{U}, 3$ to $17 \mathrm{MeV}$, was measured by Watt, ${ }^{15}$ who also reviewed measurements by others in lower ranges and proposed an empirical expression to fit the data from 0.075 to $17 \mathrm{MeV}$ :

$$
N(E)=0.484 e^{-E} \sinh (2 E)^{1 / 2} \text { neutrons } \mathrm{MeV}^{-1} \text { fission }{ }^{-1}
$$

where $N(E)$ is the fraction of neutrons per unit energy interval emitted per fission and $E$ is neutron energy in MeV. The Watt fission spectrum was widely used until Cranberg, Frye, Nereson, and Rosen ${ }^{16}$ reported new measurements from 0.18 to $12 \mathrm{MeV}$.

These results were based on time-of-flight measurements to about $8 \mathrm{MeV}$ and photographic emulsion exposures to $12 \mathrm{MeV}$. Cranberg et al. reported that 


$$
N(E)=0.453 e^{-E / 0.965} \sinh (2.29 E)^{1 / 2} \text { neutrons } \mathrm{MeV}^{-1} \text { fission }^{-1}
$$

was a more accurate fit over the entire range.

Note, however, that uncertainties in the measurements, $15 \%$ or less to $8 \mathrm{MeV}$, were $30 \%$ or more at $12 \mathrm{MeV}$ and above. Equation 2.4-5 is plotted in Fig. 2.18 and tabulated for numerical use in Ref. 14. The tabulation

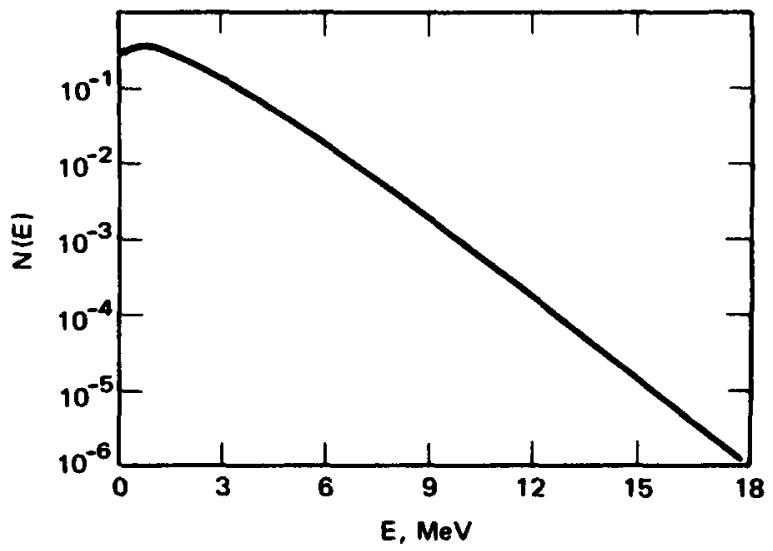

Fig. 2.18-Fraction of neutrons per $\mathrm{MeV}$ interval emitted at energy $E$ from the thermal fission of ${ }^{235} \mathrm{U}$. (From Herbert Goldstein, Reactor Handbook, Second Edition, Vol. III, Part B, Shielding, E. P. Blizard (Ed.), p. 19, Interscience Publishers, a division of John Wiley \& Sons, Inc., New York, 1962.)

also gives the fraction of neutrons above $E$ and the energy per fission carried by neutrons above $E$.

An even simpler expression that is within $15 \%$ of Eq. $2.4-5$ over its range of validity (and within $7 \%, 5<E<13 \mathrm{MeV}$ ) is due to Goldstein: ${ }^{14}$

$N(E)=1.75 e^{-0.766 E}$ neutrons $\mathrm{MeV}^{-1}$ fission $^{-1}$

$$
(4<E<14 \mathrm{MeV})
$$

This form is very convenient in analytical manipulations and adequately covers the energy range of greatest interest in many reactor shielding problems.

Both the spectrum and the total number of neutrons evolved in fission vary with the energy of the incident neutron and with the species of fissionable material. In experiments at Los Alamos, Grundl and Neuer ${ }^{17}$ and 
Leachman ${ }^{18}$ compared the spectra from the fission of ${ }^{235} \mathrm{U},{ }^{233} \mathrm{U}$, and ${ }^{239} \mathrm{Pu}$. Using the average energy, $\bar{E}$, as a parameter, they represented the spectra from all three isotopes by the formula

$$
N(E) \propto E^{1 / 2} e^{-x_{2}} \frac{E}{\bar{E}}
$$

From correlations of these and other data, Terrell ${ }^{18}$ found that $\bar{E}$ may be expressed in terms of $\bar{\nu}$, the average number of neutrons given off per fission, by

$$
\bar{E} \simeq 0.78+0.621(\bar{\nu}+1)^{1 / 2} \mathrm{MeV}
$$

Values of $\nu$ for fissions resulting from thermal-neutron absorption in various isotopes are listed in Table 2.2.

Table 2.2-NUMBER OF NEUTRONS PRODUCED BY FISSION

\begin{tabular}{cc}
\hline Fissionable isotope & $\bar{\nu}$ \\
\hline${ }^{233} \mathrm{U}$ & $2.54 \pm 0.04$ \\
$235 \mathrm{U}$ & $2.46 \pm 0.03$ \\
$239 \mathrm{Pu}$ & $2.88 \pm 0.04$ \\
\hline
\end{tabular}

The value for $\bar{\nu}$ increases almost linearly with energy as the incidentneutron energy rises above thermal. For ${ }^{235} \mathrm{U}$

$$
\bar{\nu}(E) \cong \bar{\nu}_{T}+0.15\left(E-E_{T}\right)
$$

where $\bar{\nu}_{T}$ is the value at thermal energy, $E_{T}$, and $E$ is the energy of the neutron-producing fission. From Eqs. 2.4-8 and 2.4-9, it is seen that the average emitted neutron energy, $E$, increases at a rate of about $4 \%$ of the increase in incident-neutron energy. For most practical shielding problems, this effect may be safely ignored. Variations between fissionable isotopes are more significant, however, and should be considered. The values of $\bar{\nu}$ given in Table 2.2 indicate that a breeder reactor whose power is evolved primarily from ${ }^{239} \mathrm{Pu}$ fissions will be a $20 \%$ stronger neutron source than an equivalent power reactor employing ${ }^{235} \mathrm{U}$. 


\subsubsection{Effect of Medium on Spectra}

It is evident that the shape of the neutron or gamma-ray energy distribution depends on the type of source. However, it is not so obvious that the attenuation properties of the medium through which the radiation is transmitted also have a strong influence on the spectrum. In fact, the spectrum of the neutrons or gamma rays emerging from a reactor shield is determined by the shield moderator and structure layers and not by the type of fission. The detailed reasons for this will become apparent from the discussion on interactions in Chap. 3 and on transport in Chap. 4. For the present it will suffice to illustrate the importance of the medium for two familiar media, water and lead.

Figure 2.19 shows the measured and calculated spectra from a fission-neutron source after the neutrons have penetrated various thicknesses

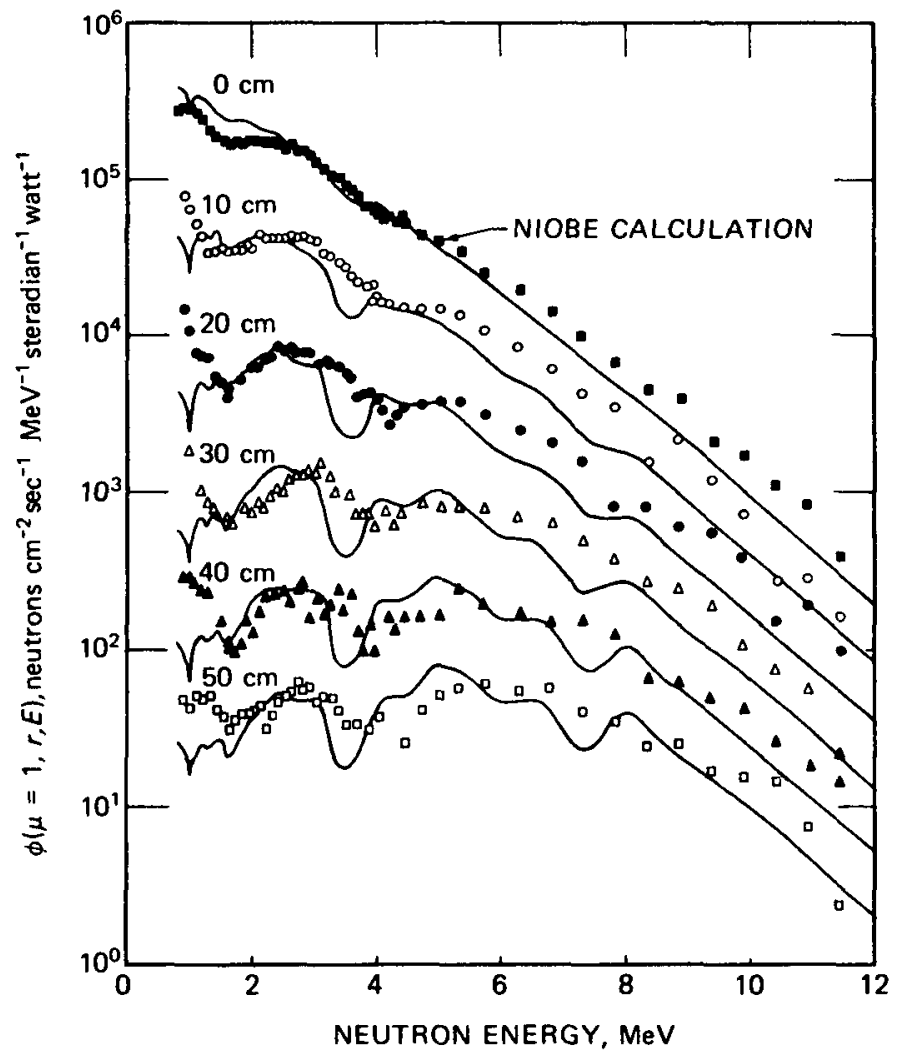

Fig. 2.19-Neutron spectrum vs. penetration through water measured in a direction normal to the slab with a collimated detector. [From V. V. Verbinski, M. S. Bokhari, J. C. Courtney, and G. E. Whitesides, Nuclear Science and Engineering, 27: 283 (1967).] 


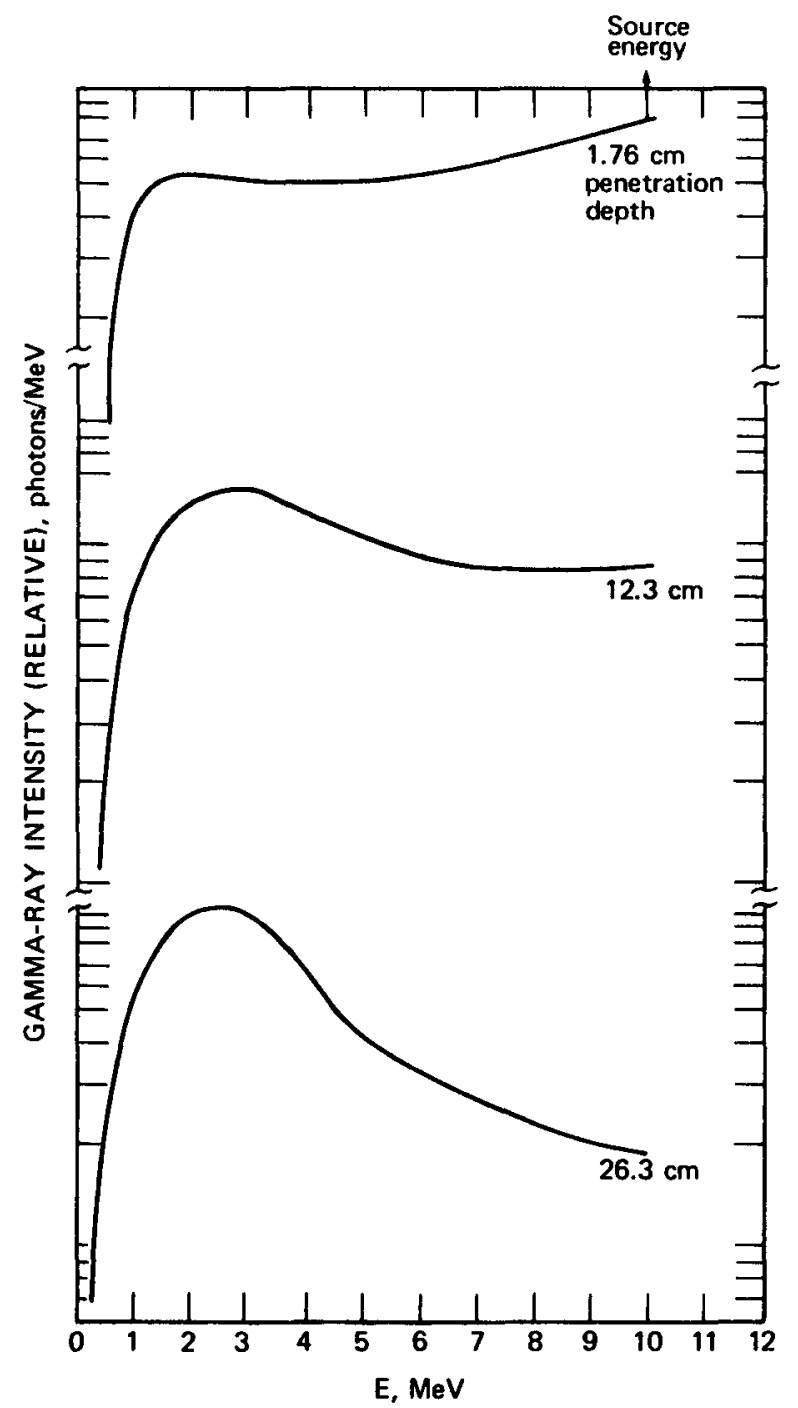

Fig. 2.20 - Differential energy spectrum for a $10-\mathrm{MeV}$ plane monodirectional gamma-ray source vs. penetration depth through a lead slab. (From Goldstein and Wilkins. ${ }^{20}$ )

of a water shield. As the thickness increases, the lower energy neutrons become less important, and the higher energy neutrons become more important; i.e., the higher energy neutrons comprise a higher percentage of the total neutron-flux density. 
Both the measurements and the calculations demonstrate the effect of the water medium. In this case the calculation is performed by direct integration of the Boltzmann equation, a technique discussed in Chap. 4.

The effect of attenuation on photons from a plane monoenergetic source through various thicknesses of lead is shown in Fig. 2.20. In this illustration the initial source spectrum is a vertical bar at $10 \mathrm{MeV}$. Despite this the distribution is almost horizontal at $1.76 \mathrm{~cm}$. Note that a pronounced peak develops between 2 and $3 \mathrm{MeV}$ as the depth increases.

\section{REFERENCES}

1. N. E. Holden, M. R. Mendelson, and T. E. Dudley, Total Gamma Energy Release Due to Thermal-Neutron Fission in ${ }^{235} \mathrm{U}, \mathrm{Nucl}$. Sci. Eng., 30: 461 (1967).

2. R. W. Peelle and F. C. Maienschein, Prompt Gamma Rays from Thermal Neutron Fission of Uranium-235, Nucl. Sci. Eng., 40:485 (1970).

3. P. N. Stevens and H. C. Claiborne, Basic Concepts of Radiation Shielding Analysis, in Weapons Radiation Shielding Handbook, Chap. 2, USAEC Report DASA-1892-5, Oak Ridge National Laboratory, June 1970.

4. International Commission on Radiation Units and Measurements, Radiation Quantities and Units, Report ICRU-11, 1968.

5. International Commission on Radiation Units and Measurements, Neutron Fluence, Neutron Spectra and Kerma, Report ICRU-13, 1969.

6. R. W. Peelle and F. C. Maienschein, The Absolute Spectrum of Photons Emitted in Coincidence with Thermal-Neutron Fission of Uranium-235, USAEC Report ORNL4457, Oak Ridge National Laboratory, 1970.

7. J. Kirkbride, The Prompt Gamma Photons from ${ }^{235} \mathrm{U},{ }^{233} \mathrm{U}$, and ${ }^{239} \mathrm{Pu}$ Due to the Absorption of a GLEEP Neutron Beam, British Report NRDC-58, Feb. 13, 1955.

8. A. B. Smith, P. R. Fields, and A. M. Friedman, Prompt Gamma Rays Accompanying the Spontaneous Fission of ${ }^{25}$ Cf, Phys. Rev., 104: 699 (1956).

9. F. C. Maienschein, Engineering Compendium on Radiation Shielding, Vol. 1, p. 76, Springer-Verlag, New York, 1968.

10. L. B. Engle and P. C. Fisher, Energy and Time Dependence of Delayed Gammas from Fission, USAEC Report LAMS-2642, Los Alamos Scientific Laboratory, July 1962.

11. J. F. Perkins and R. W. King, Energy Release from the Decay of Fission Products, Nucl. Sci. Eng., 3: 726 (1958).

12. J. F. Perkins, Decay of ${ }^{235}$ U Fission Products, Report RR-TR-63-11, U. S. Army Missile Command, Redstone Arsenal, July 1963.

13. J. F. Scoles, Calculated Gamma-Ray Spectra from ${ }^{235}$ U Fission Products, Report NARF-58-37T (FZK-9-132), Convair, Aug. 29, 1958.

14. Herbert Goldstein, Sources of Neutrons and Gamma Rays, in Reactor Handbook, 2nd ed., Vol. III, Part B, Shielding, E. P. Blizard (Ed.), Chap. 8, Interscience Publishers, a division of John Wiley \& Sons, Inc., New York, 1962. 
15. B. E. Watt, Energy Spectrum of Neutrons From Thermal Fission of ${ }^{235}$ U, Phys. Rev., 87: 1037 (1952).

16. L. Cranberg, G. Frye, N. Nereson, and L. Rosen, Fission Neutron Spectrum of ${ }^{235} \mathrm{U}$, Phys. Rev., 103: 662 (1956).

17. J. A. Grundl and J. R. Neuer, Comparison of ${ }^{235} \mathrm{U}$ and ${ }^{239} \mathrm{Pu}$ Fission Neutron Spectra, Bull. Amer. Phys. Soc., 1: 95 (Abstract N4) (1956).

18. R. B. Leachman, Neutrons and Radiations from Fission, in Proceedings of the Second United Nations International Conference on the Peaceful Uses of Atomic Energy, Geneva, 1958, Vol. 15, p. 331, United Nations, New York, 1958.

19. V. V. Verbinski, M. S. Bokhari, J. C. Courtney, and G. E. Whitesides, Measurements and Calculations of the Spectral and Spatial Details of the Fast-Neutron Flux in Water Shields, Nucl. Sci. Eng., 27: 283 (1967).

20. H. Goldstein and J. E. Wilkins, Calculations of the Penetration of Gamma Rays, USAEC Report NYO-3075, Nuclear Development Associates, Inc., June 30, 1954.

\section{EXERCISES}

2.1 The survivorship function for the time dependence of radioactive decay is given by

$$
N(t)=N_{0} e^{-\lambda t}
$$

where $N(t)$ is the number of radioactive atoms existing at time $t, N_{0}=N(0)$, and $\lambda$ is a constant for a given isotope. (a) Find the differential time distribution function $p(t)$, where $p(t) d t$ gives the number of atoms disintegrating in interval $d t$. (b) Using the definitions given in Sec. 2.2.1, obtain the cumulative distribution $F_{1}(t)$ (Eq. 2.2-4) and the fractional distributions $f_{1}(t)$ (Eq. 2.2-7). and $f_{2}(t)$ (Eq. 2.2-8). (c) Sketch these distributions. (d) Compute the number of atoms disintegrating between times $T_{1}$ and $T_{2}$.

2.2 (a) Compute the solid angle $\Omega$ subtended by a circular disk of radius $a$ at a point $P$ that is located a distance $R$ from the disk, where $P$ lies on the normal $\mathbf{n}$ passing through the center of the disk. (b) As $R \rightarrow 0$ or $a \rightarrow \infty, \Omega \rightarrow$ ?

2.3 An isotropic surface source of $S_{a}$ particles $\mathrm{sec}^{-1} \mathrm{~cm}^{-2}$ is located on the surface of a sphere of radius $R$. Assuming a nonabsorbing medium within the sphere: (a) compute the flux density at the center of the sphere; (b) compute the net current density at the center of the sphere through a midplane.

2.4 Compute (a) the flux density and (b) the current density for the geometry of Problem 2.3 when the surface source is located only on the hemisphere above the midplane.

2.5 In the geometry shown by the following sketch, the plane $P$ is parallel to the isotropic disk source $S_{1}$, which emits $S_{a}$ particles $\sec ^{-1} \mathrm{~cm}^{-2}$. Point source $S_{2}$ is isotropic and emits $S$ particles/sec. Compute (a) the flux density and (b) the net current density through plane $P$ at point $D$. 


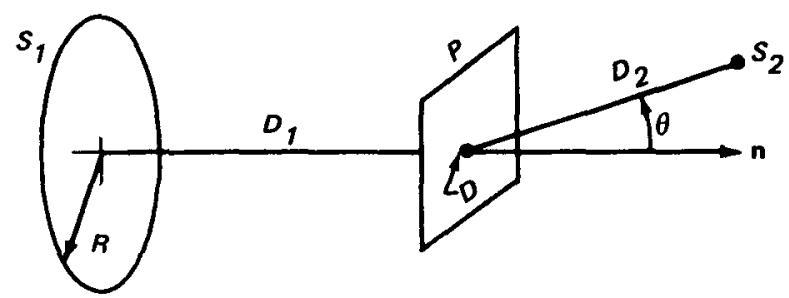

Exercise 2.5-Disk plus point source.

2.6 Derive Eq. 2.3-5 given in Sec. 2.3.2 for the flux density above a surface array of isotropic point sources; i.e., show

$$
\phi(\theta)=\frac{S}{4 \pi c^{2} \cos \theta} \text { particles } \mathrm{cm}^{-2} \sec ^{-1} \operatorname{steradian}^{-1}
$$

2.7 A slab of thickness $X$ that is infinite in the other two dimensions (see sketch for this exercise) is nonabsorbing and contains a uniformly distributed volume source of $S_{v}$ particles $\mathrm{cm}^{-3} \sec ^{-1}$. At a point $P$ on the surface of the slab, compute the following: (a) Compute the surface source differential distribution $G(\theta)$, where $G(\theta) d \Omega$ is the flux density emerging in solid-angle element $d \Omega$ about $\Omega$. ( $\Omega$ is inclined at an angle $\theta$ to the normal of the slab.) Compare the result with that of Problem 2.6 (b) Compute the differential flux-density distribution $\Phi(\theta)$, where $\Phi(\theta) d S$ is particles $\sec ^{-1}$ steradian ${ }^{-1}$ emitted into $d \Omega$ about $\Omega$ from the slab surface element $d S$. (c) Compute the total surface flux (particles $\sec ^{-1} \mathrm{~cm}^{-2}$ ) emerging from the slab. (d) What is an equivalent isotropic flux? (e) Compute the differential angle flux density $H(\theta)$, where $H(\theta) d \theta$ is the flux density of particles whose directions lie in angle element $d \theta$ about $\Omega$, integrated over all $2 \pi$ azimuthal angles. (f) Compute the relative total flux density in the following angle intervals of $\theta: 0^{\circ}$ to $10^{\circ}, 40^{\circ}$ to $50^{\circ}$, $75^{\circ}$ to $85^{\circ}, 80^{\circ}$ to $90^{\circ}$.

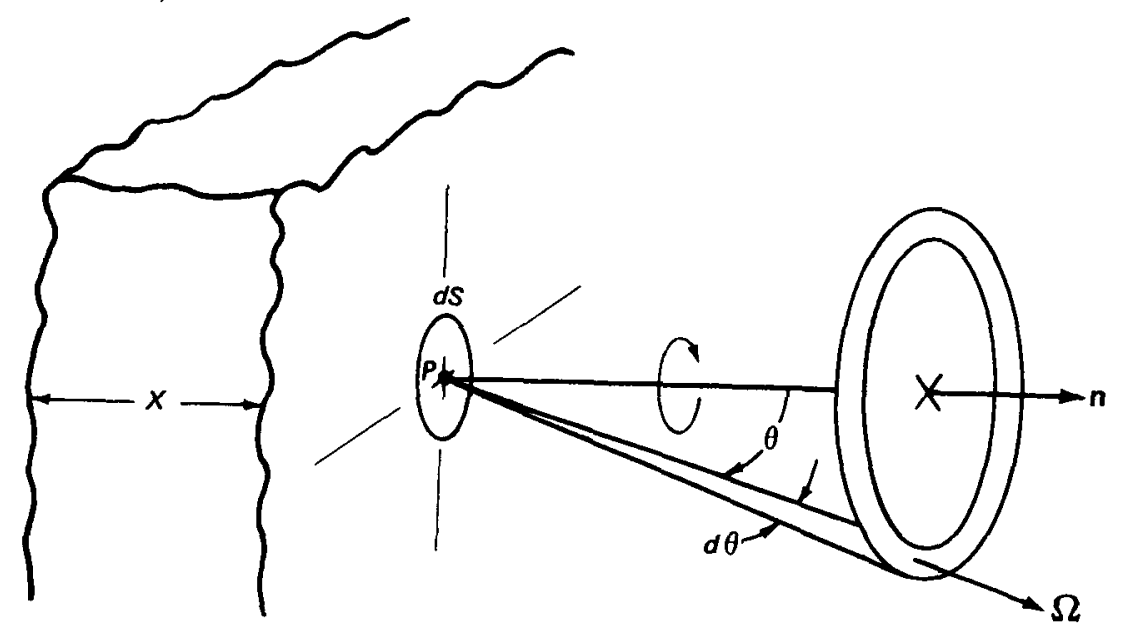

Exercise 2.7-Nonabsorbing slab source. 
2.8 A differential energy distribution for the fission spectrum is often chosen to be

$$
N(E)=0.77 E^{1 / 2} e^{-0.776 E}
$$

because it is simpler than Eq. 2.4-7 and accurate within $12 \%$ for $E<9 \mathrm{MeV}$. For this distribution, compute: (a) the most probable energy, $\hat{E}$; (b) the average energy, $\bar{E}$, and its associated speed, $v_{\bar{E}}$; and $(c)$ the average speed, $\bar{\nu}$, and its associated energy $E_{\bar{v}}$. Recall that for $E$ in $\mathrm{MeV}$ and $v$ in $\mathrm{cm}^{2} / \mathrm{sec}$,

$$
E=0.525 \times 10^{-18} v^{2}
$$




\section{Interactions of Radiation with Matter}

Once the source of radiation has been properly defined for a particular shield analysis, the second phase of the analysis can be performed. This phase involves the calculation of the intensity and distribution of the radiation that penetrates the shield or, alternatively, the attenuation afforded by the shield.

Although radiation attenuation is simply the macroscopic ramification of the microscopic interaction processes between the particle and the atoms of the medium, the transition from the individual processes to the gross behavior of the radiation field is a complex one. Not only must some consideration be given to the statistical nature of these processes but also the relative importance of the various interactions must be considered for the type of radiation and the appropriate range of energy. Thus an understanding of the energy-transfer mechanisms involved in the various interactions and of the relative probabilities among the several possible interactions is fundamental to shield analysis.

To begin a discussion of interactions, we will review the concept of cross section, the basic measure of a radiation interaction. Then we will review the atomic and nuclear processes important to photon and neutron transport and to attenuation calculations. We conclude the chapter with a discussion of some important interaction rates, namely, absorbed dose rate, kerma rate, and exposure rate, which are often used to characterize radiation fields. As in Chap. 2, for convenience we use the word particle to include photons as well as particles with mass.

\subsection{CROSS SECTIONS}

Consider a particle traversing a medium; numerous factors influence the probability of interaction with the nuclei and electrons of the medium. Some of the more important (but not necessarily independent) factors 
include (1) nuclides in the medium, (2) partial densities of each nuclide, (3) electron density within the medium, and (4) energy and direction distributions of the incident radiation.

Other factors must sometimes be considered as well. For low-energy particles, particularly thermal neutrons, the physical state of the medium may be important; for example, a nucleus bound in a crystal lattice may have a different interaction probability associated with it than an identical but unbound nucleus. Further, although we usually assume in shield analyses that target nuclei are at rest, thermal motion of the nuclei can sometimes be significant. For example, when the shield design involves the analysis of thermal-neutron transport through high-temperature regions of a reactor, the shift in value of the thermal-neutron energy must be considered.

\subsubsection{Microscopic Cross Section}

The basic measure of the probability that a neutron or photon will interact with a nucleus, an electron, or an atom is a quantity called the microscopic cross section. Microscupic cross sections are functions of all the variables listed above and traditionally are denoted by the symbol $\sigma$. Subscripts are used to denote specific interactions to which a cross section refers; e.g., $\sigma_{a}$ refers to an absorption cross section, $\sigma_{i n}$ to an inelasticscattering cross section, $\sigma_{s}$ to scattering, and $\sigma_{t}$ to a total cross section. Functional dependencies are often shown; e.g., $\sigma_{a}(E)$ represents the value of an absorption microscopic cross section as a function of the energy, $E$, of the incident radiation.

A microscopic cross section has units of area, hence the term cross section. It may be visualized as the effective projected cross-sectional area of a sphere centered about the target particle through which an incident radiation particle must pass if an interaction is to occur. In general, this effective area represents the range of the interaction force between incident particle and target nuclei and is not directly related to the size of the nucleus. The effective area for absorption $\left(\sigma_{a}\right)$ may be very different from the effective area for scattering $\left(\sigma_{s}\right)$ for the same target and incident radiation.

Calculation and measurement of microscopic cross sections have been active fields of endeavor for many years, and extensive files of evaluated data on computer tape serve as a primary source for input to radiation shielding analysis. For a given target, radiation type, and interaction process, microscopic cross sections are usually tabulated as functions of incident radiation energy. Sometimes, however, cross sections are reported as average 
values, the average being taken over a distribution in energy of the incident particle. For example, thermal-neutron cross sections are reported in this manner where the energy distribution of the neutrons is assumed to approximate a Maxwell-Boltzmann distribution.

The body of information on neutron cross sections has grown so rapidly in the past 10 years that it is no longer feasible to revise and update compilations; they have become so large as to be practicable only through the use of a computer. The National Neutron Cross Section Center at Brookhaven National Laboratory has concentrated more on developing cross-section data files on computer tape than on updating publications (for example, those cited in the section on neutron interaction later in this chapter). Evaluated nuclear data files, abbreviated ENDF/B, are available from National Neutron Cross Section Center, Brookhaven National Laboratory, Upton, New York 11973.

If very current data are not needed, the compilations of neutron cross sections cited in the following sections should be helpful. Sources of gamma-ray cross-section data are also cited.

Microscopic cross sections are usually measured in units of barns (b), millibarns (mb), and microbarns $(\mu \mathrm{b})$. A barn is $10^{-24} \mathrm{~cm}^{2}$; a millibarn, $10^{-27} \mathrm{~cm}^{2}$; and a microbarn, $10^{-30} \mathrm{~cm}^{2}$.

\subsubsection{Macroscopic Cross Section}

Although microscopic cross sections deal with probabilities of the interaction of radiation with individual targets (nuclei and electrons), macroscopic cross sections are related to probabilities for interaction with the aggregate of targets that compose the medium through which the radiation is passing. Almost invariably shield analysis requires macroscopic cross sections that, as shown in the following text, are computed from a knowledge of microscopic cross sections and the constituents of the medium. Two symbols universally used to denote macroscopic cross sections are $\mu$ and $\Sigma$; the former is most often applied to photon cross sections and the latter to neutron cross sections. As with microscopic cross sections, subscripts and functional dependencies are often used in conjunction with these symbols.

If $N$ is the target particle density (nuclei/ $\mathrm{cm}^{3}$ ), the relation between the macroscopic cross section, $\Sigma$, and the microscopic cross section, $\sigma$, for a specified interaction as a function of incident-radiation energy, $E$, is

$$
\Sigma(E)=N \sigma(E)
$$


Macroscopic cross sections as defined by Eq. 3.1-1 thus have units of reciprocal length (usually $\mathrm{cm}^{-1}$ ) and are often called linear cross sections or coefficients. (The term macroscopic cross section is used principally for neutrons; following historical precedent in attenuation of light, the term coefficient, with various modifiers to distinguish types, is used for gamma rays.) In fact, such a macroscopic cross section is the total apparent cross-sectional area of interaction presented to a radiation field per unit volume of material (i.e., $\mathrm{cm}^{2} / \mathrm{cm}^{3}$ ).

For mixtures of target nuclides, the macroscopic cross section for a specified interaction process is given by

$$
\Sigma(E)=\sum_{i} N_{i} \sigma_{i}(E)
$$

where $N_{i}$ is the volumetric density of the $i$ th nuclide and $\sigma_{i}(E)$ is the microscopic cross section for the $i$ th nuclide. It is easily shown that

$$
\Sigma(E)=\sum_{i} \frac{A_{v}}{A_{i}} \rho_{i} \sigma_{i}(E)
$$

where $A_{v}$ is Avogadro's number $\left(6.023 \times 10^{23}\right), A_{i}$ is the atomic weight of the $i$ th nuclide, and $\rho_{i}$ is the partial density $\left(\mathrm{g} / \mathrm{cm}^{3}\right)$ of the $i$ th nuclide in the mixture.

Macroscopic cross sections can be added. Thus, if $\Sigma_{s}(E)$ and $\Sigma_{a}(E)$ are scattering and absorption macroscopic cross sections, respectively, the cross section for both processes taken together is $\Sigma_{s}(E)+\Sigma_{a}(E)$.

If Eq. 3.1-1, 3.1-2, or 3.1-3 is divided by the density, $\rho$, of the medium, the apparent cross-sectional area of interaction per unit mass of material $\left(\mathrm{cm}^{2} / \mathrm{g}\right)$ is obtained. Such mass attenuation coefficients are most often encountered in photon shielding calculations and are usually denoted by $\mu / \rho$, $\mu$ being the linear macroscopic cross section.

\subsubsection{Radiation Reaction Rates}

It was noted in the discussion of measures of radiation intensity (Sec. 2.2.4, Chap. 2) that interaction rates of a radiation field with its environment are important and often-used characterizations of radiation intensity. However, further discussion of the concept was deferred to this chapter so that the various microscopic processes of interaction could first be reviewed. 
Many interaction processes can be used to measure a radiation field. With a photon field, for example, the total photon collision rate (number of photons interacting by all processes per unit volume per unit time) can be used. The rate of energy removal $\left(\mathrm{MeV} \mathrm{cm}^{-3} \mathrm{sec}^{-1}\right)$ from the primary photon beam can also be used. A third example might be the rate of local energy deposition ( $\mathrm{MeV} \mathrm{cm} \mathrm{cm}^{-3} \mathrm{sec}^{-1}$ ), which has the same dimensions but may not equal the energy removal in the local region being considered because of the energy carried away from and/or delivered to the local site by secondary processes. Rates of these types are regularly used in the calculation and evaluation of reactor shields; they are discussed later in this chapter. First, however, some fundamental considerations are in order.

The macroscopic cross section may be viewed as the reaction rate per unit number flux density of a radiation field. Thus, for a linear macroscopic cross section, the reaction-rate density is given by

$$
R R=\mu \Phi \text { reactions } \mathrm{cm}^{-3} \mathrm{sec}^{-1}
$$

if the cross section, $\mu$, is in units of $\mathrm{cm}^{-1}$ and the flux density, $\Phi$, is in units of $\mathrm{cm}^{-2} \mathrm{sec}^{-1}$. Of course, the reaction or reactions whose rates are calculated by Eq. 3.3-1 are those associated with the particular macroscopic cross section used.

In a similar manner, the reaction rate per unit mass density is given by

$$
R R=\frac{\mu}{\rho} \Phi \text { reactions } \mathrm{g}^{-1} \sec ^{-1}
$$

where $\Phi$ is again the flux density and $\mu / \rho$ is the mass macroscopic cross section. The total reaction rate over energy flux spectrum and volume, $\mathrm{V}$, is given by the integral

$$
R R=\int_{V} \int_{E} \mu(E) \Phi(\mathbf{r}, E) d E d V \text { reactions } / \mathrm{sec}
$$

in which the differential flux density, $\Phi(r, E)$, is defined by Eq. 2.2-23 of Sec. 2.2.4 and $d V$ is the differential volume element.

\subsection{RADIATION INTERACTIONS}

The physics of important neutron and photon interaction processes for radiations having energies found in fission reactors is well known, and 
thorough discussions can be found in nuclear science texts as well as in specialized treatments such as those of Foderaro, ${ }^{1}$ Blizard, ${ }^{2}$ and Goldstein. ${ }^{3}$ We will summarize the basic interactions here and make reference to sources of cross-section data for them.

\subsubsection{Photon Interactions}

The energy range of interest for gamma rays associated with fission or from fission-neutron interactions extends from the low $\mathrm{keV}$ range to about $10 \mathrm{MeV}$. There are many possible mechanisms for the interaction of gamma rays in this energy range, but only three interactions contribute in a significant way to photon attenuation. These are the photoelectric effect, pair production, and Compton scattering. Of the numerous possible references to photon-interaction data, we will mention only two: Hubbell, ${ }^{4}$ a recent tabulation with summary discussions of the processes of interest, and Hubbell and Berger, ${ }^{5}$ an article prepared a year earlier and appearing in the Engineering Compendium on Radiation Shielding.

(a) Photoelectric Effect. In the photoelectric process, a photon is absorbed and an orbital electron is emitted. The entire energy of the photon is transferred to the electron, which is ejected from its shell and emerges from the atom as a photoelectron. Momentum is conserved by recoil of the whole atom, and the more tightly bound electrons have the greatest probability of being ejected. The photoelectron is ejected from the atom with kinetic energy $T=E_{\gamma}-B_{e}$, where $B_{e}$ is the binding energy of the orbital electron. The orbital vacancy is then filled by transition of an outer electron; this transition is accompanied by emission of characteristic $\mathrm{X}$ rays called fluorescent radiation. Neither the photoelectron nor its fluorescent radiation is sufficiently energetic to be of further interest in shield analysis.

Extensive tabulations of photoelectric cross sections are given by Hubbell ${ }^{4}$ and Hubbell and Berger. ${ }^{5}$ Qualitatively the photoelectric cross section depends on atomic number, $Z$, and incident gamma-ray energy, $E_{\gamma}$,

$$
\sigma_{p e} \propto \frac{Z^{n}}{E_{\gamma}^{3}}
$$

where $n$ varies from 3 to 5 . We see that the probability of interaction is proportional to the -3 power of the photon energy and to the third to fifth power of the atomic number of the target atom. Thus the importance of the 
photoelectric effect is greater for the higher $Z$ elements and diminishes rapidly with increasing photon energy for any element.

Since the $K$-shell electrons are the most tightly bound, they have the highest probability of a photoelectric absorption. For incident-photon energy below the $K$-shell ionization energy, only $L$-shell or higher electrons are available (with a lower probability for interaction). Thus discontinuities in the cross sections for the photoelectric effect are found at the ionization energies of the various electron shells. Figure 3.1 illustrates this phenomenon for lead; both the $K$-edge and the $L$-edge are shown.

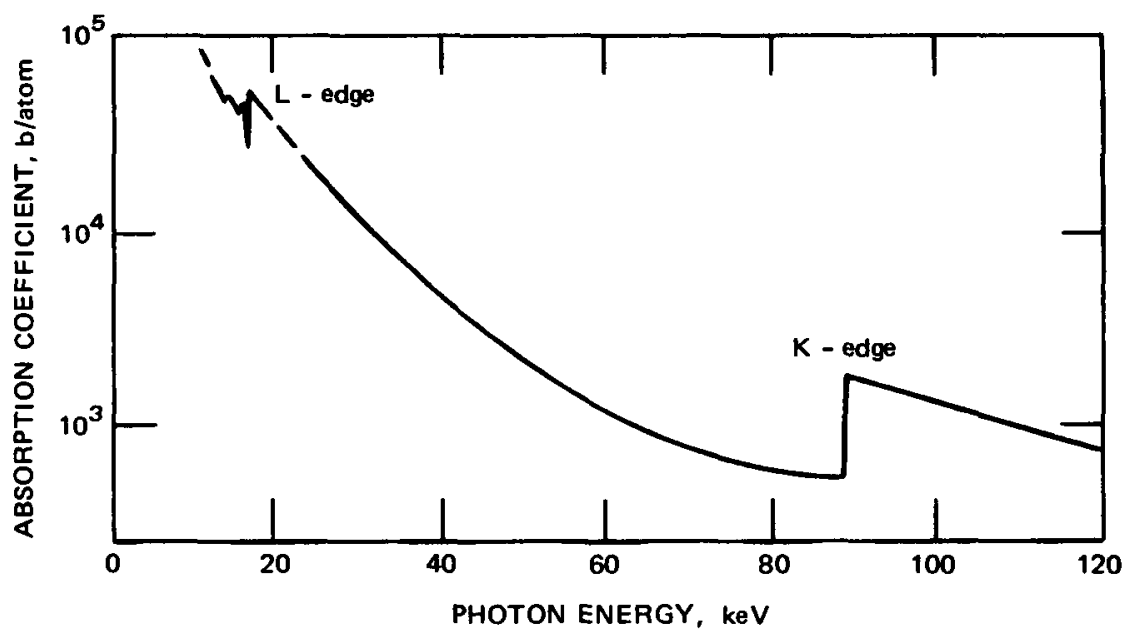

Fig. 3.1_-Photoelectric effect in lead.

The ionization energy for a given electron shell increases with increasing atomic number; the energy of the $K$-edge as a function of $Z$ is shown in Table 3.1. Also shown in Table 3.1 is the photon energy at which the photoelectric effect accounts for half the total photon interaction probability. At energies above the $E_{1 / 2}$ value, the photoelectric effect diminishes in importance. At energies below $E_{\not k}$, it becomes increasingly more important (see discussion on total attenuation coefficients at the end of this section).

As shown in Table 3.1, the energies at which the photoelectric effect predominates lie well below the region of 0.5 to $10 \mathrm{MeV}$, which is of greatest concern in reactor shielding. Although not predominant, the effect is still significant within that range. However, the greatest significance of the photoelectric effect is that it establishes a lower limit to the photon energy requiring consideration in a shield analysis. Because of the rapid increase in 
Table 3.1 - ENERGY AT WHICH THE PHOTO-

ELECTRIC EFFECT PROVIDES ONE-HALF

THE TOTAL ABSORPTION COEFFICIENT

$\left(E_{1 / 2}\right)$ AND ENERGY OF K-SHELL

IONIZATION $\left(E_{K}\right) \dagger$

\begin{tabular}{rlrl}
\hline$Z$ & Element & $E_{K}, \mathrm{MeV}$ & $E_{1 / 2}, \mathrm{MeV}$ \\
\hline 1 & Hydrogen & $1.4 \times 10^{-5}$ & $10^{-4}$ \\
4 & Beryllium & $2.2 \times 10^{-4}$ & 0.011 \\
6 & Carbon & $2.8 \times 10^{-4}$ & 0.016 \\
8 & Oxygen & $5.2 \times 10^{-4}$ & 0.025 \\
13 & Aluminum & $1.5 \times 10^{-3}$ & 0.046 \\
20 & Calcium & $4.0 \times 10^{-3}$ & 0.079 \\
26 & Iron & $6.9 \times 10^{-3}$ & 0.11 \\
42 & Molybdenum & $2.0 \times 10^{-2}$ & 0.195 \\
50 & Tin & $2.9 \times 10^{-2}$ & 0.25 \\
74 & Tungsten & $6.06 \times 10^{-2}$ & 0.42 \\
82 & Lead & $8.8 \times 10^{-2}$ & 0.50 \\
92 & Uranium & $11.6 \times 10^{-2}$ & 0.62 \\
\hline
\end{tabular}

†From Goldstein. $^{3}$

the photoelectric absorption cross section with decreasing photon energy, a point in energy is reached below which, for all practical purposes, no photons exist.

(b) Compton Effect. The Compton effect is the scattering of a photon by a free electron. The photon imparts energy to the electron and is altered in direction and energy. A very important feature of this effect is the fact that, except when the scattering angle is large, the photon emerges from the interaction with a significant fraction of the incident-photon energy. This fact accounts for much of the complexity associated with gamma-ray transport analysis.

In the analysis of the Compton effect, the electrons are assumed to be free, neither interacting among themselves nor bound within the atom. The Compton effect for an atom is therefore the additive effect of all its electrons, and the macroscopic cross section for Compton scattering is determined by the electron density. Thus the dependence of this process on atomic number is merely a linear dependence on the number of electrons per atom. Figure 3.2 illustrates the process. The photon is scattered through an angle $\theta$ and carries a portion of its incident energy. The remainder of the energy is taken up as kinetic energy by the recoiling electron, which scatters through an angle $\psi$. 


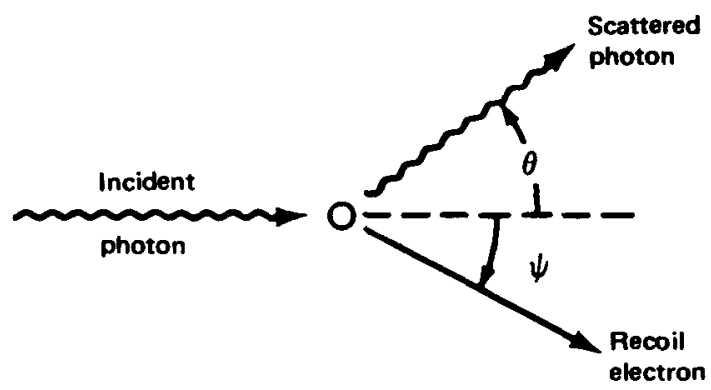

Fig. 3.2-Compton scattering.

The equations describing the angle and energy relations of the Compton process are most easily expressed when photon energies are measured either in units of the rest mass energy of an electron $\left(m_{e} c^{2}\right)$ or in units of Compton wavelengths. In these units, if $E^{*}$ is energy in units of $\mathrm{MeV}$, the energy $E$ in units of $m_{e} c^{2}$ is given by

$$
E=\frac{E^{*}}{0.511}
$$

and the Compton wavelength is given by the reciprocal:

$$
\lambda=\frac{1}{E}=\frac{0.511}{E^{*}}
$$

One unit of Compton wavelength is equal to $h / m_{e} c$, or $0.02426 \AA$, where $h$ is Planck's constant $\left(6.625 \times 10^{-27}\right.$ erg-sec). Subsequent equations in this section on Compton scattering are written in terms of these units of energy and wavelength.

From the conservation of energy and momentum, it may be shown that the relation between the incident-photon energy, $E$, scattered-photon energy, $E^{\prime}$, and scattering angle, $\theta$, for a Compton collision is

$$
E^{\prime}=\frac{E}{1+E(1-\cos \theta)}
$$

In terms of Compton wavelengths, the relation seems even simpler

$$
\lambda^{\prime}-\lambda=1-\cos \theta
$$

where $\lambda^{\prime}$ refers to the scattered photon. 
From these equations it is seen that straight-ahead scattering $(\theta=0)$ results in no energy loss for the photon; the electron recoil angle, $\psi$, is $90^{\circ}$ in this case. A lower limit to the scattered photon energy occurs in backward scattering $\left(\theta-180^{\circ}\right)$, where

$$
E_{\min }^{\prime}=\frac{E}{1+2 E}
$$

and

$$
\lambda_{\text {max }}^{\prime}=\lambda+2
$$

The electron recoil angle, $\psi$, is zero in this case. Thus the photon can scatter in any direction, but electron recoil is limited to forward (relative to the initial photon's track) directions.

The energy of the scattered photon varies from the initial energy down to the lower limit. For initial energies much greater than 1 rest mass $(\gg 0.511 \mathrm{MeV})$, the lower limit is approximately $E / 2$.

The essence of the process is contained in the Klein-Nishina formula, which describes, to a very good approximation, the probability per electron that a photon will Compton scatter into a unit solid angle about a scattering angle, $\theta$. This differential function takes on a convenient form when the microscopic electron cross section is measured in Thomson units (T.U.). One T.U. $=8 \pi / 3\left(e^{2} / m_{e} c^{2}\right)^{2}=0.665 \mathrm{~b}$. In these units the Klein-Nishina relation, expressed in terms of energy or Compton wavelength, is

$$
\sigma(\theta)=\frac{3}{16 \pi}\left(\frac{E^{\prime}}{E}\right)^{2}\left(\frac{E}{E^{\prime}}+\frac{E^{\prime}}{E}-\sin ^{2} \theta\right) \text { T.U. electron }{ }^{-1} \operatorname{steradian}^{-1}
$$

or

$$
\sigma(\theta)=\frac{3}{16 \pi}\left(\frac{\lambda}{\lambda^{\prime}}\right)^{2}\left(\frac{\lambda^{\prime}}{\lambda}+\frac{\lambda}{\lambda^{\prime}}-\sin ^{2} \theta\right) \text { T. U. electron }{ }^{-1} \operatorname{steradian}^{-1}
$$

[Since $\sigma(\theta)$ is a differential microscopic cross section (unit area per unit solid angle), some authors prefer to denote these functions by $\mathrm{d} \sigma / \mathrm{d} \Omega$ to emphasize the differential.] It should be noted that the variables $E^{\prime}, E$, and $\sin \theta$ (or $\lambda^{\prime}, \lambda$, and $\sin \theta$ ) are not independent in the preceding equations but are related through Eq. 3.2-4 (or 3.2-5). Thus $\sigma(\theta)$ is actually a function 
only of initial and scattered photon energy or initial photon energy and scattering angle. This is explored in the exercises.

The total cross section for Compton scattering by an electron may be obtained by integration of Eq. 3.2-8 over all scattering angles.

$$
\begin{aligned}
\sigma_{c}(E)=\frac{3}{4}\left\{\frac{1+E}{E^{3}}\right. & {\left[\frac{2 E(1+E)}{1+2 E}-\ln (1+2 E)\right] } \\
& \left.+\frac{1}{2 E} \ln (1+2 E)-\frac{1+3 E}{(1+2 E)^{2}}\right\} \text { T.U./electron }
\end{aligned}
$$

where $\sigma_{c}(E)$ is the microscopic cross section for Compton scattering per electron for a photon of energy $E$. For very low energies $(E \ll 1), \sigma_{c}(E)$ approaches 1 T.U. In the other extreme $(E \gg 1)$,

$$
\sigma_{c}(E) \approx \frac{3}{8 E}\left(\ln 2 E+\frac{1}{2}\right) \text { T.U./electron }
$$

As noted in the preceding text, the energy of the incident photon is divided into two parts in a Compton collision: that contained in the kinetic energy of the recoiling electron and thus deposited very close to the site of the collision, and that carried away from the collision site by the scattered photon. The fraction, $f(\theta)$, of energy deposited locally (i.e., transferred to the electron) as a function of scattering angle is given by

$$
f(\theta)=\frac{E-E^{\prime}(\theta)}{E}
$$

The average fractional energy loss per Compton collision, $\bar{f}_{c}$, is given by

$$
\bar{f}_{c}=\frac{1}{\sigma_{c}} \int f(\theta) \sigma(\theta) d \Omega
$$

where the integration is carried out over all solid angle. The quantity

$$
\sigma_{c a}(E)=\bar{f}_{c} \sigma_{c}(E)
$$

is a cross section reflecting the probability of local energy deposition in a Compton collision and is called the Compton energy absorption cross section. 
The relations between initial- and scattered-photon energies, scattering angle, Compton cross section, and energy transferred to the electron are sufficiently complex that they are not intuitively obvious. Figure 3.3 illustrates the relation between initial and scattered photon energies as a function of scattering angle. For initial photon energies of $0.1 \mathrm{MeV}$ or less, there is little energy degradation. For larger initial energies, degradation is large for large scattering angles. Figure 3.4 shows the dependence of electron recoil energy on initial photon energy and scattering angle. Figure 3.5 shows the differential Compton cross section, $\sigma(\theta)$, for several representative initial photon energies. This graph shows that in the $1-$ to $10-\mathrm{MeV}$ range the cross section is peaked in the forward direction and demonstrates that Compton scattering is highly forward. Figure 3.4 is taken from Nelms, ${ }^{6}$ who presents over 80 graphs showing the interrelations of these parameters with scales sufficiently expanded to allow reasonable accuracy. Finally, Table 3.2 lists the total Compton cross section per electron, $\sigma_{c}$, as a function of initial photon energy. Also given in this table are values of $\sigma_{c a}$ and $\sigma_{c}-\sigma_{c a}$; the former cross section relates to the energy deposited by the incident photon, and the latter is a cross section related to the energy carried off by the scattered photon.

Some gamma-ray analysis methods require that the differential crosssection data be put into some format other than that just given. For example, in many Monte Carlo calculations the integral probability distribution for scattering at an angle $\alpha \leqslant \theta$ is expressed as a function of $\theta$. Such a function has the limits of 0 probability at $\theta=0^{\circ}$ and 1 at $\theta=180^{\circ}$. All the more sophisticated methods require differential angle cross-section data in some form.

(c) Pair Production. In the process of pair production, a photon interacts with the electric field of atomic electrons or the nucleus. The incident photon is completely annihilated, and its energy is converted into the mass of an electron-positron pair. Clearly, the incident photon must have at least enough energy to create the mass of the electron-positron pair. Thus a threshold for the pair-production process is $2 m_{e} c^{2}$, or $1.022 \mathrm{MeV}$. Any excess energy of the incident photon is given to the kinetic energies of the two charged particles produced in the process and to the recoiling nucleus. As in the photoelectric effect, the nucleus plays an essential part in pair production since its recoil is necessary to conserve momentum in the process.

For a given nuclide the nuclear pair-production cross section increases rapidly from the threshold of $1.022 \mathrm{MeV}$ to $10 \mathrm{MeV}$. This is illustrated in 


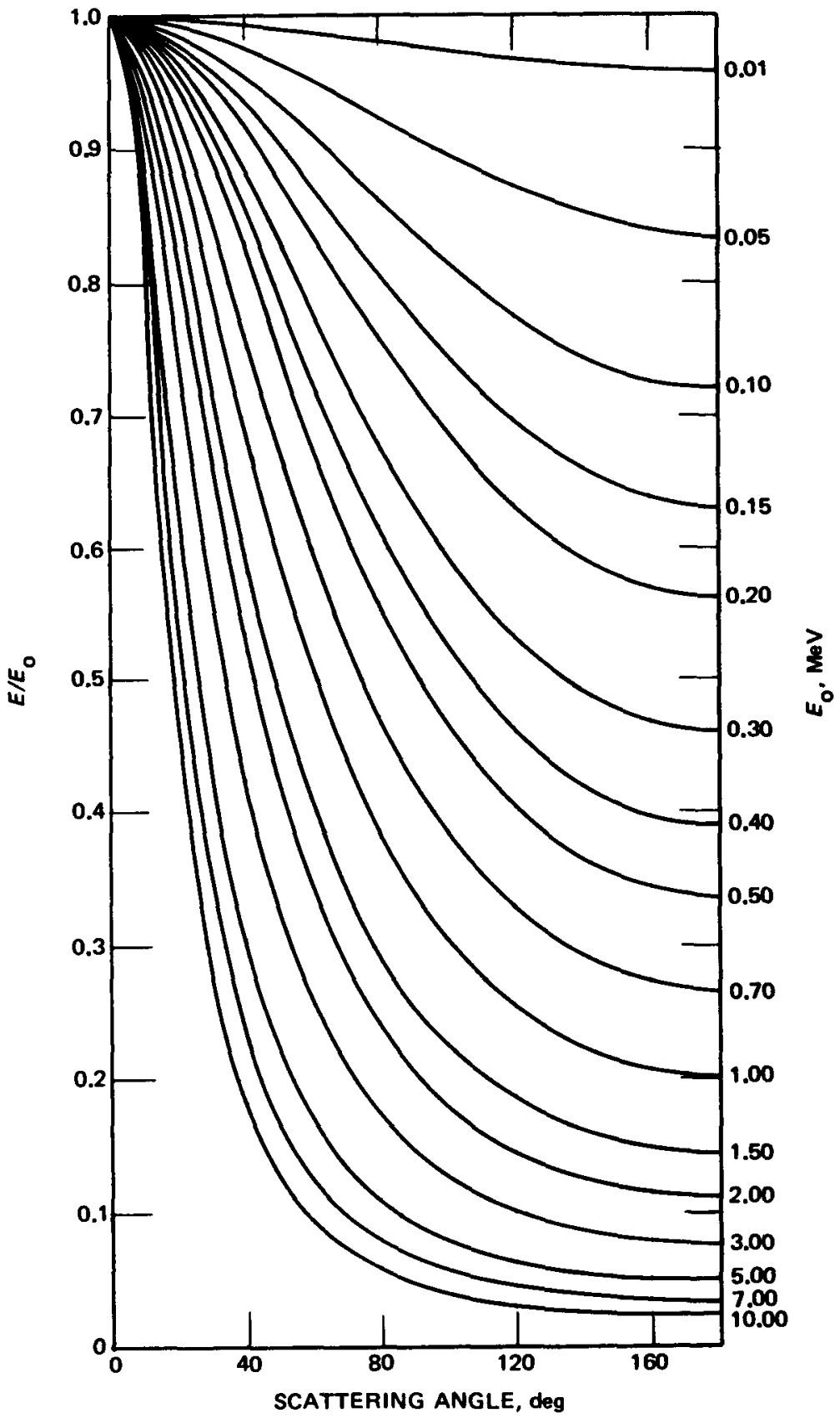

Fig. 3.3-Reduction in gamma-ray energy by Compton scattering $\left(E_{0}=\right.$ initial energy; $E=$ reduced energy). 


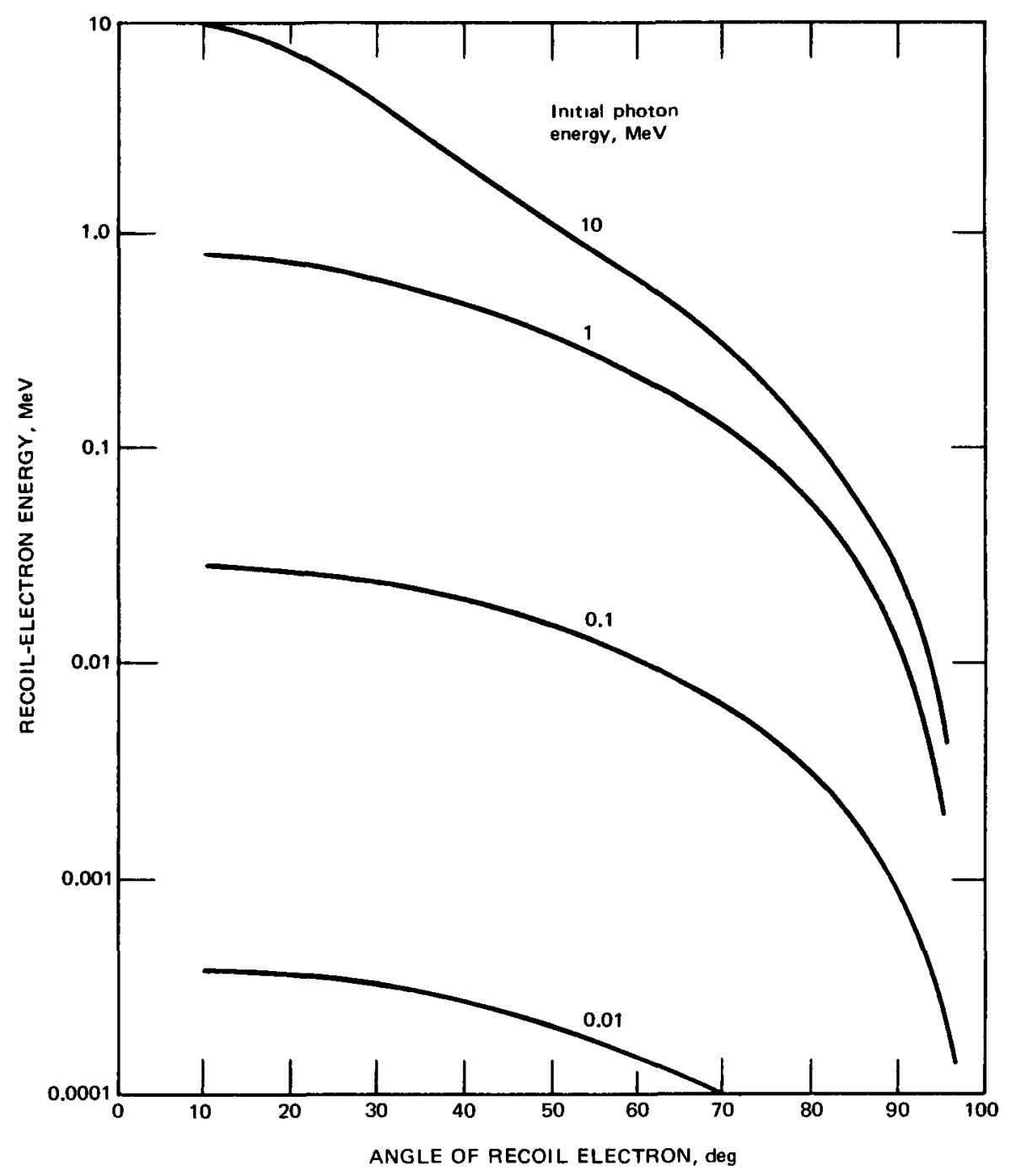

Fig. 3.4-Recoil-electron energy vs. angle of recoil. (From Nelms. ${ }^{6}$ )

Fig. 3.6, which shows the dependence of the cross section on incidentphoton energy in lead. As a function of atomic number, pair production for interactions with atomic electrons is proportional to $Z$, and that for nuclear pair production, to $Z^{2}$. This latter effect increases the dominance of nuclear pair production in reactor shield materials. In fact, below $10 \mathrm{MeV}$ the probability of pair production with atomic electrons ranges from 10 to $30 \%$ of that for nuclear pair production in hydrogen and is negligible in high- $Z$ materials such as lead. 


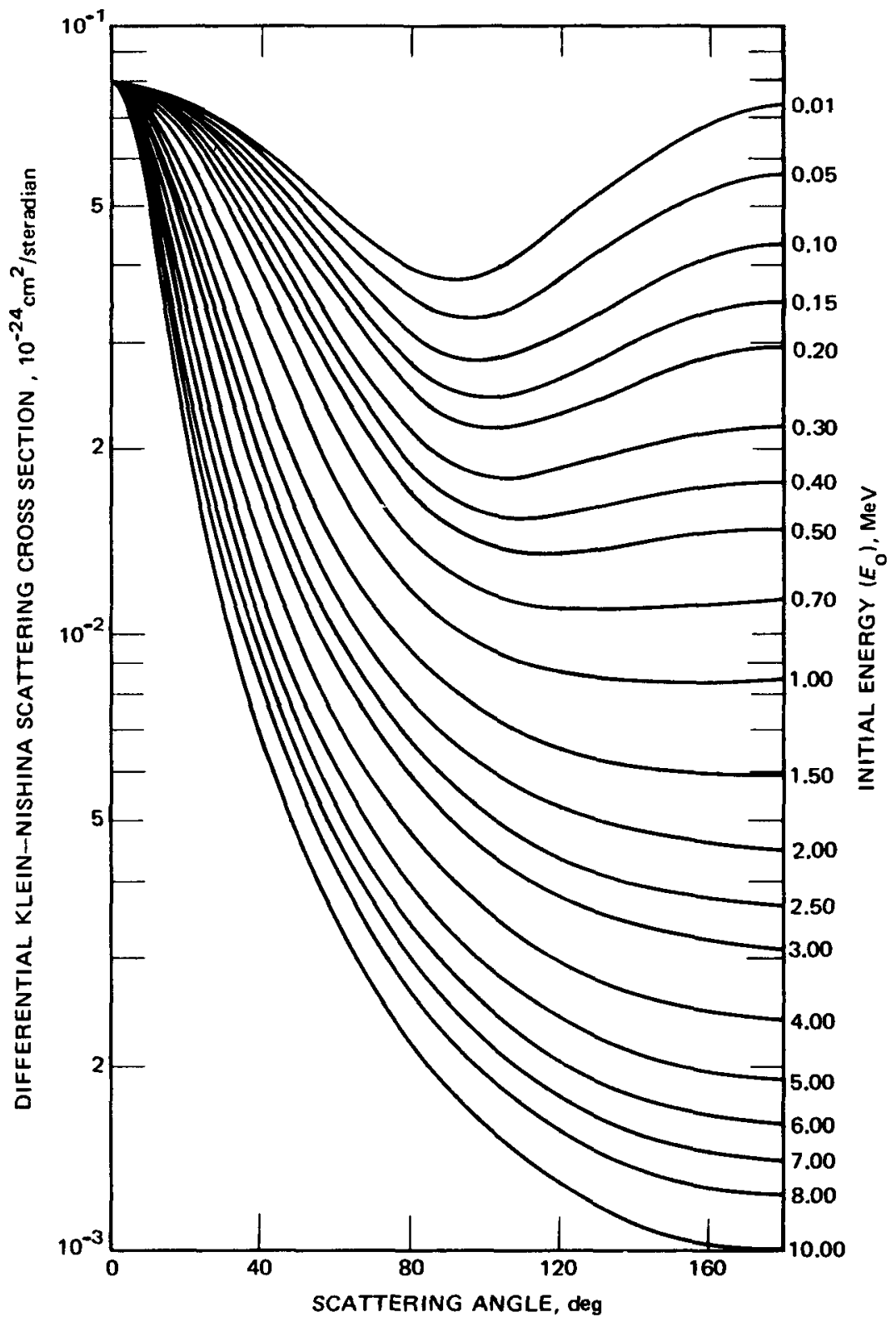

Fig. 3.5-Differential Compton scattering cross sections. 
Table 3.2-TOTAL AND ENERGY-ABSORPTION CROSS SECTIONS FOR THE COMPTON EFFECT $\dagger$

\begin{tabular}{llll}
\hline E, MeV & $\sigma_{c}, \mathrm{~b} /$ electron & $\sigma_{c a}, \mathrm{~b} /$ electron & $\sigma_{c}-\sigma_{c a}, \mathrm{~b} /$ electron \\
\hline 0.0 & 0.665 & 0 & 0.665 \\
0.01 & 0.637 & 0.0077 & 0.629 \\
0.015 & 0.627 & 0.0138 & 0.613 \\
0.020 & 0.616 & 0.0196 & 0.596 \\
0.030 & 0.596 & 0.0295 & 0.566 \\
0.040 & 0.578 & 0.0380 & 0.540 \\
0.050 & 0.561 & 0.0451 & 0.516 \\
0.060 & 0.546 & 0.0509 & 0.459 \\
0.080 & 0.517 & 0.061 & 0.456 \\
0.10 & 0.4929 & 0.0685 & 0.4244 \\
0.150 & 0.4436 & 0.0812 & 0.3624 \\
0.200 & 0.4066 & 0.0886 & 0.3180 \\
0.300 & 0.3535 & 0.0958 & 0.2577 \\
0.4 & 0.3167 & 0.0982 & 0.2185 \\
0.5 & 0.2892 & 0.0984 & 0.2866 \\
0.6 & 0.2675 & 0.0984 & 0.1691 \\
0.8 & 0.2350 & 0.0959 & 0.1391 \\
1.0 & 0.2112 & 0.0929 & 0.1183 \\
1.5 & 0.1716 & 0.0849 & 0.0867 \\
2.0 & 0.1464 & 0.0777 & 0.0687 \\
3.0 & 0.1151 & 0.0664 & 0.0487 \\
4.0 & 0.0960 & 0.0582 & 0.0378 \\
5.0 & 0.0828 & 0.0519 & 0.0309 \\
6.0 & 0.0732 & 0.0471 & 0.0261 \\
8.0 & 0.0599 & 0.0399 & 0.0200 \\
10.0 & 0.0510 & 0.0349 & 0.0161 \\
15.0 & 0.0377 & 0.0268 & 0.0109 \\
\hline
\end{tabular}

†From Goldstein. ${ }^{3}$

As discussed in the next section, the photoelectric process, the Compton process, and pair production all compete for the absorption of the energy of a photon field. However, rarely are photoelectric effect and pair production simultaneously of importance. In low- $Z$ materials, the photoelectric and Compton processes are dominant. Only in iron and higher $Z$ elements does pair production account for over one-half the energy absorption for photons with an energy less than $10 \mathrm{MeV}$. Table 3.3 lists the photon energy above which pair production accounts for one-half or more of the total energy absorption. 


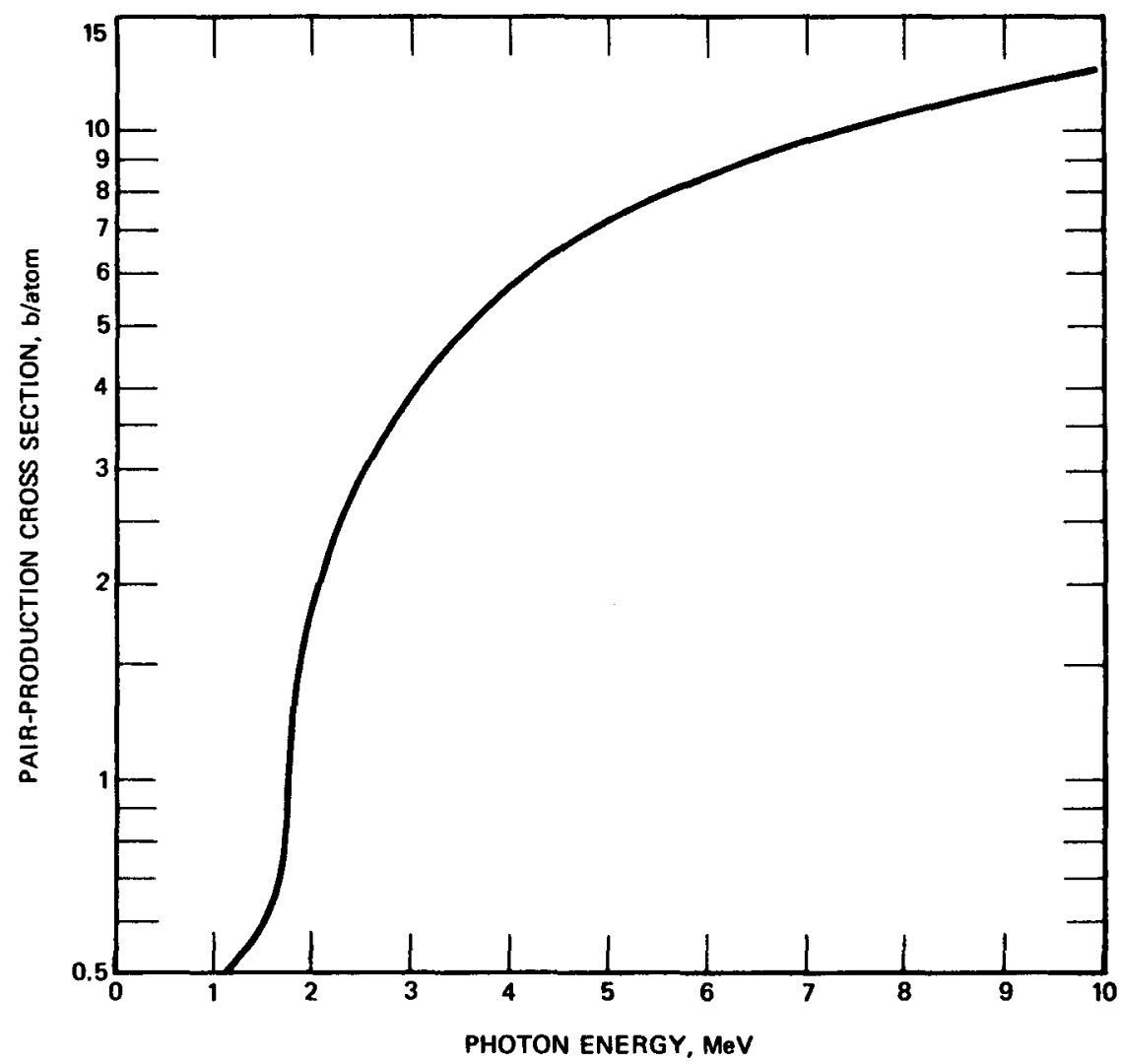

Fig. 3.6-Pair-production cross section in lead. (From Hubbell. ${ }^{4}$ )

Hubbell ${ }^{4}$ and Hubbell and Berger ${ }^{5}$ give discussions of the models used to calculate pair-production cross sections and tabulations of both nuclear and electron pair-production cross-section data for a wide range of photon energies in many elements and several compounds and mixtures.

Although pair production results in the annihilation of the incident photon and plays an important part in the attenuation of high-energy photons, it also results in a secondary photon source that should be considered in some shield analyses. The positron created in the process combines with an electron very close to the site of the pair production, and both the positron and electron are, in turn, annihilated. This gives rise to two $0.511-\mathrm{MeV}$ photons that emerge in opposite directions. For most purposes this secondary source may be considered to originate at the site of the initial pair-production event. This secondary source can account for as much as $5 \%$ of the total energy deposited. 
Table 3.3-CNERGY AT WHICH PAIR PRODUCTION PROVIDES ONE-HALF THE TOTAL ABSORPTION COEFFICIENT $\dagger$

\begin{tabular}{rlc||clc}
\hline$Z$ & Element & $E, \mathrm{MeV}$ & $Z$ & Element & $E, \mathrm{MeV}$ \\
\hline 1 & Hydrogen & 78 & 26 & Iron & 9.5 \\
4 & Beryllium & 35 & 42 & Molybdenum & 7.5 \\
6 & Carbon & 28 & 50 & Tin & 6.5 \\
8 & Oxygen & 20 & 74 & Tungsten & 5.2 \\
13 & Aluminum & 15 & 82 & Lead & 5.0 \\
20 & Calcium & 12 & 92 & Uranium & 4.8 \\
\hline
\end{tabular}

†From Goldstein. ${ }^{3}$

(d) Other Processes. Several other photon interactions, such as coherent electron scattering, Thomson nuclear scattering, and Delbrück scattering, exist; however, coherent scattering, also called Rayleigh scattering, is the only one that has any possible significance in reactor shielding situations. These other interactions are summarized in Refs. 4 and 5.

Coherent scattering is similar to Compton scattering in that the incident photon is scattered from an electron. However, in contrast to the Compton process, the binding forces of the orbital electrons are significant in coherent photon scattering. In this process the recoil momentum is assumed by the whole atom with the result that the scattered photon emerges with an energy that is, for all practical purposes, equal to that of the incident photon. Further, in the photon energy range of interest for fission sources, the photon scattering angles, $\theta$, are small, for example, less than $15^{\circ}$ at $0.1 \mathrm{MeV}$ and $2^{\circ}$ at $1.0 \mathrm{MeV}$ in aluminum. The practical effect of coherent scattering is to scatter a photon in the forward direction with no reduction in energy, and thus it may be treated as though no interaction took place.

However, if coherent scattering is ignored, care must be taken with the cross sections used in photon-attenuation calculations. Often the cross section for coherent scattering is included in tabulations of total photonattenuation coefficients. Since such use would treat the coherent scatter as an attenuating event when, in fact, it has essentially no effect on photon attenuation, the coherent portion should be subtracted from the total cross section. The tabulations in Refs. 4 and 5 list such data both with and without the coherent portion.

(e) Total Photon-Attenuation Coefficients. The three processes that contribute to photon attenuation in reactor shields are the photoelectric 
effect, Compton scattering, and pair production. Each process causes some or all of the energy of the incident photon to be deposited at the site of the interaction, and the last two cause photons of reduced energy to be emitted in new directions. Thus the total microscopic cross section per atom of atomic number $Z$ for photon attenuation is given by

$$
\sigma_{t}=\sigma_{p e}+\sigma_{z c}+\sigma_{p p}
$$

where $\sigma_{p e}$ is the cross section per atom for the photoelectric effect, $\sigma_{z c}=Z \sigma_{c}$, and $\sigma_{p p}$ is the cross section per atom for pair production. If we include coherent scattering as well as Compton scattering, we replace $\sigma_{z c}$ by $\sigma_{c o h}$. The term $\sigma_{z c}$ is sometimes written $\sigma_{i n c o h}$ to explicitly show that coherent scattering is not included. As functions of photon energy, $\sigma_{p e}$ and

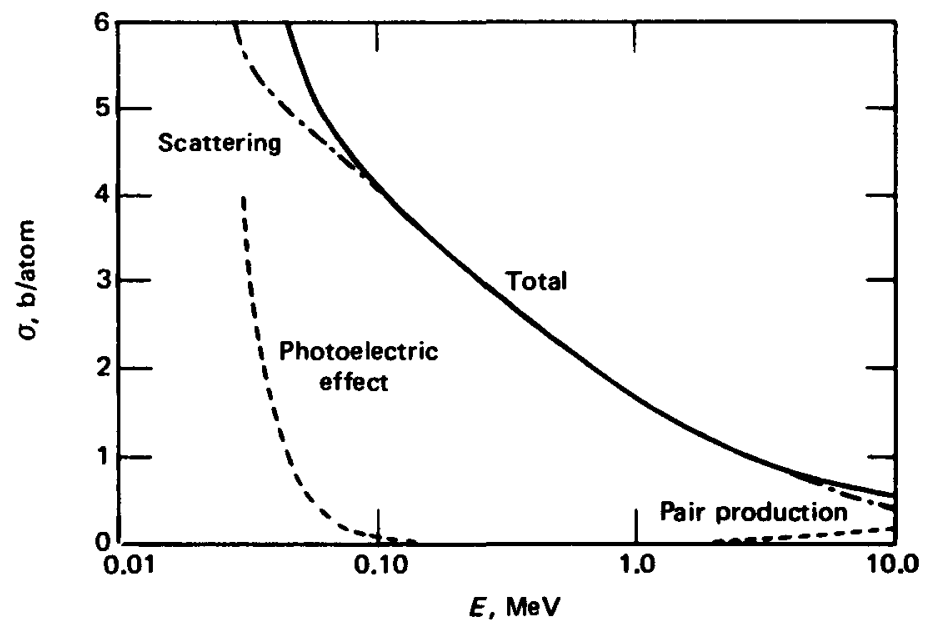

Fig. 3.7-Total gamma-ray cross sections for oxygen. (From Goldstein. ${ }^{3}$ )

$\sigma_{z c}$ decrease with increasing energy; $\sigma_{p p}$, however, increases as the incident-photon energy increases. Thus for all elements a minimum occurs in the total cross section at some energy. This behavior is illustrated in Figs. 3.7 and 3.8, where all three components and the total cross section are plotted for oxygen and lead, respectively. The Compton effect is predominant for intermediate energies, 1 to $5 \mathrm{MeV}$ for lead and other high- $Z$ materials and a wider range for low- $Z$ materials. In hydrogen the Compton effect accounts for the total cross section over the entire energy range of interest. As suggested by these two figures, the point in energy where the minimum in 


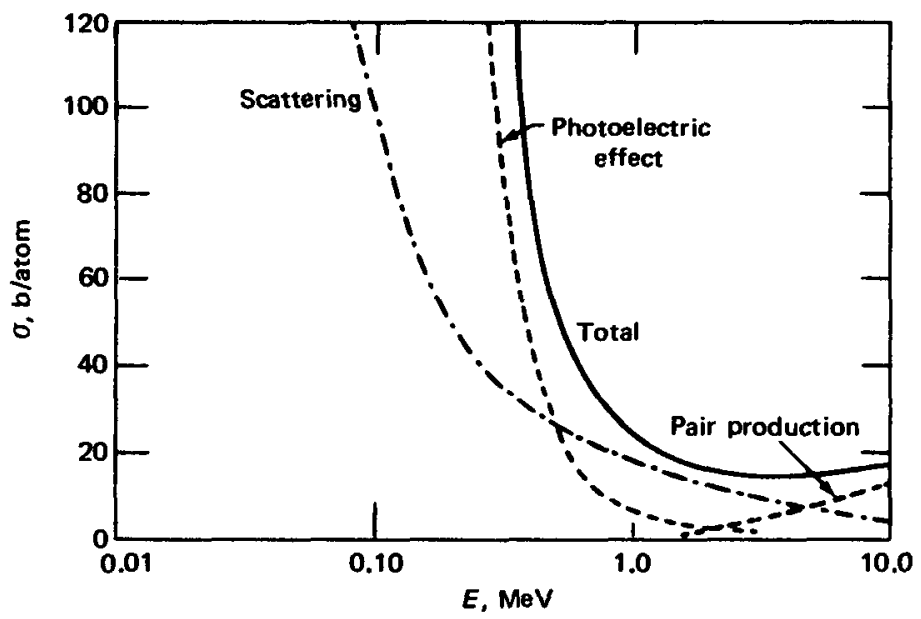

Fig. 3.8-Total gamma-ray cross sections for lead. (From Goldstein. ${ }^{3}$ )

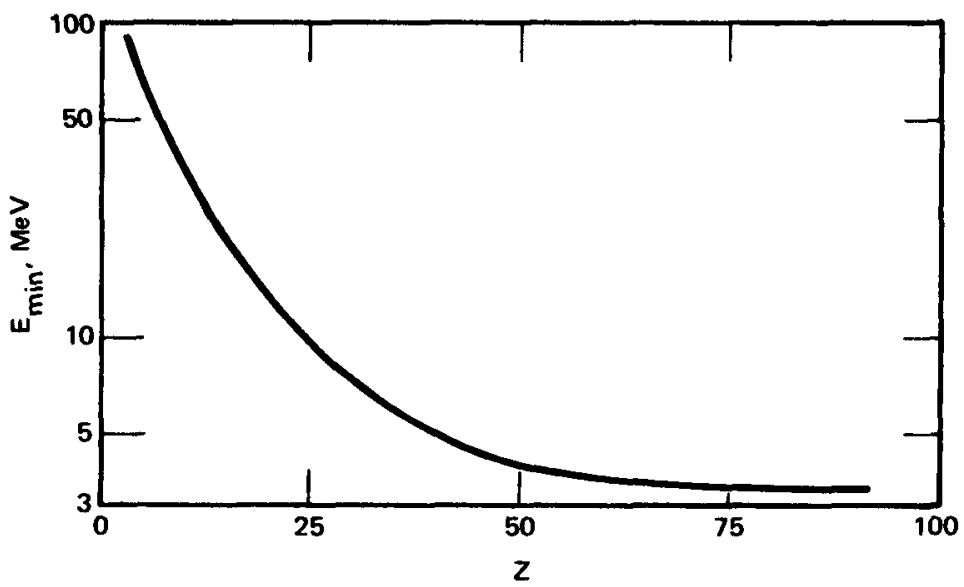

Fig. 3.9-Energy of minimum total photon cross section. (From Goldstein. ${ }^{3}$ )

total cross section occurs decreases with increasing atomic number. Figure 3.9 is a plot of the location of the minimum as a function of $Z$.

It should be noted that a macroscopic cross section computed from the total microscopic cross section of Eq. 3.2-15 is a total interaction coefficient and includes more than energy deposition by virtue of the fact that the entire Compton cross section is included. Thus these macroscopic cross sections are sometimes called narrow-beam absorption coefficients since all processes, including Compton events, effectively remove photons from a 
collimated beam. However, they are more commonly called attenuation coefficients. Thus $\mu_{t}\left(=N \sigma_{t}\right)$ is called a linear attenuation coefficient, and $\mu_{t} / \rho$, a mass attenuation coefficient. The total interaction rate is sometimes called the reaction rate. The reaction rate per unit volume is simply the product $\Phi \mu_{t}$.

Another macroscopic cross section, which actually reflects the local energy removed from a photon field by an element of atomic number $Z$, is given by

$$
\mu_{a}=N\left(\sigma_{p e}+Z \sigma_{c a}+\sigma_{p p}\right)
$$

where $N$ is the atomic density (atoms $/ \mathrm{cm}^{3}$ ) of the element and $\sigma_{c a}$, the Compton energy absorption cross section per electron, is defined by Eq. 3.2-14. The quantities $\mu_{a}$ and $\mu_{a} / \rho$ are known as linear and mass energy-deposition coefficients, respectively.

The fact that electrons are reaction products of each of the three gamma-ray interactions important to transport through shields deserves one further comment. Bremsstrahlung (Sec. 2.1.1) is produced by the decelerating electrons and represents an additional form of the initial gamma-ray energy. In this book we will usually assume that this radiation is absorbed at the point of formation, with one important exception, which is discussed in Sec. 3.3, Responses to Radiation.

\subsubsection{Neutron Reactions}

In several respects the processes associated with neutron attenuation contrast sharply with those associated with photons. For example, only three photon processes are significant at photon energies found in fission reactors, but many neutron reactions must be considered. Also, although all photon reactions of interest involve electrons, no neutron reactions are with electrons; they are all with nuclei. Most cross-section data for photons are accurately obtained by calculation (well verified by experiment); exactly the opposite is true for neutrons. The processes associated with nuclear interactions are far more complicated and, in many cases, are not well understood. Thus most neutron cross sections must be measured experimentally. Active experimental programs have been under way for many years; however, important gaps in certain data still exist. With the exception of the $K$-edge, $L$-edge, etc., in the photoelectric cross section, photon-interaction probabilities are smooth functions of both energy and atomic number. In many neutron cross sections, however, resonance and threshold phenomena 
cause abrupt excursions. Further, neutron cross sections are the ramification of complex interactions of nuclear forces, which depend on several factors in addition to simple atomic number and weight. Thus interpolations are in many instances impossible, and neutron cross sections must be determined nucleus by nucleus. It should be noted, however, that for certain neutron reactions theoretical considerations are effectively used in making interpolations. Foderaro ${ }^{1}$ discusses in detail the application of nuclear models to calculate neutron cross sections.

The neutron has a mass of 1.00898 atomic mass units, slightly greater than the proton mass of $1.00759 \mathrm{amu}$. Since it does not possess electrical charge, a neutron is not affected by the atomic electric field. Consequently it travels through matter unhindered until it passes close enough to a nucleus to interact with the short-range nuclear forces. A nuclear interaction may alter the energy and direction of the neutron or may result in its absorption into the nucleus. Scattering may be either elastic, in which the kinetic energy of the system is conserved, or inelastic, in which some of the kinetic energy is transformed into excitation energy of the nucleus. As discussed in Chap. 2, a neutron absorption can result in the emission of one or more gamma rays, charged particles, and, at times, one or more neutrons. Inelastic scattering also produces secondary emissions, and, of course, neutron absorption can cause fission in certain isotopes. Thus all neutron interactions, except elastic scattering, produce a secondary source of radiation.

As with photons, the total neutron cross section for an interaction with a specified nucleus is the sum of the absorption and scattering cross sections

$$
\sigma_{t}=\sigma_{a}+\sigma_{s} \mathrm{~b} / \text { nucleus }
$$

Most often the scattering probabilities are subdivided into elastic and inelastic portions, as are the various types of absorption reactions. Care must be used in the interpretation of the term absorption. In the present context absorption implies the disappearance of the incident neutron as a separate entity, the neutron becoming a part of the new nucleus created by the interaction. However, as mentioned in the section on inelastic reactions, certain scattering processes are also assumed to involve the absorption of a neutron by a nucleus followed by the emission of one or more secondary neutrons.

Many nuclear processes have certain characteristics that are relatively invariant over broad bands of neutron energy. It is thus convenient to divide the energy spectrum of fission-produced neutrons into four energy groups. 
Neutrons in the lowest group, ranging from 0 to about $0.4 \mathrm{eV}$, are called thermal, or slow, neutrons. Their energy distribution approximates the Maxwell-Boltzmann distribution given by

$$
f_{n}(E)=\frac{2 \pi}{(\pi k T)^{\frac{1}{2}}} E^{1 / 2} e^{-E / k T}
$$

where $f_{n}(E)$ is the fraction of neutrons per unit energy about energy $E, k$ is the Boltzmann constant $\left(8.6 \times 10^{-5} \mathrm{eV} /{ }^{\circ} \mathrm{K}\right)$, and $T$ is the absolute temperature of the medium. $f$ In the thermal range neutrons are assumed to be in equilibrium with the thermal agitation of the nuclei of the medium. Because neutron absorption often varies with the reciprocal of neutron speed in the thermal region, this region is also known as the $1 / v$ region.

The next higher energy region ranges from $0.4 \mathrm{eV}$ to about $1 \mathrm{keV}$ and is called the resonance, or epithermal, region because many cross sections exhibit one, a few, or many resonance peaks in this region. The cross sections at these peaks can have values several orders of magnitude above the base level; their effect on the average cross section across the resonance region depends on both their number and their widths. These latter quantities vary greatly among nuclei.

Depending on the nucleus, the resonance region stops somewhere from 1 to $50 \mathrm{keV}$. For convenience we call neutrons in the range from a few $\mathrm{keV}$ to $500 \mathrm{keV}$ intermediate neutrons.

The highest energy region ranges from $0.5 \mathrm{MeV}$ to the upper limit of energy of fission neutrons, about $18 \mathrm{MeV}$. In this fast-neutron region, some resonances are found, most often in the low-energy portion, but, for the most part, cross sections are relatively smooth and, in general, have lower values than those of the lower energy regions.

Consistent with our treatment of photon interaction, we will briefly describe neutron reactions in this section and refer the reader to detailed discussions elsewhere. In particular, a recent text on neutron interactions by Foderaro ${ }^{1}$ should be noted here since it is well-suited to the needs of shielding specialists.

(a) Elastic Neutron Scattering. In elastic neutron scattering both kinetic energy and momentum are conserved. Thus the simple billiard-ball collision

tStrictly speaking, the average neutron temperature equals the temperature of the medium only for nonabsorbing media. For absorbing media the average neutron temperature will be somewhat higher, and the distribution will depart from that of Maxwell-Boltzmann. 

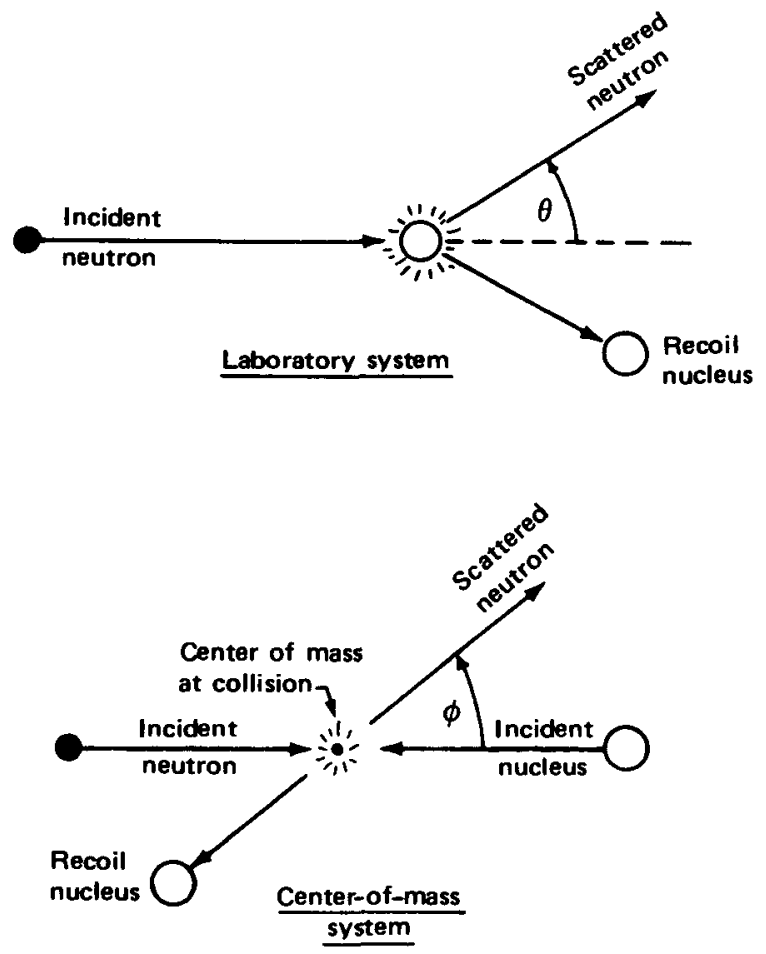

Fig. 3.10-Collision coordinate systems.

model can be used to obtain the direction of motion and the speed of the scattered neutron and recoiling nucleus relative to the direction and speed (or energy) of the incident neutron.

Actually two processes are assumed to produce elastic scattering of neutrons. The first is known as resonance, or capture, scattering. In this process the neutron is assumed to be absorbed by the target nucleus and reemitted in (possibly) another direction. The second process is called potential, or diffraction, scattering. In this process the neutron is assumed not to enter the target nucleus but rather to be elastically scattered by interaction with the potential well created around the nucleus by the short-range nuclear forces. The probability for elastic scatter is the sum of the probabilities for both processes; the cross section for elastic scattering is based on this sum and is often denoted by $\sigma_{e s}$. In adding these probabilities, we must take account of the spin states of the particles since coherent effects are involved.

Resonance elastic scattering is most important at low or intermediate neutron energies, where it causes an oscillating behavior of the elastic- 
scattering cross section. At higher energies (i.e., the fast region), almost all resonance scattering is inelastic since the compound nucleus formed by resonance scattering results in an excited nucleus following neutron reemission. Kinetic energy is therefore not conserved, and some of the kinetic energy reappears as a photon. Potential scattering, which is generally a smooth function of neutron energy, is the predominant component of elastic scattering in the fast region.

The scattering angle-energy relations for elastic interactions are given most simply in the center-of-mass coordinate system in which the total momentum is constant zero, and particle speeds after collision are equal to those before collision. Figure 3.10 illustrates the collision geometry in both the laboratory and the center-of-mass systems.

The energy, $E$, of the elastically scattered neutron is related to the energy, $E_{0}$, of the incident neutron, the atomic mass, $A$, of the target nucleus, and the scattering angle, $\phi$ (in the center-of-mass system), by the equation

$$
\frac{E}{E_{0}}=\frac{A^{2}+2 A \cos \phi+1}{(A+1)^{2}}
$$

The scattering angle, $\theta$, in the laboratory system is related to $\phi$ by the equation

$$
\cos \theta=\frac{A \cos \phi+1}{\left(A^{2}+2 A \cos \phi+1\right)^{1 / 2}}
$$

It is seen that for hydrogen scattering $(A=1)$ the neutron can lose all its energy in just one collision. Maximum energy loss occurs where $\phi=\pi$, and Eq. 3.2-19 becomes

$$
\frac{E_{\min }}{E_{0}}=\left(\frac{A-1}{A+1}\right)^{2}
$$

For low-energy neutrons elastic scattering is approximately isotropic in the center-of-mass system, and the average scattering angle in the laboratory system is given by

$$
\cos \bar{\theta}=\frac{2}{3 A}
$$


Equation 3.2-22 illustrates the point that scattering (in the laboratory system) is peaked in the forward direction $(1>\cos \vec{\theta}>0)$ and that, as $A$ increases, $\bar{\theta}$ approaches $\pi / 2$. Thus, for low energies and heavy nuclei, scattering approaches isotropy in the laboratory system as well.

However, the approximation of isotropic scattering in the center-of-mass system deteriorates as the incident-neutron energy increases, and above about $0.1 \mathrm{MeV}$ scattering is anisotropic for all except the lightest nuclei. In this energy region Goldstein ${ }^{3}$ quotes an approximate equation for $\bar{\theta}$ (where $E_{0}$ is in units of $\mathrm{MeV}$ )

$$
\bar{\theta}=\frac{200}{\left(E_{0}\right)^{1 / 2} A^{1 / 3}} \text { degrees }
$$

which indicates that for incident energies in the fast region anisotropy increases with increasing $A$.

As with photon scattering, differential angle scattering probabilities for elastic neutron scattering play an important role in neutron-transport calculations. The compilation of experimentally determined angle-distribution data by Goldberg, May, and Stehn ${ }^{7}$ is widely used.

(b) Inelastic Neutron Scattering. In inelastic neutron scattering the inelastic collision differs from an elastic event primarily in that a portion of the incident-neutron energy appears as excitation of the target nucleus. The excited nucleus subsequently decays by photon emission; thus inelastic neutron scattering was first introduced in Chap. 2 as a secondary gamma-ray source. The inelastically scattered neutron leaves the collision site usually altered in direction and generally with much-reduced energy as well. In fact, inelastic scattering is an important means of reducing fast $(>1 \mathrm{MeV})$ neutron energies in reactor shields not only because a large amount of energy can be transferred to the nucleus in one inelastic collision but also because of the increasing importance of inelastic scattering with energy in the 1- to $14-\mathrm{MeV}$ range. For most elements of interest, elastic-scattering cross sections are slowly oscillating, generally decreasing in the fast-energy region. Since inelastic scattering is a threshold reaction, the corresponding cross section usually increases with increasing energy. (An exception occurs if particle reactions are present; the inelastic cross section will decrease with energy if there are competing particle reactions.)

Inelastic scattering cannot occur unless the incident neutron has a kinetic energy somewhat greater than the first excited state of the target nucleus. Depending on the nucleus, this threshold varies from 0.1 to 4 or $5 \mathrm{MeV}$. As 
the neutron kinetic energy exceeds energy states of the nucleus above the first, these higher states may also become excited, and thus the neutron may excite the nucleus to any level up to the limit of the incident-neutron energy. As the excitation energy is increased, spacing between levels decreases until a continuum is reached. For a given nuclear species and neutron energy, there is a fixed probability for exciting each energy level. For all but the first level, there are alternate routes by which the nucleus

Table 3.4_TYPICAL CROSS SECTIONS FOR NEUTRON INELASTIC SCATTERING AT FOUR ENERGIES $t$

\begin{tabular}{clllll}
\hline & $\begin{array}{c}\text { Approximate } \\
\text { Element } \\
\text { threshold } \\
\text { energy, } \\
\text { MeV }\end{array}$ & 14 MeV & $5.16 \mathrm{MeV}$ & $2.0 \mathrm{MeV}$ & $\mathbf{1 . 0} \mathrm{MeV}$ \\
\hline Tungsten & & & & & \\
$\sigma_{n n^{\prime}}(L)$ & 0.0115 & 0 & 0.095 & 1.23 & 2.34 \\
$\sigma_{n n^{\prime}}(C)$ & 0.99 & 2.49 & 2.47 & 1.35 & 0.005 \\
Lead & & & & & \\
$\sigma_{n n^{\prime}}(L)$ & 0.57 & 0 & 0 & 7.7 & 0.34 \\
$\sigma_{n n^{\prime}}(C)$ & 3.13 & 2.52 & 2.06 & 0 & 0 \\
Nickel & & & & & 0 \\
$\sigma_{n n^{\prime}}(L)$ & 1.4 & 0 & 0 & 0.6 & 0 \\
$\sigma_{n n^{\prime}}(C)$ & 4.02 & 1.13 & 1.17 & 0 & 0 \\
Carbon & & & & & 0 \\
$\sigma_{n n^{\prime}}(L)$ & 4.91 & 0.268 & 0.0436 & 0 & 0 \\
$\sigma_{n n^{\prime}}(C)$ & 9.4 & 0.216 & 0 & 0 & 0 \\
\hline
\end{tabular}

†From Troubetzkoy et al. ${ }^{8}$

may release the excitation energy: one photon may be given off, dropping the nucleus to the ground state; or a cascade of photons may be emitted as a result of the presence of the intermediate levels. There is, therefore, one set of probabilities for exciting the various levels and another set of probabilities for alternate decay routes from each of the levels. Cross-section data that account for all these alternatives are necessarily somewhat complex.

The total cross section for all inelastic events is often split into two components: $\sigma_{n n^{\prime}}(L)$, which is the probability of excitation to any level from which discrete gamma rays are emitted, and $\sigma_{n n^{\prime}}(C)$, which is the probability for excitation to the region in which levels are so closely spaced that the emission is essentially continuous. Table 3.4 shows for four elements the neutron-energy threshold and the values of $\sigma_{n n^{\prime}}(C)$ and $\sigma_{n n^{\prime}}(L)$ 
for four energies. The general trend is for the inelastic cross section to increase as either the atomic weight of the target nucleus or the energy of the neutron increases. The threshold for inelastic scattering decreases with increasing $Z$.

Inelastic-scattering cross sections useful for detailed radiation-transport calculations are often expressed in terms of the gamma-ray spectrum emitted

Table 3 5-NUMBER OF GAMMA RAYS EMITTED PER NEUTRON PRODUCING REACTION IN LEAD†

\begin{tabular}{|c|c|c|c|c|c|c|c|c|c|c|c|c|}
\hline \multirow[b]{2}{*}{$t_{\|} \mathrm{MeV}$} & \multicolumn{12}{|c|}{$\Gamma_{\gamma}, \mathrm{MeV}$} \\
\hline & 025 & 075 & 125 & 175 & 225 & 275 & 325 & 375 & 425 & 475 & 525 & 650 \\
\hline $\begin{array}{l}1802 \times 10^{1} \\
1700 \\
1600 \\
1500 \\
1450\end{array}$ & $\begin{array}{l}0539 \\
0567 \\
0579 \\
0587 \\
0587\end{array}$ & $\begin{array}{ll}0 & 2730 \\
0 & 293 \\
0 & 301 \\
0 & 305 \\
0 & 305\end{array}$ & $\begin{array}{ll}0 & 168 \\
0 & 182 \\
0 & 188 \\
0 & 188 \\
0 & 186\end{array}$ & $\begin{array}{lll}0 & 119 \\
0 & 130 \\
0 & 134 \\
0 & 132 \\
0 & 133\end{array}$ & $\begin{array}{l}0095 \\
0099 \\
0099 \\
0095 \\
0087\end{array}$ & $\begin{array}{l}0069 \\
0075 \\
0071 \\
0063 \\
0057\end{array}$ & $\begin{array}{ll}0 & 059 \\
0 & 057 \\
0 & 053 \\
0 & 047 \\
0 & 039\end{array}$ & $\begin{array}{ll}0 & 047 \\
0 & 043 \\
0 & 035 \\
0 & 031 \\
0 & 023\end{array}$ & $\begin{array}{ll}0 & 035 \\
0 & 033 \\
0 & 025 \\
0 & 017 \\
0 & 011\end{array}$ & $\begin{array}{ll}0 & 031 \\
0 & 023 \\
0 & 015 \\
0 & 009 \\
0 & 005\end{array}$ & $\begin{array}{ll}0 & 023 \\
0 & 015 \\
0 & 009 \\
0 & 005 \\
0 & 003\end{array}$ & $\begin{array}{ll}0 & 062 \\
0 & 029 \\
0 & 009 \\
0 & 0026 \\
0 & 0019\end{array}$ \\
\hline $\begin{array}{ll}1 & 400 \\
1 & 350 \\
1 & 300 \\
1 & 250 \\
1 & 200\end{array}$ & $\begin{array}{l}0587 \\
0583 \\
0571 \\
0559 \\
0540\end{array}$ & $\begin{array}{ll}0 & 292 \\
0 & 269 \\
0 & 277 \\
0 & 261 \\
0 & 252\end{array}$ & $\begin{array}{ll}0 & 180 \\
0 & 174 \\
0 & 164 \\
0 & 1547 \\
0 & 142\end{array}$ & $\begin{array}{lll}0 & 1 & 123 \\
0 & 119 \\
0 & 103 \\
0 & 087 \\
0 & 072\end{array}$ & $\begin{array}{l}0079 \\
0069 \\
0059 \\
0045 \\
0032\end{array}$ & $\begin{array}{ll}0 & 047 \\
0 & 035 \\
0 & 027 \\
0 & 023 \\
0 & 020\end{array}$ & $\begin{array}{ll}0 & 027 \\
0 & 019 \\
0 & 011 \\
0 & 009 \\
0 & 008\end{array}$ & $\begin{array}{ll}0 & 015 \\
0 & 011 \\
0 & 009 \\
0 & 007 \\
0 & 010\end{array}$ & $\begin{array}{l}0007 \\
0005 \\
0003 \\
0003 \\
0006\end{array}$ & $\begin{array}{ll}0 & 003 \\
0 & 003 \\
0 & 001 \\
0 & 003 \\
0 & 004\end{array}$ & $\begin{array}{ll}0 & 001 \\
0 & 001 \\
0 & 001 \\
0 & 001 \\
0 & 004\end{array}$ & $\begin{array}{ll}0 & 0015 \\
0 & 0019 \\
0 & 0036 \\
0 & 0047 \\
0 & 0138\end{array}$ \\
\hline $\begin{array}{l}1150 \\
1100 \\
1050 \\
1000 \\
9500 \times 10^{0}\end{array}$ & $\begin{array}{l}0522 \\
0463 \\
0404 \\
0285 \\
0205\end{array}$ & $\begin{array}{l}0248 \\
0254 \\
0259 \\
02277 \\
0292\end{array}$ & $\begin{array}{lll}0 & 1 & 34 \\
0 & 1 & 25 \\
0 & 1 & 117 \\
0 & 1 & 112 \\
0 & 111\end{array}$ & $\begin{array}{ll}0 & 056 \\
0 & 044 \\
0 & 040 \\
0 & 053 \\
0 & 065\end{array}$ & $\begin{array}{ll}0 & 020 \\
0 & 014 \\
0 & 012 \\
0 & 018 \\
0 & 026\end{array}$ & $\begin{array}{ll}0 & 020 \\
0 & 032 \\
0 & 052 \\
0 & 081 \\
0 & 1111\end{array}$ & $\begin{array}{lll}0 & 010 \\
0 & 014 \\
0 & 020 \\
0 & 032 \\
0 & 045\end{array}$ & $\begin{array}{ll}0 & 014 \\
0 & 022 \\
0 & 034 \\
0 & 048 \\
0 & 069\end{array}$ & $\begin{array}{ll}0 & 010 \\
0 & 018 \\
0 & 028 \\
0 & 040 \\
0 & 059\end{array}$ & $\begin{array}{ll}0 & 008 \\
0 & 012 \\
0 & 020 \\
0 & 032 \\
0 & 045\end{array}$ & $\begin{array}{ll}0 & 006 \\
0 & 012 \\
0 & 018 \\
0 & 026 \\
0 & 037\end{array}$ & $\begin{array}{lll}0 & 021 \\
0 & 038 \\
0 & 058 \\
0 & 080 \\
0 & 107\end{array}$ \\
\hline $\begin{array}{l}9000 \\
8500 \\
8000 \\
7500 \\
7000\end{array}$ & $\begin{array}{lll}0 & 1 & 03 \\
0 & 054 \\
0 & 034 \\
0 & 034 \\
0 & 025 \\
0 & 021\end{array}$ & $\begin{array}{l}0319 \\
0351 \\
0 \quad 391 \\
0429 \\
0467\end{array}$ & $\begin{array}{lll}0 & 109 \\
0 & 1 & 110 \\
0 & 1112 \\
0 & 113 \\
0 & 1117\end{array}$ & $\begin{array}{ll}0 & 087 \\
0 & 112 \\
0 & 140 \\
0 & 158 \\
0 & 183\end{array}$ & $\begin{array}{ll}0 & 039 \\
0 & 050 \\
0 & 063 \\
0 & 072 \\
0 & 078\end{array}$ & $\begin{array}{ll}0 & 153 \\
0 & 200 \\
0 & 246 \\
0 & 291 \\
0 & 331\end{array}$ & $\begin{array}{ll}0 & 058 \\
0 & 062 \\
0 & 065 \\
0 & 064 \\
0 & 061\end{array}$ & $\begin{array}{ll}0 & 089 \\
0 & 106 \\
0 & 119 \\
0 & 124 \\
0 & 131\end{array}$ & $\begin{array}{ll}0 & 078 \\
0 & 098 \\
0 & 110 \\
0 & 113 \\
0 & 113\end{array}$ & $\begin{array}{ll}0 & 058 \\
0 & 075 \\
0 & 089 \\
0 & 098 \\
0 & 100\end{array}$ & $\begin{array}{ll}0 & 045 \\
0 & 054 \\
0 & 057 \\
0 & 053 \\
0 & 043\end{array}$ & $\begin{array}{lll}0 & 133 \\
0 & 141 \\
0 & 136 \\
0 & 091 \\
0 & 041\end{array}$ \\
\hline $\begin{array}{l}6500 \\
6000 \\
5500 \\
5000 \\
4500\end{array}$ & $\begin{array}{lll}0 & 022 \\
0 & 027 \\
0 & 029 \\
0 & 030 \\
0 & 026\end{array}$ & $\begin{array}{l}0491 \\
0523 \\
0553 \\
0575 \\
0623\end{array}$ & $\begin{array}{lll}0 & 120 \\
0 & 127 \\
0 & 133 \\
0 & 135 \\
0 & 137\end{array}$ & $\begin{array}{ll}0 & 196 \\
0 & 208 \\
0 & 213 \\
0 & 200 \\
0 & 182\end{array}$ & $\begin{array}{ll}0 & 073 \\
0 & 062 \\
0 & 046 \\
0 & 030 \\
0 & 018\end{array}$ & $\begin{array}{l}0370 \\
0393 \\
0 \quad 402 \\
0395 \\
0376\end{array}$ & $\begin{array}{ll}0 & 055 \\
0 & 050 \\
0 & 043 \\
0 & 035 \\
0 & 026\end{array}$ & $\begin{array}{lll}0 & 133 \\
0 & 134 \\
0 & 131 \\
0 & 112 \\
0 & 069\end{array}$ & $\begin{array}{lll}0 & 111 \\
0 & 1 & 01 \\
0 & 087 \\
0 & 060 \\
0 & 021\end{array}$ & $\begin{array}{lll}0 & 093 \\
0 & 069 \\
0 & 038 \\
0 & 010\end{array}$ & $\begin{array}{l}0026 \\
0007\end{array}$ & 0015 \\
\hline $\begin{array}{l}4000 \\
3500 \\
3250 \\
3000 \\
2750\end{array}$ & $\begin{array}{ll}0 & 018 \\
0 & 013 \\
0 & 006\end{array}$ & $\begin{array}{l}0692 \\
0745 \\
0761 \\
0870 \\
1060\end{array}$ & $\begin{array}{lll}0 & 135 \\
0 & 120 \\
0 & 108 \\
0 & 092 \\
0 & 080\end{array}$ & $\begin{array}{ll}0 & 162 \\
0 & 130 \\
0 & 115 \\
0 & 104 \\
0 & 100\end{array}$ & 0006 & $\begin{array}{ll}0 & 337 \\
0 & 281 \\
0 & 231 \\
0 & 185 \\
0 & 080\end{array}$ & 0012 & 0018 & & & & \\
\hline $\begin{array}{l}2500 \\
2250 \\
2000 \\
1750 \\
1500 \\
1250\end{array}$ & & $\begin{array}{ll}1 & 126 \\
1 & 125 \\
1 & 095 \\
1 & 062 \\
1 & 037 \\
1 & 000\end{array}$ & $\begin{array}{lll}0 & 057 \\
0 & 043 \\
0 & 0 & 27 \\
0 & 015\end{array}$ & $\begin{array}{ll}0 & 068 \\
0 & 037 \\
0 & 013\end{array}$ & & & & & & & & \\
\hline
\end{tabular}

fFrom Troubcekoy at d $d^{\text {s }}$

by the nucleus for specific incident-neutron energies. These data may include gamma rays from other nonelastic events, i.e., reactions other than elastic scattering including inelastic scattering, $(n, 2 n),(n, p)$, etc. Data of this type are shown in Table 3.5, which gives the number of gamma rays given off at various gamma-ray energies per neutron-producing reaction in lead, $\left(n, n^{\prime}\right)$ and $(n, 2 n)$. These values are not interaction cross sections but rather give the distribution of gamma rays resulting from an interaction. The data were 
obtained from the report of Troubetzkoy $e t$ al. ${ }^{8}$ which is an evaluation of calculations, measurements, and extrapolation. Multiplication of these values by the total cross section for neutron-producing events would give the gamma rays produced per unit neutron-flux density.

In most stationary power reactors with concrete shields, gamma rays from inelastic scattering are usually not a determining factor in the shield design. In cases such as mobile reactors with lead or tungsten as gamma-ray shields, however, a careful analysis of inelastic scattering is required. Also, in fast reactors with high- $Z$ materials in their structure, it may be necessary to include inelastic gamma rays from the core as a part of the source definition.

As with elastic scattering, theoretical concepts have been used to describe the nature of inelastic neutron cross sections. For the most part, however, such treatments rely on parameters that must be determined by measurement. Reference 7 contains a compilation of measured neutron inelastic-scattering angle distributions. A summary of gamma rays from inelastic neutron scattering based on both calculation and measurement is given in Appendix A.

(c) Neutron Radiative Capture. For neutron energies in the lower ranges, radiative capture competes in importance with the elastic-scattering process. In radiative capture the incident neutron is absorbed by the target nucleus; the compound nucleus is almost invariably left in an excited state and decays in a variety of ways, emitting one or more gamma rays. The total excitation energy is equal to the energy of the incident neutron plus the neutron binding energy.

The binding energy of neutrons varies from $3.2 \mathrm{MeV}$ in hydrogen to 11 $\mathrm{MeV}$ in nitrogen, averaging about $7 \mathrm{MeV}$. Thus energetic gamma rays can be emitted. The probability for the emission of such a photon is highest in light and magict nuclei. If, however, the compound nucleus formed by the absorption has energy levels lower than the combined binding and kinetic energies of the incident neutron, a gamma-ray cascade can result. Recent theoretical work in the prediction of the gamma-ray spectra from capture reactions is discussed by Foderaro, ${ }^{1}$ Troubetzkoy et al., ${ }^{8}$ Yost, ${ }^{9}$ and Howerton and Plechaty. ${ }^{10}$

Experimental work on gamma-ray yields from neutron capture is a continuing effort of a number of laboratories (notably in Canada and the USSR). A compilation of data to 1960 is given in the Shielding volume of 
the Reactor Handbook. ${ }^{2}$ More recent data can be found in the journal Nuclear Data. ${ }^{11}$ One to five gamma rays, ranging in energy from less than 1 to $12 \mathrm{MeV}$, are emitted per capture. Typical emittance spectra taken from a compilation in the Reactor Handbook ${ }^{2}$ are shown in Fig. 3.11.

The thermal-neutron cross sections for radiative capture in most elements and isotopes are given in the famous "barn book." 12 Very few measurements have been made on the $\sigma(n, \gamma)$ cross sections at neutron energies other than thermal. Generally, the capture cross section becomes quite small for neutron energies above 10 to $20 \mathrm{keV}$. Therefore the total
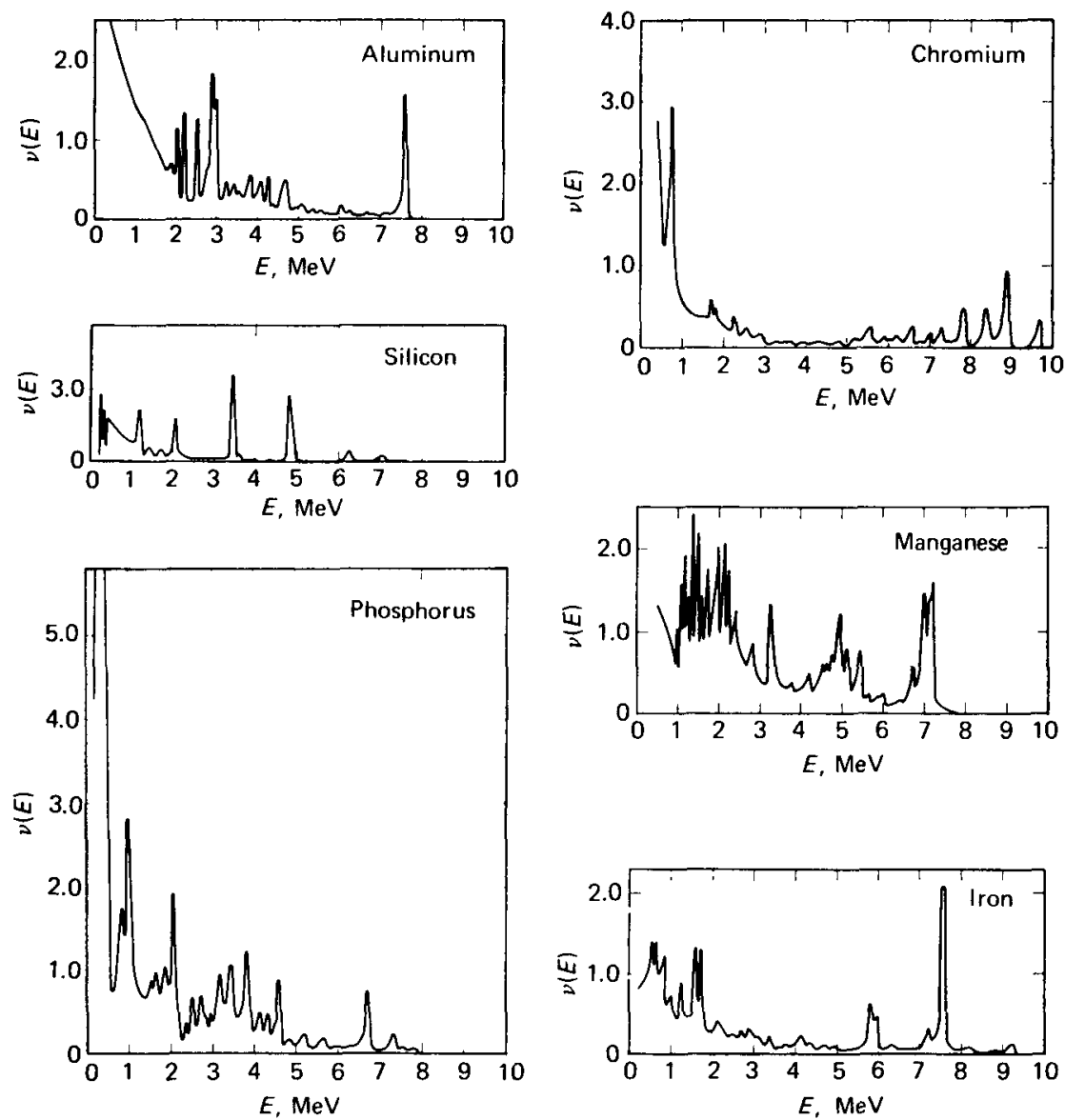

Fig. 3.11-Typical gamma-ray spectra from neutron capture, $\nu(E)$ (photons per MeV per capture) vs. gamma-ray energy, E. [From E. P. Blizard (Ed.), Reactor Handbook, 2nd ed., Vol. III, Part B, Shielding, pp. 50-51, Interscience Publishers, a division of John Wiley \& Sons, Inc., New York, 1962.] 
energy emitted as gamma rays is almost equal to the neutron binding energy because the energy of the incident neutron is generally quite small by comparison. The gamma-ray spectra resulting from neutrons captured at epithermal energies may differ significantly from the thermal capture spectra. However, since the data are frequently lacking, gamma-ray yield data for thermal neutrons are often used for all capture energies. Yost and Solomito $^{13}$ show that this assumption leads to considerable error in gamma-ray transport for many designs. Yost ${ }^{9}$ has developed a method for calculating the capture gamma-ray spectrum as a function of neutron energy.

(d) Other Nonelastic Reactions. There are reactions other than inelastic scattering in which the kinetic energy of the system is not conserved. These include $(n, 2 n),(n$, fission), and ( $n$,charged particle) reactions. The nonelastic neutron cross section, $\sigma_{n e}$, is defined by

$$
\sigma_{n e}(E)=\sigma_{t}(E)-\sigma_{e s}(E)
$$

in which $\sigma_{t}$ is the total neutron cross section and $\sigma_{e s}$ is the cross section for elastic scatter. Since $\sigma_{n e}$ is easier to measure than the inelastic-scattering cross section, $\sigma_{i n}$, it is often quoted. It should be recognized that $\sigma_{i n} \leqslant \sigma_{n e}$.

Charged-particle reactions generally are produced by neutrons with energies greater than $1 \mathrm{MeV}$. At lower energy these reactions are generally inhibited either by the energetics of the process or by the Coulomb barrier, which must be penetrated by the charged reaction products. However, some light-particle thermal-neutron reactions do occur. One of these of importance to shielding because of its high thermal-neutron absorption cross section is the ${ }^{10} \mathrm{~B}(n, \alpha)^{7} \mathrm{Li}$ reaction, which is accompanied by the emission of a relatively soft $0.5-\mathrm{MeV}$ gamma ray. Borated materials are widely used in reactor shields because ${ }^{10} \mathrm{~B}$ competes for the absorption of thermal neutrons and thus inhibits the production of more-energetic secondary gamma rays by reducing radiative capture in other isotopes.

(e) Activation Cross Sections. Many neutron interactions result in the production of excited nuclei, which decay by a variety of means. Cross sections that describe the probability of the formation of a specific radioactive nucleus are often called activation cross sections and are specified in units of barns per nucleus. Thus the total radiative capture cross section of a nucleus may be the sum of several activation cross sections. On the other hand, the total activation cross section may include processes other than radiative capture. 
Activation cross sections are useful in making estimates of secondary sources in reactor shields and materials, in radioisotope-production calculations, and in the relatively new and rapidly growing field of activation analysis. The details of decay schemes, emitted-particle energies, branching ratios, etc., can be found in several sources. Perhaps the best current source for such data is Nuclear Data Sheets. ${ }^{14}$ Activation cross sections are also found in the barn book. ${ }^{12}$

\subsection{RESPONSES TO RADIATION}

It was noted in the discussion of measures of radiation intensity (Sec. 2.2.4) that interaction rates of a radiation field with its environment are important and are often-used characterizations of radiation intensity. However, further discussion of the concept was deferred to this chapter so that the various microscopic processes of interaction could first be reviewed.

Calculations of radiation transport are fundamentally in terms of flux densities and currents (described in Chap. 2). However, the quantity most often measured and the quantity by which the shield design criteria are specified is a property of the radiation field called dose. We use the term in a generic sense to include any quantity that relates the energy deposited by the radiation field to biological damage. Therein lies the rub. Biological damage is intrinsically difficult to measure and even more difficult to predict. Fortunately we limit our shielding interest in biological effects to defining a proper interface between the shield designer and the radiobiologist.

In an ideal situation a given dose would always produce the same biological effect irrespective of the nature and energy of the radiation or of the body organ being irradiated. However, studies by radiobiologists in which doses and biological damage to specific organs have been correlated for known radiation fields have established that nature is not that simple. In addition to being a function of absorbed energy, biological responses are functions of the irradiated organ and of the type, rate, and energy of the radiation. From these studies the concept of relative biological effectiveness (RBE) has evolved. The RBE is a weighting factor that is used to compare the biological effects produced by the same physical dose (same amount of energy deposited) of a standard radiation with radiation of a different type and/or energy. When the physical dose is multiplied by the RBE, it becomes a biological dose. 
The foregoing gives some indication of the problems associated with establishing doses for shield design that are related to biological hazards. The shield designer's task is further complicated by the perturbation of the radiation field by the human body, an effect not usually included in a typical shielding calculation. Obviously the dose received at a particular location within the body is not the same as the dose in a small detector at the same location in space with the body absent. It is apparent that shielding studies should include anthropomorphic phantoms as part of the shield configuration, a theoretically possible but usually impractical consideration.

As an alternative, slab or cylindrical phantoms of a composition resembling that of the human body have been used, and the doses have been calculated or measured as a function of depth in the phantom for a given incident radiation field. The results are then used as response functions to relate an unperturbed radiation field (usually called a free field) to the dose in a human body had it been present.

The following discussion on the various quantities used to define physical and biological doses reveals the confusion that has developed in the terminology and in the definition of units, both because of the burgeoning nuclear science and technology and because radiobiologists and shield designers basically differ in their viewpoints. The International Commission on Radiation Units and Measurements (ICRU) has as its principal objective the development of internationally acceptable recommendations regarding quantities and units of radiation and radioactivity, procedures suitable for the measurement and application of these quantities, and physical data needed in the application of these procedures. The ICRU recognized the confusion that existed, and in an effort to mitigate it recommended a consistent set of definitions and units. ${ }^{15}$ Although shield designers have accepted most of the ICRU recommendations, they have continued to use terms not included in the recommendations because these terms are so ingrained in the shielding field and because the ICRU did not include all the concepts needed in shield design. In the following descriptions the ICRU recommendations and the traditional viewpoints are contrasted.

Trubey ${ }^{16}$ describes the impact of the ICRU recommendations on the shielding community.

\subsubsection{Absorbed Dose}

Absorbed dose is the energy imparted by radiation to a unit mass of matter and as such is a physical quantity as opposed to a biological effect. A 
formal definition for the energy imparted, $E_{D}$, is: The energy imparted by ionizing radiationt to the matter in a volume is the difference between the sum of the energies of all the directly and indirectly ionizing particles which have entered the volume and the sum of the energies of all those which have left it plus the energy equivalent (Q) of any decrease in rest mass that took place in nuclear or elementary-particle reactions with the volume. For all practical purposes the energy imparted is usually equal to the heating effect, but in some cases part of the energy deposited may result in changes in interatomic-bond energies.

As proposed by the ICRU, ${ }^{15}$ the energy imparted can be expressed as

$$
E_{D}=\Sigma E_{\text {in }}-\Sigma E_{e x}+\Sigma Q
$$

where $\Sigma E_{\text {in }}=$ sum of the energies (excluding rest energies) of all those directly and indirectly ionizing particles which have entered the volume

$\boldsymbol{\Sigma} E_{\text {ex }}=$ sum of the energies (excluding rest energies) of all those directly and indirectly ionizing particles which have left the volume

$\boldsymbol{\Sigma} Q=$ sum of all the energies released minus the sum of all the energies expended in any nuclear reactions, transformations, and elementary-particle processes that have occurred within the volume

The absorbed dose $(D)$ is

$$
D \equiv \frac{\Delta E_{D}}{\Delta M}
$$

where $\Delta E_{D}$ is the energy imparted by ionizing radiation to the matter in a volume element and $\Delta M$ is the mass of the matter in that volume element.

fIonizing radiation is a radiation consisting of directly or indirectly ionizing particles or a mixture of both. Directly ionizing particles are charged particles (electrons, protons, alpha particles, etc.) having sufficient kinetic energy to produce ionization by collision. Indirectly ionizing particles are uncharged particles (neutrons, gamma rays, etc.) which can liberate directly ionizing particles or can initiate a nuclear transformation.

$¥$ The notation $\Delta E_{D}$ implies that the volume element $\Delta V$ associated with the element of mass $\Delta M$ be of an appropriate size such that the limiting process $D=\Delta E_{D} / \Delta M$ yields a meaningful estimate of the absorbed dose, and we invoke the special limiting process of Sec. 2.2.4. 
The special unit of absorbed dose is the rad:

$$
1 \mathrm{rad}=100 \mathrm{ergs} / \mathrm{g}
$$

The absorbed dose rate is

$$
\text { Absorbed dose rate }=\frac{\Delta D}{\Delta t}
$$

where $\Delta D$ is the increment in absorbed dose in time, $\Delta t$. A special unit of absorbed dose rate is any quotient of the rad or its multiple or submultiple divided by a suitable unit of time ( $\mathrm{rads} / \mathrm{sec}, \mathrm{mrads} / \mathrm{hr}$, etc.).

Energy can be imparted to a volume of matter by many different particle reactions. Particles having the same initial energy do not necessarily deposit the same amount of energy because, of course, energy deposition depends not only on the initial energy but also on the type of radiation and the kinds of interactions that occur. Schematic representations of energy imparted to the matter contained within a volume element $\Delta V$ for two particles having initial energies $E_{0}^{1}$ and $E_{0}^{2}$ are shown in Fig. 3.12 (superscripts 1 and 2 identify the reactions produced by particles 1 and 2 , respectively). The corresponding equations for the energy imparted are

$$
\begin{gathered}
\Delta E_{D}^{1}=E_{0}^{1}-E_{\beta}^{1}-E_{\gamma}^{1}-Q^{1} \\
\Delta E_{D}^{2}=E_{0}^{2}-E_{\gamma}^{2}+Q^{2}
\end{gathered}
$$

where $\Delta E_{D}$ is the energy imparted to the matter within the volume element $\Delta V, \mathrm{Q}$ is the energy equivalent of any change in the rest mass due to nuclear or elementary-particle reactions within the volume, and subscripts $\beta$ and $\gamma$ refer to the type of particle leaving the volume. In reaction 1 an incident gamma ray undergoes Compton scattering within volume element $\Delta V$. The term $Q^{1}$ indicates the binding energy of the Compton electron, which is usually of negligible magnitude. The Compton electron loses some of its energy through ionization within the volume and then departs with the energy $E_{\beta}^{1}$. The scattered (degraded) gamma ray also leaves the volume. In the second reaction a neutron undergoes radiative capture and a gamma ray is produced which leaves the volume. The term $Q^{2}$ indicates the binding energy associated with neutron capture, which appears as excitation energy. Secondary collisions within the volume element were purposely omitted in 


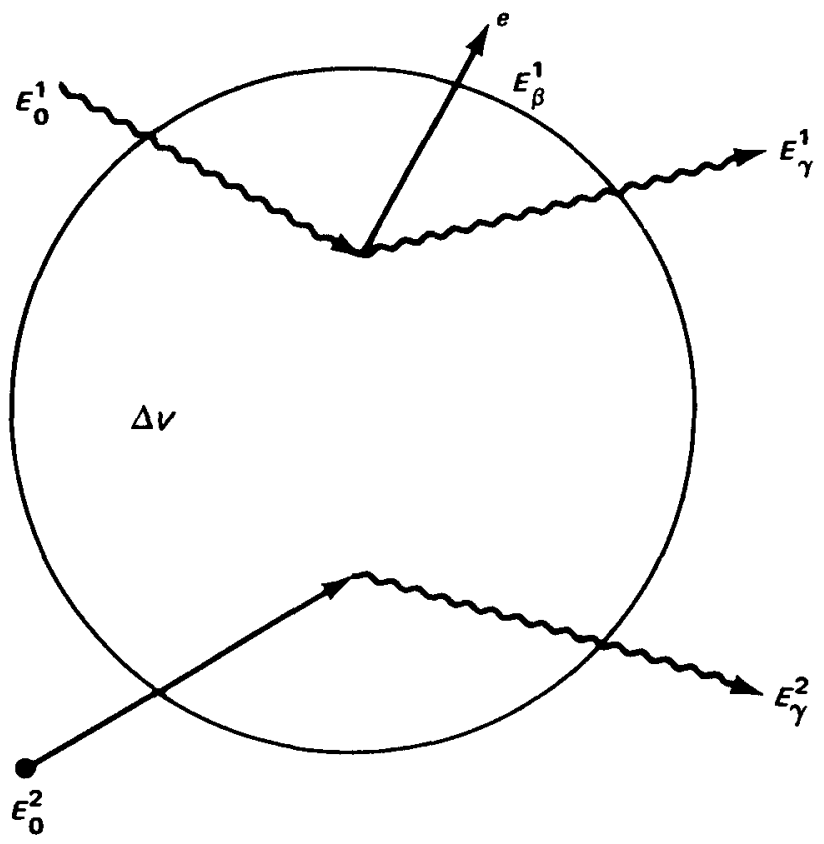

Fig. 3.12-Schematic representation of relation between absorbed dose (energy deposition) and kerma.

this schematic representation to illustrate that the volume element $\Delta V$ should be large enough for many primary interactions to occur but sufficiently small so that a primary particle and/or secondary neutrons or gamma rays usually would not suffer collisions subsequent to the initial collision by the incident particle within the volume element $\Delta V$.

The average energy imparted to the matter within the volume element $\Delta \mathrm{V}$ by all reactions of particles of a particular type and energy can be written as

$$
\bar{E}_{D}(E)=\frac{\sum_{i} R_{i}(E) E_{i}(E)}{\sum_{i} R_{i}(E)}
$$

where $\bar{E}_{D}(E)=$ average energy imparted in ergs or $\mathrm{MeV}$

$R_{i}(E)=$ rate of the $i$ th type of reaction for particles of energy $E$ within the volume element $\Delta V$

$E_{i}(E)=$ energy imparted, in ergs or $\mathrm{MeV}$, associated with a particle of energy $E$ undergoing reaction $i$ within the volume element $\Delta V$ 


\subsubsection{First-Collision Dose and Kerma}

The quantity absorbed dose discussed in the previous section is a physical variable that is closely related to the biological effect of a radiation field but for a real situation is sometimes very difficult to calculate accurately or to relate to the response in a human being. Consequently other concepts have been devised and used.

One of these concepts is the first-collision dose (also called the single-collision dose). This quantity has been subject to several interpretations that differ from one another in subtle ways. We will discuss the two that are pertinent to the present discussion:

1. The first-collision dose is the absorbed dose contributed by all particles incident on an isolated small mass (unless the term is explicitly modified to include only one component, e.g., neutrons) in which charged-particle equilibrium $\dagger$ exists.

2. The first-collision dose is the energy transferred from uncharged incident radiation, regardless of origin, to the charged particles fcrmed in a limitingly small probe that may be located anywhere. Charged-particle equilibrium in the probe is not a requirement.

The small mass specified in the first interpretation means that the probe is small enough to leave the radiation field unperturbed and the probability is negligible that the incident particles will interact with the probe more than once or that secondary gamma rays $\neq$ produced within the mass will be absorbed within it. The small probe specified in the second interpretation has the same requirements, but it can be smaller than that required for the first interpretation because charged-particle equilibrium is not necessary.

The first interpretation is widely used, particularly by experimentalists, and is often called the free-field dose, the air dose, or the free-air dose. However, in an attempt to alleviate confusion, the ICRU chose the second interpretation to use as a basis for an exact definition and called the quantity so defined the kerma (kinetic energy released in material). In fact, the ICRU $^{15}$ encourages the exclusive use of the term kerma with the implication that the term first-collision dose and its other interpretations be avoided.

tCharged-particle equilibrium may be viewed as that condition when, on the average, as many charged particles, such as electrons, enter the volume element $\Delta V$ as leak out, thereby resulting in an essentially zero net transfer of energy by the flow of electrons.

$\$$ As used here secondary gamma rays refer to those gamma rays produced by interaction of the incident neutrons with the medium (e.g., capture gamma rays and inelastic-scattering gamma rays). 
The definition of kerma, $K$, as recommended by the $\operatorname{ICRU}^{15}$ is

$$
K \equiv \frac{\Delta E_{K}}{\Delta M}
$$

where $\Delta E_{K}$ is the sum of the initial kinetic energies (ergs) of all the charged particles liberated by indirectly ionizing particles in a volume element of material $\Delta V$ and $\Delta M$ is the mass (grams) of the material contained in the volume element $\Delta V$.

The kerma rate is

$$
\text { Kerma rate }=\frac{\Delta K}{\Delta t}
$$

where $\Delta K$ is the increment in kerma in time $\Delta t$.

Since $\Delta E_{K}$ is the sum of the initial kinetic energies of all charged particles liberated by indirectly ionizing particles, it includes not only the kinetic energy these charged particles expend in collisions but also the bremsstrahlung they radiate and the energy of any charged particles that are produced in secondary processes. Note also that no restriction is placed on the volume into which these secondary radiations penetrate.

The kerma or kerma rate for a specified material in free space or at a point inside a different material will be that which would be obtained if a small quantity of the specified material were placed at the point of interest.

With the definition of kerma in mind, we have interpreted the reactions shown in Fig. 3.12 in terms of the initial kinetic energy imparted to charged particles

$$
\begin{aligned}
& \Delta E_{K}^{1}=E_{0}^{1}-E_{\gamma}^{1}-Q^{1} \\
& \Delta E_{K}^{2}=E_{0}^{2}-E_{\gamma}^{2}+Q^{2}
\end{aligned}
$$

We can see that the expression for $\Delta E_{K}^{2}$ and the expression given in Eq. 3.3-6 for $\Delta E_{D}^{2}$ are the same. However, the expression for $\Delta E_{K}^{1}$ differs from that given in Eq. 3.3-5 for $\Delta E_{D}^{1}$ because the Compton electron produced by reaction 1 did not deposit all its energy in the volume element and there was no compensating inleakage of an electron; i.e., charged-particle equilibrium did not exist, and $\Delta E_{K}^{1}>\Delta E_{D}^{1}$. If charged-particle equilibrium did exist, we 
could assume that, on the average, a recoil electron with energy $E_{\beta}^{1}$ would enter $\Delta V$ of Fig. 3.12 from an adjacent region. This would then increase $\Delta E_{D}^{1}$ and make it equal $\Delta E_{K}^{1}$ (latter is unaltered).

The choice of the second interpretation was not a popular one for experimentalists. Auxier ${ }^{17}$ expressed the issue clearly. He argues that the first interpretation should be formally recognized as first-collision dose, principally because the quantity measured with ionization chambers, proportional counters, and other devices is absorbed energy and not transferred kinetic energy.

Therefore, since measured doses cannot be interpreted as kerma, except by deduction, some dosimetrists are insisting on retaining the term first-collision dose. On the other hand, if charged-particle equilibrium exists, which it may or may not, and if bremsstrahlung radiation is ignored, then first-collision dose and kerma are equivalent. In bulk shields or large tissue masses, first-collision dose and kerma have nearly the same magnitude, but in thin layers, such as clothing or skin, they can be quite different. It is possible to find the factors by which fluence must be modified to convert to kerma. Such calculations have been made and are indispensable for comparing analytical results with measurement.

The early calculations of first-collision dose per unit fluence were performed by Snyder $^{18}$ for monoenergetic neutrons incident on a fourelement tissue model. Although the term kerma had not yet been introduced, the doses obtained by Snyder are those described by the second interpretation and thus are kerma per unit fluence. His calculations considered only neutron captures and elastic scatterings; the latter was assumed to take place isotropically in the center-of-mass system. Later, Henderson $^{19}$ made similar calculations for neutrons for a four-element tissue model and for ethylene. He reported his results as absorbed dose (in rads per hour per unit flux), but they are actually kerma rate per unit flux.

The most recent and most comprehensive calculations of neutron kerma factors (kerma per unit fluence) were made by Ritts, Solomito, and Stevens. ${ }^{20}$ They included essentially all reactions, considered anisotropic scattering in those reactions for which angular cross-section data were available, and in all cases used the most recent cross-section data. One of the models they used was an 11-element standard-man model in which all tissue organs and bone were homogenized; other models were for specific body organs. The elemental compositions used in the calculations, the reactions considered, and the neutron-fluence-to-kerma conversion factors for standard man are given in Appendix B. The kerma factor for a particular 


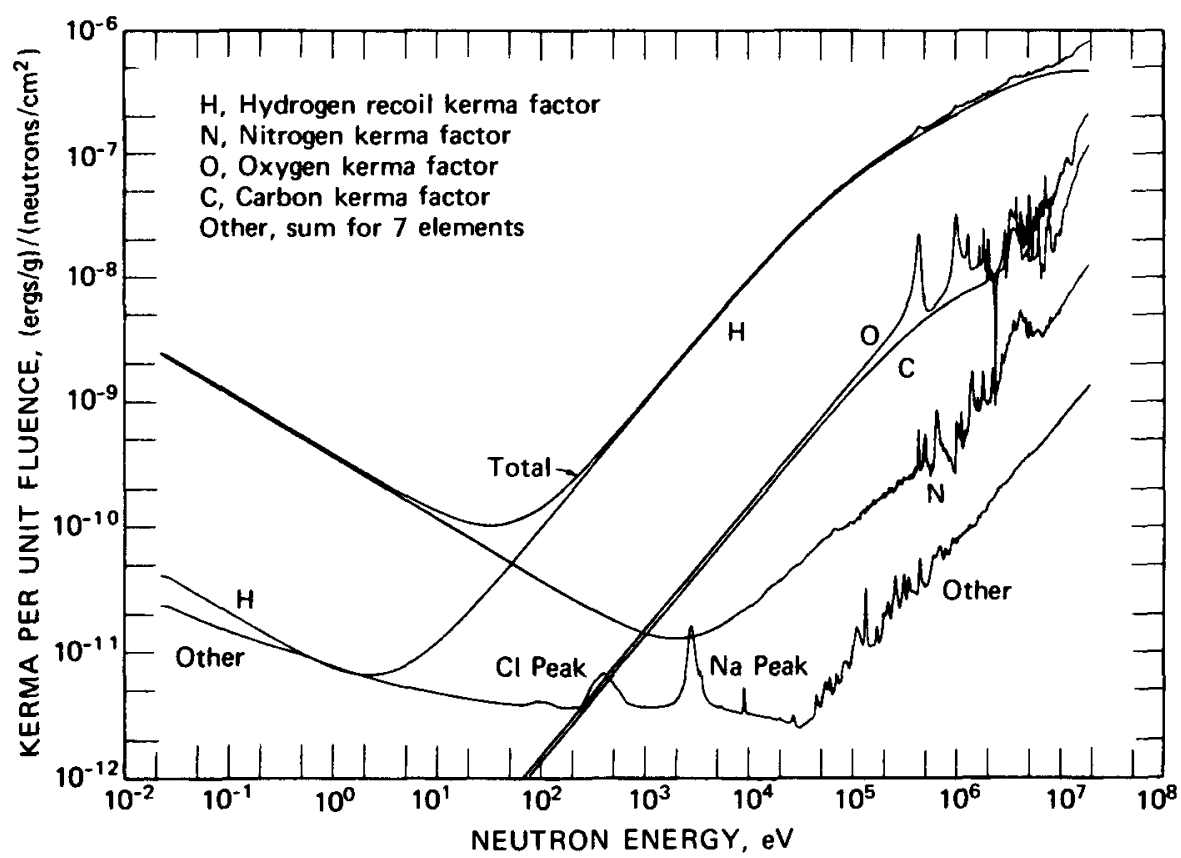

Fig. 3.13-Neutron-fluence-to-kerma factors for standard-man model and for elements contributing to standard-man model. (From Ritts et al. ${ }^{20}$ )

irradiated material can be found by summing the averages of the kinetic energies imparted to the struck nuclei and the energies associated with charged particles that are emitted.

The kerma per unit fluence can be expressed as

$$
R_{K}=\sum_{i} \sum_{j} C N_{i}(E) \sigma_{i j}(E) E_{i j}
$$

where $N_{i}=$ number density of element $i$ (atoms $/ \mathrm{g}$ )

$\sigma_{i j}=$ microscopic cross section of reaction $j$ for element $i\left(\mathrm{~cm}^{2} /\right.$ atom)

$E_{i j}=$ average energy deposited by reaction $j$ for element $i(\mathrm{MeV})$

$C=1.602 \times 10^{-6}(\mathrm{ergs} / \mathrm{MeV})$

Since the kerma values are given per unit fluence, they are fluence-tokerma conversion factors (usually referred to simply as kerma factors). Such kerma factors for the standard-man model are plotted in Fig. 3.13 and tabulated in Appendix B along with kerma factors for the individual elements making the greatest contributions to the total factor.

Kerma-rate factors for monoenergetic gamma rays incident on carbon, air, and a four-element tissue model were calculated by Henderson ${ }^{19}$ and are 


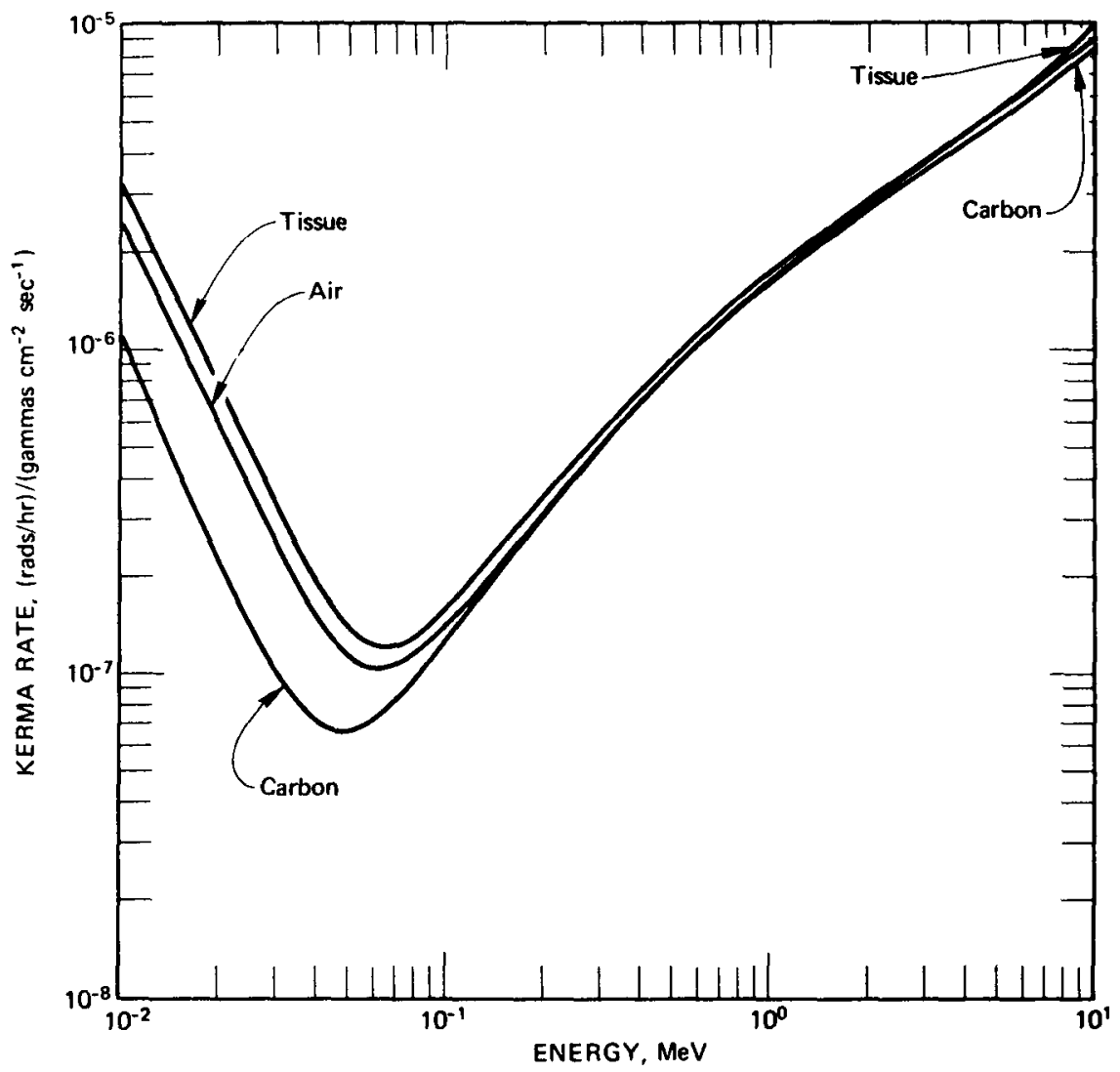

Fig. 3.14-Gamma-ray kerma-rate factors for air, carbon, and tissue. (From Henderson. ${ }^{19}$ )

plotted in Fig. 3.14. (Henderson's gamma-ray results, like his neutron results, were actually reported as absorbed dose, the term kerma not having been introduced yet.)

\subsubsection{Exposure}

Exposure is a term that should be used only for gamma rays. Formerly called exposure dose, it came into common use after the problem of specifying biological dose associated with $\mathrm{X}$ rays was first encountered. As recommended by the ICRU, exposure $(X)$ describes the deposition of energy in air by electromagnetic radiation and is defined by

$$
X \equiv \frac{\Delta Q}{\Delta M}
$$


where $\Delta Q$ is the sum of the electrical charges of all the ions of one sign produced in air when all electrons liberated by photons in a volume of air $\Delta V$ are completely stopped $\dagger$ and $\Delta M$ is the mass of the material contained in the volume element $\Delta V$. Exposure rate is given by $\Delta X / \Delta t$, where $\Delta X$ is the increment of exposure during the increment of time $\Delta t$.

The unit of exposure is the roentgen (R). It is the quantity of $X$ or gamma radiation that produces, in air, ions carrying 1 electrostatic unit (esu) of charge per $0.001293 \mathrm{~g}$ of dry air (or $2.58 \times 10^{-4}$ coulomb $/ \mathrm{kg}$ ). In terms of ergs, the roentgen is equal to 87.7 ergs per gram of air or 96 ergs per gram of tissue. For gamma rays above $3 \mathrm{MeV}$ in energy, the range of secondary electrons in low-atomic-number materials becomes comparable with the relaxation length of the gamma rays. Consequently the ionization produced in a small volume is no longer a sole measure of the intensity of the radiation at that point. The ICRU does not recommend the use of the roentgen above $3 \mathrm{MeV}$; in practice, however, it is still used above $3 \mathrm{MeV}$ with instruments calibrated in terms of energy absorption in air.

Since $1 \mathrm{rad}$ is equal to 100 ergs per gram of irradiated material, the roentgen and the rad are frequently interchanged when tissue exposure is referred to, the difference of $4 \%$ being no real consequence in shielding calculations. Strictly speaking, however, the rad should be reserved for describing an absorbed dose.

Away from boundaries the distinction between kerma and exposure loses relevance if only the three principal processes (Compton scattering, photoelectric effect, and pair production) are considered and if the photoelectric effect and pair production are assumed to be absorptions. For this simple and widely used model, $\$$ the fluence-to-exposure conversion factor is proportional to the product of the photon energy and the energy-deposition coefficient, in which case exposure, absorbed dose, first-collision dose, and kerma in air are all equal in magnitude.

In some calculations, however, a slightly more complex model is used in which pair production contributes to scattering as well as absorption. As in the preceding model the total kinetic energy of the electron pair created in the pair-production process is assumed to be absorbed at the point of its emission. However, the two $0.51-\mathrm{MeV}$ gamma rays produced by the

tThe ionization that would be produced by the bremsstrahlung associated with secondary electrons is not included in $\Delta Q$. Except for this small difference, which is insignificant for photon energies less than $15 \mathrm{MeV}$, exposure is equivalent to kerma.

$\ddagger$ For this and the subsequent model, the bremsstrahlung radiation that would be produced by the secondary electrons (and positrons) is assumed to be immediately absorbed. 
annihilation of the positron are treated as scattered gamma rays, and pair production is also viewed as a scattering process. Absorption from pair production is described in terms of a modified cross section, which is given by

$$
\mu_{p p_{m}}=\mu_{p p}\left(1-\frac{1.022}{E}\right) \quad(\text { for } E>1.022 \mathrm{MeV})
$$

where $E$ is the energy of the incident photon $(\mathrm{MeV})$.

\subsubsection{RBE Dose; Dose Equivalent}

Whenever the biological response of a human organ to radiation exposure is of concern, merely knowing the absorbed dose (energy deposited) is not sufficient to predict the biological consequence. Biological responses vary both with the nature and energy of the radiation and with the composition and function of the irradiated organs. Thus, when an organ is exposed to a mixed radiation field or to a field of radiation comprised of one type of particle with various energies, the energy deposited by each type of particle of a given energy must be weighted by some factor before the total biological dose received by the organ can be determined by a summing of the individual contributions.

For a specific biological effect in a particular mass, the weighting factor is mainly a function of the linear rate of energy transfer (LET) to the system by charged particles set in motion by the interactions of incident radiation. The LET of a material is related to the linear stopping power, but the concepts are somewhat different. The linear stopping power is the average energy loss per unit path length by a charged particle in traversing a medium regardless of where the energy is absorbed. The LET, however, refers to energy imparted within a limited volume. These two quantities are equal only in the special case when the LET includes the absorption of all secondary particles, in which case it is called LET $_{\infty}$. These quantities are described more completely by Trubey. ${ }^{16}$

The LET-dependent weighting factor is called the relative biological effectiveness (RBE) and is defined as

$$
\begin{aligned}
\mathrm{RBE}= & \text { (rads of standard radiation producing a given } \\
& \text { biological effect }) /(\text { rads of another type of radiation } \\
& \text { producing the same effect })
\end{aligned}
$$

The standard radiation referred to is $X$ rays of $250 \mathrm{keV}$ energy, and thus by definition the RBE for $250-\mathrm{keV} X$ rays is 1 . 
When a dose given in rads is weighted by an $\mathrm{RBE}$ value, the resulting dose, sometimes referred to as the RBE dose, is given in rems, a unit derived from the term roentgen equivalent man:

$\mathrm{RBE}$ dose in rems $\equiv$ dose in rads $\times \mathrm{RBE}$

It follows that for $250-\mathrm{keV} \mathrm{X}$ rays the rad dose and the rem dose are numerically equal.

\begin{tabular}{ccc}
$\begin{array}{c}\text { Table 3.6- QUALITY FACTOR AS A FUNCTION } \\
\text { OF LINEAR ENERGY TRANSFER AND } \\
\text { ION-PAIR FORMATION }\end{array}$ \\
\hline \multicolumn{3}{c}{$\begin{array}{c}\text { Average specific } \\
\text { ionization, }\end{array}$} \\
$\begin{array}{r}\text { Qverage LET, } \\
\text { ion pairs/ } \mu \text { of water } \\
\mathrm{keV} / \mu \text { of water }\end{array}$ \\
\hline 1 & $<100$ & $<3.5$ \\
2 & 200 & 7.0 \\
5 & 650 & 23 \\
10 & 1500 & 53 \\
20 & 2000 & 175 \\
\end{tabular}

The rem is quantitatively defined as the absorbed dose due to any ionizing radiation that has the same biological effectiveness as 1 rad of $X$ rays with an average specific ionization of 100 ion pairs per micron of water in terms of its air equivalent. It turns out that $\mathrm{X}$ rays and gamma rays generally do not exceed this specific ionization (LET $<3.5 \mathrm{keV} / \mu$ ), which is considered to be the boundary between low-LET and high-LET radiation and thus to be the limiting condition for $\mathrm{RBE}=1$.

In the past $R B E$ has been used in the fields of radiobiology and radiation protection, but this generated concern because of the differences in application and, to some extent, in concept. Consequently ICRU recommends that RBE be used only for correlating radiobiological experiments and that a new term, the quality factor (QF), be used in the field of radiation protection. Quality factors are actually those values of $R B E$ which are intended to embrace all effects that are hazardous to human beings. In other words, QF values are not related to specific organs of the body as are some of the RBE values. When a $Q F$ value is used to weight absorbed dose, 
the resulting dose, in rems, is identified as the dose equivalent (DE). Dose equivalents, like RBE doses, are assumed to be additive.

Although the QF is defined in purely physical terms (that is, as a function of LET only, which, in turn, is a function of the number of ion pairs produced per centimeter of travel by charged particles), the basis of the legislated value is biological. Table 3.6 shows the recommendations of the International Commission on Radiological Protection (ICRP) 1,22 for the relation between $\mathrm{QF}$ and both LET and ion-pair formation.

The ICRP recommends a QF of 1 for $X$ rays, gamma rays, electrons, and positrons of any specific ionization; ${ }^{21} \mathrm{QF}$ values for neutrons, protons, and heavy recoil nuclei vary with energy. The ICRP recommendations for $Q F$ values for neutrons between 0.01 and $1000 \mathrm{MeV}$ are plotted in Fig. 3.15. Tabulated values for lower energy neutrons are also shown. These values are

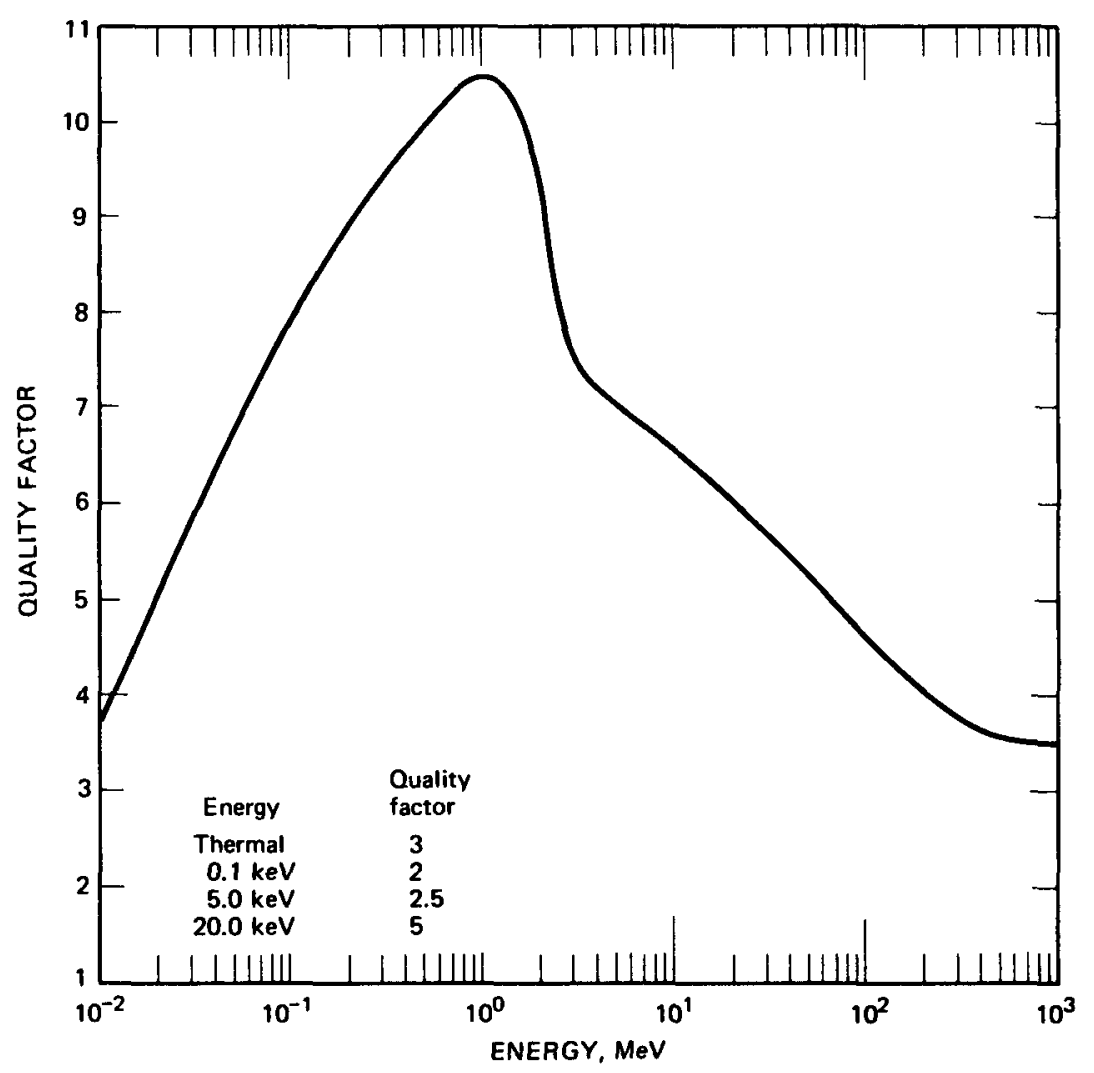

Fig. 3.15-Quality factors for neutrons. 
based on the calculated results of Snyder and Neufeld. ${ }^{23}$ As a rough and conservative approximation, a $\mathrm{QF}$ of 10 is applicable to protons and a $\mathrm{QF}$ of 20 can be used for heavy recoil nuclei.

The preceding QF values are assumed to be applicable for whole-body irradiation. When specific organs are being considered, additional modifying factors may be needed. For example, ICRP specifies that, when the lens of the eye is being irradiated, a modifying factor of 3 should be used if the $\mathrm{QF} \geqslant 10$ and a modifying factor of 1 should be used when the $\mathrm{QF}=1$. Strangely enough, no recommendations are given for $1<\mathrm{QF}<10$. Presumably, linear interpolation is to be used between these limits.

When the dose in an organ is nonuniform, a dose distribution factor (DF) should also be applied. These factors cannot be established with certainty, and only one, the relative damage factor applied in calculating the dose equivalent in bone from internal radiation, is in current use.

In general, the shielding community has accepted the use of QF and DE, as is reflected by the latest shielding literature. From a practical viewpoint the change merely amounts to replacing RBE by QF and calling the product of the quality factor and absorbed dose the dose equivalent (DE) instead of the RBE dose. Consequently no confusion should exist when the older literature is consulted. It should be kept in mind, however, that $Q F$ values are chosen by such groups as the ICRP and thus are more subject to change than would be an RBE value based on experimental data.

All quality factors and other modifying factors are intended solely for chronic exposure to low-level radiation fields, with genetic damage being the hazard considered. High accidental exposures must be assessed on the basis of particular circumstances. The ICRP has no recommendations for such exposures, but in many cases the absorbed dose will give a better indication of the biological risks than will the dose equivalent. In other words, for acute effects due to massive exposures, the QF should be taken as unity, which partially results because energy may be wasted from the standpoint of the production of biological effects. Consequently, for military applications during war, when early death or incapacitation is the effect to be considered, the dose, in rems, may be taken as numerically equal to the absorbed or physical dose until better information becomes available.

\subsubsection{Maximum Absorbed Dose; Maximum Dose Equivalent}

As was pointed out in the introduction to this section, the usual shielding calculation provides a detailed description of the unperturbed radiation field (free field): that is, perturbations of the field by a human 
body are not considered. This greatly simplifies the analysis without seriously compromising the overall accuracy. However, the results must somehow be related to the hazards to a human being if he were exposed to such a radiation field.

A correlation between the unperturbed radiation field and the dose in a human body has been accomplished through the use of slab and cylindrical phantoms, which have dimensions and compositions resembling those of the human body. Calculations (or measurements) of the absorbed dose or the biological dose were made as a function of depth in the phantom for given monoenergetic neutrons or gamma rays incident on the phantom. The incident angular distribution was a monodirectional beam or an isotropic flux. (Calculations for neutrons include the contribution to the dose by secondary gamma rays.) The maximum values in the depth-dose distributions were identified as the maximum absorbed dose and maximum dose equivalent for the absorbed (physical) and biological doses, respectively. Since these values were obtained and reported on the basis of a unit particle entering the phantom, they can be used as response functions to relate an unperturbed field of mixed radiation to the maximum dose that would be received by some part of the body. Use of these maximum values is dictated by a conservative design philosophy that does not allow the permissible dose to be exceeded at any point in the body.

(a) Neutron Doses in Phantoms. The first dose calculations in a phantom were performed by Snyder and Neufeld $^{23}$ for a beam of monoenergetic neutrons normally incident on a phantom represented by a slab of tissue. The slab, infinite in the transverse directions, was assumed to be $30 \mathrm{~cm}$ thick. Snyder and Neufeld determined the distribution of the absorbed dose through the slab and found that the maximum dose occurred at nearly the surface or within a few centimeters of the surface. In these calculations only neutron captures and elastic scatterings were considered, which limited the contribution by secondary gamma rays to capture gamma rays. The resulting maximum absorbed doses and biological doses as a function of the incident-neutron energy are shown in Table 3.7. These values have been used widely in reactor-shield design to convert neutron fluences to doses and have become virtual standards since these dose equivalents have been stipulated for use by the Federal Register. ${ }^{24}$

In similar calculations, performed later by Auxier, Snyder, and Jones, ${ }^{25}$ the infinite slab was replaced by a finite cylindrical phantom that more nearly represented a human body. The phantom was $60 \mathrm{~cm}$ high and $30 \mathrm{~cm}$ in diameter. It was divided into annular shells cut into $60^{\circ}$ angular sectors 
and five layers $15 \mathrm{~cm}$ high. This division yielded 150 volume elements. The maximum doses (averaged for a volume element) occurred in the outermost volume element at the midplane on the side facing the beam. These maximum doses are given in Appendix C, Table C.1.

Table 3.7-MAXIMUM ABSORBED DOSE AND MAXIMUM DOSE EQUIVALENT FOR MONOENERGETIC NEUTRONS INCIDENT ON A SLAB TISSUE PHANTOM $†$

\begin{tabular}{cccc}
\hline $\begin{array}{c}\text { Neutron energy, } \\
\text { MeV }\end{array}$ & $\begin{array}{c}\text { Maximum absorbed } \\
\text { dose, } \ddagger \\
\text { mrad neutron }\end{array} \mathrm{cm}^{-2}$ & $\begin{array}{c}\text { Maximum dose } \\
\text { equivalent, } \S \\
\text { mrem neutron }\end{array} \mathrm{cm}^{-2}$ & $\begin{array}{c}\text { Effective } \\
\text { QF }\end{array}$ \\
\hline $\begin{array}{c}\text { Thermal } \\
0.0001\end{array}$ & $3.2(-7) \S$ & $1.04(-6)$ & 3.25 \\
0.005 & $6.9(-7)$ & $1.39(-6)$ & 2.01 \\
0.02 & $5.7(-7)$ & $1.22(-6)$ & 2.14 \\
0.1 & $5.7(-7)$ & $2.35(-6)$ & 4.12 \\
0.5 & $1.10(-6)$ & $8.3(-6)$ & 7.55 \\
1.0 & $2.4(-6)$ & $2.30(-5)$ & 9.61 \\
2.5 & $3.8(-6)$ & $3.80(-5)$ & 10.0 \\
5.0 & $4.3(-6)$ & $3.41(-5)$ & 7.93 \\
7.5 & $5.8(-6)$ & $3.80(-5)$ & 6.55 \\
10 & $7.1(-6)$ & $4.16(-5)$ & 5.85 \\
& $7.0(-6)$ & $4.16(-5)$ & 5.94 \\
\hline
\end{tabular}

†From Snyder and Neufeld. ${ }^{23}$

$\ddagger$ Multiply by 3600 to convert to $(\mathrm{mrad} / \mathrm{hr}) /\left(\right.$ neutron $\left.\mathrm{cm}^{-2} \mathrm{sec}^{-1}\right)$ or to (mrem/hr)/(neutron $\left./ \mathrm{cm}^{-2} \mathrm{sec}^{-1}\right)$.

$\S$ Read: $3.2 \times 10^{-7}$, etc.

The effective quality factors shown in Tables 3.7 and C. 1 are the ratios of the maximum dose equivalent to the maximum absorbed dose for a given incident-neutron energy and phantom configuration. The magnitude of the effective quality factor is very close to but usually slightly less than that recommended by the ICRP (see Fig. 3.15). The differences in the values would be expected since the values recommended by the ICRP consider only the initial collision of the neutron whereas the values of the effective quality factors represent an average of all collisions experienced by the neutron within the phantom. 
(b) Gamma-Ray Doses in Phantoms. Claiborne and Trubey ${ }^{2}$ recently calculated the dose delivered to a phantom by monoenergetic gamma rays. Using the discrete-ordinates method, with some checks by the Monte Carlo method (both of these transport methods are described in the next chapter), they calculated the dose distributed in a 30 -cm-thick infinite-slab phantom having the standard-man composition shown in Appendix B, Table B.2. Their maximum dose rates, which occurred at nearly the surface or within the first $2 \mathrm{~cm}$ of the surface, are compared in Fig. 3.16 with the experimental results of Jones. ${ }^{27}$

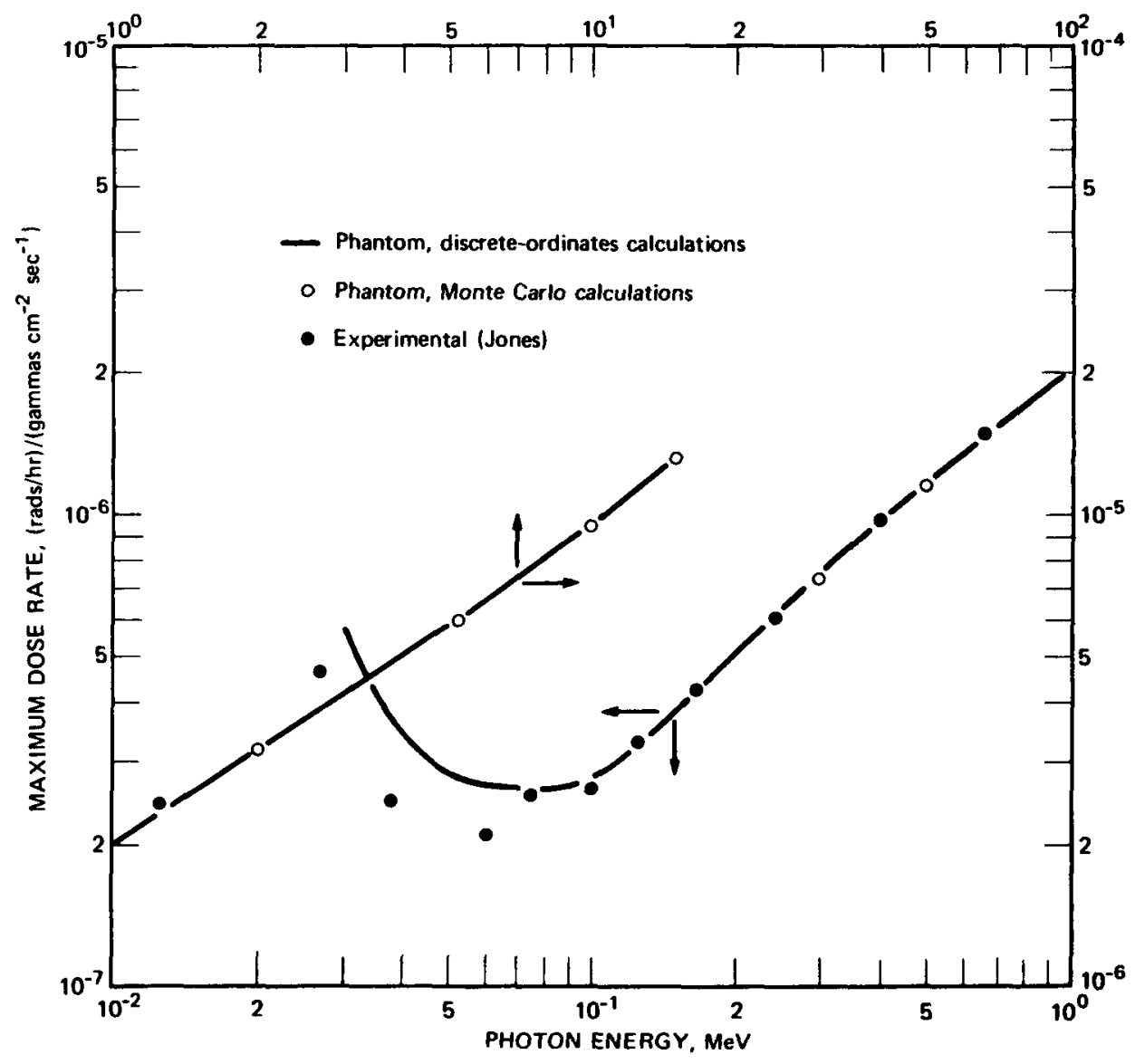

Fig. 3.16-Maximum gamma-ray dose rate in slab phantom of standard-man composition. Comparison with kerma calculations and with phantom measurements. (From Claiborne and Trubey. ${ }^{26}$ ) 


\subsubsection{Multicollision Dose}

The term multicollision dose, which until recently has been used solely for neutron exposure, is not recognized by the ICRU. The multicollisiondose concept was developed by health physicists in an effort to relate the neutron flux or fluence incident upon a configuration of tissue representing the human body to the absorbed dose or to the dose equivalent. The multicollision dose could, in turn, be related to a maximum permissible dose (MPD) established by shield-design criteria. The term has tacitly been defined by common usage in shield design as the sum of the maximum dose equivalents (or maximum absorbed doses) delivered by each neutron-energy group to a slab of tissue as defined by Snyder and Neufeld ${ }^{2} 3$ This includes the dose due to secondary gamma rays produced by the neutrons in the slab. This dose is calculated by

$$
\text { Multicollision dose }=\sum_{g} \psi_{g} D_{\max }\left(E_{g}\right)
$$

where $\psi_{g}$ is the group fluence and $D_{\max }\left(E_{g}\right)$ is the maximum dose equivalent (or maximum absorbed dose) in the slab for an incident neutron of the energy corresponding to the gth neutron group.

Gamma-ray multicollision doses are similarly calculated with Eq. 3.3-16, with, of course, $\psi_{g}$ and $D_{\max }\left(E_{g}\right)$ being the appropriate values for gamma rays.

The total dose for a mixed-radiation field incident on a tissue slab is determined by adding the neutron and gamma-ray multicollision doses obtained separately for the slab.

Although the concept of multicollision doses, or dose rate, is not recognized by the ICRU, it seems clear that, to fully implement the trend to preciseness in the fields of radiobiology and radiation protection, the dose calculated in shield design should be given recognition by some official body. Perhaps the term multicollision dose can be retained for the neutron-induced contribution, and the term total multicollision dose can be used when the incident gamma-ray contribution is also included. Or perhaps an entirely new term could be introduced; several have been suggested but none have been accepted as yet. 


\section{REFERENCES}

1. A. Foderaro, The Elements of Neutron Interaction Theory, The MIT Press, Cambridge, Mass., 1971.

2. E. P. Blizard (Ed.), Reactor Handbook, 2nd ed., Vol. III, Part B, Shielding, Interscience Publishers, a division of John Wiley \& Sons, Inc., New York, 1962.

3. H. Goldstein, Fundamental Aspects of Reactor Shielding, Addison-Wesley Publishing Company, Inc., Reading, Mass., 1959 (out of print); now available from Johnson Reprint Corporation, New York.

4. J. H. Hubbell, Photon Cross Sections, Attenuation Coefficients, and Energy Absorption Coefficients from $10 \mathrm{keV}$ to $100 \mathrm{GeV}$, Report NSRDS-NBS-29, National Bureau of Standards, 1969.

5. J. H. Hubbell and M. J. Berger, in Engineering Compendium on Radiation Shielding, R. G. Jaeger (Ed.), Vol. 1, p. 167, Springer-Verlag, New York, 1968.

6. A. T. Nelms, Graphs of the Compton Energy-Angle Relationship and the KleinNishina Formula from $10 \mathrm{keV}$ to $500 \mathrm{MeV}$, National Bureau of Standards, Circular 542, Superintendent of Documents, U. S. Government Printing Office, Washington, D. C., 1953.

7. M. D. Goldberg, V. M. May, and J. R. Stehn, Angular Distributions in NeutronInduced Reactions, USAEC Report BNL-400, Vol. 2, Brookhaven National Laboratory, October 1962.

8. E. S. Troubetzkoy, M. C. Berten, J. Celnik, H. Flesher, G. Grochowski, M. H. Kalos, and J. H. Ray, Neutron Cross Sections of ${ }^{238} \mathrm{U},{ }^{235} \mathrm{U},{ }^{237} \mathrm{U},{ }^{239} \mathrm{U},{ }^{234} \mathrm{U},{ }^{236} \mathrm{U}$,

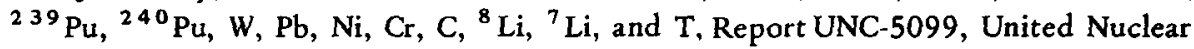
Corporation, 1964.

9. K. J. Yost, A Method for the Calculation of Neutron Capture Gamma-Ray Spectra, Nucl. Sci. Eng., 32: 62 (1968).

10. R. J. Howerton and E. F. Plechaty, A Formalism for Calculation of Neutron-Induced Gamma-Ray Production Cross Sections and Spectra, Nucl. Sci. Eng., $32: 178$ (1968).

11. Nuclear Data, A3: $367-650$ (1967); A5: 1-242 (1968); A5: 243-431 (1969).

12. D. J. Hughes and R. B. Schwartz, Neutron Cross Sections, USAEC Report BNL-325 and Supplements, 2nd ed., Brookhaven National Laboratory, 1958-1966.

13. K. J. Yost and M. Solomito, Sensitivity of Gamma-Ray Dose Calculations to the Energy Dependence of Gamma-Ray Production Cross Sections, in Neutron Cross Sections and Technology, Proceedings of a Conference, Washington, D. C., Mar. 4-7, 1968, D. T. Goldman (Ed.), NBS-SPEC.PUBL.-299 (Vol. 1), p. 53, Superintendent of Documents, U. S. Government Printing Office, Washington, D. C., 1968.

14. Nuclear Data Sheets, originally appearing as the Annual Cumulation of Nuclear Data in Nuclear Science Abstracts, now published as Nuclear Data, Section B, by Academic Press, Inc., New York.

15. International Commission on Radiation Units and Measurements, Radiation Quantities and Units, Report ICRU-11, Sept. 1, 1968.

16. D. K. Trubey, Use of ICRU-Defined Units in Shielding, USAEC Report ORNL RSIC-16, Oak Ridge National Laboratory, 1968. 
17. J. A. Auxier, Kerma Versus First Collision Dose: The Other Side of the Controversy, Health Phys., 17: 342 (1969).

18. W. S. Snyder, Protection Against Neutron Radiation up to $30 \mathrm{MeV}$, Report of the National Council on Radiation Protection and Measurements, National Bureau of Standards, Handbook 63, p. 6, Superintendent of Documents, U.S. Government Printing Office, Washington, D. C., 1967.

19. B. J. Henderson, Conversion of Neutron or Gamma Ray Flux to Absorbed Dose Rate, USAEC Report XDC-59-8-179, General Electric Company, Aug. 14, 1959.

20. J. J. Ritts, E. Solomito, and P. N. Stevens, Calculation of Neutron Fluence-to-Kerma Factors for the Human Body, USAEC Report ORNL-TM-2079, Oak Ridge National Laboratory, 1968.

21. Recommendations of the International Commission on Radiological Protection, ICRP Publication 4, Report of Committee IV (1953-1959) on "Protection Against Electromagnetic Radiation Above $3 \mathrm{MeV}$ and Electrons, Neutrons and Protons," The Macmillan Company, New York, 1964.

22. Report of the RBE Committee on the International Commission on Radiological Protection and on Radiological Units and Measurements, Health Phys., 9: 357-386 (1963).

23. W. S. Snyder and J. Neufeld, On the Passage of Heavy Particles Through Tissue, Radiat. Res., 6: 67 (1957); also reprinted in NBS Handbook 63.

24. Standards for Protection Against Radiation, Federal Register, Title 10, Part 20, 1966.

25. J. A. Auxier, W. S. Snyder, and T. D. Jones, Neutron Interactions and Penetration in Tissue, in Radiation Dosimetry, Vol. I, Chap. 6, F. H. Attix and W. C. Roesch (Eds.), Academic Press, Inc., New York, 1968.

26. H. C. Claiborne and D. K. Trubey, Dose Rates in a Slab Phantom from Monoenergetic Gamma Rays, Nucl. Appl. Technol., 8(5): 450-455 (1970).

27. A. R. Jones, Proposed Calibration Factors for Various Dosimeters at Different Energies, Health Phys., 12: 663-671 (1966).

\section{EXERCISES}

3.1 Assume that air has the following composition by volume: nitrogen, $78.5 \%$; oxygen, $20.99 \%$; and argon, $0.96 \%$. Calculate, at standard temperature and pressure $\left(273^{\circ} \mathrm{K}\right.$, $76 \mathrm{~cm} \mathrm{Hg}$ ): (a) The partial density in air of each constituent. (b) The weight percent of each constituent. (c) The atomic density in air of each constituent. (d) The electron density of air. (e) The average atomic number of air. (Densities at STP are as follows: nitrogen, $0.00125 \mathrm{~g} / \mathrm{cm}^{3}$; oxygen, $0.00143 \mathrm{~g} / \mathrm{cm}^{3}$; argon, $0.00178 \mathrm{~g} / \mathrm{cm}^{3}$; and air, $0.00129 \mathrm{~g} / \mathrm{cm}^{3}$. Atomic numbers and weights, respectively, are nitrogen, 7 and 14.0; oxygen, 8 and 16.0; and argon, 18 and 39.9.)

3.2 It is often convenient to treat mixtures such as air and compounds such as water as homogeneous media made up of one pseudoelement. For water, compute the following properties of the pseudoelement: (a) Atomic density. (b) Electron density. (c) Atomic number. (Assume the density of water to be $1.0 \mathrm{~g} / \mathrm{cm}^{3}$.) 
3.3 At 0.10 and $3.0 \mathrm{MeV}$, Hubbell ${ }^{4}$ lists the photon microscopic cross sections shown in the table for Exercise 3.3.

TABLE FOR EXERCISE 3.3, PHOTON CROSS SECTIONS

\begin{tabular}{|c|c|c|c|c|}
\hline & \multicolumn{2}{|c|}{$\sigma_{s}$, b/atom } & \multirow[b]{2}{*}{$\begin{array}{c}\sigma_{p e} \\
\text { b/atom }\end{array}$} & \multirow[b]{2}{*}{$\begin{array}{c}\sigma_{p p} \\
\text { b/atom }\end{array}$} \\
\hline & $\begin{array}{l}\text { With } \\
\text { coherent }\end{array}$ & $\begin{array}{l}\text { Without } \\
\text { coherent }\end{array}$ & & \\
\hline \multicolumn{5}{|l|}{$\mathrm{E}=0.1 \mathrm{MeV}$} \\
\hline Nitrogen & 3.54 & 3.45 & 0.041 & \\
\hline Oxygen & 4.06 & 3.94 & 0.071 & \\
\hline Argon & 9.85 & 8.87 & 3.6 & \\
\hline Hydrogen & 0.493 & 0.493 & & \\
\hline \multicolumn{5}{|l|}{$\mathrm{E}=3.0 \mathrm{MeV}$} \\
\hline Nitrogen & & 0.806 & & 0.025 \\
\hline Oxygen & & 0.921 & & 0.032 \\
\hline Argon & & 2.07 & & 0.17 \\
\hline Hydrogen & & 0.1151 & & 0.00052 \\
\hline
\end{tabular}

(a) Explain the blanks in the table.

(b) For nitrogen at STP, compute the total mass attenuation coefficient $\left(\mathrm{cm}^{2} / \mathrm{g}\right)$ and the linear attenuation coefficient $\left(\mathrm{cm}^{-1}\right)$ without coherent scattering at $0.1 \mathrm{MeV}$.

(c) Using the results of Problem 3.1, compute the total mass and linear attenuation coefficients for air at $3.0 \mathrm{MeV}$.

(d) What is the mean free path in air for $3.0 \mathrm{MeV}$ photons? Express your answer in meters.

(e) Compute the two scattering, the photoelectric, and the pair-production microscopic cross sections at 0.1 and $3.0 \mathrm{MeV}$ for the water pseudoelement. Express the results in terms of barns per molecule.

3.4 A thin $\left(0.04 \mathrm{~g} / \mathrm{cm}^{2}\right)$ aluminum disk $2 \mathrm{~cm}$ in radius is subjected on one side to a monodirectional photon flux density of $10^{10}$ photons $\mathrm{cm}^{-2} \mathrm{sec}^{-1}$, normal to the disk. The photon energy is $0.10 \mathrm{MeV}$. Hubbell ${ }^{4}$ lists the following microscopic cross sections for aluminum whose density is $2.7 \mathrm{~g} / \mathrm{cm}^{3}$ :

$$
\begin{aligned}
& \sigma_{\text {coh }}=6.79 \mathrm{~b} / \mathrm{atom} \\
& \sigma_{\text {incoh }}=6.41 \mathrm{~b} / \text { atom } \\
& \sigma_{p e}=0.78 \mathrm{~b} / \mathrm{atom}
\end{aligned}
$$

Compute: (a) The total reaction rate (photons per second) in the disk. (b) The rate at which photons are absorbed on their first collision in the disk. (c) The first-collision 
scattering rate in the disk. (d) The exit flux density of $0.1-\mathrm{MeV}$ photons. (e) Approximately what fraction of the disk's atoms are reacting with the photon beam per unit time?

3.5 For scattering angle $\theta(0 \leqslant \theta \leqslant \pi / 4)$, the differential scattering cross section for Compton scattering of $1.25-\mathrm{MeV}$ photons is closely approximated by

$$
\sigma(\theta)=\left(29.5-78.3 \cos \theta+56.6 \cos ^{2} \theta\right) \times 10^{-26} \mathrm{~cm}^{2} \text { electron }{ }^{-1} \text { steradian }^{-1}
$$

For the disk of Problem 3.4, compute the flux density of singly scattered photons at a point $P$ located $2 \mathrm{~cm}$ from the center and on the opposite side of the disk from a normally incident $1.25-\mathrm{MeV}$ photon flux density of $10^{10}$ photons $\mathrm{cm}^{-2} \mathrm{sec}^{-1}$.

3.6 The differential speed distribution of thermal neutrons is given by

$$
n(v) d v=A v^{2} e^{-m v^{2} / 2 k T} d v \text { neutrons } / \mathrm{cm}^{3} \text { whose speed is in } d v \text { about } v
$$

where $A$ and $k$ are constants, $T$ is the neutron temperature, and $m$ is the neutron mass. Show that, for a material whose cross section varies as $1 / v[\sigma(v)=C / v]$, the average cross section for the reaction rate of neutrons with this speed distribution is given by

$$
\bar{\sigma}=C\left(\frac{m \pi}{8 k T}\right)^{1 / 2} \quad(C \text { is a constant })
$$

and thus the energy for which $\sigma(v)=\bar{\sigma}$ is $4 k T / \pi$.

3.7 The accompanying table gives the densities and mass energy transfer coefficients $\mu_{k} / \rho$ for four materials. Compute the kerma rate $\left(\mathrm{ergs} \mathrm{g}^{-1} \mathrm{sec}^{-1}\right)$ in a small volume of each material ( $a, b, c, d$ ) located in a vacuum $100 \mathrm{~cm}$ from a $1-\mathrm{Ci}$ isotropic ${ }^{60} \mathrm{Co}$ point source.

TABLE FOR EXERCISE 3.7

\begin{tabular}{lcc}
\hline & $\begin{array}{c}\mu_{k} / \rho, \\
\mathrm{cm}^{2} / \mathrm{g}\end{array}$ & $\rho, \mathrm{g} / \mathrm{cm}^{3}$ \\
\hline & 0.0268 & 0.00129 \\
(a) Air & 0.0300 & 1.00 \\
(b) Water & 0.030 \\
(c) Iron & 0.0253 & 7.90 \\
(d) Lead & 0.0350 & 11.35 \\
\hline
\end{tabular}

(e) Compute the absorbed dose rate in tissue, where $\mu_{k} / \rho=0.029 \mathrm{~cm}^{2} / \mathrm{g}$. Assume that charged-particle equilibrium exists and bremsstrahlung is negligible. Give the answer in rads per hour. 
3.8 In Sec. 3.2.1, the energy-angle relation, expressed in units of Compton wavelength, was noted to be

$$
\lambda^{\prime}-\lambda=1-\cos \theta
$$

and the differential angle scattering cross section was given as

$$
\sigma(\theta)=\frac{d \sigma}{d \Omega}=\frac{3}{16 \pi}\left(\frac{\lambda}{\lambda^{\prime}}\right)^{2}\left(\frac{\lambda^{\prime}}{\lambda}+\frac{\lambda}{\lambda^{\prime}}-\sin ^{2} \theta\right) \text { T.U. electron }{ }^{-1} \text { steradian }^{-1}
$$

Show that, when reduced to its one independent variable, $\lambda^{\prime}$, the differential angle scattering distribution of photons of initial wavelength $\lambda$ is given by

(a) $\sigma(\theta)=\frac{3}{16 \pi}\left(\frac{\lambda}{\lambda^{\prime}}\right)^{2}\left[\frac{\lambda^{\prime}}{\lambda}+\frac{\lambda}{\lambda^{\prime}}+2\left(\lambda-\lambda^{\prime}\right)+\left(\lambda-\lambda^{\prime}\right)^{2}\right]$ T.U. electron ${ }^{-1}$ steradian $^{-1}$

and thus that

(b) $\sigma\left(\lambda^{\prime}\right)=\frac{d \sigma}{d \lambda^{\prime}}=\frac{3}{8}\left(\frac{\lambda}{\lambda^{\prime}}\right)^{2}$

$$
\times\left[\frac{\lambda^{\prime}}{\lambda}+\frac{\lambda}{\lambda^{\prime}}+2\left(\lambda-\lambda^{\prime}\right)+\left(\lambda-\lambda^{\prime}\right)^{2}\right] \text { T.U. electron }{ }^{-1} \text { unit wavelength }{ }^{-1}
$$

where $\sigma\left(\lambda^{\prime}\right) d \lambda^{\prime} / \sigma_{c}$ gives the probability that a photon of energy $\lambda$ which undergoes a Compton scatter has a scattered energy in wavelength interval $d \lambda^{\prime}$ about $\lambda^{\prime}$. 
8 


\section{Radiation Transport}

Radiation transport is to neutron or photon interactions what dynamics of rigid bodies is to particle kinematics. In each case the former represents the macroscopic manifestation of the latter. The principal task in shield analysis is to solve the transport equation. In the following sections we will discuss some simple concepts and then develop the Boltzmann transport equation, which is not so simple. Subsequent sections are devoted to descriptions of several techniques for solving this equation by direct analysis and by alternate approaches. The Monte Carlo method of solution has been so successful in reactor shielding that we have devoted Chap. 5 exclusively to it.

In many instances solutions are tabulated. Thus a shield designer must, at most, choose one method from several methods of solving the transport problem or, frequently, apply a predecessor's result. The selection of one of these techniques for application to a particular problem depends on the nature of the problem, the accuracy required, and, mundanely, the time and funds available. Of course, the most economical technique for handling the problem and obtaining the necessary accuracy is the optimum choice. However, there is no simple way in which to make this choice. We have attempted to describe the theory involved in the various methods in a manner that will allow the shield designer to utilize his best judgment in making a good selection. Subsequent chapters discuss the application of these methods in various practical situations.

The radiation field at a point in space removed from the source can be divided into two components. The first is the uncollided, or, as it is sometimes called, the unscattered radiation (particles that arrive at the point without having undergone any interactions with the transporting medium). The second component is composed of the collided, or scattered, particles (particles that have undergone one or more interactions that have caused a change in direction or energy or both). Primary radiation particles and those generated in secondary reactions both may be so classified, although 
secondary particles are sometimes treated as a portion of the collided component of the primary beam.

Solution of the generalized Boltzmann equation can result in the determination of both components simultaneously; however, the calculation is often divided into two parts, which are performed separately. The first is a computation of the uncollided component, and the second, a calculation of the scattered component. Analysis of the uncollided component is rather straightforward even though complexities of energy spectra and geometry sometimes require use of numerical techniques. Analysis of the scattered component, on the other hand, may become quite complicated, and it is toward this task that most of the efforts in shield analysis are devoted. Following scatter, a particle or photon will have altered interaction probabilities owing to a shift in energy, and subsequent scatters will further alter these probabilities. The distribution of absorptions within the shield is also complicated by scattering since absorptions can occur following scattering.

As the radiation penetrates deeper into the shield, a higher percentage of the total flux density will be scattered radiation. The ratio of scattered to uncollided radiation may increase to some maximum value, which is a complex function of the incident spectrum and material cross sections, or (as is usually the case) the ratio may continue to increase indefinitely. Thus the thicker the shield, the more important the scattered radiation becomes.

As will be seen in subsequent sections, most practical situations involve complicated geometries and source characteristics, and calculation of the scattered component requires the use of simplifying assumptions.

There has been an active effort to develop and refine methods for predicting scattered radiation. It is important to remain in close touch with the technical literature because new developments appear frequently.

\subsection{FUNDAMENTAL CONSIDERATIONS}

The flux density at a detector point is influenced by two factors: the geometric relation between the source and the detector (including the source angular distribution) and the character of the material between the source and the detector. These two factors combine in a multiplicative fashion in simple geometries and are illustrated in the following example.

Consider the uncollided flux density at the detector point in Fig. 4.1. Even if the two slabs of materials are identical and the source strengths (particles $\mathrm{cm}^{-2} \mathrm{sec}^{-1}$ incident on the surface of the slab) are equal, the 


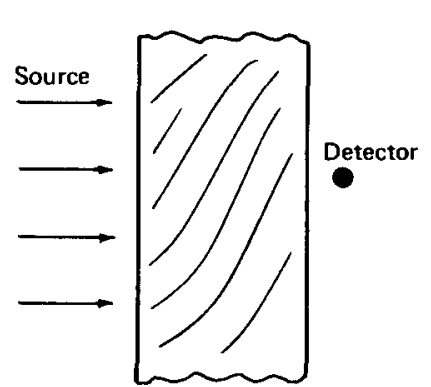

Monodirectional source

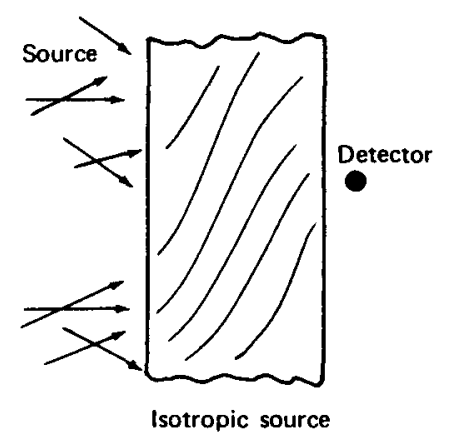

Fig. 4.1-Geometric differences due to sources.

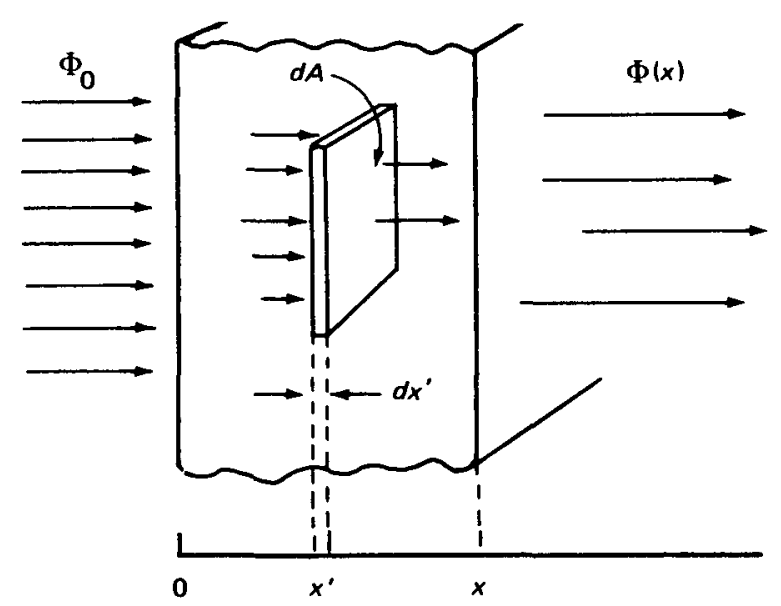

Fig. 4.2-Slab attenuation, monodirectional flux density.

radiation intensity will be different at the two detectors owing to the different source angular distributions, i.e., different geometries. On the other hand, if the material in one slab were altered, the detector response behind that slab would change with no change in source-detector geometry. This illustrates the attenuation (sometimes called barrier) factor.

In the absence of external forces, particles travel in straight lines. From this elementary statement it follows that the flux density in the beam of a plane monodirectional source does not vary with distance from the source unless, of course, it is attenuated by some material. This fact is useful in the analysis of individual components of a radiation field, and we apply it here to develop another elementary result.

Consider the slab geometry of Fig. 4.2, in which a monodirectional source of $\Phi_{0}$ particles $\mathrm{cm}^{-2} \mathrm{sec}^{-1}$ is normally incident on a slab. Every 
interaction within the slab removes a particle from the uncollided beam. We shall compute the flux density, $\Phi(x)$, of uncollided particles that emerge from the slab. Incident on the differential slab volume $d V$ of cross-sectional area $d A$ and thickness $d x^{\prime}\left(d V=d A d x^{\prime}\right)$ are $\Phi\left(x^{\prime}\right)$ particles $\mathrm{cm}^{-2} \mathrm{sec}^{-1}$, and emerging from $d V$ are $\Phi\left(x^{\prime}+d x^{\prime}\right)$ particles $\mathrm{cm}^{-2} \sec ^{-1}$. As shown in Chap. 3, the interaction rate per unit volume occurring in $d V$ is given by $\Sigma_{t} \Phi\left(x^{\prime}\right)$, where $\Sigma_{t}$ is the total linear attenuation coefficient (or total macroscopic cross section). Thus particle conservation in volume $d V$ demands that

$$
\Phi\left(x^{\prime}+d x^{\prime}\right) d A-\Phi\left(x^{\prime}\right) d A=-\Sigma_{t} \Phi\left(x^{\prime}\right) d V
$$

where the minus sign on the right side is introduced because collisions remove particles from the uncollided beam. After both sides have been divided by $d A$, Eq. 4.1-1 may be written

$$
d \Phi\left(x^{\prime}\right)=-\Sigma_{t} \Phi\left(x^{\prime}\right) d x^{\prime}
$$

or

$$
\frac{1}{\Phi\left(x^{\prime}\right)} d \Phi\left(x^{\prime}\right)=-\Sigma_{t} d x^{\prime}
$$

This familiar differential equation has the solution

$$
\Phi\left(x^{\prime}\right)=c e^{-\Sigma_{t} x^{\prime}}
$$

where $c$ is a constant. By applying the condition that $\Phi(0)=\Phi_{0}$, we obtain

$$
\Phi\left(x^{\prime}\right)=\Phi_{0} e^{-\Sigma_{t} x^{\prime}}
$$

Thus the exit flux density of the uncollided component is given by

$$
\Phi(x)=\Phi_{0} e^{-\Sigma_{t} x}
$$

This exponential attenuation law is basic to the calculation of radiation transport through matter.

Consider a point isotropic source at the center of a spherical shell of material, as shown in Fig. 4.3. With no shell material present, the flux density of uncollided particles at the detector would be given by 
$\Phi(R)=S / 4 \pi R^{2}$, where $S$ is the source strength. However, the flux density passing through the shell is reduced by a factor of $e^{-\Sigma_{t} x}$. Thus

$$
\Phi(R)=\frac{S}{4 \pi R^{2}} e^{-\Sigma_{t} x}
$$

the product of a geometric factor, $1 / 4 \pi R^{2}$, and an attenuation factor, $e^{-\Sigma_{t} x}$.

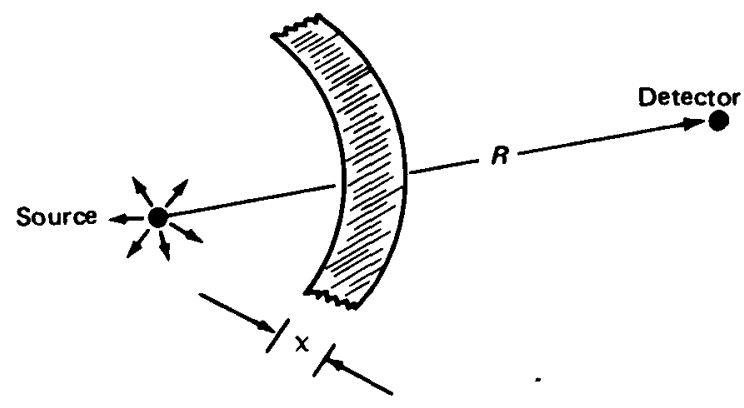

Fig. 4.3-Spherical shell attenuation.

Successively complex radiation-transport calculations occur if (1) the radiation is allowed to scatter with no change in energy, (2) scattering with energy degradation is allowed, and (3) secondary radiations are taken into account. An accurate analysis of neutrons and gamma rays from a reactor source requires that energy degradation and secondaries be considered.

Consider a material in which only scattering and absorption occur. The total macroscopic cross section, $\Sigma_{t}$, consists of two components: $\Sigma_{s}$, the scattering cross section, and $\Sigma_{a}$, the absorption cross section. For a parallel monoenergetic beam incident to a slab, one component of the penetrating radiation will be that which has not interacted with the shield, given by $\Phi_{0} e^{-\Sigma_{t} x}$. The other penetrating component will be the radiation that has scattered one or more times within the shield. This scattered component will exit the shield in all directions and over a continuous energy distribution even though the incident radiation is of only one energy and one direction.

\subsection{THE BOLTZMANN TRANSPORT EQUATION}

The complex form of radiation transport in which scattering and energy degradation occur was originally described by the differential equation due 
to Boltzmann. In a book on transport theory, Kušcer ${ }^{1}$ notes that Boltzmann formulated this equation in 1872 to calculate the coefficient of self-diffusion for a gas in which the molecules were assumed to scatter as elastic spheres. The gas problem is equivalent to the radiation problem of interest here. Kušcer states that a solution of the equation was not achieved until early this century and contrasts the ease with which transport problems are formulated with the difficulties encountered in obtaining solutions. Basically this equation is a "bookkeeping" statement that accounts for additions to and subtractions from the radiation in a given increment of space, energy, and direction. Particles may be interpreted as either neutrons or gamma rays in the derivation.

The differential flux density is a function of seven independent variables: three that define the spatial position of the detector point, two that define direction of particle motion, one that measures particle energy, and one that measures time. In vector notation these can be reduced to three quantities: a vector defining spatial position, a vector defining momentum (specifying both direction and energy), and one scalar time variable. A radiation field is completely defined (i.e., the radiation-transport problem of interest is solved) when the particle or energy-flux density at every spatial point within the region of interest is known.

For shield analysis a steady state is ordinarily assumed, and the time variable is not required. It is also customary to use a unit vector for direction and an independent scalar variable for energy (or its equivalents, speed or wavelength). Thus we define

$$
\Phi(\mathbf{r}, \Omega, E) d \Omega d E \quad \text { particles unit area }{ }^{-1} \sec ^{-1}
$$

as the flux density (or track length per unit volume per second) of particles at spatial point $\mathbf{r}$ whose directions of travel lie in the solid angle $d \Omega$ about $\boldsymbol{\Omega}$ and whose energies lie in the increment $d E$ about $E$. We derive the Boltzmann equation using particle conservation in a differential volume $d V d \Omega d E$ of six scalar dimensions, in which $d V$ is a differential spatial volume element, as illustrated in Fig. 4.4. The six-dimensional system, of which $d V d \Omega d E$ is a differential element, is sometimes called a six-dimensional phase space, and the differential element, a differential phase-space cell. Care must be taken when using spherical coordinates for the spatial coordinate system; the polar $\left(\theta_{r}\right)$ and azimuthal $\left(\psi_{r}\right)$ angles defining the direction of $r$ must not be confused with the polar $(\theta)$ and azimuthal $(\psi)$ angles defining unit vector $\Omega$, which meas'res particle direction. 


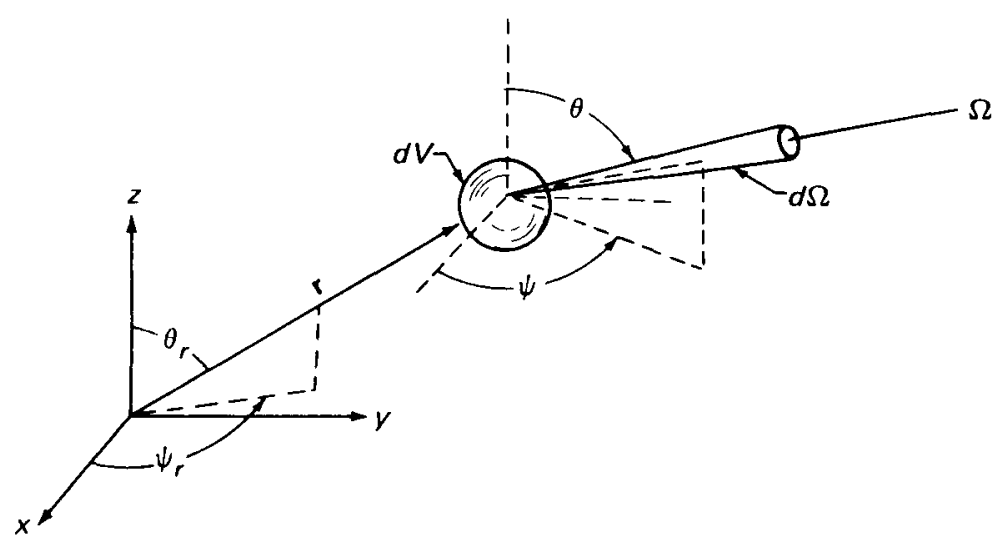

Fig. 4.4-Volume element in phase space.

Particles of the appropriate energy $(d E$ about $E$ ) and direction ( $d \Omega$ about $\Omega$ ) can be introduced into $d V d \Omega d E$ by the following processes:

1. They can be born in $d V$ at the appropriate energy and direction by a source located there.

2. They can flow into $d V$ with the proper energy and direction from adjacent spatial regions.

3. Particles bearing other directions or energies or both can undergo an interaction within $d V$ such that the scattered particle is in $d V d \Omega d E$ (the inscatter source).

In a similar fashion, particles within $d V d \Omega d E$ can be removed from that differential element by the following processes:

1. They can undergo an interaction that causes them to be absorbed or changed in direction or energy or both.

2. They can flow out of $d V$ into adjacent spatial regions.

In a steady state the losses from $d V d \Omega d E$ must equal the gains. Further, the leakage gains and losses into and out of $d V$ can be combined into one term: net leakage. Thus

$$
\text { Net leakage }+ \text { interactions }=\text { inscattering }+ \text { source }
$$

where, by convention, net leakage is defined as being positive in the outward direction (a loss).

The net leakage term, $N L$, is equal to $\boldsymbol{\nabla} \cdot \boldsymbol{\Omega} \Phi(\mathbf{r}, \boldsymbol{\Omega}, E) d V d \Omega d E$ particles/ sec, a fact that we will demonstrate. Consider a small spatial volume element 
$\Delta V$ enclosed by surface $\Delta S$ (Fig. 4.5); $d S$ is a surface differential area on $\Delta V$ with outward normal $\mathbf{n}$, and $d A$ is the projection of $d S$ normal to $\boldsymbol{\Omega}$. The particle flow through $d S$ and $d A$ in direction $\boldsymbol{\Omega}$ is given by $\boldsymbol{\Phi}(\mathbf{r}, \boldsymbol{\Omega}, E) d \Omega d E d A$ particles/sec, which may be written

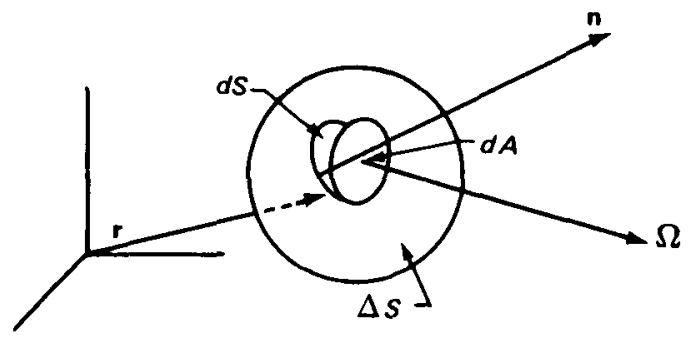

Fig. 4.5-Differential surface element.

$\boldsymbol{\Phi}(\mathbf{r}, \boldsymbol{\Omega}, E) d \boldsymbol{\Omega} d E(d A / d S) d S$. However, since $d A / d S=(\mathbf{n} \cdot \boldsymbol{\Omega})$, the net leakage, $d(N L)$, through $d S$ is

$$
d(N L)=\Phi(\mathbf{r}, \boldsymbol{\Omega}, E) d \Omega d E(\mathbf{n} \cdot \boldsymbol{\Omega}) d S
$$

[Proof that Eq. 4.2-1 gives net rather than outward leakage through $d S$ is given as one of the problems in the exercises.] The vector property $(\mathbf{u} \cdot \mathbf{v})=\mathbf{v} \cdot \mathbf{u}$ enables us to write

$$
d(N L)=[\boldsymbol{\Omega} \Phi(\mathbf{r}, \boldsymbol{\Omega}, E) d \boldsymbol{\Omega} d E] \cdot \mathbf{n} d S
$$

The total net leakage from $\Delta V$ is then given by

$$
\Delta N L=\int_{\Delta S} d(N L)=\int_{\Delta S}[\boldsymbol{\Omega} \Phi(\mathbf{r}, \Omega, E) d \Omega d E] \cdot \mathbf{n} d S
$$

But $\Delta V, \Delta S$, and $\Omega \Phi(r, \Omega, E) d \Omega d E$ meet the conditions for application of the divergence theorem (stated in Appendix $\mathrm{D}$ ), and the net leakage from $\Delta V$ is thus

$$
\Delta N L=\int_{\Delta V} \nabla \cdot \Omega \Phi(\mathrm{r}, \Omega, E) d \Omega d E d V
$$

Now consider the limit as $\Delta V$ approaches $d V$. The function $\nabla \cdot \Omega \Phi(r, \Omega, E)$ $d \Omega d E$ becomes a constant with respect to $\mathbf{r}$, and

$$
\lim _{\Delta V \rightarrow d V} \Delta N L=[\nabla \cdot \Omega \Phi(\mathbf{r}, \boldsymbol{\Omega}, E) d \Omega d E] \lim _{\Delta V \rightarrow d V} \int_{\Delta V} d V
$$


The limit of the integral is simply $d V$. Thus the net leakage, $N L$, from $d V$ is given by

$$
N L=\boldsymbol{\nabla} \cdot \boldsymbol{\Omega} \Phi(\mathbf{r}, \boldsymbol{\Omega}, E) d V d \boldsymbol{\Omega} d E \text { particles } / \mathrm{sec}
$$

Every interaction within $d V$ (except for coherent scattering without a change in direction) removes particles initially in $d V d \Omega d E$ from that differential element. Thus the interaction loss term, $I L$, is given by

$$
I L=\Sigma_{t}(\mathbf{r}, E) \Phi(\mathbf{r}, \boldsymbol{\Omega}, E) d V d \Omega d E \text { particles } / \mathrm{sec}
$$

The macroscopic loss coefficient is taken to be the total attenuation coefficient, $\Sigma_{t}$.

The source term is handled formally. If $S(\mathbf{r}, \boldsymbol{\Omega}, E)$ is defined to be the differential source distribution (particles per second per unit volume, solid angle, and energy), the total source in $d V d \Omega d E$ is

$$
S=S(\mathbf{r}, \boldsymbol{\Omega}, E) d V d \Omega d E \text { particles/sec }
$$

The remaining term expresses the inscattering. Let $p\left(E^{\prime} \rightarrow E, \Omega^{\prime} \rightarrow \boldsymbol{\Omega}\right)$ $d \Omega d E$ be the probability that a particle of energy $E^{\prime}$ and direction $\Omega^{\prime}$ scatters into $d E$ about $E$ and $d \Omega$ about $\boldsymbol{\Omega}$. The rate at which scattering events in $d V d \Omega^{\prime} d E^{\prime}$ occur is given by $\Sigma_{s}\left(\mathbf{r}, E^{\prime}\right) \Phi\left(\mathbf{r}, \Omega^{\prime} E^{\prime}\right) d V d \Omega^{\prime} d E^{\prime}$ particles/sec, in which $\Sigma_{s}\left(r, E^{\prime}\right)$ is the macroscopic scattering coefficient. Inscattering from $d V d \Omega^{\prime} d E^{\prime}$ into $d V d \Omega d E$ is then

$$
\Sigma_{s}\left(\mathbf{r}, E^{\prime}\right) p\left(E^{\prime} \rightarrow E, \boldsymbol{\Omega}^{\prime} \rightarrow \boldsymbol{\Omega}\right) d \Omega d E \boldsymbol{\Phi}\left(\mathbf{r}, \boldsymbol{\Omega}^{\prime}, E^{\prime}\right) d V d \Omega^{\prime} d E^{\prime}
$$

The total inscattering rate, $I S$, is given by

$$
\begin{aligned}
I S=\int_{E^{\prime}} \int_{\Omega^{\prime}} \Sigma_{s}\left(\mathbf{r}, E^{\prime}\right) p\left(E^{\prime} \rightarrow E, \Omega^{\prime} \rightarrow \Omega\right) d \Omega & d E \Phi\left(\mathbf{r}, \Omega^{\prime}, E^{\prime}\right) \\
& \times d V d \Omega^{\prime} d E^{\prime} \text { particles/sec }
\end{aligned}
$$

Conservation requires that losses equal gains: $N L+I L=I S+S$ or

$$
\begin{aligned}
& \boldsymbol{\nabla} \cdot \boldsymbol{\Omega} \Phi(\mathrm{r}, \boldsymbol{\Omega}, E)+\Sigma_{t}(\mathrm{r}, E) \Phi(\mathrm{r}, \boldsymbol{\Omega}, E) \\
& \quad=\int_{E^{\prime}} \int_{\boldsymbol{\Omega}^{\prime}} \Sigma_{s}\left(\mathrm{r}, E^{\prime}\right) p\left(E^{\prime} \rightarrow E, \boldsymbol{\Omega}^{\prime} \rightarrow \boldsymbol{\Omega}\right) \Phi\left(\mathbf{r}, \boldsymbol{\Omega}^{\prime}, E^{\prime}\right) d \Omega^{\prime} d E^{\prime}+S(\mathbf{r}, \boldsymbol{\Omega}, E)
\end{aligned}
$$


in which the common factor $d V d \Omega d E$ has been cancelled from each term. Equation 4.2-8 is the integro-differential form of the Boltzmann equation and is that most often used as a starting point in the solution of the radiation transport problem, although other forms (i.e., purely integral) are sometimes encountered. Equation 4.2-8 is applicable to both neutron and photon transport. Simple transformations (some are given in the exercises) allow the Boltzmann equation to be expressed in terms of wavelength $\lambda(\lambda=h \nu / E)$ rather than energy and differential energy-flux densities $I(\mathbf{r}, \Omega, E)$ and $I(\mathbf{r}, \Omega, \lambda)$ rather than number-flux densities. In photon transport, because of the relatively simple expressions that result, the form involving $I(\mathbf{r}, \Omega, \lambda)=$ $\lambda \Phi(r, \Omega, \lambda)(\lambda$ in units of Compton wavelength) is convenient.

The appearance of the Boltzmann equation varies considerably with the coordinates involved, the type of radiation being considered, and whether energy degradation is allowed. For this discussion the vector notation is used, and various symbols representing interaction parameters are defined. Appendix $\mathrm{D}$ lists the transformation between the three common coordinate systems and defines various vector operators, functions, and theorems helpful in deriving and manipulating the Boltzmann equation.

A direct, analytic solution of the Boltzmann equation can be found only in a few very simple and highly idealized cases. For the most part, approximate solutions are all that are possible in practical situations. Approximate solutions can be obtained in two ways: simplifying assumptions can be made which alter the Boltzmann equation so that an analytic solution is obtainable or numerical methods can be used. An example of the first type is the elementary one-speed diffusion equation of reactor physics which, although usually derived differently, can be shown to be a form of Eq. 4.2-8 with the proper assumptions invoked.

Successful numerical solutions to the Boltzmann equation have been obtained with difference methods and iterative procedures. These methods evolved in parallel with, and were influenced by, the development of high-speed computing machines, which are a necessary tool in their application.

The approach generally used involves the derivation of a difference approximation to the Boltzmann equation for each point of a mesh filling the shield volume. Steps in such a calculation include:

1. Choosing the division points between energy groups.

2. Choosing a method of representing the differential scattering cross section and the angular dependence of the flux density. 
3. Integrating the Boltzmann equation over each energy group (cross sections must be suitably averaged over each group).

4. Approximating the equations relating the spatial derivatives of the differential (angle and energy) flux density as functions of $r$ by a finite difference system at each mesh point.

5. Solving the resulting system of equations by an iterative method.

The various difference methods used in solving the Boltzmann equation differ primarily in their method of representing the angular dependence, although they may also differ in the iteration scheme indicated in step 5 .

Before proceeding to a review of various solution methods, we should point out that Eq. 4.2-8 includes some undefined functions, in particular those contained in the functions $S(\mathbf{r}, \boldsymbol{\Omega}, E)$ and $p\left(E^{\prime} \rightarrow E, \boldsymbol{\Omega}^{\prime} \rightarrow \boldsymbol{\Omega}\right)$. To solve the Boltzmann equation, we must replace these expressions with functions that describe the processes involved in the problem being considered.

\subsection{SPHERICAL HARMONICS METHOD}

Although the method of spherical harmonics is not currently in widespread use, it was one of the first developed and embodies concepts used in other techniques in this chapter. The method as applied to the solution of the Boltzmann transport equation consists in representing the various angle-dependent terms as expansions in the spherical harmonics polynomials. These polynomials, commonly called associated spherical harmonics, $t$ vary in definition from one reference to another. We use the polynomials described by Weinberg and Wigner. ${ }^{2}$

Applying the spherical harmonics technique to the general transport problem is inherently complex. However, a simplified and lucid illustration of the method can be shown for a steady-state (no time dependence), one-speed (no energy dependence), one-dimensional (slab geometry with azimuthal symmetry), homogeneous (constant system parameters) neutron transport problem. Consistent with these simplifications, the general Boltzmann transport equation can be written $¥$

$$
\mu \frac{\partial \Phi(x, \mu)}{\partial x}+\Sigma_{t} \Phi(x, \mu)=S(x, \mu)+\int_{-1}^{+1} \Sigma_{s}\left(\mu, \mu^{\prime}\right) \Phi\left(x, \mu^{\prime}\right) d \mu^{\prime}
$$

†Definitions and theorems of associated spherical harmonics and the related Legendre functions are given in Appendix $\mathrm{D}$.

$¥$ The derivation of Eq. 4.3-1 is given as a problem in the exercises. 
where $\Phi(x, \mu)$ = angular flux density (neutron-flux density per unit $\mu$ )

$x=$ spatial variable in slab geometry, the direction of which is specified by the unit vector $\mathbf{i}$

$\mu=$ direction cosine with respect to the $x$-axis

$=\boldsymbol{\Omega} \cdot \mathbf{i}=\cos \theta$

$\Sigma_{t}=$ total macroscopic cross section

$\Sigma_{s}\left(\mu, \mu^{\prime}\right) d \mu=$ a scattering cross section that describes the probability that a neutron with an incident direction cosine $\mu^{\prime}$ will be scattered so that its emergent direction has a direction cosine in $d \mu$ about $\mu$

$\Sigma s\left(\mu, \mu^{\prime}\right)=\int_{0}^{2 \pi} \Sigma_{s}\left(\Omega, \Omega^{\prime}\right) d \psi$

$\Sigma_{s}\left(\boldsymbol{\Omega}, \boldsymbol{\Omega}^{\prime}\right)=$ the macroscopic differential scattering cross section

$S(x, \mu)=$ source particles per unit $\mu$, volume, and time

The angle-dependent terms of Eq. 4.3-1 can be represented as a series of spherical harmonics of the first kind, the Legendre polynomials $P_{j}(\mu)$. Expanding the angular flux density and source term in terms of these polynomials yields

$$
\begin{gathered}
\Phi(x, \mu)=\sum_{j=0}^{\infty} \Phi_{j}(x) P_{j}(\mu) \\
S(x, \mu)=\sum_{j=0}^{\infty} S_{j}(x) P_{j}(\mu)
\end{gathered}
$$

where $\Phi_{j}(x)=$ position-dependent Legendre coefficients corresponding to the flux density:

$$
\Phi_{j}(x)=\frac{2 j+1}{2} \int_{-1}^{+1} \Phi(x, \mu) P_{j}(\mu) d \mu \quad(j=0,1,2, \ldots)
$$

and $S_{j}(x)=$ position-dependent Legendre coefficients corresponding to the source term:

$$
S_{j}(x)=\frac{2 j+1}{2}=\int_{-1}^{+1} S(x, \mu) P_{j}(\mu) d \mu \quad(j=0,1,2, \ldots)
$$

Since for most practical situations the differential scattering cross section depends only on the change in direction given by $\mu_{0}=\boldsymbol{\Omega} \cdot \boldsymbol{\Omega}^{\prime}$, the series expansion for $\Sigma_{s}\left(\boldsymbol{\Omega}, \boldsymbol{\Omega}^{\prime}\right)$ is made in terms of the Legendre polynomials $P_{i}\left(\mu_{0}\right):$ 


$$
\Sigma_{s}\left(\Omega, \Omega^{\prime}\right)=\sum_{i=0}^{\infty} \eta_{i} P_{i}\left(\mu_{0}\right)
$$

where the values of $\eta_{i}$ are the Legendre coefficients $(i=0,1,2, \ldots, \infty)$ corresponding to the differential scattering cross section

$$
\eta_{i}=\frac{2 i+1}{2} \int_{-1}^{+1} \Sigma_{s}\left(\Omega, \Omega^{\prime}\right) P_{i}\left(\mu_{0}\right) d \mu_{0}
$$

The spherical harmonics form of the Boltzmann equation is obtained by introducing the preceding series representations for $\Phi(x, \mu), S(x, \mu)$, and $\Sigma_{s}\left(\Omega, \Omega^{\prime}\right)$ into Eq. 4.3-1, multiplying each term by the Legendre polynomial $P_{n}(\mu)$, and integrating over all $\mu(-1$ to +1$)$. When Eqs. 4.3-2, 4.3-3, and 4.3-6 are substituted into Eq. 4.3-1 and the orthogonality property of Legendre polynomials is used along with the addition theorem and a recursion relation, $t$ the following set of coupled differential equations is obtained:

$$
\begin{aligned}
\frac{n+1}{2 n+3} \frac{d}{d x} \Phi_{n+1}(x)+\frac{n}{2 n-1} \frac{d}{d x} \Phi_{n-1}(x)=\frac{4 \pi}{2 n+1} \eta_{n} \Phi_{n}(x) \\
\quad-\Sigma_{t} \Phi_{n}(x)+S_{n}(x) \quad(\text { for } n=0,1,2, \ldots, \infty)
\end{aligned}
$$

This set of equations, which no longer involves the directional variables and therefore is more amenable to solution than Eq. 4.3-1, is called the second (or spherical-harmonics component) form of the Boltzmann equation by Weinberg and Wigner ${ }^{2}$ and others.

Practical methods of solution require that the series representations of $\Phi(x, \mu)$ be limited to a finite number of terms, say $(n+1)$ terms; $n$ is commonly called the truncation number, and the corresponding calculation is called the $P_{n}$ approximation. The $P_{1}$ approximation is equivalent to diffusion theory (see Sec. 4.6) and involves only a linear representation of the flux density, which restricts its application to situations wherein the neutron-flux density is nearly isotropic, a severe limitation for deep-penetration problems.

The accuracy of the spherical-harmonics calculation is also influenced by the number of terms used to represent the differential scattering cross section. Only a few terms are necessary for nearly isotropic scattering, but a

tThese properties are given in Appendix D. The use of the addition theorem makes possible the evaluation of the inscattering-integral term containing $\Sigma_{s}\left(\boldsymbol{\Omega}, \boldsymbol{\Omega}^{\prime}\right)$, which is necessarily expanded in terms of $P_{j}\left(\mu_{0}\right)$ rather than $P_{j}(\mu)$. 
large number of terms are required for adequate treatment of anisotropic scattering; in the past this has limited the use of the spherical harmonics treatment. However, recent advances in cross-section technology and increased computer capacity have for all practical considerations removed this limitation.

Shure ${ }^{3}$ found that a multigroup $P_{3}$ approach in one dimension for calculating spatial and spectral neutron distributions in metal hydrogenous reactor shields yielded satisfactory estimates of neutron attenuation for reasonable amounts of computer time. Further, Lanning ${ }^{4}$ recognized that for some design problems the low-order approximations were sufficiently accurate. He successfully calculated the spatial distribution of the gamma-ray energy-flux density in one-dimensional slab geometry.

\subsection{DISCRETE-ORDINATES $S_{n}$ METHOD $\dagger$}

The discrete-ordinates $S_{n}$ method is a means of effecting a numerical solution of the Boltzmann transport equation. The most recent versions of the method permit anisotropic scattering to be included and thus make it suitable for both neutron and gamma-ray deep-penetration calculations in a wide variety of shielding problems. Since the method is fundamentally formulated as a finite-difference equation, a minimum number of limiting assumptions are required, and the solutions apparently approach the exact solution of the Boltzmann equation as the space, angle, and energy mesh approach differential size. The method can be applied without significant restrictions to the problem of calculating criticality, and it can be used for both homogeneous and laminated shields with a variety of source configurations, including surface- and volume-distributed sources.

We should note that a finite-difference equation is defined as an algebraic statement relating values of the variable (in this instance, the discrete ordinate flux density) from point to point in phase space. To obtain the difference equations, we section phase space into a finite number of discrete points and relate the flux density at each point to the flux densities at adjacent points. As will be shown, this is accomplished by integrating the conservative form of the Boltzmann equation over a finite-difference cell in phase space. This procedure replaces the Boltzmann integro-differential equation with a system of simultaneous difference equations. The latter may then be solved numerically by an iterative technique.

tThis section is primarily the work of F. R. Mynatt. 
Early applications of discrete ordinates, such as the Wick ${ }^{5}$-Chandrasekhar ${ }^{6}$ method, were limited to simple problems, such as the transport of monoenergetic neutrons isotropically scattered in one-dimensional slabs. The fundamental assumption in the Wick-Chandrasekhar method was that the integral in the Boltzmann equation could be approximated by a Gaussian quadrature formula; consequently functions involved in the integral had to be evaluated only at the angles corresponding to the Gaussian zeros. Although this original discrete-ordinates method could be extended to anisotropic scattering, it was limited to slab geometry.

A discrete-ordinates technique that could be extended to spherical and cylindrical geometries was introduced by Carlson; ${ }^{7}$ this method is commonly called the discrete-ordinates $S_{n}$ method.

Other approaches that can be classified as discrete-ordinates methods are the direct numerical integration techniques employed by the $\mathrm{NIOBE}^{8}$ and other codes, but these techniques have not been so widely used for shielding problems.

Early versions of the $S_{n}$ method assumed that the angular flux density varies with angle as connected line segments in an even number of equally spaced angular increments. This representation, although reasonably accurate for homogeneous one-dimensional systems, was found to be unsuitable for the general problem. Recursions involving many terms are required, and an extension of the method to two-dimensional geometries is most difficult. These shortcomings are largely alleviated by the use of the diamond difference technique described by Carlson, Lee, and Worlton, ${ }^{9}$ which relates the angular flux density within each particular angular increment in a general way to the end-point values of the increment. With the diamond difference method, the Boltzmann equation can be integrated over an angular increment, yielding, for the derivative terms, a two-point difference equation involving the angular flux density evaluated at the increment end points.

The linear Boltzmann equation is a flow balance for a differential phase-space cell, treating the events causing an increase or a decrease in the number of particles contained in the cell. The discrete-ordinates difference equations can be formulated in an equivalent manner considering a finite-difference cell (it is presented this way in most references). For some time it was not clear that the difference equations would, in general, approach the analytic form of the Boltzmann equation as the finite-difference phase-space cell approached differential size. Lathrop ${ }^{10}$ showed that they would for the one-dimensional geometries, and this is established implicitly in the following paragraphs in which the difference equations for 
spherical geometry are derived directly from the analytic Boltzmann equation. Spherical geometry, although simple, serves to illustrate all the characteristics of the discrete-ordinates equations except for discrete ray streaming, which occurs only in two- or three-dimensional geometry.

\subsubsection{Transport Equation and Phase-Space Geometry}

The derivation of the discrete-ordinates equations is given for the special case of spherical symmetry in spherical (position) coordinates. With only slight modifications these results can be made to apply to slab and infinite-cylinder geometries. This discussion also embodies the central features of two-dimensional derivations while avoiding much of the complexity. The differential phase-space cell is defined by three variables: the scalar value of the radius, $r$ (Fig. 4.4), the cosine of the angle of the particle direction relative to the radius, $\mu=(1 / r) \mathbf{r} \cdot \Omega$, and the energy of the particle, $E$; that is,

Differential phase-space cell $=d V d \mu d E$

$$
=4 \pi r^{2} d r d \mu d E
$$

The finite-difference cell is obtained by integrating Eq. 4.4-1 over selected finite intervals of radius, angle, and energy; it is given by

Finite-difference cell $=V_{I} \Delta \mu_{D} \Delta E_{G}$

$$
=\frac{4 \pi}{3}\left(r_{i+1}^{3}-r_{i}^{3}\right)\left(\mu_{d+1}-\mu_{d}\right)\left(E_{g+1}-E_{g}\right)
$$

The following subscript notation is used throughout this section: subscripts $I, D$, and $G$ denote functions whose values are associated with the Ith space interval, $D$ th angular interval, and $G$ th energy group, respectively; $i$ and $i+1$ refer to a function evaluated at the lower and upper limits of the Ith space interval, $d$ and $d+1$ refer to a function evaluated at the lower and upper limits of the $D$ th angular interval, and $g$ and $g+1$ refer to a function evaluated at the lower and upper limits of the Gth energy group.

For this problem (one-dimensional spherical geometry), the following two analytic forms t of the Boltzmann transport equation can be considered: 


$$
\begin{aligned}
\mu \frac{\partial}{\partial r} \Phi(r, \mu, E) & +\frac{\left(1-\mu^{2}\right)}{r} \frac{\partial}{\partial \mu} \Phi(r, \mu, E)+\Sigma_{t} \Phi(r, \mu, E) \\
& =S(r, \mu, E)+\int_{-1}^{+1} \int_{0}^{\infty} \Sigma_{s}\left(r, E^{\prime} \rightarrow E, \mu_{0}\right) \Phi\left(r, E^{\prime}, \mu^{\prime}\right) d E^{\prime} d \mu^{\prime}
\end{aligned}
$$

and

$$
\begin{array}{r}
\frac{\mu}{r^{2}} \frac{\partial}{\partial r}\left[r^{2} \Phi(r, \mu, E)\right]+\frac{1}{r} \frac{\partial}{\partial \mu}\left[\left(1-\mu^{2}\right) \Phi(r, \mu, E)\right]+\Sigma_{t}(r, E) \Phi(r, \mu, E) \\
=S(r, \mu, E)+\int_{-1}^{+1} \int_{0}^{\infty} \Sigma_{s}\left(r, E^{\prime} \rightarrow E, \mu_{0}\right) \Phi\left(r, E^{\prime}, \mu^{\prime}\right) d E^{\prime} d \mu^{\prime}
\end{array}
$$

where $\Phi(r, \mu, E)=$ particle track length per unit volume (flux density) about $r$, per unit time, per unit energy about $E$, and per unit direction cosine about $\mu$

$\Sigma_{t}(r, E)=$ position- and energy-dependent macroscopic total cross section

$\Sigma_{s}\left(r, E^{\prime} \rightarrow E, \mu_{0}\right) d E^{\prime} d \mu^{\prime}=$ differential scattering cross section describing the probability that a particle with an initial energy $E^{\prime}$ and direction cosine $\mu^{\prime}$ undergoes a collision at $r$, resulting in a change of flight direction described by the cosine of the scattering angle $\mu_{0}$, which places it in a new direction that lies in $d \mu$ about $\mu$ with a new energy in $d E$ about $E$

$\mu_{0}=$ cosine of the scattering angle $=\boldsymbol{\Omega} \cdot \boldsymbol{\Omega}^{\prime}$

$\boldsymbol{\Omega}, \boldsymbol{\Omega}^{\prime}=$ final and initial flight direction unit vectors, respectively

$S(r, \mu, E)=$ source particles per unit volume about $r$, per unit time, per unit energy about $E$, and per unit direction cosine about $\mu$

Equation 4.4-4 is called the conservative form of the transport equation, and its integration over any phase-space volume results in interface terms, which may be identified as leakage terms, that satisfy the divergence theorem exactly. As a consequence the conservative equation is the preferred formal basis for numerical analyses.

For convenience we number the terms in Eq. 4.4-4 consecutively so that it becomes

$$
T_{1}+T_{2}+T_{3}=T_{4}+T_{5}
$$




\subsubsection{Derivation of Finite-Difference Equation}

The discrete-ordinates difference equation is obtained by applying the following integral operator to the transport equation (Eq. 4.4-4) in a manner consistent with the classical technique for obtaining difference equations:

$$
\text { Integral operator }=\int_{r \epsilon V_{I}} \int_{\mu \epsilon \Delta \mu_{D}} \int_{E \epsilon \Delta E_{G}} 4 \pi r^{2} d r d \mu d E
$$

This operator integrates each term of the transport equation over the difference cell. [The integration limits are expressed symbolically by $x \in X$, which implies a definite integral with respect to the variable $x$ over the interval $X$.] Application of the operator to the first term of Eq. 4.4-4 gives

$T_{1}=\int_{r \epsilon V_{I}} \int_{\mu \epsilon \Delta \mu_{D}} \int_{E \epsilon \Delta E_{G}} \frac{\mu}{r^{2}} \frac{\partial}{\partial r}\left[r^{2} \Phi(r, \mu, E)\right] 4 \pi r^{2} d r d \mu d E$

which when rearranged becomes

$$
T_{1}=4 \pi \int_{r \in V_{I}} \int_{\mu \epsilon \Delta \mu_{D}} \mu \frac{\partial}{\partial r}\left[r^{2} \int_{E \epsilon \Delta E_{G}} \Phi(r, \mu, E) d E\right] d \mu d r
$$

The integral of the flux density over the energy group $G$ may be identified as the group angular flux

$$
\Phi_{G}(r, \mu) \equiv \int_{E \epsilon \Delta E_{G}} \Phi(r, \mu, E) d E
$$

in which case Eq. 4.4-7 becomes

$$
T_{1}=4 \pi \int_{\mu \epsilon \Delta \mu_{D}} \mu d \mu \int_{r \epsilon V_{I}} \frac{\partial}{\partial r}\left[r^{2} \Phi_{G}(r, \mu)\right] d r
$$

The volume integral in Eq. 4.4-9 can be modified and evaluated in the following manner:

$$
\begin{aligned}
\int_{r \in V_{I}} \frac{\partial}{\partial r}\left[r^{2} \Phi_{G}(r, \mu)\right] d r & =\int_{r \in V_{I}} d\left[r^{2} \Phi_{G}(r, \mu)\right] \\
& =r_{i+1}^{2} \Phi_{G, i+1}(\mu)-r_{i}^{2} \Phi_{G, i}(\mu)
\end{aligned}
$$

where $\Phi_{G, i+1}(\mu)=\Phi_{G}\left(r_{i+1}, \mu\right)$ and $\Phi_{G, i}(\mu)=\Phi_{G}\left(r_{i}, \mu\right)$. 
Substitution of Eq. 4.4-10 into Eq. 4.4-9 yields the following expression for the first term:

$T_{1}=4 \pi \int_{\mu \epsilon \Delta \mu_{D}} \mu r_{i+1}^{2} \Phi_{G, i+1}(\mu) d \mu-4 \pi \int_{\mu \in \Delta \mu_{D}} \mu r_{i}^{2} \Phi_{G, i}(\mu) d \mu$

It follows from the mean-value theorem that any integral can be approximated by

$\int_{x_{1}}^{x_{2}} x f(x) d x \cong \bar{x} f(\bar{x}) \Delta x \quad\left(\right.$ for $x_{1}<\bar{x}<x_{2}$, and $\left.\Delta x=x_{2}-x_{1}\right)$

The parameter $\bar{x}$ may be adjusted to give the equality; for well-behaved functions, the closer $\bar{x}$ is to the true mean, the better the approximation. Applying the mean-value theorem to Eq. 4.4-11 to evaluate the solid-angle intervals results in

$$
T_{1}=4 \pi\left(\bar{\mu}_{D} r_{i+1}^{2} \Phi_{G, i+1, D} \Delta \mu_{D}-\bar{\mu}_{D} r_{i}^{2} \Phi_{G, i, D} \Delta \mu_{D}\right)
$$

where $\Phi_{G, i, D} \equiv \Phi_{G, i}\left(\bar{\mu}_{D}\right)$ and $\bar{\mu}_{D}$ is a mean-value approximation for the direction cosine over the direction increment $\Delta \mu_{D}$. Identifying the surface areas of the volume increment by

$$
\begin{aligned}
A_{i} & =4 \pi r_{i}^{2} \\
A_{i+1} & =4 \pi r_{i+1}^{2}
\end{aligned}
$$

yields the final form for the first term:

$$
T_{1}=\bar{\mu}_{D} \Delta \mu_{D}\left(A_{i+1} \Phi_{G, i+1, D}-A_{i} \Phi_{G, i, D}\right)
$$

The integral operator (Eq. 4.4-5) is next applied to the second term in Eq. 4.4-4, and the result is rearranged:

$$
T_{2}=4 \pi \int_{r \in V_{I}} \int_{\mu \epsilon \Delta \mu_{D}} r \frac{\partial}{\partial \mu}\left[\left(1-\mu^{2}\right) \int_{E \epsilon \Delta E_{G}} \Phi(r, \mu, E) d E\right] d \mu d r
$$

If we introduce the group angular flux as before, Eq. 4.4-16 becomes

$$
T_{2}=4 \pi \int_{r \in V_{I}} r d r \int_{\mu \epsilon \Delta \mu_{D}} \frac{\partial}{\partial \mu}\left[\left(1-\mu^{2}\right) \Phi_{G}(r, \mu)\right] d \mu
$$


The integration over $\mu$ is accomplished according to the procedure suggested by Eq. 4.4-10 with the following result

$$
\begin{aligned}
T_{2}=4 \pi\left[\int_{r \epsilon V_{I}}\left(1-\mu_{d+1}^{2}\right) \Phi_{G, d+1}(r)\right. & r d r \\
& \left.-\int_{r \epsilon V_{I}}\left(1-\mu_{d}^{2}\right) \Phi_{G, d}(r) r d r\right]
\end{aligned}
$$

The remaining integration over the radius variable is performed using the mean-value approximation (Eq. 4.4-12)

$$
T_{2}=4 \pi\left[\left(1-\mu_{d+1}^{2}\right) \Phi_{G, d+1,1} \bar{r}_{I} \Delta r_{I}-\left(1-\mu_{d}^{2}\right) \Phi_{G, d, I} \bar{r}_{I} \Delta r_{I}\right]
$$

Equation 4.4-19 reduces to a two-point difference in the angle index

$$
T_{2}=\left(B_{I, d+1} \Phi_{G, d+1, I}-B_{I, d} \Phi_{G, d, I}\right)
$$

if a curvature coefficient, $B_{I, d}$, is defined by the expression

$$
B_{I, d} \equiv 4 \pi \bar{r}_{I} \Delta \bar{r}_{I}\left(1-\mu_{d}\right)
$$

Consistent with the conservation property of the technique, Eq. 4.4-19 or Eq. 4.4-20 gives an overall neutron balance. This is apparent since a summation of Eq. 4.4-19 over $M$ direction increments $\Delta \mu_{D}$ yields $\left[\left(1-\mu_{M+1}^{2}\right) \Phi_{I, G, M+1}-\left(1-\mu_{1}^{2}\right) \Phi_{I, G, 1}\right]$, which is identically zero since $\mu_{M+1}=-1$ and $\mu_{1}=+1$ ( $M$ is the total number of angle increments). Equation 4.4-21, which defines the curvature coefficients, can be recast in the form of a recursion relation that involves the coefficients $B_{I, d+1}$ and $B_{I, d}$. First, $B_{I, d}$ is subtracted from $B_{I, d+1}$ (where $B_{I, d}$ and $B_{I, d+1}$ are given by Eq. 4.4-21):

$$
B_{I, d+1}-B_{I, d}=-4 \pi \bar{r}_{I} \Delta r_{I}\left(\mu_{d+1}^{2}-\mu_{d}^{2}\right)
$$

We assume that $\bar{r}_{I}$ in Eq. $4.4-22$ is the arithmetic mean; that is,

$$
\bar{r}_{I}=\frac{r_{i+1}+r_{i}}{2}
$$

Then it follows that

$$
\bar{r}_{I} \Delta r_{I}=\frac{r_{i+1}^{2}-r_{i}^{2}}{2}
$$


Following similar arguments, the factor $\left(\mu_{d+1}^{2}-\mu_{d}^{2}\right)$ can be expressed as

$$
\left(\mu_{d+1}^{2}-\mu_{d}^{2}\right)=2 \bar{\mu}_{D} \Delta \mu_{d}
$$

Introducing Eqs. 4.4-24 and 4.4-25 into Eq. 4.4-22 yields the following recursion relation:

$$
B_{I, d+1}-B_{I, d}=-4 \pi\left(r_{i+1}^{2}-r_{i}^{2}\right) \bar{\mu}_{D} \Delta \mu_{D}
$$

The final form for the recursion relation is obtained by introducing the cell areas $A_{i+1}$ and $A_{i}$ (from Eq. 4.4-14) and rearranging:

$$
B_{I, d+1}=B_{I, d}-\bar{\mu}_{D} \Delta \mu_{D}\left(A_{i+1}-A_{i}\right)
$$

where $B_{I, M+1}=0$. Equation 4.4-27 is the form of the curvature coefficient found in the literature. The only approximation made in the preceding derivation is in the application of mean values.

When the integral operator, Eq. 4.4-5, is applied to the fifth term of Eq. 4.4-4 (the inscattering integral), the result is

$$
\begin{aligned}
T_{5}=\int_{r e V_{I}} \int_{\mu \in \Delta \mu_{D}} \int_{E \epsilon \Delta E_{G}} \int_{-1}^{+1} \int_{0}^{\infty} \Sigma_{s}\left(r, E^{\prime} \rightarrow E, \mu_{0}\right) \\
\times \Phi\left(r, E^{\prime}, \mu^{\prime}\right) d E^{\prime} d \mu^{\prime} 4 \pi r^{2} d r d \mu d E
\end{aligned}
$$

The differential scattering cross section can be approximated by a truncated Legendre polynomial expansion in the cosine of the scattering angle:

$$
\Sigma_{s}\left(r, E^{\prime} \rightarrow E, \mu_{0}\right)=\frac{1}{2} \sum^{N} \Sigma^{n}\left(r, E^{\prime} \rightarrow E\right) P_{n}\left(\mu_{0}\right)
$$

where the $\Sigma^{n}$ values are Legendre coefficients of the expansion. The Legendre polynomial, $P_{n}\left(\mu_{0}\right)$, of the scattering angle cosine, $\mu_{0}$, is related to the initial and final angle direction cosines, $\mu, \mu^{\prime}$, by the addition theorem for Legendre polynomials, $\uparrow$ which for spherically symmetric geometry is simply

$$
P_{n}\left(\mu_{0}\right)=P_{n}(\mu) P_{n}\left(\mu^{\prime}\right)
$$

tThe addition theorem is quoted in Appendix D. 
In adapting Eq. 4.4-28 to a multigroup calculation, the integrals over all incident energies and all incident angles are replaced by sums of integrals over the primed phase-space cell. Symbolically this is denoted by

$$
\begin{aligned}
& \int_{0}^{\infty} f\left(E^{\prime}\right) d E^{\prime}=\sum_{G^{\prime}=1}^{L} \int_{E^{\prime} \epsilon \Delta E_{G^{\prime}}} f\left(E^{\prime}\right) d E^{\prime} \\
& \int_{-1}^{+1} f\left(\mu^{\prime}\right) d \mu^{\prime}=\sum_{D^{\prime}=1}^{M} \int_{\mu^{\prime} \in \Delta \mu^{\prime} D} f\left(\mu^{\prime}\right) d \mu^{\prime}
\end{aligned}
$$

where $L$ and $M$ signify the number of energy groups and the number of angular increments, respectively.

Combining Eqs. 4.4-29 and 4.4-30 with Eq. 4.4-28, expressing the incident energy and angle integrals by Eq.4.4-31, and evaluating all remaining integrals by the mean-value theorem yields (after considerable rearrangement of terms) the following forms for the inscattering integral:

$$
\begin{aligned}
T_{5} & =\frac{V_{I} \Delta \mu_{D}}{2} \sum_{G^{\prime}=1}^{L} \sum_{n=0}^{N} P_{n}\left(\bar{\mu}_{D}\right) \Sigma_{G^{\prime} \rightarrow G}^{n, I} \sum_{D^{\prime}=1}^{M} \Phi_{I, G^{\prime}, D^{\prime}} P_{n}\left(\bar{\mu}_{D^{\prime}}\right) \Delta \mu_{D^{\prime}} \\
& =\frac{V_{I} \Delta \mu_{D}}{2} \sum_{G^{\prime}=1}^{L} \sum_{n=0}^{N} P_{n}\left(\mu_{D}\right) \Sigma_{G^{\prime} \rightarrow G}^{n, I} j_{I, G^{\prime}}^{n}
\end{aligned}
$$

where $\Sigma_{G}^{n, I} \rightarrow G$ is the $n$th Legendre moment of the multigroup scattering cross section (multigroup macroscopic transfer coefficient), defined by

$$
\begin{aligned}
\Sigma_{G}^{n, I} \rightarrow G & {\left[4 \pi \int_{r \in V_{I}} \int_{E \in \Delta E_{G}} \int_{E^{\prime} \epsilon \Delta E_{G^{\prime}}} \Sigma^{n}\left(r, E^{\prime} \rightarrow E\right)\right.} \\
& \left.\times \int_{-1}^{+1} \Phi\left(r, E^{\prime}, \mu^{\prime}\right) P_{n}\left(\mu^{\prime}\right) d \mu^{\prime} d E^{\prime} d E r^{2} d r\right] \\
& \times\left[\int_{r \in V_{I}} \int_{E^{\prime} \in \Delta E_{G^{\prime}}} \int_{-1}^{+1} \Phi\left(r, E^{\prime}, \mu^{\prime}\right) P_{n}\left(\mu^{\prime}\right) d \mu^{\prime} d E^{\prime} r^{2} d r\right]^{-1}
\end{aligned}
$$

and $j_{I, G^{\prime}}^{n}$ is the $n$th Legendre coefficient of the angular dependence of the group flux density, calculated from

$$
j_{I, G^{\prime}}^{n}=\sum_{D^{\prime}=1}^{M} \Phi_{I, G^{\prime}, D^{\prime}} P_{n}\left(\bar{\mu}_{D^{\prime}}\right) \Delta \mu_{D^{\prime}}
$$

Application of the integral operator (Eq. 4.4-5) to the removal term (third term) of Eq. 4.4-4 gives

$$
T_{3}=\int_{r \epsilon V_{I}} \int_{\mu \epsilon \Delta \mu_{D}} \int_{E \epsilon \Delta E_{G}} \Sigma_{t}(r, E) \Phi(\mathbf{r}, \mu, E) 4 \pi r^{2} d r d \mu d E
$$


The evaluation of Eq. 4.4-35 requires some effort to avoid the assumption of angle-energy separability in the weighting of the multigroup cross sections. As the first step in evaluating Eq. 4.4-35 in terms of a cross section that is independent of angle, the energy integral of Eq. 4.4-35 is written as

$$
\begin{aligned}
T_{3}^{E} & =\int_{E \epsilon \Delta E_{G}} \Sigma_{t}(r, E) \Phi(r, \mu, E) d E \\
& =\Sigma_{G}^{t}(r) \Phi_{G}(r, \mu)-R
\end{aligned}
$$

where $R$ is the correction factor that is to be determined and $\Sigma_{G}^{t}(r)$ is the flux-density weighted group- $G$ total cross section defined by

$$
\Sigma_{G}^{t}(r) \equiv \frac{\int_{E \epsilon \Delta E_{G}} \Sigma_{t}(r, E) j^{0}(r, E) d E}{\int j^{0}(r, E) d E}
$$

in which $j^{0}(r, E)$ is the zeroth Legendre coefficient of the angular dependence of the flux density; $j^{0}(r, E)$ is identical to the differential flux density, $\Phi(r, E)$.

Rearrangement of Eq. 4.4-36 provides an explicit expression for $R$ :

$$
R=\Sigma_{G}^{t}(r) \Phi_{G}(r, \mu)-\int_{E \epsilon \Delta E_{G}} \Sigma_{t}(r, E) \Phi(r, \mu, E) d E
$$

The correction factor, $R$, is determined by expressing the angular flux densities as truncated Legendre series and then combining the two terms that comprise Eq. 4.4-38. The truncated Legendre series representation of the flux density is

$$
\Phi(r, \mu, E) \cong \sum_{n=0}^{N} \frac{2 n+1}{2} j^{n}(r, E) P_{n}(\mu)
$$

When Eq. 4.4-39 is substituted into Eq. 4.4-38, the result is

$$
\begin{aligned}
R=\Sigma_{G}^{t}(r) \sum_{n=0}^{N} \frac{2 n+1}{2} j_{G}^{n}(r) P_{n}(\mu)-\int_{E \epsilon \Delta E_{G}} \Sigma_{t}(r, E) \\
\\
\times \sum_{n=0}^{N} \frac{2 n+1}{2} j^{n}(r, E) P_{n}(\mu) d E
\end{aligned}
$$


which can be written as

$$
R=\sum_{n=0}^{N} \frac{2 n+1}{2}\left[\Sigma_{G}^{t}(r)-\Sigma_{G}^{t n}(r)\right] j_{G}^{n}(r) P_{n}(\mu)
$$

where

$$
j_{G}^{n}(r)=\int_{E \epsilon \Delta E_{G}} j^{n}(r, E) d E
$$

and the energy moments of the cross section are defined by

$$
\Sigma_{G}^{t n}(r) \equiv \frac{\int_{E \epsilon \Delta E_{G}} \Sigma_{t}(r, E) j^{n}(r, E) d E}{j_{G}^{n}(r)}
$$

Substitution of Eq. 4.4-41 into Eq. 4.4-36 yields the final form for the energy integral:

$$
\begin{aligned}
T_{3}^{E}=\Sigma_{G}^{t}(r) \Phi_{G}(r, \mu) & \sum_{n=0}^{N} \frac{2 n+1}{2} j_{G}^{n}(r) P_{n}(\mu) \\
& -\sum_{n=0}^{N} \frac{2 n+1}{2}\left[\Sigma_{G}^{t}(r)-\Sigma_{G}^{t n}(r)\right] j_{G}^{n}(r) P_{n}(\mu)
\end{aligned}
$$

With this form for the energy integral in Eq. 4.4-35, the remaining integrals are evaluated by the mean-value approximation; the result is

$$
\begin{aligned}
T_{3}=V_{I} \Delta \mu_{D}\left[\Sigma_{G, I}^{t} \Phi_{G, I, D}\right. & \\
& \left.-\sum_{n=0}^{N} \frac{2 n+1}{2}\left(\Sigma_{G, I}^{t}-\Sigma_{G, I}^{t n}\right) j_{G, I}^{n} P_{n}\left(\bar{\mu}_{D}\right)\right]
\end{aligned}
$$

The series in Eq. 4.4-44 is very similar in form to the inscattering integral term $\left(T_{5}\right)$ and may be included there by replacing $\Sigma_{G \rightarrow G}^{n, I}$ in Eq. 4.4-32 with

$$
\Sigma_{G \rightarrow G}^{n, I(\bmod )}=\Sigma_{G \rightarrow G}^{n, I}+(2 n+1)\left(\Sigma_{G, I}^{t}-\Sigma_{G, I}^{t n}\right) \delta_{G, G^{\prime}}
$$

where $\delta_{G, G^{\prime}}=1$ if $G^{\prime}=G$ and 0 if $G^{\prime} \neq G$. The modified removal term then has the desired form

$$
T_{3}=V_{I} \Delta \mu_{D} \Sigma_{G, I}^{t} \Phi_{G, I, D}
$$


Application of the integral operator to the source term of Eq. 4.4-4 is straightforward since, with the exception of multigroup constants, the mean-value approximation is used for all variables. The final result for a general fixed source is

$$
T_{4}=V_{I} \Delta \mu_{D} S_{I, G, D}
$$

If multiplication (fission) is present, the source term is represented by

$$
S(r, \mu, E)=\frac{1}{k_{e f f}} \chi(E) \int_{0}^{\infty} \nu \Sigma_{f}\left(r, E^{\prime}\right) j^{0}\left(r, E^{\prime}\right) d E^{\prime}
$$

which gives

$$
T_{4}=V_{I} \Delta \mu_{D} \frac{\chi_{G}}{k_{e f f}} \sum_{G^{\prime}=1}^{L} \nu \Sigma_{I, G^{\prime}}^{f} j_{I, G^{\prime}}^{0}
$$

where $k_{\text {eff }}=$ effective multiplication constant of the system

$\Sigma f_{I, G}=$ macroscopic fission cross section at energy $G^{\prime}$

$\nu=$ number of neutrons per fission by neutrons of energy $G^{\prime}$

$\chi_{G}=$ fission spectrum defined by

$$
\begin{gathered}
\chi_{G} \equiv \int_{E \epsilon \Delta E_{G}} \chi(E) d E \\
\nu \Sigma_{I, G^{\prime}}^{f}=\frac{\int_{E^{\prime} \epsilon \Delta E_{G^{\prime}}} \nu \Sigma_{I}^{f}\left(E^{\prime}\right) j_{I\left(E^{\prime}\right)}^{0}}{j_{I, G^{\prime}}^{0}}
\end{gathered}
$$

The discrete-ordinates difference equation is obtained by substituting the derived expressions for each of the five terms into Eq. 4.4-4 and then dividing through by $\Delta \mu_{D}$. The result is

$$
\begin{aligned}
& \bar{\mu}_{D}\left(A_{i+1} \Phi_{G, i+1, D}-A_{i} \Phi_{G, i, D}\right)+ \frac{1}{\Delta \mu_{D}}\left(B_{d+1} \Phi_{G, I, D+1}-B_{d} \Phi_{G, I, d}\right) \\
&\left.+V_{I} \Sigma_{G, I}^{t} \Phi_{G, I, D}=V_{I} S_{G, I, D}+\frac{V_{I}}{2} \sum_{n=0}^{N} P_{n}\left(\bar{\mu}_{D}\right) \sum_{G^{\prime}=1}^{L} \sum_{G^{\prime} \rightarrow G}^{n, I(m o d}\right) \\
& \times \sum_{D^{\prime}=1}^{M} \Phi_{I, G^{\prime}, D^{\prime}} P_{n}\left(\bar{\mu}_{D^{\prime}}\right) \Delta \mu_{D^{\prime}}
\end{aligned}
$$


Although derived for spherical geometry, that is, for $A_{i}=4 \pi r_{i}^{2}$ and $V_{I}=$ $(4 \pi / 3)\left(r_{i+1}^{3}-r_{i}^{3}\right)$, Eq. 4.4-50 is the general discrete-ordinates difference equation for one-dimensional geometries. The equations for the other geometries can be obtained from Eq. 4.4-50, with $A_{i}=1.0$ and $V_{I}=\Delta r_{I}$ for a slab and $A_{i}=2 \pi r_{i}$ and $V_{I}=\pi\left(r_{i+1}^{2}-r_{i}^{2}\right)$ for a cylinder.

\subsubsection{Numerical Solution of the Discrete-Ordinates Equation}

Equation 4.4-50 contains discrete flux-density variables having both centered and end-point subscripts. This in effect increases the number of unknowns such that an insufficient number of determining relations are available for their solution. This difficulty can be resolved by relating the centered and end-point flux densities in some consistent fashion. The diamond difference technique is the most widely used method for this purpose and includes two relations for the spatial variable,

$$
\Phi_{G, I, D}=A \Phi_{G, i+1, D}+(1-A) \Phi_{G, i, D} \quad(\text { for } \mu>0)
$$

and

$$
\Phi_{G, I, D}=(1-A) \Phi_{G, i+1, D}+A \Phi_{G, i, D} \quad(\text { for } \mu<0)
$$

and a single expression for the angular variable

$$
\Phi_{G, I, D}=B \Phi_{G, I, d+1}+(1-B) \Phi_{G, I, d}
$$

where $A$ and $B$ are constants that can be assigned values of the interval $(1 / 2,1)$. When $A=B=1 / 2$, Eqs. 4.4-51 and 4.4-52 are the same for all values of $\mu$ and, together with Eq. 4.4-53, are known as the ordinary diamond difference equations, which we rewrite:

$$
\Phi_{G, i+1, D}=2 \Phi_{G, I, D}-\Phi_{G, i, D} \quad\left(\text { for } \bar{\mu}_{D}>0\right)
$$

or

$$
\Phi_{G, i, D}=2 \Phi_{G, I, D}-\Phi_{G, i+1, D} \quad\left(\text { for } \bar{\mu}_{D}<0\right)
$$

and

$$
\Phi_{G, I, d+1}=2 \Phi_{G, I, D}-\Phi_{G, I, d}
$$


These equations form the basis for most current computer solutions. For a spatial sweep when $\mu_{D}>0$, Eqs. 4.4-54 through 4.4-56 are combined to provide the following explicit expression for the centered discrete-ordinate flux, $\Phi_{G, I, D}$ :

$$
\begin{aligned}
\Phi_{G, I, D}= & {\left[\bar{\mu}_{D}\left(A_{i+1}+A_{i}\right) \Phi_{G, i, D}+\left(1 / \Delta \mu_{D}\right)\left(B_{I, d+1}+B_{I, d}\right) \Phi_{G, I, d}\right.} \\
& \left.+V_{I} S_{G, I, D}^{\prime}\right]\left[\bar{\mu}_{D}\left(2 A_{i+1}\right)+\left(2 B_{I, d+1} / \Delta \mu_{D}\right)+V_{I} \Sigma_{G, I}^{t}\right]^{-1}
\end{aligned}
$$

where the source term $S_{G, I, D}^{\prime}$ includes the fixed source and all inscattering sources. For a typical spatial mesh sweep $\left(\bar{\mu}_{D}>0\right)$, Eq. 4.4-57 is used to solve for the centered flux $\Phi_{G, I, D}$. Then the end-point fluxes $\Phi_{G, i+1, D}$ and $\Phi_{G, I, d+1}$ are calculated by Eqs. 4.4-54 and 4.4-55, respectively. The next centered flux $\Phi_{G, I+1, D}$ is then calculated again with Eq. 4.4-57 and so on. If the flux is decreasing so rapidly that the centered flux $\Phi_{G, I, D}$ is less than one-half of either previous end-point flux, $\Phi_{G, i, D}$ or $\Phi_{G, I, d}$, then the newly calculated end-point flux, $\Phi_{G, i+1, D}$ or $\Phi_{G, I, d+1}$, will be negative. This phenomenon is called diamond difference breakdown and will result in a meaningless positive-negative oscillation of the calculated fluxes. The calculation may be modified by refining the space or angle mesh or both to remedy this, but this would necessarily increase the computational time. However, most problems are reasonably well behaved except possibly for a few points. A technique called negative flux fix-up has been used where negative values occur. The troublesome fluxes are immediately recalculated with the step difference equations, which always yield positive fluxes.

If $A=B=1$ in Eqs. 4.4-51 through 4.4-53, the step-function relation is obtained which equates the centered fluxes to the appropriate end-point fluxes. The step difference equations are:

$$
\Phi_{G, i+1, D}=\Phi_{G, I, D} \quad\left(\text { for } \bar{\mu}_{D}>0\right)
$$

or

$$
\Phi_{G, i, D}=\Phi_{G, I, D} \quad\left(\text { for } \bar{\mu}_{D}<0\right)
$$

and

$$
\Phi_{G, I, d+1}=\Phi_{G, I, D}
$$


An explicit expression for the centered flux $\Phi_{G, I, D}$ is obtained for the $\mu_{D}>0$ spatial mesh sweep by substituting Eqs. 4.4-58 and 4.4-60 into Eq. 4.4-50. The result is

$$
\Phi_{G, I, D}=\frac{\bar{\mu}_{D} A_{i} \Phi_{G, i, D}+\left(1 / \Delta \mu_{D}\right)\left(B_{I, d} \Phi_{G, I, d}\right)+V_{I} S_{G, I, D}^{\prime}}{\bar{\mu}_{D} A_{i+1}+\left(B_{I, d+1} / \Delta \mu_{D}\right)+V_{i} \Sigma \Sigma_{G, I}}
$$

These difference equations are solved by the same calculational sequence described earlier for the ordinary diamond difference equations. The step-function relation is less accurate than the ordinary diamond difference scheme for the same mesh; however, it has the advantage of always giving positive flux densities for positive sources.

The choice of the discrete directions plays an important role in the discrete-ordinates $S_{n}$ method. It does not appear that a most accurate (or best) quadrature scheme for a specific problem can be selected in advance. The efficiency of a given set of discrete directions (quadrature set) depends on problem parameters, such as geometry, optical thickness, energy-group structure, spatial mesh size, etc., and a generalization of these dependencies is not possible.

The discrete directions and associated weights (which represent solid angle) define the quadrature used in the inscattering integral; the directions also define the mean values for the angles, such as $\bar{\mu}_{D}$, and thus affect the approximations in the convection term.

In all $S_{n}$ codes the discrete directions are represented as points on the surface of a unit sphere located at the point in space for which the flux density is to be defined and oriented in a fixed manner with respect to the coordinate system. The points or directions are located on the sphere symmetrically with respect to the three planes defining an octant such that the point description of one octant defines the whole sphere. This is not an absolute necessity but is usually required because of reflecting boundaries.

The more recent $S_{n}$ codes allow specification of direction weights as well as the directions themselves. Lee ${ }^{9}$ developed an elegant method of areas which computes directions and direction weights that are symmetric with respect to rotational interchange of the axes of the unit sphere. Although the directions and weights in this method are somewhat adjustable, the best results occur with the recommended values, which satisfy various approximate moment conditions and asymptotic theories. The area method has the advantage of rotation symmetry and the important advantage of all positive weights for any order of $S_{n}$. 
Although rotation-reflection symmetry has desirable qualities, only three-dimensional calculations would benefit from full symmetry; twodimensional problems thus require twofold symmetry, and one-dimensional problems require no symmetry conditions within the octant. Thus for oneand two-dimensional geometries, and especially for problems where other conditions outweigh the symmetry considerations, some liberty in choosing directions may be exercised.

The selection of a set of directions and the weights (biasing scheme) is particularly important and is geometry dependent. The recommendation of the programmer should be followed when his machine program is used. A bad direction set will lead to implausible flux densities in particular directions.

A typical computer solution of the discrete-ordinates problem is a procedure for iterating the solution to some prescribed degree of convergence. The sweep of the mesh points (i.e., the sequence for moving through the discrete points) is carefully ordered to follow the neutrons (or gamma rays). For shielding problems all particles undergoing scattering will always be degraded in energy. Therefore the calculation will begin with the highest energy group $(G=1)$ and progress sequentially through the lower energy groups. The angular sweep is performed in the direction of increasing $D$ (decreasing $\bar{\mu}_{D}$ ) beginning with $D=1$, which for most penetration problems corresponds to the most important direction. The spatial sweep begins at a boundary along which the inwardly directed flux values are specified, and the sweep is made to the other boundary and then repeated for the next angle. The spatial sweep for negative $\bar{\mu}_{D}$ begins at the other boundary, at which point the reentrant fluxes are usually specified as zero, and proceeds to the source boundary. After the spatial sweep has progressed through all angles at one energy, the next lower energy group is treated in a similar manner and so on.

In the solution of Eq. 4.4-57 or Eq. 4.4-61 for the centered flux $\Phi_{G, I, D}$, some of the required discrete-ordinate fluxes have not yet been calculated; e.g., the within group scattering involves some as yet undetermined fluxes, those which correspond to angles $\bar{\mu}_{D^{\prime}}$, where $D^{\prime} \geqslant D$. Therefore the solution is obtained through the process of inner iteration, whereby values for the unknown fluxes are taken as their previous iterate estimates. Details of the various iteration schemes and of the related convergence problem are omitted here. Several iteration schemes are described by Mynatt ${ }^{11}$ as part of a detailed development and experimental evaluation of the two-dimensional equation in his dissertation. An iterative technique called the synthetic 
method was developed by Kopp ${ }^{12}$ to calculate neutron transport and has been adapted by Gelbard and Hageman ${ }^{13}$ to accelerate convergence of $S_{n}$ calculations.

\subsubsection{Advantages and Disadvantages}

From the results of calculations made with $S_{n}$ codes, the discreteordinates methods appear to have the following advantages for shielding applications:

1. Depending somewhat on the sophistication desired, the $S_{n}$ calculations are easy to prepare.

2. The method is not stochastic, and flux-density errors at deep penetration are systematic rather than statistical.

3. A series of problems having similar characteristics benefit from knowledge of flux densities calculated in a similar case.

4. Secondary gamma rays can be calculated by the same method, either as a second calculation or simultaneously with neutrons. The gamma-ray yield distribution may also be made a function of the energy of the captured neutron.

5. The range of neutron energies from highest fission erergies to thermal, including upscattering in energy, can be calculated by the same method.

6. The one-dimensional calculations are much faster (in computer time) than similar Monte Carlo calculations (see Chap. 5). In two dimensions the type of problem and the desired answers determine whether $S_{n}$ or Monte Carlo is better.

The following disadvantages are evident, but proponents believe that additional development can alleviate or eliminate them:

1. Convergence of an iteration method is not always uniform and well-defined. The best method currently used is to determine from each iterate the maximum deviation in the scalar flux density at any point in space relative to the previous iterate value. Iterations proceed until the maximum deviation falls below a specified limit.

2. Flux-density aberrations are frequently observed in two dimensions owing to localized sources and the propagation of neutrons in discrete directions (this is sometimes called the ray effect).

3. No basic ground rules exist to define for a particular problem the best direction set, space mesh, multigroup structure, and polynomial expansion limit. 


\subsection{MOMENTS METHOD}

The method of moments was formulated by Spencer and Fano ${ }^{14}$ and was the first technique to be successfully applied to the Boltzmann equation for solutions useful to reactor shielding. In addition to its historical prominence, this technique has some important advantages not shared by other methods, one being that foreknowledge about the behavior of the solution can be incorporated analytically in a very natural way, thereby often reducing the effort required to achieve a specific result or a desired accuracy or both. Another is that the type of recursion relation developed precludes a truncation at a crucial part of the calculation; that is, a finite number of moments can be calculated exactly (ignoring errors due to the numerical solution) without considering the influence of higher moments.

In the moments method one considers first the formal definition for the moments and the manner in which they relate to some system parameter of interest, say $f(x)$. If $f(x)$ is defined for all $x$ within the interval $A \leqslant x \leqslant B$, then the $n$th moment of $f(x)$ is

$$
M_{n}=\int_{A}^{B} x^{n} f(x) d x
$$

provided the integral exists. Only nonnegative integer values of $n$ are considered in practical applications.

Definite interpretations may be associated with the various moments. For example, the zeroth moment is a normalizing number, and the first, second, third, and fourth moments are closely related to the mean value, variance, skewness, and kurtosis, respectively. In the physics of statics and dynamics, the first moment of the mass is the center of gravity and the second is the moment of inertia.

No such particular meanings are given to the moments as they are used in the solution of radiation-transport problems (although the second moment of the flux density is proportional to the Fermi age). Rather, they are regarded as a transform, much the same as Laplace, Fourier, or finite trigonometric transforms. The major portion of the calculation is performed in terms of the transform (moments) space; then, by an appropriate inversion, the desired answer is reconstructed.

The application of the moments method to the solution of the Boltzmann transport equation is limited with respect to the source-shield configuration. It is usually applied only to infinite homogeneous media with 
plane, line, or point sources. The method as applied to gamma rays has been described by Fano, Spencer, and Berger ${ }^{15}$ and by Goldstein and Wilkins, ${ }^{16}$ and, as applied to neutrons, by Goldstein. ${ }^{17}$ The technique is basically the same for both neutrons and gamma rays, and a description for one should suffice for the other. The most significant differences lie in the treatment of the scattering integral and in the more complex nature of the neutron cross sections. The following description is for slab geometry in terms of the simpler gamma-ray problem in which the dependent variable is the angular energy-flux density, $I(x, \lambda, \mu)$ and the Compton wavelength is taken as the energy variable.

Consider the following specialized form of the Boltzmann equation: $\dagger$

$$
\begin{aligned}
\mu \frac{\partial I}{\partial x}+\Sigma(\lambda) I(x, \lambda, \mu)= & \int_{0}^{\lambda} \int_{4 \pi} I\left(x, \lambda^{\prime}, \mu^{\prime}\right) \epsilon k\left(\lambda^{\prime}, \lambda\right) \\
& \times \frac{\delta\left(1+\lambda^{\prime}-\lambda-\Omega \cdot \Omega^{\prime}\right)}{2 \pi} d \Omega^{\prime} d \lambda^{\prime}+S(\lambda, \mu) \delta(x)
\end{aligned}
$$

where $I(x, \lambda, \mu) d E d \mu=$ energy-flux density ( $\mathrm{MeV}$ per unit area and time) due to gamma rays with energies in $d E$ about $E$ and direction cosines that lie in $d \mu$ about $\mu$

$x=$ spatial coordinate in slab geometry

$\lambda=$ gamma-ray energy after scattering expressed in terms of its Compton wavelength

$\lambda^{\prime}=$ gamma-ray wavelength prior to scattering

$\mu=$ direction cosine with respect to the $x$-axis

$\Sigma(\lambda)=$ total macroscopic cross section evaluated at the energy corresponding to the gamma-ray wavelength, $\lambda$

$\boldsymbol{\Omega} \cdot \boldsymbol{\Omega}^{\prime}=\cos \theta=\operatorname{cosine}$ of the scattering angle between initial and final gamma-ray directions, where $\boldsymbol{\Omega}^{\prime}$ and $\boldsymbol{\Omega}$ are the initial and final unit direction vectors, respectively

$\epsilon=$ electron density

$k\left(\lambda^{\prime}, \lambda\right)=2 \pi\left(\lambda / \lambda^{\prime}\right) \sigma\left(\lambda^{\prime}, \theta\right)$

$\sigma\left(\lambda^{\prime}, \theta\right)=$ microscopic cross section per electron for Compton scattering given by the Klein-Nishina formula 
$\delta(x)=$ Dirac delta function $\dagger$ that locates the plane at $x=0$ $\delta\left(1+\lambda^{\prime}-\lambda-\Omega \cdot \Omega^{\prime}\right)=$ Dirac delta functiont that prescribes that the angular change $\left(\boldsymbol{\Omega} \cdot \boldsymbol{\Omega}^{\prime}\right)$ be consistent with the change in wavelength $\left(\lambda-\lambda^{\prime}\right)$ as given by the Compton scattering equation, $\lambda-\lambda^{\prime}=1-\Omega \cdot \Omega^{\prime}$

$S(\lambda, \mu) d E d \mu=$ plane source of gamma rays (energy emission per unit area and time of gamma rays with energies in $d E$ about $E$ and direction cosines that lie in $d \mu$ about $\mu)$.

Solving Eq. 4.5-2 by the moments method is similar to using the spherical-harmonics treatment (see Sec. 4.3) in that the angular energy-flux density is first expanded as a Legendre polynomial series:

$$
I(x, \lambda, \mu)=\sum_{j=0}^{\infty} \frac{2 j+1}{2} I_{j}(x, \lambda) P_{j}(\mu)
$$

where the Legendre coefficients are given by

$$
I_{j}(x, \lambda)=\int_{-1}^{+1} I(x, \lambda, \mu) P_{j}(\mu) d \mu
$$

It can be shown that $I_{0}(x, \lambda)$ is the energy-flux density and that $I_{1}(x, \lambda)$ is the energy-current density.

With this series representation for the angular energy-flux density, the integro-differential equation (Eq. 4.5-2) with the dependent variable $I(x, \lambda, \mu)$ and three continuous independent variables can be transformed into a sequence of integro-differential equations for the variables $I_{j}(x, \lambda)$, which are dependent on only two independent variables. This desired result is obtained by multiplying Eq. 4.5-2 by the Legendre polynomial $P_{j}(\mu)$ and integrating over all solid angle. The result (after some manipulation) is the following sequence of equations:

$$
\begin{array}{r}
\frac{j+1}{2 j+1} \frac{\partial I_{j+1}}{\partial x}+\frac{j}{2 j+1} \frac{\partial I_{j-1}}{\partial x}+ \\
\quad \Sigma(\lambda) I_{j}(x, \lambda)=S_{j}(\lambda) \delta(x)+\int_{0}^{\lambda} P_{j}\left(1+\lambda^{\prime}-\lambda\right) \\
\times \in k\left(\lambda^{\prime}, \lambda\right) I_{j}\left(x, \lambda^{\prime}\right) d \lambda^{\prime} \quad(j=0,1,2, \ldots, \infty)
\end{array}
$$


The spatial variable in this sequence of equations is eliminated by applying the moments of the Legendre coefficients of the angular energyflux density, which are defined as

$$
b_{n j}(\lambda) \equiv \frac{\sum_{0}^{n+1}}{n !} \int_{-\infty}^{+\infty} I_{j}(x, \lambda) x^{n} d x
$$

where $\Sigma_{0}$ is the total macroscopic cross section evaluated at the source energy. The equations satisfied by $b_{n j}(\lambda)$ are obtained by multiplying Eq. 4.5-6 by $\sum_{0}^{n+1}\left(x^{n} / n !\right)$ and integrating with respect to $x$ from $-\infty$ to $+\infty$. Because of this integration over all space, the application of the moments method to the transport problem becomes restricted to the infinite-medium geometry. With the use of Eq. 4.5-6, the original Boltzmann equation can be reduced to the following doubly indexed sequence of linear integral equations (the variable upper limit in the integral classifies it as the Volterra type):

$$
\begin{array}{r}
\Sigma(\lambda) b_{n j}(\lambda)=\int_{0}^{\lambda} k\left(\lambda^{\prime}, \lambda\right) P_{j}\left(1+\lambda^{\prime}-\lambda\right) b_{n j}\left(\lambda^{\prime}\right) d \lambda^{\prime}+\frac{\Sigma_{0}}{2 j+1}\left[(j+1) b_{n-1, j+1}\right. \\
\left.+j b_{n-1, j-1}\right]+\mu_{0} S_{j}(\lambda) \delta_{n 0}
\end{array}
$$

where $j=0,1,2, \ldots, \infty$, and $n=0,1,2, \ldots, \infty$. The Kronecker delta function $\delta_{n 0}=1$ if $n=0, \delta_{n 0}=0$ if $n \neq 0$.

The moments for a given problem can be evaluated by a straightforward numerical solution of Eq. 4.5-7. The ease of numerical calculation depends on the form of the source function $S_{j}(\lambda)$. Many problems involve monoenergetic sources, and the $S_{j}(\lambda)$ are given by $\lambda_{0} S_{j} \delta\left(\lambda-\lambda_{0}\right)$, where $\lambda_{0}$ is the Compton wavelength of the source energy. Since the presence of the delta function is undesirable for machine calculation, the following transformation is made:

$$
b_{n j}(\lambda)=B_{n j}(\lambda)+\lambda_{0} \delta\left(\lambda-\lambda_{0}\right) C_{n j}
$$

Introducing the transformation defined by Eq. 4.5-8 into Eq. 4.5-7 yields the defining equations for $B_{n j}$ and $C_{n j}$ :

$$
\begin{aligned}
\Sigma B_{n j}=\int_{\lambda_{0}}^{\lambda} \epsilon k\left(\lambda^{\prime}, \lambda\right) P_{j}\left(1+\lambda^{\prime}-\lambda\right) B_{n j}\left(\lambda^{\prime}\right) d \lambda^{\prime}+\frac{\Sigma_{0}}{2 j+1}[(j+1) \\
\left.\times B_{n-1, j+1}+j B_{n-1, j-1}\right]+\lambda_{0} k\left(\lambda_{0}, \lambda\right) P_{j}\left(1+\lambda_{0}-\lambda\right) C_{n j}
\end{aligned}
$$




$$
\Sigma C_{n j}=\Sigma_{0}\left(\frac{j+1}{2 j+1} C_{n-1, j+1}+\frac{j}{2 j+1} C_{n-1, j-1}\right)+\Sigma_{0} S_{j} \delta_{n 0}
$$

The equations that define $C_{n j}$ are similar in form to the equations that define $b_{n j}$ and $B_{n j}$ except that the inscattering integral does not appear. It follows that $\lambda_{0} \delta\left(\lambda-\lambda_{0}\right) C_{n j}$ are the moments for the unscattered-energy-flux density. Therefore the transformation given by Eq. 4.5-8 separates the unscattered-energy-flux density (energy-flux density corresponding to $C_{n j}$ ) from the total energy-flux density (energy-flux density corresponding to $\left.b_{n j}\right)$. The solution to Eq. 4.5-9 requires values of $C_{n j}$ as input, and the calculated moments $B_{n j}$ are associated only with the scattered-energy-flux density. This is convenient since the uncollided angular energy-flux density, $I^{0}(x, \lambda, \mu)$, is easily calculated, and values of $C_{n j}$ are then uniquely determined.

For a typical calculation, the quantity of greatest interest is usually the total, or scalar, energy-flux density, $I_{0}(x, \lambda)$. Therefore only the moments $B_{n \mathbf{0}}(n=0,1,2, \ldots, N)$ are required. However, the calculation of a given $B_{n j}$ requires the prior calculation of $B_{n-1, j+1}$ and $B_{n-1, j-1}$; therefore moments other than the $B_{n 0}$ moments must be calculated. In general, the moments $B_{0 j}(j=0,1,2, \ldots, J)$ can be calculated directly, but a $B_{n j}$ moment cannot be calculated until calculations have been made of all the $B_{n^{\prime}} j^{\prime}$ 's for which $(n+j)-\left(n^{\prime}+j^{\prime}\right)$ is a nonnegative even integer (including zero) and $n^{\prime}<n$. Table 4.1 illustrates a typical calculation sequence (for $n=5$ ). As noted, all

Table 4.1-SEQUENCE OF MOMENTS CALCULATION FOR $n=5$

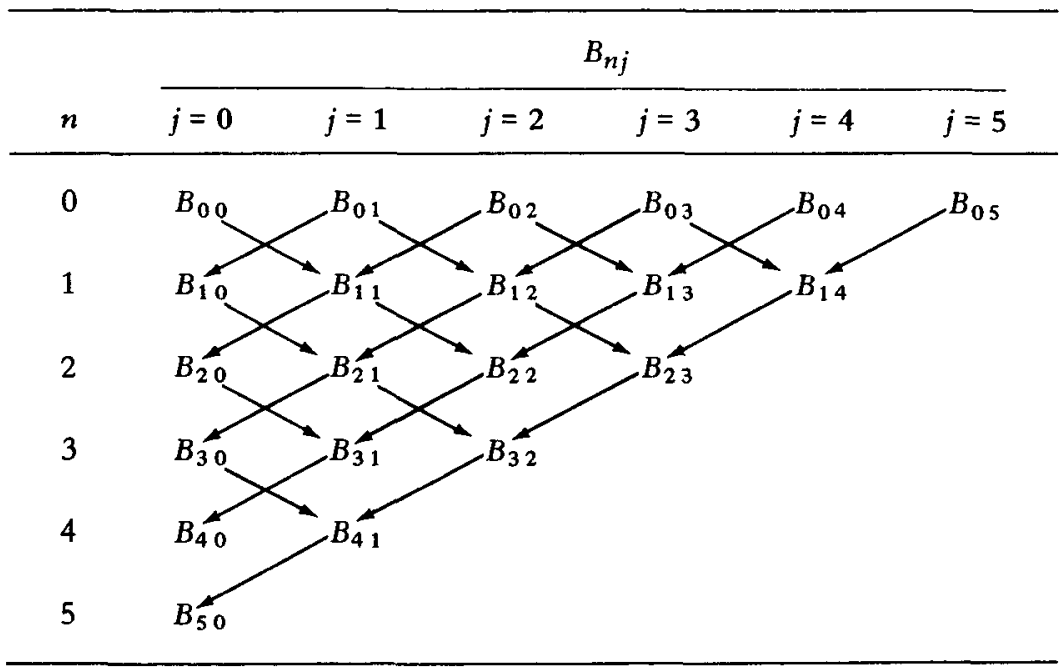


the moments shown in the table must be calculated to determine $B_{n_{0}}$ for $n \leqslant 5$.

We rewrite the Eq. 4.5-9 integral in the following form:

$$
\Sigma\left(\lambda_{n}\right) v\left(\lambda_{n}\right)=\int_{\lambda_{0}}^{\lambda_{n}} H\left(\lambda_{n}, \lambda^{\prime}\right) v\left(\lambda^{\prime}\right) d \lambda^{\prime}+T\left(\lambda_{n}\right)
$$

where $\lambda_{n}=\lambda_{0}+n \Delta \lambda$

$$
\begin{aligned}
\Delta \lambda & =\text { arbitrary increment of wavelength } \\
v\left(\lambda_{n}\right) & =B_{n j}\left(\lambda_{n}\right) \\
H\left(\lambda_{n}, \lambda^{\prime}\right) & =\epsilon k\left(\lambda^{\prime}, \lambda_{n}\right) P_{j}\left(1+\lambda^{\prime}-\lambda_{n}\right) \\
T\left(\lambda_{n}\right) & =\left[\Sigma_{0} /(2 j+1)\right]\left[(j+1) B_{n-1, j+1}+j B_{n-1, j+1}\right]+\lambda_{0} H\left(\lambda_{n}, \lambda_{0}\right) C_{n j}
\end{aligned}
$$

This type of integral equation (Volterra type) is characterized by (1) the limit of integration being the independent variable $\lambda_{n} ;(2)$ the value of the dependent variable $v\left(\lambda_{n}\right)$ depending on the values of $v\left(\lambda^{\prime}\right)$ if $\lambda^{\prime}<\lambda_{n}$ but not if $\lambda^{\prime}>\lambda_{n}$; and (3) $T\left(\lambda_{n}\right)$ involving only known or previously calculated quantities. A numerical evaluation of the integral is required, and several schemes are available. Regardless of which scheme is used, there are coefficients $M_{n k}$ such that Eq. 4.5-11 can be rewritten as

$$
\begin{aligned}
\Sigma\left(\lambda_{n}\right) v\left(\lambda_{n}\right)=\sum_{k=0}^{n-1} H\left(\lambda_{n}, \lambda_{k}\right) v\left(\lambda_{k}\right) & M_{n k} \\
& +H\left(\lambda_{n}, \lambda_{n}\right) v\left(\lambda_{n}\right) M_{n n}+T\left(\lambda_{n}\right)
\end{aligned}
$$

where

$$
H\left(\lambda_{n}, \lambda_{n}\right)=k\left(\lambda_{n}, \lambda_{n}\right) P_{j}\left(1+\lambda_{n}-\lambda_{n}\right)=\frac{3}{4}
$$

Trapezoidal rule is used for the $(n=1)$ interval for which $M_{n n}=\Delta \lambda / 2$, and the following explicit expression for $v\left(\lambda_{1}\right)$ is obtained:

$$
v\left(\lambda_{1}\right)=\frac{T\left(\lambda_{1}\right)+H\left(\lambda_{1}, \lambda_{0}\right) v\left(\lambda_{0}\right)(\Delta \lambda / 2)}{\Sigma\left(\lambda_{1}\right)-(3 \Delta \lambda / 8)}
$$

For $n>1, M_{n n}=\Delta \lambda / 3$, and Eq. $4.5-12$ is rewritten

$$
v\left(\lambda_{n}\right)=\frac{T\left(\lambda_{n}\right)+\sum_{k=0}^{n-1} H\left(\lambda_{n}, \lambda_{k}\right) v\left(\lambda_{k}\right) M_{n k}}{\Sigma\left(\lambda_{n}\right)-(\Delta \lambda / 4)}
$$


The coefficients $M_{n k}$ for $n$ even are determined by Simpson's rule. For $n=3$, the following coefficients are used: $M_{30}=5 / 16, M_{31}=1$, and $M_{32}=5 / 4$; for $n$ odd but greater than 3 , the integral from $\lambda_{0}$ to $\lambda_{3}$ is evaluated using the special $(n=3)$ coefficients, and the integral from $\lambda_{3}$ to $\lambda_{n}$ is evaluated by Simpson's rule. The gamma-ray scattering process is such that

$$
H\left(\lambda_{n}, \lambda_{k}\right)=0 \quad\left(\text { when } \lambda_{n}>\lambda_{k}+2\right)
$$

Therefore the sums on the index $k$ involve only a fixed number of terms. Also

$$
H\left(\lambda_{n}, \lambda_{0}\right)=0 \quad\left(\text { when } \lambda_{n}>\lambda_{0}+2\right)
$$

and the second term in $T\left(\lambda_{n}>\lambda_{0}+2\right)$ also vanishes; $H\left(\lambda_{n}, \lambda_{k}\right)$ can be calculated directly since only the Klein--Nishina formula and the Legendre polynomials are involved.

At this point we assume that the moments for a given problem can be calculated and consider the problem of reconstructing the flux densities. It should be emphasized that the calculation to this point can be performed with very few approximations, $\dagger$ excluding the approximations involved in the numerical procedures. The major source of error will lie in the subsequent reconstruction process since only a finite number of moments are available. In fact, for a finite number of moments, there is an infinite number of allowable functions. The problem is basically one of choice: the selection of a functional form that will come as close as possible to describing the spatial dependence of $I_{j}(x, \lambda)$.

Two methods have been used to reconstruct the flux densities: the polynomial expansion method and the method of undetermined parameters. Both were developed by Spencer and Fano. ${ }^{14} \mathrm{~A}$ more recent description is given by Spencer. ${ }^{18}$ The polynomial expansion method assumes that $I_{j}(\rho, \lambda)$ behaves roughly as some trial function $f(\rho)$, where $\rho$ is measured in mean free paths at the initial energy; i.e., $\rho \equiv \Sigma_{0} x$. Then

$$
I_{j}(\rho, \lambda)=f(\rho) g_{j}(\rho, \lambda)
$$

TThese few assumptions can be very limiting, however, since they include the assumptions of homogeneity and infinite extent of the transporting medium. 
where $g_{j}(\rho, \lambda)$ contains the $\lambda$ dependence of the $j$ th coefficient and provides a correction for the $\rho$ dependence. If a reasonable choice of $f(\rho)$ can be made, then $g_{j}(\rho, \lambda)$, need be only a gently varying smooth function of $\rho$; for example, a polynomial of degree $N$ in $\rho$ when $(N+1)$ moments are available. The $g_{j}(\rho, \lambda)$ could be represented as an infinite series with respect to a set of orthogonal polynomials of degree $n$ :

$$
g_{j}(\rho, \lambda)=\sum_{n=0}^{\infty} A_{n j}(\lambda) p_{n}(\rho) \dagger
$$

The orthogonality relation is given by

$$
\int_{-\infty}^{+\infty} P_{n}(x) P_{m}(x) f(x) d x=\delta_{n m}
$$

where $f(x)$ is a weighting function as well as the trial function $f(\rho)$. In practice this representation of $g_{j}(\rho, \lambda)$ is limited to a finite number of terms since, given $(N+1)$ moments, only $(N+1)$ values of $A_{n j}$ can be obtained.

The approximation for $I_{j}^{s}(\rho, \lambda)$ can then be written

$$
I_{j}^{s}(\rho, \lambda)=f(\rho) \sum_{n=0}^{N} A_{n j}(\lambda) P_{n}(\rho)
$$

where $I_{j}^{S}(\rho, \lambda)$ is the $j$ th Legendre coefficient of the scattered component of the angular flux density. This assumes that values of $(N+1)$ moments $B_{n j}$ are available for the reconstruction of $I_{j}^{s}(\rho, \lambda)$, which is accomplished by evaluating the $(N+1)$ coefficient $A_{n j}$ in terms of the known $(N+1)$ moments $B_{n j}$ for a given value of $j$. To this end $I_{j}^{S}(\rho, \lambda)$ is multiplied by $p_{m}(\rho) d \rho$, and the product is integrated from $-\infty$ to $+\infty$ :

$$
A_{n j}\left(\lambda^{\prime}\right)=\int_{-\infty}^{+\infty} I_{j}^{s}(\rho, \lambda) p_{n}(\rho) d \rho
$$

The polynomial $p_{n}(\rho)$ can be written

$$
p_{n}(\rho)=\sum_{i=0}^{n} a_{i} \rho^{i}
$$

where the $a_{i}$ 's are known parameters for a given type of polynomial. The expression for $A_{n j}(\lambda)$ then becomes

$$
A_{n j}(\lambda)=\sum_{j=0}^{n} a_{i} \int_{-\infty}^{+\infty} I_{j}^{S}(\rho, \lambda) \rho^{i} d \rho
$$

tThe polynomial set $\left(p_{n}\right)$ is any orthogonal set, one of which is the Legendre polynomial set. 
The moments $B_{i j}$ are defined as

$$
B_{i j}(\lambda) \equiv \frac{1}{i !} \int_{-\infty}^{+\infty} I_{j}^{S}(\rho, \lambda) \rho^{i} d \rho
$$

Elimination of the integrals between the preceding expressions for $A_{n j}(\lambda)$ and $B_{i j}(n)$ provides the desired relation

$$
A_{n j}(\lambda)=\sum_{i=0}^{n}(i !) a_{i} B_{i j}
$$

Practical considerations will usually restrict accurate calculation to the differential energy-flux density $I_{0}^{S}(\rho, \lambda)$, and then only the $A_{n_{0}}(\lambda)$ 's are required; that is,

$$
I_{0}^{S}(\rho, \lambda)=f(\rho) \sum_{n=0}^{N} A_{n 0}(\lambda) p_{n}(\rho)
$$

where

$$
A_{n 0}(\lambda)=\sum_{i=0}^{n}(i !) A_{i} B_{i 0}
$$

In principle, $A_{n j}(\lambda)$ for $j>0$ can be calculated. However, since the angular flux density $I(x, \lambda, \mu)$ is usually highly peakec in the forward direction, the series

$$
I^{s}(x, \lambda, \mu)=\sum_{j=0}^{J} \frac{2 j+1}{2} I_{j}^{s}(x, \lambda) p_{j}(\mu)
$$

converges slowly, thereby requiring a large number of values of $I_{j}^{s}(x, \lambda)$, which, in turn, would require a large number of moments. Finally, unscattered-energy-flux densities are easily obtained for most simple geometries and combined with the scattered energy-flux densities:

$$
I_{0}(x, \lambda)=I_{0}^{0}(x, \lambda)+I_{0}^{s}(x, \lambda)
$$

The polynomial expansion method described in the preceding text is most often used for reconstructing the energy-flux density of the gamma-ray problem. This is partly for historical reasons and partly due to the ability of the method to make full use of large numbers of moments within the same systematic framework of analysis. For the neutron problem the selection of 
a suitable weighting (or test) function is not obvious, and the method loses much of its flexibility.

When the method of undetermined parameters is used to reconstruct the flux densities, $I_{j}(\rho, \lambda)$ is represented as

$$
I_{j}(\rho, \lambda)=\sum_{i} a_{i j}(\lambda) h_{i j}(\rho)
$$

where $h_{i j}(\rho)$ is a function having the general expected behavior of $I_{j}(\rho, \lambda)$ but containing one or more undetermined parameters, and the $a_{i j}$ are undetermined parameters that include the $\lambda$ dependence. In particular, let $j=0$ and assume that $(N+1)$ values of the $B_{n 0}(\lambda)$ moments are known; then

$$
I_{0}^{s}(\rho, \lambda)=\sum_{i} a_{i 0}(\lambda) h_{i 0}(\rho)
$$

The moments corresponding to $j=0$ can be written as

$$
B_{n 0}(\lambda)=\frac{1}{n !} \int_{-\infty}^{+\infty} I_{0}^{S}(\rho, \lambda) \rho^{n} d \rho \quad(n=0,1,2, \ldots, N)
$$

Substituting Eq. 4.5-31 into 4.5-32 yields the following set of $(N+1)$ equations:

$$
B_{n 0}(\lambda)=\frac{1}{n !} \sum_{i} a_{i 0}(\lambda) \int_{-\infty}^{+\infty} h_{i 0}(\rho) \rho^{n} d \rho \quad(n=0,1,2, \ldots, N)
$$

Values of $h_{i 0}(\rho)$ should be selected so that the above integration can be evaluated either analytically or numerically, and, if $(N+1)$ moments are available, then a total of $(N+1)$ undetermined parameters are allowed.

Problems not amenable to other methods can sometimes be solved by the method of undetermined parameters because of the much greater choice that can be made in the $h_{i j}(\rho)$ values. As a result this method has been more widely applied to the neutron-penetration problem. A characteristic of the method is when it fails, it fails catastrophically, leaving no doubt about its applicability. Usually not all the moments available are needed to obtain a satisfactory solution. The surplus moments can be used to check the accuracy by constructing moments corresponding to the unused moments, a feature not so easily accomplished by the other methods.

The application of the moments method to neutron-transport problems is similar in many respects to the gamma-ray formulation. The primary differences are the use of differential neutron-scattering cross sections rather 
than Compton scattering cross sections, particle-flux density, $\Phi(\mathbf{r}, E, \boldsymbol{\Omega})$, rather than energy-flux density, and the use of neutron lethargy in performing integrals over energy.

The spatial moments in the neutron case are defined as

$$
I_{n l}(u)=\frac{1}{n !} \int_{-\infty}^{\infty} I_{l}(u, x) x^{n} d x
$$

and the set of interlocked integral equations has the form

$$
\begin{aligned}
\sigma_{t}(u) I_{n l}(u)=2 \pi \sum_{i} & \int_{-1}^{1} I_{n l}\left(u^{\prime}\right) \sigma_{n, i}\left(u^{\prime}\right) f_{i}\left(u^{\prime}, \mu_{i}\right) P_{l}\left(\alpha_{i}\right) d \mu_{i} \\
& +\frac{1}{2 l+1}\left[(l+1) I_{n-1, l+1}+l I_{n-1, l-1}\right]+S_{n l}(u)
\end{aligned}
$$

where $u$ is a variable based on the neutron energy, $u=\ln \left(E / E_{0}\right) ; \mu$ is the cosine of the scattering angle in the center-of-mass system; and $\alpha$ is the cosine of the scattering angle in the laboratory system.

Differences also appear in the means of reconstructing the flux density from the moments. For neutrons the results are more sensitive to the choice of $f(\rho)$.

Cross sections change more rapidly with energy for neutrons, and the scattering integral limits vary from element to element. For hydrogen the scattering integral may be integrated directly by numerical means as was done for gamma rays. For all other elements, three optional approaches are available, depending on whether energy degradation in scattering is treated exactly or approximately or ignored entirely.

For sufficiently large nuclei, the energy degradation may be ignored. The minimum mass for which this approximation can be made will depend on the other constituents of the material. For example, energy degradation by oxygen may be ignored in the presence of hydrogen but not in a thick shield consisting primarily of heavy elements.

The approximate treatment consists in ignoring degradation for heavy elements but accounting for it with hydrogen scattering. This treatment has been found to give results within 20\% of the exact treatment for a mass of 6 or $7 \mathrm{amu}$ where hydrogen accounts for most of the slowing down. Similar accuracy is obtained at 10 to 20 mean free paths for atomic masses above 20 in the absence of lower weight elements. A method for a more exact treatment of degradation was developed by Certaine. ${ }^{19}$ 


\subsection{APPLICATION OF DIFFUSION THEORY}

An approach to the particle-transport problem which neglects the detailed directional aspects of particle motion comes from diffusion theory. If we consider the neutron balance in a medium, the equation of continuity is a statement that the neutron gains are equal to the losses in a given volume element. We assume no energy degradation and that all neutrons have one velocity.

$$
\begin{aligned}
{\left[\begin{array}{c}
\text { rate of gain } \\
\text { per unit volume }
\end{array}\right]=} & {\left[\begin{array}{c}
\text { source per } \\
\text { unit volume }
\end{array}\right] } \\
& -\left[\begin{array}{c}
\text { absorption } \\
\text { per unit volume }
\end{array}\right]-\left[\begin{array}{c}
\text { leakage } \\
\text { per unit volume }
\end{array}\right]
\end{aligned}
$$

In four-dimensional phase space, $(r, t)$, this statement becomes

$$
\frac{\partial n(\mathbf{r}, t)}{\partial t}=S(\mathbf{r}, t)-\Sigma_{a} \Phi(\mathbf{r}, t)-\nabla \cdot J(\mathbf{r}, t)
$$

where $n(r, t)=$ neutron density (neutrons $/ \mathrm{cm}^{3}$ )

$\Sigma_{a}=$ macroscopic absorption cross section $\left(\mathrm{cm}^{-1}\right)$

$S(\mathbf{r}, t)=$ general source term (neutrons $\mathrm{cm}^{-3} \mathrm{sec}^{-1}$ )

$\Phi(\mathbf{r}, t)=$ total neutron-flux density (neutrons $\mathrm{cm}^{-2} \sec ^{-1}$ )

$J(\mathbf{r}, t)=$ net neutron-current density (neutrons $\mathrm{cm}^{-2} \mathrm{sec}^{-1}$ )

$\partial n / \partial t=$ time rate of change of the neutron density (neutrons $\mathrm{cm}^{-3}$ $\sec ^{-1}$ )

$\Sigma_{a} \Phi(\mathrm{r}, t)=$ loss of neutrons due to absorption (neutrons $\mathrm{cm}^{-3} \mathrm{sec}^{-1}$ )

$\nabla \cdot J(\mathbf{r}, t)=$ loss of neutrons due to convection (neutrons $\mathrm{cm}^{-3} \mathrm{sec}^{-1}$ )

Equation 4.6-1 can be regarded as a precise relation that can be applied without restriction to the general problem of particle transport. However, a basic limitation in its use is that, except for certain very restricted situations, a tractable form for the net neutron current, $J(\mathrm{r}, t)$, does not exist. If we assume that absorption is small compared with scattering and confine ourselves to steady-state (time-independent) conditions, the net current density depends on the change in neutron flux with position

$$
J(\mathbf{r})=-D \nabla \Phi(\mathbf{r})
$$

where $D$ is the position-independent diffusion coefficient $(\mathrm{cm})$ and $\nabla \Phi(\mathbf{r})$ is the gradient of the total neutron flux. It is noted that with the steady-state 
assumption, phase space has been reduced to three position variables denoted by the vector (r). Equation 4.6-2 is known as Fick's law, which simply states that the net diffusion of particles (or molecules) in liquids and gases will be from regions of high particle density to regions of low particle density, with the gradient of the particle-flux density as the driving potential.

Substitution of Eq. 4.6-2 into the steady-state form of Eq. 4.6-1 leads to the diffusion equation

$$
D \nabla^{2} \Phi(\mathbf{r})-\Sigma_{a} \Phi(\mathbf{r})+S(\mathbf{r})=0
$$

Equation $4.6-3$ has the same form as the steady-state form of the $P_{1}$ approximation to the spherical-harmonics treatment of the Boltzmann equation (see Sec. 4.3).

Certain limitations are inherent to diffusion theory: (1) the scattering process is assumed to be isotropic in the laboratory frame of reference (the use of a "transport-corrected" diffusion coefficient, $D=1 / 3 \Sigma_{t r}$, removes this limitation); (2) the directional distribution of the particle-flux density is nearly isotropic; (3) the diffusing medium must be a poor absorber, i.e., $\Sigma_{a} \ll \Sigma_{s}$; and (4) the results are invalid for regions within two to three mean free paths of boundaries, strong sources, and strong sinks. The existence of these limitations is a clear indication of the approximate nature of diffusion theory insofar as the physical situation is concerned. In reality, the preceding conditions of applicability for diffusion theory are seldom satisfied. However, with the judicious selection of system parameters, the diffusiontheory solutions of certain problems $\dagger$ compare favorably with solutions obtained with more exact theories or with the physical situation itself.

A neutron shielding problem would involve a continuous energy spectrum over a wide energy range (typically from a low keV region to $10 \mathrm{MeV}$ ); thus a group approach is required to adequately describe the diffusion process. The energy range is divided into $G$ energy groups with the gth group corresponding to the energy width $E_{g+1}-E_{g}$.

The group-diffusion equation for the $g$ th group is given by

$$
D_{g} \nabla^{2} \Phi_{g}(\mathbf{r})-\Sigma g(\mathbf{r})+S_{g}(\mathbf{r})=0
$$

tFor example, diffusion theory is used in fast reactor shielding design since the leakage spectrum peaks below $0.5 \mathrm{MeV}$ and the materials involved are nonhydrogenous (sodium and graphite). Also, the small source (reactor core) requires two-dimensional calculations, which are much simpler with diffusion theory. 
where $D_{g}=$ group-averaged diffusion coefficient

$$
=\frac{\int_{E_{g}}^{E_{g+1}} D(E) \Phi(r, E) d E}{\Phi_{g}(r)}
$$

$\Phi_{g}(\mathbf{r})=$ total flux density corresponding to the gth energy group $(g=$ $1,2, \ldots, G)$

$$
=\int_{E_{g}}^{E_{g+1}} \Phi(\mathbf{r}, E) d E
$$

$\Sigma \&=$ group-averaged macroscopic absorption cross section

$$
=\frac{\int_{E_{g}}^{E_{g+1}} \Sigma_{a}(E) \Phi(\mathbf{r}, E) d E}{\Phi_{g}(\mathbf{r})}
$$

$$
\begin{aligned}
S_{g}(\mathbf{r}) & =\text { general source term } t \text { for the } g \text { th group } \\
& =\int_{E_{g}}^{E_{g+1}} S(\mathbf{r}, E) d E
\end{aligned}
$$

However, the typical neutron shielding problem is not amenable to solution by the straightforward application of diffusion theory, because the neutrons are on the average very energetic and possess a strong forward directional bias. The limitations of diffusion theory under these conditions are clearly violated, and results thus obtained would be meaningless. However, when applied to certain special problems in combination with other methods, diffusion theory has proved useful. Applications of diffusion theory to the neutron shielding problem are discussed further in Sec. 4.8.

The use of diffusion theory to predict gamma-ray energy-flux densities seems to be unjustified on superficial examination of the gamma-ray-transport phenomenon. Certainly deep penetration by gamma radiation cannot be described by diffusion theory, because the resultant gamma-ray-flux density is due to photons that have maintained a strong directional correlation. However, diffusion theory seems to be adequate for small-to-moderate penetrations relatively near the source under conditions where the lowenergy end of the spectrum predominates and the scattering is more nearly isotropic. These restricted conditions exist, for example, for most gamma-ray heating calculations.

fObserve that the source term includes both particles born in energy group $\left(E_{g} E_{g+1}\right)$ and particles that have scattered into that energy interval from other groups, Thus the set of equations $4.6-4$ $(g=1,2, \ldots, G)$ is coupled through the source term. 


\subsection{INVARIANT IMBEDDING METHOD}

The method of invariant imbedding is not another method for solving the Boltzmann transport equation; rather, it is a different fundamental approach to the mathematical description of particle transport. The method has for its historical basis the early works of a Russian astrophysicist, Ambarzumian, ${ }^{20}$ whose interest was in the transport problems of astrophysics. Recent investigations of Bellman, Kalaba, and Wing ${ }^{21}$ and Wing ${ }^{22}$ have shown that the invariant imbedding approach can be applied with high-speed computers to a much broader class of problems, including the neutron- and gamma-ray-transport problems encountered in radiation shielding.

The dependent variables of the invariant imbedding formulation are the reflection and transmission functions, with the region dimensions (shield thickness) and the energy and direction of the particle comprising a six-dimensional phase space. In this context a particular shielding problem is viewed as being imbedded in a more general class of shields having different dimensions. Characteristically, and in contrast with solutions of the Boltzmann transport equation, the invariant imbedding method provides transmission and reflection information for a large variety of shields as well as for the specific problem of interest. However, the detailed behavior of the radiation during transport through the shield is not explicit during the analysis and for that reason is unavailable, a not too serious shortcoming, if not a real advantage, for the typical shielding problem. The pertinent equations can be derived by applying the usual conservation principles of radiation transport to a shield system, the dimensions of which are allowed to vary by differential amounts. For simplicity and greater clarity, the derivations are performed in slab geometry with azimuthal symmetry. Phase space becomes three dimensional: the shield thickness, $X$, the energy variable, $E$, and the direction variable, $\mu(=\cos \theta)$. A schematic representation of this configuration is shown in Fig. 4.6.

The reflection function $R\left(X ; \mu, E ; \mu_{0}, E_{0}\right) d \mu d E$ is defined as the number of particles reflected from a slab of thickness $X$ with energies in $d E$ about $E$ and directions that lie in $d \mu$ about $\mu$ per incident particle with energy $E_{0}$ and direction $\mu_{0}$; the function can be regarded as an angular flux density within the differential slab thickness $d X$. The reflection equation describes the change in the reflection function due to changes in the shield thickness and is formulated without involving the transmission function. The derivation is accomplished by equating the difference in the reflection functions for slabs 


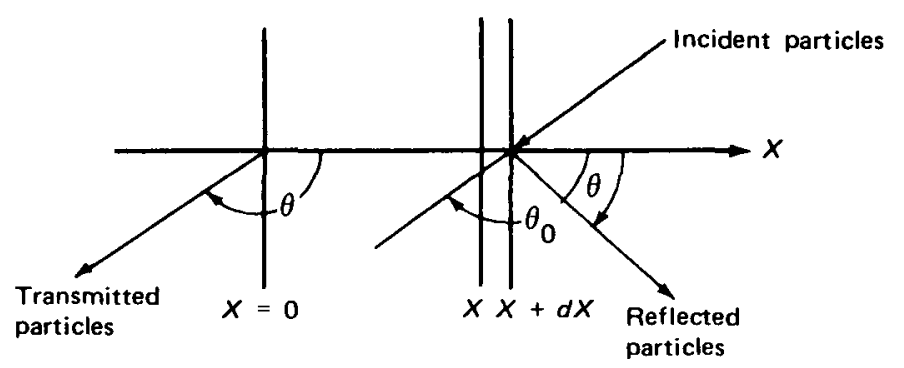

Fig. 4.6-Geometry for invariant imbedding technique.

of thicknesses $X+d X$ and $X$ with the net change in the reflection function which results from collisions suffered by the particles within the differential slab $d X$ :

$R\left(X+d X ; \mu, E ; \mu_{0}, E_{0}\right) d \mu d E-R\left(X ; \mu, E ; \mu_{0}, E_{0}\right) d \mu d E$

$$
\begin{aligned}
= & -\Sigma_{t}\left(X, E_{0}\right) R\left(X ; \mu, E ; \mu_{0}, E_{0}\right) \frac{d X}{\mu_{0}} d \mu d E \\
& -\Sigma_{t}(X, E) R\left(X ; \mu, E ; \mu_{0}, E_{0}\right) \frac{d X}{\mu} d \mu d E+\frac{d X}{\mu_{0}} \Sigma_{s}\left(X ; \mu, E ; \mu_{0}, E_{0}\right) \\
& +\int_{-1}^{0} d \mu^{\prime} \int_{0}^{\infty} d E^{\prime} \Sigma_{s}\left(X ; \mu^{\prime}, E^{\prime} ; \mu_{0}, E_{0}\right) R\left(X ; \mu, E ; \mu^{\prime}, E^{\prime}\right) \frac{d X}{\mu_{0}} d \mu d E \\
& +\int_{0}^{1} \frac{d \mu^{\prime}}{\mu^{\prime}} \int_{0}^{\infty} d E^{\prime} R\left(X ; \mu^{\prime}, E^{\prime} ; \mu_{0}, E_{0}\right) \Sigma_{s}\left(X ; \mu, E ; \mu^{\prime}, E^{\prime}\right) d X d \mu d E \\
& +\int_{0}^{1} \frac{d \mu^{\prime}}{\mu^{\prime}} \int_{-1}^{0} d \mu^{\prime \prime} \int_{0}^{\infty} d E^{\prime} \int_{0}^{\infty} d E^{\prime \prime} R\left(X ; \mu^{\prime}, E^{\prime} ; \mu_{0}, E_{0}\right) \\
& \times \Sigma_{s}\left(X ; \mu^{\prime \prime}, E^{\prime \prime} ; \mu^{\prime}, E^{\prime}\right) R\left(X ; \mu, E ; \mu^{\prime \prime}, E^{\prime \prime}\right) d X d \mu d E
\end{aligned}
$$

where $\Sigma_{t}\left(X, E_{0}\right)$ is the position-dependent total macroscopic cross section evaluated at the particle energy $E_{0}$ and $\Sigma_{s}\left(X ; \mu^{\prime}, E^{\prime} ; \mu_{0}, E_{0}\right) d \mu^{\prime} d E^{\prime}$ represents the position-dependent differential scattering cross section which describes the probability that a particle with an initial energy $E_{0}$ and an initial direction $\mu_{0}$ undergoes a scattering collision that places it into a direction which lies in $d \mu^{\prime}$ about $\mu^{\prime}$ with a new energy in $d E^{\prime}$ about $E^{\prime}$. 
The first and second terms on the right-hand side of Eq. 4.7-1 represent the particle losses due to collisions within $d X \dagger$ (any collision is presumed to alter the particle's energy and direction). The first term is the loss of incident particles scattered within $d X$ such that they do not enter the slab of thickness $X$, and the second term is the loss of particles that are scattered within $d X$ such that they are prevented from emerging from the slab of thickness $X+d X$. The third, fourth, fifth, and sixth terms represent the inscattering gains due to scattering collisions within $d X$. The fourth term is the gain from particles that scatter from $d X$ into the slab of thickness $X$ with energies in $d E^{\prime}$ about $E^{\prime}$ and directions $d \mu^{\prime}$ about $\mu^{\prime}$ and then are reflected from the slab of thickness $X$ with the proper emergent angle and direction. The fifth term is the gain from particles that scatter from the slab of thickness $X$ into $d X$ with energies in $d E^{\prime}$ about $E^{\prime}$ and directions $d \mu^{\prime}$ about $\mu^{\prime}$ and then are scattered within $d X$ with the proper emergent energy and direction. The sixth term is the gain from particles that scatter from the slab of thickness $X$ into $d X$ with energies in $d E^{\prime}$ about $E^{\prime}$ and direction $d \mu^{\prime}$ about $\mu^{\prime}$, are scattered back into the slab of thickness $X$ with energies in $d E^{\prime \prime}$ about $E^{\prime \prime}$ and direction $d \mu$ " about $\mu^{\prime \prime}$, and are finally reflected from the slab of thickness $X$ with the proper emergent energy and direction. A rearrangement of terms leads to the usual form of the reflection equation

$$
\begin{aligned}
& \frac{d}{d X} R\left(X ; \mu, E ; \mu_{0}, E_{0}\right)=\frac{1}{\mu_{0}} \Sigma_{s}\left(X ; \mu, E ; \mu_{0}, E_{0}\right)-\left[\frac{\Sigma_{t}\left(X, E_{0}\right)}{\mu_{0}}+\frac{\Sigma_{t}(X, E)}{\mu}\right] \\
& \quad \times R\left(X ; \mu, E ; \mu_{0}, E_{0}\right)+\int_{-1}^{0} d \mu^{\prime} \int_{0}^{\infty} d E^{\prime} \Sigma_{s}\left(X ; \mu^{\prime}, E^{\prime} ; \mu_{0}, E_{0}\right) \\
& \quad \times R\left(X ; \mu, E ; \mu^{\prime}, E^{\prime}\right) \frac{1}{\mu_{0}}+\int_{0}^{1} \frac{d \mu^{\prime}}{\mu^{\prime}} \int_{0}^{\infty} d E^{\prime} R\left(X ; \mu^{\prime}, E^{\prime} ; \mu_{0}, E_{0}\right) \\
& \quad \times \Sigma_{s}\left(X ; \mu, E ; \mu^{\prime}, E^{\prime}\right)+\int_{0}^{1} \frac{d \mu^{\prime}}{\mu^{\prime}} \int_{-1}^{0} d \mu^{\prime \prime} \int_{0}^{\infty} d E^{\prime} \int_{0}^{\infty} d E^{\prime \prime} \\
& \quad \times R\left(X ; \mu^{\prime}, E^{\prime} ; \mu_{0}, E_{0}\right) \Sigma_{s}\left(X ; \mu^{\prime \prime}, E^{\prime \prime} ; \mu^{\prime}, E^{\prime}\right) R\left(X ; \mu, E ; \mu^{\prime \prime}, E^{\prime \prime}\right)
\end{aligned}
$$

with the initial condition that

$$
R\left(0 ; \mu, E ; \mu_{0}, E_{0}\right)=0
$$

†The flight paths within the volume element $d X$ are $d X / \mu_{0}$ and $d X / \mu$ for the first and second terms, respectively. 
The transmission function $T\left(X ; \mu, E ; \mu_{0}, E_{0}\right) d \mu d E$ is defined as the number of particles that are transmitted through a slab of thickness $X$ and emerge with energies in $d E$ about $E$ and directions in $d \mu$ about $\mu$ per incident particle with energy $E_{0}$ and direction $\mu_{0}$.

The transmission equation is derived in a manner similar to that used to derive the reflection equation, making use of the fact that the difference in the transmission functions for slabs of thickness $X+d X$ and $S$ is due to collisions suffered by the particles within the differential slab $d X$. A familiar form of the transmission equation is

$$
\begin{aligned}
\frac{d}{d X} T\left(X ; \mu, E ; \mu_{0}, E_{0}\right)= & -\frac{\Sigma_{t}\left(X, E_{0}\right)}{\mu_{0}} T\left(X ; \mu, E ; \mu_{0}, E_{0}\right) \\
& +\int_{-1}^{0} d \mu^{\prime} \int_{0}^{\infty} d E^{\prime} \Sigma_{s}\left(X ; \mu^{\prime}, E^{\prime} ; \mu_{0}, E_{0}\right) \\
& \times T\left(X ; \mu, E ; \mu^{\prime}, E^{\prime}\right) \frac{1}{\mu_{0}}+\int_{0}^{1} \frac{d \mu^{\prime}}{\mu^{\prime}} \int_{-1}^{0} d \mu^{\prime \prime} \int_{0}^{\infty} d E^{\prime} \int_{0}^{\infty} d E^{\prime \prime} \\
& \times R\left(X ; \mu^{\prime}, E^{\prime} ; \mu_{0}, E_{0}\right) \Sigma_{s}\left(X ; \mu^{\prime \prime}, E^{\prime \prime} ; \mu^{\prime}, E^{\prime}\right) \\
& \times T\left(X ; \mu, E ; \mu^{\prime \prime}, E^{\prime \prime}\right)
\end{aligned}
$$

with the initial condition that

$$
T\left(0 ; \mu, E ; \mu_{0}, E_{0}\right) d \mu d E=\delta\left(\mu-\mu_{0}\right) \delta\left(E-E_{0}\right) d \mu d E
$$

The first term on the right-hand side of Eq. 4.7-4 represents the loss of incident particles due to collisions suffered within $d X$ (any collision is assumed to alter particle energy and direction). The second and third terms represent the inscattering gains due to scattering collisions within $d X$. The second term is the gain from particles that scatter from $d X$ into the slab of thickness $X$ with energies in $d E^{\prime}$ about $E^{\prime}$ and directions in $d \mu^{\prime}$ about $\mu^{\prime}$ and finally emerge with energies in $d E$ about $E$ and directions in $d \mu$ about $\mu$. The third term is the gain from particles that are reflected from the slab of thickness $X$ into $d X$ and are then scattered back into the slab of thickness $X$, finally emerging with energies in $d E$ about $E$ and directions in $d \mu$ about $\mu$.

The reflection equation (Eq. 4.7-2) and the transmission equation (Eq. 4.7-4) are both nonlinear integro-differential equations that for the radiation-transport problems of nuclear engineering form problems of the initial-value type. The reflection equation involves only the reflection 
function as the dependent variable; thus it can be solved without consideration of the transmission equation. The transmission equation appears simpler in form (fewer terms) but contains the reflection function, which must be known before a solution can be effected. Therefore a typical shielding transmission problem (initial value) would involve the solution of a coupled pair of nonlinear integro-differential equations. This is in contrast to the Boltzmann equation, which is a linear integro-differential equation and for the same application forms a boundary-value-type problem.

Analytical solutions of the reflection and transmission equations for practical problems are not possible because of their complexity. Consequently all useful solutions are numerical in nature and are accomplished with digital computers. The numerical techniques are similar to those used to solve the Boltzmann equation by the discrete-ordinates technique, in which a specific combination of the independent variables defines discrete values of the neutron flux $\Phi_{G, I, D}$ (see Sec. 4.4 for a more complete description).

In invariant imbedding specific combinations of the energy and direction of the particle define the $i$ th particle state. In this context the discrete reflection variable $R_{i j}(X)$ is the number of particles in state $i$ reflected by a slab of thickness $X$ due to a unit source of particles in state $j$ that are incident on the slab. The discrete transmission variable $T_{i j}(X)$ is the number of particles in state $i$ that penetrate a slab of thickness $X$ due to a unit source of particles in state $j$ that are incident on the slab. The reflection and transmission equations in discrete-variable notation, along with a general description of the numerical techniques used in their solution, are given by Mathews, Hansen, and Mason. ${ }^{23}$

The paper of Mathews et al. also describes the application of invariant imbedding to practical energy-dependent neutron shielding problems, such as for a thick water shield and a thinner heterogeneous iron-polyethyleneiron shield. A very detailed set of reflection and transmission equations in particle-state notation for the monoenergetic-neutron-transport problem in slab geometry is given by Mingle, ${ }^{24}$ who includes applications of the method of escape probabilities, blackness coefficients, and critical-size determinations. Solutions for the gamma-ray-transport problem in slab geometry, including results for slabs of iron, water, lead, and concrete, are given by Shimizu and Mizuta. ${ }^{25}$

The advantages and disadvantages of the invariant imbedding method relative to other techniques should strongly influence the extent and direction of future applications. The advantages of the method are that it yields very detailed solutions (gives energy and angular distributions), is 
efficient for deep penetrations with reasonably short computer times, is well suited for heterogeneous shield configurations, the effects of boundaries are implicitly and exactly included in the solution, and it has the computational advantages of being an initial-value problem. The disadvantages of the method are that it is inefficient for thin shields (the method is very slow during initial phases of solution), difficult to apply to other than slab geometries, and does not generate detailed particle-state information within the shield (actually an advantage from a computational point of view). Furthermore, the basic equations are nonlinear (not too serious if the solution is obtained numerically), and the calculational techniques and computer programs are not as advanced as those for the solutions of the Boltzmann equation.

\subsection{KERNEL TECHNIQUE}

The kernel technique, which in the language of mathematical physics is known as the method of Green's functions, is one of the more widely used methods for the solution of both gamma-ray and neutron shielding problems. The point kernel used in shielding, $K\left(\left|\mathbf{r}-\mathbf{r}^{\prime}\right|\right)$, is formally the solution to the unit point-source problem and is defined as the desired response of a detector (particle-flux density, energy-flux density, dose, or energy absorption) at the space point $r$ due to a unit point source of radiation at the space point $\mathbf{r}^{\prime}$. This kernel provides the means for solving a variety of problems that involve distributed sources. As an illustration of the procedure, consider the surface-source problem. In terms of the point kernel, the detector response at a distance $\left|\mathbf{r}-\mathbf{r}^{\prime}\right|=R$ away from a differential source area $d A(R)$ of intensity $S_{A}(R)$ (particles $\mathrm{cm}^{-2} \mathrm{sec}^{-1}$ ) is

$$
d \Phi=\left[S_{A}(R) d A(R)\right] K(R)
$$

The differential area $d A(R)$ is selected so that the term $\left[S_{A}(R) d A(R)\right]$ can be considered as a point source located at a distance $R$ from the detector. The total detector response (the desired answer to the surface-source problem) is obtained by integrating over the entire source surface:

$$
\Phi=\int d \Phi=\int_{A} S_{A}(R) K(R) d A(R)
$$

The utility of the method is considerably enhanced if the integral can be evaluated analytically. 
Kernels that are used in practical shielding calculations almost invariably result from solutions (either analytical or numerical) for infinite homogeneous media. Consequently applications of these kernels to finite-geometry configurations require corrections for boundary effects.

Kernel techniques for gamma-ray-penetration analysis have evolved somewhat differently than those for neutron-penetration analysis. For photon transport the parameters within the kernels have been developed primarily by numerical methods (predominantly Monte Carlo and moments calculations) and were validated by experimental measurement. On the other hand, most parameters used in neutron-attenuation kernels historically have been empirical quantities obtained by measurement. For certain cases, calculations have been made to verify the experimental results, and more recently Monte Carlo and moments calculations have also been effectively applied to the neutron problem.

It is convenient to divide the discussion of kernel techniques into two parts. The following two sections discuss the basic concepts of kernel methods for photons and neutrons, respectively. Applications of these techniques are further discussed in Chaps. 6 and 8.

\subsubsection{Gamma-Ray Calculations $\dagger$}

In the analysis of gamma-ray-transport problems, the uncollided-flux density (i.e., the flux density due to source gamma rays that arrive at the point of interest without suffering an interaction) is usually easily calculated. For a monoenergetic point isotropic source in an infinite medium, the uncollided-flux density (gamma rays $\mathrm{cm}^{-2} \mathrm{sec}^{-1}$ ) is given by

$$
\Phi^{0}(R)=S \frac{e^{-\mu(E) R}}{4 \pi R^{2}}
$$

where $S=$ source strength (gamma rays $/ \mathrm{sec}$ )

$\mu(E)=$ macroscopic total cross section evaluated at the initial gamma-ray energy, $E\left(\mathrm{~cm}^{-1}\right)$

$e^{-\mu(E) R}=$ material attenuation factor, which is the probability that a gamma ray of energy $E$ travels a distance $R(\mathrm{~cm})$ without suffering a collision

$\left(4 \pi R^{2}\right)^{-1}=$ geometric attenuation for a point source $\left(\mathrm{cm}^{-2}\right)$

IIn this section we will not need direction cosines, and we will return to the usual notation $\mu$ for gamma-ray macroscopic cross section. 
Calculation of the scattered-flux density is, in general, much more complex. The scattered component can be handled by introducing a buildup factor, which accounts for the increase (i.e., buildup) in the flux density at some point $\mathbf{r}$ that is due to the scattered gamma rays. This buildup factor, defined as

$B \equiv[$ some desired property (particle-flux density, energy-flux density, dose, etc.) of the total gamma-ray-flux density at $R$ ]/[same property due to the uncollided-flux density at $R$ ]

serves as the basis for formulating the point kernels required for gamma-ray shield analysis. For the calculation of dose, the kernel is given by

$$
\begin{aligned}
K_{r}(R) & =\frac{\mu_{a t}(E) E \Phi^{0}(R)}{\rho_{t} S} B_{r} \\
& =\frac{\mu_{a t}(E) E e^{-\mu(E) R}}{4 \pi R^{2} \rho_{t}} B_{r}
\end{aligned}
$$

where $\mu_{a t}(E)$ is the macroscopic energy-absorption cross section for tissue evaluated at the initial gamma-ray energy $E ; \rho_{t}$ is the density of tissue (usually taken as $1.0 \mathrm{~g} / \mathrm{cm}^{3}$ ); and $B_{r}$ is the exposure buildup factor, $\dagger$ which is the ratio of the actual dose at $R$ to the uncollided dose at $R$. Similarly, if the desired property is the energy absorbed per unit mass, the kernel is given by

$$
\begin{aligned}
K_{a}(R) & =\frac{\mu_{a}(E) E \Phi^{0}(R)}{S \rho} B_{a} \\
& =\frac{\mu_{a}(E) E e^{-\mu(E) R}}{4 \pi R^{2} \rho} B_{a}
\end{aligned}
$$

where $\mu_{a}(E)$ is the macroscopic energy-absorption cross section evaluated at the initial gamma-ray energy $E$ for the material in which the energy is absorbed, and $B_{a}$ is the energy-absorption (or energy-deposition) buildup factor, which is the ratio of the actual energy absorbed at $R$ to the uncollided energy absorbed at $R$.

†Formerly called dose buildup factor. Away from boundaries for energies pertinent to reactor shields, gamma-ray absorbed dose, kerma, and exposure are numerically equal (see Chap. 2). 
The results of many accurate gamma-ray-attenuation calculations are reported in terms of buildup factors, which in combination with the kernel technique provide a relatively simple and, in many cases, accurate calculational method. The primary source of buildup data is the collection of moments-method data reported by Goldstein and Wilkins ${ }^{16}$ for point isotropic and plane monodirectional sources. The point-source data are perhaps the most widely used because of their application to point-kernel solutions. The infinite-plane data also find use in approximations such as predicting the gamma-ray penetration through the side of a large cylindrical or rectangular shield surrounding a large, diffuse source.

Tables of the exposure buildup factor vs. atomic number for various penetration depths and initial energies are shown in Appendix $E$ for a point isotropic source and infinite-medium geometry.

A variety of functions have been derived to fit the buildup data. Some of the more commonly used functions are:

1. The linear form

$$
B(E, \mu R)=1+A_{1}(E) \mu R
$$

with only one constant, $A_{1}$, to be evaluated as a function of energy. This form has the advantage of simplicity but does not provide a consistently accurate fit.

2. The quadratic form

$$
B(E, \mu R)=1+A_{2}(E) \mu R+b(E)(\mu R)^{2}
$$

should be applied to deep-penetration calculations.

3. The form due to Taylor ${ }^{26}$ is

$$
B(E, \mu R)=A e^{-\alpha_{1}(E) \mu}+(1-A) e^{-\alpha_{2}(E) \mu R}
$$

4. The polynomial form is

$$
B(E, \mu R)=\sum_{n=0}^{3} \beta_{n}(E)(\mu R)^{n}
$$

Capo ${ }^{27}$ published a set of the coefficient $\beta_{n}(E)$ for a wide range of materials and gamma-ray energies.

5. The form due to Berger ${ }^{28}$ is

$$
B(E, \mu R)=1+C(E) \mu R e^{D(E) \mu R}
$$


This two-parameter formula is only slightly more complicated than the linear form but provides better accuracy.

Trubey ${ }^{29}$ published a review and evaluation of these and other empirical equations which contains values of the fitted parameters and comparisons of the expressions with the data of Goldstein and Wilkins. ${ }^{16}$ Some of the conclusions of his study are:

1. The linear form is generally not adequate.

2. The best fit is provided by the four-term polynomial fit by Capo.

3. For most purposes the function proposed by Berger is recommended because it is simpler to apply and provides accuracy approaching that of the more complicated forms.

Values of the coefficients for all these forms of the buildup factor are given for a variety of materials, photon energies, and source-detector distances in Appendix F. Data in all these tables are for a point isotropic source geometry. Applications of buildup factors to gamma-ray transport problems are discussed in Sec. 6.4.1 and to gamma-ray heating in Sec. 8.1.1.

There are many other possible forms of buildup factors, such as those cited by Hubbell, ${ }^{30}$ but they are generally more complicated than the forms given here. Hubbell's power-series form, for example, converges adequately at short distances only and thus usually requires many terms, but it has the advantage of allowing separation of the variables dependent on the medium properties, geometry, and thickness.

Sometimes solutions to photon shielding problems can be reasonably approximated from attenuation data for a disk or rectangular source and a slab shield. These simple plane-source problems are amenable to analytic or numerical solution by integrating a point kernel of the form of Eq. 4.8-3 over the source area. The results when tabulated or plotted are directly usable in practical applications.

Consider, for example, a detector shielded from a plane disk source that is uniformly emitting $S$ monoenergetic photons $\mathrm{cm}^{-2} \mathrm{sec}^{-1}$ isotropically in $4 \pi$ steradians (see Fig. 4.7). When the point kernel as given by Eq. 4.8-3 is applied, the unscattered dose rate along the disk axis is

$$
\Gamma\left(\mu t, r_{0}, z\right)=S G(E) \int_{0}^{r_{0}} \frac{e^{-\mu t \sec \theta}(2 \pi r) d r}{4 \pi R^{2}}
$$

in which $G(E)$ is a particle-flux-density to dose-rate conversion function. Its units will determine the units of the dose calculated. 


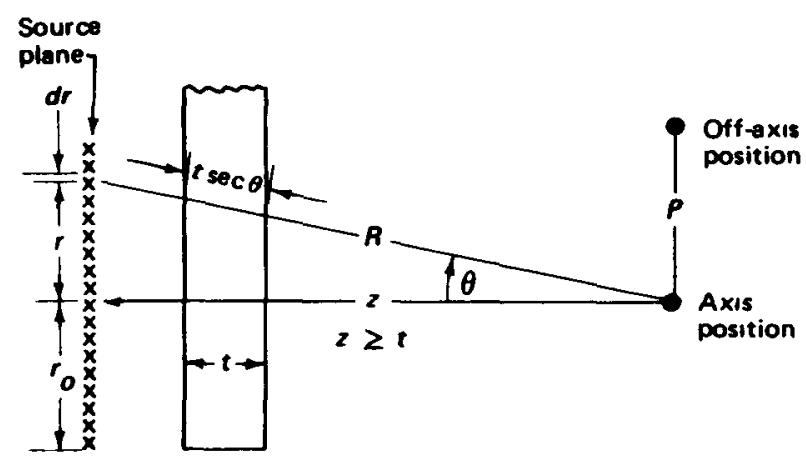

Fig. 4.7-Schematic diagram of disk source.

Since $R^{2}=r^{2}+z^{2}$, Eq. $4.8-11$ can be transformed to

$$
\Gamma\left(\mu t, z / r_{0}\right)=\frac{S G(E)}{2} \int_{\mu t}^{\mu t \sqrt{1+\left(r_{0} / z\right)^{2}}} \frac{e^{-y}}{y} d y
$$

where $y \equiv \mu t \sec \theta$. When integrated, Eq. 4.8-12 becomes

$$
\Gamma\left(\mu t, z / r_{0}\right)=\frac{S G(E)}{2}\left\{E_{1}(\mu t)-E_{1}\left[\mu t \sqrt{1+\left(r_{0} / z\right)^{2}}\right]\right\}
$$

where $E_{1}$ is the exponential integral function $t$ of the first order and is defined by

$$
E_{1}(x) \equiv \int_{x}^{\infty} \frac{e^{-y}}{y} d y
$$

In general,

$$
E_{n}(x)=x^{n-1} \int_{x}^{\infty} e^{-y} y^{-n} d y
$$

[Equation 4.8-13, as well as the following equations for computing uncollided doses, can be used to determine the total dose (uncollided plus scattered) by using the Taylor form of the buildup factor.]

For an isotropic flux density $\Phi(0)$ at the source plane, which is equivalent to a current density with a $\cos \theta$ angular distribution (as shown in Chap. 2), the unscattered dose rate is

tGraphs of this function (as well as $e^{-x}, E_{2}$, and $E_{3}$ ) are given in Appendix $G$. 


$$
\frac{e^{-\mu t \sec \theta} \cos \theta(2 \pi r) d r}{4 \pi R^{2}}
$$

which integrates to

$$
\begin{aligned}
\Gamma\left(\mu t, z / r_{0}\right)=\frac{\Phi(0) G(E)}{2}\left\{E_{2}(\mu t)\right. & \\
& \left.\quad-\frac{1}{\sqrt{1+\left(r_{0} / z\right)^{2}}} E_{2}\left[\mu t \sqrt{1+\left(r_{0} / z\right)^{2}}\right]\right\}
\end{aligned}
$$

where $E_{2}$ is the exponential function of the second order.

The angular current density $J(\theta)$ equivalent to $\Phi(0)$ in the forward hemisphere is $[\Phi(0) / 4 \pi] \cos \theta$, defined as the number of photons per unit solid angle crossing a unit area on the source plane in the directions within the interval $-1 \leqslant \cos \theta \leqslant 1$ as measured normal to the source plane. Therefore

$J(0)=\int_{\Omega / 2} \frac{\Phi(0)}{4 \pi} \cos \theta d \Omega=\int_{0}^{1} \frac{\Phi(0) \cos \theta}{4 \pi} 2 \pi$

$$
x d(\cos \theta)=\frac{\Phi(0)}{4}
$$

If the current density in the forward direction is used, $4 J(0)$ must be substituted for $\Phi(0)$ in Eq. 4.8-16.

In general, for the $\cos ^{n} \theta$ angular distribution in the forward direction,

$$
\Phi(\theta)=\frac{(n+1) \Phi^{\prime}(0) \cos ^{n} \theta}{2 \pi}
$$

where $\Phi^{\prime}(0)$ is the total or scalar flux density in the forward direction only, and

$$
\begin{aligned}
& \Gamma\left(\mu t, z / r_{0}\right)=\frac{(n+1) \Phi(0) G(E)}{2}\left\{E_{n+2}(\mu t)\right. \\
& \left.-\frac{E_{n+2}\left[\mu \hbar \sqrt{1+\left(r_{0} / z\right)^{2}}\right]}{\left[\sqrt{1+\left(r_{0} / z\right)^{2}}\right]^{n+1}}\right\}
\end{aligned}
$$


For the off-axis position at a distance $\rho$ measured perpendicularly to the disk axis (see Fig. 4.7), integrations must be done numerically. Hubbell, Bach, and Herbold ${ }^{31}$ integrated an expression similar to Eq.4.8-11, the isotropic source case, for off-axis positions and tabulated the results in terms of the parameters $\mu t, z / r_{0}$, and $\rho / r_{0}$. These results are shown in Appendix $\mathrm{H}$. The quantity tabulated is $4 \pi \Gamma\left(\mu t, z / r_{0}, \rho / r_{0}\right) /[S G(E)]$, which is the same as $4 \pi \Phi^{0}\left(\rho / r_{0}\right) / S$, where $\Phi^{0}\left(\rho / r_{0}\right)$ is the uncollided-flux density at $\rho / r_{0}$.

Similarly, Trubey ${ }^{32}$ determined the data for an isotropic flux density. The results are given in Appendix $\mathrm{H}$ as $2 \Gamma\left(\mu t, z / r_{0}, \rho / r_{0}\right) /[\Phi(0) G(E)]$, which is the same as $2 \Phi^{0} / \Phi(0)$.

Certain circular aperture and disk-source configurations to which these results might be applied are shown in Fig. 4.8.
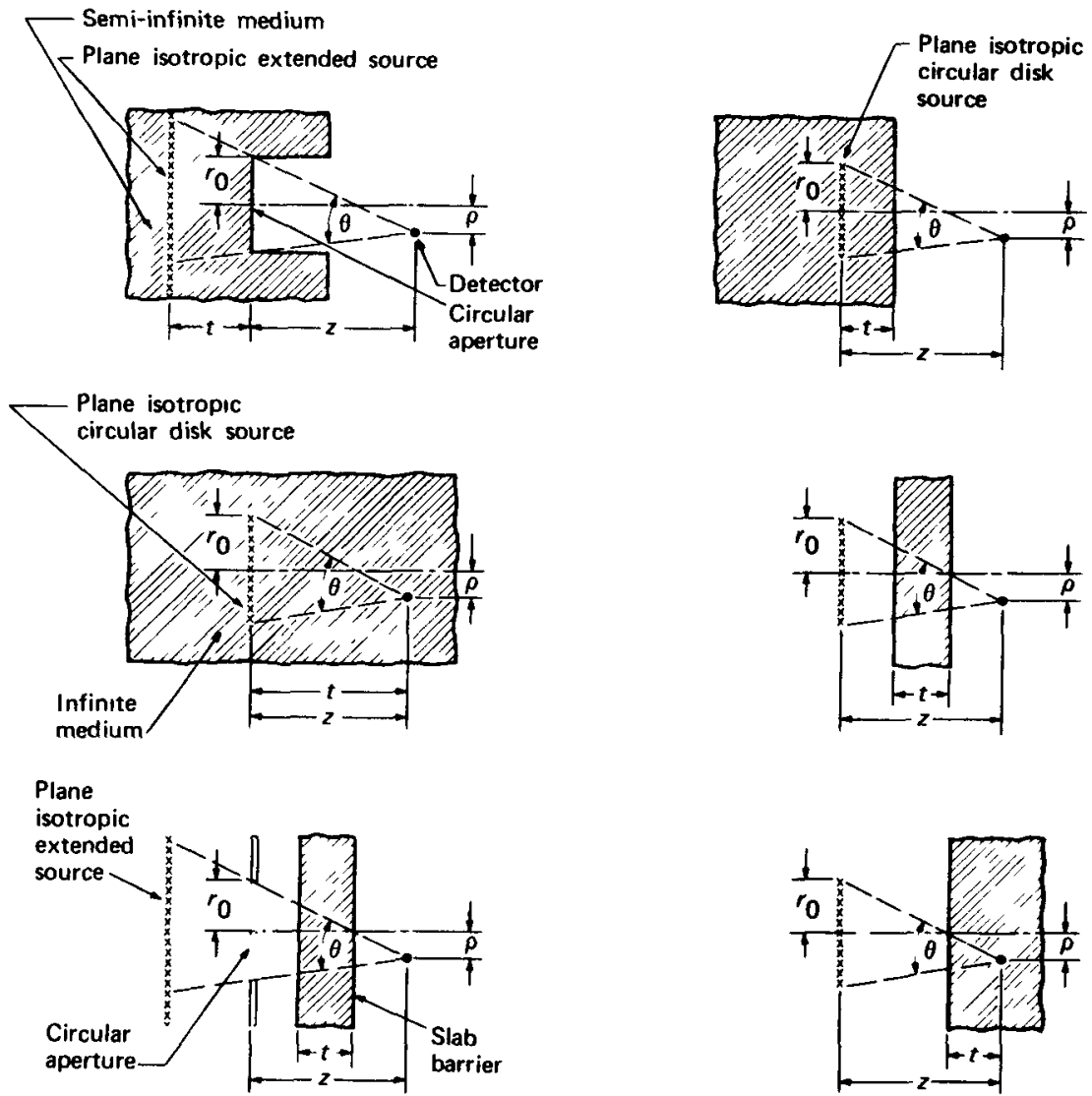

Fig. 4.8-Some circular aperture or disk-source shield configurations to which point kernels are applicable (From Hubbell et al. ${ }^{31}$ ) 
A solution was developed by Hubbell, Bach, and Lamkin ${ }^{33}$ for the uncollided-flux density at a distance $z$ from a plane isotropic rectangular source. Expressed as the product of separable source and geometry functions, the uncollided-flux density is given by

$$
\Phi^{0}(a, b)=\sum_{n=0}^{\infty} \frac{2 n+1}{2} g_{n} p_{n}(a, b)
$$

where $g_{n}$ and $p_{n}(a, b)$ are Legendre coefficients of the source and geometry functions, respectively.

If $a=H / z$ and $b=W / z$, where $H$ and $W$ are the height and width, respectively, of the source plane (see Fig. 4.9), then Eq. 4.8-20 gives the flux density at the corner position, that is, the flux density at a distance $z$ along the normal to the corner of the rectangular source. It follows that the use of

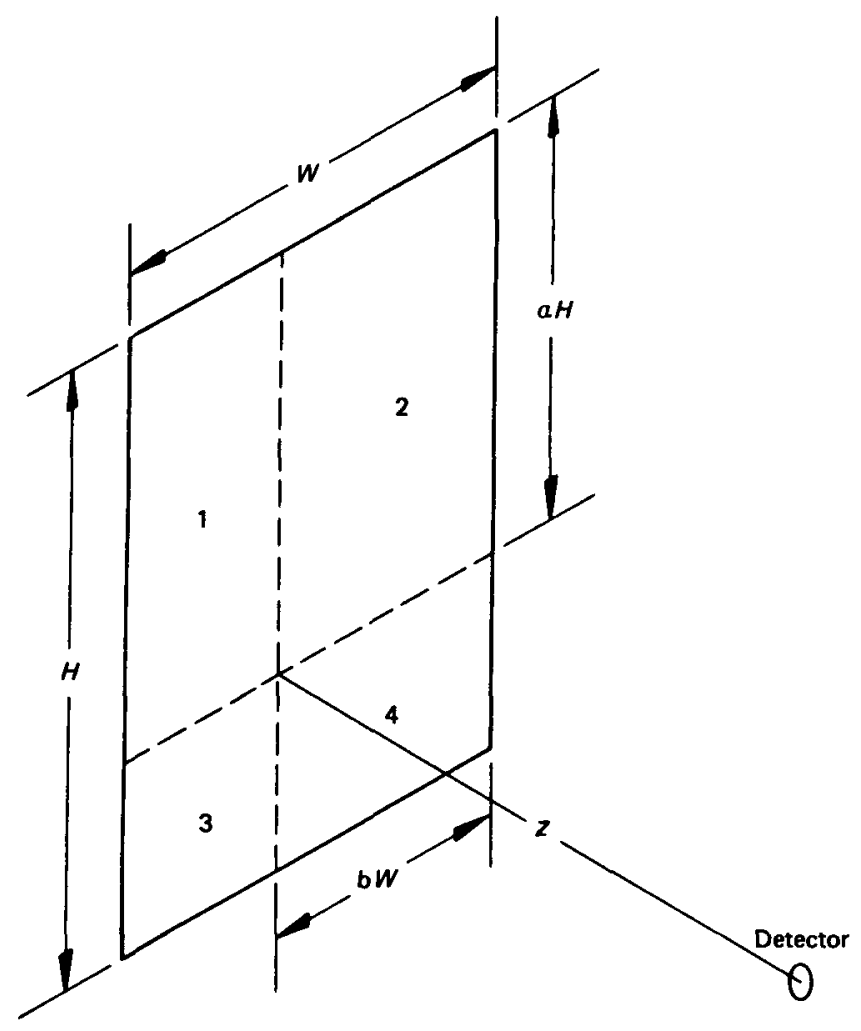

Fig. 4.9-Schematic diagram demonstrating the use of the corner position of a rectangular source to calculate dose at an arbitrary position by point-kernel techniques. 
the half-height and half-width gives one-fourth of the flux density at $z$ along a normal to the center of the source plane. The source function is

$$
g_{n}=\int_{-1}^{1} g(\cos \theta) P_{n}(\cos \theta) d(\cos \theta)
$$

where $g(\cos \theta)$ represents the angular flux density at the source plane for the case of a slab shield of thickness $t(z \geqslant t)$ located between the source and the detector at a distance $z$ from the source; that is,

$$
g(\mu t, \cos \theta)=\frac{S e^{-\mu t / \cos \theta}}{4 \pi \cos \theta}
$$

Substituting Eq. 4.8-22 into Eq. 4.8-21, Hubbell et al. ${ }_{0}^{33}$ evaluated $g_{n}$ and $p_{n}$ numerically and solved Eq. 4.8-20. The results for a corner position $(a=H / z, b=W / z)$ are given in Appendix $\mathrm{H}$ as $4 \pi \Gamma /[S G(E)]$, or $4 \Phi^{\circ} / S$, in terms of the parameters $\mu t, a$, and $b$.

Similarly, Trubey ${ }^{32}$ numerically evaluated the equivalent of Eq. 4.8-20 for an isotropic flux density (cosine distribution of the angular current density), that is, for

$$
g(\mu t, \cos \theta)=\frac{\Phi(0) e^{-\mu t / \cos \theta}}{4 \pi}
$$

These results for a corner position are given in Appendix $\mathrm{H}$ as $2 \Gamma /[\Phi(0) G(E)]$, or $2 \Phi^{0} / \Phi(0)$, which is the same quantity tabulated for the disk source in Appendix H. For a square, the dose will be slightly greater than that for a disk of radius $W$.

Although these results relate directly to the response of a detector in a corner position, they are also applicable to any arbitrary position lying within the projection of the source plane. It is obvious from Fig. 4.9 that the dose rate at the detector is

$$
\begin{aligned}
\Gamma(H / z, W / z)= & \Gamma_{1}\left[\frac{\alpha H}{z}, \frac{(1-\beta) W}{z}\right] \\
& +\Gamma_{2}\left(\frac{\alpha H}{z}, \frac{\beta W}{z}\right)+\Gamma_{3}\left[\frac{(1-\alpha) H}{z}, \frac{(1-\beta) W}{z}\right] \\
& +\Gamma_{4}\left[\frac{(1-\alpha) H}{z}, \frac{\beta W}{z}\right]
\end{aligned}
$$




\subsubsection{Neutron Techniques}

As noted previously, the use of buildup factors in the attenuation function, or kernel, for neutrons has not developed to a large extent, primarily because neutron interactions are more complex than gamma-ray interactions. However, a simple kernel developed by Albert and Welton, ${ }^{34}$ which uses an energy-dependent hydrogen cross section, has been widely applied to hydrogenous shields. This section contains a description of the Albert-Welton kernel and a discussion of removal cross sections that are required in the kernel when other shield materials are used in conjunction with the hydrogenous medium.

Other kernels that can be used to calculate differential energy spectra of neutrons in hydrogenous media can be developed from the moments method or Monte Carlo calculations. For example, the moments-method code RENUPAK was used ${ }^{32}$ to calculate the differential energy-flux density as a function of distance from a point fission source in an infinite medium of lithium hydride, and an empirical attenuation function based on the results was then incorporated in the point-kernel code QAD by Solomito and Stockton $^{35}$ for use in space-reactor shield designs. Since these kernels are for an infinite medium, some caution should be exercised in their use. However, simple neutron-dose attenuation functions determined from such calculations can be used for preliminary shield-design applications, and some of these functions for concrete and several other materials are included in this section.

(a) Removal Cross Sections. Measurements in the Oak Ridge National Laboratory (ORNL) Lid Tank Shielding Facility ${ }^{36}$ showed that the insertion of relatively thin slabs of material between a fission source and a thick water shield gives an effect that can be correlated by a simple exponential attenuation factor that is characteristic of absorption processes alone. This behavior might not be expected since nonabsorption effects predominate in fast-neutron attenuation. However, the large thickness of water (at least $6 \mathrm{~g} / \mathrm{cm}^{2}$ ) filters out the neutrons deflected by the sample and thereby effects their complete removal. Therefore the effect of slabs of shield materials when followed by large thicknesses of hydrogenous material can be described by an equivalent absorption cross section averaged over energy, called the removal cross section.

An ideal way to experimentally determine the validity of the concept would be to use a plane monodirectional source of fission neutrons incident on a tank of water. For such a configuration the removal-cross-section 
concept would be valid if the doses measured at the source distance $z$ in water could be correlated by

$$
D_{2}(z)=D_{1}(z) e^{-\Sigma_{R} t}
$$

where $D_{1}(z)$ is the observed neutron dose attenuated through a distance $z$ of water, $D_{2}(z)$ is the observed neutron dose attenuated through a slab of material of thickness $t$ (inserted between source and water) plus water of thickness $z$, and $\Sigma_{R}$ is the macroscopic removal cross section.

In the experimental shielding facility where this concept was originally tested, the source was a finite isotropic disk rather than a plane monodirectional source. However, with a few simple assumptions about the behavior of neutron penetration, an analog to Eq. 4.8-25 was derived and used in obtaining removal cross sections from experimental data. ${ }^{37}$

Values of microscopic removal cross sections $\left(\sigma_{R}\right)$ determined from measurements at the Lid Tank Shielding Facility for several elements and compounds are shown in Table 4.2. Empirical functions useful for interpolation in the experimental data have been derived by Zoller: ${ }^{38}$

$$
\begin{aligned}
\frac{\Sigma_{R}}{\rho} & =0.19 Z^{-0.743} \mathrm{~cm}^{2} / \mathrm{g} \quad(\text { for } Z \leqslant 8) \\
& =0.125 Z^{-0.565} \mathrm{~cm}^{2} / \mathrm{g} \quad(\text { for } Z>8) \\
\frac{\Sigma_{R}}{\rho} & =0.206 A^{-1 / 3} Z^{-0.294} \approx 0.206(A Z)^{-1 / 3}
\end{aligned}
$$

where $Z$ is the atomic number. Most of the macroscopic removal cross sections given in Table 4.3 were obtained with Eq. 4.8-26.

It should be emphasized that most of the removal cross sections determined by experiment were obtained for a slab-type configuration with water following the shield material and may not be applicable to other configurations. Trubey and Chapman ${ }^{39}$ reported that in a homogeneous medium the removal cross section for oxygen is $0.75 \pm 0.05 \mathrm{~b}$ rather than $0.99 \pm 0.10 \mathrm{~b}$ as shown in Table 4.2. They also note that the removal cross section may vary with sample thickness (the value for oxygen obtained from the homogeneous-medium measurements increased from $0.72 \mathrm{~b}$ at a distance $90 \mathrm{~cm}$ from the source to $0.79 \mathrm{~b}$ at a distance $140 \mathrm{~cm}$ from the source). 
Table 4.2-MICROSCOPIC REMOVAL CROSS SECTIONS OF VARIOUS ELEMENTS AND COMPOUNDS MEASURED AT THE ORNL LID TANK SHIELDING FACILITY $\dagger$

\begin{tabular}{lc||lc}
\hline \multicolumn{1}{c||}{ Material } & $\sigma_{R}, \mathrm{~b} /$ atom & \multicolumn{1}{c}{ Material } & $\sigma_{R}$, b/atom \\
\hline Aluminum & $1.31 \pm 0.05$ & Tungsten & 3.36 \\
Beryllium & $1.07 \pm 0.06$ & Zirconium & $2.36 \pm 0.12$ \\
Bismuth & $3.49 \pm 0.35$ & Uranium & $3.6 \pm 0.4$ \\
Boron & $0.97 \pm 0.10$ & Boric oxide, $\mathrm{B}_{2} \mathrm{O}_{3}$ & $4.30 \pm 0.41$ \\
Carbon & $0.81 \pm 0.05$ & Boron carbide, $\mathrm{B}_{4} \mathrm{C}$ & $4.7 \pm 0.3$ \\
Chlorine & $1.2 \pm 0.8$ & Fluorothene, $\mathrm{C}_{2} \mathrm{~F}_{3} \mathrm{Cl}$ & $6.66 \pm 0.8$ \\
Copper & $2.04 \pm 0.11$ & Heavy water, $\mathrm{D}_{2} \mathrm{O}$ & $2.76 \pm 0.11$ \\
Fluorine & $1.29 \pm 0.06$ & Hevimet $(90$ wt.\%, & \\
Iron & $1.98 \pm 0.08$ & 6 wt.\% $\mathrm{Ni}, 4 \mathrm{wt} . \% \mathrm{Cu})$ & $3.22 \pm 0.18$ \\
Lead & $3.53 \pm 0.30$ & Lithium fluoride, $\mathrm{LiF}$ & $2.43 \pm 0.34$ \\
Lithium & $1.01 \pm 0.05$ & Oil, CH & $2.84 \pm 0.11$ \\
Nickel & $1.89 \pm 0.10$ & Paraffin, $\mathrm{C}_{30} \mathrm{H}_{62}$ & $80.5 \pm 5.2$ \\
Oxygen & $0.99 \pm 0.10$ & Perfluoroheptane, $\mathrm{C}_{7} \mathrm{~F}_{16}$ & $26.3 \pm 0.8$ \\
\hline
\end{tabular}

†From E. P. Blizard (Ed.), Reactor Handbook, 2nd ed., Vol. III, Part B, Shielding, p. 83, Interscience Publishers, a division of John Wiley \& Sons, Inc., New York, 1962.

There is really no reason to expect the removal cross section to remain constant with sample thickness since the removal concept is the result of a crude application of theoretical principles; however, the variation should not be very great up to about five relaxation lengths.

Another point to be emphasized is that the removal cross section for a material can be applied only when that material is used in conjunction with a hydrogenous shield since hydrogen is required to moderate and absorb the scattered neutrons, as occurred in the experiments for determining the removal cross sections. Thus a minimum thickness of hydrogenous material following the sample is required to validate the use of removal theory. When the hydrogenous material is water, moments-method calculations have shown that about $60 \mathrm{~cm}$ is required, whereas experimental results indicate that about $45 \mathrm{~cm}$ is adequate. If the hydrogen thickness is less than that provided by this amount of water, the neutrons interacting with the heavier elements will not be adequately "removed", and the effective removal cross section will be decreased.

It follows from the removal-cross-section concept that the removal cross sections of elements in a series of slabs or mixed together should be additive; i.e., the number of relaxation lengths becomes 


$$
\sum_{i} \Sigma_{R_{i}} t_{i}
$$

where the index $i$ refers to the various elements. This additive property has been generally accepted, even though some discrepancies have been noted, particularly in regard to compounds.

Removal cross sections can be predicted theoretically. Phenomenologically, the removal process can be considered equivalent to the total reaction rate minus the forward component of the scattering process. This suggests that an estimate of the removal cross section could be obtained from the transport cross section. As it turns out, $\Sigma_{R} \cong \Sigma_{t r}$ for neutrons between 6 and $8 \mathrm{MeV}$; therefore

$$
\Sigma_{R}=\Sigma_{t r}=\Sigma_{t}-\Sigma_{s} \overline{\cos } \theta
$$

where $\cos \theta$ is the average cosine of the neutron scattering angle per collision in the laboratory system.

Removal cross sections can also be estimated from

$$
\Sigma_{R} \cong \frac{2}{3} \bar{\Sigma}_{t}
$$

where $\bar{\Sigma}_{t}$ is the average total macroscopic cross section between 6 and $8 \mathrm{MeV}$, and from

$$
\frac{\Sigma_{R}}{\rho}=0.21 A^{-0.58}
$$

where $\rho$ is the density and $A$ is the atomic weight. Figure 4.10 compares graphs of measured values of $\Sigma_{R} / \rho$ and $\Sigma_{t} / \rho$ at $8 \mathrm{MeV}$ as a function of atomic weight. A reasonably good fit to the curve for $A>10$ is obtained by Eq. 4.8-30.

(b) Removal Cross Sections for Hydrogen-Deficient Shields. The traditional removal cross section as discussed in the preceding section is limited in application to a shield configuration that has a hydrogen density of at least $6 \mathrm{~g} / \mathrm{cm}^{2}$ in its outer layer. Recognizing the usefulness of a removal cross section that could be applied for shields that contain less hydrogen, Dudziak and Schmucker ${ }^{40}$ performed a series of calculations to investigate the effect on the removal cross section of varying the surface density of the hydrogen. Using a simplified $P_{3}$ approximation to the transport equation, they 
Table 4.3-FAST-NEUTRON REMOVAL CROSS SECTIONS AND MASS ATTENUATION COEFFICIENTS †

\begin{tabular}{|c|c|c|c|c|c|}
\hline Element & $\begin{array}{l}\text { Atomic } \\
\text { number }\end{array}$ & $\begin{array}{c}\rho, \\
\mathrm{g} / \mathrm{cm}^{3}\end{array}$ & $\begin{array}{c}\Sigma_{R} / \rho \\
\text { (calc.) } \\
\mathrm{cm}^{2} / \mathrm{g}\end{array}$ & $\mathrm{cm}_{\mathrm{R}_{\mathrm{i}}}^{\Sigma}$ & $\begin{array}{c}\Sigma_{R} / \rho \\
(\exp ) \\
\mathrm{cm}^{2} / g\end{array}$ \\
\hline Aluminum & 13 & 2.699 & 0.0293 & 0.0792 & $0.0292 \pm 0.0012$ \\
\hline Antimony & 51 & 6.691 & 0.0136 & 0.0907 & \\
\hline Argon & 18 & & 0.0244 & & \\
\hline Arsenic & 33 & 5.730 & 0.0173 & 0.0993 & \\
\hline Barium & 56 & 3.500 & 0.0129 & 0.0450 & \\
\hline Beryllium & 4 & 9.013 & 0.0678 & 0.1248 & $0.0717 \pm 0.0043$ \\
\hline Bismuth & 83 & 9.747 & 0.0103 & 0.1003 & $0.010 \pm 0.0010$ \\
\hline Boron & 5 & 3.330 & 0.0575 & 0.1914 & $0.0540 \pm 0.0054$ \\
\hline Bromine & 35 & 3.120 & 0.0168 & 0.0523 & \\
\hline Cadmium & 48 & 8.648 & 0.0140 & 0.1213 & \\
\hline Calcium & 20 & 1.540 & 0.0230 & 0.0354 & \\
\hline Carbon & 6 & 1.670 & 0.0502 & 0.0838 & $0.0407 \pm 0.0024$ \\
\hline Cerium & 58 & 6.900 & 0.0126 & 0.0870 & \\
\hline Cesium & 55 & 1.873 & 0.0130 & 0.0243 & \\
\hline Chlorine & 17 & & 0.0252 & & $0.020 \pm 0.014$ \\
\hline Chromium & 24 & 6.920 & 0.0208 & 0.1436 & \\
\hline Cobalt & 27 & 8.900 & 0.0194 & 0.1728 & \\
\hline Copper & 29 & 8.940 & 0.0186 & 0.1667 & $0.0194 \pm 0.0011$ \\
\hline Dysprosium & 66 & 8.562 & 0.0117 & 0.1003 & \\
\hline Erbium & 68 & 4.770 & 0.0115 & 0.0550 & \\
\hline Europium & 63 & 5.166 & 0.0120 & 0.0621 & \\
\hline Fluorine & 9 & & 0.0361 & & $0.0409 \pm 0.0020$ \\
\hline Gadolinium & 64 & 7.868 & 0.0119 & 0.0938 & \\
\hline Gallium & 31 & 5.903 & 0.0180 & 0.1060 & \\
\hline Germanium & 32 & 5.460 & 0.0176 & 0.0963 & \\
\hline Gold & 79 & 19.320 & 0.0106 & 0.2045 & \\
\hline Hafnium & 72 & 13.300 & 0.0112 & 0.1484 & \\
\hline Helium & 2 & & 0.1135 & & \\
\hline Holmium & 67 & & 0.0116 & & \\
\hline Indium & 49 & 7.280 & 0.0139 & 0.1009 & \\
\hline lodine & 53 & 4.930 & 0.0133 & 0.0654 & \\
\hline Iridium & 77 & 22.420 & 0.0107 & 0.2408 & \\
\hline Iron & 26 & 7.865 & 0.0198 & 0.1560 & $0.0214 \pm 0.0009$ \\
\hline Krypton & 36 & & 0.0165 & & \\
\hline Lanthanum & 57 & 6.150 & 0.0127 & 0.0783 & \\
\hline Lead & 82 & 11.347 & 0.0104 & 0.1176 & $0.0103 \pm 0.0009$ \\
\hline Lithium & 3 & 0.534 & 0.0840 & 0.0449 & $0.094 \pm 0.007$ \\
\hline Lutetium & 71 & & 0.0112 & & \\
\hline Magnesium & 12 & 1.741 & 0.0307 & 0.0535 & \\
\hline Manganese & 25 & 7.420 & 0.0203 & 0.1505 & \\
\hline Mercury & 80 & 13.546 & 0.0105 & 0.1424 & \\
\hline Molybdenum & 42 & 10.200 & 0.0151 & 0.1543 & \\
\hline
\end{tabular}

†From L. K. Zoller, Nucleonics, 22(8): 129 (1964) 
Table 4.3-(Continued)

\begin{tabular}{|c|c|c|c|c|c|}
\hline Element & $\begin{array}{l}\text { Atomic } \\
\text { number }\end{array}$ & $\begin{array}{c}\rho, \\
\mathrm{g} / \mathrm{cm}^{3}\end{array}$ & $\begin{array}{l}\Sigma_{R} / p \\
\left.\left(c_{1} a l_{c}\right)\right) \\
\mathrm{cm}^{2} / \mathrm{g}\end{array}$ & $\underset{\mathrm{cm}^{-\mathrm{i}}}{\Sigma}$ & $\begin{array}{c}\Sigma_{R} / \rho \\
(\exp .) \\
\mathrm{cm}^{2} / \mathrm{g}\end{array}$ \\
\hline Neodymium & 60 & 6.960 & 0.0124 & 0.0861 & \\
\hline Neon & 10 & & 0.0340 & & \\
\hline Nickel & 28 & 8.900 & 0.0190 & 0.1693 & $0.0190 \pm 0.0010$ \\
\hline Niobium & 41 & 8.400 & 0.0153 & 0.1288 & \\
\hline Nitrogen & 7 & & 0.0448 & & \\
\hline Osmium & 76 & 22.480 & 0.0108 & 0.2432 & \\
\hline Oxygen & 8 & & 0.0405 & & $0.031 \pm 0.002$ \\
\hline Palladium & 46 & 12.160 & 0.0144 & 0.1747 & \\
\hline Phosphorus & 15 & 1.820 & 0.0271 & 0.0493 & \\
\hline Platinum & 78 & 21.370 & 0.0107 & 0.2279 & \\
\hline Potassium & 19 & 6.475 & 0.0237 & 0.1533 & \\
\hline Praseodymium & 59 & 6.500 & 0.0125 & 0.0812 & \\
\hline Radium & 88 & 5.000 & 0.0100 & 0.0498 & \\
\hline Rhenium & 75 & 20.530 & 0.0109 & 0.2238 & \\
\hline Rhodium & 45 & 12.440 & 0.0145 & 0.1810 & \\
\hline Rubidium & 37 & 1.532 & 0.0163 & 0.0249 & \\
\hline Ruthenium & 44 & 12.060 & 0.0147 & 0.1777 & \\
\hline Samarium & 62 & 7.750 & 0.0121 & 0.0941 & \\
\hline Scandium & 21 & 3.020 & 0.0224 & 0.0676 & \\
\hline Selenium & 34 & 4.800 & 0.0170 & 0.0818 & \\
\hline Silicon & 14 & 2.420 & 0.0281 & 0.0681 & \\
\hline Silver & 47 & 10.503 & 0.0142 & 0.1491 & \\
\hline Sodium & 11 & 0.971 & 0.0322 & 0.0313 & \\
\hline Strontium & 38 & 2.540 & 0.0160 & 0.0407 & \\
\hline Sulfur & 16 & 2.070 & 0.0261 & 0.0540 & \\
\hline Tantalum & 73 & 16.600 & 0.0111 & 0.1838 & \\
\hline Tellurium & 52 & 6.240 & 0.0134 & 0.0837 & \\
\hline Terbium & 65 & & 0.0118 & & \\
\hline Thallium & 81 & 11.860 & 0.0104 & 0.1238 & \\
\hline Thorium & 90 & 11.300 & 0.0098 & 0.1111 & \\
\hline Thulium & 69 & & 0.0114 & & \\
\hline Tin & 50 & 6.550 & 0.0137 & 0.0898 & \\
\hline Titanium & 22 & 4.500 & 0.0218 & 0.0981 & \\
\hline Tungsten & 74 & 19.300 & 0.0110 & 0.2120 & $0.0082 \pm 0.0018$ \\
\hline Uranium & 92 & 18.700 & 0.0097 & 0.1816 & $0.0091 \pm 0.0010$ \\
\hline Vanadium & 23 & 5.960 & 0.0213 & 0.1267 & \\
\hline Xenon & 54 & & 0.0131 & & \\
\hline Ytterbium & 70 & & 0.0113 & & \\
\hline Yttrium & 39 & 3.800 & 0.0158 & 0.0599 & \\
\hline Zinc & 30 & 7.140 & 0.0183 & 0.1306 & \\
\hline Zirconium & 40 & 6.440 & 0.0156 & 0.1001 & \\
\hline
\end{tabular}




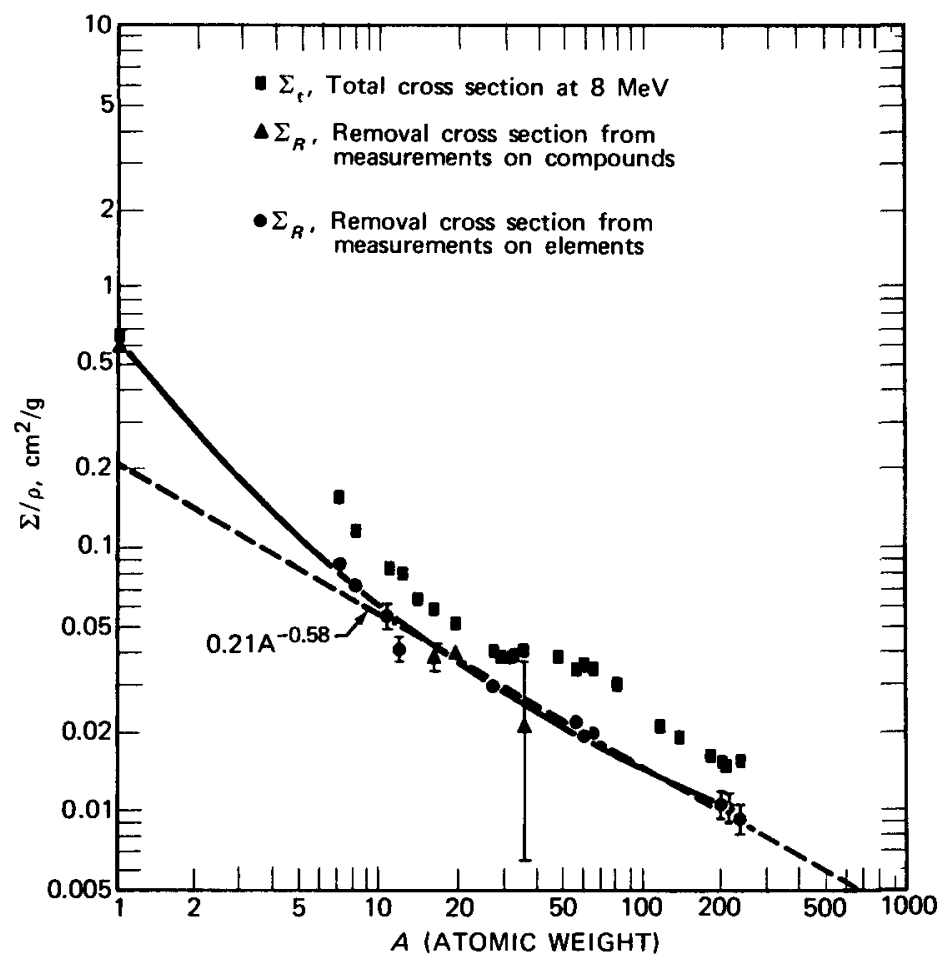

Fig. 4.10-Removal cross sections per unit mass for fission neutrons as a function of atomic weight. (From Chapman and Storrs. ${ }^{36}$ )

calculated the transport of neutrons from a Po-Be source through lead followed by varying amounts of polyethylene in spherical geometry. They showed that removal cross sections for large thicknesses of polyethylene approached the asymptotic value of $0.116 \pm 0.01 \mathrm{~cm}^{-1}(3.53 \pm 0.30 \mathrm{~b})$ reported from the Lid Tank Shielding Facility measurements with a fission source. They also found that by extrapolating the cross-section curve back to a zero thickness of polyethylene they obtained a value that was very close to the value of $0.128 \mathrm{~cm}^{-1}$ for lead alone reported by Price and Dunn ${ }^{41}$ on the basis of experiments with Po-Be neutrons.

Dudziak ${ }^{4}$ later performed calculations with better cross sections, more neutron-energy groups, and an $\mathrm{S}_{8} \mathrm{P}_{3}$ approximation and reached essentially the same conclusions. The results of the calculations by Dudziak and Schmucker ${ }^{40}$ are shown in Table 4.4. The macroscopic removal cross sections given in the table were fit to within $1.4 \%$ by a least-squares procedure to an analytical expression given by 


$$
\Sigma_{R}(t)=0.1106\left(1-0.9836 e^{-0.109 t}\right)
$$

where $t$ is the polyethylene thickness in centimeters.

In calculations similar to those of Dudziak and Schmucker, but for a fission source, Shure, O'Brien, and Rothberg ${ }^{4}$ determined removal cross sections for both iron and lead followed by polyethylene thicknesses up to $50 \mathrm{~cm}$. They used two cutoff energies, $E_{c}, 302 \mathrm{keV}$ and $0.625 \mathrm{eV}$, in their dose calculations. The resulting microscopic removal cross sections, shown in Table 4.5, indicate that, at least for small thicknesses of polyethylene, the removal cross sections are relatively insensitive to the cutoff energy chosen. Shure et al. also found that the asymptotic values for iron and lead are in very good agreement with the experimental values of $1.98 \pm 0.08 \mathrm{~b}$ and $3.53 \pm 0.30 \mathrm{~b}$, respectively, obtained from Lid Tank measurements. ${ }^{3} 7$ When no polyethylene was present, the value for lead of $0.74 \mathrm{~b}\left(0.0243 \mathrm{~cm}^{-1}\right)$ obtained for the fission source is nearly twice that for the Po-Be source, which could be expected since the Po-Be spectrum is harder. The fact that the asymptotic removal cross sections are in agreement for the two different sources shows that the inelastic scattering in lead apparently degrades the high-energy part of the spectra sufficiently to produce spectra that equilibrate about equally after traversing approximately $10 \mathrm{~cm}$ of polyethylene.

Table 4.4-EFFECTIVE MACROSCOPIC REMOVAL CROSS SECTIONS FOR LEAD FOLLOWED BY VARIOUS THICKNESSES OF POLYETHYLENE (Po-Be SOURCE) $f$

\begin{tabular}{cc}
\hline $\begin{array}{c}\text { Polyethylene } \\
\text { thickness, } \\
\text { cm }\end{array}$ & $\begin{array}{c}\text { Lead } \\
\text { removal cross } \\
\text { section, cm }\end{array}$ \\
\hline 3 & 0.0328 \\
5 & 0.0473 \\
7 & 0.0597 \\
9 & 0.0701 \\
15 & 0.0901 \\
21 & 0.0999 \\
25 & 0.1036 \\
31 & 0.1069 \\
35 & 0.1081 \\
41 & 0.1093 \\
\hline
\end{tabular}

†From Dudziak and Schmucker. ${ }^{40}$ 
Table 4.5-EFFECTIVE MICROSCOPIC REMOVAL CROSS SECTIONS FOR IRON AND LEAD FOLLOWED BY VARIOUS POLYETHYLENE THICKNESSES (FISSION SOURCE) $t$

\begin{tabular}{|c|c|c|c|c|}
\hline \multirow{3}{*}{$\begin{array}{c}\text { Polyethylene } \\
\text { thickness, } \\
\text { cm }\end{array}$} & \multicolumn{4}{|c|}{ Removal cross section, b } \\
\hline & \multicolumn{2}{|c|}{ Iron } & \multicolumn{2}{|c|}{ Lead } \\
\hline & $E_{c}=302 \mathrm{keV}$ & $E_{c}=0.625 \mathrm{eV}$ & $E_{c}=302 \mathrm{keV}$ & $E_{c}=0.625 \mathrm{eV}$ \\
\hline 0 & 0.76 & 0.61 & 0.88 & 0.74 \\
\hline 1 & 0.94 & 0.78 & 1.17 & 1.00 \\
\hline 2 & 1.08 & 0.91 & 1.41 & 1.22 \\
\hline 5 & 1.37 & 1.24 & 1.92 & 1.74 \\
\hline 10 & 1.66 & 1.60 & 2.47 & 2.36 \\
\hline 15 & 1.84 & 1.79 & 2.83 & 2.77 \\
\hline 20 & 1.91 & 1.89 & 3.06 & 3.03 \\
\hline 25 & 1.95 & 1.95 & 3.20 & 3.19 \\
\hline 30 & 1.97 & 1.97 & 3.29 & 3.28 \\
\hline 40 & 1.99 & 1.99 & 3.37 & 3.37 \\
\hline 50 & 1.99 & 1.99 & 3.41 & 3.41 \\
\hline
\end{tabular}

fFrom K. Shure, J. A. O'Brien, and D. M. Rothberg, Nuclear Science and Engineering, 35: 373 (1969).

Shure et al. also investigated the use of these removal cross sections for hydrogen-deficient shields with the technique that is normally used when removal cross sections are applied to large thicknesses of hydrogenous shielding following the laminations of nonhydrogenous material. The normal expression used to estimate the neutron dose transmitted through $t \mathrm{~cm}$ of hydrogenous material and several slabs of nonhydrogenous materials is

$$
D_{l a m}(x, t)=D_{c a l c}(0, t) \exp \left(-\sum_{i} N_{i} \sigma_{i} t_{i}\right)
$$

where $D_{c a l c}=$ calculated dose for the hydrogenous material alone

$N_{i}=$ density

$\sigma_{i}=$ removal cross section

$t_{i}=$ thickness of the $i$ th nonhydrogenous material

Equation 4.8-32 was applied to three different shield configurations using lead and iron followed by various thicknesses of polyethylene. Values of $D_{\text {calc }}$ were obtained, and values of $\sigma_{i}$ from Table 4.5 were used. A comparison of the resulting dose rates with dose rates obtained with a separate, complete transport calculation showed that the frequently used design technique exemplified by Eq. 4.8-32 does provide good estimates 
(within 10\%) of the dose rates when the removal cross sections for hydrogen-deficient shields are used.

(c) Albert-Welton Kernel. The experimentally determined removal cross section provides a simple method for determining the attenuation through nonhydrogenous portions of shield material if there is a hydrogenous portion of the shield. Albert and Welton ${ }^{34}$ developed a semiempirical theory of neutron attenuation which provides a simple method for calculating neutron attenuation through the complete shield; this theory is based on the removal-cross-section concept. Basic to the Albert-Welton model is the assumption that any collision with hydrogen has the effect of an absorption. This, in effect, neglects the buildup of scattered neutrons that have undergone only small-angle scatterings by hydrogen. Inelastic scatterings with heavier nuclei are also regarded as absorptions because of the characteristically large energy loss. Other collisions are mainly small-angle elastic scatterings within the forward peak of the angular distribution, which amount to virtually no collisions. Attenuation through the materials in the shield is described in terms of removal cross sections. For hydrogen the removal cross section is taken to be its energy-dependent total cross section, and for the heavier nuclides it is taken to be an empirical energy-independent removal cross section, such as the removal cross sections described in the preceding paragraphs. Thus the Albert-Welton model provides a theoretical basis for the removal-cross-section concept.

The Albert-Welton formulation for fission neutrons from a plane monodirectional source which penetrate through a mixture of water and heavy materials is given by

$$
\Phi(r) \propto \exp \left(-\sum_{i} f_{i} \Sigma_{R_{i}} r\right) \int_{0}^{\infty} S(E) e^{-\Sigma_{H}(E) r} d E
$$

where $\Phi(r)=$ number flux density at a distance $r$ from the source

$\Sigma_{R_{i}}=$ macroscopic removal cross section of $i$ th element (other than hydrogen)

$f_{i}=$ volume fraction of $i$ th nonhydrogenous material

$S(E) d E=$ fraction of fission neutrons at $E$ in interval $d E$ for a total source of 1 fission $\mathrm{cm}^{-2} \mathrm{sec}^{-1}$

$\Sigma_{H}=$ total macroscopic cross section for hydrogen

The proportionality constant included in the original Albert-Welton derivation has been removed from Eq. 4.8-33 to avoid the implication that the actual number flux density can be computed from this relation. 
Integration of Eq. 4.8-33 yields the original Albert-Welton kernel for the hydrogenous portion of the shield, which is included in the first set of braces in the following relation:

$$
\begin{aligned}
\Phi(r) \propto\left\{\left(f_{w} r\right)^{0.29} \exp [-\right. & \left.\left.0.928\left(f_{w} r\right)^{0.58}\right]\right\} \\
& \times \exp \left[-f_{w} r \Sigma_{R_{0}}-\left(1-f_{w}\right) r \sum_{i} f_{i} \Sigma_{R_{i}}\right]
\end{aligned}
$$

where $f_{w}=$ volume fraction of water

$\Sigma_{R_{0}}=$ removal cross section of oxygen

$\Sigma_{R_{i}}=$ removal cross section of nonhydrogenous materials other than the oxygen in the water

$r=$ distance $($ in $\mathrm{cm})$ from source

Although the derivation was for a plane source, Eq. 4.8-34 holds for a point source when multiplied by the geometric factor $1 / 4 \pi r^{2}$, and the integral of $S(E)$ is normalized to 1 fission/sec. The relation is also valid when slabs of heavy material are laminated with the water. A minimum of about 50 or $60 \mathrm{~cm}$ of water is required between the dose point and the last of the heavy materials (whether as slabs or in a mixture) to comply with the limitations of the removal-cross-section concept.

On the basis of more recent experimental results, Casper ${ }^{44}$ evaluated new constants for the Albert-Welton kernel. The result for a point fission-spectrum source is

$4 \pi r^{2} D(r)=2.78 \times 10^{-5}\left[\left(f_{w} r\right)^{0.349} \mathrm{e}^{-0.422\left(f_{w} r\right) 0.698}\right] \mathrm{e}^{-0.0308 f_{w} r}$

where $D(r)$ is the neutron dose rate $r$ centimeters from the source [in $\left(\right.$ rads hr$\left.{ }^{-1}\right) /\left(\right.$ neutrons sec $\left.\left.{ }^{-1}\right)\right]$. When shield materials are inserted between the water and the fission source, Eq. 4.8-35 is multiplied by

$$
\exp \left[-\left(1-f_{w}\right) \sum_{i} \Sigma_{R_{i}} f_{i}\right]
$$

to obtain the dose rate at the shield surface.

\subsection{COMBINATION REMOVAL-DIFFUSION METHODS}

The removal-cross-section concept described in Sec. 4.8.2 provides a method for calculating the dose rate due to high-energy neutrons that 
penetrate a hydrogenous shield; however, the technique cannot be used to predict the dose rate due to neutrons that have been moderated to epithermal and thermal energies or to predict the thermal-neutron-flux density, which is used to obtain the capture gamma-ray source distribution within the shield. $\dagger$

The energy and spatial distributions of the moderated neutrons throughout a shield have sometimes been calculated by using the elementary theories of neutron diffusion and moderation (see Sec. 4.6). However, these methods of reactor physics are normally used to predict the average behavior of neutrons involved in reactor criticality problems, and, in the typical shielding problem, the neutron of significance is the unusual fission neutron that is born with an energy much greater than the average and contributes very little to reactor criticality. This unusual neutron penetrates into regions deep within the shield.

The inadequacy of both the removal concept and the elementary methods of reactor-core physics in calculations of the whole shielding problem has resulted in neutron transport's being regarded as a two-step process: a step in which a high-energy neutron penetrates to a position deep within the shield, where it suffers a collision that degrades its energy significantly, and a step in which the resulting low-energy neutron enters a diffusion process. Characteristically, the distance traveled by the neutron during the diffusion process is very much shorter than the distance it traveled as a fast neutron, and, once it has entered this second phase, the methods of elementary reactor physics conceivably could apply. Such reasoning prompted the first-flight correction to the age in Fermi age theory. ${ }^{2}$ This correction was necessary because a neutron cannot enter a process described as continuous slowing down (as required by Fermi age theory) until it has had at least one collision.

The development of high-speed computers and the resulting extensive use of multigroup diffusion theory for reactor criticality problems made the development of a technique that utilized diffusion theory even more attractive. In one of the first attempts to develop such a technique, Haffner $^{45}$ in 1958 used diffusion theory to calculate thermal-neutron-flux densities within a reactor shield and then normalized the results at each space point according to the fast-neutron dose rate obtained with the Albert-Welton kernel (see Sec. 4.8.2). Anderson and Shure ${ }^{46}$ used a similar technique when they applied a known pure-water kernel to normalize 
diffusion (actually $P_{1}$ multigroup) results for a metal-water mixture. In general, they obtained good results for laminated iron-water shields. (Shure $e^{3,47}$ later showed that a straightforward $P_{3}$ calculation without the use of a kernel also gave good results.) The main assumption in the Anderson-Shure technique is that the multigroup procedure correctly calculates the ratio between the flux densities in water and those in a metal-water mixture.

After several attempts had been made to develop a technique by correcting diffusion-theory results, a different approach to the problem evolved: a first-flight correction was made before the diffusion-theory calculation was performed. In the early calculations this was done by computing the singly scattered neutron-flux density from the uncollided-flux density and then using it as a source for the diffusion-theory calculation. A difficulty inherent in this procedure, especially for hydrogenous media, is that the penetrating component does not consist of uncollided neutrons alone but rather is composed largely of neutrons that have had one or more collisions and have suffered only small angular deflections. When these neutrons were accounted for, the first successful two-step model for neutron-penetration calculations became available. The method, in which the fast-neutron removal concept and age-diffusion theory are combined, is commonly referred to as the Spinney method, after its chief developer. The remainder of this section is devoted to a description of the original version of this removal-age-diffusion method and subsequent variations of it.

\subsubsection{The Spinney Method}

The Spinney method as first described by Avery, Bendall, Butler, and Spinney ${ }^{48}$ is characterized by the following basic physical assumptions:

1. The penetrating component of the source neutrons consists of the high-energy neutrons that suffer only small energy loss through small-angle elastic collisions and the uncollided neutrons.

2. Neutrons that suffer large energy loss through either wide-angle elastic or inelastic scattering are regarded as being removed from the fast beam.

3. The removed neutrons are degraded in energy in accordance with age theory and do not travel significantly from the point of removal.

4. The removed neutrons have a spectral and spatial distribution closely described by the conventional age-diffusion theory near the source.

5. Neutrons removed after they have penetrated deep into a homogeneous medium develop an equilibrium spectrum and are attenuated at the same rate as the penetrating component. 
6. The equilibrium spectrum of the degraded neutrons is disturbed near the boundaries between dissimilar media. [Note: Assumptions 4, 5, and 6 are not required in recent versions of the Spinney method.]

The neutron-flux density (removal flux) that corresponds to the penetrating component of the source neutrons is given by the kernel

$$
\Phi^{0}(r)=\frac{S_{0} \mathrm{e}^{-\Sigma_{R} r}}{4 \pi r^{2}}
$$

where $S_{0}$ is the source strength of high-energy neutrons of energy $E, \Sigma_{R}$ is the removal cross section evaluated at the source energy $E$ (determined experimentally or approximated by the transport cross section (see Sec. 4.8.2), and $r$ is the distance traveled by the neutron to its first collision.

The removed neutrons are regarded as a local source of degraded neutrons, the behavior of which can be adequately described by diffusion theory. The intensity of this source is given by

$$
S(r)=\Phi^{0}(r) \Sigma_{R}=\frac{S_{0} \Sigma_{R} \mathrm{e}^{-\Sigma_{R} r}}{4 \pi r^{2}}
$$

These neutrons (i.e., the removed neutrons) are then introduced into the highest energy group of an appropriate set of multigroup diffusion equations to calculate the distribution of the low-energy neutron-flux density. The equations comprising the multigroup set are given by

$$
\begin{aligned}
\nabla^{2} \Phi_{1}(r)-k_{1}^{2} \Phi_{1}(r)-\frac{\Sigma_{a 1}}{D_{i}} \Phi_{1}(r)+\frac{S(r)}{D_{i}}=0 & (i=1) \\
\nabla^{2} \Phi_{i}(r)-k_{i}^{2} \Phi_{i}(r)-\frac{\Sigma_{a i}}{D_{i}} \Phi_{i}(r)+\frac{D_{i-1} k_{i-1}^{2} \Phi_{i-1}(r)}{D_{i}}=0 & (i>1)
\end{aligned}
$$

where $\Phi_{i}=$ group flux density for the $i$ th group

$\Sigma_{a i}=$ group-averaged macroscopic absorption cross section

$D_{i}=$ group-averaged diffusion coefficient

$k_{i}^{-1}=$ slowing-down length for the $i$ th group

The slowing-down length is calculated according to age theory and for the $i$ th group is given by

$$
\left(\frac{1}{k_{i}}\right)^{2}=\int_{E_{i-1}}^{E_{i}} \frac{d E}{3 \xi(E) \Sigma_{s}(E) \Sigma_{t r}(E) E}
$$


where $\xi(E)$ is the average change in lethargy per collision for neutrons of energy $E, \Sigma_{s}(E)$ is the macroscopic scattering cross section for neutrons of energy $E$, and $\Sigma_{t r}(E)$ is the macroscopic transport cross section for neutrons of energy $E$.

In the original formulation of the Spinney method, five energy groups were taken for the multigroup diffusion calculation. The bottom group, which was a thermal group, had an upper energy of $2.81 \mathrm{kT}$ $\left(k=8.61 \times 10^{-5} \mathrm{eV} /{ }^{\circ} \mathrm{K}\right)$, and the highest group $(i=1)$ had an upper energy of $2 \mathrm{MeV}$. All removed source neutrons were assumed to be placed directly into the highest group. Solution of the group-diffusion equations, of course, required that boundary conditions be specified at the inner and outer surfaces of the shield. A zero reentrant condition was imposed at the outer boundary: this was stated in terms of the extrapolated boundary condition, which requires that the flux densities vanish at a distance $3.13 D_{i}$ beyond the physical boundary. The boundary conditions at the inner surface of the shield were established by requiring that the flux densities and current densities be equal to those determined from reactor-core calculations.

The original formulation was used with some success in predicting the distribution of low-energy neutrons in concrete shields for existing graphitemoderated reactors, but it was not suited for general application. Some of its inadequacies were that (1) all the removed neutrons were placed in one group, which neglected any additional diffusion-type transport that could have been accomplished at energies greater than $2 \mathrm{MeV} ;(2)$ not enough groups were used to adequately represent the continuous slowing-down process: and (3) the transfer of neutrons from one energy group to the next lower group did not describe the large energy losses experienced by neutrons that had suffered an inelastic scattering or a collision with hydrogen.

\subsubsection{Variations of the Spinney Method}

Many modifications and variations of the Spinney method have been developed, such as the RASH E, MAC, NRN, SABINE, and ATTOW codes. $\dagger$ In the RASH $\mathrm{E}$ formulation of Bendall ${ }^{9}$ and Butler, ${ }^{50}$ the modifications include an increase in the number of groups to 16 and a broader energy range ( 0 to $10 \mathrm{MeV}$ ). Also, the multigroup equations have been modified to include

tRASH E is the latest member of the RASH family of codes utilizing the Spinney method. RASH E is included in a FORTRAN code package known as COMPRASH and can be obtained from the Radiation Shielding Information Center. Oak Ridge National Labolatory, Oak Ridge, Tenn Code pachages tor MAC, NRN, SABINE, and ATTOW are also avalable from this center 
a direct source of removed neutrons into the nine highest energy groups. The equations so modified are as follows:

$$
\begin{gathered}
\nabla^{2} \Phi_{1}(r)-k_{1}^{2} \Phi_{1}(r)-\frac{\Sigma_{a 1}}{D_{1}} \Phi_{1}(r)+\frac{\psi_{1}(r)}{D_{1}}=0 \quad(i=1) \\
\nabla^{2} \Phi_{i}(r)-k_{i}^{2} \Phi_{i}(r)-\frac{\Sigma_{a i}}{D_{i}} \Phi_{i}(r) \\
+\frac{D_{i-1} k_{i-1}^{2}}{D_{i}} \Phi_{i-1}(r)+\frac{\psi_{i}(r)}{D_{i}}=0 \quad(i=2,3, \ldots, 9) \\
\nabla^{2} \Phi_{i}(r)-k_{i}^{2} \Phi_{i}(r)-\frac{\Sigma_{a i}}{D_{i}} \Phi_{i}(r) \\
+\frac{D_{i-1} k_{i-1}^{2}}{D_{i}} \Phi_{i-1}(r)=0 \quad(i=10,11, \ldots, 15) \\
\nabla^{2} \Phi_{T}(r)-\frac{\Sigma_{a}^{T}}{D_{T}} \Phi_{T}(r)+\frac{D_{15} k_{15}^{2}}{D_{T}} \Phi_{15}(r)=0 \quad(i=16)
\end{gathered}
$$

where $T$ corresponds to $i=16$ and designates the thermal-group flux density.

The source term for the $i$ th group resulting from removed neutrons is designated as $\psi_{i}(r)$ and is determined in the following manner. The fission spectrum is divided into 18 energy bands of $1-\mathrm{MeV}$ width. Neutrons removed from the $j$ th energy band are given by

$S_{j}(r)=S_{0} \int_{E_{j}}^{E_{j+1}} \frac{F(E) \Sigma_{R}(E) \mathrm{e}^{-\Sigma_{R}(E) r}}{4 \pi r^{2}} d E \quad(j=1,2, \ldots, 18)$

where $S_{0}$ is a magnitude factor determined by the power level of the reactor and $F(E)$ is the normalized fission spectrum.

The neutrons from each of the removal bands in the energy range 0 to $8 \mathrm{MeV}(j=18,17,16, \ldots, 11)$ are introduced into the energy group whose upper energy limit corresponds to the mid-energy of the band. Neutrons from all the bands above $8 \mathrm{MeV}(j=10,4,8, \ldots, 1)$ have a mean energy of about $10 \mathrm{MeV}$ and are all introduced into the highest energy group (group 1), which has an upper energy of $10.5 \mathrm{MeV}$. This transfer scheme, along with the removal-band and energy-group structures for RASH E, is given in Table 4.6 . 
Table 4.6-REMOVAL-BAND AND ENERGY-GROUP STRUCTURES USED IN RASH E $\dagger$

\begin{tabular}{|c|c|c|c|c|c|c|}
\hline \multicolumn{3}{|c|}{ Removal bands } & \multicolumn{3}{|c|}{ Diffusion groups } & \multirow{3}{*}{$\begin{array}{l}\text { Band-to } \\
\text { group } \\
\text { transfer } \\
\text { scheme }\end{array}$} \\
\hline \multirow{2}{*}{$\begin{array}{l}\text { Band } \\
\text { No. }\end{array}$} & \multicolumn{2}{|c|}{ Energy limits, $\mathrm{MeV}$} & \multirow{2}{*}{$\begin{array}{c}\text { Group } \\
\text { No. }\end{array}$} & \multicolumn{2}{|c|}{ Energy limits, MeV } & \\
\hline & Upper & Lower & & Upper & Lower & \\
\hline 1 & 18 & 17 & 1 & $1.05 \times 10^{1}$ & $7.5 \times 10^{0}$ & $1 \rightarrow 1$ \\
\hline 2 & 17 & 16 & 2 & $7.5 \times 10^{0}$ & $6.5 \times 10^{0}$ & $2 \rightarrow 1$ \\
\hline 3 & 16 & 15 & 3 & $6.5 \times 10^{0}$ & $5.5 \times 10^{0}$ & $3 \rightarrow 1$ \\
\hline 4 & 15 & 14 & 4 & $5.5 \times 10^{0}$ & $4.5 \times 10^{0}$ & $4 \rightarrow 1$ \\
\hline 5 & 14 & 13 & 5 & $4.5 \times 10^{0}$ & $3.5 \times 10^{0}$ & $5 \rightarrow 1$ \\
\hline 6 & 13 & 12 & 6 & $3.5 \times 10^{0}$ & $2.5 \times 10^{0}$ & $6 \rightarrow 1$ \\
\hline 7 & 12 & 11 & 7 & $2.5 \times 10^{0}$ & $1.5 \times 10^{0}$ & $7 \rightarrow 1$ \\
\hline 8 & 11 & 10 & 8 & $1.5 \times 10^{0}$ & $5.0 \times 10^{-1}$ & $8 \rightarrow 1$ \\
\hline 9 & 10 & 9 & 9 & $5.0 \times 10^{-1}$ & $5.0 \times 10^{-2}$ & $9 \rightarrow 1$ \\
\hline 10 & 9 & 8 & 10 & $5.0 \times 10^{-2}$ & $5.0 \times 10^{-3}$ & $10 \rightarrow 1$ \\
\hline 11 & 8 & 7 & 11 & $5.0 \times 10^{-3}$ & $5.0 \times 10^{-4}$ & $11 \rightarrow 2$ \\
\hline 12 & 7 & 6 & 12 & $5.0 \times 10^{-4}$ & $5.0 \times 10^{-5}$ & $12 \rightarrow 3$ \\
\hline 13 & 6 & 5 & 13 & $5.0 \times 10^{-5}$ & $5.0 \times 10^{-6}$ & $13 \rightarrow 4$ \\
\hline 14 & 5 & 4 & 14 & $5.0 \times 10^{-6}$ & $5.5 \times 10^{-7}$ & $14 \rightarrow 5$ \\
\hline 15 & 4 & 3 & 15 & $5.5 \times 10^{-7}$ & $7.0 \times 10^{-8}$ & $15 \rightarrow 6$ \\
\hline 16 & 3 & 2 & 16 & \multicolumn{2}{|c|}{ Thermal } & $16 \rightarrow 7$ \\
\hline 17 & 2 & 1 & & & & $17 \rightarrow 8$ \\
\hline 18 & 1 & 0 & & & & $18 \rightarrow 9$ \\
\hline
\end{tabular}

†From Bendall. ${ }^{49}$

In the MAC formulation of Peterson ${ }^{51}$ and Canali, Ilsemann, and Preusch, 52 the number of energy groups for the group-diffusion calculation is increased to 31 over an energy range from 0 to $10 \mathrm{MeV}$. Again the fission spectrum is divided into 18 removal bands of $1-\mathrm{MeV}$ width. The flux density for the source of the removed neutrons (usually called renoval flux) corresponding to the $g$ th removal band, which is introduced into the $i$ th energy group, is given by

$$
\Phi_{g i}^{0}=S_{0} \int_{E_{g}}^{E_{g+1}} \frac{F(E) \mathrm{e}^{-\Sigma_{R}(E) r}}{4 \pi r^{2}} d E
$$

The removed neutrons are introduced into the five highest energy groups only. The transfer scheme, along with the removal-band and energy-group structures, is given in Table 4.7 . 
Table 4.7-REMOVAL-BAND AND ENERGY-GROUP STRUCTURES USED IN MAC $t$

\begin{tabular}{|c|c|c|c|c|c|c|}
\hline \multicolumn{3}{|c|}{ Removal bands } & \multicolumn{3}{|c|}{ Diffusion groups } & \multirow{3}{*}{$\begin{array}{l}\text { Band-to } \\
\text { group } \\
\text { transfer } \\
\text { scheme }\end{array}$} \\
\hline \multirow{2}{*}{$\begin{array}{l}\text { Band } \\
\text { No. }\end{array}$} & \multicolumn{2}{|c|}{ Energy limits, $\mathrm{MeV}$} & \multirow{2}{*}{$\begin{array}{c}\text { Group } \\
\text { No. }\end{array}$} & \multicolumn{2}{|c|}{ Energy limits, MeV } & \\
\hline & Upper & Lower & & Lower & Upper & \\
\hline 1 & 18 & 17 & 1 & $6.065 \times 10^{0}$ & $1.000 \times 10^{-1}$ & $1 \rightarrow 1$ \\
\hline 2 & 17 & 16 & 2 & $3.679 \times 10^{0}$ & $6.065 \times 10^{0}$ & $2 \rightarrow 1$ \\
\hline 3 & 16 & 15 & 3 & $2.231 \times 10^{0}$ & $3.679 \times 10^{\circ}$ & $3 \rightarrow 1$ \\
\hline 4 & 15 & 14 & 4 & $1.353 \times 10^{0}$ & $2.231 \times 10^{0}$ & $4 \rightarrow 1$ \\
\hline 5 & 14 & 13 & 5 & $8.208 \times 10^{-1}$ & $1.353 \times 10^{0}$ & $5 \rightarrow 1$ \\
\hline 6 & 13 & 12 & 6 & $3.876 \times 10^{-1}$ & $8.208 \times 10^{-1}$ & $6 \rightarrow 1$ \\
\hline 7 & 12 & 11 & 7 & $1.830 \times 10^{-1}$ & $3.876 \times 10^{-1}$ & $7 \rightarrow 1$ \\
\hline 8 & 11 & 10 & 8 & $6.733 \times 10^{-2}$ & $1.830 \times 10^{-1}$ & $8 \rightarrow 1$ \\
\hline 9 & 10 & 9 & 9 & $2.600 \times 10^{-2}$ & $6.733 \times 10^{-2}$ & $9 \rightarrow 1$ \\
\hline 10 & 9 & 8 & 10 & $2.000 \times 10^{-2}$ & $2.600 \times 10^{-2}$ & $10 \rightarrow 1$ \\
\hline 11 & 8 & 7 & 11 & $9.118 \times 10^{-3}$ & $2.000 \times 10^{-2}$ & $11 \rightarrow 1$ \\
\hline 12 & 7 & 6 & 12 & $3.355 \times 10^{-3}$ & $9.118 \times 10^{-3}$ & $12 \rightarrow 1$ \\
\hline 13 & 6 & 5 & 13 & $1.234 \times 10^{-3}$ & $3.355 \times 10^{-3}$ & $13 \rightarrow 2$ \\
\hline 14 & 5 & 4 & 14 & $4.540 \times 10^{-4}$ & $1.234 \times 10^{-3}$ & $14 \rightarrow 2$ \\
\hline 15 & 4 & 3 & 15 & $3.199 \times 10^{-4}$ & $4.540 \times 10^{-4}$ & $15 \rightarrow 2$ \\
\hline 16 & 3 & 2 & 16 & $2.255 \times 10^{-4}$ & $3.199 \times 10^{-4}$ & $16 \rightarrow 3$ \\
\hline 17 & 2 & 1 & 17 & $1.120 \times 10^{-4}$ & $2.255 \times 10^{-4}$ & $17 \rightarrow 4$ \\
\hline \multirow[t]{14}{*}{18} & 1 & 0 & 18 & $6.147 \times 10^{-5}$ & $1.120 \times 10^{-4}$ & $18 \rightarrow 5$ \\
\hline & & & 19 & $3.374 \times 10^{-5}$ & $6.147 \times 10^{-5}$ & \\
\hline & & & 20 & $1.515 \times 10^{-5}$ & $3.374 \times 10^{-5}$ & \\
\hline & & & 21 & $1.016 \times 10^{-5}$ & $1.515 \times 10^{-5}$ & \\
\hline & & & 22 & $4.565 \times 10^{-6}$ & $1.016 \times 10^{-5}$ & \\
\hline & & & 23 & $1.375 \times 10^{-6}$ & $4.565 \times 10^{-6}$ & \\
\hline & & & 24 & $9.214 \times 10^{-7}$ & $1.375 \times 10^{-6}$ & \\
\hline & & & 25 & $6.716 \times 10^{-7}$ & $9.214 \times 10^{-7}$ & \\
\hline & & & 26 & $4.140 \times 10^{-7}$ & $6.716 \times 10^{-7}$ & \\
\hline & & & 27 & $2.775 \times 10^{-7}$ & $4.140 \times 10^{-7}$ & \\
\hline & & & 28 & $1.860 \times 10^{-7}$ & $2.775 \times 10^{-7}$ & \\
\hline & & & 29 & $1.247 \times 10^{-7}$ & $1.860 \times 10^{-7}$ & \\
\hline & & & 30 & $7.595 \times 10^{-8}$ & $1.247 \times 10^{-7}$ & \\
\hline & & & 31 & 0 & $7.595 \times 10^{-8}$ & \\
\hline
\end{tabular}

†From Peterson. ${ }^{51}$

The MAC formulation differs from the original Spinney method in two major respects: (1) the removal flux density is added directly to the group-diffusion flux density after the diffusion calculation has been performed, and the combined flux density is then used to calculate source 
neutrons for the lower energy groups; and (2) the general treatment of the downscatter transfer of neutrons allows for a more accurate representation of inelastic scattering and collisions with hydrogen.

The highest energy group $(i=1)$ in the diffusion part of the calculation is not actually treated as a diffusion group. The collision density,

$$
\Sigma_{1 k}\left[\sum_{g=1}^{12} \Phi_{g^{1}}^{0}(\mathrm{r})\right]
$$

which is based on the removal flux densities (corresponding to the energy bands 1 to 12), provides neutrons by downscattering from the first group into the $k$ th group, $k=2,3, \ldots, K$. The kinds of possible interactions, as described by their respective group-to-group removal cross section, $\Sigma_{1 k}$, will determine the extent of the downscatter. A diffusion calculation is then performed on the second group, the neutrons removed from group 1 being used as the source. Solution of the group 2 diffusion equation

$D_{2} \nabla^{2} \Phi_{2}(\mathbf{r})-\sum_{k=3}^{K} \Sigma_{2 k} \Phi_{2}(\mathbf{r})-\Sigma_{a 2} \Phi_{2}(\mathbf{r})+\Sigma_{12}\left(\sum_{g=1}^{12} \Phi_{g 1}^{0}\right)=0$

yields the group-diffusion flux density $\Phi_{2}(r)$. The group 2 removal flux densities are then added to the diffusion flux density so that the downscatter source of neutrons from group 2 into the lower energy groups can be calculated. The downscatter source into group $k(k=3,4, \ldots, K)$ is given by

$$
\Sigma_{2 k}\left[\Phi_{2}(\mathbf{r})+\sum_{g^{=13}}^{15} \Phi_{g^{2}}^{0}(\mathbf{r})\right]
$$

The calculation proceeds in a similar fashion from one group to the next lower group and so on. In general, for $i>2$ the group-diffusion equations are given by

$$
\begin{aligned}
D_{i} \nabla^{2} \Phi_{i}(\mathrm{r})-\sum_{k=i+1}^{K} & \Sigma_{i k} \Phi_{i}(\mathbf{r})-\Sigma_{a i} \Phi_{i}(\mathbf{r}) \\
& +\sum_{i=i-1}^{1} \Sigma_{j i}\left[\Phi_{j}(\mathbf{r})+\sum_{g} \Phi_{g j}^{0}\right]=0 \quad(i=3,4, \ldots)
\end{aligned}
$$

and the downscatter source term from the $i$ th group into the $k$ th group is

$$
\Sigma_{i k}\left[\Phi_{i}(\mathrm{r})+\Phi_{g i}^{0}\right]
$$

In the NRN formulation of Hjarne ${ }^{53}$ and Hjarne and Leimdorfer, ${ }^{54}$ the energy structure for the removal bands and energy groups differs signifi- 
cantly from that used in the RASH and MAC formulations. The group structure for the group-diffusion calculation consists of 24 groups over an energy range 0 to $18 \mathrm{MeV}$, and the fission spectrum is divided into 30 bands of varying widths. The removal-band and energy-group structures are given in Table 4.8.

Table 4.8-REMOVAL-BAND AND ENERGY-GROUP STRUCTURES USED IN NRN†

\begin{tabular}{|c|c|c|c|c|c|}
\hline \multicolumn{3}{|c|}{ Removal bands } & \multicolumn{3}{|c|}{ Diffusion group } \\
\hline \multirow{2}{*}{$\begin{array}{c}\text { Band } \\
\text { No. }\end{array}$} & \multicolumn{2}{|c|}{ Energy limits, MeV } & \multirow{2}{*}{$\begin{array}{c}\text { Group } \\
\text { No. }\end{array}$} & \multicolumn{2}{|c|}{ Energy limits, MeV } \\
\hline & Upper & Lower & & Upper & Lower \\
\hline 1 & $1.8 \times 10^{1}$ & $1.43 \times 10^{1}$ & 1 & $1.8 \times 10^{1}$ & $1.35 \times 10^{1}$ \\
\hline 2 & $1.43 \times 10^{1}$ & $1.136 \times 10^{1}$ & 2 & $1.35 \times 10^{1}$ & $1.0 \times 10^{1}$ \\
\hline 3 & $1.136 \times 10^{1}$ & $9.021 \times 10^{0}$ & 3 & $1.0 \times 10^{1}$ & $7.8 \times 10^{0}$ \\
\hline 4 & $9.021 \times 10^{0}$ & $7.166 \times 10^{0}$ & 4 & $7.8 \times 10^{0}$ & $5.9 \times 10^{0}$ \\
\hline 5 & $7.166 \times 10^{0}$ & $5.692 \times 10^{0}$ & 5 & $5.9 \times 10^{0}$ & $4.4 \times 10^{0}$ \\
\hline 6 & $5.692 \times 10^{0}$ & $4.521 \times 10^{0}$ & 6 & $4.4 \times 10^{0}$ & $3.4 \times 10^{0}$ \\
\hline 7 & $4.521 \times 10^{0}$ & $3.591 \times 10^{0}$ & 7 & $3.4 \times 10^{0}$ & $2.6 \times 10^{0}$ \\
\hline 8 & $3.591 \times 10^{0}$ & $2.853 \times 10^{0}$ & 8 & $2.6 \times 10^{0}$ & $2.0 \times 10^{0}$ \\
\hline 9 & $2.853 \times 10^{0}$ & $2.267 \times 10^{0}$ & 9 & $2.0 \times 10^{0}$ & $1.5 \times 10^{0}$ \\
\hline 10 & $2.267 \times 10^{0}$ & $1.800 \times 10^{0}$ & 10 & $1.5 \times 10^{0}$ & $1.2 \times 10^{0}$ \\
\hline 11 & $1.800 \times 10^{0}$ & $1.430 \times 10^{0}$ & 11 & $1.2 \times 10^{0}$ & $9.0 \times 10^{-1}$ \\
\hline 12 & $1.430 \times 10^{0}$ & $1.136 \times 10^{0}$ & 12 & $9.0 \times 10^{-1}$ & $7.0 \times 10^{-1}$ \\
\hline 13 & $1.136 \times 10^{0}$ & $9.021 \times 10^{-1}$ & 13 & $7.0 \times 10^{-1}$ & $5.1 \times 10^{-1}$ \\
\hline 14 & $9.021 \times 10^{-1}$ & $7.166 \times 10^{-1}$ & 14 & $5.1 \times 10^{-1}$ & $3.8 \times 10^{-1}$ \\
\hline 15 & $7.166 \times 10^{-1}$ & $5.692 \times 10^{-1}$ & 15 & $3.8 \times 10^{-1}$ & $3.0 \times 10^{-1}$ \\
\hline 16 & $5.692 \times 10^{-1}$ & $4.521 \times 10^{-1}$ & 16 & $3.0 \times 10^{-1}$ & $1.0 \times 10^{-1}$ \\
\hline 17 & $4.521 \times 10^{-1}$ & $3.591 \times 10^{-1}$ & 17 & $1.0 \times 10^{-1}$ & $3.10 \times 10^{-2}$ \\
\hline 18 & $3.591 \times 10^{-1}$ & $2.853 \times 10^{-1}$ & 18 & $3.10 \times 10^{-2}$ & $1.10 \times 10^{-2}$ \\
\hline 19 & $2.853 \times 10^{-1}$ & $2.267 \times 10^{-1}$ & 19 & $1.10 \times 10^{-2}$ & $1.10 \times 10^{-3}$ \\
\hline 20 & $2.267 \times 10^{-1}$ & $1.800 \times 10^{-1}$ & 20 & $1.10 \times 10^{-3}$ & $1.10 \times 10^{-4}$ \\
\hline 21 & $1.800 \times 10^{-1}$ & $1.430 \times 10^{-1}$ & 21 & $1.10 \times 10^{-4}$ & $1.10 \times 10^{-5}$ \\
\hline 22 & $1.430 \times 10^{-1}$ & $1.136 \times 10^{-1}$ & 22 & $1.10 \times 10^{-5}$ & $1.10 \times 10^{-6}$ \\
\hline 23 & $1.136 \times 10^{-1}$ & $9.021 \times 10^{-2}$ & 23 & $1.10 \times 10^{-6}$ & $1.05 \times 10^{-7}$ \\
\hline 24 & $9.021 \times 10^{-2}$ & $7.166 \times 10^{-2}$ & 24 & \multirow{2}{*}{\multicolumn{2}{|c|}{ Thermal }} \\
\hline 25 & $7.166 \times 10^{-2}$ & $5.692 \times 10^{-2}$ & & & \\
\hline 26 & $5.692 \times 10^{-2}$ & $4.521 \times 10^{-2}$ & & & \\
\hline 27 & $4.521 \times 10^{-2}$ & $3.591 \times 10^{-2}$ & & & \\
\hline 28 & $3.591 \times 10^{-2}$ & $2.853 \times 10^{-2}$ & & & \\
\hline 29 & $2.853 \times 10^{-2}$ & $2.267 \times 10^{-2}$ & & & \\
\hline 30 & $2.267 \times 10^{-2}$ & $1.80 \times 10^{-2}$ & & & \\
\hline
\end{tabular}

†From Hjarne. ${ }^{53}$ 
The NRN method allows for the transfer of removed neutrons from each removal band to many diffusion groups. The source for the $i$ th diffusion group arising from all removal collisions is

$$
\sum_{g} \Sigma_{g i}^{0} \Phi_{g}^{0}
$$

where $\Phi_{g}^{\mathbf{0}}$ is the removal flux density in the $g$ th energy band and $\Sigma_{g i}^{\mathbf{0}}$ is the energy-averaged removal cross section for the transfer of neutrons from the $g$ th removal band into the $i$ th energy group.

The calculation also allows transfer from each diffusion group to all lower energy diffusion groups. The group-diffusion equation for the $i$ th group is given by

$$
\begin{aligned}
D_{i} \nabla^{2} \Phi_{i}(\mathbf{r})-\sum_{k=i+1}^{K} \Sigma_{i k} \Phi_{i}(\mathbf{r})-\Sigma_{a i} & \Phi_{i}(\mathbf{r}) \\
& +\sum_{j=i-1}^{1} \Sigma_{j i} \Phi_{j}(\mathbf{r})+\Sigma_{g^{i}}^{\mathbf{0}} \Phi_{g}^{0}=0
\end{aligned}
$$

where the various diffusion-theory parameters have conventional definitions.

The SABINE code, described by Ponti, Preusch, and Schubart ${ }^{55}$ and by Nicks, Perline, and Ponti, ${ }^{56}$ represents later European technology than RASH and NRN. SABINE uses 19 removal groups and 26 diffusion groups. The code is also one dimensional and can solve problems in slab, cylindrical, and spherical geometries. Particular attention has been paid to the coupling of the removal flux with the diffusion equations. The assumed model in SABINE makes use of the same transfer matrix for band-to-group and group-to-group transfers, i.e.,

$$
\Sigma_{j i} \equiv \Sigma_{g i}
$$

where the removal band $g$ and diffusion group $i$ correspond to the same energy and $j \neq i$. Within-group transfer of removal neutrons to the diffusion group of the same energy range is also included.

Gamma-ray transport is accomplished by using seven energy groups and empirical region-dependent buildup factors based on transport calculations. Both primary sources (fission gamma rays) in the reactor core and secondary sources (capture and inelastic-scattering gamma rays) generated within the shield can be included.

The ATTOW code is a recent British development described by Collier and Curtis $^{57}$ and by Avery and Curtis. ${ }^{58}$ It is a two-dimensional (finite 
cylinder or $2 \mathrm{D}$ rectangular) diffusion code that can accept removal sources prepared by a built-in subroutine. The removal-source subroutine calculates sources at points determined by the routine and fits two-dimensional polynomials, of order chosen by the user, to the results. These data are put on tape in a form that can be used by ATTOW. The spatial integration over the reactor core is performed by a Gaussian scheme using a stored table of zeros and weights.

Removal cross sections $\Sigma_{g i}$ are input to the program, allowing the user control of the removal assumptions. The full group-to-group transfer matrix $\Sigma_{j i}$ is also assumed for the diffusion treatment.

The ATTOW code has been used extensively in the United Kingdom for solving problems associated with fast breeder reactors. It was found that the results were some what sensitive to the energy-group structure chosen. ${ }^{58} \mathrm{~A}$ 23-group structure was judged best. This sensitivity is most pronounced when materials such as graphite are present and is attributed to the continuous slowing-down (age theory) assumption, although even a fullscatter matrix cannot preserve the energy-angle correlation in diffusion theory. These difficulties should not be apparent in hydrogenous materials, which generally have short diffusion lengths.

\subsubsection{Differences in Current Methods}

A comparison of the preceding formulations shows that, with respect to the removal-band and energy-group schemes, RASH E and MAC are similar in concept and identical in many respects. The NRN, SABINE, or ATTOW approach is more general and should provide the most accurate model if the required removal and transfer cross sections are known.

With regard to removal cross sections, RASH E and MAC use the cross sections suggested by the original Spinney formulation, which has the general form

$$
\Sigma_{R}=\Sigma_{t}-f \Sigma_{e l}
$$

where $\Sigma_{R}=$ removal cross section

$\Sigma_{t}=$ total macroscopic cross section

$\Sigma_{e l}=$ elastic-scattering cross section

$f=$ fraction of elastic collisions that can be regarded as glancing

If $f$ is taken to be the average cosine of scattering in the laboratory system, $\bar{\mu}_{0}$, the removal cross section becomes the transport cross section originally used by Spinney. In general, the parameter $f$ cannot be determined 
intrinsically, and thus a value must be assumed or determined empirically. This has been done for a large variety of typical shield configurations, and the removal cross sections thus determined are used with a high degree of confidence.

The NRN removal cross sections are obtained by experimentally determining the angles of scatter above which elastic collisions can be considered as removals. The removal cross section is given by

$$
\Sigma_{R}=\Sigma_{t}-2 \pi \int_{\cos \theta_{R}}^{1} \sigma(\theta) d(\cos \theta)
$$

where $\sigma(\theta)$ is the differential elastic-scattering cross section per unit solid angle about the scattering angle $\theta$ in the center-of-mass system and $\theta_{R}$ is the scattering angle above which the collision is considered to be a removal. The value of $\theta_{R}$ is determined by comparing predicted neutron reaction rates with experimental values. Aalto ${ }^{59}$ obtained a best value of $\cos \theta_{R}=0.45$ for hydrogen, and $\cos \theta_{R}=0.60$ was obtained for other nuclides. With these values of $\theta_{R}$, a full set of removal cross sections can be derived.

The NRN removal cross sections may not have any advantage over the Spinney cross sections since each scheme involves only a single adjustable parameter, $\theta_{R}$ and $f$, respectively.

The MAC scheme for transferring removed neutrons into energy groups differs significantly from that used by either RASHE or NRN. The procedure in MAC is to add the removal flux density to the newly calculated group-diffusion flux density to establish the group-to-group downscatter source. In contrast, RASH E and NRN introduce the removed neutrons into given groups as source neutrons to that group, a more natural procedure for the group-diffusion calculation. The RASH E has a very restricted transfer scheme wherein the removed neutrons from a given removal band are introduced into a prescribed energy group and into no other. The NRN provides for a much more general scheme, using a removal matrix to describe the transfer of removed neutrons from a given removal band into any of the lower energy groups.

Of the methods mentioned, the slowing-down model embodied in NRN, and especially in SABINE, gives the most accurate description of the slowing-down process. It involves a general-group to any lower energy-group transfer matrix using detailed elastic and inelastic scattering cross sections for all nuclides. A similar scheme is employed by MAC; however, some inaccuracy is allowed in the description of the nonhydrogen elastic scattering. 
The RASH E uses a group-to-group transfer cross section based on the continuous slowing-down (age) model, which allows transfer to the next lower energy group only. This could lead to serious inaccuracics, particularly with respect to inelastic scatterings and collisions with hydrogen.

In conclusion, we should note that the Spinney method, used much more in Europe than in the United States, provides reliable results. As with any empirical method, it should be applied with some caution. Before being used extensively for design, removal-diffusion results should be compared with rigorous calculational methods or experiments for similar configurations. The simplicity and speed of computation give the Spinney method a significant advantage over more-sophisticated methods in design work.

\section{REFERENCES}

1. I. Kušcer, Developments in Transport Theory, E. Inönü and P. F. Zweifel (Eds.), p. 243, Academic Press, London, 1967.

2. A. M. Weinberg and E. P. Wigner, The Physical Theory of Neutron Chain Reactors, University of Chicago Press, Chicago, 1958.

3. K. Shure, P-3 Multigroup Calculations of Neutron Attenuation, Nucl. Sci. Eng., 19: 310 (1964).

4. W. D. Lanning, Application of the Spherical Harmonics Technique to Problems in Gamma Transport, Nucl. Sci. Eng., 15: 259-267 (1963).

5. G. C. Wick, Über ebene Diffusionprobleme, Physick, 121 : 702 (1943).

6. S. Chandrasekhar, Radiative Transfer, Clarendon Press, Oxford, 1950.

7. B. G. Carlson, Solution of the Transport Equation by the $S_{n}$ Method, USAEC Report LA-1891, Los Alamos Scientific Laboratory, 1955.

8. S. Preiser, G. Rabinowitz, and E. deDufour, A Program for the Numerical Integration of Boltzmann Transport Equation, NIOBE, Report ARL-TR-60-314, Nuclear Development Corp. of America, Aeronautical Research Laboratories, 1960.

9. B. Carlson, C. Lee, and J. Worlton, The DSN and TDC Neutron Transport Codes, USAEC Report LAMS-2346, Los Alamos Scientific Laboratory, Feb. 12, 1960; C. E. Lee, The Discrete $S_{n}$ Approximation to Transport Theory, USAEC Report LA-2595, Los Alamos Scientific Laboratory, Mar. 9, 1962.

10. K. D. Lathrop, DTF-IV, A FORTRAN-IV Program for Solving the Multigroup Transport Equation with Anisotropic Scattering, USAEC Report LA-3373, Los Alamos Scientific Laboratory, July 15, 1965.

11. F. R. Mynatt, A User's Manual for DOT, A Two-Dimensional Discrete Ordinates Transport Code with Anisotropic Scattering, informal notes included in RSIC Computer Code Collection CCC-89, Oak Ridge National Laboratory; see also F. R. Mynatt, F. J. Muckenthaler, and P.N. Stevens, Development of Two-Dimensional Discrete Ordinate Transport Theory for Radiation Shielding, USAEC Report CTC-INF-952 (AD-692168), Union Carbide Corporation, August 1969. 
12. H. J. Kopp, Synthetic Method Solution of the Transport Equation, Nucl. Sci. Eng., 17: 65 (1963).

13. E. M. Gelbard and L. A. Hageman, The Synthetic Method as Applied to the $S_{n}$ Equation, Nucl. Sci. Eng., 37: 288 (1969).

14. L. V. Spencer and U. Fano, Calculation of Spatial Distributions by Polynomial Expansion, J. Res. Nat. Bur. Stand., 46: 446 (1951).

15. U. Fano, L. V. Spencer, and M. J. Berger, The Penetration and Diffusion of X-Rays, Encyclopedia of Physics, S. Flügge (Ed.), p. 724, Springer-Verlag, New York, 1959.

16. H. Goldstein and J. E. Wilkins, Jr., Calculations of the Penetrations of Gamma Rays, Final Report, USAEC Report NYO-3075 (NDA-15C-41), Nuclear Development Associates, Inc., 1954.

17. H. Goldstein, Fundamental Aspects of Reactor Shielding, Addison-Wesley Publishing Company, Inc., Reading, Mass., 1959.

18. L. V. Spencer, in Radiation Shielding, Vol. 1, W. R. Kimel (Ed.), Office of Civil Defense, Technical Report TR-40, 1966.

19. J. Certaine, A Solution of the Neutron Transport Equation. Introduction and Part I, USAEC Report NYO-3081 (NDA-15C-43), Nuclear Development Associates, Inc., July $25,1954$.

20. V. A. Ambarzumian, Diffusion of Light by Planetary Atmosphere, Astron. Zh., 19: 30 (1942).

21. R. Bellman, R. Kalaba, and G. M. Wing, Invariant Imbedding and Mathematical Physics. I. Particle Processes, J. Math. Phys., 1(4): 280 (1960).

22. G. M. Wing, An Introduction to Transport Theory, John Wiley \& Sons, Inc., New York, 1962.

23. D. R. Mathews, K. F. Hansen, and E. A. Mason, Deep Penetration of Radiation by the Method of Invariant Imbedding, Nucl. Sci. Eng., 27: 263 (1967).

24. J. O. Mingle, Applications of the Invariant Imbedding Method to Monoenergetic Neutron Transport Theory in Slab Geometry, Nucl. Sci. Eng., 28: 177 (1967).

25. A. Shimizu and H. Mizuta, Application of Invariant Imbedding to the Reflection and Transmission Problems of Gamma Rays (II), J. Nucl. Sci. Technol. (Tokyo), 3: 10 (1966); also Nucl. Eng. Des., 32: 184-194 (1968).

26. J. J. Taylor, Application of Gamma Ray Build-Up Data to Shield Design, USAEC Report WAPD-RM-217, Westinghouse Electric Corp., Atomic Power Division, June 28, 1954.

27. M. A. Capo, Polynomial Approximation of Gamma Ray Buildup Factors for a Point Isotropic Source, USAEC Report APEX-510, General Electric Co., Atomic Products Division, August 1959.

28. M. J. Berger, in Proceedings of Shielding Symposium Held at the U.S. Naval Radiological Defense Laboratory, October 17-19, 1956, U. S. Naval Radiological Defense Laboratory Reviews and Lectures No. 29.

29. D. K. Trubey, A Survey of Empirical Functions Used to Fit Gamma-Ray Buildup Factors, USAEC Report ORNL-RSIC-10, Oak Ridge National Laboratory, February 1966.

30. J. H. Hubbell, A Power-Series Buildup Factor Formulation Application to Rectangular and Off-Axis Disk Source Problems, J. Res. Nat. Bur. Stand., C, 67: 291 (1963). 
31. J. H. Hubbell, R. L. Bach, and R. J. Herbold, Radiation Field from a Circular Disk Source, J. Res. Nat. Bur. Stand., C, 65: 249 (1961).

32. D. K. Trubey, Oak Ridge National Laboratory, unpublished calculations.

33. J. H. Hubbell, R. L. Bach, and J. C. Lamkin, Radiation Field from a Rectangular Source, J. Res. Nat. Bur. Stand., C, 64: 121 (1960).

34. R. D. Albert and T. A. Welton, A Simplified Theory of Neutron Attenuation and Its Application to Reactor Shield Design, USAEC Report WAPD-15, Westinghouse Electric Corp., Atomic Power Division, Nov. 30, 1950.

35. E. Solomito and J. R. Stockton, Modifications of the Point Kernel Code QAD-P5A: Conversion to the IBM-360 Computer and Incorporation of Additional Geometry Routines, USAEC Report ORNL-4181, Oak Ridge National Laboratory, 1968.

36. G. T. Chapman and C. L. Storrs, Effective Neutron Removal Cross Sections for Shielding, USAEC Report ORNL-1843 (AECD-3978), Oak Ridge National Laboratory, 1955.

37. E. P. Blizard (Ed.), Reactor Handbook, 2nd ed., Vol. III, Part B, Shielding, Interscience Publishers, a division of John Wiley \& Sons, Inc., New York, 1962.

38. L. K. Zoller, Fast-Neutron-Removal Cross Sections, Nucleonics, 22(8): 128 (1964).

39. D. K. Trubey and G. T. Chapman, Effective Neutron Removal Cross Sections for Carbon and Oxygen in Continuous Mediums, USAEC Report ORNL-2197, Oak Ridge National Laboratory, Sept. 19, 1958.

40. D. J. Dudziak and J. E. Schmucker, Hydrogenous-Material-Dependent Removal Cross Section of Lead for Fast Nelitron Biological Dose, USAEC Report WAPD-TM-662, Westinghouse Electric Corp., June 1968.

41. W. J. Price and W. L. Dunn, Dose Attenuation of Polonium-Beryllium Neutrons by Iron, Aluminum, and Lead, Air Force Institute of Technology, Tech. Report 57-7, 1967; also USAEC Report ORNL-2497 (Vol. 1), pp. 1L-12L, Oak Ridge National Laboratory.

42. D. J. Dudziak, Fast-Neutron Biological Dose Attenuation by Lead and Polyethylene Shields, Nucl. Appl., 6: 63 (1969).

43. K. Shure, J. A. O'Brien, and D. M. Rothberg, Neutron Dose Rate Attenuation by Iron and Lead, Nucl. Sci. Eng., 35: 371 (1969).

44. A. W. Casper, Modified Fast Neutron Attenuation Functions, USAEC Report XDC-60-2-76, General Electric Co., Atomic Products Division, Feb. 3, 1960.

45. J. W. Haffner, Neutron Energy Spectrum Calculations in Reactor Shields, Preprint V-84, Nuclear Engineering and Science Conference, April 6-9, 1959, Cleveland, Ohio, Engineers Joint Council, New York.

46. D. C. Anderson and K. Shure, Thermal Neutron Flux Distributions in MetalHydrogenous Shields, Nucl. Sci. Eng., 8: 260 (1960).

47. K. Shure, Few-Group and Multigroup Calculations of Neutron Penetration, Nucl. Sci. Eng., 27: 468 (1967).

48. A. F. Avery, D. E. Bendall, J. Butler, and K. T. Spinney, Methods of Calculation for Use in the Design of Shields for Power Reactors, British Report AERE-R-3216, February 1960.

49. D. E. Bendall, RASH D-A Mercury Programme for Neutron Shielding Calculations, British Report AEEW-M-261, August 1962. 
50. J. Butler, The Status of Theoretical Methods for Reactor Shield Design, British Report AEEW-R-361, March 1964.

51. E. G. Peterson, MAC-A Bulk Shielding Code, USAEC Report HW-73381, Hanford Atomic Products Operation, April 1962.

52. U. Canali, H. Ilsemann, and H. Preusch, MAC-RAD, A Reactor Shielding Code, Report EUR-2152.e, European Atomic Energy Community, 1964.

53. L. Hjarne (Ed.), A User's Manual for the NRN Shield Design Method, Report AE-145, AB Atomenergi, June 1964.

54. L. Hjarne and M. Leimdorfer, A Method for Predicting the Penetration and Slowing Down of Neutrons in Reactor Shields, Nucl. Sci. Eng., 24: 165 (1966).

55. C. Ponti, H. Preusch, and H. Schubart, SABINE: A One-Dimensional Bulk Shielding Program, Italian Report EUR-3636.e, European Atomic Energy Community, 1967.

56. R. Nicks, G. Perline, and C. Ponti, SABINE: A One-Dimensional Shielding Programme Which Employs Experimental Removal Cross Sections, in Proceedings of the Conference on the Physics Problems of Reactor Shielding, September 1967, British Report AERE-R-5773, Vol. 1, pp. 5-40, 1968.

57. W. D. Collier and G. C. Curtis, ATTOW, A Two-Dimensional Shielding Program, British Report TRG-1466(R), 1967.

58. A. F. Avery and C. C. Curtis, Neutron Attenuation Studies in Fast Reactor Shielding, in Proceedings of the Conference on the Physics Problems of Reactor Shielding, September 1967, British Report AERE-R-5773, Vol. 2, pp. 515-553, 1968.

59. E. Aalto, The Fine Adjustment of the Neutron Penetration in the NRN Method, Nucl. Sci. Eng., 22: 443 (1965).

\section{EXERCISES}

4.1 Explain why the expression

$$
\Phi(r, \Omega, E) d \Omega d E(\mathbf{n} \cdot \Omega) d S
$$

in Eq. 4.2-1 gives the net (outward minus inward) leakage through the differential surface $d S$.

4.2 Transform the particle-flux-density form of the Boltzmann equation with energy as an independent variable (Eq. 4.2-8) into its equivalent form with the energy-flux density, $I=E \Phi$, as the dependent variable and the wavelength, $\lambda$, as an independent variable. [Hint: $I(\lambda)=I(E)$.]

4.3 Starting with the Boltzmann equation (Eq. 4.2-8), show that for a one-speed one-dimensional slab geometry with azimuthal :ymmetry the equation reduces to

$$
\mu \frac{\partial \Phi(x, \mu)}{\partial x}+\Sigma_{t} \Phi(x, \mu)=S(x, \mu)+\frac{1}{2 \pi} \int_{0}^{2 \pi} \int_{0}^{2 \pi} \int_{1}^{1} \Sigma_{s}\left(\boldsymbol{\Omega}, \boldsymbol{\Omega}^{\prime}\right) \Phi\left(x, \boldsymbol{\Omega}^{\prime}\right) d \mu^{\prime} d \phi^{\prime} d \phi
$$

in which $\mu=\cos \theta$, where $\theta$ is the polar angle of $\Omega$ with respect to the $x$-axis, thus verifying Eq. 4.3-1. 
4.4 Show that for the one-dimensional spherical geometry the Boltzmann equation can be reduced to

$$
\begin{aligned}
\mu \frac{\partial \Phi(r, \mu, E)}{\partial r}+\frac{\left(1-\mu^{2}\right)}{r} \frac{\partial \Phi(r, \mu, E)}{\partial \mu} & +\Sigma_{t} \Phi(r, \mu, E) \\
& +S(r, \mu, E)+\int_{-1}^{1} \int_{0}^{\infty} \Sigma_{s}\left(r, E^{\prime} \rightarrow E, \mu_{0}\right) \Phi\left(r, E^{\prime}, \mu^{\prime}\right) d E^{\prime} d \mu^{\prime}
\end{aligned}
$$

thus proving Eq. 4.4-3.

4.5 Using the form of the Boltzmann equation obtained as a result in problem 4.2, show that for a one-dimensional slab geometry photon transport is described by

$$
\begin{aligned}
\mu \frac{\partial I(x, \lambda, \mu)}{\partial x}+\Sigma_{t}(\lambda) & I(x, \lambda, \mu)=S(\lambda, \mu) \delta(x) \\
& +\int_{0}^{\lambda} \int_{4 \pi} I\left(x, \lambda^{\prime}, \mu^{\prime}\right) \Sigma_{s}\left(\lambda^{\prime}, \Omega^{\prime} \cdot \Omega\right) \frac{\lambda}{\lambda^{\prime}} \delta\left(1-\lambda+\lambda^{\prime}-\Omega^{\prime} \cdot \Omega\right) d \Omega^{\prime} d \lambda^{\prime}
\end{aligned}
$$

in which $\mu=\Omega \cdot \mathrm{i}$ ( $\mathrm{i}$ is the unit vector along the $x$-axis), $\Sigma_{t}(\lambda)$ is the total attenuation coefficient, and $\Sigma_{s}\left(\lambda^{\prime}, \Omega^{\prime} \cdot \Omega\right)$ is the differential macroscopic Compton cross section for scattering from wavelength $\lambda^{\prime}$ through an angle whose cosine is $\Omega^{\prime} \cdot \boldsymbol{\Omega}$. This proves Eq. 4.5-2. 
8

0 


\section{Monte Carlo Methods for Radiation Transport}

Monte Carlo techniques have been so important in shield analysis in the past decade that a separate chapter on this subject is warranted. For a more formal and detailed development of general Monte Carlo techniques, the reader is referred to one of the texts devoted to the subject. ${ }^{1-3}$ We will be concerned primarily with the step-by-step development of radiationtransport applications of the method based on a knowledge of microscopic cross sections and elementary probability theory. A brief discussion on use of the method to solve integral equations is given as an aid to understanding the equivalence of this method with other means of solving the Boltzmann transport equation.

We assume that the reader has some grasp of the elementary concepts of probability, namely, that the probability of one of several possible events occurring will be approximately equal to the ratio of the number of times the desired event occurs to the total number of events observed in an unbiased manner. As the number of observations increases, this ratio should more closely approximate the true probability. Beyond this simple concept, more specific definitions will be developed as they are required.

Much of the information available on the physics of individual nuclear interactions is obtained experimentally by observing the fate of large numbers of particles. In like manner, Monte Carlo may be considered as a means of repeatedly applying interaction probability data to individual particles selected randomly until a sufficient number of particles have been observed to allow conclusions to be drawn concerning the macroscopic multicollision behavior of the total population of particles within a material region. Dependence on such a mathematical experiment for the macroscopic probabilities (shield leakage, particle absorption density, etc.) is in many ways analogous to the laboratory determination of microscopic interaction cross sections. To be sure, the laboratory experiment may be based on the observation of a much larger sample than can be processed economically in 
the mathematical game, but the principles are the same. Research on the problem of increasing the sample size or employing other methods to reduce the uncertainty associated with Monte Carlo data has led to the development of special techniques combining analytical estimates with random particle tracking.

It is possible to show how the various steps involved in a Monte Carlo solution correspond to terms of the Boltzmann transport equation. Although Monte Carlo may be considered a means of solving the Boltzmann equation, it is more properly a modeling of the principles from which the Boltzmann equation was developed; that is, the method may be developed and properly applied to radiation-transport problems without reference to a differential transport equation. The general course followed in this chapter is to develop individually, and in a logical sequence, the steps required to solve a radiation-transport problem by the Monte Carlo method.

Before getting into the detailed operations involved in generating a particle track, we will first discuss the general organization of the tasks. Approaches to accomplishing these tasks then will be developed through the chapter. Since our objective is to explain the logical processes involved in applying the Monte Carlo method, little space is devoted to explaining how these operations are programmed for the computer. Programming is a strong function of the nature of the computer, its auxiliary equipment, and the language used. Further, a large number of general-purpose computer codes using the Monte Carlo method for shielding applications are readily available, and the probability is rather high that developing a new code for a particular application will not be required.

Simply stated, the Monte Carlo approach requires that we construct case histories of the travel of individual particles through the geometry and then analyze these histories to derive relevant data, such as flux density and dose rate. One particle history includes the birth of a particle at its source, its random walk through the transporting medium as it undergoes various scattering interactions, and its death, which terminates the history. A death can occur when the particle becomes absorbed, leaves the geometric region of interest, or loses significance owing to other factors (e.g., low energy).

If we assume for the moment that the source-shield geometry has been mathematically modeled, the major steps involved in generating a particletrack history are shown in Fig. 5.1.

The loop 2 through 4 is continued until the particle parameters fall outside some predetermined limit of values, such as geometrical bounds, minimum energy, or minimum statistical weight (a concept to be developed 
later). This process only generates particle histories. Separate provision must be made for estimating desirable output quantities, such as flux density, current density, or interaction densities. The first three operations in Fig. 5.1 involve the selection of parameters at random from a probability distribution of all possible values of these parameters. Thus a sufficiently large number of

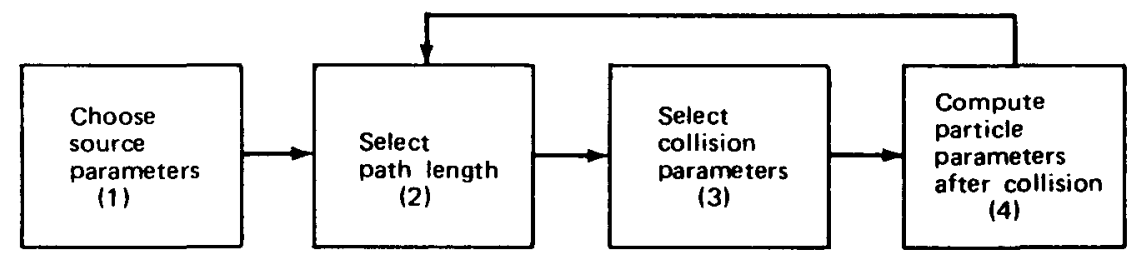

Fig. 5.1--History generator.

selections of a given parameter would be distributed in the same manner as the corresponding probabilities. The steps in making a random selection from such probability distributions are based on the use of numbers randomly distributed between the limits of 0 and 1 .

\subsection{SAMPLING FROM PROBABILITY DISTRIBUTION FUNCTIONS}

As suggested earlier, all physical processes, including the emission of radiations from sources and their subsequent transport through material, are probabilistic; that is, one cannot predict, with certainty, exactly what will occur for every individual particle in the process. Nevertheless, such stochastic, or random, processes can be effectively characterized and predictions can be made by describing the average behavior of many elements or by estimating with a known degree of confidence (but never with certainty) the behavior of one element. Mathematically this characterization is accomplished through the use of various probability functions. Processes dependent on one or many independent variables may be so handled.

An event is a physical occurrence, for example, a coin toss resulting in a head or a photon assuming a specific encrgy $E_{0}$. An event space is the set of all possible (mutually exclusive) events within the process under consideration. Event spaces, sometimes called sample spaces, may be finite and discrete (the coin-toss problem where it contains only two points, heads and tails), continuous (the visible electromagnetic spectrum), and/or infinite in extent. 
Clearly, events such as heads and tails require numerical representation to be handled effectively from a mathematical standpoint. Thus events are mapped onto real numbers by a function called a random variable. For example, a coin-toss random variable might be a function that assigns 0 to tails and 1 to heads. To be precise, one must define the random-variable functions used. For the subject matter of this text, however, the event and the value assigned it by the random variable (e.g., a photon produced at energy $E$ is assigned the value $E$ ) will be quite clear, and we will not explicitly define the mapping function.

Inherent in the Monte Carlo procedure is the concept of the probability density function (PDF) (also called frequency functions and probability distributions). This concept is an extension of differential distribution functions introduced in Sec. 2.2.1. The probability density function, $f(x)$, describes the relative frequency of occurrence of its random variable, $x$. Its domain (all possible values of $x$ ) constitutes the event space.

Let $P\left(x<x_{0}<x+\Delta x\right)$ be the probability that a random variable $x_{0}$ lies within the interval $(x, x+\Delta x)$ of its event space. The PDF $f(x) \dagger$ is related to this probability by the equality

$$
P\left(x<x_{0}<x+\Delta x\right)=f(x) \Delta x
$$

as $\Delta x$ becomes vanishingly small. Thus the PDF is central to the prediction problem, and knowledge of the PDF enables one to obtain the probability of occurrence of a specific event. Equation 5.1-1 shows that the PDF $f(x)$ gives the probability of occurrence per unit interval of the random variable $x$.

Over larger intervals PDF's are used with differentials. Thus

$$
P(a<x<b)=\int_{a}^{b} f(x) d x
$$

Since PDF's describe relative frequencies of occurrences of events within an event space, two properties of PDF's emerge; namely,

$$
0 \leqslant f(x)
$$

and

$$
\int_{-\infty}^{\infty} f(x) d x=1
$$

†As with differential distributions, the concept is easily extended to two or more variables and may be changed to phase-space notation by substituting $P$ for $x$, where $P$ represents $(\mathrm{r}, \Omega, E)$. 
Equation 5.1-4 states that the probability of finding a chosen event somewhere within its event space must be unity, and Eq. 5.1-3 accrues from the fact that relative frequencies (i.e., probability values) must be positive.

The cumulative distribution function (CDF) gives the probability that the random variable $x$ has a value less than or equal to some fixed value. It is given by

$$
P\left(x \leqslant x_{0}\right)=F\left(x_{0}\right)=\int_{-\infty}^{x_{0}} f(x) d x
$$

Two restrictions placed on $F(x)$ that follow from Eqs. 5.1-3 and 5.1-4 are

$$
\lim _{x \rightarrow \infty} F(x)=1
$$

and

$$
\lim _{x \rightarrow-\infty} F(x)=0
$$

The probability $P(a<x<b)$ of $x$ lying within an interval $(a, b)$ is thus given by

$$
P(a<x<b)=\int_{a}^{b} f(x) d x=F(b)-F(a)
$$

Clearly, if the variable $x$ can only take on values within some finite range, then one may substitute the lower bound of that range for $-\infty$ and the upper bound for $+\infty$ in the preceding expressions.

Various distribution functions may be encountered which are not probability density functions. For example, a variable may be limited to the region $2 \leqslant x \leqslant 4$ and be distributed proportional to $x^{2}$ in that region. The function $x^{2}$ violates both of the preceding restrictions on probability values but may, with proper normalization, be converted to a PDF. In this case the $\operatorname{PDF} f(x)$ is given by

$$
f(x)=\frac{x^{2}}{\int_{2}^{4} x^{2} d x}
$$

and

$$
F\left(x_{0}\right)=\frac{\int_{2}^{x_{0}} x^{2} d x}{\int_{2}^{4} x^{2} d x}
$$


Or, stated more generally, where $q(x)$ is any function of $x$ that is positive for all values of $x$,

$$
f(x)=\frac{q(x)}{\int_{-\infty}^{\infty} q(\mathbf{x}) d x}
$$

and

$$
F\left(x_{0}\right)=\frac{\int_{-\infty}^{x_{0}} q(x) d x}{\int_{-\infty}^{\infty} q(x) d x}
$$

Where the event space is discrete, the random variable can only take on selected values, and the PDF $f\left(x_{i}\right)$ is defined by

$$
P\left(x_{i-1}<x_{i}<x_{i+1}\right)=f\left(x_{i}\right) \quad(i=1,2, \ldots)
$$

where

$$
\sum_{i} f\left(x_{i}\right)=1
$$

The CDF becomes

$$
P\left(x_{i}<x_{n}\right)=F\left(x_{n}\right)=\sum_{i=1}^{n} f\left(x_{i}\right)
$$

The mean value of a distribution is given by

$$
\vec{x}=\mu_{x}=\sum_{i=1}^{n} x_{i} f\left(x_{i}\right)
$$

in the discrete case, or by

$$
\bar{x}=\mu_{x}=\int_{-\infty}^{\infty} x f(x) d x
$$

in the continuous case. Since the average of a large sample of values of $x$ tends to this point, $\mu$ is sometimes called the expected value of $x$.

The expected value of $g(x)$ is then

$$
\bar{g}=\sum_{i=1}^{n} g\left(x_{i}\right) f\left(x_{i}\right)
$$


and in the continuous case

$$
\bar{g}=\int_{-\infty}^{\infty} g(x) f(x) d x
$$

Note that

$$
\overline{x^{2}}=\sum_{i=1}^{n} x_{i}^{2} f\left(x_{i}\right)
$$

The variance of a distribution function is a measure of how closely the distribution is grouped about the mean. The variance, denoted $\sigma^{2}$, is defined as

$$
\sigma_{x}^{2}=\sum_{i=1}^{n}\left(x_{i}-\mu\right)^{2} f\left(x_{i}\right)
$$

in the discrete case or as

$$
\sigma_{x}^{2}=\int_{-\infty}^{\infty}(x-\mu)^{2} f(x) d x
$$

in the continuous case. A little manipulation will show that

$$
\sigma_{x}^{2}=\bar{x}^{2}-\mu_{x}^{2}
$$

in either of the preceding expressions. The standard deviation is often used instead of the variance as a measure of the dispersion about the mean. The standard deviation, $\sigma_{x}$, is given, logically enough, by

$$
\sigma_{x}=\left(\sigma_{x}^{2}\right)^{1 / 2}
$$

In analyzing and solving problems involving random processes, one must randomly select events from distributions for which the PDF's are known, exactly or approximately. Thus the problem is first to choose a value of $f(x)$ [or its equivalent, $F(x)$ ] by some random process. Then, having obtained $f(x)$, find $x$, which, of course, defines the desired event. Comprehending this inverse process is often difficult for the beginner, who is accustomed to calculating values of $f(x)$, given $x$. It is common practice to use random numbers equally distributed between 0 and 1 to obtain random samples of a variable that will be distributed in the same manner as $f(x)$. If a random 
number $n(0<n<1)$ is chosen, the value of the associated random variable $x$ may be obtained by solving the inverse of the equation

$$
n=F(x)
$$

for $x$, where $F(x)$ is a cumulative distribution function. We emphasize that the random number $n$ is a value chosen from the range of the function $F(x)$, whereas the desired random variable $x$ is a value within the domain of $F(x)$.

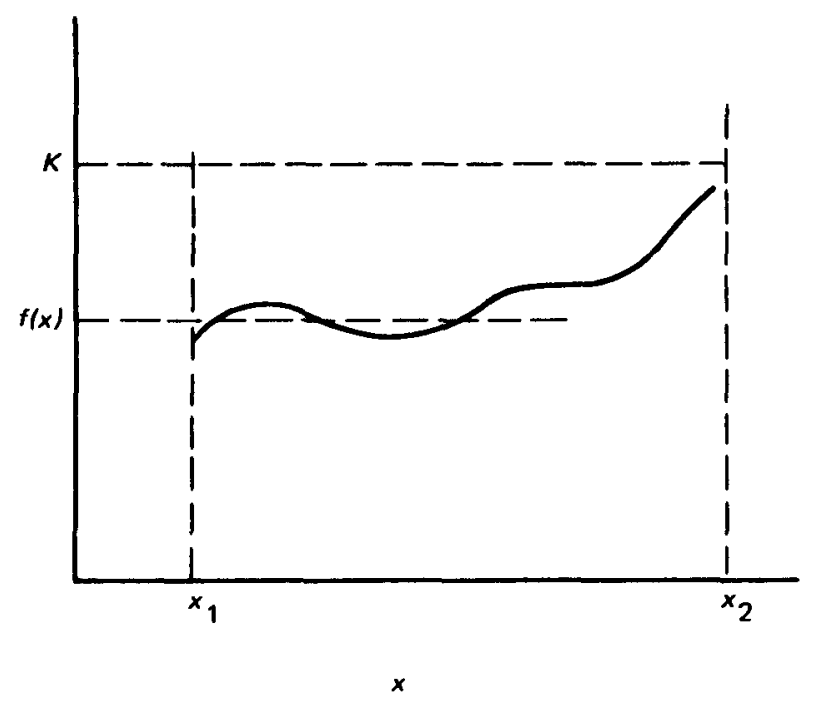

Fig. 5.2-Rejection technique.

It is also possible to select randomly from a continuous probability distribution by a powerful method known as a rejection technique. Consider the function $f(x)$ as plotted in Fig. 5.2. The objective is to generate a sequence of values of $x$ such that $f(x)$ is approximated by the relative frequency of occurrence of values of $x$ within the collection formed by the sequence.

We first choose a value $K$ that exceeds all values of $f(x)$ within the region. Then, using appropriate techniques, we obtain a random number $n$, which is used to select a value $x_{i}\left(x_{1}<x_{i}<x_{2}\right)$ by the relation

$$
x_{i}=x_{1}+n\left(x_{2}-x_{1}\right)
$$


The value of $f\left(x_{i}\right)$ is evaluated at the selected value of $x_{i}$. Taking another random number $n^{\prime}$, we next select a number $N$ from a random distribution between 0 and $K$ by setting

$$
N=n^{\prime} K
$$

If $N>f\left(x_{i}\right)$, the value of $x_{i}$ is rejected. The process is repeated by generating new values of $x_{i}$ and $N$ until $N \leqslant f\left(x_{i}\right)$. When this occurs the value of $x_{i}$ is accepted and used as the random sample. The probability of a particular $x_{i}$ being accepted is given by $f\left(x_{i}\right) / K$ and the probability of rejection is $\mid K-$ $\left.f\left(x_{i}\right)\right] / K$. Thus, after a large number of determinations for various values of $x$, the distribution in $x$ of the values accepted would approximate the function $f(x)$.

The efficiency of this tcchnique for selecting from $f(x)$ is given by the ratio of the area under the curve to the total area, or

$$
E=\frac{\int_{x_{1}}^{x_{2}} f(x) d x}{K\left(x_{2}-x_{1}\right)}
$$

For highest efficiency, the value $K$ should be the smallest possible value that always exceeds $f(x)$ in the region.

Within a computer program, random numbers may be taken from tables called into the machine memory or they may be gencrated by a subroutine as necded. Random-number-gencrator routines are available as standard software with most gencral-purpose computers. Numbers obtained in either way arc more properly termed pseudorandom because they are systematically obtained.

Despite their availability and short length, the codes that gencrate pscudorandom numbers are not trivial. In fact, a precise test to verify the randomness of a generator has yet to be defined. Various criteria can be applied which test for necessary conditions; the test for sufficiency awaits further research. It is sound practice when selecting a random-numbergenerator code to examine the tests to which it has been put. In fact, some generators that had been used for years were found to be not so random after all when sophisticated tests were applied. Appendix I is an introduction to random-number generators, including descriptions of some in general use and a discussion of tests for randomness. 


\subsection{THE EVALUATION OF INTEGRALS}

A theorem of statistics called the law of large numbers provides the basis for evaluating integrals with the Monte Carlo method. To illustrate this theorem, we will consider the integral

$$
\bar{Z}=\int_{-\infty}^{\infty} Z(x) f(x) d x
$$

where $f(x)$ is a probability distribution. The term $\bar{Z}$ is called the expected value of the function $Z(x)$. The law of large numbers states that this integral may be approximated by

$$
\hat{\eta}_{N}=\frac{1}{N} \sum_{1}^{N} Z\left(x_{i}\right)
$$

and that $\hat{Z}_{N}$ will approach $\bar{Z}$ as a limit as $N$ approaches $\infty$. The $x_{1}, x_{2}, \ldots, x_{N}$ are randomly selected from the $\operatorname{PDF} f(x)$ (the rejection technique could be used), and $\hat{Z}_{N}$ is called an estimate of $\bar{Z}$.

Biasing may be defined as any means of distorting the sampling technique to advantage and may be introduced into the evaluation of the preceding integral by means of a biasing function, $h(x)$

$$
\bar{Z}=\int_{\infty}^{\infty} \frac{Z(x) f(x) h(x)}{h(x)} d x
$$

This equation is then evaluated by sampling from a PDF given by the product $[f(x) h(x)]$, and each sample is adjusted by $1 / h(x)$. A reasonable choice of the function $h(x)$ is one that will encourage choices of $x$ most likely to give values of $Z$ lying at or near the expected value; that is, the variance of the distribution $[f(x) h(x)]$ should be less than that of $f(x)$. A goal is that the product $[f(x) h(x)]$ approach a constant such that there is zero variance and

$$
\bar{Z}=\frac{Z(x)}{h(x)} \text { for any choice of } x
$$

The integral Boltzmann transport equation may be expressed in a form similar to the preceding equation for $\bar{Z}$. To evaluate the density $\Phi(\mathbf{P})$ of 
particles emerging from collision in a unit of phase space $d \mathbf{P}$, (e.g., $d \mathbf{P}=d E$ $d \Omega d \mathbf{r}$ ), we can write the Boltzmann equation

$$
\Phi(\mathbf{P})=\int K\left(\mathbf{P}^{\prime} \rightarrow \mathbf{P}\right) \Phi\left(\mathbf{P}^{\prime}\right) d \mathbf{P}^{\prime}+S(\mathbf{P})
$$

The term $K\left(\mathbf{P}^{\prime} \rightarrow \mathbf{P}\right) d \mathbf{P}$ is the transfer kernel giving the probability of a particle emerging from a collision in increment $d \mathbf{P}$ about $\mathbf{P}$ given that one particle emerged from a collision at $\mathbf{P}^{\prime} ; S(\mathbf{P})$ is the density of the source generating particles in $d \mathbf{P}$ directly. In generating random-particle tracks through a medium, we are essentially generating a $\Phi\left(\mathbf{P}^{\prime}\right)$ by straightforward sampling. Performing a statistical estimation of the contribution to $d \mathbf{P}$ from each collision point is a means of evaluating the kernel $K\left(\mathbf{P}^{\prime} \rightarrow \mathbf{P}\right)$. Evaluation of the estimates from each collision point then is equivalent to performing the integral over volume, energy, and angle. The transfer kernel may be decomposed into the form

$$
K\left(\mathbf{P}^{\prime} \rightarrow \mathbf{P}\right)=T\left(\mathbf{r}^{\prime} \rightarrow \mathbf{r} \mid E^{\prime}, \Omega^{\prime}\right) C\left(E^{\prime} \rightarrow E, \Omega^{\prime} \rightarrow \Omega \mid \mathbf{r}\right)
$$

That is, the transfer kernel $K$ is expressed as a product of $T$, a transport kernel, and $C$, a collision kernel. The vertical line in the arguments of the two functions indicates quantities that are held constant; in $T, \mathbf{r}^{\prime} \rightarrow \mathbf{r}$ for a given $E^{\prime}, \Omega^{\prime}$; in $C$, both $E^{\prime} \rightarrow E$ and $\Omega^{\prime} \rightarrow \Omega$ for a given $\mathrm{r}$. The function $T$ would contain a delta function that vanishes everywhere except where $r$ lies along the ray $\Omega^{\prime}$; otherwise it is essentially the probability of interaction per unit length along the ray.

Functions that are introduced into this equation to provide biasing of parameters are often called importance functions because they provide sampling in those areas most important to the answer. For example, an importance function depending on position alone may be introduced into the function $\mathrm{T}$ by defining

$$
T^{*}=T\left(\mathbf{r}^{\prime} \rightarrow \mathbf{r} \mid E^{\prime}, \Omega^{\prime}\right) \frac{I(\mathbf{r})}{I\left(\mathbf{r}^{\prime}\right)}
$$

The $I(\mathbf{r})$ function often assumes an exponential form in penetration problems. This function can then be combined in whole or in part with the exponential in the transport kernel. A Monte Carlo application of position biasing is discussed in the section on selection of path lengths. 
Other importance functions of energy, angle, or position may be introduced into the equation in a comparable manner. Goertzel and Kalos ${ }^{4}$ developed the Monte Carlo application to radiation transport in phase-space notation.

A more systematic and generally successful approach to importance sampling is to use the value function, a solution to the equation adjoint to the Boltzmann equation. The value function has been shown by Coveyou, Cain, and Yost $^{5}$ to be a very good, sometimes optimum, importance function for biasing the Monte Carlo procedure. Cain ${ }^{6}$ has shown that the value function can be readily obtained by $S_{n}$ methods and applied to the Monte Carlo problem.

\subsection{SOURCE PARAMETERS}

With this background in selecting from distributions, we are now ready to consider the first block in the history-generator routine: choose source parameters. The source parameters will be assumed to include the energy, spatial point of origin, and direction of motion of the source particle, as well as biasing parameters.

These parameters may be independent, or they may be interrelated in various ways. For example, where the energy distribution is dependent on the direction of motion, the order of selection would call first for selection of the initial angle and then for selection from the energy distribution applicable to that angle. When biasing parameters are used, they are tied to onc or more of the other parameters.

\subsubsection{Selection from an Energy Distribution}

The most convenient form for expressing an energy distribution depends on the nature of the distribution. Some of the forms of distribution that may be available for input to the problem are discussed in Chap. 2. Where possible, a cumulative energy distribution should be input at frequent energy points such that interpolation may be used with reasonable accuracy. Most distributions can be input in this form without an undue amount of data handling. The source-energy selection process proceeds as shown in Fig. 5.3. For some types of problems, energy groups may be defined by using boundary values: $E_{1}, \ldots, E_{n+1}$ for $n$ groups. The particles may then be classified both by their energies and by the $n$ groups to which they belong. This procedure adds one parameter which must be specified at the source 
but which does not necessarily alter the procedure for selecting the source energy.

\subsubsection{Selection of Spatial Point of the Source Particle}

The problem of locating and tracking particles from point to point in material regions is primarily one of geometry. Physics becomes involved only

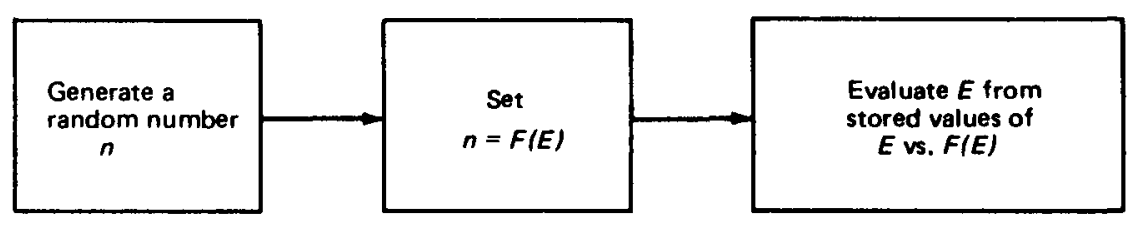

Fig. 5.3-Selection of initial energy.

in the determination of track lengths or in the fate of the particle at the interaction point.

The means of expressing geometric parameters depends on the choice of the coordinate system; for example, infinite-medium calculations lend themselves to spherical geometry, but many reactor shielding problems are best handled in cylindrical or rectangular geometry. Some examples of the selection of source spatial position are discussed in the following paragraphs.

For sources uniformly distributed on a disk, the probability density function describing the distribution of source points as a function of radius is $2 \pi r / \pi R_{1}^{2}$, where $R_{1}$ is the radius of the disk, and the cumulative probability distribution is

$$
P(r)=\int_{0}^{r} f\left(r^{\prime}\right) d r^{\prime}=\frac{r^{2}}{R_{1}^{2}}
$$

Here a random number $n$ used to select a value $P(r)$ can be used to select a random value of $r$ directly. Since

$$
n=P(r)=\frac{r^{2}}{R_{1}^{2}}
$$

it follows that

$$
r=R_{1} n^{\text {h }}
$$


At a given $r$ the source positions are equally distributed in azimuthal angle $\phi$; thus

$$
n=P(\phi)=\frac{\phi}{2 \pi}
$$

and

$$
\phi=2 \pi n
$$

If $\phi$ is to be measured only to $\pm \pi$, then

$$
\phi=\pi(2 n-1)
$$

and

$$
n=\frac{\phi+\pi}{2 \pi}
$$

This may be extended to the case of a uniform cylindrical volume distribution by next selecting a $Z$ coordinate equally distributed between the limits on $\%$.

For a nonuniform spherical-shell distribution, consider a spherical shell bounded by radius limits $r_{1}$ and $r_{2}$ in which the volume density of source particles is given by

$$
\rho=k r
$$

The distribution function in $r$ is given by

$$
f(r)=\frac{4 \pi r^{2}(k r)}{\pi k\left(r_{2}^{4}-r_{1}^{4}\right)}
$$

The cumulative distribution is given by

$$
P(r)=\frac{\int_{r_{1}}^{r} f\left(r^{\prime}\right) d r^{\prime}}{\int_{r_{1}}^{r_{2}} f\left(r^{\prime}\right) d r^{\prime}}=\frac{r^{4}-r_{1}^{4}}{r_{2}^{4}-r_{1}^{4}}
$$

And the randomly selected radius is given by

$$
r=\left[r_{1}^{4}+n\left(r_{2}^{4}-r_{1}^{4}\right]^{4 / 4}\right.
$$


The corresponding $\theta$ and $\phi$ coordinates would be selected for uniform distribution over the shell at radius $r$ (Sec. 5.3.3).

A varicty of other distributions may be expressed analytically so that coordinates may be selected from a continuous distribution. In practical application it may not be possible to cxpress the distribution analytically. In such cases the distribution may be numerically integrated to provide a

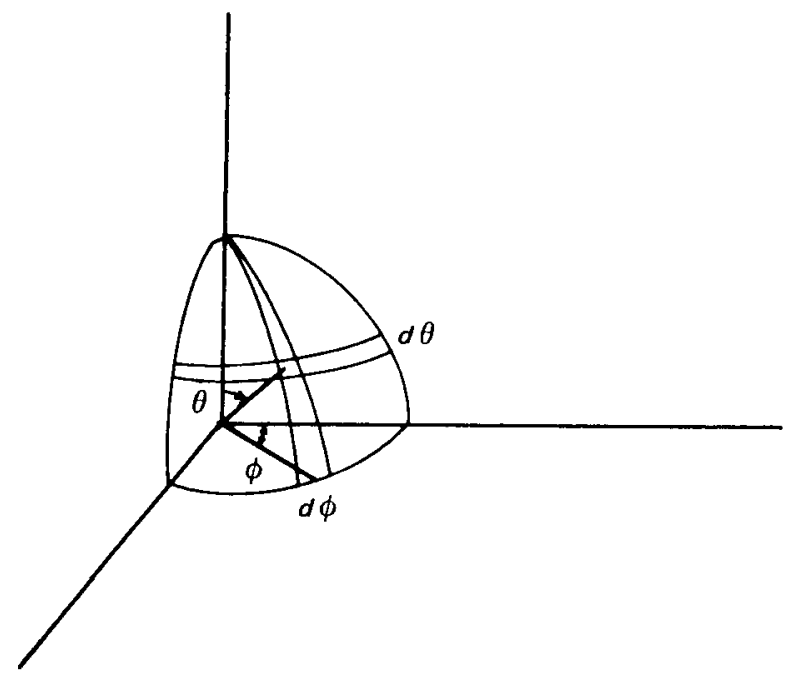

Fig. 5.4-Integral for isotropic distribution.

cumulative distribution in each dimension. Where the dimensional probabilities cannot be separated, i.e., where $P(r, \theta, \phi)$ cannot be expressed in terms of $P(r) P(\theta) P(\phi)$, it may be necessary to specify $P(\theta)$ and $P(\phi)$ or $P(\theta, \phi)$ for each interval in $r$. Two approximations are then possible in making a random selection: either a value of $r$ is classificd as being within a given $\Delta r$ increment and the $P(\theta)$ and $P(\phi)$ values for that increment are selected or the $P(\theta)$ and $P(\phi)$ values are interpolated between the two adjacent values of $r$ for which they are input.

\subsubsection{Selection of Initial Direction of Source Particle}

We turn next to the problem of assigning a direction to a source particle. Consider first sources that are emitting isotropically. Picking a uniformly distributed direction is tantamount to selecting unit vectors terminating uniformly on the surface of a unit sphere. The cumulative probability function is therefore given by the integral over the spherical surface area shown schematically in Fig. 5.4. 
The distribution in $\theta$ is given by

$$
n=P(\theta)=\frac{\int_{0}^{\theta} 2 \pi \sin \theta^{\prime} d \theta^{\prime}}{\int_{0}^{\pi} 2 \pi \sin \theta^{\prime} d \theta^{\prime}}=\frac{1}{2}(1-\cos \theta)
$$

and

$$
\theta=\cos ^{-1}(1-2 n)
$$

The angle $\phi$ will be uniformly distributed between 0 and $\pm \pi$, or

$$
\phi=\pi(2 n-1)
$$

Note that the direction cosines are functions of these two angles and can be computed once the angles are known.

For a surface source emitting isotropically, the direction would be selected randomly from a $2 \pi$ half-space, or

$$
n=\frac{\int_{0}^{\theta} 2 \pi \sin \theta^{\prime} d \theta^{\prime}}{\int_{0}^{\pi / 2} 2 \pi \sin \theta^{\prime} d \theta^{\prime}}=1-\cos \theta
$$

and

$$
\theta=\cos ^{-1}(1-n)
$$

or, since $(1-n)$ is distributed in the same manner as $n$,

$$
\theta=\cos ^{-1} n
$$

In like manner, for a surface source emitting a cosine distribution, the PDF is given by $\sin \theta^{\prime} \cos \theta^{\prime}$ and the angles $\theta$ would be selected from

$$
\cos ^{2} \theta=n
$$

or

$$
\theta=\cos ^{-1} \sqrt{n}
$$

Any arbitrary distribution in direction may be put in terms of tabulated values of $P(\theta)$ and $P(\phi)$. When various source distributions are to be 
considered incident on the same shield configuration, it may be advantageous to run individual unit source problems. The results obtained from the unit source problems can then be combined with various weightings to stimulate a variety of source distributions.

\subsubsection{Source-Biasing Parameters}

In certain calculations it may be desirable to prejudice the selection of one or more source parameters to favor those most likely to contribute to

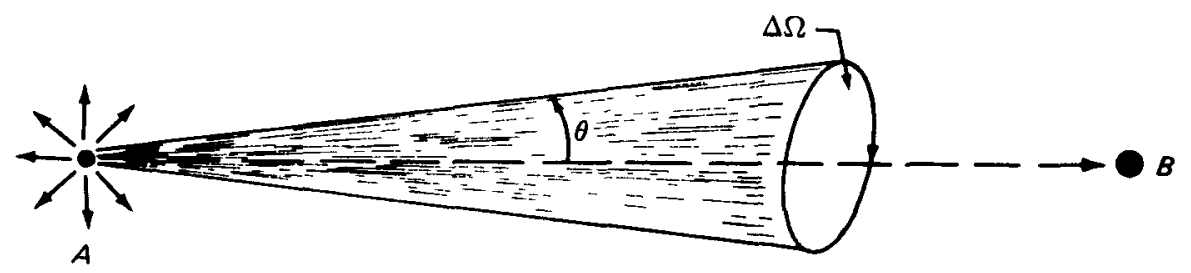

Fig. 5.5-Source direction biasing.

the quantity of interest, such as shicld leakage or detector response. This can be done by selecting a larger number of the important source particles and assigning to each particle a number, called its weight, to adjust for the bias that was introduced. Figure 5.5 illustrates this concept.

Consider an isotropic source located at $A$ and a detector at $B$. Clearly, source particles leaving $A$ in the direction of $B$ will contribute more to the flux density at $B$ than those leaving in opposite directions. (We assume, of course, that scattering is permitted in the transporting medium.) Suppose, in following 10,000 source particles, we estimate that 2000 would lie in $\Delta \Omega$ in the unbiased isotropic case. However, because of their greater importance, we desire to force 6000 of the 10,000 to lie in $\Delta \Omega$. The weight assigned to them is $1 / 3$ to remove this bias. Further, the weight of the remaining 4000 outside of $\Delta \Omega$ must be increased to 2 since each history in the biased case represents two particles in the unbiased case. Table 5.1 summarizes these facts. As each particle history is generated, its contribution, $\phi_{i}$, to the total flux density at $B$ can be determined. This total flux density is given by

$$
\Phi=K \sum_{i=1}^{10,000} w_{i} \Phi_{i}
$$

where $W_{i}$ is the weight ( $1 / 3$ or 2 ) of the particle contributing $\Phi_{i}$ and $K$ is a source-normalization constant. (For a unit source strength, $K=1 / 10,000$.) 
When biasing is used, the summation of particle weights equals that of the unbiased case where the weights are identically 1 . However, because of the altered statistical sample, a more representative answer is usually obtained for a fixed total number of source particles.

Table 5.1-WEIGHTS FOR BIASING ILLUSTRATION

\begin{tabular}{|c|c|c|c|c|}
\hline & \multicolumn{2}{|c|}{ Unbiased case } & \multicolumn{2}{|c|}{ Biased case } \\
\hline & $\begin{array}{l}\text { No. of } \\
\text { histories }\end{array}$ & $\begin{array}{c}\text { Particle } \\
\text { weight }\end{array}$ & $\begin{array}{c}\text { No. of } \\
\text { histories }\end{array}$ & $\begin{array}{c}\text { Particle } \\
\text { weight }\end{array}$ \\
\hline Histories in $\Delta \Omega$ & 2,000 & 1 & 6,000 & $1 / 3$ \\
\hline Histories outside $\Delta \Omega$ & 8,000 & 1 & 4,000 & 2 \\
\hline Total No. of histories & 10,000 & & 10,000 & \\
\hline
\end{tabular}

To amplify the example, suppose the $180^{\circ}$ range of $\theta$ is divided into 18 intervals of $10^{\circ}$ each and the cumulative probability emission of a source particle in the $j$ th increment is given by $\Delta P_{j}(\theta), j=1,2, \ldots, 18$ :

$$
\sum_{j=1}^{18} \Delta P_{j}(\theta)=1=P_{18}(\theta)
$$

This distribution may be adjusted in any arbitrary manner by assigning probability values $\Delta P_{j}^{\prime}(\theta)$ and weights $W_{j}$ to each angular group such that

$$
w_{j} \Delta P_{j}^{\prime}(\theta)=\Delta P_{j}(\theta)
$$

provided one does not violate the rule of probability that $\Delta P_{j}^{\prime}(\theta)<1$ and that

$$
\sum_{j=1}^{18} \Delta P_{j}^{\prime}(\theta)=1
$$

As a second example, consider the case of a plane isotropic source where it is desired to bias the histories toward directions near the normal to the plane. The unbiased cumulative angular distribution at $\theta=10^{\circ}$ (measured from the normal) as computed by Eq. 5.3-14 is 0.015 . This value is to be increased by a factor of 5 such that

$$
P_{1}^{\prime}(\theta)=0.075
$$


Particles generated within this interval will have a weight 0.2 , and particles generated in all other categories will have probability increments

$$
P_{j}^{\prime}(\theta)=P_{j}(\theta) \frac{1-P_{1}^{\prime}\left(10^{\circ}\right)}{1-P_{1}\left(10^{\circ}\right)} \cong 0.939 P_{j}
$$

and will have weights given by

$$
W_{i}=\frac{P_{j}}{P_{j}^{\prime}}=\frac{1-P_{1}}{1-P_{1}^{\prime}} \cong 1.065
$$

Thus the total particle weight generated by selecting $N$ particles will be

$$
0.2(0.075) N+1.065(1-0.075) N=N
$$

Situations where biasing might be used include:

1. Selecting more source points near the periphery of a reactor.

2. Selecting more particles with an initial direction toward the shield or detector.

3. Selecting more particles with a high energy or with an energy corresponding to a low total cross section.

Probability and weight adjustments can be developed for biasing the starting position or energy for a particular situation. It is important to remember that an adjustment in one category (e.g., angle interval or energy group) affects not only that group but the entire distribution.

In summary, if the number of particles within a particular category is increased by a factor $k$, then their weight is decreased by a factor $1 / k$. The weight of particles in other categories is increased such that the summation of particle weights is equivalent to the number of particles generated.

\subsection{PATH LENGTH}

Thus far the geometry has been defined and a set of source-particle parameters has been selected. Next we determine the particle path length from the source to the point of interaction. The path length along with the parameters of initial direction defines the point at which an interaction occurs. 
Assume initially a single infinite region through which particles are being tracked. If the total macroscopic cross section of the region is $\Sigma_{t}(E)$, then the number of particles of energy E penetrating to depth $L$ out of $N_{0}$ trials is

$$
N_{0} \mathrm{e}^{-\Sigma_{t}(E) L}
$$

and the probability of penetration to depth $L$ is

$$
P(L)=\frac{\text { number penetrating to } L}{\text { number of trials }}=\mathrm{e}^{-\Sigma_{t}(E) L}
$$

The probability of particle interaction at some path length $\leqslant L$ is thus given by

$$
\left.P^{\prime}(L)=\mid 1-\mathrm{e}^{-\Sigma_{t}(E) L}\right\}
$$

If path lengths $L$ to first collision are to be sampled at random, the random number, $n$, is set equal to $P^{\prime}(L)$, or

$$
n=1-\mathrm{e}^{-\Sigma_{t}(E) L}
$$

from which

$$
L=\frac{1}{\Sigma_{t}(E)} \ln (1-n)=\frac{1}{\Sigma_{t}(E)} \ln n
$$

since $1-n$ is distributed in the same manner as $n$.

Suppose now that a single region is bounded such that the boundary is encountered at path length $L=A$. If the randomly selected path length excecds the value $A$, then the particle will be assumed to have escaped the system. Thus values of $L$ may be selected as shown above for an infinite medium and tested to determine whether they exceed $A$. If they do the particle has escaped and the history is terminated; if they do not, a collision is assumed to have occurred at the selected point, and collision parameters are calculated. The value of $A$ will be a function of the initial direction of the particle and the geometry of the region boundary, and it should be calculated after the source direction has been selected so that it will be available for the test against $L$.

Consider next a multiregion geometry with each region homogeneous. A ray extending in the direction of particle motion encounters region 
thicknesses in the various regions given by $A_{1}, A_{2}, \ldots, A_{x}$ with associated total cross sections given by $\Sigma_{t 1}, \Sigma_{t 2}, \ldots, \Sigma_{t x}$.

The effect of successive probabilities is such that the escape probability from the entire system is equal to the product of the individual

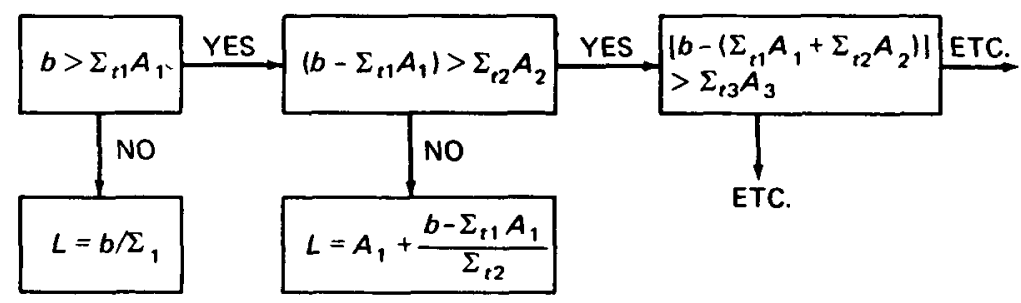

Fig. 5.6-Selection of path length.

zone-penetration probabilities. Thus the fate of the particle along the track could be determined in the following way:

Select a path length at random assuming an infinite medium with cross section $\Sigma_{t_{1}}$.

If $L_{1}$ exceeds $A_{1}$, move the particle to the point where its track intersects the boundary between region 1 and region 2 .

Select a path length at random, in the original direction, assuming an infinite medium with cross section $\Sigma_{t 2}$.

If $L_{2}$ excecds $A_{2}$, translatc the particle to the region 2 - region 3 boundary. This procedure is repeated until one of the $L_{i}<A_{i}$ or until the particle escapes.

The total escape probability will be given by

$$
P_{E}=\exp -\left(\Sigma_{t 1} A_{1}+\Sigma_{t 2} A_{2} \ldots \Sigma_{t x} A_{x}\right)
$$

Instcad of selccting path lengths in each of the materials successively, as described above, it would perhaps be simpler to decide the fate of the particle by selecting only one random number. For this purpose we will define $b=\sum_{i} \Sigma_{t i} A_{i}$, where $A_{i}$ is the thickness of material $i$ lying along the particle path between the source point and the first collision. The value of $b$ is selected at random from

$$
b=\ln n
$$

The process of finding the path length corresponding to the selected value of $b$ is symbolized in Fig. 5.6. This search is continued until $L$ is defined. Flow 
charts for calculating boundary-intercept distances for several types of geometry are given by Cashwell and Everett. ${ }^{3}$

Biasing may be introduced into the path-length selection in a manner similar to that in source-particle selection; i.e., the true distribution is distorted in some arbitrary fashion, and the particle weight is adjusted to account for this distortion. The mechanics of track-length biasing depend on the motive for biasing. Where it is desired to improve the statistics on collisions internal to a region, particles may be prevented from escaping by picking collision points from a cumulative distribution which becomes 1.0 at the boundary; the weight of the colliding particle is then reduced by subtracting the fraction that would ordinarily escape the region. Where it is desired to improve the statistical data on particles penetrating a thick shield, particle track lengths may be stretched in a direction favoring penetration and reduced to the opposite direction with a commensurate adjustment in particle weight. In some cases the approximation is made to replace transport in an adjacent region by a surface effect or albedo, and one forces collisions to occur only at the boundary.

To illustrate the first type of biasing in its simplest form, we return to the case of a single limited region. If the initial weight of a source particle is $W_{0}$ and the path length to escape is $A_{1}$, then a particle of weight $\mathrm{W}_{e}=W_{0}$ $\mathrm{e}^{-A_{1} \Sigma_{t}}$ is allowed to escape, and a particle of weight

$$
W=W_{0}\left(1-\mathrm{e}^{-\Sigma_{t} A_{1}}\right)
$$

is forced to have a collision prior to escape. The cumulative probability of collision $P(L)$ is given by

$$
P(L)=n P\left(A_{1}\right)=n\left(1-e^{-\Sigma_{t} A_{1}}\right)
$$

where $P(L)$ is given by $P(L)=1-\mathrm{e}^{-\Sigma_{t} L}$. Solving for $L$,

$$
L=-\frac{1}{\Sigma_{t}} \ln \left[1-n\left(1-\mathrm{e}^{-\Sigma_{t} A_{1}}\right)\right]
$$

In a multiregion problem, this procedure could obviously be repeated for paths beyond the first, forcing the particle to remain within the region of interest until the history is terminated by minimum-weight or minimumenergy criteria. 
Biasing of track length to affect deeper penetration can be done by sampling from a linear rather than an exponential distribution with track length. For example, for a thick-slab shield with the incident radiation normal to the shield, the first-collision depth could be sampled from $L=n A_{1}$, where $L$ is the depth to first collision, $n$ is a random number, and $A_{1}$ is the total shield thickness. For the linear distribution the probability of collision between some arbitrary path length $L_{1}$ and $L_{1}+d L$ is given by $d L / A_{1}$, whereas the probability in the true distribution is $\Sigma_{t} e^{-\Sigma_{t} L_{1}} d L$. A weighting function must be assigned to the particles such that the product of the number colliding times their weight is equivalent in the two systems, or

$$
\frac{d L}{A_{1}} w=\Sigma_{t} \mathrm{e}^{-\Sigma_{t} L_{1}} d L w_{0}
$$

From this equality the weighting function

$$
\frac{I V}{W_{0}}=A_{1} \Sigma_{t} \mathrm{e}^{-\Sigma_{t} L_{1}}
$$

is given, where $W$ is the weight assigned to particles at depth $L_{1}$ and $W_{0}$ is the initial particle weight in the unbiased system. Although the particle weight is less, a larger fraction of source particles penetrates to great depths, which increases the accuracy of the averaging there.

Similarly, any other arbitrary distribution of collision densities may be used with the appropriate weighting schemes. One of these, the exponential transform, is designed to maintain approximately the same population of particles at any point within the shield. Since the particle population and flux density decrease approximately exponentially with travel through the shield, these quantities are weighted by a function that increases exponentially with shield penetration. In this scheme a solution is sought for the quantity

$$
\Phi(\mathrm{r}, \omega, E)=N(\mathrm{r}, \omega, E) \mathrm{e}^{g(\mathrm{r}, \omega, E)}
$$

where $N=$ unbiased flux density

$r=$ spatial position

$\boldsymbol{\omega}=$ direction of particle motion

$E=$ particle energy 
In a source-free region this amounts to biasing of track lengths only, but in a distributed source the same importance function is applied both to source starting positions and to track length.

We will consider only the implications to initial track-length selection and particle weight in this section.

For an exponential adjustment we may define a pseudo cross $\operatorname{section} \Sigma_{t}^{*}$ given by

$$
\Sigma_{t}^{*}=\Sigma_{t}(E)-g(\mathbf{r}, \omega, E)
$$

and select from a distribution of first collisions given by

$$
f(L)=\Sigma_{t}^{*} \mathrm{e}^{-\Sigma_{t}^{*} L}
$$

The weight applicable to a particle suffering a first collision at depth $L$ can be obtained by equating the products of first-collision density times the weight in the biased and unbiased systems

$$
w(L) \Sigma_{t}^{*} \mathrm{e}^{-\Sigma_{t}^{*} L} d L=W_{0} \Sigma_{t} \mathrm{e}^{-\Sigma_{t} L} d L
$$

from which

$$
\frac{W(L)}{W_{0}}=\frac{\Sigma_{t}}{\Sigma_{t}-g(\mathbf{r}, \omega, E)} e^{-g(\mathrm{r}, \omega, E) L}
$$

Thus, for positive values of $g$, the following observations may be made concerning the collisions occurring at a large value of $L$ :

1. A larger number of particles are available for interaction.

2. The number of interactions occurring per unit flux density is smaller.

3. The weight of interacting particles is smaller unless $\Sigma_{t}^{*} \ll 1$.

One form of the transform, first proposed by Kahn, ${ }^{7}$ is given by

$$
g(\mathbf{r}, \omega, E)=\epsilon \cdot \omega C
$$

where $\epsilon$ is a unit vector in the direction for which penetration is to be studied, $\boldsymbol{\omega}$ is the direction of particle motion, and $C$ is a constant governing the magnitude of the transforming factor. In this form the magnitude of the transform is proportional to the cosine of the angle between $\epsilon$ and $\omega$ [i.e., 
$g(\mathbf{r}, \boldsymbol{\omega}, E)=C$ when the particle motion is in the direction $\boldsymbol{\epsilon}$ and $g(\mathrm{r}, \omega, E)=-C$ in the opposite direction $]$.

Eriksson ${ }^{8}$ has proposed a form

$$
g(\mathrm{r}, \omega, E)= \pm C\left[\left(x-x_{0}\right)^{2}+\left(y-y_{0}\right)^{2}+\left(z-z_{0}\right)^{2}\right]^{1 / 2}
$$

which would give spherically inward or outward biasing (depending on the selection of sign) about the point $\left(x_{0}, y_{0}, z_{0}\right)$. Perkins and Burrell ${ }^{9}$ have proposed the form

$$
\Sigma_{t}^{*}=\Sigma_{t}[1-M(\omega \cdot \epsilon)]
$$

and have made efficiency comparisons between this and the form

$$
\Sigma_{t}^{*}=\Sigma_{t}-C(\omega \cdot \epsilon)
$$

No clear preference was indicated for either of these two methods from their study.

One of the most persistent difficulties in applying the exponential transform is the possibility that the effective cross section $\Sigma *$ may become negative. Some users ${ }^{8-10}$ have prevented this by restricting the choice of $g$ so that $\Sigma_{t}$ is positive for the minimum value of $\Sigma_{t}$ encountered in the problem. However, such a restriction in the choice of $g$ may result in a severe limitation on the efficiency of application of the transform.

Leimdorfer ${ }^{11}$ proposes two alternatives. The first is to set

$$
\Sigma^{*}=\Sigma^{\prime}>0
$$

whenever $\Sigma^{*}$ becomes less than zero, where the value $\Sigma^{\prime}$ is arbitrarily chosen by the user. The second method is simply to accept the negative value and select from the resulting distribution. This method is generally restricted to problems that have finite external boundaries at distance $T_{1}$ in the direction of transport. Negative values are then allowed only for penetration $\leqslant T_{1}$; beyond the boundary they are replaced by an arbitrary positive value as above. Thus, along a particular track crossing the boundary, two weighting functions might be applied, one for each of the segments lying on either side of the boundary. 
Extending these concepts to a track along which $\Sigma^{*}$ varies with the track length and negative valucs are permissible, the track-length distribution is given by

$$
I^{\prime}(L)=\left|\Sigma_{t}^{*}(L)\right| \exp \left[-\int_{0}^{L} \Sigma_{t}^{*}(S) d S\right]
$$

where $\Sigma_{*}^{*}(S)$ is the total cross section as a function of track length, or, if collision is to be forced between some limits $0<L<T$, then

$$
I(L)=\frac{\left|\Sigma_{t}^{*}(L)\right| \exp \left[-\int_{0}^{L} \Sigma_{t}^{*}(S) d S\right]}{\int_{0}^{T}\left\{\left|\Sigma_{t}^{*}(L)\right| \exp \left[-\int_{0}^{L} \Sigma_{t}(S) d S\right]\right\} d L}
$$

As in previous cases, the weighting function is given by

$$
W=\frac{F_{0}(L) W_{0}}{F(L)}
$$

where the subscript zero refers to the unbiased values.

Clark $^{12}$ has prepared an excellent revicw of the exponential transform in which he discusses the efficiency of the method as a function of the form and magnitude of $g(\mathbf{r}, \omega, I:)$. For high-energy ( $7 \mathrm{MeV})$ gamma-ray sources uniformly distributed through a $100-\mathrm{cm}$ slab of ordinary concrete and for the transform given by

$$
g=\omega \cdot \epsilon C
$$

he found that the estimated relative crror $\dagger$ reached a minimum near $C / \Sigma=1$. Where the sources were distributed with slab thickness, $Z$, in accord with the function $e^{-k Z}$ and Leimdorfer's first scheme for treating negative values of $\Sigma$ was used, the minimum relative error was obtained at values of $C / \Sigma$ lying on either side of 1.0 (e.g., 0.6 and 1.6) over a wide range of values of the parameter $k(0.5 \leqslant k \leqslant \infty)$. As slab thickness is increased, the minimum error again occurs near $C / \Sigma=1.0$, although this minimum is a larger value owing to the increased penetration. When negative cross sections were dealt with and Leimdorfer's second scheme was used, increased efficiency was noted, and the minimum relative error in all cases was $C / \Sigma=1$. 
With various particle-weighting techniques, it occasionally happens that a particle arrives deep within the shicld with a weight far in excess of the average weight of particles at that depth. The presence of these high-weight particles adversely affects the convergence of the value of flux density per unit source particle for the region. This can be prevented by use of the technique of splitting, which decreases the particle weight and increases the number of particles by the same factor. Splitting may be instituted at the point of collision or where previously defined boundarics are crossed. A parallel problem is that of particles scattering back into a region where their weight is so far below the average weight that further tracking would have little effect on the average flux density. This is adjusted by a game of Russian roulette. Whether or not the particle survives is determined by the ratio of particle weight to some arbitrary weight typical of the region. As discussed in the following text, a random number is used to determine the future of such particles.

One may define certain minimum and maximum permissible particleweight values for a given region. When a particle appears with $w^{\prime}<w_{\min }$, a random number is obtained and compared with $W / w_{\text {min }}$. If $n<w / w_{\text {min }}$, the particle is saved and its weight is increased by the ratio $W_{\mathrm{min}} / W$ so that it has weight $\mathfrak{W}_{\min }$. If $n>W / W_{\min }$, the particle perishes; i.e., its history is terminated. Any arbitrary value $\bar{W}$ between $W_{\min }$ and $W_{\max }$ (e.g., an average of the two limits) could be set as the weight of particles surviving Russian roulette provided the corresponding ratio $(\mathcal{W} / \bar{W})$ is permitted to survive. When $W>W_{\min }$, a further test is made to determine whether $W>W_{\max }$. If it is, the particle is split into a number of particles given by the integer value of the ratio $W / w_{\max }$, denoted as $\left.\mid w / w_{\max }\right\rfloor$. Each particle resulting from the split is assigned a lower weight, $W_{0}$, given by

$$
W_{0}=\frac{W}{\left|I / W_{\max }\right|}
$$

and then each is tracked separately to its death.

The boundaries at which splitting and Russian roulette occur may be arbitrarily defined without regard to region boundaries. The values of $W_{\max }$ and $W_{\mathrm{min}}$ assigned to regions should be based on the expected behavior of the flux density. Where strong biasing is used in a particular direction, the flow of particles in the opposite direction may be adequately controlled by the biased track lengths so that Russian roulette is not necessary. Splitting may still be required, however, to cover cases where the weight-adjustment 
quantity $\left(\Sigma_{t} / \Sigma_{t}^{*}\right) \mathrm{e}^{-g L}$ becomes very large owing to approximately equal values of $\Sigma_{t}$ and $g$.

An alternative to the splitting and Russian roulette techniques is a technique that also performs the biasing function. In this method boundaries are defined at which particles crossing in one direction are automatically split into an arbitrary multiple, $M$, and those passing in the opposite direction suffer a fractional mortality equivalent to $1-(1 / M)$.

For example, such boundaries could be defined at surfaces where the flux density would be expected to decrease by a factor of 2 , and the particles crossing the boundary toward the reduced-flux-density region would be doubled. In this manner the particle population would remain approximately at the same level through the shield, and the average particle weight would reflect the degree of attenuation. The efficiency of this method would depend in part on how well the flux-density behavior is predicted at the outset.

\subsection{COLLISION PARAMETERS}

When it has been determined on the basis of the total material cross section that a collision has occurred, it is then necessary to determine which of the possible nuclear species was involved and which of the possible interactions of that species took place. For this determination both the total and the individual interaction cross sections must be available to the computer for each nuclide over the energy range of interest.

In a material containing several elements of atomic density $\rho_{i}$ (nuclei/ $\left.\mathrm{cm}^{3}\right)$, and total cross section $\sigma_{t i}\left(\mathrm{~cm}^{2} /\right.$ nucleus), the total material macroscopic cross section is given by

$$
\Sigma_{t}=\rho_{1} \sigma_{t 1}+\rho_{2} \sigma_{t 2}+\ldots+\rho_{n} \sigma_{t n}
$$

and the probability of a particular species' being involved is given by the fraction of the total cross section represented by that species $\rho_{i} \sigma_{t i} / \Sigma_{t}$. The nucleus involved in a collision can be obtained by selecting a random number $r$ and comparing it to a cumulative distribution,

$$
P(k)=\frac{\sum_{i=1}^{k} \rho_{i} \sigma_{t i}}{\Sigma_{t}}=r
$$

and solving for $k$, where $k$ can be any integer between 1 and $n$, inclusive. 
In a like manner, the type of interaction can be determined by comparing another random number to

$$
P(l)=\frac{\sum_{j=1}^{l} \sigma_{i j}}{\sigma_{t i}}=r
$$

where the $\sigma_{i j}$ are the various components of the different interactions making up the total cross section of element $i$.

For neutrons the $\sigma_{i j}$ would ordinarily be the cross sections for elastic scatter, absorption, fission, $\left(n, n^{\prime}\right),(n, 2 n)$, and $(n, 3 n)$. Other identifiable reaction types can ordinarily be classed into one of these categories; e.g., $(n, p),(n, \alpha),(n, d)$, etc., are absorptions, and $\left(n, n^{\prime}, \alpha\right)$ could be classed as $\left(n, n^{\prime}\right)$ since the resulting charged particles are of no interest except as they may affect the energy of the neutron given off.

Secondary neutrons and gamma rays are associated with most of these interactions. Ordinarily the secondary neutrons would be included by tracking them in turn after the history of the incident neutron is terminated. The secondary gamma-ray source data would be stored for later processing by a gamma-ray transport program.

For gamma rays only three cross-section components, photoelectric effect, compton scattering, and pair production, would be involved. Two of these, photoelectric effect and pair production, would terminate the history, although pair production would result in secondary gamma rays from positron annihilation, which should be included as a new isotropic source of $0.511-\mathrm{MeV}$ gamma ray.

At first glance the choice of collision parameters would appear to be one of the simpler tasks in tracking a particle. This is deceptive, however, because the task of amassing the required cross-section data in the proper format can dwarf that of coding the problem. A distinguishing feature of the better Monte Carlo codes is the degree to which the cross-section preparation task has been automated (or the availability of an auxiliary code for that purpose). In addition to data at individual energy points, provision must be made (through interpolation or analytic fit) to cover all possible energies of interest. Schemes often used in this task include linear or logarithmic interpolations or polynomial fits to the data within certain energy bounds.

An additional complicating factor is the use of biasing in the selection of the interaction type. For example, incident neutrons may be assumed to scatter at every interaction point, and the weight of each after scattering will be reduced by the fraction $\Sigma_{s} / \Sigma_{t}$, the ratio of the scattering to the total 
cross section. In this manner the average scattering kernel will be the same as though the proper number of neutrons were allowed to scatter with no weight degradation, but the computing efficiency will generally be greater because histories will not be terminated by capture events.

In practice the entire transport problem can be run with only scattering interactions being allowed to occur (making the proper weight reductions) and with the collision parameters at each interaction being stored for future processing. The collision parameters are then analyzed to determine absorption density, to make flux-density estimates (as discussed later in this chapter), or to calculate energy-absorption density.

\subsection{PARTICLE PARAMETERS AFTER COLLISION}

The next task is the determination of the parameters of the particle(s) that survive an interaction. These parameters include the type, number, energy, and direction of the surviving incident particle and of any secondaries created in the interaction. Depending on the purpose of the calculation, one may also wish to determine the energy deposited in the system. The determination of these parameters involves the application of differential cross-section data in even greater detail in the selection of the interaction type. The details of the method will necessarily depend on the format used in specifying cross sections.

Rather than catalog the large number of possible nuclear interactions involving neutrons and gamma rays and the optional methods that may be employed in treating these in a Monte Carlo code, we will give examples illustrating the principles involved.

Reference should be made to Chap. 3 for a discussion of the types of interactions and of some of the expressions for particle energy following collision.

\subsubsection{Neutron Elastic Scattering}

We recall that the angular distribution of elastically scattered neutrons is approximately isotropic in the center-of-mass system for the lighter elements and becomes more complicated for the heavier elements in which forward (and occasionally backward) scattering is usually favored. The anisotropic behavior generally intensifies with increasing energy of the incident neutron. Most Monte Carlo programs use scattering angles measured in the center-ofmass system for selecting the scattering angle and computing energy after 
scattering. The angle of the scattered neutron is then converted to the laboratory system for tracking.

The scattering angle $\psi$ in the center-of-mass system, measured from the direction of travel of the neutron, will be selected randomly from an isotropic distribution by the formula

$$
\cos \psi=\mu=2 n-1
$$

where $n$ is a random number. This is identical to choosing the polar angle of emission from an isotropically emitting source.

Where the isotropic scattering approximation cannot be used, the differential angular scattering cross sections, $\sigma(\psi, E)$, must be used to determine the probability distribution of scattering angle. A customary procedure is to tabulate values of the cumulative scattering probability

$$
P(\psi, E)=\frac{\int_{0}^{\psi} \sigma(\psi, E) d \psi}{\int_{0}^{\pi} \sigma(\psi, E) d \psi}
$$

at discrete values of $\psi$ and $E$ and use interpolation in both dimensions. There are disadvantages to this method, however, from the standpoint of interpolation inaccuracies and machine-storage requirements. As an alternative, the differential cross section $\sigma(\psi, E)$ may be fit by a function such as

$$
\sigma(\psi, E)=A_{E}+B_{E} \cos \psi+C_{E} \cos ^{2} \psi
$$

and the selection may be performed using the differential probabilities in conjunction with a rejection technique.t Values of the coefficients $A_{E}, B_{E}$, and $C_{E}$ may be input for various values of energy where the function provides an adequate fit. Where the function cannot provide an adequate fit, the differential form may still be used by selecting values at fixed values of $\psi$ and $E$ and using interpolation in conjunction with a rejection technique. This latter method would be inferior to using the cumulative distribution where computing time is concerned, although input requirements for the differential form are simpler.

Once the scattering angle $\psi$ has been obtained in the center-of-mass system, the energy loss can be computed from the energy and momentum

tWe should note that $\sigma(\psi, E)$ is often given by Legendre polynomial coefficients. providing an alternative to Eq. 5.6-3. 


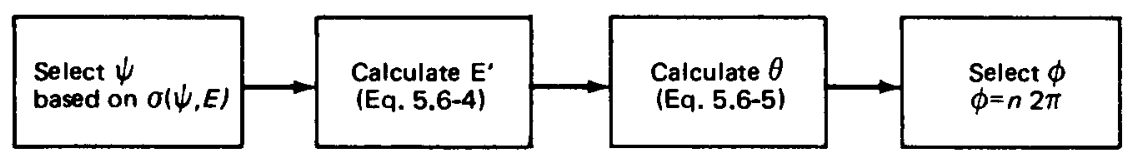

Fig. 5.7-Elastic scattering.

balance equations and the laboratory scattering angle can be calculated. The equations involved in these two operations are given in Sec. 3.2.2. If, as before, we assume that the mass ratio of the target nucleus to the neutron is $A$,

$$
\frac{E^{\prime}}{E}=\frac{1+A_{i}^{2}+2 A_{i} \cos \psi}{\left(A_{i}+1\right)^{2}}
$$

and

$$
\cos \theta=\frac{1+A_{i} \cos \psi}{\left(1+A_{i}^{2}+2 A_{i} \cos \psi\right)^{1 / 2}}
$$

where $E^{\prime}=$ energy after scatter

$E=$ energy before scatter

$A_{i}=$ mass of a nucleus of type $i$ (neutron mass $=1$ )

$\theta=$ scattering angle in the laboratory system

$\psi=$ scattering angle in the center-of-mass coordinates

With $E^{\prime}$ and $\theta$ known, the only parameter needed to resume tracking the neutron is the azimuthal angle $\phi$, which is, in all cases (except where polarization is considered), selected from a distribution equally probable in the region $0 \leqslant \phi \leqslant 2 \pi$, or $\phi=n 2 \pi$.

In summary, the basic operations in the neutron elastic-scattering routine are illustrated by Fig. 5.7. The directions of the neutron may then be translated into direction cosines taken relative to the geometry axes based on the known direction of the incident neutron and the deflection $(\theta, \phi)$ from that direction.

\subsubsection{Neutron Inelastic Scattering}

An added parameter is included for neutron inelastic scattering, i.e., the amount of energy deposited in the target nucleus. The loss of energy by this means prevents the energy of the neutron after collision from being a unique 
function of the scattering angle. The equations derived from energy and momentum conservation $\dagger$ corresponding to the elastic-scattering equations (Eqs. 5.6-4 and 5.6-5) then become

$$
\begin{gathered}
\frac{E^{\prime}}{E}=\frac{1}{\left(A_{i}+1\right)^{2}}\left[1+A_{i}^{2}\left(1-\frac{\epsilon}{E}\right)+2 A_{i} \cos \psi\left(1-\frac{\epsilon}{E}\right)^{3 / 2}\right] \\
\cos \theta=\frac{1+A_{i} \cos \psi\left(1-\frac{\epsilon}{E}\right)^{3 / 2}}{\left[1+A_{i}^{2}\left(1-\frac{\epsilon}{E}\right)+2 A_{i} \cos \psi\left(1-\frac{\epsilon}{E}\right)^{1 / 2}\right]^{1 / 2}}
\end{gathered}
$$

where $\epsilon$ is the excitation energy of the target nucleus. These equations reduce to the elastic-scattering relations when $\epsilon=0$. For $\epsilon>0$ and for $A_{i}$ large, they become approximately $E^{\prime}=E-\epsilon$ and $\cos \theta=\cos \psi$. As with elastic scattering, the distribution in $\psi$ must be an input quantity.

A particular nucleus may have various excited states, each with its own excitation energy, $\epsilon$. The neutron energy required for excitation of a particular level is $E>[(A+1) / A] \epsilon$. When the energy of the neutron is sufficiently high that any one of several levels may become excited, the interaction probabilities may be expressed in a total inelastic-scattering cross section, $\sigma_{n n^{\prime}}(E)$, with an associated probability distribution $P(E)$ among the levels, or in terms of separate cross sections $\sigma_{n n^{\prime}}(E, \epsilon)$ associated with the levels. In light nuclei the levels may be dealt with individually. This may also be possible for some levels in heavy nuclei, but in many cases (notably at higher excitation energies) a large number of closely spaced levels may appear which require an alternate treatment. One procedure is to split the possible values of incident and deflected neutron energy into bands and use experimentally determined probabilities for group-to-group transfer. These are expressed as cumulative probabilities over the possible $E^{\prime}$ (energy after scatter) groups for a given incident group. Then $E^{\prime}$ is selected by search and interpolation as discussed previously. An alternative approach that can be used in some cases is to assume a distribution in the continuum region of $E^{\prime}$

$$
f\left(E^{\prime}\right)=\frac{E^{\prime}}{T^{2}} \mathrm{e}^{-E^{\prime} / T}
$$


where $T$ is a constant (or a function of $E$ ). This is a result of the statistical model of the nucleus developed by Blatt and Weisskopf $\mathrm{f}^{13}$ and frequently used in neutron-cross-section estimates. This function could be integrated to obtain $P\left(E^{\prime}\right)$, but it would be more appropriate to select $E^{\prime}$ from the $f\left(E^{\prime}\right)$ distribution function using a rejection technique.

More than one secondary energy law may be used for particles of a given incident energy. For example, the probability of exciting any one of several widely spaced levels may be 0.3 , and the corresponding probability of falling into the continuous range may be 0.7 . By generating a random number, the code could choose the technique to be used in calculating $E^{\prime}$.

\subsubsection{Compton Scattering}

The treatment of Compton scattering collisions is similar to that for neutron elastic scattering in that the scattering angle is ordinarily picked from stored distributions and the photon energy is computed as a function of incident energy and scattering angle. Center-of-mass coordinates are no simpler in this case; thus laboratory coordinates are used.

The photon energy after collision (discussed in Sec. 3.2.1) is given by

$$
E^{\prime}=\frac{E}{1+E(1-\cos \theta)}
$$

where both $E$ and $E^{\prime}$ are expressed in terms of the electron rest-mass energy $\left(m c^{2}=0.511 \mathrm{MeV}\right)$. Extensive graphs and tables of the angular distribution are available ${ }^{14,15}$ for input to the program. Some investigators have elected to select scattering angles exactly by a rejection technique, using the Klein-Nishina formula (see Chap. 3) as the probability density function. Methods of making such an exact selection are given by $\mathrm{Kahn}^{16}$ and by Cashwell and Everett. ${ }^{3}$

\subsubsection{Particle Absorptions}

There are no particle parameters to be calculated following an absorption interaction. This event simply terminates the particle history. However, a record of the particle parameters at the time of collision may be desired for collision analysis.

As with neutrons, it may be desirable to increase the average number of photon scatterings per particle by forcing a scattering at each interaction 
point and making the appropriate weight adjustment to account for the portion of photons that, in reality, are absorbed.

In an absorption event secondary particles of the same type radiation as the incident particle may be tracked from the absorption point in the same calculation. If the secondary particle is of another type (e.g., gamma rays from neutron capture), the capture parameters will be used in formulating a source distribution for a separate calculation of the transport of the secondary particles.

Fission of heavy nuclei is a special case of absorption in which secondary neutrons and gamma rays will be generated with a known yield, $\nu_{0}$, per interaction and a known energy distribution. With secondary neutrons, account must be taken of the weight, $W$, of the neutron initiating the fission. For example, one neutron could be generated with a weight $\nu_{0} W$. Alternately, a number of neutrons could be selected from a distribution of integral values whose average is $\nu_{0}$ and each of these assigned a weight $W$. In both cases the neutron energy would be determined by selection from a cumulative energy distribution, and the direction would be chosen from an isotropic distribution.

\subsubsection{Calculation of Emergent-Direction Cosines}

Usually particle directions are defined by recording the direction cosines of the particle-track vector. As discussed previously, scattering angles at a collision point are characterized by calculating the polar angle $\theta$ (determined by the differential scattering cross section) and the azimuthal angle $\phi$ (picked at random between 0 and $2 \pi$ ) of the scattered track relative to the incident track. If $(\alpha, \beta, \gamma)$ are the direction cosines of the incident particle, the direction cosines of the scattered particle $\left(\alpha^{\prime}, \beta^{\prime}, \gamma^{\prime}\right)$ are given by the set of equations:

$$
\begin{aligned}
& \alpha^{\prime}=\alpha \cos \theta+\gamma \alpha \frac{\sin \theta \cos \phi}{\left(1-\gamma^{2}\right)^{1 / 2}}-\beta \frac{\sin \theta \sin \phi}{\left(1-\gamma^{2}\right)^{1 / 2}} \\
& \beta^{\prime}=\beta \cos \theta+\gamma \beta \frac{\sin \theta \cos \phi}{\left(1-\gamma^{2}\right)^{1 / 2}}+\alpha \frac{\sin \theta \sin \phi}{\left(1-\gamma^{2}\right)^{1 / 2}} \\
& \gamma^{\prime}=\gamma \cos \theta-\left(1-\gamma^{2}\right)^{1 / 2} \sin \theta \cos \phi
\end{aligned}
$$


except in the case where $1-\gamma^{2}$ approaches zero, in which case the degenerate form

$$
\begin{aligned}
& \alpha^{\prime}=\sin \theta \cos \phi \\
& \beta^{\prime}=\sin \theta \sin \phi \\
& \gamma^{\prime}=\gamma \cos \phi
\end{aligned}
$$

is used.

\subsection{PARTICLE SCORING}

The discussions to this point have concerned the generation of source particles, tracking to the first collision, and the determination of the new particle direction and energy following collision. The process of picking track lengths and analyzing collisions may be continued until the particle history is terminated. The termination may, as previously mentioned, be the result of exceeding predetermined bounds placed upon energy, spatial position, and/or particle weight.

We next consider the output of the Monte Carlo calculation. Specifically, answers are required for the following questions: What is the desired form of the output data? How do particle histories contribute to obtaining these answers?

Consider the case of a point source and an infinite-slab shield of thickness $T$. Typical of the results that might be desired are the following:

1. Flux density as a function of position, direction, and/or energy inside the shielding material.

2. The penetrating dose or flux density.

3. The energy and angular distribution of the penetrating particles.

4. The distribution of penetrating particles relative to the number of collisions encountered before penetrating.

5. The distribution in time of arriving particles.

There are obviously many other possibilities.

The flux density per unit source particle incident per unit area inside the infinite slab can be obtained by the sum

$$
\Phi=\frac{1}{n} \sum_{i} \frac{w_{i}}{\cos \theta_{i}}
$$


of particles crossing a plane surface parallel to the sides of the slab, where $\theta$ is the angle between the particle direction and the normal to the plane. The result is normalized to a unit source by dividing by the number of case histories, $n$.

The flux density at a particular location can be estimated by describing a volume region about that location and calculating the weight times the track length per unit volume of particles passing through the region. The flux

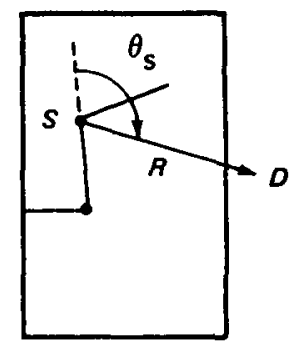

Fig. 5.8-Statistical estimation.

density at a point can be obtained by a method known as statistical estimation. This technique is used to estimate the scattered-radiation contribution to point detectors from scattering events within the shield and is often used with the forced-scattering technique discussed in Sec. 5.4. Consider, for example, a track within the shield in Fig. 5.8. The flux density at point $D$ is estimated by determining the particle wcight with which the particle would arrive at $D$ (within a unit spherical detector) if it were forced to scatter in that direction from each point of interaction, $S$. If the angle between the incident-particle direction and the line of sight $(S$ to $D)$ is $\theta_{S}$ and the particle is incident on $S$ with energy $E$, then the weight $W_{i}$ of the particle forced to scatter to $D$ is

$$
w_{i}=w_{0} \frac{\sigma_{s}(E)}{\sigma_{t}(E)} f\left(\theta_{s}, E\right)
$$

where $W_{0}=$ weight of the incident particle (at $S$ )

$\sigma_{s}(E)=$ total scattering cross section of the element involved in the collision

$\sigma_{t}(E)=$ total cross section of that element

$f\left(\theta_{s}, E\right)=$ probability density per steradian of scattering through angle $\theta_{s}$

Note that $\int f\left(\theta_{s}, E\right) d \Omega=1$ and that the product $\sigma_{s} f(\theta, E)$ is equivalent to the differential angular scattering cross section $\sigma(\theta, E)$. 
The contribution $\Phi_{i}$ of the scattered particle to the flux density at $D$ is given by

$$
\Phi_{i}=W_{i} \frac{\mathrm{e}^{-\Sigma_{t}\left(E^{\prime}\right) R}}{R^{2}}
$$

where $\Sigma_{t}\left(E^{\prime}\right)$ is the total macroscopic cross section of the scattered particle. The flux density at point $D$ is estimated by summing the contributions from all scattering interactions:

$$
\Phi=\frac{1}{n} \sum_{i} \Phi_{i}
$$

This estimate is independent of the particle-tracking procedure and does not terminate the history. Ordinarily the only calculation common to both the estimator and the tracking routine is the determination of which nuclear species in the material was involved in the collision. In the estimating process, every collision is assumed to be a scatter in the direction of the detector, but, for tracking purposes, completely independent determinations are made of interaction type and particle parameters after collision. A better justification for this technique is given in Sec. 5.2.

The problems of convergence using statistical estimation of this type have been examined by Kalos. ${ }^{17}$ He shows that an infinite variance and a preferential tendency to converge from below (as the number of histories is increased) result. Nevertheless the process does converge, and it has been a very useful technique in many situations.

Where surface detectors are of primary interest, a different form of estimator may be used. In this form flight-path length and direction are selected normally just as in statistical estimation, but estimates are made by extending flight paths in a straight line until a surface intersection is obtained. In one form, known as the last-flight estimate, this estimator consists in stretching every flight path that will provide an intersection. In another form, known as the first-flight estimate, the first-flight path length is adjusted, where possible, such that all collisions lie on the surface. Of course, appropriate weight adjustments must be made.

To illustrate the last-flight estimator, let us consider a point source in an infinite medium with a spherical surface detector at radius $R$. Collision points in Fig. 5.9 are assumed at points $A$ to $D$, and estimates are made by stretching each track until an intersection with the sphere is obtained. Note 
that intersection is always possible for tracks inside the sphere. Projection of tracks from collision points outside the sphere may not intersect, but, when they do, there will be a double score since the particle penetrates the sphere

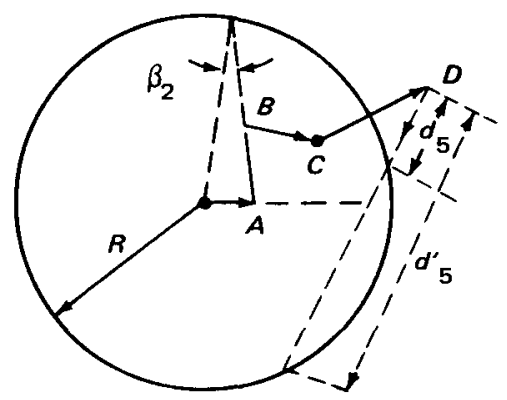

Fig. 5.9-Last-flight estimator.

twice. The estimate of flux density, $\Phi_{i}(R)$, at the sphere for one particle flight will be given by

$$
4 \pi R^{2} \Phi_{i}(R)=\frac{W_{i} \mathrm{e}^{-\Sigma_{i} d_{i}}}{\left|\cos \beta_{i}\right|}
$$

where $W_{i}=$ particle weight on the $i$ th flight

$d_{i}=$ total distance along the selected flight direction to the point of intersection

$\beta_{i}=$ angle between the normal to the surface and the track at the point of crossing

$\Sigma_{i}=$ total cross section of the medium for particles on the $i$ th flight

Thus the weight of the particle is adjusted by the attrition probability of the total flight to the surface. For the path originating at point $D$ (the 5th flight), the estimator will be

$$
4 \pi R^{2} \Phi_{5}(R)=\frac{W_{5}\left(\mathrm{e}^{-\Sigma_{5} d_{5}}+\mathrm{e}^{-\Sigma_{5} d_{5}^{\prime}}\right)}{\left|\cos \beta_{5}\right|}
$$

to account for the double encounter with the surface. Here again the tracking continues independently of the estimates, but particle parameters after collision are used as a basis for making the estimates.

The first-flight estimator is similar to the last-flight estimator except that adjustments are made in the first flight so that, where possible, each collision point lies on the surface of the sphere. In Fig. 5.10, the simulated tracks 
involved in the estimate for each collision in the history shown in (a) are shown in (b) through (d). In (b) the first flight is adjusted to make the first collision point lie on the surface. In (c) the first flight is extended so that the randomly determined end point of the second flight is shifted (parallel to the

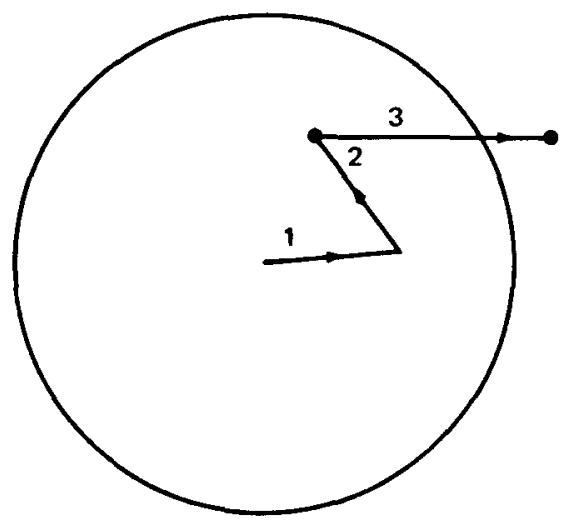

(a)

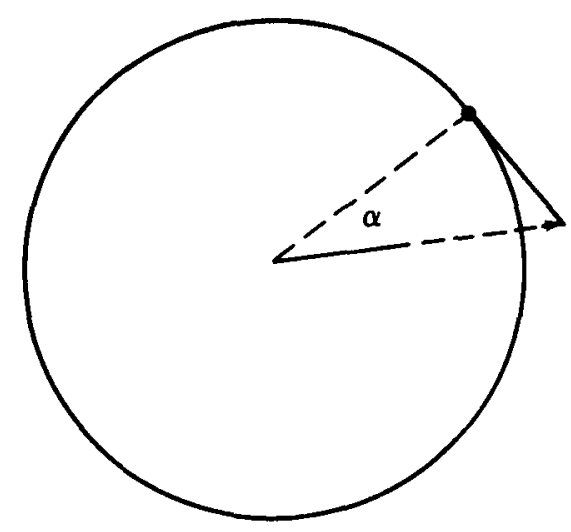

(c)

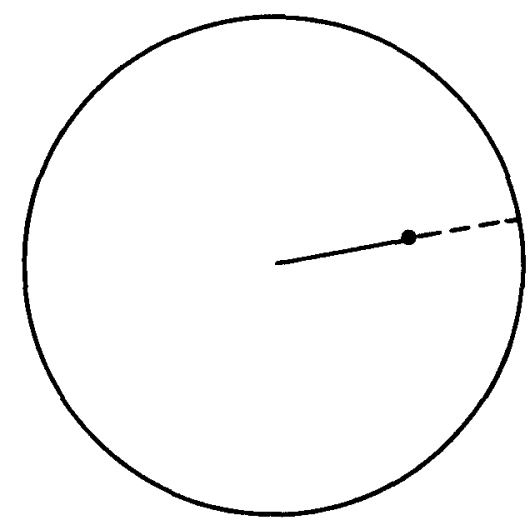

(b)

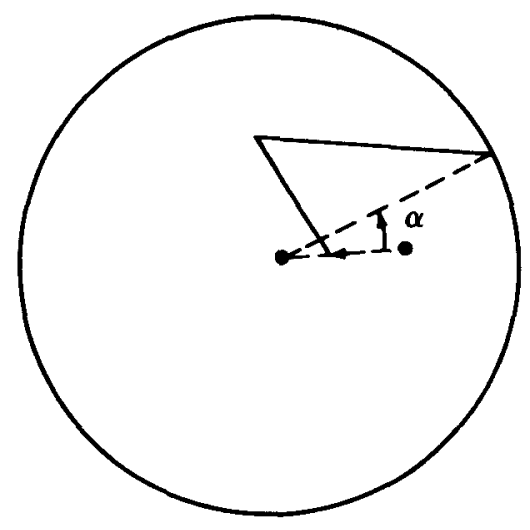

(d)

Fig. 5.10-First-flight estimator. (a) True particle track. (b) Adjustment for first flight. (c) Adjustment for second flight. (d) Adjustment for third flight.

first flight path) until it coincides with the surface. In (d) the first-flight length is reduced so that the third collision lies on the sphere. These tracks have been confined to a plane for ease of illustration, but adjustment of collision points lying outside the plane must still be in a direction parallel to the first flight. 
Numerically, the flux value of the particle scored in each case is

$$
4 \pi R^{2} \Phi_{i}(R)=\frac{W_{i} \Sigma_{0} \mathrm{e}^{-\Sigma_{0} d_{f}}}{\Sigma_{i}|\cos \alpha|}
$$

where $d_{f}=$ first-flight length after adjustment

$\alpha=$ angle between the first-flight path and the normal at the point of intersection

$\Sigma_{0}=$ total cross section at the source particle energy

$\Sigma_{i}=$ total cross section on the $i$ th flight

This expression is similar to the last-flight estimator in that the path-length weighting factor is $e^{-\Sigma d}$. Primary differences are that the angle of intersection with the sphere is determined by the first flight rather than the last flight and that the weight is adjusted by $\Sigma_{0} / \Sigma_{i}$ to account for the fact that interaction occurs at energy during the $i$ th flight rather than the source energy. In Fig. 5.10 (d) the interaction point is outside the sphere; thus the first flight must be adjusted backward to provide intersection. The estimate cannot be made for points external to the sphere if projection in line with the first flight does not intersect the sphere or if the adjustment requires a negative first flight. The estimator on the first leg [Fig. 5.10(b)] will be numerically equivalent whether the first- or last-flight estimators are used.

Trubey $^{18}$ performed a series of calculations for point sources of fission neutrons and 14-MeV neutrons in water to compare the results of these two estimators. He concluded that either of the two forms may be applied with equal ease, but the first-flight estimator appeared to give better results, particularly in the low-energy $(0.01$ to $4 \mathrm{MeV})$ fission-neutron range.

\subsection{STATISTICAL VARIANCE}

The number of histories that must be followed to yield acceptable results in a given problem depends to a large extent on the nature of the answer desired. For example, a calculation could yield a value of total dose per source particle accurate to $1 \%$ and at the same time have a very large uncertainty associated with the current of neutrons in the range $3 \mathrm{MeV}<E<4 \mathrm{MeV}$ and $10^{\circ}<\theta<30^{\circ}$.

A theorem of mathematical statistics that may be invoked as a means of estimating statistical uncertainty is the central limit theorem. Consider an experiment that can result in $K$ different ways, designated as $W_{1} \ldots W_{K}$ with 
associated probabilities $P_{1} \ldots P_{K}$, where $\sum_{i=1} P_{i}=1$. In this case the average (or mean) result may be defined as $\mu=\Sigma P_{K} W_{K}$ and the dispersion (variance about the expected value) as

$$
\sigma_{e}^{2}=\sum P_{K}\left(W_{K}-\mu\right)^{2}=\left(\sum P_{K} W_{K}^{2}\right)-\mu^{2}
$$

Suppose now that in a Monte Carlo penetration calculation $N$ source particles are started and that the total penetrating weight (summed over $N$ trials) of particles in a certain category is $M$. The central limit theorem states that

$$
P\left(\left|\frac{M}{N}-\mu\right|<\epsilon\right)=\operatorname{erf} \frac{t}{\sqrt{2}}+\rho_{N}
$$

This relates the probability that our answer will fall within some variation $\epsilon$ about the true average to the number of trials and the desired maximum value of $\epsilon$

$$
\begin{aligned}
\operatorname{erf}(x) & =\frac{2}{\sqrt{\pi}} \int_{0}^{x} e^{-u^{2}} d u \\
t & =\epsilon N^{1 / 2} \sigma_{e}^{-2} \\
\rho_{N} & \rightarrow 0 \text { as } N \rightarrow \infty
\end{aligned}
$$

The value of $\rho_{N}$ would be given in terms of the third moment of the $W$ distribution and for large $N$ would not be a significant contribution. Thus, for a given level of probability (given value of $t$ ), the size of $\epsilon$ is directly proportional to $\sqrt{\sigma_{e} / N}$. Thus doubling the number of histories decreases the value of $\epsilon$ only by a factor of $2^{-\frac{1}{2}}$. This is a very general result; i.e., Eq. 5.8-2 is true provided only that $\mu$ and $\sigma_{e}$ exist. Spanier and Gelbard ${ }^{1}$ discuss this and other aspects of statistical variance in some detail.

In cases involving direct analog calculations with no particle weighting, it may be prohibitively expensive to increase $N$ sufficiently to bring $\epsilon$ within acceptable limits. Then an alternate means of decreasing $\epsilon$ would be to decrease the dispersion, $\sigma_{e}$, by introducing biasing and weighting schemes that increase the number of samples for a particular output category per source particle, or give contributions that on the average lie closer to the average value, or that do both. 
To illustrate how $\sigma_{e}$ might be estimated in a particular problem, let us suppose $N$ source particles are generated and their contributions to penetrating particles of a certain category are $X_{1}, X_{2}, \ldots, X_{N}$. The average of this sample is defined as

$$
U=\frac{1}{N} \sum_{i} X_{i}
$$

and the sample variance is given by

$$
\begin{aligned}
\boldsymbol{\sigma}^{2} & =\frac{1}{N} \sum_{i}\left(X_{i}-U\right)^{2} \\
& =\frac{1}{N} \sum_{i} X_{i}^{2}-\left(\frac{1}{N} \sum_{i} X_{i}\right)^{2} \\
& =\overline{X^{2}}-U^{2}
\end{aligned}
$$

It should be noted that $U$ is not the truc expected value; it is a computed average for the sample, which should approach the expected value for large $N$ if the problem has been formulated properly. Thus Eq. 5.8-6 is only an estimate of the variance.

The variance $\sigma_{e}^{2}$ about the expected value $a$ is related to the variance $\sigma^{2}$ about the average $U$ of a series of observations by

$$
\frac{\sigma^{2}}{N}=\sigma_{e}^{2}
$$

Thus an estimate of $\sigma_{e}^{2}$ is given by

$$
\sigma_{e}^{2}=\frac{1}{N^{2}} \sum_{i} X_{i}^{2}-\frac{1}{N^{3}}\left(\sum_{i} X_{i}\right)^{2}
$$

In some Monte Carlo programs, the $X_{i}$ and $X_{i}^{2}$ are tabulated for use in calculating the variance of the answers obtained in one or more categories. In others qualitative judgments of the output are made on the basis of convergence and stability of the average as $N$ increases.

Some investigators have shown apparent reductions in the estimated variance by grouping the data, although this is not a real improvement in the certainty of the answer. Suppose, for example, a penetration calculation is to 
be made with 4000 particle histories. The data may be subdivided into 40 groups of 100 histories each and averages, $\mu_{i}$, computed. The variance of these $\mu_{i}$ about an overall average, $\bar{\mu}$, would ordinarily be much smaller than the variance of the individual data.

The question frequently arises concerning the effect on variance of some biasing scheme. This question is not easily answered. As noted in Sec. 5.2, the optimum importance function gives zero variance, but we do not know it is optimum unless we have prior knowledge of the result we seek to calculate. Obviously, the importance function must reduce the variance of the result below that obtained from an unbiased calculation or it is useless. Furthermore, there are cases where the apparent (computed) variance is reduced, but the answer is clearly erroneous. Consequently the task of proving or demonstrating the adequacy of the importance function rests heavily on the shoulders of its advocate. Numerous schemes are used, e.g., comparisons with nonstochastic calculations, with measurement, and with unbiased, straight analog Monte Carlo.

Systematic sampling is a technique used to obtain more representative answers when only a few histories are to be run-such as may be done in the check-out of a code. In this system the cumulative probability distribution of an occurrence (e.g., source angle, track length, or scattering angle) may be divided into increments, and, instead of the random selection of variable values, selections are made by systematically rotating through the increments. Instead of

$$
F(X)=\int_{-\infty}^{x} f(X) d X=n
$$

one may use

$$
F(X)=\frac{i-(1 / 2)}{N}
$$

where $i=1,2, \ldots, N$ and $N$ is the number of probability increments defined between 0 and 1.0. To relieve any bias that may be introduced by sampling from the midpoint of the increment, one may select from

$$
F(X)=\frac{i-n}{N}
$$

where $i=1,2, \ldots, N$ and $n$ is a random number. In this system the increments are rotated but the position within an increment is selected at random. 


\subsection{DEMONSTRATION MONTE CARLO PROGRAM}

The previous sections of this chapter introduce a number of concepts used in a Monte Carlo calculation. A simple and obvious way to interrelate various segments of the problem is to formulate a Monte Carlo program. Following standard practice in devising a computer program, we will define the problem and list the major divisions of the program; in Appendix J we give a logic flow chart and a FORTRAN listing of the program. Appendix $J$ also lists the input-data requirements and presents a test problem, including in put and output, which an interested reader can use to verify that his copy of this program is running properly. Although this program does not include all the techniques discussed in earlier sections, its use should provide a first-hand appreciation of Monte Carlo methods. We have compiled and run this program on a small ( $8 \mathrm{~K}$ core of 16 -bit words) computer with only the card reader and printer and its FORTRAN compiler.

The program listing contains a number of comments that describe what each set of statements accomplishes. Although the computer will ignore these comments (and they need not be included in working copies), they will be useful to the inexperienced programmer as explanatory notes to relate to the text.

\section{PROBLEM DEFINITION}

Neutron source:

Point, monoenergetic, cosine current located on the face of the shield.

Shield:

Homogeneous semi-infinite slab of thickness $t$ composed of a light element of atomic weight A.

Assumptions:

(1) Only capture and isotropic (center of mass) scattering are allowed.

(2) No biasing.

(3) No energy or geometry cutoff.

(4) Cross-section handling will provide $\Sigma_{t}(E)$ and $\Sigma_{s}$ on request.

(5) A random-number-generating routine will provide random numbers on request.

(6) Appropriate values of constants, such as source energy, shield thickness, maximum number of histories, flux-density-to-dose-rate conversion factors, and energy-dependent macroscopic cross sections, are input data. 
Output requirements: Leakage energy and angle-dependent current, fluence, and dose for both shield surface and neutrons absorbed as a function of energy and $z$ position within the shield. Neutrons leaving the shield at $z=t$ are called transmitted neutrons, and those leaving at $z=0$ are called reflected neutrons.

Geometry:

Fig. 5.11.

Symbols used:

\begin{tabular}{|c|c|}
\hline$n$ & random number \\
\hline$\theta$ & incident polar angle \\
\hline$\phi$ & incident azimuthal angle \\
\hline & $\begin{array}{l}\text { cutoff angle below which } \theta \text { is as- } \\
\text { sumed to be zero }\end{array}$ \\
\hline & source energy \\
\hline & energy after scattering \\
\hline & $\begin{array}{l}\text { cosine of scattering angle in center- } \\
\text { of-mass system }\end{array}$ \\
\hline$v_{s}$ & laboratory scattering angle \\
\hline$\phi_{s}$ & azimuth of scattered direction \\
\hline 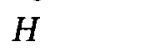 & history number \\
\hline$H_{\max }$ & number of histories to be run \\
\hline & path length \\
\hline$(E, \theta)$ & number of leakage neutrons \\
\hline$(E, \theta)$ & flux density of leakage neutrons \\
\hline$(E, \theta)$ & dose transmitted by leakage neutron \\
\hline$N_{a}(E, \theta)$ & number of absorbed neutrons \\
\hline$\alpha, \beta, \gamma$ & direction cosines of particle \\
\hline & $\begin{array}{l}\text { coordinate of particle in direction } \\
\text { normal to shield }(z=0 \text { is the } \\
\text { shield surface on which particles } \\
\text { are incident) }\end{array}$ \\
\hline$(E)$ & $\begin{array}{l}\text { Flux-density-to-dose-rate conversion } \\
\text { factors }\end{array}$ \\
\hline
\end{tabular}

No attempt is made to account for particle positions in the $x$-y plane. The only information desired from the leakage particles is their energy and the angle at which they penetrate eitier shield surface. Calculation of the $x, y$ coordinates could easily be added to the diagram, if desired, since the direction cosines and track length are known for each particle flight. 
The cross-section handling subroutine, XSEC, and the random-numbergenerating routine, RAND, which are included, are intended only for illustrative purposes and to allow the reader to duplicate results obtained in the text. The subroutine XSEC reads a set of cross-section data the first time

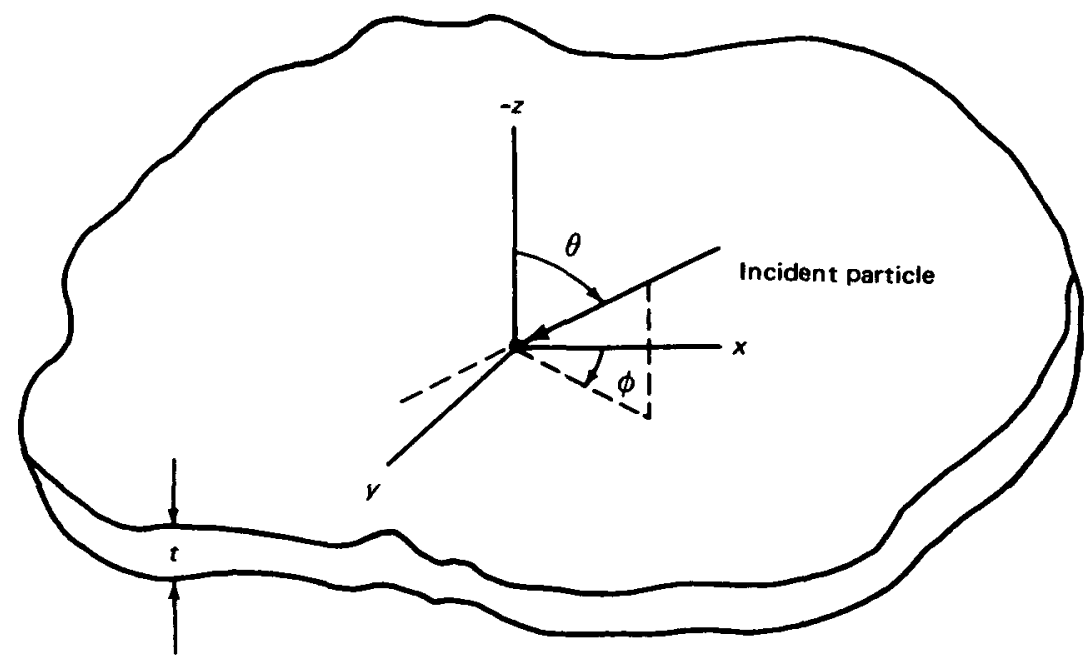

Fig.5.11 — Geometry for demonstration Monte Carlo program.

it is called. Then a linear interpolation is performed to determine the total and scattering cross sections as the energy transferred to XSEC through its argument list. After the first call of XSEC, no data are read, and only the interpolation is performed. The subroutine RAND is actually not a random-number 'generator but a table of 23 numbers and a stepping procedure to alter the sequence of using them. For a realistic computation, XSEC could be used provided the cross-section variation is approximately linear with energy; however, RAND should be replaced with an actual random-number generator.

Major divisions of the program logic are numbered below in the same way as the flow chart (shaded connector symbols) in Appendix $\mathrm{J}$ and may be categorized as follows:

1. Source-particle generation
A. Select direction angles $\theta$ and $\phi$.
B. Calculate direction cosines $\alpha, \beta$, and $\gamma$.
C. Step history counter.
D. Test for history completion.
E. If all histories are complete, branch to print area. 
2. Path-length determination
A. Calculate path length $L$.
B. Calculate $z$ coordinate.
C. Test for penetration through either slab surface.
D. If penetration occurs, store results and terminate history.

3. Collision-parameter calculations
A. Select scatter or absorption.
B. If absorption, store absorption parameters and terminate history.
C. If scattering, calculate scattering angle $\mu$.
D. Calculate neutron encrgy $E^{\prime}$ after collision.
E. Calculate direction angles $\theta_{s}$ and $\phi_{s}$ of scattered neutron.

4. New-particle-direction calculation

A. Determine new direction cosines $\alpha^{\prime}, \beta^{\prime}$, and $\gamma^{\prime}$ using coordinate rotation equations (see second chart in Appendix J).

B. Return to (2) for next flight calculations.

5. Analysis of leakage radiation
A. Calculate dose rate per bin.
B. Print out flux density and dose rate.
C. Print out absorptions per increment.

A listing of the FORTRAN coding of this problem is shown in Appendix J. Designation of input variables has been changed in some cases from those previously given for ease of coding. A table has been included to define input variables, limits, and input format requirements. Finally, the test problem is defined with specimen input and output.

\subsection{PROGRAMMING SUGGESTIONS}

A few additional remarks are in order concerning use of the Monte Carlo method for solving shielding problems.

Monte Carlo codes tend to become quite large and complex as the various options on geometry, biasing, and analysis are incorporated. The programs are more manageable if they are broken into subroutines which are called into the computer fast memory only when needed (unless the fast memory is large enough to contain the whole program and working data). They have also been coded as completely separate routines linked by data tapes. For example, one code generates source-particle parameters and stores these on tape. Another code reads the source parameters and generates 
collision histories. A third code analyzes the tape output of the collisionhistory processor and calculates the desired output data, such as flux density, dose, and current. Each of those programs must have compatible features and use overlapping input data for geometry description, cross sections, etc.

Checking or debugging Monte Carlo codes is a tedious and difficult task since the nondeterministic nature of the calculation may mask errors large enough to influence the answer but not large enough to make the answer appear unreasonable. Debugging is the step that separates the men from the boys. It has been found useful to debug each subroutine separately and in various aggregates prior to assembling the entire program. Simple problems can be devised to check individual calculations by allowing only onc interaction or by inputting an extreme in geometry or energy. Additional output features can be inserted into the program for use during debugging and complete particle historics can be printed out. If the sequence of path lengths, particle weights, and parameters after collision is available, errors arc easicr to detect.

The encrgy cutoff specified in a fast-ncutron calculation to a large extent will determine the average computing time required per history and thus should not be set lower than required by the accuracy of the problem. In problems designed to calculate fast-neutron dose, an encrgy cutoff near 0.1 $\mathrm{MeV}$ is ordinarily used.

A reasonably economical calculation can be made of thermal-neutron transport and diffusion from a source of thermal neutrons, but the epithermal region between fast and thermal is much more difficult to treat. The application of Monte Carlo to reactor criticality studics has been quite limited. The Monte Carlo method cannot compete with analytical and semicmpirical methods because of the large number of collisions required to thermalize fast neutrons. It has found limited application, ${ }^{19,20}$ however, because of the ability to treat complex three-dimensional geometries. Monte Carlo has been successfully used to determine the thermal slowing-down density for the input to a thermal-neutron diffusion or transport code. The choice of a low-energy cutoff point is less important for gamma rays because of the rapid rise in the photoelectric cross section with decreasing energy. There is usually little or no incentive to track photons with energies below $0.01 \mathrm{MeV}$.

Monte Carlo techniques may be designed to reproduce a physical model in as much detail as is necessary and so provide a powerful tool for solving 
problems with very few compromises with the physics. The Monte Carlo method can incorporate any geometry. To use Monte Carlo successfully, however, one generally must invest a considerable amount in analysis, programming, and computer machine time. The importance of machine time is often overemphasized, and analysis and programming are underemphasized. The user should keep in mind that a well-developed theory exists which specifies, in principle, a near-optimum procedure for solving a given problem. This procedure consists in obtaining the best possible approximation to the value function for the problem and then using this function to obtain parameters for importance-sampling techniques or to guide development of new sampling techniques.

As an aid to the programmer, the concept of a Monte Carlo programming system was developed. For example, the 05R system ${ }^{21}$ and its updated version 06R can, in principle, be used to solve any neutron-transport problem. The framework is there (cross-section handling, geometry-solving routines, random-walk procedures, etc.), but the programmer must incorporate the special features he desires by adding subroutines to the framework.

More recently a highly versatile and easy to use multipurpose neutronand gamma-ray-transport code, the MORSE code, ${ }^{22}$ has been developed at the Oak Ridge National Laboratory. Some of its features include the ability to treat the transport of either neutrons or gamma rays or to simultaneously treat the transport of neutrons and secondary gamma rays; the incorporation of multigroup cross sections; an option for solving either the forward or adjoint problem; modular input-output; cross section, analysis, and geometry modules; debugging routines; time dependence for both shielding and criticality problems, and albedo option at any material boundary; one-, two-, and three-dimensional geometry packages; and several types of optional importance sampling.

Traditionally, Monte Carlo codes for solving neutron- and gamma-raytransport problems have frequently been separate codes because of the physics of the interaction processes and the corresponding cross-section information required. However, when multigroup cross sections are used, the energy group-to-group transfers contain the cross sections for all processes. Also, for anisotropic scattering each group-to-group transfer has an associated angular distribution that is a weighted average over the various cross sections involved in the energy-transfer process. Thus these multigroup cross sections have the same format for both neutrons and gamma rays. In addition, the generation of secondary gamma rays may be considered as just 
another group-to-group transfer. Therefore, when multigroup cross sections are used, the logic of the random-walk process (the process of being transported from one collision to another) is identical for both neutrons and gamma rays.

In general, the Monte Carlo method is not the best method for one-dimensional problems since discrete-ordinates codes are likely to be much faster than Monte Carlo codes. For two-dimensional problems, Monte Carlo and discrete-ordinates methods are somewhat comparable, but for three-dimensional or two-dimensional time-dependent problems, there is no competitor to Monte Carlo for accurate solutions to transport problems.

\section{REFERENCES}

1. J. Spanier and E. M. Gelbard, Monte Carlo Principles and Neutron Transport Problems, Addison-Wesley Publishing Company, Inc., Reading, Mass., 1969.

2. H. G. Tucker, An Introduction to Probability and Mathematical Statistics, Academic Press, Inc., New York, 1962.

3. E. Cashwell and C. Everett, A Practical Manual on the Monte Carlo Method for Random Walk Problems, Pergamon Press, Inc., New York, 1959.

4. G. Goertzel and M. H. Kalos, Monte Carlo Methods in Transport Problems, in Progress in Nuclear Energy, Physics and Mathematics, Series I, Vol. 2, p. 35, Pergamon Press, Inc., New York, 1958.

5. R. R. Coveyou, V. R. Cain, and K. J. Yost, Adjoint and Importance in Monte Carlo Application, Nucl. Sci. Eng., 27: 219(1967).

6. V. R. Cain, Application of $\mathrm{S}_{\mathrm{N}}$ Adjoint Flux Calculations to Monte Carlo Biasing, Trans. Amer. Nucl. Soc., 10: 399(1967).

7. H. Kahn, Random Sampling (Monte Carlo) Techniques in Neutron Attenuation Problems, I and II, Nucleonics, 6(5): 27-37 and 6(6): 60-65(1950).

8. B. Eriksson, On the Use of Importance Sampling in Particle Transport Problems, Swedish Report AE-190, June 1965.

9. J. Perkins and M. Burrell, Efficiency of Some Biased Sampling Schemes in Monte Carlo Calculations, in Nuclear Engineering and Science Conference, April 6-9, 1959, Cleveland, Ohio, Engineers Joint Council, New York.

10. M. Kalos, Methods in Monte Carlo Solution of the Radiation Transport Equation, Report UNC-5014 (NDL-TR-36), United Nuclear Corporation, May 31, 1962.

11. M. Leimdorfer, A Monte Carlo Method for the Analysis of Gamma Radiation Transport from Distributed Sources in Laminated Shields, Nukleonik, 6(2): 58-65 (March 1964).

12. F. H. Clark, The Exponential Transform as an Importance-Sampling Device, USAEC Report ORNL-RSIC-14, Oak Ridge National Laboratory, January 1966. 
13. J. M. Blatt and V. F. Weisskopf, Theoretical Nuclear Physics, John Wiley \& Sons, Inc., New York, 1952.

14. A. Nelms, Graphs of the Compton Energy-Angle Relationship and the Klein-Nishina Formula from $10 \mathrm{keV}$ to $500 \mathrm{MeV}$, Report NBS-542, National Bureau of Standards, August 1953.

15. R. Latter and H. Kahn, Gamma-Ray Absorption Coefficients, Report R-170 (RAND), Rand Corporation, Sept. 19, 1949.

16. H. Kahn, Applications of Monte Carlo, USAEC Report AECU-3259 (RM-1237-AEC), Rand Corporation, April 1956.

17. M. Kalos, On the Estimation of Flux at a Point by Monte Carlo, Nucl. Sci. Eng., 16: III (1963).

18. D. K. Trubey and M. B. Emmett, A Comparison of First- and Last-Flight Expectation Values Used in the 05R Monte Carlo Calculation of Neutron Distributions in Water, USAEC Report ORNL-RSIC-3, Oak Ridge National Laboratory, May 1965.

19. Proceedings of Conference on the Application of Computing Methods to Reactor Problems, May 17-19, 1965, Argonne National Laboratory, USAEC Report ANL7050, August 1965.

20. H. Rief and H. Kschwendt, Reactor Analysis by Monte Carlo, Nucl. Sci. Eng., 30: 295-418 (1967).

21. D. C. Irving, R. M. Freestone, Jr., and F. B. K. Kam, 05R, A General-Purpose Monte Carlo Neutron Transport Code, USAEC Report ORNL-3622, Oak Ridge National Laboratory, February 1965.

22. E. A. Straker, P. N. Stevens, D. C. Irving, and V. R. Cain, MORSE Code: A Multigroup Neutron and Gamma-Ray Monte Carlo Transport Code, USAEC Report ORNL-4585, Oak Ridge National Laboratory, September 1970.

\section{EXERCISES}

5.1 Define the event (sample) space and specify a typical event for the following: (a) One die randomly tossed once. (b) Two dice randomly tossed together. (c) One die randomly tossed twice in sequence. (d) The fission-neutron spectrum. (e) A photon undergoing a Compton scatter.

5.2 For $5.1(\mathrm{a}), 5.1(\mathrm{~b})$, and $5.1(\mathrm{c})$, describe and plot: (a) The probability density function (PDF). (b) The cumulative distribution function (CDF). (c) Were you forced to use a random-variable function? (Hint: Suppose the six sides of a die were labeled a,b,c,d,e,f rather than with spots).

5.3 Figures 2.1 and 2.4 in Chap. 2 present spectral data. (a) Is either function a PDF? (b) Can the other functions be converted to a PDF? How?

5.4 Using 1-MeV intervals, compute and plot the CDF for your answer to 5.3(a).

5.5 A PDF is known to have the form $f(x)=C \sin x$ over the integral $(0<x<\pi)$, where $C$ is a constant. (a) Compute $C$. Does the PDF satisfy Eqs. 5.1-3 and 5.1-4? (b) Compute the CDF. Does it satisfy Eqs. 5.1-6 and 5.1-7? (c) On one set of axes, plot the PDF and CDF. (d) Rather than generate random numbers, select an evenly distributed set of numbers in the interval (0.1) (e.g., at 0.1 intervals). Using the CDF 
plotted in 5.5(c), obtain the values of $x$ for each selected number. Use $n=F(x)$ (Eq. 5.1-24). (e) Is the distribution of events obtained in 5.5(d) consistent with the PDF? (f) Would it have been so if the numbers had been selected randomly? (g) Note: $5.5(\mathrm{~d}), 5.5(\mathrm{e})$, and $5.5(\mathrm{f})$ illustrate the principle of biasing as well as random sampling. Why?

5.6 The following experiment illustrates the rejection technique. As with any random process, no two experimenters should obtain exactly the same answer. One side of a die is labeled $A$, two sides $B$, and the remaining three sides $C$. The accompanying graph is a proper distribution function for one toss of such a die, as the student should verify.

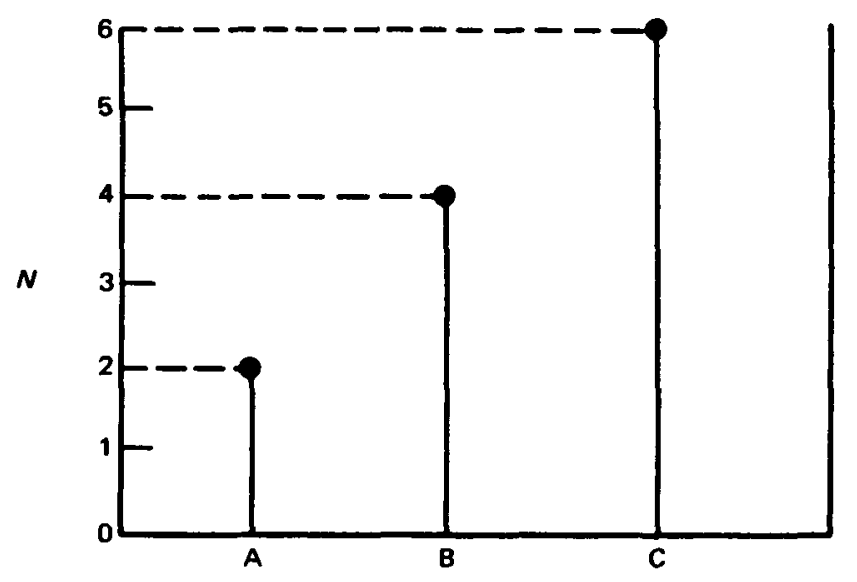

Graph for Exercise 5.6(a). Distribution function for one toss of a die.

(a) Is this a graph of a PDF? Why? (b) Use a normal die to randomly select with equal probability a letter from $A, B$, or $C$. For example, let 1 or $2=A, 3$ or $4=B$, and 5 or $6=C$. From the graph find $N$ associated with the selected letter. Then roll the normal die again to obtain $N^{\prime}$, a number from 1 to 6 . If $N^{\prime} \leqslant N$, accept the letter choice. If $N^{\prime}>N$, reject it. Repeat the process until a sequence of accepted letters has been established. Tabulate the distribution within the sequence. If both you and your die were honest, the ratio of $A$ 's to $B$ 's to $C$ 's should approach $2: 4: 6$. 
8 


\section{Shield Attenuation Calculations}

Now we can turn to some of the practical considerations for applying data and empirical formulas to attenuation problems. Two previously mentioned observations are worth repeating. There are several levels of attenuation calculations, ranging from elegant and precise to improvised and approximate. Since no one method satisfies all applications, the reader should become familiar with a number of techniques so that he can select the one most appropriate for a particular application. The second observation is that for most of these techniques a good bit of the calculation has already been done; a particular application usually reduces to assembling the appropriate data matrix from the existing literature and integrating over the particular shield geometry and composition of interest.

\subsection{ANALYSIS OF THE SOURCE}

Although this topic was discussed in an earlier chapter, some additional remarks are pertinent. There are, in general, two approaches to the analysis of the radiation source depending on the methods to be used in analyzing the shield. The direct approach is to treat the source and shield as an entity and to obtain as a single calculation the distributions of radiations leaving the shield which originated within the source, thus solving the sourcedescription and shield-penetration problems simultaneously. The direct approach is used for a "one-shot" calculation where the system can be precisely defined and only one set of answers is required. An example would be a final, detailed analysis of a reactor shield "as built."

The alternate approach is to split the source description and shield penetration into two distinct phases. The first phase involves calculating the radiation leakage from the source region to obtain an energy and angular distribution incident to the shield. This distribution can then be applied to 
shield-attenuation calculations. This method provides a useful means for comparing several designs based on parametric data and is more economical than the direct method where a number of designs are to be analyzed or where the effect of small variations in shield design is to be studied.

The task of analyzing the radiation emitted by a reactor source is often as complex as the shield-attenuation calculation because of a plurality of sources. The source density must be determined for fission neutrons and gamma rays, fission-product gamma rays, secondary gamma rays from nonfission neutron interactions, and, in some instances, secondary neutrons from photoneutron interactions. This analysis requires a detailed knowledge of the density of fission events, the material compositions, and the neutron spectrum as a function of position within the reactor.

For example, the local source strength of gamma rays (except for the fission-product source) in an energy group $\Delta E_{k}$ will be given by

$$
F G_{k}+\sum_{i} \sum_{j} \Phi_{i} N_{j} \sigma_{i j} Y_{i j k}
$$

where $F=$ local fission density

$G_{k}=$ yield of gamma rays per fission within $\Delta E_{k}$

$\Phi_{i}=$ local flux density of neutrons in energy group $\Delta E_{i}$

$N_{j}=$ atomic density of element $j$

$\sigma_{i j}=$ local microscopic cross section for radiative interaction of neutrons of energy $i$ with element $j$

$Y_{i j k}=$ yield of gamma rays per interaction

To this sum the fission-product sources should be added.

The method used in evaluating fission-product sources will vary with their relative importance and with the operating history of the reactor. Their intensity will increase with time of operation of the reactor at a given power level until some maximum or "saturation" level is attained. It is common practice to combine the saturation fission-product activity with the fission gamma-ray source and consider only this maximum condition. A source spectrum for this condition is given in Chap. 2.

The fission density and low-energy neutron spectrum, as a function of position within the reactor core and reflector, are usually derived in an iterative process using transport and diffusion theory. These data, obtained in the process of reactor design, are ordinarily available to the shield designer. If only the fission density is available, the low-energy neutron-fluxdensity spectrum may be calculated using a combination of transport and diffusion calculations, or an $S_{n}$ (or $P_{n}$ ) calculation. In general, the methods 
used in shield analysis are not adequate for the task of determining the distribution of fissions within the reactor core or for determining criticality. The reverse is also true, most reactor analysis codes do not do an adequate job of predicting the radiation leakage from the reactor. Although the theory is analogous in reactor and shielding codes, the emphasis is different and the accuracy with which a particular reaction type is analyzed varies significantly. Two methods that do provide adequate results in both reactor and shield analysis are the Monte Carlo and the discrete ordinate $S_{n}$.

Once the position, energy, and intensity of all sources within the reactor have been evaluated, reactor leakage can be analyzed. The form used in expressing the energy and spatial distribution of the sources will vary with the intended method of performing the leakage calculation. The methods used in performing the leakage calculation are the same as those used for the analysis of shield penetration as discussed below.

For a direct Monte Carlo solution, a separate source generator routine is used to generate particles with the appropriate distribution in energy, angle, and position. Biasing can be used to generate more of the particles expected to be important to shield penetration, such as those born at high energy, traveling in a radial direction, and/or originating near the periphery. In a discrete-ordinate $S_{n}$ calculation, the spatial variation of source strength can be expressed analytically. If existing data sets, either experimental or analytical, are used, then it is sometimes desirable to express the source as an equivalent simple source, such as a disk, an infinite plane, or a series of point sources.

\subsection{DIRECT SOLUTIONS}

One means of obtaining shield-attenuation data is the direct application of Monte Carlo, moments method, $S_{n}$, or other similar methods that directly use the material cross sections. Chapters 4 and 5 are devoted to the general theory of the methods involved in direct solutions. Applications of these methods invariably involve the use of rather sophisticated computer programs and an expenditure that some problems may not justify. Consequently direct solutions are not widely used in shield design and evaluation studies. They are mentioned here only to call attention to the fact that they may be applied directly to a shield-attenuation problem.

To some extent a judgment based on analogy with similar designs or with the available basic data will assist in the selection of a method by determining which penetrating components are likely to be most important. 
For example, where low-energy neutrons are not expected to be significant in heating or in the penetrating-radiation component, a Monte Carlo approach may provide the most accurate analysis. If gamma rays from thermal-neutron capture are expected to be the primary factor, then a moments method, $S_{n}$, or a combination of transport and diffusion methods may be selected.

\subsection{APPLICATION OF PARAMETRIC DATA}

A variety of data is available from calculations and experiments that have been performed to determine the penetration of radiation from basic, typical sources of neutrons and gamma rays through materials of general interest. These data may be scaled to approximate the solution to a variety of attenuation problems.

The concept of the unit source, introduced in Chap. 2, is integral to the understanding and application of parametric data. The term unit source generally refers to a source of unit strength, i.e., 1 particle/sec, 1 watt $\mathrm{cm}^{-3}$, $1 \mathrm{MeV} \mathrm{sec}{ }^{-1} \mathrm{~cm}^{-2}$, etc. All the radiation evolved from such a source need not necessarily come from the same point, be traveling in the same direction, or be of the same energy. Distributions in each of the independent variables (position, angle of emission, and energy) commonly chosen for unit-source characterization are listed below. Various combinations of the listed spatial, angular, and energy distributions have been used.

The most frequently used spatial distributions are point, plane, disk, and line; angular distributions are usually isotropic, monodirectional, cosine, and $(\text { cosine })^{n}$; energy distributions are usually monoenergetic, uniformly distributed, and fission spectra.

For a given shield material, the number of dimensions involved in the parametric data is determined by the calculational method used to obtain the data, the shield geometry, and the type of detector assumed. For example, in the geometries represented by a point source in a spherical shield or an infinite-plane source and shield, the data can be applied to any point at a given radius or thickness. On the other hand, for a point source and slab geometry, the position on the exit face also becomes a parameter. Source energy and angle and exit energy and angle may also be parameters. Thus parametric data available for application to shield analysis vary from one-dimensional sets of dose vs. thickness to nine-dimensional sets involving all the parameters mentioned (two source angles, source energy, material thickness, two exit coordinates, two exit angles, and exit energy). In 
specialized studics one might include additional parameters, such as the number of collisions suffered by the particle before exiting the shield or the time of flight from source to detector.

In addition to direct applications of parametric-data matrices, interpolation (linear, logarithmic, or other) is often required between particular values of one or more of the parameters to improve accuracy. Graphical interpolation is one of the most widely practiced methods because together with simplicity it provides a more direct view of the behavior of the data with respect to a particular parameter. Computer programs have been written for complex interpolations between two or more variables where nonautomated methods would become quite tedious. In addition, some of the data sets have been fit with analytical functions. With these functions analytical representations of the parameters can be used for calculating transmission or reflection probabilities at any desired point.

Occasionally the shape of an energy or angular distribution does not change with distance within some range. Such a distribution is said to be in equilibrium for that range. It is always desirable to observe such equilibrium conditions because of the resulting simplifications in representing the data. The existence of equilibrium with distance is an indication that the distance variable can be separated in the distribution function.

The three types of data most likely to be available for application to a shielding problem include data resulting from moments-method calculations, Monte Carlo analyses, and experiments. These three categories are reviewed in the following sections. Other types of analytical data, particularly from $S_{n}$ calculations, may be available and just as applicable to a particular shielding problem. In such cases the methods of application are analogous to those discussed in the following sections but have to be adjusted to the number and form of the variables considered.

\subsubsection{Moments-Method Differential Energy Spectra}

(a) Neutrons. The moments method has been applied to neutron penetration through a variety of materials. Data from these calculations have been summarized by Goldstein ${ }^{1}$ and more recently by Krumbein and O'Reilly. ${ }^{2}$ Studies have included penetration of point isotropic fission sources through water, hydrogen, lithium, lithium hydride, lithium hydroxide, beryllium, carbon, and various combinations of carbon and hydrogen. In addition, data were obtained for penetration from monoenergetic sources through water and hydrogen. 


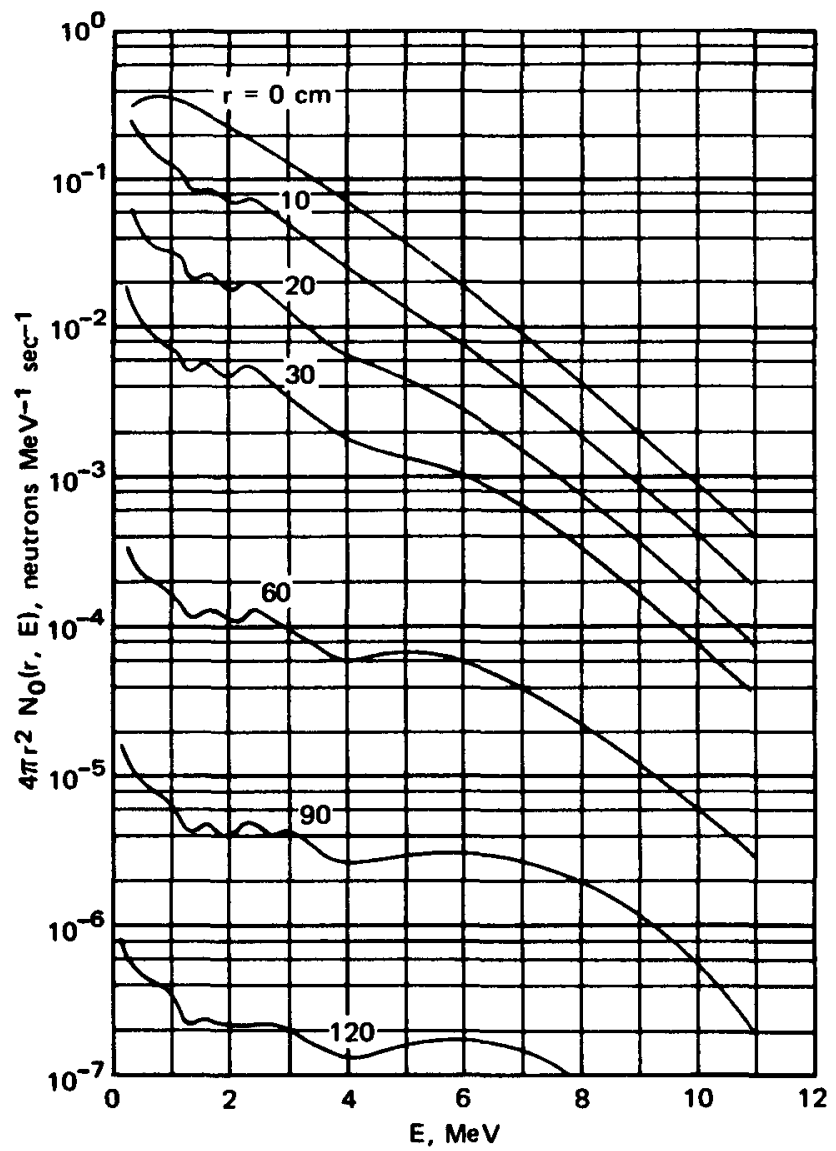

Fig. 6.1-Differential number spectrum of neutrons in water from a point isotropic fission source emitting 1 neutron $\sec ^{-1}$. (From Goldstein. ${ }^{1}$ )

A sample of the data from these calculations is given in Fig. 6.1, which is a graph of the differential neutron spectrum for various penetration depths in water resulting from a point isotropic fission source of 1 neutron $\mathrm{sec}^{-1}$.

The slope of the curves in Fig. 6.1 decreases with increasing penetration, indicating a hardening of the spectrum (increasing fraction of higher energy particles). This trend is followed in all materials containing significant concentrations of hydrogen and is a consequence of the dependence of the hydrogen cross section on neutron energy. As the neutron energy increases, the hydrogen cross section decreases, and higher energy neutrons then suffer fewer collisions in crossing a given thickness of hydrogenous material than lower energy neutrons. The energy loss in hydrogen is so great that neutrons will usually be thermalized very near their point of initial interaction. For 


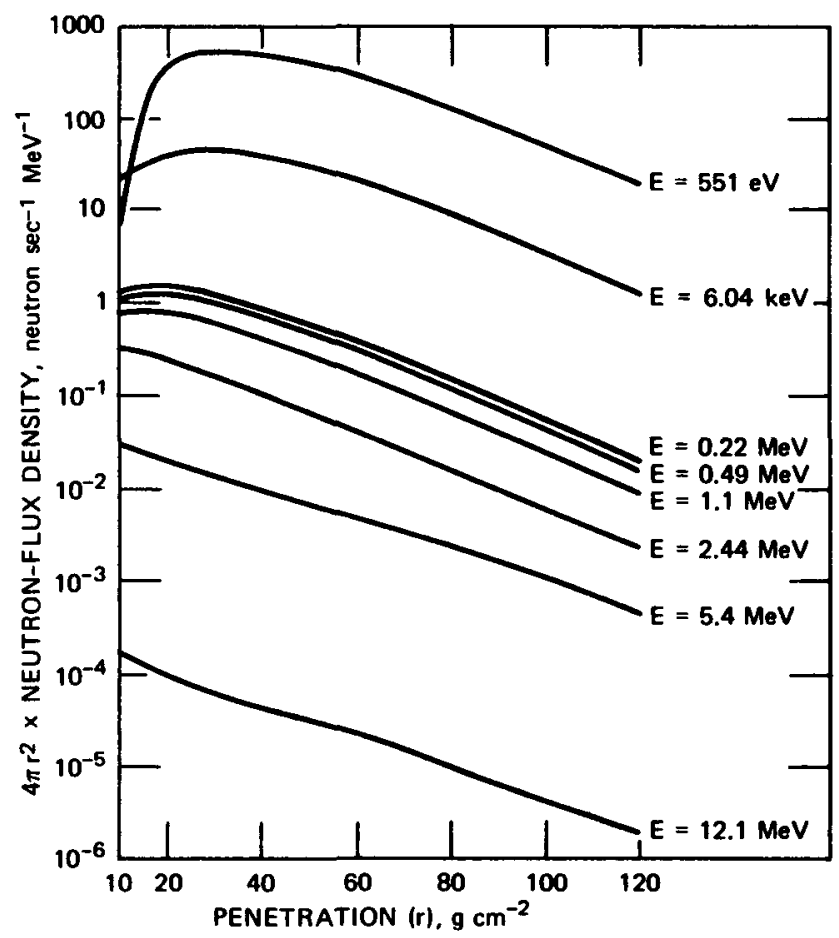

Fig. 6.2-Differential neutron-flux density $\left(x 4 \pi r^{2}\right)$ vs. penetration $(r)$ in carbon due to unit point isotropic fission-neutron source. (From Goldstein. ${ }^{1}$ )

this reason the initial flight is quite important to the distribution deep within the shield. A larger fraction of the higher energy will be transmitted and will thus contribute to a reduction in slope (or hardening) of the energy distribution.

Figure 6.2 shows similar graphs for carbon except that the parameters have been switched so that the flux density, as a function of depth, is plotted for various energies. Here the slopes are essentially equal beyond $30 \mathrm{~g} \mathrm{~cm}^{-2}$, which indicates that an equilibrium spectrum is established. Moments-method neutron-penetration data for several materials are given in Appendix $\mathrm{K}$ in tabular form.

In all these calculations the point source is assumed to be surrounded by an infinite medium. Thus particle spectra calculated at a given depth include radiation that has been scattered back to that depth from more distant points. For a shield whose outer surface corresponds to that depth, the flux density at the surface is overpredicted by the infinite-medium data (particularly at lower energy where a large percentage of the flux density is 
due to backscattering); thus corrections are necessary for applications of infinite-medium data to shield layers. A severe limitation of the momentsmethod data is that angular-distribution information on the flux density is not given.

This type of data can be applied to surface sources by breaking the surface into small increments and treating each increment as an equivalent point source. In like manner leakage from a volume-distributed source is usually represented by volume-distributed point sources or by points on the surface of the volume source. As an application of these data, consider the following problem:

Find the flux density of neutrons lying above $2 \mathrm{MeV}$ at a penetration depth of $60 \mathrm{~cm}$ in water from a small (assumed point) fission source generating 1 watt of power. The solution is obtained by multiplying the 60-cm differential spectrum curve (Fig. 6.1) by the source strength (converted to neutrons $\mathrm{sec}^{-1}$ ) and then integrating over energies above $2 \mathrm{MeV}$ :

$\mathrm{S}$ (source strength, neutrons $\left.\sec ^{-1}\right)=\left(3.1 \times 10^{10}\right.$ fissions $\sec ^{-1}$ watt $\left.^{-1}\right)$

$$
\begin{array}{r}
\times\left(2.47 \text { neutrons fission }{ }^{-1}\right) \\
=7.56 \times 10^{10} \text { neutrons sec }^{-1} \\
\Phi_{60}=\frac{7.56 \times 10^{10}}{4 \pi(60)^{2}} \int_{2}^{E_{\max } 4 \pi r^{2} N_{0}(r, E) d E}
\end{array}
$$

where the integral is carried out over the 60-cm curve given in Fig. 6.1.

(b) Gamma Rays. The gamma-ray data generated by infinite-medium moments-method calculations have found wide application in shielding calculations. These data are quite extensive both in the number of materials and the variety of source types that have been considered. Extensive discussions of these calculations, their results, and their application are given by Goldstein. ${ }^{1.3}$ The gamma-ray penetration data are more extensive and also more precise than the neutron data. Goldstein has estimated the overall accuracy of the data at $\pm 20 \%$. Since backscattering for gamma rays is much less than for neutrons, interface effects are less important for gamma rays, and infinite-medium data can be applied to finite layers to a good approximation.

Examples of the differential spectra are shown in Appendix $L$ for the penetration of gamma rays of various energies through water and lead. One significant difference between these data and the neutron data discussed in 
the preceding section is that the photon data at a given range have been multiplied by $4 \pi r^{2} e^{\mu_{0} r}$, where $\mu_{0}$ is the linear absorption coefficient of the material at the source energy. Since the exponential factor accounts only for the attenuation of the unscattered component, the flux density at a given energy falls off less rapidly than $\mathrm{e}^{-\mu_{0} r}$. Thus these data, which have been multiplied by $4 \pi r^{2} e^{\mu_{0} r}$, increase with increased penetration.

These data have also been integrated over a flux-density-to-dose-rate response function and expressed as a ratio of total dose rate to the dose rate due to unscattered photons alone. These ratios, called dose buildup factors, are a more compact representation of the data that are useful for applications where biological dose is of primary importance and the spectrum is of only secondary interest. Buildup factors were discussed in Sec. 4.8.1 and values are found in Appendixes E and F. Applications of dose buildup factors are discussed in Sec. 6.4.1.

The data for water indicate that an equilibrium spectrum is established at very shallow penetrations and does not vary greatly, even at penetration depths approaching 20 mean free paths. The spectra for lead exhibit a wider variation in distribution with penetration depth. Differences in the shape of the $\mu_{0}$ vs. $E$ curves for water and lead are instructive in understanding the relative spectral shifts (see Chap. 3 ).

Differential spectra for gamma rays are applied to shield attenuation calculations in much the same manner as the neutron data. An added benefit in the case of gamma rays is the broader range of materials for which data are available and the apparent smooth variation of the data with atomic number, which allows interpolation between elements for which data are available. A variety of computer programs have been written to perform this interpolation, also to interpolate between source energies and penetration depths. One method of performing this interpolation is discussed in Sec. 6.3.4. These programs also integrate over a complex source geometry and estimate the combined effect of a mixture of elements in a shield. Integrations over a complex source are performed numerically by breaking the source into individual point sources representing volume increments and by breaking the source spectrum into individual monoenergetic sources representing energy increments. The programs are limited in that they consider only the materials lying in the straight-line path from a source point to the detector and also in that they must apply infinite-medium data and cannot account for the effects of interfaces along the path. The limitations are not severe in that interface effects are small $(\sim 10 \%)$ and may be compensated with appropriate corrections. 


\subsubsection{Monte Carlo}

The Monte Carlo method has an advantage over the moments method in that Monte Carlo techniques can accept a wider range of source types and can predict the angular distribution of the neutrons and gamma rays at the exterior surface or at any arbitrary internal point in the material. This feature, however, spreads the existing calculations through a wider range of possible parameter variations and thus decreases the probability of finding an existing set of data to fit a particular need.

With few exceptions, Monte Carlo has been used more for obtaining precise answers to individual problems than for the generation of parametric data. This is probably the most economical approach since, as mentioned previously, it would be difficult and expensive to obtain data for all foreseeable parameter combinations. Data have been generated, however, for the penetration of unit sources through finite slabs and through infinite media of some materials.

Monte Carlo neutron data have probably found wider application than gamma-ray data. This is due partially to the fact that the momentsmethod gamma-ray data have proven to be widely adaptable and have compared favorably with experiments and other analyses, thus obviating the need for Monte Carlo photon data.

With Monte Carlo techniques very complex penetration and reflection probabilities can be obtained. For example, consider a monoenergetic monodirectional beam incident on a laterally infinite slab at polar angle $\theta_{i}$ and azimuthal angle $\phi_{j}$ (Fig. 6.3). Penetration probability values, $\mathrm{P}\left(\theta_{i}, t, E_{k}, \phi_{j} ; R, \beta, \theta, \phi, E\right)$, and reflection probability values, $\alpha\left(\theta_{i}, E_{k}, \phi_{j}\right.$; $\left.R^{\prime}, \beta^{\prime}, \theta^{\prime}, \phi^{\prime}, E^{\prime}\right)$, are needed. In general practice, however, data of this nature are more expensive to obtain and more complex than most applications warrant. Furthermore, in any Monte Carlo study, as the number of variables increases, the accuracy of the differential data degenerates for a given total number of histories. Consequently the reflected energy $E^{\prime}$ can be eliminated by tabulating only the exit dose rate or the distance $R^{\prime}$ can be eliminated by integrating over the surface, or, indeed, all the exit parameters can be eliminated and only the total exit dose rate calculated.

If the calculational model described in the preceding text is being used to simulate a collimated narrow beam of radiation incident on a shield, the variation of intensity with the position of exit is sometimes an important parameter to the design of the shield. The same model can be used for broad-beam incidence with the principle of reciprocity. The reciprocal relation of adjacent entrance and exit points indicates that the integral of the 


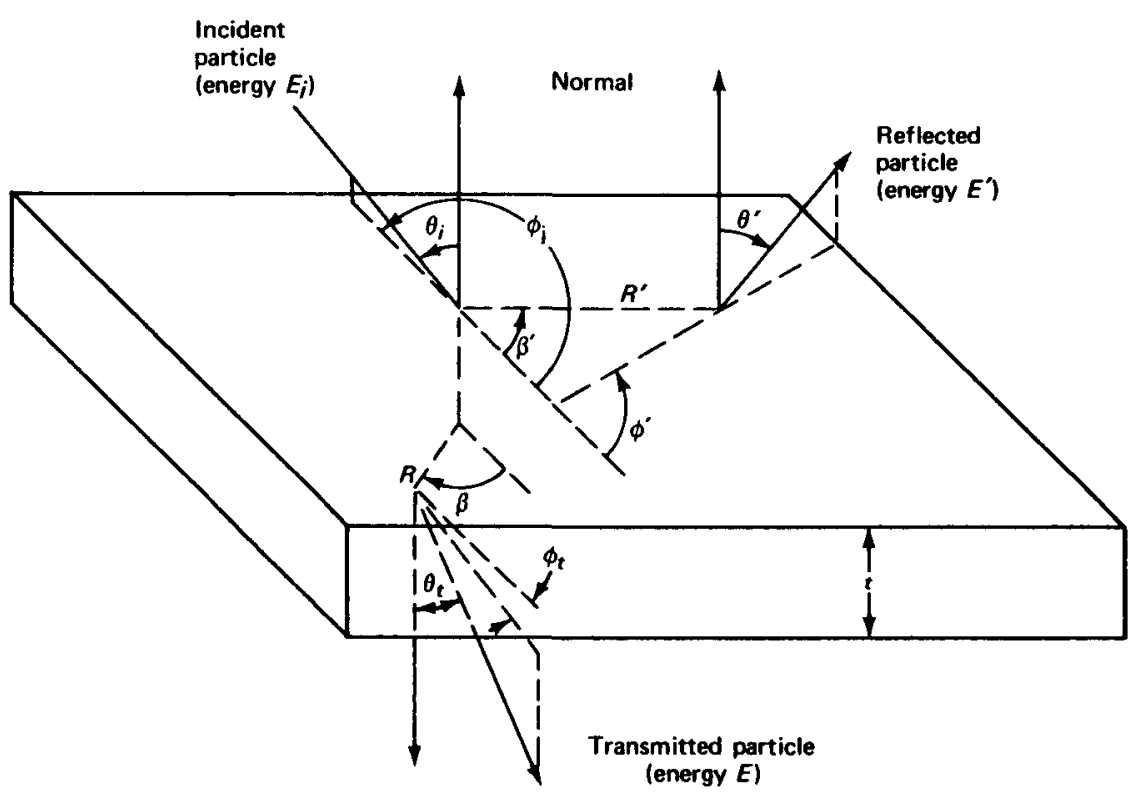

Fig. 6.3-Particle history parameters.

current density from a $1-\mathrm{cm}^{2}$ beam is equivalent to the leakage per square centimeter from a broad beam of the same intensity per square centimeter as the collimated beam. Thus the integral

$$
\int_{\substack{\text { over exit } \\ \text { surface }}} P\left(\theta_{i}, t, E_{k}, \phi_{j} ; R, \beta, \phi, E\right) d A
$$

where the transmission probabilities in the integral are for a collimated source of 1 particle/sec, yields the probability of leakage per square centimeter due to a broad-beam source of 1 particle $\mathrm{sec}^{-1} \mathrm{~cm}^{-2}$.

The parameter $E$ can be eliminated by integrating the leakage radiation to obtain a total particle current density, energy current density, or dose current. All these quantities are assumed to be dependent on the exit angle. There is little or no advantage to performing a parametric study by Monte Carlo unless the angular dependence is retained. The term dose current is used for the quantity that is given by the product of the differential angular current density (particles $\mathrm{cm}^{-2} \mathrm{sec}^{-1}$ steradian $^{-1}$ ) and the flux-density-todose-rate conversion factor. This quantity is a convenient way of expressing the radiation intensity in terms of a dose response while maintaining the angular dependence. Dose current, $D\left(\theta^{\prime}, \phi^{\prime}\right)$, is given by 


$$
D\left(\theta^{\prime}, \phi^{\prime}\right)=\int J\left(\theta^{\prime}, \phi^{\prime}, E\right) R(E) d E
$$

where $J\left(\theta^{\prime}, \phi^{\prime}, E\right)$ is the current density per steradian about the direction $\left(\theta^{\prime}, \phi^{\prime}\right)$ and $R(E)$ is the flux-density-to-dose-rate conversion factor. An application of the data reported in this form is illustrated by the fact that the dose rate at a point outside the shield from a differential area, $d A$, of the surface is

$$
D=\frac{1}{r^{2}} J\left(\theta^{\prime}, \phi^{\prime}, E\right) R(E) d E d A=\frac{D\left(\theta^{\prime}, \phi^{\prime}\right)}{r^{2}} d A
$$

where the detector is at distance $r$ in the direction $\left(\theta^{\prime}, \phi^{\prime}\right)$. This quantity is integrated over the surface of the shield to obtain a total dose rate at the external point. The variation of the direction of the detector with position on the shield surface usually necessitates a numerical integration. The directional energy-current density or particle-current density can be applied in the same manner as discussed for the dose current. However, the total energy or particle-flux density at a detector is not generally as important as the biological dose rate.

Sample results of a typical Monte Carlo study are shown in Fig. 6.4 from a report by Allen, Futterer, and Wright. ${ }^{4}$ These investigators calculate the total neutron-energy distribution (integrated over angle) and a total angular distribution (integrated over energy) but not doubly differential data, i.e., energy distribution within a particular angular interval. The data result from a calculation for $2-\mathrm{MeV}$ neutrons incident on a $60-\mathrm{cm}$-thick laterally infinite slab of water at an angle of $45^{\circ}$ from the slab normal. In this analysis the calculation is performed for a thick slab, and the neutrons are tagged on their first and subsequent penetrations through a given thickness plane. In this manner both initial and multiple penetrations are tallied, and flux density vs. thickness and flux density vs. depth are calculated simultaneously. The initial penetrations correspond to the flux density vs. thickness as though the slab had nothing beyond the plane; thus there is zero probability of scattering back into the material once the neutrons penetrate. Of course, the multiple-penetration calculations do contain the effects of backscattering. Figure 6.4 shows the curves of total flux density vs. thickness and flux density vs. depth resulting from these calculations. The data for flux density vs. thickness remain 30 to $50 \%$ below the data for flux density vs. depth over a wide range of material thickness, illustrating the effect at each depth into the slab of backscattering from deeper regions. 


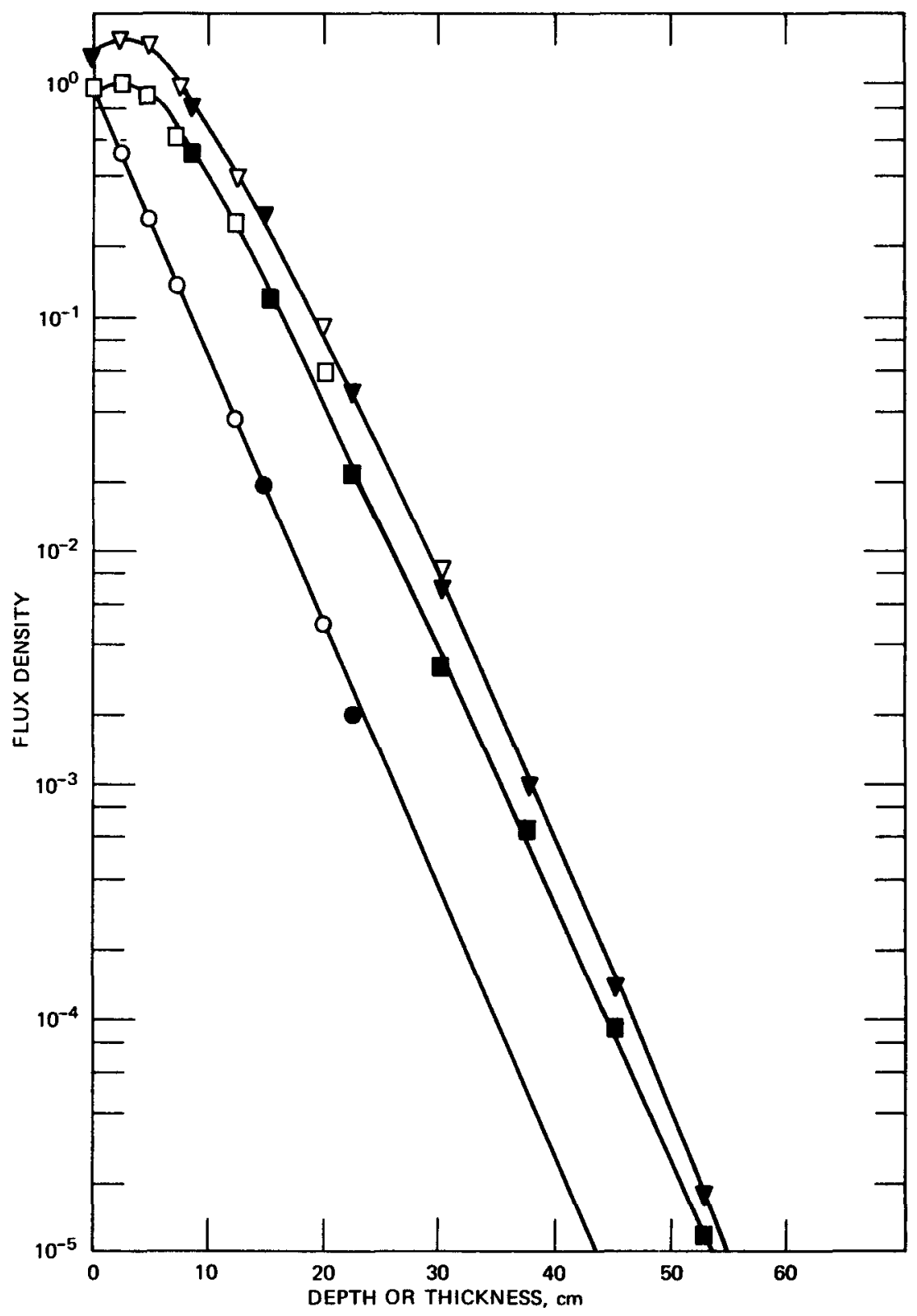

Fig. 6.4-Energy-dependent and total flux density vs. depth and total flux density vs. thickness. Slab, $60 \mathrm{~cm}$ water; incident energy, $2 \mathrm{MeV}$; angle of incidence, $0^{\circ}$. Flux density vs. depth: $\circ, 2 \mathrm{MeV} ; \nabla, 10 \mathrm{eV}$ to $2 \mathrm{MeV}$. Flux density vs. thickness: $\square, 10 \mathrm{eV}$ to $2 \mathrm{MeV}$. Hollow symbols, run 1; solid symbols, run 2. (From Allen, Futterer, and Wright. $^{4}$ ) 


\subsubsection{Measured Data}

Experimental data can be applied directly to some shielding problems by properly scaling source intensities and by adjusting for geometry differences. Experiments are essential for the verification of an analysis or design and for obtaining cross-section information. In the early days of nuclear technology, empirical data were widely used either directly in design work or as the basis of semiempirical analysis methods (see, for example, Sec. 6.4.2).

The source energies available naturally limit the parameter range of experimental results. Other parameters of the experiment, such as incident angle, exit angle, and energy at the detector, can be controlled or measured fairly accurately. Reactor-shield experiments are generally performed with either reactor sources or the cleaner source of fission neutrons and gamma rays from a fission-source plate. Other neutron sources include various pseudo-fission spectra (obtained in accelerators from boil-off in heavyelement bombardment) and a few monoenergetic sources obtained from low-energy charged-particle interactions. Notable among the more useful particle interactions are the $\mathrm{d}-\mathrm{t}$ interaction, obtained by accelerating deuterons into a tritium target, and the $\mathrm{d}-\mathrm{d}$ interaction, obtained by accelerating deuterons into a deuterium target. The $Q$ values (kinetic energy release) of these interactions are 17.58 and $3.26 \mathrm{MeV}$, respectively, and the neutrons evolved are approximately 14 and $2.5 \mathrm{MeV}$, respectively. Relatively low intensity neutron sources formed by combining $\alpha$ - or $\gamma$-emitting isotopes with light elements, such as deuterium, beryllium, lithium, boron, and fluorine, have been used for shield experimentation on a limited basis. Neutrons from these interactions are generally of little interest for shield measurements because they comprise mixed energies that do not approximate any spectrum to which the shield will be subjected.

Gamma-ray sources available for shielding experiments include fission gamma rays, fission-product gamma rays, and individual gamma-ray-emitting isotopes. Difficulties are encountered with fission gamma-ray sources because of the interference of secondary gamma rays from neutron interactions. The spectrum from fission products gives a lower average energy but is useful in testing containers for spent fuel elements and in weapons-fallout shielding analyses. Individual isotopes can provide useful monoenergetic photon sources for obtaining penetration data in the range below $1.5 \mathrm{MeV}$; however, most of the sources emitting gamma rays of energy above $1 \mathrm{MeV}$ emit undesirable background energies. Three of the isotopes frequently used for shield measurements are ${ }^{60} \mathrm{Co}$, which emits one 1.17 $\mathrm{MeV}$ and one $1.33-\mathrm{MeV}$ gamma ray per disintegration; ${ }^{137} \mathrm{Cs}$, whose 
daughter, ${ }^{137} \mathrm{Ba}$, emits one $0.662-\mathrm{MeV}$ gamma ray per disintegration; and ${ }^{144} \mathrm{Ce}$, which emits 17 gamma rays of energy $0.134 \mathrm{MeV}$ per 100 disintegrations accompanied by 2 at $0.1 \mathrm{MeV}, 2$ at $0.08 \mathrm{MeV}$, and 17 at $0.04 \mathrm{MeV}$.

Some of the experimental data quoted here were originally reported before the adoption of the terms kerma and absorbed dose (as defined in Chap. 2). We have converted the obsolete unit rep to the kerma unit on the basis that $1 \mathrm{rep}=100 \mathrm{ergs} / \mathrm{g}$. Examples of data obtained with fission sources, either in fission plates or reactors, are shown in Figs. 6.5 through 6.7. Figure 6.5 shows the neutron dose rate vs. distance through water as measured along the center line of the Lid Tank Shielding Facility. ${ }^{5}$ This curve has been widely used as a check on the validity of methods for calculating neutron penetration through hydrogenous media. Figure 6.6 shows typical results from an experiment using the Aerospace Shield Test Reactor $^{6}$ housed in an aboveground tank. The angular distribution of neutrons exiting slabs of borated polyethylene was measured as a function of slab thickness. A narrowly collimated detector was rotated on a circle of 4.9-m radius centered at a point on the exit face. The reactor neutrons incident on the shield were precollimated into a $15.2-\mathrm{cm}$ beam. Figure 6.7 shows the variation in thermal-neutron-flux density as a function of range in air from the Tower Shielding Reactor. ${ }^{7}$ The influence of the ground is seen as an increase in the thermal-neutron-flux density as the detector height was decreased from 46 to $1.0 \mathrm{~m}$.

Figure 6.8 is an example ${ }^{8}$ of time-of-flight neutron measurements in which a boil-off spectrum from a lead target in a linear accelerator was used. Spectra are shown for the source and for the neutrons penetrating slabs of zirconium hydride of various thicknesses. With this method a very fine angular resolution of the penetrating neutrons can be obtained. The spectra shown are for penetrations normal to the slab along the source-shielddetector axis.

Data from experiments to determine the transport of ${ }^{60} \mathrm{Co}$ gamma rays through air from an isotropic source are shown in Fig. 6.9, taken from Burton. ${ }^{9}$ A collimated detector was used for measuring the relative gamma-ray spectra for a variety of detector polar angles about the source-detector axis. The data are therefore on a per-unit-solid-angle basis, which means that the total counts per angular increment were obtained by dividing by the solid angle intercepted by the increment. Figure 6.10 shows the geometry and results obtained from measurements by Serduke and $\mathrm{Smith}^{10}$ of ${ }^{60} \mathrm{Co}$ gamma-ray penetration through iron. Here the angular 


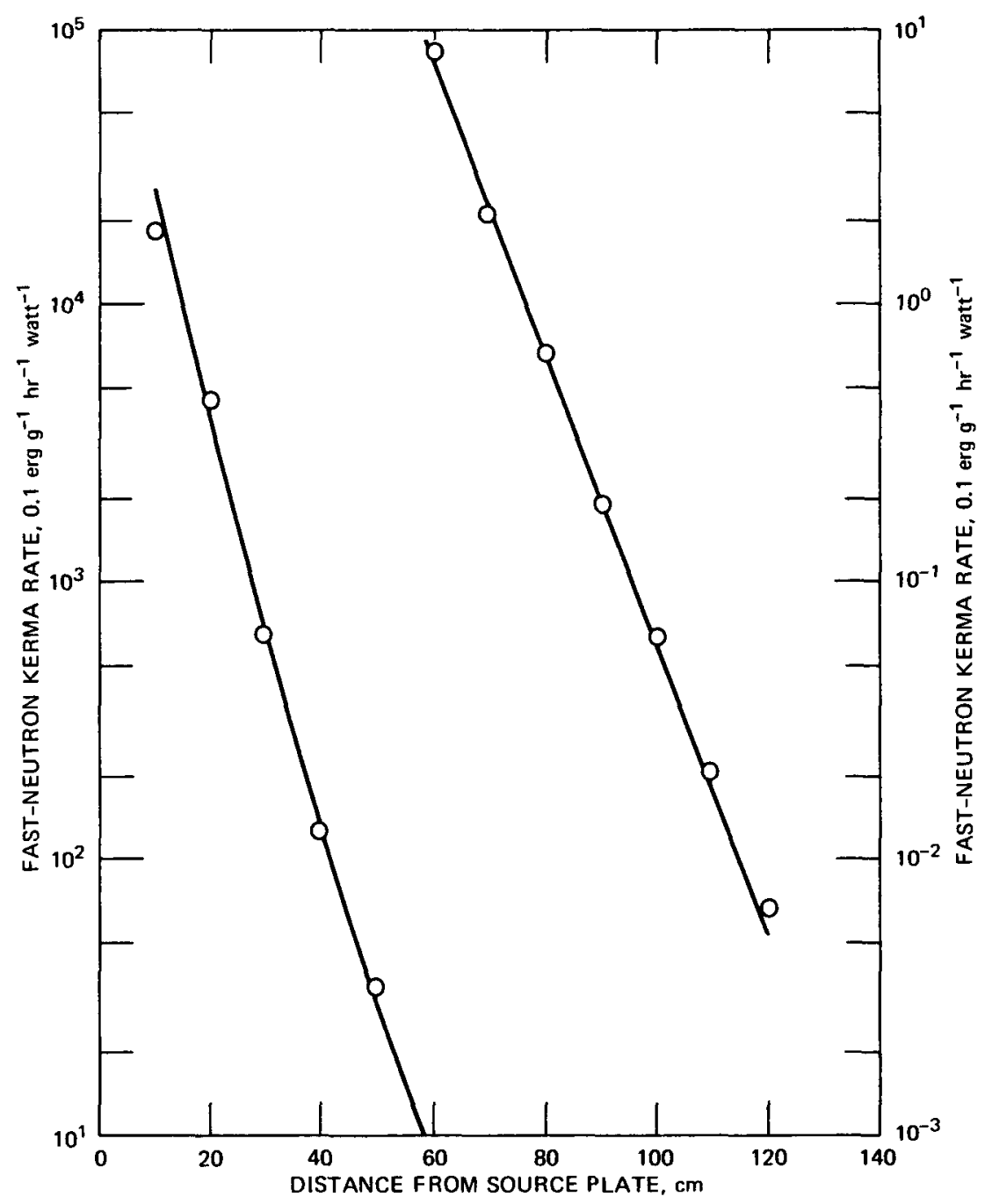

Fig. 6.5-Fast-neutron kerma rates measured in $\mathrm{H}_{2} \mathrm{O}$ vs. distance along the center line of the ORNL Lid Tank Shielding Facility. (From Casper. ${ }^{5}$ )

distribution is also given but this time as a cumulative distribution. This represents the integral of flux density over solid angle

$$
\int_{\pi / 2}^{\theta} \Phi\left(\theta^{\prime}\right) 2 \pi \sin \theta^{\prime} d \theta^{\prime}
$$

as the value of $\theta^{\prime}$ ranges from $\pi / 2$ to $\theta$. 


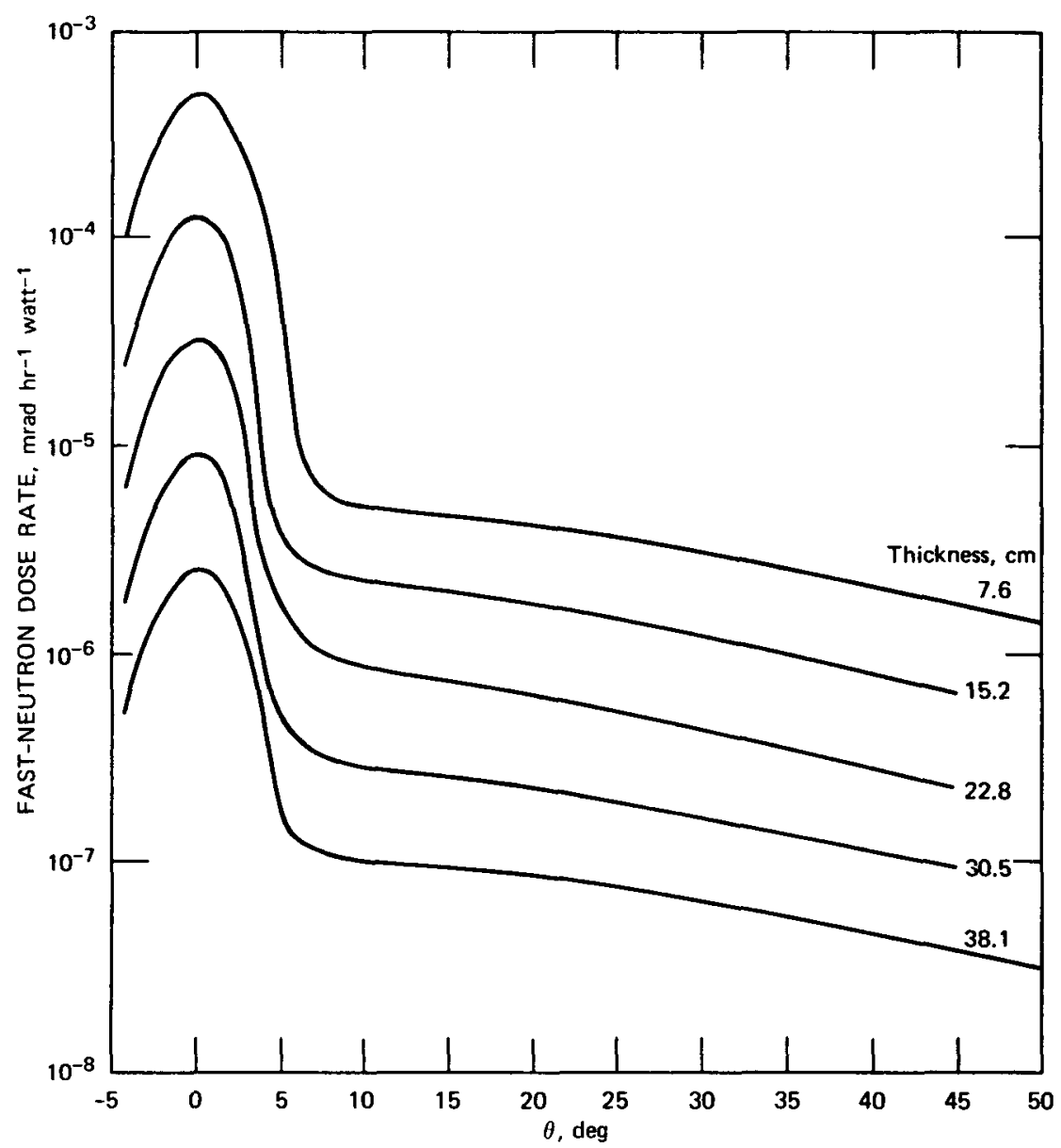

Fig. 6.6-Angular distribution of fast-neutron dose rate from particular thicknesses of $3 \%$ borated polyethylene slabs. (From Western. ${ }^{6}$ )

\subsubsection{Fitted-Parameter Data}

The data from a parametric shield analysis or experiment may comprise a bewildering collection of numbers that can be laborious to apply in design situations. It is, therefore, desirable to find some means of reducing the bulk of information without losing detail or accuracy. One solution is to fit analytical functions to the data. An advantage offered by this approach, in addition to the reduction of data, is that it may provide a means of analytically integrating over one or more parameters. As an example, consider the variation of exit current density with slab penetration thickness. 


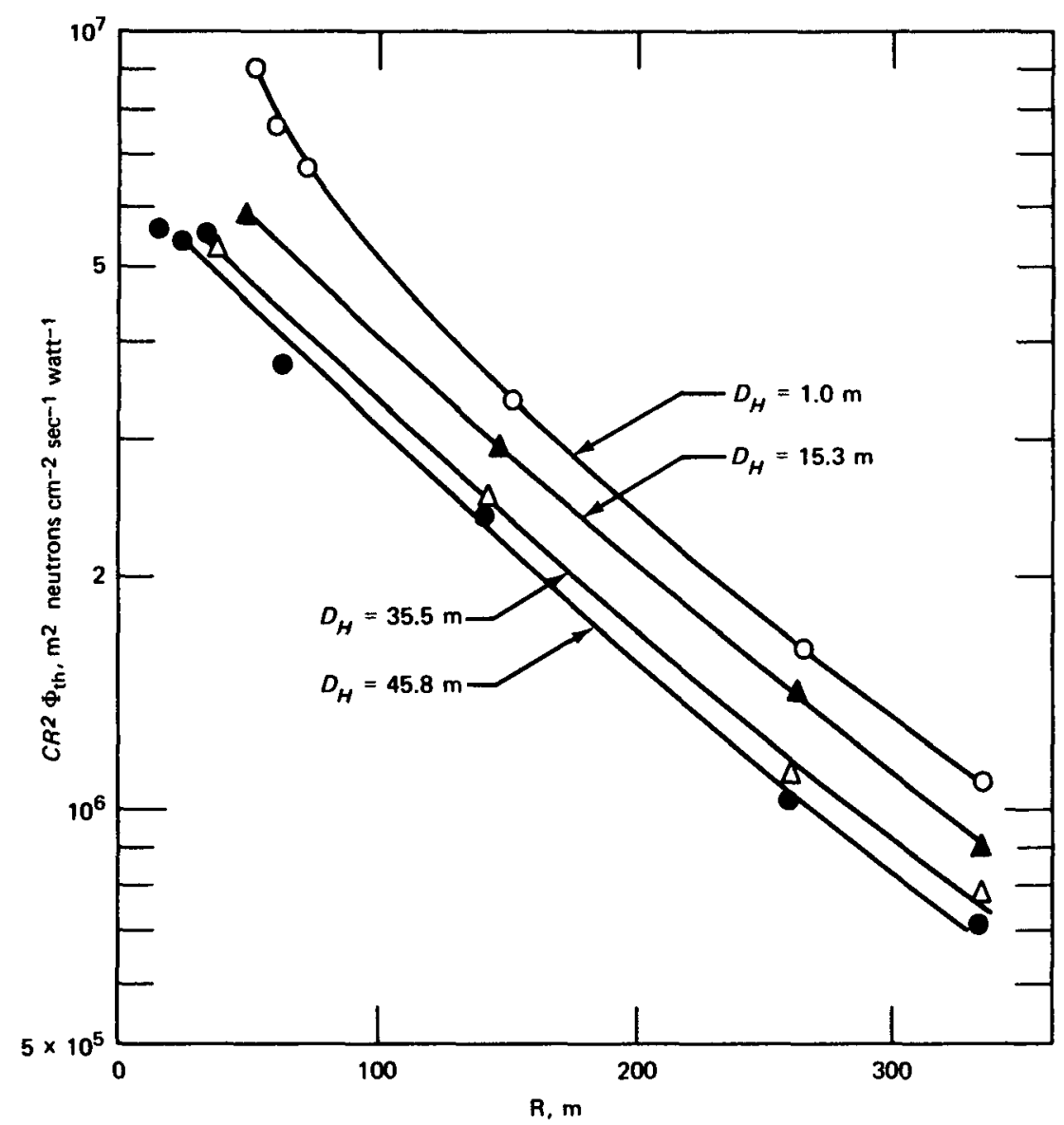

Fig. 6.7-CR ${ }^{2} \Phi_{t h}$ as a function of slant range (R) and detector height $\left(D_{H}\right)$. Reactor height, approximately $53 \mathrm{~m} ; C=4 \pi$ (0.093). (From Muckenthaler, Holland, and Maerker. ${ }^{7}$ )

This often fits an exponential function of the form

$$
J(t)=J_{0} e^{-a t+b} \text { particles } \mathrm{cm}^{-2} \sec ^{-1}
$$

or in rare cases by a simpler exponent $(b=0)$. The variation with exit angle often approximates a function of the form

$$
J(t, \theta)=J(t) k\left[a+b(\cos \theta)^{c}\right] \text { particles } \sec ^{-1} \operatorname{steradian}^{-1}
$$

where the constant $k$ normalizes to unity the integral of the function over the $2 \pi$ solid angle at the exit face, that is, 


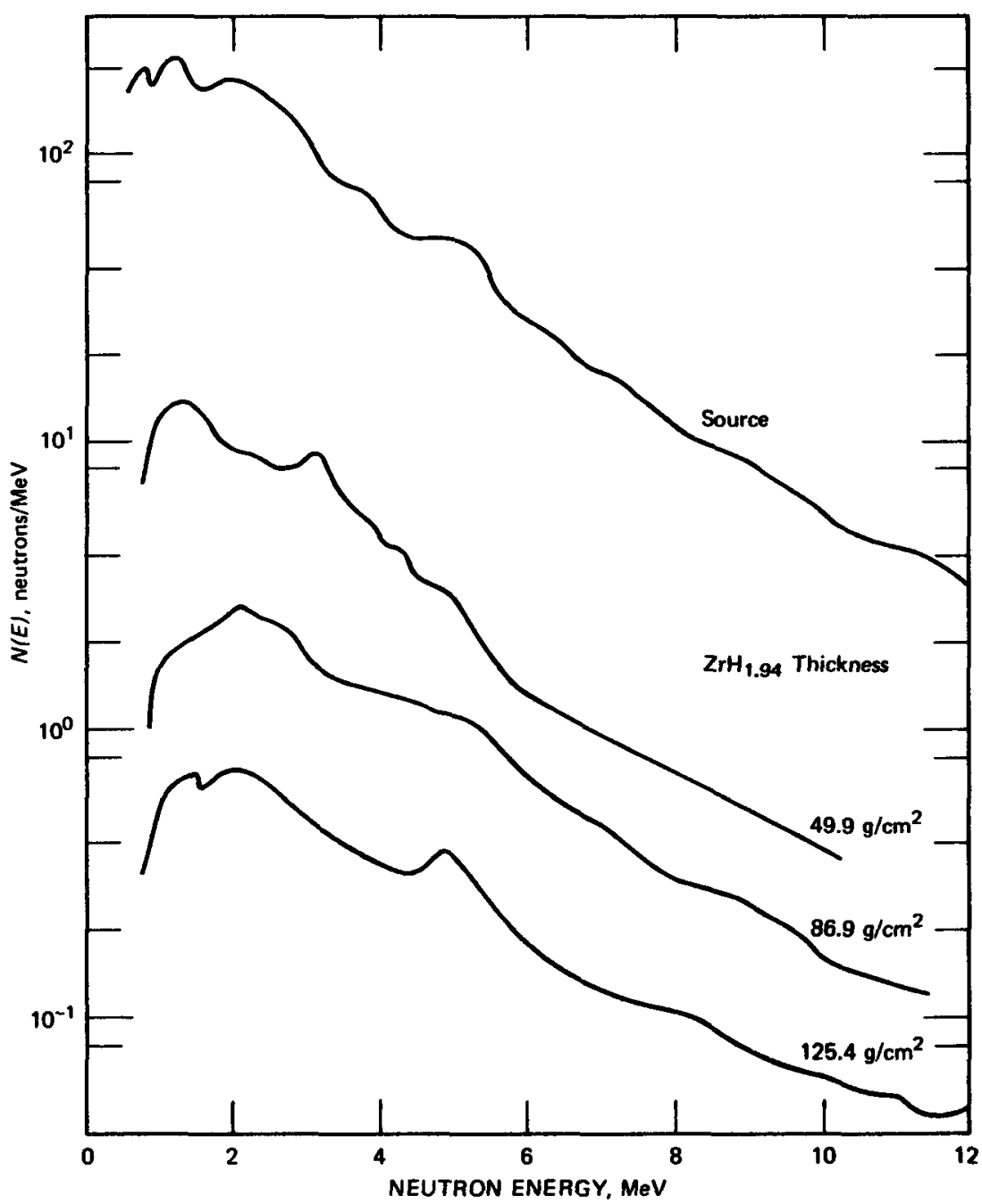

Fig. 6.8-Time-of-flight measurement of neutron spectra penetrating zirconium hydride. [From W. E. Selph, Transactions of the American Nuclear Society, 7(1): 42 (June 1964).]

$$
k \int_{0}^{\pi / 2}\left[a+b(\cos \theta)^{c}\right] 2 \pi \sin \theta d \theta=1
$$

Sometimes the angular distribution has been observed to be a function of penetration. When this occurs, the constants in the angular-distribution equation must be evaluated at each depth. At other times the distribution 


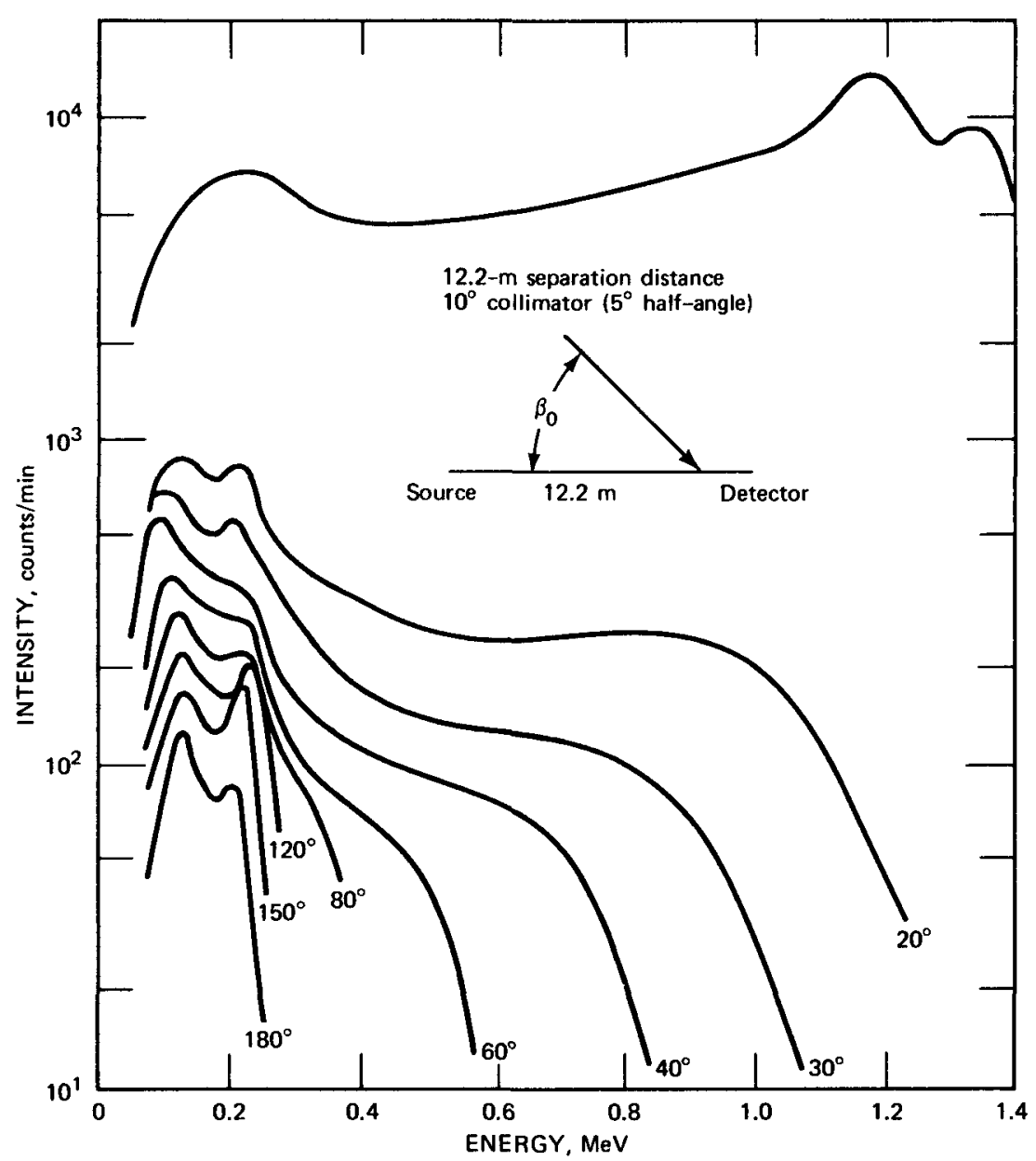

Fig. 6.9-Measured gamma-ray spectra and angular distribution from a ${ }^{60} \mathrm{Co}$ source penetrating through $12.2 \mathrm{~m}$ of air. (From Burton. ${ }^{9}$ )

has reached an equilibrium after penetrating a depth of one relaxation length or less, which suffices for most requirements.

The energy spectrum of the penetrating radiation is likely to require a more complex fitting function than the other distributions and consequently is usually left in numerical or graphical form.

The problem of fitting a function to the energy spectrum is avoided if the calculation requires only the total particle-current density, energy-flux density, or dose rate of the exit radiation. Use of these units may also involve an angular distribution. For example, an angular distribution of the 


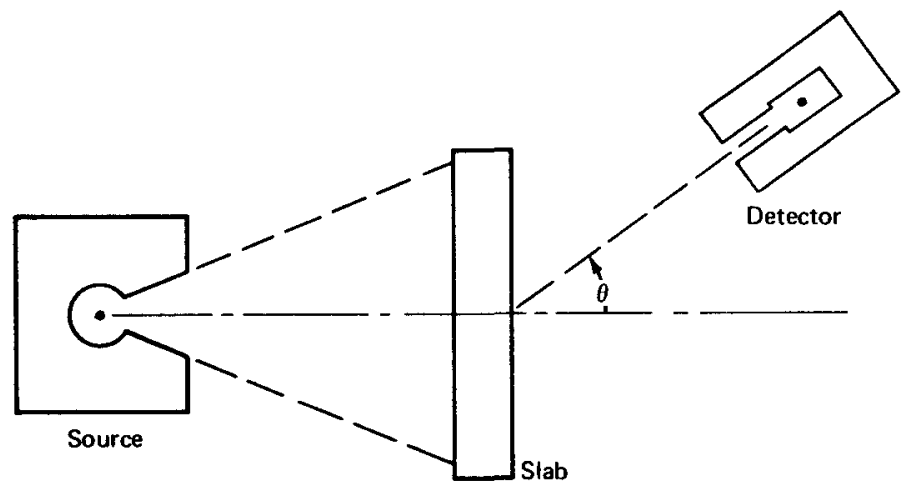

(a)

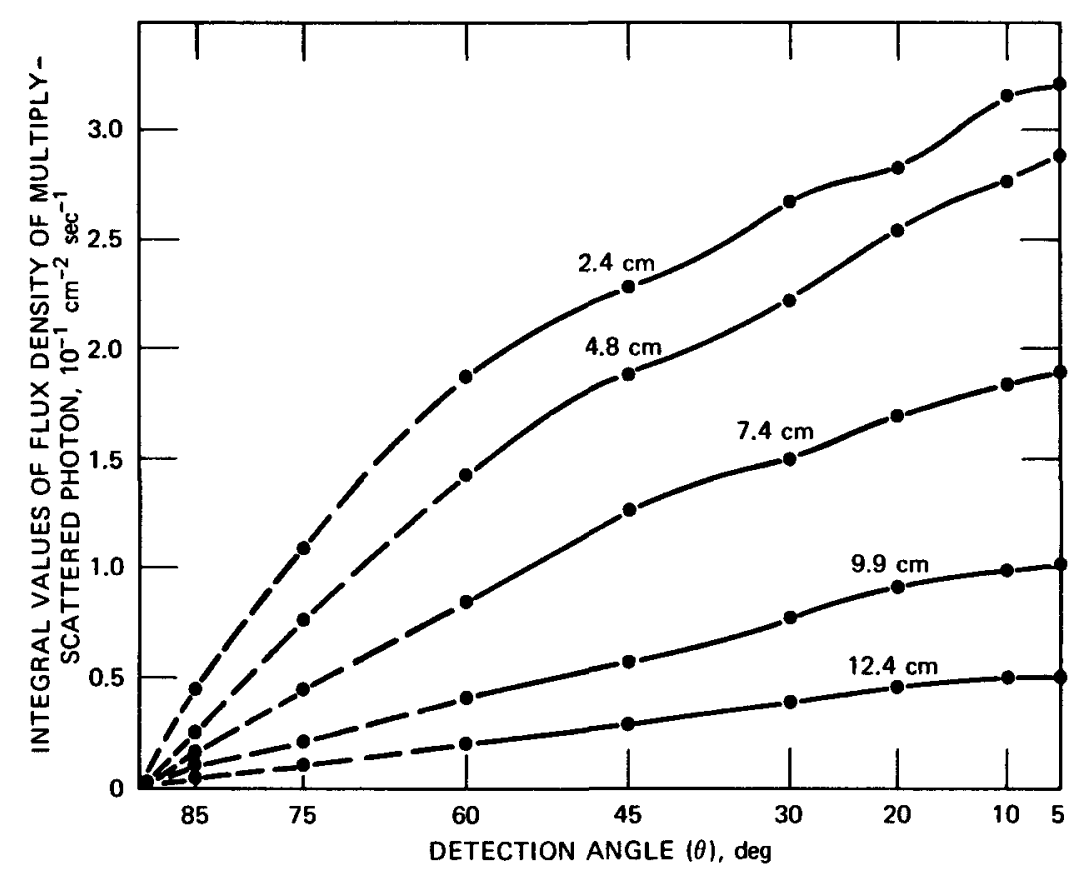

(b)

Fig. 6.10-A family of integral curves for five mean free paths of iron with ${ }^{60} \mathrm{Co}$ as the irradiation source. Integral values of multiply-scattered photon number-flux density are plotted as a function of detection angle. (a) Geometry. (b) Measurements. (From Serduke and Smith. ${ }^{10}$ ) 
dose rate can be obtained from that of the flux density or that of the current density. The dose rate due to flux density at angle $\theta$ is given by

$$
D(\theta)=\int_{E_{\min }}^{E_{\max }} \Phi(\theta, E) R(E) d E
$$

where $\Phi(\theta, E)$ is the flux density at energy $E$ per unit solid angle about the polar angle $\theta$ (azimuthal symmetry is assumed) and $R(E)$ is the flux-densityto-dose-rate conversion factor.

In addition, the quantity called dose current (Sec. 6.3.2) is defined as

$$
D_{c}(\theta)=\int_{E_{\min }}^{E_{\max }} J(\theta, E) R(E) d E
$$

where $J(\theta, E)$ is the current density per steradian per $\mathrm{MeV}$ exiting at polar angle $\theta$.

A set of data giving dose rate vs. depth and dose rate vs. angle is much more compact than triple differential data in depth, energy, and angle, particularly if one angular distribution can be used at most depths.

The variations of dose rate with thickness and angle can be combined into a single equation. In rare cases a function can be found that fits dose rate vs. angle, thickness, and energy of the incident particle.

In an attempt to fit all the parameters adequately, the empirical equations can become so complex and so difficult to apply that the advantage over raw tabular or graphical data is lost for hand calculations. Such complex equations may still have an advantage for computer applications in that the computer memory required for storing constants and evaluating the equations generally is less than that required for storing the original data and interpolating between data points.

An example of such a fitted function is one developed by Peterson to represent the moments-method differential gamma-ray-energy spectra for use in the $\mathrm{C}-17$ point-kernel computer program. ${ }^{11}$ The probability, $f$, of gamma rays' of source energy $E_{0}$ being degraded to penetration energy $E$ in penetrating a thickness $\mu_{1} t$ relaxation lengths was fitted by a quadratic in the variables $\mu_{1} t$ and the effective atomic number, $Z_{\mathrm{eff}}$, of the shield material. The form of the equation is

$$
\begin{aligned}
\ln \left[\frac{f\left(E_{0}, E\right)}{\mu_{1} t}\right]=A_{1}\left(\mu_{1} t\right)^{2}+A_{2}\left(\mu_{1} t\right)+A_{3}\left(\mu_{1} t\right) Z_{\text {eff }} & \\
& +A_{4} Z_{\text {eff }}+A_{5}\left(Z_{\text {eff }}\right)^{2}+A_{6}
\end{aligned}
$$


where a set of coefficients, $A_{i}$, is given for each combination of source and final energy. For particular initial and final energies, this equation provides a continuous probability distribution over penetration thickness and material composition. The effective atomic number, $Z_{\mathrm{eff}}$, is found by computing the quantity

$$
\mu=\frac{\sum_{i} \frac{\mu_{i} Z_{i}}{\sum_{i} Z_{i}}}{2}
$$

summing over the constituents of the material. This value can be used to select a $Z_{\text {eff }}$ from a plot of $\mu_{i}$ vs. $Z$. For most cases the $Z_{\text {eff }}$ so determined will be very close to the average atomic number of the material.

Although this form of curve fit results in a rather extensive library of coefficients, it is more efficient in application than attempting to store all the moments-method data. It can be compared to the use of the equation of a surface as opposed to a numerical listing of points on the surface.

Equations can also be derived to fit parameters appearing in equations for dose rate vs. material thickness. Discussions in Sec. 4.8.1 and the following section on the buildup-factor approach to the moments-method gamma-ray data present examples of such equations.

\subsection{SIMPLIFIED SOLUTIONS}

It is natural that attempts should be made to simplify the solution to problems as complex as those associated with radiation transport. It is also inevitable that most simplifications result in some sacrifice in the number of parameters or in the precision associated with the solution.

Simplifications of radiation-transport analyses can generally be classified as those resulting from (1) analysis of detailed analytical or experimental data which reveals a simpler means of obtaining some gross quantity, such as dose rate or total flux density, or (2) attempts to simplify the mathematical models of radiation-transport physics and thereby obtain (in an easier manner) data which are a little less precise but which are possibly as detailed as the more complex solutions. The use of gamma-ray-dose buildup factors and removal theory fall into the first category; the other methods are broadly classed in the second category. 


\subsubsection{Applications of Gamma-Ray Buildup Factors}

The definitions and theory of exposure and energy-deposition buildup factors were presented in Sec. 4.8.1. In this section we describe some typical applications.

For a thin shield and narrow beam (Fig. 6.11), the buildup factor is unity, $B=1$, because scattering events deflect the gamma rays out of the

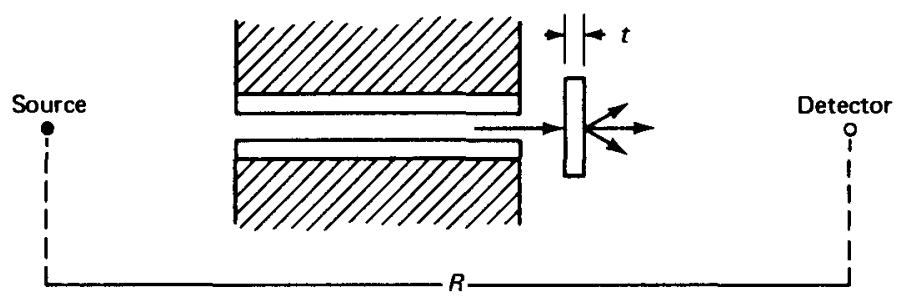

Fig. 6.11-Narrow-beam geometry.

beam and thus away from the detector. Where $t$ is small and the detector size can be neglected, the gamma-ray-flux density at the detector is given by

$$
\Phi=\frac{s}{4 \pi R^{2}} e^{-\mu(E) t}
$$

where $s$ is the source strength, $\mu(E)$ is the linear attenuation coefficient $\left(\mathrm{cm}^{-1}\right)$, and $t$ is the shield thickness $(\mathrm{cm})$.

For the geometry of Fig. 6.12, as the thickness of the shield is increased so that multiple scatters become more prevalent or as the width of the beam

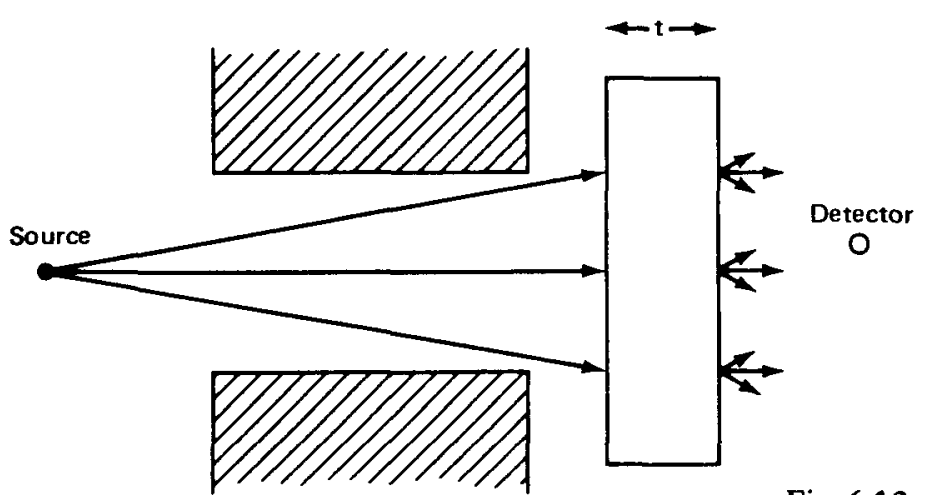

Fig. 6.12 - Wide-beam geometry. 
is increased so that single scatters may reach the detector, the buildup factor increases to values greater than 1 . The value of $B$ continues to increase with increasing beam width or shield thickness. A saturation point is reached when the size of the beam forms a $2 \pi$ solid-angle intercept at the detector.

The gamma-ray-flux density at the detector in this case is

$$
\Phi=\frac{s}{4 \pi R^{2}} B\left(E_{0}, \mu t\right) e^{-\mu\left(E_{0}\right) t}
$$

Note that the value of $B$ is dependent on the angular distribution of the source incident on the shield, the shield thickness, and the energy of the gamma rays. We should also recognize that this buildup factor applies only to the photon-flux density. As mentioned previously, the concept of buildup can be applied to any detector response, but, for a given set of conditions, the dose buildup factor, flux-density buildup factor, or energy-flux-density buildup factor are not necessarily numerically equal. To be generally useful therefore, buildup factors must be identified as to source-shield geometry, source energy, shield thickness, shield material, and detector response. An example is the dose buildup factor for an infinite-plane collimated source of 2-MeV gamma rays shielded by an infinite-plane slab of lead four mean free paths thick.

As an example of the application of buildup factors, consider the penetration of gamma rays from an infinite-plane source (Fig. 6.13). The source is assumed to emit monoenergetic gamma rays isotropically with a source strength of $S_{0}$ photons $\mathrm{cm}^{-2} \mathrm{sec}^{-1}$.

The dose rate at the detector is given by

$$
D_{z}=S_{0} F(E) \int_{0}^{\infty} \frac{e^{-\mu R}}{4 \pi R^{2}} B(E, \mu R) 2 \pi r d r
$$

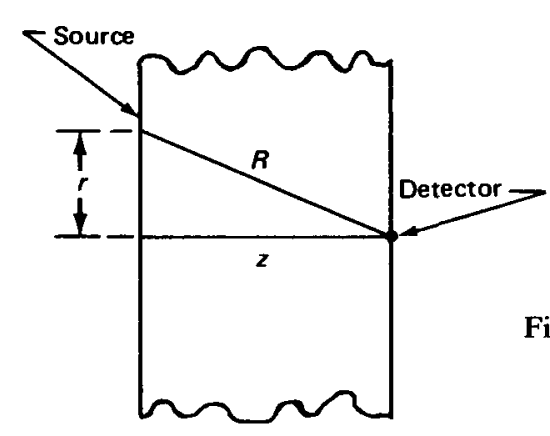

Fig. 6.13-Buildup from infinite-plane source. 
where $r$ is the radius along the source plane to the source element, $z$ is the distance of the detector from the source plane, and $F(E)$ is the flux-densityto-dose-rate conversion function. By changing the variable from $r$ to $R$ and replacing $B(E, \mu R)$ with the Berger formula, $B(E, \mu R)=1+C(E) \mu R e^{D(E) \mu R}$ (see Sec. 4.8.1), the expression becomes

$$
D_{z}=\frac{S_{0} F(E)}{2} \int_{z}^{\infty} e^{-\mu R}\left(1+C \mu R e^{D \mu R}\right) \frac{d R}{R}
$$

Introducing the exponential integral

$$
E_{n}(x)=x^{n-1} \int_{x}^{\infty} e^{-y} y^{-n} d y
$$

the expression becomes

$$
D_{z}=\frac{S_{0} I(E)}{2}\left[E_{1}(\mu z)+\frac{C}{1-D} e^{-(1-D) \mu z}\right]
$$

Graphs of the function $E_{n}(x)$ for $n=1,2$, and 3 are available in Appendix G.

\subsubsection{Applications of Neutron-Removal-Theory Kernels}

In the discussion of neutron-removal-theory kernels (Sec. 4.8.2), the Albert-Welton kernel ${ }^{12}$ was discussed as one method of handling neutron attenuation in hydrogenous shields. The Albert-Welton kernel is especially useful in the following two applications:

1. This kernel can be used to correct measured or calculated data when small changes are made in the heavy elements of a shicld. For example, suppose that a lead layer and a water layer surround a point source. If the lead layer is increased and the water thickness remains the same, the new dose rate is given by

$$
4 \pi r_{2}^{2} D\left(r_{2}\right)=4 \pi r_{1}^{2} D_{1}\left(r_{1}\right) e^{-\Sigma_{R} t}
$$

where $\Sigma_{R}=$ removal cross section for lead

$t=$ change in lead thickness $=r_{1}+t$

$r_{2}=$ new distance from source

$D\left(r_{2}\right)=$ new dose rate

$r_{1}=$ original distance

$D_{1}\left(r_{1}\right)=$ original dose rate 
Notice that the assumption is made that the water thickness (and its effect) remains unchanged. Consequently the dose rates are evaluated at different positions.

2. The kernel can be used to correct results obtained for one hydrogenous medium so that they apply for another hydrogenous medium. The assumption is that the hydrogen effect remains constant for a given hydrogen length, with the effects of other elements accounted on the basis of removal cross sections. Thus the hydrogen attenuation kernel in one medium is set equal to the hydrogen attenuation in the other, which gives

$$
4 \pi r_{2}^{2} D\left(r_{2}\right) e^{\Sigma_{R_{2}} r_{2}}=4 \pi r_{1}^{2} D_{1}\left(r_{1}\right) e^{\Sigma_{R_{1}} r_{1}}
$$

with the constraint, to ensure the equivalence of the hydrogen cffect, of

$$
\rho_{2} r_{2}=\rho_{1} r_{1}
$$

where $\rho_{2}=$ hydrogen density in medium for which $D\left(r_{2}\right)$ is unknown

$\rho_{1}=$ hydrogen density in reference medium for which $D_{1}\left(r_{1}\right)$ is known

$\Sigma_{R_{2}}=$ removal cross section for all clements except hydrogen in the medium bcing analyzed

$\Sigma_{R_{1}}=$ removal cross section for all elements except hydrogen in the reference medium

Combining the preceding equations yields

$$
D\left(r_{2}\right)=D_{1}\left(\rho_{2} r_{2} / \rho_{1}\right)\left(\frac{\rho^{2}}{\rho^{1}}\right)^{2} \exp \left(\Sigma_{R_{1}} \frac{\rho_{2} r_{2}}{\rho_{1}}-\Sigma_{R_{2}} r_{2}\right)
$$

A word of caution is appropriate here. The preceding equations represent a simple model of rather complex phenomena, and rather large errors are possible.

Although the differences are not great, values of the removal cross section measured in homogeneous-hydrogenous mixtures are slightly lower than those measured in slabs preceding hydrogenous materials. The value would be expected to vary slightly with the ratio of atomic densities of hydrogen to the material being measured. Table 6.1 shows the extent of these variations in some particular measurements given in the shielding volume of the Reactor Handbook. ${ }^{13}$ 
Table 6.1-COMPARISONS OF $\Sigma_{R}$ AND $\Sigma_{D R}$

\begin{tabular}{lccc}
\hline & $\Sigma_{R}$ measured in slab & \multicolumn{2}{c}{ Measured in homogeneous mixture } \\
\cline { 3 - 4 } Element & preceding $\mathrm{H}_{2} \mathrm{O}$ & $\Sigma_{D R}$ & Mixture \\
\hline Carbon & $0.81 \pm 0.05$ & $0.72 \pm 0.05$ & $\mathrm{C}_{12} \mathrm{H}_{22} \mathrm{O}_{11}-\mathrm{H}_{2} \mathrm{O}$ \\
& & $0.7-0.8$ & $\begin{array}{c}\mathrm{CH}_{2} \text { and } \mathrm{CH}_{2} \text { (moments- } \\
\text { method calculation) }\end{array}$ \\
Lithium & $1.01 \pm 0.05$ & 0.9 & LiH \\
Oxygen & $0.99 \pm 0.01$ & $0.92 \pm 0.05$ & Oil and water \\
\hline
\end{tabular}

In both neutron and gamma-ray calculations in all types of media, estimates of the approximate change in detector response with material thickness can occasionally be made with an effective relaxation length, $\lambda_{\text {eff }}$, for the material. The effective relaxation length is defined as the material thickness required to reduce the response by a factor of $e^{-1}$, or

$$
\frac{R}{R_{0}}=e^{-t / \lambda_{\text {eff }}}
$$

neglecting any differences in geometric attenuation. The value of $\lambda_{\mathrm{eff}}$ is determined from an examination of basic experimental or analytical data. The selection of a value for $\lambda_{\text {eff }}$ is fraught with hazards because it may be a function of many variables, such as distribution in energy, angle, and position of the source and the total thickness of the shielding material. Also, slight differences may be observed in the response as a function of thickness or in the response as a function of depth due to spectral shift with increasing penetration. Provided that data can be obtained under analogous circumstances, use of an effective relaxation length can provide a quick and inexpensive estimate.

\subsubsection{Other Point-Kernel Applications}

The point-kernel method has been discussed in preceding sections, but not all applications of the method have been covered. It should be emphasized that this method is not a means of obtaining basic penetration data but is rather a widely used means of applying these data. This method can be used in combination with appropriate point-source, infinite-medium data to determine either dose rate or spectrum at a point due to a distributed source. It can also be used in combination with total-cross-section data to obtain the uncollided component at a point due to a distributed source. 


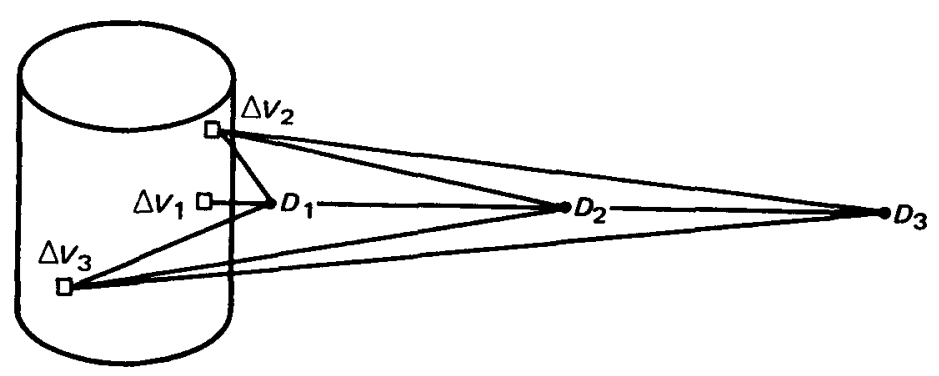

Fig. 6.14-Cylindrical point-kernel model.

Other applications are distinguished by the nature of the source being analyzed. In the last-collision method (to be discussed at the end of this section), the source is proportional to the local scattering density. In the analysis of secondary radiation, the source is proportional to the local density of radiative interactions.

(a) Numerical Techniques. The major steps in performing a numerical integration over the source volume are: (1) divide the source into increments that may be considered as point sources, (2) determine the source strength to be associated with each increment, (3) determine the attenuation along each source-detector ray, and (4) sum the contributions from individual sources to obtain a total detector response.

These tasks are considered in order in the following discussion. As with all forms of numerical integration, the number and size of the increments selected and the means of averaging within an increment are critical to the accuracy that can be obtained. Consider the three detector positions near the cylindrical source shown in Fig. 6.14. Assuming uniform distribution of the source strength throughout the cylinder, the nearest region will account for most of the response at detector $D_{1}$. Consequently nearby increments should be smaller in size than those at a greater distance so that the averaging in this region is at least as precise as that for more distant points. The increment size selected should be such that the distance from any point in the increment to the detector would be a very weak function of position in the increment. In addition, the material attenuation across the increment should be small. Under these conditions the midpoint of the volume element can be used as a representative average of all source points within the increment. The maximum acceptable increment size is therefore influenced both by the distance to the detector and by the relaxation length of the source region. For detectors at great distances from the source, the increment size is determined primarily by material relaxation length. For 
nearby or internal detectors, particular caution should be exercised in laying out local increments lest a source point be so nearly coincident with the detector that its effect overshadows all others. $f$ Other factors that will influence increment layout include source-density variations and shield inhomogeneities. The estimated relative importance of a given source region to total detector response should determine the precision in layout of the region.

The source strength to be associated with each of the assumed central point sources is given by the integral of source density over the increment or, if the source density is fairly uniform, by the product of source density times volume associated with the increment:

$$
n=\int_{\Delta v} \rho d v=\bar{\rho} \Delta v
$$

The attenuation along each source-detector ray is generally determined from a set of basic penetration information. The material thickness along the ray $\left(\mathrm{g} \mathrm{cm}^{-2}\right)$ is the criterion used in normalizing to the basic data. The effect of a material mixture or laminate along the ray is assumed to be the same as that of each of the component materials taken individually and in sequence with no perturbation due to interface effects.

Any of the various types of attenuation data that have been discussed in Secs. 4.8 and 6.3 may be used in this manner. The choice depends on whether spectrum or dose response is required at the detector and also on the availability of data for the elements involved. Depending on the type of attenuation data used, the summation of the contributions from the individual source points may be as simple as adding dose-rate values or as complicated as adding contributions to individual energy and angular increments.

(b) Attenuation Kernels from Monte Carlo Calculations. Results from Monte Carlo calculations (or from other transport methods) for dose-rate transmission through slab shields with an incident beam of neutrons can be quite useful when expressed in terms of attenuation kernels, i.e., as plots of transmission factors or dose-rate attenuation as a function of slab thickness. With such graphs it is a simple matter to estimate the fraction of the dose rate that is transmitted for each incident-energy group of neutrons, the total dose rate being the sum of the dose rates from all energy groups.

$\dagger 1 / 4 \pi r^{2}$ gets very large as $r$ gets very small. 
Clark, Betz, and Brown ${ }^{14}$ performed Monte Carlo calculations for monoenergetic beams of neutrons normally incident on slabs of ordinary concrete and also on a semi-infinite medium (half-space) of concrete. The neutron energies were $0.7,1.3,2,3,4,6,8,10,12$, and $14 \mathrm{MeV}$. The density of the concrete was assumed to be $2.43 \mathrm{~g} \mathrm{~cm}^{-3}$, and its composition, other than its water content, was representative of that given for ordinary concrete 01 in Report ANL-5800 (Ref. 15). The resulting curves for dose attenuation are shown in Appendix M (Figs. M-1 through M-10). In addition to being useful directly, these results can be helpful in adjusting the large body of infinite-medium neutron-attenuation data for concrete (for example, Trubey and Emmett ${ }^{16}$ ) to finite layers. After one or two relaxation lengths, the penetrating characteristics of neutrons in an infinite medium of concrete should differ very little from those of neutrons in a semi-infinite medium. Therefore data such as is shown in Figs. M-1 to M-10, which in all other respects appear to be appropriate for application to a particular situation, can be adapted to a finite system by correcting the data in proportion to the ratio of the curve for the semi-infinite medium (dashed curve) to the curve for the slab configuration (solid curve) at the proper penetration distance and energy.

Other useful results were obtained by Allen and Futterer, ${ }^{17}$ who determined the attenuation of the multicollision dose rate in the materials listed in Table M-1 due to monoenergetic neutron beams incident at various angles. The neutron energies used were $5,3,2,1$, and $0.5 \mathrm{MeV}$, and the results are plotted in Figs. M-11 through M-15. In order to use these curves, we must know the multicollision dose rate at the inner surface of a slab of one of these materials due to neutrons incident in a broad beam at an angle (or angle band) and energy (or energy band) close to the angle and energy for which the attenuation data are given. The attenuation factor appropriate to the material, thickness, energy, and angle is read from the curve, and the incident dose rate multiplied by that factor should approximate the dose rate that has penetrated the slab.

(c) Formulas Derived from Kernel Integrations. Transformations of source geometries with kernel integrations are elucidated by Blizard, Foderaro, Goussev, and Kovalev. ${ }^{18}$ They have derived equations to relate dose rate (or other nondirectional radiation quantities) from one type of source geometry to that of another. For example, the dose rate from a finite disk source may be related to either that from a point source or that from an infinite-plane source. A cylindrical source may be shown equivalent to a line source buried within the cylinder. Additionally, the attenuation of various 
sources in passing through slab shields can often be expressed in terms of standard integral equations whose values are well known as a function of the parameters involved. The formulas derived in this manner are useful in the evaluation of the recurring regular geometry problems that may require only a preliminary estimate of shield effectiveness. The exponential integral functions (Eq. 6.4-5) are involved in the formulas and are given for reference in Appendix G. The function

$$
F(\theta, \mu t)=\int_{0}^{\theta} e^{-\mu t \sec \theta^{\prime}} d \theta^{\prime}
$$

is known as Sievert's integral and is occasionally involved. Numerical values (detailed plots) are given by Blizard et al. ${ }^{13,18}$ We will summarize some useful cases using the following symbols for source intensities:

Volume sources $\left(S_{v}\right)$, particles $\mathrm{cm}^{-3} \sec ^{-1}$

Surface sources $\left(S_{a}\right)$, particles $\mathrm{cm}^{-2} \mathrm{sec}^{-1}$

Line sources $\left(S_{l}\right)$, particles $\mathrm{cm}^{-1} \mathrm{sec}^{-1}$

Point sources $\left(S_{0}\right)$, particles $\sec ^{-1}$

Shield penetration depth is denoted by $(\mu t)$ as though only one material were involved. Where there is more than one shield material, the symbol should be interpreted as $\Sigma \mu_{i} t_{i}$.

Basic point-source kernels are

$$
\Phi=\frac{S_{0}}{4 \pi R^{2}}
$$

for the direct-beam (uncollided) component of an unshielded source,

$$
\Phi=\frac{S_{0} e^{-\mu t}}{4 \pi R^{2}}
$$

for the direct-beam component with an intervening absorbing shield, and

$$
\Phi=S_{0} B(\mu t) \frac{e^{-\mu t}}{4 \pi R^{2}}
$$

for the total (direct and scattered) component with an intervening absorbing and scattering shield where $B(\mu t)$ is the buildup factor for shield thickness $(\mu t)$ at the source energy. 
An element $d l$ in an unshielded line source (Fig. 6.15) gives

$$
d \Phi=\frac{S_{l} d l}{4 \pi(a \sec \theta)^{2}}
$$

but, since

$$
\begin{gathered}
d l=a \sec ^{2} \theta d \theta \\
\Phi=\frac{S_{l}}{4 \pi a}\left(\theta_{1}+\theta_{2}\right)
\end{gathered}
$$

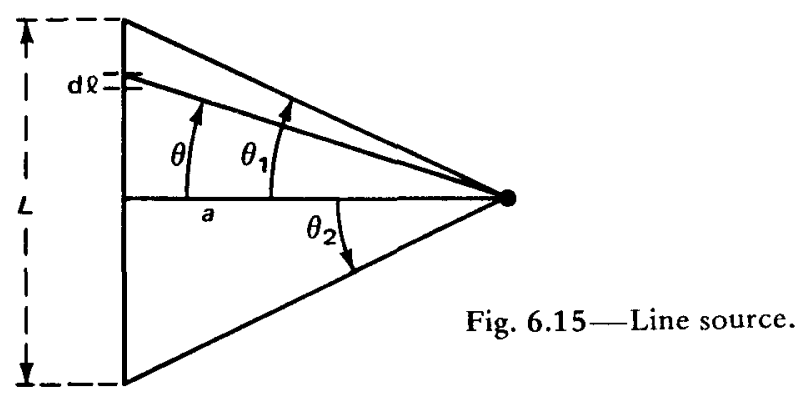

If a shield is placed between the line source and the detector, the uncollided flux density is given by

$$
\begin{gathered}
d \Phi=\frac{S_{l} d l e^{-\mu t \sec \theta}}{4 \pi(a \sec \theta)^{2}} \\
=S_{l} \frac{e^{-\mu t \sec \theta}}{4 \pi a} d \theta \\
\Phi=\frac{S_{l}}{4 \pi a}\left(\int_{0}^{\theta_{1}} e^{-\mu t \sec } d \theta+\int_{0}^{\theta_{2}} e^{-\mu t \sec \theta} d \theta\right) \\
=\frac{S_{l}}{4 \pi a}\left[F\left(\theta_{1}, \mu t\right)+F\left(\theta_{2}, \mu t\right)\right]
\end{gathered}
$$

In like manner the integral over a disk source (Fig. 6.16) can be evaluated in terms of the exponential functions, $E_{n}(x)$. 


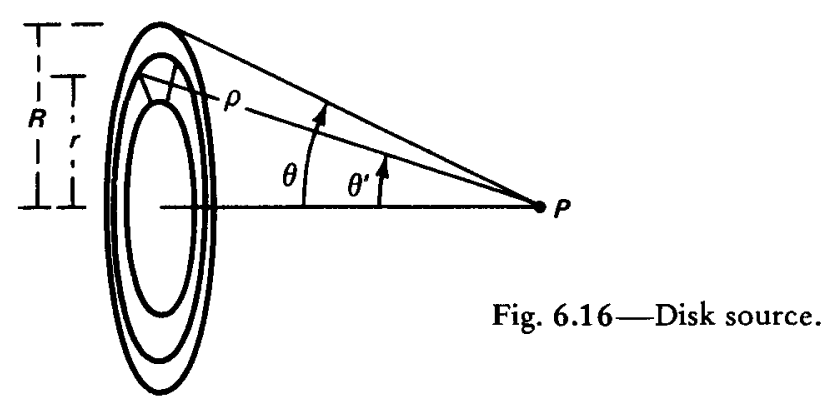

At point $P$ on the axis of the disk, the uncollided flux-density contribution of the source increment in a differential ring at radius $r$ from the center is given by

$$
d \Phi=\frac{S_{a}(2 \pi r d r)}{4 \pi \rho^{2}} e^{-\mu t \sec \theta^{\prime}}
$$

Substituting $\rho^{2}=r^{2}+a^{2}, \rho d \rho=r d r$, and $\sec \theta^{\prime}=\rho / a$

$$
d \Phi=\frac{S_{a}}{2} \frac{d \rho}{\rho} e^{-\mu t \rho / a}
$$

or, if $\mu t \rho / a \equiv \mathrm{t}$,

$$
\begin{aligned}
\Phi & =\frac{S_{a}}{2} \int_{\mu t}^{\mu t \sec \theta} \frac{e^{-t}}{t} d t \\
& =\frac{S_{a}}{2}\left[E_{1}(\mu t)-E_{1}(\mu t, \sec \theta)\right]
\end{aligned}
$$

Similar although more complex expressions can be derived for volumedistributed sources, such as a cylinder or an infinite slab. ${ }^{18}$

(d) Special Applications. A first estimate of the scattered radiation can be obtained by performing a kernel integration over the density of first collisions within a medium. Consider Fig. 6.17 for a point source and slab shield. 


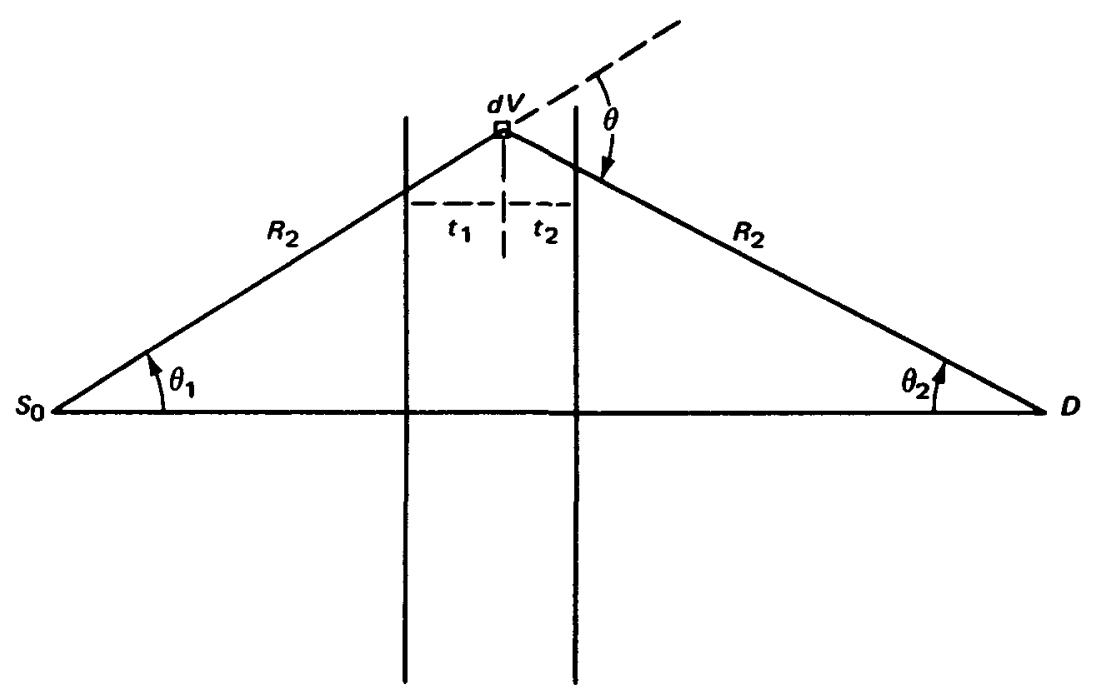

Fig. 6.17-Single-scattering geometry.

The uncollided flux density at $d V$ is

$$
\Phi_{1}=S_{0} \frac{e^{-\Sigma_{t} t_{1} \sec \theta_{1}}}{4 \pi R_{1}^{2}}
$$

where $\Sigma_{t}$ is the total cross section.

The density of first collisions within $d V$ is

$$
\Sigma_{s} \Phi_{1} d V=\frac{S_{0} \Sigma_{s} d V}{4 \pi R_{1}^{2}} \exp \left(-\dot{\Sigma}_{t} t_{1} \sec \theta_{1}\right)
$$

and the flux density at $D$ due to single scatters within $d V$ is

$$
d \Phi_{0}=\Phi_{1} d V \frac{\Sigma_{s}(\theta)}{R_{2}^{2}} \exp \left(-\Sigma_{t} t_{1} \sec \theta_{2}\right)
$$

where $\Sigma_{s}(\theta)$ is the value of the angular-distribution function (macroscopic differential-scattering cross section) for scattering at angle $\theta$, $\Sigma_{s}(\theta)=N \sigma_{s}(\theta)$. Integrating Eq. 6.4-30 over the entire scattering volume 
yields the singly scattered flux density at point $D$. This method gives a reasonable estimate of the total scattered flux density in cases where the scattering medium is thin with respect to the relaxation length of the radiation, i.e., where there is a small probability of multiple scattering within the medium. In such cases, especially for problems of air scattering, it is common practice to neglect the attenuation within the scattering medium of both the unscattered and the singly scattered radiation, which approximately corrects for the buildup of the multiply scattered component.

The last-collision method ${ }^{19}$ can be used in estimating the angular distribution of the radiation penetrating a shield. Prior knowledge of the approximate flux density vs. shield thickness must be available from other sources, however. The approach is similar to that for the single-scattering model except that a total flux density (rather than an uncollided flux density) is used in determining the density of scatters. The total-fluxdensity-vs.-penetration data are taken from any of the basic sources discussed in Sec. 6.3. In this method we assume that the total flux density within the slab is collimated in the direction of the incident beam and is of the same energy as the source. For a broad-beam flux density $\Phi_{0}$ normally incident on a slab (Fig. 6.18), where the flux density vs. penetration depth

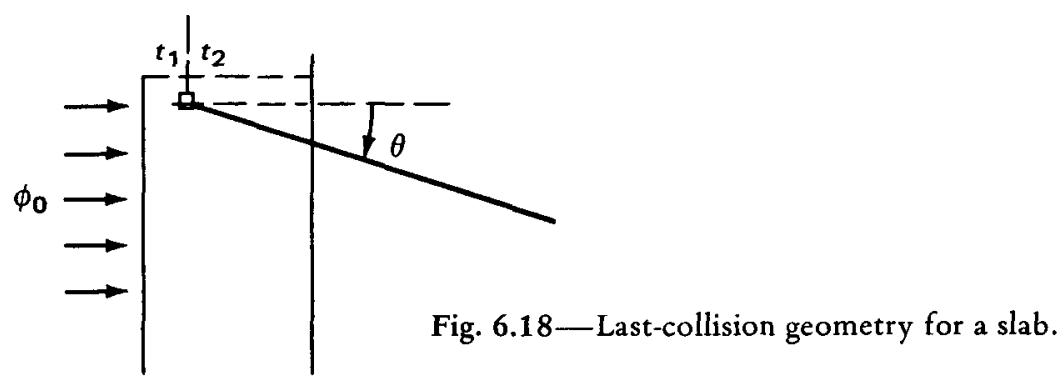

can be expressed in terms of an exponential function utilizing the removal cross section, $\exp \left[-\Sigma_{R}\left(E_{0}\right) t\right]$, the local scattering density is given by

$$
\Sigma_{s} \Phi d V=\Sigma_{s}\left(E_{0}\right) \Phi_{0} \exp \left[-\Sigma_{R}\left(E_{0}\right) t_{1}\right] d V
$$

If we assume that the energy after scatter, $E$, is given by an elastic scatter of particles at the initial energy and that the cross section for scattering into a unit solid angle about the direction of the detector is $\Sigma_{s}\left(E_{0}, \theta\right)$, then the flux density at the detector due to scattering in $d V$ is given by 


$$
d \Phi=\frac{\Sigma_{s}\left(E_{0}, \theta\right)}{r^{2}} \Phi_{0} \exp -\left[\Sigma_{R}\left(E_{0}\right) t_{1}+\Sigma_{t}(E) t_{2}\right] d V
$$

The term $\Sigma_{s}\left(E_{0}, \theta\right)$ is the differential scattering cross section. Equation 6.4-32 can be integrated over that portion of the shield lying within solid-angle increments as seen by the detector. A differential angular distribution at the detector can be obtained by expressing $d \Phi$ on a per-unit solid-angle basis. This is done by dividing by the differential solid angle $(2 \pi \sin \theta d \theta)$ and integrating along $r$.

The last-collision method can be applied to any geometry using numerical techniques, and in certain simple geometries the integration can be carried out analytically. In the slab-geometry broad-beam normal-incidence case, the flux density per steradian incident at angle $\theta$ per unit incident flux density at energy $E_{0}$ is

$$
\begin{aligned}
\phi\left(\theta, E_{0}\right)=\frac{\Sigma_{s}\left(E_{0}, \theta\right) \exp \left[-\Sigma_{R}\left(E_{0}\right) t_{m}\right]}{\Sigma_{t}(E)-\Sigma_{R}\left(E_{0}\right) \cos \theta} \\
\quad \times\left(1-\exp \left\{-\sec \theta\left[\Sigma_{t}(E)-\Sigma_{R}\left(E_{0}\right) \cos \theta\right] t_{m}\right\}\right)
\end{aligned}
$$

where $t_{m}$ is the slab thickness. Evaluation of this equation at representative angles provides an estimate of the differential angular distribution at the detector. Where a spectrum of radiation is incident, an evaluation can be made at each angle for each incident-energy group. The energy spectrum at the detector is estimated by logging the contributions from the particular incident-energy-scattering-angle combinations into appropriate energy bins at the detector. This approach ignores the spectral shift likely to be encountered as the radiation penetrates the shield. However, energy loss due to the last collision is included.

Consider another geometry: a detector on the axis of a cylindrical shield as shown in Fig. 6.19. Broad-beam incidence is assumed with all particles incident at angle $\beta$ with respect to a plane through the axis of the cylinder. In this case the scattering angle is $\theta$ and the reference angle at the detector is $\alpha$. The integral equation for scattered flux density per steradian per incident neutron at energy $E_{0}$ and $\operatorname{dircction} \beta$ is 


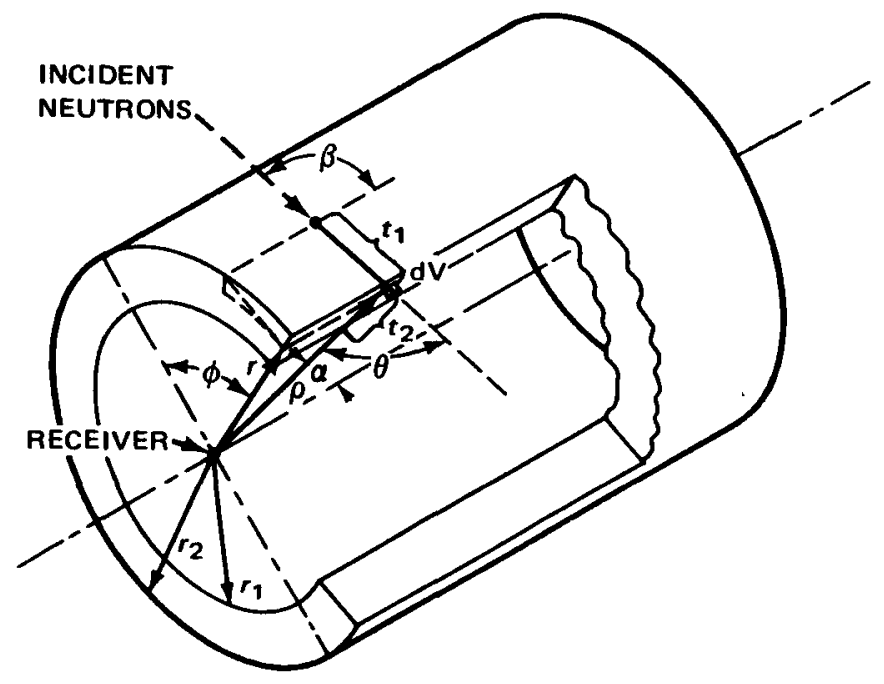

Fig. 6.19-Last collision for cylindrical geometry.

$$
\begin{aligned}
\Phi(\alpha)_{E_{0}, \beta}=\frac{1}{\pi \sin \alpha} \int_{r_{1}}^{r_{2}} \int_{0}^{\phi_{\max }} \Sigma_{s}\left(E_{0}, \theta\right) \exp \{ & {\left[\Sigma_{R}\left(E_{0}\right) t_{1}\right.} \\
& \left.\left.+\Sigma_{t}(E) t_{2}\right]\right\} d \phi d r
\end{aligned}
$$

where $t_{1}=\left[\left(r_{2}^{2}-r^{2} \sin ^{2} \phi\right)^{1 / 2}-r \cos \phi\right] \csc \beta$

$$
\begin{aligned}
t_{2} & =\left(r-r_{1}\right) \csc \alpha \\
\phi_{\max } & =\frac{\pi}{2}+\cos ^{-1}\left(r_{1} / r_{2}\right)
\end{aligned}
$$

In stating Eqs. 6.4-33 and 6.4-34, the flux density vs. penetration depth was given in terms of an exponential function. The method can also be applied with a basic set of spectrum-vs.-depth data, although the integrations could no longer be carried out analytically but would require a numerical solution. French ${ }^{19}$ demonstrates that the simplified approach (ignoring spectral shift) gives results in reasonable agreement with experiment and Monte Carlo analyses for $\mathrm{LiH}$ shields of about 10 - and $20-\mathrm{cm}$ thickness.

\subsubsection{Methods for Estimating Low-Energy Neutron-Flux Density}

Estimating the thermal-neutron-flux density as a function of position inside the shield presents a dilemma. The diffusion methods for analyzing 
neutrons are generally inadequate for predicting the transport of higher energy components, which are the source of thermal neutrons deep within the shield. Likewise, some of the transport methods are extremely inefficient for obtaining thermal-flux density because of the many decades of energy degradation that must be considered. Various combinations of transport and diffusion methods have been used to resolve this dilemma. The assumption is made that at some energy around $1 \mathrm{keV}$ the neutron angular distribution changes from forward-peaked, which is best treated by transport theory, to nearly isotropic, which is characteristic of diffusion. One of the earliest corrections to diffusion theory was the first-flight correction to the age in Fermi age theory. ${ }^{20}$ The correction is applied because the neutron cannot enter a process described by continuous slowing down (as Fermi age theory requires) until it has collided at least the first time.

A calculational model formulated by Blizard ${ }^{21}$ equates the slowingdown source to the rate of removal from the fast beam. The resulting equation for the equilibrium thermal-neutron-flux density (ignoring diffusion at thermal) is

$$
\Phi_{\mathrm{th}}(z)=\frac{J(z) e^{\tau / \lambda^{2}}}{\lambda \Sigma_{a}}
$$

where $\Phi_{\mathrm{th}}(z)=$ thermal-neutron-flux density

$J(z)=$ fast-neutron current density

$\lambda=$ relaxation length of $J(z)$

$\Sigma_{a}=$ thermal-neutron-absorption cross section

$\tau=$ average age of neutrons from removal to thermal

Obvious limitations of this model are the need for empirically determined values of $J(z), \lambda$, and $\tau$. These values are dependent on penetration depth, material, and geometry; thus values from one measurement do not necessarily apply to another set of conditions.

The development of more sophisticated multigroup diffusion models made it possible to obtain reasonably good estimates of the thermal-to-fastneutron ratio in a material, even though the penetration of the fast component could not be treated in this way. Haffner ${ }^{2}$ used such an approach and normalized the results to the fast-neutron distribution given by the Albert-Welton kernel. In this approach the corrected thermal-neutron flux is given by 


$$
\Phi_{\mathrm{th}}=D(R) \frac{\int K_{1}(E) \Phi(E, R) d E}{\int K_{2}(E) \Phi(E, R) d E}
$$

where $K_{1}(E)=$ response of detector used to measure thermal-neutron-flux density

$K_{2}(E)=$ response of a fast-neutron dosimeter

$D(R)=$ Albert - Welton kernel dose rate at position $R$

$\Phi(E, R)=$ flux density computed by the multigroup diffusion code

Good results were obtained with this method, but it is limited to cases where a reliable $D(R)$ kernel is available.

A refinement of the preceding method is to perform a multigroup diffusion calculation using as a source the neutrons recoiling from first collisions within the shield. Although this method has been used with some success by Schreiber and Kodras ${ }^{23}$ in predicting thermal-neutron distributions in beryllium oxide, difficulties are encountered in hydrogenous media owing to neutrons which have suffered one or more small-angle collisions but which have retained their directional character. Spinney ${ }^{24}$ used a removal kernel rather than an uncollided kernel for the diffusion source to solve this difficulty. The removal concept was applied to individual source energies in order to approximate a transport kernel for individual energy groups which accounted for both the uncollided and the small-angle scattered components. Spinney first used age theory to predict slowing down of a removed neutron and later used the concept in multigroup diffusion calculations. ${ }^{25}$ This combination of removal and diffusion theory has come to be known as the Spinney method, the theory of which is discussed in Sec. 4.9.

Trubey $^{2} 6$ proposed a direct coupling of a multigroup diffusion code to the results of a transport code such as RENUPAK (moments method), NIOBE (numerical integration of the Boltzmann equation), or Monte Carlo codes. In a trial calculation of the transfusion (transport diffusion) method, Trubey chose the RENUPAK transport code for fast-neutron calculations and the MODRIC diffusion code for the analysis of neutrons below $1 \mathrm{keV}$. MODRIC is a one-dimensional multigroup diffusion code written for the IBM 7090 computer. With RENUPAK, an energy spectrum of neutrons was calculated at various spatial points between 0 and $160 \mathrm{~cm}$. These values were then interpolated to provide spectra at 226 spatial points. Two separate determinations were made with lower energy limits of 1 and $10 \mathrm{keV}$ to test sensitivity. The spectral values were written on magnetic tape and used as a source distribution for the low-energy portion of the transfusion calculation. 
Variation of the cutoff energy from 1 to $10 \mathrm{keV}$ affected the thermal-flux density by only 1 to $5 \%$. Figure 6.20 compares the thermal-neutron-flux density calculated by Trubey using the transfusion method with measurements in water at the Lid Tank Shielding Facility. The experimental data have been converted to an equivalent point source, and all data have been normalized to unit-source strength. The transfusion method provides a much closer fit to the measurements than does the application of diffusion theory alone.

\subsection{APPLICATION OF KERNEL TECHNIQUE TO CALCULATIONS OF SECONDARY GAMMA-RAY DOSE $\dagger$}

Often a large fraction of the radiation dose rate behind reactor and shelter shields is the gamma-ray dose rate due to neutron capture and, in some instances, to inelastic scattering within the shield. If the spatial distribution of the neutron-flux density is known, the gamma-ray dose rate can be calculated for a large number of configurations by integrating the dose kernel over the source volume. With the kernel technique as exemplified by Eq. 4.8-4 and the slab geometry shown in Fig. 6.21, the dosc rate, $\Gamma(t, a, b)$, on the shield surface due to a distributed monoenergetic isotropic gamma-ray source, $S(x)$, bounded by planes at $a$ and $b$ is given by

$$
\Gamma(t, a, b)=G(E) \int_{a}^{b} S(x) d x \int_{0}^{\infty} B_{r}(\mu R) \frac{e^{-\mu R}}{4 \pi R^{2}} 2 \pi \rho d \rho
$$

where $x=$ one-dimensional spatial coordinate measured from the reference plane

$t=$ shield thickness

$\rho=$ radial distance to source point measured from the detector axis

$R=$ distance from the source point to the detector

$\mu=$ total macroscopic cross section for gamma rays of source energy $E$ $G(E)=$ flux density to dose rate in tissue conversion factor, which for conversion to rads/hr is $5.767 \times 10^{-5}\left[\mu_{a}(E) / \rho\right] E$, where $\mu_{a} / \rho$ is the mass energy absorption coefficient for tissue

$B_{r}(\mu R)=$ dose buildup factor for gamma rays of energy $E$

†This section by P. A. Stevens and D. K. Trubey is adapted from Methods for Calculating Neutron and Gamma Ray Attenuation, in Weapons Radiation Shielding Handbook, Chap. 3, USAEC Report DASA-1892-3, Oak Ridge National Laboratory, 1968. 


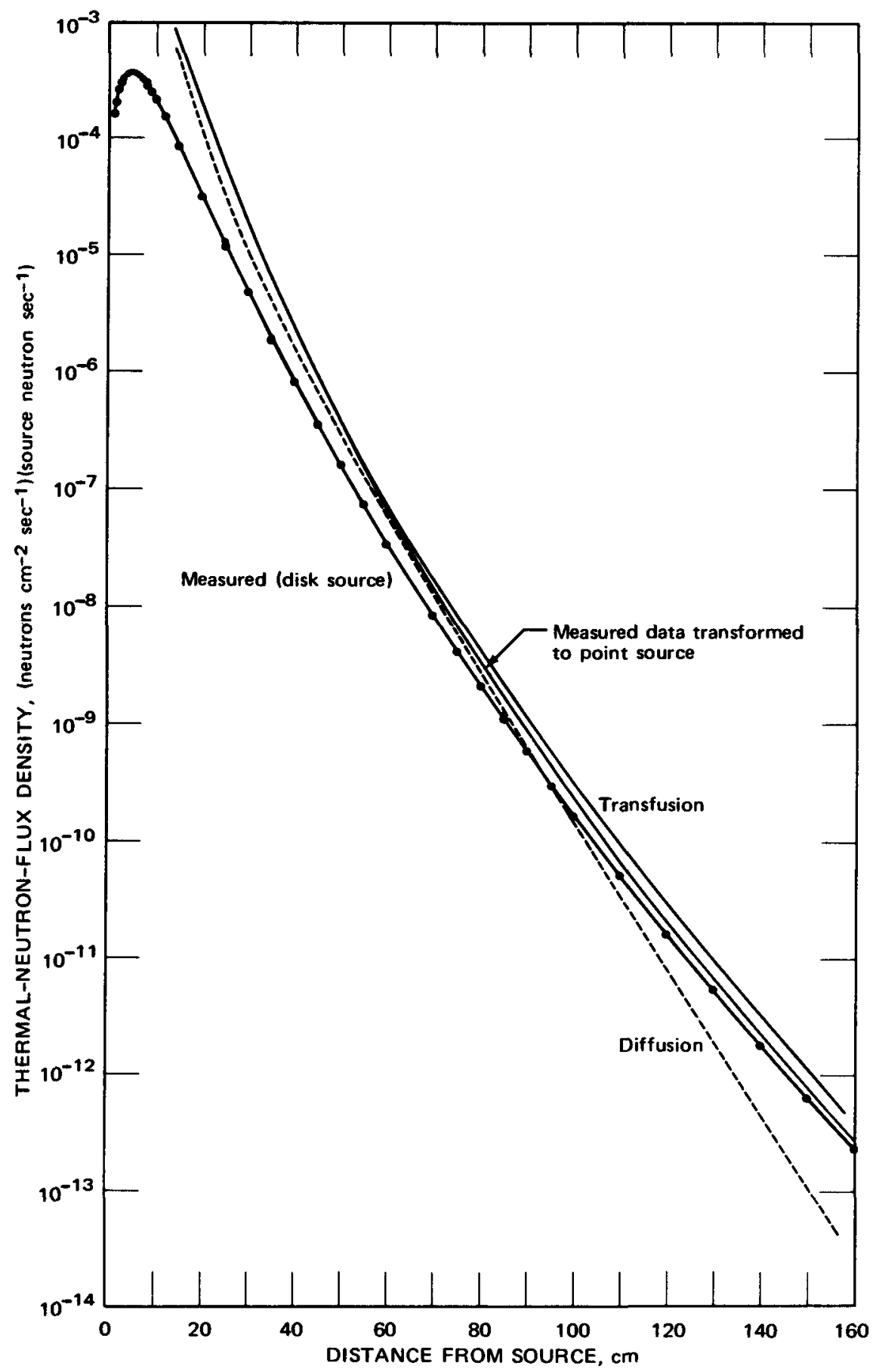

Fig. 6.20-Comparison of measured and calculated thermal-neutron-flux densities as a function of distance from the source. (From Trubey. ${ }^{26}$ ) 


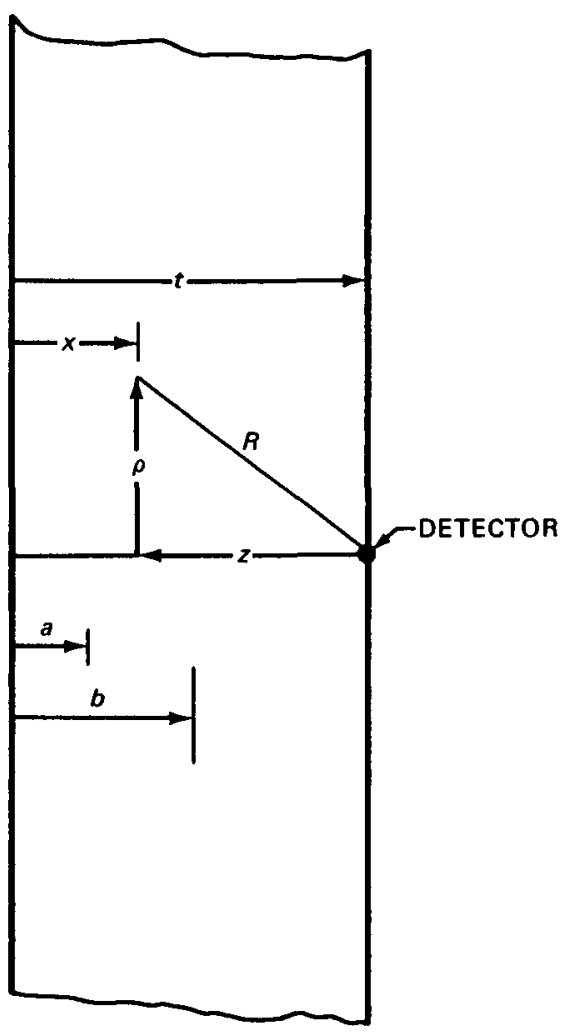

Fig. 6.21 - Geometry for integration over exponential source distribution.

Since $R^{2}=\rho^{2}+z^{2}$,

$$
\Gamma(t, a, b)=\frac{G(E)}{2} \int_{a}^{b} S(x) d x \int_{i-x}^{\infty} B_{r}(\mu R) \frac{e^{-\mu R}}{R} d R
$$

The gamma-ray source term usually can be represented quite well either by fitting with several terms or by piecewise fitting of the thermal-neutronflux density distribution f (or of the fast-flux density distribution if inelastic scattering is being considered) with a function of the form

$$
S(x)=S_{a} e^{-k x}
$$

tThe production of secondary gamma rays by the capture of nonthermal neutrons is usually insignificant for thermal reactors. It can be important in fast reactors and in thermal reactors where absorbing layers deplete the thermal flux. 
where $S_{a}$ is the gamma-ray source at $x=0$, which gives $S(x)$ in the interval $(a, b)$, and $k$ is the reciprocal of the effective neutron relaxation length. Usually $S_{a}$ can be calculated by

$$
S_{a}=y \Phi(a) \Sigma
$$

where $y$ is the number of photons of energy $E$ released per neutron capture (or per inelastic scattering), $\Phi(a)$ is the neutron-flux density (usually thermal-flux density for capture and fast-flux density for inelastic scattering), and $\Sigma$ is the macroscopic neutron cross section for thermal-neutron capture (or for inelastic scattering).

When exponential or polynomial forms of the buildup factor are used (see Sec. 4.8.1) together with the source description given by Eq. 6.5-3, then Eq. 6.5-2 can be integrated analytically, and very useful results can be obtained. In the following paragraphs examples of such integrations are given for two cases of interest: a slab shield of finite thickness $t$ and a semi-infinite shield $(t=\infty)$, the latter corresponding to a real problem in which the shield is very thick.

\subsubsection{Calculation for Slab Shield}

Trubey ${ }^{2}$ calculated the secondary gamma-ray dose rate for a slab shield using the Berger form of the buildup factor

$$
B_{r}(E, \mu R)=1+C(E) \mu R e^{D \mu R}
$$

used in Eq. 6.5-2. The equation then becomes

$$
\begin{aligned}
\Gamma(t, a, b)=\frac{G(E)}{2} S_{a} \int_{a}^{b} e^{-k x} d x & {\left[\int_{\mu(t-x)}^{\infty} \frac{\mathrm{e}^{-\mu R}}{\mu R} \mathrm{~d}(\mu \mathrm{R})\right.} \\
& \left.+\int_{\mu(t-x)}^{\infty} C(E) e^{\mu(D-1) R} \mathrm{~d}(\mu \mathrm{R})\right]
\end{aligned}
$$

where the uncollided dose ratc, $\Gamma_{0}(t, a, b)$, is represented by the first term and the scattered dose rate, $\Gamma_{s}(t, a, b)$, is given by the second term.

Letting $\mu(t-x)=y$ and integrating the first term of Eq. 6.5-6 by parts, we find the dose rate from the uncollided gamma-ray dose rate is given by 


$$
\begin{aligned}
\Gamma_{0}(t, a, b)= & \frac{G(E) S_{a} e^{-\alpha \mu t}}{2 \alpha \mu}\left(\left.e^{\alpha y} E_{1}(y)\right|_{\mu(t-b)} ^{\mu(t-a)}+\int_{\mu(t-b)}^{\mu(t-a)} e^{a y} \frac{e^{-y}}{y} d y\right) \\
= & \frac{G(E) S_{a} e^{-\alpha \mu t}}{2 \alpha \mu}\left\{e^{\alpha \mu(t-a)} E_{1}[\mu(t-a)]-E_{1}[(1-\alpha) \mu(t-a)]\right. \\
& \left.+E_{1}[(1-\alpha) \mu(t-b)]-e^{\alpha \mu(t-b)} \quad E_{1}[\mu(t-b)]\right\}
\end{aligned}
$$

where $\alpha=k / \mu$ and $E_{1}$ is an exponential function of the first order and is defined by

$$
E_{1}(x)=\int_{x}^{\infty} \frac{e^{-y}}{y} d y
$$

Appendix $G$ contains graphs and other details of the exponential functions. If $\alpha=1$ or 0 (case of uniform source distribution) or if $b=t$, indeterminate forms result which may be resolved by l'Hospital's rule, by series expansions, or by integrating Eq. 6.5-6 for $k=\mu, k=0$, and $b=t$, respectively. These cases are as follows:

For $b<t$ and $\alpha=0$

$$
\begin{aligned}
& \Gamma_{0}(t, a, b)=\frac{G(E) S_{a}}{2 \mu}\left\{\mu(b-a) E_{1}[\mu(t-b)]\right. \\
&\left.+\mu t E_{1}[\mu(t-a)]-\mu t E_{1}[\mu(t-b)]\right\}
\end{aligned}
$$

For $b<t$ and $\alpha=1$

$$
\begin{aligned}
\Gamma_{0}(t, a, b)=\frac{G(E) S_{a}}{2 \mu}\left\{e^{-\mu a} E_{1}[\mu(t-a)]\right. & \\
& \left.-e^{-\mu b} E_{1}[\mu(t-b)]+e^{-\mu t} \ln \frac{t-a}{t-b}\right\}
\end{aligned}
$$

For $b=t$ and $\alpha \neq 0$ or 1

$$
\begin{aligned}
& \Gamma_{0}(t, a, t)=\frac{G(E) S_{a}}{2 \mu \alpha}\left(e^{-\alpha \mu a} E_{1}[\mu(t-a)]\right. \\
& \left.-e^{-\alpha \mu t}\left\{E_{1}[\mu(1-\alpha)(t-a)]+\ln |1-\alpha|\right\}\right)
\end{aligned}
$$


For $b=t$ and $\alpha=0$

$$
\Gamma_{\mathbf{0}}(t, a, t)=\frac{G(E) S_{a}}{2 \mu}\left\{1+\mu(t-a) E_{1}[\mu(t-a)]-e^{-\mu(t-a)}\right\}
$$

For $b=t$ and $\alpha=1$

$$
\Gamma_{0}(t, a, t)=\frac{G(E) S_{a}}{2 \mu}\left\{e^{-\mu a} E_{1}[\mu(t-a)]-e^{-\mu t} \ln \gamma \mu(t-a)\right\}
$$

where $\boldsymbol{\gamma}=0.577215665 . .$, , Euler's constant.

For the special case of $b=t$ and $a=0$, Eq. 6.5-11 can be represented by

$$
\Gamma_{0}(t, 0, t)=\frac{G(E) S_{a}}{\mu} \psi_{0}(\mu t, \alpha)
$$

where

$$
\psi_{0}(\mu t, \alpha)=\frac{1}{2 \alpha}\left(E_{1}(\mu t)-e^{-\alpha \mu t}\left\{E_{1}[(1-\alpha) \mu t]+\ln |1-\alpha|\right\}\right)
$$

Equation 6.5-15 is plotted in Fig. N.1 as a function of the number of mean-free-paths $\mu t$ with $\alpha$ as a parameter. [Equations 6.5-9 through 6.5-15 can be used to calculate the total gamma-ray dose (uncollided + scattered) when the Taylor form of the buildup factor is used (see Sec. 4.8.1).]

Since $z=t-x$, the scattered dose rate behind a slab shield can be determined by expressing the second term of Eq. 6.5-6 (i.e., the Berger term) as

$\Gamma_{s}(t, a, b)=\frac{G(E) C(E) S_{a} e^{-\alpha \mu t}}{2} \int_{t-b}^{t_{-} a} d z \int_{z}^{\infty} e^{\alpha \mu z} e^{-(1-D) \mu R} d(\mu R)$

Integrating Eq. 6.5-16 gives

$$
\Gamma_{s}\left(t, a, b=\frac{G(E) C(E) S_{a} e^{-\alpha \mu t}}{2(1-D)(1-D-\alpha) \mu}\left[e^{-(1-D-\alpha) \mu(t-b)}-e^{-(1-D-\alpha) \mu(t-a)}\right]\right.
$$




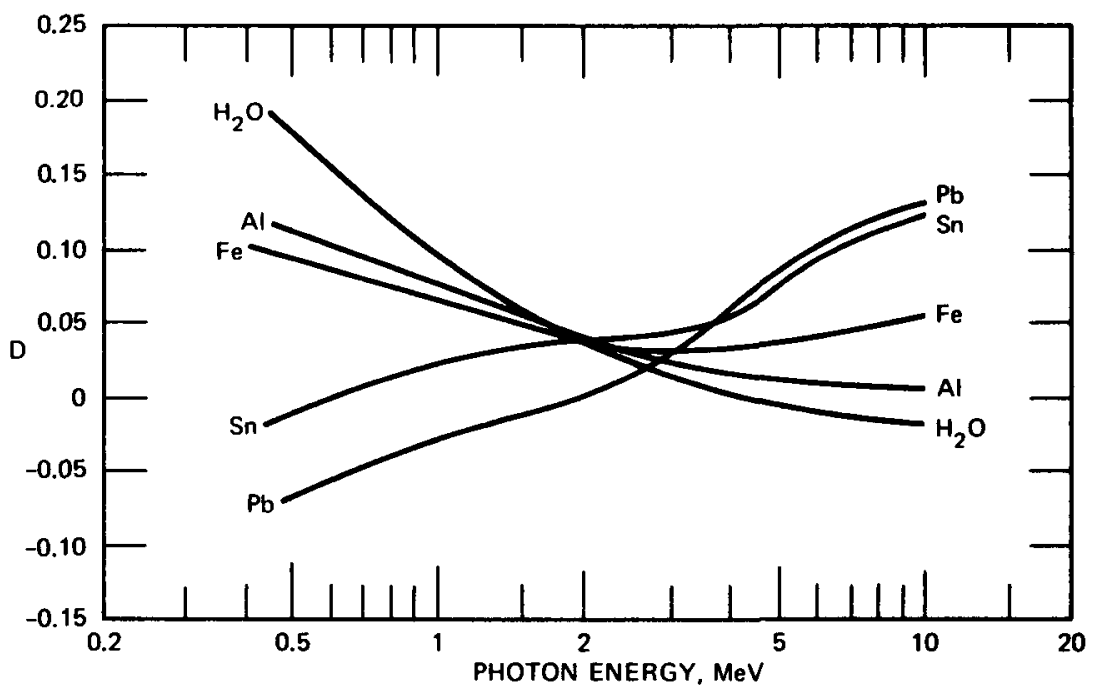

Fig. 6.22-The coefficient $D$ for the Berger form of the gamma-ray-dose buildup factor. (Based on data from Chilton. ${ }^{28}$ )

Equations 6.5-16 and 6.5-17 reveal that, unless $D$ is less than 1, negative dose rates are obtained. However, $D$ is always significantly less than 1 , as is shown in Fig. 6.22 from the work of Chilton. ${ }^{28}$

When $\alpha+D=1$, Eq. 6.5-17 gives an indeterminate form, which, when resolved, becomes

$$
\Gamma_{s}(t, a, b)=\frac{G(E) C(E) S_{a} e^{-\alpha \mu t}(b-a)}{2(1-D)}
$$

For the special case when $b=t$ and $a=0$, Eq. 6.5-18 can be expressed as

$$
\Gamma_{s}(t, 0, t)=\frac{G(E) C(E) S_{a} e^{D \mu t}}{\mu(1-D)} \psi_{1}\left(\mu t, \alpha^{\prime}\right)
$$

where

$$
\psi_{1}\left(\mu t, \alpha^{\prime}\right)=\frac{e^{-\alpha^{\prime} \mu t}\left[1-e^{-\mu t\left(1-\alpha^{\prime}\right)}\right]}{2}
$$

and $\alpha^{\prime}=\alpha+D$. The function given by Eq. 6.5-20 is shown in Fig. N.2. 


\subsubsection{Calculation for Semi-Infinite Shield}

Solutions of Eq. 6.5-2 for a semi-infinite shield, i.e., for $b=\infty$, give useful results that are generally applicable for the special case in which $a=0$, particularly if one is interested in a gamma-ray heating rate within a shield. Using the polynomial form of the buildup factor

$$
B_{r}(\mu R)=\sum_{n=0}^{3} A_{n}(\mu R)^{n}
$$

Claiborne ${ }^{29}$ determined solutions to Eq. 6.5-2 for this case which were all in the form

$$
\Gamma(\mu x)=\frac{G(E) S_{a}}{\mu} \sum_{n=0}^{3} n ! A_{n} \psi_{n}
$$

The dose rate from the uncollided-flux density is represented by the first term and is given by

$$
\psi_{0}(\mu t)=\frac{1}{2 \alpha}\left(E_{1}(\mu t)-e^{-\alpha \mu t}\left\{E_{1}[(1-\alpha) \mu t]-\ln \left|\frac{1+\alpha}{1-\alpha}\right|\right\}\right)
$$

and the sum of the next three terms represents the scattered contribution. The terms are

$$
\begin{gathered}
\psi_{1}(\mu t)=\frac{e^{-\alpha \mu t}}{2}\left[\frac{1-e^{-(1-\alpha) \mu t}}{1-\alpha}+\frac{1}{1+\alpha}\right] \\
\psi_{2}(\mu t)=\frac{4 e^{-\alpha \mu t}-(1+\alpha)^{2}(2-\alpha) e^{-\mu t}-(1+\alpha)^{2}(1-\alpha) \mu t e^{-\mu t}}{4(1-\alpha)^{2}(1+\alpha)^{2}} \\
\psi_{3}(\mu t)=\frac{2}{3} \psi_{2}+\left[\frac{1}{(1-\alpha)^{3}}+\frac{1}{(1+\alpha)^{3}}\right] \frac{e^{-\alpha \mu t}}{6} \\
-\left[\frac{(\mu t)^{2}}{2(1-\alpha)}+\frac{\mu t}{(1-\alpha)^{2}}+\frac{1}{(1-\alpha)^{3}}\right] \frac{e^{-\mu t}}{6}
\end{gathered}
$$

When $\alpha=0$, an indeterminate form occurs in Eq. 6.5-23, and, when $\alpha=1$, indeterminate forms occur in Eqs. 6.5-23 through 6.5-26. The following equations result when the indeterminate forms are evaluated: 
For $\alpha=0 \quad \psi_{0}(\mu t)=1+\frac{\mu t}{2} E_{1}(\mu t)-\frac{e^{-\mu t}}{2}=1-\frac{1}{2} E_{2}(\mu t)$

For $\alpha=1$

$$
\begin{gathered}
\psi_{0}(\mu t)=\frac{E_{1}(\mu t)}{2}+\frac{e^{-\mu t}}{2} \ln (2 \gamma \mu t) \\
\psi_{1}(\mu t)=\left(\mu t+\frac{1}{2}\right) \frac{e^{-\mu t}}{2} \\
\psi_{2}(\mu t)=\frac{\psi_{1}}{2}+\left[2(\mu t)^{2}+1\right] \frac{e^{-\mu t}}{16} \\
\psi_{3}(\mu t)=\frac{2 \psi_{2}}{3}+\left[\frac{(\mu t)^{3}}{3}+\frac{1}{4}\right] \frac{e^{-\mu t}}{12}
\end{gathered}
$$

The functions given by Eqs. 6.5-28 through 6.5-31 for the semi-infinite medium are plotted in Figs. N.3 and N.4 for various values of $\alpha$. These figures can be compared with Figs. N.1 and N.2, which are the corresponding functions evaluated for a slab shield.

These solutions contain the contribution from the gamma-ray sources between the detector position at $t$ and infinity since integration of Eq. 6.5-2 from $x=0$ to $x=\infty$ produces two integrals: one gives the contribution from the interval $0 \leqslant x \leqslant t$ and the other gives the contribution from the interval $t \leqslant x \leqslant \infty$. In the usual shield, however, the contribution from the second interval at deep penetrations is small, and the gamma-ray dose rate outside a shield of thickness $\mu t$ will be only slightly less than that calculated for a distance $\mu t$ within a semi-infinite shield.

If Eq. 6.5-22 is used for gamma-ray heating calculations within a shield, the coefficients $A_{n}$ must correspond to the polynomial fit of the energy-absorption buildup factor, and the conversion factor for expressing the heating rate (in watts $\mathrm{g}^{-1}$ ) becomes

$$
G(E)=1.6 \times 10^{-13} \frac{\mu_{a}}{\rho} E
$$

where $\mu_{a} / \rho$ is the mass energy absorption coefficient of the material in which heat is generated. 


\section{REFERENCES}

1. H. Goldstein, Fundamental Aspects of Reactor Shielding, Addison-Wesley Publishing Company, Inc., Reading, Mass., 1959 (out of print); now available from Johnson Reprint Corporation, New York.

2. A. D. Krumbein and B. D. O'Reilly, Neutron Attenuation in Hydrogenous Media by the Method of Moments, in Engineering Compendium on Radiation Shielding, Vol. 1, Sec. 3.3.1, R. G. Jaeger (Ed.), Springer-Verlag, New York, 1968.

3. H. Goldstein and J. E. Wilkins, Jr., Calculations of the Penetrations of Gamma Rays, USAEC Report NYO-3075 (NDA-15C-41), Nuclear Development Associates, Inc., June 1954.

4. F. J. Allen, A. Futterer, and W. Wright, Neutron Reflection and Flux Versus Depth for Water, Report BRL-1204, Ballistic Research Laboratories, June 1963.

5. A. W. Casper, Modified Fast Neutron Attenuation Functions, USAEC Report XDC-60-2-76, General Electric Company, Feb. 3, 1960.

6. G. T. Western, Energy and Angular Distribution Experiment, Angular Distribution of Reactor Radiation from Slabs and of Emergent Secondary Gamma Rays, Report NARF-62-16T, Volume I, General Dynamics, Dec. 31, 1962.

7. F. J. Muckenthaler, L. B. Holland, and R. E. Maerker, In-Air Measurements in the Vicinity of the Tower Shielding Reactor II, USAEC Report ORNL-3288, Oak Ridge National Laboratory, April 1963.

8. W. E. Selph, Experimental Determination of Neutron Attenuation in Zirconium Hydride, Trans. Amer. Nucl. Soc., 7(1): 42 (June 1964).

9. B. S. Burton, Jr., Air Scattering of $\mathrm{Co}^{60}$ Gamma Radiation, Report FZM-1016, General Dynamics Corporation, October 1957.

10. J. T. Serduke and C. V. Smith, Penetration of Plane Normal Gamma Rays Through Slabs of Aluminum and Steel, Report USNRDL-TR-730, Radiological Defense Laboratory, Feb. 17, 1964.

11. D. M. Peterson, Shield Penetration Programs C-17 and L-63, Report NARF-61-39T, General Dynamics Corporation, Dec. 29, 1961.

12. R. D. Albert and T. A. Welton, A Simplified Theory of Neutron Attenuation and Its Application to Reactor Shield Design, USAEC Report WAPD-15, Westinghouse Electric Corporation, Nov. 15, 1950.

13. E. P. Blizard (Ed.), Reactor Handbook, 2nd ed., Vol. III, Part B, Shielding, Interscience Publishers, a division of John Wiley \& Sons, Inc., New York, 1962.

14. F. H. Clark, N. A. Betz, and J. Brown, Monte Carlo Calculations of the Penetration of Normally Incident Neutron Beams Through Concrete, USAEC Report ORNL-3926, Oak Ridge National Laboratory, January 1967.

15. Argonne National Laboratory, Reactor Physics Constants, USAEC Report ANL-5800 (2nd ed.), July 1963.

16. D. K. Trubey and M. B. Emmett, Some Calculations of the Fast-Neutron Distribution in Ordinary Concrete from Point and Plane Isotropic Fission Sources, USAEC Report ORNL-RSIC-4, Oak Ridge National Laboratory, June 1965.

17. F. J. Allen and A. T. Futterer, Neutron Transmission Data, Nucleonics, 21(8): 120 (1963). 
18. E. P. Blizard, A. Foderaro, N. G. Goussev, and E. E. Kovalev, Extended Radiation Sources (Point Kernel Integrations), in Engineering Compendium on Radiation Shielding, Vol. 1, Chap. 6, R. G. Jaeger (Ed.), Springer-Verlag, New York, 1968.

19. R. L. French, A Last Collision Approach to Calculating the Angular Distribution of Fast Neutrons Penetrating a Shield, Nucl. Sci. Eng., 23: 28-33 (1965).

20. A. M. Weinberg and E. P. Wigner, The Physical Theory of Neutron Chain Reactions, The University of Chicago Press, Chicago, 1958.

21. E. P. Blizard, Nuçlear Radiation Shielding, in Nuclear Engineering Handbook, Chap. 7-3, pp. 7-89, H. Etherington (Ed.), McGraw-Hill Book Company, Inc., New York, 1958.

22. J. W. Haffner, Use of Program G-2 for Shielding Calculations, USAEC Report DC-59-7-170, General Electric Company, July 20, 1959.

23. P. W. Schreiber and F. D. Kodras, Measured and Calculated Radiation Levels Within and Behind Beryllium Oxide, USAEC Report XDC-61-1-149, General Electric Company, Dec. 15, 1960.

24. K. T. Spinney, Neutron Attenuation in Concrete, British Report AERE-T/R-2507, 1957.

25. A. T. Avery, D. E. Bendall, J. Butler, and K. T. Spinney, Methods of Calculations for Use in the Design of Shields for Power Reactors, British Report AERE-R-3216, 1960.

26. D. K. Trubey, Calculation of Fission-Source Thermal-Neutron Distribution in Water by the Transfusion Method, USAEC Report ORNL-3487, Oak Ridge National Laboratory, August 1964.

27. D. K. Trubey, A Survey of Empirical Functions Used to Fit Gamma-Ray Buildup Factors, USAEC Report ORNL-RSIC-10, Oak Ridge National Laboratory, February 1966.

28. A. B. Chilton, Two-Parameter Formula for Point Source Buildup Factors, Nucleonics, 23(8): 119 (1965).

29. H. C. Claiborne, Analytical Solutions for Heat Generation Distributions in Regular Geometries, in Engineering Compendium on Radiation Shielding, Vol. 1, Sec. 7.3, J. G. Jaeger (Ed.), Springer-Verlag, New York, 1968.

\section{EXERCISES}

These exercises can be done with data given in this and previous chapters and the appendixes.

6.1 Two isotropic fission point sources, $A$ and $B$, are contained in a tank of water, as shown by the plan view in the accompanying sketch. Sources $A$ and $B$ both emit $10^{10}$ neutrons/sec. (a) Estimate the spectra at points 1 and 2 at the edge of the tank. (b) Estimate the total energy-flux density ( $\mathrm{MeV} \mathrm{cm}^{-2} \mathrm{sec}^{-1}$ ) at points 1 and 2. (c) Make a rough estimate of the thermal-flux density at points 1 and 2 .

6.2 An ordinary concrete slab is to be used to shield a 15.25 -cm-diameter beam tube from a fission reactor. The fast-neutron-flux density at the face of the core is known to be isotropic with a value of $10^{12}$ neutrons $\mathrm{cm}^{-2} \mathrm{sec}^{-1}$ at the core face. Estimate the thickness $x$ required to reduce the neutron dose rate at point $A$ to $1 \mathrm{mrad} / \mathrm{hr}$. Neglect scattering in the walls of the beam tube. 


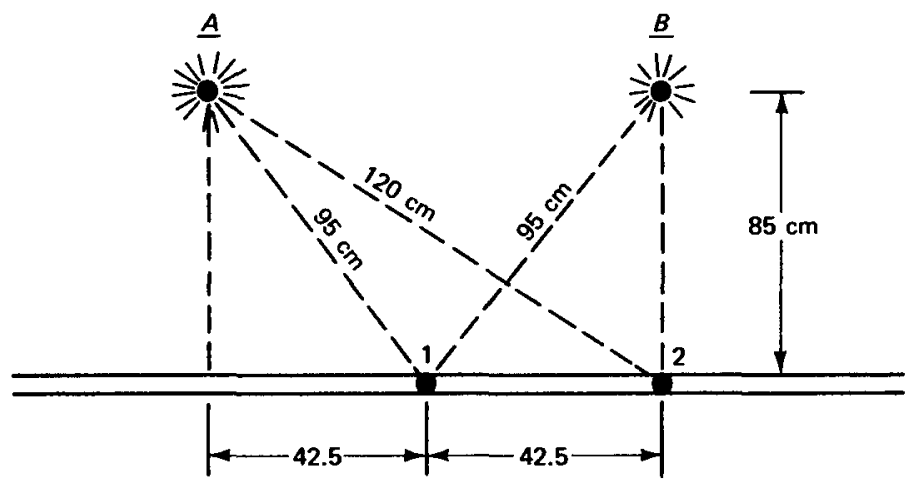

Geometry of Exercise 6.1.

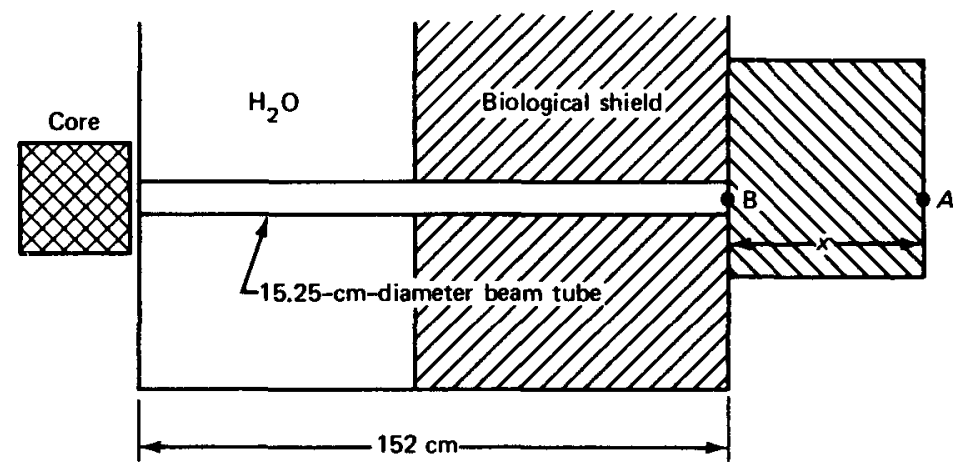

Beam-tube geometry, Exercise 6.2

6.3 In the beam-tube slab shield of Problem 6.2, estimate the thermal-flux density emerging at point $A$.

6.4 A thin ${ }^{60} \mathrm{Co}$ disk source $10 \mathrm{~cm}$ in radius with a strength of $5000 \mathrm{Ci}$ is shielded in a large pool of water. Using the Berger form of the buildup factor: (a) Compute the dose rate (DR) at a point $75 \mathrm{~cm}$ from the disk along its axis. (b) Compute the buildup factor for this situation. Note: At $1.25 \mathrm{MeV},\left(\mu_{t} / \rho\right) \mathrm{H}_{2} \mathrm{O}=0.064 \mathrm{~cm}^{2} / \mathrm{g}$ and $\mu_{a} / \rho=0.03 \mathrm{~cm}^{2} / \mathrm{g}$.

6.5 It was decided to add $5 \mathrm{~cm}$ of lead to the inside face of the concrete slab covering the beam tube of Problem 6.2 to lower the gamma dose rate. How does this addition affect the fast-neutron dose rate calculated in Problem 2? Use removal theory.

6.6 Approximately what was the prompt fission gamma-ray dose-rate reduction that resulted from adding the $5 \mathrm{~cm}$ of lead in Problem 6.5? 


\section{Albedos, Ducts, and Voids}

We turn now to a number of subjects we might euphemistically call special topics. They are special only in the sense that they are necessities. In contrast to previous chapters that deal fundamentally with one topic, transport in dense media, this chapter and Chap. 8 deal with a number of topics equally important to shield design. We might call them engineering considerations, perhaps another euphemism. At any rate some of the following considerations will arise in any reactor-shield design.

In this chapter we are concerned exclusively with an interface between two media, usually air and a shield material. The geometrical shape of the interface may be an infinite plane, a wall of a room, a cylindrical tube with several bends, or a spherical cavity.

Because the most successful methods for analysis of these interfaces use an albedo concept, the first half of the chapter is devoted to the definition and description of albedos. The most important application of an albedo for a reactor-shield designer is the analysis of ducts that penetrate the shield. Accordingly, duct analysis occupies most of the last half of the chapter. A brief final section covers a closely related topic, voids.

On the assumption that these topics are considered to be advanced in shielding and on our opinion that they are still in a state of development, we present this material in review form with a discussion of approaches taken by several laboratories. Experimental results play an important role here and are included in greater number than in earlier subjects for comparison with analysis and to illustrate trends.

\subsection{INTRODUCTION TO ALBEDOS}

Backscattering of radiation incident to a dense medium has been treated with some success as a reflection phenomenon. Borrowing the optical concept of albedo, we represent the scattering from interfaces, such as ducts 
and walls, strictly as a surface effect. So long as the surface dimensions are large compared with the relaxation length of radiation in the medium, the approximation is valid and useful.

When applied to nuclear radiation, the definition of albedo is much broader than the traditional one used in optics. Nuclear-radiation albedos include radiation that is scattered at depths of the order of a relaxation length rather than just from the surface of the medium since particles scattering from these depths contribute significantly to the total radiation emerging from the surface. The tacit assumption made in most applications of albedo theory is that particles emerge from the medium at the same points on the surface at which they were incident. Some nuclear-radiation albedos refer to mixed radiations; i.e., emergent particles are sometimes of a type different from the incident particles. Such albedos more properly carry the modifier effective, the emergent radiation being identified as a particular type of secondary radiation. For example, effective capture gamma-ray albedos are albedos specifying the gamma-ray dose emerging from a medium which is caused by incident neutrons being captured within the medium. This type of albedo is especially important since for some duct configurations the capture gamma-ray doses can exceed the scattered-neutron doses.

Theoretically, calculations of nuclear-radiation albedos should be straightforward since a large body of information is available on interaction probabilities, the angular distribution of scattered radiation, and the emergent energy vs. scattering angle for a variety of incident energies and materials. However, even though the single-event probabilities are well known, the solution of the macroscopic multicollision albedo problem becomes complex. Consequently the value of the single-event probabilities lies primarily in their usefulness in predicting trends. For example, if the ratio of the scattering cross section to the absorption cross section is high, as it is for neutrons diffusing in concrete, the resulting albedo will tend to be high. If, however, the scattering is predominantly in the forward direction, as it is for high-energy gamma rays, the albedo will be low. The albedo properties of concrete have been thoroughly investigated since this material is used in virtually every type of fixed shield. Other materials have been studied, such as water, iron, lead, borated polyethylene, aluminum, and various soils, but the albedo data for each are generally more limited. Investigations have consisted primarily of calculations with the Monte Carlo machine programs. In addition, a number of measurements have been made to establish the validity of the calculated data. In nearly all cases the calculated results have been fitted to empirical expressions. 


\subsection{DEFINITIONS}

Traditionally, albedo refers to the ratio of the radiation current reflected from a surface to the current incident on that surface. Consider a monodirectional source of radiation of energy $E_{0}$ incident on a surface at polar angle $\theta_{0}$ (see Fig. 7.1). The reflected current of energy $E$ per unit energy per unit solid angle at polar angle $\theta$ and azimuthal angle $\phi$ is given by

$$
J(E, \theta, \phi)=J\left(E_{\mathbf{0}}, \theta_{0}\right) \alpha\left(E_{\mathbf{0}}, \theta_{\mathbf{0}}, E, \theta, \phi\right)
$$

where $J\left(E_{0}, \theta_{0}\right)$ is the incident current and $\alpha\left(E_{\mathbf{0}}, \theta_{0}, E, \theta, \phi\right)$ is the albedo.

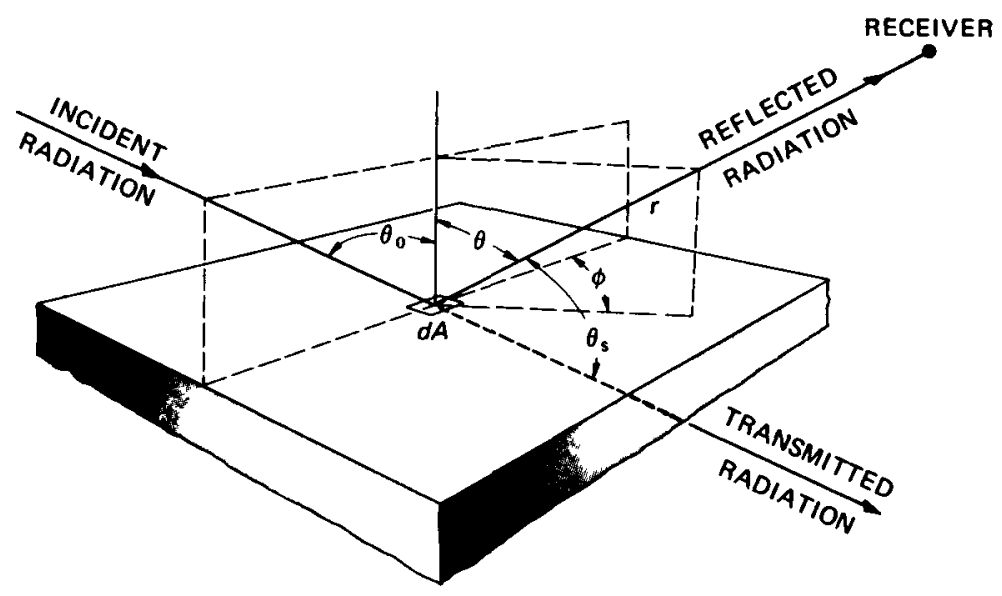

Fig. 7.1 - Geometry for calculating neutron and gamma-ray reflection from a surface.

When applied to nuclear radiation, albedo is not always expressed as the ratio of the reflected current per incident current but instead may be given as the ratio of reflected current per unit incident flux density, of reflected dose per unit incident current, of reflected dose per incident dose, etc. Neutron and gamma-ray albedos are available in several forms. The form used in Eq. 7.2-1 is doubly differential; i.e., it is differential with respect to both the reflected energy, $E$, and the reflected direction (as determined by $\theta$ and $\phi)$. A singly differential albedo is integrated over either energy or direction. Dose albedos obtained experimentally as a function of exit directions are also examples of singly differential albedos since dose is an integral quantity with respect to energy. The term total albedo always implies that integration has been performed over both energy and direction. 
In this text differential albedos are denoted by the symbol $\alpha$, as in Eq. 7.2-1, and total albedos by the symbol $A$.

Three different types of differential and total albedos have been used by various investigators in reporting their results on material reflectivity. The first type, denoted here by the subscript 1 , is an albedo that represents an incident flux density of particles and an emergent current. The second and third types, denoted by subscripts 2 and 3 , are albedos for which the incident and emergent particles are considered to be the same, current for the type 2 albedo and flux density for the type 3 albedo. Flux densities and currents are related functionally by the cosine of the entrance or exit angles. For example, if $\Phi(E, \theta, \phi)$ is the reflected differential flux density per unit energy per steradian, then the reflected current per unit energy per steradian, $J(E, \theta, \phi)$, is equal to $\Phi(E, \theta, \phi) \cos \theta$.

If the albedo being considered represents some weighting of the particle-flux density, such as dose or energy-flux density, then the subscripted letter $D$ or $E$ will precede the numerical subscript. In the following albedo definitions, the term dose or dose rate is used in the generic sense. The albedo definitions are not affected by the various types or names of doses that are used. The choice of the dose definition and the flux-to-dose conversion ratio govern whether the quantity is exposure, absorbed dose, kerma, or dose equivalent. A functional notation is used in conjunction with the albedo symbols to designate the independent variables for the particular albedo being considered.

\subsubsection{Differential-Dose Albedos}

The three types of differential albedos for which the particle-flux density has been weighted by a dose response function are defined as follows:

The form $\alpha_{D_{1}}\left(E_{0}, \theta_{0}, \theta, \phi\right)$ represents differential current out (in dose units) per incident flux density (in dose units). If the dose due to particles of energy $E_{0}$ incident at angle $\theta_{0}$ is $D_{0}$, then the particle current (in dose units) per steradian reflected in the direction $\theta, \phi$ is given by $D_{0} \alpha_{D_{1}}$. The reflected-particle current in dose units (or dose current) has no physical meaning but is merely a computational convenience. It is expressed mathematically by

$$
D(\theta, \phi)=\int K(E) J(E, \theta, \phi) d E
$$

where $J(E, \theta, \phi)=\phi(E, \theta, \phi) \cos \theta$ and $K(E)$ is the flux-to-dose conversion 
factor for particles of energy $E$. The current measuring plane in these definitions is the material-interface plane.

The form $\alpha_{D_{2}}\left(E_{0}, \theta_{0}, \theta, \phi\right)$ is used for differential current out (in dose units) per incident current (in dose units). If the dose due to particles of energy $E_{0}$ incident at angle $\theta_{0}$ is $D_{0}$ (flux density in dose units), then the particle current (in dose units) per steradian reflected in the direction $\theta, \phi$ is given by $D_{0} \cos \left(\theta_{0}\right) \alpha_{D_{2}}$, where $D_{0} \cos \theta_{0}$ is the incident-particle current in dose units. This type differs from the traditional current albedo only in that the current is weighted by a dose response function.

The form $\alpha_{D_{3}}\left(E_{0}, \theta_{0}, \theta, \phi\right)$ is used for differential flux density out (in dose units) per incident flux density (in dose units). If the dose due to particles of energy $E_{0}$ incident at angle $\theta_{0}$ is $D_{0}$, then the dose per steradian due to particles reflected in the direction $\theta, \phi$ is $D_{0} \alpha_{D_{3}}$. If the incidentparticle current per unit surface area is $J\left(E_{0}\right)$, then $D_{0}=K\left(E_{0}\right) J\left(E_{0}\right) \sec \theta_{0}$. If the reflected-particle current per unit surface area is $J(E, \theta, \phi)$, then the reflected differential dose is

$$
D(\theta, \phi)=\int K(E) J(E, \theta, \phi) \sec \theta d E
$$

where $D(\theta, \phi)$ is the dose per steradian due to particles reflected in the direction $\theta, \phi$.

These three types of albedos are related by

$$
\alpha_{D_{1}}=\alpha_{D_{2}} \cos \theta_{0}=\alpha_{D_{3}} \cos \theta
$$

\subsubsection{Total-Dose Albedos}

Total-dose albedos are obtained by integrating differential-dose albedos over the solid angle represented by the exit hemisphere. Thus the three types of total-dose albedos corresponding to the differential albedos described in the preceding section are defined by

$$
\begin{aligned}
A_{D_{1}}\left(E_{0}, \theta_{0}\right) & =\int \alpha_{D_{1}}\left(E_{0}, \theta_{0}, \theta, \phi\right) d \Omega \\
A_{D_{2}}\left(E_{0}, \theta_{0}\right) & =\int \alpha_{D_{2}}\left(E_{0}, \theta_{0}, \theta, \phi\right) d \Omega \\
& =\sec \theta_{0} \int \alpha_{D_{1}}\left(E_{0}, \theta_{0}, \theta, \phi\right) d \Omega
\end{aligned}
$$




$$
\begin{aligned}
A_{D_{3}}\left(E_{0}, \theta_{0}\right) & =\int \alpha_{D_{3}}\left(E_{0}, \theta_{0}, \theta, \phi\right) d \Omega \\
& =\int \alpha_{D_{1}}\left(E_{0}, \theta_{0}, \theta, \phi\right) \sec \theta d \Omega \\
& =\cos \theta_{0} \int \alpha_{D_{2}}\left(E_{0}, \theta_{0}, \theta, \phi\right) \sec \theta d \Omega
\end{aligned}
$$

where $d \Omega=\sin \theta d \theta d \phi$ and the limits of integration are from $\theta=0$ to $\pi / 2$ and from $\phi=0$ to $2 \pi$.

Differential data must be available when data for $A_{D 3}$ are being compared with the other two types of total albedos, whereas $A_{D_{1}}$ and $A_{D_{2}}$ are directly related; i.e., $A_{D_{1}}=\cos \theta_{0} A_{D_{2}}$.

\subsubsection{Other Albedos}

Particle flux density or current- and energy-flux density or current albedos, which refer either to particle or energy flow, have also been used. In keeping with the previous nomenclature, these are

$A_{1}$ or $\alpha_{1}=$ particle current out per unit particle flux density in

$A_{2}$ or $\alpha_{2}=$ particle current out per unit particle current in

$A_{3}$ or $\alpha_{3}=$ particle flux density out per unit particle flux density in

For the case in which energy flow is considered, these particle-flow quantities are weighted by the energy and $A_{E_{1}}$ or $\alpha_{E_{1}}=$ energy current out per unit energy-flux density in, etc.

All the parameters involved in these albedo definitions are the same as in the dose albedo definitions except that neither the incident nor the reflected flux density (or current) is converted to dose units.

An effective albedo may be defined for the emergence of secondary gamma rays from a material due to incident neutrons. Examples are capture gamma-ray albedo, inelastic gamma-ray albedo, or activation gamma-ray albedo.

\subsection{NEUTRON ALBEDOS}

The fundamental mechanisms that result in neutron backscattering are elastic and inelastic scattering, the two processes being distinguished by the condition of the target nucleus following its collision with the neutron. 
Owing to the relatively weak variation of the dose response function with energy, neutron-dose albedos are more nearly proportional to the particle-flux-density albedo than to the total-energy albedo. Calculations of neutron albedos have been successfully performed and experimentally verified in a number of cases. Investigations fall into three major categories, distinguished by the energies of the reflected neutrons: fast neutrons, intermediate-energy neutrons, and thermal neutrons. Studies of thermalneutron albedos have been further categorized as relating to those resulting from incident thermal neutrons and those resulting from neutrons incident at energies higher than thermal energy. Since neutrons incident at thermal energy scatter in a more orderly process than do higher energy neutrons, this category has yielded to direct analytical approach more readily than the other categories.

\subsubsection{Fast-Neutron Albedos}

Major contributions to the data on fast-neutron albedos have resulted from studies made at Oak Ridge National Laboratory (ORNL) by Maerker and Muckenthaler ${ }^{1}$ and at the U.S. Army Ballistic Research Laboratories (BRL) by Allen, Futterer, and Wright. ${ }^{2}$ Both groups performed detailed Monte Carlo calculations to determine the reflection of fast neutrons as a function of incident energy and angle. The results of Maerker and Muckenthaler are more detailed than those of Allen et al. in that the reflection data are differential with respect to both the reflected direction (see Fig. 7.1) and to the reflected energy. The data of Allen et al. are differential with respect to the direction only. The ORNL results are limited to concrete; the BRL studies include several additional materials.

The calculations by Maerker and Muckenthaler were performed as part of an extensive analytical and experimental program that covered a wide range of neutron energies (see Secs. 7.3.2 and 7.3.3) and included an investigation of secondary gamma-ray albedos (see Sec.7.5). In the measurements a $23-\mathrm{cm}$-thick concrete slab that was reinforced with steel bars at a depth of $3.8 \mathrm{~cm}$ from either side was used. For the fast-neutron calculations the steel was not considered, and the concrete composition was assumed to be a typical concrete of the composition shown in Table 7.1.

Calculations were performed for six incident-energy bands covering the energy range between 0.2 and $8 \mathrm{MeV}$. In a given problem neutrons incident to the concrete were sampled uniformly from each incident-energy band, and a statistical estimation technique was used to obtain estimates of the 
Table 7.1-COMPOSITION OF CONCRETE USED IN MONTE CARLO CALCULATIONS

\begin{tabular}{lcc}
\hline & \multicolumn{2}{c}{$\begin{array}{c}\text { Composition, } \\
10^{21} \text { atoms cm }^{-3}\end{array}$} \\
\cline { 2 - 3 } & $\begin{array}{c}\text { Maerker and } \\
\text { Muckenthaler }\end{array}$ & Allen et al. ${ }^{2}$ \\
\hline Element & & \\
Hydrogen & 9.43 & 13.75 \\
Oxygen & 47.6 & 45.87 \\
Silicon & 11.85 & 20.15 \\
Calcium & 7.8 & \\
Density, $\mathrm{g} \mathrm{cm}^{-3}$ & 2.35 & 2.26 \\
\hline
\end{tabular}

current emerging from the surface at various angles from a normal to the surface.

Emergent angles were determined by the intersection points of a grid formed by nine space-fixed polar angles and six azimuthal angles. The results, obtained for distinct values of $\theta_{0}, \theta$, and $\phi$, were grouped into energy bands $\Delta E_{0}$ and $\Delta E$. There were 10 reflected-energy bands, which, like the incident-energy bands, covered the range between 0.2 and $8 \mathrm{MeV}$. (Albedos that include neutrons reflected at energies less than $0.2 \mathrm{MeV}$ were determined separately and are discussed in Secs. 7.3.2 and 7.3.3.)

The differential albedo $\alpha_{D_{2}}\left(E_{0}, \theta_{0}, E, \theta, \phi\right)$ calculated by Maerker and Muckenthaler is in units of reflected current (in single-collision dose units) per $\mathrm{MeV}$ per steradian per incident current (in single-collision dose units) of a "gun-barrel" beam source. The average statistical uncertainty associated with the Maerker-Muckenthaler data is about $10 \%$ for the doubly differential albedos and about $3 \%$ for singly differential albedos.

Results from these calculations are shown in Figs. 7.2 through 7.5. Figure 7.2 shows the variation of the total albedo (integrated over both the reflected energy and the reflected angle) as a function of the incident angle and incident-energy band. Figures 7.3 and 7.4 show the dependence of the $\mathrm{d}$ fferential-dose albedo on the reflection angles $\theta$ and $\phi$; Fig. 7.4 clearly illustrates that an assumption of no dependence on the reflected azimuthal angle $\phi$ can lead to considerable error in the differential albedo for some conditions. The dependence on the azimuthal angle is strongest for high-energy neutrons at grazing angles of incidence and emergence; it becomes very weak for low-energy neutrons or for values of $\phi$ greater than $45^{\circ}$. This trend is consistent with what would be expected since the first 


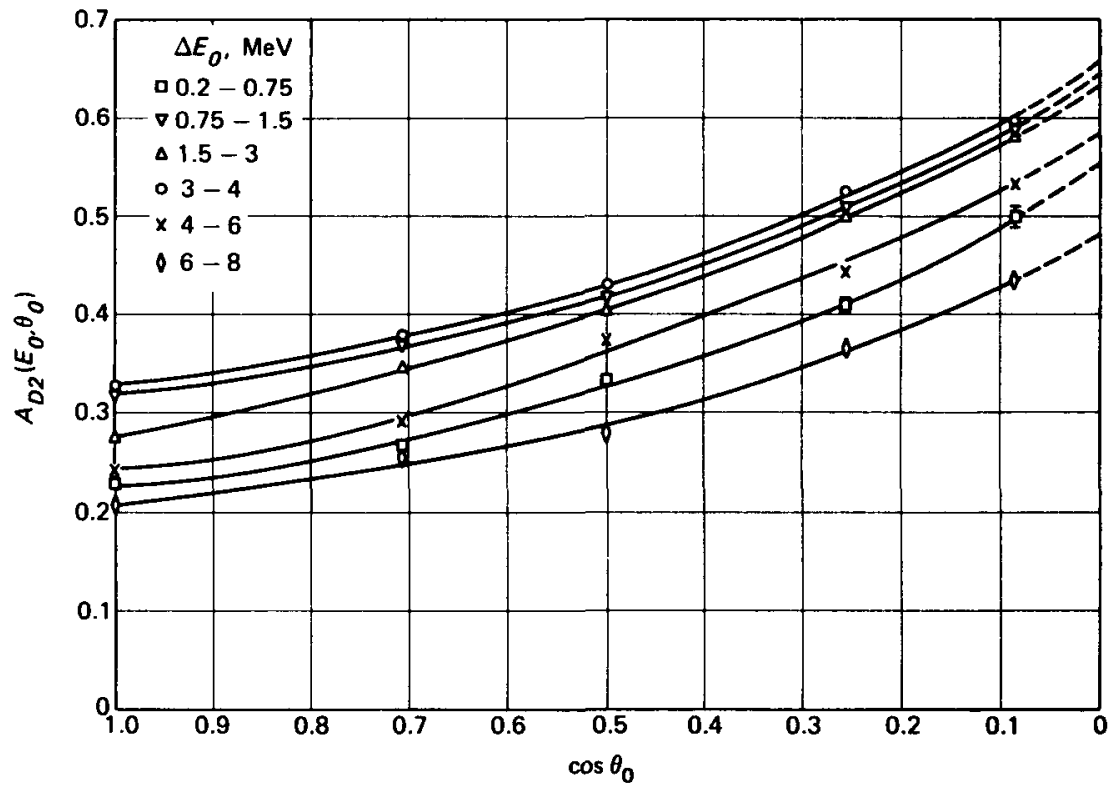

Fig. 7.2-Total single-collision dose albedo as a function of $\cos \theta_{0}$ and $\Delta E_{0}$ for fast neutrons $(>0.2 \mathrm{MeV})$ reflected from concrete. [From R. E. Maerker and F. J. Muckenthaler, Nuclear Science and Engineering, 22: 458 (1965).]

scatterings of high-energy neutrons are in the forward direction and since neutrons that have scattered more than once tend to have "forgotten" their initial direction and thus emerge from the material in a random manner. Figure 7.5 shows how the ratio of the total-dose albedo for singly scattered neutrons to the total-dose albedo for singly plus multiply scattered neutrons increases with increasing values of the polar angle of incidence.

Maerker and Muckenthaler developed an expression to fit their angular differential-dose-albedo data which reproduces their Monte Carlo results within $10 \%$. The expression involves two terms: the first accounts only for singly scattered neutrons and the second includes all multiply scattered neutrons; it is assumed that the configuration is effectively a semi-infinite body of concrete. The expression is

$$
\begin{aligned}
\alpha_{D_{2}}\left(\Delta E_{0}, \theta_{0}, \theta, \phi\right)= & \frac{\cos \theta}{\cos \theta+K_{1}\left(\Delta E_{0}\right) \cos \theta_{0}} \\
& \times \sum_{m=0}^{M} G_{m}\left(\Delta E_{0}\right) P_{m}\left(\cos \theta_{s}\right)+\frac{\cos \theta}{\cos \theta+K_{2}\left(\Delta E_{0}, \theta_{0}, \theta\right)} \\
& \times \sum_{k=0}^{K} B_{k}\left(\Delta E_{0}\right) P_{k}\left(\cos \theta_{s}\right)
\end{aligned}
$$


where $\cos \theta_{s}=\sin \theta_{0} \sin \theta \cos \phi-\cos \theta_{0} \cos \theta ; P_{m}$ and $P_{k}$ are Legendre polynomials of orders $m$ and $k$;

$$
K_{2}\left(\Delta E_{0}, \theta_{0}, \theta\right)=\sum_{i=0}^{I}(\cos \theta)^{i} \sum_{j=0}^{J} a_{i j}\left(\Delta E_{0}\right) \cos j \theta_{0}
$$

and the constants $G_{m}, B_{k}, K_{1}$, and $a_{i j}$ are given in Appendix O, Table O.1.

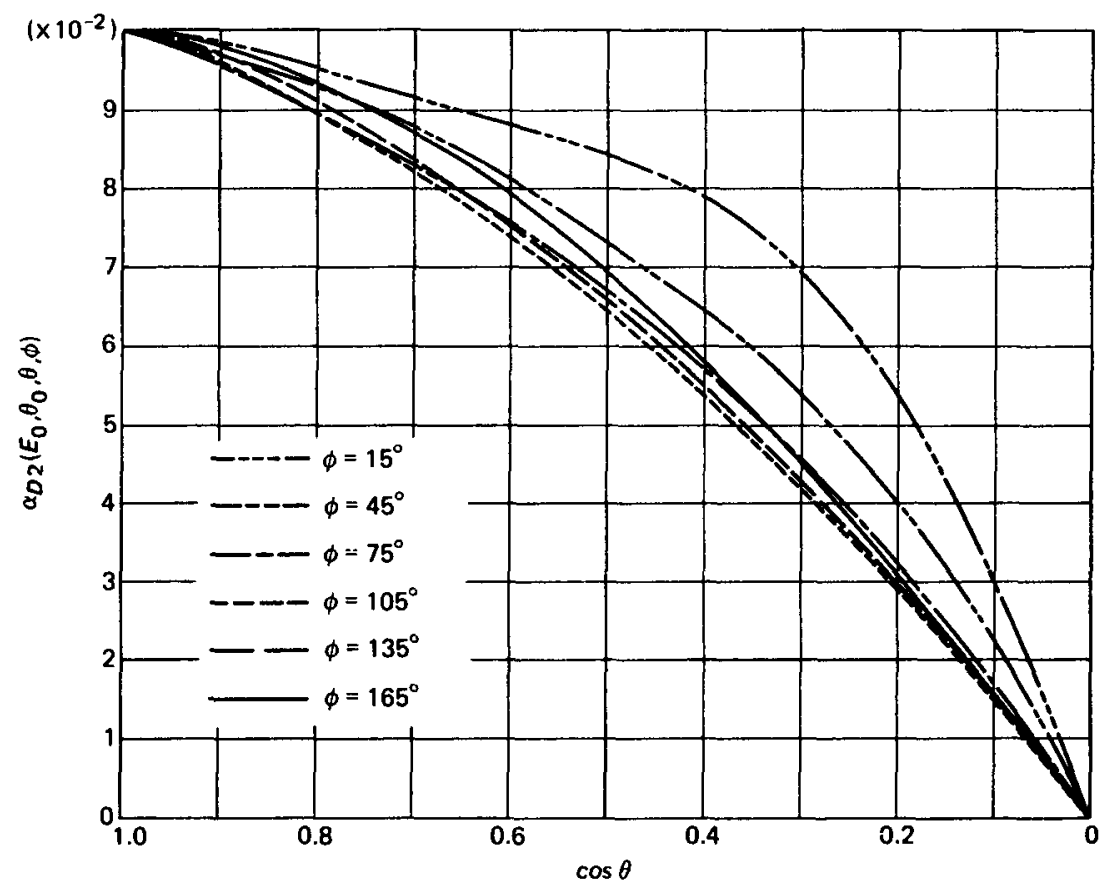

Fig. 7.3-Differential single-collision dose albedo per steradian as a function of $\cos \theta$ and $\phi$ for 1.5- to 3-MeV neutrons incident on concrete at $\theta_{0}=60^{\circ}$. [From R. E. Maerker and F. J. Muckenthaler, Nuclear Science and Engineering, 22: 458 (1965).]

To provide experimental verification of these data, Maerker and Muckenthaler used their differential albedos to predict reflected fast-neutron doses from a collimated beam of reactor neutrons incident at various angles to a 1.8-m-square, 23-cm-thick concrete slab and compared calculations with corresponding measurements at the ORNL Tower Shielding Facility (TSF). The values of the incident angles $\theta_{0}$ covered in the calculations and the experiment were $0^{\circ}, 45^{\circ}, 60^{\circ}$, and $75^{\circ}$. The calculations were weighted by an incident spectrum that had been previously measured at the TSF, and the incident dose rate used was the dose rate determined by integrating the 
measured dose rates over the effective cross-sectional area of the incident beam.

Agreement was considered good in that the root-mean-square deviation between the predicted and measured values was $3.1 \%$ and the largest single deviation was $9 \%$. Maerker and Muckenthaler subsequently incorporated these results in a duct calculation for comparison with a series of

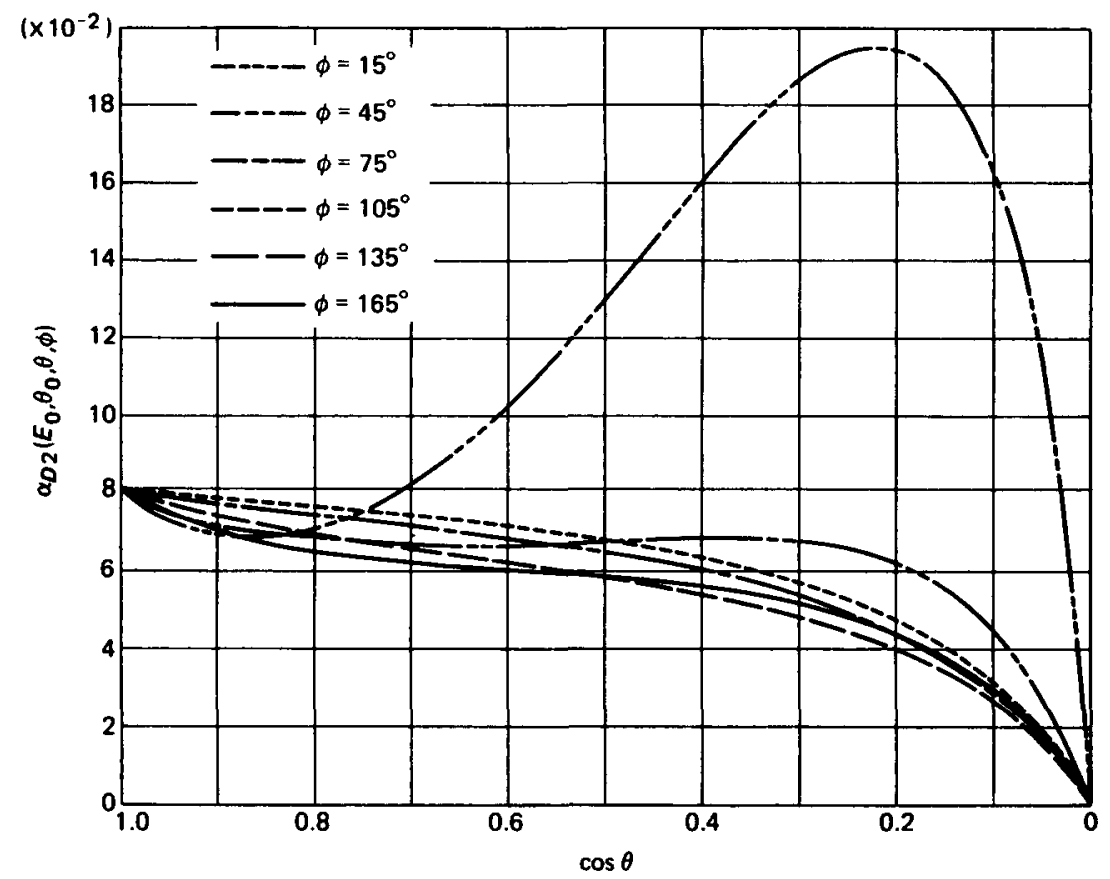

Fig. 7.4-Differential single-collision dose albedo per steradian as a function of $\cos \theta$ and $\phi$ for 6- to 8-MeV neutrons incident on concrete at $\theta_{0}=85^{\circ}$. [From R. E. Maerker and F. J. Muckenthaler, Nuclear Science and Engineering, 22: 458 (1965).]

measurements in a three-legged duct. Their results are described later in this chapter.

The Monte Carlo calculations performed by Allen et al. ${ }^{3}$ were used to determine the fraction of neutrons from monoenergetic sources that was transmitted through and reflected from infinite slabs of various materials, including concrete. The source energies were $0.10,0.25,0.50,1.0,2.0,3.0$, 5.0 , and $14 \mathrm{MeV}$, and the angles of incidence were $0^{\circ}, 30^{\circ}, 45^{\circ}$, and $70^{\circ}$ from the normal to the slab. The low-energy cutoff was $10 \mathrm{eV}$. Transmitted and reflected neutrons were accumulated in energy and angle intervals, the reflected data yielding the multicollision-dose albedos $\alpha_{D_{3},}\left(E_{0}, \theta_{0}, \theta\right)$ and 


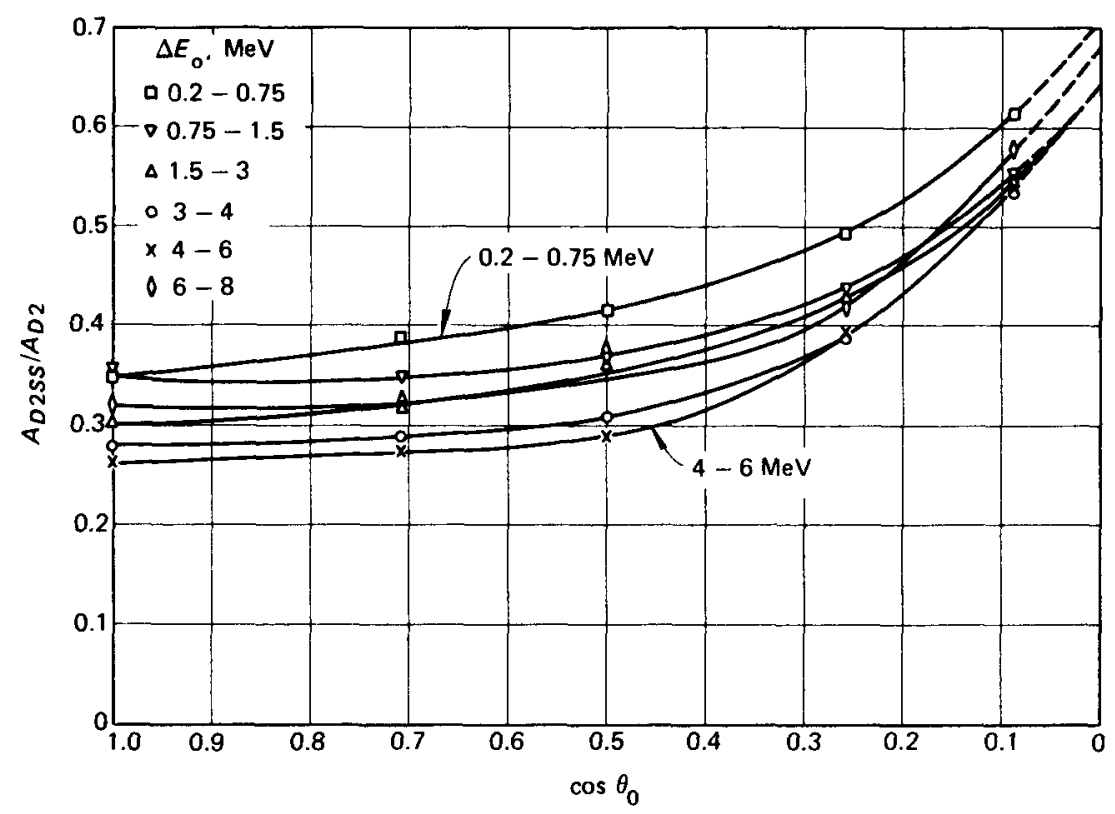

Fig. 7.5-Ratio of total single-collision dose albedo for fast neutrons (>0.2 MeV) singly scattered from concrete to total albedo for singly plus multiply scattered neutrons as a function of $\cos \theta_{0}$ and $E_{0}$. [From R. E. Maerker and F. J. Muckenthaler, Nuclear Science and Engineering, 22: 458 (1965).]

$A_{D_{3}}\left(E_{0}, \theta_{0}\right)$. Energy spectra of the reflected flux were also determined for each incident energy-angle combination, but the albedo was not calculated as a function of the energy of reflected particles.

In addition to concrete, the materials included in the calculations were water, iron, borated polyethylene $\left(8 \% \mathrm{~B}_{4} \mathrm{C}\right.$ by weight), and three Nevada Test Site (NTS) soils that differed only in moisture content. The densities and elemental content of the materials are given in Appendix O, Table O.2. In all cases the slabs were assumed to be sufficiently thick to yield albedo data approximating those for a semi-infinite geometry.

Some results from the calculations of Allen et al. ${ }^{3}$ are shown in Figs. 7.6 through 7.9. Figures 7.6 through 7.8 are plots of the total-dose albedos for the various materials as a function of the hydrogen content for incident source energies of $0.1,2.0$, and $14.0 \mathrm{MeV}$. Figure 7.9 gives the angular distributions of reflected neutrons from a $1.0-\mathrm{MeV}$ source.

French and Wells ${ }^{4}$ analyzed the differential data of Allen et al. ${ }^{3}$ and obtained a fit that is a function of the incident and reflected polar angles only. The lesser detail of the BRL results led to the conclusion that the 


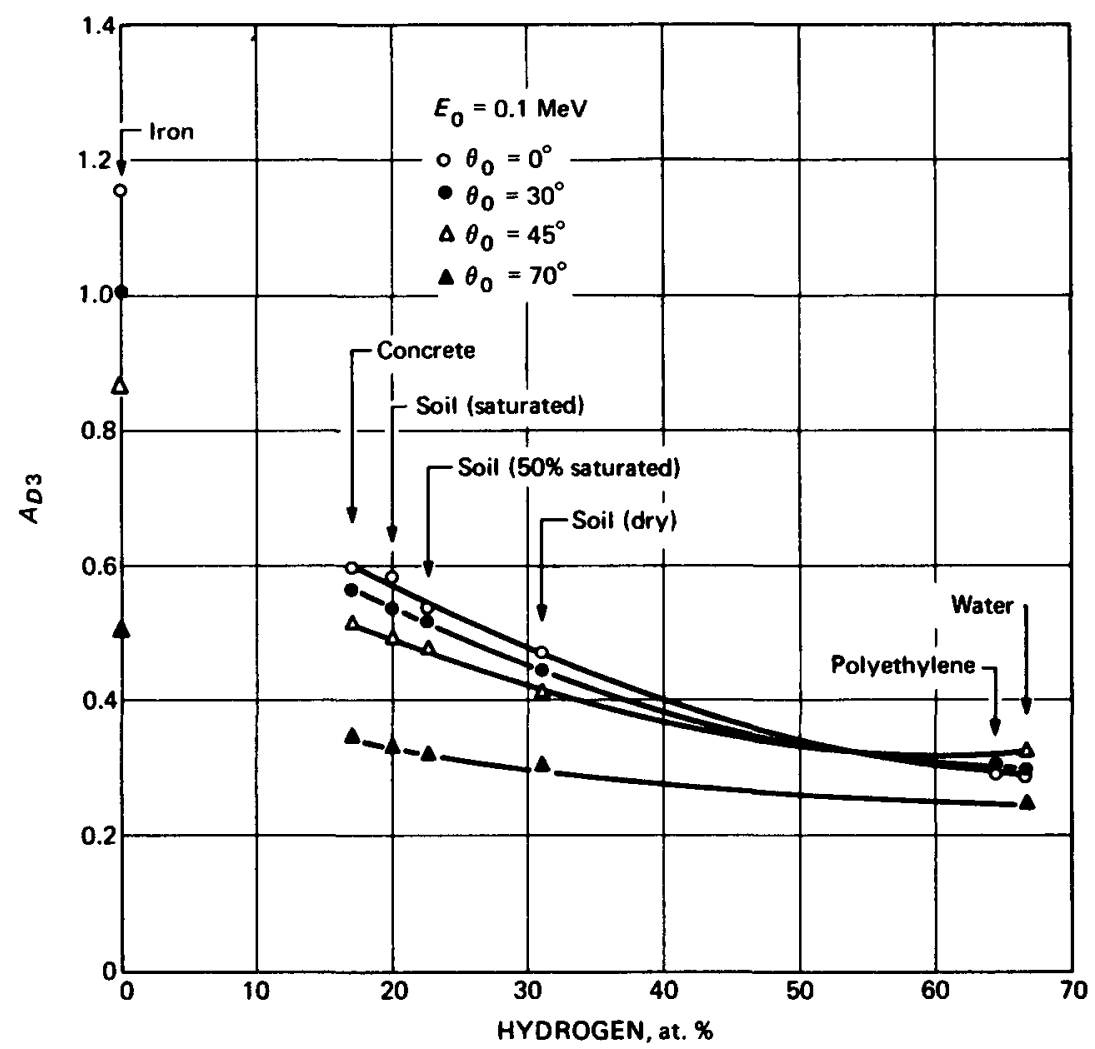

Fig. 7.6-Total multicollision dose albedo for $0.1-\mathrm{MeV}$ neutrons incident on various materials. (From Allen, Futterer, and Wright. ${ }^{3}$ )

dependence of the reflected azimuthal angle $\phi$ was weak and for the most part irregular; thus the dose reflection data were averaged over $\phi$. The dependence on the reflected polar angle was found to fit a $\cos \theta$ function, and the dependence on the incident angle was approximated by $\cos ^{3} \theta_{0}$, which yielded an expression of the form $\dagger$

$$
\alpha_{D_{1}}=k\left(E_{0}\right) \cos 3 / 3 \theta_{0} \cos \theta
$$

where $k\left(E_{0}\right)$ is a normalizing constant that includes the effect of incident energy and reflecting material. Values of $k\left(E_{0}\right)$ are shown in Appendix $O$, Table O.3, for concrete, the three NTS soils, and iron for eight monoen-

tThe original data of Allen et $a .^{3}$ were converted by French and Wells to a type 1 albedo (see Sec. 7.2). 


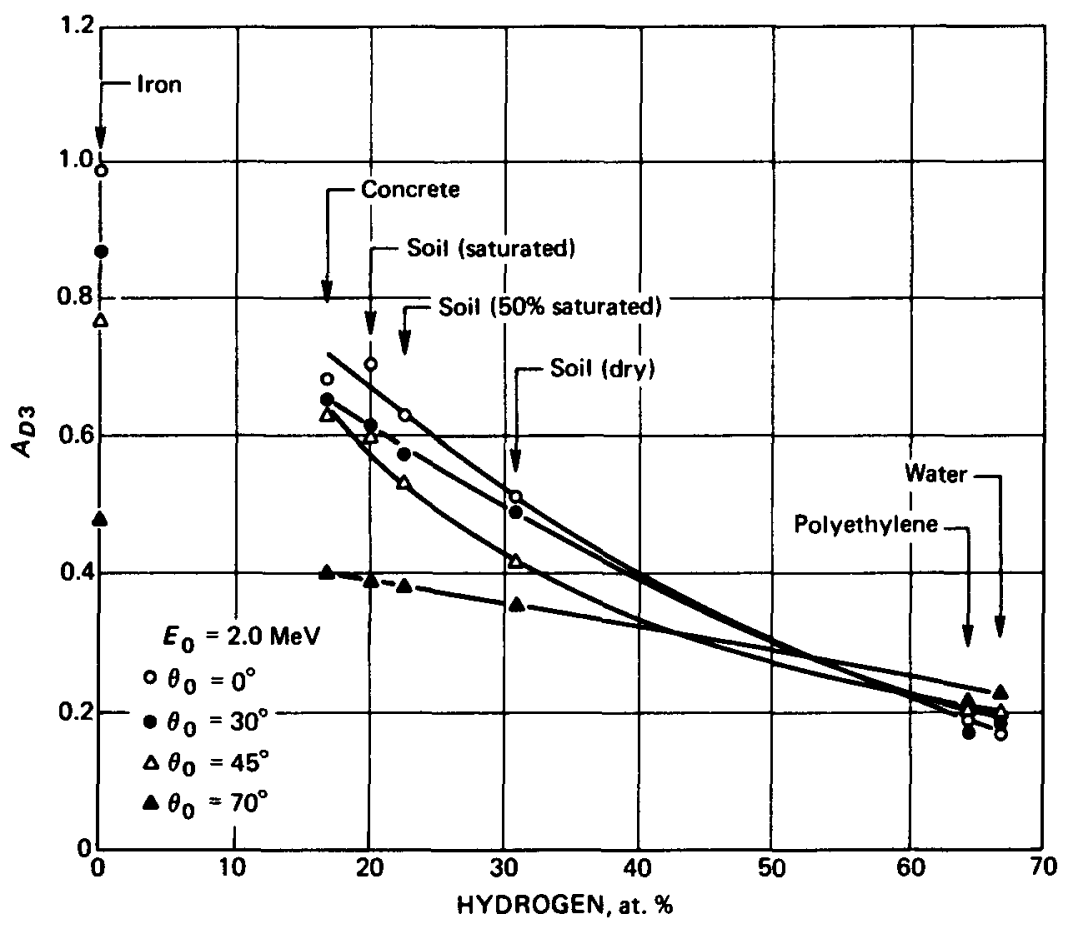

Fig. 7.7-Total multicollision dose albedo for 2.0-MeV neutrons incident on various materials. (From Allen, Futterer, and Wright. ${ }^{3}$ )

ergetic scurces and a fission source. Equation 7.3-3 is assumed to be valid for all materials of low to moderate hydrogen content $\left(\Sigma_{H} / \Sigma_{t}<0.5\right)$. (The water data of Allen et al. show a less pronounced dependence on $\theta_{0}$ and are not represented by Eq. 7.3-3.)

French and Wells found that, except for incident energies near cross-section peaks of the elements in the material, the total-dose-albedo data of Allen et al. ${ }^{3}$ could be correlated by a linear function of the ratio of the macroscopic hydrogen cross section of the material to its macroscopic total cross section. There is also an excellent correlation when the total albedo is averaged over the fission-neutron spectrum, as is shown in Fig. 7.10 for normally incident and normally reflected fission neutrons. This should be a useful correlation in extrapolating to other materials for which calculations have not been performed.

Song ${ }^{5}$ used the Monte Carlo data of Allen et al. ${ }^{3}$ to obtain values of an energy-dependent parameter that would give the best fit to a semiempirical formula he had derived for the fast-neutron differential-dose albedo for 


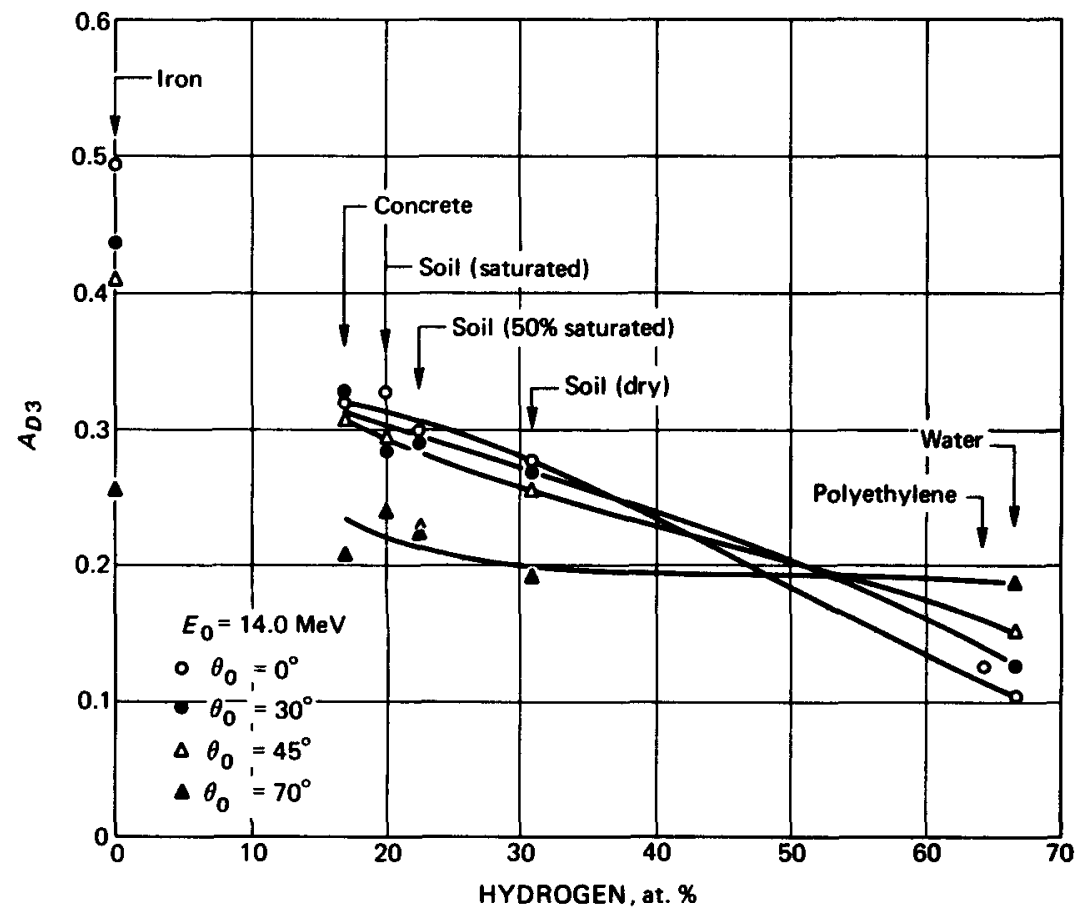

Fig. 7.8-Total multicollision dose albedo for 14.0-MeV neutrons incident on various materials. (From Allen, Futterer, and Wright. ${ }^{3}$ )

concrete. The formula, analogous to that used by Chilton and Huddleston ${ }^{6}$ for gamma rays (given in Sec. 7.4), is

$$
\alpha_{D_{2}}\left(E_{0}, \theta_{0}, \theta\right)=\frac{F\left(E_{0}\right) \cos \theta}{\cos \theta_{0}+\cos \theta}
$$

where $F\left(E_{0}\right)$ is the energy-dependent parameter. Song obtained values of this parameter from a least-squares analysis of the Monte Carlo data for concrete which gave the best fit to the equation. The values were then empirically correlated as a function of energy by

$$
F\left(E_{0}\right)=E_{0} \exp \left(0.9719-2.895 \sqrt{E_{0}}+0.3417 E_{0}\right)
$$

Another investigation of fast-neutron albedos was performed by Henry, Mooney, and Prevost, ${ }^{7}$ who studied the reflection of fast neutrons normally 


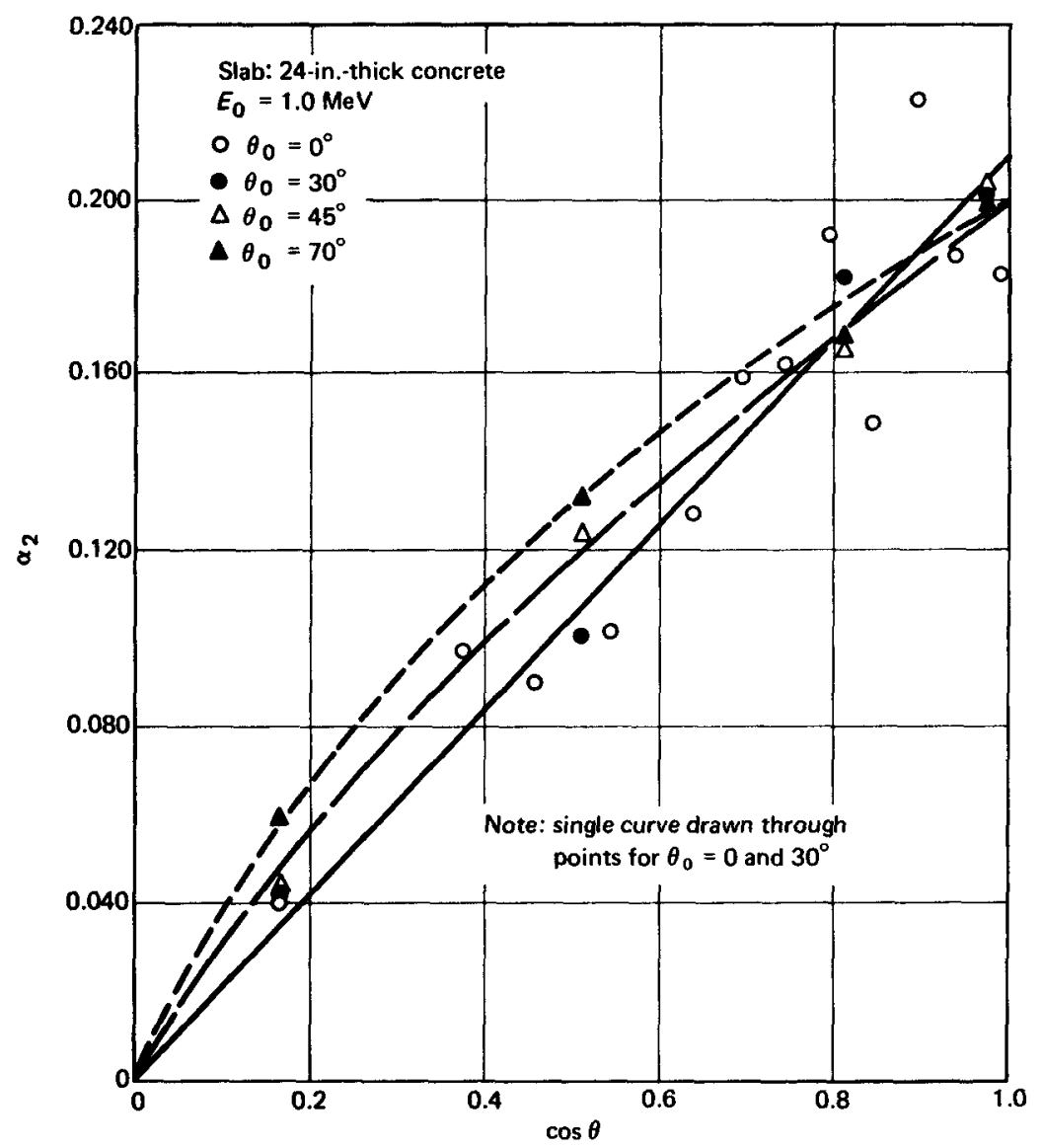

Fig. 7.9-Angular distributions of fast neutrons reflected from concrete. (From Allen, Futterer, and Wright. ${ }^{3}$ )

incident on various thicknesses of steel and $6 \%$ borated polyethylene. Their work included both Monte Carlo calculations and experiments utilizing a well-collimated reactor beam. In the experiments total-dose albedos $\left(A_{D_{3}}\right)$ were evaluated from data obtained by traversing the beam area with a dosimeter in a plane adjacent to the slab and repeating the traverse in the same plane with the slab removed. Figure 7.11 shows experimental and calculated results.

The data of Henry et al. are of particular interest because they show the dependence of the albedo on material thickness. In addition, Fig. 7.12 shows the reduction of the steel albedo caused by facing the steel slab with various thicknesses of polyethylene. In Fig. 7.11 it appears that the albedo for steel is approaching a value of nearly 0.6 , which is lower than the value of 0.84 


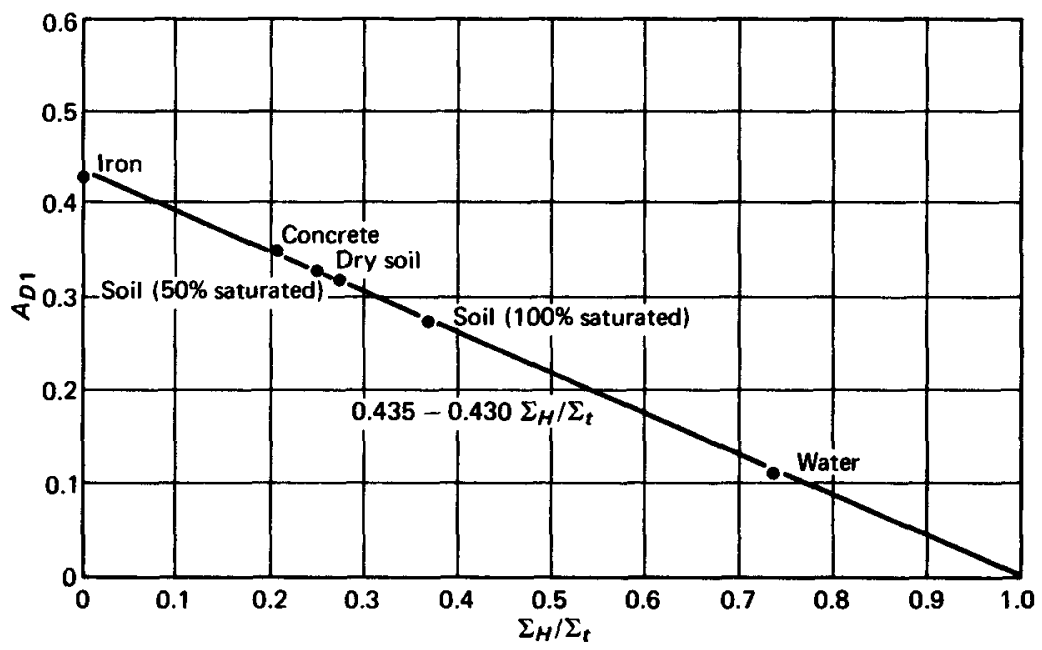

Fig. 7.10-Dependence of fast-neutron total multicollision dose albedo on hydrogen content of reflecting material (fission neutrons, $E_{0}>0.2 \mathrm{MeV}$ ). (From French and Wells. ${ }^{4}$ )

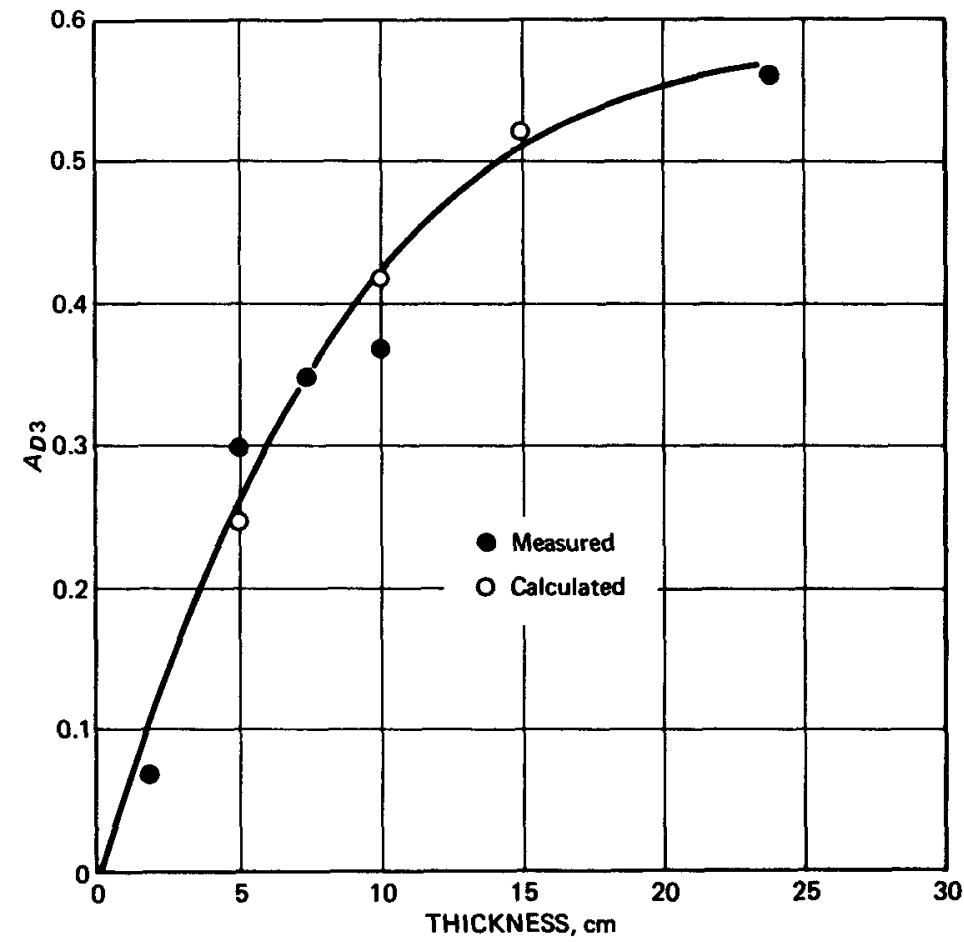

Fig. 7.11 - Total single-collision dose albedos for fission neutrons normally incident on steel slabs: comparison with experiment. (From Henry, Mooney, and Prevost. ${ }^{7}$ ) 
obtained when the data of Allen et al. are put in the $A_{D_{3}}$ form. The finite detector used in the experiments to traverse the interface approximates the slab detector assumed in the calculations of Allen et al. except for the low-energy cutoff, which was $0.2 \mathrm{MeV}$ in the experiment and in the calculations performed by Henry et al. but was $10 \mathrm{eV}$ in the calculations by

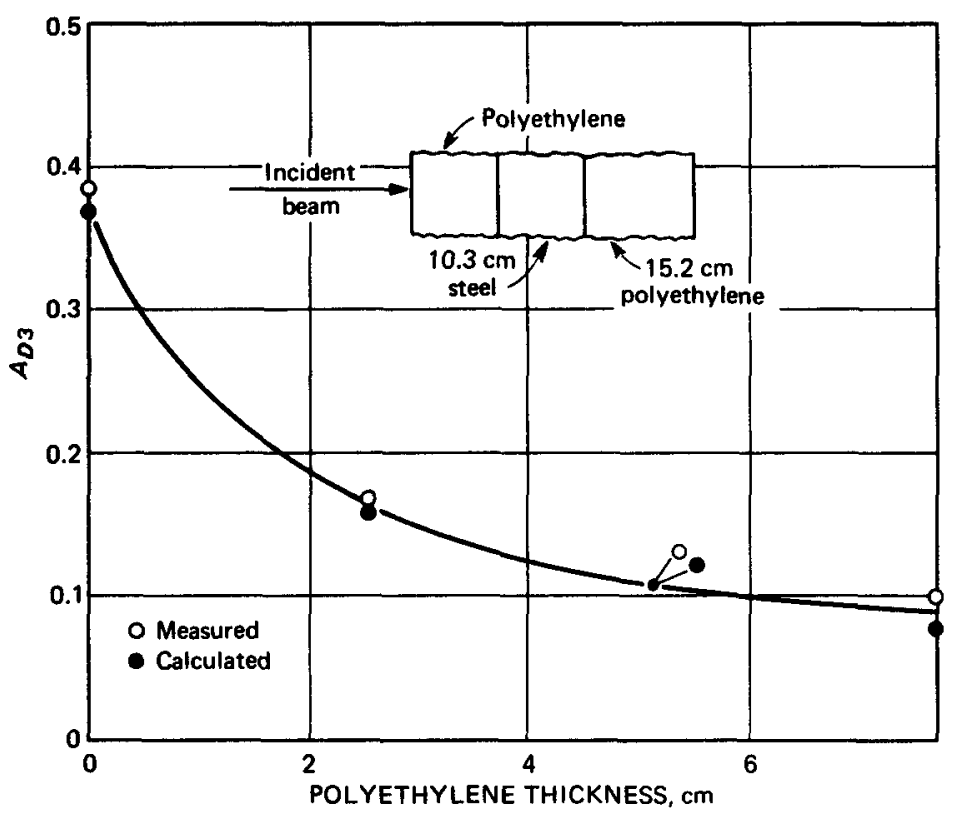

Fig. 7.12-Comparison with experiment of total single-collision dose albedos for fission neutrons normally incident on a laminated slab of steel and $6 \%$ borated polyethylene. (From Henry, Mooney, and Prevost. ${ }^{7}$ )

Allen et al. When the contribution below $0.2 \mathrm{MeV}$ is subtracted from the data of Allen et al. for representative energy groups, good agreement with the results of Henry et al. is obtained.

The Monte Carlo results of Maerker and Muckenthaler ${ }^{1}$ are the most complete available for neutron scattering from concrete, especially for grazing angles of incidence and emergence of high-energy neutrons where the dependence on azimuthal angle was found to be important. For water, soil, iron, and polyethylene, the Monte Carlo data of Allen et al. ${ }^{3}$ can be used.

The best accuracy is obtained when the Monte Carlo data are used directly in calculations; if the requirements on accuracy are not too stringent, the empirical expressions for the data can be applied, especially when an azimuthal dependence is not expected to be great. The analytical fits will generally provide less information on the distribution of the 
scattered radiation, but they will allow the reflected dose to be expressed in compact form, which will greatly reduce the bulk of data needed for calculations. A less precise but expedient calculation can be made by using the fission-spectrum total albedo shown in Fig. 7.10 with an assumed $\cos ^{2} \theta_{0} \cos \theta$ angular dependence.

\subsubsection{Intermediate-Neutron Albedos}

Coleman, Maerker, Muckenthaler, and Stevens ${ }^{8}$ calculated albedos for neutrons whose incident and reflected energies both are in the intermediateenergy range for steel-reinforced concrete. Using a Monte Carlo technique similar to the one used for the fast-neutron albedos, ${ }^{1}$ they determined the distribution in energy and angle of neutrons reflected from the concrete for 5 incident directions and 10 incident-energy groups in the energy range $0.5 \mathrm{eV}$ to $200 \mathrm{keV}$. The reflected distributions are given in terms of a doubly differential albedo for each of 54 different emergent directions for each group lying between and including the incident group and the lowest group $(0.5$ to $1.8 \mathrm{eV})$.

Reinforced concrete was used in these calculations because they were a part of the ORNL albedo program mentioned earlier. In the experiment a 23-cm-thick concrete slab that had steel reinforcing bars at a depth of $3.8 \mathrm{~cm}$ from either side was used; therefore all the calculations except the fast-neutron calculations were performed for a mock steel configuration in which each depth interval containing steel reinforcing rods was taken as a homogenized region of concrete and steel that was $2.5 \mathrm{~cm}$ thick. The result was a five-region slab that had ordinary concrete and reinforced-concrete layers. The effect of the iron on the intermediate-energy-neutron albedos was found to be negligible.

A complete tabulation of the doubly differential albedos calculated by Coleman $e t$ al. is too lengthy to be included here. The results, however, were numerically integrated over all exit energies to produce singly differential albedos and over all exit energies and angles to produce total albedos. The integrated results were fitted by the following expressions to within $15 \%$ for differential albedos and to within $3 \%$ for total albedos:

$$
\begin{aligned}
\alpha_{2}\left(\Delta E_{0}, \theta_{0}, \theta, \phi\right)= & \frac{\mu\left[\epsilon_{1}+\epsilon_{2} \mu_{0}+\mu\left(\beta_{1}+\beta_{2} \mu_{0}\right)\right]}{\mu+\gamma_{1}+\gamma_{2} \mu_{0}} \\
& \times\left\{1+(1-\mu)\left(1-\mu_{0}\right)\left[a\left(2 \cos ^{2} \phi-1\right)\right.\right. \\
& \left.\left.+b \cos \phi+c \cos ^{3} \phi\right]\right\}
\end{aligned}
$$


and

$$
A_{2}\left(\Delta E_{0}, \theta_{0}\right)=\delta_{1}+\delta_{2} \mu_{0}
$$

where $\mu=\cos \theta, \mu_{0}=\cos \theta_{0}$, and the constants are given in Appendix $O$, Table 0.4 , for each incident-energy group. When the expression for the differential albedo was determined, all exit neutrons were assumed to have a $1 / E$ energy distribution within each energy group. The expression for the total albedo was obtained by integrating Eq. 7.3-6 over all exit angles.

\subsubsection{Thermal-Neutron Albedos}

As mentioned previously, two types of thermal-neutron albedos can be considered: the purely thermal albedo, for which both the incident and the reflected neutrons are at thermal energy, and the albedo for emergent thermal neutrons that result from the moderation of neutrons that are incident at energies higher than thermal. These two categories are treated separately.

(a) Neutrons Incident at Thermal Energy. Various approximations to the purely thermal albedo have been derived analytically, with isotropic scattering and capture being the only interactions allowed. In some approximations only the total albedo is derived, and it is expressed as a function of the incident angle, assuming that the reflected neutrons will emerge with isotropic or cosine distributions. In other approximations differential albedos that are functions of both the incident angle and the exit angle are obtained.

In analytical treatments of purely thermal scattering, the exit current is independent of the azimuthal angle by virtue of the assumption of isotropic scattering. Monte Carlo calculations made with this assumption have shown reasonable agreement with the other forms of analysis; however, Monte Carlo calculations in which anisotropic scattering was assumed for hydrogen contained in the material have shown that the albedo exhibits an azimuthal dependence, although to a lesser extent than was shown for fast neutrons. Results from Monte Carlo calculations in which both types of scattering assumptions were used follow the discussion of various analytical approaches.

Probably the first investigation of thermal-neutron reflection was performed by Fermi, ${ }^{9}$ who showed that for large values of $N\left(N=\Sigma_{t} / \Sigma_{a}\right.$, the ratio of the total cross section to the absorption cross section) the total 
albedo for thermal neutrons incident on an infinitely thick isotropically scattering medium bounded by a plane is given approximately by

$$
A_{2}\left(\theta_{0}\right)=\frac{\sqrt{N}-1}{\sqrt{\bar{N}}+\sqrt{3} \cos \theta_{0}}
$$

where $A_{2}\left(\theta_{0}\right)$ is the number of thermal neutrons reflected per incident thermal neutron and $\theta_{0}$ is the angle of incidence.

A rigorous calculation was carried out later by Halpern, Lueneburg, and Clark, ${ }^{10}$ who obtained the formula

$$
A_{2}=1-\frac{k}{\sqrt{N+1}}
$$

where $\mathrm{k}=2.91,2.31$, and 2.48 for normal, isotropic, and cosine angular distributions of incidence, respectively.

Glasstone and Edlund ${ }^{1}{ }^{1}$ derived a formula by use of diffusion theory which is given by

$$
A_{2}=\frac{1-2 K D}{1+2 K D}
$$

where $K$ is the reciprocal of the thermal-neutron diffusion length and $D$ is the diffusion coefficient.

Chandrasekhar ${ }^{12}$ treated radiation backscattering by a method that can, in general, be applied to any type of radiation that scatters isotropically with a relatively constant cross section, a condition that is approximately met by thermal-neutron scattering. The resulting backscattering angular distribution can be written in the form

$$
\alpha_{2}\left(\theta_{0}, \theta, p\right)=\frac{p}{2} \frac{\cos \theta}{\cos \theta+\cos \theta_{0}} H\left(p, \cos \theta_{0}\right) H(p, \cos \theta)
$$

where $p$ is the probability of nonabsorption in a single interaction ( $p=\Sigma_{s} / \Sigma_{t}$, the ratio of the scattering cross section to the total cross section) and Chandrasekhar's $H$ function is tabulated ${ }^{12}$ for various values of $p$ and $\cos \theta_{0}$. The total albedo as a function of $\theta_{0}$ and $p$ is obtained by integrating Eq. 7.3-11 over the exit angle $\theta$. This integration yields

$$
A_{2}\left(\theta_{0}, p\right)=1-\sqrt{1-p} H\left(p, \cos \theta_{0}\right)
$$


Spencer, Diaz, and Moses ${ }^{13}$ have applied Chandrasekhar's albedo to neutron duct penetration and compared it with the Monte Carlo data.

Mockel $^{14}$ studied alternate approaches for determining the total thermal-neutron albedo for strongly absorbing media as a function of slab thickness. His objective was to develop a method that would be readily adaptable to computer usage and still provide good results with less

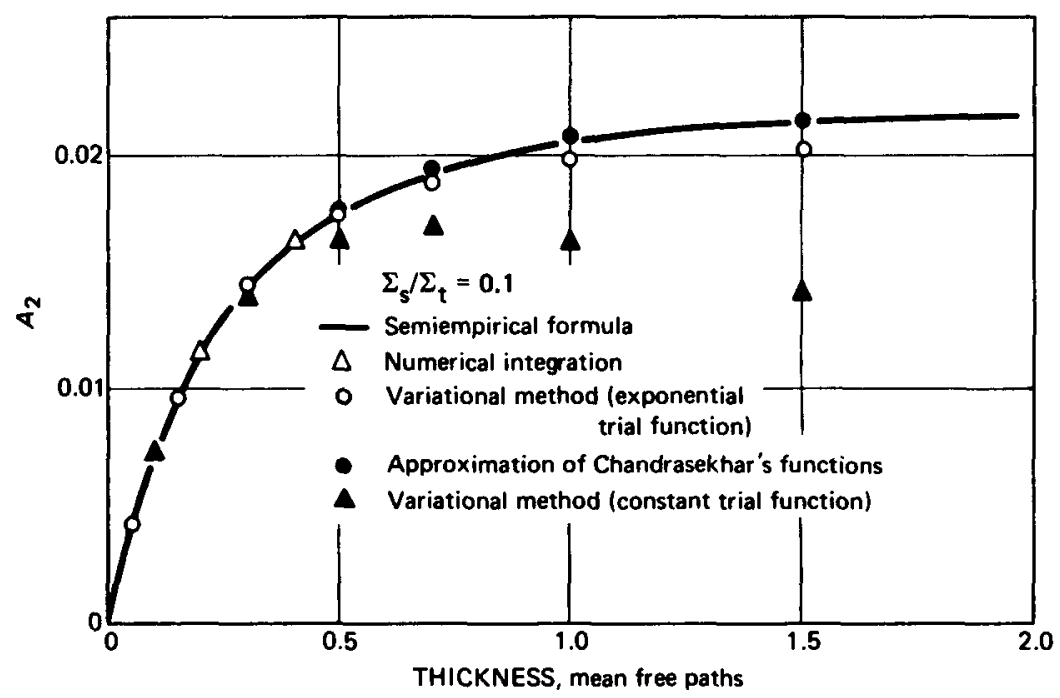

Fig. 7.13 - Total albedos obtained by various methods for thermal neutrons isotropically incident on a strongly absorbing medium $\left(\Sigma_{s} / \Sigma_{t}=0.1\right)$. [From A. Mockel, Nuclear Science and Engineering, 22: 346 (1965).]

computing time than is required for integration of the Boltzmann equation. A variational method with a constant trial function was found to give poor results except for thin slabs. In a search for better methods, three solutions were tried which were based on an approximation to the moments of the Chandrasekhar function, a variational solution with an exponential trial function, and a diffusion-like semiempirical formula. Figures 7.13 and 7.14 compare the results from the three methods for isotropic incidence and two values of $\Sigma_{s} / \Sigma_{t}$. Also shown are results obtained with a constant trial function and with numerical integration of the Boltzmann equation.

The semiempirical formula provides a fit within $2 \%$ for an isotropically incident current (plane isotropic source) and within $1 \%$ for a current either normally incident or incident with a cosine distribution (isotropically incident flux). It has the form 


$$
A_{2}=\frac{1-e^{-2 \alpha x}}{1-A e^{-2 \beta x}}\left[\frac{1-f(p)}{1+f(p)}+g(p)\right]
$$

where $p=\Sigma_{s} / \Sigma_{t}$, as before, $x$ is slab thickness in mean free paths, and values of the coefficients and functions are given in Appendix O, Table O.5, for three conditions of neutron incidence.

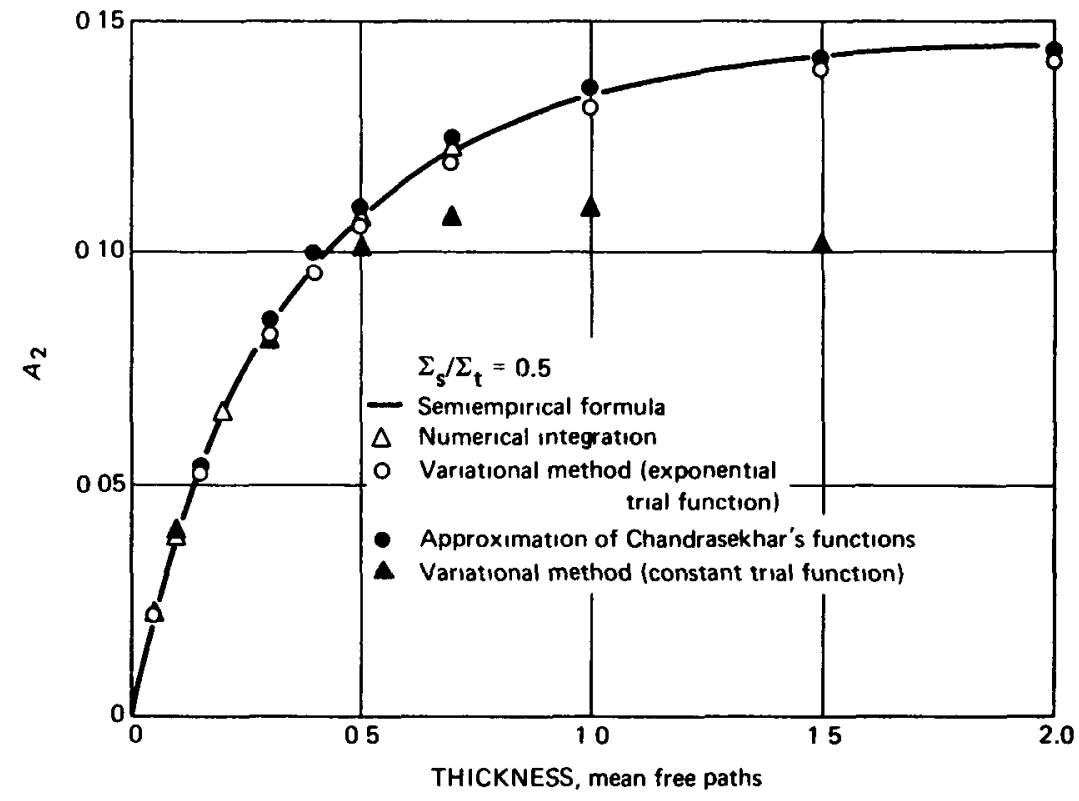

Fig. 7.14-Total albedos obtained by various methods for thermal neutrons isotropically incident on a strongly absorbing medium $\left(\Sigma_{s} / \Sigma_{t}=0.5\right)$. [From A. Mockel, Nuclear Science and Engineering, 22: 346 (1965).]

Pomraning $^{15}$ proposed a variational solution with an exponential trial function which yields for the total albedo the expression

$$
A_{2}=\frac{2}{(1+\nu) \ln \left(1-\nu^{2}\right)}[\ln (1+\nu)-\nu]
$$

for normally incident thermal neutrons and the expression

$$
A_{2}=-\frac{4}{\nu^{2} \ln \left(1-\nu^{2}\right)}[\ln (1+\nu)-\nu]^{2}
$$


for isotropically incident thermal neutrons. Here $\nu$ is a positive quantity satisfying the transcendental equation

$$
\frac{2 \nu}{c}=\ln \left(\frac{1+\nu}{1-\nu}\right)
$$

and $c$ is the mean number of secondaries per collision.

Values obtained with these relatively simple formulas compare very favorably with the results of exact solutions by Chandrasekhar, ${ }^{12}$ the difference being generally less than $2 \%$.

Equations 7.3-14 and 7.3-15 are derived from the more general expression

$$
A_{2}=\frac{2 \Gamma}{\ln \left(1-\nu^{2}\right)}[\ln (1+\nu)-\nu]
$$

where

$$
\Gamma=\frac{\int_{0}^{1} d \mu \frac{\mu B(\mu)}{1+\mu \nu}}{\int_{0}^{1} \mu B(\mu) d \mu}
$$

in which $\mu$ is the cosine of the incident angle and $B(\mu)$ is the general expression for the angular distribution of the incident flux. The success of Eqs. 7.3-14 and 7.3-15 for normal and isotropic incidence suggests that Eq. 7.3-17 could be applied to other angular distributions with equal success.

Wells ${ }^{16}$ determined total and differential thermal-neutron albedos for portland concrete by analyzing Monte Carlo results obtained in a calculation that he had originally performed to establish the distribution of capture gamma-ray sources in concrete and air due to thermal neutrons incident on the concrete. ${ }^{17}$ Expressions that he derived to fit the Monte Carlo results are

$$
A_{1}=0.66 \cos ^{2 / 3} \theta_{0}
$$

and

$$
\alpha_{1}=0.21 \cos ^{2 / 3} \theta_{0} \cos \theta
$$

which, when converted to type 2 albedos, are given by

$$
A_{2}=0.66 \cos ^{-1 / 3} \theta_{0}
$$


and

$$
\alpha_{2}=0.21 \cos ^{-1 / 3} \theta_{0} \cos \theta
$$

A type 3 total albedo is obtained by integrating the differential albedo over the exit hemisphere as follows:

$$
\begin{aligned}
A_{3}(\text { portland }) & =\int \alpha_{1} \sec \theta d \Omega \\
& =\int_{0}^{\pi / 2} \int_{0}^{2 \pi} 0.21 \cos ^{2 / 3} \theta_{0} \sin \theta d \theta d \phi \\
& =1.32 \cos ^{2 / 3} \theta_{0}
\end{aligned}
$$

Expression 7.3-23 is to be compared with an expression obtained by Wells for a particular concrete used in structures built at the ORNL Tower Shielding Facility (TSF):

$$
A_{3}(T S F)=1.3 \cos \theta_{0}
$$

In both the preceding calculations the thermal neutrons were assumed to scatter isotropically in the center-of-mass system with no energy loss, and histories were terminated after a fixed number of interactions. Statistical estimates were made for each interaction point. The concrete compositions used in these calculations are given in Table 7.2.

For their thermal-neutron albedos, Maerker and Muckenthaler ${ }^{18}$ per-

\begin{tabular}{|c|c|c|}
\hline Element & $\begin{array}{l}\text { Portland concrete, } \\
10^{21} \text { atoms } \mathrm{cm}^{-3}\end{array}$ & $\begin{array}{l}\text { TSF concrete, } \\
10^{21} \text { atoms cm }\end{array}$ \\
\hline Hydrogen & 2.868 & 15.6 \\
\hline Oxygen & 43.260 & 39.6 \\
\hline Carbon & 6.507 & 5.42 \\
\hline Magnesium & & 0.40 \\
\hline Aluminum & & 1.32 \\
\hline Silicon & 9.899 & 10.00 \\
\hline Calcium & 8.736 & 7.40 \\
\hline Iron & & 0.31 \\
\hline
\end{tabular}
formed single-velocity Monte Carlo calculations for a 23-cm-thick steelreinforced concrete slab. Two different scattering laws were used for the

Table 7.2-ASSUMED CONCRETE COMPOSITIONS USED IN MONTE CARLO CALCULATION BY WELLS ${ }^{16}$ 
water contained in the concrete: (1) isotropic scattering in the laboratory system and (2) anisotropic scattering with an approximation based on experiments by Greenspan and Baksys ${ }^{19}$ at $0.0358 \mathrm{eV}$. Scattering from all other constituents in the concrete was assumed to be isotropic in the laboratory system.

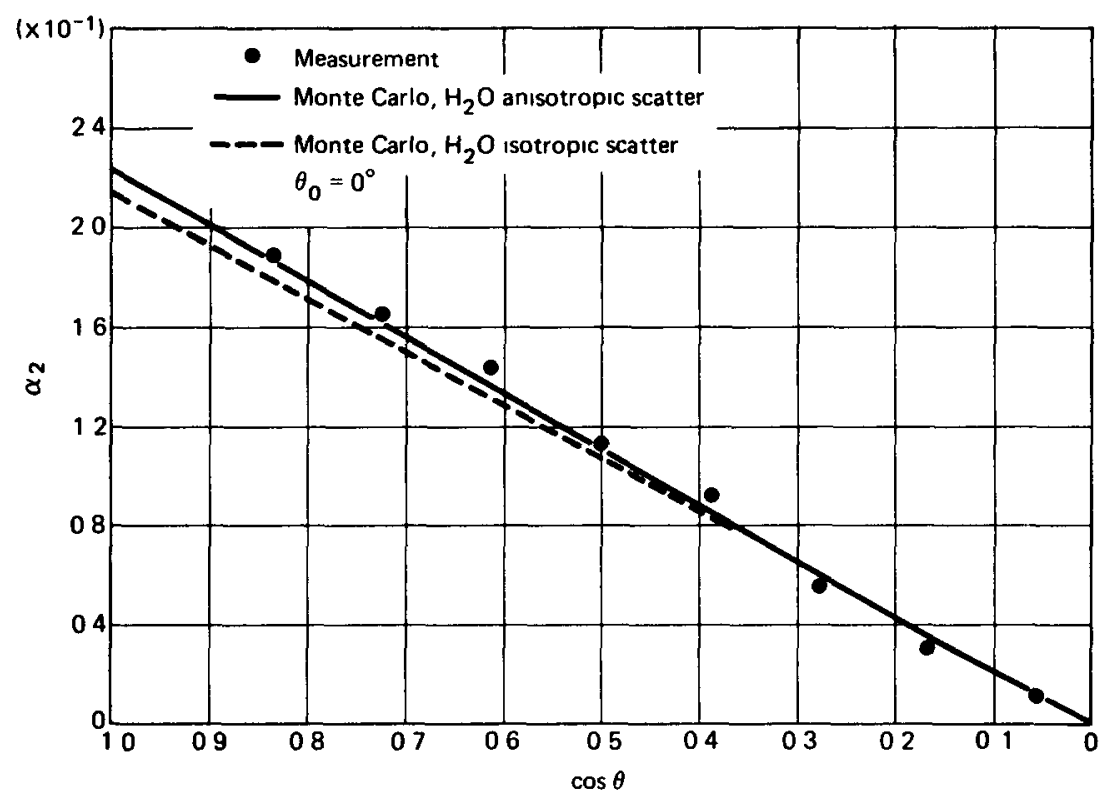

Fig. 7.15-Differential albedos for thermal neutrons normally incident on steelreinforced concrete. A comparison of single-velocity Monte Carlo calculations and ORNL TSF experiments. [From R. E. Maerker and F. J. Muckenthaler, Nuclear Science and Engineering, 26: 345 (1966).]

Typical results from the calculations are shown in Figs. 7.15 and 7.16, both of which illustrate that the best fit to the experimental data is obtained when the scattering in water is assumed to be anisotropic. Figure 7.16 also shows that even for thermal neutrons there is some dependence of the albedo on the azimuthal angle.

From an analysis of their data, Maerker and Muckenthaler fitted their results for the differential albedo as follows:

$$
\begin{aligned}
\alpha_{2}= & \frac{0.0875 \mu}{\mu+\mu_{0}}(1+1.28 \mu)\left(1+1.62 \mu_{0}-0.42 \mu_{0}^{2}\right) \\
& \times\left[1+\left(1-\mu_{0}\right)(1-\mu)\left(-0.10+0.43 \cos \phi+0.20 \cos ^{2} \phi\right)\right]
\end{aligned}
$$


where $\mu=\cos \theta$ and $\mu_{0}=\cos \theta_{0}$. A much simpler fit was obtained for the total thermal-neutron albedo (integrated over all exit angles):

$$
A_{2}=0.86-0.19 \cos \theta_{0}
$$

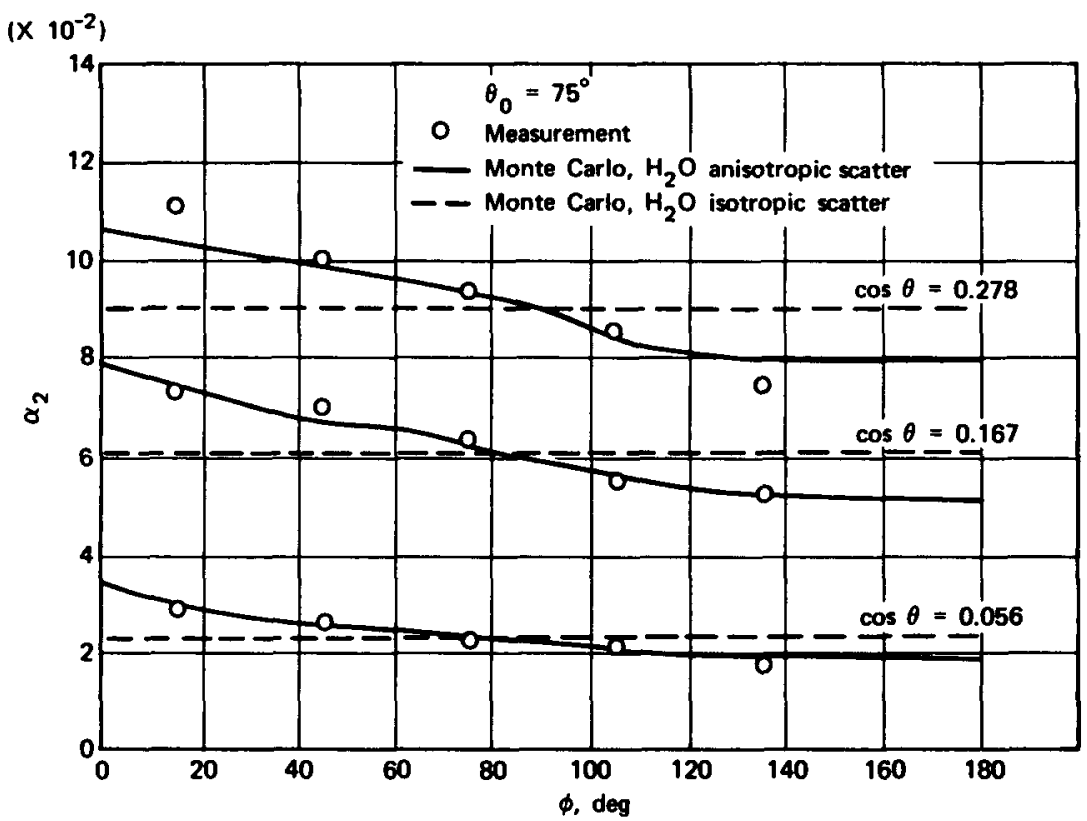

Fig. 7.16-Differential albedos of thermal neutrons incident at $75^{\circ}$ on steel-reinforced concrete. A comparison of single-velocity Monte Carlo calculations and ORNL TSF experiments. [From R. E. Maerker and F. J. Muckenthaler, Nuclear Science and Engineering, 26: 346 (1966).]

Results obtained for concrete with the various equations for thermalneutron albedos are compared in Table 7.3. Comparisons are made between the total-current albedo for various conditions of incidence. For all cases except $\theta_{0}=75^{\circ}$, there would appear to be $\pm 10 \%$ agreement between all the values. The formula due to Wells should be limited to angles of $\theta_{0}<$ approximately $60^{\circ}$ because of its tendency to overpredict at grazing incidence.

(b) Neutrons Incident at Nonthermal Energies. The only estimates available for albedos of reflected thermal neutrons resulting from incident neutrons of higher energy are those from the Monte Carlo calculations of Coleman et al. ${ }^{8}$ for $0.5-\mathrm{eV}$ to $200-\mathrm{keV}$ neutrons incident on the same steel-reinforced concrete described previously (see especially Sec. 7.3.2). 
Expressions that reproduce the Monte Carlo values to within $\pm 15 \%$ for the differential albedos and to within $\pm 10 \%$ for the total albedos are given in Appendix O, Table O.6. The reflected angular distributions (differential albedos) for the six highest energy groups have a shape that is independent of $\mu_{0}$ (the cosine of the incident polar angle) and identical to the shape

Table 7.3-COMPARISON OF VARIOUS VALUES OF THE TOTAL ALBEDO FOR THERMAL-NEUTRON REFLECTION FROM PORTLAND CONCRETE $†$

\begin{tabular}{|c|c|c|c|c|c|c|c|}
\hline \multirow[b]{2}{*}{ Source } & \multicolumn{7}{|c|}{ Values of $A_{2}$ obtained with the formulas of } \\
\hline & $\begin{array}{l}\text { Maerker } \\
\text { and } \\
\text { Mucken- } \\
\text { thaler }^{18}\end{array}$ & Wells $^{16}$ & $\begin{array}{c}\text { Pom- } \\
\text { raning }\end{array}$ & $\begin{array}{l}\text { Halpern } \\
\text { et al. }\end{array}$ & $\begin{array}{l}\text { Glasstone } \\
\text { and } \\
\text { Edlund }^{11}\end{array}$ & Fermi $^{9}$ & $\begin{array}{l}\text { Chandra- } \\
\text { sekhar }^{12}\end{array}$ \\
\hline $\begin{array}{l}\text { Isotropic } \\
\text { distribution }\end{array}$ & 0.78 & 0.792 & 0.8 & 0.719 & 0.74 & & 0.79 \\
\hline $\begin{array}{l}\text { Cosine } \\
\text { distribution } \\
\text { Monodirec- } \\
\text { tional } \\
\text { source }\end{array}$ & 0.734 & & & 0.698 & & & \\
\hline $\begin{array}{l}\theta_{0}=0^{\circ} \\
\theta_{0}=45^{\circ} \\
\theta_{0}=75^{\circ}\end{array}$ & $\begin{array}{l}0.67 \\
0.72 \\
0.855\end{array}$ & $\begin{array}{l}0.66 \\
0.74 \\
0.996\end{array}$ & 0.698 & 0.645 & & $\begin{array}{l}0.745 \\
0.78 \\
0.82\end{array}$ & $\begin{array}{l}0.691 \\
0.730 \\
0.82\end{array}$ \\
\hline
\end{tabular}

$\dagger \Sigma_{s} / \Sigma_{t}=0.9849$ except for the Maerker-Muckenthaler results, which are based on the five-region concrete slab discussed in the text.

derived by $\mathrm{Fermi}^{9}$ for the emergent angular distribution from a plane surface in the simplified case of thermal neutrons diffusing in a noncapturing and isotropically scattering semi-infinite medium.

Some contribution is also made to the emergent thermal-neutron current from the moderation of incident neutrons with energies greater than $200 \mathrm{keV}$. Coleman et al. accounted for these higher energy neutrons by extrapolating the results of Table 0.6 to obtain expressions for energies up to $9.57 \mathrm{MeV}$ (see Appendix O, Table O.7). For the spectrum of neutrons from the ORNL Tower Shielding Reactor II, which was the source used in verifying the calculations, it was estimated that a consistent error of $20 \%$ in the extrapolated results would lead to an error of only about $8 \%$ in the predicted values of the differential thermal-neutron albedos. 


\subsection{GAMMA-RAY ALBEDOS}

The primary interaction that contributes to the backscattering or albedo of gamma rays is Compton scattering from electrons. In this interaction the photon rebounds with an energy that is directly dependent on the scattering angle and the incident energy and is given by the Klein-Nishina formula (Sec. 3.2.1). The higher the incident energy, the more strongly will the forward direction be favored. Thus the gamma-ray albedo has an inverse relation with the incident-photon energy. Owing to the strongly peaked forward scattering, the gamma-ray albedo also has a strong dependence on the azimuthal angle $\phi$; i.e., the scattering angle $\theta_{s}$ (see Fig. 7.1) at $\phi=0^{\circ}$ is smaller than the scattering angle at $\phi=180^{\circ}$, and hence the albedo decreases with increasing $\phi$. The magnitude of the difference increases with increasing values of the incident polar angle $\theta_{0}$; thus at grazing incidence the difference lies between forward scattering $\left(\phi=0^{\circ}\right)$ and backscattering $\left(\phi=180^{\circ}\right)$.

Another interaction that contributes to the reflected energy for incident gamma rays of high energy is pair production (Sec. 3.2.1). The positron created in this reaction is annihilated by combining with an atomic electron and releasing energy in the form of two new gamma rays. This reaction is possible only if the energy of the incoming gamma ray is greater than $1.022 \mathrm{MeV}$, and it is predominant only at energies above about $5 \mathrm{MeV}$. Each of the photons created has an energy of $0.511 \mathrm{MeV}$, which is greater than the maximum energy possible for gamma rays scattered backward $\left(\theta_{s}=180^{\circ}\right)$ by Compton scattering.

Leimdörfer ${ }^{20}$ investigated the relative contributions by positron annihilation and Compton scattering to the total gamma-ray albedo for concrete in a calculation that considered single scattering only. The results for normally incident gamma rays are shown in Fig. 7.17, in which the fraction of the albedo due to annihilation is plotted as a function of the incident gamma-ray energy. Also plotted is the same fraction from a calculation by Wells ${ }^{21}$ in which multiple scattering was considered.

As with neutrons, most of the studies of gamma-ray albedos have been carried out either by Monte Carlo analysis or by experiments and most of them have been for concrete. Where both experimental and calculated data are available, there is good agreement (within 10\%); however, experiments have been limited to low gamma-ray energies $(<2 \mathrm{MeV})$ that can be obtained from isotope sources, and definitive Monte Carlo calculations have been performed for only a few materials. 
One of the earliest Monte Carlo calculations was performed by Berger and Doggett, ${ }^{22}$ who obtained the total-dose albedo, $A_{D_{3}}$, for monoenergetic sources incident on iron, tin, lead, and water. From these calculations a quantitative measure of the dependence of the albedo on the thickness of

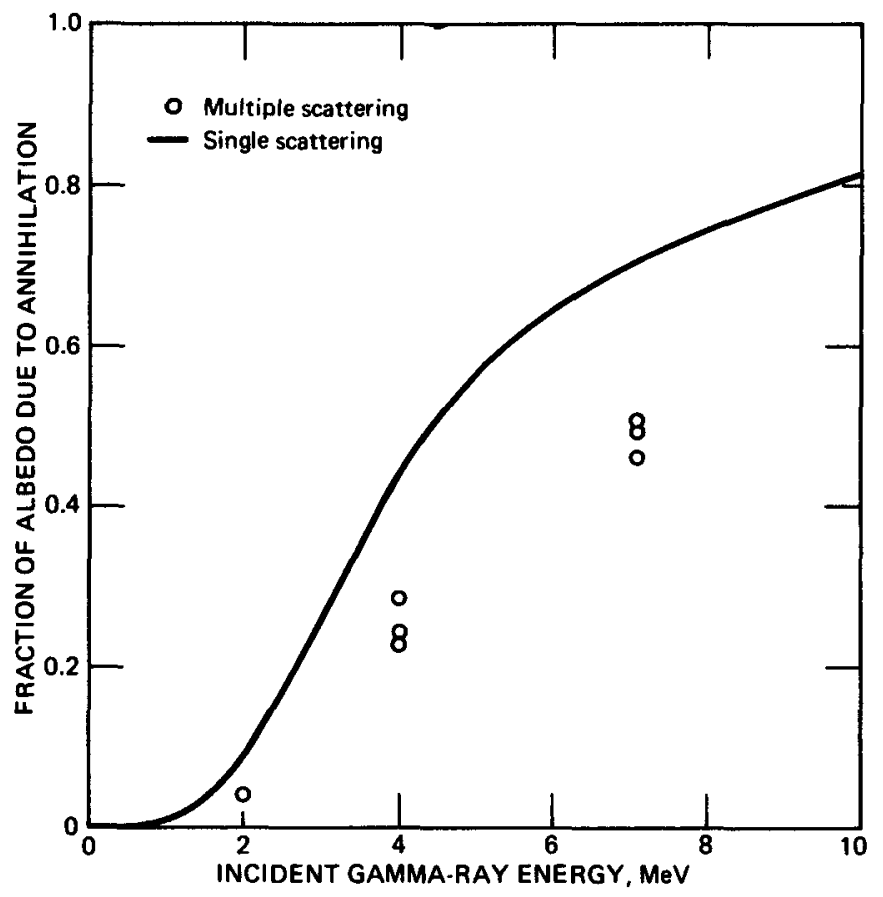

Fig. 7.17 - Ratio of pair-production annihilation albedo to total albedo for gamma rays normally incident on concrete. (From Leimdörfer ${ }^{20}$ and Wells. ${ }^{21}$ )

the scattering material was obtained. This dependence is illustrated for iron and water in Table 7.4.

Berger and Raso ${ }^{2,24}$ carried out an extensive series of Monte Carlo calculations to determine the total-energy albedo for monoenergetic gamma rays incident on a variety of materials, and in some cases they obtained energy and angular distributions. An interesting result of their work is the analysis of the variation in the total albedo with the atomic number of the scattering material. Plots of the albedo for normally incident gamma rays are shown in Fig. 7.18. These data may be fitted by smooth curves; however, data at intermediate $Z$ values would greatly increase confidence in interpolation, particularly at the $2.0-\mathrm{MeV}$ level. 
Raso $^{25}$ performed additional calculations for concrete for source energies of $0.5,1,2,4,6$, and $10 \mathrm{MeV}$ and polar angles of incidence of $\cos \theta_{0}=0.10,0.25,0.50,0.75$, and 1.0 . The emergent gamma rays were divided into 8 polar and 12 azimuthal angular increments, and differentialdose albedos, $\alpha_{D_{2}}\left(E_{0}, \theta_{0}, \theta, \phi\right)$, were obtained for all exit-angle combinations. Total albedos, $A_{D_{2}}\left(E_{0}, \theta_{0}\right)$, were obtained for each incident energy and

Table 7.4-DEPENDENCE OF GAMMA-RAY TOTAL-DOSE ALBEDO, $A_{D 3}$, ON MATERIAL THICKNESS $\dagger$

\begin{tabular}{cccccc}
\hline & & & \multicolumn{3}{c}{$\begin{array}{c}\text { Fraction of reflected } \\
\text { photons reflected } \\
\text { within depth of }\end{array}$} \\
\cline { 3 - 6 } Material & MeV & deg & $\begin{array}{c}\text { 0.5 } \\
\text { mean free } \\
\text { paths }\end{array}$ & $\begin{array}{c}1.0 \\
\text { mean free } \\
\text { paths }\end{array}$ & $\begin{array}{c}2.0 \\
\text { mean free } \\
\text { paths }\end{array}$ \\
\hline $\mathrm{H}_{2} \mathrm{O}$ & 0.66 & 0 & 0.65 & 0.88 & 0.99 \\
& 0.66 & 60 & 0.61 & 0.96 & 1.0 \\
Iron & 1.0 & 0 & 0.79 & 0.93 & 1.0 \\
& 1.0 & 60 & 0.89 & 0.98 & 1.0 \\
\hline
\end{tabular}

†Adapted from Berger and Doggett. ${ }^{2}$

angle. Values of the differential- and total-dose albedos for normal incidence are shown in Figs. 7.19 and 7.20 , respectively.

Figures 7.19 and 7.20 also show Monte Carlo results obtained by Wells, ${ }^{21}$ who calculated differential- and total-dose albedos for gamma-ray reflection from concrete by analysis of prior Monte Carlo data ${ }^{26}$ on gamma-ray scattering in air and concrete. The results of Raso and Wells are essentially in agreement on the total albedo for energies of $2 \mathrm{MeV}$ and below; there are, however, some differences in the differential albedos at these energies. At energies above $2 \mathrm{MeV}$, there is a substantial disagreement between Raso and Wells on the magnitude of the total albedo, as shown in Fig. 7.20. The divergence of agreement with increasing energy could be attributed to the different manner in which pair production was treated in the two calculations.

As is apparent from Fig. 7.19, the incident energies used in the calculations by Wells were $0.6,1,2,4$, and $7 \mathrm{MeV}$. The incident polar angles were $0,30,45,60$, and $75^{\circ}$. The emergent angles varied with the individual problems, and no regularly spaced grid was used; however, by extrapolation 


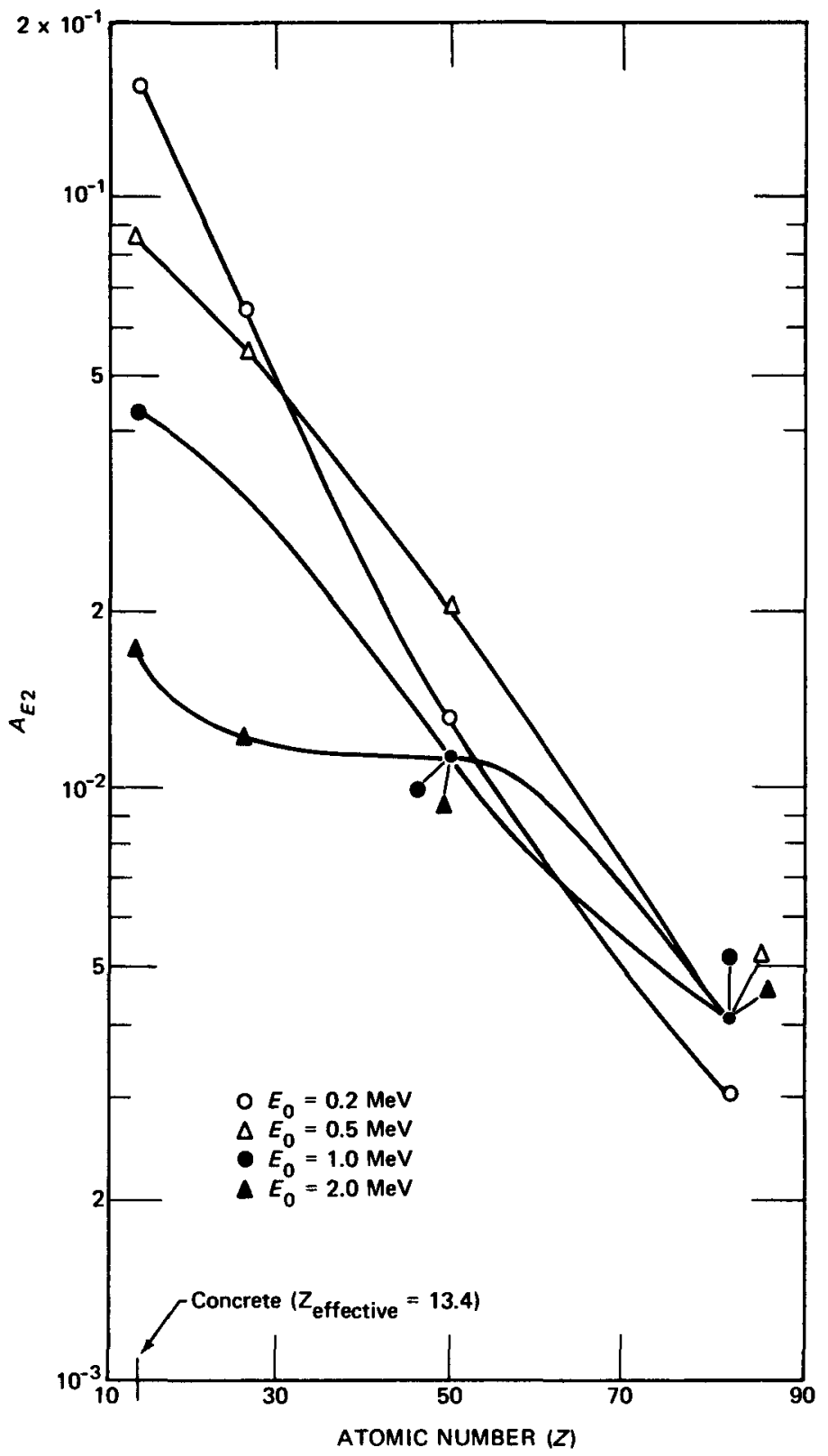

Fig. 7.18 - Total-energy albedo for normally incident monoenergetic gamma rays as a function of the atomic number of the material. (From Berger and Raso. ${ }^{23,24}$ ) 


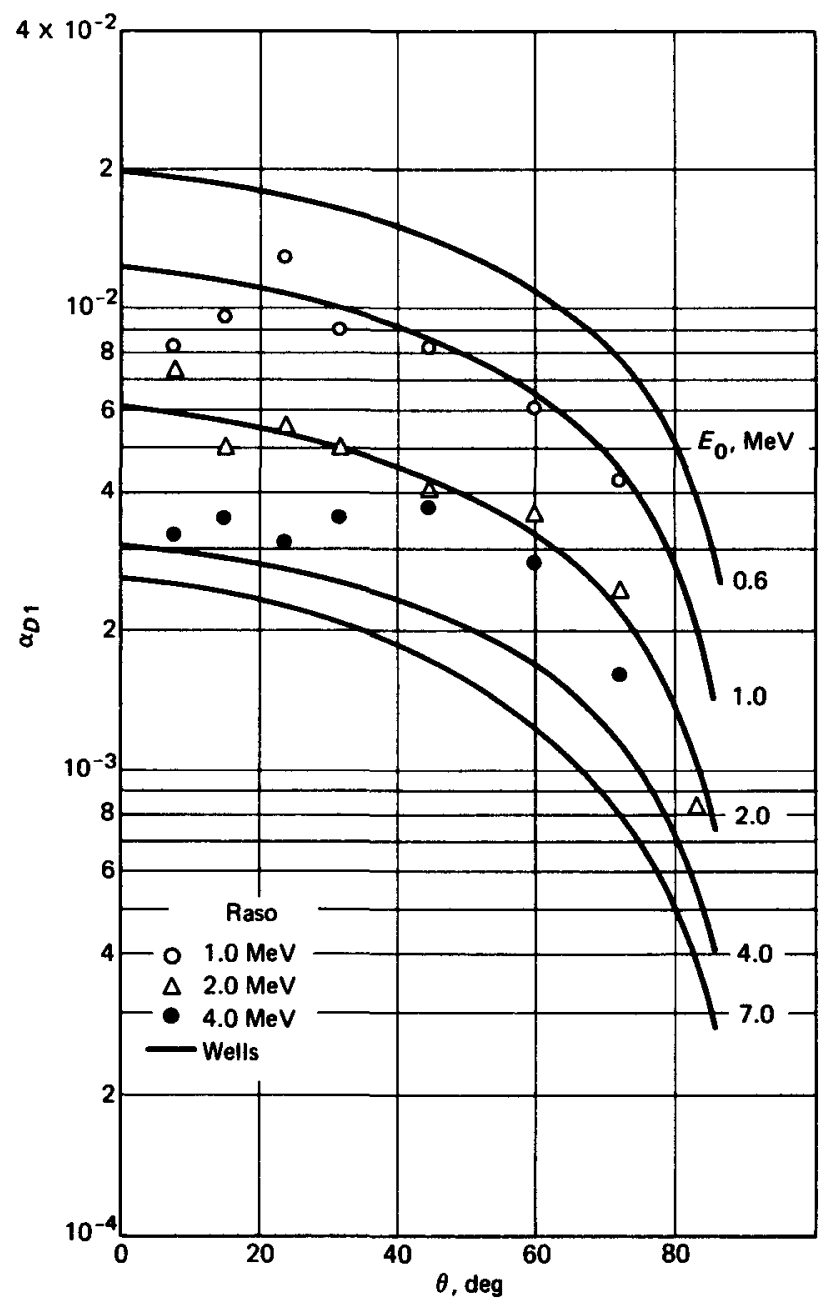

Fig. 7.19-Differential-dose albedos for gamma rays normally incident on concrete. (From Wells ${ }^{21}$ and Raso. ${ }^{25}$ )

and interpolation, smooth curve fits were obtained for $\alpha_{D_{1}}$ vs. $\theta$ for azimuthal angles of 0 and $180^{\circ}$. Complete data sets are given in Ref. 21 .

Chilton and Huddleston ${ }^{6}$ developed a semiempirical formula for the differential-dose albedo for concrete of the form

$$
\alpha_{D_{2}}\left(E_{0}, \theta_{0}, \theta, \phi\right)=\frac{C K\left(\theta_{s}\right) \times 10^{26}+C^{\prime}}{1+\left[\left(\cos \theta_{0}\right) /(\cos \theta)\right]}
$$


where $K\left(\theta_{s}\right)$ is the Klein-Nishina differential-energy scattering coefficient for the scattering angle $\theta_{s}$, and $C$ and $C^{\prime}$ are adjustable parameters that are dependent on the initial energy. Values of $K\left(\theta_{s}\right)$ can be obtained from Eq. 3.2-8 $\left[K\left(\theta_{s}\right)=\sigma(\theta)\right]$. Values of $C$ and $C^{\prime}$ were obtained by Chilton,

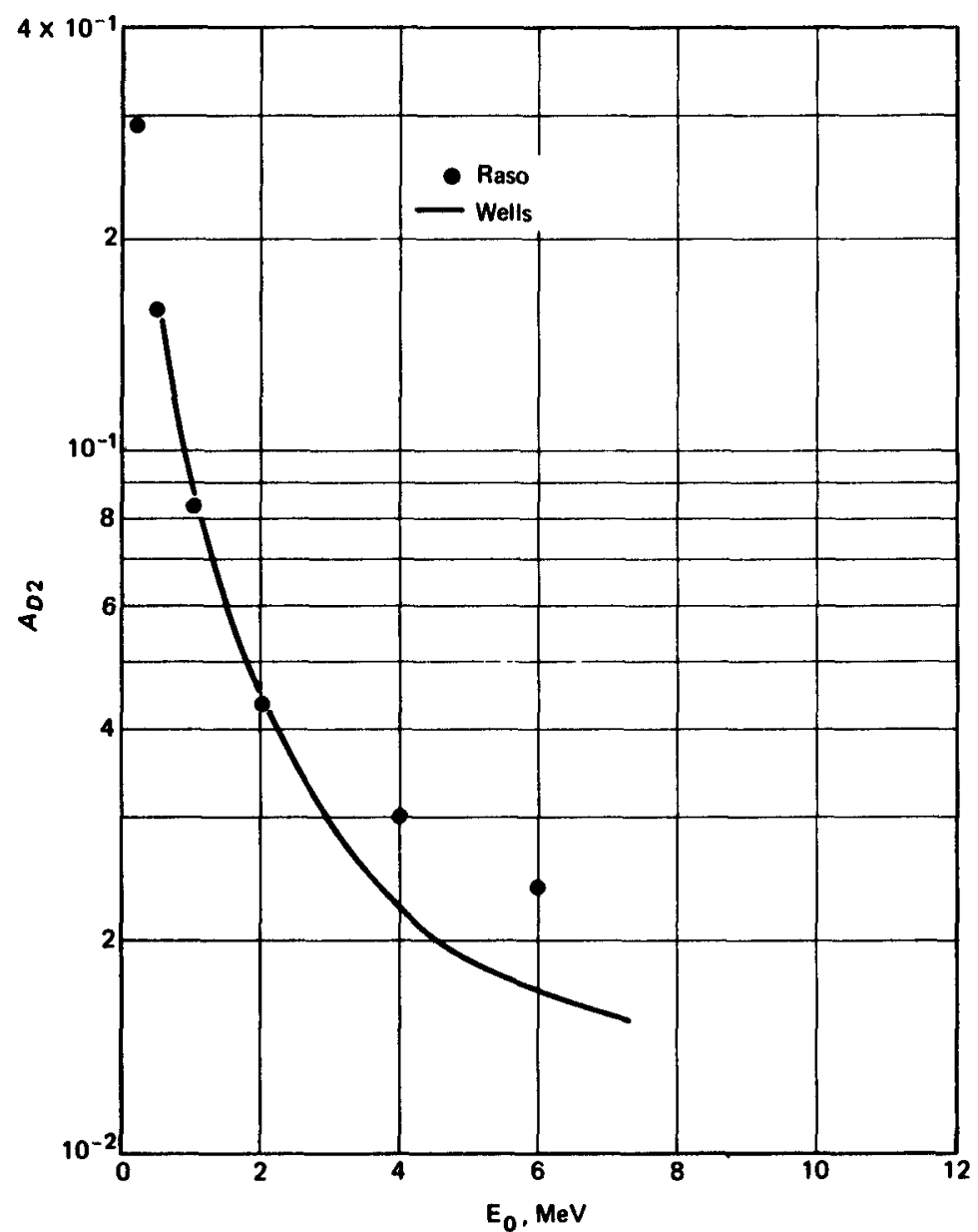

Fig. 7.20-Total-dose albedos for gamma rays normally incident on concrete. (From Wells ${ }^{21}$ and Raso. ${ }^{25}$ )

Davisson, and Beach ${ }^{2}$ from an analysis of earlier Monte Carlo calculations performed by Davisson and Beach. ${ }^{28}$ Parameters were ubtained by normalization to the total calculated albedo rather than to the differential data. The resulting values of $C$ and $C^{\prime}$ are given in Appendix $O$, Table 0.8 , for water, concrete, iron, and lead. Chilton ${ }^{29}$ later revised Eq. 7.4-1 by altering the 
denominator and multiplying by a fraction with five adjustable constants. The newer formula has been applied only to concrete but is accurate within $10 \%$ for ${ }^{137} \mathrm{Cs}$ and ${ }^{60} \mathrm{Co}$. Chilton's formula is given in Table O.9.

In most of the experimental studies of gamma-ray albedos, the sources used were ${ }^{60} \mathrm{Co}$ and ${ }^{137} \mathrm{Cs}$. Examples are the data obtained for concrete by

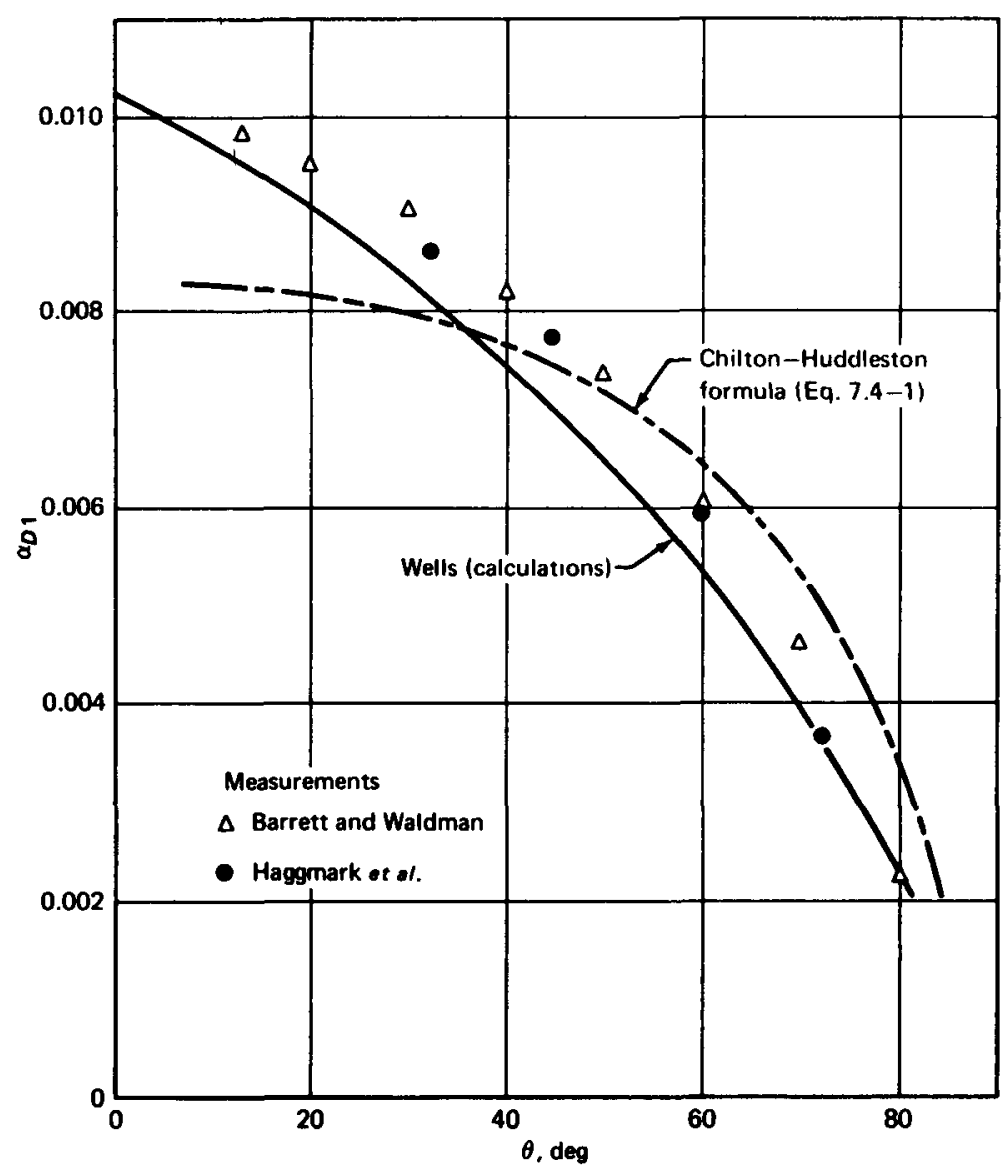

Fig. 7.21 - Comparison between calculated and measured differential-dose albedos for ${ }^{60} \mathrm{Co}$ gamma rays $(1.25 \mathrm{MeV})$ normally incident on concrete. (From Wells. ${ }^{21}$ )

Clifford; ${ }^{30}$ Haggmark, Jones, Scofield, and Gurney; ${ }^{31}$ Barrett and Waldman; ${ }^{32}$ and Berger and Morris. ${ }^{33}$ Comparisons of typical data from these investigations with results from some of the calculations discussed previously are shown in Figs. 7.21 through 7.23. For these figures all the values were converted to type 1 albedos, and, where necessary, the analytical data were interpolated to match the experimental energies. 
Except for small angles of reflection, the experimental and calculated values generally are within $10 \%$. For the single case shown in Fig. 7.22, the weighted values of Raso and the calculations by Wells are very similar. Figure 7.23 points up the strong dependence on $\phi$ for obliquely incident radiation.

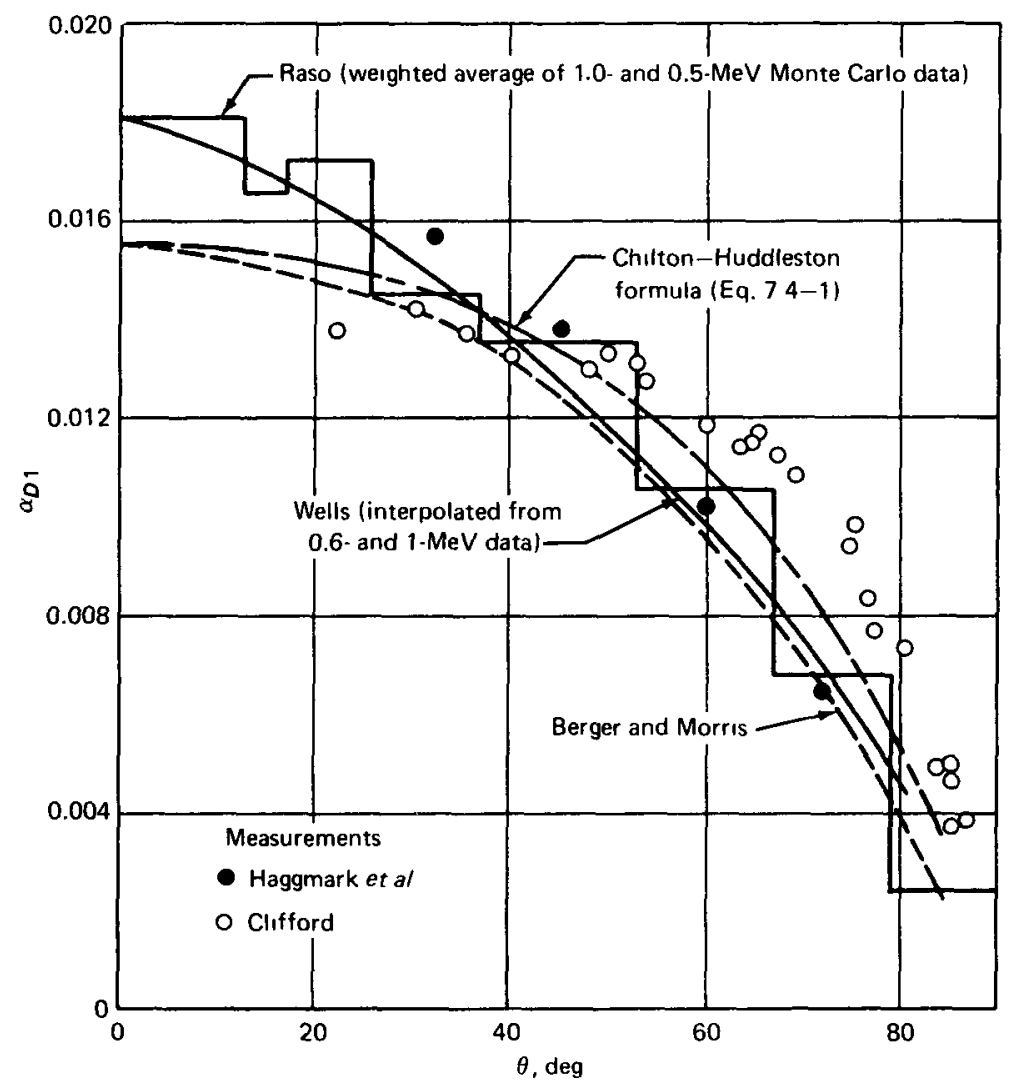

Fig. 7.22-Comparison between calculated and measured differential-dose albedos for ${ }^{137} \mathrm{Cs}$ gamma rays $(0.662 \mathrm{MeV})$ normally incident on concrete. (From Wells. ${ }^{21}$ )

Haggmark et al. ${ }^{31}$ found that their experimental data on differentialdose albedos for concrete, iron, and aluminum could all be represented by the expression

$$
\alpha_{D_{3}}\left(E_{0}, \theta_{0}, \theta_{s}\right)-b\left(E_{0}, \theta_{0}\right)=e^{-\pi \theta_{s}}
$$

where $\theta_{s}$ is the effective scattering angle shown in Fig. $7.1 ; \alpha_{D_{3}}$ is the differential-dose albedo described in Sec. 7.2 except that the exit direction is 
expressed in terms of $\theta_{s}$, which is a function of $\theta$ and $\phi$; and $b$ is a constant for a given $\theta_{0}, E_{0}$, and material. Figure 7.24 shows a comparison of Eq. 7.4-2 with the experimental data for iron, concrete, and aluminum reduced by the appropriate $b$ value given in Appendix O, Table O.10.

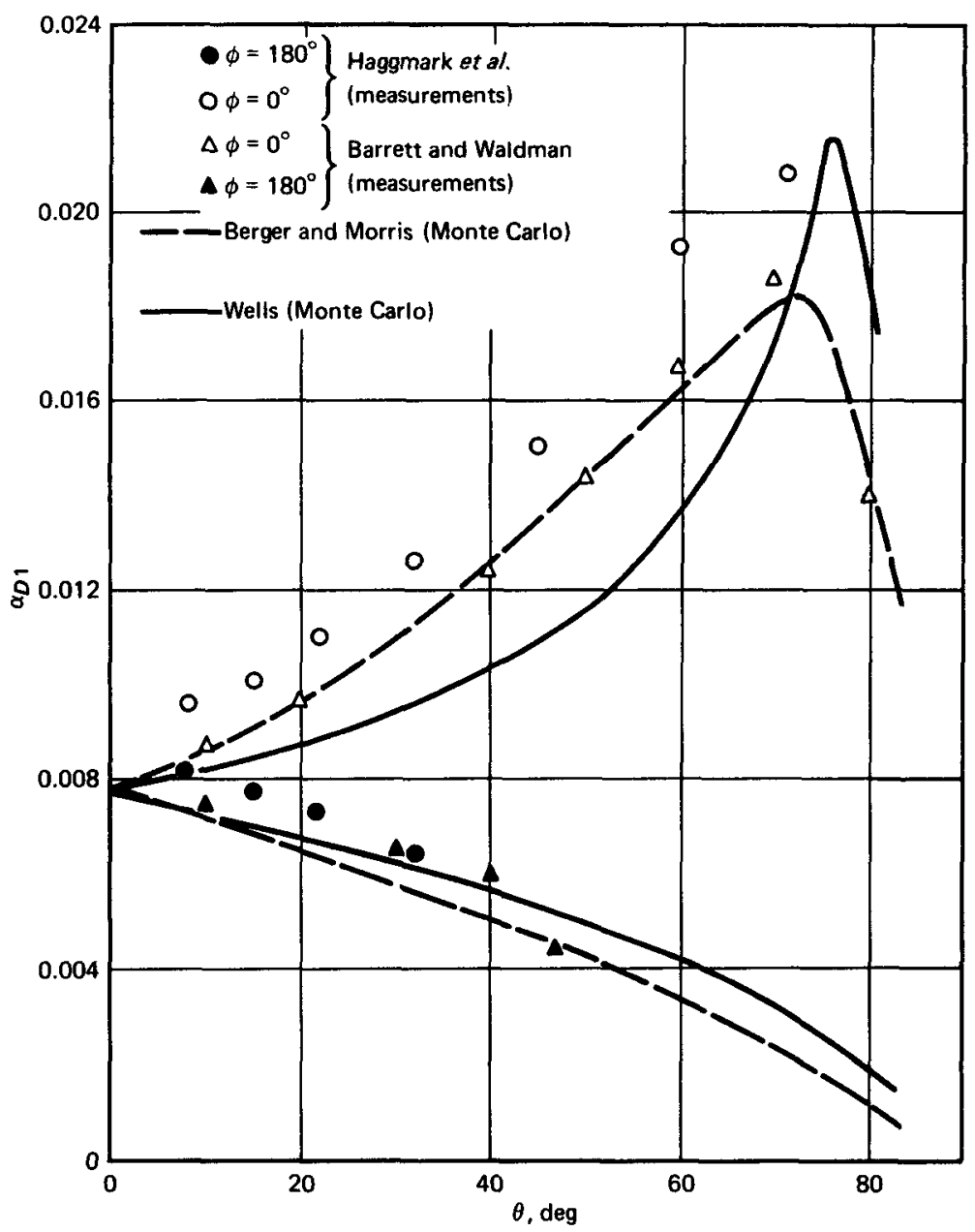

Fig. 7.23-Comparison between calculated and measured differential-dose albedos for ${ }^{60} \mathrm{Co}$ gamma rays $(1.25 \mathrm{MeV})$ incident on concrete at $60^{\circ}$. (From Wells. ${ }^{21}$ )

Equation 7.4-2 is a useful tool for extrapolating albedo data, but it is not valid for grazing angles since the measurements do not include $\theta_{s}<30^{\circ}$.

Leimdorfer used the Monte Carlo method for calculating the total gamma-ray-energy-flux albedo from concrete slabs ${ }^{20}$ and also from spherically concave concrete walls. ${ }^{34}$ An interesting result of the latter studies is shown 
in Fig. 7.25, which gives the energy-flux albedo, $A_{E_{3}}$, for $1-\mathrm{MeV}$ gamma rays from a concrete wall surrounding a spherical cavity as a function of the radius of curvature of the wall. The point isotropic source is located in the center of an evacuated cavity in an infinite concrete medium. The $A_{E}{ }_{3}$ is defined as the ratio of the collided to the uncollided energy-flux density at

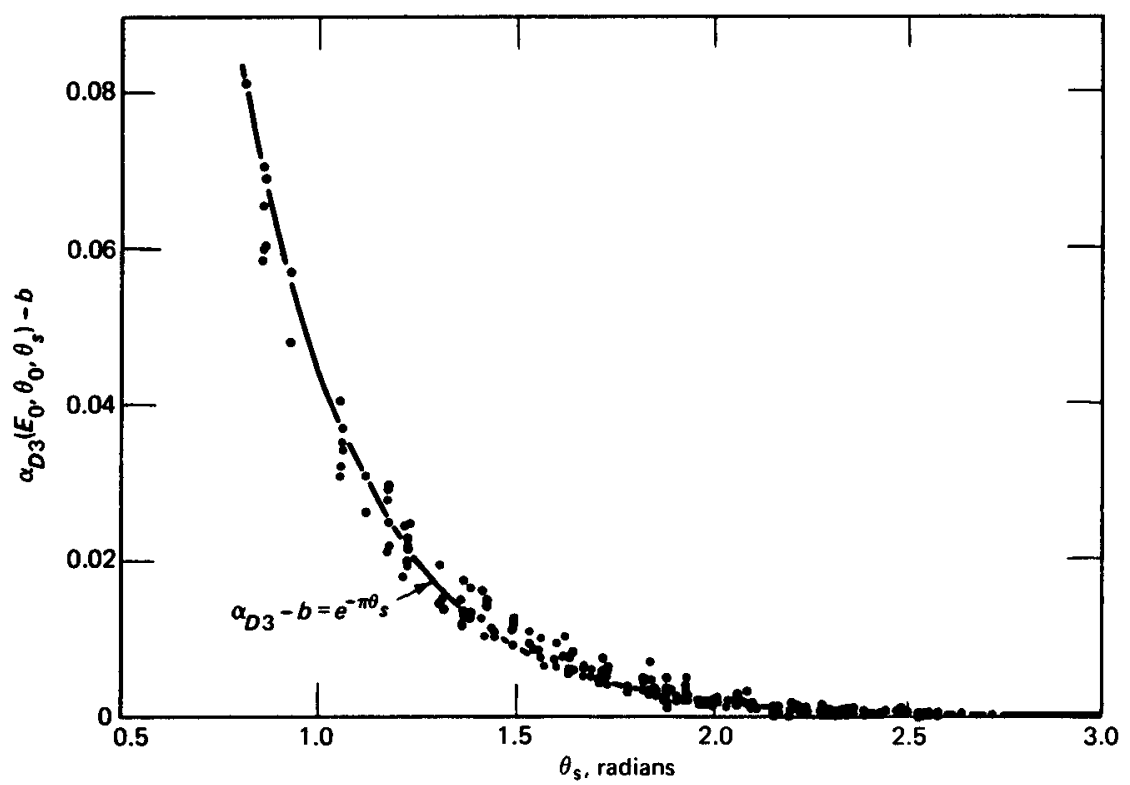

Fig. 7.24-Gamma-ray differential-dose albedo for aluminum, iron, and concrete as a function of the scattering angle $\theta_{s}$. [From L. G. Haggmark, T. H. Jones, N. E. Scofield, and W. J. Gurney, Nuclear Science and Engineering, 23: 145 (1965).]

the wall surface. The infinite-radius (flat-surface) albedo is calculated to be only about $2.5 \%$ higher than the albedo for a $500-\mathrm{cm}$ radius spherical cavity. Other interesting conclusions drawn by Leimdörfer from these calculations are that reflections beyond the third or fourth order do not contribute appreciably to the energy-flux albedo in the spherical geometry and that at least $90 \%$ of the reflections occur within the first $24 \mathrm{~g} \mathrm{~cm}^{-2}$ thickness for gamma-ray energies below $10 \mathrm{MeV}$.

\subsection{SECONDARY-GAMMA-RAY ALBEDOS}

: Secondary gamma rays, i.e., gamma rays produced by the interactions of neutrons with nuclei, can appreciably increase the number of gamma rays 
reflected from a material surface. The most important neutron interaction involved is radiative capture, described in Chap. 3 , which results in capture gamma rays. Since these gamma rays usually create the greatest problem for shield designers, the few studies of secondary-gamma-ray albedos that have been performed involve captures. Activation gamma rays and inelastic-

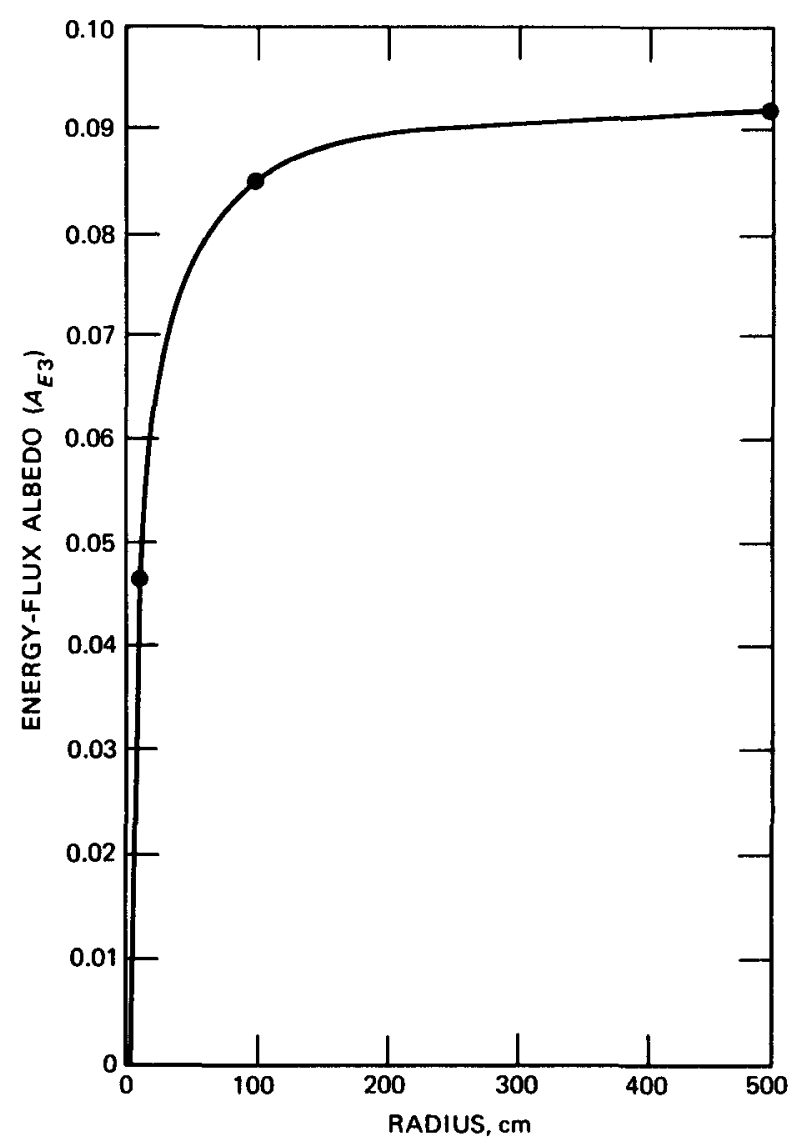

Fig. 7.25-Gamma-ray-energy flux albedo as a function of the radius of the concrete reflecting wall (1-MeV source). (From Leimdörfer. ${ }^{34}$ )

scattering gamma rays could be of concern in special situations. Albedo definitions given in Sec. 7.2 do not apply precisely to secondary-gamma-ray albedos. To emphasize that the incident particles are neutrons and the emergent particles are gamma rays, we have included the notation $(n \gamma)$ in the subscripts associated with the albedo symbols. All albedos are based on an incident-particle current or flux density and an emergent gamma-ray dose current. 
For capture gamma-ray albedos to be determined, the capture gammaray source density within a medium must be known. The density at a point is given by

$$
S_{Y}\left(E^{\prime}\right)=\sum_{i} \int \sigma_{c i}(E) Y_{i}\left(E^{\prime}\right) N_{i} \Phi(E) d E
$$

where $\sigma_{c i}(E)=$ the capture cross section of element $i$ for neutrons of energy E $N_{i}=$ the atomic density of element $i$ $\Phi(E)=$ the scalar neutron-flux density at energy $E$ $Y_{i}\left(E^{\prime}\right)=$ the yield per capture in material $i$ of gamma rays of energy $E^{\prime}$

Equation 7.5-1 displays the quantities that must be known as a function of position within the absorbing material for a calculation of the gamma-ray production in the medium It also illustrates that a number of parameters must be duplicated if secondary-gamma-ray data obtained for one situation is to be applicable to another situation. In many cases sufficient accuracy can be obtained by considering only the thermal-neutron-flux-density profile and the capture cross sections averaged over the thermal group.

Data on capture gamma-ray albedos are quite limited. Wells ${ }^{16}$ performed a series of Monte Carlo calculations from which he obtained capture gamma-ray albedos for the portland and TSF concrete compositions given in Table 7.2. Assuming that only thermal neutrons were incident, he arrived at the following expressions for the differential-dose albedo, which is given in rads $\mathrm{hr}^{-1}$ steradian $^{-1}$ per unit thermal-neutron-flux density incident at angle $\theta_{0}$ :

$$
\alpha_{D_{1}(n, \gamma)}\left(\theta_{0}, \theta\right)=6.986 \times 10^{-8} \cos \theta_{0} \cos \theta
$$

for portland concrete and

$$
\alpha_{D_{1(n, \gamma)}}\left(\theta_{0}, \theta\right)=1.046 \times 10^{-7} \cos ^{5 / 4} \theta_{0} \cos ^{2 / 3} \theta
$$

for TSF concrete. In calculating the capture gamma-ray dose rate along the axis of a concrete-lined cylindrical hole, Wells found that the results obtained with the TSF albedo were higher by a factor of 40 to $50 \%$ than those obtained with the albedo for portland concrete, which demonstrates that variations in local aggregates cause variations in the capture gamma-ray albedo because of changes in both capture density and gamma-ray yield per capture. 
Maerker and Muckenthaler ${ }^{18}$ also obtained capture gamma-ray dose albedos from Monte Carlo calculations for thermal neutrons incident on concrete. In their case the concrete was the same five-region slab containing steel in two regions. The differential-albedo data were fitted to the expression

$$
\alpha_{D_{2}(n, \gamma)}=\mu^{2 / 3}\left(1.01+1.67 \mu_{0}-0.56 \mu_{0}^{2}\right) \times 10^{-7}
$$

where $\alpha_{D_{2}}$ is given in rads $\mathrm{hr}^{-1}$ steradian ${ }^{-1}$ per incident unit thermal-neutron current and $\mu_{0}$ and $\mu$ are the cosines of the incident and reflected polar angles, respectively. The corresponding expression for the total albedo is

$$
A_{D_{2}(n, \gamma)}=3.77 \times 10^{-7}\left(1.01+1.67 \mu_{0}-0.56 \mu_{0}^{2}\right)
$$

given in rads hr-1 per incident unit thermal-neutron current. These equations fit the Monte Carlo data to within 15\%.

Figure 7.26 compares the differential albedos obtained with the Maerker-Muckenthaler expression with those obtained with the Wells expression for TSF concrete, the latter being converted to type 2 albedos. The data differ by a factor of approximately 2 , which may have resulted from several causes. In the calculations by Wells, neutron histories were terminated after 50 collisions, whereas 200 collisions were allowed in the calculations by Maerker and Muckenthaler. According to additional studies performed by Maerker and Muckenthaler ${ }^{18}$ and later by Coleman et al., ${ }^{8}$ this would account for no more than $20 \%$ of the difference. Other differences may appear in the gamma-ray yields, the minimum gamma-ray energy considered, and the concrete composition. In particular, the layered concrete slab used by Maerker and Muckenthaler had more iron nearer the surface than did the homogenized system used by Wells. The additional iron at this position probably resulted in more iron-capture gamma rays emerging from the surface.

Coleman et al. ${ }^{8}$ calculated, also by Monte Carlo methods, the capture gamma-ray albedos resulting from the slowing down and capture of neutrons incident on concrete at intermediate energies $(0.5 \mathrm{eV}$ to $200 \mathrm{keV})$. As was the case for the other calculations by Maerker and Muckenthaler, the concrete was the same five-region slab containing two regions with iron; however, at most the iron contributed only $20 \%$ of the capture gamma-ray dose.

The empirical equations representing the results of Coleman et al. are shown in Appendix O, Table O.11. The departure from unity of the last 
term in parentheses in the expressions for the differential albedos reflects the contributions from captures occurring at nonthermal energies. These expressions reproduce the Monte Carlo values to within $15 \%$ for the differential albedos and to within $10 \%$ for the total albedos.

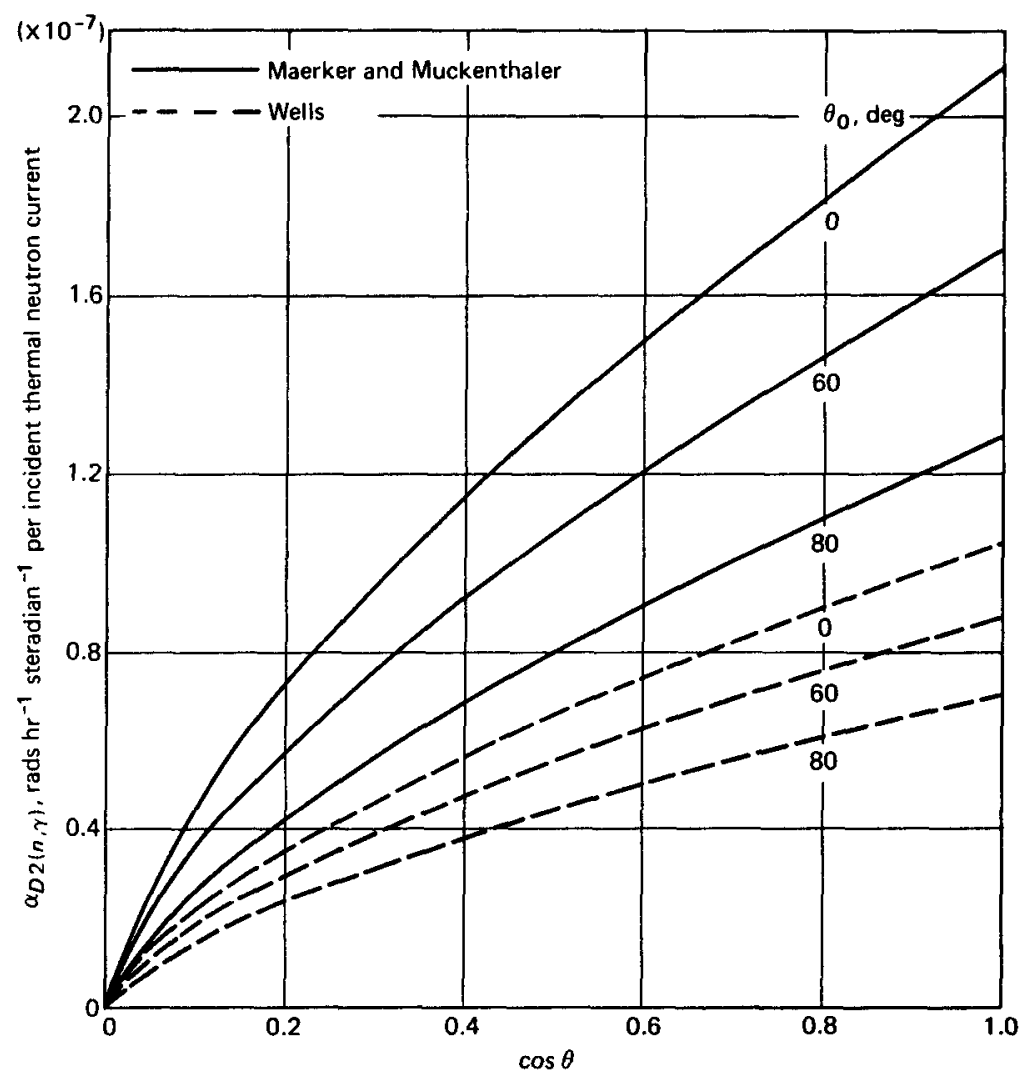

Fig. 7.26-Capture gamma-ray differential albedos for thermal neutrons incident on concrete. (From Wells ${ }^{16}$ and Maerker and Muckenthaler. ${ }^{18}$ )

Using thermal-neutron fluxes measured in TSF concrete, French, Wells, and Schaeffer ${ }^{35}$ calculated the capture gamma source strength as a function of depth and obtained the resulting gamma-ray leakage from the concrete with a Monte Carlo code. The resulting angular dose rates were found to fit

$$
D(\theta)=6.14 \times 10^{-8} \cos \theta
$$

where $D(\theta)$ is the dose rate in rads $h^{-1} \operatorname{steradian}^{-1}$ per unit thermalneutron-flux density at the concrete surface. 
To approximate the capture gamma dose rate from this concrete, we integrate over the solid angle that the surface subtends at the detector

$$
D=\int_{\Omega} \Phi D(\theta) d \Omega
$$

where $d(\theta)$ is given by Eq. 7.5-6 and $\Phi$ is the thermal-neutron-flux density.

\subsection{APPLICATIONS OF ALBEDOS}

Albedos are invaluable in estimating the streaming through ducts, as will be seen in the remainder of this chapter. They are also useful in dealing with

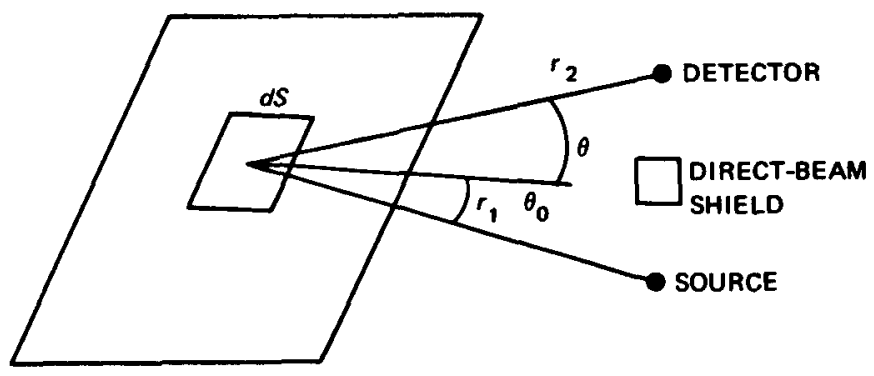

Fig. 7.27-Wall scattering.

other interfaces, such as scattering from the ground in an air-ground geometry or scattering from the inside surfaces within a shielded room. As an illustration, consider the scattering from a wall, as shown in Fig. 7.27, where the detector is shielded from the direct radiation but is exposed to a wall that is irradiated by the source.

We assume a monoenergetic isotropic point source of $N$ particles/sec. The dose rate incident to wall-area increment $d S$ is

$$
D_{0}=\frac{N K\left(E_{0}\right)}{4 \pi r_{1}^{2}}
$$

where $K\left(E_{0}\right)$ is the flux-to-dose conversion factor. If we use type 1 dose albedo data, then the dose reflected to the detector from the area increment will be

$$
\Delta D=\frac{D_{0} \alpha_{D_{1}}\left(E_{0}, \theta_{0}, \theta, \phi\right)}{r_{2}^{2}}
$$


The dose due to scattering from the entire surface will be

$$
D=\int_{S} \frac{D_{0} \alpha_{D 1}\left(E_{0}, \theta_{0}, \theta, \phi\right) d S}{r_{2}^{2}}
$$

The integration over surface area in these equations would ordinarily be carried out numerically. The dose at the wall surface has two components, the uncollided dose arriving from the source and the scattered dose from the wall:

$$
\begin{aligned}
D & =D_{0}\left[1+A_{D_{3}}\left(E_{0}, \theta_{0}\right)\right] \\
& =D_{0}\left[1+\int_{0}^{2 \pi} \int_{0}^{\pi / 2} \alpha_{D_{3}}\left(E_{0}, \theta_{0}, \theta, \phi\right) \sin \theta d \theta d \phi\right]
\end{aligned}
$$

The interface effect is then expressed by the integral.

The albedo of radiation from the floor, ceiling, and walls is often significant to the total dose inside shielded compartments. For example, the neutron dose at the center of an underground shielded compartment due to neutrons penetrating the cover shield is almost a factor of 2 higher than would be predicted on neutrons traveling directly from the shield to the detector. Most of this factor can be predicted by performing an albedo calculation over the inside walls.

\subsection{DUCTS}

Passages through the bulk attenuation layers of a reactor shield are ubiquitous. The working fluid must enter and leave the core. Controls and instruments must have mechanical or electrical conduits leading outside. Maintenance implies access to every region, and access implies door, plug, or labyrinth. The shield designer is inevitably faced with the problem of maintaining the integrity of the shield while providing workable means for the necessary communicating pathways to the outside world. Each shield penetration may be considered a duct, whether it is a $3-\mathrm{m}$ length of $1-\mathrm{cm}$ tubing through the primary shield or a 1 - by $2-\mathrm{m}$ passageway with three turns into the control room.

Design and analysis of a straight duct is relatively uncomplicated. The line-of-sight component is obviously the most important. Radiation entering the duct from surrounding shield materials, either uncollided or scattered, is 
another potentially significant component. Generally these components may be treated with a ray-analysis technique (described in Sec. 7.9).

More-sophisticated approaches are needed for treating ducts with two or more legs (one or more bends) where the scattered component predominates. Because of the importance of duct analysis in a shield design, a number of techniques have been advanced which range from simpleminded (with limited success) to quasi-mystical (adjust enough parameters and it will fit) to thoroughgoing, detailed procedures.

As with other facets of shielding, notably those involving three spatial dimensions, Monte Carlo seems the best method for accurate results. However, a complete analog Monte Carlo calculation for a multibend duct geometry is usually an expensive undertaking, especially if parameter variations are sought in an effort to optimize the design. Consequently modified Monte Carlo programs have been developed which follow particle paths only within the duct (not in the surrounding shield medium) and employ albedo theory to estimate reflectance from the duct walls. In fact, some simplified albedo methods that do not require Monte Carlo (or any computer programs) have been devised with useful results.

We shall review the current literature on each of these methods to provide some examples of the alternative approaches. The applicability and validity of each method is usually demonstrated by comparison with measurements.

\subsection{LINE-OF-SIGHT COMPONENT}

For a duct that penetrates straight through a thick shield and has dimensions that are large compared with the mean free path of the penetrating radiations, the line-of-sight radiation (that which travels directly from the source to the detector through the duct) usually is the most significant portion of the total radiation reaching the detector. If one assumes a plane source at the duct opening and a ratio of the duct length to the diameter $>1$, all line-of-sight points are approximately equidistant from the other end of the duct and may be treated collectively as an equivalent point source. If the plane source is treated as having isotropic current, the equivalent point intensity is the product $S N_{0}$, where $N_{0}$ is the particle emission rate per unit area and time and $S$ is the area on the source plane that is viewed through the duct from the point of interest. For nonisotropic emitters each differential area element must be weighted by the 
angular distribution when integration is performed over the source area. For many geometries and source angular distributions that can be expressed analytically, it is possible to derive formulas for the line-of-sight current through an opening.

Several methods for computing the line-of-sight current in simple ducts of various geometries have been developed. The source is assumed to be a plane normal to the axis of the duct that covers the duct entrance. Two types of source angular current distributions are considered: an isotropic distribution and cosine distributions. Here isotropic distribution (as used in the previous paragraphs) refers to radiation that is given off in all directions in the forward hemisphere with equal probability but with no emission in the backward hemisphere. Thus, if a surface emission is $N_{0} \mathrm{~cm}^{-2} \mathrm{sec}^{-1}$, then the number emitted into any unit solid angle is $N_{0} / 2 \pi \mathrm{cm}^{-2}$ steradian $^{-1}$ sec. $^{-1}$

For cosine distributions the radiation leakage through a unit area on the surface of a self-absorbing volume-distributed source is most intense in the direction normal to the surface, and the variation with the angle $\theta$ from the normal is approximated by a cosine function, in which case the number emitted per unit solid angle is $\left(N_{0} \cos \theta\right) / \pi \mathrm{cm}^{-2} \operatorname{steradian}^{-1} \sec ^{-1}$.

Other functions have been used to describe sources that have more strongly peaked angular distributions than that given by $\cos \theta$. In general, most of these can be represented by $\cos ^{n} \theta$, where $n$ may be as large as 20 . The $\cos ^{n} \theta$ function has been useful in representing a current (or a flux) at a shield surface from an absorbing source region in which the activity per unit volume increases with depth.

Two types of plane sources have been used in analytical expressions derived for the transmission of uncollided particles through ducts. In one type the source strength is defined by an emission rate, which we designate $N_{0}\left(\mathrm{~cm}^{-2} \sec ^{-1}\right)$. In the other type the source strength is defined by a flux density that will be designated here as $\Phi_{0}\left(\mathrm{~cm}^{-2} \mathrm{sec}^{-1}\right)$. The latter source is the more natural one for use in shield design.

When calculating the flux density in a duct, one must take care to ensure that the proper source angular distribution is used in a given situation since there are two basic source descriptions. A particle emission rate is a current and therefore is referenced to a unit of the source area, whereas a flux density is always referenced to a unit of area normal to the particle direction. Thus, to correct for the different orientation of the flux-source reference area, one must multiply the flux density passing through that area 
by $\cos \theta$ to project the reference area on the source plane; i.e., the relation between the angular current $J(\theta)$ and the angular flux $\Phi(\theta)$ is

$$
J(\theta)=\Phi(\theta) \cos \theta
$$

The effect of the difference in the two source descriptions can be shown by the following example. The uncollided flux density in a duct is to be calculated for a given angular distribution $g(\cos \theta) \mathrm{cm}^{-2}$ steradian $^{-1} \mathrm{sec}^{-1}$ specified on the source plane. If $g(\cos \theta)$ is the normalized angular current [the normalization condition on $g(\cos \theta)$ is $\int_{0}^{1} g(\cos \theta) 2 \pi d(\cos \theta)=1$ ], then the flux density at any point in the duct is

$$
\int_{S} N_{0} g(\cos \theta) \frac{d S}{r^{2}}
$$

where $N_{0}$ is the total emission rate, $d S$ is a differential area on the source plane, $S$, and $r$ is the distance between $d S$ and the detector. If, on the other hand, $g(\cos \theta)$ is the normalized angular flux density, then the flux density in the duct is

$$
\int_{S} \Phi_{0} g(\cos \theta) \frac{\cos \theta}{r^{2}} d S
$$

where $\Phi_{0}$ is the total or scalar flux density at the source plane.

A general relation between the emission rate and the flux density can be derived for the very useful case of the $\cos ^{n} \theta$ distribution by defining the angular emission rate (angular current) as

$$
J(\cos \theta)=\frac{n+1}{2 \pi} N_{0} \cos ^{n} \theta
$$

That Eq. $7.8-4$ is properly normalized is shown by calculating the total emission rate:

$$
\int_{0}^{1} \frac{n+1}{2 \pi} N_{0} \cos ^{n} \theta 2 \pi d(\cos \theta)=N_{0}
$$

The corresponding expression for the total flux density, $\Phi_{0}$, at the source plane is then 


$$
\Phi_{0}=\int_{0}^{1} \frac{n+1}{2 \pi} N_{0} \frac{\cos ^{n} \theta}{\cos \theta} 2 \pi d(\cos \theta)=\frac{n+1}{n} N_{0}
$$

It is evident that Eq. 7.8-6 does not hold when $n=0$; in fact, the flux cannot be defined at the source plane when the emission rate is isotropic.

For all the different duct geometries included in the following discussion, the flux density, $\Phi$, or leakage current, $J$, at the exit plane of the duct is expressed in the same units as the source strength (particles $\mathrm{cm}^{-2}$ $\sec ^{-1}$ ). To obtain the total leakage flux density or current through the exit plane of the duct, one must integrate $\Phi$ or $J$ over the exit area.

\subsubsection{Rectangular Ducts}

The work of Hubbell, Bach, and Lamkin ${ }^{36}$ on radiation from uniform rectangular sources with arbitrary angular distributions is directly applicable to the calculation of the uncollided flux density along the axis of a rectangular duct. Radiation crossing a plane $S$ (cross-sectional area of duct normal to axis) is assumed to be known and is designated by an arbitrary function, $g(\cos \theta)$, which is the angular flux density and represents the number of particles traveling in the direction $\theta$ with respect to the duct axis per unit solid angle, unit time, and unit area normal to direction $\theta$ (neutrons $\mathrm{cm}^{-2} \sec ^{-1}$ steradian $\left.^{-1}\right)$. The flux density at any point along the axis is expressed by

$$
\Phi=\int_{S} g(\cos \theta) d \Omega_{s}(\theta)
$$

where $d \Omega_{s}(\theta)$ is the differential solid angle subtended from an isotropic detector by the surface $d S$ (see Fig. 7.28) and is $\cos \theta d S / r^{2}$.

A solution to Eq. 7.8-7 was developed by Hubbell et al. in terms of completely separable source and geometry functions and is expressed as

$$
\Phi=\sum_{l=0}^{\infty} \frac{2 l+1}{2} g_{l} p_{l}(a, b)
$$

where $a$ is the ratio of the half height $(H / 2)$ to the length $(Z)$ of the duct, $b$ is the ratio of the half width $(W / 2)$ to the length, and $g_{l}$ and $p_{l}$ are coefficients of Legendre polynomial expansions of the source and geometry functions, respectively. With these definitions of $a$ and $b$, Eq. 7.8-8 gives the flux at a corner for the quarter plane limned in Fig. 7.28. The flux density at the 


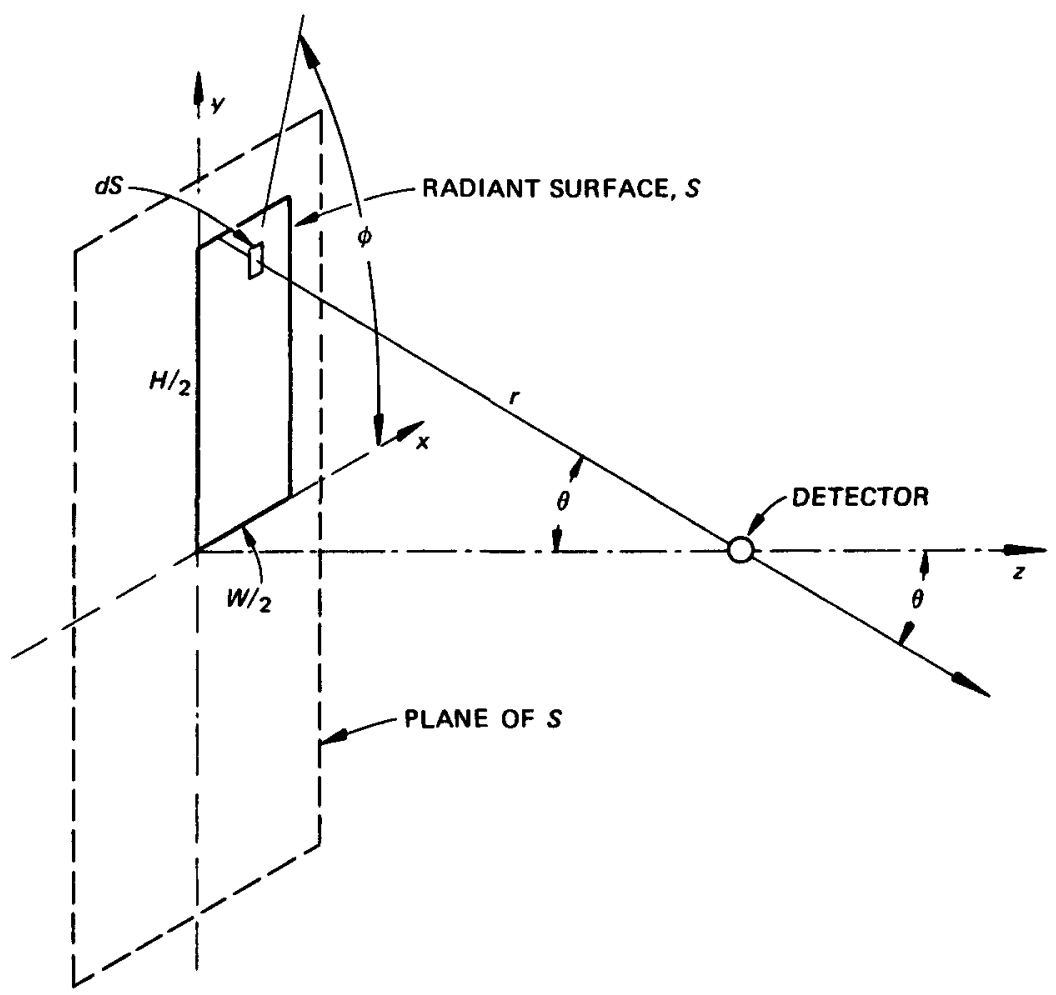

Fig. 7.28-Schematic drawing of source plane and detector position for a rectangular duct.

center line for the full plane will be four times as great as that given by Eq. 7.8-8.

The coefficients are calculated by

$$
g_{l}=\int_{-1}^{+1} g(\cos \theta) P_{l}(\cos \theta) d(\cos \theta)
$$

and

$$
p_{l}(a, b)=\int_{S} P_{l}(\cos \theta) d \Omega_{s}(\theta)
$$

where the values of $P_{l}(\cos \theta)$ are the Legendre polynomials.

Although the solution of Eq. $7.8-10$ is in terms of a finite series, the terms become progressively more complicated with increasing $l$, and only the first four $p_{l}$ values were evaluated analytically. Hubbell et al. numerically evaluated $p_{l}$ values for $\theta \leqslant l \leqslant 13$ over a grid of $a$ and $b$ values of 
$0.1 \leqslant b \leqslant a \leqslant 20$. With these tabulated values and the $g_{l}$ values determined for any arbitrary $g(\cos \theta)$, the flux density along the axis or at the corner of a duct can be calculated with Eq. 7.8-8.

For the special and useful case of the $\cos ^{n} \theta$ distribution,

$$
g(\cos \theta)=\frac{(n+1) \Phi_{0}}{2 \pi} \cos ^{n} \theta
$$

where $\Phi_{0}$ is the source strength that is represented by a scalar flux density obtained by integrating the angular flux density over all angles in the direction of the duct entrance. Equation 7.8-11 is properly normalized since

$$
\int_{0}^{1} \frac{n+1}{2 \pi} \cos ^{n} \theta 2 \pi d(\cos \theta)=1
$$

For integral values of $n$, Eq. 7.8-11 can be expanded into a finite number of Legendre polynomials, and, in principle, an analytical solution to Eq. 7.8-8 can be obtained. However, the solution rapidly becomes unwieldy for progressively higher values of $n$. The flux densities along the axis of a rectangular duct for the isotropic source $(n=0)$ and for more forwardly peaked sources ( $n=1$ and 2) from Maerker, Claiborne, and Clifford ${ }^{37}$ are given below. The flux density at a corner of a rectangle with dimensions $W / 2 \times H / 2$ is one-fourth that calculated by Eqs. 7.8-13 through 7.8-15.

For $n=0$ :

$$
\Phi=\frac{2 \Phi_{0}}{\pi} \tan ^{-1} \frac{a b}{\sqrt{1+a^{2}+b^{2}}}
$$

For $n=1$ :

$$
\Phi=\frac{2 \Phi_{0}}{\pi}\left(\frac{a}{\sqrt{1+a^{2}}} \tan ^{-1} \frac{b}{\sqrt{1+a^{2}}}+\frac{b}{\sqrt{1+b^{2}}} \tan ^{-1} \frac{a}{\sqrt{1+b^{2}}}\right)
$$

For $n=2$ :

$$
\Phi=\frac{3 \Phi_{0}}{\pi}\left[\tan ^{-1} \frac{a b}{\sqrt{1+a^{2}+b^{2}}}+\frac{a b}{\sqrt{1+a^{2}+b^{2}}}\left(\frac{1}{1+a^{2}}+\frac{1}{1+b^{2}}\right)\right]
$$

As the distance between the source and detector becomes large with respect to cross-section dimensions, the inverse tangent in Eq.7.8-13 
approaches $a b$ and the terms in the parentheses in Eq. 7.8-14 and in the brackets in Eq. 7.8-15 approach $2 a b$ or $W H / 2 Z^{2}$. In general, it can be shown that for long thin ducts of rectangular cross section ${ }^{35}$

$$
\Phi=\frac{(n+1) W H \Phi_{0}}{2 \pi Z^{2}}
$$

Equation 7.8-16 represents a lower limit for a point source (emitting only into the forward hemisphere) and also for a plane source (for a detector far away from the duct entrance) since the flux scattered from the wall of the duct is not included. In many practical cases the contribution to the dose by the scattered flux will be of the same order or smaller than the contribution by the uncollided flux. (The dose is calculated by summing the product of the flux density and the appropriate dose response function for each energy group of neutrons.)

\subsubsection{Rectangular Slots}

The geometry used in the derivations of the approximate equations for a rectangular slot is shown in Fig. 7.29. The dimensions of the duct adjacent to the source plane are $W$ by $H$, and the distance from the source plane to the exit end of the slot is $Z$. When $H \gg W$ (say, $H / W>5$ ), the exposed source can be approximated by a line source, and the line-of-sight flux density, $\Phi$, and the leakage current, $J$, at any point $P$ along the horizontal axis on the exit end of the slot can be approximated by

$$
\begin{gathered}
\Phi_{i}=\frac{N_{0} W}{2 \pi Z}\left(\theta_{1}+\theta_{2}\right) \\
J_{i}=\frac{N_{0} W}{2 \pi Z}\left(\sin \theta_{1}+\sin \theta_{2}\right) \\
\Phi_{c}=\frac{N_{0} W}{\pi Z}\left(\sin \theta_{1}+\sin \theta_{2}\right) \\
J_{C}=\frac{N_{0} W}{4 \pi Z}\left[\sin 2 \theta_{1}+\sin 2 \theta_{2}+2\left(\theta_{1}+\theta_{2}\right)\right]
\end{gathered}
$$

where $N_{0}$ is source emission rate (or current) per unit area, $\theta$ is in radians, and the subscripts $i$ and $c$ refer to isotropic and cosine sources, respectively. 


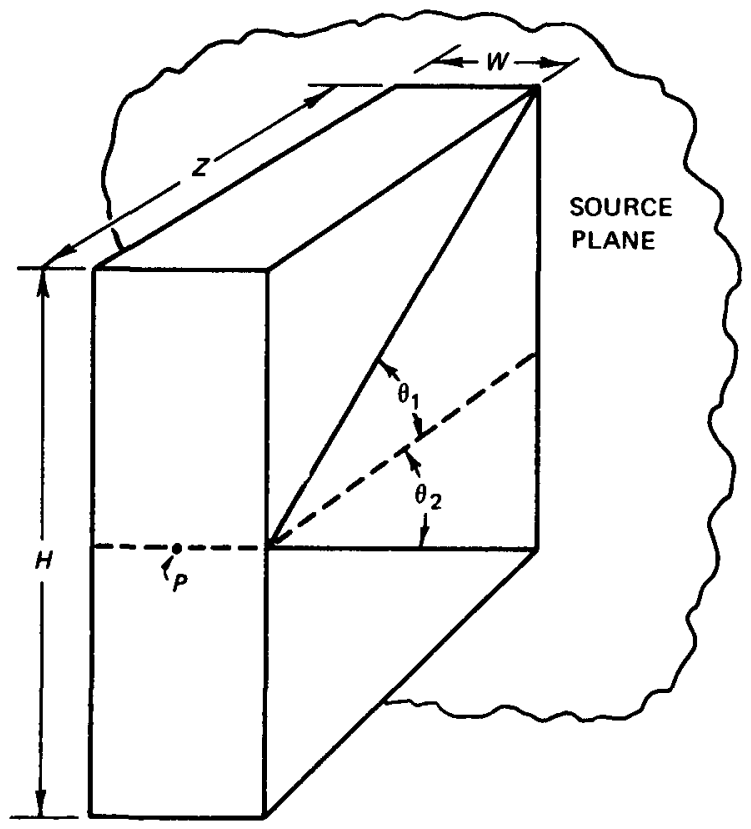

Fig. 7.29-Rectangular slot geometry. Source plane and exit plane are normal to $Z$ direction.

If the slot dimensions are such that $\theta_{1}+\theta_{2} \rightarrow \pi$, the preceding equations become

$$
\begin{gathered}
\Phi_{i}=\frac{N_{0} W}{2 Z} \\
J_{i}=\frac{N_{0} W}{\pi Z} \\
\Phi_{c}=\frac{2 N_{0} W}{\pi Z} \\
J_{c}=\frac{N_{0} W}{2 Z}
\end{gathered}
$$

The rectangular slot can be considered a special case of the rectangular duct for the condition when $W \rightarrow 0$, and Eq. 7.8-19 can be derived by taking the limit of Eq. 7.8-13 as $W \rightarrow 0$. In the limiting process an apparent anomaly 
occurs; the answer is in terms of $\Phi_{0}$, the flux density at the source. However, the flux densities on a line source for both isotropic and cosine distributions are always infinite. Consequently, when the limit of Eq. $7.8-13$ is taken as $W \rightarrow 0, \Phi_{0}$ should be converted to an emission rate $\left(2 N_{0}\right)$.

\subsubsection{Cylindrical Ducts}

Consider a cylindrical duct of radius a normal and adjacent to a plane source with the detector a distance $Z$ from the source plane and on the duct

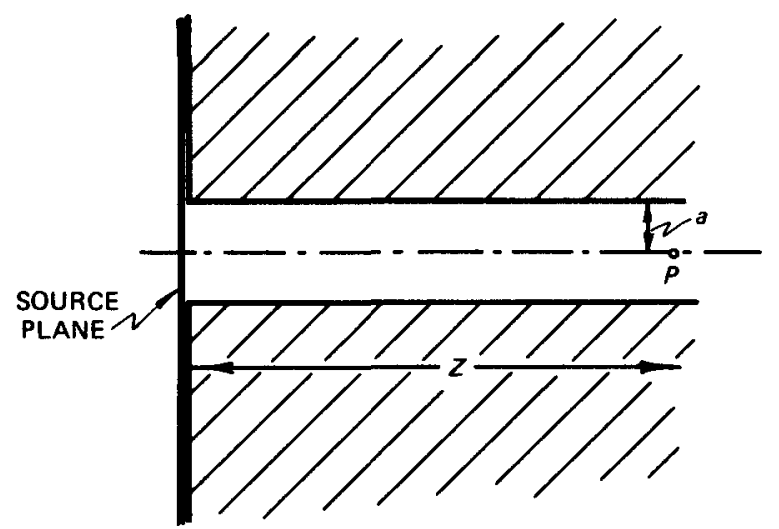

Fig. 7.30-Geometry for cylindrical duct adjacent to plane source.

axis (Fig. 7.30). For an isotropic current or emission rate on the source plane, the line-of-sight flux density at any point $P$ is given by

$$
\Phi_{i}=\frac{N_{0}}{2} \ln \left[1+\left(\frac{a}{Z}\right)^{2}\right]
$$

and the current parallel to the axis of the cylinder is given by

$$
J_{i}=N_{0}\left[1-\frac{1}{\sqrt{1+(a / Z)^{2}}}\right]
$$

For a cosine current source

$$
\Phi_{c}=2 N_{0}\left[1-\frac{1}{\sqrt{1+(a / Z)^{2}}}\right]
$$


and

$$
J_{c}=\frac{N_{0} a^{2}}{Z^{2}\left[1+(a / Z)^{2}\right]}
$$

Equations 7.8-27 and 7.8-28 can be expressed in terms of an isotropic flux density at the source plane by letting $2 N_{0}=\Phi_{0}$.

For $Z \gg a$, say, $Z / a>5$, these equations approach

$$
\Phi_{i} \cong J_{i} \cong \frac{N_{0}\left(\pi a^{2}\right)}{2 \pi Z^{2}} \cong \frac{N_{0} a^{2}}{2 Z^{2}}
$$

which is the same result for a point source of strength $N_{0}\left(\pi a^{2}\right)$, and

$$
\Phi_{c} \cong J_{c} \cong \frac{N_{0}\left(\pi a^{2}\right)}{\pi Z^{2}} \cong \frac{N_{0} a^{2}}{Z^{2}}
$$

If the condition of a long duct of small cross-sectional area is met, Eqs. 7.8-29 and 7.8-30 will also hold for a straight duct of any crosssectional geometry if $\pi a^{2}$ is replaced by the cross-sectional area of the duct.

\subsubsection{Cylindrical Annulus}

The geometry for a long cylindrical annular duct is shown in Fig. 7.31. The duct is bounded by two cylindrical surfaces having radii $a_{1}$ and $a_{2}$. For the conditions $Z \gg a_{2}-a_{1}, a_{2} \gg a_{2}-a_{1}$, and an isotropic source $N_{0}$, the average line-of-sight flux density, $\bar{\Phi}$, along the duct axis can be approximated by

$$
\bar{\Phi}=\frac{M N_{0}}{2 \pi Z^{2}}\left[\left(2 a_{2}^{2}-a_{1}^{2}\right) \cos ^{-1}\left(\frac{a_{1}}{a_{2}}\right)-a_{1} \sqrt{a_{2}^{2}-a_{1}^{2}}\right]
$$

The average leakage current, $\bar{J}$, for the isotropic source $N_{0}$ can also be obtained from Eq.7.8-31 by setting $M=1$. The equation given by Price, Horton, and Spinney ${ }^{38}$ for the flux density at any radius $r\left(a_{1} \leqslant r \leqslant a_{2}\right)$ within the annulus is

$$
\begin{aligned}
\Phi(r, Z)=\frac{M N_{0} a_{2}^{2}}{\pi Z^{2}}\left\{1-\left(\frac{a_{1}}{a_{2}}\right)^{2} \cos ^{-1}\left(\frac{a_{1}}{r}\right)\right. \\
\\
\left.\quad+\cos ^{-1}\left(\frac{a_{1}}{a_{2}}\right)-\left(\frac{a_{1}}{a_{2}}\right)\left[1-\left(\frac{a_{1}}{a_{2}}\right)^{2}\right]^{1 / 2}\right\}
\end{aligned}
$$


where $M=1$ for an isotropic source, $M=2$ for a cosine source, and $r$ and $Z$ are the cylindrical coordinates of the point $P$ at which the flux density is given. Equation 7.8-32 is equivalent to

$$
\Phi(r, Z)=\frac{M N_{0} S}{2 \pi Z^{2}}
$$

where $S$ is the area of the annulus on the source plane that is viewed from the point $P(r, Z)$ (Ref. 38).

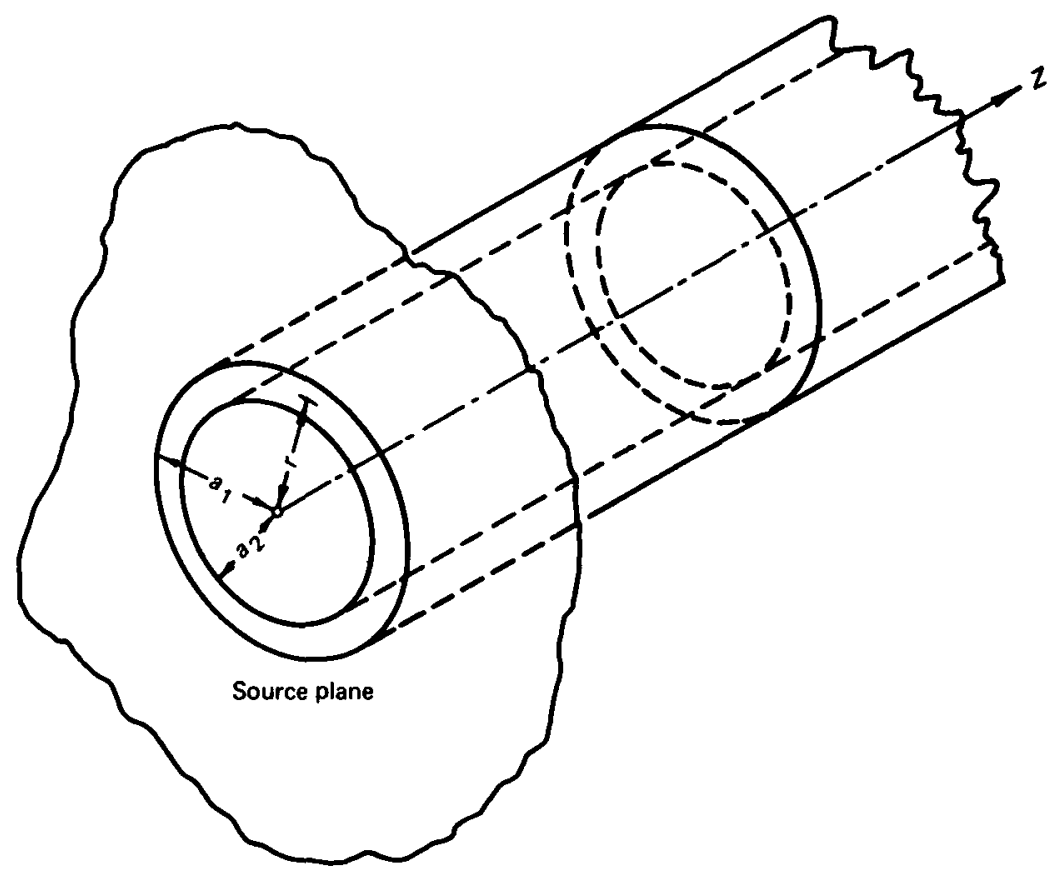

Fig. 7.31-Geometry for cylindrical annulus adjacent to plane source.

\subsection{WALL-PENETRATION COMPONENT}

In addition to the line-of-sight radiation, the flux reaching a detector located near the exit end of a simple duct includes radiation that enters the duct through its walls. $t$ This contribution consists of two components:

†Radiation that enters the mouth of the duct in a direction other than the direction of the detector but subsequently scatters in the duct wall toward the detector also contributes to the flux. For simple ducts where the solid angle subtended by the walls at the detector is less than that subtended by the source, the contribution from the walls is usually small, but for ducts with bends it becomes the dominant component (see Sec. 7.10). 
radiation that travels directly from the source to the detector through shielding material without an interaction (uncollided flux) and radiation that scatters in the direction of the detector as a result of interactions with the shield. Ray analysis is a useful method for calculating these components.

The assumption of the ray-analysis technique (actually a point-kernel method) is that the radiation transmission is a function only of the path

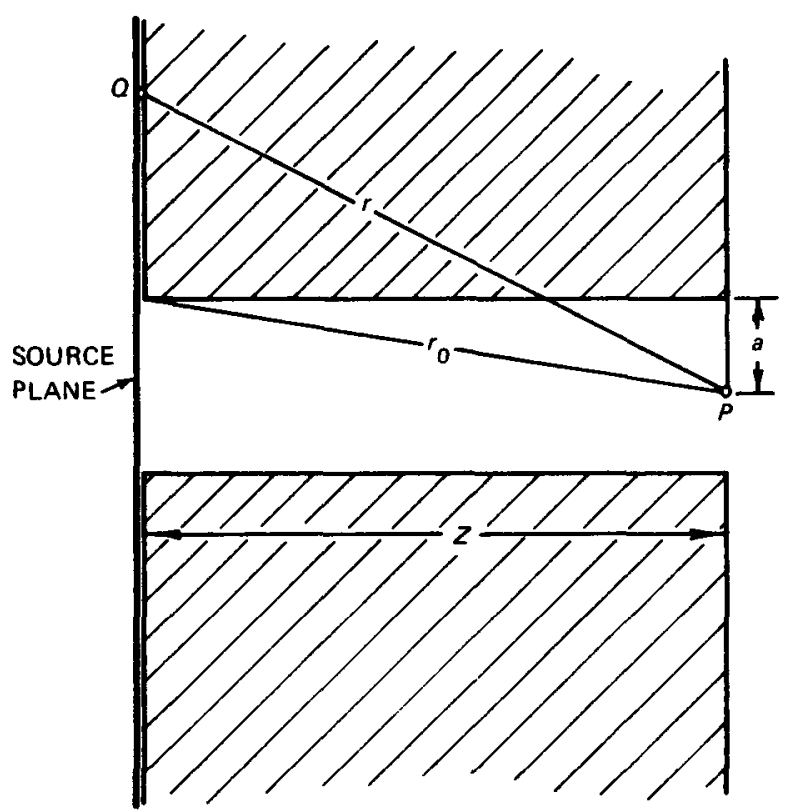

Fig. 7.32-Geometry for ray-analysis calculation of radiation component arriving through wall of duct.

lengths through each material or void encountered along a straight line between the source point and the detector. This assumption is entirely correct for predicting the uncollided flux density; for geometries in which the scattered flux density becomes important, the collided flux densities can be estimated to a first approximation by using buildup factors for gamma rays and removal cross sections for neutrons. Although the accuracy of the method is highly dependent on the particular configuration being considered, an approximation of limited accuracy for the scattered component can be applied to simple ducts since the radiation reaching the detector consists largely of the uncollided component.

To illustrate the method, we will consider a point detector $P$ removed a distance $r$ from a point source at $Q$, where the straight-line path between the two points passes through both shielding material and void (see Fig. 7.32). 
The flux density at the detector is given by

$$
\Phi_{P}=\frac{N_{p}}{4 \pi r^{2}} K
$$

where $N_{p}$ is the point-source strength and $K$ is the material attenuation kernel for all the materials located between the source and the detection point.

If only the uncollided flux density at $P$ is considered,

$$
K=\exp \left(-\sum_{i} t_{i} \frac{1}{l_{i}}\right)
$$

where $l_{i}$ is the mean free path of a particle and $t_{i}$ is the straight-line path through the $i$ th material. When the radiation being considered consists of neutrons, $1 / l_{i}$ is usually expressed in terms of the total macroscopic cross section, $\Sigma_{t}\left(\mathrm{~cm}^{-1}\right)$, and $t_{i}$ is given in centimeters. When gamma rays are being treated, $1 / l_{i}$ is expressed as $\mu_{i}$.

Equation 7.9-2 can be modified to include an approximation of the scattered gamma radiation by multiplying the right-hand side by a buildup factor:

$$
K=B\left(t_{1}, t_{2}, \ldots\right) \exp \left(-\sum_{i} t_{i} \frac{1}{l_{i}}\right)
$$

The equation can be modified similarly to include an approximation of scattered neutrons by substituting the neutron removal cross section $\Sigma_{R}$ for $\Sigma_{t}$ :

$$
K=\exp -\Sigma \Sigma_{R_{i}} t_{i}
$$

In this discussion we assume that the flux density at the point $P$ is the same as it would be if all the particles traveling from $Q$ to $P$, including those that are scattered, encountered the amount of attenuating material that exists along the line from $Q$ to $P$. If significantly less material is encountered by some particles, the total flux density at $P$ determined by Eq. 7.9-1 may

tRemoval cross sections are valid only when the shielding materials are followed by a thick layer of water or, to a lesser extent, when mixtures containing hydrogenous materials are used (see Chap. 6). 
be underestimated. Conversely, if significantly more material is encountered, the flux density may be overestimated. Also, Eq. 7.9-1 neglects the effects of material--void interfaces or interfaces between dissimilar materials encountered along the ray and in the surrounding regions.

When the ray-analysis method is used for integration over a source plane or source volume, any inaccuracy involved in the contribution of a source region may be masked by the contributions from other points. There are, of course, complex geometries and material combinations for which the simple ray-analysis technique does not predict the radiation fluxes with a satisfactory degree of accuracy. However, ray analysis is adequate in many cases and is easily applied.

There are also cases in which the accuracy requirements and the complexity of the geometry may prohibit the use of the approximations given here except as first estimates. In such situations numerical integration can be used to obtain a ray-analysis solution either by hand or by machine calculations.

\subsubsection{Application to Cylindrical Ducts}

If the duct shown in Fig. 7.32 were a cylindrical duct of radius $a$ penetrating an infinite-slab shield adjacent to an infinite-plane isotropic source, the uncollided flux density at $P$ that would arrive through the duct walls would be

$$
\Phi_{i}=N_{0} \int_{r_{0}}^{\infty} e^{-\mu l(r)} \frac{d r}{r}
$$

where $N_{0}=$ surface emission rate or current

$l(r)=$ path length in the shield that lies along $r$

$$
\begin{aligned}
=r\left(1-\frac{a}{\sqrt{r^{2}-Z^{2}}}\right) \\
\mu=\text { linear attenuation coefficient }
\end{aligned}
$$

The corresponding flux density for a cosine source is given by

$$
\Phi_{c}=2 N_{0} \int_{r_{0}}^{\infty} e^{-\mu l(r)} \frac{Z}{r^{2}} d r
$$


Equations 7.9-5 and 7.9-6 are not amenable to straightforward analytical solutions, although values of the integrals can be found in tables of mathematical functions. Certain approximations, based on a Taylor expansion of $l(r)$, have been given by Chase, ${ }^{39}$ but they are valid only for large values of $a / Z$. Trubey ${ }^{40}$ evaluated the equations numerically for a unit-surface-source intensity (for $N_{0}=1$ ). The results are shown in Appendix P, Tables P.1 and P.2, as a function of the ratio of the duct radius to the length $(a / Z)$ and the shield thickness $(\mu Z)$, where $\mu Z$ is measured in relaxation lengths (or mean free paths determined from material cross sections).

The data for the uncollided flux density given in Tables P.1 and P. 2 can be used to estimate the total radiation arriving through the duct wall provided that the parameters $a / Z$ and $\mu Z$ are measured in terms of an effective relaxation length, $\lambda=1 / \mu$, which accounts for the radiation scattered in the direction of $P$ by the shield. Relaxation lengths that include scattering can be obtained from experimental data or from basic calculations of penetration through the material. Relaxation lengths are necessarily a function of the radiation spectrum as well as of the material surrounding the duct. Neutron removal cross sections or gamma-ray buildup factors, both of which are derived from basic penetration data, can be used in obtaining values of $\lambda$. In this use of the data, the approximation is made that the total attenuation is exponential; i.e., the relaxation length does not vary with penetration depth.

Figures 7.33 and 7.34 , which are based on data by Trubey, ${ }^{40}$ show the variation with shield thickness and duct size of the total flux density, including the line-of-sight component, reaching the center of the duct exit. All parameters are given in terms of the shield-material relaxation length. The flux density is given for an infinite-plane source of unit source strength (e.g., 1 particle $\mathrm{cm}^{-2} \mathrm{sec}^{-1}$ ).

The relative importance of the line-of-sight radiation discussed in Sec. 7.8 and of the uncollided radiation that leaks through a duct wall is illustrated by some additional calculations by Trubey in Appendix P, Table P.3. From these calculations it is apparent that the ratio of the line-of-sight component to the total uncollided flux density depends strongly on the ratio of the duct radius to the length $(a / Z)$ and on the shield thickness $(\mu Z)$. These ratios represent an upper limit since the buildup of the flux due to scattering has not been considered. The ratios are applicable to either fast neutrons or gamma rays provided the shield thickness is defined in terms of the number of relaxation lengths. Data in Table P.3 show, as one would expect, that the radiation that penetrates the side wall dominates over that from the source 


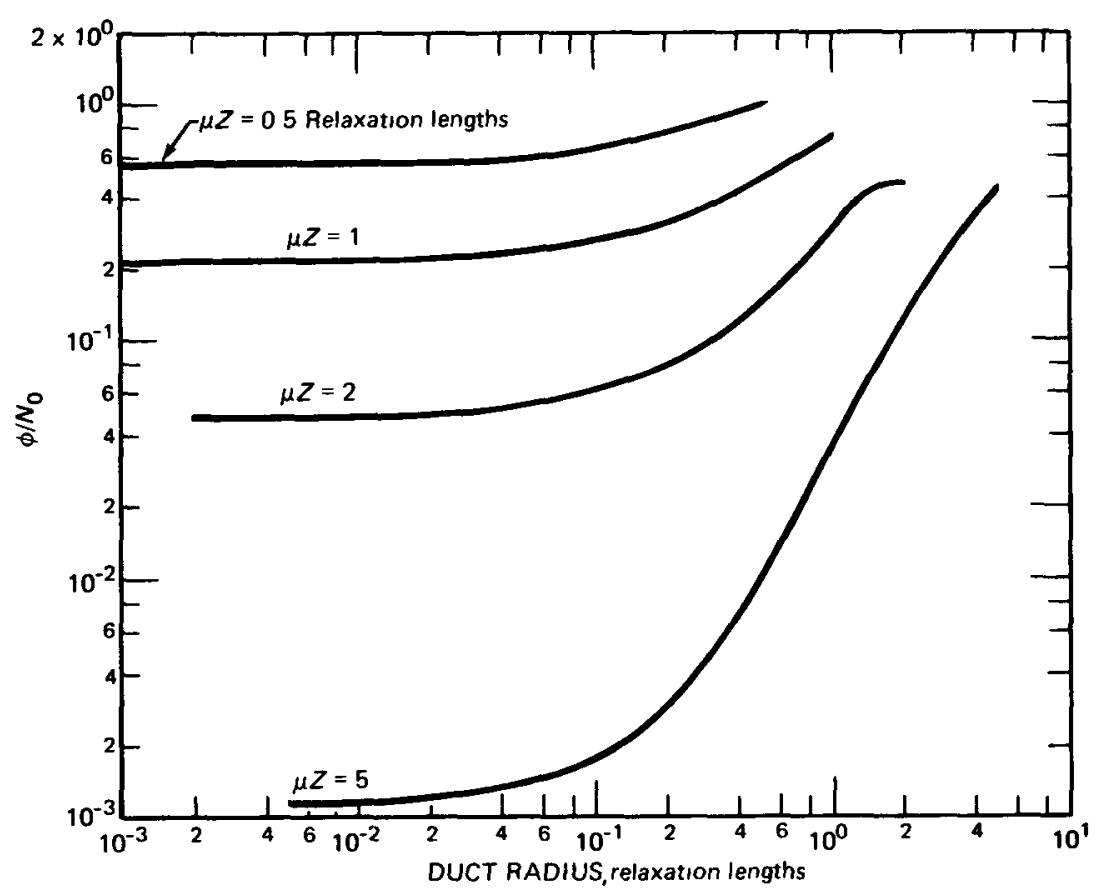

Fig. 7.33-Effect of duct radius and shield thickness on total uncollided flux density reaching exit of cylindrical duct (isotropic source).

in the mouth of the duct for small diameters penetrating thin shields. As the duct diameter increases or the shield thickness increases, or both, the line-of-sight component increases in importance.

If the duct is filled with a lightly attenuating medium, formulas similar to those in the preceding sections could be derived which include exponential attenuation through the material filling the duct in the kernel integration. This condition would tend to decrease further the importance of the source at the mouth of the duct.

\subsubsection{Application to Partially Penetrating Cylindrical Ducts}

The radiation transmitted through the walls of a cylindrical duct that penetrates only part of the shield and is not adjacent to the source can be calculated by the ray-analysis technique in much the same manner as for a completely penetrating duct. Consider the duct shown in Fig. 7.35(a). Equations 7.9-5 and 7.9-6 can be applied to this geometry merely by changing the lower limit of integration from $r_{0}$ to $Z$. As before, $l(r)$ is that portion of $r$ lying inside the shield. 


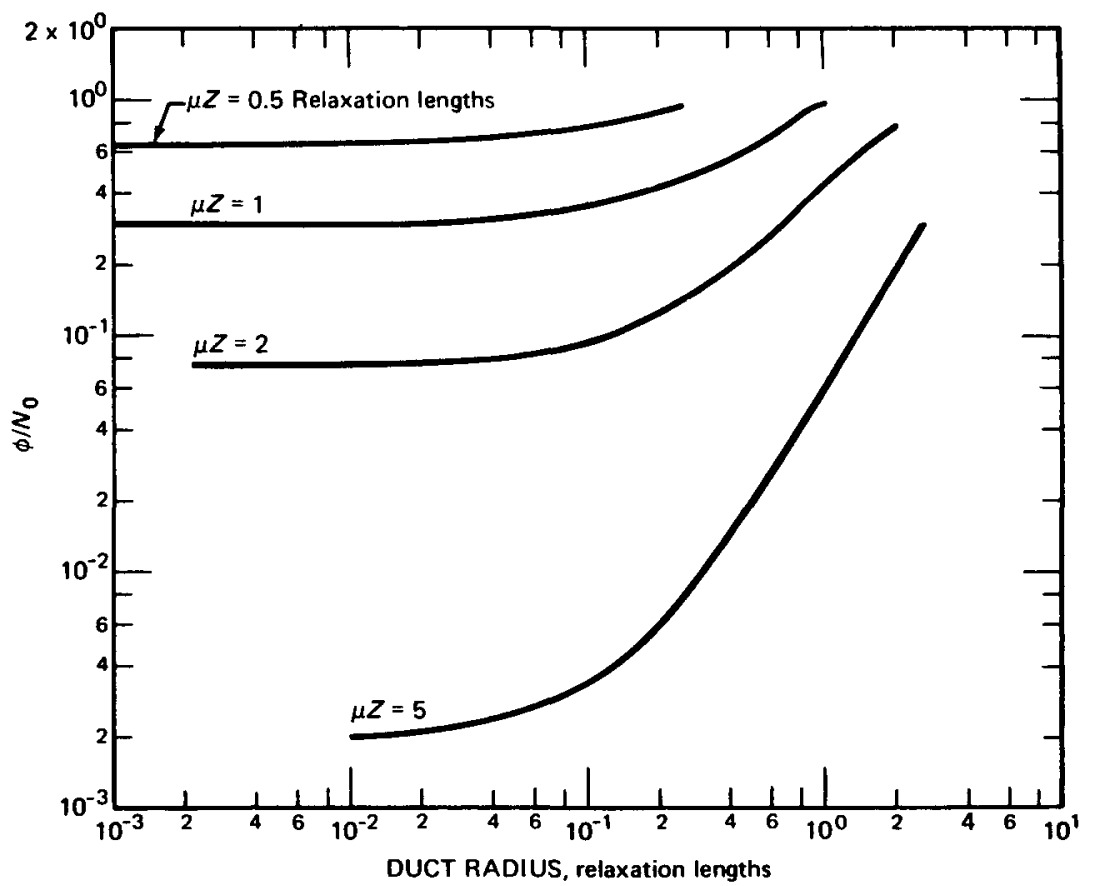

Fig. 7.34-Effect of duct radius and shield thickness on total uncollided flux density reaching exit of cylindrical duct (cosine source).

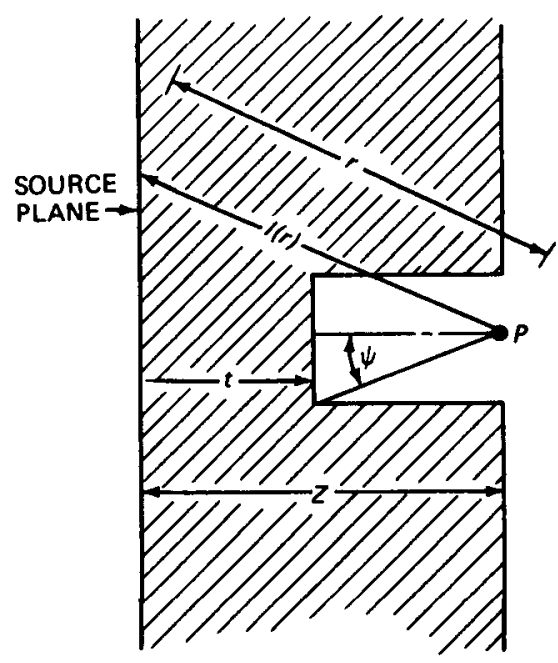

(a)

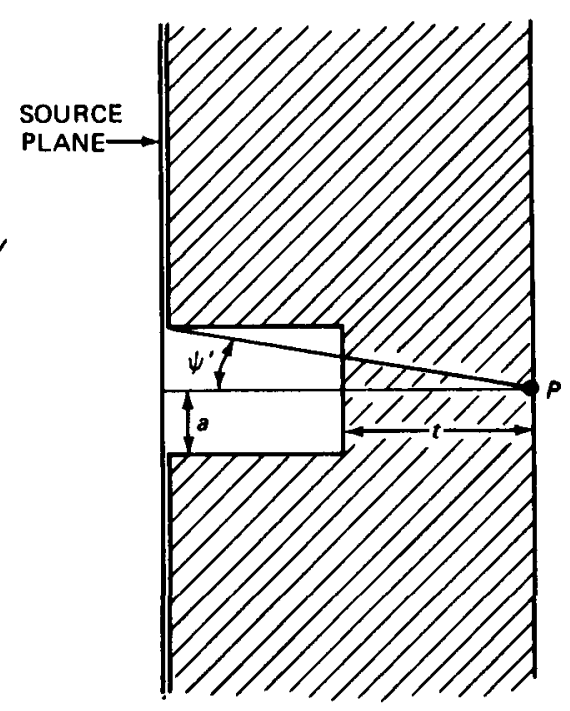

(b)

Fig. 7.35-Geometries for ray-analysis calculations of radiation component arriving through walls of ducts that partially penetrate shield. 
An alternative to numerical integration of these equations for each case would be to determine the flux density penetrating an infinite slab of thickness $t$ (see Sec. 7.11.1), use this as a source-strength input to the ducted shield of thickness $Z-t$, and evaluate using the data given in Figs. 7.33 and 7.34. In the absence of more definitive data, a more forwardly peaked distribution, such as a cosine distribution, should be used at the artificial interface if $t$ is greater than one relaxation length. Even when the original source is isotropic, the radiation will have taken on a more directional character after penetrating one relaxation length.

Simpler equations may be given for the flux density through partially penetrating ducts if the assumption is made that only the radiation penetrating the end of the particle duct need be considered. ${ }^{39}$ In this case the flux density at $P$ in Fig. 7.35(a) can be estimated by

$$
\Phi=N_{0}\left[E_{1}(\mu t)-E_{1}(\mu t \sec \Psi)\right]
$$

where $\Psi=\tan ^{-1}[a /(Z-t)]$. Similarly, the flux density at $P$ due to radiation entering the mouth of a duct that penetrates only part of the shield but is adjacent to the source [see Fig. 7.35(b)] is given by

$$
\Phi=N_{0}\left[E_{1}(\mu t)-E_{1}\left(\mu t \sec \Psi^{\prime}\right)\right]
$$

where $\Psi^{\prime}=\tan ^{-1}(a / Z)$.

For the same shield and duct dimensions, the angle $\Psi^{\prime}$ is smaller than $\Psi$ and the peak flux density increase at $P$ due to the presence of the duct is greater in case $b$ than in case $a$. For the approximation $\mu t a^{2} \ll 2 Z^{2}$, the integrated emergent current can be approximated by $\pi a^{2} N_{0} e^{-\mu t}\left[a^{2} / 2(Z-\right.$ $t)^{2}$ ] for either case $a$ or $b$. Thus, although the peak current tends to be greater in case $b$, the integrated current increase due to the void is approximately the same in both cases.

\subsubsection{Comparison with Experiment}

The ray-analysis technique has been used successfully many times in predicting measured values for both gamma-ray and neutron transmission in solid unpenetrated shields. In complex geometries involving ducts, the technique should give better results for gamma rays than for neutrons since neutrons on the average undergo more scatterings before they are absorbed or escape. However, as is demonstrated by the following comparisons, the 
technique yields good results for neutrons transmitted through straight ducts abutting a reactor.

Benenson and Fasano ${ }^{41}$ used the ray-analysis technique for analyzing experiments at the Brookhaven National Laboratory Shielding Facility on the transmission of fast neutrons through straight cylindrical ducts in a water shield. Neutrons from a fission source plate in the shielding facility entered the bottom of a water tank in which a duct had been vertically positioned, and neutrons that reached the detector at the opposite end of the duct arrived there either through the base of the duct or through its walls after passing through the surrounding water. The ducts were 5.1 to $40 \mathrm{~cm}$ in diameter. The ${ }^{32} \mathrm{~S}(n, p)^{32} \mathrm{P}$ reaction was used to measure the fast-neutron-flux density, and an experimentally determined relaxation length was used to analyze the penetration of neutrons above the ${ }^{32} \mathrm{~S}$ threshold from the fission source. Figure 7.36, which is typical of the results of this work, shows that the ray-analysis method apparently yields good results when applied to streaming of very penetrating radiation from a diffuse source through simple ducts.

Piercey and Bendall ${ }^{42}$ also used the ray-analysis technique for calculating the flux density of fast neutrons transmitted through straight cylindrical ducts. Their calculations corresponded to measurements made for 2.5-, 5-, and 9.5-cm-diameter aluminum ducts positioned in the water of the LIDO Shielding Facility at Harwell, England. The duct lengths were up to 200 duct radii. As in the Brookhaven experiment, the fast flux density was determined by the ${ }^{32} \mathrm{~S}(n, p){ }^{32} \mathrm{P}$ reaction. The calculations predicted the absolute sulfur reaction rate to within a factor of 2 over the experimental values. The poorest agreement occurred in the region of the duct nearest the reactor. Piercey and Bendall concluded that most of the discrepancy resulted because of the inhomogeneity of the system and particularly because of the aluminum layer between the duct face and the reactor. Figure 7.37 shows a comparison of the calculated and experimental results as an example of this work.

\subsection{WALL-SCATTERED COMPONENT}

We have seen in the preceding sections that for straight ducts the most important components contributing to the flux density at the detector are line-of-sight radiation and radiation that enters the duct through its wall at the proper angle to reach the detector. For more complicated geometries 


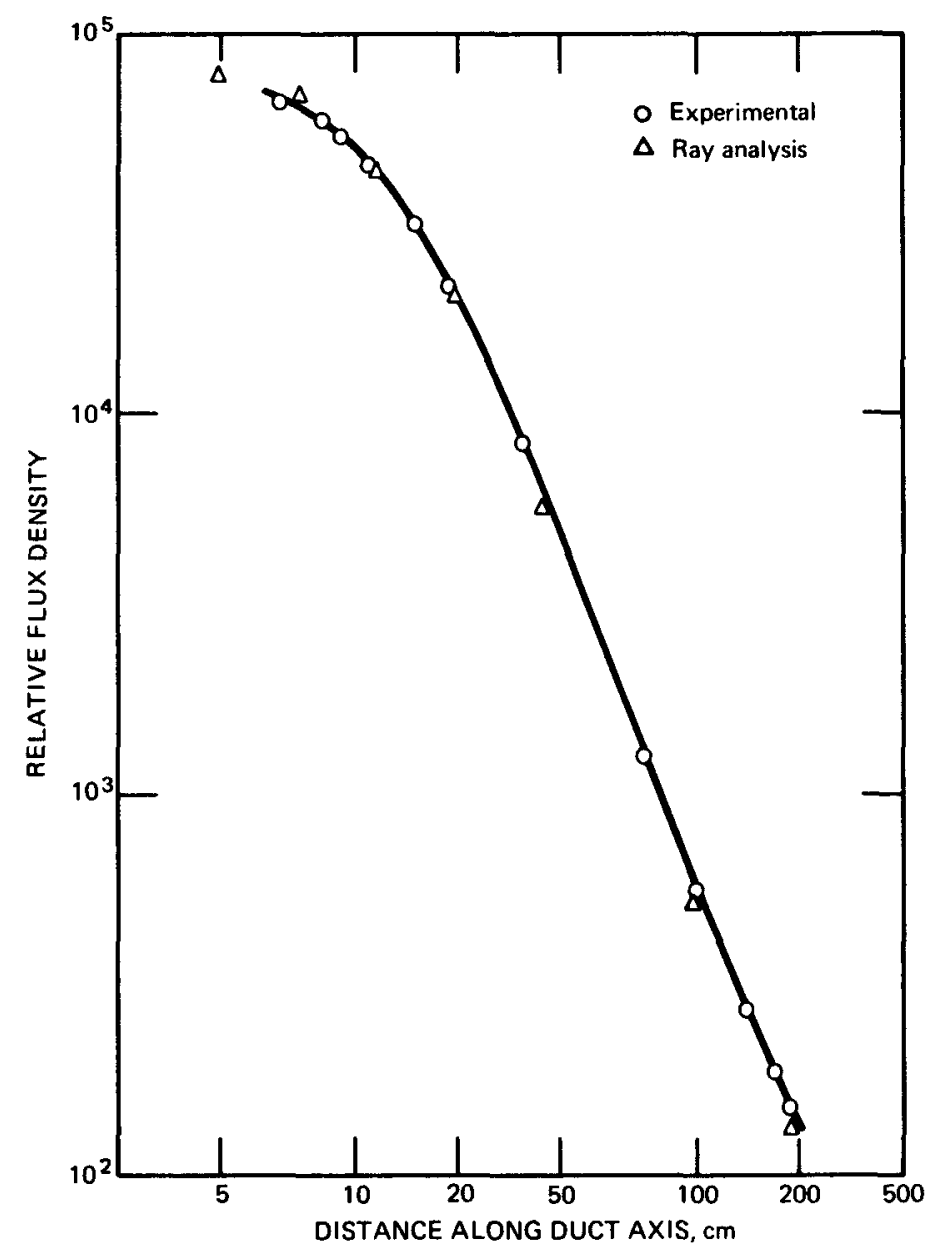

Fig. 7.36-Comparison of ray-analysis calculations with measurements of fast-neutronflux density along axis of $20-\mathrm{cm}$-diameter straight cylindrical duct in a water shield; fission source at duct mouth. (From Benenson and Fasano. ${ }^{41}$ )

involving ducts with bends, the line-of-sight component disappears, and the dominant component becomes radiation that is transmitted through the duct by successive scatterings from the surrounding shield. Calculations of this contribution require more involved techniques than those described previously since on the average many scatterings are involved and several boundaries are crossed.

\subsubsection{Analog Montc Carlo Calculations}

Two types of Monte Carlo methods can be applied to duct transmission problems: analog Monte Carlo methods, described here, and albedo Monte 


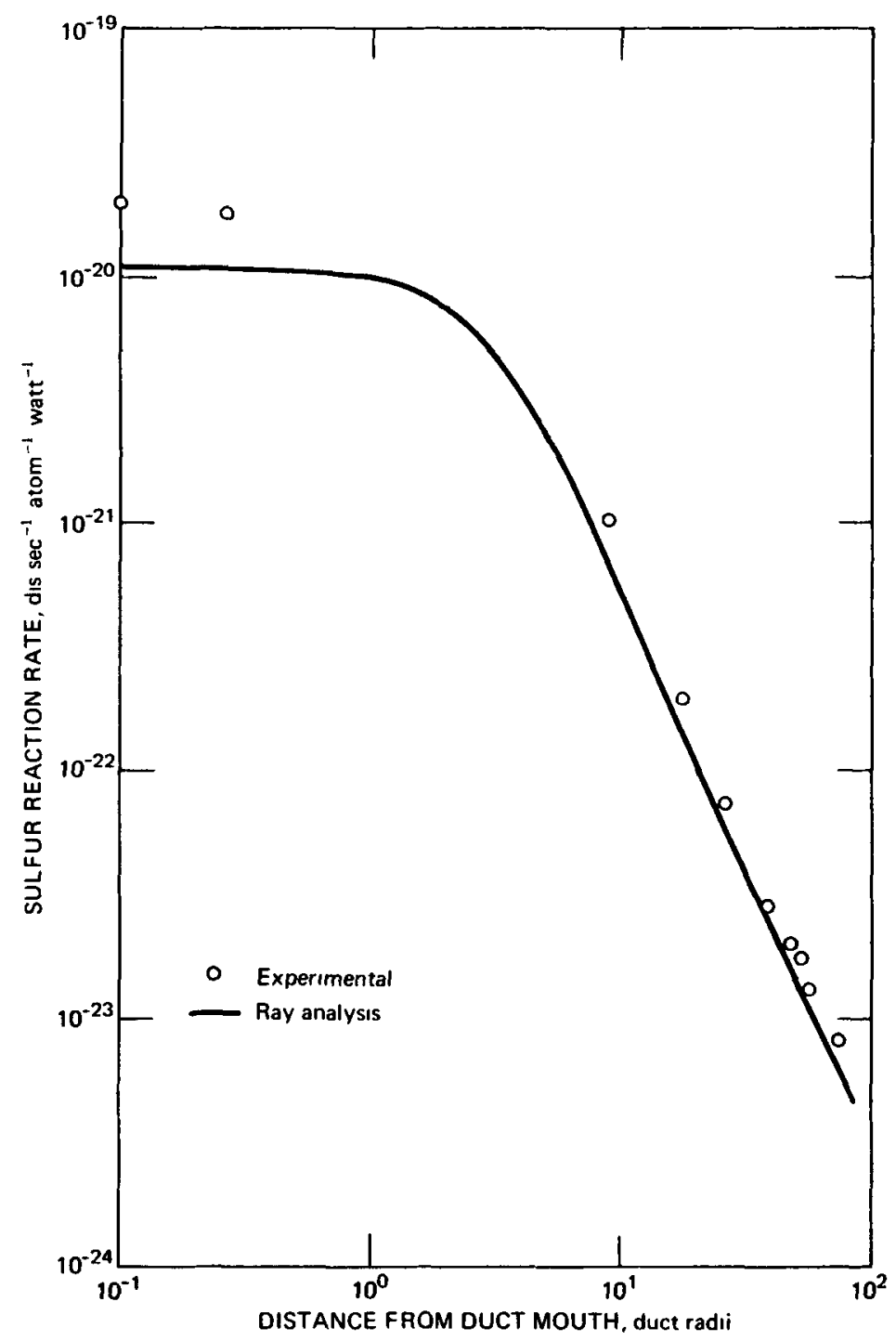

Fig. 7.37-Comparison of ray-analysis calculations with measurements of fast-neutronflux density along axis of $9.5-\mathrm{cm}$-dia meter straight cylindrical duct in a water shield; duct mouth adjacent to reactor core. (From Piercey and Bendall. ${ }^{42}$ )

Carlo methods, described in the next section. The term analog is used to designate calculations in which the model solved is an analog of the interactions occurring as the particles traverse the duct and confining walls as opposed to the albedo calculations in which the particles traversing a duct are followed by a random-walk process but wall interactions are represented by a reflection coefficient. 
The analog Monte Carlo method is not always practical because of the machine computing time required; nevertheless, several machine programs that have been developed are applicable to this type of calculation.

Collins and McCleary ${ }^{43}$ used a Monte Carlo code (called LO5) to calculate the transmission of neurrons from a Po-Be source $\left(3.08 \times 10^{7}\right.$ neutrons $/ \mathrm{sec}$ ) through $30-\mathrm{cm}$ long, $7.6-$ and $15.2-\mathrm{cm}$-diameter straight

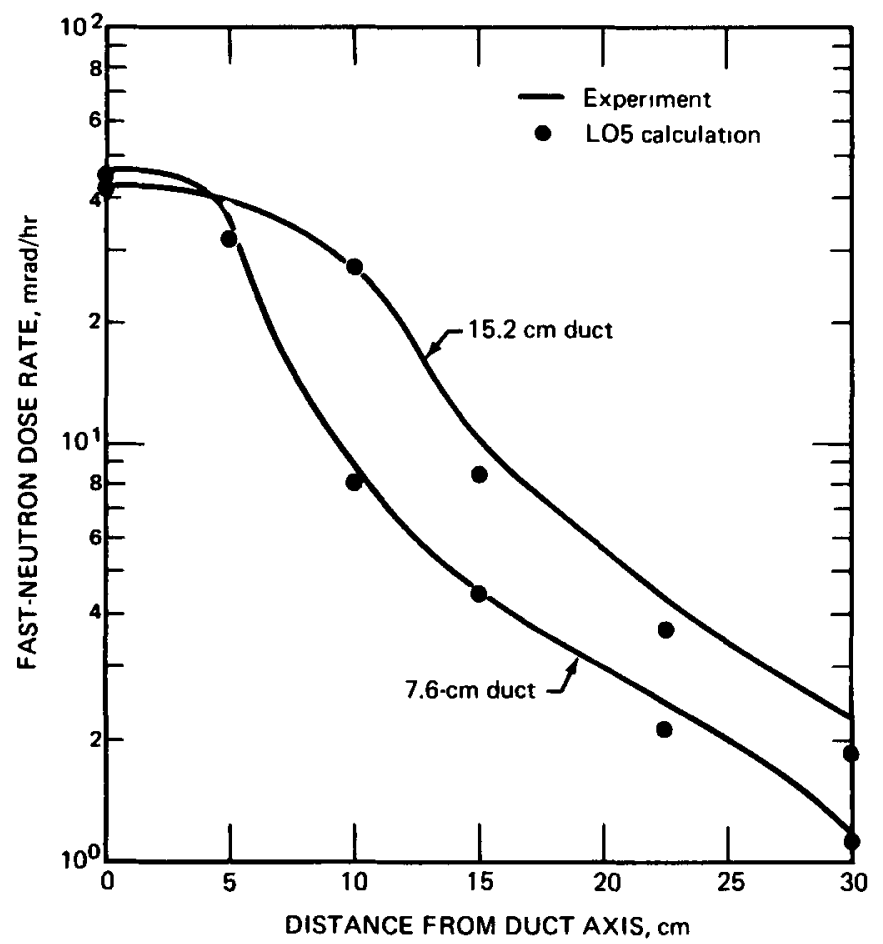

Fig. 7.38-Comparison of LO5 analog Monte Carlo calculations with measurements of fast-neutron dose rates along the axes of 30-cm-long, 7.6- and 15.2-cm-diameter straight cylindrical ducts in water; Po-Be neutron source. (From Collins and McCleary. ${ }^{43}$ )

cylindrical ducts in water. The detector was positioned $7.6 \mathrm{~cm}$ from the duct mouth. The agreement they obtained between the measured and calculated data (Fig. 7.38) shows that the LO5 procedure predicts correctly for this simple case.

The LO5 code was then used by Marshall ${ }^{44}$ for calculations corresponding to measurements made of radiation transmitted through one-bend cylindrical ducts in water. The ducts were reconstructed of 7.6-cm-diameter aluminum tubing and had one bend midway along their 
lengths; the bend angle varied between 30 and $60^{\circ}$. The sources were 14-MeV neutrons and ${ }^{60} \mathrm{Co}$ gamma rays positioned on the duct axis $7.6 \mathrm{~cm}$ from the mouth. The detector at the opposite end of the duct was also $7.6 \mathrm{~cm}$ from the duct mouth.

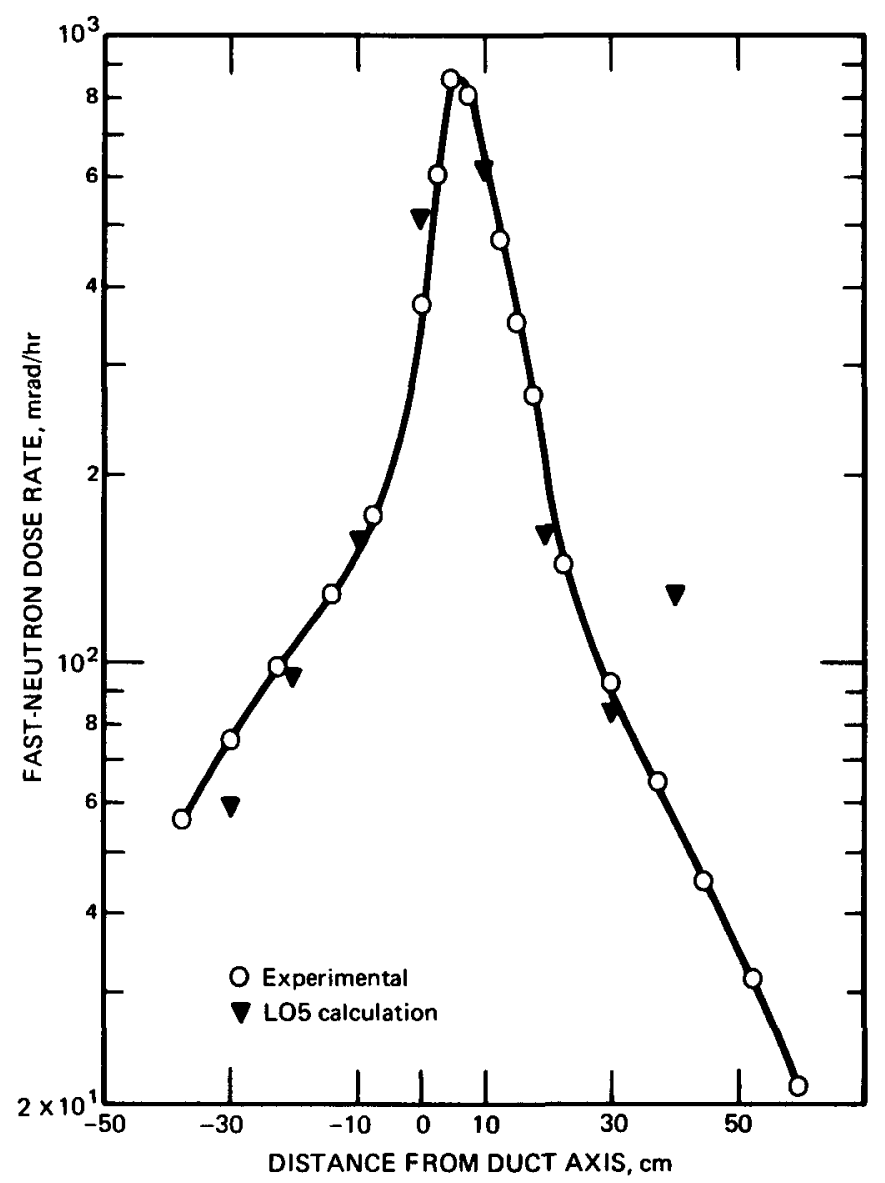

Fig. 7.39-Comparison of LO5 analog Monte Carlo calculations with measurements of the fast-neutron dose rate transmitted through a bent $\left(30^{\circ}\right)$ duct $(7.6 \mathrm{~cm}$ in diameter by $46 \mathrm{~cm}$ long) in a water shield; $14-\mathrm{MeV}$ neutron source. (From Marshall. ${ }^{44}$ )

Figures 7.39 and 7.40 compare some typical experimental data and calculated results for a duct bent $30^{\circ}$ which penetrates a $46-\mathrm{cm}$ water shield. In general, Marshall found that for shield thicknesses that exceeded $46 \mathrm{~cm}$ the calculated dose rates were in good agreement with the experimental data in shape but were too low in magnitude. 


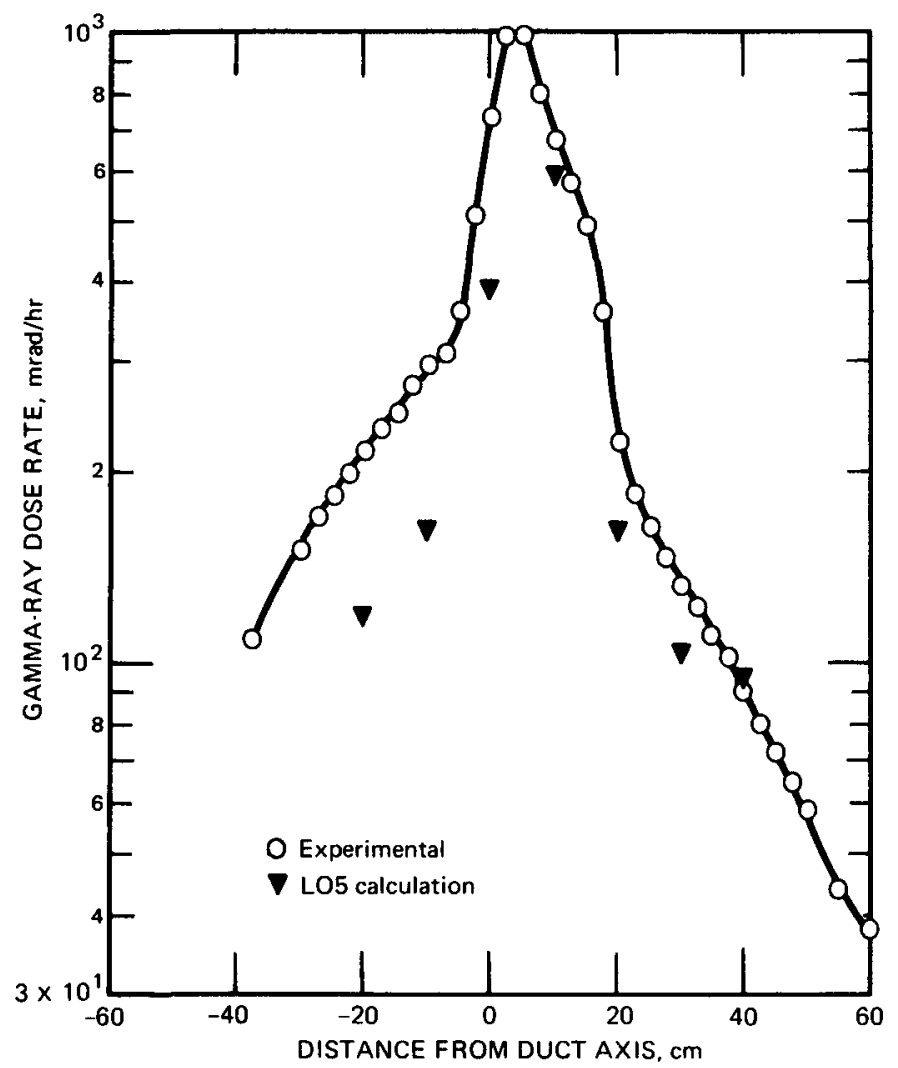

Fig. 7.40-Comparison of LO5 analog Monte Carlo calculations with measurements of the gamma-ray dose rate transmitted through a bent $\left(30^{\circ}\right)$ duct $(7.6 \mathrm{~cm}$ in diameter by $46 \mathrm{~cm}$ long) in a water shield; ${ }^{60} \mathrm{Co}$ gamma-ray source. (From Marshall. ${ }^{44}$ )

\subsubsection{Albedo Methods}

Perhaps the simplest albedo application to a duct is the case where only one reflection need be considered betweer the source and the detector. The albedo is integrated over duct surfaces that have an unobstructed line of sight to both the source and the detector. Consider the point source and simple duct shown in Fig. 7.41. The dose at $P$ due to first-order reflection from the wall is

$$
D=\int_{\substack{\text { wall } \\ \text { surface }}} \frac{N p R\left(E_{0}\right) \alpha_{D_{2}}\left(E_{0}, \theta_{0}, \theta, \phi\right) \cos \theta_{0} d S}{4 \pi r_{1}^{2} r_{2}^{2}}
$$


where $\quad N_{p}=$ source strength

$E_{0}=$ source energy

$R\left(E_{0}\right)=$ flux-to-dose conversion factor

$\alpha_{D_{2}}\left(E_{0}, \theta_{0}, \theta, \phi\right)=$ differential-dose albedo (reflected current per incident current) (see definitions in Sec. 7.2)

$\phi=$ azimuth angle separating the incident and reflected rays

and $r_{1}, r_{2}, \theta_{0}$, and $\theta$ are defined in Fig. 7.41. The reflected dose must, of course, be added to the line-of-sight dose to obtain the total.

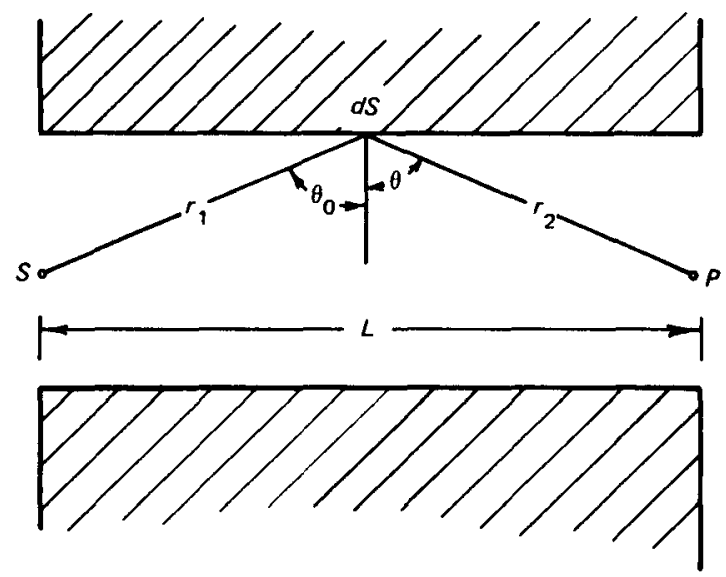

Fig. 7.41 - Geometry for albedo calculation of singly reflected radiation in a simple duct (point source).

The Simon and Clifford ${ }^{45}$ equations for neutrons reflected through ducts having cylindrical geometry (discussed later in this section) resulted from an integration of Eq. 7.10-1 for an assumed functional form for $\alpha$. For cases in which use of their expression for $\alpha$ is possible, the Simon-Clifford equations can be used. Other expressions can be derived which would allow the integration to be performed for simple geometries.

If either the nature of $\alpha$ or the geometry prohibits analytical integration, Eq. 7.10-1 can be integrated numerically by dividing the duct wall into small scattering areas and assuming that all points within an elemental scattering area are the same distance from the source or detector and that they have the same angles of incidence and reflection. A numerical evaluation performed by LeDoux and Chilton ${ }^{46}$ led to a set of equations for calculating singly reflected gamma rays propagated through bent rectangular ducts. (The LeDoux-Chilton technique is discussed later in this section.) 


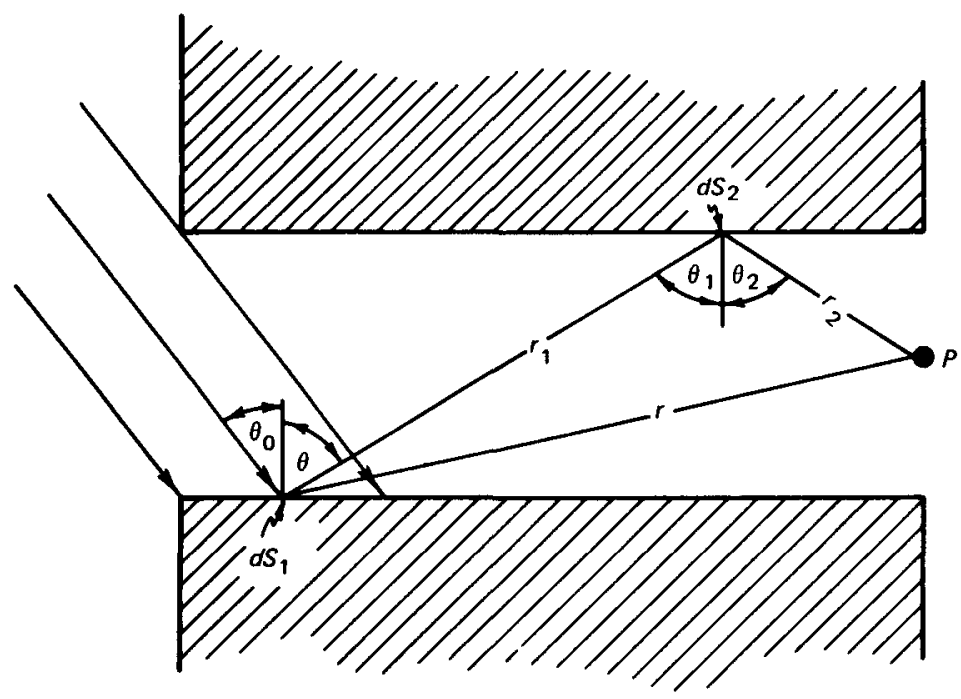

Fig. 7.42 - Geometry for albedo calculation of doubly reflected radiation in a simple duct (broad-beam source).

For a broad-beam source incident on the duct at an angle such as that shown in Fig. 7.42, no line-of-sight radiation reaches the detector and all reflected radiation must be evaluated, including that which has been reflected more than once. The singly reflected component at $P$ is given by

$$
D_{P_{1}}=\int_{\substack{\text { illuminated } \\ \text { area }}} \frac{D_{i} \alpha_{D_{2}}\left(E_{0}, \theta_{0}, \theta, \phi\right) \cos \theta_{0} d S_{1}}{r^{2}}
$$

where $D_{i}$ is the incident dose and $r$ is the distance from the surface area element to the detector. The doubly reflected component is estimated by

$$
\begin{aligned}
& D_{P_{2}}=\int_{\substack{\text { illuminated } \\
\text { area }}} \int_{\substack{\text { all } \\
\text { area }}}\left\{\left[D_{i} \alpha_{D_{2}}\left(E_{0}, \theta_{0}, \theta, \phi_{1}\right) \cos \theta_{0} d S_{1}\right.\right. \\
&\left.\left.\times \alpha_{D_{2}}\left(E_{1}, \theta_{1}, \theta_{2}, \phi_{2}\right) \cos \theta_{1} d S_{2}\right]\left(r_{1}^{2} r_{2}^{2}\right)\right\}^{-1}
\end{aligned}
$$

Complexities of the problem will almost always require the use of a computer program for numerical evaluation of these equations. Cain ${ }^{47}$ has shown that for low-energy neutrons numerical evaluation of these integrals becomes impractical because the number of reflection events that must be 
considered to adequately calculate the penetrating radiation is so large. As an alternative, a method was developed that traces particles through successive reflection events by an albedo Monte Carlo technique (discussed later in this section).

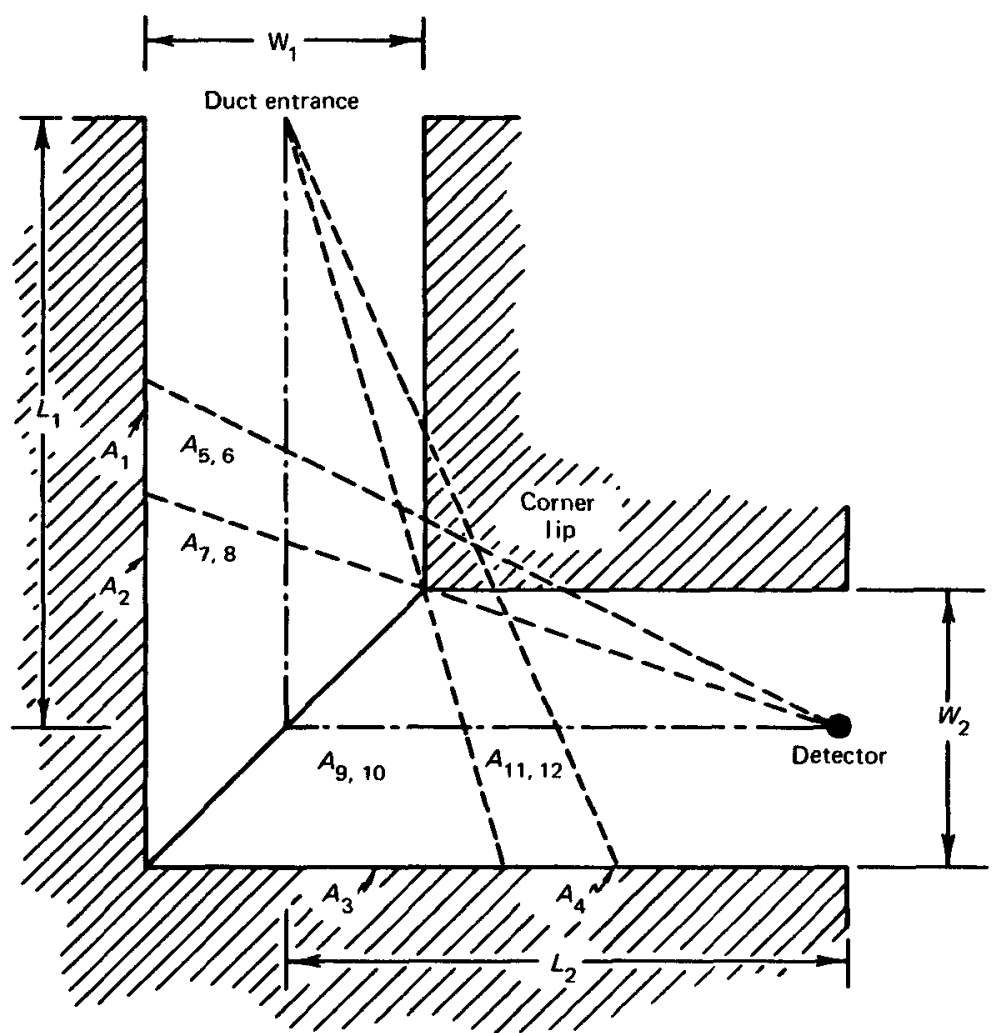

Fig. 7.43-Geometry for determining corner-lip transmission effect in two-legged rectangular duct. This geometry also illustrates the LeDoux-Chilton technique (discussed later in this section).

In most albedo calculations of the transmission of radiation through rectangular ducts with bends, the corner lip (the corner formed by the intersection of two legs) is assumed to be opaque to radiation in the main parts of the calculation, and a correction is made for its effect with a separate approximate calculation. This correction is usually broken down into two contributions: a transmission effect and an inscattering effect.

Corner-lip transmission is illustrated in Fig. 7.43 by the dashed line that originates at the duct entrance and passes through the inside corner. The radiation penetrating the lip scatters from the opposite wall and contributes 
to the dose at the detector. In accounting for this radiation, the albedo integrations of Eqs. 7.10-2 and 7.10-3 can be performed with the incident dose represented by a variable that depends on the path length in the duct-wall material of a ray that is parallel to the initial direction of the radiation and terminates at the scattering point. An approximation can be made by assuming that all particles that penetrate the material with a path length less than about a relaxation length are unattenuated and that all particles that penetrate more than a relaxation length are completely absorbed. (LeDoux and Chilton used the energy-absorption coefficient for estimating this effective relaxation length for gamma rays.) On this basis, the effect of the corner-lip penetration can be approximated by an increase in the number of scattering areas beyond the primary scattering areas. The areas designated as $A_{1}, A_{4}, A_{5}, A_{6}, A_{11}$, and $A_{12}$ in Fig. 7.43 show some of these additional scattering surfaces.

Corner-lip inscattering is the process by which radiation is redirected toward the detector by scattering in the corner. This component can be evaluated with the last-collision technique of French, ${ }^{48}$ for which the geometry is shown in Fig. 7.44. The flux density at a distance $t_{1}$ in the corner lip is given by

$$
\Phi\left(t_{1}\right)=\Phi_{0} e^{-\left[t_{1} / \lambda\left(E_{0}\right)\right]}
$$

where $\lambda\left(E_{0}\right)$ is the effective relaxation length for the incident radiation in the material. The radiation per unit solid angle scattered toward $P$ by the ith element in the material is given by $\Phi K_{i}(E, \theta) N_{i} d v$, where $K_{i}(E, \theta)$ is the differential angular scattering cross section of material $i$ for radiation incident at energy $E$ and $N_{i}$ is the atomic density of element $i$ in the duct-wall material. The flux density at $P$ due to radiation of energy $E$ scattering in the lip is then

$$
\Phi=\int_{V}\left(d v \Phi _ { 0 } e ^ { - [ t _ { 1 } / \lambda ( E _ { 0 } ) ] ) } \sum _ { i } K _ { i } ( E _ { 0 } , \theta ) N _ { i } \left(e^{\left.-\left[t_{2} / \lambda\left(E_{1}\right)\right]\right)} \frac{1}{R^{2}}\right.\right.
$$

where $E_{1}$ is the energy after scattering and $V$ is the scattering volume. This technique is analogous to integrating a point kernel with the strength of the source point being used as the differential scattering density at that point. The scattering volume includes that portion of the corner for which $t_{1}+t_{2}$ is between one and two relaxation lengths. 
(a) Simon-Clifford Technique for Cylindrical Ducts. The albedo method of Simon and Clifford ${ }^{45}$ is one of the least complicated of the methods available for calculating the transmission of thermal-and intermediate-energy neutrons through cylindrical ducts. One considers only the neutrons entering the open end of the duct, and transmission is assumed to

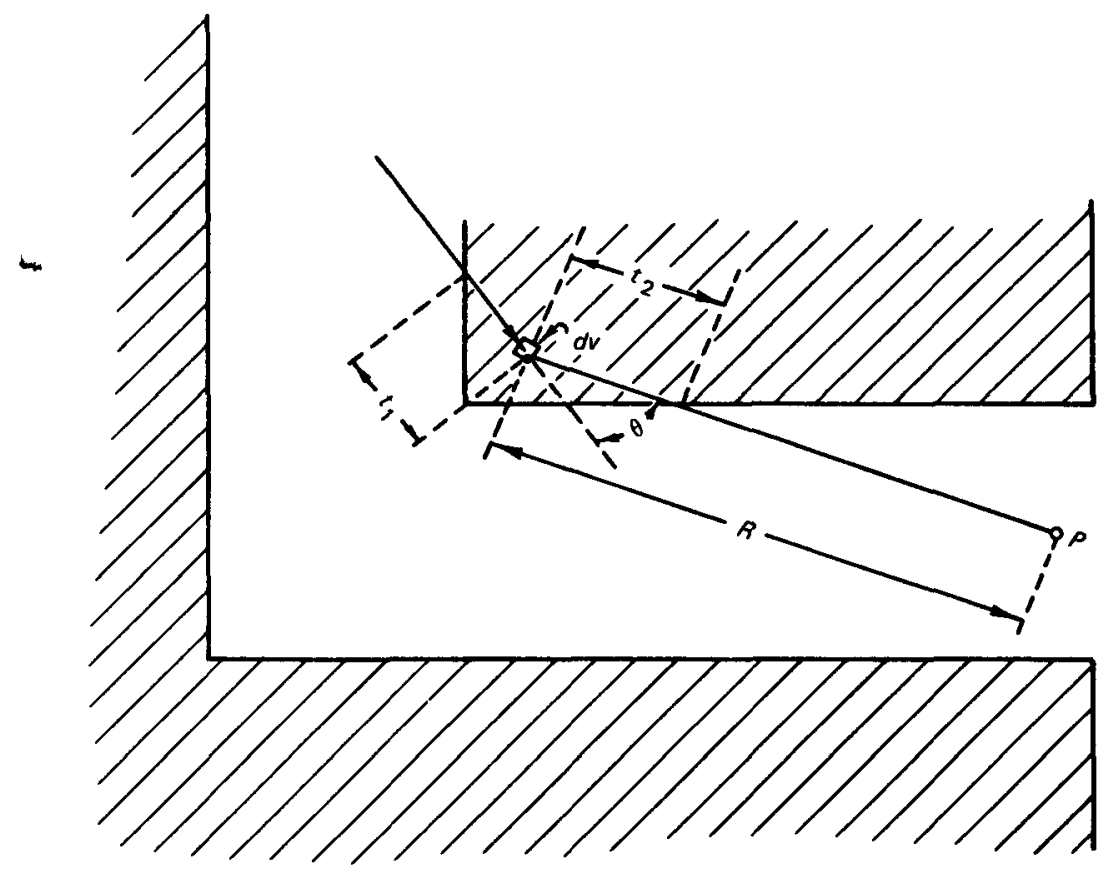

Fig. 7.44-Geometry for last-collision calculation of corner-lip inscattering effect in two-legged rectangular duct.

be either by line of sight or by scattering from the duct walls. The wall scattering is calculated with a single-energy spectrum-averaged material albedo.

For a straight cylindrical duct, the albedo, $A_{2}$, is defined as the fraction of incident neutrons that is scattered from the wall (see Sec. 7.2). In this form of the albedo, the reflected radiation has been integrated over all exit directions, the reflected angular distribution being expressed in the general form $(\beta+2 \gamma \cos \theta) / 2 \pi$, where $\beta$ is the fraction scattered isotropically, $\gamma$ is the fraction scattered with a cosine distribution, and $\beta+\gamma=1$. The flux density at distance $Z$ along a duct of radius $a$ is then given by 


$$
\Phi(Z)=\frac{N_{0}}{2}\left(\frac{a}{Z}\right)^{2}\left[1+\left(\frac{A_{2}}{1-A_{2}}\right)\left(\beta+\frac{4 \gamma a}{Z}\right)\right]
$$

where $\Phi(Z)$ is in the same units as the surface source strength $N_{0}$.

The first term in this equation is the line-of-sight component given in Sec. 7.8. The second term, which accounts for the wall-scattering component, is a strong function of the material albedo. Since $A_{2}$ is of the order of 0.1 for fast neutrons and the second term is small for long thin ducts $(a \ll Z)$, Eq. 7.10-6 simplifies to that for the uncollided flux density. However, for thermal neutrons, which have an albedo of about 0.8 , the second term becomes important, and the scattered flux can dominate.

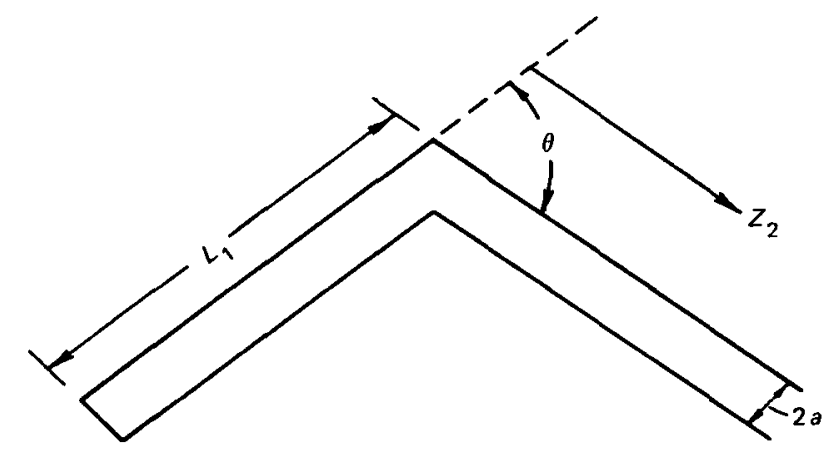

Fig. 7.45-Geometry for calculating neutron transmission through cylindrical duct by Simon-Clifford method.

Artigas and Hungerford ${ }^{49}$ improved the results of Simon and Clifford by developing a more accurate version of Eq. 7.10-6 in terms of integral functions of the parameter $\lambda=a^{2} / Z^{2}$. The newer results are particularly better for values of $\lambda$ greater than about 0.1 .

Simon and Clifford ${ }^{4}$ also covered the case of a bent cylindrical duct (see Fig. 7.45) by assuming that the albedo (in the area of the bend) of the neutrons transmitted through the first leg of the duct is the source for calculating transmission through the second leg. When both legs are of equal diameter and their angular separation is $\theta$, the total transmission at a distance $Z_{2}$ along the second leg is given by

$$
\Phi\left(Z_{2}\right)=\frac{N_{0} A_{2}^{\prime}}{4}\left(\frac{a}{L_{1}}\right)^{2}\left(\frac{a}{Z_{2}}\right)^{2}\left(\frac{\beta+2 \gamma \sin \theta}{\sin \theta}\right)
$$

where $A_{2}^{\prime}$ is an empirically determined albedo parameter. 
If a series of bends is involved, the flux density at a distance $Z$ from the last bend may be expressed as

$$
\begin{aligned}
\Phi\left(Z_{n}\right)=\frac{N_{0}}{2}\left(\frac{a}{Z_{n}}\right)^{2}\left[A_{2}^{\prime}\left(\frac{a}{L_{1}}\right)^{2}\left(\frac{\beta+2 \gamma \sin \theta_{1}}{\sin \theta_{1}}\right)\right] \\
\ldots\left[A_{2}^{\prime}\left(\frac{a}{L_{n-1}}\right)^{2}\left(\frac{\beta+2 \gamma \sin \theta_{n-1}}{\sin \theta_{n-1}}\right)\right]
\end{aligned}
$$

where the duct segments are of lengths $L_{1}, L_{2}, \ldots, L_{n}$ and are separated by angles $\theta_{1}, \theta_{2}, \ldots, \theta_{n-1}$.

Ducts that follow a smooth curve through the shield may be treated by this method provided the radius of curvature is large with respect to the radius of the duct. ${ }^{38}$ The curved path may be divided into a series of equivalent straight sections of length equal to the maximum chord that can be drawn internal to the duct. The angular separation between successive chords can then be used as the angle between the equivalent sections. Horton $^{50}$ treated helical ducts with an albedo approach similar to the Simon-Clifford method. For a helical path divided into $n$ equivalent straight sections of radius $a$ and length $l$ deviating successively by constant angle $\psi$, the flux density is approximated by

$$
\Phi=\frac{N_{0}}{2^{n}}\left(\frac{a}{l}\right)^{2 n}\left(A_{2}^{\prime} \operatorname{cosc} \psi\right)^{n-1}
$$

This can be compared with Eq.7.10-8, which would be the equivalent Simon-Clifford expression provided all values of $\theta$ equal $\psi$ and all values of $L$ equal $l$. The usefulness of this and other semiempirical albedo techniques depends on the availability of suitable values of the albedos and angular distributions of the reflected neutrons.

Horton and Halliday ${ }^{51}$ used the Simon-Clifford albedo method for calculating the transmission of neutrons through straight cylindrical ducts. The calculations corresponded to an experiment in which 5-, 10-, and 20 -cm-diameter steel-lined ducts penetrated a water shield that was adjacent to an extended plane source of thermal neutrons. A current albedo, $\beta$, of 0.55 was used, and a cosine distribution was assumed. The comparison shown in Fig. 7.46 demonstrates that the Simon-Clifford equation gives good agreement with experiments in which the transmission of thermal neutrons through cylindrical ducts is measured provided that reasonable assumptions are made for the thermal-neutron albedos. 


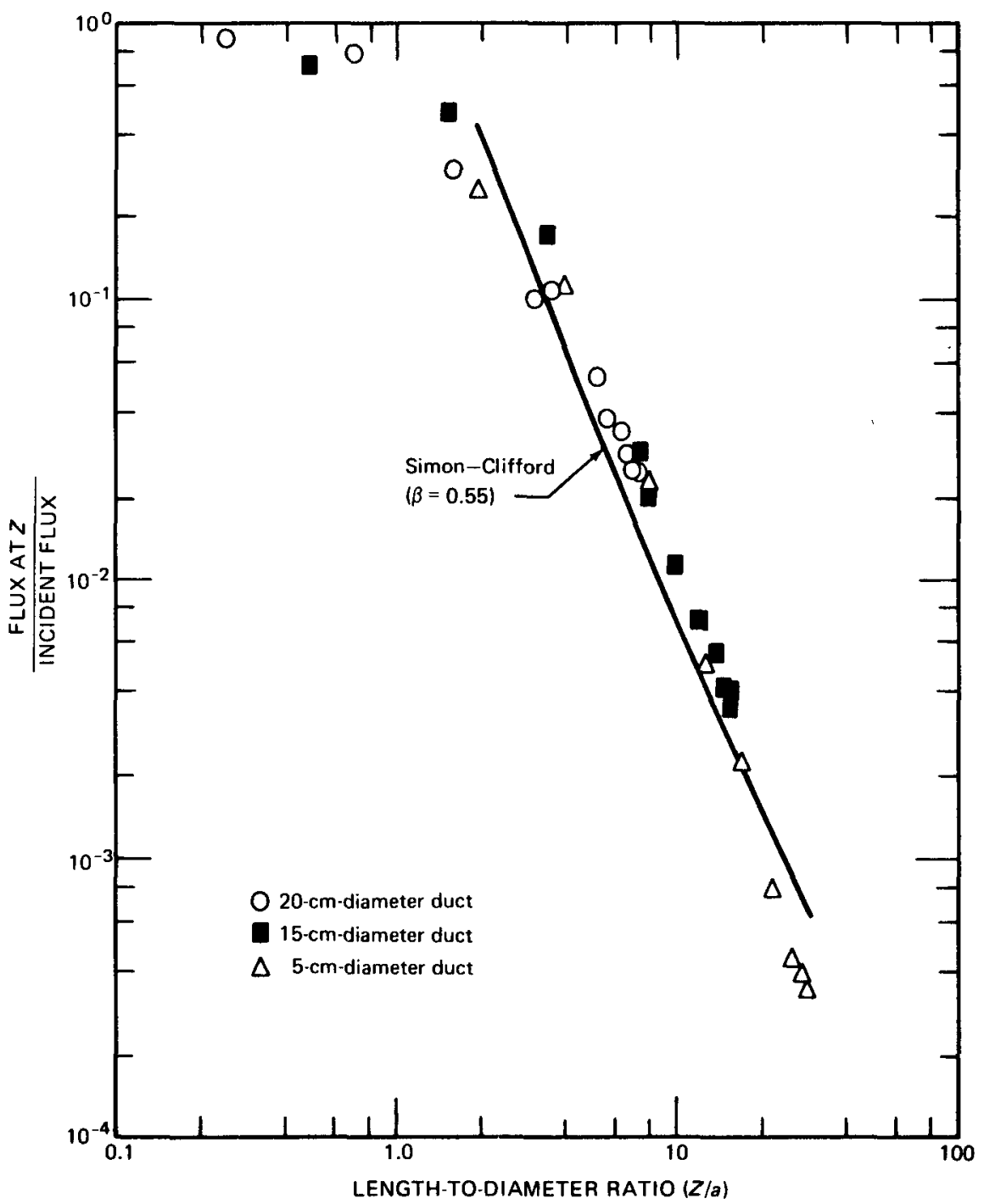

Fig. 7.46-Comparison of albedo calculations by Simon-Clifford technique with thermal-neutron-flux densities measured in straight cylindrical steel-wall ducts through a water shield; plane source of thermal neutrons. (From Horton and Halliday. ${ }^{51}$ )

(b) LeDoux-Chilton Technique for Rectangular Ducts. A method of calculating gamma-ray transmission through two-legged rectangular ducts was formulated by LeDoux and Chilton, ${ }^{46}$ extended by Chapman, and the same general technique was used by Song ${ }^{5}$ for neutron transmission. This group of investigators has applied a maximum of insight with a minimum of input parameters to obtain reasonable agreement between calculation and 
measurement. A review of this work is instructive because the role of each component of the calculation is easy to follow, and the necessity for adding second- and third-order contributions becomes apparent. Equations were derived for calculating radiation streaming down two-legged concrete ducts based on (1) a single-reflection albedo for surfaces that are viewed by both the source and the detector and (2) scattering by material in the corner lip of the duct.

In the absence of sufficient differential albedo data at the time of this work, a total albedo was used, and radiation was assumed to emerge isotropically. To simplify the equations, they assumed that all points on a major scattering area (numbered areas of Fig. 7.43) could be considered to have the same angle of incidence from the source and the same reflection angle to the detector. Formulas were then written for the detector response due to first-order scattering from each of the surfaces. Later work ${ }^{25,52.53}$ with more accurate differential albedo data showed that this method tends to underpredict because multiple reflections are neglected. That the method predicts measurements as well as it does is attributed to a compensating overprediction caused by the assumption of isotropic scattering.

Chapman ${ }^{54}$ extended the LeDoux-Chilton approach to include double reflections and second-order effects, such as a wall backscatter followed by a corner-lip inscatter. In place of the total albedos with the assumption of isotropy of the reflected radiation used by LeDoux and Chilton, Chapman substituted the semiempirical formula for the differential-dose albedo that was derived by Chilton and Huddleston ${ }^{6}$ (described earlier in this chapter). The two parameters in this formula were determined by a least-squares fit to the Monte Carlo data of Raso. ${ }^{25}$

The number of the interaction combinations was enlarged (e.g., one backscatter, one inscatter, one backscatter plus one penetration, two backscatters, and one backscatter plus one inscatter).

The singly reflected component was evaluated by integrating over each of the numbered areas in Fig. 7.43. When penetration through the corner lip was evaluated, the assumption was made that rays penetrating less than one relaxation length of material are unattenuated and that those encountering more than one relaxation length of material do not penetrate. Scattering in the corner lip was calculated with the singly reflected formula that LeDoux and Chilton derived on the basis of Klein-Nishina differential scattering probabilities.

To perform the double-reflection calculations, Chapman divided the duct into a larger mesh consisting of 12 scattering areas as shown in Fig. 7.47, and combinations that would allow transport from the source to the detector 
with two scatters were considered. Combinations whose fractional contribution to the total dose was considered to be small were deleted. Specifically, these were reflection from surface 1 to surfaces 10 and 12 and reflection from surfaces 2 and 4 to surfaces 10,11 , and 12 . The incidence of singly reflected radiation on the corner lip of the duct was combined with

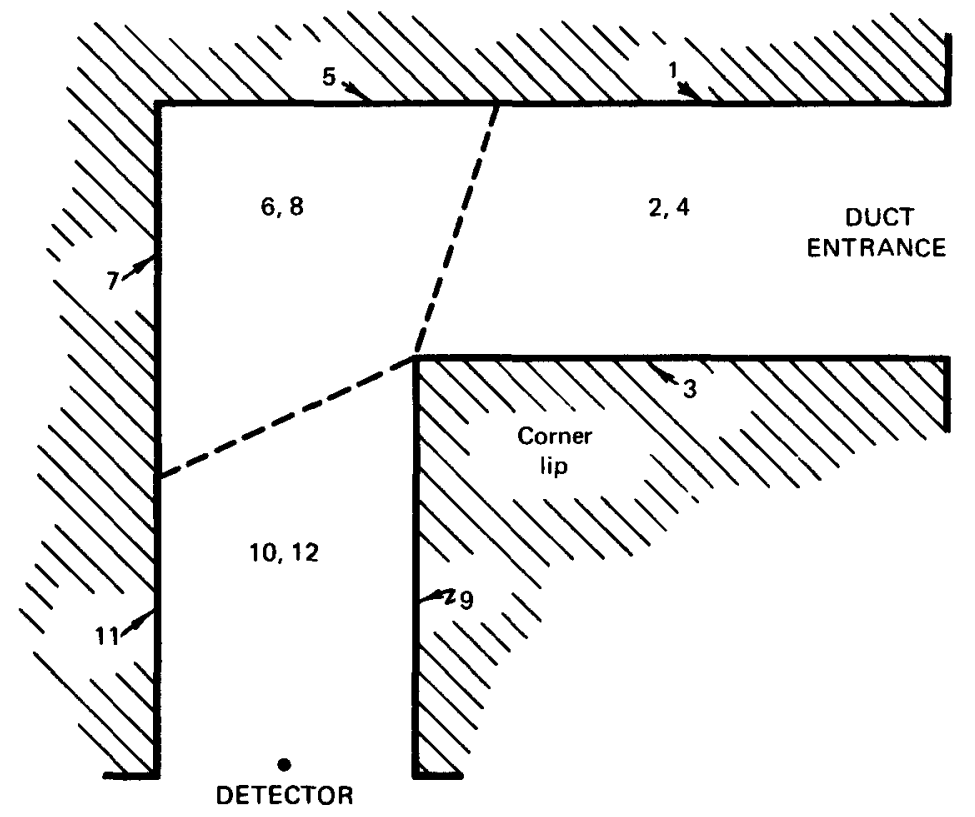

Fig. 7.47--Scattering areas for albedo calculation of doubly reflected gamma rays in two-legged rectangular duct.

the lip inscatter calculation to determine the contribution due to one backscatter plus one inscatter. In all cases the energy degradation in a reflection was assumed to be that of a single Compton scattering.

Comparisons with a number of measurements were made by Chapman, ${ }^{54}$ who used the extended LeDoux-Chilton technique for calculating gamma-ray dose rates at the exit end of two-legged $L$-shaped rectangular ducts, the source in each case being a gamma-ray-emitting isotope at the mouth of the duct. Chapman also calculated the gamma-ray dose rates along the axes of two- and three-legged rectangular ducts, and comparisons are shown in Figs. 7.48 through 7.50 with the dose rates measured at several points along the duct axes for three sources of different energy. 5-5 7 


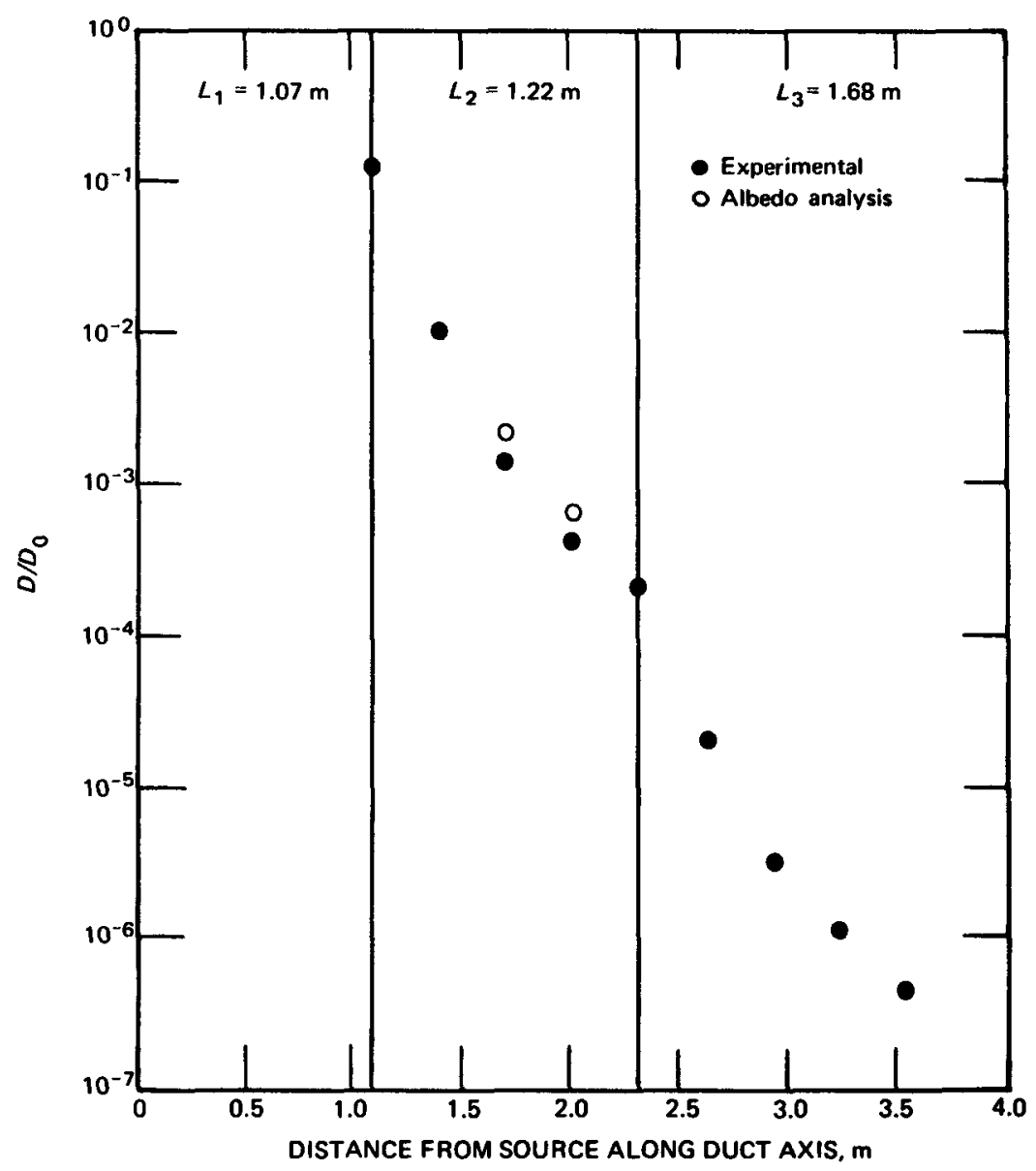

Fig. 7.48-Comparison of albedo calculations with measurements of gamma-ray dose rates along the axis of a $0.3-\mathrm{m}$-square $U$-shaped concrete duct; $80-\mathrm{Ci}{ }^{137} \mathrm{Cs}$ gamma-ray source. (Experimental data from Terrell et al. ${ }^{55}$; calculations from Chapman. ${ }^{54}$ )

Figure 7.49 also includes results of an empirical formula developed by Ingold and Huddleston ${ }^{58}$ from the experimental and analytical data of LeDoux, Chilton, and Chapman. By data fitting, the formula was found to be

$$
\frac{D}{D_{0}}=3.44 \frac{(H / W)^{0.907} W^{2.864}}{L_{1}^{2.534} L_{2}^{2.667} E_{0}^{0.710}}
$$




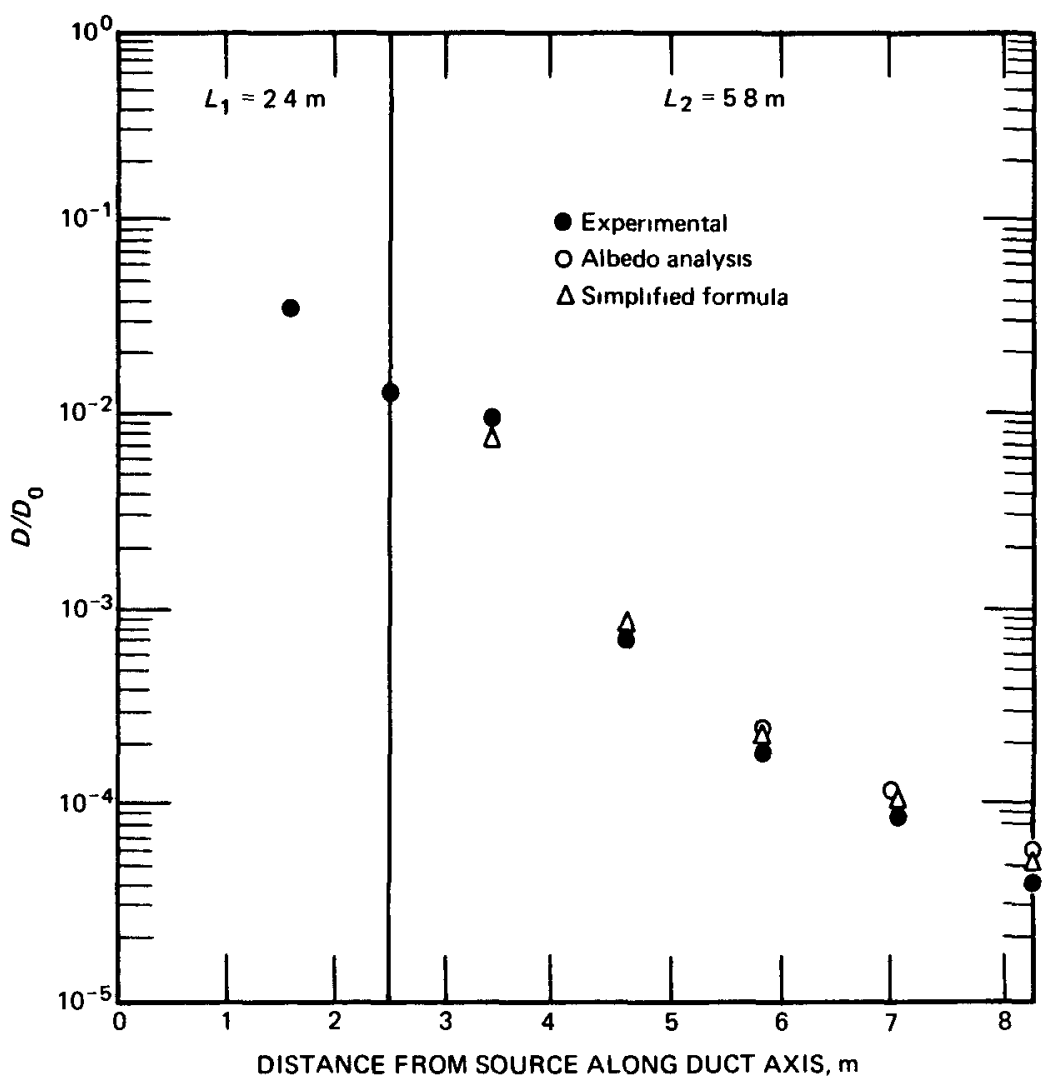

Fig. 7.49-Comparison of albedo calculations with measurements of gamma-ray dose rates along the axis of a $1.8-\mathrm{m}$-square $L$-shaped concrete duct; $3.67-\mathrm{Ci}{ }^{60} \mathrm{Co}$ source. The results designated simplified formula correspond to the Ingold-Huddleston equation (Eq. 7.10-10). (Experimental data from Terrell et al. ${ }^{55}$; calculations from Chapman. ${ }^{54}$ )

where $D=$ dose rate on axis of duct at point of interest

$D_{0}=$ dose rate $0.30 \mathrm{~m}$ from the source

$H=$ height of duct (m)

$W=$ width of duct $(\mathrm{m})$

$L_{1}=$ length of first leg measured from the source to the center of the first corner $(\mathrm{m})$

$L_{2}=$ length of second leg measured from the center of the corner to the detector $(\mathrm{m})$

$E_{0}=$ gamma-ray source energy $(\mathrm{MeV})$

Use of this formula is limited to problems that meet the following criteria: Point source at duct entrance, $0.662 \mathrm{MeV} \leqslant E_{0} \leqslant 6.0 \mathrm{MeV}$, 


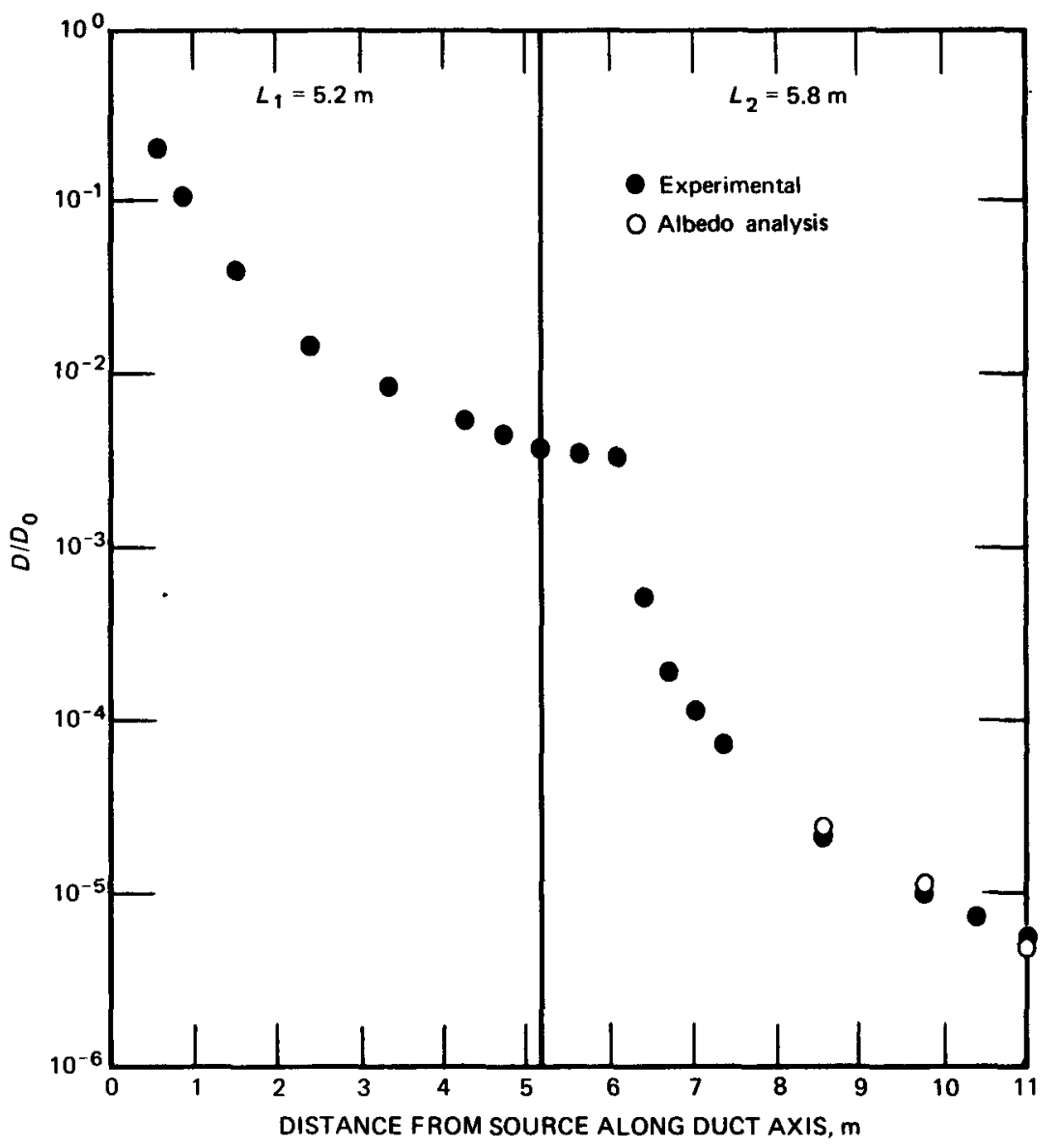

Fig. 7.50-Comparison of albedo calculations with measurements of gamma-ray dose rates along the axis of a $1.8-\mathrm{m}$-square $L$-shaped duct; $4.2-\mathrm{Ci}^{24} \mathrm{Na}$ source. (Experimental data from Terrell et al. ${ }^{57}$; calculations from Chapman. ${ }^{54}$ )

$0.3 \leqslant H \leqslant 1.8 \mathrm{~m}, \quad 0.3 \leqslant W \leqslant 1.8 \mathrm{~m}, \quad 0.6 \leqslant L_{1} \leqslant 11 \mathrm{~m}, \quad 1 \leqslant H / W \leqslant 2$, $L_{1} / H \leqslant 6, L_{2} / H \leqslant 6, L_{1} / W \geqslant 2, L_{2} / W \geqslant 2$. Although this formula is useful for point sources, it should be applied with caution to a broad-beam source.

In calculating neutron transmission through rectangular ducts, Song ${ }^{5}$ used Chapman's modification of the LeDoux-Chilton method. The reflected neutron spectrum was assumed to consist of two neutron energy groups, the number and energy in each group being based on the Monte Carlo data of Allen et al. ${ }^{2}$ In one analysis the duct was assumed to be $0.91 \mathrm{~m}$ square in cross section, and the sources considered were 14.7 - and 2.5-MeV neutrons. The analysis included all single and double scattering events to determine the 
energy and angular dependence of the neutron-dose albedo. A comparison of the results with measurements made by Doty ${ }^{59}$ (Fig. 7.51) shows good agreement. (The solid line represents the calculations for both the 2.5- and the 14.7-MeV sources since there was very little difference between the two sets of results.) Song derived a semiempirical formula for use in the calculations in terms of a single-energy parameter for the differential

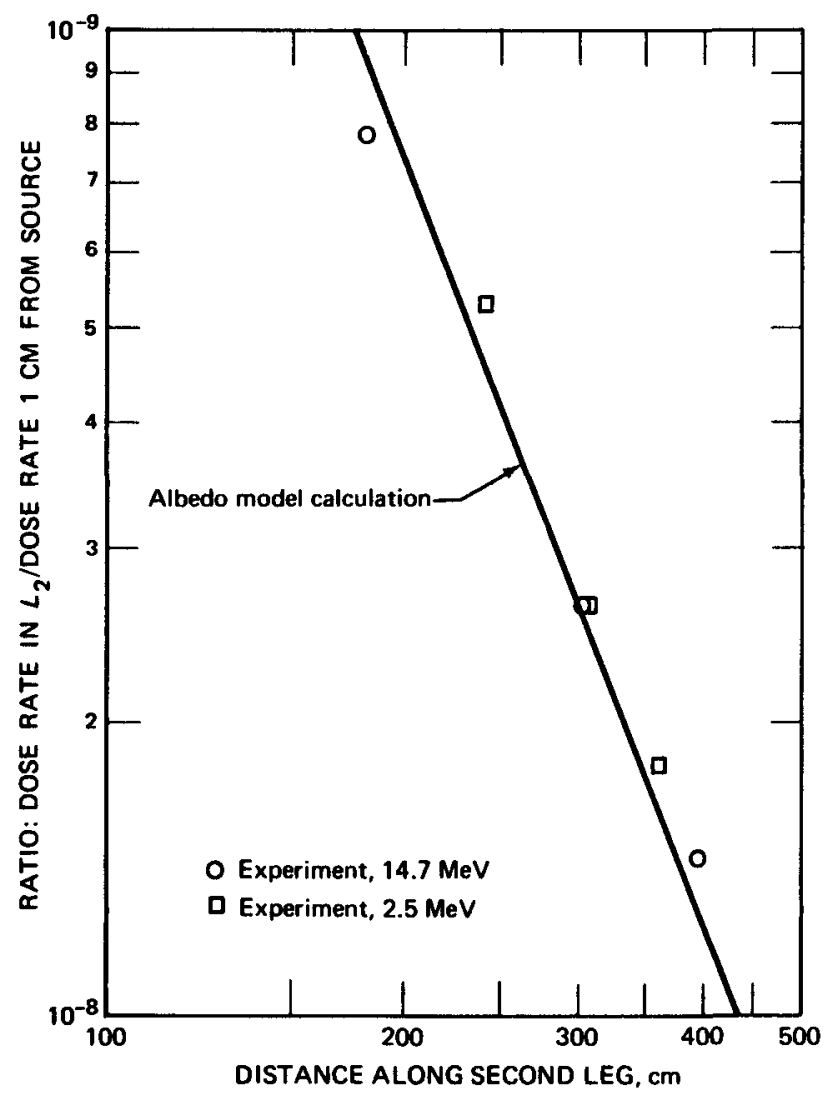

Fig. 7.51-Comparison of albedo calculations with measurements of neutron dose rates in the second leg of a $0.91-\mathrm{m}$-square two-legged concrete duct; $2.5-$ and $14.7-\mathrm{MeV}$ neutron sources. In the experiment the source was $30 \mathrm{~cm}$ outside the duct and $L_{1}=4.6 \mathrm{~m}$; in the calculation the source was assumed to be at the duct entrance and $L_{1}=4.9 \mathrm{~m}$. (From Song. ${ }^{5}$ )

neutron-dose albedo in a manner analogous to that used by Chilton and Huddleston $^{6}$ in their gamma-ray calculations. Values for the energydependent parameter were obtained by a least-squares analysis that gave the best fit to the data of Allen et al. ${ }^{2}$ 
(c) Albedo Monte Carlo Methods. Experiments and calculations have shown that gamma rays produced by neutron absorptions in a concrete shield surrounding a rectangular duct make a significant contribution to the total dose in the duct, and analysis of this component requires a knowledge of the distribution of low-energy neutrons throughout both the duct and the shield. These distributions can be determined with accuracy by analog Monte Carlo calculations, but, as pointed out in Sec. 7.10.1, the machine time required to trace neutrons until they approach thermal equilibrium can become excessive. To circumvent this, Cain developed a technique ${ }^{47}$ that uses a random-walk Monte Carlo approach in which particles are traced by selecting random paths inside the duct but prohibits particle penetration into the shielding material. Thus tracking through a large number of interactions prior to emergence from the wall material is averted, and no time is wasted in tracking particles that never emerge. Each time a particle encounters the wall, it is assumed to reemerge with a reduced weight (given by the incident weight times the albedo of the wall). Flux densities at various points along the duct are then calculated either by making a statistical estimate for each encounter to various point detectors or by determining the track length per unit volume in a detector region. The most satisfactory detector was found to be a thin region extending across the duct and perpendicular to the duct axis.

The method was modified by Maerker and Cain ${ }^{60}$ to include doubly differential thermal-neutron current and fast-neutron-dose albedo data (differential in reflected solid angle and energy) previously determined ${ }^{1,8,18}$ by Monte Carlo calculations for infinite slabs of concrete (described in Sec. 7.3). In addition, a provision was included for calculating the capture-gamma-ray dose rate arising from neutron capture in the duct walls.

In the modified code the incident neutrons are reflected with reduced weight into various energy groups and directions, the probabilities for which are predetermined from the doubly differential albedo data. The neutrons are followed in the random-walk procedure until they either escape out the front or the rear end of the duct or are killed by Russian roulette (Chap. 5) when their weight falls below a predetermined value. Statistical estimations of the fluxes to point detectors are made to obtain the results.

Good accuracy is obtained when the calculations are done separately for three energy ranges: (1) Neutrons with energies from $200 \mathrm{keV}$ to $8 \mathrm{MeV}$ are divided into six energy groups and are sampled to obtain the fast flux contribution to the dose rate. (2) The dose rate from neutrons of intermediate energy $(0.5 \mathrm{eV}$ to $200 \mathrm{keV})$, including all neutrons that have 
slowed down to intermediate and thermal energies from higher energy groups, is determined with a 13-group calculation that involves sampling the entire energy range from thermal to $8 \mathrm{MeV}$ but scoring only those neutrons with energies below $200 \mathrm{keV}$. (3) The dose rate from source thermal neutrons (subcadmium, $<0.5 \mathrm{eV}$ ) is then determined in a separate calculation using a single-velocity model.

Monte Carlo albedo data of Maerker, Muckenthaler, Coleman, and Stevens ${ }^{1,8,18}$ are used in each energy range as described earlier in the chapter. To recapitulate, five sets of albedo data are used: one for each of the three neutron-energy ranges given in the preceding paragraph plus two for capture gamma rays (for incident thermal neutrons and for incident intermediate neutrons). In this method no neutron is allowed to penetrate a corner during the course of its random walk, although a statistical estimate of the flux that includes a corner-lip penetration in the last flight can be made.

Maerker and Muckenthaler ${ }^{\mathbf{8 . 6 1 . 6 2}}$ carried out an extensive series of experiments and calculations to test the accuracy of the Monte Carlo albedo method as programmed in the AMC code. ${ }^{60}$ Three configurations of a 0.91-m-square duct were used: a straight duct $13.7 \mathrm{~m}$ long; a two-legged duct with a right-angle bend located $4.6 \mathrm{~m}$ down the first leg, the second leg being $9.8 \mathrm{~m}$ in length; and a three-legged duct with two right-angle bends, the two bends being located $4.6 \mathrm{~m}$ down the first and second legs and the third leg being $6.1 \mathrm{~m}$ in length. In the experiments a collimated neutron beam from the Tower Shielding Reactor II entered each configuration through a 30 - by $30-\mathrm{cm}$ area located at the geometric center of the duct mouth. The neutrons were incident at an angle $45^{\circ}$ to one of the duct walls. This angle of incidence was chosen to serve as a rigorous test of the calculation since the fluxes and doses for detector locations greater than about a meter from the duct mouth would then be due entirely to wall-scattered radiation. The measured absolute energy spectrum of the beam was used for the source spectrum in the calculations.

Figure 7.52 shows a comparison of the calculated and measured results for fast neutrons. In general, the agreement averages better than $20 \%$ through five orders of fast-neutron dose attenuation. For subcadmium or thermal fluxes that result from an incident beam of subcadmium neutrons (Fig. 7.53), the agreement is within $20 \%$ in the second and third legs and within $5 \%$ for most of the straight ducts.

Calculations were also made of the thermal-neutron-flux density resulting from all source neutrons with energies above cadmium cutoff to test 
the accuracy of the slowing-down model used in the code. The comparison between calculations and experiment shown in Fig. 7.54 indicates that the agreement is about the same as that obtained for incident subcadmium neutrons. The statistical errors, number of wall backscatterings, and running times for the same number of source histories were comparable in the two

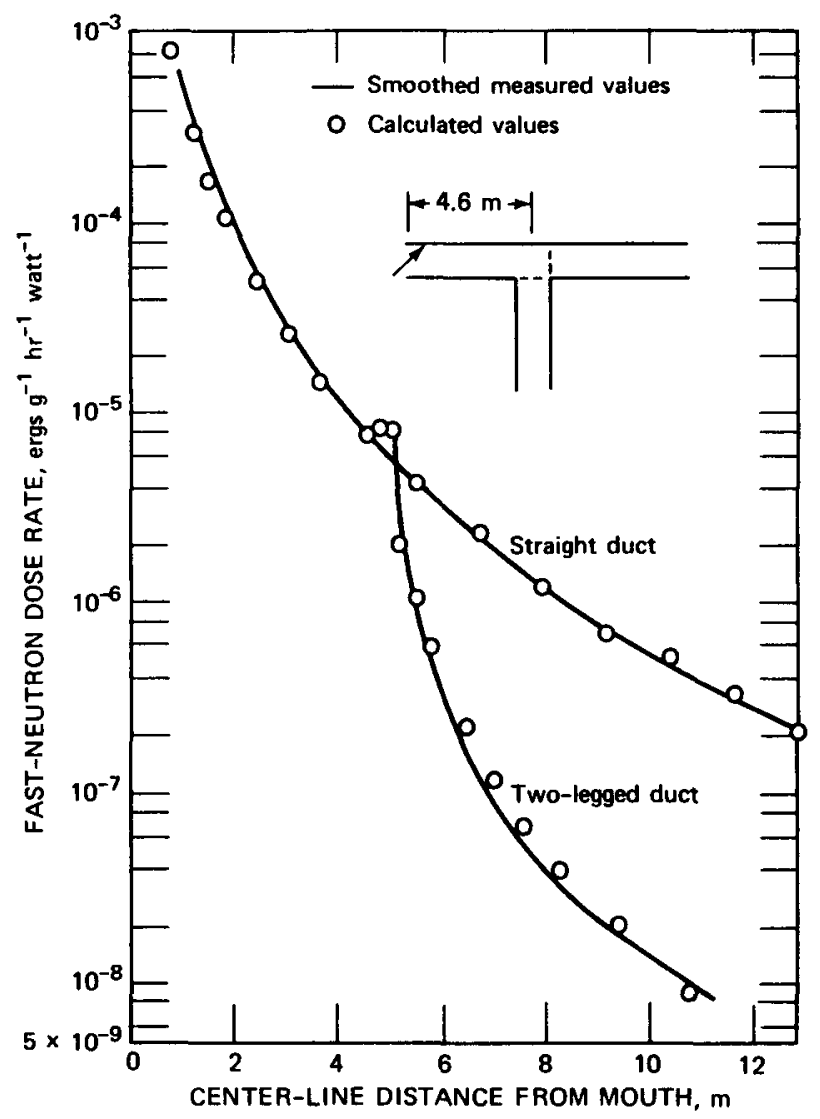

Fig. 7.52-Comparison of AMC albedo calculations with measurements of fast-neutron dose rates in one- and two-legged 0.91 -m-square concrete ducts. (From Maerker and Muckenthaler. ${ }^{62}$ )

calculations. It should be noted that the calculations were normalized in each case to the measured number of neutrons incident on the wall of the duct from the source beam.

Calculations were also made of the epicadmium or nonthermal spectra at several locations along the center line of the three-legged duct, and these 


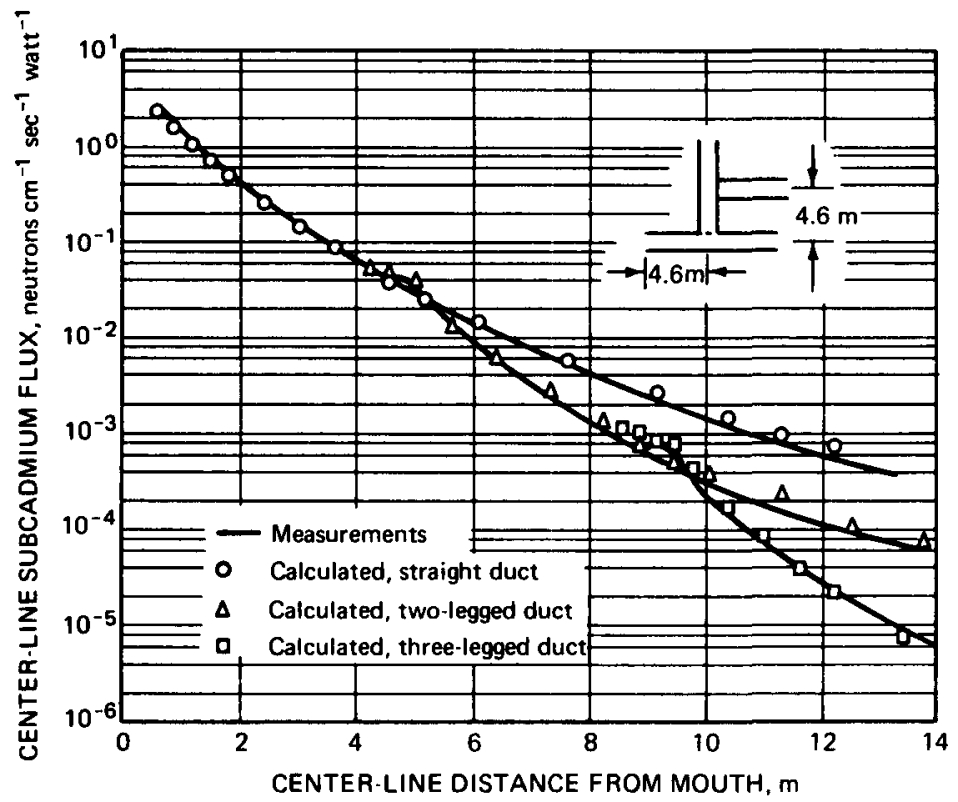

Fig. 7.53-Comparison of AMC albedo calculations with measurements of thermalneutron-flux densities in one-, two-, and three-legged 0.91 -m-square concrete ducts due to incident subcadmium neutrons. (From Maerker and Muckenthaler. ${ }^{62}$ )

spectra, together with the flux-to-multicollision dose factors of Snyder and Neufeld, ${ }^{63}$ were used to calculate the epicadmium multicollision dose rates at the same locations. The results are compared in Table 7.5 with measurements made with a dosimeter designed to give a response proportional to the Snyder-Neufeld multicollision dose curve over the range of interest. The dosimeter was a spherical $\mathrm{BF}_{3}$ counter placed inside a 25- or 30-cm-diameter polyethylene ball covered with cadmium.

The method of Cain and Maerker (AMC) may be extended to cover any other duct arrangements by providing an appropriate geometry analysis routine. Calculations in cylindrical geometry have been made by Selph, Newell, and Bayer. ${ }^{64}$

\subsubsection{Additional Experimental Investigations}

A selection of experimental data has been presented in the preceding discussions for the purpose of validating analytical or empirical methods of duct analysis. Some additional experiments can be cited which help to illustrate principles involved in duct penetration. 


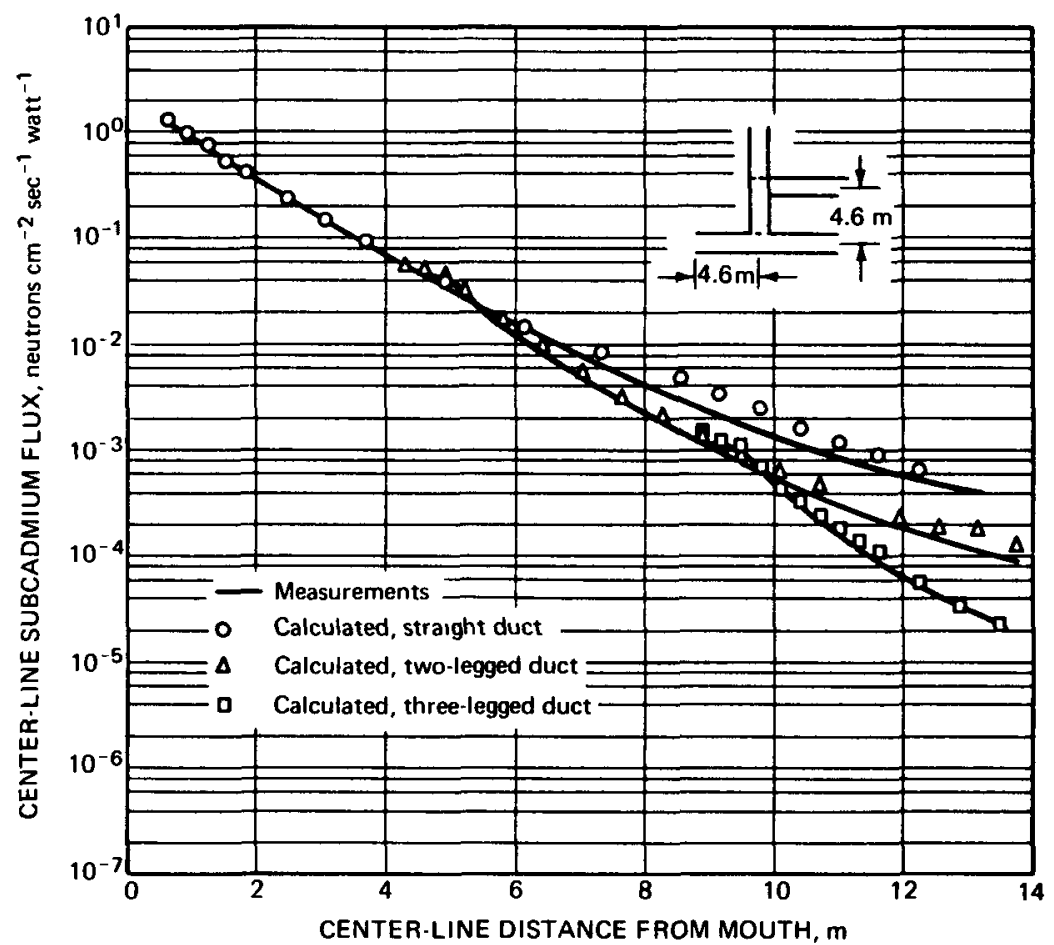

Fig. 7.54-Comparison of AMC albedo calculations with measurements of thermalneutron-flux densities in one-, two-, and three-legged 0.91 -m-square concrete ducts due to neutrons having energies above cadmium cutoff. (From Maerker and Muckenthaler. ${ }^{62}$ )

Table 7.5-CALCULATED VS. MEASURED EPICADMIUM MULTICOLLISION DOSE RATES IN A THREE-LEGGED DUCT

\begin{tabular}{ccccc}
\hline & $\begin{array}{c}\text { Distance } \\
\text { from mouth } \\
\text { to detector, }\end{array}$ & \multicolumn{4}{c}{ Dose rate, ergs $\mathrm{g}^{-1} \mathrm{hr}^{-1}$ watt $^{-1}$} \\
\cline { 3 - 5 } $\begin{array}{c}\text { Duct } \\
\text { leg }\end{array}$ & $\mathrm{m}$ & $25-\mathrm{cm}$ ball & Measured & Calculated \\
\hline 2 & 5.8 & $2.5 \times 10^{-6}\left(2.1 \times 10^{-6}\right)$ & $1.35 \times 10^{-6}\left(1.1 \times 10^{-6}\right)$ & $1.9 \times 10^{-6}$ \\
2 & 7.0 & $5.5 \times 10^{-6}\left(4.9 \times 10^{-7}\right)$ & $2.85 \times 10^{-7}\left(2.45 \times 10^{-7}\right)$ & $4.5 \times 10^{-7}$ \\
2 & 8.8 & $1.28 \times 10^{-7}\left(1.2 \times 10^{-7}\right)$ & $6.6 \times 10^{-7}\left(6.1 \times 10^{-8}\right)$ & $1.05 \times 10^{-7}$ \\
2 & 10.0 & $1.45 \times 10^{-8}\left(1.2 \times 10^{-8}\right)$ & $6.4 \times 10^{-9}\left(4.8 \times 10^{-9}\right)$ & $9.2 \times 10^{-9}$ \\
3 & 12.2 & $1.08 \times 10^{-9}\left(9.0 \times 10^{-10}\right)$ & $6.0 \times 10^{-10}\left(5.1 \times 10^{-10}\right)$ & $6.5 \times 10^{-10}$ \\
\hline
\end{tabular}

tThe dose rates in parentheses are those obtained when the center of detection was assumed to be at the center of the polyethylene ball rather than at its leading edge. 
Terrell, Jerri, and Lyday ${ }^{55}$ performed experiments to investigate the effect of geometry and source energy on the attenuation of gamma rays through rectangular concrete ducts with two right-angle bends. They found that for $1.8-\mathrm{m}$-square concrete ducts it made little difference whether the gamma-ray source was ${ }^{60} \mathrm{Co}$ or ${ }^{137} \mathrm{Cs}$ or whether the right-angle bends formed a $U$-shape (Fig. 7.55a) or a $Z$-shape (Fig. 7.55b). These data, plotted in Fig. 7.55 c, show a maximum deviation of a factor of 1.5 when properly

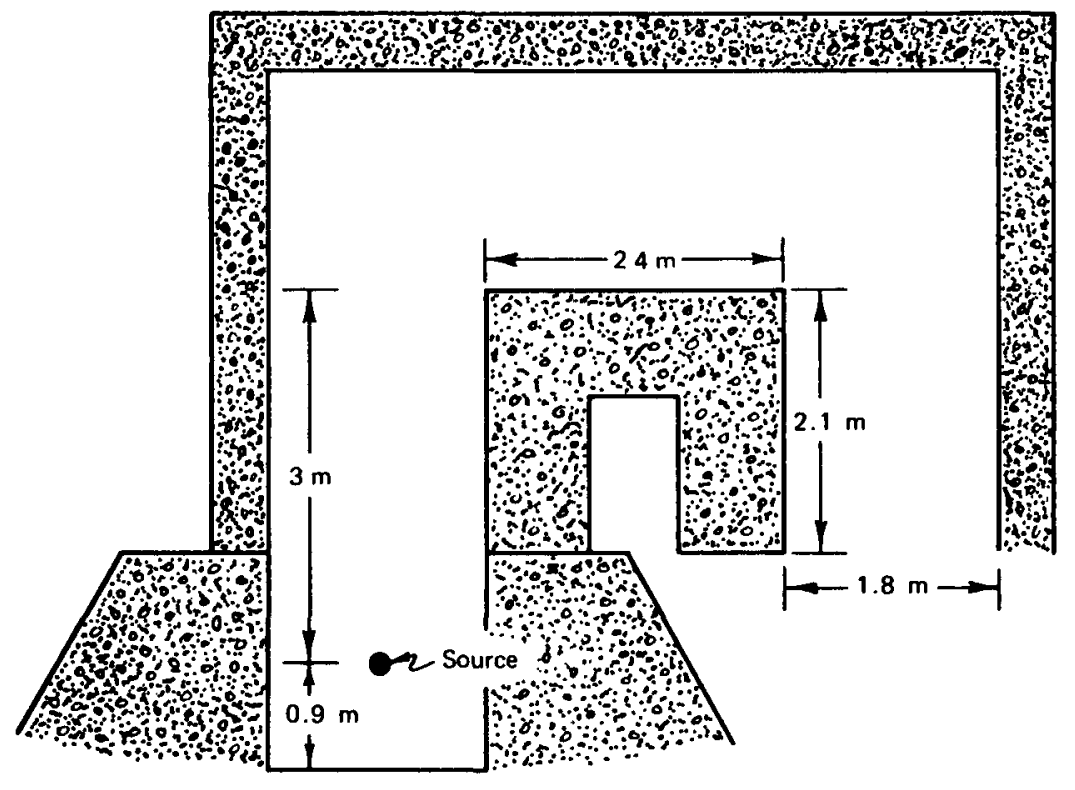

Fig. 7.55a-Geometry of $U$-shaped duct.

normalized. The effect of geometry and energy becomes more noticeable for a 30-cm-square duct.

Chapman ${ }^{65}$ calculated the contribution of a given scattering area on the gamma-ray dose rate in an $L$-shaped 0.91 -m-square concrete duct. The scattering area chosen was the one labeled $S_{1}$ in Fig. 7.56a. Gamma rays from a ${ }^{60} \mathrm{Co}$ source at the mouth of the duct were scattered from this area to the detector at the exit end of the duct. The dose rate due to gamma rays that were initially scattered from $S_{1}$ was calculated to be $0.379 \mathrm{mr} / \mathrm{hr}$, which compares favorably with a measured dose rate of $0.404 \mathrm{mr} / \mathrm{hr}$.

During the experiment Chapman measured the gamma-ray spectrum at the exit end of the duct both with and without the scattering area $S_{1}$ shadowed from the source. The results are shown in Fig. 7.56b. Shielding of 
$S_{1}$ had a more pronounced effect on the higher energy radiation than on the multiply reflected low-energy component. This effect would be expected since the percentage reduction in the single-reflected area that results from blocking $S_{1}$ is greater than the percentage reduction in possible combinations of multiple-reflection areas.

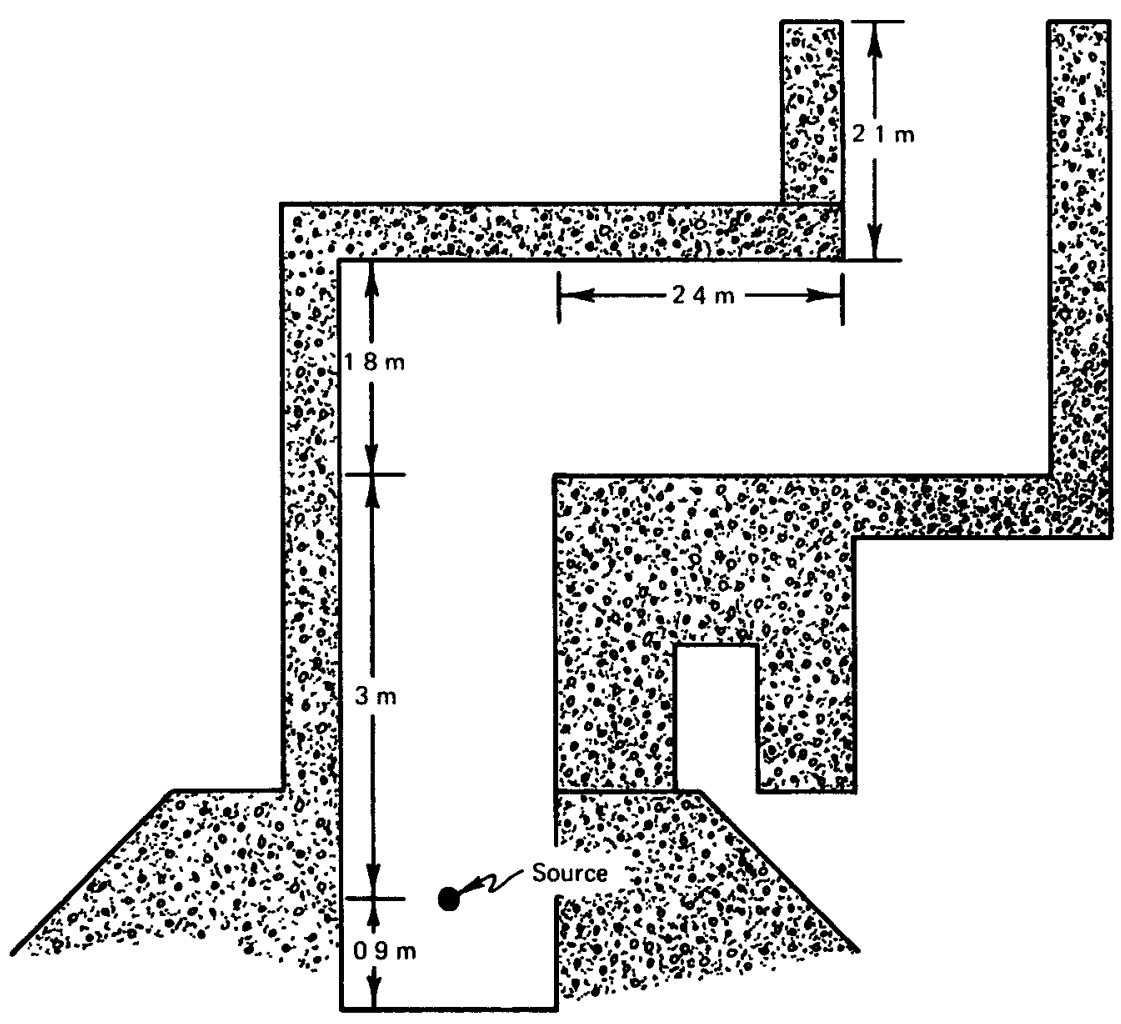

Fig. 7.55b-Geometry of $Z$-shaped duct.

In a subsequent experıment Chapman and Grant ${ }^{66}$ investigated the relative effectiveness of coplanar and noncoplanar three-legged rectangular ducts. For the cases investigated $\left(28-\mathrm{cm}\right.$-square duct, ${ }^{60} \mathrm{Co}$ source, $\left.L_{1}=75 \mathrm{~cm}, L_{2}=80 \mathrm{~cm}, L_{3}=91 \mathrm{~cm}\right)$, the attenuation provided by the noncoplanar configuration was found to be greater by a factor of 2 than that provided by the coplanar configuration.

Clifford ${ }^{52}$ used differential albedos ${ }^{25}$ to calculate dose rates corresponding to those measured in experiments designed to determine the importance of multıple reflection on the transmission of ${ }^{137} \mathrm{Cs}$ gamma rays through 20-, 


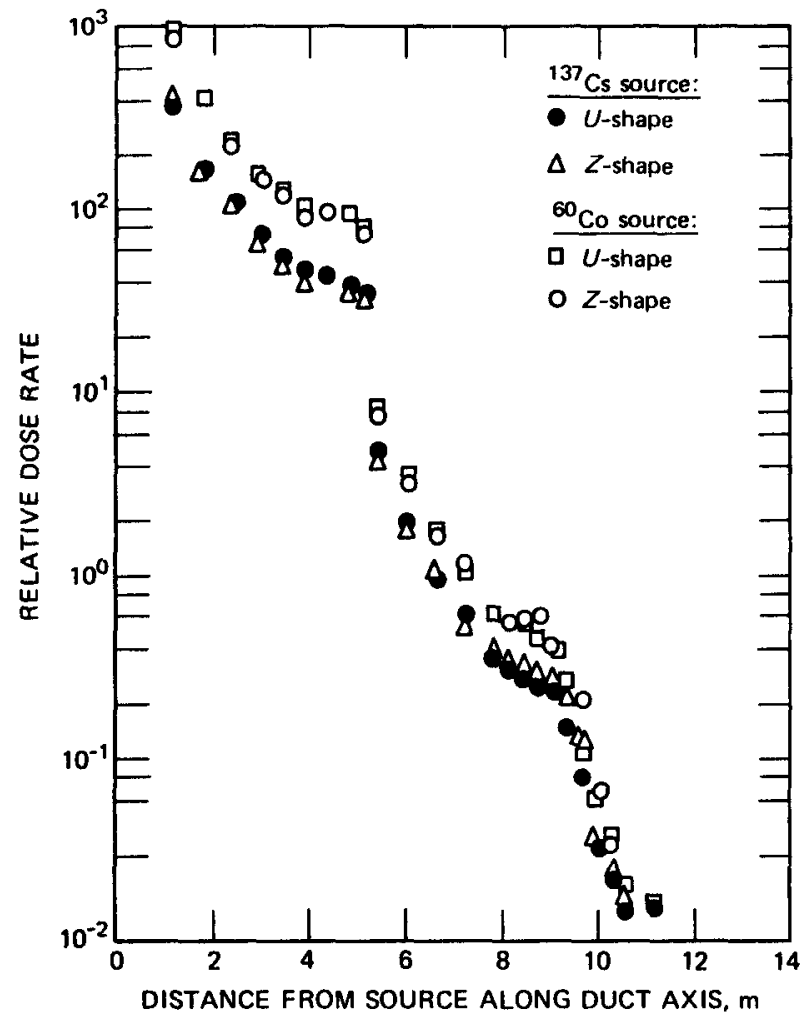

Fig. $7.55 \mathrm{c}-$ Gamma-ray dose rates measured in 1.8 -m-square $U$ - and $Z$-shaped concrete ducts; ${ }^{60} \mathrm{Co}$ and ${ }^{137} \mathrm{Cs}$ gamma-ray sources. (From Terrell et al. ${ }^{55}$ )

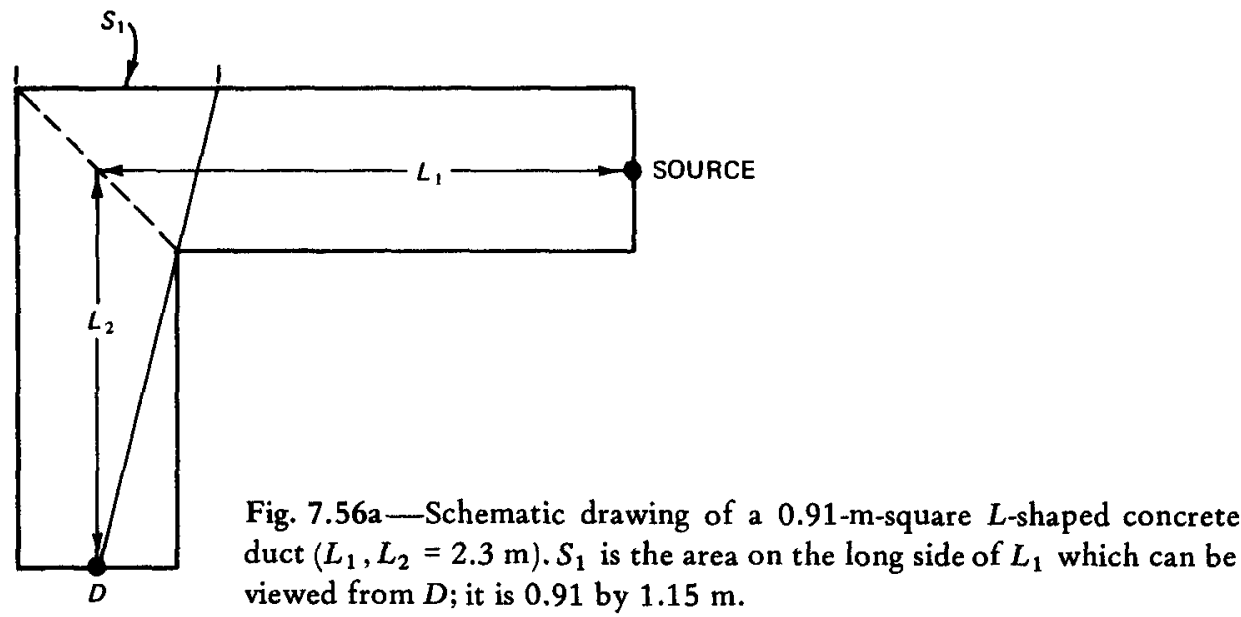




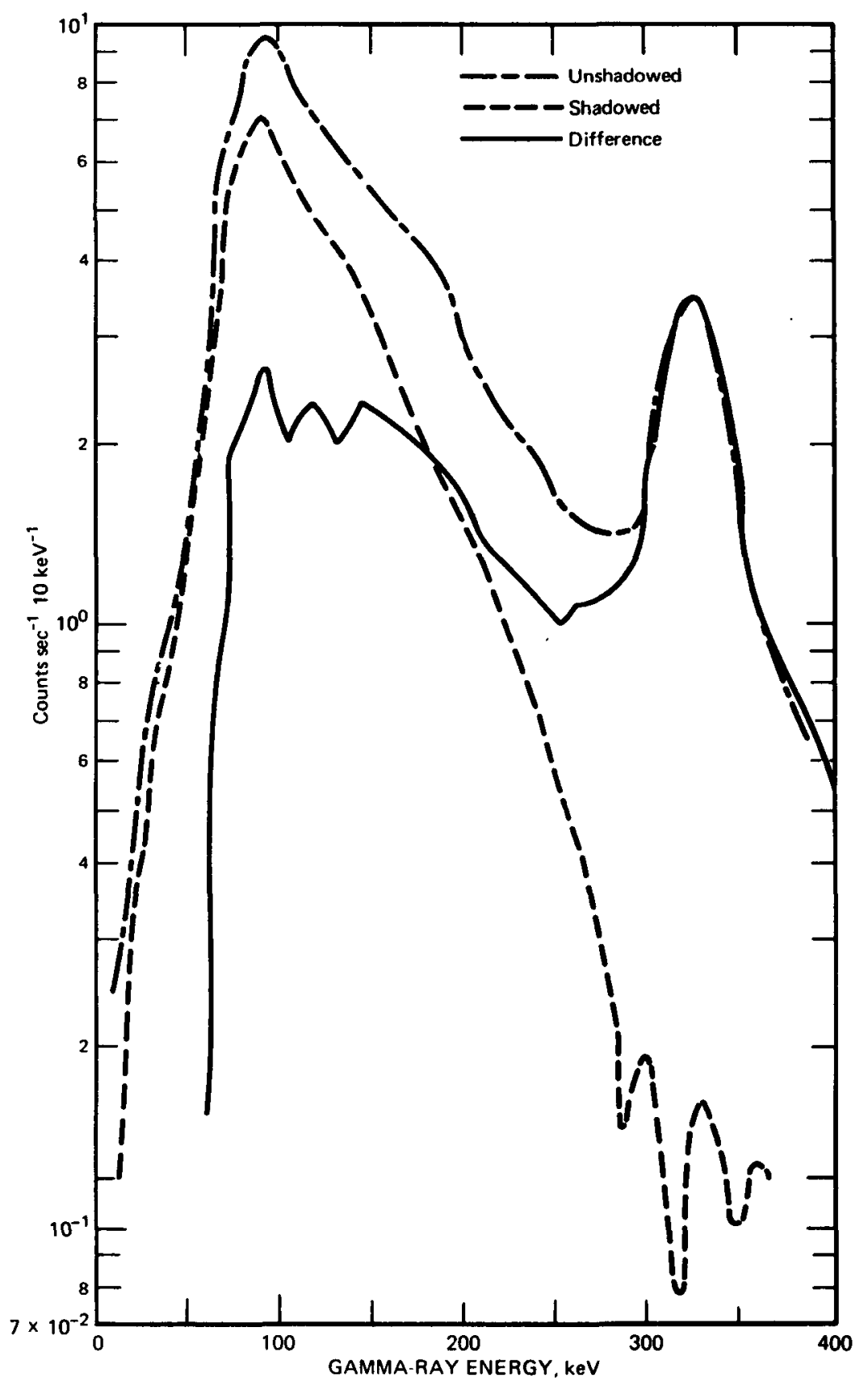

Fig. 7.56b - Gamma-ray energy spectrum at exit of 0.91 -m-square $L$-shaped duct with and without scattering area $S_{1}$ [see Fig. 7.56(a)] being shadowed from ${ }^{60}$ Co source. (From Chapman. ${ }^{65}$ ) 
$30-$, and $91-\mathrm{cm}$-square concrete ducts. In the experiments collimated gamma rays were incident on the duct entrance at an angle to the duct axis such that the area in which the first reflection could occur was near the entrance of the duct (see Fig. 7.57a). The dose rate along the duct center lines was measured first with the unmodified concrete (condition $A$ ). The second measurement (condition $B$ ) was with a lead lining over all inside surfaces except the first scatter area. The third measurement (condition $C$ ) was with a lead lining over the entire inside surface. Figure $7.57 \mathrm{~b}$ shows the results of the measurements for all three conditions in the $20-\mathrm{cm}$ duct together with a single-reflection analysis based on the differential albedo for condition $B$, and Fig. 7.58 shows the fraction of the total dose which is due to reflection from surfaces not exposed to the primary radiation for all the ducts. It is apparent from the results of all three duct sizes that the multiply reflected component increases to become as much as 20 to $30 \%$ of the total dose as the duct length is increased.

\subsection{1 vOIDS}

An irregularity that increases the radiation penetration through a shield but does not extend to either surface is called a void. Under this definition we consider single voids in a shield, such as an air bubble in a concrete pour, or multiple small voids created by filling a container with lead shot. Interest in an accidental void is likely to be centered about repair or determining the patch size to restore shield integrity.

\subsubsection{Single Voids}

The technique most frequently applied to single voids is the ray-analysis technique, which is described in Sec. 7.8 in connection with duct transmission problems. As is pointed out there, the assumption of the ray-analysis technique is that the radiation transmission is a function only of the path lengths through each material or void encountered along a straight line between the source point and the point of interest. When applied to voids the increase in the flux density at the shield surface is determined by the difference in intensities calculated with and without the void.

Consider a disk-shaped void in an infinite-slab shield adjacent to an infinite-plane gamma-ray source with the planes of the disk parallel to the slab planes as shown in Fig. 7.59. Let $\Phi(L)$ be the flux density of energy $E$ at a point on the surface of the shield with no void and $\Phi(L-t)$ be the flux 

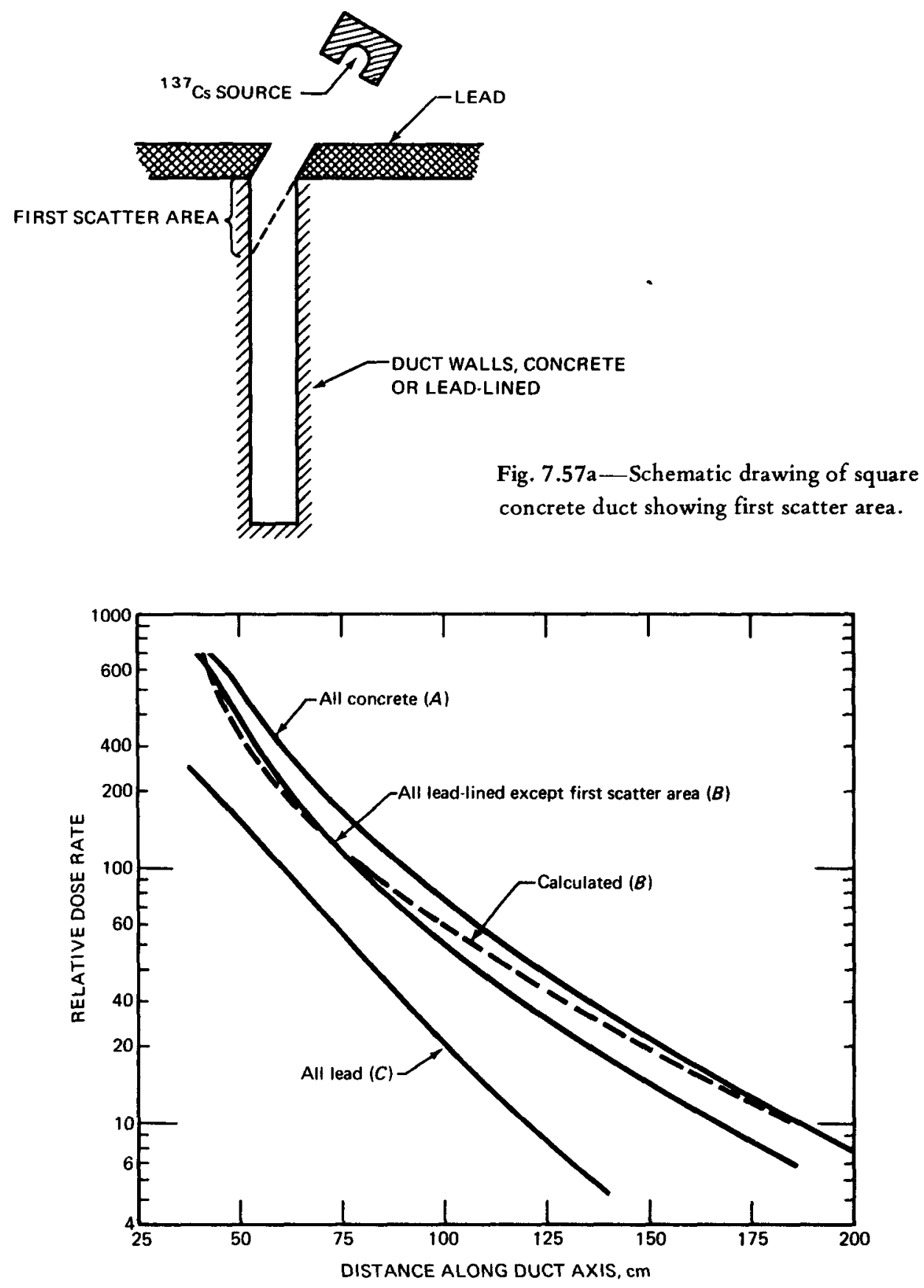

Fig. 7.57b-Measurements and single-reflection analysis of gamma-ray dose rates along the axis of a $20-\mathrm{cm}$-square concrete duct with and without lead linings; ${ }^{137} \mathrm{Cs}$ source. (From Clifford. ${ }^{52}$ ) 


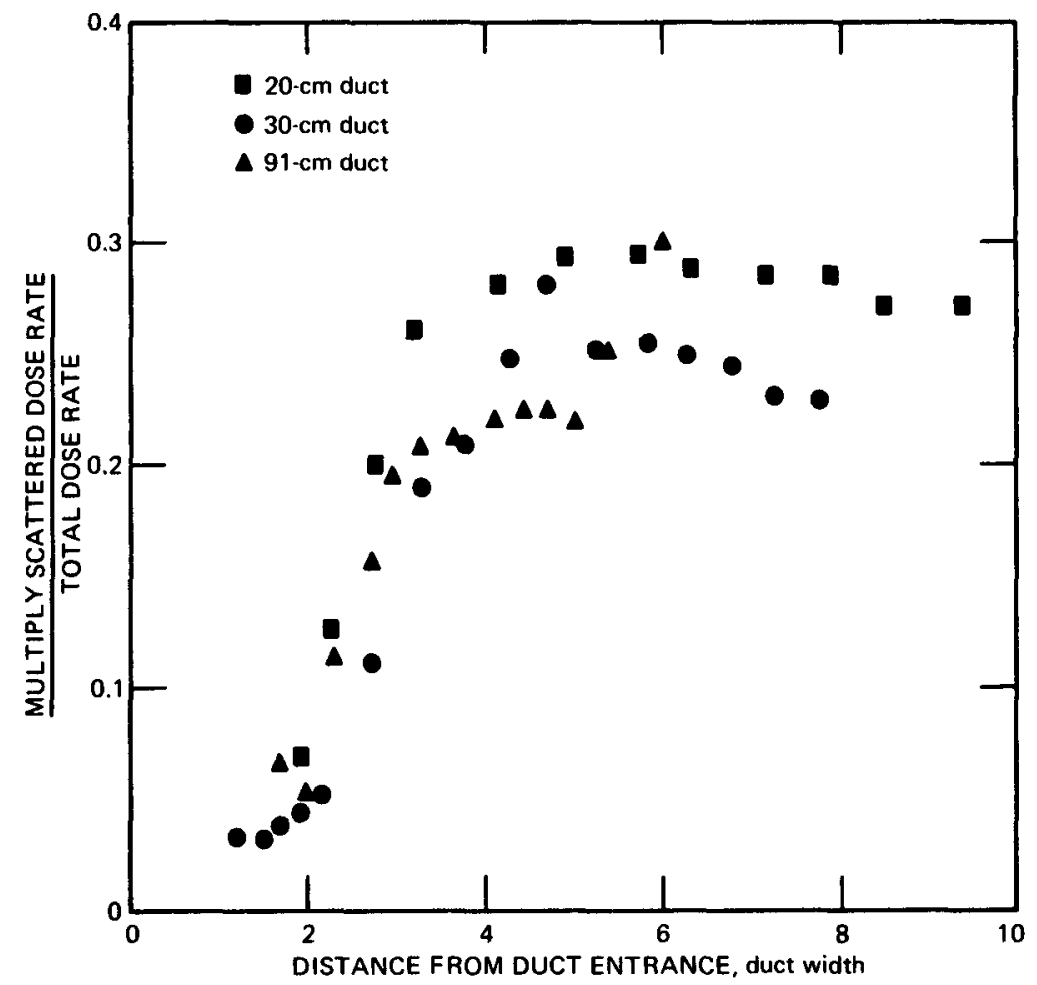

Fig. 7.58 - Ratio of multiply scattered gamma-ray dose rate of total dose rate along the axes of 20-, 30-, and 91-cm-square concrete ducts; ${ }^{137} \mathrm{Cs}$ source. (From Clifford..$^{52}$ )

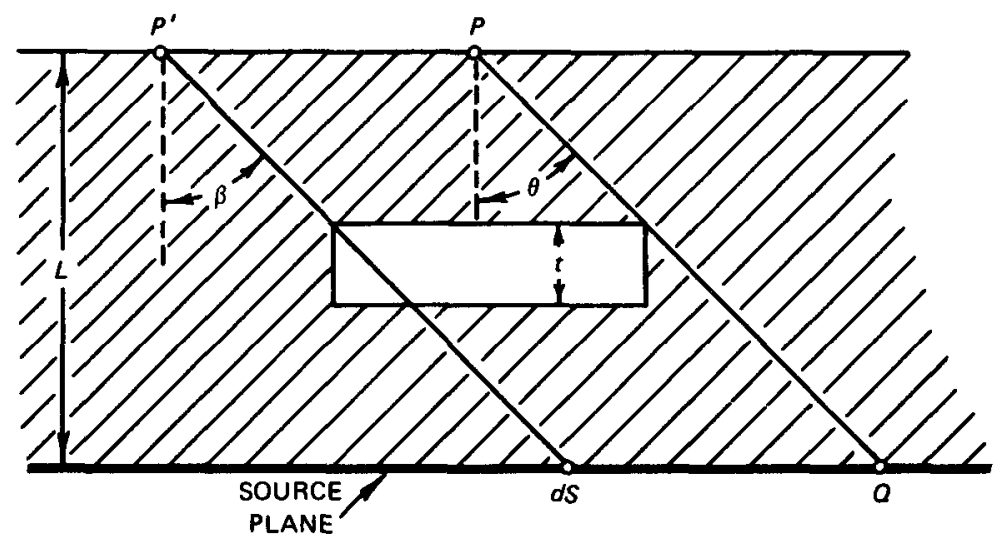

Fig. 7.59-Geometry for calculating the effect of a disk-shaped void on radiation transmission through a shield. 
density with the void present. For the latter, the point of interest is the intersection of the center line of the void with the shield surface. The influence of the void is estimated by performing a point-kernel integration over the source plane to obtain $\Phi(L)$ and $\Phi(L-t)$. To calculate the flux density for the plane shield of thickness $L$, define $b_{1}=\sum \mu_{i} t_{i}$ over $L$ normally through the shield. The contribution of a ring-shaped source with a particle-emission rate of $N_{0}$ at an angle $\theta$ from the detector would then be

$$
d \Phi=\frac{N_{0}}{2} \frac{d r}{r} e^{-b_{1} \sec \theta}
$$

Integrating over the plane source to an angle $\theta$ gives

$$
\Phi(\theta)=\frac{N_{0}}{2} \int_{b_{1}}^{b_{1} \sec \theta} \frac{e^{-t}}{t} d t
$$

where $t=b_{1} \sec \theta$. Since by definition

$$
E_{1}(x)=\int_{x}^{\infty} \frac{e^{-t}}{t} d t
$$

then

$$
\Phi(\theta)=\frac{N_{0}}{2}\left[E_{1}\left(b_{1}\right)-E_{1}\left(b_{1} \sec \theta\right)\right]
$$

integrating over the entire source plane gives

$$
\Phi(L)=\frac{N_{0}}{2} E_{1}\left(b_{1}\right)
$$

If the gamma-ray number buildup through $b_{1}$ is $B_{1}$, then $\Phi(L)$ may be estimated by

$$
\Phi(L)=\frac{N_{0} B_{1}}{2} E_{1}\left(b_{1}\right)
$$

The buildup factor used here as a simple multiplier is only symbolic since the buildup factor is really part of the kernel that is integrated over all space. 
As is pointed out in Chap. 4, when the Taylor form of the buildup factor is used, the form of these equations is unchanged, and the buildup effect is simply included by modifying the arguments of the exponential functions. Actually in these problems it is sufficiently accurate to use the equation as it stands and to determine $B_{1}$ only on the basis of the slant penetration $(Q$ to $P$ ) that grazes the void. For $B_{2}$, an average of $B_{1}$ and of the buildup factor based on the minimum path length normal to the void is usually adequate. Use of the arithmetic mean will always produce a conservative result since this gives equal weight to the buildup factor from a region of lesser importance.

To calculate the flux density for a shield in which a disk-shaped void intercepts at an angle $\theta$ from the point $P$, define $b_{2}=\Sigma \mu_{i} t_{i}$ over $(L-t)$. Then for angles less than $\theta$

$$
\Phi_{1}=\frac{N_{0} B_{2}}{2}\left[E_{1}\left(b_{2}\right)-E_{1}\left(b_{2} \sec \theta\right)\right]
$$

and for angles greater than $\theta$

$$
\Phi_{2}=\frac{N_{0} B_{1}}{2} E_{1}\left(b_{1} \sec \theta\right)
$$

Thus the total flux density is

$$
\begin{aligned}
\Phi(L-t)=\Phi_{1}+\Phi_{2}=\frac{N_{0} B_{1}}{2} E_{1}\left(b_{1} \sec \theta\right) \\
\\
+\frac{N_{0} B_{2}}{2}\left[E_{1}\left(b_{2}\right)-E_{1}\left(b_{2} \sec \theta\right)\right]
\end{aligned}
$$

and the difference $\Phi(L-t)-\Phi(L)$ is the increase in the flux density at point $P$ due to the presence of the void. Since $P$ is centered over the void, it will be the point of maximum increase.

For points offset from the center, for example, for point $P^{\prime}$ in Fig. 7.59, Eq.7.11-9 will not be valid. If the source is isotropic, the increase in the contribution at $P^{\prime}$ by a source surface increment $d S$ caused by the void is given by

$$
\Phi_{i}=\frac{N_{0} d S}{2 \pi(L \sec \beta)^{2}}\left[B_{3} e^{-\mu(L-t) \sec \beta}-B_{4} e^{-\mu L \sec \beta}\right]
$$


where $B_{3}$ and $B_{4}$ are the buildup factors along the path $d S \rightarrow P^{\prime}$ with and without the void, respectively. If the source is a cosine source, the increase is given by

$$
\Phi_{c}=\frac{N_{0} d S \cos \beta}{\pi(L \sec \beta)^{2}}\left[e^{-\mu L \sec \beta}-e^{-\mu(L-t) \sec \beta}\right]
$$

The integral of Eq. $7.11-10$ or 7.11-11 over the appropriate source area will give the total increase at $P^{\prime}$ due to the presence of the void. The appropriate source area includes all points for which a ray $P^{\prime}$ passes through the void. In most practical situations this integral must be evaluated numerically. The value of the integral at representative points will serve to determine the shape and thickness of the shield patch required to negate the effect of the void.

An approximate form of ray analysis given by Tonks ${ }^{67}$ can be applied to certain problems involving voids or depressions in a very thick shield. With this method we first calculate the flux density at a point on the surface of a uniform infinite-slab shield whose thickness is equal to the ray of minimum length through a void or depression in the shield. We then account for the reduction in flux density due to the longer material path of nearby rays terminating at the same point. The method is based on the fact that the formula for the flux density through an infinite slab

$$
\Phi=\frac{N_{0}}{2} E_{1}(\mu t)
$$

can be approximated by

$$
\Phi \cong \frac{N_{0}}{2 \mu t} e^{-\mu t}
$$

for $\mu t \gg 1$, with an error that is approximately equal to $1 / \mu t$.

The application of Tonks' approximation is as follows: Consider a slab shield with a void such as that shown in Fig. 7.60. Let the ray passing through $Q$ and $P$ be the path of minimum material penetration, $t_{0}$, and let it coincide with the $z$-axis. In many cases nearby rays may be expressed as

$$
t \cong t_{0}+\frac{1}{2} a \theta_{x}^{2}+\frac{1}{2} b \theta_{y}^{2}
$$




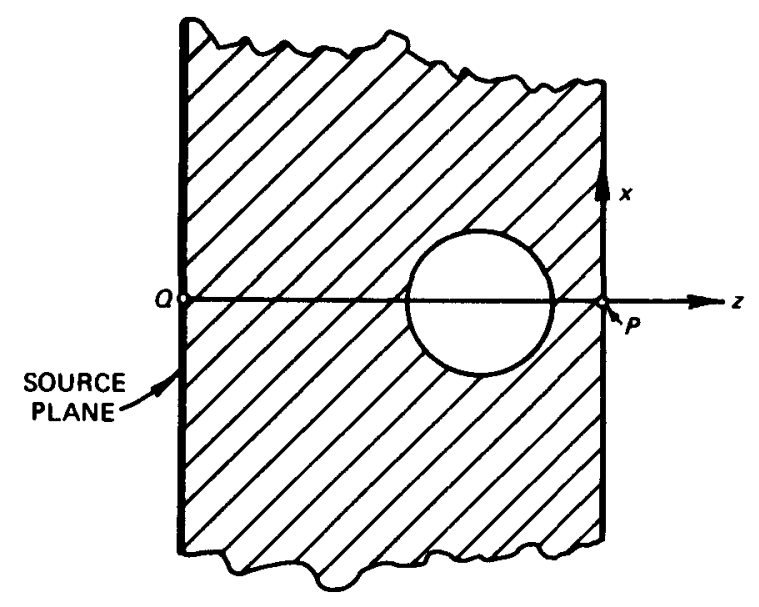

Fig. 7.60-Geometry for calculating by Tonks' approximation the effect of a void in a shield.

where $\theta_{x}$ and $\theta_{y}$ are the angles between the minimum ray and a nearby ray projected onto the $x-y$ and $y-z$ planes, respectively, and $a$ and $b$ are determined by the specific geometry. Substituting Eq. 7.11-14 into an approximation analogous to Eq. 7.11-13 yields for the flux density $\Phi^{\prime}$ at $P$

$$
\Phi^{\prime}=N_{0} \frac{e^{-\mu t_{0}}}{\mu \sqrt{ } a b}
$$

if both $a$ and $b$ are greater than zero. The ratio of the flux density estimated by Eq. 7.11-15 to that estimated by Eq. 7.11-13 (for a slab of minimum penetration) is

$$
\frac{\Phi^{\prime}}{\Phi}=\frac{t_{0}}{\sqrt{a b}}
$$

which, provided $\sqrt{a b}>1$, gives the increase in effectiveness at $P$ of the shield with a void over that of a simple slab whose thickness is equivalent to the minimum ray.

The limit of $\theta_{x}$ and $\theta_{y}$ for which Tonks' approximation is valid is set by $\theta_{x}-\theta_{y}<\alpha$, where $\alpha$ is given by $\alpha=\sqrt{2 / \mu t_{0}}$. As an example, for a spherical void of radius $R_{v}$ whose center lies at a depth $R$ from the outside surface of a slab shield of thickness $T$, it can be shown that the length of a ray near the minimum ray is given by 


$$
t=t_{0}+\left(\frac{T}{2}+\frac{R^{2}}{R_{v}}\right) \theta_{x}^{2}+\frac{t_{0}}{2} \theta_{y}^{2}
$$

and the increased effectiveness at the minimum ray terminus is given by

$$
\frac{\Phi^{\prime}}{\Phi}=\frac{t_{0}}{\left[\left(\frac{T}{2}+\frac{R^{2}}{R_{v}}\right) \frac{t_{0}}{2}\right]^{1 / 2}}
$$

Other expansions of $t=t_{0}+f\left(\theta_{x}, \theta_{y}\right)$ may be derived which would lead to equations of similar form to Eq. 7.11-15. Kouts ${ }^{68}$ treated the problem of

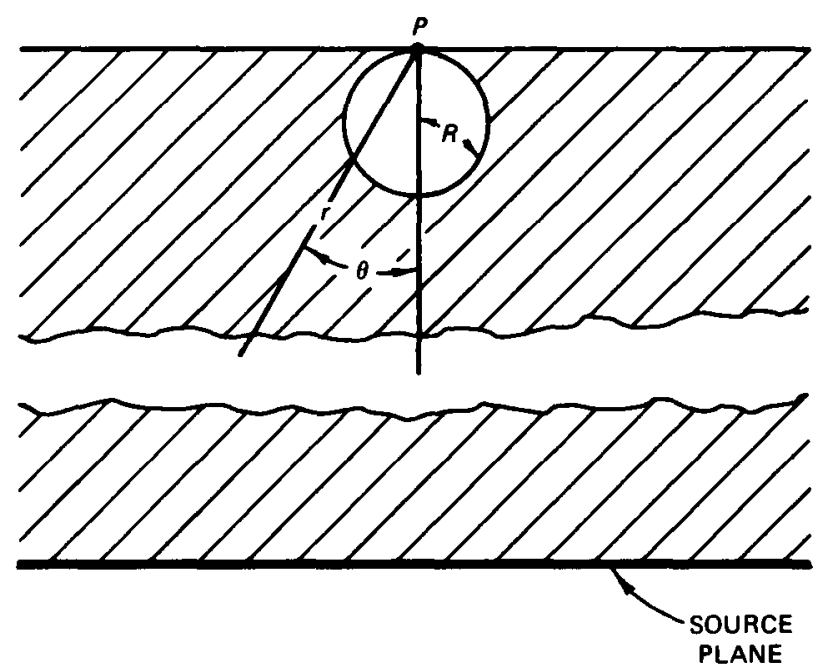

Fig. 7.61-Geometry for calculating by Kouts' technique the effect of a void in a shield.

voids on the basis of one-velocity transport theory. Instead of using the boundary conditions of the differential transport equation to describe the void, he accounted for it by the way in which the kernel of the integral equation is defined. The effect of the void is then calculated as a perturbation of the flux density in a homogeneous medium (no-void case). The method is applicable to either gamma rays or neutrons.

Using this approximation to the transport equation, Kouts investigated the effect of spherical voids in water and obtained good agreement with experiment when he chose certain constants in the solution by fitting to the experimental data at one point. The expression derived for a spherical void in the geometry shown in Fig. 7.61 has the form 


$$
\Phi(P)=\frac{B V}{2} \int_{0}^{1} d \mu \mu^{k} \exp \left(\frac{2 R \mu^{2}}{L}\right)
$$

where $\Phi(P)=$ flux density at point $P$

$B=$ constant in the approximation to the angular distribution of the scattered flux used by Kouts, i.e.,

$$
\begin{aligned}
d \Omega \Phi(\Omega) \Sigma_{t} p\left(\Omega^{\prime} \rightarrow \Omega\right) & =B \cos \theta \quad\left(0<\theta \leqslant \frac{\pi}{2}\right) \\
& =0\left(\frac{\pi}{2}<\theta<\pi\right)
\end{aligned}
$$

$\Sigma_{t}=$ total macroscopic cross section

$P\left(\Omega^{\prime} \rightarrow \Omega\right)=$ probability of scattering from direction $\Omega^{\prime}$ to direction $\Omega$

$V=$ parameter in the expression for flux distribution through the shield in the absence of voids given by $\Phi_{0}=V \Phi(\Omega) e^{-3 / b}$

$R=$ radius of void

$L=$ shield thickness

$b=$ number of relaxation lengths in the shield material

$\mu=\cos \theta$

A best value of $k=4.5$ was chosen by Kouts by fitting experimental data for gamma rays at $2 R / L=1$.

Results obtained with this equation for a water shield are compared in Fig. 7.62 with experimental data for gamma rays.

\subsubsection{Small Random Voids}

For a large shield containing small randomly spaced voids (small diameter relative to the relaxation length of the radiation, e.g., a gamma-ray shield consisting entirely of iron shot), the effect of the voids on the radiation transmission is obtained simply by increasing the relaxation length used in the calculation of the shield attenuation by the ratio of the average density of the shield to the density of the solid material used in the shield.

When the voids are not negligibly small, however, it can be shown by a statistical argument that the penetrating radiation will be greater than would be predicted by using an average density. Consider two normal rays through the shield, one that is the average penetration $t$ plus an increment $\delta$ and one 
that is shorter than average by the same increment. Their average penetration is

$$
\frac{1}{2}\left[e^{-\mu(t+\delta)}+e^{-\mu(t-\delta)}\right]=e^{-\mu t} \cosh \delta \mu>e^{-\mu t}
$$

Thus, although their average penetration distance is $t$, their average penetration is greater than $e^{-\mu t}$.

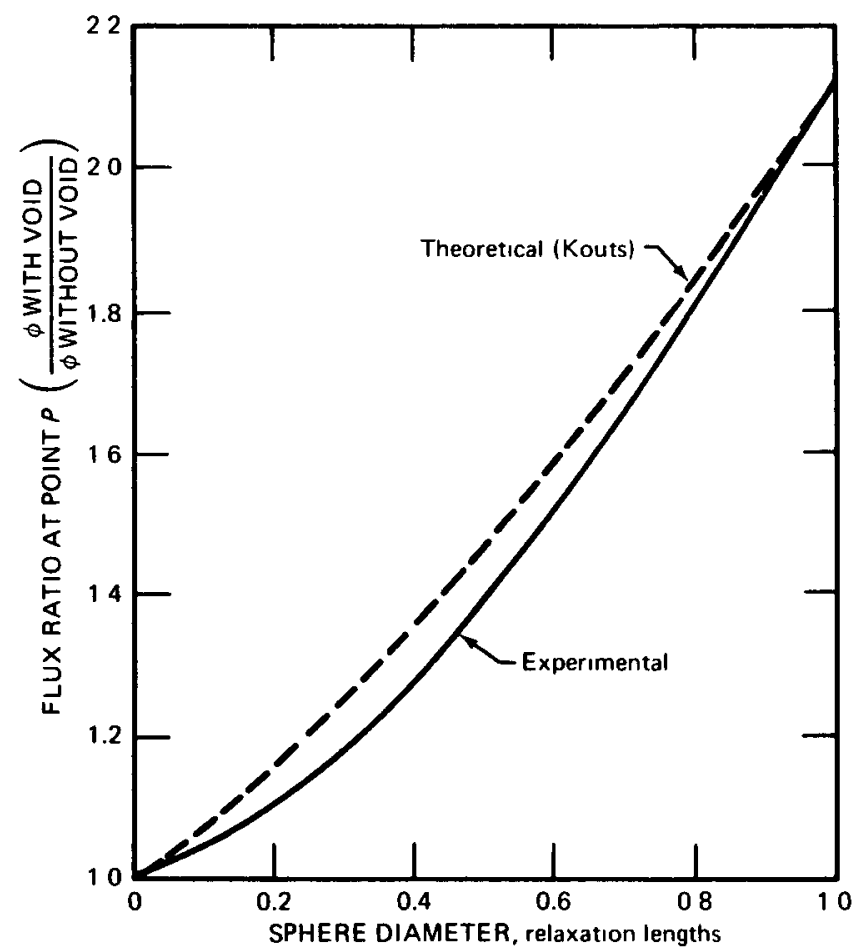

Fig. 7.62 - Comparison of flux increase due to spherical void in a water shield calculated by Kouts' technique with that determined by experiment. (From Kouts. ${ }^{68}$ )

This problem has been treated statistically by Smith. ${ }^{69}$ From his work an effective thickness, $t_{e}$, can be derived:

$$
t_{e}=\frac{t(1-v)}{1+0.55 s v^{2}}
$$

where $t$ is the actual thickness, $v$ is the fraction of the volume that is void, and $s$ is the average distance between voids measured in relaxation lengths. 
An equivalent expression for an effective attenuation coefficient is

$$
\mu_{e}=\frac{\mu(1--\nu)}{1+0.55(s / \mu) \nu^{2}}
$$

or, generalized to the case where the voids contain a material of attenuation coefficient $\mu^{\prime}$,

$$
\mu_{e}=\frac{\mu^{\prime}+(1-v)\left(\mu-\mu^{\prime}\right)}{1+0.55(s / \mu)\left(\mu-\mu^{\prime}\right) v^{2}}
$$

\section{REFERENCES}

1. R. E. Maerker and F. J. Muckenthaler, Calculation and Measurement of the Fast-Neutron Differential Dose Albedo for Concrete, Nucl. Sci. Eng., 22: 455 (1965).

2. F. J. Allen, A. Futterer, and W. Wright, Neutron Reflection and Flux Versus Depth for Nevada Test Site Soil, Report BRL-1190, January 1963; . . for Concrete, Report BRL-1189, January 1963; ... for Iron, Report BRL-1199, March 1963; Neutron Reflection and Flux Versus Depth for Water, with an Appendix: Comparison with Results of National Bureau of Standards, Report BRL-1204, June 1963; Neutron Reflection and Flux Versus Depth for Aluminum, with an Appendix: Neutron Dose Transmission Versus Thickness, Report BRL-1238, February 1964, Ballistic Research Laboratories.

3. F. J. Allen, A. Futterer, and W. Wright, Dependence of Neutron Albedos upon Hydrogen Content of a Shield, Report BRL-1224, Ballistic Research Laboratories, October 1963.

4. R. L. French and M. B. Wells, An Angular Dependent Albedo for Fast-Neutron Reflection Calculations, USAEC Report RRA-M31, Radiation Research Associates, Inc., November 1963.

5. Y. T. Song, Fast Neutron Streaming Through Two-Legged Concrete Ducts, Report AD-457746 (TR-354), Naval Civil Engineering Laboratory, Feb. 2, 1965.

6. A. B. Chilton and C. M. Huddleston, A Semiempirical Formula for Differential Dose Albedo for Gamma Rays on Concrete, USAEC file number NP-12455 (TR-228), Naval Civil Engineering Laboratory, Nov. 16, 1962; also Nucl. Sci. Eng., 17: 419 (1963).

7. R. L. Henry, L. G. Mooney, and R. J. Prevost, Study of Radiation Penetration and Reflection from Shield Materials, Report FZK-183, General Dynamics Corporation, August 1964.

8. W. A. Coleman, R. E. Maerker, F. J. Muckenthaler, and P. N. Stevens, Calculation of Doubly Differential Current Albedos for Epicadmium Neutrons Incident on Concrete and Comparison of the Reflected Subcadmium Component with Experiment, Nucl. Sci. Eng., 27: 411 (1967). 
9. E. Fermi, On the Motion of Neutrons in Hydrogenous Substances, USAEC file number NP-2385 [translated from Ric. Sci., VII(2): 13 (1936)].

10. O. Halpern, R. Lueneburg, and O. Clark, On Multiple Scattering of Neutrons. 1. Theory of the Albedo of a Plane Boundary, Phys. Rev., 53: 173 (1938).

11. S. Glasstone and M. C. Edlund, The Elements of Nuclear Reactor Theory, D. Van Nostrand Co., Inc., New York, 1952.

12. S. Chandrasekhar, Radiative Transfer, Clarendon Press, Oxford, England, 1950.

13. L. V. Spencer, J. A. Diaz, and E. Moses, Neutron Penetration in Cylindrical Ducts, Report NBS-8542, National Bureau of Standards, Sept. 1, 1964.

14. A. Mockel, Reflection and Transmission by a Strongly Absorbing Slab, Nucl. Sci. Eng., 22: 339 (1965).

15. G. C. Pomraning, The Albedo Problem, Nucl. Sci. Eng., 21 : 265 (1965).

16. M. B. Wells, Reflection of Thermal Neutrons and Neutron-Capture Gamma Rays from Concrete, USAEC Report RRA-M44, Radiation Research Associates, Inc., June 1964.

17. M. B. Wells, Gamma Dose Rates Resulting from Neutron Capture in Air and Concrete, USAEC Report NARF-59-31T, Convair, September 1959.

18. R. E. Maerker and F. J. Muckenthaler, Measurements and Single-Velocity Calculations of Differential Angular Thermal-Neutron Albedos for Concrete, Nucl. Sci. Eng., 26: 339 (1966).

19. H. Greenspan and I. G. Baksys, Addenda to Newsletters Nos. 3 and 4, Argonne National Laboratory Newsletter No. 9, USAEC Report TID-18481, March 1963.

20. M. Leimdorfer, The Backscattering of Gamma Radiation from Plane Concrete Walls, Swedish Report AE-92, December 1962.

21. M. B. Wells, Differential Dose Albedos for Calculation of Gamma-Ray Reflection from Concrete, USAEC Report RRA-T46, Radiation Research Associates, Inc., December 1964.

22. M. J. Berger and J. Doggett, Reflection and Transmission of Gamma Radiation by Barriers: Semianalytic Monte Carlo Calculations, J. Res. Nat. Bur. Stand., 56: 89 (1956).

23. M. J. Berger and D. J. Raso, Monte Carlo Calculations of Gamma-Ray Backscattering, Radiat. Res., 12: 20 (1960).

24. M. J. Berger and D. J. Raso, Backscattering of Gamma Rays, Radiat. Res., 12(1): 20-37 (1960).

25. D. J. Raso, Monte Carlo Calculations on the Reflection and Transmission of Scattered Gamma Rays, Nucl. Sci. Eng., 17: 411 (1963).

26. M. B. Wells, Air and Concrete Scattering of Gamma Rays, USAEC Report NARF-59-11T (MR-N-229), Convair, March 1959.

27. A. B. Chilton, C. M. Davisson, and L. A. Beach, Parameters for C-H Albedo Formula for Gamma Rays Reflected from Water, Concrete, Iron, and Lead, Trans. Amer. Nucl. Soc., 8: 656 (1965).

28. C. M. Davisson and L. A. Beach, A Monte Carlo Study of Backscattered Gamma Radiation, Trans. Amer. Nucl. Soc., 5: 391 (1962).

29. A. B. Chilton, A Modified Formula for Differential Exposure Albedo for Gamma Rays Reflected from Concrete, Nucl. Sci. Eng., 27: 481 (1967). 
30. C. E. Clifford, Differential Dose Albedo Measurements for $0.66 \mathrm{MeV} \gamma$ 's Incident on Concrete, Iron and Lead, Canadian Report DRCL-412, August 1963.

31. L. G. Haggmark, T. H. Jones, N. E. Scofield, and W. J. Gurney, Differential Dose-Rate Measurements of Backscattered Gamma Rays from Concrete, Aluminum and Steel, Nucl. Sci. Eng., 23: 138 (1965).

32. M. J. Barrett and J. Waldman, Experimental Gamma-Ray Backscattering by Various Materials, Report TO-B-64-68, Technical Operations Research, July 1964.

33. M. J. Berger and E. E. Morris, Dose Albedo and Transmission Coefficients for Cobalt-60 and Cesium-137 Gamma Rays Incident on Concrete Slabs, Report NBS-9071, National Bureau of Standards, July 5, 1966.

34. M. Leimdörfer, Backscattering of Gamma Radiation from Spherical Concrete Walls. Swedish Report AE-93, January 1963.

35. R. L. French, M. B. Wells, and N. M. Schaeffer, Penetration of Neutron and Gamma Radiation Through the Openings of Underground Structures, USAEC Report RRA-T41, Radiation Research Associates, Inc., Nov. 30, 1964; also Neutron and Gamma Radiation Penetration into Concrete-Shielded Underground Structures, USAEC Report RRA-T51, Radiation Research Associates, Inc., May 1965.

36. J. H. Hubbell, R. R. Bach, and J. C. Lamkin, Radiation Field from a Rectangular Source, J. Res. Nat. Bur. Stand., C 64: 121 (1960).

37. R. E. Maerker, H. C. Claiborne, and C. E. Clifford, Neutron Attenuation in Rectangular Ducts, in Engineering Compendium on Radiation Shielding, R. G. Jaeger (Ed.), Vol. 1, Springer-Verlag, New York, 1968.

38. B. T. Price, C. C. Horton, and K. T. Spinney, Effects of Ducts and Voids in Shields, in Radiation Shielding, Sec. 4.12, p. 202, Pergamon Press, Inc., New York, 1957.

39. D. M. Chase, Ducts and Voids in Shields, in Reactor Handbook, Vol. III, Part B, Shielding, E. P. Blizard (Ed.), Chap. 12, Interscience Publishers, a division of John Wiley \& Sons, Inc., New York, 1962.

40. D. K. Trubey, A Calculation of Radiation Penetration of Cylindrical Duct Walls, USAEC Report CF-63-2-64, Oak Ridge National Laboratory, Feb. 28, 1963.

41. R. E. Benenson and A. N. Fasano, The Transmission of Fission Neutrons Having Energy Above the $\mathrm{S}^{32}(\mathrm{n}, \mathrm{p}) \mathrm{P}^{32}$ Threshold by Straight Cylindrical Ducts in Water, Report WADC-TR-57-89, Wright Air Development Center, February 1957.

42. D. C. Piercey and D. E. Bendall, The Transmission of Fast Neutrons Along Air Filled Ducts in Water, British Report AEEW-R-69, June 1962.

43. D. G. Collins and L. W. McCleary, A Systemization and Penetration Study for Straight Cylindrical Ducts, Report NARF-63-3T, General Dynamics, May 1963.

44. J. D. Marshall, Analysis of Radiation Streaming Through Single-Bend Ducts, Report AD-613480 (WL-TR-64-136), Air Force Weapons Laboratory, January 1965.

45. A. Simon and C. E. Clifford, The Attenuation of Neutrons by Air Ducts in Shields, Nucl. Sci. Eng., 1: 156-166 (1956).

46. J. C. LeDoux and A. B. Chilton, Attenuation of Gamma Radiation Through Two-Legged Rectangular Ducts and Shelter Entranceways-An Analytical Approach, Report AD-264022 (TN-383), Naval Civil Engineering Laboratory, January 1961.

47. V. R. Cain, Calculations of Thermal-Neutron Flux Distributions in Concrete-Walled Ducts Using an Albedo Model with Monte Carlo Techniques, USAEC Report ORNL-3507, Oak Ridge National Laboratory, January 1964. 
48. R. L. French, A First/Last Collision Model of the Air/Ground Interface Effects on Fast-Neutron Distributions, Nucl. Sci. Eng., 19: 151 (1964).

49. R. Artigas and H. E. Hungerford, A Numerical Treatment of the Attenuation of Neutrons by Air Ducts in Shields, Nucl. Sci. Eng., 36: 295 (1969).

50. C. C. Horton, The Shielding of Helical Ducts, Nucl. Sci. Eng., 6: 525-529 (1959).

51. C. C. Horton and D. B. Halliday, The Attenuation of Thermal Neutrons in Cylindrical Ducts Through a Water Shield, British Report AERE-SWP/P-28, January 1956.

52. C. E. Clifford, Gamma Shielding Provided by Ducts, Canadian Report DRCL-370, May 1962.

53. W. C. Ingold, Some Applications of a Semiempirical Formula for Differential Dose Albedo for Gamma Rays on Concrete, Report AD-291133 (TN-469), Naval Civil Engineering Laboratory, November 1962.

54. J. M. Chapman, Computer Calculation of Dose Rates in Two-Legged Ducts Using the Albedo Concept, USAEC file number NP-13652 (TR-264), Naval Civil Engineering Laboratory, Oct. 24, 1963.

55. C. W. Terrell, A. J. Jerri, and R. O. Lyday, Jr., Radiation Streaming in Ducts and Shelter Entranceways, Report ARF-1158-A02-7, Armour Research Foundation, April 1962.

56. C. W. Terrell, A. J. Jerri, R. O. Lyday, and D. Sperber, Radiation Streaming in Shelter Entranceways, Report ARF-1158-12, Armour Research Foundation, October 1960.

57. C. W. Terrell and A. J. Jerri, Radiation Streaming in Shelter Entranceways, Final Report, Report ARF-1158-A01-5, Armour Research Foundation, July 1961.

58.W. C. Ingold and C. M. Huddleston, An Empirical Formula for Calculating Gamma-Ray Dose Attenuation in Concrete Ducts, Report AD-609049 (TR-349), Naval Civil Engineering Laboratory, November 1964.

59. D. R. Doty, Dose Measurements for Neutron Streaming in Ducts, USAEC file number NP-13805 (AD-436183; TR-282), Naval Civil Engineering Laboratory, March 1964.

60. R. E. Maerker and V. R. Cain, AMC: A Monte Carlo Code Utilizing the Albedo Approach for Calculating Neutron and Capture Gamma-Ray Distributions in Rectangular Concrete Ducts, USAEC Report ORNL-3964, Oak Ridge National Laboratory, August 1967.

61. R. E. Maerker and F. J. Muckenthaler, Calculation of the Fast-Neutron Dose Rate Along the Center Line of Both a Straight and a Two-Legged Square Concrete Duct Using the Albedo Concept and Comparison with Experiment, Trans. Amer. Nucl. Soc., 9: 147 (1966).

62. R. E. Maerker and F. J. Muckenthaler, Monte Carlo Calculations Using the Albedo Concept of Fast-Neutron Dose Rates Along the Center Lines of One- and Two-Legged Square Concrete Open Ducts and Comparison with Experiment, USAEC Report ORNL-TM-1557, Oak Ridge National Laboratory, September 1966.

63. W. S. Snyder and J. Neufeld, Calculated Depth Dose Curves in Tissue for Broad Beams of Fast Neutrons, USAEC Report ORNL-1872, Oak Ridge National Laboratory, Apr. 13, 1955.

64. W. E. Selph, J. Newell, and C. E. Bayer, Albedo Monte Carlo Analysis of Fast Neutron Transport in a Missile Launch Tube, Trans. Amer. Nucl. Soc., 11: 1 (June 1968). 
65. J. M. Chapman, Gamma-Ray Dose Rates and Energy Spectra in a 3-Foot Square Duct, Report AD-450421 (TR-325), Naval Civil Engineering Laboratory, October 1964.

66. J. M. Chapman and J. S. Grant, Gamma Ray Attenuation in Coplanar and Noncoplanar Three-Legged Concrete Ducts, Report AD-453022 (TN-658), Naval Civil Engineering Laboratory, November 1964.

67. L. Tonks, Enhancement of Leakage by Internal Voids in a Shield, USAEC Report KAPL-107, Knolls Atomic Power Laboratory, January 1949.

68. H. J. Kouts, Theory of Flux Perturbations by Voids in Shields, USAEC Report BNL-1357, Brookhaven National Laboratory, Sept. 15, 1952.

69. N. M. Smith, Jr., The Absorption and Scattering of Radiation in Random Aggregates of Pebbles, USAEC Report CNL-21, Oak Ridge National Laboratory, March 1948. 


\section{Shield Heating, Air Transport, Shield Materials, and Shield Optimization}

L. G. MOONEY and N. M. SCHAEFFER

8

Four additional design considerations-shield heating, air transport, shield materials, and shield optimization-should be added to the special topics needed to supplement the transport calculations in the design of a shield. The grouping of the four topics is a matter of convenience and has no special significance.

\subsection{SHIELD HEATING}

Heat transfer may be said to dictate the design of a reactor. Materials selection is based on a compromise between thermal and nuclear properties. Within the core the kinetic energy of the fission fragments is the principal heat source. However, the design of the reactor structure, core vessel, and thermal and biological shield is determined by the energy deposition of radiation emerging from the core. Although significant heating rates may be generated by neutrons in the reflector, control rods, and inner shield regions, most of the heating in the shield results from energy degradation and absorption of gamma rays. Generally speaking, neutrons are considered in shield heating only insofar as they produce gamma rays. Energy deposited by charged particles is a consideration only in a limited number of materials.

Methods for calculating energy deposition are identical to those for obtaining attenuation. Thus all the techniques and data described in Chaps. 4 through 6 are pertinent here. The principal difference between attenuation and heating-rate analyses is the quantity of interest. For an attenuation calculation, we are primarily interested in the quantity transmitted. In shield heating we are interested in the quantity absorbed. This distinction makes the shield-heating calculation less demanding. The first orders of attenuation are obviously most important to heating rates; thus approximate methods can be used to better advantage for shield heating than for radiation transmission. 
We should not oversell the acceptability of approximate results, however. An underestimate of a biological dose rate by a factor of 2 is a nuisance: working times are more limited or exclusion areas must be enlarged. Consequences of a similar error in heating-rate estimates in the shield could be severe; excessive temperature rises could wreck the reactor structure at design power levels. A prudent design approach would therefore be to estimate the heating rates two ways: one that is known to overpredict and one that is known to underpredict.

It is evident that a calculation of heating rates is simply another response function of the flux density. If one preserves his flux-density data from the shield analysis, then the heating rate can be obtained simply by converting the flux density to energy deposition.

\subsubsection{Gamma-Ray Heating $\dagger$}

The principal interactions of a gamma ray are pair production, photoelectric absorption, and Compton scattering. In Chap. 3 the Compton energy absorption cross section, $\sigma_{c a}$, was defined on the basis of the average energy loss per collision (Eq. 3.2-14). Since the other two processes involve complete absorption, $\sigma_{c a}$ is used with them to define a macroscopic cross section (Eq. 3.2-16, $\mu_{a}=N\left[\sigma_{p e}+Z \sigma_{c a}+\sigma_{p p}\right]$ ) that represents local energy deposition. The photon-energy deposition gives rise to a heating rate

$$
H(\mathbf{r})=C \int_{E_{\min }}^{E_{\max }} E \mu_{a}(E, \mathbf{r}) \Phi(E, \mathbf{r}) d E
$$

where $H(\mathbf{r})=$ heat generation rate at spatial position $\mathbf{r}\left(\right.$ watts $\left.\mathrm{cm}^{-3}\right)$

$E=$ gamma-ray energy $(\mathrm{MeV})$

$\Phi(\mathrm{E}, \mathrm{r})=$ photon-flux density at energy $E$ (photons $\mathrm{cm}^{-2} \mathrm{sec}^{-1} \mathrm{MeV}^{-1}$ )

$\mu_{a}=$ energy-absorption coefficient or macroscopic cross section for energy deposition $\left(\mathrm{cm}^{-1}\right)$

$C=$ conversion factor $\left(1.60 \times 10^{-13}\right.$ watt-sec $\left.\mathrm{MeV}^{-1}\right)$

We should emphasize that $\Phi(E, \mathbf{r})$ includes the contributions from secondary as well as from primary gamma rays.

Implicit in the use of the energy-absorption coefficient as defined here is the assumption that some other possible second-order processes are negligible and that the small amount of radiation that survives the photoelectric effect and pair production through fluorescence, bremsstrahlung, or annihilation radiation is absorbed where it is produced. Such

†This section follows a recent review by Claiborne.' 
assumptions are justified for reactor design because of the low photon energies involved in these secondary processes.

Calculation of the heating rate due to gamma rays is then reduced to obtaining the spectrum $\Phi(E, r)$, the subject of Chaps. 4 through 6 . We will discuss buildup factors and mention some other methods in general use for calculating heating rates.

(a) Buildup Factors. Because they are manageable with hand calculations as well as with machine application, buildup factors represent the most popular technique, and they have been validated by comparison with experiment. Perhaps the most valuable feature of the buildup-factor method is the relative certainty with which the sign of the error in the calculated heating is known. Even for extreme multilayered construction (assuming that other sources of error are reasonably small), it is possible to bracket the true answer by separate calculations using the largest and the smallest individual buildup factors for the effective buildup factor of the composite shield.

Recalling that buildup factors can be used as a type of point kernel, we measure the path length along the line of sight from the source to the receiver. The uncollided flux density at the receiver (the point of energy deposition) is then multiplied by the buildup factor to account for the scattered contribution. If the source is distributed (i.e., a reactor core), we represent it as an array of volume elements, each represented by a point source, and integrate the point kernel over the source volume for each source energy. The integral over volume is then

$$
H(\mathbf{r})=E_{0} \mu_{a}\left(E_{0}\right) \int_{V} \frac{S_{v}\left(\mathbf{r}^{\prime}\right) B_{a}(\mu x) e^{-\mu x}}{4 \pi x^{2}} d V
$$

where $H(\mathbf{r})=$ energy deposition per unit volume $\left(\mathrm{MeV} \mathrm{sec} \mathrm{scm}^{-1}\right)$

$S_{v}\left(\mathbf{r}^{\prime}\right)=$ isotropic source intensity in photons per unit time per unit volume at energy $E_{0}\left(\mathrm{sec}^{-1} \mathrm{~cm}^{-3}\right)$

$V=$ source volume

$E_{0}=$ energy of gamma ray emitted by source

$\mu_{a}=$ energy-absorption coefficient of material at the point of energy deposition

$B_{a}(\mu x)=$ energy-deposition buildup factor

$\mu=$ total attenuation coefficient at energy $E_{0}$ 
$x=\left|\mathbf{r}-\mathbf{r}^{\prime}\right|$, the distance between the source and the point of energy deposition

$r=$ point of energy deposition

$\mathbf{r}^{\prime}=$ location of source in volume $V$

We note that $B_{a}(\mu x)$ is an energy-deposition (formerly called energyabsorption) buildup factor and not the energy-fluence buildup factor. The latter would give the total energy flux, which would result in an overestimate of the heating rate.

All we need to obtain heating rates for photons is a proper set of buildup factors. For infinite homogeneous media we can use the energy-deposition buildup factors given by Goldstein and Wilkins. ${ }^{2}$ For finite media and, more realistically, for the multilayered media to be found in any reactor shield, we need other buildup factors or an additional assumption. The pitfalls encountered in applying infinite-media data to finite geometries, described briefly in the discussion of differential energy spectra in Sec. 6.3.1, apply here as well.

The infinite-media data (either buildup factors or differential energy spectra) contain the effect of the medium beyond the receiver point as well as that between the source and the receiver. Therefore a receiver at the far edge of a slab will intercept an intensity differing from the infinite-medium case depending on the difference in backscattering from the adjacent medium. If the adjoining medium is air, the infinite-medium buildup factor produces an overestimate. If it is another dense medium, the infinite-medium buildup factor will give more or less, depending on the relative albedo of the two media. Since the albedo depends on the ratio of scattering to absorption cross sections, a greater ratio in the adjoining medium would imply that the infinite-medium buildup factor gives an underestimate of the actual intensity at the receiver. A lower ratio would imply an overestimate. The saving grace is that the magnitude of the error is usually of the order of a few percent for photons, reaching 20 to $30 \%$ only in unusual cases.

Assuming that errors of this magnitude are acceptable, we can use infinite-medium buildup factors. Equation 8.1-2 must be modified to accept this compromise. Making the further assumption that the source energy distribution is divided into groups and that $\bar{E}_{j}$ is the mean energy for the $j$ th group, we can write 


$$
H(\mathbf{r})=\bar{E}_{j} \mu_{a}\left(\bar{E}_{j}\right) \int_{V} \frac{S_{v}\left(\mathbf{r}^{\prime}\right) B_{a}\left(\sum_{i} \mu_{i} t_{i}\right) \exp \left(-\sum_{i} \mu_{i} t_{i}\right) d V}{4 \pi x^{2}}
$$

where $t$ is the thickness of each region and $i$ refers to the number of regions of different composition interposed in the line-of-sight path $x$ between a source point and any particular point of energy deposition. The sums over $i$ imply that we set the path length equal to the cumulative sum of mean free paths in each material to the point at which the heating rate is to be obtained.

The buildup factor can be selected according to several rules of thumb. For homogeneous or near-homogeneous mixtures (regions significantly smaller than the mean free path), the so-called equivalent $Z$ (atomic number) method $^{3}$ has been used with good effect. In this method the equivalent atomic number is obtained by a recipe with a sound theoretical basis. Briefly, this method involves comparing the shape of the curve obtained by plotting the total attenuation coefficient per electron of the mixture as a function of energy to the corresponding curves for the individual elements until a reasonable match is found for energies below source energy. To be completely equivalent, the ratio of total to scattering cross section should be the same in the two cases, which usually occurs to a good approximation when the shapes are matched.

Extension of the buildup-factor method to structures with multiple layers leans more heavily on rules of thumb and physical intuition. When the outermost layer exceeds two or three mean free paths, the buildup factor of the outermost material is generally recommended, but based on the total number of mean free paths along the line of sight through all materials. This seems a reasonable procedure since the gamma spectrum would readjust to the new medium and tend to approach the spectrum that would exist if the whole structure consisted of the outermost material.

In the most extreme case where the structure is multilayered and each layer is less than two mean free paths thick, no clear-cut procedure is available. Some Monte Carlo calculations have been made on energy deposition in multilayered shields, but correlations for design use have generally been lacking. Based on Monte Carlo studies of heat deposition in one- and two-layer lead slabs in water by a plane monodirectional beam, Bowman and Trubey ${ }^{4}$ suggested that combinations of the Goldstein-Wilkins energy-deposition buildup factors could be used in the relation representing 
the buildup factor for stratified slabs. This effective buildup factor is expressed by

$$
\begin{aligned}
B_{a}\left(\mu_{1} t_{1}, \mu_{2} t_{2}\right)= & B_{a_{1}}\left(\mu_{1} t_{1} \sec \theta, E_{0}\right) B_{a_{2}}\left(\mu_{2} t_{2} \sec \theta, E_{0}\right) \\
& \times \exp \left(-\mu_{2} t_{2} \sec \theta\right)+B_{a_{2}}\left[\left(\mu_{1} t_{1}\right.\right. \\
& \left.\left.+\mu_{2} t_{2}\right) \sec \theta, E_{0}\right]\left[1-\exp \left(-\mu_{2} t_{2} \sec \theta\right)\right]
\end{aligned}
$$

where $\theta$ is the angle between the direction of the incident gamma ray and the normal to the slab. Subscripts 1 and 2 refer to the first and second materials, respectively.

For a double-layer shield whose outer layer is less than two or three mean free paths or a multilayer shield with the exterior layer more than two or three mean free paths in thickness, use of Eq. 8.1-4 seems intuitively justified for most purposes. The angular dependence $(\theta$ now becomes the angle that each path makes with the normal to the shield) does complicate the integral when Eq. 8.1-4 is substituted into Eq. 8.1-3; however, the numerical integration presents no problem when electronic computers are used, and in some cases it would not be too arduous for hand calculations.

For conditions that are significantly different from the preceding cases, some other method should be used if it is desired to do other than bracket the answer by using techniques that will give results known to be high or low. For instance, in a multilayered shield with all layers less than two mean free paths, a high result is obtained by using the largest individual buildup factor as the effective buildup factor for the composite shield, and a low result is obtained when the smallest individual buildup factor is used.

Constants for the energy-deposition buildup factors in the Berger form are given in Appendix $F$ for water, aluminum iron, tin, and lead. Tobias, Vondy, and Lietzke ${ }^{5}$ have interpolated the results of Goldstein and Wilkins to obtain energy-deposition buildup factors for even atomic numbers from 2 to 78 and have obtained coefficients for best fit to the third-degree polynomial

$$
B\left(\mu x, E_{\mathbf{0}}\right)=1+a(\mu x)+b(\mu x)^{2}+c(\mu x)^{3}
$$

Values of $a, b$, and $c$ for seven energies from 0.5 to $10 \mathrm{MeV}$ are given in Appendix F, Table F.4, and can be used for materials not explicitly given elsewhere. Because they are regarded as most accurate (Sec. 4.8.1), the Capo $^{6}$ polynomial expressions with regions of applicability are given in 
Appendix F, Table F.5. This set includes the same materials as Appendix F, Table F.3.

Walker and Grotenhuis ${ }^{7}$ have examined the effective atomic numbers of concretes and found them to range from 11 for ordinary concrete to 27 for a barytes aggregate. Buildup factors for a particular concrete can be obtained by interpolating available data to the effective atomic number. Trubey ${ }^{8}$ published coefficients for the Berger form of the buildup factor for ordinary, magnetite, and barytes concretes as well as for sand, wood, air, and lithium hydride. These are reproduced in Appendix F, Table F.6.

The buildup-factor method has been widely used and is a versatile tool for shield design. Calculations with this method have generally checked experimental measurements to within $35 \%$. It is reasonable to expect similar accuracy when this method is carefully used in design calculations for shields or reactor structures that do not involve extreme multilayered construction with each region less than two mean free paths in thickness. Even for multilayered construction, however, when the outermost layer is more than two or three mean free paths in thickness, particularly when the low- $Z$ material follows the high- $Z$ material, the method should be quite good since the spectrum tends to readjust to the new medium.

(b) Other Methods for Gamma-Ray Heating. Every method devised for gamma-ray attenuation has been applied to obtain heating rates. Greenhow, Mueller, and Sabian ${ }^{9}$ devised a recipe with some success for applying any neutron multigroup diffusion code to gamma-ray heating by proper exchange of cross sections. French ${ }^{10}$ applied differential energy spectra from the Goldstein-Wilkins moments results to carry the gamma-ray spectral description through multilayered shields. This gives additional detail to the results, but it is essentially no more accurate and is considerably more involved than the buildup-factor techniques based on the same moments data.

Lathrop ${ }^{11}$ has modified a standard LASL one-dimensional $S_{n}$ code (DTF) to include up to 10 moments $\left(P_{10}\right)$ of the group-to-group scattering cross sections. A subroutine cross-section code produces scattering cross sections by performing group averages of the Legendre moments of the Klein-Nishina differential cross section. At the option of the user, cross-section data can be flux weighted, source weighted, or unweighted. Test calculations with this code generally compare favorably with Monte Carlo calculations. Engle ${ }^{12}$ also developed a one-dimensional $S_{n}-P_{l}$ code (ANISN) which in principle can handle any number of moments of the 
group cross sections; a two-dimensional version (DOT) was developed by Mynatt, Muckenthaler, and Stevens. ${ }^{13}$

Trubey, Penny, and Lathrop ${ }^{14}$ compared the results of an $\mathrm{S}_{16}-P_{3}$ calculation with Monte Carlo and buildup-factor methods (the latter is equivalent to the moments method for the homogeneous medium) for a sphere of iron $70 \mathrm{~cm}$ in radius containing a unit gamma-ray fission source distributed uniformly within the inner $25-\mathrm{cm}$ radius. The Monte Carlo results were obtained with the FMC code. ${ }^{15}$ No importance sampling was used, and a total of 5000 case histories was calculated. When the buildup-factor method was applied, the usual point kernel was integrated over source and energy with each buildup factor obtained by a Lagrangian interpolation of the buildup factors in the paper by Goldstein and Wilkins. ${ }^{2}$ As shown in Fig. 8.1, the agreement between the three methods is excellent; in fact, it is rather surprising that the agreement is good all the way to the edge. The Monte Carlo results are typical in that the statistics get bad as the distance from the source increases, and the net result is that the flux is usually underestimated. At the origin the poor statistics resulted because the scoring volume was too small. Toward the edge the decrease in the $S_{n}$ results with respect to the moments method most probably is a boundary effect. The $S_{n}$ calculation assumed a vacuum beyond $70 \mathrm{~cm}$; use of buildup factors implies an infinite region.

For deep penetrations of the order involved in shielding calculations, it had been generally felt that a $P_{3}$ approximation was insufficient to account for the anisotropy of gamma scattering. In some preliminary studies by Lathrop, ${ }^{11}$ however, the difference in results between the $P_{0}$ and $P_{1}$ approximation was quite pronounced, but only small improvement resulted from the use of higher order approximations in place of the $P_{1}$ approximation.

For multilayered shields it is apparent that Monte Carlo or discreteordinate methods are the best available, and they should be used where accuracy requirements exceed the capability of buildup factors.

\subsubsection{Neutron Heating}

Direct heating effects are potentially important for elastic scattering of fast neutrons in low- $Z$ materials. For equal numbers of $1-\mathrm{MeV}$ neutrons and gamma rays, the contribution of elastic neutron scattering to the heating rate varies widely with the atomic number of the medium. Foderaro, Hoover, and Marable $^{16}$ show that the ratio of neutron to gamma-ray heating is 5 to 1 in 


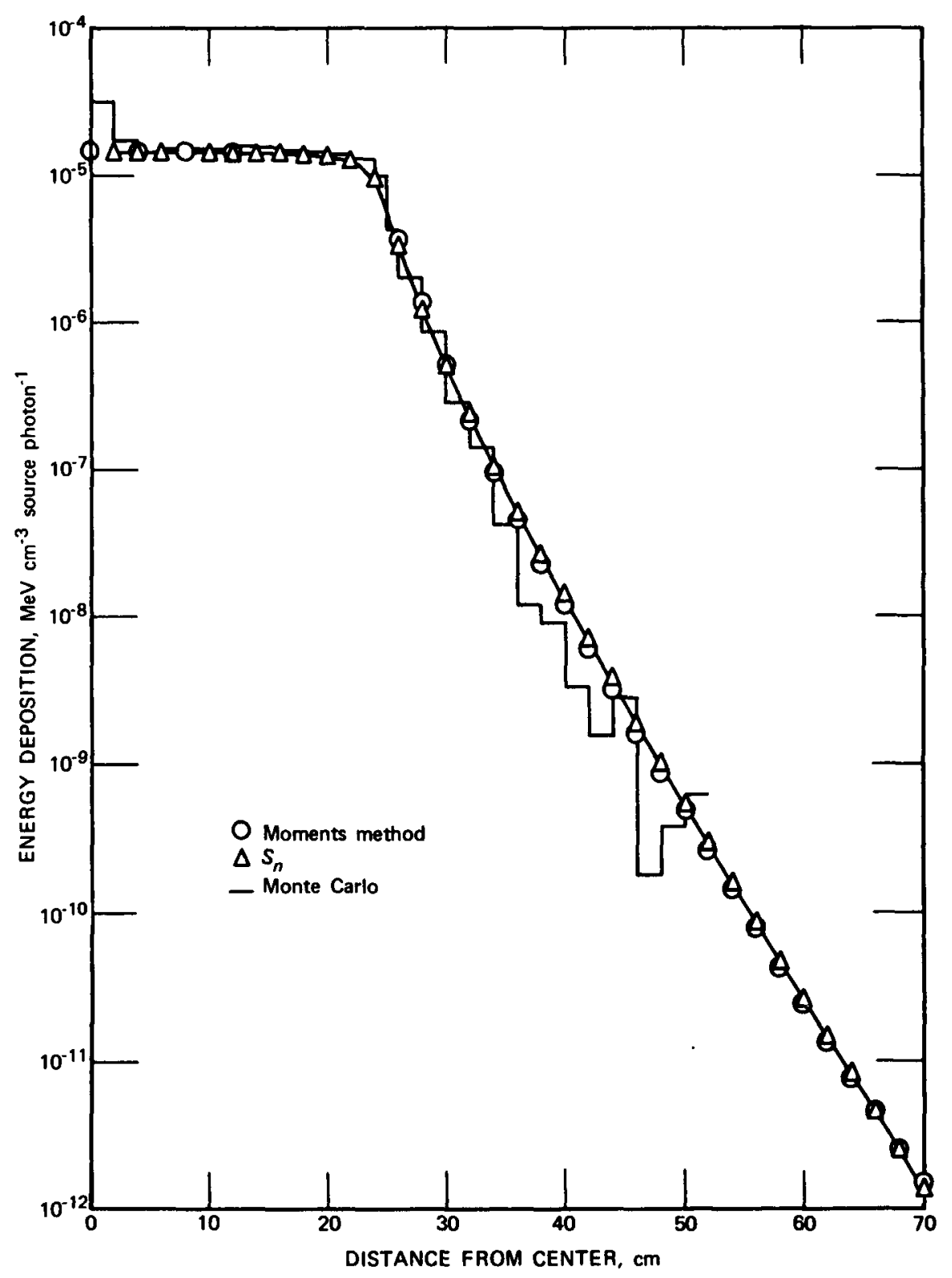

Fig. 8.1-Comparison of three methods for calculating heating in iron from 25-cm spherical source. (From Trubey et. al. ${ }^{14}$ ) 
water, 6 to 10 in carbon, and 1 to 50 in iron. We can show this by developing the neutron analog to Eq. 8.1-3. From Sec. 3.2.2, a neutron of energy $E$ initially and energy $E^{\prime}$ after an elastic collision is

$$
E^{\prime}=E\left[\frac{A^{2}+2 A \cos \theta+1}{(A+1)^{2}}\right]
$$

where $A$ is the mass number of the target nucleus and $\theta$ is the scattering angle (in center-of-mass coordinates). The energy loss is

$$
\Delta E(E, \theta)=E-E^{\prime}=E\left(\frac{2 A}{(A+1)^{2}}\right)(1-\cos \theta)
$$

We need the average energy loss. If the average scattering angle is given by $\overline{\cos \theta}$,

$$
\overline{\Delta E(E)}=E\left(\frac{2 A}{(A+1)^{2}}\right)(1-\overline{\cos \theta})
$$

We can now write the heating rate caused by elastic scattering in a material with $N_{i}$ atoms $/ \mathrm{cm}^{3}$

$$
H_{n, n}=K N_{i} \int_{E} \overline{\Delta E(E)} \sigma_{i}(E) \Phi(E) d E
$$

where $H_{n, n}=$ heating rate (watts $\mathrm{cm}^{-3}$ )

$K=$ conversion constant $\left(K=1.6 \times 10^{-13}\right.$ watt-sec $\left.\mathrm{MeV}^{-1}\right)$

$\sigma_{i}(E)=$ elastic-scattering cross section of the $i$ th material

$\Phi=$ flux density of neutrons in $d E$ about $E$

$E=$ incident neutron energy

Foderaro et al. ${ }^{16}$ use the orthogonality property of Legendre polynomials to show that the average value of the cosine of the scattering angle is

$$
\overline{\cos \theta}=f_{1 i}(E)
$$

where $f_{1} i(E)$ is the first expansion coefficient of the Legendre polynomial expansion of the differential elastic-scattering cross sections of the $i$ th 
element. Since these cross sections are commonly expressed in terms of Legendre polynomial expansions, this is a very convenient result. Values of $f_{1}(E)$ for selected materials are quoted by Foderaro et al. and are given in most compilations of differential elastic-scattering distributions. The average energy loss is then

$$
\overline{\Delta E(E)}=E \frac{2 A_{i}}{\left(A_{i}+1\right)^{2}}\left[1-f_{1 j}(E)\right]
$$

The significant term is $A_{i} /\left(A_{i}+1\right)^{2}$. It tells us that neutron heating decreases rapidly with the mass of the target nucleus and supports the conclusion that fast-neutron heating in heavy materials is unimportant in the presence of comparable numbers of gamma rays. For $A<12$, both neutrons and gamma rays tend to contribute significantly.

A corollary to these conclusions relates to the importance of inelastic scattering of fast neutrons. Since inelastic-scattering cross sections in the energy range of interest $(E<6 \mathrm{MeV})$ are significant only in heavy nuclei, inelastic neutron scattering is usually unimportant in heat-generation computations. Even where inelastic scattering is significant, it is generally less than elastic scattering. Thus where elastic scattering is unimportant for heating considerations so is inelastic scattering.

Indirect neutron heating through production and absorption of gamma rays is significant where there is appreciable radiative capture and conceivably where there is appreciable gamma-ray production by inelastic events.

\subsubsection{Charged-Particle Heating}

Three nuclei account for most of our interest in neutron-induced charged-particle heating, ${ }^{6} \mathrm{Li},{ }^{10} \mathrm{~B}$, and ${ }^{14} \mathrm{~N}$. Because the cross sections tend to be in the millibarn range in the $\mathrm{MeV}$ region, charged-particle-out reactions are not important in heating except at thermal-neutron energies. Table 8.1 summarizes the reactions of interest. ${ }^{17}$

Note that three isotopes of uranium are included in Table 8.1. The large energy release per fission would make these isotopes important heat generators if they were used in a shield.

For any of these reactions, say $(n, \alpha)$, the heating rate due to neutrons in energy interval $\Delta E$ is simply

$$
H_{n, \alpha}=K N \sigma_{n, \alpha}(E) \Phi(E)(E+Q) \Delta E
$$


where $K=$ same conversion constant given before $\left(1.6 \times 10^{-13}\right.$ watt-sec $\mathrm{MeV}^{-1}$ )

$N=$ number of atoms of target material per cubic centimeter

$\sigma_{n, \alpha}(E)=(n, \alpha)$ cross section at energy $E$

$\Phi(E)=$ neutron-flux density at neutron energy $E$

$Q=$ energy release for the reaction

Table 8.1 —CHARGED-PARTICLE REACTIONS IMPORTANT IN HEATING $†$

\begin{tabular}{llcc}
\hline Nuclide & Reaction & $\begin{array}{c}\text { Thermal-neutron } \\
\text { cross section, b }\end{array}$ & $\begin{array}{c}\text { Energy emitted, } \\
\mathrm{MeV}\end{array}$ \\
\hline${ }^{6} \mathrm{Li}$ & $(n, \alpha)$ & 950 & 4.8 \\
${ }^{10} \mathrm{~B}$ & $(n, \alpha)$ & 3840 & $2.3,0.48$ \\
${ }^{14} \mathrm{~N}$ & $(n, \rho)$ & 1.8 & 0.63 \\
$238 \mathrm{U}$ & Fast fission & $0.58(3 \mathrm{MeV})$ & $\begin{array}{c}\text { Average total energy } \\
\text { from fission, }\end{array}$ \\
${ }^{235} \mathrm{U}$ & Thermal fission & 582 & $\sim 190 \mathrm{MeV}$ \\
${ }^{233} \mathrm{U}$ & Thermal fission & 527 & \\
\hline
\end{tabular}

†From C. N. Klahr, Reactor Handbook, Second Edition, Vol. III, Part B, Shielding, E. P. Blizard (Ed.), p. 174, Interscience Publishers, a division of John Wiley \& Sons, Inc., New York, 1962.

\subsection{AIR TRANSPORT}

Although transport of neutrons and gamma rays through air is not usually an important consideration in the design of shields for fixed power plants, it is an important shielding consideration for any portable or mobile system. Laboratory experiments involving reactors, isotopes, or accelerators also frequently require some assessment of air transport. Actually two programs have accounted for most of the research in air transport that has been done: nuclear-powered aircraft and weapons radiation studies. Interest from the weapons standpoint is obvious; all the initial radiation from a nuclear weapon detonated within the atmosphere arrives at a distant receiver via the atmosphere. In analysis of exposure to fallout radiation, the transport through air from airborne or ground-deposited activity is the primary concern. The reasons for interest in air transport from the standpoint of nuclear aircraft are also evident; weight limitations give additional incentive to tight design, and transport of radiation by air and structure from reactor shield to passengers and crew becomes a crucial design factor. 
The processes involved in air transport are identical to those in dense media. The difference that makes air a separate topic is entirely in the geometry. Relaxation lengths in air are of the order of a hundred meters as compared with relaxation lengths in dense materials of the order of a tenth of a meter. Put in a more familiar way, the density of air at standard conditions is $1.29 \mathrm{mg} \mathrm{cm}^{-3}$; the density of most solids is in the range 1 to $10 \mathrm{~g} \mathrm{~cm}^{-3}$.

The geometry of interest includes the ground and the cover of air extending out to large distances in space. The importance of the boundary effects at the air-ground interface depends on the ratio $a / h$, the sourcedetector separation divided by the height of source or detector above the ground. For $a / h \geqslant 1$, the presence of the ground significantly affects the amount of scattered radiation arriving at the detector. For $a / h<1$, the relative importance of the ground effect depends on the height of the source and the detector. If the height of either the source or the detector is less than a mean free path in air, the ground effect is likely to be significant.

Consider the case where both the detector and the source are located in the upper regions of the atmosphere. Here we have what can be described as an infinite medium within which neutron and gamma-ray mean free paths are extremely long and within which the density of material varies significantly over distances of interest. The density variation must be included if the accuracy of the air-transport calculation is to be acceptable. As the source and the detector are lowered in the atmosphere toward the earth's surface, the density increases, and the density gradient must be considered until the gradient becomes unimportant over several mean-freepath distances. Air-transport calculations in the lower atmosphere at heights from 3 to 10 mean free paths or so above the ground surface are very similar to infinite-medium calculations for other materials of uniform density.

As the source and the detector are lowered further toward the ground surface, the effects of the ground become noticeable, and the air-ground interface effects become most important when both the source and the detector are on the surface. If either the source or the detector is raised above the surface, the interface effects diminish.

The radiation transport in an air-over-ground geometry is generally calculated to obtain the radiation distribution in the air. For such problems the ground is considered as a perturbing medium. The perturbation consists in absorption and reflection of the incident radiations as well as the contribution of secondary gamma rays produced by neutron interactions in the ground. Only for special problems, such as ground activation and heating 
rates, is there a need for neutron and gamma-ray distributions within the ground proper.

\subsubsection{Infinite Air Medium}

As mentioned in the preceding text, the transport of neutrons and gamma rays in an infinite air medium can be calculated in much the same manner as an infinite medium of any other material provided the density is uniform. In fact, probably more infinite-medium calculations have been performed for air than for any other material.

Air transport has been calculated with moments, discrete-ordinates, and Monte Carlo methods in recent years. The advantages of the Monte Carlo and discrete-ordinate methods in providing angular distributions at the receiver have resulted in their use almost exclusively. Early definitive calculations of air scattering of gamma rays were done by Lynch, Benoit, Johnson, and Zerby ${ }^{18}$ using the Monte Carlo method. Similar calculations for neutrons were reported by Wells ${ }^{19}$ and Zerby. ${ }^{20}$

Trubey ${ }^{21}$ showed that for source-detector separation distances less than about $30 \mathrm{~m}$ a single-scattering calculation gives an excellent approximation of the intensity at a detector but does not give an adequate representation of the energy or angular distributions at the receivers.

Figure 8.2 illustrates recent comparative calculations of the neutron dose as a function of distance from a point isotropic $14-\mathrm{MeV}$ neutron source by Straker and Gritzner, ${ }^{22}$ Keith and Shelton, ${ }^{23}$ and Webster. ${ }^{24}$ Straker's results were obtained by discrete ordinates, and the remainder, by Monte Carlo. Straker's data are lower in magnitude because of his use of different cross-section data in the high-energy region. Webster and Keith and Shelton used identical cross-section data, and the difference in their calculations at larger distances is probably due to poor statistics in the Monte Carlo output. The abscissa and ordinate of this graph deserve mention. By plotting the dose per source neutron times $4 \pi R^{2}$ ( $R$ the source-receiver separation) as the ordinate, we can remove the inverse-square geometric effect. Using slant range in density units $\left(\mathrm{g} \mathrm{cm}^{-2}\right)$ for the abscissa, we can avoid dependence on the air density. This graph is then applicable to any uniform air density, and the separation (or slant range) in centimeters is obtained by dividing the slant range in $\mathrm{g} / \mathrm{cm}^{2}$ by the air density in $\mathrm{g} / \mathrm{cm}^{3}$. For convenience, Table 8.2 gives the conversion to meters for sea-level air density.

Figure 8.3 shows similar data from Straker and Webster for a point isotropic fission-neutron source. The differences noted in the 14-MeV source 


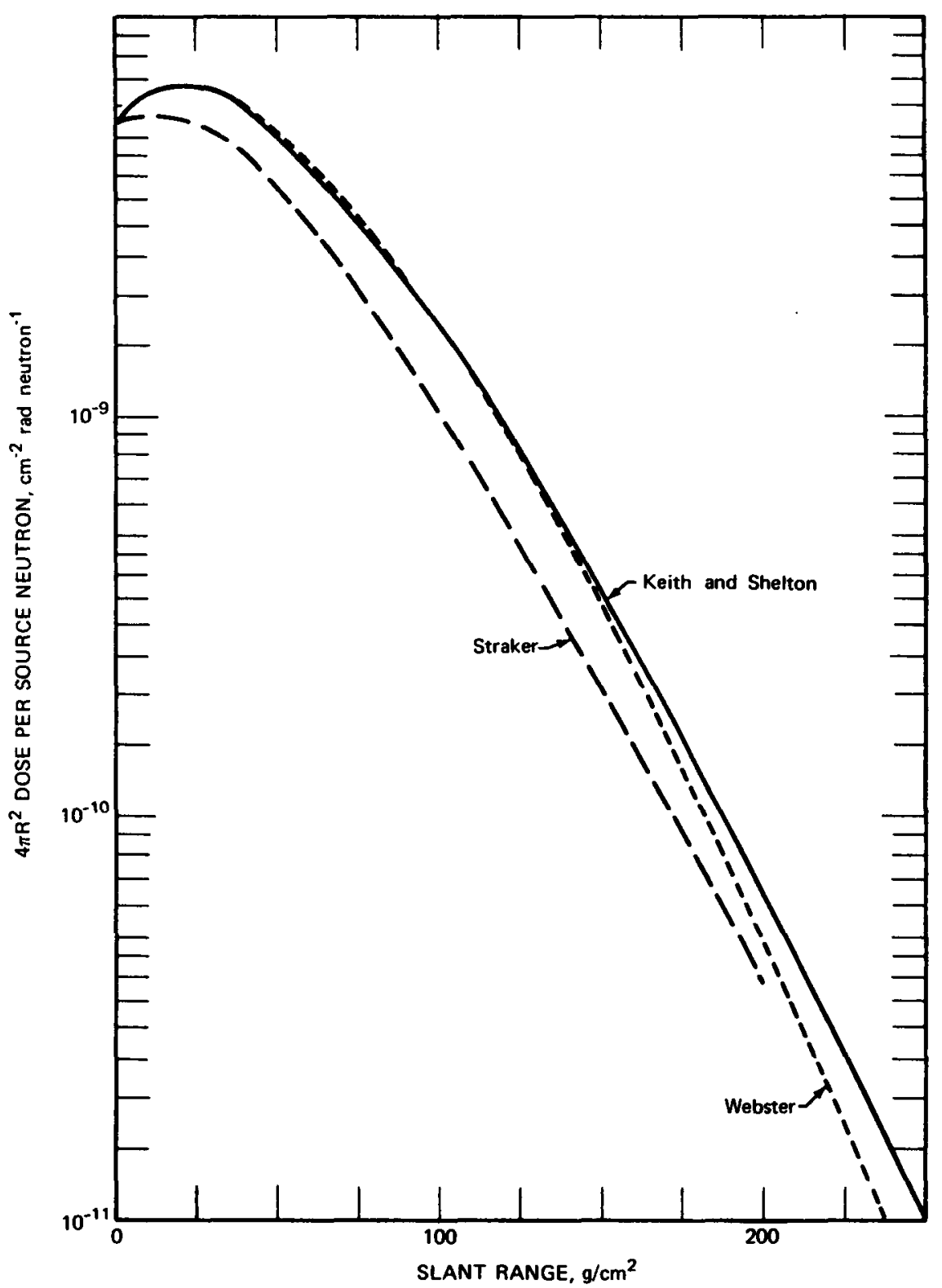

Fig. 8.2-Comparison of the neutron dose in infinite air from a 14-MeV neutron source calculated by Straker, ${ }^{22}$ by Keith and Shelton, ${ }^{23}$ and by Webster. ${ }^{24}$ 


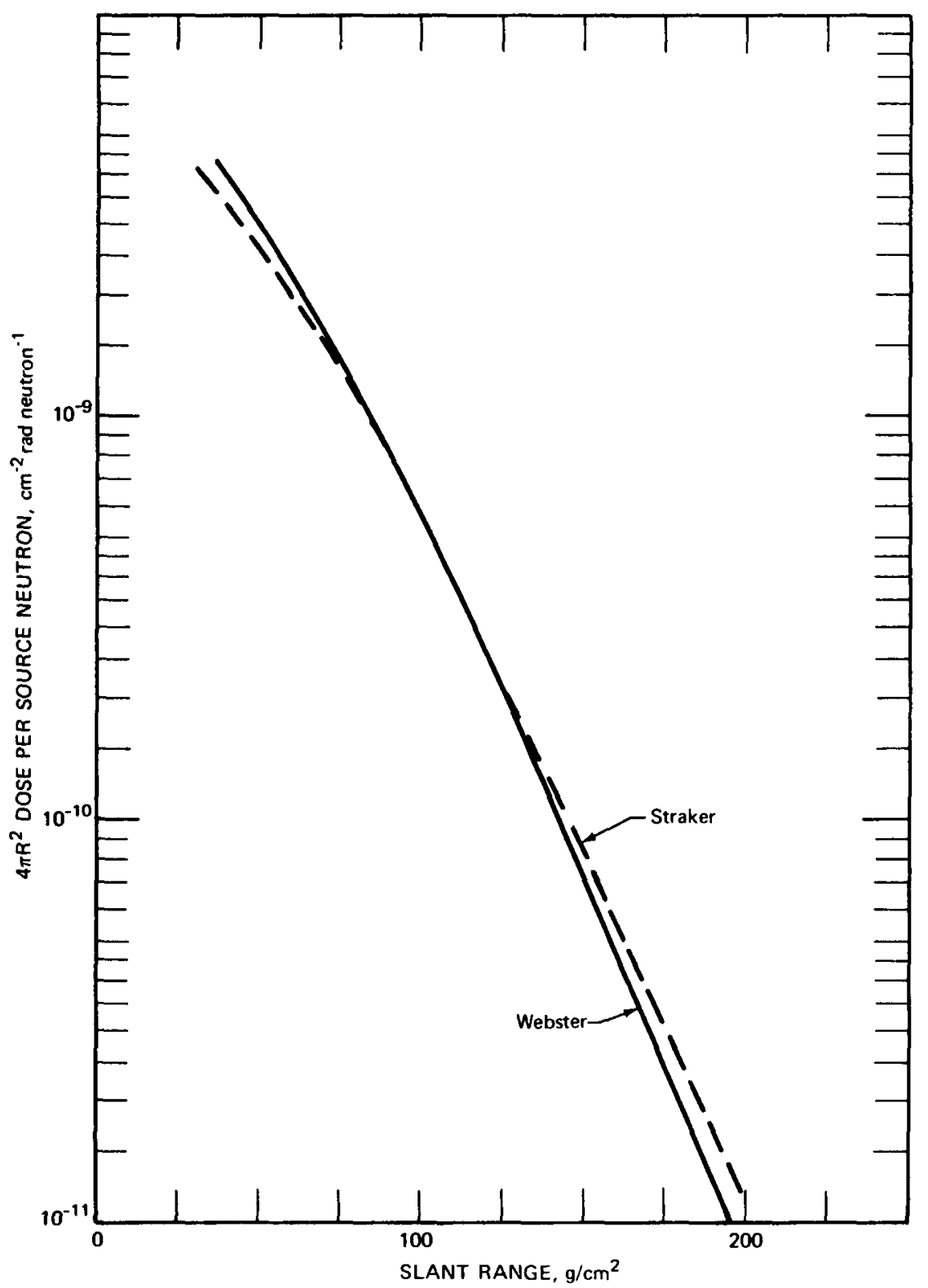

Fig. 8.3 - Comparison of the neutron dose in infinite air from a fission-neutron source as calculated by Straker2 2 and by Webster. ${ }^{24}$ 
data are seen to wash out for the fission source as a result of the smaller number of high-energy neutrons in the fission distribution. Some differences in slope are evident, however. Comparisons of calculated and measured data for an infinite air medium are shown later in this chapter.

Figure 8.4 compares the results of three calculations for gamma rays in an infinite air medium. Two are based on the moments method: a

Table 8.2-AREAL-DENSITY CONVERSION FOR SEA-LEVEL AIR DENSITY $\left(\rho=1.293 \mathrm{mg} / \mathrm{cm}^{3}\right)$

\begin{tabular}{rc}
\hline Range, $\mathrm{m}$ & Range, $\mathbf{g} / \mathrm{cm}^{2}$ \\
\hline 50 & 6.5 \\
77 & 10.0 \\
100 & 12.9 \\
200 & 25.9 \\
387 & 50.0 \\
500 & 64.6 \\
773 & 100.0 \\
1000 & 129.3 \\
1160 & 150.0 \\
1547 & 200.0 \\
\hline
\end{tabular}

calculation for air by Vogel ${ }^{25}$ in 1963 and a direct application of the 1954 buildup factors for water. The two sets of data are close together except at $0.5 \mathrm{MeV}$, where the older results are based on an interpolation. The third set of data shown is from a Monte Carlo calculation by Marshall and Wells ${ }^{26}$ in 1966. This set of data is widely used because it is recent and because it is differential in angle and energy at the receiver, which makes it suitable for application to shielded detectors. ${ }^{27}$

Unfortunately a doubly differential data set is needed to convey useful working information: for 10 angle groups and 10 scattered energy groups, a 10-by-10 matrix is required for each initial energy. Thus one complete data set includes about $10^{3}$ entries. All this is preliminary to stating that we do not have the luxury of including such data in this text. We respectfully refer the reader to the references and information centers.

The effect of air density on air scattering is straightforward and simply related to source-receiver separation distance. Zerby ${ }^{28}$ showed that the flux density or dose rate from an anisotropic point source in an infinite homogeneous medium at a given air density can be transformed to the 


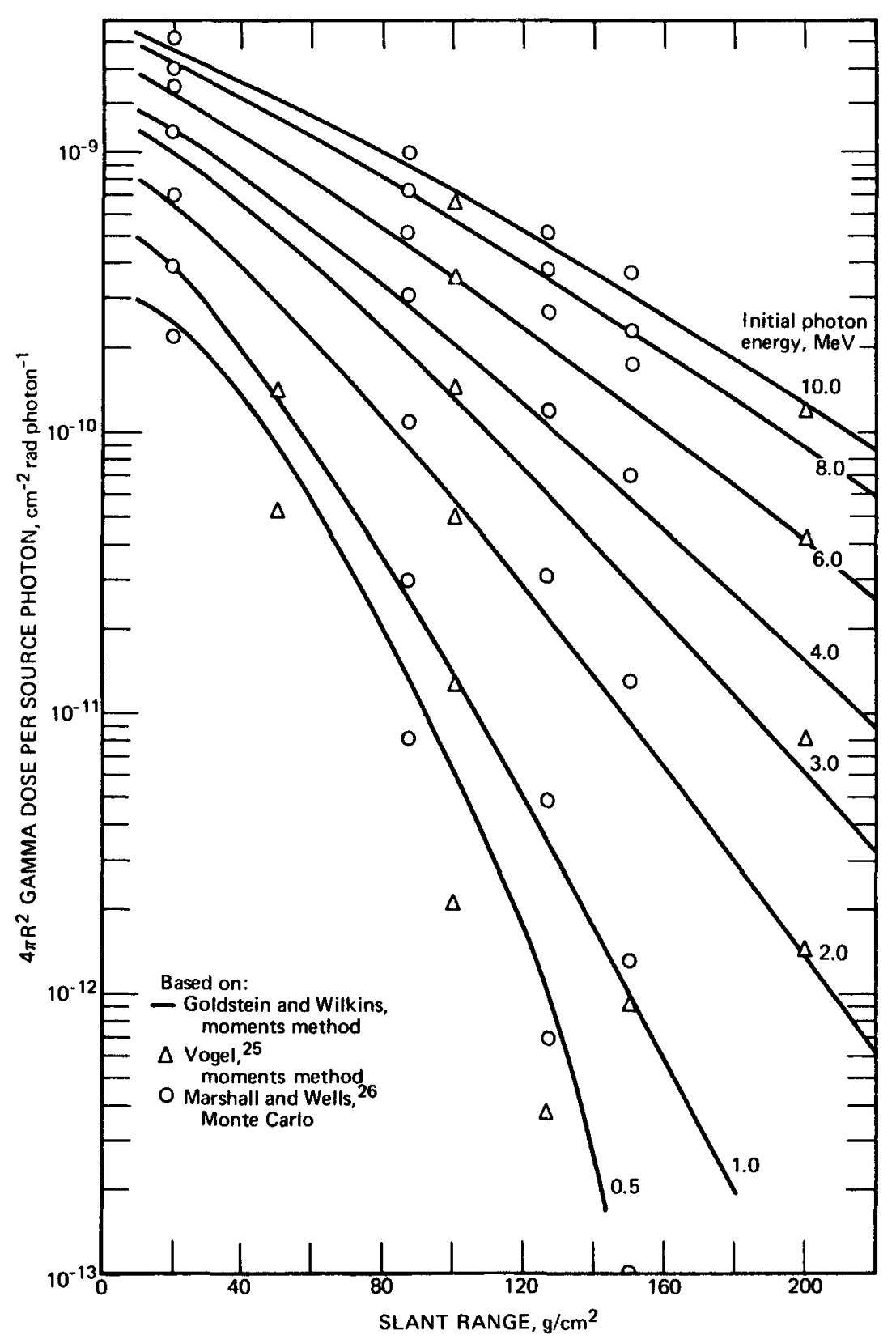

Fig. 8.4-Comparison of the calculated gamma-ray dose as a function of range in an infinite air medium. 
corresponding quantity at any other air density in the medium:

$$
\begin{aligned}
& \Phi\left(\rho_{2}, R_{2}\right)=\frac{\rho_{2}^{2}}{\rho_{1}^{2}} \Phi\left(\rho_{1}, R_{1}\right) \\
& D\left(\rho_{2}, R_{2}\right)=\frac{\rho_{2}^{2}}{\rho_{1}^{2}} D\left(\rho_{1}, R_{1}\right)
\end{aligned}
$$

where $\rho$ is the air density, $R$ is the separation distance, and

$$
R_{2}=\frac{\rho_{1}}{\rho_{2}} R_{1}
$$

This relation can be used only when the source and the receiver are at the same air density.

The question sometimes arises whether or not the scattering in air is affected by moisture (rain, fog, or water vapor). The possibility is most simply resolved by considering the relative densities. A cubic meter of air at standard conditions weighs about $1.3 \mathrm{~kg}$. Saturated water vapor at $0^{\circ} \mathrm{C}$ weighs $5 \mathrm{~g} / \mathrm{m}^{3}$; even at $35^{\circ} \mathrm{C}\left(95^{\circ} \mathrm{F}\right)$ saturated water vapor weighs $40 \mathrm{~g} / \mathrm{m}^{3}$. During a heavy rainstorm of $5 \mathrm{~cm}$ per hour, the water density is only about $5 \mathrm{~g} / \mathrm{m}^{3}$. A heavy fog yields a water density in the same range. Thus the water density never exceeds a few percent of the air density, and such a water content would have a comparable effect (of no more than a few percent) on the scattered intensity.

\subsubsection{Air-Over-Ground Calculations}

Calculations of the neutron and secondary gamma-ray transport in an air-over-ground geometry are difficult to compare since interface effects depend on the source and the detector height, the separation distance, and the composition of the ground used in the calculation.

Probably the first definitive air-over-ground calculation for gamma rays to reach the open literature was the Monte Carlo study of Berger. ${ }^{29}$ The point isotropic source was $1.28 \mathrm{MeV}$ gamma rays, and the results were given in terms of boundary correction factors, which made them convenient to apply to fallout radiation studies. Air-over-concrete calculations for point monoenergetic and monodirectional gamma-ray ${ }^{30}$ and neutron ${ }^{31}$ sources were reported by Wells. These data were to be applied for the nuclear aircraft with a reactor $4 \mathrm{~m}$ above a concrete ramp. 


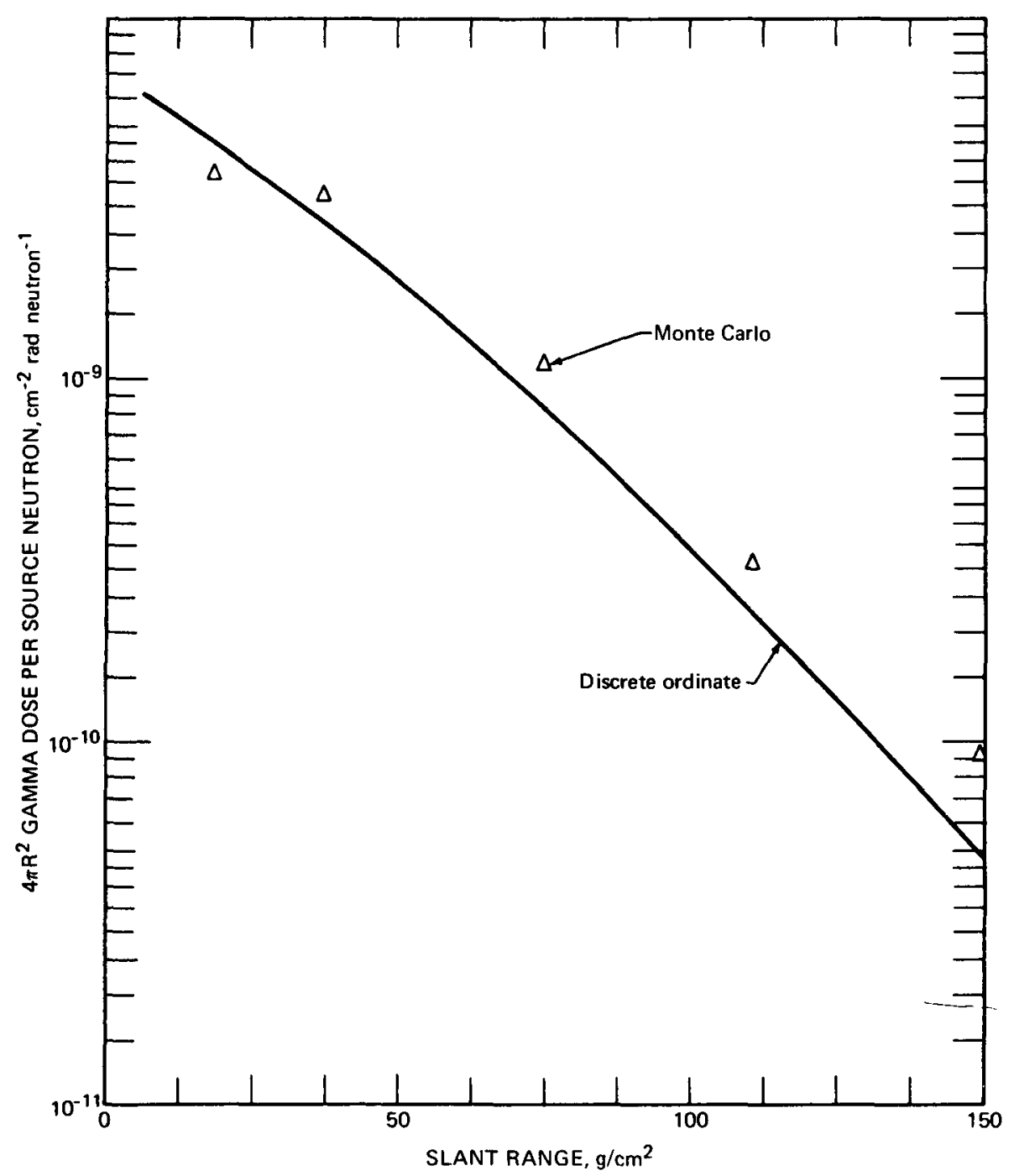

Fig. 8.5-Comparison of the neutron dose vs. range as reported by Straker $^{22}$ and by Marcum $^{32}$ for a 14-MeV type source in an air-over-ground geometry.

A comparison of more recent results is shown in Fig. 8.5, where the discrete-ordinate data of Straker ${ }^{22}$ are compared to the Monte Carlo data of Marcum $^{32}$ for an identical neutron source composed largely of 14-MeV neutrons. Differences in the data are probably due to cross sections used for nitrogen and oxygen since several new sets were published in the intervening 


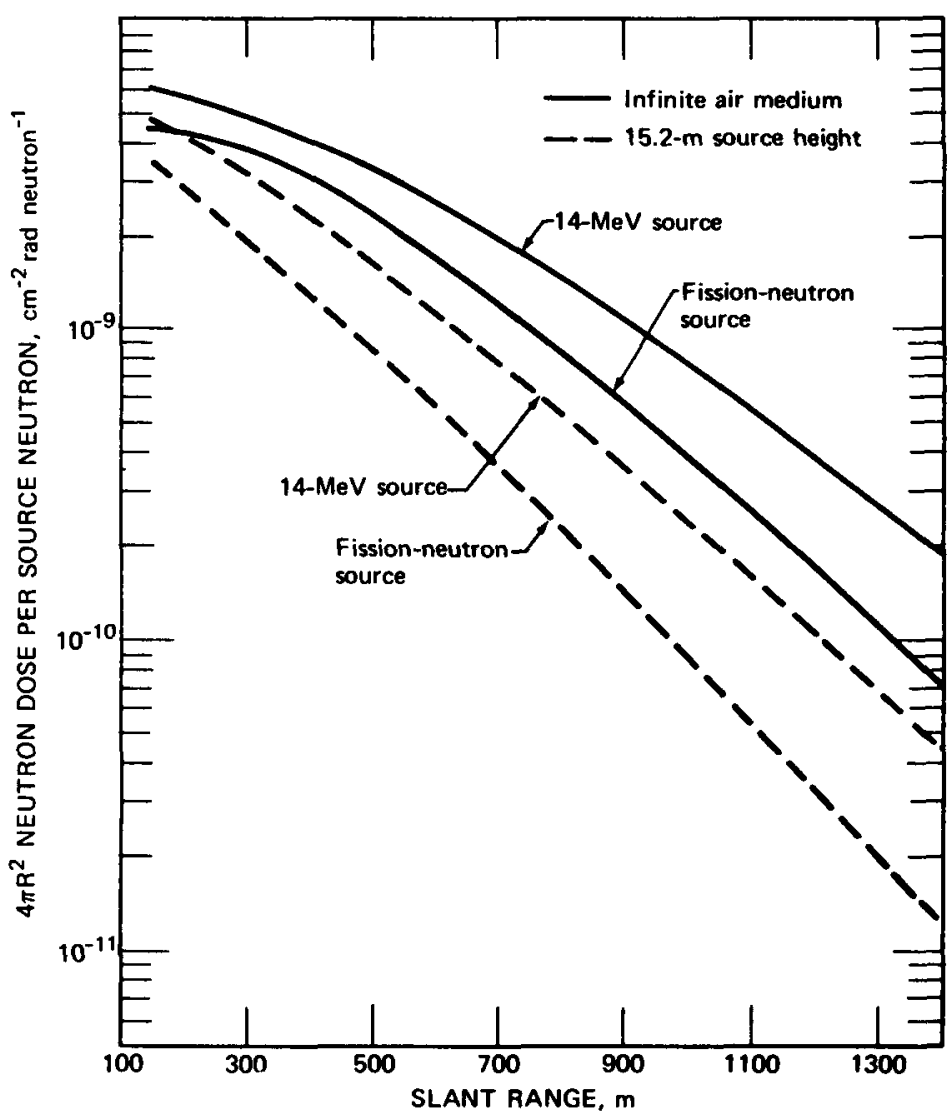

Fig. 8.6-Comparison of the neutron dose as a function of range for a fission-neutron source and a 14-MeV neutron source in an infinite air medium and for a source height of 15.2 m. (From Straker. ${ }^{22}$ )

3 years. Both sets of data were for detectors at the ground, but the Straker set is for a $15-\mathrm{m}$ source height and the Marcum set for a ground-level source. This difference does not account for the differences in the results.

\subsubsection{Air-Ground Interface Effects}

Figure 8.6 compares the neutron dose in infinite air and in an air-over-ground geometry for both a high-energy $14-\mathrm{MeV}$ neutron source and a fission-neutron source. These data are from the same set by Straker 2 quoted previously. The neutron dose is appreciably lower for the air-over- 


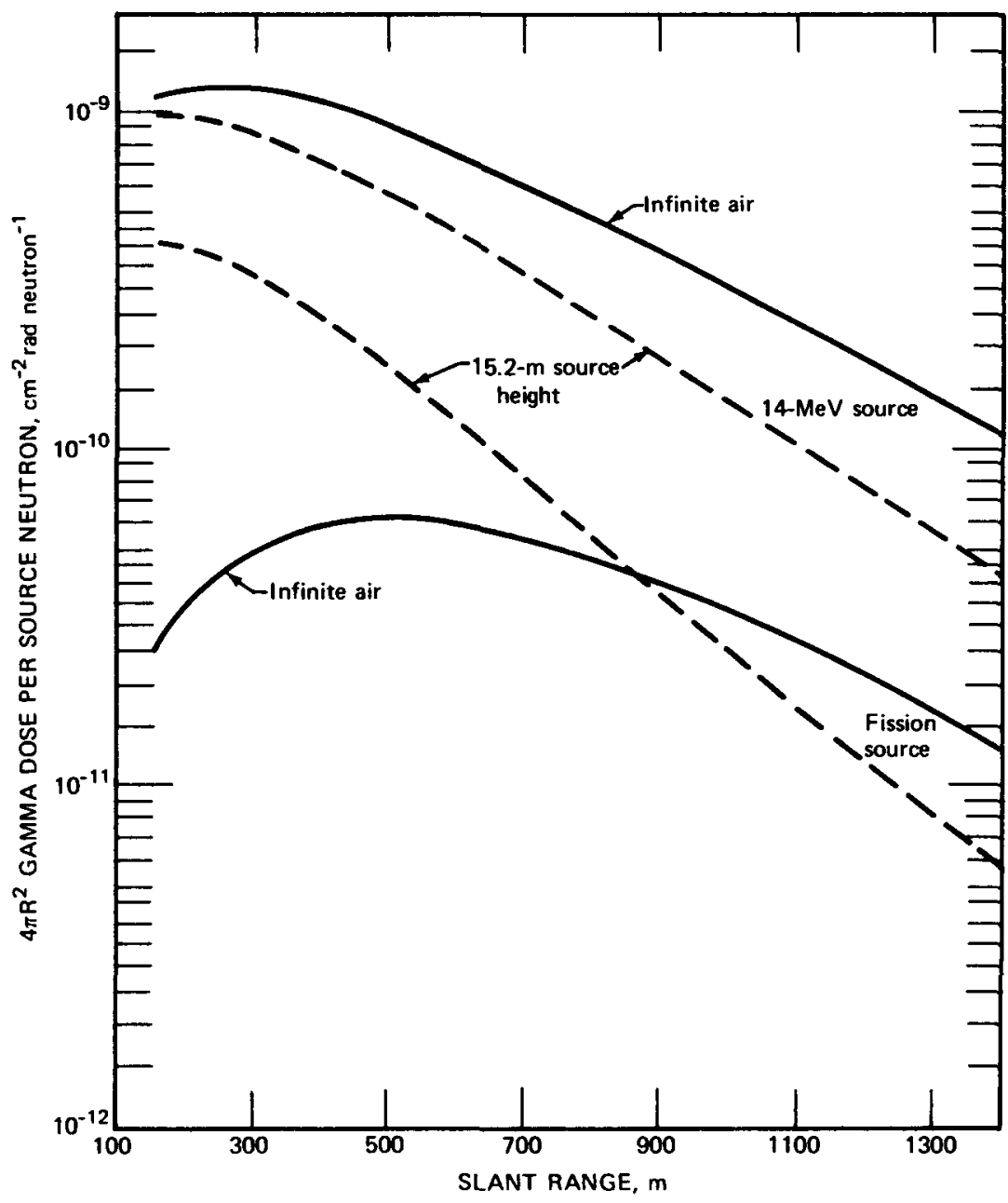

Fig. 8.7-Comparison of the secondary gamma dose from a fission-neutron source and a 14-MeV neutron source in an infinite air medium and for a source height of $15.2 \mathrm{~m}$. (From Straker. ${ }^{2}$ )

ground geometry than for infinite air, and the effect appears to be somewhat dependent on the neutron source energy. This effect would be much more evident at larger ranges. Figure 8.7 shows the effects of the interface on the secondary gamma-ray dose for the same conditions as those given for the neutron dose. Here the effects are highly dependent on both the source height and the neutron-energy distribution.

Some indication of the accuracy of the infinite-air and air-over-ground transport data can be obtained from Figs. 8.8 and 8.9, which compare 


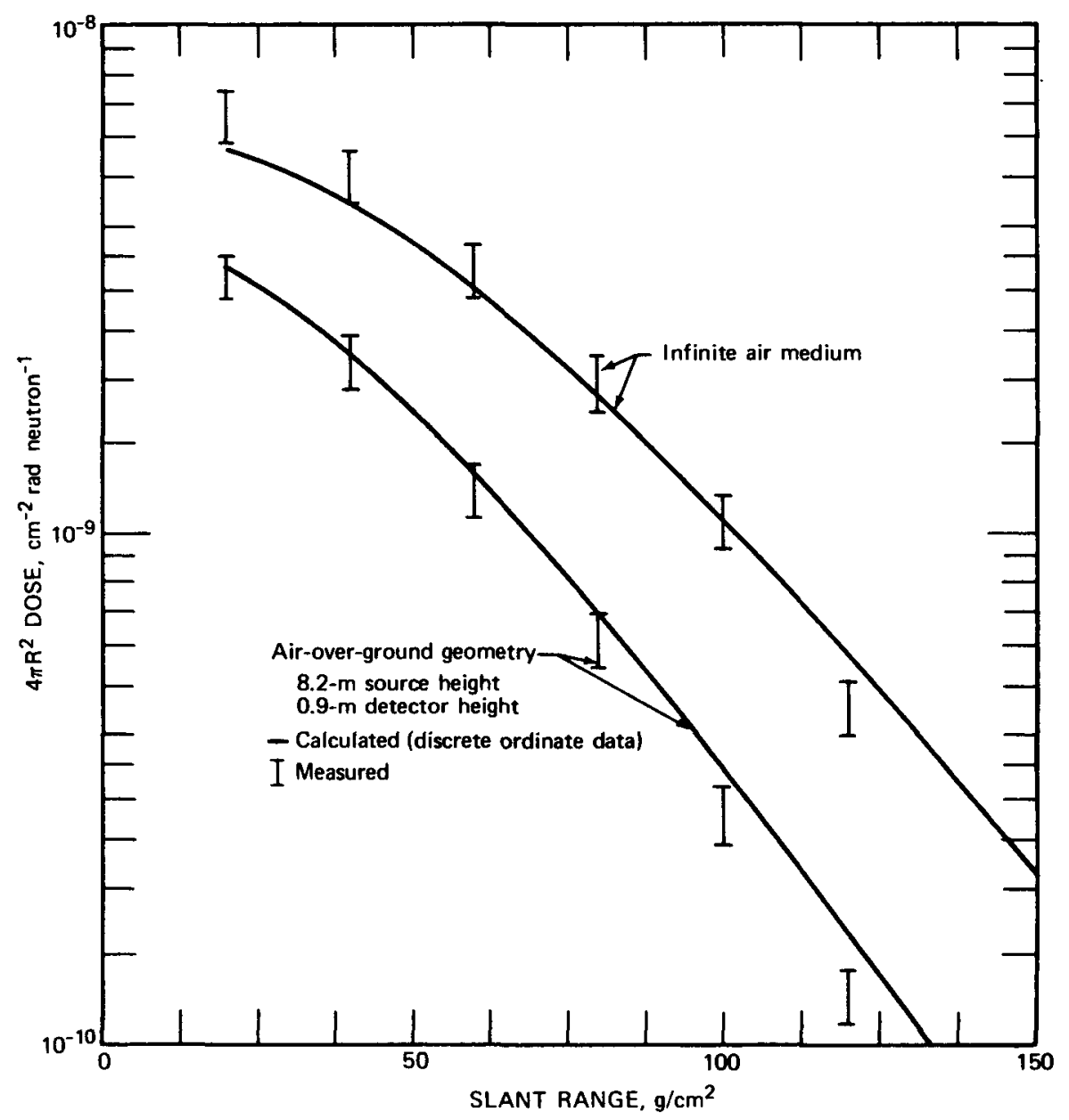

Fig. 8.8-Comparison of the measured ${ }^{33}$ and calculated ${ }^{22}$ neutron dose in an infinite air medium and in an air-over-ground geometry resulting from the HENRE neutron source.

Straker's data and measurements made during Operation HENRE ${ }^{33}$ at the Nevada Test Site. Operation HENRE (High Energy Neutron Reaction Experiment) was designed to provide a high-intensity source of $14-\mathrm{MeV}$ neutrons from an accelerator using the $\mathrm{D}-\mathrm{T}$ reaction. The accelerator was mounted on a 457-m tower and could be raised to approximately $366 \mathrm{~m}$ to approximate an infinite air medium. Neutron and secondary gamma-ray doses were measured as a function of range for the source and the detector at $336 \mathrm{~m}$ (simulating an infinite air medium) and for the source at an $8.2-\mathrm{m}$ height and the detector on the ground surface. ${ }^{34.35}$ Results of the measurements are compared with calculated values in Figs. 8.8 and 8.9. The 


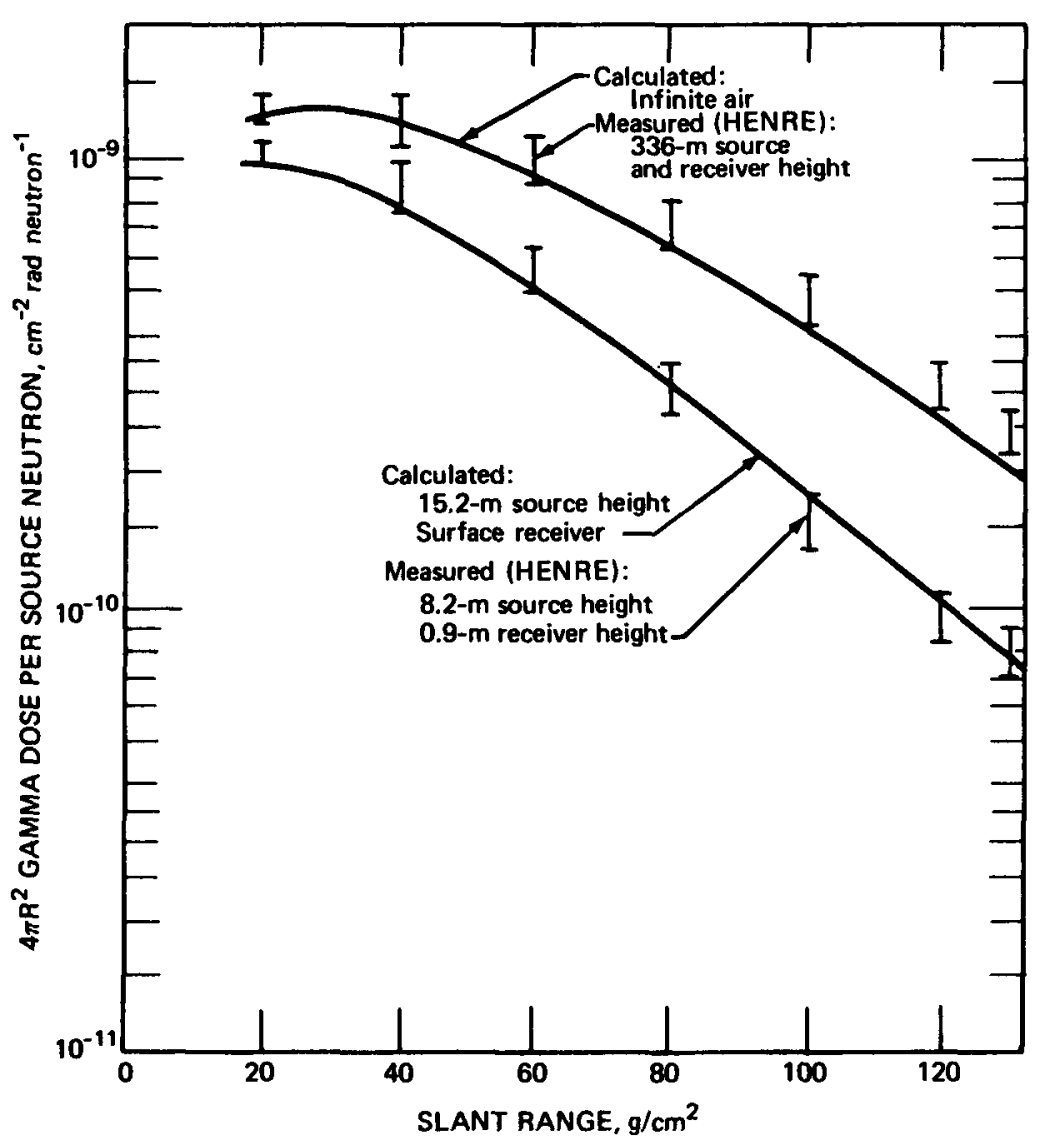

Fig. 8.9-Comparison of the measured ${ }^{33}$ and calculated ${ }^{22}$ secondary gamma dose in an infinite air medium and in an air-over-ground geometry resulting from the HENRE neutron source.

vertical bars describing the measurements represent uncertainties in the measurements caused by shadowing of the neutron source by components of the accelerator with a consequent departure from isotropy; the actual source intensity was therefore uncertain.

Limited success has been achieved in developing theoretical models to describe the effects of the air-ground interface. One such model, called the First-Last Collision Model, devised by French $^{\mathbf{3 6}}$ has been reasonably successful in describing interface effects on the integrated neutron dose. The model was originally conceived as a possible means of adjusting infinite-airmedium transport data to account for the perturbation resulting from an interface. The model presupposes that interface effects operate primarily on 
the first collision of neutrons near the source and on the last collision of neutrons near the detector. Perturbation fractions are calculated, which represent the effects of the interface on the number of first and last collisions, as the source, or detector, or both approach the ground surface. The model accounts only for the scattered-neutron component and is used only for separation distances where multiple scattering predominates. Results with the first-last collision model also indicate that the interface effects are limited to those neutrons which arrive at the detector from the lower $2 \pi$ hemisphere below the detector.

An experiment for testing this last conclusion was devised and carried out during Operation HENRE. The $14-\mathrm{MeV}$ neutron source was fixed at a height of $34 \mathrm{~m}$ and the detector height was varied from 0.2 to $21 \mathrm{~m}$. A $2 \pi$ shield was sandwiched between two detectors to separate the neutron dose arriving from the upper $2 \pi$ hemisphere from that arriving from the lower. A third detector was positioned on a boom attached to the shield to measure the total free-field dose as a function of distance above the ground. The entire assembly was suspended at a horizontal distance of $305 \mathrm{~m}$ from the source tower. More details of the experimental arrangement are given in Sec. 9.3.1.

Measurements were made on all three detectors as a function of distance above the ground. Appropriate corrections were made to the measured data to account for backscattering of the neutrons by the shield and for ground shadowing by the shield.

Figure 8.10 compares the experimental results and the values calculated from the model. The free-field measured and calculated neutron doses were normalized at the maximum height of $21 \mathrm{~m}$ since the absolute source strength could not be established with sufficient accuracy. In general, the agreement is excellent, and the experimental results can be considered a reasonable verification of the model.

\subsection{SHIELD MATERIALS}

The selection of materials is obviously an important part of reactor shield technology. Materials selection and a related task, optimization, together offer the greatest potential for weight or cost savings. Nevertheless, materials selection has not been reduced to an analytical approach and has received rather less attention by shielding investigators than other more elegant aspects of shield design. Of course, there has been an active nuclear-data collection program, and extensive properties handbooks are 


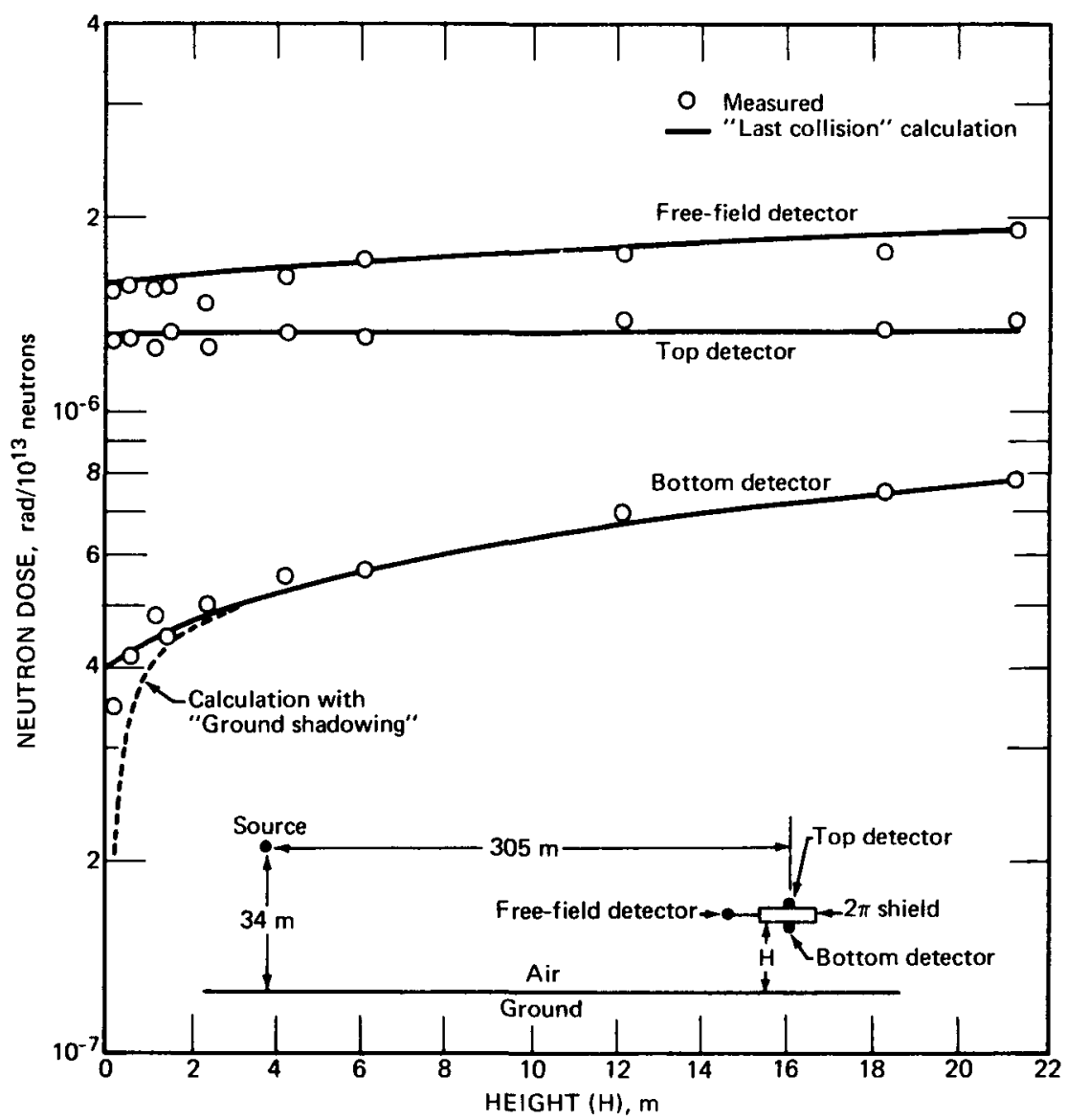

Fig. 8.10-Comparison of measured and calculated scattered fast-neutron dose near air-ground interface $305 \mathrm{~m}$ from 14-MeV neutron source. [From R. L. French and L. G. Mooney, Nucl. Sci. Eng., 43: 279 (1971).]

available. ${ }^{37}$ Virtually every accelerator laboratory has been engaged in measuring some interaction cross sections, most research reactors have been used to measure attenuation constants, and numerous national and international organizations are engaged in collecting, compiling, and evaluating the resulting data. But all this effort has been devoted to nuclear data; relatively little effort has been spent relating these results to other physical and chemical properties important to the selection of shield materials.

Perhaps the deficiency arises because the materials task requires a variety of skills: nuclear interaction, heat transfer, chemical interaction, structural characteristics, other physical properties, and economics must all be applied 
in a thoroughgoing analysis. It is evident that the selection of shield materials deserves additional attention.

\subsubsection{Considerations in Materials Selection}

The primary nuclear properties to be considered are obviously neutron and gamma-ray attenuation. As a rule, good gamma-ray attenuators also produce secondary gamma rays from neutron inelastic scattering and radiative capture; thus the production of secondaries is also a consideration. For shields requiring several orders of attenuation, volume and mass affect materials choices. Structural strength at operating temperatures is also involved. Some low-strength materials having excellent attenuation properties may be rejected because the structure necessary to support them offsets their primary advantage.

Inner layers of the shield usually require some thermal protection; thus cooling must be considered. Good gamma-ray attenuators are heavy metals and are usually the highest density material in the shield. To conserve weight and cost, these materials are usually placed nearest the core, which gives rise to a cooling requirement.

Different design objectives lead to the selection of different materials. For a fixed power-reactor system, cost is a primary consideration; thus the local concrete aggregate becomes a baseline for comparison with other materials. Higher attenuation substitutes may be considered only if some cost saving results.

For mobile systems, total weight becomes the primary consideration, and more exotic materials can be considered. We will discuss materials in both the exotic and conventional categories.

In a recent Russian book on nuclear power plants, Komarovskii ${ }^{38}$ has summarized in wry fashion the contradictory properties required of biological shield materials. We quote directly from the translation:

In general, protective materials used for biological (and radiation) shielding must have the following properties and ensure the following:

1. Maximum possible density (i.e., maximum specific or bulk weight) to ensure maximum attenuation of gamma radiation from the core and for the absorption of the secondary gamma radiation caused by the capture of neutrons by the material of the shield. Materials of higher density also slow down fast neutrons efficiently, transforming their energy into epithermal and thermal neutrons which are then more easily absorbed. (This statement evidently refers to the effect of inelastic scattering in heavy materials.)

2. Constant density and homogeneity of the shielding material (along all or part of the shielding). 
3. A definite, economically justified content of hydrogen (usually contained in chemically bound water) for an efficient slowing down of epithermal neutrons to the thermal level. It is desirable for the hydrogen content of the shielding material to be independent of the temperature of the material.

4. Minimum possible energy of capture gamma radiation forming on absorption and slowing down neutrons.

5. Satisfactory resistance of the material to radiation effects.

6. Low residual radioactivity, i.e., minimum amount of long-living radioisotopes which might accumulate in the shielding under the effect of radiation.

7. Mechanical strength (primarily compressive strength) and three-dimensional stability of the structure. The latter requirement may be explained by the fact that the shielding against external radiation is in most cases also the carrying structure of the nuclear plant (cover, walls of the reactor tank adjoining the shielding, etc.); the shielding must not have continuous seams and joints or local caverns, holes, or traversing cracks through which radiation might easily penetrate.

8. Relatively low modulus of elasticity thus permitting reduction of tensile stresses in the outer zone of the shielding as a result of its unilateral heating (heating from one side).

9. Maximum possible heat conductivity so as to reduce the temperature gradient over the thickness of the shielding and thus the tensile stresses in the outer zone of the shielding.

10. Minimum thermal expansion of the material, also enhancing reduction of thermal stresses.

11. Minimum thermal hydration during hardening (of concrete).

12. Minimum contraction (shrinkage) during the construction of the shielding and later, since upon contraction the various apertures, pipes, and conduits in the shielding may become displaced.

13. Refractoriness and thermal stability, and if the material catches fire, the combustion rate should be as low as possible with the flame tending to self-quenching as the fire source is removed. Particular demands as to refractoriness are put on materials used to shield the reactor core directly. The shielding materials should not melt even at emergency temperature in the reactor. This is of particular importance for fast-neutron reactors.

14. Waterproofness.

15. Gasproofness.

16. Safe (in terms of effects of the evolution of gases from the material during its heating).

17. Chemical inertness and first of all resistance to the chemical action of the coolant (this refers particularly to sodium-cooled reactors in which the shielding should not include water-metal mixes).

18. Noncorrosive with respect to metal (steel, lead, aluminum, etc.).

19. Easy construction as well as easy repair and if necessary, replacement.

20. Low cost. 
In nature there are no materials which satisfy all these requirements. Artificial materials (such as concrete) must therefore be used, or in some cases a shield consisting of layers of different materials must be constructed. In selecting the proper protective materials allowance must be made for the ratio of neutron-flux intensity to gamma-ray intensity.

Many of the above requirements are conflicting. When using materials of high density, for example, the requirement of minimum cost cannot be satisfied. High hydrogen content conflicts with the requirement of maximum content of heavy elements. In this case a compromise must be found by using a mixture of heavy and hydrogen-containing compounds (e.g., concrete) or, which is less frequently done, by constructing the shield in alternating layers of heavy and light materials.

In selecting the construction materials for the shielding all the engineering and economic advantages and disadvantages of the various materials must be weighed carefully and the operating conditions of the particular nuclear plant must be taken into account. Preference should therefore be given to materials of suitable properties. When constructing new premises for a reactor plant, it is usually possible to create a shielding of large volume but of less heavy materials. On the other hand, when building a reactor in already existing premises the thickness of the material often has to be reduced due to restricted access conditions, and in this case more expensive materials of higher density have to be used.

Now let us examine the properties of some materials actually in use for the shielding of mobile and stationary systems and compare some of their characteristics with the preceding requirements.

\subsubsection{Shield Materials for Stationary Reactor Systems}

Concrete is the most commonly used radiation shielding material for fixed reactors and accelerators. Since concrete is a mixture of elements, the variation in composition is large. Therefore, when referring to concrete as a shielding material, we should state the specific composition in order for its characteristics to have meaning. Concretes are generally referred to as ordinary or heavy. Ordinary (portland) concrete has a density range from 2.2 to $2.4 \mathrm{~g} / \mathrm{cm}^{3}$ and is largely composed of oxygen and silicon or calcium (or both), depending on the aggregates (granite, sandstone, or limestone). Heavy concretes with densities ranging from 3 to $6 \mathrm{~g} / \mathrm{cm}^{3}$ are those in which the aggregate consists of iron or iron ore, baryte (barium sulfate), steel shot, punchings, sheared bars, or other metal additives. There is obviously an added cost factor for heavy concretes.

Other materials that have been used in large quantity for shielding are compacted soil, iron, lead, and water. Experience has shown, however, that 
heavy-concrete shielding has many advantages over these materials. ${ }^{37,39,40}$ It is also evident that these materials satisfy few of the preceding desired properties.

Probably more than $90 \%$ of the stationary reactor systems designed for power production are shielded with concrete. As an illustration, of twenty-three test-reactor shields described in a 1968 survey published by the Radiation Shielding Information Center, ${ }^{41}$ six used both heavy and ordinary concrete, eleven used only ordinary concrete, and five used only heavy concrete. Only one shicld is described without concrete. Fourteen contained water: eleven were swimming pool reactors, two were boiling-water reactors, and one was a homogeneous solution. Three others included heavy water. Other materials mentioned were iron, steel, wet sand, boral, graphite, borated graphite, polyethylene, earth, and boric acid. Most of the materials used in the experimental reactor shields, other than concrete, such as iron, lead, and graphite, individually have poor structural or radiation shielding properties but, when combined in laminated configurations, result in extremely good shields.

Let us look at these materials in more detail. To compare nuclear properties of materials, we need a set of standards for comparison. Despite the universal use of concrete, we shall use lead for gamma rays and water for neutrons as standards for comparison of attenuation properties. Both are frequently used and are regarded as excellent attenuators. Both are pure substances (and relatively free from impurities in practice) and so do not present the multiplicity (and variability) of constituents with which we must contend with concrete.

(a) Water. Because of the success of the swimming pool reactor as a research tool and of its derivative, the pressurized water reactor, as a pioneer power source, water layers are found in reactor-shield analyses almost as often as concrete. Water is an excellent neutron attenuator because of its large hydrogen content. It is an inferior attenuator of gamma rays because of its low electron density. Since oxygen has no thermal-neutron capture and the 330-mb thermal-neutron capture cross section of hydrogen gives only $2.2-\mathrm{MeV}$ gamma rays, water is relatively low in secondary gamma-ray production.

Considerations when water is used as a shield material include containment, temperature control, corrosion, and purification (demineralization).

(b) Lead. Best of the conventional materials for gamma-ray attenuation, lead is exceeded only by uranium. Lead bricks are to be found in almost 
every radiation laboratory as portable shield bricks. Lead suffers creep at high temperatures (above $260^{\circ} \mathrm{C}$ ) and must be constrained to avoid downward flow.

Lead shot makes good filler for cavities in plugs and doors in shields. If provision is made for extracting the filler in place, it becomes a great convenience should it become necessary to dismantle or remove the door. Lead has relatively poor neutron properties and produces $7.4-\mathrm{MeV}$ gamma rays on neutron capture. Impurities in commercial lead can cause significant activation problems. Reference 37 gives additional physical properties.

(c) Graphite. A good neutron moderator and reflector material, graphite is used extensively in reactor design. Since it is available in very pure form, it exhibits few secondary gamma rays. Like water, it is a poor gamma-ray attenuator. Besides its use as a reflector-moderator, it is also found in thermal shields and occasionally as a primary shield material (see the discussion of the Enrico Fermi power plant in Chap. 10). The excellent high-temperature properties of graphite are additional reasons for its application.

(d) Iron. Iron is almost always present in the reactor structure as steel and is used for thermal shields and pressure vessels. Iron produces a number of secondary gamma rays up to $10 \mathrm{MeV}$, most of them at $7.6 \mathrm{MeV}$. Not surprisingly, it is intermediate in gamma-ray attenuation between water, carbon, and boron at the low end and tungsten, lead, and uranium at the high end. Neutron attenuation per unit thickness of iron is good; it is intermediate between the same materials mentioned for gamma rays. The isotope ${ }^{58} \mathrm{Fe}$ is easily activated by thermal neutrons producing ${ }^{59} \mathrm{Fe}$, with a 59-day half-life, which emits a $1.5-\mathrm{MeV}$ gamma ray. This activation of iron may limit accessibility to a reactor after shutdown. ${ }^{38}$ All steels embrittle after exposure to thermal-neutron fluence of the order of $10^{19}$ to $10^{20}$ neutrons $/ \mathrm{cm}^{2}$, showing hardness increases from 50 to $100 \%$, depending on the alloy and heat treatment. (See Chap. 23 of Ref. 37.)

(e) Boron. This element is used principally to absorb thermal neutrons because of the cross section of $3840 \mathrm{~b}$ for the ${ }^{10} \mathrm{~B}(n, \alpha \gamma)$ reaction. The alphas are easily absorbed (producing a heating problem for large incident flux densities); the gamma ray at $0.5 \mathrm{MeV}$ should be considered in borated water and polyethylene. Boron is available in a number of useful forms: as boric oxide $\left(\mathrm{B}_{2} \mathrm{O}_{3}\right)$, as boron carbide $\left(\mathrm{B}_{4} \mathrm{C}\right)$, as elemental boron, and as a sandwich material called boral. Boral consists of an aluminum $-\mathrm{B}_{4} \mathrm{C}$ mixture 
clad with pure aluminum. Pure boron has also been used as an additive to polyethylene and graphite to add neutron absorption properties to good neutron attenuators. It has also been added to some steels to reduce secondary gamma-ray productions and activation problems of iron.

(f) Concrete. Since concrete is a mixture of materials, the mixture can be tailored to provide optimum structural and shielding properties. Table 8.3 gives the elemental composition of ordinary portland concrete and several concretes fabricated for improved shielding properties. ${ }^{37}$ Table 8.3 contains only a small sample of the many custom-designed concretes available. References 37, 42, and 43 contain information on mixing proportions, placement techniques, costs, etc. There is no reported standard concrete composition since variation in normal concretes is due primarily to the aggregate. One can, however, use the ordinary portland concrete as a basis for comparing the improved structural and shielding properties of heavy concretes.

The ferrophosphorus concrete is of high density and has excellent bonding qualities. The aggregate is ferrophosphorus ore. The iron-limonite is a heavy concrete containing the iron-ore limonite and steel punchings as aggregates. Limonite is composed of goethite and hematite, both ironbearing ores. This concrete is similar to that used to shield the Brookhaven graphite reactor.

The iron-portland and several baryte concretes were all developed for shielding use. The iron-portland concrete is of high density. Lumnite is a cement (as is portland) which is better described as calcium aluminate hydraulic cement. Lumnite has a high aluminum and calcium content. Colemanite, used as an aggregate, is hydrated calcium borate with a high fixed-water and boron content. Baryte is a barium sulfate ore used as an aggregate in heavy concretes. The baryte concretes contain boron for neutron absorption and hydrogen for neutron attenuation, and the baryte ore serves as a good gamma-ray attenuator. The densities vary from 3 to $3.2 \mathrm{~g} / \mathrm{cm}^{3}$, which is lower than iron-aggregate concretes.

Neutron and gamma-ray attenuation per unit mass vary only slightly from one of these mixes to the next. The principal advantage in the heavier mixes lies in the smaller volume needed for a given total attenuation.

\subsubsection{Shield Materials for Mobile Reactor Systems}

Weight rather than cost is generally the principal design criteria in the application to mobile reactor systems. The emphasis, therefore, shifts to the 
Table 8.3-ELEMENTAL COMPOSITION OF CONCRETES $\dagger$

\begin{tabular}{|c|c|c|c|c|c|c|c|c|c|}
\hline \multirow[b]{2}{*}{ Element } & \multicolumn{9}{|c|}{ Composition, wt.\% } \\
\hline & $\begin{array}{l}\text { Portland } \\
\text { concrete }\end{array}$ & $\begin{array}{l}\text { Mag- } \\
\text { netite }\end{array}$ & $\begin{array}{c}\text { Ferro- } \\
\text { phosphorus } \\
\text { concrete }\end{array}$ & $\begin{array}{c}\text { Iron- } \\
\text { limonite }\end{array}$ & $\begin{array}{l}\text { Iron- } \\
\text { portland }\end{array}$ & $\begin{array}{l}\text { Colemanite- } \\
\text { baryte }\end{array}$ & $\begin{array}{l}\text { Boron } \\
\text { frits- } \\
\text { baryte }\end{array}$ & $\begin{array}{l}\text { Lumnite- } \\
\text { colemanite- } \\
\text { baryte }\end{array}$ & $\begin{array}{l}\text { Lumnite- } \\
\text { portland- } \\
\text { colemanite- } \\
\text { baryte }\end{array}$ \\
\hline Iron & 1.4 & 50.5 & 61.2 & 72.1 & 87.50 & 1.03 & 2.19 & 3.07 & 1.87 \\
\hline Hydrogen & 1.0 & 0.3 & 0.5 & 0.05 & 0.33 & 0.85 & 0.56 & 1.09 & 1.10 \\
\hline Boron & & & & & & 0.98 & 1.04 & 0.88 & 1.02 \\
\hline Oxygen & 52.9 & 32.0 & 10.4 & 18.0 & 5.82 & 34.89 & 33.80 & 36.95 & 36.98 \\
\hline Magnesium & 0.2 & 0.6 & 0.2 & 0.2 & 0.13 & 0.22 & 0.23 & 0.14 & 0.20 \\
\hline Calcium & 4.4 & 0.7 & 4.2 & 6.1 & 3.96 & 8.46 & 6.26 & 5.48 & 7.67 \\
\hline Barium & & & & & & 40.70 & 40.13 & 38.59 & 38.03 \\
\hline Silicon & 33.7 & 3.5 & 3.4 & 1.4 & 0.91 & 1.76 & 3.31 & 0.96 & 1.49 \\
\hline Sodium & 1.6 & & & & & 0.11 & 1.21 & 0.11 & 0.11 \\
\hline Potassium & 1.3 & & & & & & 0.10 & & \\
\hline Aluminum & 3.4 & 2.9 & 0.4 & 0.5 & 0.33 & 0.61 & 0.64 & 1.76 & 1.32 \\
\hline $\begin{array}{l}\text { Manganese } \\
\text { Chlorine }\end{array}$ & & 0.07 & & 1.6 & 0.35 & 0.01 & 0.02 & 0.12 & 0.04 \\
\hline Carbon & 0.1 & & & & & & & & \\
\hline Phosphorus & & 0.17 & 19.7 & & & & & & \\
\hline Sulfur & & 1.07 & & 0.1 & 0.05 & 9.63 & 9.15 & 9.06 & 8.97 \\
\hline Zinc & & & & & & & 0.66 & & \\
\hline Fluorine & & & & & & & 0.23 & & \\
\hline Titanium & & 2.8 & & & & & & 1.27 & 0.071 \\
\hline Density, & & & & & & & & & \\
\hline $\mathrm{g} / \mathrm{cm}^{3}$ & 2.3 & 3.45 & 4.8 & 4.27 & 5.8 & 3.2 & 3.1 & 3.1 & 3.1 \\
\hline
\end{tabular}

†From H. E. Hungerford, Reactor Materials, Vol. I, Materials, C. R. Tipton, Jr. (Ed.), p. 1086, Interscience Publishers, a division of John Wiley \& Sons, Inc., New York, 1960. 
improved shielding provided by the so-called exotic materials used in layered combination.

The approach in designing minimum-weight shields for reactor systems usually is to select the most efficient of the neutron shielding materials compatible with the design and to combine this with the most efficient of the gamma-ray shielding materials rather than to select a single bulk attenuator medium. These two component materials are usually layered in some manner that yields minimum weight by providing the proper balance between primary and secondary radiations and geometric effects on material volume.

The efficiency of a neutron attenuator can be correlated to its hydrogen density, and the efficiency of a gamma-ray attenuator can be correlated to the total density of the material. Thus minimum-weight shield component materials are such combinations as lead plus polyethylene, tungsten (wolfram) alloys plus lithium hydride, or depleted uranium plus titanium hydride.

Shields of this nature placed in proximity to a propulsion reactor invariably create a thermal problem because of the high-energy absorption. Internal temperature rise can be limited by cooling the materials with a forced coolant flow between thin layers of the material or through internal cooling channels. Some of the high-density gamma-ray shielding materials, such as wolfram and depleted uranium, have a relatively high thermal conductivity and can tolerate a fairly high operating temperature if clad to protect against oxidation and corrosion. In contrast, most materials that are high in hydrogen content have low thermal conductivity and cannot tolerate high operating temperatures without melting or decomposing.

Although submarines were the first application for nuclear propulsion, the application to surface ships is also well known. Two such examples are the U.S. merchant ship N.S. Savannah and the Russian icebreaker Lenin. The reactor shield of the N.S. Savannah is composed of light water, lead, polyethylene, standard concrete, and heavy concrete (described in Chap. 10). The reactor shields of the Lenin are composed of water, steel, and heavy concrete. ${ }^{41}$

In addition to mobile reactor systems, other mobile or portable sources of radiation require shielding, e.g., neutron sources fabricated from plutonium-beryllium or polonium-beryllium and radioisotopes (usually gamma-ray emitters) resulting from high-intensity neutron exposures in reactors.

Shipping and storage casks must be provided for sources of this type, and they are usually constructed of lead for gamma-ray shielding and contain 
paraffin or other hydrogenous material for neutron shielding. These sources are usually small and present few design problems. An exception to this is found in spent-fuel-element shipping casks where large burnups have been experienced. ${ }^{44}$ These elements contain sizeable quantities of ${ }^{242} \mathrm{Cm}$, ${ }^{244} \mathrm{Cm}$, and ${ }^{241} \mathrm{Am}$, all of which fission spontaneously, giving rise to a fission-neutron distribution (as well as the accompanying prompt gamma-ray spectrum) in addition to the long-term fission-product gamma rays. Shield design of shipping casks for these fuel elements presents nearly all the problems of a reactor shield: radiation heating (forced cooling is necessary), good neutron and gamma-ray shielding, and minimum size and weight (usually limited by the largest available truck size for transport).

Weight-limited shields are also used for isotope power supplies, which use the heat generated by the decay of radioactive nuclides to produce electricity. Such sources have been used to power orbiting satellites and experimental equipment placed on the moon. Shields for these systems must obviously be efficient.

As mentioned, among the top contenders in materials for mobile reactor systems are lead, polyethylene, tungsten alloys, lithium hydride, depleted uranium (238), and titanium hydride. The properties of some of these materials are given in the following paragraphs. Some physical properties of the metals discussed are summarized in Table 8.4 and of the hydrogenous materials, in Table 8.5.

(a) Polyethylene. This plastic is a pure hydrocarbon $\left(\mathrm{CH}_{2}\right)_{n}$ with a density of $0.92 \mathrm{~g} / \mathrm{cm}^{3}$ which actually contains $18 \%$ (by volume) more hydrogen than does water (both contain more hydrogen per unit volume than liquid hydrogen). Water contains $6.7 \times 10^{22}$ hydrogen atoms $/ \mathrm{cm}^{3}$, and polyethylene has about $8 \times 10^{22}$ hydrogen atoms $/ \mathrm{cm}^{3}$. Polyethylene does not activate, but it does soften at $110^{\circ} \mathrm{C}$, and it supports combustion. A more-dense version of polyethylene has a higher softening point $\left(200^{\circ} \mathrm{C}\right)$ and slightly higher density $\left(0.96 \mathrm{~g} / \mathrm{cm}^{3}\right)$. Either material suffers radiation damage and must be replaced before decomposition reduces its effectiveness (probably well above $10^{11}$ ergs/g energy deposition). ${ }^{45}$ As mentioned under the discussion on boron, polyethylene can be borated to produce the added benefit of high neutron absorption. Linear thermal expansion is large, and thermal conductivity is very small; both properties must be borne in mind.

(b) Tungsten Alloys. Tungsten powder blended with binder metals, such as nickel, copper, and iron, has been sintered to form very dense machineable alloys with densities varying from 16.5 to $18.5 \mathrm{~g} / \mathrm{cm}^{3}$. The 
Table 8.4-PHYSICAL PROPERTIES OF DENSE SHIELD MATERIALS

\begin{tabular}{|c|c|c|c|c|c|}
\hline & $\begin{array}{c}\text { Iron } \\
\text { (stainless steels) }\end{array}$ & Lead & Tungsten & Uranium & Concretes \\
\hline Density, $\mathrm{g} / \mathrm{cm}^{3}$ & 7.9 to 8.0 & 1.34 & 19.3 & 18.5 to 19.04 & 2.5 to 5.0 \\
\hline $\begin{array}{l}\text { Specific heat, } \\
\text { cal g }^{-1} \mathrm{deg}^{-1}\end{array}$ & 0.12 & 0.0309 & 6.0 & 0.02 to 0.04 & 0.16 to 0.2 \\
\hline Melting point, ${ }^{\circ} \mathrm{C}$ & 1370 to 1425 & 327.4 & 3410 & 857 & \\
\hline $\begin{array}{l}\text { Thermal conductivity, } \\
\text { cal sec } \mathrm{sm}^{-1} \mathrm{cme}^{-1} \\
\text { Coefficient of thermal }\end{array}$ & 0.03 to 0.05 & 0.083 & 0.40 to 0.46 & 0.6 to 1.0 & $(6$ to 11$) 10^{-3}$ \\
\hline $\begin{array}{l}\text { Coefticient of thermal } \\
\text { expansion, } \text { deg }^{-1}\end{array}$ & $(14$ to 20$) 10^{-6}$ & $29.1\left(10^{-6}\right)$ & $(4.4$ to 5.0$) 10^{-6}$ & $(8$ to 10$) 10^{-6}$ & $(6$ to 10$) 10^{-6}$ \\
\hline $\begin{array}{l}\text { Tensile strength, } \\
\mathrm{kg} / \mathrm{cm}^{2}\end{array}$ & $(4$ to 6$) 10^{3}$ & 160 to 180 & $(8.1) 10^{3}$ & $(7.7$ to 9.1$) 10^{3}$ & \\
\hline
\end{tabular}

Table 8.5-PHYSICAL PROPERTIES OF NEUTRON SHIELD MATERIALS

\begin{tabular}{|c|c|c|c|c|c|}
\hline & Graphite & Polyethylene & $\begin{array}{l}\text { Lithium } \\
\text { hydride }\end{array}$ & $\begin{array}{l}\text { Titanium } \\
\text { hydride }\end{array}$ & $\begin{array}{l}\text { Zirconium } \\
\text { hydride }\end{array}$ \\
\hline $\begin{array}{l}\text { Density, } \mathrm{g} / \mathrm{cm}^{3} \\
\text { Sublimation, }{ }^{\circ} \mathrm{C}\end{array}$ & $\begin{array}{l}1.5 \text { to } 1.9 \\
3650\end{array}$ & 0.92 to 0.96 & 0.82 & 3.9 & 5.6 \\
\hline $\begin{array}{l}\text { Melting (softening) } \\
\text { point, }{ }^{\circ} \mathrm{C} \\
\text { Coefficient of thermal } \\
\text { expansion linear, } \\
\text { deg }^{-1}\end{array}$ & $(3.5$ to 8$) 10^{-6}$ & $\begin{array}{l}110 \text { to } 200 \\
\text { (16 to } 18) 10^{-5}\end{array}$ & 680 & 400 (decomposes) & 430 (decomposes) \\
\hline $\begin{array}{l}\text { Thermal conductivity, } \\
\text { cal sec } \mathrm{cm}^{-1} \mathrm{deg}^{-1} \\
\text { Specific heat, } \\
\text { cal g }^{-1} \mathrm{deg}^{-1}\end{array}$ & 0.1 to 0.3 & $(6$ to 8$) 10^{-4}$ & & & \\
\hline
\end{tabular}


more common of these have a density of about 16.9 to $17.1 \mathrm{~g} / \mathrm{cm}^{3}$. Fabrication is limited by currently available equipment to pieces with a maximum dimension of about $1 \mathrm{~m}$. Although this material has good strength properties, it is still likely to be held in place by a steel framework because of the limit on piece size. Tungsten falls just under lead in gamma-ray attenuation per unit mass. It is much better than lead per unit thickness for neutron attenuation and worse for secondary production.

(c) Depleted Uranium. The separation of uranium isotopes to produce $235 \mathrm{U}$-enriched material for reactor and weapons uses has reacted a supply of depleted uranium that is essentially pure ${ }^{238} \mathrm{U}$. This material has a high density (18.7 to $19.08 \mathrm{~g} / \mathrm{cm}^{3}$ ) and good strength properties. It may be cast, rolled, forged, extruded, and machined (with environmental precautions).

Uranium oxidizes in air to form first a brown and then a black surface layer. In moist air corrosion will continue beyond this stage as the coating layer spalls off and a new layer forms. The metal from this process can be protected by cladding or alloying or both. Alloying with a $2 \%$ molybdenum has proven satisfactory from the corrosion standpoint and also serves to improve the mechanical properties of the material. There is a slight activity associated with depleted uranium, but this does not severely restrict its use. We should note, however, that depleted uranium always has a residual amount of ${ }^{235} \mathrm{U}$ that should not be neglected in estimating radiation heating, secondary gamma-ray production, and activation. Precautions are required in machining the metal because of its pyrophoricity and the hazards of internal toxicity and internal radiation exposure.

It is unlikely that any structural support or framework would be required for ${ }^{238} \mathrm{U}$ shields in that they can be manufactured in about any size and geometry and they possess strength properties on a par with mild steel.

Uranium is the best attenuator for gamma rays on a mass absorption basis as well as on a thickness basis, and it provides roughly the same neutron attenuation as lead.

(d) Lithium Hydride. Lithium hydride ( $\mathrm{LiH})$, the best known neutron attenuator, ${ }^{46}$ contains approximately $12.6 \mathrm{wt} . \% \mathrm{H}_{2}$, has a density of 0.78 $\mathrm{g} / \mathrm{cm}^{3}$, and has a melting point of $683.5^{\circ} \mathrm{C}$. It is difficult to fabricate, normally requires surface protection at all temperatures, combines actively with water, and requires containment, venting, and structural support. Weight-conscious shield designers like to recommend its use; mechanical designers shudder at the thought. The structure and cost penalties in applying this material should be analyzed carefully in considering its use. 
Lithium has no capture gamma ray. Much consideration, including experimentation work, has been given to this material for reactor shields in space applications.

(e) Titanium Hydride. Titanium hydride $\left(\mathrm{TiH}_{2}\right)$, with a density of 3.76 $\mathrm{g} / \mathrm{cm}^{3}$ and a maximum useful temperature of approximately $600^{\circ} \mathrm{C}$, has a maximum $\mathrm{H}_{2}$ content of $3.9 \mathrm{wt} . \%$. This hydride is self-supporting. It needs no surface protection in air but will evolve hydrogen at high temperatures.

\subsubsection{Comparison of Attenuation Properties}

The efficiency of various materials for the attenuation of gamma rays increases with the average atomic number of the constituents. Figure 8.11 shows various material thicknesses required to reduce gamma-ray intensities by a factor of 10 as a function of the incident gamma-ray energies. These are narrow-beam conditions. When extrapolated to broad-beam conditions (to include buildup), the separation between the lines would become even greater because the more-dense materials inhibit buildup.

Neutron attenuation is greater in low- $Z$ materials, particularly those containing large quantities of hydrogen. Figure 8.12 shows the neutron-dose attenuation in infinite media of normal concrete, ${ }^{47}$ hydrous iron-ore concrete, ${ }^{47}$ water, ${ }^{47}$ a simple hydrocarbon and boron mixture,,${ }^{47}$ and lithium hydride 48 for a point isotropic source of fission neutrons. The concrete compositions are given in Table 8.6 as types $\mathrm{A}$ and $\mathrm{H}$. The lithium hydride data of Kam and Clark ${ }^{48}$ in Fig. 8.12 have been normalized to the data of Nichols ${ }^{4}$ for the other materials at $R=0 \mathrm{~cm}$.

Figure 8.13 shows the capture-gamma-ray dose from the fission-neutron source in water, concrete, and the hydrocarbon and boron mixture ${ }^{47}$ in Fig. 8.12. Concrete does not thermalize neutrons as rapidly as water, and it is also a better gamma-ray attenuator. The effect of the boron is evident in the lower curve.

The effect of water content in concrete on the neutron-dose attenuation can be seen in Fig. 8.14 for concretes A, B, C, and D. ${ }^{47}$ The compositions are given in Table 8.6. Concrete type $A$ is called magnetite $A$ in Volume III of the Reactor Handbook. ${ }^{49}$ Concretes B, C, and D are the same concrete with three-fourths, one-half, and one-fourth the water content of concrete A, respectively. The effect of decreasing water content on the dose rates, all shown as solid lines in the figure, is very pronounced. The limonite concrete (E) has a relatively low density but a high water content; dose rates 


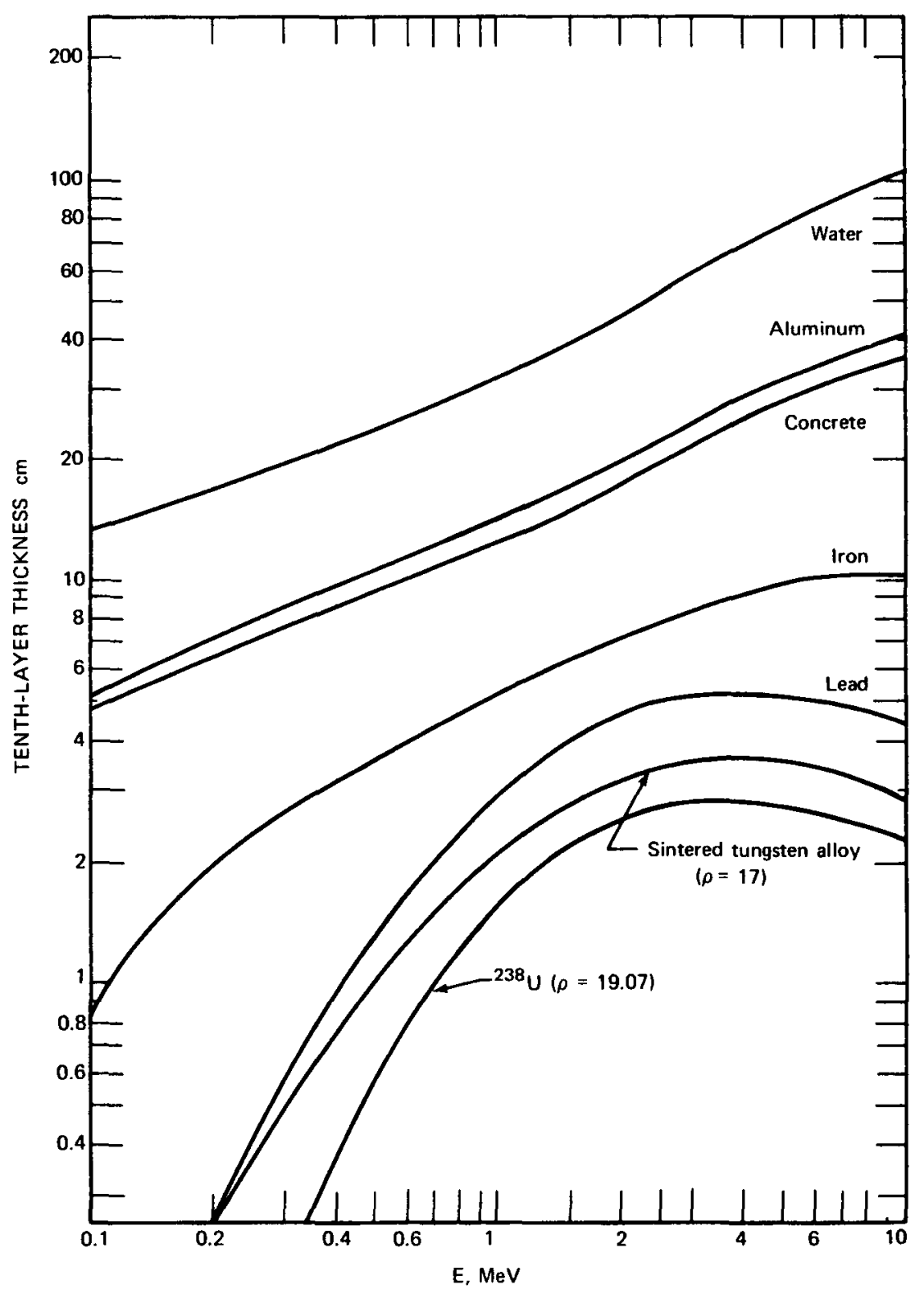

Fig. 8.11 -Narrow-beam gamma-ray absorption for various materials. 


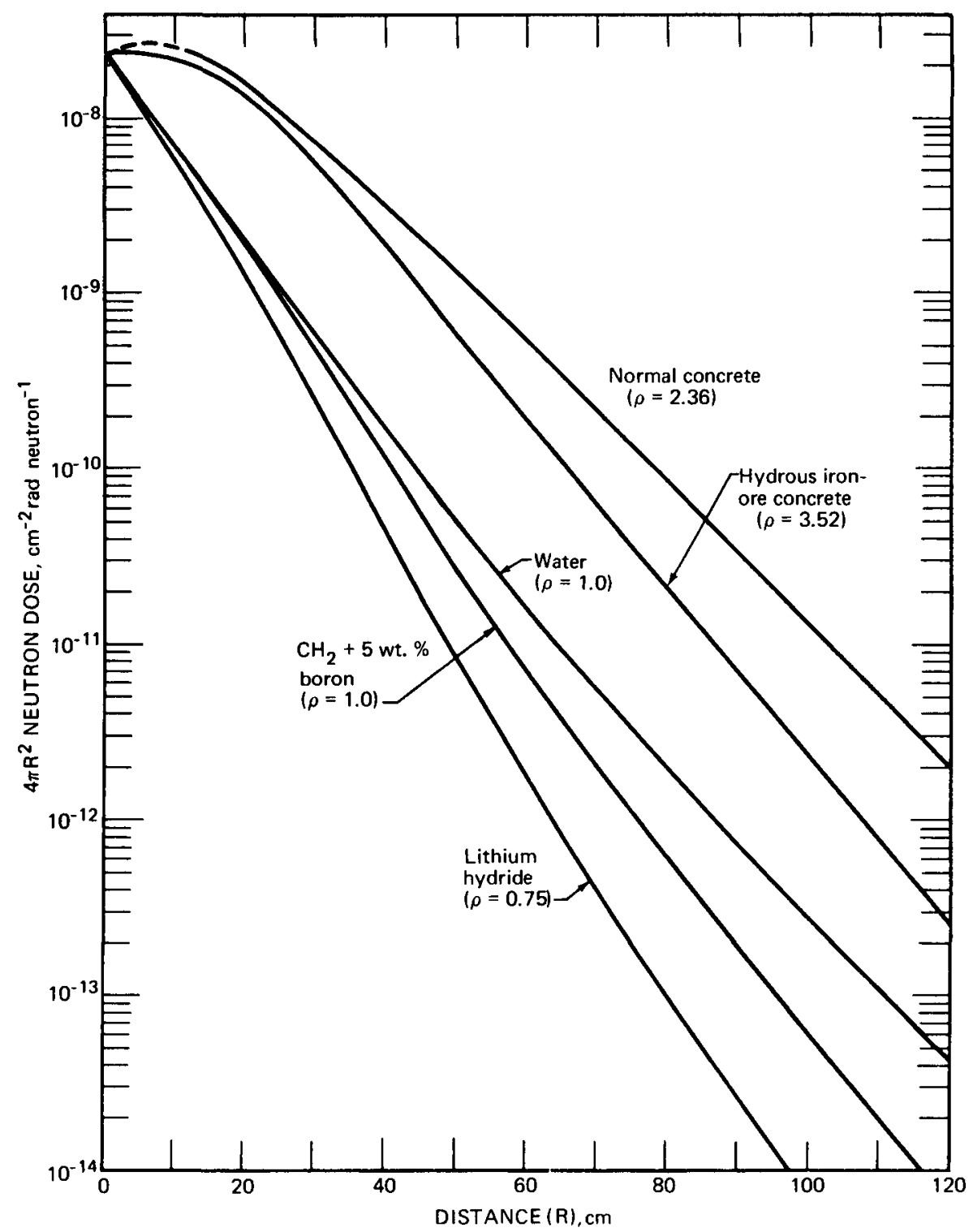

Fig. 8.12-Neutron dose vs. depth in the indicated materials fission-neutron source. (Based on data from Nichols ${ }^{47}$ and Kam and Clark. ${ }^{48}$ ) 
Table 8.6-ELEMENTAL DENSITY $\left(\mathrm{g} / \mathrm{cm}^{3}\right)$ OF CONCRETES ASSUMED FOR SHIELD ANALYSIS $\dagger$

\begin{tabular}{|c|c|c|c|c|c|c|c|c|}
\hline \multirow[b]{2}{*}{ Element } & \multicolumn{8}{|c|}{ Type of concrete } \\
\hline & $\begin{array}{l}\text { A } \\
\text { Hydrous } \\
\text { iron ore }\end{array}$ & $\begin{array}{l}\text { B } \\
\text { Hydrous } \\
\text { iron ore }\end{array}$ & $\begin{array}{l}\text { C } \\
\text { Hydrous } \\
\text { iron ore }\end{array}$ & $\begin{array}{l}\text { D } \\
\text { Hydrous } \\
\text { iron ore }\end{array}$ & $\begin{array}{c}E \\
\text { Limonite }\end{array}$ & $\begin{array}{c}\text { F } \\
\text { Ferrophosphate- } \\
\text { serpentine }\end{array}$ & $\begin{array}{c}\text { G } \\
\text { Magnetite- } \\
\text { serpentine }\end{array}$ & $\begin{array}{c}\mathrm{H} \\
\text { Normal }\end{array}$ \\
\hline $\mathrm{H}$ & 0.0215 & 0.0160 & 0.0108 & 0.00538 & 0.0370 & 0.0208 & 0.0122 & 0.0200 \\
\hline $\bar{O}$ & 1.304 & 1.261 & 1.218 & 1.176 & 1.113 & 0.7805 & 1.180 & 1.116 \\
\hline$\overline{\mathrm{Fe}}, \mathrm{Cr}, \mathrm{Mn}, \mathrm{V}$ & 1.960 & 1.960 & 1.960 & 1.960 & 1.189 & 1.355 & 1.181 & \\
\hline$\overline{\mathrm{Si}}, \mathrm{Al}, \mathrm{Mg}, \mathrm{S}, \mathrm{P}$ & 0.108 & 0.108 & 0.108 & 0.108 & 0.165 & 1.044 & 0.457 & 0.491 \\
\hline \multirow{2}{*}{$\frac{\mathrm{Ca}}{\mathrm{C}}, \mathrm{Ti}$} & 0.128 & 0.128 & 0.128 & 0.128 & 0.227 & 0.196 & 0.458 & 0.612 \\
\hline & & & & & & & & 0.118 \\
\hline Total & 3.522 & 3.473 & 3.425 & 3.377 & 2.731 & 3.396 & 3.288 & 2.357 \\
\hline
\end{tabular}

†From Nichols. ${ }^{4} 7$

$\$$ Cross sections for the underlined elements were used in the fast-neutron dose calculation. 


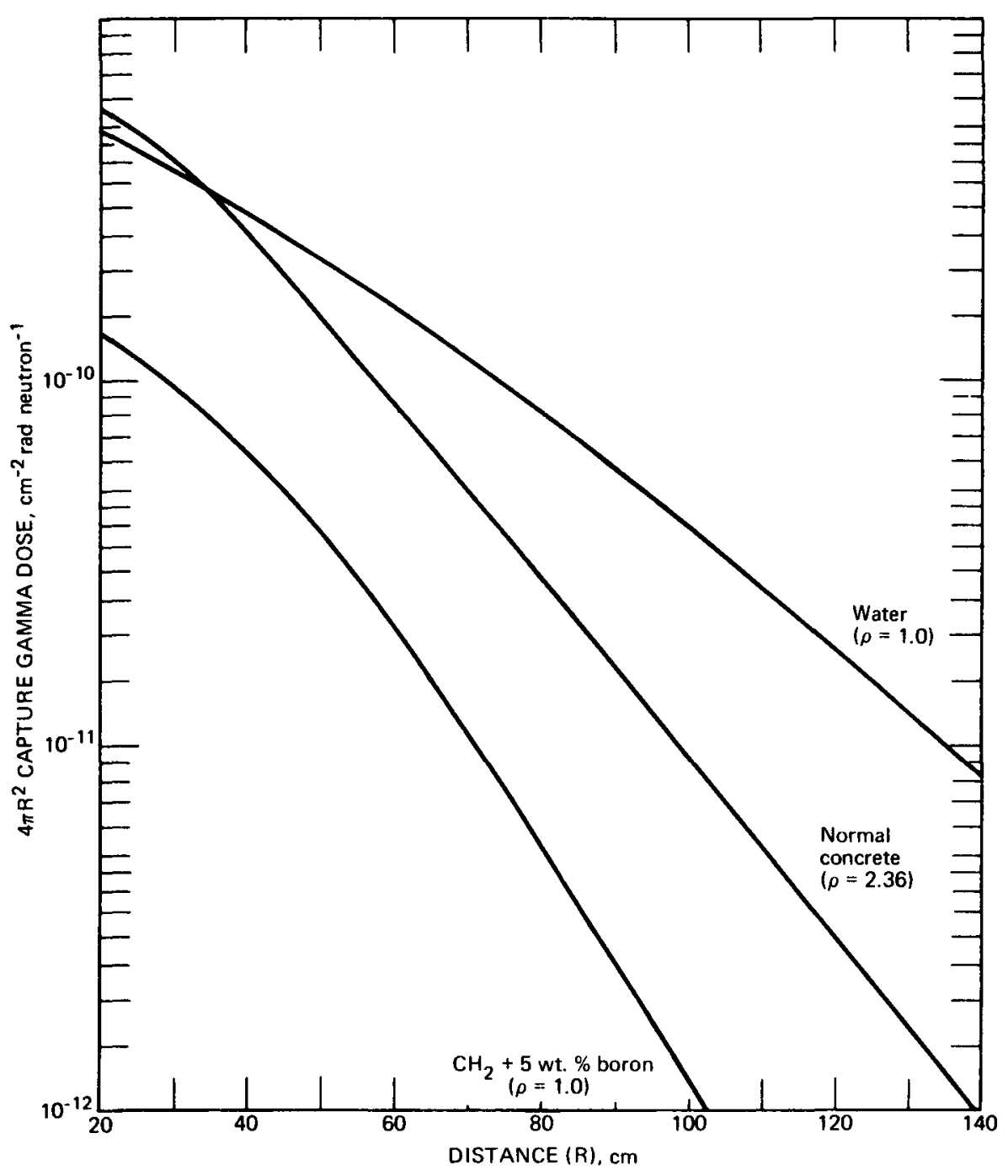

Fig. 8.13 -Capture-gamma-ray dose for the indicated materials fission-neutron source.

are approximately the same as those for concrete $A$. Concretes $F$ and $G$ are fictitious mixtures of aggregates.

Figure 8.15 shows the effect, from an analysis by Lahti, ${ }^{50}$ of placing various thicknesses of tungsten between the fission-neutron source and the lithium hydride medium. The data show the neutron dose as a function of distance in natural lithium hydride after first passing through the indicated thicknesses of tungsten. Even though tungsten is a heavy material, it provides good neutron attenuation per unit thickness. 


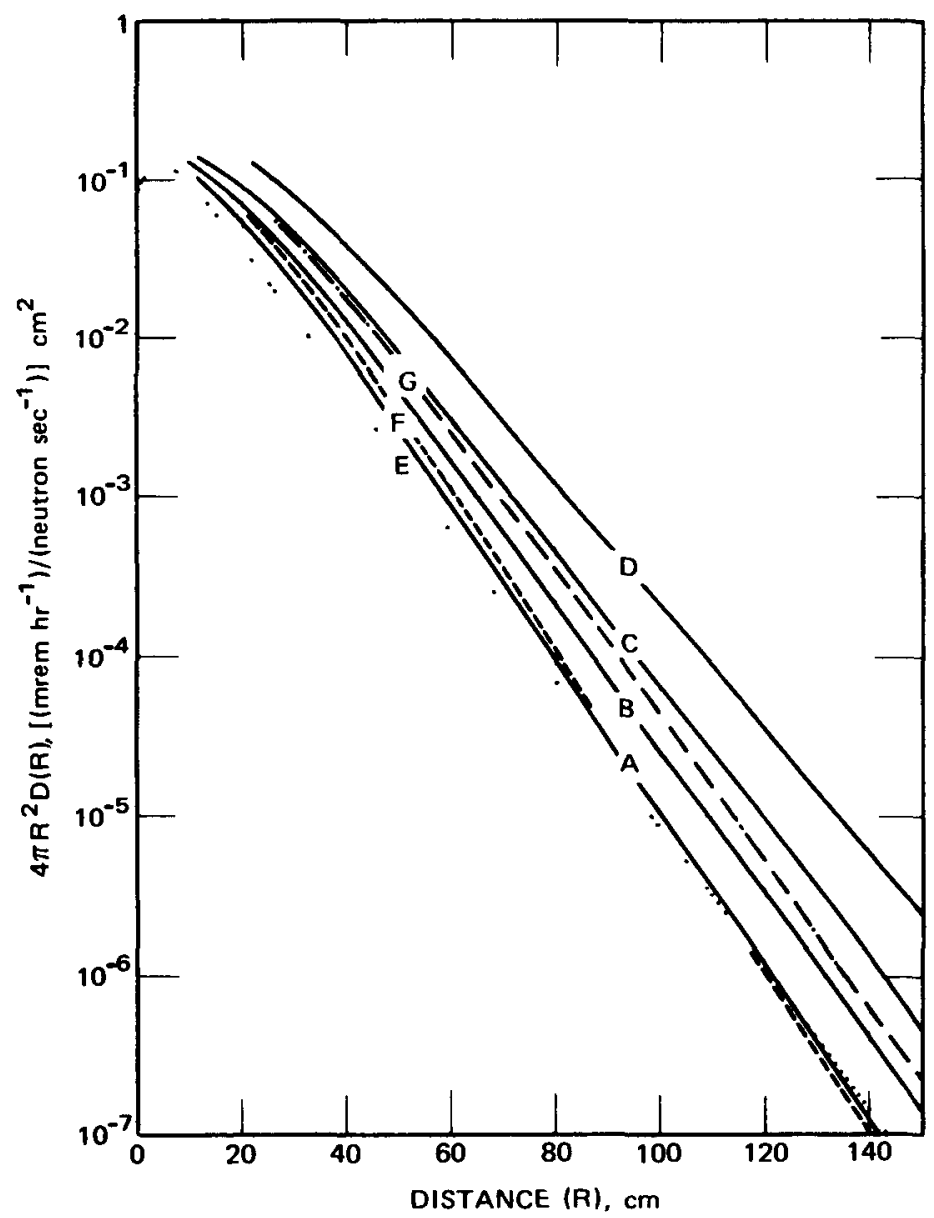

Fig. 8.14-Fast-neutron dose rate in various concretes as a function of the distance from a unit point isotropic fission source of ${ }^{235} \mathrm{U}$. (From Nichols. ${ }^{47}$ )

$\begin{array}{ccc}\text { Density, } & \begin{array}{c}\text { Hydrogen } \\ \text { content, } \\ \mathrm{g} / \mathrm{cm}^{3}\end{array}\end{array}$
A Hydrous iron ore
$3.52 \quad 0.022$
B Hydrous iron ore
3.47
0.016
C Hydrous iron ore
3.42
0.011
D Hydrous iron ore
3.38
0.0054
E Limonite
2.73
0.037
F Ferrophosphate- serpentine
3.40
0.021
G Magnetite- serpentine
3.29
0.020 


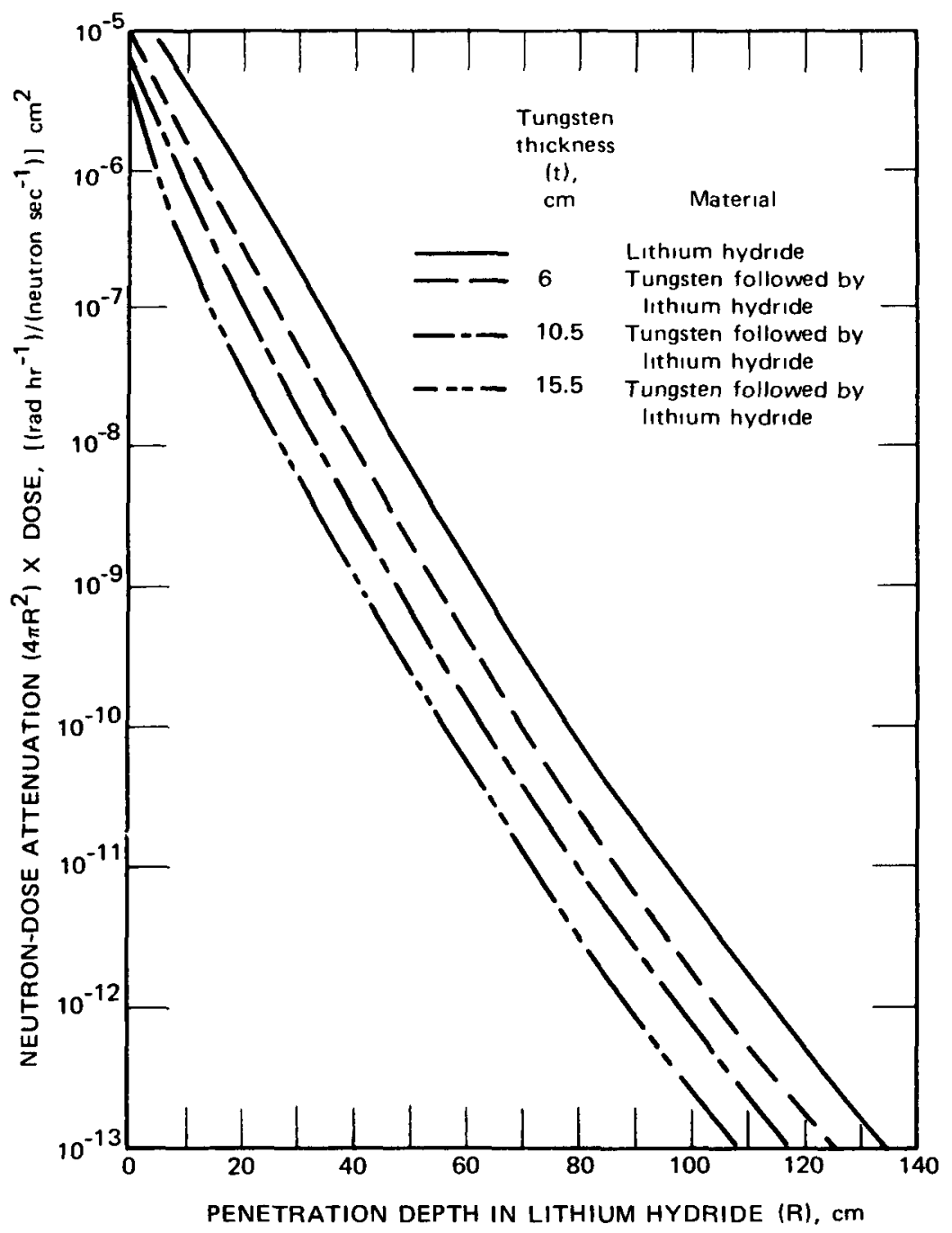

Fig. 8.15-Discrete-ordinates calculation of fission neutrons in tungsten followed by lithium hydride (From Lahti. ${ }^{50}$ )

\subsection{SHIELD OPTIMIZATION}

The problem of finding the optimum materials arrangement for a shield that satisfies a given set of design criteria is the most intriguing in the area of reactor shielding. It has challenged many investigators, ${ }^{4-60}$ and, to this writing, a general solution has not been found and may not be. Assuredly, a few workable schemes have been developed, and each study has contributed 
some insight to the subject. A few have published evidence (parameter studies or claimed weight savings) intended to substantiate their methods. The complex of phenomena involved in expressing the performance of the shield invariably requires simplifying or limiting assumptions that detract from the result. The problem intrigues the shield designer for several reasons. The classical mathematical analyses of Lagrange and Euler give us the guidelines for deriving the optimum conditions. Variational calculus and the relations for determining stationary values of an integral are the usual starting point. Since shield weight is usually a significant fraction of the total system weight, the potential for gain seems large. Even in systems where weight is not important, optimization with respect to cost is important and provides ample incentive for pursuing the problem.

Most frequently optimization is with respect to weight. Other conditions that must be met, such as volume restrictions, dose-rate requirements, or number of shield layers, are called constraints. These constraints, together with the boundary conditions, help determine the parameters arising from the optimization procedure.

The optimization proceeds in at least two phases. The first is to apply the necessary conditions for a minimum value of the weight function. The analogue in elementary calculus is to find the stationary values of the function $y(x)$. The function has a stationary or extremum value (both include maxima and minima) at $x=a$ if $y^{\prime}(a)=0$. To find the value $a$, one simply takes the derivative of $y$ with respect to $x$, sets it equal to zero, and solves for $a$.

The second phase is to determine the sufficient conditions for a minimum as opposed to a maximum or a local minimum (saddle point). This second phase usually involves proof of the existence of the minimum. To complete the analogy with differential calculus, the sufficient condition that $y^{\prime}(a)$ is a minimum is that $y^{\prime \prime}(a)>0$ [for a maximum, that $\left.y^{\prime \prime}(a)<0\right]$ and that $y^{\prime}(x)$ is continuous in the neighborhood of $x=a$.

The variational approach to shield optimization involves the generalization of these simple conditions to apply to functions of several variables with accessory constraint conditions. The variational problem is to find a function such that a related definite integral has a minimum value.

Troubetzkoy ${ }^{56}$ provides a recent example of optimization work done in this country in a method called shield synthesis. His objective was to devise a

\footnotetext{
†Variational calculus, Lagrange's method of undetermined multipliers, and Euler's equation are found in texts on mathematical physics, for example, Morgenau and Murphy ${ }^{61}$ or Butkov. ${ }^{62}$
} 
design technique that yields the selection and arrangement of materials giving minimum weight for a spherical shield given reactor power and total dose. He uses simplified models for neutron and gamma-ray attenuation and secondary gamma-ray production. Application of the variational conditions to the model leads to restrictions on the choice of possible materials and the relation of materials in adjacent layers. He concludes that the innermost and outermost of $n$ arbitrary concentric layers must be pure materials; any other layer may contain a radius-dependent mixture of no more than two materials. Regions adjacent to such mixtures must be pure materials identical to one of the two components of the mixture. Troubetzkoy states that the approximations due to the simplified attenuation model can be offset by performing an exact numerical analysis of the shield (presumably by Monte Carlo or discrete ordinates) to obtain its performance to a better accuracy. He reports that shield synthesis gives considerable weight savings over more conventional designs.

This approach yields a procedure for greatly narrowing the choice of materials and mixtures that can be used. The approximation due to assumptions of simplified, manageable attenuation models does not affect the accuracy of the design of the shield, but it probably does affect the determination of the optimum. However, studies have shown that these minima are usually rather broad; thus, if one is indeed near the actual optimum, most of the potential weight saving has been realized.

So argue the Russian authors Suvorov and Fedorenko, ${ }^{60}$ who have recently reported a somewhat different approach to optimization of a reactor shield. They also use a variational approach but determine the optimum variations of the location of heterogeneous layers with constant concentrations of material within each layer. Theirs is also a one-dimensional approach (using a cylindrical shield), but they use linear perturbation theory. By altering the number of layers and the choice of materials, they are able to compare minima so derived to observe the effect of these choices. It is noteworthy that their test for having reached an optimum set of thickness is that comparable variations in each layer give equal variations in the dose outside the shield. This technique enables them to show the effect of adding boron to an iron-water (or lead-water) shield either as a homogeneous mixture or constrained as additional layers. They also show the advantage of a ten-layer two-material shield over a five-layer shield of the same materials. This technique uses a five-group neutron attenuation in a $P_{1}$ approximation, but exponential gamma-ray attenuation using infinitemedium buildup factors. Neutron-produced secondaries are added to the 
primary gamma-ray distribution. Weight savings of the order of 20 to $40 \%$ are shown over straightforward designs. From the published description of the technique, it is evident that a great deal of high-speed computer time is required for applications of this method.

The Troubetzkoy method clearly gives more guidance in the selection of materials, but the Suvorov-Fedorenko method would seem to give more realistic results.

Sheffield ${ }^{53}$ and Miller and Cranford ${ }^{54}$ in an earlier study used gradient nonlinear programming to obtain minimum weights of divided crew shield-reactor shield geometries appropriate to aircraft or rockets. In this case the dose in the crew compartment is the primary constraint, and the effect of air transport from reactor shield to crew compartment must be included in the optimization procedures. This method places emphasis on the shape of the reactor shield rather than on the number of layers or composition. It is interesting that the optimization procedure in one phase on the computer is to test at each step for the most effective sector on the reactor or crew compartment to place a given increment of shield material. The increment is then placed where it gives the greatest reduction in dose.

Aronson, Klahr, Steinberg, and Held ${ }^{5}$ developed a procedure for optimizing the placement of shadow shields (shield splitting) in space vehicles. In a paper given at a conference in Harwell, Enginol ${ }^{58}$ describes an interesting variational technique that can use any available method for computing the attenuation through the shield and optimizes with respect to cost or weight or any linear combination of parameters.

Another recent program, ASOP, performs detailed one-dimensional discrete-ordinate transport calculations with ANISN (described in Chap. 4) for each step in the iterative optimization. ${ }^{63}$ This feature distinguishes ASOP from its forerunners since others involve a simplified transport approximation. This treatment leads to significant improvements in the optimization technique since weight savings of $25 \%$ are estimated. ${ }^{64}$

\section{REFERENCES}

1. H. C. Claiborne, Survey of Methods for Calculating Gamma-Ray Heating, USAEC Report ORNL-RSIC-8, Oak Ridge National Laboratory, June 1965.

2. H. Goldstein and J. E. Wilkins, Jr., Calculations of the Penetrations of Gamma Rays, Final Report, USAEC Report NYO-3075 (NDA-15C-41), Nuclear Development Associates, Inc., June 30, 1954. 
3. H. Goldstein, Fundamental Aspects of Reactor Shielding, p. 221, Addison-Wesley Publishing Company, Inc., Reading, Mass., 1959 (out of print); now available from Johnson Reprint Corporation, New York.

4. L. A. Bowman and D. K. Trubey, Deposition of Gamma-Ray Heating in Stratified Lead and Water Slabs, USAEC Report CF-58-7-99, Oak Ridge National Laboratory, July $28,1958$.

5. M. L. Tobias, D. R. Vondy, and M. P. Lietzke, Nightmare -an IBM 7090 Code for the Calculation of Gamma Heating in Cylindrical Geometry, USAEC Report ORNL-3198, Oak Ridge National Laboratory, Feb. 26, 1962.

6. M. A. Capo, Polynomial Approximation of Gamma-Ray Buildup Factors for a Point Isotropic Source, USAEC Report APEX-510, General Electric Company, August 1959.

7. R. L. Walker and M. Grotenhuis, A Summary of Shielding Constants for Concrete, USAEC Report ANL-6443, Argonne National Laboratory, November 1961.

8. D. K. Trubey, Gamma-Ray Buildup Factor Coefficients for Concrete and Other Materials, Nucl. Appl. Technol., 9: 439 (170).

9. C. R. Greenhow, G. O. Mueller, and G. E. Sabian, Use of Diffusion Theory Codes to Predict Gamma Heating, USAEC Report KAPL-M-DNA-5, Knolls Atomic Power Laboratory, 1963.

10. R. L. French, Reactor Shield Heating Calculations from Gamma Rays, USAEC file number NP-9354(FZM-1076), Convair, June 1958.

11. K. D. Lathrop, DTF-IV, A Fortran-IV Program for Solving the Multigroup Transport Equation with Anisotropic Scattering, USAEC Report LA-3373, Los Alamos Scientific Laboratory, July 15, 1965.

12. W. W. Engle, Jr., A Users Manual for ANISN, A One-Dimensional Discrete Ordinates Transport Code with Anisotropic Scattering, USAEC Report K-1693, Union Carbide Corporation, Mar. 30, 1967.

13. F. R. Mynatt, F. J. Muckenthaler, and P. N. Stevens, Development of Two-Dimensional Discrete Ordinates Transport Theory for Radiation Shielding, USAEC Report CTC-INF-52, Union Carbide Corporation, August 1969.

14. D. K. Trubey, S. K. Penny, and K. D. Lathrop, A Comparison of Three Methods Used to Calculate Gamma-Ray Transport in Iron, USAEC Report ORNL-RSIC-9, Oak Ridge National Laboratory, 1965.

15. J. J. Loechler and J. E. MacDonald, Flexible Monte Carlo Programs FMC-N and FMC-G, USAEC Report APEX-706, General Electric Company, Apr. 28, 1961.

16. A. Foderaro, L. J. Hoover, and J. H. Marable, Heat Generation by Neutrons, in Engineering Compendium on Radiation Shielding, Sec.7.2, R. G. Jaeger (Ed.), Springer-Verlag, New York, 1968.

17. C. N. Klahr, Heat Generation in Shields, in Reactor Handbook, 2nd ed., Vol. III, Part B, Shielding, E. P. Blizard (Ed.), Interscience Publishers, a division of John Wiley \& Sons, Inc., New York, 1962.

18. R. E. Lynch, J. W. Benoit, W. P. Johnson, and C. D. Zerby, A Monte Carlo Calculation of Air-Scattered Gamma Rays, USAEC Report ORNL-2292 (Vol. I), Oak Ridge National Laboratory, Sept. 10, 1958.

19. M. B. Wells, Studies in Shielding-III. Monte Carlo Calculations of Neutron Scattering in Air, Report NARF-57-49-T (FZK-9-120), Convair, Nov. 4, 1957. 
20. C. D. Zerby, A Monte Carlo Calculation of Air-Scattered Neutrons, USAEC Report ORNL-2277, Oak Ridge National Laboratory, May 24, 1957.

21. D. K. Trubey, The Single-Scattering Approximation to the Solution of the Gamma-Ray Air-Scattering Problem, Nucl. Sci. Eng., 10: 102-116 (1961).

22. E. A. Straker and M. L. Gritzner, Neutron and Secondary Gamma-Ray Transport in Infinite Homogeneous Air, USAEC Report ORNL-4464, Oak Ridge National Laboratory, December 1969.

23. J. R. Keith and F. H. Shelton, 14-MeV Neutron Transport Results, Report KN-774-68-1, Kaman Aircraft Corporation, September 1968.

24. W. M. Webster, Neutron Air Transport Calculations, USAEC Report UCRL-50570, Lawrence Radiation Laboratory, January 1969.

25. R. E. Vogel, A Fortran II Program for the Calculation of the Transport of Gamma Rays by the Method of Moments, Report KN-63-76(R), Kaman Aircraft Corporation, June 1, 1963.

26. J. D. Marshall and M. B. Wells, The Effect of Cutoff Energy on Monte Carlo Calculated Gamma-Ray Dose Rates in Air, USAEC Report RRA-M67, Radiation Research Associates, Inc., Oct. 31, 1966.

27. N. M. Schaeffer, Energy and Angle Distributions in Air for Gamma Rays, in Engineering Compendium on Radiation Shielding, Vol. I, Sec. 4.5.2.1, R. G. Jaeger (Ed.), Springer-Verlag, New York, 1968.

28. C. D. Zerby, Radiation Flux Transformation as a Function of Density of an Infinite Medium with Anisotropic Point Sources, USAEC Report ORNL-2100, Oak Ridge National Laboratory, Oct. 9, 1956.

29. M. J. Berger, Calculation of Energy Dissipation by Gamma Radiation Near the Interface Between Two Media, J. Appl. Phys., 28: 1502 (1957).

30. M. B. Wells, Air and Concrete Scattering of Gamma Rays, Report NARF-59-11T(MR-N-229), Convair, Mar. 20, 1959.

31. M. B. Wells, Air and Concrete Scattering of Neutrons. Monte Carlo Calculations, Report NARF-58-15T(MR-N-197), Convair, Apr. 2, 1958.

32. J. I. Marcum, Monte Carlo Calculations of the Transport of 14-MeV Neutrons in the Atmosphere, Report RM-3531-PR, RAND Corporation, 1963.

33. F. F. Haywood, T. G. Provenzano, and J. A. Auxier (Eds.), Operations Plan-Operation HENRE, USAEC Report CEX-65.03, Oak Ridge National Laboratory, 1965.

34. A. E. Fritzsche, N. E. Lorimier, and Z. G. Burson, Measured High-Altitude Neutron and Gamma Dose Distributions Due to a 14-MeV Neutron Source, USAEC Report EGG-1183-1438, EG\&G, May 1969.

35. A. E. Fritzsche, N. E. Lorimier, and Z. G. Burson, Measured Low-Altitude Neutron and Gamma Dose Distributions Due to a 14-MeV Neutron Source, USAEC Report EGG-1183-1449, EG\&G, Aug. 21, 1969.

36. R. L. French, A First-Last Collision Model of the Air/Ground Interface Effects on Fast-Neutron Distributions, Nucl. Sci. Eng., 19: 151-157 (1964).

37. H. E. Hungerford, The Nuclear. Physical and Mechanical Properties of Shielding Materials, in Reactor Handbook, Vol. I, Materials, C. R. Tipton, Jr. (Ed.), Chap. 51, Interscience Publishers, a division of John Wiley \& Sons, Inc., New York, 1960. 
38. A. N. Komarovskii, Design of Nuclear Plants, translated from Storoitel'stvo Yadernykh Ustanovok, Atomizdat, Moscow, 1965 (Russian), USAEC Report AEC-tr-6722, 1965.

39. H. S. Davis, How to Choose and Place Mixes for High-Density Concrete Reactor Shields, Nucleonics, 13: 60 (June 1955).

40. R. L. Walker and M. Grotenhuis, Concrete Shielding Constants, Nucleonics, 20: 141 (1962).

41. Oak Ridge National Laboratory, Compilation of Data on Experimental Shielding Facilities and Tests of Shields of Operating Reactors, USAEC Report ORNL-RSIC-24, November 1968.

42. H. M. Glen, Materials of Biological Shielding, American Society of Mechanical Engineers Paper No. 57-NESC-51, March 1957.

43. W. Q. Hullings, Materials Data for Nuclear Systems, Report NARF-61-24T (FZK-9-162), General Dynamics/Fort Worth, July 1961.

44. F. P. Roberts and H. H. Van Tuyl, Chemical Analysis of Spent Power Reactor Fuels, USAEC Report BNWL-SA-503, Battelle-Northwest, Dec. 27, 1965.

45. A. Shapiro, Radiation Chemistry of Polymeric Systems, Vol. 15, High Polymer Series, Interscience Publishers, a division of John Wiley \& Sons, Inc., New York, 1962.

46. R. Van Houten and W. G. Baxter, High Temperature-High Hydrogen Content Shield Materials, Transactions of the American Nuclear Society Topical Meeting, Nuclear Materials for Space Application, Cincinnati, Ohio, April 1963.

47. J. P. Nichols, Calculations of Concrete Shields for Fission Sources, USAEC Report ORNL-TM-1167, Oak Ridge National Laboratory, June 17, 1965.

48. F. B. F. Kam and F. H. S. Clark, Fission Neutron Attenuation and Gamma-Ray Buildup Factors for Lithium Hydride, Nucl. Appl. Technol., 3: 433-435 (July 1967).

49. E. P. Blizard (Ed.), Reactor Handbook, 2nd ed., Vol. III, Part B, Shielding, Interscience Publishers, a division of John Wiley \& Sons, Inc., New York, 1962.

50. Gerald P. Lahti, Fission Neutron Attenuation in Lithium-6, Natural Lithium Hydride, and Tungsten, Report NASA-TN-D-4684, National Aeronautics and Space Administration, 1968.

51. H. Goldstein and E. P. Blizard, A Criterion for the Experimental Optimization of Two-Component Unit Shields, USAEC Report TID-5018, Nuclear Development Associates, Inc., May 10, 1951.

52. H. Hurwitz, Jr., Note on a Theory of Minimum Weight Shields, USAEC Report KAPL-1441, Knolls Atomic Power Laboratory, 1959.

53. R. D. Sheffield, Shield System Optimization_Gradient Nonlinear Programming, Report NARF-57-62T(MR-N-207), Convair, 1957.

54. R. A. Miller and W. Cranford, Shield System Optimization, Report NARF-58-24T(MR-N-207), Convair, June 16, 1958.

55. R. Aronson, C. Klahr, H. Steinberg, and K. Held, Effect of Shield Splitting and Placement in Nuclear-Propelled Space Vehicles, Report N-65-22849 (NASA-CR-62456; TRG-136-FR), Technical Research Group, Inc., Mar. 1, 1961.

56. E. S. Troubetzkoy, Minimum Weight Shield Synthesis, USAEC Report UNC-5017(Pt. A), United Nuclear Corporation, Oct. 15, 1962. 
57. S. Sasse, Weight Optimization for Radiation Shields of Large Nuclear Reactors, USAEC Report ORNL-tr-1525, translated from Report ABS-THH-1023 (German), Oak Ridge National Laboratory, 1966.

58. T. B. Enginol, A Procedure for Shield Optimization, in Proceedings of the Conference on the Physics Problems of Reactor Shielding, British Report AERE-R-5773 (Vol. 5), pp. 1269-1287, May 1968.

59. G. P. Lahti and P. F. Hermann, Comparison of Tungsten and Depleted Uranium in Minimum-Weight, Layered Shields for a Space Power Reactor, Report NASA-TM-X-1874 (N69-35817), National Aeronautics and Space Administration, 1969.

60. A. P. Suvorov and R. P. Fedorenko, Selection of Optimum Metal-Water Shielding for Reactors, Problems in the Physics of Reactor Shielding, No. 3, D. L. Broder, A. P. Suvorov, and S..G. Tsypin (Eds.), Report JPRS-49745, Joint Publications Research Service, Superintendent of Documents, U.S. Government Printing Office, Washington, 1970.

61. H. Morgenau and G. M. Murphy, The Mathematics of Physics and Chemistry, 2nd ed., D. Van Nostrand Company, Inc., Princeton, N. J., 1956.

62. E. Butkov, Mathematical Physics, Addison-Wesley Publishing Company, Inc., Reading, Mass., 1968.

63. W. W. Engle, Jr., A Users Manual for ASOP ANISN Shield Optimization Program. USAEC Report CTC-INF-941, Union Carbide Corporation, Sept. 8, 1969.

64. W. W. Engle, Jr., and F. R. Mynatt, Development of One-Dimensional Shield Optimization Techniques, in Neutron Physics Division Annual Progress Report for Period Ending May 31, 1968, USAEC Report ORNL-4280, Oak Ridge National Laboratory, 1968. 
$\delta$

ค 


\section{Experimental Shielding}

Mensuration marks the first and last steps in executing a shield system. Not that the shield designer starts his task with a dosimeter, but he does start with a measured fission spectrum and a large body of measured cross sections. The final step in shield design consists in a radiation survey of the completed system to verify that the design objectives have been met. Furthermore, all the steps between the first and last are based in some manner on previous measurements, either by direct input of empirical data or by use of an analytical method that has withstood the test of experimental verification. So it is with any technology; no uniqueness claim can be made for shielding, only that this technology is relatively young and still has very close ties with experiment. Our dependence on measurement is evident; key experimental results have been quoted in each topic where they substantiate a method or provide needed input. In this chapter the presentation of shielding data is incidental; our purpose rather is to convey something of the flavor of experimental shielding by describing a selection of measurements and facilities chosen to represent the range of these investigations.

Radiation detectors are the tools of this trade, and our discussion will begin with a brief description of them. Shielding experiments may approximately be classified in three categories: materials properties, phenomenological studies, and shield-system tests. This is an arbitrary, nonexclusive classification. Some experiments could obviously be classified in more than one of the three. Investigations of shield materials include those studies whose objective is to characterize the transport properties of bulk materials or combinations of materials. These include slab penetration measurements and cross-section studies. Phenomenological studies involve a particular geometry; they include air-transport, air-ground interface, albedo, and duct-penetration studies. Shield-system studies include mock-up experi- 
ments and proof tests. The system test, or mock-up, is always connected to a particular design; the other two types of experiments usually have more general applicability. Shield-system tests are discussed in Chap. 10 (Shield Design), where the tests are described in the context of their related designs.

It is evident that an experiment in any one of our three classes might serve several purposes. As already stated, some quantities have not been predicted and have only been obtained by measurement. Some geometries are sufficiently complex to defy accurate analytical description, and some experiments are performed to verify a calculation or a design method. These various purposes will be clarified by the description of experiments to follow.

\subsection{DETECTORS FOR SHIELDING EXPERIMENTS}

Measurements have meaning only to the extent that detector outputs can be interpreted. Thus it is imperative that the user be familiar with the response, intensity range of operation, general characteristics, and limitations of the detector used. In the following sections, we discuss the detectors commonly used in shielding measurements. References 1 through 6 contain more complete descriptions.

\subsubsection{Active Neutron Detectors}

Two types of detectors are used to determine the neutron environment: (1) active electronic detectors, which provide data directly while the neutron source is operating, and (2) passive detectors, which are installed before an experiment, exposed to neutrons, then retrieved and read after the source has ceased operating.

The Hurst fast-neutron dosimeter is a proportional counter consisting of a cylindrical chamber lined with polyethylene and containing a hydrogenous gas, usually methane. The counter is operated with a pulse-height weighting circuit. Since the proton-recoil pulse-height distribution depends on incident-neutron energy, proper pulse weighting produces a total count proportional to fast-neutron kerma in the counter material. A low-energypulse bias voltage (corresponding to 100 - to $200-\mathrm{keV}$ neutron energy) is necessary to reject gamma-ray pulses in a mixed neutron and gamma-ray field. The bias succeeds in rejecting gamma-ray pulses below 10 to $20 \mathrm{R} / \mathrm{hr}$ exposures. Above this level, gamma-ray sensitivity must be corrected. Small neutron sources are used for pretest and posttest calibration. Neutron 
sensitivity ranges from $0.5 \mathrm{mrad} / \mathrm{hr}$ to $0.5 \mathrm{rad} / \mathrm{hr}$. Special pulse-counting procedures have been used to extend sensitivity to lower intensities.

Fission chambers are ionization chambers coated with ${ }^{237} \mathrm{~Np},{ }^{238} \mathrm{U}$, ${ }^{232} \mathrm{Th},{ }^{239} \mathrm{Pu}$, or ${ }^{235} \mathrm{U}$. The first four are used for counters which respond only to neutrons whose energy is above their fast-fission threshold $(0.6,1.5$, 1.75 , and $0.01 \mathrm{MeV}$, respectively) and which measure the total flux above that threshold. There are two problems: thermal-neutron reactions in the other contaminating isotopes normally found with these isotopes (even a few parts per million of ${ }^{235} \mathrm{U}$ in ${ }^{238} \mathrm{U}$ can be troublesome) and with gamma-ray sensitivity. Frequently, data are obtained with bare and cadmium-covered ${ }^{235} \mathrm{U}$ chambers to provide an indication of the thermal-neutron flux (actually subcadmium) rather than an integral over all energies. Fission chambers can be compensated for gamma rays by incorporating two regions: one containing no fissionable material and thus responding only to gamma rays and the other containing fissionable material and responding to both neutrons and gamma rays. The difference in output of the two regions is therefore proportional to the neutron fluence.

Detectors containing boron either in the form of a gas (usually boron trifluoride) or coated on the walls of the chamber are widely used. A matched set of bare and cadmium-covered detectors provides subcadmium $\left(E_{\mathrm{n}}<0.4 \mathrm{eV}\right)$ or thermal-neutron flux via the ${ }^{10} \mathrm{~B}(n, \alpha)$ reaction. Gamma-ray compensation also can be used with these detectors. Sensitivity is varied by varying the gas pressure in the initial filling, by varying the enrichment of the boron isotope, by varying coating thickness, or by varying the size of the detector. Boron detectors can be operated in either the pulse mode or the current mode with the latter having the advantage of giving essentially instantaneous readout. The current mode is particularly helpful when a spatial traverse is being made since detector output can be monitored with a chart recorder showing detector output vs. a position variable. A considerable variety of boron chambers are commercially available.

Helium-3-filled detectors take advantage of the large $(n, p)$ cross section of ${ }^{3} \mathrm{He}$ to measure neutron flux. Fairly elaborate electronics are required to convert the out put to a flux, but the detector is quite sensitive.

A number of recently developed liquid scintillators, such as NE-211 and 213, can be used to provide neutron spectra if appropriate shielding and collimation are provided around the detector as well as appropriate electronics and response functions for converting the output to a true spectrum. 
Much interest has developed in recent years in the use of solid-state detectors, such as lithium-drifted silicon and lithium-drifted germanium, for measurements of neutron spectra, flux, and dose. These detectors generally have good resolutions but low efficiency. They make use of such neutron reactions as ${ }^{3} \mathrm{He}(n, p),{ }^{1} \mathrm{H}(n, n){ }^{1} \mathrm{H},{ }^{6} \mathrm{Li}(n, \alpha)$, and ${ }^{10} \mathrm{~B}(n, \alpha)$. The addition of drifted lithium to silicon and germanium increases the detection efficiency for high-energy neutrons. Solid-state detectors are sensitive to light, X rays, gamma rays, and charged particles; thus they must be adequately shielded and compensated.

\subsubsection{Passive Neutron Detectors}

A number of passive detectors, nonelectronic devices, are commercially available for the measurement of neutron intensities. These detectors can be placed within a shield, exposed to neutrons, recovered, and later read to determine the neutron intensity at the time of exposure. These devices have the advantage of small size, and they can be located in otherwise difficult-to-monitor situations. Among them are the following:

Small thin specimens (foils) of a great variety of materials become radioactive when exposed to neutrons. If the composition and mass of the foil are known, as well as the half-life, cross section, decay energy of the radioactive species, the irradiation interval, and the specific activity of the foil (disintegrations per second per gram), one can determine the neutron flux. Some foils respond primarily to low-energy neutrons (gold and dysprosium), some to resonance-energy neutrons, and some only to neutrons above a threshold energy. Foils necessarily must be of high purity and have half-lives neither too long nor too short. In general, they are reusable. Many of the fission foils must be enclosed in boron to eliminate thermal-neutroninduced response.

Several $p-n$ junction (solid-state) devices have been developed in which neutrons change the electrical characteristics in a measurable and reproducible fashion. Most of these are cumulative (i.e., they cannot be reset to zero) and so can be reused only by determining the difference in total dose registered by them before and after exposure. These devices are almost as small as foils, but they can be stored for some time before readout.

A relatively new device is the damage track-length detector. ${ }^{2}$ This device makes use of the fact that chemical etching of a plastic material penetrated by fission fragments greatly enlarges the tracks produced by the fragments so that their lengths can be determined and the number of fissions occurring in 
foils of fissionable isotopes having a variety of thresholds can be found; this, in turn, provides the neutron fluence. These plastic track-length detectors are one to two orders of magnitude more sensitive than fission foils and have a long shelf life.

Nuclear emulsions are still occasionally used for determining neutron spectra external to radiation shields. Collimation must be used along with suppression of the gamma-ray field at the emulsion to reduce gamma-ray fogging.

\subsubsection{Active Gamma-Ray Detectors}

Both active and passive detectors have been used to obtain experimental gamma-ray data of interest in reactor shielding. A number of ionization chambers have been used, varying in size and sensitivity as well as in the maximum temperatures to which they may be exposed, their neutron responsiveness, and the dynamic range over which they can be used. These devices are usually calibrated with standard isotope sources, such as ${ }^{60} \mathrm{Co}$ and ${ }^{137} \mathrm{Cs}$. Ionization chambers mounted on traversing devices can be read continuously and thus can map an entire region since the position variable can be one of the variables on a chart recorder and the dose rate the other.

Anthracene scintillation dosimeters (ASD) have often been used for measuring gamma-ray dose rates since the output of a photomultiplier tube attached to the scintillation crystal is proportional to the gamma-ray dose rate at the crystal. Neutron captures in the detector can provide a spurious response, and temperature sensitivity is also a problem.

The widespread use of scintillation detectors as gamma-ray spectrometers has been made possible by the availability of large single crystals of $\mathrm{NaI}(\mathrm{Tl})$ and the development of end-window photomultiplier tubes with high resolution. Except for edge effects created when secondary photons from pair production escape the crystal, essentially all the incident gamma-ray energy is converted to light via the photoelectric effect, Compton effect, or pair-production process, and the light pulse energy is proportional to the primary gamma-ray energy.

A number of high-resolution solid-state gamma-ray spectrometers have been developed which can be used in some situations; however, neutron sensitivity can be a very serious problem. Of particular importance is lithium-drifted germanium. Resolution at high energies is much better with these devices than with the scintillation spectrometers, but more elaborate spectrum unfolding techniques and electronics are required. 


\subsubsection{Passive Gamma-Ray Detectors}

One of the earliest gamma-ray detectors was photographic film. It is still used since it is relatively inexpensive and can be read automatically. Sensitivity can be varied over a wide range. Most emulsions exhibit neutron sensitivity, but this can sometimes be compensated. Also film does not have a spectral response approximating tissue. Various absorbers can help reduce this difficulty.

Chemical indicators of ionization have also been used as gamma-ray detectors in shielding experiments, although these have largely been replaced by easier-to-read chemically activated glass detectors and the newer thermoluminescent detectors. The latter devices (usually called TLD's) come in a variety of sizes and shapes, including powder, micro and mini chips, rods, and hollow tubes. Most TLD's were developed and calibrated for situations in which only gamma rays would be present and where great accuracy was not required. Automatic or semiautomatic readers have become available, although careful calibration is required. The detectors must be heated before reuse; some are temperature-sensitive in the ambient range and lose some of the dose indication if an extended time period intervenes between exposure and readout.

\subsubsection{Interpretations of Detector Output}

Considerable caution must be exercised when detectors developed for a particular set of circumstances are used under conditions that are quite different. For example, small thermoluminescent gamma-ray dosimeters were developed initially for use in pure gamma-ray fields produced by isotope sources or accelerators wherein the energy of the gamma rays was usually well below $2 \mathrm{MeV}$. Some care is required in obtaining reasonably precise data in a mixed field of neutrons and both high- and low-energy gamma rays. The lithium in LiF TLD's responds to neutrons to an extent determined primarily by the concentration of ${ }^{6} \mathrm{Li}$, which has a high neutron-capture cross section, and the ratio of the neutron to gamma-ray intensity. Manganese-activated calcium fluoride used in a TLD may also have a significant response to neutrons as a result of captures in manganese, which has a high capture cross section for resonance-energy neutrons. Detectors of $\mathrm{CaF}$ are also frequently exposed in small tantalum covers, which serve to flatten the energy response of the TLD to make it similar to that of tissue. These covers present no particular problem in a pure gamma-ray field similar to that in which the detectors are calibrated; however, tantalum has very 
high thermal- and resonance-neutron absorption cross sections. The detectors, when exposed to a mixed field of neutrons and gamma rays, may therefore be responding in part to the gamma rays produced in the covers by either neutron capture or subsequent decay of the activated tantalum.

For high-energy gamma rays, the efficiency of the TLD, particularly the very small micro dosimeter, may be reduced with increased energy because the range of the electrons produced by the gamma rays may be greater than the dimensions of the detector.

There have also been instances in which the preamplifier attached to a gamma-ray detector was more sensitive to radiation than the detector itself and produced a signal even when the detector was disconnected. The long cables required for remotely controlled experiments can also significantly change detector-response systems. Calibrations should be made with the detector attached to the cable to be used in the field.

Thermal-neutron detectors, calibrated in a thermal column where the ratio of the number of thermal neutrons to the number of epithermal neutrons may be 40/60, may give quite misleading thermal fluxes in a situation in which there are few thermal neutrons and many epithermal ones, such as in a thick region of aluminum outside a highly enriched intermediate-spectrum reactor.

In short, detectors almost invariably provide data, but frequently considerable effort is required to ensure that the data are related to the quantity one is trying to determine.

\subsection{SHIELDMATERIAL MEASUREMENTS}

The following experiments are only a sample of many performed in recent years to characterize the transport properties of bulk materials or combinations of materials. They were selected as well-designed experiments that are representative of those performed in materials studies. Experiments are discussed for reactors, accelerators, and fixed sources. The purpose and plan of the investigations are emphasized, but sample data are included to indicate the utility of the results. A recent survey of reactor shielding facilities has been reported by the Radiation Shielding Information Center. ${ }^{7}$

\subsubsection{Reactors}

An extensive series of slab-penetration measurements were performed in a specially designed facility called the Outside Test Tank (OTT). These 
measurements compared the relative effectiveness of various arrangements of a given set of materials and of different sets of materials. The facility was also used for testing mock-ups of designs for various shields. The tests were performed at the Nuclear Aerospace Research Facility at General Dynamics, Fort Worth, Texas, and are described by Belcher and Zoller. ${ }^{8}$ The source of radiation was the Ground Test Reactor (GTR) which fits into the moderator tank shown in Fig. 9.1. The OTT provided an unusual capability for the testing of a wide variety of shield configurations of interest in the development of minimum-weight airborne or space nuclear systems since earlier measurements required that slabs and detectors be submerged under water or oil, and it permitted measurements in air without extraneous liquid layers. The power level of the reactor used in the OTT was high enough (up to $500 \mathrm{kw}$ ) that active dosimetry measurements could be made in air to distances of $30 \mathrm{~m}$ or more from the reactor. The shields tested were sufficiently thin compared to the water and lead surrounding the reactor to ensure that only radiation penetrating the shields reached the external detectors with no contribution from air or ground scattering. The tank itself was $4 \mathrm{~m}$ high by $4.5 \mathrm{~m}$ in diameter and could be rotated remotely. Two independent dosimeter traversing mechanisms were also remotely controlled from an underground control room.

Several hundred shield configurations consisting of either laminated slabs or boxes containing semiporous shields were tested in the shield compartment adjacent to the reactor moderator tank. A few of these shields were mock-ups of proposed shield designs, but the great majority were part of a systematic study of the effects on external dose rates of varying the quantity or position of a given material either by itself or within an array of another material. Typically, one might have 2 to $10 \mathrm{~cm}$ of gamma shielding, such as tungsten, steel, or depleted uranium, in an array of neutron shielding, such as lithium hydride, beryllium, or beryllium oxide, with or without boral layers adjacent to the higher density materials. Borated stainless steel, zirconium hydride, lead, and boron carbide were also tested. A number of simulated ducts were investigated, as were various thicknesses of neutron shields with no gamma-ray shielding. Samples of a great many different materials used in aircraft turbines and structures were activated in various neutron spectra obtained by changing the composition of the materials between the samples and the reactor to give a solid basis for estimates of component activation.

Many measurements made at distances of 3 to $30 \mathrm{~m}$ from various reactor shield configurations are reported by Friedman. ${ }^{9}$ Figure 9.2 is typical of the data obtained. The gamma-ray dose rates at 2.7 and $15.2 \mathrm{~m}$ from the reactor 
center are plotted as a function of both the thickness of a tungsten alloy and as a function of the amount of $\mathrm{LiH}$ between the reactor and the tungsten. Secondary gamma rays were shown to be of considerable importance. Secondary gamma-ray production decreased as the amount of neutron shielding between the tungsten and the core was increased. However, for most situations increasing the inner radius of heavy gamma-ray shielding material would also increase shield weight. The addition of boron to the heavy material or the use of thin protective layers of boral was expected to reduce the number of thermal-neutron captures in the gamma shield significantly. The number of captures of epithermal or resonance-energy neutrons should not be reduced significantly, and the production of gamma rays by inelastic scattering of fast neutrons $(E>200 \mathrm{keV})$ should not be affected. The data shown in Fig. 9.3 were obtained in an attempt to differentiate between these various sources of gamma rays. The data showed that adding boron to stainless steel is more effective in suppressing secondary gamma-ray production than placing boral between the steel and the reactor, even with a good moderating material in front of the steel. These same experiments also provided neutron-flux density and gamma-ray dose rate within the shields for comparison with predictions as well as gamma-ray spectra outside the tank.

Foil exposures between the slabs for the same configurations as shown in Fig. 9.2 were made as a function of tungsten thickness with ${ }^{27} \mathrm{Al}$ $(E>8.6 \mathrm{MeV}),{ }^{32} \mathrm{~S}(E>2.9 \mathrm{MeV})$, and ${ }^{63} \mathrm{Cu}(E<0.4 \mathrm{eV})$ foils. Measurements were made for four thicknesses of tungsten, 2.5, 5.1, 7.6, and $10.2 \mathrm{~cm}$, inserted where the tungsten layer is shown in Fig. 9.2. Foils were placed between slabs separated by gaskets. The configuration was compressed to eliminate the space at the tungsten location and expanded as the tungsten slabs were inserted.

Foil activities (counts $\min ^{-1} \mathrm{~g}^{-1}$ watt ${ }^{-1}$ ) are shown in Fig. 9.4 at reactor shutdown as a function of tungsten thickness for the copper foils in terms of cadmium differences. Note that for a tungsten thickness of 0 the LiH slabs are moved left to the $\mathrm{BeO}$ and boral slabs. As the tungsten slabs are inserted, the LiH slabs are moved to the right. An effect on the thermal activity is evident. As the $\mathrm{LiH}$ is replaced by tungsten, a valley begins to form, which indicates the much higher absorption cross section for tungsten. The valley deepens and widens as the tungsten thickness is increased. The sulfur and aluminum activities (not shown) are reduced by a factor of 2 in the $\mathrm{LiH}$ region when $\mathrm{LiH}$ is replaced by $10 \mathrm{~cm}$ of tungsten. This reduction 


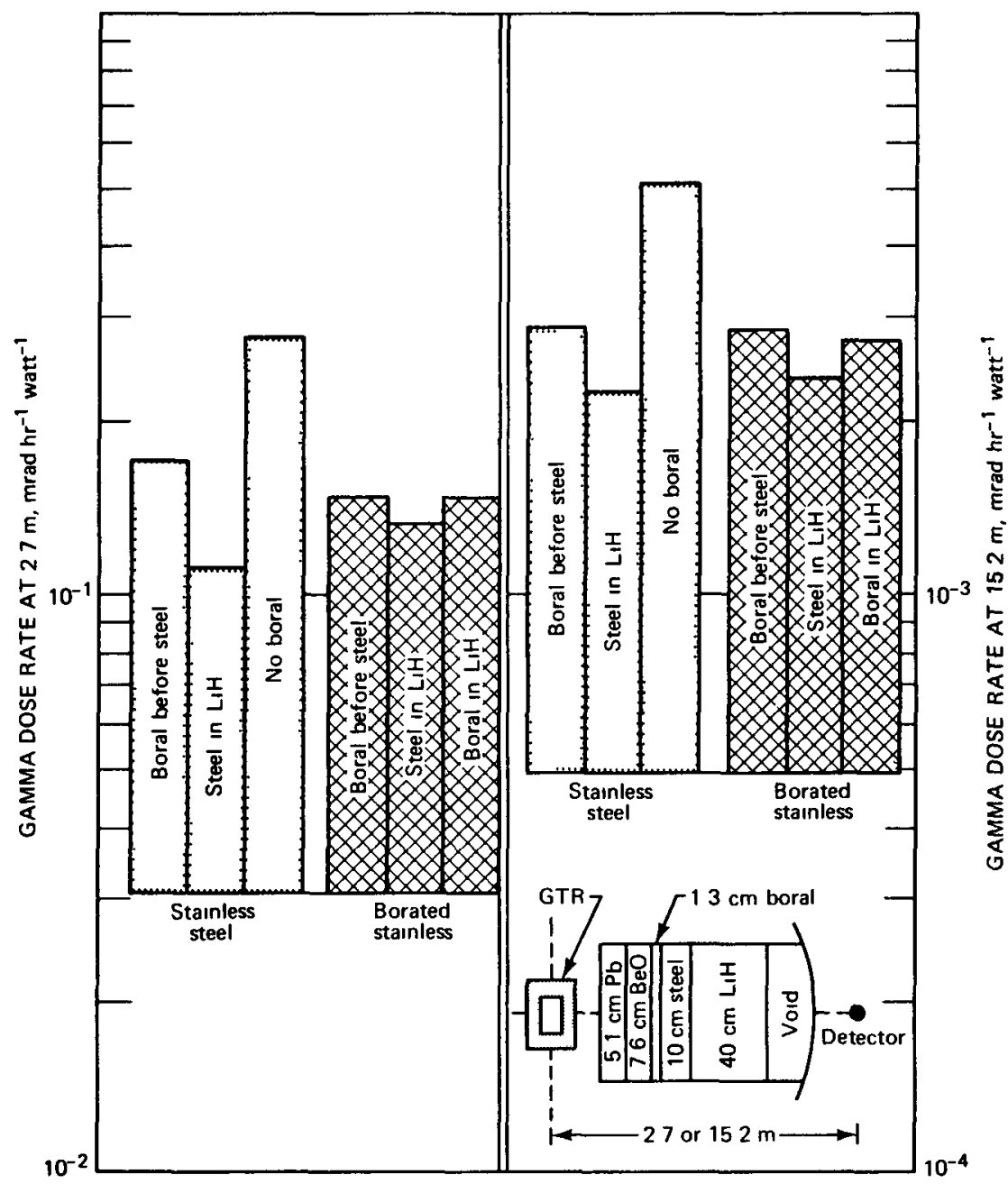

Fig. 9.3-Gamma dose rates in air behind stainless steel and borated stainless steel (From Friedman. ${ }^{9}$ )

demonstrates the effectiveness of adding a high- $\angle$ inelastic scatterer to a low- $Z$ elastic scatterer.

Verbinski, Bokharı, Courtney, and Whitesides ${ }^{10}$ describe a reactor experiment that yielded measurements in bulk water shields at Oak Ridge National Laboratory (ORNL). The source of neutrons was the Bulk Shieldıng Reactor I (BSR-I) described by the Radiation Shielding Information Center. ${ }^{7}$ This was a series of measurements of the spectral and spatial details of the fast-neutron flux density penetrating water shields. The purpose was to obtain data for evaluating neutron-transport codes that 


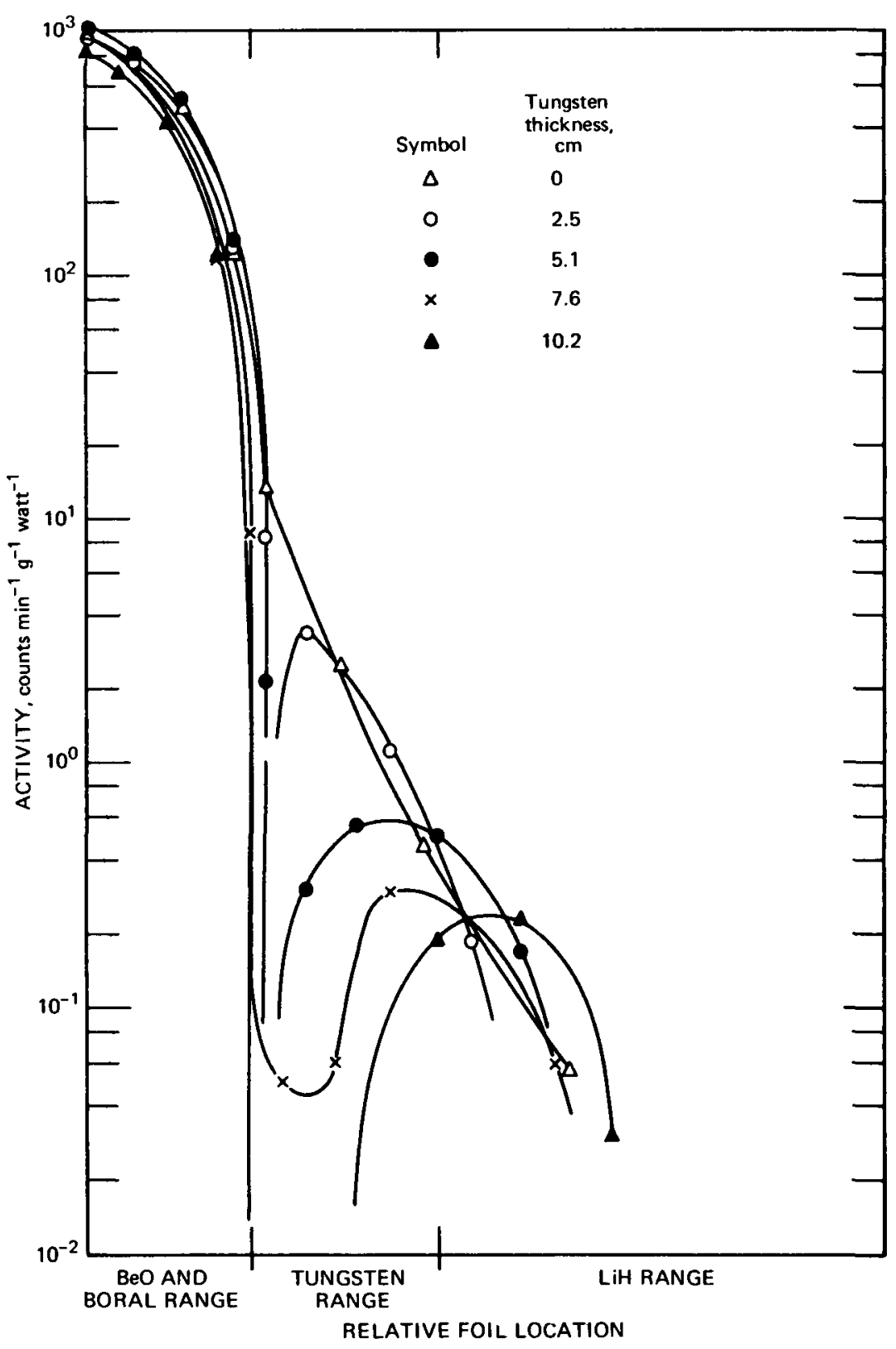

Fig. 9.4-Measured ${ }^{63} \mathrm{Cu}$ activity (cadmium differences) as a function of foil location for the indicated thicknesses of tungsten. 
predict the variation of flux density with energy, angle, and penetration depth in a shield. Two basically different shielding codes were compared with the measurements. Two different types of experiments were performed to provide a broad basis for evaluating the codes. In one a large source with adjacent shield was used; in the other a small source well-separated from the shielding slab roughly approximated a good-geometry neutron-scattering arrangement. This discussion is concerned with the first experiment: the large source with adjacent shield. The small-source experiment will be discussed later. The first experiment was performed at ORNL and consisted in the mapping of the absolute spectral intensity in the water shield of a pool-type reactor (the BSR-I) as a function of both angle and distance from the source. In conjunction with these measurements, both the absolute reactor power and the distribution of the neutron-source strength throughout the reactor were measured. The results of the reactor-power calibration were then used as input to the neutron-transport codes so that the codes could be evaluated for the complete task of predicting the neutron-transport pattern, beginning with an absolute fission-source distribution and ending with spectral intensities at a large number of points and directions in the shield.

The fuel loading of the BSR-I used for the absolute measurements of spectral intensity is shown in Fig. 9.5. It is a water-reflected rectangular configuration ( 38 by 38 by $60 \mathrm{~cm}$ ) of aluminum-clad elements with two additional elements in the rear which were required for criticality. For each spectral measurement the relative power of the reactor was determined to within $2 \%$ by means of two sulfur foils (actually pellets) located $35 \mathrm{~cm}$ above and $38 \mathrm{~cm}$ east of the reactor centerline. The sulfur activation was later related to the absolute total reactor power as well as to the absolute neutron-source density throughout the reactor volume. The absolute power was determined to an accuracy of about $5 \%$.

A shielded diode spectrometer, designed especially for these measurements, was positioned to measure the spectral intensity at a distance $r$ from the face of the reactor, along the center line of the reactor midplane, and at an angle $\theta$ with respect to the center line as shown in Fig. 9.5. The spectrometer consists of a very thin layer of ${ }^{6} \mathrm{LiF}$ between, but not in contact with, two silicon-gold diodes. Neutrons traversing the spectrometer collimator strike the ${ }^{6} \mathrm{Li}$ foil, and the alpha particle and triton emitted in the resulting ${ }^{6} \mathrm{Li}(n, \alpha) \mathrm{T}$ reaction $(\mathrm{Q}=4.78 \mathrm{MeV})$ are detected in coincidence in the two diodes. Shadow shielding in the collimator protects the diodes from 


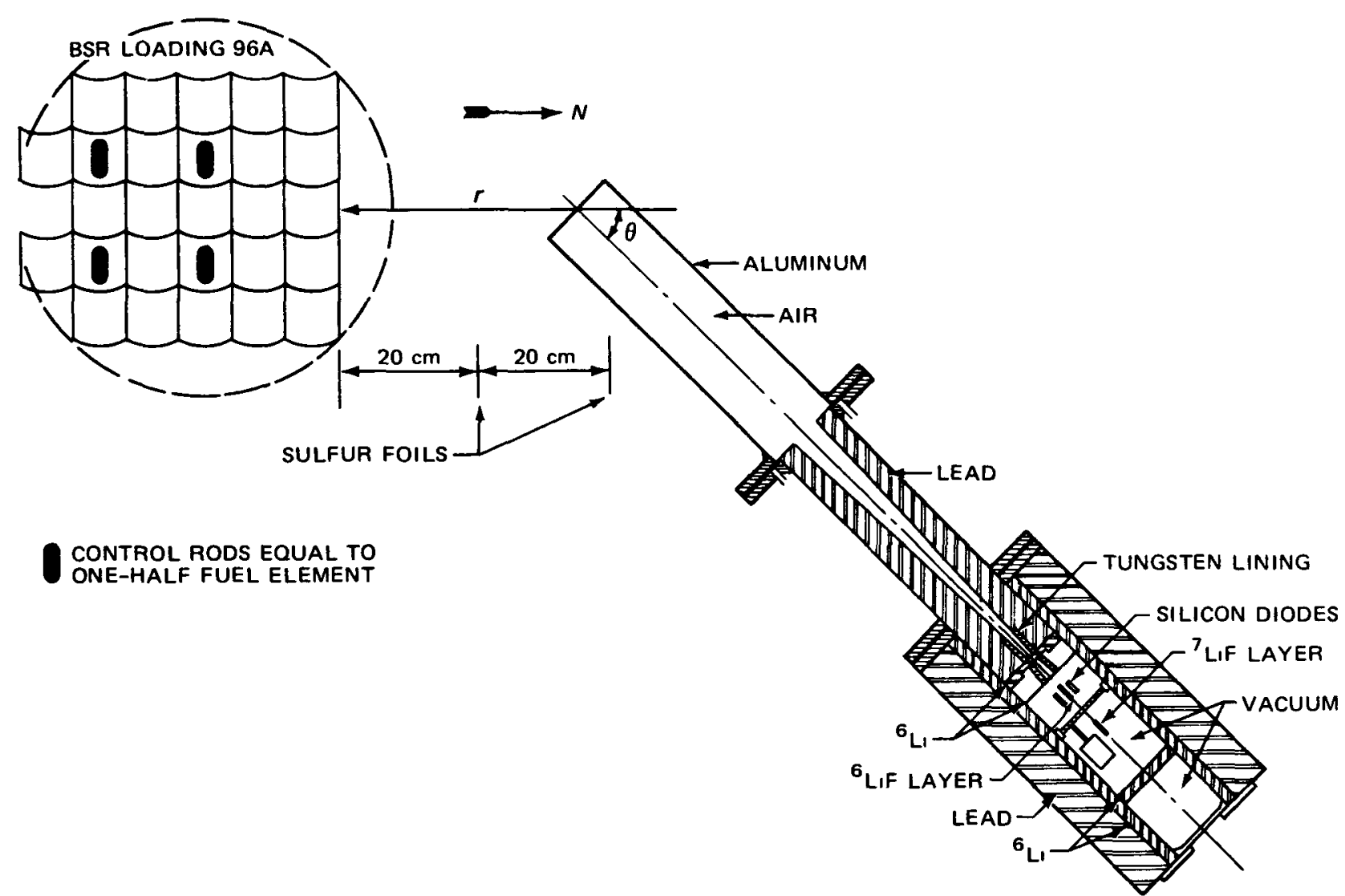

Fig. 9.5-Experimental arrangement showing relative positions of reactor and spectrometer for measuring neutron spectra in BSR-I water shield. The dashed circle represents the spherical source region used for the calculations, for which the distance $r$ is measured from the surface of the sphere. [From V. V. Verbinski, M. S. Bokhari, J. C. Courtney, and G. E. Whitesides, Nuclear Science and Engineering, 27(2): 284 (1967).] 
direct reactor radiations. Background measurements are made by substituting a ${ }^{7} \mathrm{LiF}$ foil for the sensitive ${ }^{6} \mathrm{LiF}$ element.

The spectrometer has operated satisfactorily in gamma-ray fluxes from the BSR-I as high as $10^{6} \mathrm{R} / \mathrm{hr}$ at the point in the water shield where the neutron flux was being sampled.

The calculations corresponding to this experiment were performed with the NIOBE ${ }^{11}$ and the DTK codes (DTK is a version of DTF, ${ }^{12}$ which is discussed in Chap. 4, Sec. 4.4). Limitations on the geometries that could be used with these codes decreed that the reactor core be simulated by a sphere. The radius of the spherical source region was taken as $26 \mathrm{~cm}$ and is shown by the dashed circle in Fig. 9.5, all values of $r$ in the calculations being given in terms of distance from the surface of this source sphere. The spectral intensity of neutrons was measured at angles $(\theta)$ of $0^{\circ}, 41^{\circ}$ and $52^{\circ}$ with respect to the extended midplane center line of the reactor. The distance $r$ from the reactor face was varied in increments of $10 \mathrm{~cm}$ out to a distance of $50 \mathrm{~cm}$. All measured (and calculated) fluxes are given for a total reactor power of 1 watt.

The spectral intensities obtained at $\theta=0^{\circ}$ are shown in Fig. 2.19, along with predictions of the NIOBE transport code. (In Fig. 2.19 and succeeding figures in this chapter, $\mu=\cos \theta$.) It is evident that the code predicts the absolute magnitude with good accuracy and reproduces some of the general features of spectral shape. The strong dip in the spectra at $3.5 \mathrm{MeV}$ is caused by a broad peak in the oxygen cross section. The measured spectra suggest that a similar dip may be present in the $20-$ to $50-\mathrm{cm}$ spectra but at an energy about $0.5 \mathrm{MeV}$ higher. However, the present model of the spectrometer was designed to provide only moderate energy resolution in order to achieve good sensitivity. Therefore the discussion of detailed spectral shapes will be deferred to the section on LINAC measurements (Sec. 9.2.2).

The $0^{\circ}$ measurements are compared with the predictions of the DTK transport code in Fig. 9.6. The agreement between the DTK calculations and measurements near the source region is about the same as that with the NIOBE calculations. The dashed lines between 5 and $7.5 \mathrm{MeV}$ show the improvement in the predictions obtained when the calculation was repeated with updated cross sections.

The measurements for $\theta=52^{\circ}$ were made at distances of $10,20,30$, and $40 \mathrm{~cm}$ from the face of the reactor. The results are compared in Figs. 9.7 and 9.8 with the predictions of the NIOBE and DTK codes, respectively. Overall agreement appears to be fair except that at $r=10$ and $20 \mathrm{~cm}$ the experimental results are higher than both calculations at high energies, where 


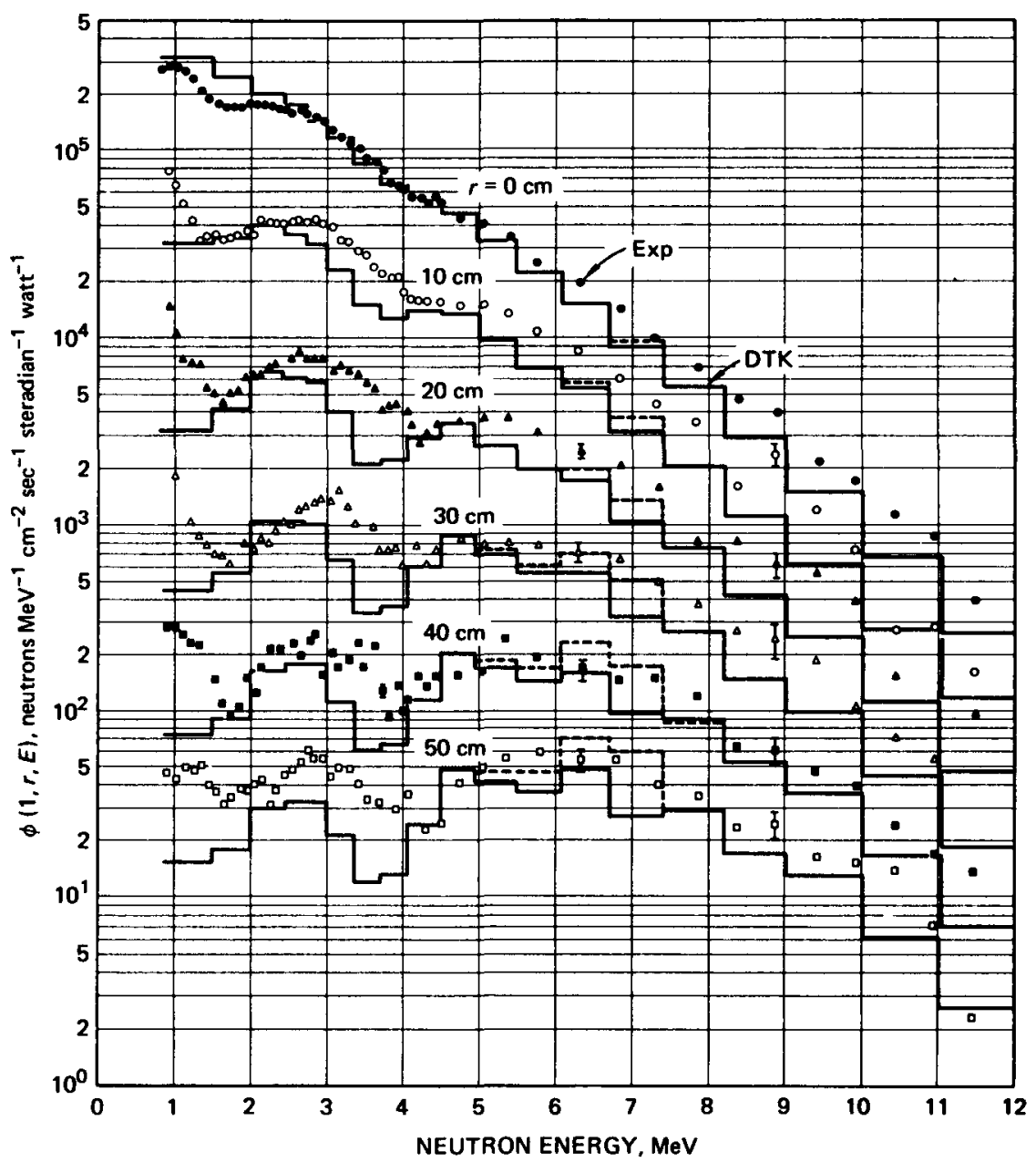

Fig. 9.6-Measured and DTK-calculated neutron spectral intensities in the BSR-I water shield at $0^{\circ}$. For the calculation the value of $\mu$ is 0.978 rather than 1 . [From V. V. Verbinski, M. S. Bokhari, J. C. Courtney, and G. E. Whitesides, Nuclear Science and Engineering, 27(2): 286 (1967).]

the neutrons are more penetrating. This must be caused by uncollided plus small-angle-scattered neutrons coming from the corner of the reactor, which is within view of the spectrometer collimator at $r=10 \mathrm{~cm}$ and almost within view at $r=20 \mathrm{~cm}$. This corner does not, of course, exist for the spherical source used for the calculations. At $r=30$ and $40 \mathrm{~cm}$, the difference in the calculational and experimental source geometries is relatively unimportant since the corner of the reactor is farther from the line of sight of the 


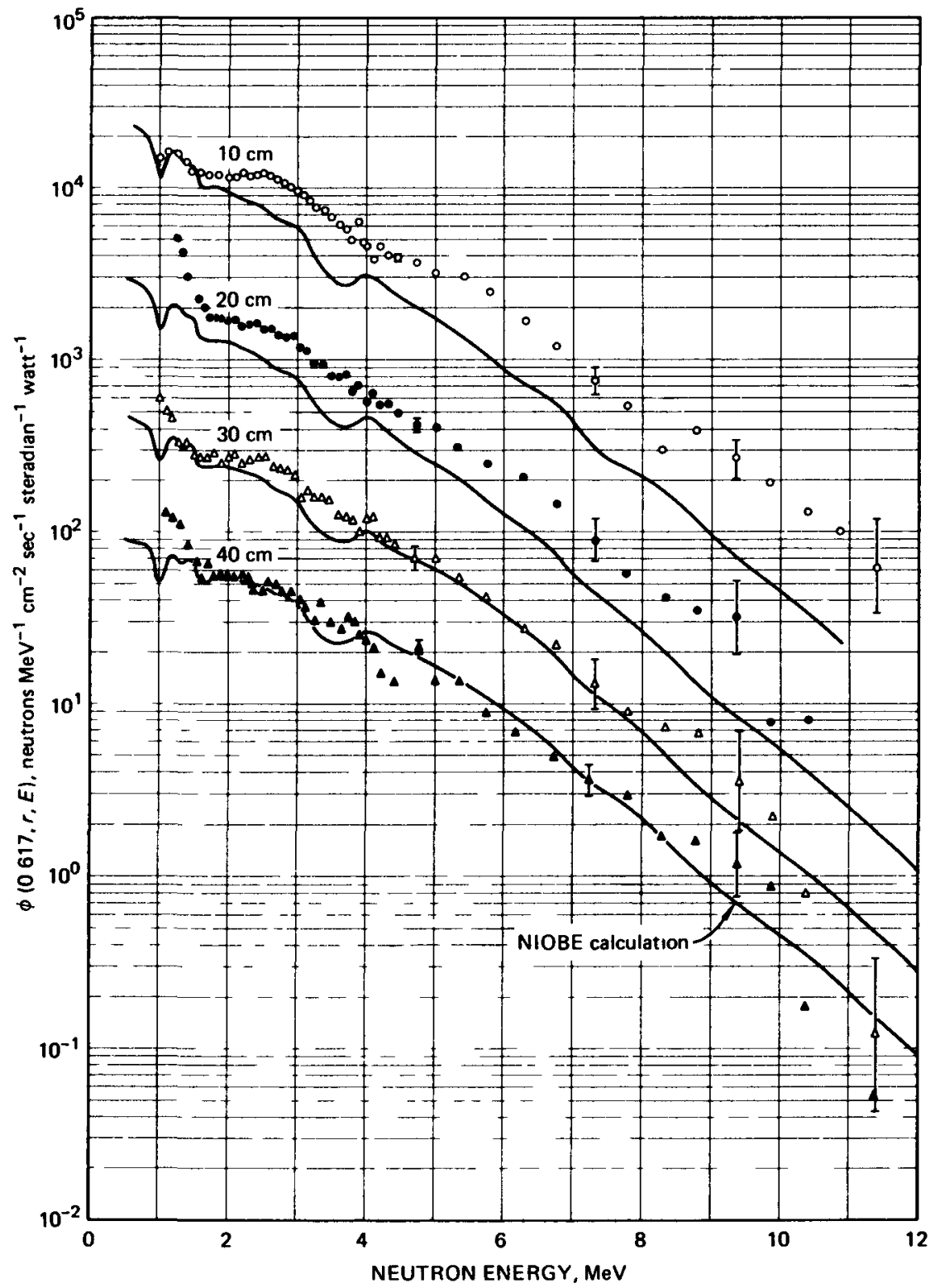

Fig. 9.7-Measured and NIOBE-calculated neutron spectral intensities in the BSR-I water shield at $52^{\circ}$. [From V. V. Verbinski, M. S. Bokhari, J. C. Courtney, and G. E. Whitesides, Nuclear Science and Engineering, 27(2): 287 (1967). 1 


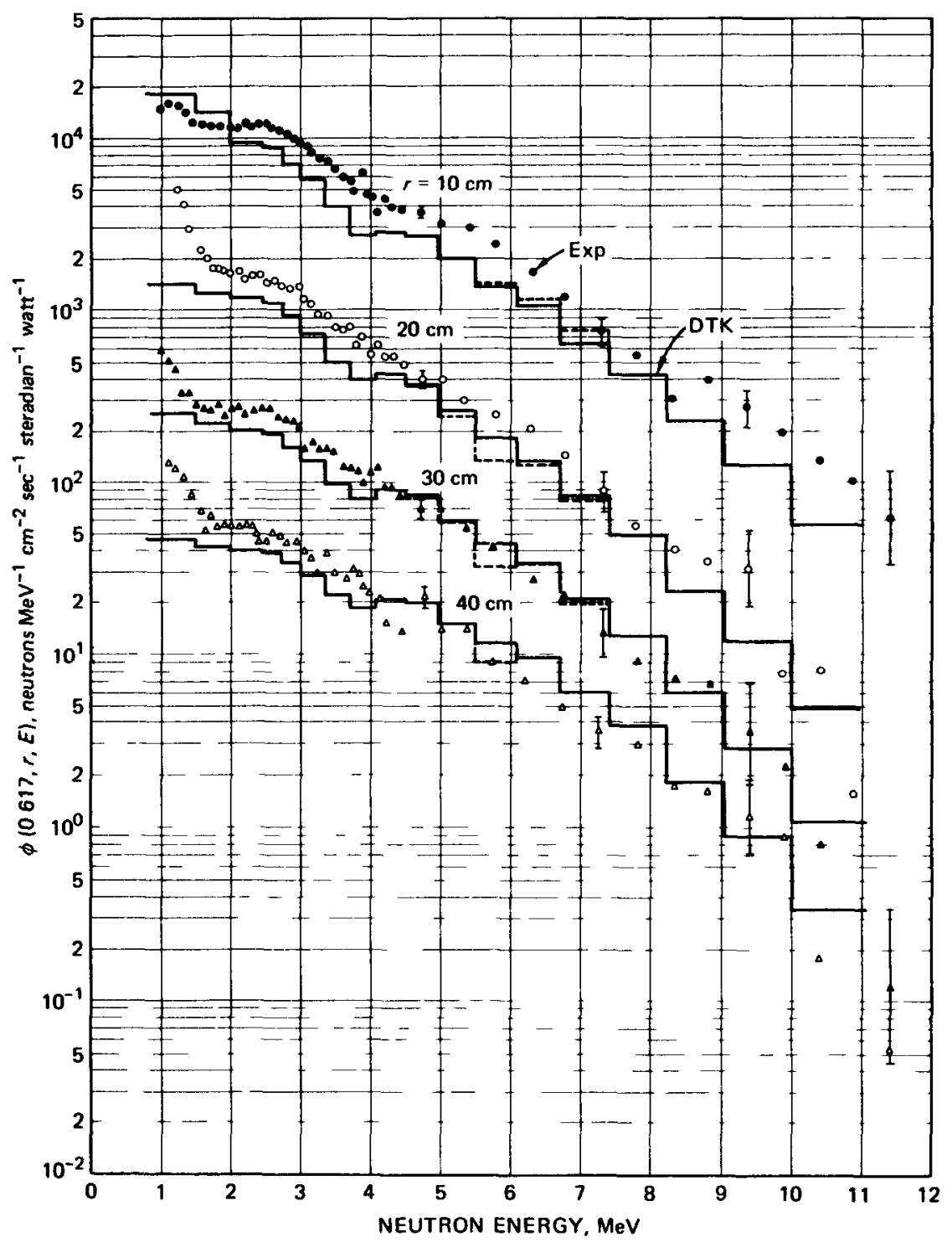

Fig. 9.8-Measured and DTK calculated neutron spectral intensities in the BSR-1 water shield at $52^{\circ}$. For the calculation the value of $\mu$ is 0650 rather than 0.617 . [From V. V. Verbinski, M. S. Bokhari, J. C. Courtney, and G. E. Whitesides, Nuclear Science and Engineering, 27(2): 287 (1967).] 
spectrometer collimator. Much better overall agreement with the experiment is realized here except that the codes predict fluxes that are somewhat low at low neutron energies. Note that at $52^{\circ}$ the transport calculation using updated oxygen total cross sections (dashed lines) produced little, if any, overall improvement in the $5-$ to $7.5-\mathrm{MeV}$ region where the older cross-section values are evidently too high.

Measured and calculated spectral intensities were also obtained for $\theta=41^{\circ}$ at $r=20$ and $40 \mathrm{~cm}$, but, since they show the same general features as those obtained for $\theta=52^{\circ}$, no further comparisons are given here.

An additional reactor experiment, described by Clifford, Muckenthaler, Maerker, Straker, and Mynatt ${ }^{13}$ and by Clifford, Straker, Muckenthaler, Verbinski, Freestone, Henry, and Burrus, ${ }^{14}$ was performed to evaluate total cross sections of several materials over the energy range of interest in reactor shielding. The experiment was carried out at the Oak Ridge National Laboratory Tower Shielding Facility (TSF). This investigation was performed with the reactor on the ground and has since been called the broomstick experiment because of the long, thin samples (Fig. 9.9).

The measurements consisted of spectra of the uncollided flux of neutrons transmitted through thick samples of shielding materials. Because the samples were quite thick, the transmitted spectra were influenced most strongly by the minima in the total cross sections. The spectrum incident on the sample and the transmitted spectra were measured with an NE-213 neutron spectrometer covering the energy range from 0.8 to $11 \mathrm{MeV}$. The total cross sections were evaluated by a direct comparison of calculated and experimental data. The uncollided spectra were calculated with the most detailed cross-section data available and then folded with the response function of the detector. Although the energy resolution of the spectrometer is rather broad, this technique still allows a determination of the errors in the very narrow minima in the total cross section since the total number of transmitted neutrons is measured to within a few percent. Because the geometry of the experiment was very good, no correction for multiple scattering was required; therefore the calculations were quite simple and straightforward, involving only the exponential attenuation of the incident spectra using fine-mesh data for the total cross section. Experiments were done to demonstrate that multiple scattering was indeed negligible. The sample diameter was varied by a factor of 2 with no measurable change in the transmitted spectra.

Good agreement between the measured and calculated spectra indicates that the valleys are adequately represented in a cross-section set. Where 


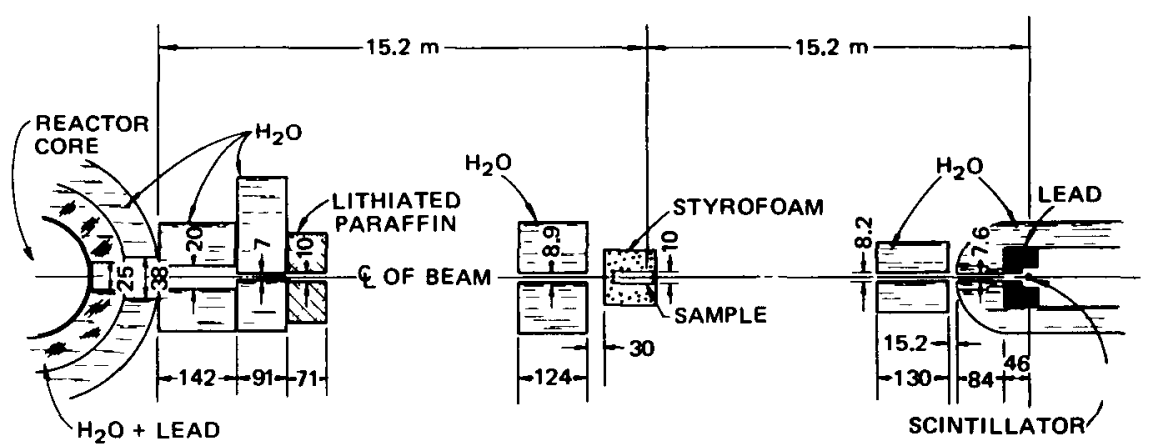

NOT TO SCALE. ALL DIMENSIONS IN CENTIMETERS EXCEPT AS NOTED. BEAM CENTER LINE, $\sim 2 \mathrm{M}$ ABOVE CONCRETE PAD.

Fig. 9.9-Arrangement of broomstick experiments. ${ }^{13,14}$

disagreement exists, detailed information about the specific region of disagreement is not possible, unless the minima are well separated, because of the poor energy resolution of the spectral measurements. Where a number of minima are close together, only the general energy range in which the disagreement exists can be determined. Because of this these measurements can in no way be described as a measurement of the total cross section but simply as an experimental evaluation of the existing cross-section data.

The spectrometer uses a 5 - by 5 -cm liquid organic scintillator (NE-213) and has an efficiency that ranges from 0.15 to 0.4 for neutrons in the $\mathrm{MeV}$ energy range. Thus it can be used in flux densities that are several orders of magnitude lower in intensity than those measurable with other spectrometers, all of which are limited by low efficiencies $\left(1 \sigma^{4}\right.$ to $\left.1 \sigma^{6}\right)$. The validity of the experimental technique was checked by measurements on thick samples of carbon, lead, and uranium, which could be easily compared with calculations since the total cross sections for these elements are well known.

So that the ratio of the uncollided flux at the detector to the collided flux could be maximized, the detector was located at a distance of $30 \mathrm{~m}$ from the shield of the reactor and the sample was placed at the approximate midpoint between the detector and the reactor shield. The arrangement is shown in Fig. 9.9. Most of the samples were cylinders that were positioned with their axes coincident with the axis of the neutron beam. Oxygen and nitrogen samples in liquid form were contained in either Dewar vessels or styrofoam-insulated brass cans.

Because neutrons scattered by the air and ground become important when the scattering sample is thick, both the detector and the neutron beam were shielded and very tightly collimated. The neutron beam incident on the 
sample passed through two collimators: one which was adjacent to the reactor shield and another which was adjacent to the sample position and limited the beam to $9 \mathrm{~cm}$ in diameter. These collimators also served to reduce the air-scattered background at the detector without producing any observable change in the measured spectra incident to the samples.

Measurements were made through some eighteen different materials. We show only the results for iron, which are particularly interesting because of considerable uncertainty that existed at the time in the fine structure of the iron cross section.

Since the experiment was primarily one of cross-section evaluation, data from the 05R, ${ }^{15} \mathrm{KFK},{ }^{16} \mathrm{BNL},{ }^{17}$ and $\mathrm{ENDF} / \mathrm{B}^{18}$ files were all examined. Data from the 05R Monte Carlo cross-section library and the ENDF/B (Evaluated Nuclear Data File) were considered the best for iron from the experimental results. Figure 9.10 shows comparisons of the measured and calculated energy spectra from the $05 \mathrm{R}$ and $\mathrm{ENDF} / \mathrm{B}$ cross-section data. Good agreement is noted in the data for energies above $2.0 \mathrm{MeV}$, but both sets of cross sections are too high for lower energies.

This technique of evaluating minima in total cross sections has been very useful in indicating which evaluated set of total cross sections is adequate for shielding calculations and thereby permitting an efficient updating of cross-section libraries by updating only those cross sections which are not adequate. It also provided information about the energy regions in which new cross-section measurements should be made.

In the foregoing sample of reactor shielding experiments, we have omitted many performed with the reactors mentioned as well as many more with other research reactors. A recent survey ${ }^{7}$ of shield facilities gives a complete list of shielding reactors and the types of investigations performed. The Lid Tank Shielding Facility, although no longer used in shielding investigations, deserves mention. Originally developed at ORNL, later duplicated elsewhere, the lid tank was successfully used for measuring removal cross sections and other materials attenuation parameters. A thermal column from a reactor core (at ORNL, the X-10 reactor) extended to a plate of fissile material attached to the wall of a tank. The fission plate (or source plate) produced a plane source of fast neutrons with an effective power of a few watts. Since the fission plate is removed some distance from the reactor, the background radiation is greatly reduced. The tank could be filled with water, oil, or a solution of interest, and materials arrangements could be lowered into the tank adjacent to the source. All the neutron removal cross sections given in Chaps. 4 and 6 were derived from lid tank measurements. 


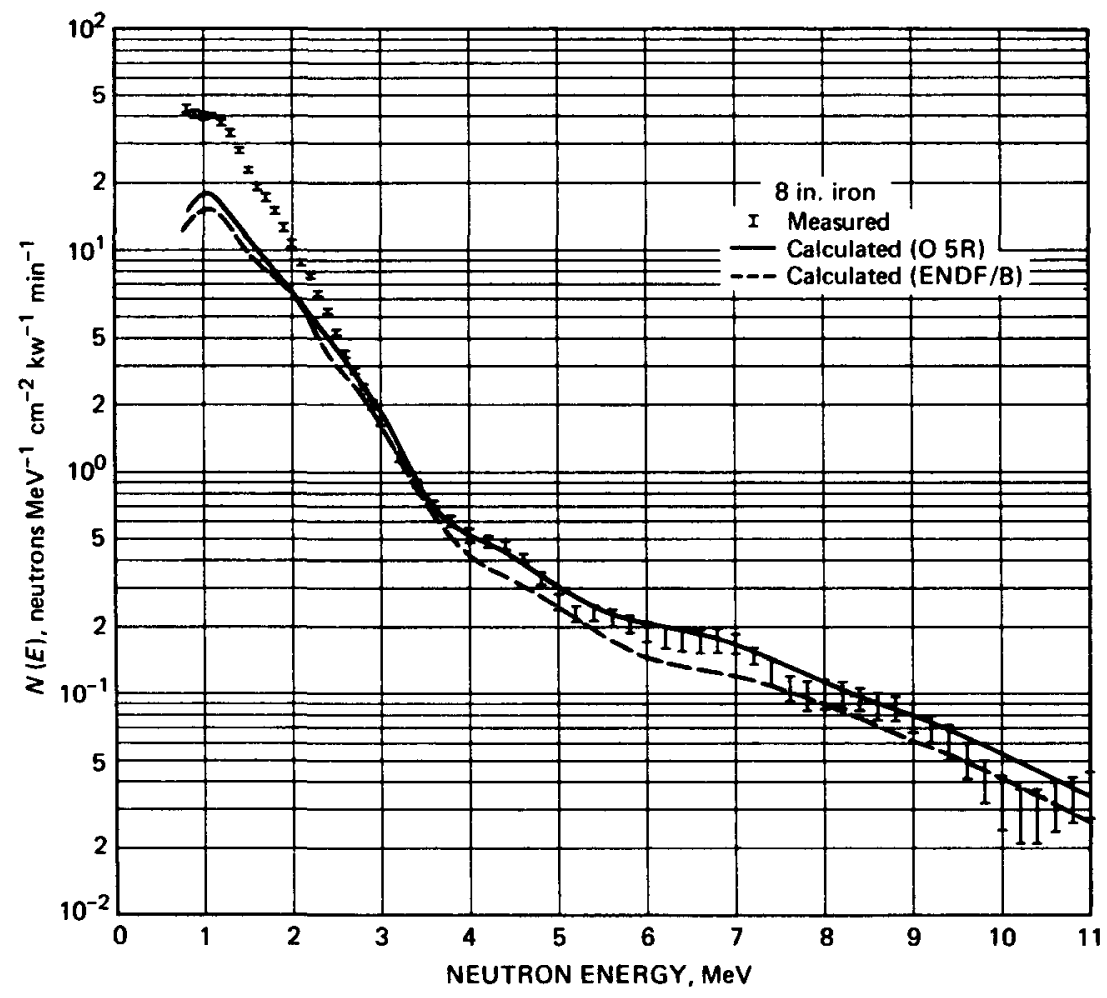

Fig. 9.10-Iron penetration results. (From Clifford et al. ${ }^{13}$ )

Although the lid tank produced many useful measurements, limitations in intensity and flexibility caused it to be superseded by the reactor facilities now in use.

\subsubsection{Accelerators}

The development of accelerators and generators capable of producing high yields of neutrons and gamma rays has provided the shielding experimentalist with opportunities for measurements previously impossible in reactor radiation fields. These sources, coupled with detectors of good sensitivity and resolution, permit cross-section measurements and evaluations, gamma-ray energy-spectra determinations, basic shield-material evaluations, and other useful measurements.

Experiments with accelerators generally exhibit a flavor different from those with reactors. Better-defined geometry is usually possible, the source is pure neutron, gamma, or charged-particle without the complex background 
associated with a reactor. Of greatest importance, however, is that with some accelerators the energy of the source radiation can be selected either directly or by time-of-flight measurement.

In the discussion of the broomstick experiment of Clifford et al., 13,14 we mentioned that appreciable uncertainties were found in the reported cross sections for iron at energies below $2.5 \mathrm{MeV}$. Those differences were attributed to poor resolution in the valleys of the total cross section. An experiment with similar purpose but entirely different approach was performed at Gulf General Atomic (GGA) by Cerbone, Miller, and Profio, ${ }^{19}$ who used the linear accelerator (LINAC) with a time-of-flight method 20,21 as a source of neutrons.

The geometry comprised a 76.30-cm-diameter iron sphere (Fig. 9.11), at the center of which was located the uranium target for photoneutron production by bremsstrahlung from incident electrons. In Fig. 9.11 the electronbeam tube is the $9.5-\mathrm{cm}$-diameter port hole, port $\mathrm{A}$ is for monitor foils, and the stepped 10.02- to $12.7-\mathrm{cm}$-diameter port is for the $0^{\circ}$.measurements. The through port at $R=23 \mathrm{~cm}$ is for off-zero internal angular-flux measurements. The neutron spectrum and angular distribution are measured by extracting a collimated beam from the experimental assembly and sorting the counts with their time of arrival at a detector placed $50 \mathrm{~m}$ distant. Time-zero is defined by pulsing the neutron source. In the iron sphere, the time to slow down and migrate to the beam extraction point is small compared to the corresponding 50-m flight time and can be neglected.

The spectrum was measured from 0.5 to $15 \mathrm{MeV}$ with NE-211 organic scintillators and from $0.6 \mathrm{keV}$ to $0.5 \mathrm{MeV}$ with an NE-908 lithium glass scintillator. Spectra were measured for several thicknesses of iron at several angles off the target-detector axis. Figure 9.12 shows the $0^{\circ}$ measured spectra at radius values of 19.05 and $38.1 \mathrm{~cm}$ (surface). The fine structure of the iron cross section is evident in the spectral changes. Since this is a good geometry experiment with fair resolution, the results are adequate for evaluating iron cross sections. Discrete-ordinate calculations of the spectra for conditions specified in Fig. 9.12 were made with the $1 \mathrm{DF}$ code (a version of DTF-IV ${ }^{2}$ ) using 29 energy groups. Group cross sections were prepared from the ENDF/B library with careful attention given to minima in the cross-section structure. An asymmetrical $S_{16}$ quadrature and a $P_{3}$ crosssection expansion were used. Wide discrepancies with the measurements were found. Possible causes were errors in the inelastic and elastic cross sections or the result of skipping valleys and neglecting self-shielding of the 


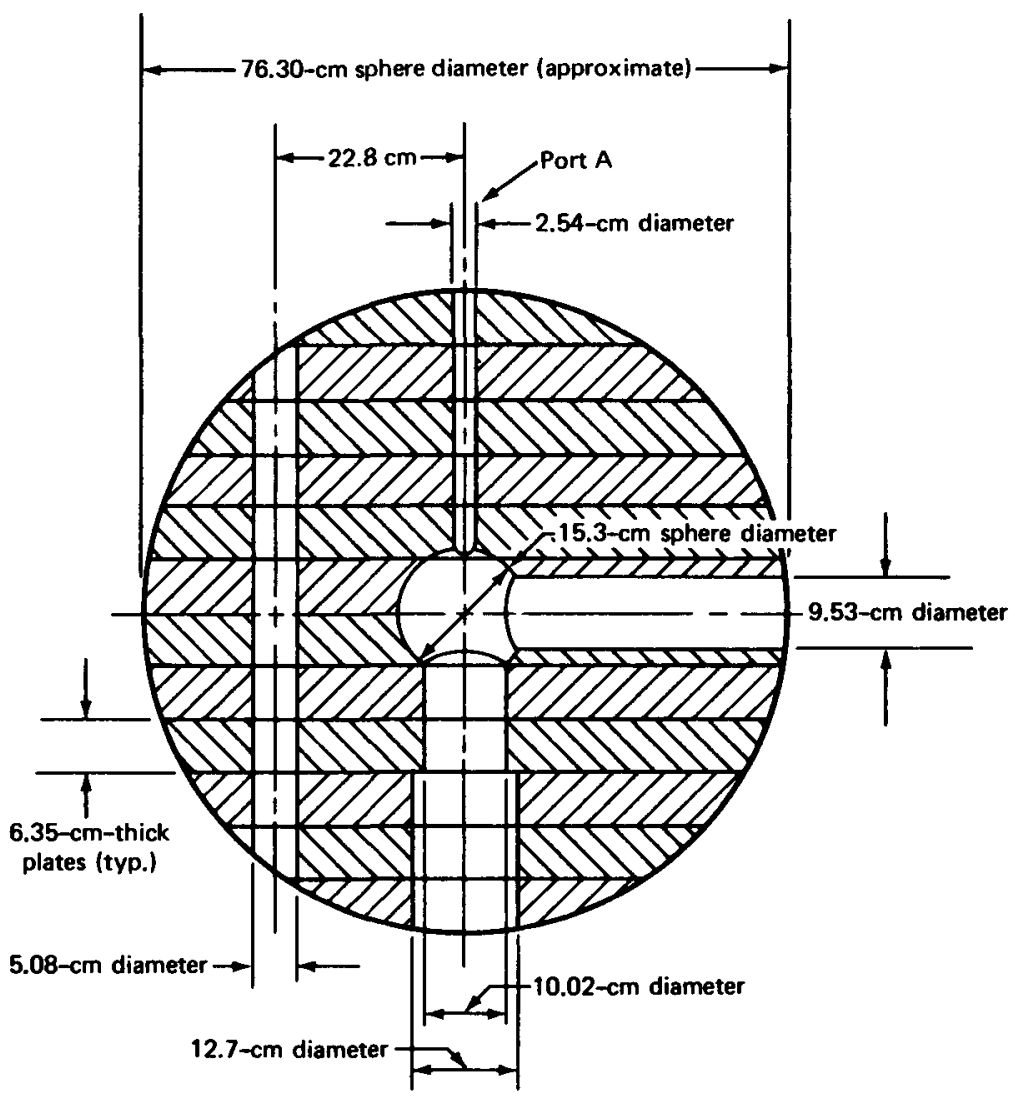

Fig. 9.11 - Iron sphere for LINAC experiment. (From Cerbone et al ${ }^{19}$ )

resonances, which often happen when too few energy groups are used to describe complex fine-structure cross sections.

A second calculation was made using 49 energy groups. Figure 9.12 shows results of this calculation compared with measurements for $R=19.05$ and $38.1 \mathrm{~cm}$. Agreement is still quite poor, which strongly suggests that errors exist in the ENDF/B point cross-section data for iron.

Thus the results of Clifford et al. ${ }^{13.14}$ and Cerbone et al. ${ }^{19}$ agreed to the extent that reported values of the iron cross section appeared to be entirely inadequate for shielding calculations.

Carlson, Cerbone, and Willoughby ${ }^{2} 3$ state:

... recently, much concern has been expressed about the adequacy of the measurements of the total-neutron cross section of iron. Numerous examples of 


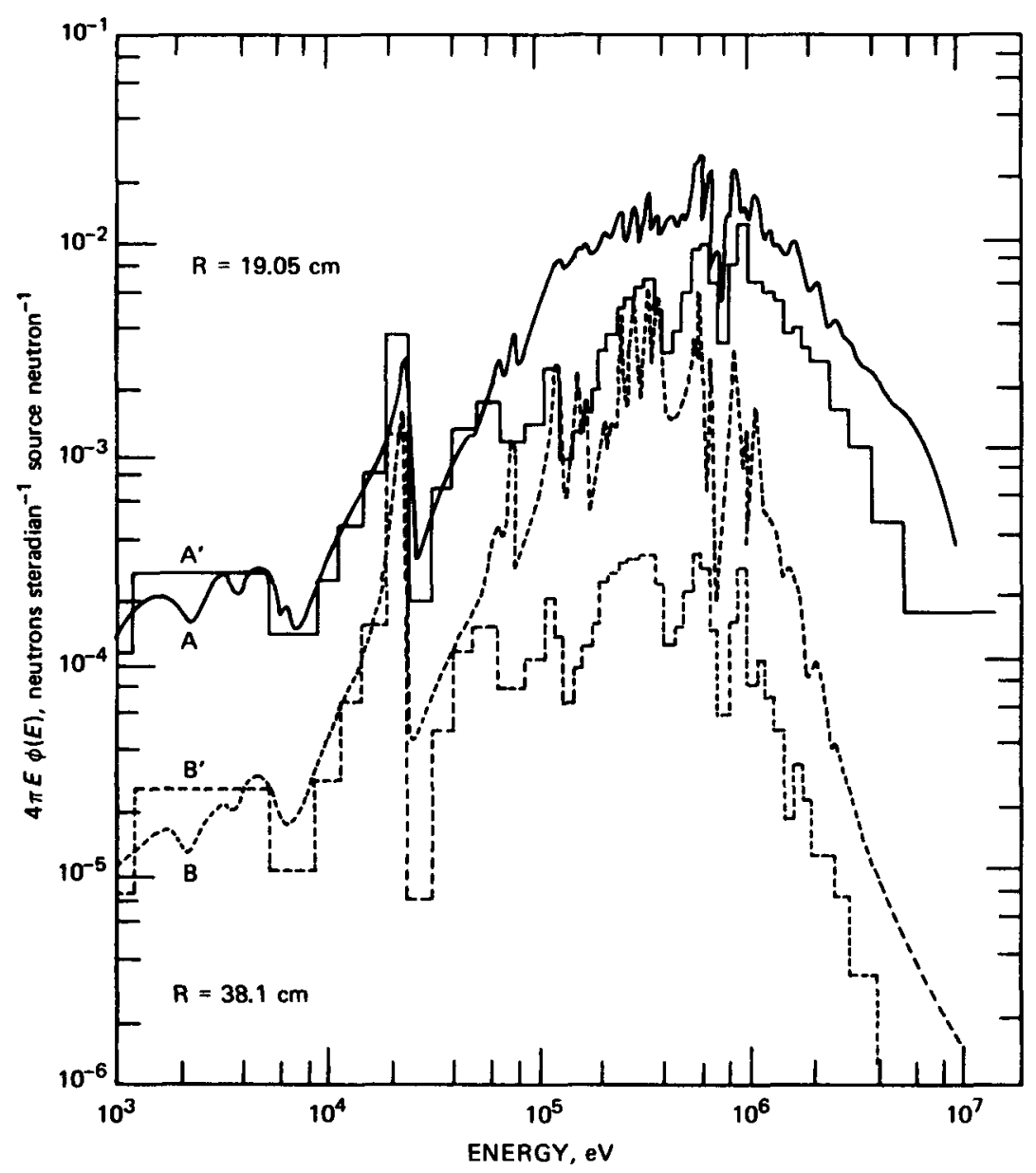

Fig. 9.12-Comparison between 49-group $1 \mathrm{DF}$ calculated $\left(\mathrm{A}^{\prime}, \mathrm{B}^{\prime}\right)$ and measured $(A, B) 0^{\circ}$ neutron angular spectra for iron at $R=19.05\left(A^{\prime}, A\right)$ and $38.1 \mathrm{~cm}\left(B^{\prime}, B\right)$. (From Cerbone et al. ${ }^{19}$ )

the questionable state of this cross section are evident. For some energy regions, ${ }^{24}$ there are large discrepancies in the measurements and energy scales; also considerably more structure than was previously seen has been observed in a recent set of measurements made by Cierjacks, Forti, Kopsch, Kropp, Nebe, and Unseld. $^{25}$

Many measurements have been made of this cross section which is of prime importance for shielding applications. However, in almost all cases the resolution was inadequate. For iron, the level spacing and level widths in the $\mathrm{MeV}$ neutron energy region are such that a multilevel fit for resonance parameters is extremely difficult; therefore, it is essential that measurements be made with very high resolution if the cross sections are to be used for shielding applications. It thus 
became clear that a new set of iron cross-section data taken with high-energy resolution and covering a large energy range was necessary.

For these reasons the iron total-neutron cross sections have been measured with high-energy resolution for the energy interval from 0.5 to $9 \mathrm{MeV}$.

The iron cross-section measurements were made by Carlson et al. ${ }^{23}$ at GGA with the same accelerator used by Cerbone et al. These transmission measurements were also made using time-of-flight (TOF) techniques but with a 226.75-m flight path. An overall plan of the geometry is shown in Fig. 9.13. High-energy neutrons are produced by electron bombardment (the bremsstrahlung produces photoneutrons) of a spherical uranium target having a radius of $3.8 \mathrm{~cm}$. The neutrons travel through an evacuated drift tube to a defining collimator at the $100-\mathrm{m}$ station. This collimator restricts the size of the neutron beam to a circular area having a radius of $6.3 \mathrm{~cm}$. In front of the collimator are two ${ }^{3} \mathrm{He}$ detectors that are used to monitor the neutron flux and a 3.1-cm-thick uranium filter that reduces the effect of the gamma flash on the neutron detector. The transmission samples and the sample changer are located behind the collimator. With the sample changer the samples can be placed accurately in a position such that the detector (at the 220-m station) is completely shadowed from the source. This mechanism is operated remotely from the data-taking area. The monitor counting rates were measured with and without the samples in place to ensure that neutrons scattered from the samples did not affect the ${ }^{3} \mathrm{He}$ monitors. No detectable difference was observed. The transmitted neutrons enter an evacuated drift tube that connects the $100-\mathrm{m}$ and the $220-\mathrm{m}$ flight-path stations. At the 220-m station the neutrons are detected in a 5 -cm-thick by 2.5-cm-radius cylindrical NE-211 scintillator coupled to a 56 AVP photomultiplier tube. The incident-neutron flux is perpendicular to the axis of the scintillator. The detector is surrounded by a lead shield that reduces the

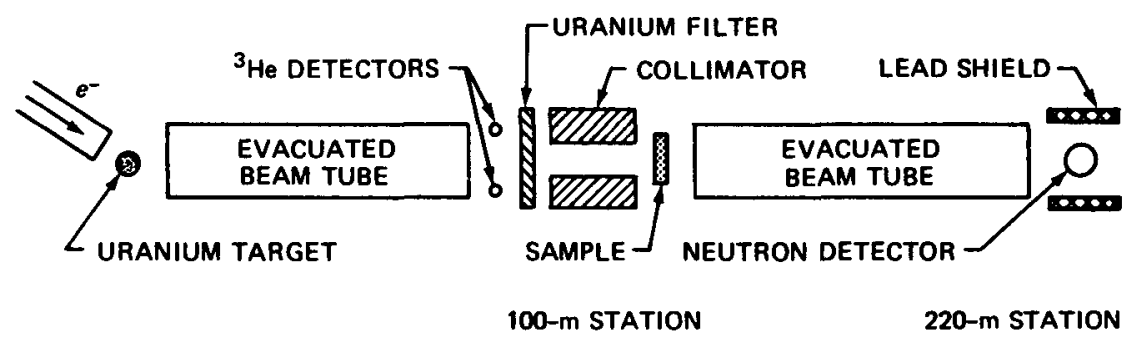

Fig. 9.13-Diagram of flight path and experimental setup used in the total-cross-section measurements of Carlson et al. ${ }^{23}$ 
ambient background. The contribution to the counting rate from neutrons that scatter off the detector into the lead shield and then back again into the detector was found to be negligible.

The iron sample was composed of standard Armco iron (99.94\% iron). The measured density agreed with the accepted value for the density of iron. The sample thickness was 0.386 nuclei/b.

Although measurements were made from 0.5 to $9.0 \mathrm{MeV}$, only a limited portion will be shown here. The complete set is given in Ref. 23 .

Figure 9.14 shows the measurements of Carlson et al. ${ }^{23}$ from 0.7 to $1.0 \mathrm{MeV}$. The curve is merely a guide obtained by connecting the points. The measurement of the cross section in this energy region where the cross section varies sharply with energy requires the utmost in energy resolution. Figure 9.15 compares the data with the data of Cierjacks et al. ${ }^{25}$ and of Schwartz, Schrack, and Heaton. ${ }^{26}$ Of the available iron total-cross-section measurements, those of Cierjacks et al. agree best with Carlson's data with respect to the presence of structure and the neutron-energy scale. Carlson's measurements, which have a somewhat smaller energy spread than those of Cierjacks $(0.035 \mathrm{nsec} / \mathrm{m}$ vs. $0.043 \mathrm{nsec} / \mathrm{m})$, resolve the structure somewhat better. The measurements of Schwartz et al. are in agreement with Carlson's data; however, a considerable amount of structure has been unresolved in these data, which were obtained with a resolution of $0.3 \mathrm{nsec} / \mathrm{m}$.

The iron total cross sections obtained in the study by Carlson et al. ${ }^{23}$ have provided much needed information about the cross-section valleys. It had previously been conjectured that deeper valleys are needed in the total cross section to obtain good agreement between the broomstick transmission measurements of Clifford et al. ${ }^{13,14}$ and calculations based on the total cross sections. The latest measurements show a trend toward deeper valleys than had been previously observed, which is primarily (it is believed) the result of better resolution.

In our discussion of the transport measurements in water reported by Verbinski et al., ${ }^{10}$ we mentioned that a small-source experiment with good geometry was also performed. The source was the LINAC described previously. In these measurements a slab shield was positioned at a distance of $40 \mathrm{~cm}$ from the source, which resulted in a nearly-good-geometry scattering configuration. Consequently the effects of the sharp resonances in the oxygen cross section on the spectral shapes could be observed and compared with predictions of shielding codes that have a fine energy mesh. The spectral measurements did not yield information on the attenuation of 


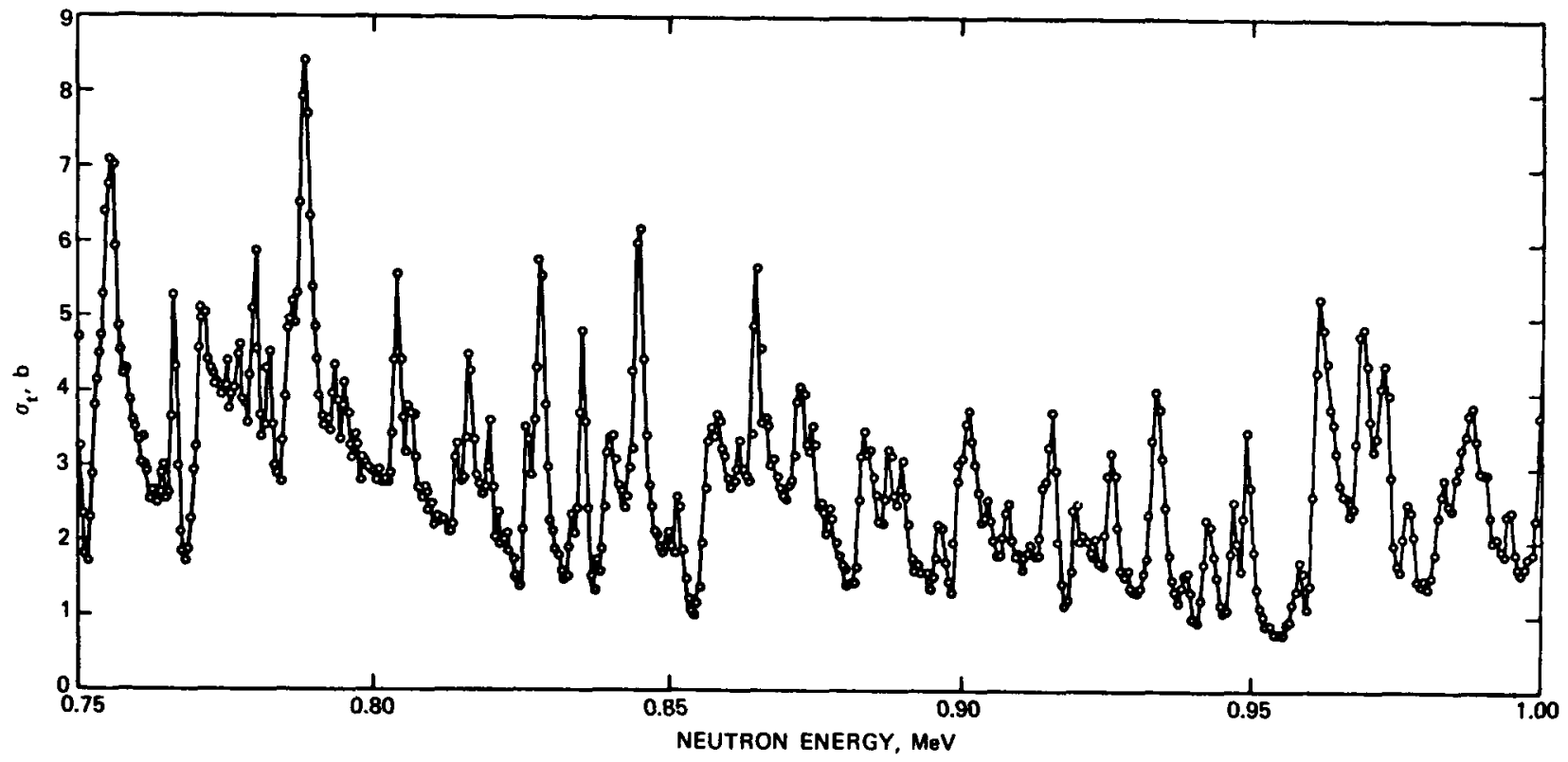

裂

Fig. 9.14-Total neutron cross section of iron (0.7 to $1 \mathrm{MeV}$ ). (From Carlson et al. ${ }^{23}$ ) 


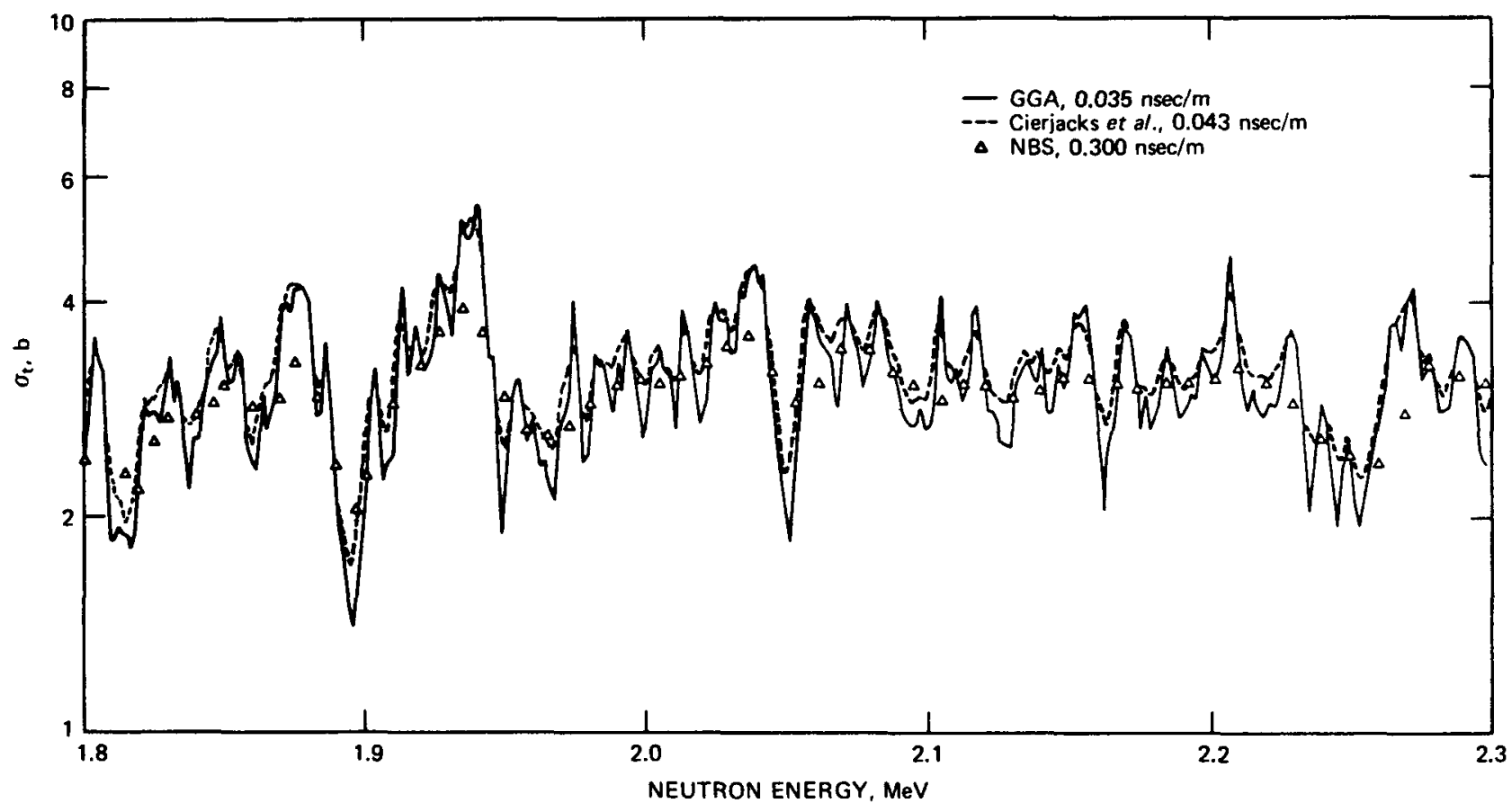

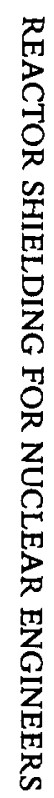

Fig. 9.15-Total neutron cross section of iron (1.8 to $2.3 \mathrm{MeV}$ ). (From Carlson et al. ${ }^{23}$ ) 
flux, but the activation of sulfur pellets placed on both the source side and the exit side of the slab provided a measure of the attenuation.

The physical arrangement for the measurements with the pulsed-neutron source is shown in Fig. 9.16. The LINAC provided short bursts of electrons (from $10 \mathrm{nsec}$ for thin shields at $0^{\circ}$ to $50 \mathrm{nsec}$ for the thickest shield at $30^{\circ}$ )

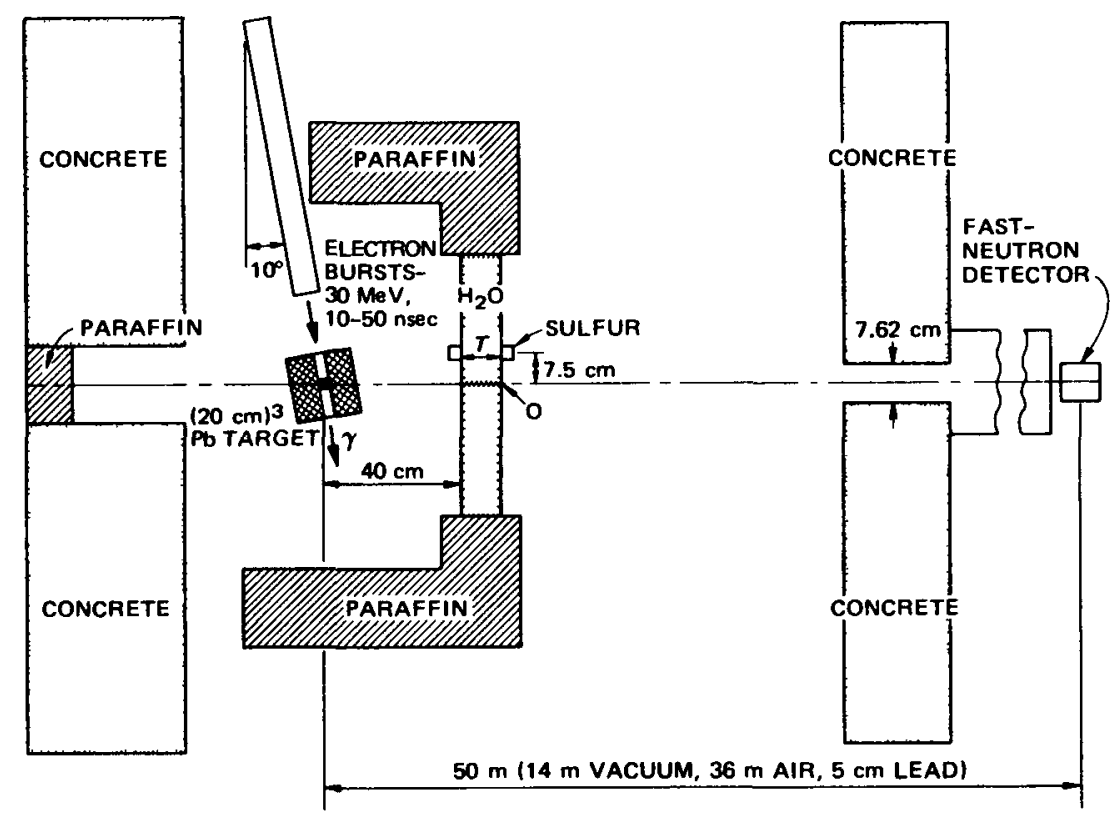

Fig. 9.16--Experimental arrangement at LINAC for measuring spectra of neutrons leaking from a water slab at $0^{\circ}$. For $30^{\circ}$ measurements, the slab-source configuration was rotated about the point "O." The positions of the sulfur pellets used for transmission measurements are also shown. [From V. V. Verbinski, M. S. Bokhari, J. C. Courtney, and G. E. Whitesides, Nuclear Science and Engineering, 27(2): 289 (1967).]

which irradiated a small lead target (5-cm-diameter by $2-\mathrm{cm}$ disk) and thus produced bremsstrahlung radiation which, in turn, produced prompt photoneutrons with a near-fission spectrum. The target was centered in a 20-cm lead housing, which was needed to suppress the bremsstrahlung radiation that would otherwise have incapacitated the neutron detector. Some of the neutrons escaping the lead housing penetrated the water shield and passed down a 50-m flight path to a 5- by 5-in. encapsulated NE-213 liquid scintillator neutron detector. Figure 9.16 shows the arrangement for measuring the $0^{\circ}$ (straight-through) spectrum for a water slab of thickness $T$. For some of the measurements, the neutron source and water slab were 
rotated $30^{\circ}$ about the point $O$ shown on the exit side of the slab. The sulfur pellets shown were used for measuring the neutron attenuation.

The configuration shown in Fig. 9.16 was calculated with both the NIOBE and the DTK codes. Since the NIOBE code cannot use a small-source geometry, the neutron source for the calculation was taken to be a 20-cm-diameter sphere with constant source density having the same spectrum as that measured with the water slab shown in Fig. 9.16 removed. Unfortunately, in this measurement not all the source could be viewed because of the limitation of the 7.62-cm-diameter collimator shown in Fig. 9.16. Comparisons of measurements with the NIOBE and DTK calculations using the same false-source geometry were equally poor.

Additional DTK calculations were performed for which the source was assumed to be a 5-cm-diameter sphere, which was a better simulation of the experimental source. The lead housing around this small source was treated as additional shielding. The spectrum used in these calculations, shown as the top curve in Fig. 9.17, was the same as that measured with the lead housing and water slab removed. The two lower curves in Fig. 9.17 compare the resulting calculated spectra with the measured $0^{\circ}$ spectra for slab thicknesses of $T=20$ and $40 \mathrm{~cm}$. The calculated results at $0^{\circ}$ are very similar to the NIOBE results ${ }^{10}$ except for the dashed-line sections at about 5 to $7.5 \mathrm{MeV}$, which were obtained with updated oxygen cross sections. The calculations for the $30^{\circ}$ configuration are compared with the measured spectra in Fig. 9.18. Here the calculated spectra are quite different from the NIOBE results, the overall agreement in Fig. 9.18 being much better. From this it is concluded that the false-source configuration used for NIOBE does not adequately approximate the experimental geometry and that reasonable care must be exercised in reproducing the source geometry in calculations that are being compared with small-source measurements of fast neutrons.

The solid line in Fig. 9.17, which represents the initial small-source DTK calculations, falls short of reproducing the broad transmission peak observed in the measured leakage spectra at $0^{\circ}$. Since the geometry for this measurement roughly approximates the good-geometry configurations used in measuring total neutron cross sections, the cross sections used in the calculations became suspect. Cross sections for the calculated solid lines in Fig. 9.17 were taken from the 1958 version ${ }^{27}$ of BNL-325. The dashed lines were calculated using the updated cross sections ${ }^{28}$ reported in the 1964 Supplement No. 2 of BNL-325. Much improvement is noted when the newer cross sections are used. 


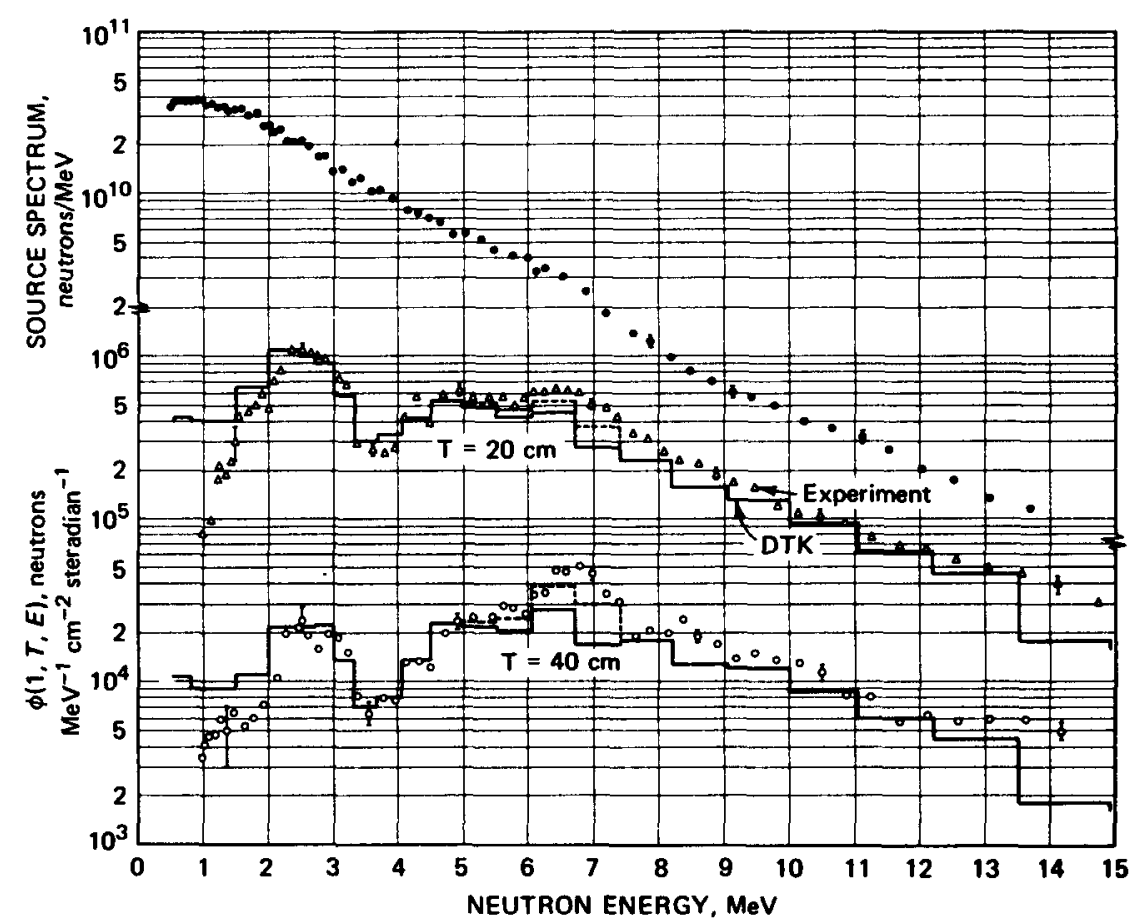

Fig. 9.17-Measured and DTK-calculated spectra of neutrons leaking from 20- and 40 -cm-thick water slabs at $0^{\circ}$. The calculational source spectrum, shown at the top of the figure, was taken as an isotropic source of $3.9 \times 10^{12}$ neutrons at the center of the lead housing shown in Fig. 9.16. Experimental spectra have been individually normalized to the DTK code results. [From V. V. Verbinski, M. S. Bokhari, J. C. Courtney, and G. E. Whitesides, Nuclear Science and Engineering, 27(2): 291 (1967).]

\subsubsection{Fixed Sources}

Fixed isotope sources provide an excellent tool for the measurement of materials if the whole reactor energy distribution is not needed. The work of Alberg, Beck, O'Brien, and McLaughlin ${ }^{2} 9$ provides a case in point. Their measurements with ${ }^{137} \mathrm{Cs}$ gamma rays provide differential energy and angle spectra from a point isotropic source in an effectively infinite medium of water for gamma-ray penetrations of one, two, three, and four mean free paths at $15^{\circ}$ intervals. The objective of this experiment was to obtain a definitive set of differential distributions in an infinite medium which could be used as a standard for comparison with transport predictions.

Figure 9.19 shows the experimental arrangement. The detector was a 5.08- by $5.08-\mathrm{cm} \mathrm{NaI}(\mathrm{Tl})$ crystal, and an EMI-9552 photomultiplier tube and 


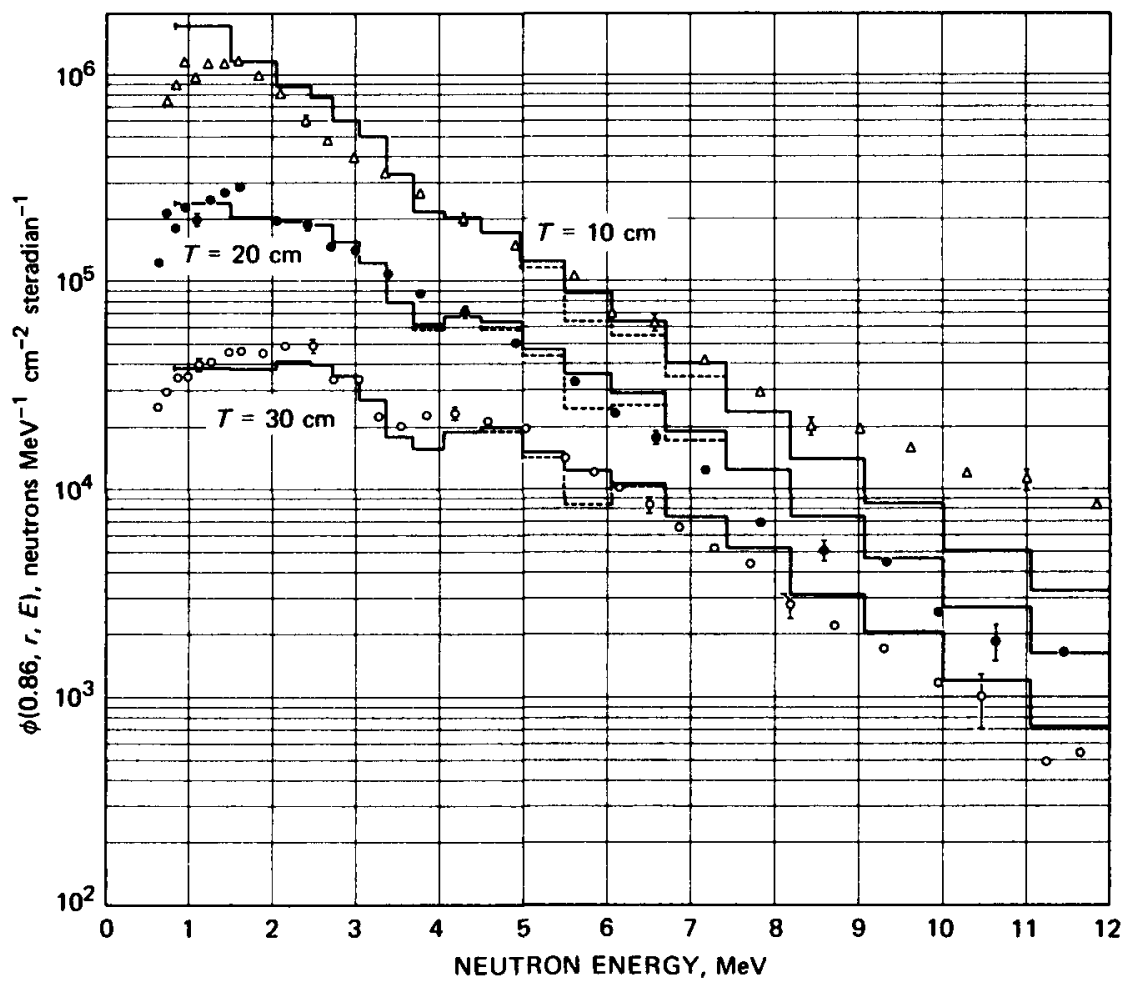

Fig. 9.18-Measured and DTK-calculated spectra of neutrons leaking from 10-, 20-, and 30 -cm-thick water slabs at $30^{\circ}$. Experimental spectra have been individually normalized to the DTK code results. [From V. V. Verbinski, M. S. Bokhari, J. C. Courtney, and G. E. Whitesides, Nuclear Science and Engineering, 27(2): 292 (1967).]

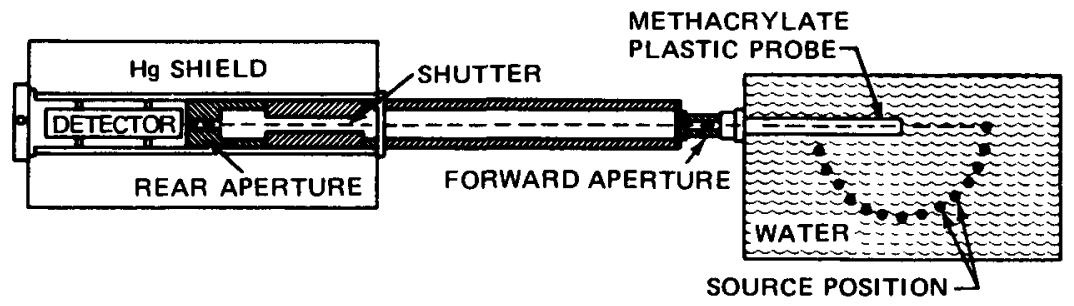

Fig. 9.19-Schematic diagram of point source ${ }^{137} \mathrm{Cs}$ differential measurements. The distance from the back edge of the rear aperture to the front edge of the forward aperture of the collimator is $80 \mathrm{~cm}$; to the end of the methacrylate plastic probe, it is $163 \mathrm{~cm}$. The aperture diameters are $1.0 \mathrm{~cm}$. [From M. Alberg. H. Beck, K. O'Brien, and J. E. McLaughlin, Nuclear Science and Engineering, 30(1): 67 (1967).] 
a 512-channel analyzer were used. About 700 liters of distilled water was used to fill a 48 - by $97-$ by $145-\mathrm{cm}$ methacrylate plastic tank. This size was chosen so that both the source and the detector would always be at least two mean free paths from the edge of the box. The ${ }^{137} \mathrm{Cs}$ source was calibrated to $\pm 5 \%$.

The detector assembly was located outside the medium to minimize displacement effects. A probe and collimator were used that were based on a design by Skarsgard and Johns. ${ }^{30}$ The defining apertures of the collimator were located outside the tank. A hollow plastic probe of $2.5 \mathrm{~cm}$ inside diameter was inserted into the tank and attached to the front end of the collimator with a vacuum and pressuretight seal. The path between the methacrylate plastic window in front of the crystal and the end of the probe was then evacuated. Pulse-height distributions corresponding to various angles between the source and the detector for a given penetration were obtained by moving the source around a semicircle of fixed radius located on the midplane of the tank. The collimator was equipped with a shutter so that background readings could be taken at each angle with the source in position.

The solid angle subtended by the crystal was determined by the method described by Skarsgard and Johns. ${ }^{30}$ In this scheme a sphere is constructed whose center lies on the collimator axis at the end window of the probe and whose radius is just large enough to include all points on the window which subtend a finite solid angle at the crystal. It is convenient to regard this sphere as the source of scattered gamma rays, even though most of the radiation seen by the crystal will have been scattered through the sphere from other points in the medium. A cross section is taken through the sphere at the window, and the solid angle seen by each differential element on this cross section is calculated. An integration over these elements yields the total solid angle seen by the crystal.

Eight sources were used to calibrate the spectrometer: ${ }^{95} \mathrm{Zr}-{ }^{95} \mathrm{Nb}$ $(750 \mathrm{keV}),{ }^{137} \mathrm{Cs}(662 \mathrm{keV}),{ }^{7} \mathrm{Be}(477 \mathrm{keV}),{ }^{113} \mathrm{Sn}(393 \mathrm{keV}),{ }^{51} \mathrm{Cr}$ $(322 \mathrm{keV}),{ }^{47} \mathrm{Sc}(160 \mathrm{keV}),{ }^{109} \mathrm{Cd}(88 \mathrm{keV})$, and ${ }^{85} \mathrm{Sr}(514 \mathrm{keV})$. Onequarter of the multichannel analyzer, 128 channels, was used to obtain a standard pulse-height distribution for each source. The eight standard pulse-height distributions were fitted with energy-dependent functions describing the photopeak, escape peak, Compton tail, and backscatter peak. These functions yielded interpolated pulse-height distributions for energies 
lying in the range covered by the standard sources. When suitably normalized the set of 124 interpolated pulse-height distributions equally spaced over the interval 0.0198 to $0.9382 \mathrm{MeV}$ formed a 124 by 124 response matrix for the spectrometer.

Pulse-height distributions were measured for separations between the source and the detector which correspond to one, two, three, and four mean free paths of water. The angle between the source and the detector was varied in $15^{\circ}$ intervals. The pulse-height distributions were unfolded to yield the differential energy and angle distributions for the various separation distances.

These spectra are normalized to a source strength of 1 photon/sec. The differential energy and angle spectrum $I$ represents the energy carried by photons of energy $E\left(\mathrm{MeV}\right.$ steradian $\left.^{-1}\right)$, which in unit time cross a unit area whose normal is in the angular direction specified. The ordinate is a product of $I$ and the factor $4 \pi r^{2} e^{\mu_{0} r}$, where $r$ is the penetration depth in centimeters and $\mu_{0}$ is the absorption coefficient at the source energy. These units not only facilitate comparison among spectra taken at different penetration depths but also allow comparison of spectra taken in different media.

Marshall and Wells ${ }^{31}$ have reported comparisons of their Monte Carlo calculated ${ }^{137} \mathrm{Cs}$ transport data in infinite air with these measured infinite-water-media data. They made comparisons of the data differential in energy and angle as well as integrated over angle. The double-differential data compared favorably in shape. Energy spectra integrated over angle for the four distances are shown in Fig. 9.20, for which the measured data have been divided by a factor of 1.2. Agreement in shape and relative magnitude is good.

In addition to the measurements, Alberg et al. ${ }^{29}$ carried out multigroup transport calculations of differential energy spectra in water for a ${ }^{137} \mathrm{Cs}$ source using the latest values of the attenuation coefficients for water. ${ }^{32}$ The spherical-harmonics technique ${ }^{33}$ (described in Sec. 4.3) was used, in which the transport equation is solved directly by expanding the angular flux in polynomials, and a matrix equation is used to solve the resulting set of multigroup equations. For these calculation expansions in Legendre polynomials up to degree seven were used. Comparisons of the calculated and measured energy spectra integrated over angle for the four mean-free-path values are shown in Fig. 9.21. The overall agreement is good. The point scatter in the experimental curves at higher energies is caused by poor counting statistics. 


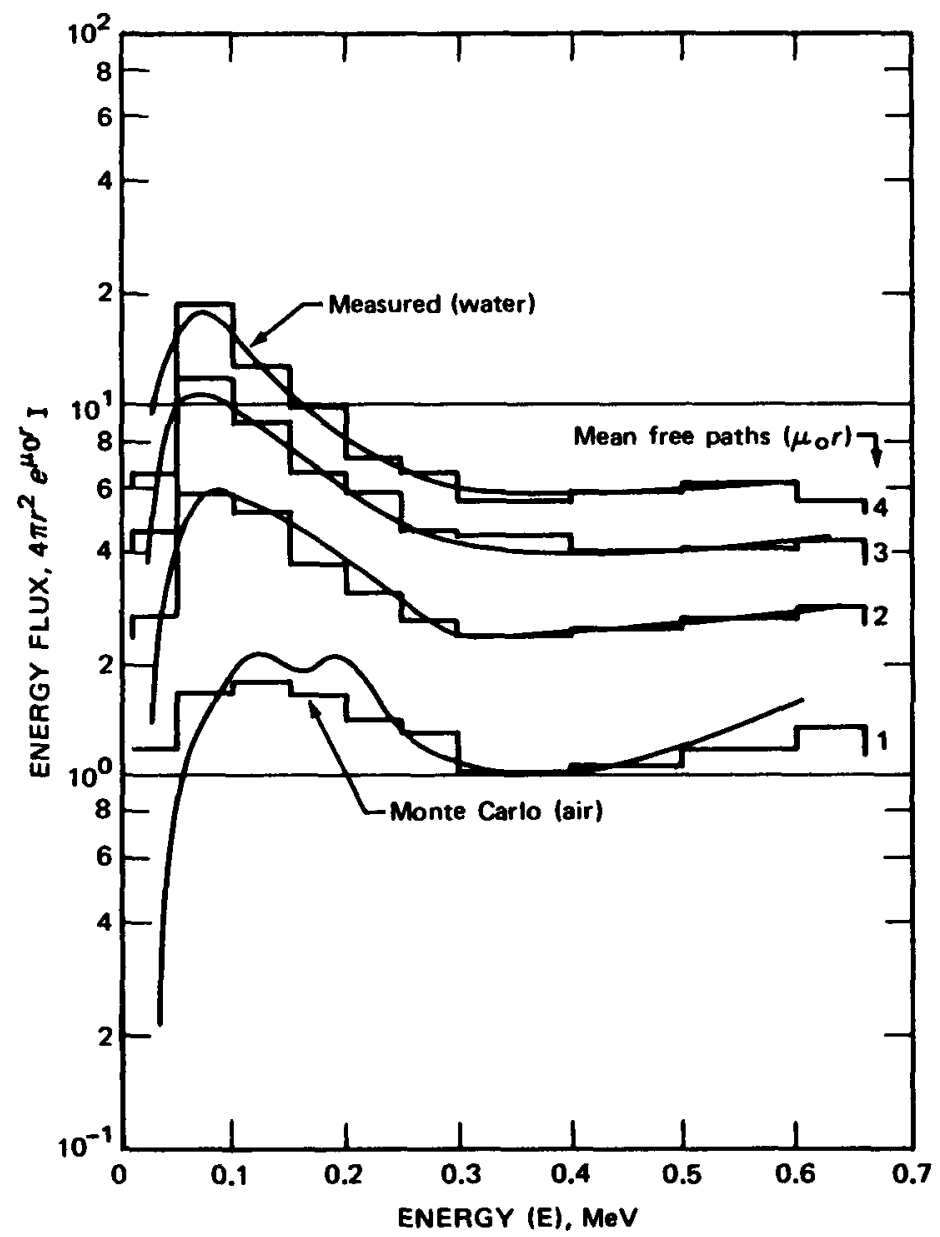

Fig. 9.20-Point isotropic ${ }^{137} \mathrm{Cs}$ source total-energy spectra. (From Marshall and Wells. ${ }^{31}$ )

\subsection{PHENOMENOLOGICAL MEASUREMENTS}

Phenomenological problems are usually geometric in nature, i.e., they are characterized by a particular geometry. Under this general heading we include air transport, air-over-ground interface effects, albedos, and duct penetration.

\subsubsection{Air-Transport and Air-Ground Interface Experiments}

In Secs. 8.2.2 and 8.2.3 we described a series of air-transport measurements made on a tower in Nevada with a $14-\mathrm{MeV}$ neutron source. It 


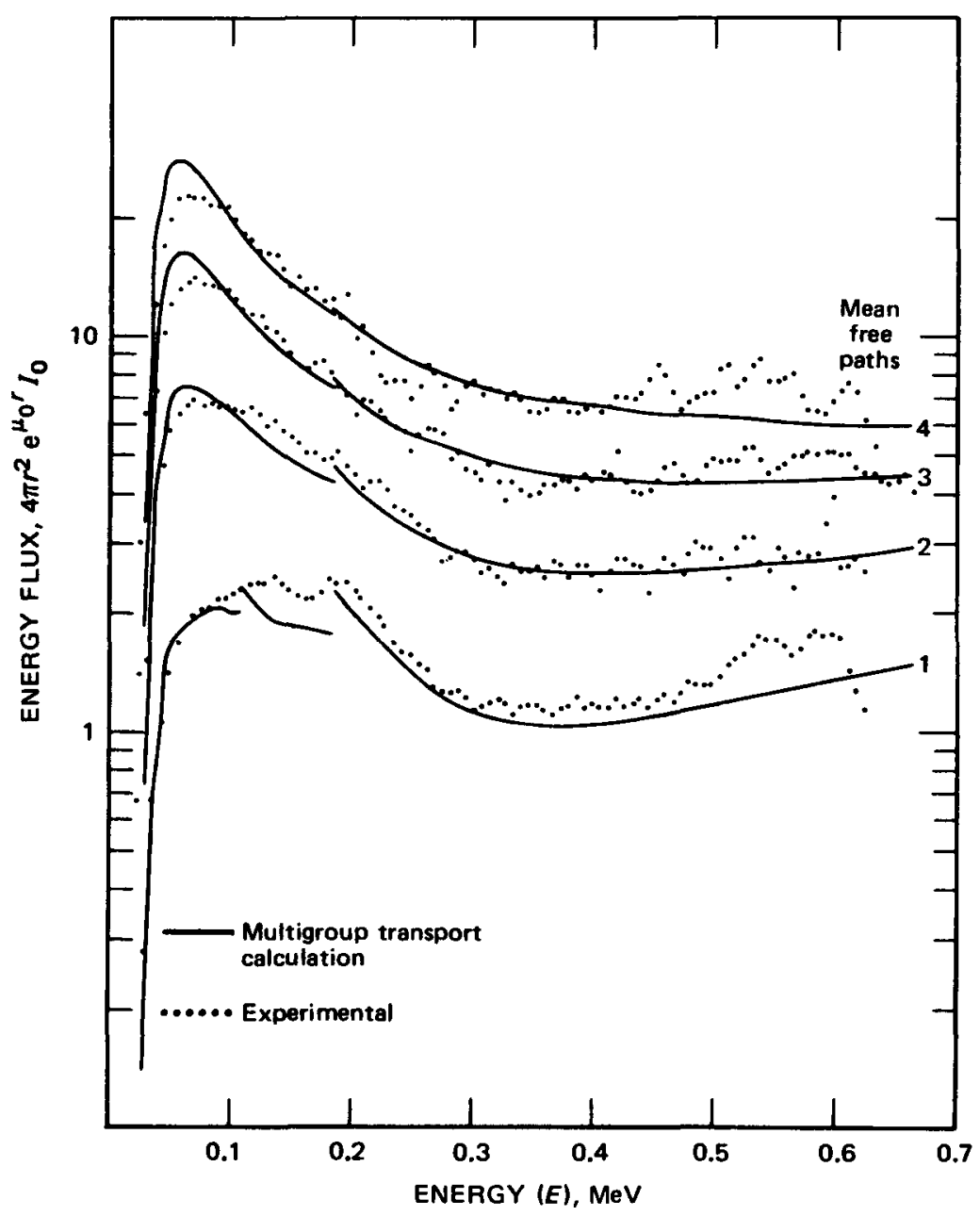

Fig. 9.21-Comparison of the experimentally determined differential energy spectra $\left(I_{0}\right)$ in water with multigroup transport calculations. The $I_{0}$ is obtained from an integration of the differential energy and angle spectrum $I$ over all angles. [From M. Alberg, H. Beck, K. O'Brien, and J. E. McLaughlin, Nuclear Science and Engineering, 30(1): 71 (1967).]

is interesting to look briefly at other measurements in this series, which involved both a reactor and a ${ }^{60} \mathrm{Co}$ source. There were several purposes for this series of measurements. The primary objective was to obtain definitive air-transport data at large distances $(>1 \mathrm{~km})$ for the source energies used. Source height and detector height were varied to obtain the effect of the air-ground interface. Although performed primarily to support nuclear- 
weapons-effects studies, measurements such as these are frequently useful in safety analysis of reactors.

Neutron and gamma-ray measurements were made at the Nevada Test Site (NTS) during Operation BREN (Bare Reactor Experiment Nevada), which is described by Sanders, Haywood, Lundin, Gilley, Cheka, and Ward ${ }^{34}$ and by Auxier, Haywood, and Gilley. ${ }^{35}$ The BREN facility consisted of a 465-m-high steel tower (Fig. 9.22). Two radiation sources were supported by an aluminum hoist car that could be moved along the face of the tower. The two sources were the ORNL Health Physics Research Reactor (HPRR) ${ }^{36}$ (shown on the tower in Fig. 9.22) and a ${ }^{60}$ Co source whose effective activity was approximately $800 \mathrm{Ci}$. The HPRR is a small fast reactor that produces a nearly isotropic angular distribution of fission neutrons and is neither moderated nor cooled.

From the extensive array of measurements made during Operation BREN, we show only dose rate vs. distance for neutrons, secondary gamma rays, and ${ }^{60} \mathrm{Co}$ gamma rays reported by Haywood, Auxier, and Loy. ${ }^{37}$

Figure 9.23 shows the neutron dose as a function of slant range for source heights of $8.2,91,343$, and $457 \mathrm{~m}$ and a detector height of $0 \mathrm{~m}$. Since radiation entering the ground beneath the source is partially absorbed, the dose at a fixed distance away should increase as the source is raised because of less ground absorption. Figure 9.23 shows that the neutron dose does indeed increase with source height.

Figure 9.24 shows the measured gamma-ray dose as a function of slant range for reactor heights of $8.2,91$, and $343 \mathrm{~m}$ and a detector height of $0 \mathrm{~m}$. The gamma-ray dose is seen to increase also as the source height increases, but the mechanism responsible for this is not necessarily the same as that for the neutron-dose increase. Some of the gamma-ray dose is caused by secondary gamma rays resulting from neutron interactions, a portion of which come from the ground.

Figure 9.25 shows the gamma-ray dose from the ${ }^{60} \mathrm{Co}$ source as a function of slant range for source heights of $8.2,91$, and $343 \mathrm{~m}$ and a detector height of $0 \mathrm{~m}$. The behavior is identical to that for neutrons in that the ${ }^{60}$ Co dose increases with source height.

A differential measure of the air-ground interface effects on the neutron dose reported by French and Mooney ${ }^{38}$ was based on data obtained during the 14-MeV measurements. described in Secs. 8.2.2 and 8.2.3 to test the concepts of the first-last collision model. The model was tested (in relation to last scatters only) by placing a slab of polyethylene on a hoisting mechanism to separate the neutron dose arriving at a detector into fractions 


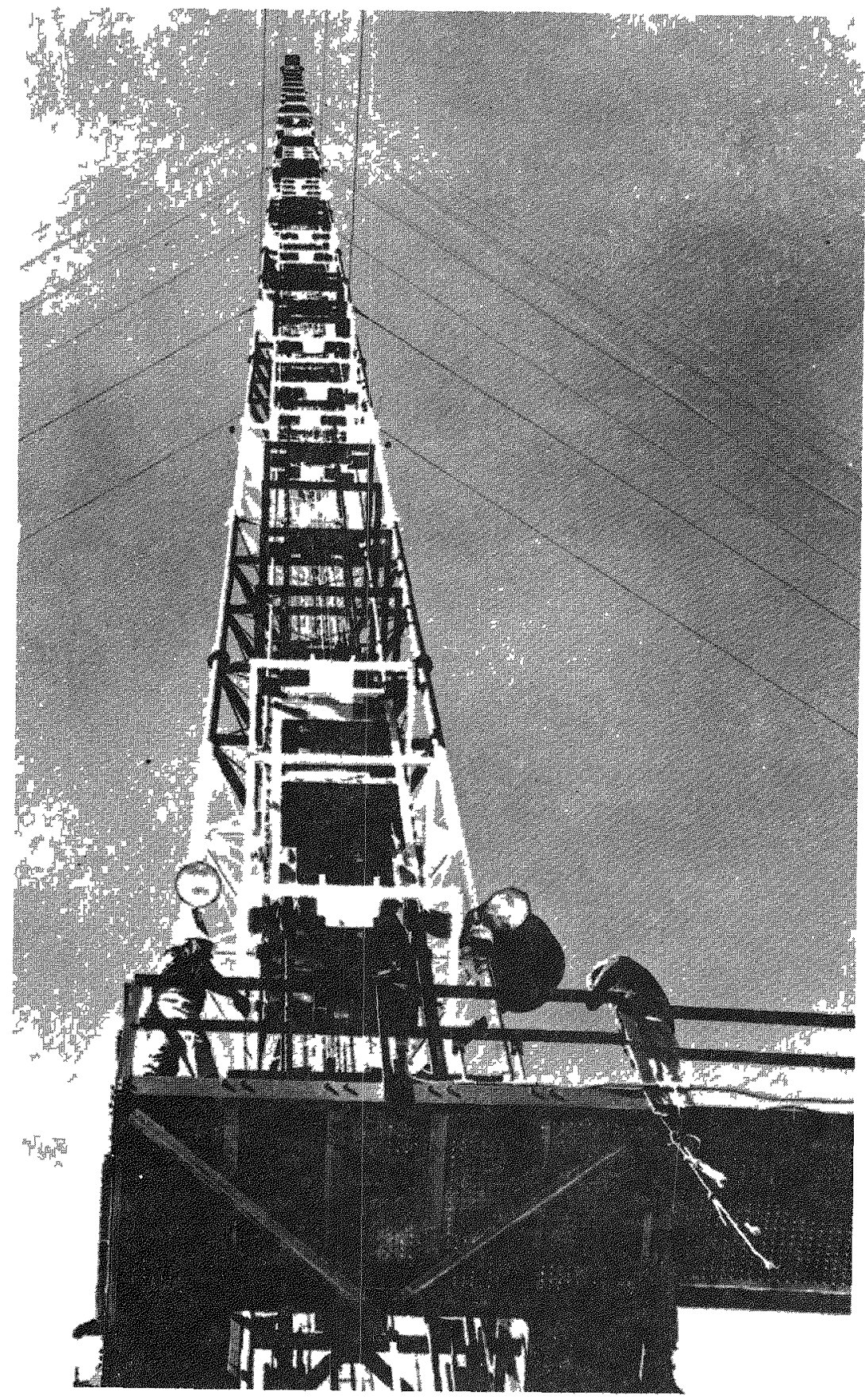

Fig 922 -The BREN tower and the reactor (HPRR) positioned in the hoisting mechanism (From Haywood et $a^{37}$ ) 


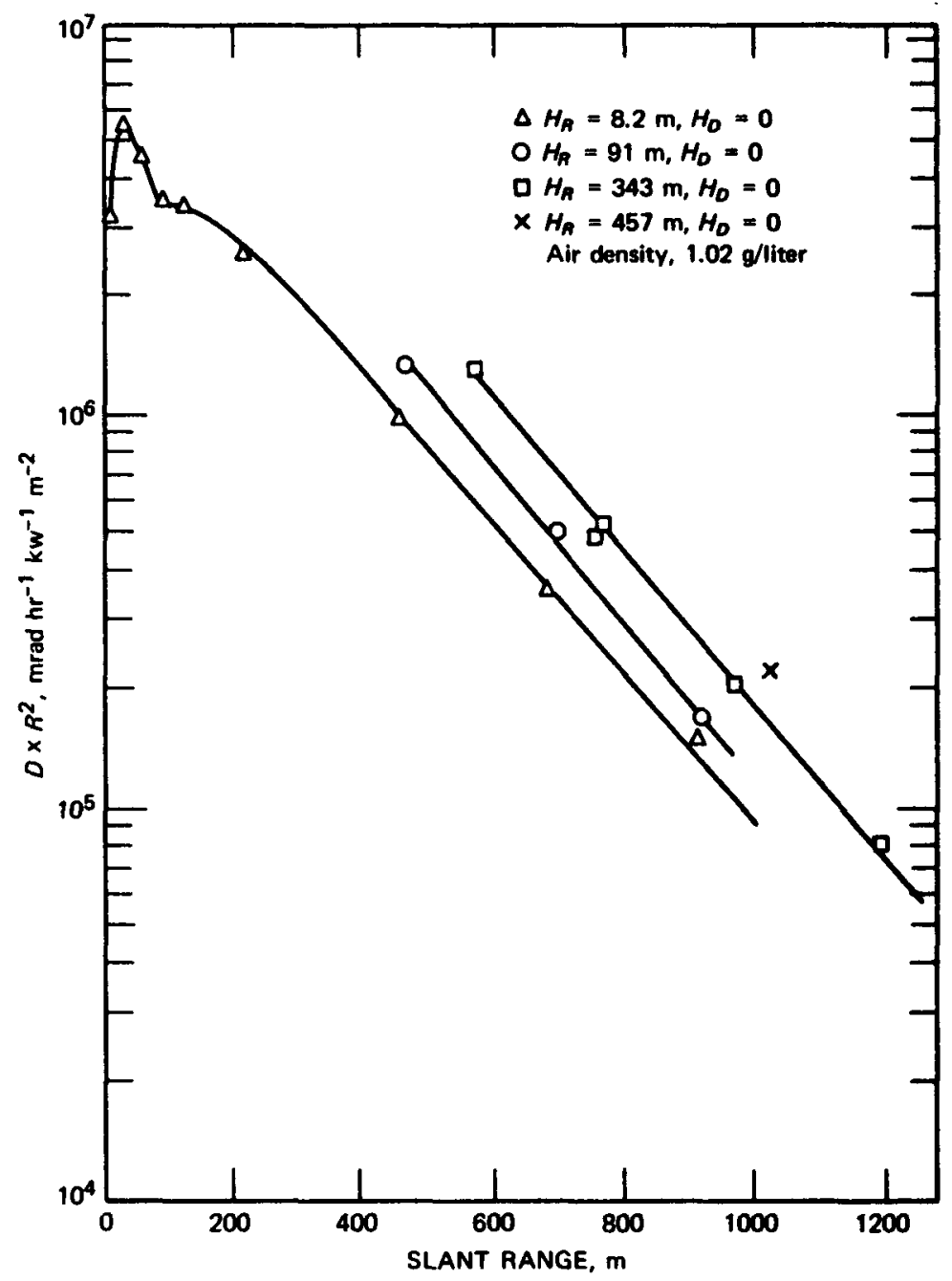

Fig. 9.23-Neutron dose times slant range squared $\left(D \times R^{2}\right)$ as a function of slant range (R) for a detector height $\left(H_{D}\right)$ of $0 \mathrm{~m}$ and various reactor heights $\left(H_{R}\right)$. (From Haywood et al. $^{37}$ )

from the upper and lower hemispheres. The slab assembly was designed to move from the ground surface to a height of $70 \mathrm{ft}$ above the ground. Hurst-type fast-neutron dosimeters were mounted on the top and bottom of the slab and on a boom for measurements of the free-field dose.

The experimental setup was located at a horizontal distance of $1000 \mathrm{ft}$ from the base of the tower supporting the 14-MeV accelerator. The source was fixed at a height of $112 \mathrm{ft}$ above the ground for the entire test. 


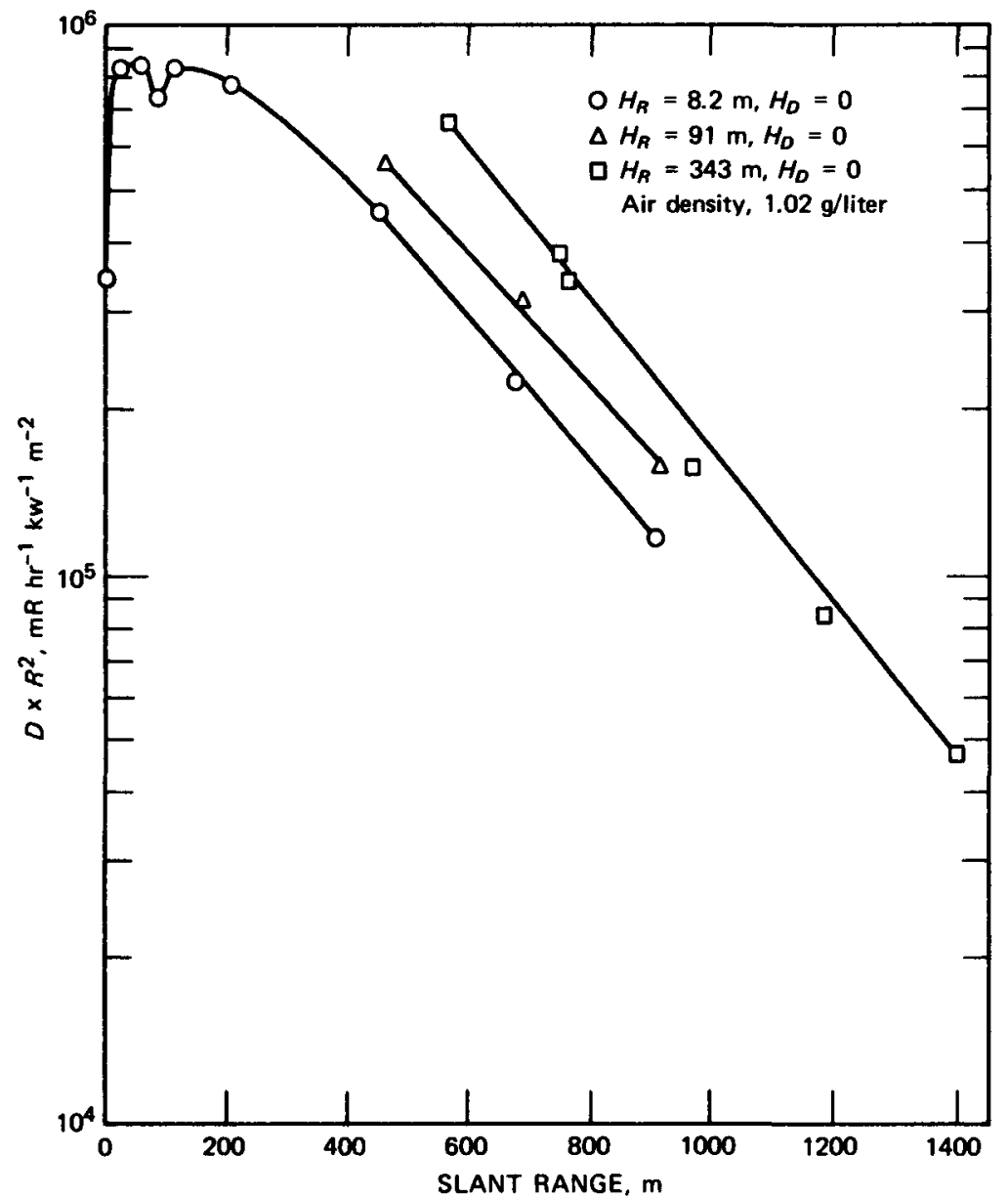

Fig. 9.24-Gamma-ray dose times slant range squared $\left(D \times R^{2}\right)$ as a function of slant range $(R)$ for a detector height $\left(H_{D}\right)$ of $0 \mathrm{~m}$ and several reactor heights $\left(H_{R}\right)$. (From Haywood et al. ${ }^{37}$ )

Figure 9.26 shows the experimental setup looking toward the BREN tower. The lower detector is attached to the bottom center of the slab, the free-field detector is on the boom extending from the slab toward the tower, and the top detector (not visible) is mounted atop the slab. Fast-neutrondose measurements were made on the three detectors for slab heights from 0.23 to $21 \mathrm{~m}$. The uncollided dose, seen only by the top and free-field detectors, was calculated and subtracted from the measured doses. The uncollided dose is not affected by the interface and is therefore ignored in the first-last collision model. In addition to functioning as a $2 \pi$ shield, the slab backscatters neutrons into the detector and reduces the neutron flux at 


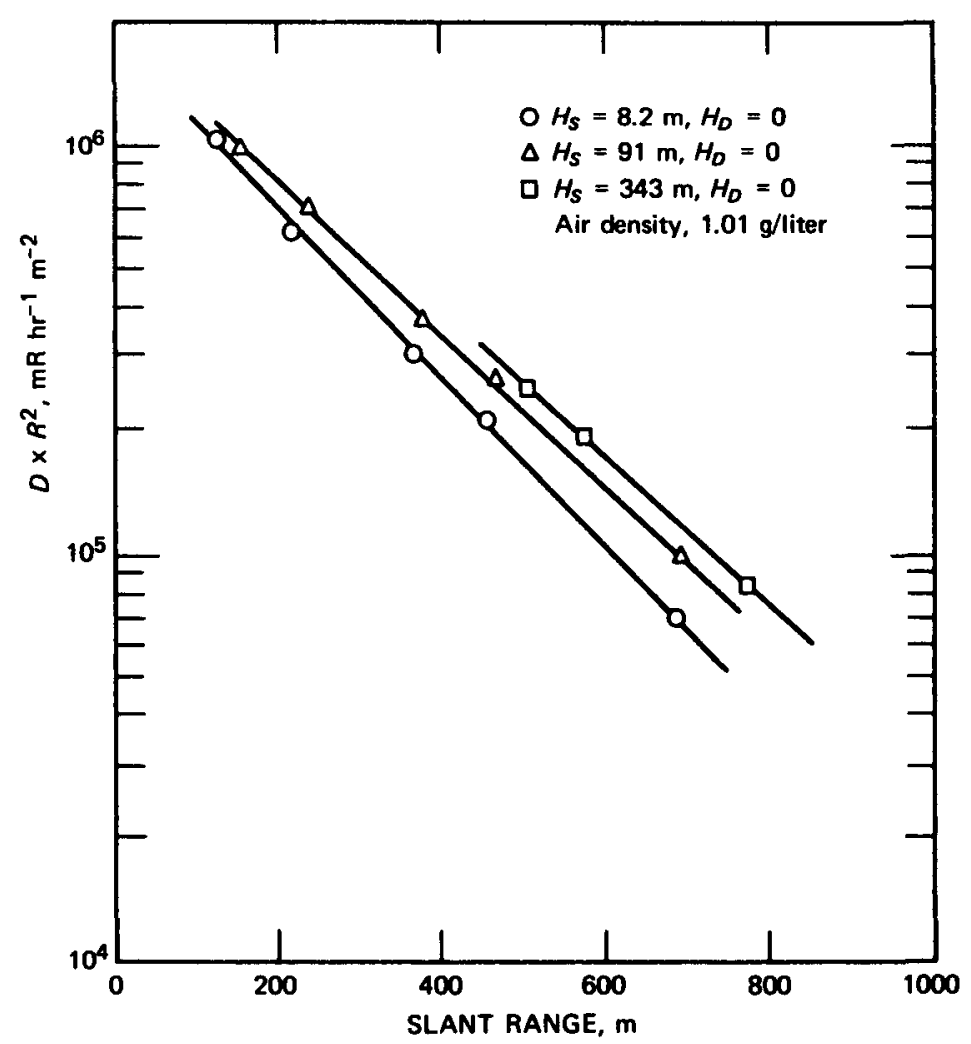

Fig. 9.25-Gamma-ray dose times slant range squared $\left(D \times R^{2}\right)$ as a function of slant range $(R)$ for a detector height $\left(H_{D}\right)$ of $0 \mathrm{~m}$ and various ${ }^{60}$ Co source heights $\left(H_{S}\right)$. (From Haywood et al. ${ }^{37}$ )

the ground in an area dependent on the slab height. These effects were calculated and subtracted from the measured dose. Results were shown in Sec. 8.2.3.

To make quantitative comparisons of the calculated and measured interface effects, the calculated total last-collision dose was normalized to the measured free-field detector dose at a height of $21 \mathrm{~m}$. This height was selected for the normalization because the interface effect should be least at this height. Comparison of the normalized results shows the extent to which the theoretical model estimates the fraction of the $21-\mathrm{m}$ free-field dose observed at lower heights.

Examination of the scattered doses shown in Fig. 8.10 shows that the dose at the top detector is relatively independent of height and that the dose at the bottom detector increases by a factor of more than 2 as the height is increased from 0.23 to $21 \mathrm{~m}$. 


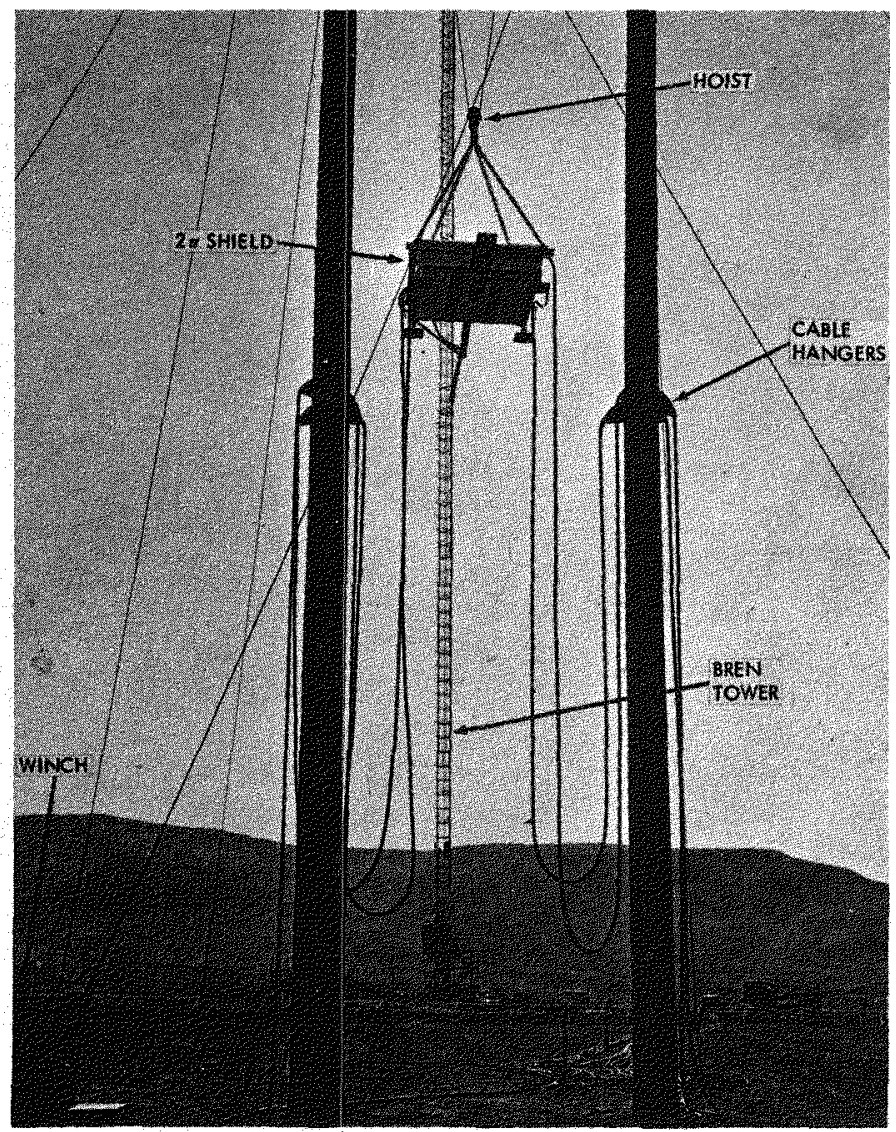

Fig. 9.26-Arrangement for $2 \pi$ shield looking toward the neutron source. (From French and Mooney. ${ }^{38}$ )

\subsubsection{Duct Penetration}

The streaming of neutrons and gamma rays through entranceways, ducts, vents, cable-route openings, portholes, and other openings has always been a major problem in shield design. Many of the efforts at devising methods for calculating streaming are reviewed in Chap. 7. Clifford et al. ${ }^{13}$ report the comparison of measurements and calculations of neutron streaming through multilegged concrete ducts from an experiment performed at the Tower Shielding Facility at ORNL. Their results have already been described in Chap. 7 , but it is interesting to see the experimental arrangement for a one-bend duct (Fig. 9.27). A 7.6-cm collimated beam of neutrons was incident to the opening of the $0.91-\mathrm{m}$-square duct at an angle of $45^{\circ}$. The 


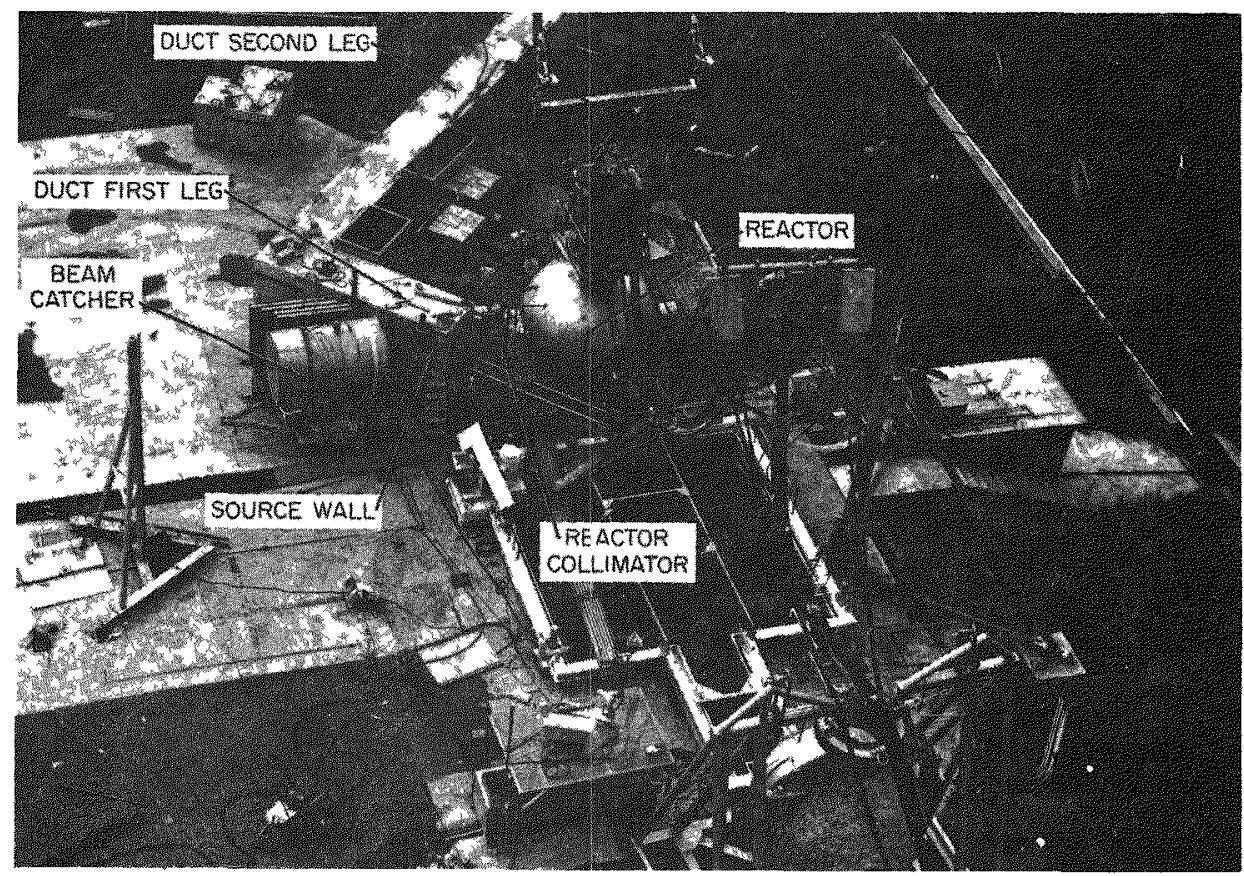

Fig. 9.27-Photograph of experiment for duct with one bend. (From Clifford et al. ${ }^{13}$ )

source of neutrons was the Tower Shielding Reactor II. The fast-neutron spectrum of the beam was measured by Bokhari, Verbinski, and Todd ${ }^{39}$ using the shielded diode spectrometer described previously. The slow-neutron spectra were obtained from measurements with a boron-shielded $\mathrm{BF}_{3}$ detector $^{40}$ having ${ }^{10} \mathrm{~B}$ filters with 23 different thicknesses.

Three duct configurations were involved in the test: a straight duct, a duct with one bend, and a duct with two bends. Principal results have been shown in Figs. 7.52 through 7.54. The measured absolute energy spectrum of the beam was used for the source spectrum in the calculations. In general, the agreement with calculations averages better than $20 \%$ through five orders of fast-neutron dose attenuation. Calculations were made of the thermal flux resulting from all source neutrons with energies above cadmium cutoff to test the accuracy of the slowing-down model used in the code. In general, the agreement is also within $20 \%$ in the second and third legs and within $5 \%$ for most of the straight ducts.' For the three-legged duct, it was necessary to follow approximately 18 wall backscatterings to obtain $90 \%$ of the flux contribution in the middle of the second leg and 25 wall backscatterings in the middle of the third leg. 
Even though we have described widely different shield experiments, we have not done justice to the diversity of arrangements in the literature. The variety of experiments performed is as great as the variety of problems the shield designer has faced since radiation sources were first used. Sources of radiation being measured have included reactors of all kinds (power, research, pulsed, and critical assemblies), as well as source plates, isotopes, neutron generators, and a variety of accelerators. Environments in which reactor sources and detectors have been placed range from the bottom of swimming pool facilities to towers 200 and $1500 \mathrm{ft}$ high, to aircraft flying at $35,000 \mathrm{ft}$, and to satellites in orbit. Detectors have been located in aircraft crew compartments, underground shelters, spacecraft payloads, ducts, armored tanks, submarines, and surface vessels.

\section{REFERENCES}

1. W. J. Price, Nuclear Radiation Detection, 2nd ed., McGraw-Hill Book Company, Inc., New York, 1964.

2. C. M. Huddleston (Ed.), Detectors for Shielding Research, in Proceedings of the Session on Detectors for Shielding Research, held at American Nuclear Society, Toronto, Canada, June 1968, Report ANS-SD-10.

3. J. Greenborg (Ed.), Proceedings of the Special Session on Fast Neutron Spectroscopy, Presented at the 1964 Winter Meeting, San Francisco, Calif., Dec. 1, 1964, Report ANS-SD-2, American Nuclear Society, February 1965.

4. H. Murphy, Summary of Neutron and Gamma Dosimetry Techniques, Report AFWL-TR-66-111, Air Force Weapons Laboratory, September 1967.

5. D. D. Glover, Experimental Reactor Analysis and Radiation Measurements, McGrawHill Book Company, Inc., New York, 1965.

6. S. T. Friedman, J. Rathbun, and R. Kaufmann, Use of the Transport Code DTK for Shielding Design and Analysis, Trans. Amer. Nucl. Soc., 7: 2 (December 1964).

7. Oak Ridge National Laboratory, Compilation of Data on Experimental Shielding Facilities and Tests of Shields of Operating Reactors, USAEC Report ORNL-RSIC-24, November 1968.

8. J. A. Belcher and L. K. Zoller, The Outside Test Tank and Associated Hardware, USAEC Report XDC-59-11-154, General Electric Company, Nov. 6, 1959.

9. S. T. Friedman, Shielding Tests Performed in the General Electric Outside Test Tank in the Period March-June 1958, USAEC Report APEX-581, General Electric Company, February 1961.

10. V. V. Verbinski, M. S. Bokhari, J. C. Courtney, and G. E. Whitesides, Measurements and Calculations of the Spectral and Spatial Details of the Fast-Neutron Flux in Water Shields, Nucl. Sci. Eng., 27(2): 283-298 (1967).

11. D. Yetman, B. Eisenman, and G. Rabinowitz, Description of Input Preparation and Operating Procedures for 9-NIOBE and IBM-7090 Code, in Advanced Shield Calculational Techniques, I, Report UNUCOR-631, United Nuclear Corporation, 
1963; S. Preiser, G. Rabinowitz, and E. deDufour, A Program for the Numerical Integration of the Boltzmann Transport Equation-NIOBE, in Advanced Shield Calculational Techniques, II, Report UNUCOR-632, United Nuclear Corporation, 1963.

12. D. K. Trubey and B. F. Maskewitz (Eds.), A Review of the Discrete Ordinates $S_{n}$ Method for Radiation Transport Calculations, USAEC Report ORNL-RSIC-19, Oak Ridge National Laboratory, March 1968.

13. C. E. Clifford, F. J. Muckenthaler, R. E. Maerker, E. A. Straker, and F. R. Mynatt, Neutron Transport Studies in Ducts and Simple Shields, in Proceedings of the Conference on the Physics Problems of Reactor Shielding held at the Atomic Energy Research Establishment, Harwell, Sept. 26-29, 1967, British Report AERE-R-5773 (Vol. 4), p. 937, 1968.

14. C. E. Clifford, E. A. Straker, F. J. Muckenthaler, V. V. Verbinski, R. M. Freestone, K. M. Henry, and W. R. Burrus, Measurements of the Spectra of Uncollided Fission Neutrons Transmitted Through Thick Samples of Nitrogen, Oxygen, Carbon, and Lead, Investigation of the Minima in Total Cross Sections, Nucl. Sci. Eng., 27(2): 299-307 (1967).

15. D. C. Irving, 05R Cross Section Library, Memo 2, Code Package CCC-17, Oak Ridge National Laboratory, 1965.

16. S. Cierjacks, P. Forti, D. Kopsch, L. Kropp, and H. Unseld, A Novel Method for Very High Resolution Cross-Section Measurements, German Report KFK-453, 1966.

17. J. R. Stehn, M. D. Goldberg, B. A. Magurno, and R. Wiener-Chasman, Neutron Cross Sections, Vol. I, 2nd ed., Suppl. 2, USAEC Report BNL-325, Brookhaven National Laboratory, 1964.

18. Brookhaven National Laboratory, ENDF/B Tape 102, Cross Section Library.

19. R. J. Cerbone, J. E. Miller, and E. A. Profio, Angular Neutron and Gamma-Ray Spectrum Measurements in a Bulk Iron Assembly, USAEC Report GA-9149(Vol. I), Gulf General Atomic, Mar. 27, 1969.

20. A. E. Profio, H. Antunez, and T. Williamson, Measurements of the Neutron Angular Flux Spectrum in Graphite, Report GA-7470, General Dynamics Corporation, Oct. 30, 1966.

21. A. E. Profio and R. J. Cerbone, Measurements of Neutron Angular Flux Spectrum in Water, Report GA-8355 (DASA Report 2056), General Dynamics Corporation, 1967.

22. K. D. Lathrop, DTF-IV, A FORTRAN-IV Program for Solving the Multigroup Transport Equation with Anisotropic Scattering, USAEC Report LA-3373, Los Alamos Scientific Laboratory, July 15, 1965.

23. A. D. Carlson, R. J. Cerbone, and D. F. Willoughby, Measurement of Neutron Penetration Standards, Vol. II, High Resolution Measurements of the Total Neutron Cross Section of Nitrogen and Iron, Report GA-9149 (DASA Report 2289), Gulf General Atomic, March 1969.

24. M. D. Goldberg, S. F. Mughabghab, B. A. Magurno, and V. M. May, Neutron Cross Sections, Vol. IIA, $\mathrm{Z}=21$ to 40, USAEC Report BNL-325, 2nd ed., Brookhaven National Laboratory, 1966.

25. S. Cierjacks, P. Forti, D. Kopsch, L. Kropp, J. Nebe, and H. Unseld, High Resolution Total Neutron Cross Sections Between 0.5-30 MeV, German Report EURFNR-591 [KFK-1000; EUR-3963; EANDC(E)-111-U], June 1968. 
26. R. B. Schwartz, R. A. Schrack, and H. T. Heaton II, Total Neutron Cross Sections of Carbon, Iron and Lead in the MeV Region, in Neutron Cross Sections and Technology, Proceedings of a Conference, Washington, D. C., Mar. 4-7, 1968, D. T. Goldman (Ed.), NBS-SPEC. PUBL.-299, Vol. 2, p. 771, Superintendent of Documents, U. S. Government Printing Office, Washington, D. C., 1968.

27. D. J. Hughes and R. B. Schwartz, Neutron Cross Sections, USAEC Report BNL-325, 2nd ed., Brookhaven National Laboratory, 1958.

28. J. R. Stehn, Murrey D. Goldberg, Benjamin A. Magurno, and Renate Wiener-Chasman, Neutron Cross Sections, USAEC Report BNL-325, Suppl. No. 2, Brookhaven National Laboratory, 1964.

29. M. Alberg, H. Beck, K. O'Brien, and J. E. McLaughlin, An Investigation of Differential Energy and Angle Spectra for ${ }^{137} \mathrm{Cs}$ Gamma Rays in Water and Air, Nucl. Sci. Eng., 30: 65 (1967).

30. L. D. Skarsgard and H. E. Johns, Spectral Flux Density of Scattered and Primary Radiation Generated at $250 \mathrm{kV}$, Radiat. Res., 14: 231 (1961).

31. J. D. Marshall and M. B. Wells, A Comparison of Monte Carlo Calculated and Experimentally Determined Energy and Angle Spectra for ${ }^{137} \mathrm{Cs}$, USAEC Report RRA-M-74, Radiation Research Associates, Inc., June 1967.

32. J. H. Hubbell and M. J. Berger, Photon Attenuation and Energy Transfer Coefficients, Tabulations and Discussion, Report NBS-8681, National Bureau of Standards, May 10, 1965.

33. W. D. Lanning, Application of the Spherical Harmonics Technique to Problems in Gamma Transport, Nucl. Sci. Eng., 15: 259 (1963).

34. F. W. Sanders, F. F. Haywood, M. I. Lundin, L. W. Gilley, J. S. Cheka, and D. R. Ward, Operation Plan and Hazards Report-Operation BREN, USAEC Report CEX-62.02, Oak Ridge National Laboratory, January 1962.

35. J. A. Auxier, F. F. Haywood, and L. W. Gilley, General Correlative StudiesOperation BREN, USAEC Report CEX-62.03, Oak Ridge National Laboratory, 1963.

36. F. F. Haywood, Spatial Dose Distribution in Air-Over-Ground Geometry, Health Phys., 11(3): 185-192 (March 1965).

37. F. F. Haywood, J. A. Auxier, and E. T. Loy, An Experimental Investigation of the Spatial Distribution of Dose in an Air-Over-Ground Geometry, USAEC Report CEX-62.14, October 1964.

38. R. L. French and L. G. Mooney, Differential Measurements of Fast-Neutron Air-Ground Interface Effects, Nucl. Sci. Eng., 43: 273-280 (1971).

39. M. S. Bokhari, V. V. Verbinski, and H. A. Todd, Measurement of the Fast-Neutron Beam Spectrum from the Tower Shielding Reactor, II, in Neutron Physics Division Annual Progress Report for Period Ending August 1, 1963, USAEC Report ORNL-3499 (Vol. 1), pp. 105-108, Oak Ridge National Laboratory, 1963.

40. T. V. Blosser and R. M. Freestone, Jr., Development of an Epithermal Neutron Spectrometer, in Neutron Physics Division Annual Progress Report for Period Ending August 1, 1965, USAEC Report ORNL-3858 (Vol. I), Oak Ridge National Laboratory, 1965. 


\section{Shield Design}

To integrate the numerous design considerations in an attempt to gain a perspective of the whole process of shield engineering, we will proceed in two different ways. First, from an entirely philosophical standpoint, we will describe a set of iterations in a shield design based on the experience of Hungerford ${ }^{1}$ in the course of developing the shield system for the Enrico Fermi Nuclear Plant. His recollection of the tasks is both interesting and instructive since it is based on actual design experience and has subsequently been refined by his lectures in radiation shielding at Purdue University. Dr. Hungerford has also formulated six principles of reactor-shield design; these are given below. A second way to obtain a perspective of the design process is to examine the shields of several operating reactors and to review the approaches used by their designers. We will discuss several widely differing types; particular attention will be given to special problems encountered in each case. We have limited the selertion of shield design for this discussion to cases now in the literature, particularly those which include publication of measurements on shield performance as well as design description. Proof of shield performance is the final step in the design process; a design is incomplete without it. This limitation means generally that systems available to us for this discussion were completed in the mid-sixties. This, in turn, implies shield analyses that date from the late fifties and early sixties. Consequently the analysis methods used in the work we will study are not as advanced as the latest ones described in Chaps. 4 and 6. There is an 8- to 10-year period in the cycle from first concept to start-up tests, and shield technology has changed markedly in the interval.

To return to our first viewpoint, the philosophical viewpoint, consider the following shield design principles that are based on experience developed over the years. They are guides to be followed in the conception and development of a reactor plant and shield system. 
Hungerford's six principles of shield design as stated in Ref. 1 are:

1. Reactor-Shield Unity: A shield is an integral part of a reactor system and must be designed at the same time as, and as an entity with, the overall reactor system.

2. Shield Integrity: Adjacent parts of a shield, having the same design criteria, must be designed with equal performance characteristics.

a. Two adjoining parts of a shield which are made of different materials must have the same radiation attenuation characteristics.

b. Shields around voids, gaps, and irregularities must be designed to give the same overall attenuation of the radiation as the bulk shield.

3. Shield Safety: Because the reactor shield is a safety device and must be considered as a part of the safety system of the reactor, there can be no compromise with expediency in its design.

a. Sloppy design and construction techniques in sensitive areas which may affect the safety of the reactor cannot be tolerated.

b. Use of cheap materials and other cost-cutting practices may be more costly in the long run, and moreover may jeopardize the safety of the reactor.

4. Shield Accommodation: A shield should be adapted to provide for the mechanical requirements of the reactor, its supporting structure, and its component systems, without sacrificing the principles of reactor-shield unity, shield integrity, or shield safety.

5. Shield Economy: The best possible shield should be designed at the lowest possible cost, consistent with the overall reactor design, without sacrificing safety, integrity, or performance.

a. An economical shield is a must in the design of large nuclear power plants for the production of electric energy.

b. This principle is subservient to the first four principles.

c. An application of this principle is the concept of shield optimization, whereby a shield may be optimized according to some parameter such as weight, size, cost, or some nuclear property, etc.

6. Shield Simplicity: A shield should be designed to be as simple in configuration as possible, with the minimum number of voids, ducts, and cutouts for the reactor components and auxiliary systems, consistent with the principle of shield accommodation.

a. An application of this principle would be to allow all pipes and cables serving the reactor to pass through the shield in one penetration of the shield (or as few as possible).

b. Simpler shields will have more satisfactory performance, less maintenance, and will cost less.

\subsection{ITERATIONS IN THE SHIELD DESIGN}

The job of creating a reactor-shield design proceeds in three distinct phases: (1) the development of a preliminary conceptual design; (2) the 
correlation of the preliminary conceptual design with the mechanical design to obtain a final conceptual design; and (3) the translation of the final conceptual design into a detailed engineering design. Each of these steps has a distinct subset of problems. The development of the conceptual design includes the consideration of the selection and availability of materials and problems with the use of these materials in the shield. Let us follow in detail the development of the design and later of the shield construction to see what types of problems may arise in each of these phases.

\subsubsection{Preliminary Conceptual Design}

The preliminary conceptual shield design begins with a rough sketch of the plant layout showing where the reactor and every auxiliary system and component is to be placed. It is important here for the shield designer to have a voice in the plant layout since this will affect the whole design and future operation of the plant. From this sketch the designation of all areas to be shielded and their interrelation is made. Once this is done the shield design criteria for each area with respect to personnel protection must be worked out. Next, rough sketches of each shield or shield area must be made so that the mechanical requirements as well as the shield requirements can be considered and developed. Close coordination with the mechanical design people is a necessity.

In most reactor design and construction projects, a time schedule for developing each design will have been set up, hopefully enough ahead of the construction schedule to give adequate time for the development of the design. Probably the shield designs for several areas will have to be developed simultaneously.

(a) Preliminary Selection of Shield Materials. Once the type of shielding and overall requirements of the shield for each area have been worked out, a preliminary selection of shield materials is made. The properties of the selected shield materials are investigated thoroughly with respect to the anticipated environment to ensure that the materials will be able to stand up to the environment and perform reliably over the lifetime of the plant. The shields, like much of the permanent structure of the plant, are not amenable to replacement during plant operation.

(b) Shield Calculations. Only after the shield materials have been selected, or the choice is at least narrowed to two or three, should the shield calculations begin in earnest. The model for the shield calculations can be set up. The preliminary design sketches may contain several different configura- 
tions based partly on the types of materials chosen and partly on economics and other considerations. Rough calculations should be made for each shield region to help narrow down the choice of materials by evaluating shield performance of each of the selected materials. Considerations should include preliminary calculations of radiation attenuation, nuclear heating, radiation effects, temperature rises, and internal stress generation. The next set of calculations should include a parametric study to determine the best arrangement of the shield materials with respect to the overall mechanical design and arrangement of the structure.

(c) Final Materials Selection. Firm and final selection of each shield material should await the results of definite tests on samples of the material exposed to a heat and radiation environment equivalent to plant operating conditions and total exposure equivalent to the plant lifetime if this information is not already known. One of the biggest mistakes that can be made in shield design is to choose an untested material that is acceptable on paper but whose integrity and reliability are questionable.

Once the shield material for a given area has been tentatively selected, the simple conceptual design of the bulk shield can be developed from the results of the calculations already at hand. The problems associated with this stage of the design are the difficulties in selecting the shield material, in developing the calculational model, or in executing the calculations.

\subsubsection{Detailed Conceptual Design}

The next step is to divide the shield areas into a number of regions to check deviations from the model of the initial calculations. An example would be that the mechanical design calls for a thinner or thicker shield in some particular area, with perhaps a change or addition of materials. Shield thicknesses for each of these regions should be calculated separately. The results of all these calculations are then brought together and assembled into a preliminary conceptual design.

At this point the design problems begin in earnest since the preliminary conceptual design must be compared with the mechanical design and the two fitted together. The preliminary design, as we have stated, includes only the most important shield regions and perhaps only those at the most important elevations or cuts through the shield. The detailed conceptual design must show clearly at every elevation and cut through the shield the exact material to be used and the broad features and configuration of the material in every area of the shield. The main penetrations through the shield and the 
shielding around them must be shown. Special calculations must be considered for the areas of penetration and areas of shielding around mechanical objects attached to the reactor vessel. Such items as support structure, coolant lines, detectors, experimental tubes, pipe hangers, cable trays, sheaths containing thermocouples, or other types of sensors are all examples of objects that the shield must be designed around.

(a) Special Calculations-Radiation Streaming. To calculate radiation streaming, one must divide the shield into a large number of special areas. In each area judgment must be passed as to the allowable leakage radiation. Calculations must be performed to determine the proper amount of shield material. Many of the calculations will involve streaming in voids, gaps, circular and rectangular ducts, and annuli. These areas in a shield present the greatest challenges because one must not only create calculational models to simplify a complex mechanical design but must also exercise good judgment in estimating the streaming and in interpreting the results.'

(b) Nuclear Heating. Another problem of great concern during this design stage is nuclear heating. Earlier analysis of materials should indicate those regions where there might be excessive heating in the bulk shield. If detailed calculations in any given area show that there is indeed a heating problem, then proper steps must be taken to change the material, add energy-absorbing (thermal) shields, or call for a shield coolant system (whichever is most appropriate). The concern here is therefore not with the shield material but with heating or radiation effects on structural material, equipment, or system components located in each region. If the heating induces excessive temperature rises or if the neutron fluence is above the radiation-damage limit, additional coolant or shield material may be needed. If the problem in a given region is too severe or if there is no room to add any more material, more drastic action must be taken, e.g., change the mechanical design or reevaluate the shielding material initially selected. This may mean upgrading the shielding properties of the material in this region, if possible, by adding more absorber into the shield material (such as increasing the boron content of borated graphite) or by replacing it with a more efficient material. Any of these actions, of course, may have economic reverberations.

(c) Economic Considerations. Once the various shield regions have been analyzed, the materials chosen, and the thicknesses and configuration for the regions decided on, an economic analysis should be performed. Grades or 
quality of the material must be specified. If the calculated costs exceed the budget allowance, the shield should be reviewed again to determine if there are areas that could be simplified or if the material could be changed.

Since all budgets are tight (by definition), there will be a great temptation during this stage of the design to try to dictate upper limits of allowed costs and, by inference, the quality or grade of material to be used. Certainly, the use of an expensive material cannot be justified if there is a less expensive one that will do the same job and positively stand up to the anticipated environment during the plant lifetime. On the other hand, if there are circumstances that call for a certain material because it is the only safe and reliable material for the job, then no substitutions can be considered. Decisions on questions of safety vs. economy must obviously favor safety.

(d) Completed Conceptual Design. When all aspects of the shield design have been evaluated, the materials selected, and the necessary modifications made in the preliminary design, the detailed conceptual design is complete. Documentation should be in the form of a report with drawings at every important elevation and penetration through the shield showing for each shielded area the basic configuration and materials.

\subsubsection{Final Engineering Design}

The conceptual design must be translated into engineering drawings. Detailed drawings of the reactor and auxiliary systems and components must be analyzed and redrawn to show the proper shield modifications. It is here that all the fine detail of the shield structure comes to light.

For instance, the reactor is built at room temperature, but it may be operated at $540^{\circ} \mathrm{C}$. The reactor, its support structure, the coolant piping, any auxiliary gas coolant lines or cover-gas lines, all mechanical appendages, sensing devices, etc., will have to operate at temperatures at or near the operational temperature of the reactor.

The mechanical system will have been designed to flex, shift, and move with temperature to relieve the stresses on the system. The coolant pumps and the motion of the coolant flowing within the system will create vibrations, and other movements of the system components. The shield cannot be erected around the reactor and its systems without regard for these expansions and movements. The engineering design therefore must include allowances for expansion and motion. 
The drawings must show or indicate clearly how close to the vessel and its systems the shield can be erected, and all clearances must be detailed. It may even be necessary at this late date to rerun shield calculations, taking into account the clearances, and perhaps to specify changes in the shielding material.

One of the problems arising at this stage of the design is the possible conflict that may arise between the shielding requirements and the mechanical requirements. A cooperative effort between the shield designer and the mechanical engineer is necessary for a satisfactory compromise solution to such problems. If this type of cooperation is not achieved before the construction phase, costly rework may result.

The engineering design, besides reflecting the requirements of the conceptual design, must also take into consideration the requirements for field erection. Therefore on each drawing all necessary instructions and notes must be carefully made in language intelligible to construction crews. The completed engineering design will be a series of elaborate drawings showing all the requisite detail for proper erection.

(a) Field Drawings. The architect-engineer delivers a set of engineering drawings to the plant constructors, who copy each drawing and prepare field drawings by adding pertinent information for field workers, e.g., welding instructions, construction tolerances, and erection sequence. It would seem that the shield design job should be complete by the time the design reaches this stage, but this is not the case, especially in the matters of construction tolerances and erection sequences. For example, if a shield is to be erected out of a series of graphite blocks that are graded in boron content, the manufacturer must color-code his blocks or identify the percent boration by some other easily recognized means, and the color code or identification instruction must appear on the field drawings.

(b) Construction Follow-Up. Each of the color-coded graphite blocks will have the specified dimensions and a dimensional tolerance in accordance with the material specifications given to the manufacturer. The erection crews will be expected to lay these blocks to form the shield with a specified construction tolerance. In most jobs the erection tolerances are generous to keep down the cost of construction. The designer's task here is to ascertain that the construction clearances and the manufacturer's dimensional tolerances when taken together do not lead to unexpected streaming paths through the shield. Even here he should be prepared to rerun some shielding calculations covering the affected region as a check to determine the effect 
of excessive clearances if these have not already been included in his previous calculations. As a result of these checks, the designer should be prepared to take one of the following actions: (1) modify the configuration of the blocks, (2) issue orders that gaps greater than a given length and width be filled, or (3) issue orders that overall erection tolerances be cut to more severe limits. He should consult and obtain the cooperation of the field engineer to ensure that the erection crew will follow the spirit as well as the specification of the changes.

(c) Construction with Concrete. In the pouring and curing of concrete, simple field procedures can be set up to ensure cleanliness of the aggregate, proper concrete mixing ratios, and uniformity of density. The poured material must be worked by hand or with mechanical vibrators to ensure closing of voids, proper joining at the boundaries between one pour and another, and setting and curing of the completed pour. For poured shields, the allowed clearances must include the forming tolerances. Normally these cannot be considered to be less than \pm 1 in. Therefore concrete to be poured around ducts and penetrations should have permanent imbedded steel sleeves, and the tolerances or clearance limitations will therefore be on the sleeve itself rather than on the concrete; the concrete must be vibrated to ensure close joining between the sleeve and the concrete shield.

(d) Testing Vendor-Supplied Materials. Quality control of all materials is essential during erection to ensure that materials received from the vendor are within the limits specified. If borated material is being used, for instance, the material must have at least the minimum boron content specified since that figure determines the proper shield thicknesses. With graphite, tests of conductivity and other properties must be run to ensure that the product is really graphite and not (for example) graphite particles bonded together with a cement. An impurity analysis should be run on samples of each material to ensure that the impurity level is within specifications, especially wherever impurities can be a detriment to shield performance. Impurities in graphite, for instance, accelerate its oxidation by hundreds of times at temperatures exceeding $800^{\circ} \mathrm{F}$ (the oxidation threshold).

(e) Field Testing and Inspection. A shield cannot be erected successfully without proper liaison with the erection crew. The shield is a three-dimensional structure to be erected from two-dimensional drawings. In some instances the blueprints are very difficult to interpret to obtain a three-dimensional concept. Thus someone knowledgeable with the shield design must be available to interpret the drawings. Inspectors are necessary 
at all phases in the erection of a shield to ensure that tolerances are held and directions are followed.

Radiation surveys of the completed shield system serve as the proof-test. For power reactors surveys are usually conducted first at low power, then at one or more intermediate levels, and finally at full power. Detailed surveys are made in each instance to ensure that design criteria are met. Results of several of these surveys are discussed later in this chapter.

\subsection{FAST BREEDER: ENRICO FERMI}

The Enrico Fermi reactor is a prototype of the sodium-cooled fast breeder concept. Shielding of a fast reactor system poses some special problems that thermal systems do not have:

1. Leakage of fast neutrons into the shield is high because fast-neutron cores operate at high power density with relatively thin breeder blankets. $\mathrm{keV}$.

2. The neutron leakage spectrum is harder, it peaks between 50 and 500

3. The absence of a thermal-neutron peak makes the secondary gammaray problem more complex because of the increased importance of resonance-neutron absorption.

4. The system components are more subject to radiation damage.

5. Moderating materials are not usually allowed within the reactor vessel because of safety considerations.

6. Liquid-metal coolants reach a high specific activity, which poses the double problem of shielding the coolant system and contending with the high temperatures.

An unusually detailed shield test program was carried out on the Enrico Fermi reactor because of its prototype nature. Aside from obtaining operating data to ensure that the shield system would permit safe operation up to the maximum design power level of $500 \mathrm{Mw}(\mathrm{t})$, the test program was also aimed at getting sufficiently accurate data to make meaningful comparisons between calculations and measurements. Because of the detailed test program and the unusually complete documentation, we will describe the design features of this system in detail and limit discussion of other reactor systems to their unusual features. The following discussion of the Enrico Fermi shield system is taken from the shield design report by Beaudry, Hungerford, Montey, and Chaltron ${ }^{2}$ and the report on the shield test program by Beaudry. ${ }^{3}$ 
The plant shielding can be classified in two main categories: the shield system for the reactor and primary coolant systems in the reactor building and the shield system for the service and fuel-handling systems in the various auxiliary buildings. The shield for the reactor is discussed in detail in Sec. 10.2.3. A general description of the plant is given in the next section. General information on the shield design of fast reactor systems is given by Hungerford, ${ }^{4}$ including brief descriptions of the shield systems of other fast reactors: Experimental Breeder Reactor No. 1 (EBR-1), ${ }^{5}$ Experimental Breeder Reactor No. 2 (EBR-2), ${ }^{6}$ Rapsodie, ${ }^{7}$ Dounreay, ${ }^{8}$ and the BR-5. ${ }^{9}$

\subsubsection{The Reactor Plant}

The Enrico Fermi Atomic Power Plant reactor ${ }^{10}$ is a sodium-cooled fast breeder designed for a maximum power of $430 \mathrm{Mw}(\mathrm{t})$. A plot plan showing the various supporting facilities is shown in Fig. 10.1, and an elevation of the reactor containment vessel is given in Fig. 10.2.

The reactor vessel and its associated structures are shown in perspective in Fig. 10.3. The stainless-steel reactor vessel is composed of four parts: lower reactor vessel, transfer-rotor container, upper reactor vessel, and rotating shield plug. The entire vessel is located in the lower compartment of the reactor building, below grade, in a nitrogen atmosphere within the primary shield tank.

The reactor vessel contains the reactor core, which is composed of fuel and the inner-radial-blanket and outer-radial-blanket subassemblies. Liquid-sodium coolant flows into the reactor vessel through three equispaced 35-cm-diameter nozzles. The sodium flows upward through the outer-radial-blanket subassemblies and through the core and inner-radial-blanket subassemblies. Heat is extracted from each subassembly as the coolant flows upward into the upper sodium plenum. Sodium leaves the upper reactor vessel through three equispaced $76-\mathrm{cm}$-diameter nozzles. The hold-down mechanism, which is mounted on the rotating shield plug, is used to prevent the core and inner-radial-blanket subassemblies from being lifted by the rapid flow of sodium through the core.

The reactor vessel is contained within a carbon-steel liner that is leaktight up to the coolant outlet lines and designed to carry any sodium leakage to detectors located at the bottom. All this is contained within the primary shield tank, the space between the vessel wall and shield-tank wall being filled by the primary shield. The construction of the primary shield tank is such that an adequate height of sodium over the core will be maintained should the reactor vessel leak. 


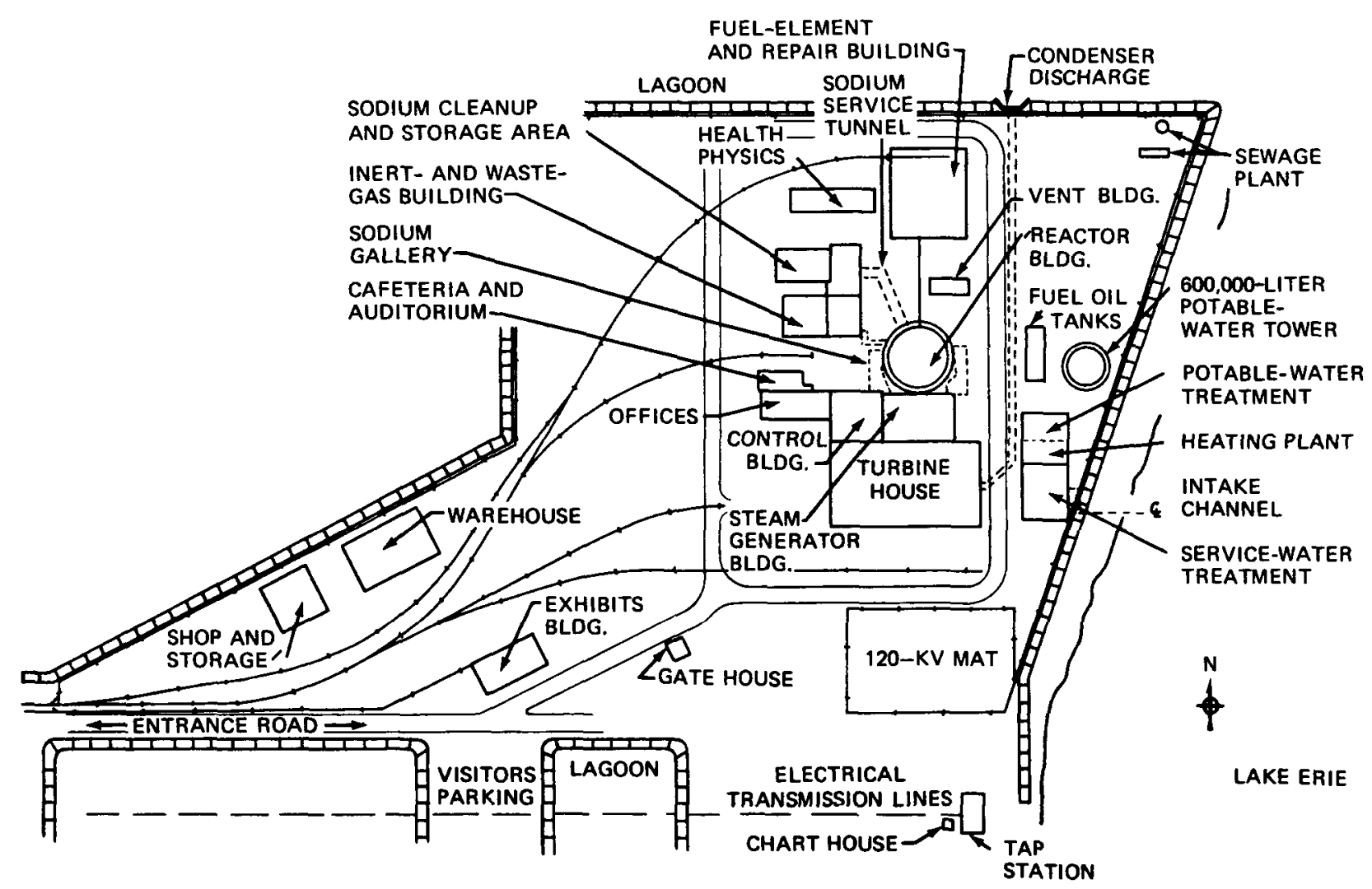

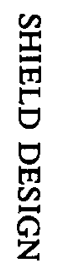

Fig. 10.1—Enrico Fermi plot plan. (From Beaudry. ${ }^{3}$ ) 


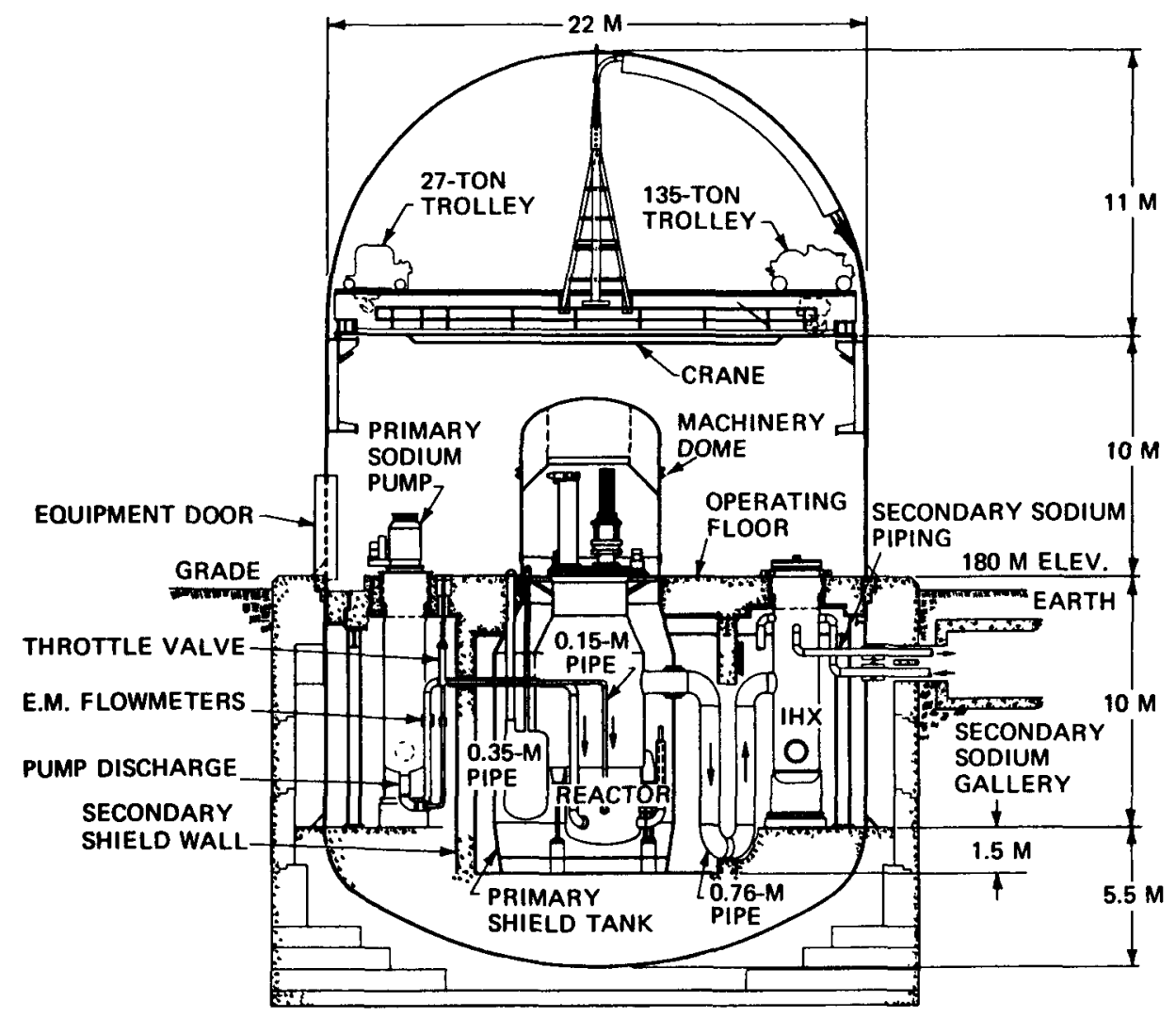

Fig. 10.2-Elevation of reactor containment vessel. (From Beaudry et al. ${ }^{2}$ )

The machinery dome, which is attached to the top flange of the primary shield tank, consists of two parts: (1) the lower machinery dome, which forms a sealed enclosure over the top of the primary shield tank, and (2) the upper machinery dome, which contains the aluminum energy absorber, the control mechanisms, and other plug-mounted equipment.

The reactor is an assembly of 870 removable and, to an extent, interchangeable units, all assembled on a square lattice unit spacing of 6.83 $\mathrm{cm}$ and contained in the lower reactor vessel.

The fuel subassemblies, which also contain the upper and lower axial blankets, occupy a region (roughly cylindrical) in the center of the lattice. The entire core region is about $79 \mathrm{~cm}$ in diameter and $79 \mathrm{~cm}$ in height, and each axial blanket section is $43 \mathrm{~cm}$ in height. Together the core and the radial and axial blanket regions approximate a cylinder $203 \mathrm{~cm}$ in diameter and $178 \mathrm{~cm}$ in height. 


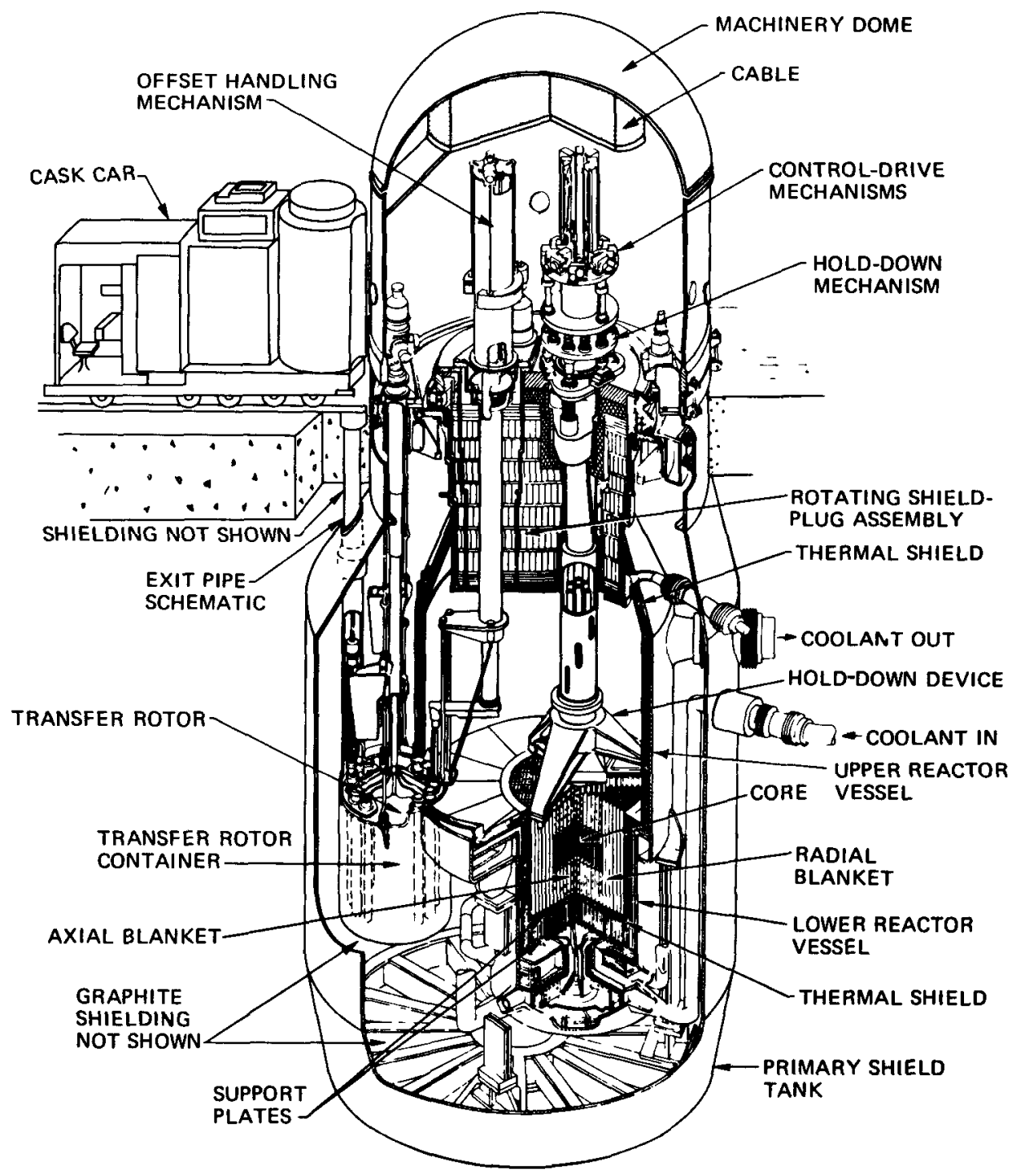

Fig. 10.3-Perspective view of reactor. (From Beaudry et al. ${ }^{2}$ )

The reactor is controlled by two operating rods and eight safety rods. Fission counters and ionization chambers for monitoring the neutron flux are located in the six neutron-counter tubes embedded in the graphite shield that surrounds the reactor vessel. An Sb-Be neutron source is located at the core-blanket interface to maintain a sufficient flux for control purposes during reactor start-up and in the shutdown condition. 
The heat-removal system comprises three primary coolant loops and three secondary coolant loops. Heat removed from the reactor core and blanket by the primary sodium coolant is transferred to the secondary sodium coolant in three parallel intermediate heat exchangers located in the reactor building. The secondary sodium system transfers the heat to the steam and feedwater system in three once-through-type steam generators.

A primary sodium service system, located in the shielded sodium service building, stores and purifies the highly radioactive primary coolant. The major components of this system are three storage tanks, a cold trap, a plugging indicator, and a primary-system overflow tank.

A separate secondary sodium system (for which shielding is unnecessary) is located in the steam-generator building. Both systems are blanketed with argon gas.

\subsubsection{Shield Design Criteria}

The design criteria for the shield system include limitations on the biological dose, radiation damage to materials, and radiation heating. Maintenance philosophy and safety were also considered in establishing these criteria.

(a) Biological Dose. A maximum dose rate from both neutrons and gamma rays was set at $0.75 \mathrm{mrem} / \mathrm{hr}$ for all unlimited access areas. Based on a 40 -hr work week, this is $30 \%$ of the average weekly exposure permitted by federal regulations ${ }^{11}$ governing workers in nuclear facilities.

Many areas of the reactor plant do not need to be occupied on an unlimited basis; consequently the shields for certain areas of the plant were designed for only limited access, i.e., access controlled and limited to specified time periods. The design criterion for these areas was based on estimates of the required working times.

(b) Radiation Damage. Radiation-damage criteria were set for the stainless-steel reactor vessel, the graphite primary shields, and the various organic materials, such as gaskets and diaphragms. For concrete, it developed that radiation heating effects were more severe than damage effects, and consequently heating dictated the exposure limitation.

On the basis of an expected 20-year plant life, a maximum fast-neutron fluence of $10^{22}$ neutrons $/ \mathrm{cm}^{2}$ (estimates indicated this value to be conservative) was used as the fast-neutron exposure limit of the stainlesssteel reactor vessel. This limitation was a principal factor in determining the thickness of the thermal shielding within the reactor vessel. 
Energy storage through lattice distortion in graphite (Wigner effect $)^{12}$ becomes a problem at room temperature for fluences of the order of $2 \times 10^{21}$ neutrons $/ \mathrm{cm}^{2}$. Studies of the Wigner effect in the graphite primary shield indicated that setting a minimum operating temperature of $127^{\circ} \mathrm{C}$ in the graphite would anneal out the energy storage for the expected fluences of $10^{18}$ to $4 \times 10^{21}$ neutrons $/ \mathrm{cm}^{2}$.

Most of the organic materials subject to radiation damage are in the auxiliary equipment, where the radiation sources are gamma rays from the spent-fuel assemblies and the primary sodium coolant. A dose limit of $10^{8}$ rads over the plant life was set for organics except for a few cases that required more stringent limits, such as Teflon at high temperatures.

(c) Radiation Heating Limitations. Concrete shields were limited to an incident-radiation energy flux ${ }^{13}$ of $4 \times 10^{10} \mathrm{MeV} \mathrm{cm}^{-2} \mathrm{sec}^{-1}$. This limit ensured that the temperature rise due to nuclear heating within the shield would not exceed $5.5^{\circ} \mathrm{C}$. Local rises greater than this value can create large gradients that may cause thermal stresses that result in cracking, spalling, and void formation.

Temperatures in excess of $93^{\circ} \mathrm{C}$ cause concrete to rapidly lose its bound water and hence decrease its neutron-attenuation properties. Therefore as a design criterion the maximum ambient temperature around the primary neutron shields of ordinary concrete was limited to $82^{\circ} \mathrm{C}$ with an internal maximum of $88^{\circ} \mathrm{C}$.

The maximum allowable temperature gradient in all shields was limited to $73^{\circ} \mathrm{C} / \mathrm{m}$ to prevent excessive thermal stresses. Concrete used only for gamma-ray shielding was allowed a temperature limit of $182^{\circ} \mathrm{C}$. The temperature limit for serpentine concrete shielding was set at $445^{\circ} \mathrm{C}$.

(d) Remote-Maintenance Consideration. Maintenance considerations were important in setting requirements for the shield system. These considerations had a strong influence on certain of the design details of the shields. Some areas in which inspection is necessary require shielded manhole access or other means of entrance through or around shields. Shields must allow the removal and handling of activated equipment by remote means. So that induced activity in these limited-access areas could be kept at a minimum, the neutron-flux-density limit was set at $10^{4}$ neutrons $\mathrm{cm}^{-2} \mathrm{sec}^{-1}$, low enough to prevent significant activation of the equipment.

The Fermi plant philosophy on remote transfer and maintenance of very large radioactive equipment was that the reactor would be shut down. The plant would be evacuated or all personnel sent to designated safe areas 
except those necessary for the plant safety and those involved in the remote operations. This philosophy avoided the requirement for shielding all work areas, offices, etc. Remote maintenance of large equipment is an operation apart from the routine affairs of the plant and is necessary only a few times during the plant lifetime; therefore the expense of shielding every area was unwarranted.

(e) Safety Design Standards. Precaution was taken in the design of the Fermi shields to ensure that the plant would be safe and also free from defects leading to unsafe conditions. Each shield design was analyzed from a safety point of view, including the designs for all penetrations for piping, cables, and ducts, to ensure that in the event of any predictable accident condition the plant would be safe.

The graphite for the primary shield was specified as nuclear-grade graphite with acceptable physical properties between predetermined ranges. Dimensional tolerances of the graphite blocks were limited to about $2 \%$ to keep the total void volume in the primary shield at a predetermined value. Gaps between blocks of adjacent layers or rows were offset to minimize radiation streaming. All graphite within the rotating plug was canned in rectangular steel containers; weep holes were provided in the tops of the containers to allow gases to escape, and room was allowed within the cans for expansion under radiation.

Concrete for shields had to meet density and strength standards, and no defects between various pours of the concrete were allowed. Curing periods up to 28 days were imposed on neutron-shield concrete to ensure proper hydration. All concrete and other shield structures underwent rigid inspection during erection. All concrete shields and other concrete in the compartments below the operating floor (which house the primary coolant system) were lined with a layer of steel to prevent spallation of the concrete and load shifting should a sodium leak occur in the primary sodium system.

\subsubsection{Reactor-Shield Systems}

The reactor shielding can be divided into three main sections: (1) the primary shield, located within the primary shield tank; (2) a secondary concrete shield wall that surrounds the primary shield tank; and (3) a biological shield that forms the operating floor and also surrounds the containment building. Elevation and plan views of these shields are shown in Figs. 10.4 and 10.5 . 


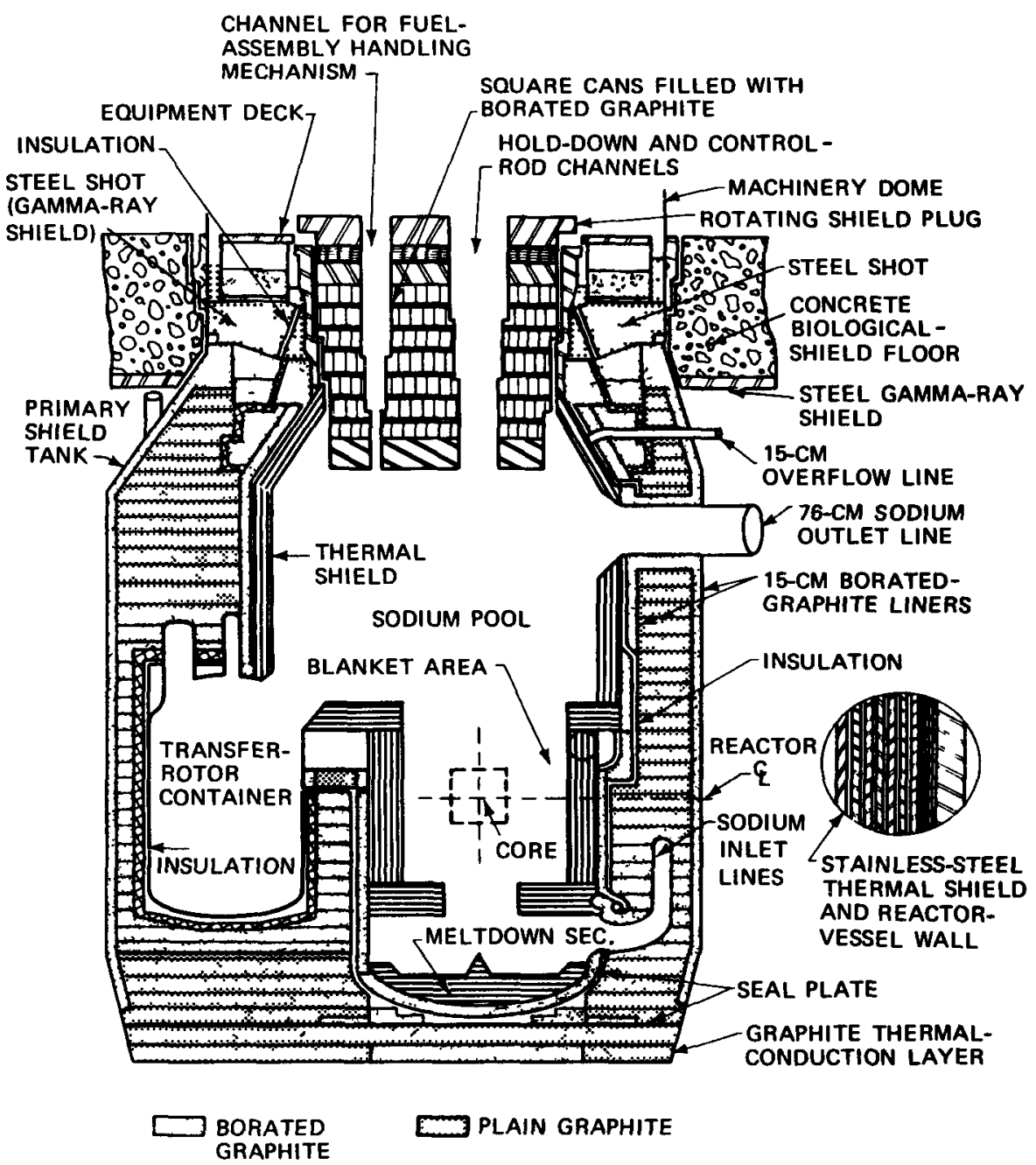

Fig. 10.4-Primary shield system. (From Beaudry et $a l_{.}{ }^{2}$ )

(a) Primary Neutron Shield. The primary shield system has three main parts: (1) a stainless-steel thermal shield within the reactor vessel, (2) a graphite and borated-graphite neutron shield outside the reactor vessel, and (3) a steel and graphite rotating-plug shield that fits into the top of the reactor vessel and acts as a biological shield. The upper part of the primary tank extends around the rotatıng plug and is also a biological shield containing steel as well as graphite. 


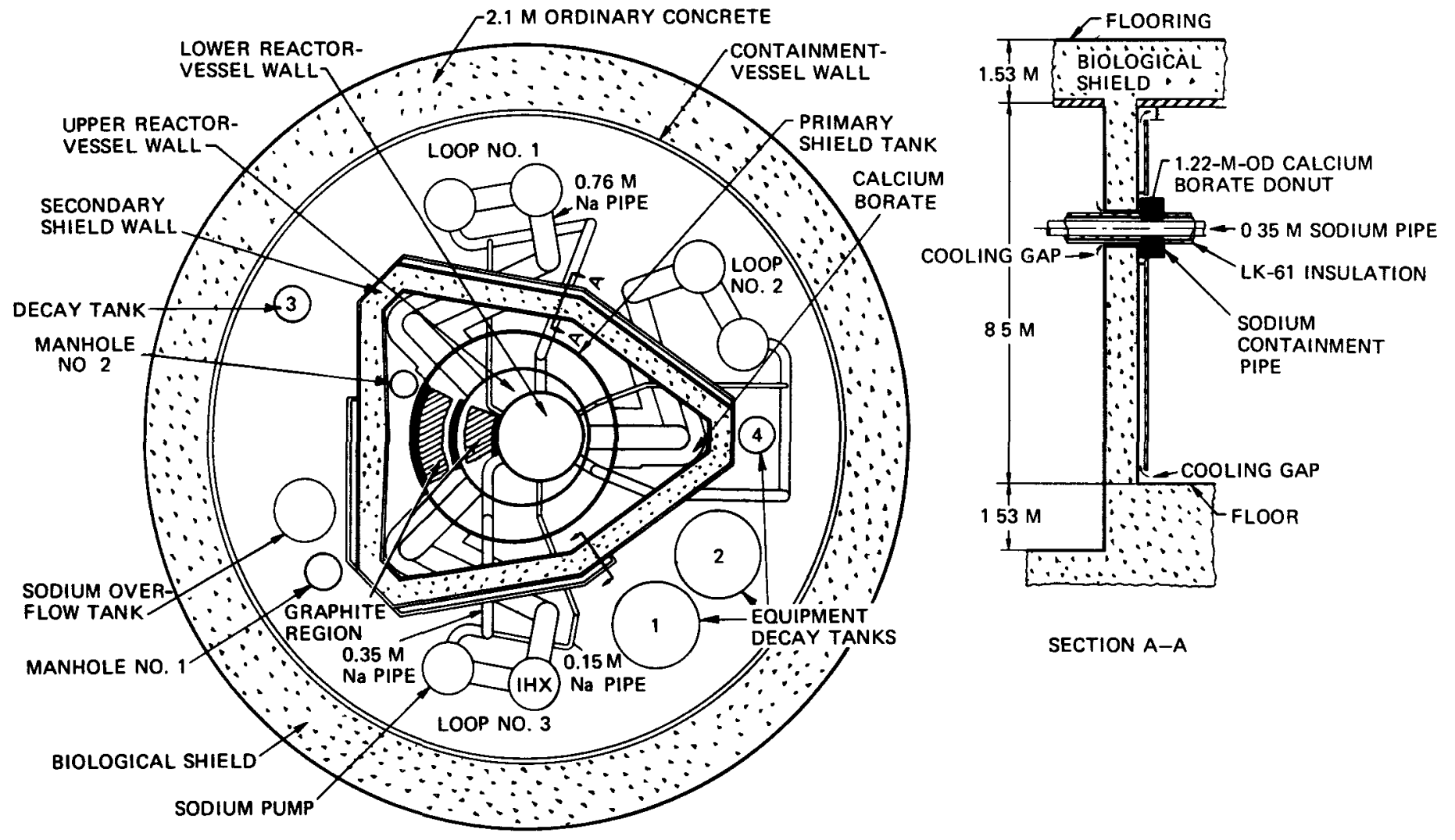

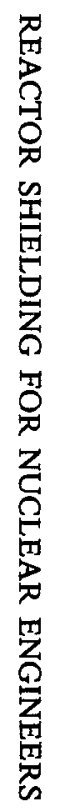

Fig. 10.5 - Reactor building shield system. (From Beaudry et al. ${ }^{2}$ ) 
The thermal shield is located between the breeder blanket and the vessel wall (Fig. 10.4) and consists of 304 stainless-steel bars and plates. Sodium coolant flows in the spaces between adjacent layers to remove the heat generated within the steel. The thermal shield is $30 \mathrm{~cm}$ thick in the lower region and $15 \mathrm{~cm}$ thick in the upper region. The functions of the thermal shield are: (1) attenuate gamma rays and reduce the heat load to the reactor vessel, (2) prevent radiation damage to the reactor vessel by lowering the energy of the high-energy neutrons by inelastic scattering, (3) act as a reflector and reduce the core leakage, and (4) protect the reactor vessel from thermal shocks caused by temperature transients in the coolant.

The graphite shield is composed of layers of graphite and borated graphite $(5 \%)$. The main portion is located outside the pressure vessel but inside the primary shield tank (Fig. 10.4). In conjunction with the thermal shield, the graphite shield is designed principally to reduce the neutron leakage to levels low enough so that material outside this shield will not be damaged or highly activated. The problems and costs of erecting the graphite shield led to the use of two block sizes, 33 by 33 by $10 \mathrm{~cm}$ and 30.5 by 15.2 by $7.6 \mathrm{~cm}$ (with a $2 \%$ dimensional tolerance). All blocks were canned and stacked in staggered arrays. They were fitted together as tightly as possible, and in areas where it was necessary to maintain a rigid structure, the blocks were fastened together.

Owing to the eccentricity of the reactor vessel with respect to the primary shield tank, the thicknesses of the graphite and borated graphite vary greatly, being about $86 \mathrm{~cm}$ wide at the narrowest part and about $152 \mathrm{~cm}$ wide at the maximum. At an elevation corresponding to the central plane of the core, the minimum distance between the core center and the outer edge of the primary shield is $2.3 \mathrm{~m}$ and the maximum distance is about $3.8 \mathrm{~m}$. The basic conceptual design specified a $15-\mathrm{cm}$-thick layer of $5 \%$ borated graphite next to the reactor vessel followed by a 7.6-cm-thick layer of insulation. Adjacent to the insulation is a region of plain graphite of varying thickness. A final $15.2-\mathrm{cm}$ layer of $5 \%$ borated graphite is located next to the inner surface of the primary shield tank. This design was arranged to provide a predetermined amount of neutron attenuation, which was established by the magnitude of the neutron flux leaving the reactor vessel and by the required neutron-flux levels on the outside of the primary shield.

The original concept as described had to be modified in many details as design progressed to accommodate the necessary structural members, instrumentation, piping, and auxiliary systems. A cross-sectional view of the 
actual shield at the approximate elevation of the reactor center is shown in Fig. 10.6. The designs of the reactor vessel, the associated sodium piping, and the primary-shield-tank arrangement were frozen at an early stage; these represented the best mechanical arrangement. Many large components were already fixed in position. In addition, many items were installed during the detailed shield design and construction, such as gas lines, instrumentation

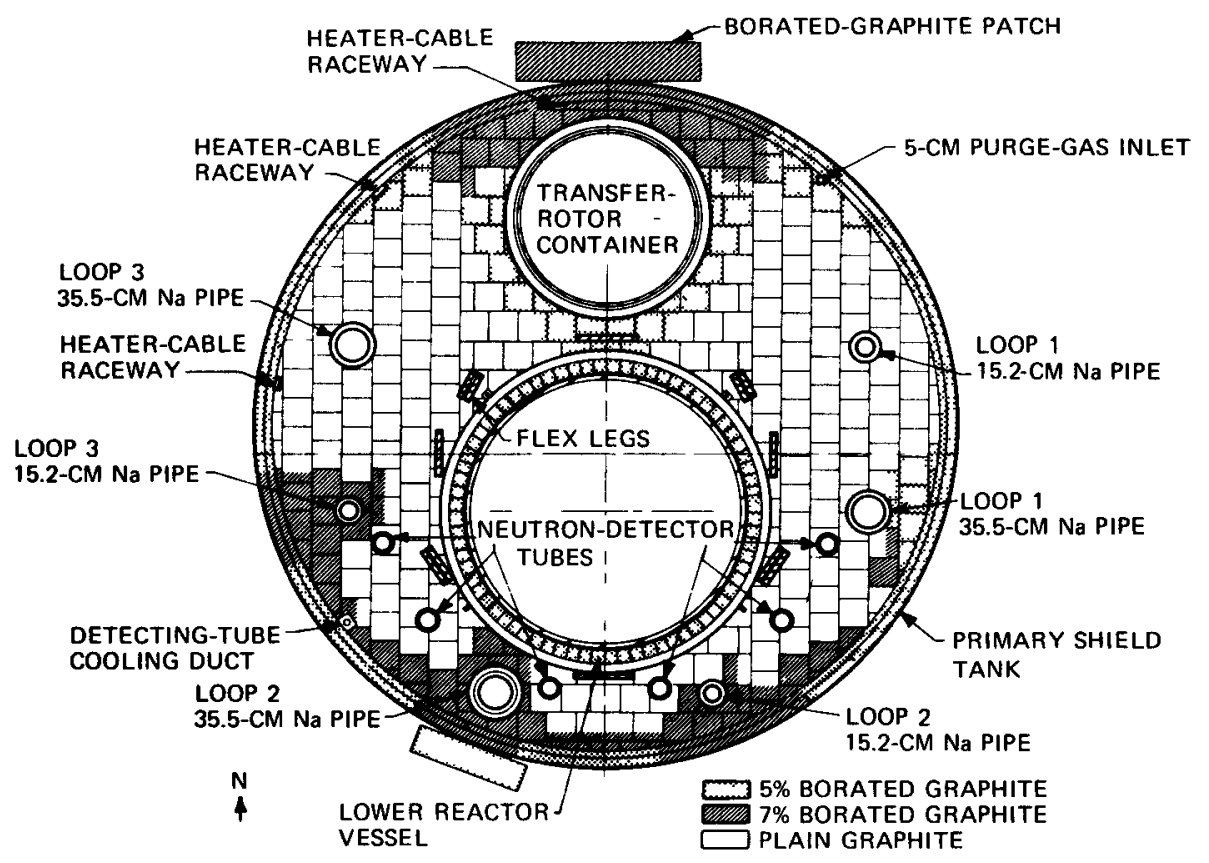

Fig. 10.6-Plan view of graphite-shield layout at 172-m elevation. (From Beaudry et $a .^{2}$ )

tubes, heaters and cables, strain gages, thermocouples, pipe hangers and supports, and cable trays. Where possible, with these late design additions, advantage was taken to arrange components conveniently for shielding reasons. Such changes included the bending and routing of cables and gas lines, alterations in structural details, reduction in size, changes in gap size, etc.

The presence of objects inside the primary shield tank caused local shielding problems by decreasing the amount of available shield material and creating streaming paths owing to the gaps that were allowed around these objects to accommodate thermal expansion and erection tolerances. It is 
estimated that at least 500 separate regions occurred for which shielding had to be specially designed.

Shielding of the primary sodium lines within the tank was very difficult since their large volume, combined with the additional insulation and expansion gaps, resulted in very large holes in the shield. A patch of extra borated graphite was installed outside the primary shield tank (but inside the secondary shield wall) to compensate for loss of shielding from voids. Borated-calcium "donut" shields were hung directly on the upper end of the sodium pipe and large blocks of steel were installed opposite the lower elbow to reduce neutron streaming along the pipes within the shield. Each of the six neutron instrument tubes created a 25 -cm-diameter hole in the shield, which resulted in excess streaming up to the operating floor. As compensation, large donuts of borated graphite were fastened to the upper ends of the tubes, and special blocks of graphite were cut to fit inside and around the electrical cables within the tubes.

Although gamma rays were of little consequence in most areas of the graphite shield, the gamma rays formed by neutron capture in the upper portions of the reactor vessel and thermal shield were found to be of great importance at the top of the primary shield near the rotating plug. The entire upper section of the shield was filled with a mixture of steel shot and borated graphite (installed in special compartments) to attenuate the gamma rays to a level of $0.75 \mathrm{mrem} / \mathrm{hr}$ at the top of the shield (the operating floor). In certain regions where the shield was very thin, the boration was increased to 7\%. The complexity of the upper shield design is illustrated in Fig. 10.4.

The primary shield was designed so that it would not require any auxiliary or internal cooling. About $85 \%$ of the total nuclear heat in the shield (approximately $77 \mathrm{kw}$ at $500 \mathrm{Mw}$ reactor power) is generated in the inner layer of borated graphite. The location of the insulation is such that the thermal gradient is toward the reactor vessel; thus most of the heat generated is returned to the vessel and carried away by the sodium coolant. Very little heat is conducted through the insulation to the outer graphite regions. The heat generated in the outer regions is about $12 \mathrm{kw}$. It is conducted to the outside of the primary shield and removed by the subfloor ventilation system.

The rotating-plug shield (see Fig. 10.4) serves both as a biological shield for the operating floor and as a means of access to the reactor vessel. The plug is $3.7 \mathrm{~m}$ thick, and its cylindrical surface has two steps or offsets. The top of the plug is slightly above the level of the operating floor; the bottom is located in the upper part of the sodium pool so that nuclear heat 
generated in the lower part of the plug will be removed by the primary coolant. The plug was designed to attenuate neutrons, primary sodium gamma rays, and gamma rays produced by neutron absorption in the steel of the plug to levels corresponding to an overall dose rate of $0.75 \mathrm{mrem} / \mathrm{hr}$.

The shell of the plug is made of stainless steel. A 45-cm layer of stainless steel is located at the bottom of the plug. This layer moderates the higher energy neutrons at the top of the sodium pool. Above the stainless steel are six 28-cm-thick layers of plain graphite capped by one 28-cm-thick layer of $1.5 \mathrm{wt} . \%$ borated graphite. Each of the graphite layers is separated from the other by a $5-\mathrm{cm}$ thickness of steel. The 1.8-m zone of plain graphite acts as a thermal column, which moderates the neutrons so that they are captured in the final layer of borated material. Directly above the layer of borated graphite is a $30-\mathrm{cm}$ layer of carbon-steel gamma-ray shielding, which attenuates the high-energy gamma rays produced by neutron capture in steel and stainless steel. A $30-\mathrm{cm}$ layer of stainless-steel insulating material and a 28-cm cover plate of solid carbon steel complete the shield. The layer of insulation enables the plug to be maintained at a relatively high temperature without overheating the steel cover plate, which bears the weight of the plug. The graphite used was in the form of canned 7.6- by $7.6-$ by $28-\mathrm{cm}$ blocks. Any large gaps or spaces between cans were filled with thin wedges of steel. Boron-steel castings were used in regions close to the access ports for the hold-down mechanism and offset handling mechanism to depress the neutron-streaming flux in these areas.

The rotating plug contains a large number of penetrations and access passages to the interior of the vessel. Among these are the large bathtubshaped access passages for the fuel-handling mechanism and the large circular penetration for the core hold-down device and for the control- and safety-rod channels. Other smaller penetrations include access ports (plugs that can be removed to allow access to the interior of the vessel), the sweep mechanism (an instrument for ensuring that all fuel subassemblies are correctly seated), and surveillance tubes (small penetrations that allow the introduction of experimental gear or materials within the reactor). Shielding for the fuel-handling mechanism matches as nearly as possible the shielding in the main part of the plug. The very complicated annular void area between the plug and this shield required the construction of special shield rings and the minimizing of clearances. Patch shielding of steel and graphite was used above the plug in this area to reduce the streaming to acceptable levels. The hold-down mechanism also contains the control- and safety-rod channels. The hollow stainless-steel control- and safety-rod guide tubes 
contain special shields of stainless steel and boron steel. Areas between the channels were partially packed with a special powdered shielding mix containing iron powder (34\%), graphite powder $(57 \%)$, and powdered boron carbide (9\%) compacted to a density of $1.8 \mathrm{~g} / \mathrm{cm}^{3}$.

The core and blanket assemblies are transported into and out of the reactor through the exit pipe (see Fig. 10.3), which is an 18-cm-diameter tube that extends downward from the operating floor to the transfer-rotor container. Subassemblies stored there during reactor operation undergo fission and contribute to the dose in the operating floor. Gamma rays reaching this area during operation come from the sodium coolant and from neutron capture in upper portions of the reactor vessel. As the fuel assemblies are raised upward through the tube, their fission-product gamma rays become increasingly important. Figure 10.7 shows the design that was evolved to cope with these radiation sources.

A shield plug, about $2.1 \mathrm{~m}$ long, fits into the top of the exit port, which is recessed about $20 \mathrm{~cm}$ into the operating floor. The plug remains in place except during fuel transfer. Shielding within the plug consists of $1.5 \mathrm{~m}$ of $1.5 \%$ borated graphite capped by $0.6 \mathrm{~m}$ of steel. The shielding outside the plug consists of borated graphite, steel, lead, serpentine concrete, dry-pack serpentine, and ordinary concrete. Vertical streaming is minimized by a series of sliding borated-graphite rings located between the inner exit-port tube and an outer tube that is part of the primary-shield-tank structure. The rings are placed in alternating layers; the smaller rings are attached to the inner tube, and the larger rings are attached to the outer tube. Additional steel and lead shielding is located both above and partially around these disks, and a steel-and-graphite plug is tightly fitted inside the inner tube.

Since the upper part of the exit tube must be heated during fuel transfer to prevent the sodium from freezing and clogging the valving, dry-packed serpentine aggregate is used for shielding in this area. A $0.6-\mathrm{m}$ removable plug located on top of the exit port completes the special shield.

(b) Secondary Shield Wall. The function of the secondary shield wall (Fig. 10.5) is to reduce the neutron-leakage flux coming from the primary shield tank to an average value of not more than $1 \times 10^{4}$ neutrons $\mathrm{cm}^{-2}$ $\sec ^{-1}$ within the equipment compartment, a level that will not appreciably activate the secondary coolant or the primary coolant equipment.

The secondary shield wall is composed of prepacked ordinary concrete with a density of $2.3 \mathrm{~g} / \mathrm{cm}^{3}$ and it has a lining of $1.3-\mathrm{cm}$ steel plate on each side. The concrete thickness is mainly $76 \mathrm{~cm}$, but it widens to $100 \mathrm{~cm}$ near 


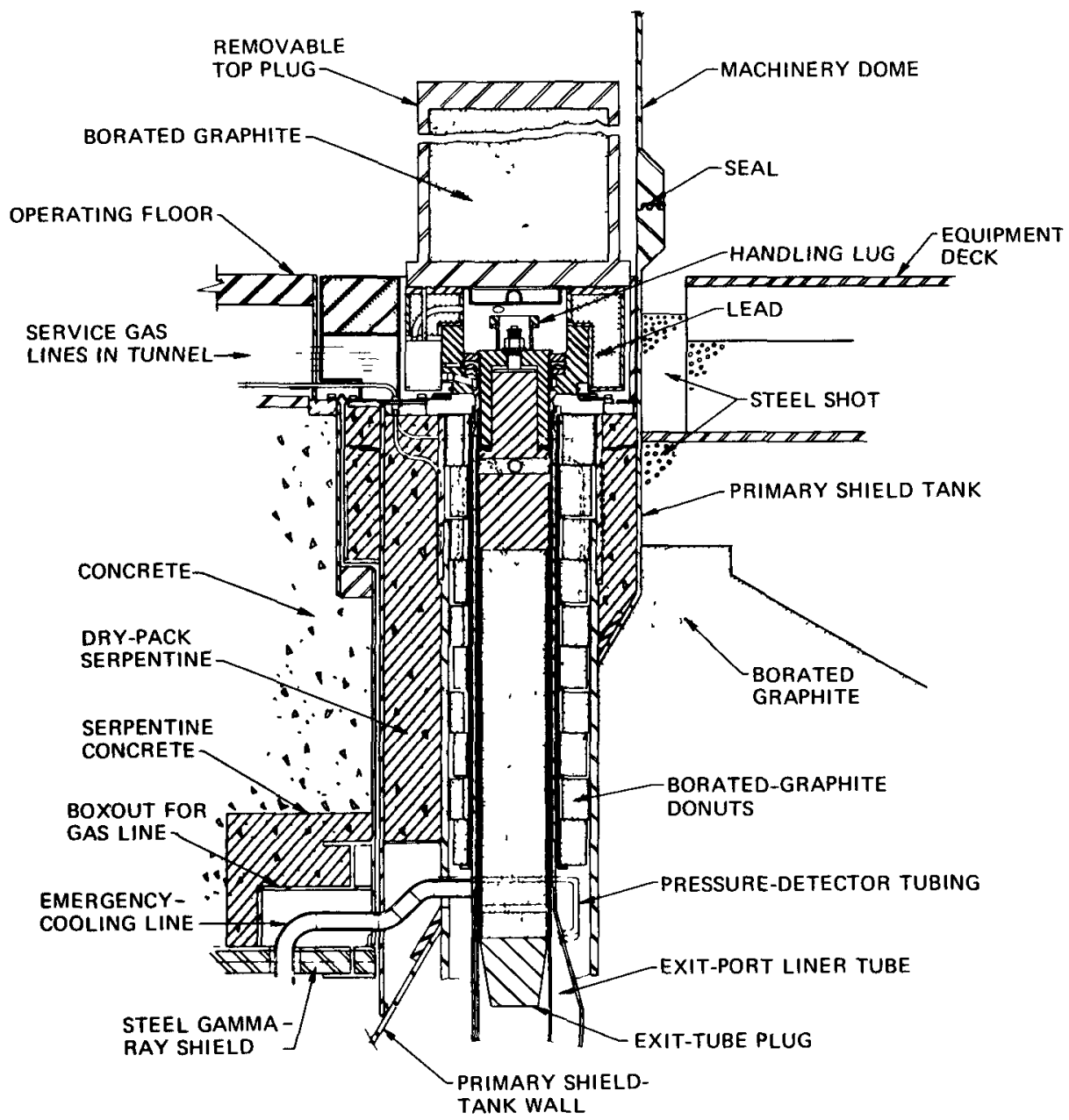

Fig. 10.7-Fuel exit-port assembly. (From Beaudry et al. ${ }^{2}$ )

the location of the transfer rotor. The increase in thickness here is necessary because when the transfer rotor is filled with spent subassemblies the neutron leakage from the primary shield in this area is of higher magnitude than the design leakage value.

A prepacked construction method whereby the aggregate is laid in first and the cement pressure-grouted into the aggregate was used to ensure uniformity in the density and water content of the completed shield. Heavy reinforcing steel bars on a $15-\mathrm{cm}$ mesh formed two planes of reinforcement for the secondary shield.

A 5-cm-thick steel thermal shield was placed around the wall in the high-gamma-flux areas to prevent excess heating and drying out of the 


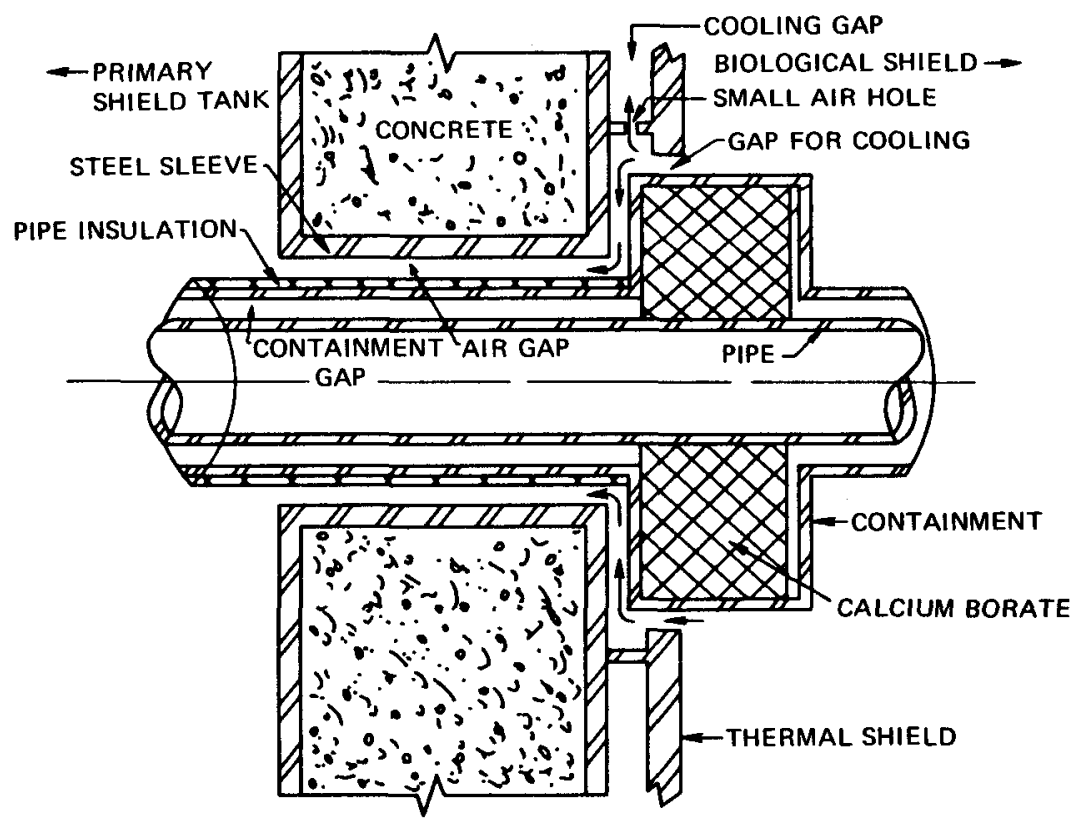

Fig. 10.8 - Schematic view of typical sodium-pipe penetration through secondary shield wall. (From Beaudry et al. ${ }^{2}$ )

concrete by gamma rays emitted from the sodium. Cooling was accomplished by ducting the lower building ventilation system to the $12.7-\mathrm{cm}$ gap between the wall and the thermal shield.

Special shielding problems were created by the various penetrations of the secondary shield wall for the primary coolant inlet pipes, primary sodium overflow pipe, gas lines, and electric cables. Electric- and gas-line penetrations were kept small; they pierced the wall at angles close to tangential with the primary shield tank. The 76-cm-diameter sodium exit lines pass under the shield wall and require special shielding within the reactor compartment and in the areas where they penetrate the floor of the equipment compartment. Because of mechanical considerations, the sodium inlet lines were required to pass through the shield wall in directions nearly perpendicular to the wall's surface.

The design of a typical sodium-pipe penetration through the secondary shield wall is shown in Fig. 10.8. The penetration contains not only the primary sodium pipe but also its outer containment pipe and an annular cooling gap. Cooling is required within the penetration to remove the nuclear heat deposited in the steel through the pipe insulation so that the concrete will not become overheated. Neutron streaming through the annular void 
regions was minimized by placing donut shields around the primary sodium pipe.

Shielding for the primary sodium overflow line, which is a normally empty $15.2-\mathrm{cm}$ pipe, was handled by passing the pipe through the shield at a $45^{\circ}$ angle with the horizontal. This arrangement ensured that the gamma radiation passing through the penetration would be scattered at least once through an angle more than $90^{\circ}$, which reduces the intensity by more than an order of magnitude.

(c) Operating Floor and Radial Biological Shield. The biological shield for the operating room, i.e., the floor (see Fig. 10.4), and the radial biological shield surrounding the lower section of the containment building are designed to allow continuous access during full-power operation. The radial shield is ordinary concrete with a thickness of $2.1 \mathrm{~m}$. The floor shield has a total thickness of $1.5 \mathrm{~m}$ (concrete and steel) in all areas except under the cask-car tracks, where the thickness is $2.1 \mathrm{~m}$ to support the 180 -ton cask car. Magnetite aggregate with a density of $5.1 \mathrm{~g} / \mathrm{cm}^{3}$ was added to the concrete to maintain a minimum dry density of $2.4 \mathrm{~g} / \mathrm{cm}^{3}$ in the operating-floor concrete. As a result of detailed studies of the possible radiation paths, the steel plate on the underside of the floor varies in thickness from 8.9 to $25 \mathrm{~cm}$. The final design thicknesses at each location are shown in Fig. 10.9. Concrete with serpentine aggregate (density, 2.1 $\mathrm{g} / \mathrm{cm}^{3}$ ) was used for high-temperature areas around the floor plugs for the primary heat exchange and the sodium pumps. The reduced density in the areas where serpentine concrete was used required that the thickness of the steel in the shield be correspondingly increased.

There were numerous penetrations of the operating floor for the reactor nuclear instrumentation, primary sodium pumps, and heat exchangers and for electric cables, gas lines, and other miscellaneous equipment. Each penetration required a separate shield design, which was handled in a manner similar to the previously described special shield designs.

(d) Intermediate-Heat-Exchanger and Sodium-Pump Shield Plugs. The outer shells of the primary sodium pump and intermediate heat exchangers rise through the operating-floor shield. The shells are stepped within the floor shield, and removable stepped shield plugs fit into the shells at the floor level.

The equipment is designed so that there is a double annular gap around each of the plugs, which allows clearance for expansion caused by heat and permits removal for maintenance. A sketch of the shield design around a 


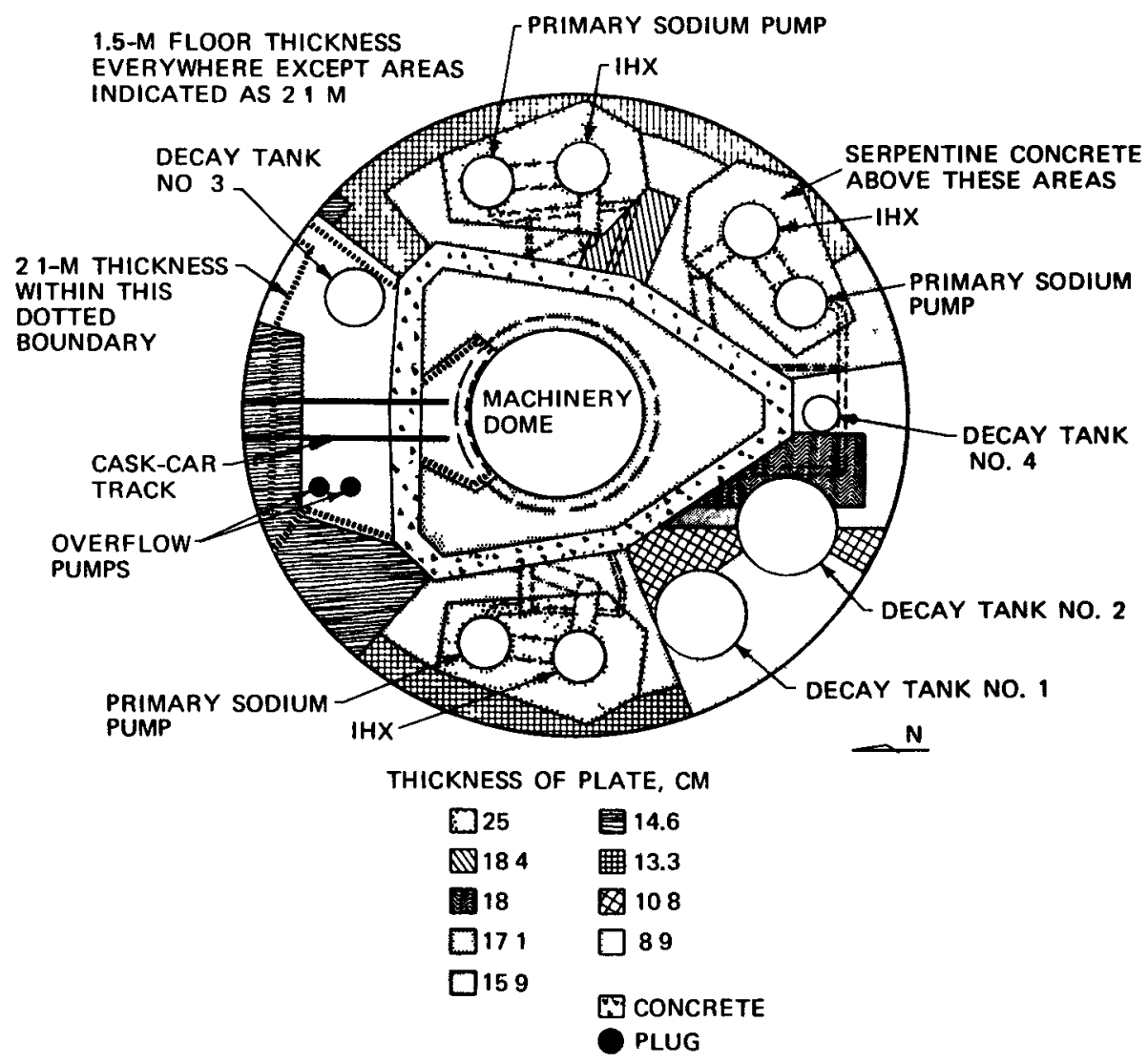

Fig. 10.9-Plan view of operating floor showing steel requirements beneath the concrete. (From Beaudry et al. ${ }^{2}$ )

heat exchanger is shown in Fig. 10.10. Since the offsets in the floor penetrations were fixed before a detailed analysis of the necessary shielding was made, a dual-purpose shielding material was needed to reduce to proper levels both the neutron and gamma radiation levels within the gaps and annuli around the plug shields. Boron steel (containing $1 \%$ boron) was chosen for this purpose and was installed below the stepped annuli around the intermediate heat exchanger. Plain carbon steel was used around the sodium-pump floor penetrations.

Because of high temperatures in this area, each of the three intermediate-heat-exchanger plugs and three pump plugs were filled with dry-packed serpentine. A $15.2-\mathrm{cm}$ layer of steel shot was added to each pump plug for additional gamma-ray shielding. 


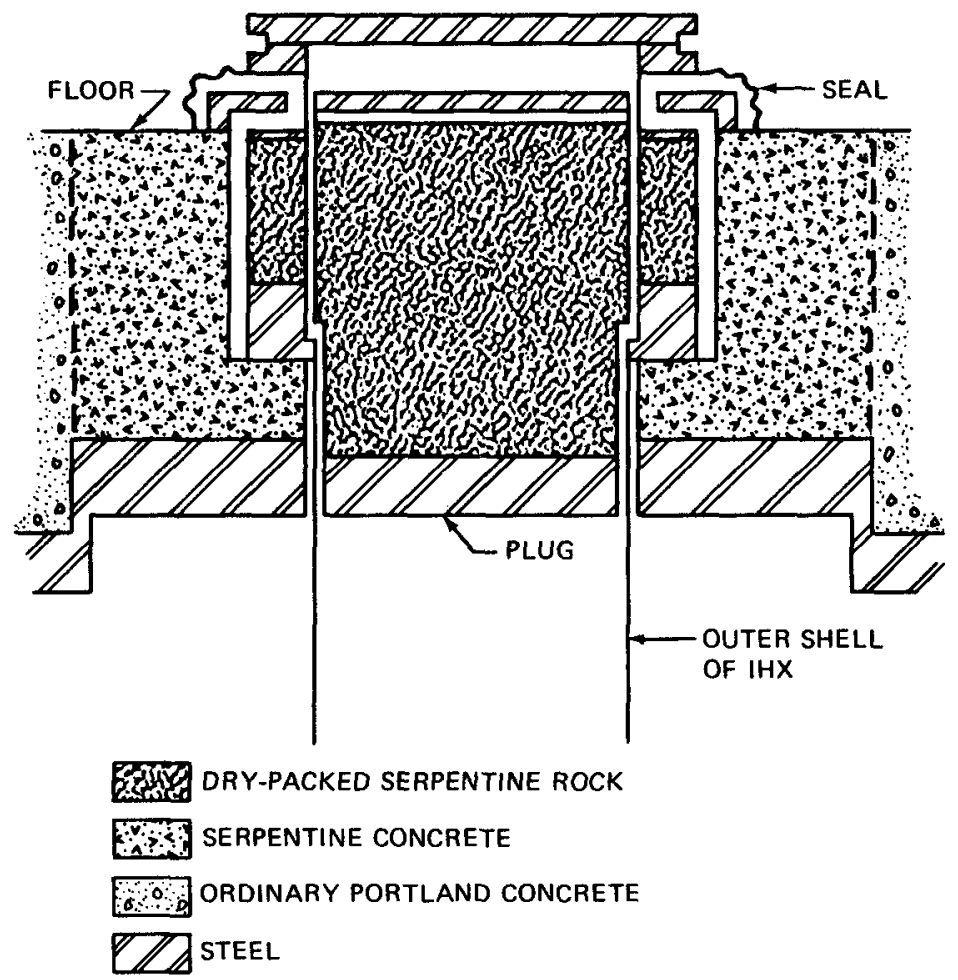

Fig. 10.10 - Sketch of intermediate-heat-exchanger shield plug. (From Beaudry et al. ${ }^{2}$ )

(e) Primary Sodium-Pipe Shields. Special considerations were given to the problem of neutron leakage from the 76-cm-diameter primary sodium pipes. A calcium borate mineral with the approximate formula $2 \mathrm{CaO}$. $3 \mathrm{~B}_{2} \mathrm{O}_{3} \cdot 2 \mathrm{H}_{2} \mathrm{O}$ in an asbestos matrix (trade name Sigma $\mathrm{K}$ ) with a density of $1.1 \mathrm{~g} / \mathrm{cm}^{3}$ was chosen as shielding for these lines. This material contains $12 \mathrm{wt} . \%$ boron and withstands temperatures up to $1100^{\circ} \mathrm{C}$ in high-radiation environments.

The problem of designing a proper pipe shield was complicated because the pipes were surrounded by 0.9 -m-diameter secondary containment pipes for protection against a sodium leak. Other factors in the design of the shields for these pipes were the type and design of insulation, heaters, and construction gaps. Allowances for pipe movements due to thermal expansions had to be considered. The thickness of the calcium borate around the three $76-\mathrm{cm}$ pipe loops varied from 23 to $28 \mathrm{~cm}$. The design of these shields is shown in Fig. 10.11. Four donut or ring shields of calcium borate were 


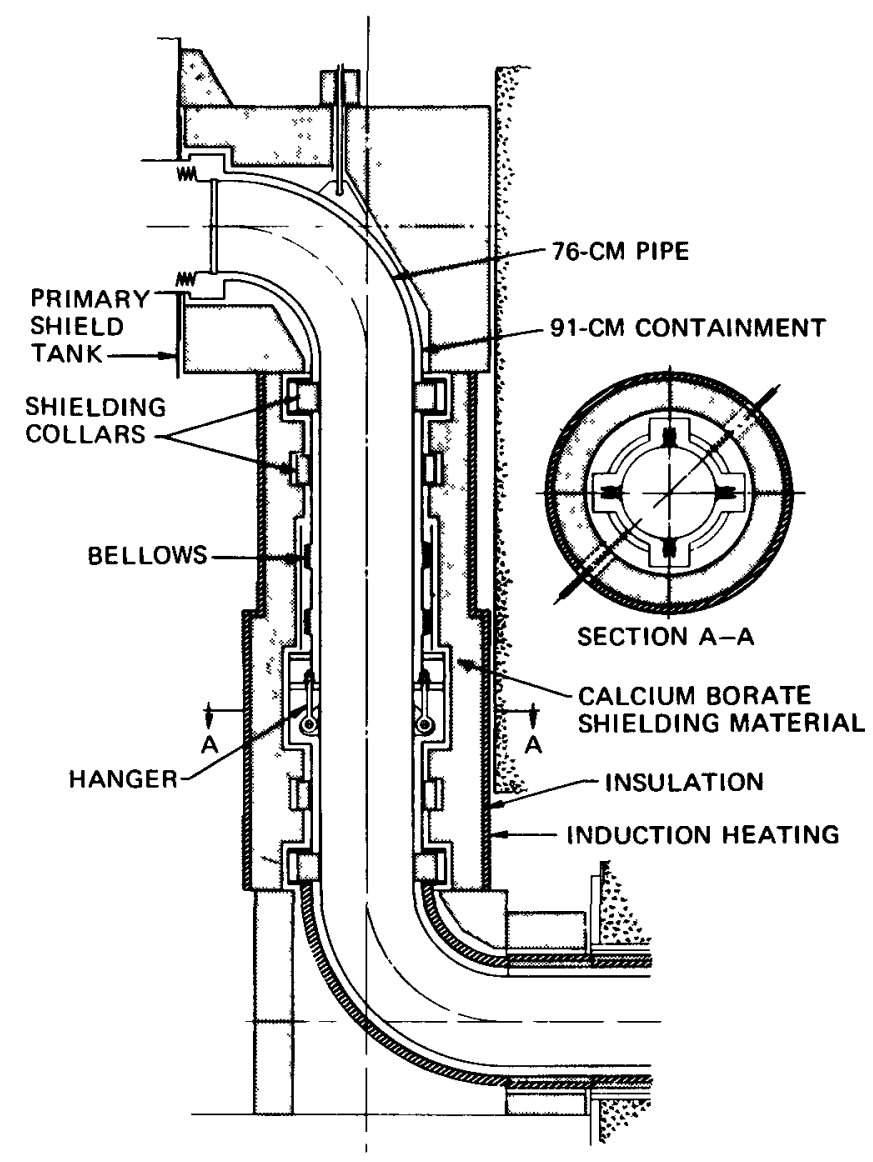

Fig. 10.11 - Calcium borate shield for primary sodium pipe. (From Beaudry et al. ${ }^{2}$ )

installed directly on the pipe and on its containment to prevent neutron streaming through the annuli around the pipes.

(f) Shield Systems for the Auxiliary Facilities. The auxiliary facilities requiring shielding are the fuel and repair building, the sodium service building and its associated pipe tunnel, the inert- and waste-gas building, the secondary sodium galleries, and the control building.

In these areas shielding against gamma rays only was required. Most of the shields were of ordinary concrete, but steel shot, steel plate, sand, gravel, and lead were used in special situations. Design criteria and construction considerations for these auxiliary facilities parallel those of the primary reactor shields and will not be described here. 


\subsubsection{Shield Costs}

The type of material was an important consideration for each shield, and the cost was highly influential in the final selection.

The main shield materials were ordinary concrete, steel, stainless steel, lead, and graphite. The more unusual or exotic materials used were (1) magnetite and serpentine aggregates in concrete, by themselves, or in mixes in a dry-packed condition; (2) steel shot; (3) boron steel; (4) boron carbide; (5) borated graphite (boron carbide in graphite); (6) calcium borate; and (7) uranium (depleted). Each material was selected on its merits for the intended use. Earth, sand, and gravel were used to advantage.

The total cost of all shields $(\$ 5,581,300)$ was approximately $5.5 \%$ of the entire cost of the project. Of this amount, $\$ 1,000,000$, or $18 \%$, was for research and development. The remainder $(\$ 4,581,300)$ was for the construction of the shields. The shield research and development cost was slightly more than $3 \%$ of the research and development cost for the entire plant.

Table 10.1 shows a breakdown of the shield costs for the Fermi plant.

\subsubsection{Calculational Techniques}

The calculation of neutron attenuation presented the greatest uncertainties in the shield design and followed the availability of improved transport techniques. The original attenuation calculations were done before 1960 with removal theory. The design of the secondary shield wall is an example of the application of removal theory and shows the conservatism incorporated. When the thicknesses of concrete required to reduce the flux in the equipment compartment to $10^{4}$ neutrons $\mathrm{cm}^{-2} \mathrm{sec}^{-1}$ were determined, calculations along various paths (see Fig. 10.12) indicated concrete thicknesses ranging from $81 \mathrm{~cm}$ at $0^{\circ}$ (opposite the thin primary shield tank) to a maximum of $100 \mathrm{~cm}$ at $180^{\circ}$ (opposite the transfer rotor). An average thickness of $91 \mathrm{~cm}$ was chosen for the final design except for the north wall opposite the transfer rotor, which was set at $100 \mathrm{~cm}$. In the final design stages, the AIM-6 diffusion-theory $\operatorname{code}^{14}$ was primarily used with some checks being with the NIOBE ${ }^{15}$ numerical integration, the TDC ${ }^{16}$ (twodimensional $S_{n}-P_{1}$ ), the $\mathrm{DTK}^{17}$ (one-dimensional $S_{n}-P_{1}$ ), and the $\mathrm{CRAM}^{18}$ (one- and two-dimensional diffusion theory) machine codes. Monte Carlo or the removal-diffusion techniques, which are used in design work today, were not used since the state of the art did not appear sufficiently advanced at the time. 
Table 10.1 -APPROXIMATE SHIELD COSTS, ENRICO FERMI ATOMIC POWER PLANT $t$

\begin{tabular}{|c|c|c|}
\hline Area & Item & Cost \\
\hline \multirow[t]{12}{*}{ Reactor building } & Concrete, poured & $\$ 45,000 \ddagger$ \\
\hline & Concrete, prepacked & 113,200 \\
\hline & Steel plates and sleeves & 294,000 \\
\hline & Primary-shield-tank shell & 110,000 \\
\hline & Primary-shield-tank graphite & $2,200,000 \S$ \\
\hline & $\begin{array}{l}\text { Reactor-vessel thermal } \\
\text { shield (stainless steel) } \\
\text { Offset-handling-mechanism }\end{array}$ & 137,500 \\
\hline & shielding & 26,200 \\
\hline & Rotating-plug shielding & 235,000 \\
\hline & $\begin{array}{l}\text { Sodium-pump plug shields ( } 3 \text { ) } \\
\text { Intermediate-heat-exchanger }\end{array}$ & 234,000 \\
\hline & plug shields (3) & 395,000 \\
\hline & Calcium borate pipe shields ( 3 ) & 280,000 \\
\hline & Total reactor building & $\$ 4,070,800$ \\
\hline \multirow[t]{2}{*}{ Auxiliary facilities } & $\begin{array}{l}\text { Concrete } \\
\text { Steel }\end{array}$ & $\begin{array}{l}398,500 \ddagger 9 \\
112,000\end{array}$ \\
\hline & Total auxiliary shields & $\$ 510,500$ \\
\hline \multirow[t]{2}{*}{ Plant total } & $\begin{array}{l}\text { Total material and erection } \\
\text { Research and development }\end{array}$ & $\begin{array}{l}4,581,300 \\
1,000,000^{* *}\end{array}$ \\
\hline & Grand total shield costs & $\$ 5,581,300$ \\
\hline
\end{tabular}

$\dagger$ From Beaudry et al. ${ }^{2}$

$\$$ Includes forming and reinforcing steel.

$\S$ About $\$ 1,750,000$ of this amount is for the removal of subgrade graphite and its replacement with high-quality material. This procedure was very much more expensive than the original installation.

IIncludes prorated additional structural costs owing to the presence of the shield.

**Prorated from total research and development costs.

Generally the AIM-6 diffusion code was used with the 18-group neutron-cross-section set of Mills $^{19}$ which was revised and updated. Occasionally the newer CRAM diffusion code was used, in both one and two dimensions, with a special 24-group set of cross sections. Little attempt was made to investigate the effect of different cross-section sets or the energy-group structure. It was felt that errors due to the calculational methods or uncertainties inherent in the mock-up of the physical problem 
would override errors due to the nuclear cross sections since diffusion theory uses the approximation of isotropic scattering. It should be pointed out, however, that, for deep-penetration calculations with the modern transport codes that allow for anisotropic scattering, the result is very sensitive to the nuclear cross section.

Most of the calculations were one-dimensional, but in later work some two-dimensional CRAM calculations were made. It was not possible to mock-up the entire primary shield in a single two-dimensional problem because of the large size and asymmetry of the system. A simplified computer model used in cylindrical geometry calculations is shown in Fig. 10.13. The corresponding regional descriptions and material densities are shown in Table 10.2. This model represents a section of the primary shield from the core center upward to the rotating plug and outward to the primary shield tank through the narrowest part of the graphite shield. The safety rods are assumed to be in their raised positions above the subassembly handling heads.

Neutron streaming through the various shield penetrations, such as pipes, ducts, and offset plugs, was a potential hazard that required considerable design study. In general, the streaming equations for the various geometries were used, but they were modified with the Fisher ${ }^{20}$ formula to include the effects of scattering. These streaming equations for the various geometries are tabulated by Hungerford. ${ }^{4}$ Gamma-ray attenuation was calculated with point kernels and ray-tracing techniques as described by Rockwell. ${ }^{21}$

Some of these techniques were programmed for computer calculations for more detailed analysis, such as determining the optimum steel and concrete requirements of the operating floor at various locations, as shown in Fig. 10.9.

For this study the below-floor primary system was divided into radioactive sources of several different geometries. A computer program was written which calculated the radiation levels at various positions on the operating floor through the shield. The program calculated the contribution from each source and summed overall sources at each point on the floor. The points were located on a grid system of radial lines marked every $10^{\circ}$ between $0^{\circ}$ and $360^{\circ}$ starting at north and circumferential lines that were about $0.91 \mathrm{~m}$ apart. The results of the computer program were used to determine the final balance between concrete thickness and steel thickness in each broad area. 


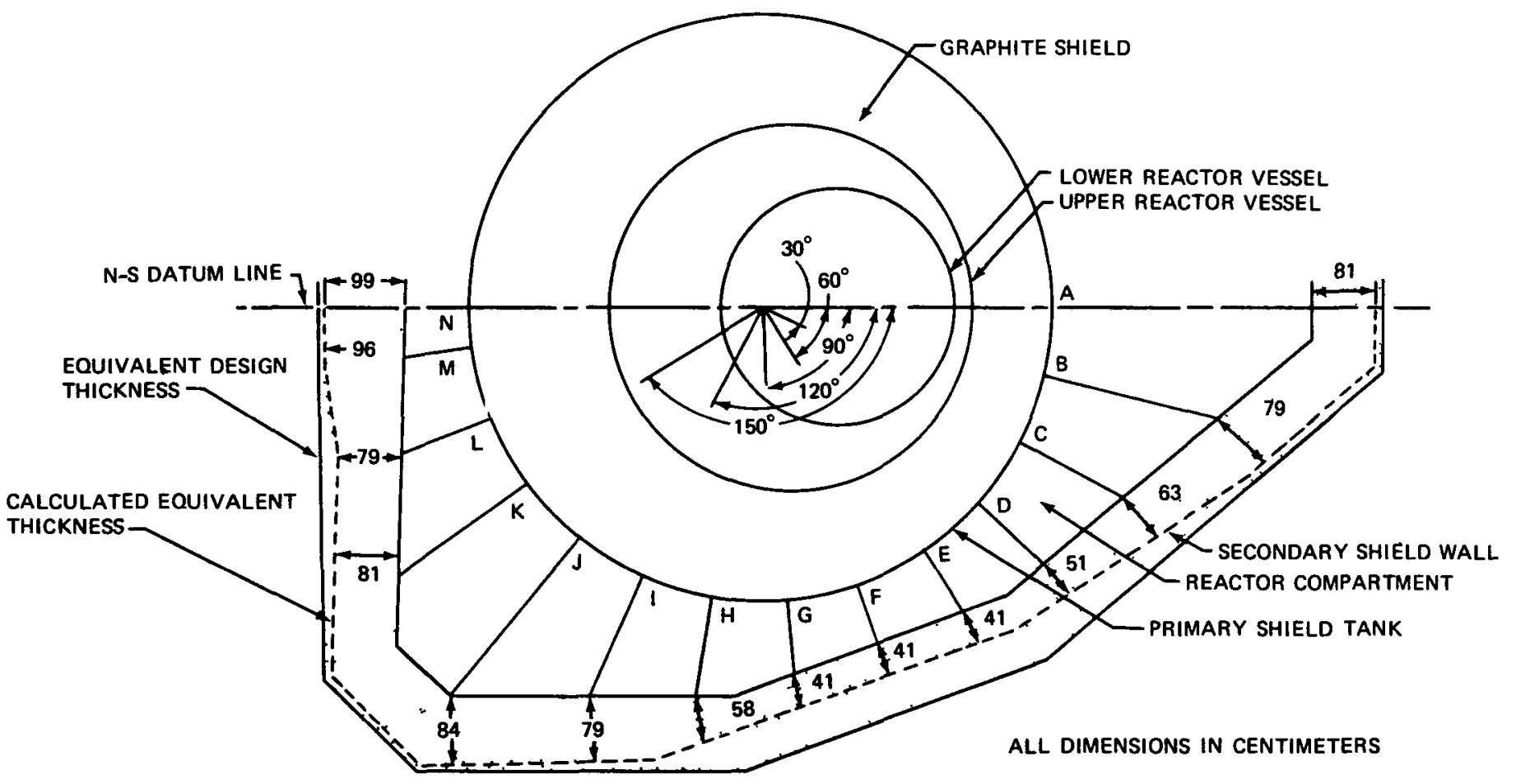

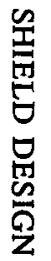

Fig. 10.12-Minimum calculated design thicknesses of secondary shield wall. (From Beaudry. ${ }^{3}$ ) 


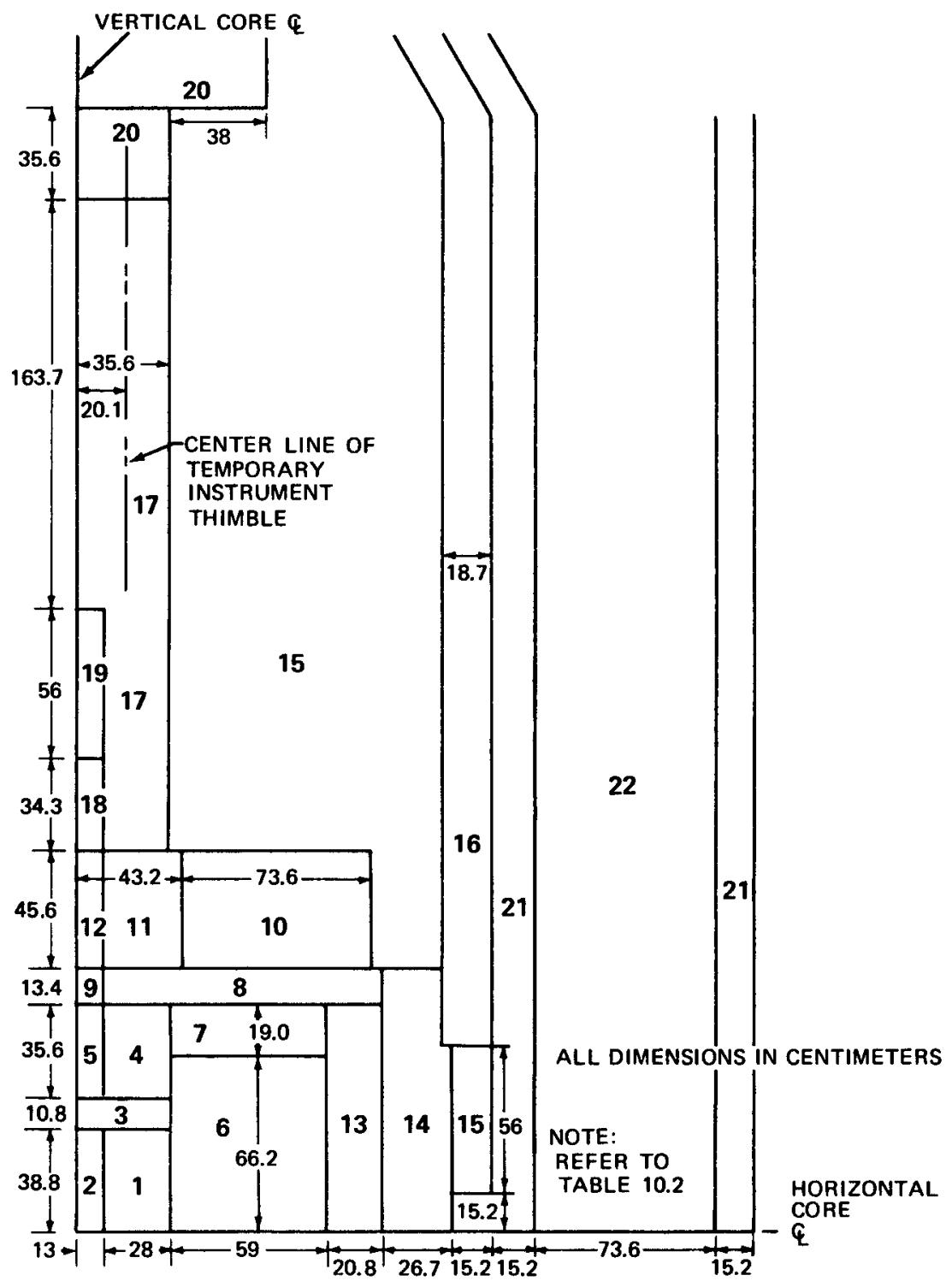

Fig. 10.13-Primary shield: Simplified layout of zones for computer problems. See Table 10.2 for key to description and composition of reactor zones. (From Beaudry. ${ }^{3}$ ) 
Table 10.2-REGIONAL DESCRIPTION AND COMPOSITION OF REACTOR ZONES FOR COMPUTATIONS

\begin{tabular}{|c|c|c|c|c|c|c|c|c|c|}
\hline \multirow[b]{2}{*}{$\begin{array}{c}\text { Zone } \\
\text { number }\end{array}$} & \multirow[b]{2}{*}{ Zone name } & \multicolumn{8}{|c|}{ Number denvities for isotopes, $10^{24}$ atoms $/ \mathrm{cm}^{3}$} \\
\hline & & ${ }^{235} \mathrm{U}$ & ${ }^{238} \mathrm{U}$ & Mo & $\mathbf{Z} \mathbf{r}$ & $\mathbf{F e}$ & $\mathbf{N a}$ & $\begin{array}{l}\text { Narural } \\
\text { Boron }\end{array}$ & $\mathbf{C}$ \\
\hline 1 & Core & 0.00360 & 0.009632 & 0.0035 & 0.00196 & 0.0148 & 0.0106 & & \\
\hline 2 & Control- and safety-rod region & 0.00215 & 0.006164 & 0.00223 & 0.00125 & 0.0141 & 0.0136 & 0.0012 & \\
\hline 3 & End gap & & & & 0.00172 & 0.017 & 0.0179 & & \\
\hline 4 & Axial blanket & 0.000039 & 0.01101 & 0.0008 & & 0.014 & 0.0139 & & \\
\hline 5 & Safety-rod region of blanket & 0.000025 & 0.00705 & 0.00051 & & 0.0194 & 0.0148 & & \\
\hline 6 & Radial blanket & 0.000066 & 0.019 & 0.0013 & & 0.0158 & 0.0092 & & \\
\hline 7 & Axial gap & & & & & 0.017 & 0.0189 & & \\
\hline 8 & Handling heads & & & & & 0.017 & 0.0189 & & \\
\hline 9 & Safety rods and hold-down & & & & & 0.018 & 0.0172 & 0.0104 & \\
\hline 10 & Hold-down (outer region) & & & & & 0.00043 & 0.0223 & & \\
\hline 11 & Hold-down (inner region) & & & & & 0.023 & 0.0172 & & \\
\hline 12 & Safety rods (poison section) & & & & & 0.0219 & 0.0161 & 0.0104 & \\
\hline 13 & Steel shield bars & & & & & 0.0721 & 0.0034 & & \\
\hline 14 & Steel thermal shield & & & & & 0.0648 & 0.00584 & & \\
\hline 15 & Sodium plenum & & & & & & 0.0236 & & \\
\hline 16 & Steel thermal shield & & & & & 0.0632 & 0.0063 & & \\
\hline 17 & Hold-down column & & & & & 0.0020 & 0.023 & & \\
\hline 18 & Safety rods (poison section) & & & & & 0.0085 & 0.0198 & 0.0104 & \\
\hline 19 & Safety rods (poison section) & & & & & 0.0098 & 0.0009 & 0.0104 & \\
\hline 20 & Steel of rotating plug & & & & & 0.085 & & & \\
\hline 21 & Borated graphite & & & & & & & 0.0045 & 0.0769 \\
\hline 22 & Plain graphite & & & & & & & & 0.0803 \\
\hline
\end{tabular}

tFrom Beaudry.

\subsubsection{Comparison of Measurements and Calculations}

The shield test program for the Enrico Fermi reactor plant was unusually complete and detailed because of the prototype nature of the reactor. The test program principally covered regions inside the reactor building, and the major emphasis was on neutron measurements, although the initial emphasis was in measuring the induced ${ }^{24} \mathrm{Na}$ activity because of its importance in the overall plant design.

Neutron measurements were made throughout the reactor vessel and the primary shield tank. Threshold detectors and ${ }^{235} \mathrm{U}$ and ${ }^{238} \mathrm{U}$ fission foils were used in the core and axial and radial blankets. Neutron fluxes were measured in the upper sodium pool up to the bottom of the rotating plug. Vertical ${ }^{235} \mathrm{U}$ fission distributions were obtained at the side of the pool in a surveillance tube near the reactor-vessel wall. A temporary instrument thimble located near the center of the pool was used to obtain vertical traverses $\left({ }^{234} \mathrm{U},{ }^{235} \mathrm{U},{ }^{236} \mathrm{U},{ }^{238} \mathrm{U},{ }^{239} \mathrm{Pu}\right.$, and $\left.{ }^{240} \mathrm{Pu}\right)$ with both foils and absolute fission counters. In the primary graphite shield, both ${ }^{235} \mathrm{U}$ foils and various neutron detectors were used. Vertical distributions were obtained from the flex-leg regions (LRV) up to the machinery deck (in the surveillance tubes) or up to the operating floor (in the neutron-counter 
tubes). The neutron-flux levels in the reactor compartment (between the primary shield tank and the secondary shield wall) were measured at 33 locations with ${ }^{235} \mathrm{U}$ foils and at one azimuthal position with a fission counter and a spherical moderating dosimeter.

At various times after criticality, radiation surveys were made throughout the entire above-floor area of the reactor building, including the rotating plug and machinery dome, and throughout the various service buildings.

The gamma-ray surveys were conducted with area survey monitors. The neutron surveys used portable proton-recoil counters, $\mathbf{B F}_{3}$ counters, and a set of multisize spherical polyethylene dosimeters. In general, the detecting instruments were capable of measuring radiation levels above $0.01 \mathrm{mR} / \mathrm{hr}$ for gamma rays and 0.02 to $0.05 \mathrm{mrem} / \mathrm{hr}$ for neutrons.

Although a large number of measurements were made and compared with calculations, we will summarize them with a very general comparison in the following section and with a few detailed comparisons subsequently.

(a) Overall Comparisons. No shield regions were found to be underdesigned, but some areas were found to be significantly overdesigned, which was to be expected. A summary of the principal test results is shown in Table 10.3. The original design calculations for the ${ }^{22} \mathrm{Na}$ and ${ }^{41} \mathrm{~A}$ activities

Table 10.3 - SUMMARY OF PRINCIPAL RESULTS OF THE ENRICO FERMI SHIELD TEST PROGRAM

\begin{tabular}{|c|c|c|c|}
\hline & $\begin{array}{l}\text { Measured, } \\
\mu \mathrm{Ci}^{-3} \mathrm{Mw}(\mathrm{t})^{-1}\end{array}$ & $\begin{array}{c}\text { Original design } \\
\text { calculation, } \\
\mu \mathrm{Ci} \mathrm{cm}{ }^{-3} \mathrm{Mw}(\mathrm{t})^{-1}\end{array}$ & $\begin{array}{c}\text { Most recent } \\
\text { calculation, } \\
\mu \mathrm{Ci} \mathrm{cm}^{-3} \mathrm{Mw}(\mathrm{t})^{-1}\end{array}$ \\
\hline $\begin{array}{l}\text { Sodium- } 24 \text { activity } \\
\text { Sodium- } 22 \text { activity } \\
\text { Argon-41 activity (STP) } \\
\text { Secondary }{ }^{24} \mathrm{Na} \text { activity } \\
\text { Radiation streaming } \\
\text { Component activation in } \\
\text { reactor vessel } \\
\text { Uranium-235 fission rates: } \\
\text { Sodium pool } \\
\text { Transfer rotor } \\
\text { Radial blanket and reflector } \\
\text { Neutron-counter tubes } \\
\text { Foil-sample tubes } \\
\text { Reactor compartment } \\
\text { Equipment compartment }\end{array}$ & \multicolumn{3}{|c|}{$\begin{array}{l}\text { No radiation measured above reactor-building floor at } 110 \mathrm{Mw}(\mathrm{t}) \text {. } \\
\text { Measured and calculated dose rates agree within a factor of } 2 \text {. } \\
\text { Measured values within } 50 \% \text { of calculated values. } \\
\text { Measured values are less than } 1 / 10 \text { of calculated values. } \\
\text { Measured values higher than calculated by } 50 \% \text { (in reflector). } \\
\text { Measured values within a factor of } 3 \text { of calculated values. } \\
\text { Measured values within a factor of } 2 \text { of calculated values. } \\
\text { Measured values less than original calculations. } \\
\text { Measured values less than original calculations. }\end{array}$} \\
\hline
\end{tabular}

†From Beaudry. ${ }^{3}$ 
were extremely conservative, but later more careful calculations with better data produced good agreement. In general, with the complexities involved, the overall agreement can be considered good. In spite of concern about radiation streaming, nothing significant was detected on the operating floor. The principal reason for overestimating streaming was undoubtedly due to the original use of estimates of the radiation levels underneath the rotating plug which were too high. In the early calculations of neutron streaming, a neutron-flux value of $2 \times 10^{13}$ neutrons $\mathrm{cm}^{-2} \mathrm{sec}^{-1}$ under the plug was used, which is equivalent to $4 \times 10^{10}$ neutrons $\mathrm{cm}^{-2} \mathrm{sec}^{-1} \mathrm{Mw}^{-1}$. The best present estimate of the neutron flux at the base of the plug is about $4 \times 10^{7}$ neutrons $\mathrm{cm}^{-2} \mathrm{sec}^{-1} \mathrm{Mw}^{-1}$, a factor of 1000 lower. Secondary reasons were: (1) the use of conservative streaming formulas and (2) the assumption of gaps larger than those which actually existed.

(b) Measurements in the Temporary Instrument Thimble. Vertical flux traverses with ${ }^{238} \mathrm{U},{ }^{239} \mathrm{Pu}$, and ${ }^{235} \mathrm{U}$ fission counters were made through the temporary instrument thimble (see Fig. 10.14). This instrument thimble, which extended down the vertical axis of the reactor core, replaced the

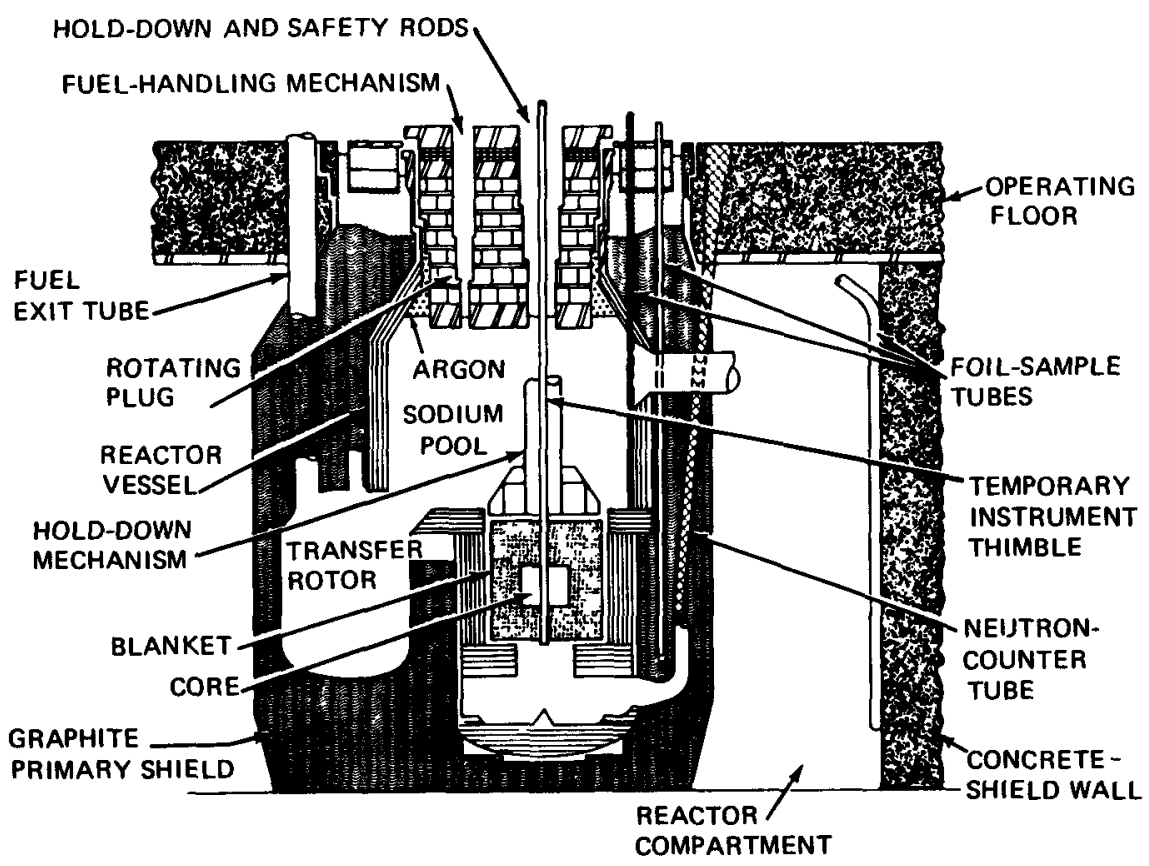

Fig. 10.14-Elevation of primary shield system showing detector locations. (From Beaudry. ${ }^{3}$ ) 


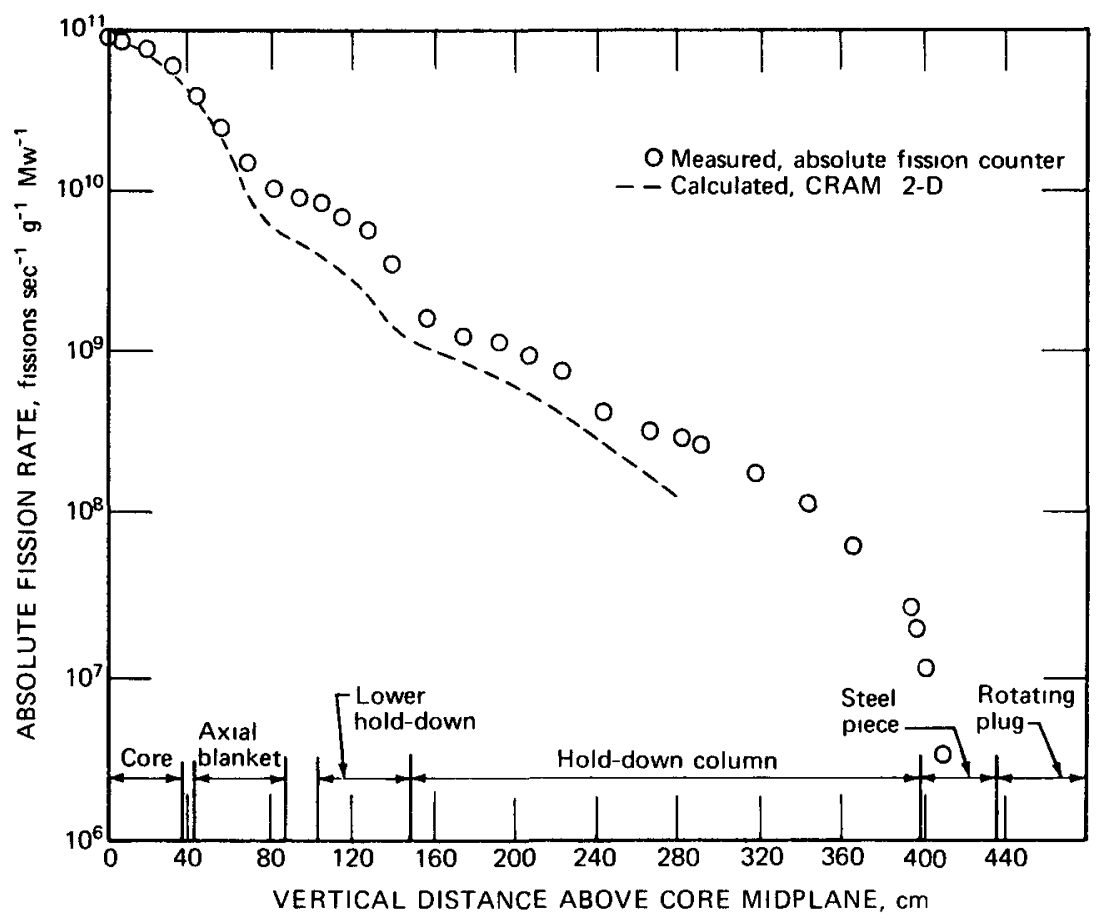

Fig. 10.15-Plutonium-239 fission distribution in the temporary instrument thimble. (From Beaudry. ${ }^{3}$ )

central safety rod. The thimble, which was $5 \mathrm{~cm}$ in diameter, was sealed against sodium at the bottom and open to the containment building at the top. Comparisons between the measurements and two-dimensional CRAM calculations for the ${ }^{239} \mathrm{Pu}$ and ${ }^{235} \mathrm{U}$ counters are shown in Figs. 10.15 and 10.16, respectively. All measurements and calculations were normalized to the same value at the core center. In the CRAM calculations the 24-group set previously mentioned was contracted to 9 neutron groups. The sharp dip in data at about the $400-\mathrm{cm}$ distance is caused by a piece of steel that completely surrounds the upper part of the thimble. The agreement is generally good, with ${ }^{235} \mathrm{U}$ measurements being in better agreement with the calculations than the ${ }^{239} \mathrm{Pu}$ data. This probably results because the ${ }^{239} \mathrm{Pu}$ cross sections in the thermal and near-thermal ranges cannot be as well represented as ${ }^{235} \mathrm{U}$ with the small number of neutron groups that were used. It is probable that, if the calculations were carried beyond the $280-\mathrm{cm}$ mark, the agreement would become poor since diffusion theory would become an inadequate model at deeper penetrations, particularly around a heavy absorber such as steel. 


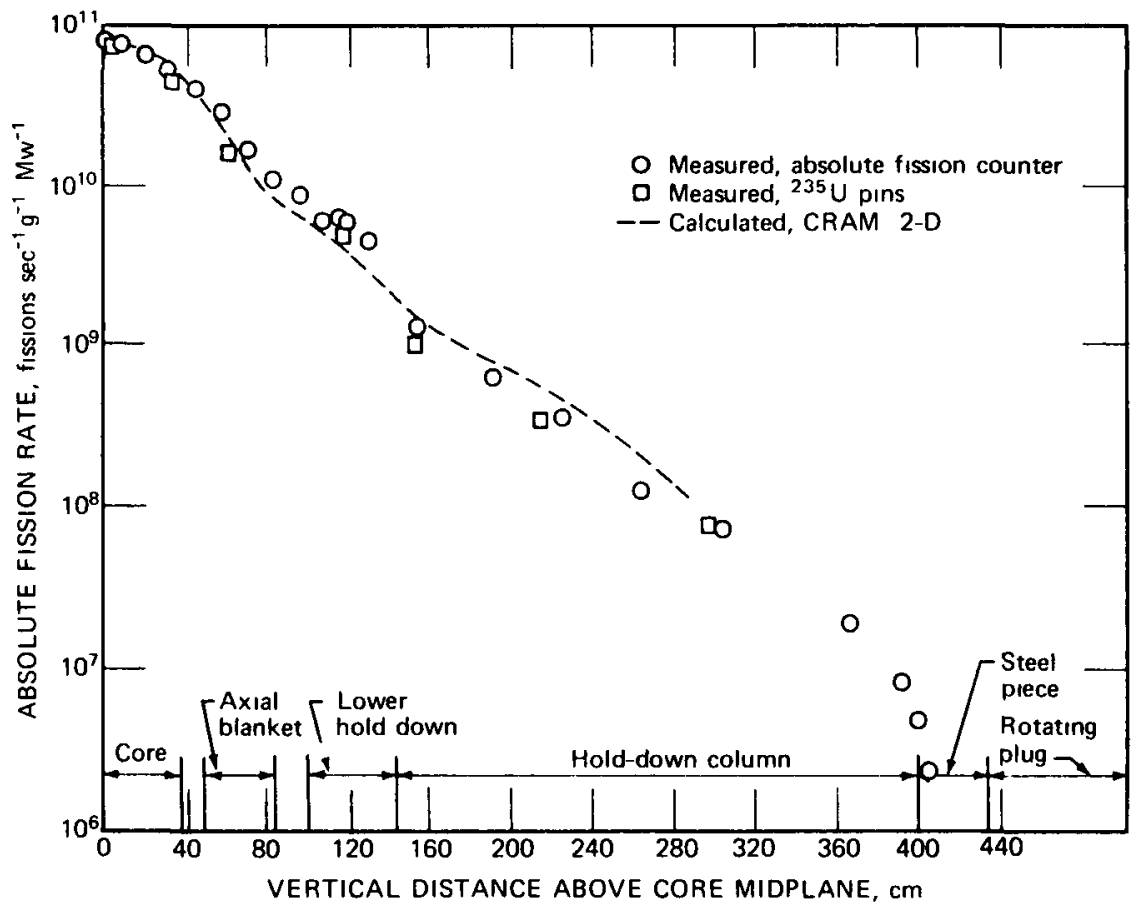

Fig. 10.16-Uranium-235 fission rate in the temporary instrument thimble. (From Beaudry. ${ }^{3}$ )

A large number of one-dimensional calculations were made with the AIM-6 and CRAM codes, and a number of cross-section sets were used. Figure 10.17 shows the results of some of these one-dimensional calculations in comparison with the measurements (curve $B$ ) and the two-dimensional CRAM results (curve $A$ ), the measurements and CRAM results being the normalized values from Fig. 10.16. In the 18-group AIM-6 calculation (curve E), the handling head and hold-down regions were considered to be described simply by a 20 -cm-thick section of steel and sodium, followed directly by a large thickness of pure sodium. This hold-down region is much thinner than the actual hold-down region, and no attempt was made to include the safety rods. This is the type of problem mock-up that had originally been used in the majority of the shielding problems run for the shielding design of the upper vessel regions (e.g., the rotating plug, shot tanks, and upper reactor vessel). Figure 10.17 shows that the use of this simplified type of geometry is quite conservative. The lack of agreement between this calculation and the measurements appears to be caused more by an inadequate geometrical mock-up than by the use of spherical 


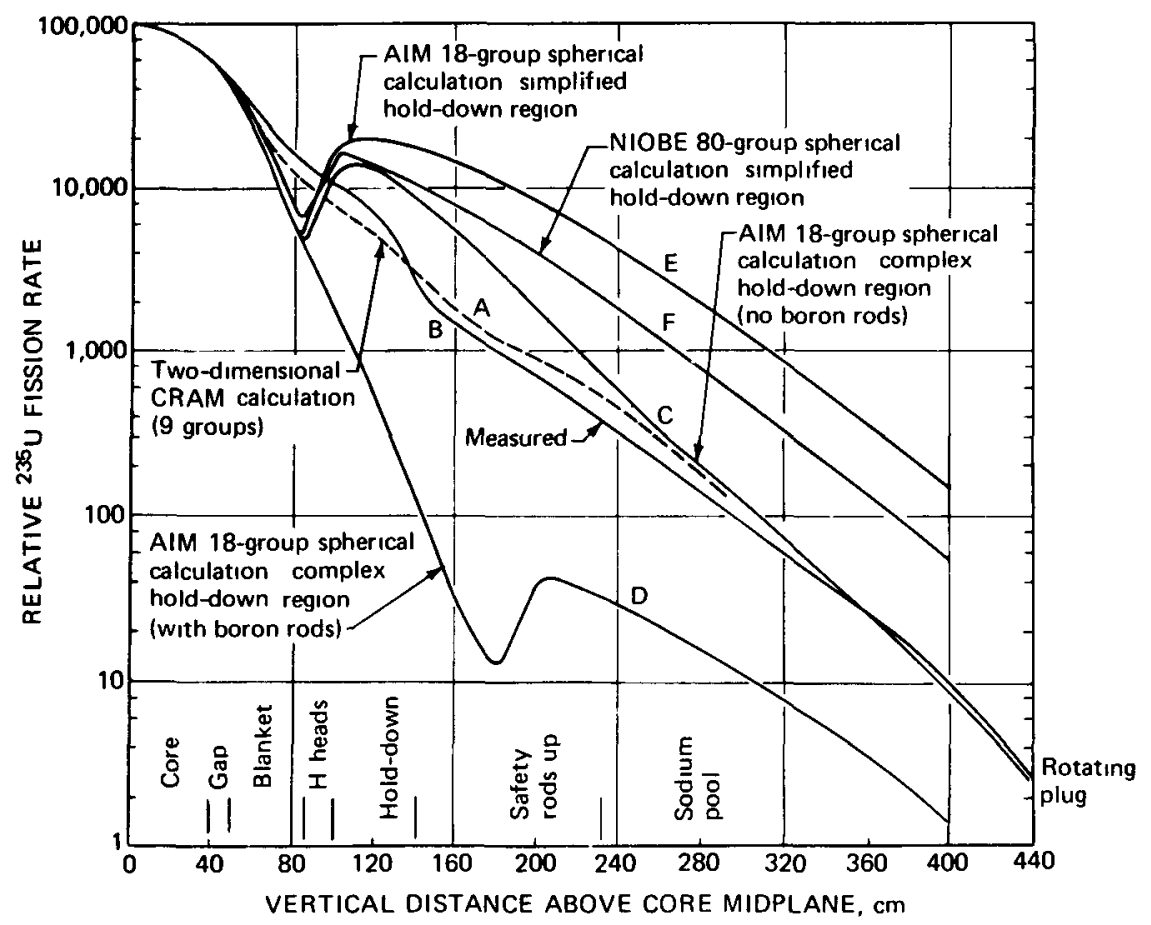

Fig. 10.17-Uranium-235 fission-rate traverse in the temporary instrument thimble. (From Beaudry. ${ }^{3}$ )

geometry. This is more evident from curves C and D of Fig. 10.17, which were obtained from spherical 18-group AIM calculations. In each of these and in the two-dimensional CRAM problem (curve A), however, the same detailed hold-down configuration was used. The geometric model used for calculating curve $\mathrm{D}$ included the safety rods with their full boron content, whereas the geometric mock-up for curve $\mathrm{C}$ omitted the boron altogether. The strong poisoning effect of these safety rods accounts for the much lower values of curve $D$, and this is apparent for the entire distance from the axial blankets out to the rotating plug. Although the fission-rate data shown in curve $\mathrm{C}$ are most different from the measured data in the region of the safety rods, they match the measured response (curve B) farther out in the sodium pool (all the way to the rotating plug).

A more complete study of the differences obtained from various cross-sectional sets also involved such items as mesh spacing, fluxconvergence criteria, group-collapsing schemes, and possible differences between the AIM- 6 and the CRAM codes. Spherical geometry was used. As tentatively found by the study, there were essentially no differences between 
the results from the AIM- 6 and the CRAM codes per se. However, the use of the various cross-sectional sets did result in great differences in the ${ }^{235} \mathrm{U}$ fission response. Again these differences first became pronounced above the axial blanket, where the twin effects of spectrum softening and the presence of large amounts of steel are encountered.

All these problems have clearly demonstrated that a one-dimensional problem mock-up can be quite incorrect in those regions of the hold-down where there are abrupt changes in absorbing materials. The general shielding calculations (as exemplified by curve E of Fig. 10.17) used in the basic shield design appear to be on the conservative side even without considering any additional correction factors. Note, however, that this entire discussion has been based primarily on the ${ }^{235} \mathrm{U}$ fission rate, which is mainly an index of thermal and near-thermal fluxes.

(c) Reactor-Compartment Measurements. The neutron flux in the reactor compartment next to the secondary shield wall is the source term in the design of that shield. A number of measurements with a fission counter and a spherical dosimeter were made directly underneath manhole No. 2 (see Fig. 10.5). All the measurements by the two different instruments were in close agreement, but no detailed comparisons with calculations were made.

In a number of regions of the reactor compartment, neutron-flux measurements were made with ${ }^{235} \mathrm{U}$ foils. When the measured foil counts were converted to fluxes on the inside of the secondary shield wall with an adjusted AIM-6 spectra, all were within the maximum design value of $10^{8}$ neutrons $\mathrm{cm}^{-2} \mathrm{sec}^{-1}$.

\subsection{FAST BREEDER: DOUNREAY FAST REACTOR}

This British power plant is contemporary with the Enrico Fermi plant, and the two plants share a number of design features. The Dounreay Fast Reactor (DFR) is a sodium-potassium-cooled fast breeder system; the reactor shield contains mostly graphite (pure and borated) and steel. The rotating top shield is designed to provide access channels for charging the core and breeder regions as well as eighteen $5-\mathrm{cm}$ penetrations for control purposes. Figures 10.18 and 10.19 show the general arrangement and materials details of the rotating top shield. The removable top shield (Fig. 10.18) was not in place for the experiments we will describe. A material called Resilon, which is a hydrogenous resin-bonded glass fiber, is used in the top shield. 


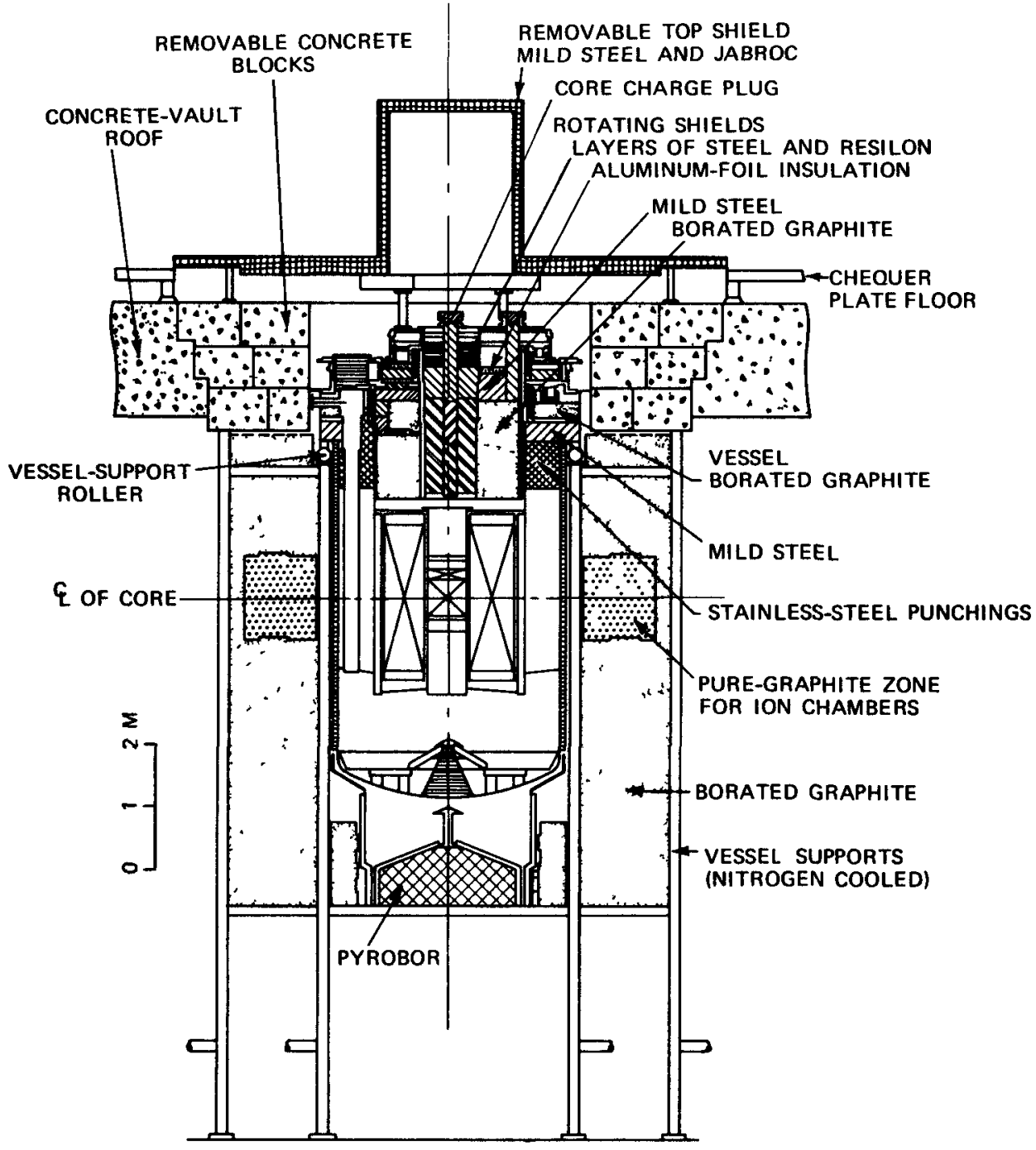

Fig. 10.18-General arrangement of the DFR shield. (From Adamson et al. ${ }^{22}$ )

Adamson, Judd, and $\mathrm{McNair}^{2}{ }^{2}$ report a study of the neutron flux in the top shield of DFR to test the validity of removal-diffusion theory in calculating the bulk shield attenuation for a fast reactor shield. Although the method had been applied successfully to large thermal reactors where the principal attenuating medium is concrete, it had not been applied to a relatively small fast reactor where the medium is principally borated graphite. We will describe some of their results for comparison with the 


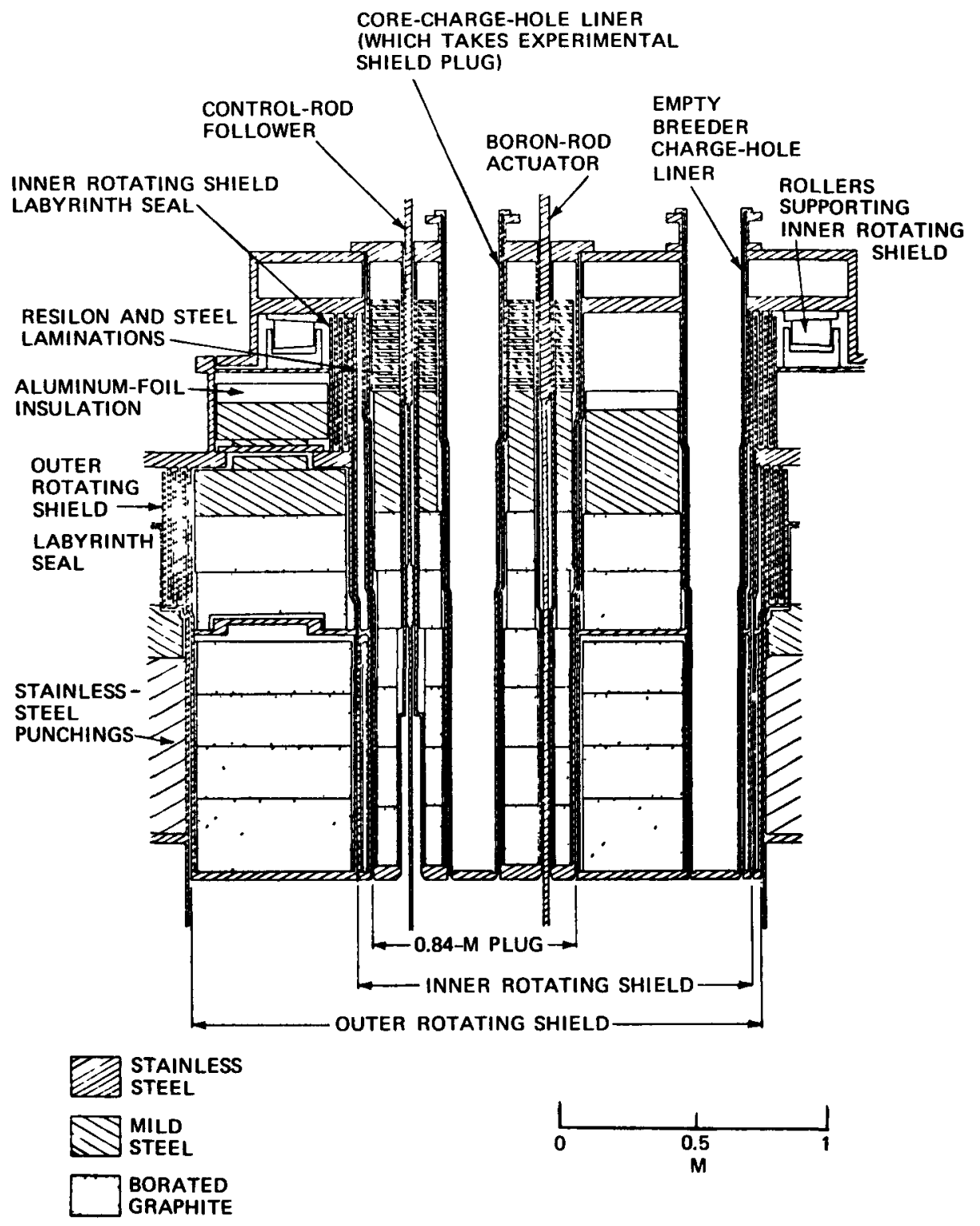

Fig. 10.19-Details of the rotating shields. (From Adamson et al. ${ }^{22}$ ) 
corresponding work shown in the preceding sections on the Enrico Fermi plant.

\subsubsection{Calculational Model for Bulk Shield}

The models of the core, blanket, and top shield used for the calculation are shown in Fig. 10.20. The Spinney removal-diffusion method and

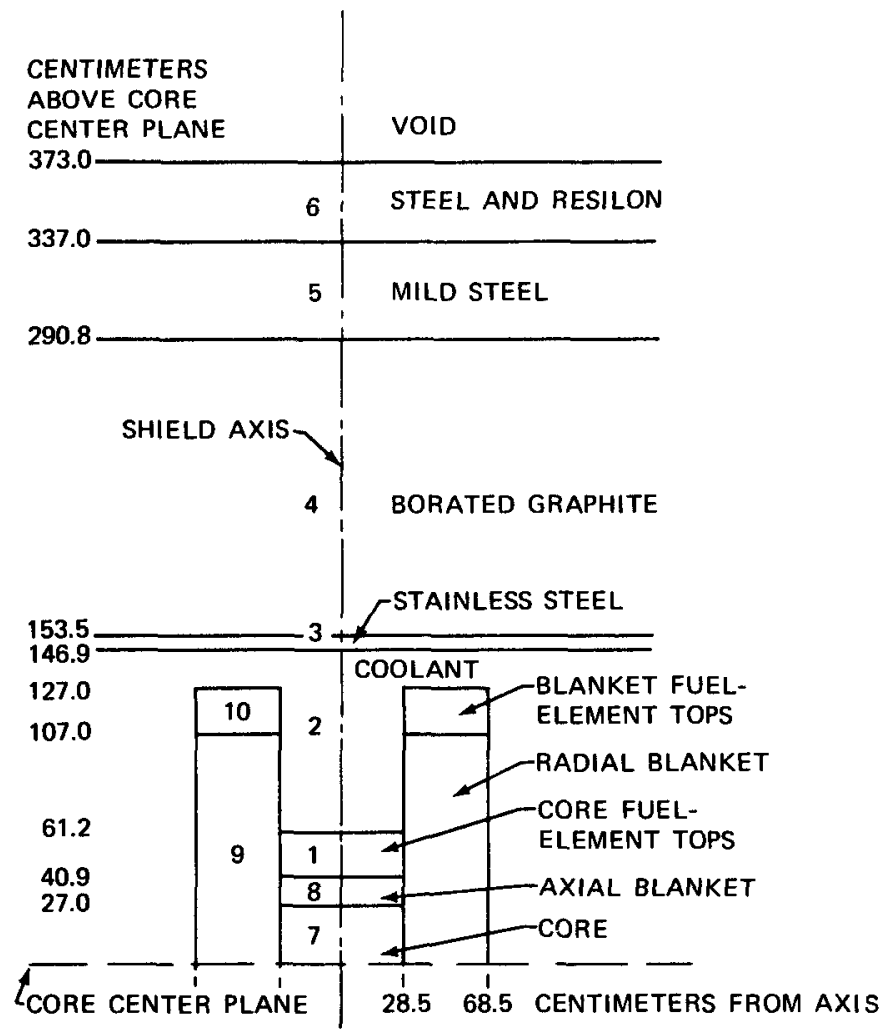

Fig. 10.20-Model of reactor and shield used for RASH D calculations. (From Adamson et $a .^{22}$ )

variations, including RASH E, are described in Sec. 4.9. At the time of this study (1964), the previous version, RASH D, was in use. The removal source is in effect the density of first collisions of neutrons born in fission. A discrete-ordinate calculation, $\mathrm{TDC}^{16}{ }^{6}$ was used to define the boundary condition flux densities at the core-shield interface. The shield comprised regions 1 through 6 in Fig. 10.20. The one-dimensional RASH D calculation was done in infinite-slab geometry. 


\subsubsection{Measurements}

An experimental shield plug was designed to fit within close tolerance into the core charge hole of the top shield (Fig. 10.19) so that foils and fission chambers could be mounted at appropriate positions in the shield plug and positioned in the core charge hole within the shield. Close-fitting blocks of shield material were assembled within the plug to match adjacent materials in the surrounding shield. Detectors are summarized in Table 10.4.

Table 10.4-DETAILS OF MEASURED REACTIONS $†$

\begin{tabular}{|c|c|c|}
\hline Reaction & $\begin{array}{l}\text { Energy of neutron } \\
\text { flux involved }\end{array}$ & $\begin{array}{c}\text { Means of } \\
\text { measurement }\end{array}$ \\
\hline${ }^{27} \mathrm{Al}(n, \alpha){ }^{24} \mathrm{Na}$ & $\begin{array}{l}\text { Fast flux, threshold } \\
\text { about } 9 \mathrm{MeV}\end{array}$ & $\begin{array}{l}\text { Activation of alu- } \\
\text { minum metal }\end{array}$ \\
\hline${ }^{32} \mathrm{~S}(n, p){ }^{32} \mathrm{P}$ & $\begin{array}{l}\text { Fast flux, threshold } \\
\text { about } 3 \mathrm{MeV}\end{array}$ & $\begin{array}{l}\text { Activation of ele- } \\
\text { mental sulfur }\end{array}$ \\
\hline${ }^{232} \mathrm{Th}(n, f)$ & $\begin{array}{l}\text { Fast flux, threshold } \\
\text { about } 1.5 \mathrm{MeV}\end{array}$ & $\begin{array}{l}\text { Thorium fission } \\
\text { counter }\end{array}$ \\
\hline${ }^{237} \mathrm{~Np}(n, f)$ & $\begin{array}{l}\text { Fast flux, threshold } \\
\text { about } 0.5 \mathrm{MeV}\end{array}$ & $\begin{array}{l}\text { Neptunium fission } \\
\text { counter }\end{array}$ \\
\hline${ }^{55} \mathrm{Mn}(n, \gamma){ }^{56} \mathrm{Mn}$ & $\begin{array}{l}\text { Thermal and epi- } \\
\text { thermal flux }\end{array}$ & $\begin{array}{l}\text { Activation of man- } \\
\text { ganese foils, un- } \\
\text { protected and in } \\
\text { cadmium capsules }\end{array}$ \\
\hline$\left.{ }^{197} \mathrm{Au}(n, \gamma)\right)^{198} \mathrm{Au}$ & $4.9 \mathrm{eV}$ & Gold sandwich foils \\
\hline
\end{tabular}

†From Adamson et al. ${ }^{22}$

Results for the ${ }^{32} \mathrm{~S}$ measurements are given in Fig. 10.21, and for the ${ }^{55} \mathrm{Mn}$, in Fig. 10.22. We have picked these two since the ${ }^{32} \mathrm{~S}$ results are typical of the fast-neutron fluxes and the ${ }^{55} \mathrm{Mn}$ of the thermal and epithermal fluxes. The ${ }^{32} \mathrm{~S}$ results (as well as ${ }^{27} \mathrm{Al}$, not shown) compare very well with the removal-diffusion approximation. Results of some earlier measurements reported by $S_{m i t h}{ }^{3}$ are also shown in Fig. 10.22, and they match the present data rather well.

Agreement between the low-energy measurements with the removaldiffusion calculations is poor, particularly in the upper regions of the shield and in air above the shield. This indicates an appreciable flux of neutrons above the top of the shield which have not diffused through the shield but which streamed through the various ducts formed by control penetrations and are reflected back from above. 


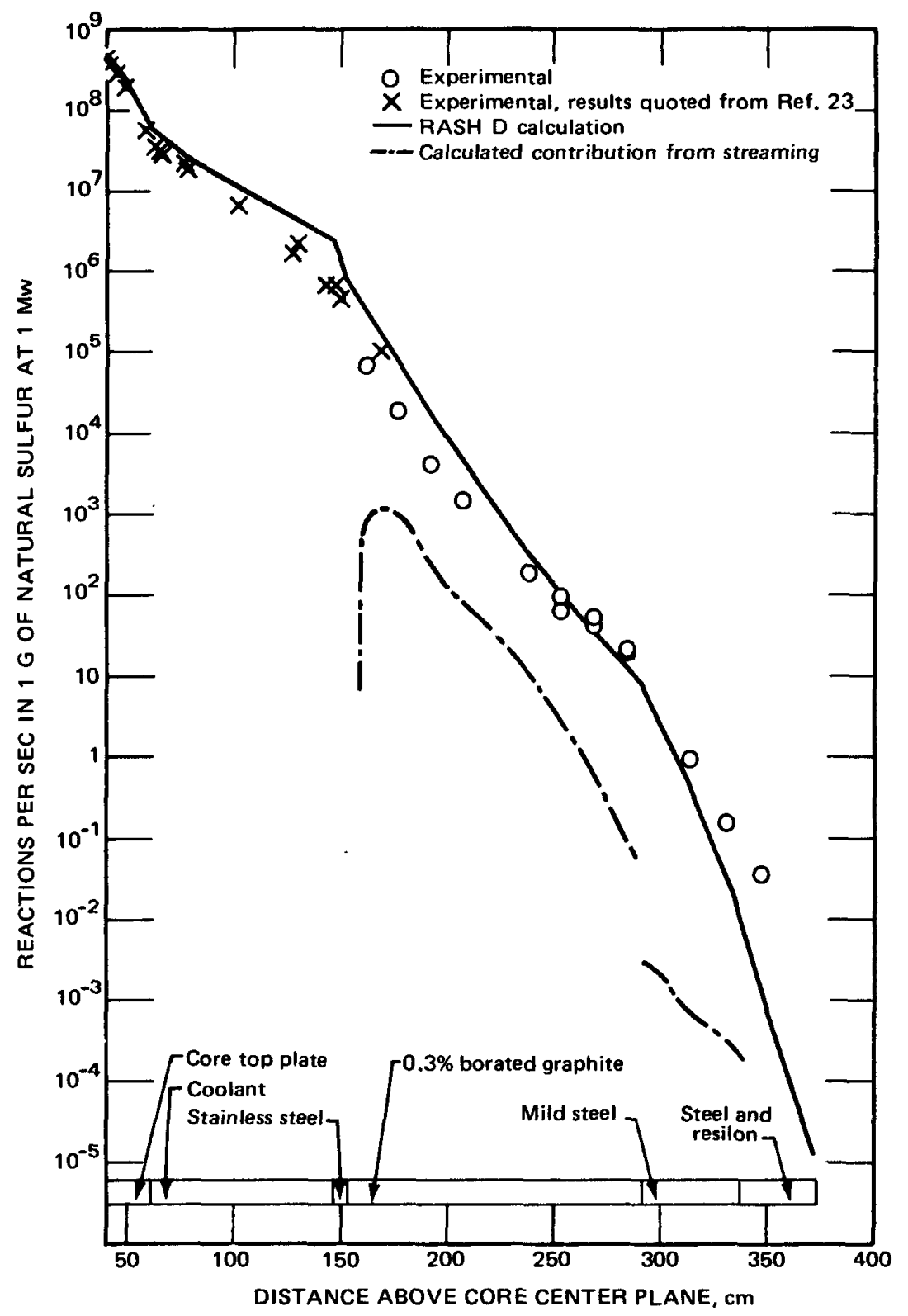

Fig. 10.21-Rate of ${ }^{32} \mathrm{~S}(n, p)^{32} \mathrm{P}$ reaction. (From Adamson et al. ${ }^{22}$ ) 


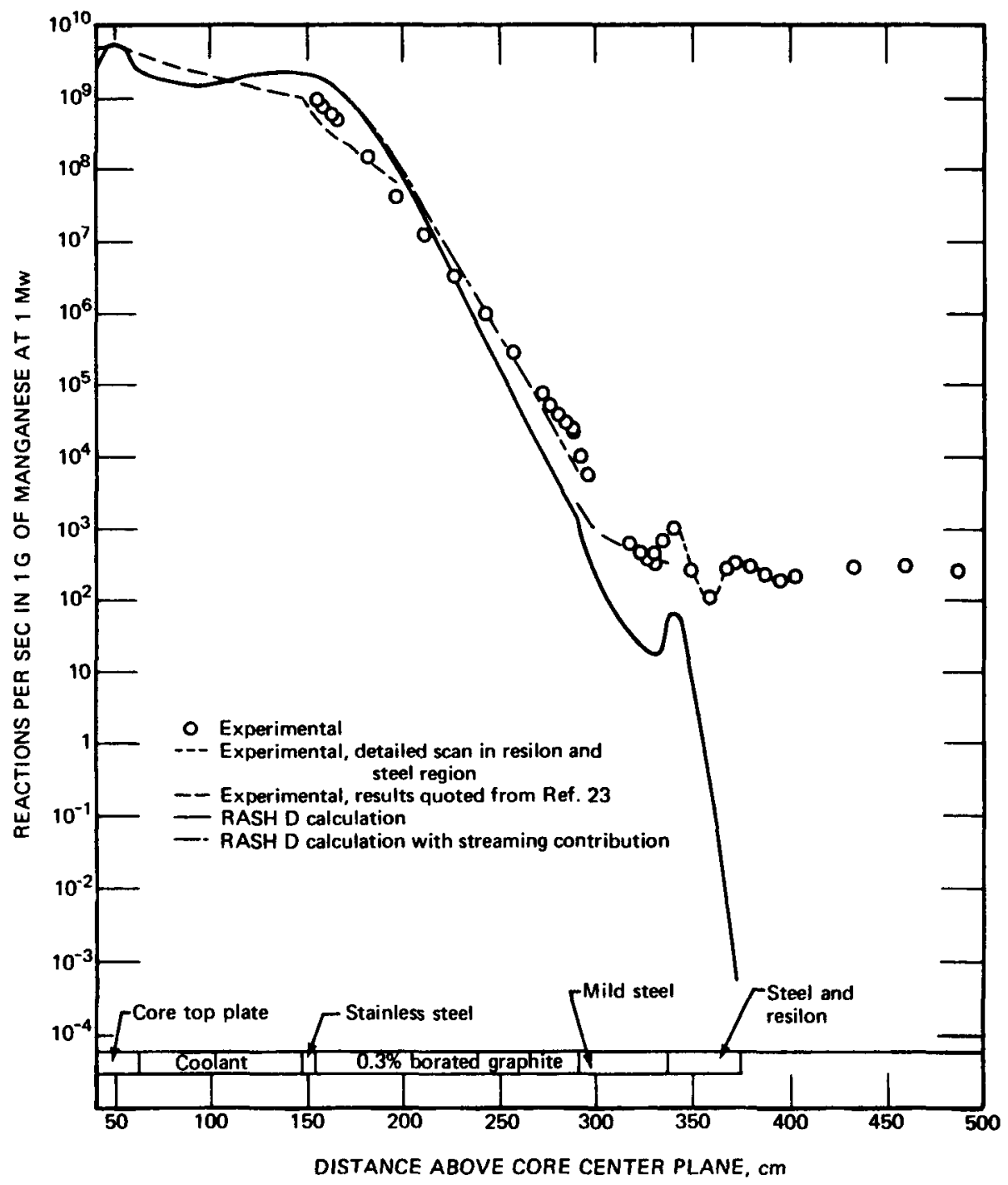

Fig. 10.22-Rate of ${ }^{55} \mathrm{Mn}(n, \gamma)^{56} \mathrm{Mn}$ reaction. (From Adamson et al. ${ }^{22}$ )

\subsubsection{Effect of Streaming}

Adamson et al. ${ }^{22}$ attempted to estimate the streaming through the 18 control ducts and the breeder charge hole (Fig. 10.19). Each duct was represented as a sink (negative source) at the end of the duct and a line of point sources along the duct axis. Age theory was used to obtain the distribution from each source in the surrounding medium. Since this is 
applicable only in infinite homogeneous media, it was not intended to give a reliable estimate at interfaces between different shield regions and at the top of the duct. Thus the streaming estimate cannot be expected to give the characteristic hump at the graphite-steel or steel-Resilon interfaces. Nevertheless, the streaming estimate when added to the RASH D bulk shield result does agree well with the ${ }^{55} \mathrm{Mn}$ measurements (and those for the gold resonance at $4.9 \mathrm{eV}$, not shown). In fact, Adamson et al. insist that the agreement for ${ }^{55} \mathrm{Mn}$ is fortuitous because of the imprecise nature of the streaming estimate. It does confirm, however, that streaming through the ducts vitiates the otherwise acceptable bulk shielding properties of the top shield. Had the attenuation of the top shield been of the same order as that of the streaming neutrons, it would have been no less effective and perhaps more economical.

\subsection{HEAVY WATER, NATURAL URANIUM: ÅGESTA}

The Agesta reactor is the first power-reactor prototype to be built in Sweden, and it has some extremely interesting features. It is also the first pressurized heavy-water natural-uranium reactor in the world. Most of the power produced is used for heating in a suburb of Stockholm. This reactor type is particularly important in countries not possessing uraniumenrichment facilities since it uses natural uranium. The demand for $\mathrm{D}_{2} \mathrm{O}$ is expected to grow rapidly because of the desirability of this system in many countries. Comparisons of measured and predicted radiation levels at Agesta are reported by Aalto, Sandlin, and Krell. ${ }^{24}$

\subsubsection{Description of Reactor and Shield}

The reactor with cooling system, service facilities, and auxiliary equipment is installed in a series of underground caverns excavated in a rock outcrop, as shown in Fig. 10.23. The rock medium provides a great deal of natural shielding and containment. The design philosophy that led to the layout shown was to provide for full access to as much of the plant as possible even during full power. This led to the compartmented arrangement where systems are separated into functional units isolated by shielding from nearby units and accessible by shielded passageways. In such an excavated plant, there is an obvious premium on space; thus magnetite concrete was used in the main shields to conserve space. 


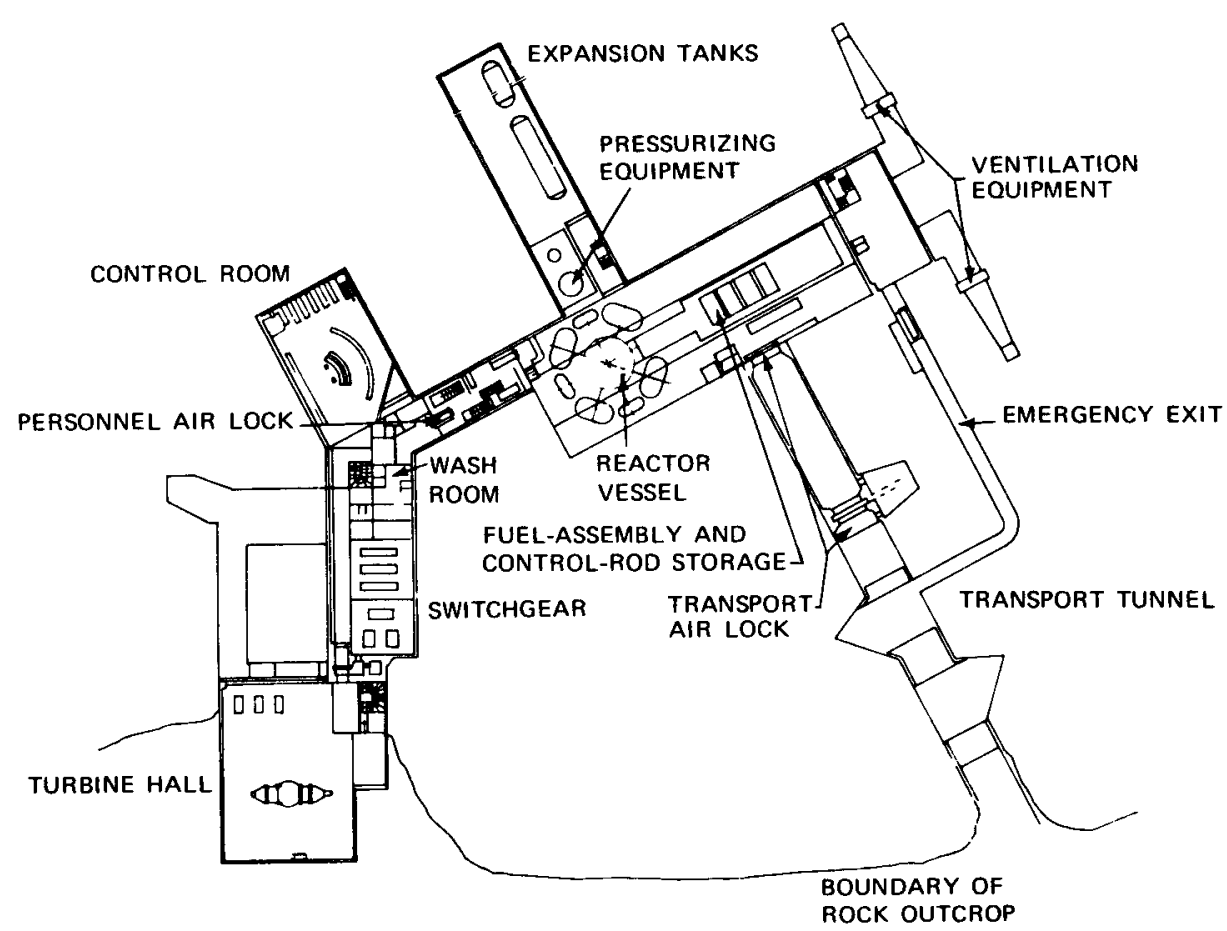

Fig. 10.23 - A gesta plant containment layout showing the relative positions of the major components in the reactor part of the plant. (From Aalto et al. ${ }^{24}$ )

A cross section of the reactor and the primary shields is shown in Fig. 10.24. The pressure vessel has an inside diameter of $4.55 \mathrm{~m}$ and a height of $4.68 \mathrm{~m}$. The core has a diameter of about $3.6 \mathrm{~m}$ and a height of $3.0 \mathrm{~m}$. The reflector layer of $\mathrm{D}_{2} \mathrm{O}$ surrounding the core is about $30 \mathrm{~cm}$ thick. Beyond the reflector are two steel thermal shields $(5$ and $10 \mathrm{~cm})$ separated by cooling spaces. The steel pressure vessel has a wall thickness of $7 \mathrm{~cm}$.

The radial shield consists of a 2.2-m layer of magnetite concrete.

\subsubsection{Calculational Model}

Aalto et al. ${ }^{24}$ mention three sets of calculations: a first set of simple hand calculations in 1958 with which the shield dimensions were set; a second set using the British RASH removal-diffusion method several years later; and a set in 1955 based on the NRN method of Hjärne and Leimdörfer. ${ }^{25}$ The latter is also a variation of the Spinney method and is described in Sec. 4.9.2. 


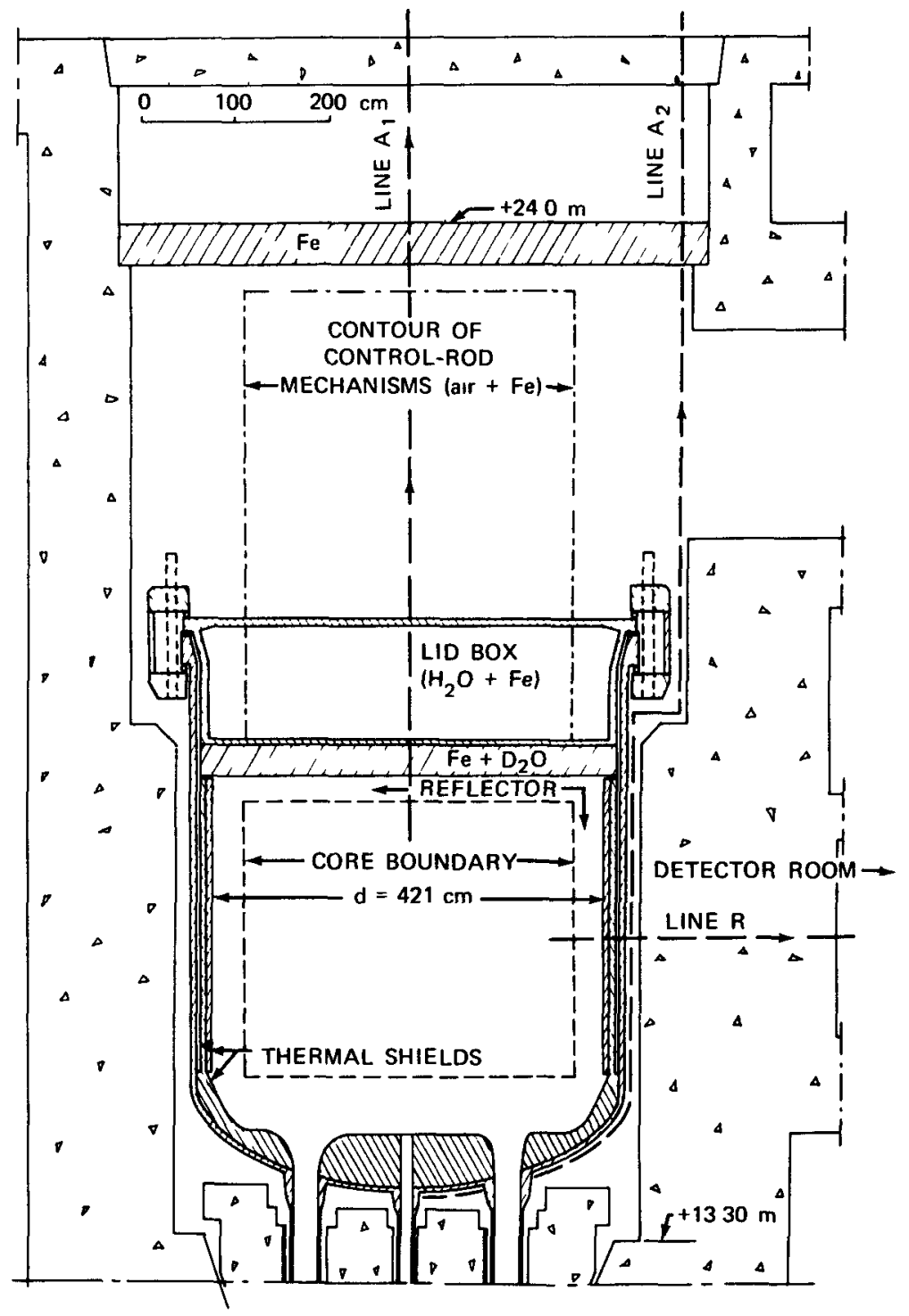

Fig. 10.24-A simplified vertical cross section of the Agesta reactor and the primary shields. (From Aalto et al. ${ }^{24}$ )

From the geometry of the shield shown in Fig. 10.24 and our recent review of the Dounreay analysis, it will become apparent that attenuation in the radial direction is governed entirely by massive unbroken layers, and the removal-diffusion method should be adequate. In the axial direction, however, the large annular duct surrounding the pressure vessel should be 


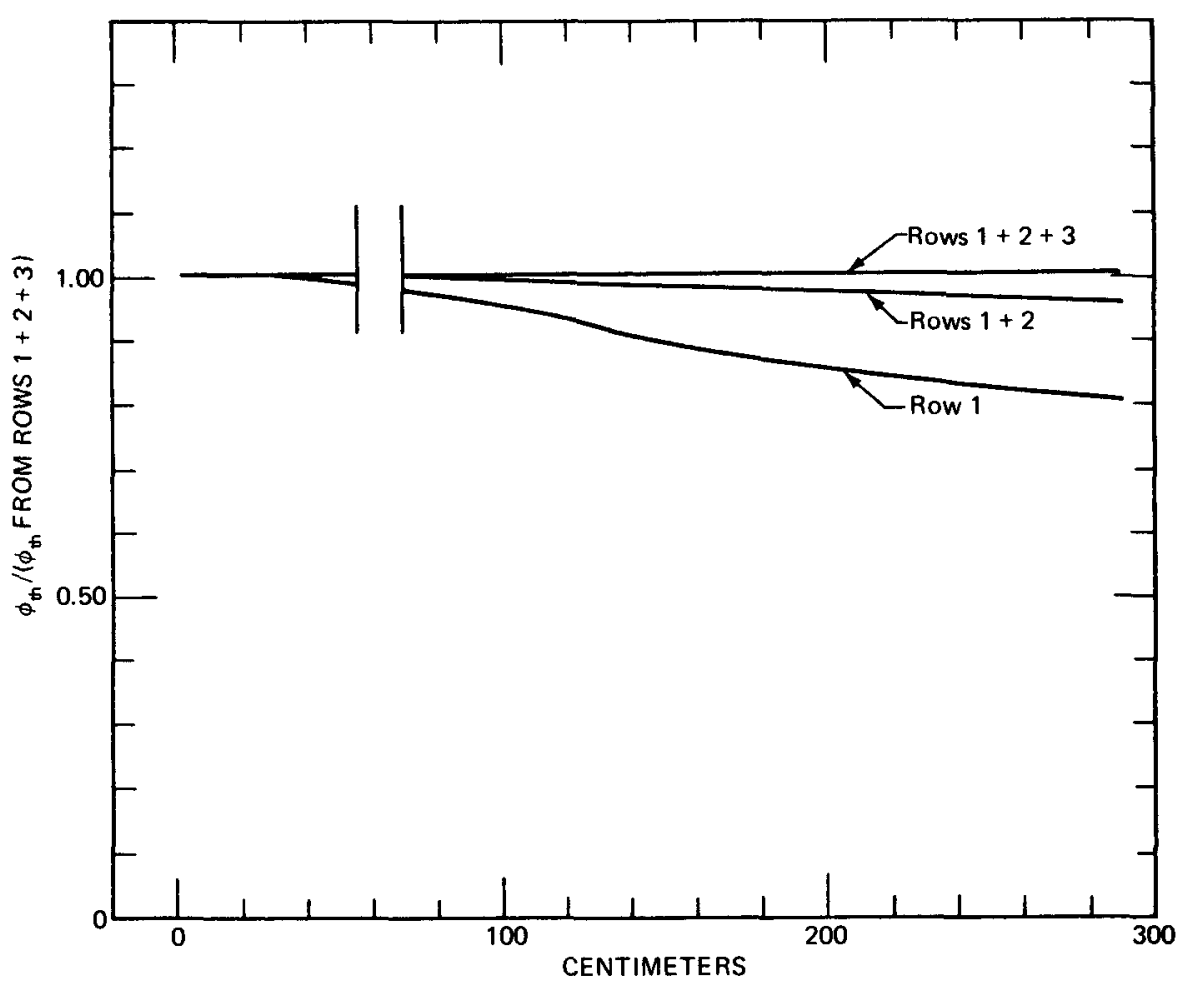

Fig. 10.25-Ratio of the radial thermal fluxes from individual fuel channels. (From Aalto et al. ${ }^{24}$ )

important in determining the radiation levels above the reactor in the region of the control-rod drives.

Radial NRN predictions were made for the first, second, and third rows of fuel elements nearest the shield to illustrate the diminishing influence of elements deeper in the core. These are shown for the thermal flux densities in Fig. 10.25 in terms of the ratio of the thermal flux density contributed by each row to the total at a distant point in the radial shield. The relative positions of the elements and their power contribution are shown in Fig. 10.26. The removal source from the third row of elements adds only $4 \%$ to the flux density and the second row contributes only $15 \%$, even though rows 3 and 2 account for about $5 x$ and $2 x$, respectively, the power in the first row. These results show clearly that the true power-density profile, particularly the density at the outermost regions of the core, is extremely important in obtaining an accurate calculation of the radiation penetrating 


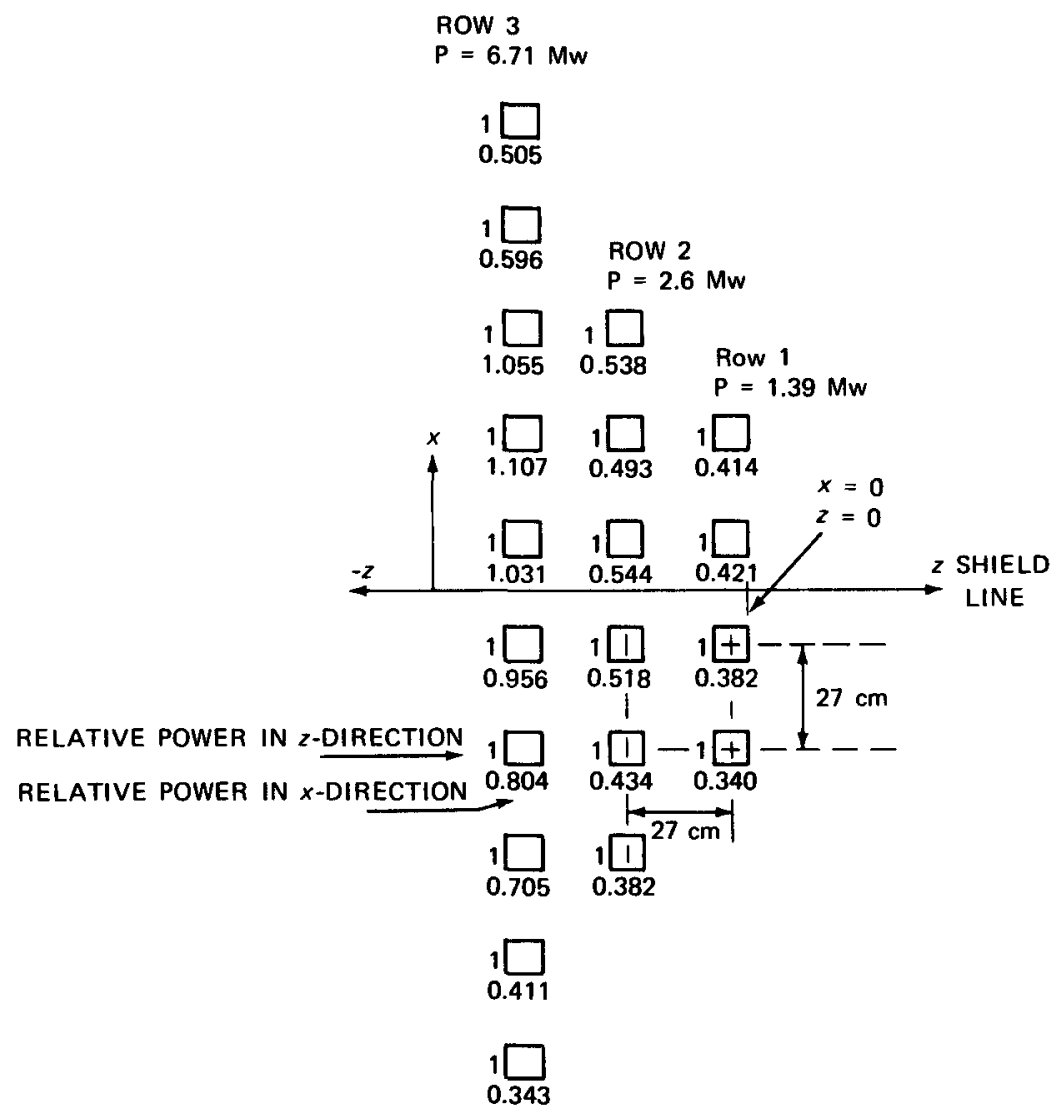

Fig. 10.26-Core configurations used when calculating the removal source in the radial NRN prediction. (From Aalto et al. ${ }^{24}$ )

the shield. This is well known as an important source of error in shielding calculations.

The actual power distribution in this case did deviate from the one on which the shield predictions were based, and it affected comparisons with attenuation measurements accordingly.

\subsubsection{Measurements}

This study was performed as a shield survey during commissioning of the plant. No special provisions had been made for locating detectors within the fixed shield; thus measurements were made only on available surfaces. This limitation accounts for the paucity of intermediate values in the radiation levels to be discussed. 
The range of magnitude of fluxes to be measured is worth noting: $10^{10}$. Since reactor power could be varied from $10 \mathrm{kw}$ to $50 \mathrm{Mw}$, a range of $5 \times 10^{3}$, the detector sensitivity range had to be sufficient to cover the remainder, about $10^{6}$. The limitation to available surfaces meant that the measurements were made mainly at the extremes of this range.

For high neutron-flux densities, thermal and epithermal measurements were made by double-foil (gold-copper) and cadmium-ratio (manganese) methods. Sulfur, with its 3-MeV threshold, was used for fast-neutron-flux measurements. The low fluxes outside the primary shields were measured with boron counters.

Gamma-ray measurements were made with glass dosimeters, ion chambers, and Geiger-Mueller detectors.

Calibration foils on the outer face of the tank at the core midline (along line R in Fig. 10.24) served as power-level monitors. They also served as an automatic correction for the variation of neutron attenuation in the radial direction because of varying moderator temperature. Neutrons degrade to the temperature of the moderator, and a change from $28^{\circ} \mathrm{C}$ to $220^{\circ} \mathrm{C}$ alters the observed "thermal" flux by a factor of 3 .

Power distribution varied $\pm 20 \%$ from the mean in the radial direction and was about $30 \%$ lower at the outer radius of the core than had been predicted. The axial distribution was asymmetrical; typically of reactors with top-mounted control rods, the maximum in the flux occurs well below the midplane of the core. The power level at the top of the core was $20 \%$ lower than predicted. These deviations translate directly to the measurements.

One additional source of error is the detailed structure near the detector. In this instance some thermal insulation and steel conduit used to support the detector were not included in the predictions, but they may have affected the thermal flux measurement by a factor of 2 . The predicted radial thermal flux densities are shown in Fig. 10.27. Only one measurement is shown (at the edge of the tank). The intensity beyond the concrete was below the minimum observable. The epithermal fluxes are similar and are not shown.

The axial variation of the thermal flux density is shown in Fig. 10.28 tor two vertical lines: $A_{1}$ and $A_{2}$ in Fig. 10.24. The calculations (solid and dashed curves) apply to $A_{1}$ and do not include an estimate of the streaming around the sides of the tank; this is shown separately, and obviously it controls the intensities above the tank in the same manner as was shown for Dounreay. The dotted lines in Fig. 10.28 connect the measurements. 


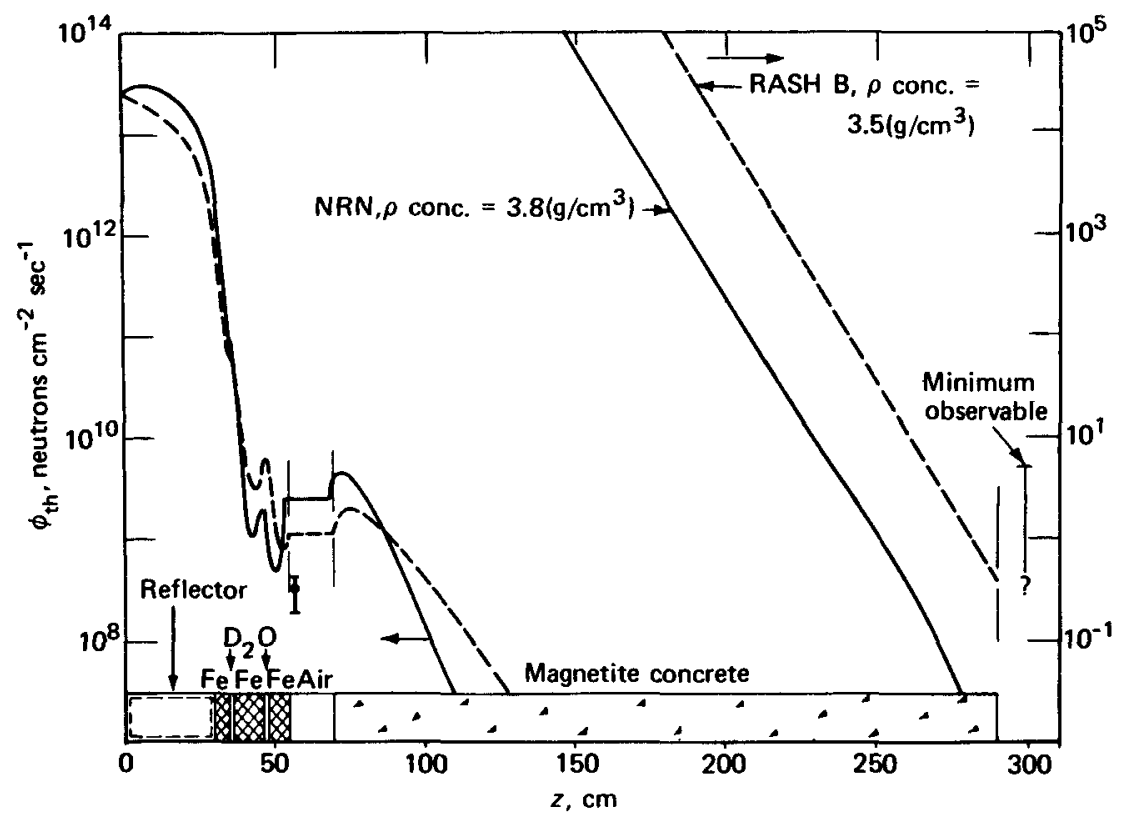

Fig. 10.27-Predicted and observed radial thermal fluxes (line R, Fig. 10.24). (From Aalto et al. ${ }^{24}$ )

Aalto et al. ${ }^{24}$ do not describe their method for calculating the streaming (which agrees very well with the observed intensities). However, their mention of multiple reflections would indicate an albedo calculation possibly coupled with Monte Carlo (see Sec. 7.10.2).

Gamma-ray dose rates are summarized in Table 10.5, and sulfur fast-neutron reaction rates (proportional to flux densities), in Table 10.6. The gamma-ray dose rate outside the pressure vessel is two to three times the predicted value, probably because of activation of the nearby steel from previous high-power runs. Since the shield design is very conservative, the only critical neutron intensities are those in the vicinity of the control-rod drives because these levels control servicing procedures. However, the original streaming estimate was in excellent agreement with the measurements; thus no additional problems developed.

Aalto et al. ${ }^{24}$ conclude that, although local flux predictions often differ from observations by a factor of 2 , these are easily accounted by the error sources mentioned. They also point out that the romplexities of a power 


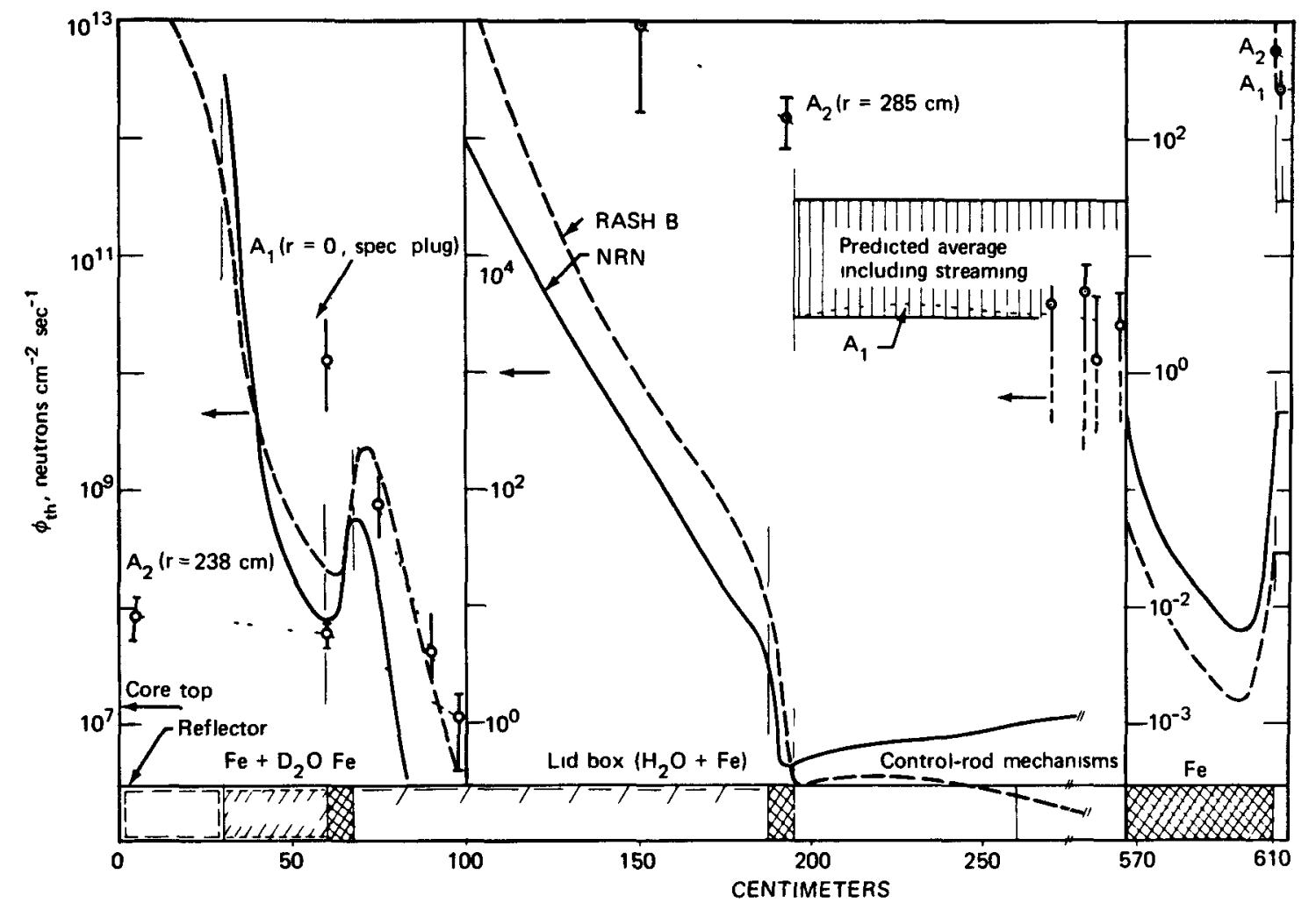

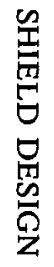

Fig. 10.28 - Predicted and observed axial thermal fluxes (lines $A_{1}$ and $A_{2}$, Fig. 1024 ). (From Aalto et al. ${ }^{24}$ ) 
Table 10.5-ÅGESTA GAMMA-RAY DOSE RATES $\dagger$

\begin{tabular}{|c|c|c|c|}
\hline $\begin{array}{l}\text { Measuring line, def- } \\
\text { inition of point }\end{array}$ & $\begin{array}{c}\text { Coordinate } \\
\mathrm{cm}\end{array}$ & $\begin{array}{l}\text { Predicted, } \\
\text { mrem } / \mathrm{hr}\end{array}$ & $\begin{array}{c}\text { Measured, } \\
\mathrm{mrem} / \mathrm{hr}\end{array}$ \\
\hline \multicolumn{4}{|l|}{ Axial, upward $\left(A_{1}\right)$} \\
\hline $\begin{array}{l}\text { Control-rod mech- } \\
\text { anisms }\end{array}$ & 200 to 570 & $\left(\sim 10^{3}\right)+$ & $7.5 \times 10^{4}$ \\
\hline $\begin{array}{l}\text { Below the concrete } \\
\text { lid }\end{array}$ & 610 to 760 & $(\sim 10)$ & 1.5 to 3.0 \\
\hline Hall floor & 810 & $\leqslant 0.7$ & 0.2 \\
\hline \multicolumn{4}{|l|}{ Radial $(R)$} \\
\hline $\begin{array}{l}\text { Outside pressure } \\
\text { vessel }\end{array}$ & 54 & $3 \times 10^{7}$ & $8 \times 10^{7}$ \\
\hline $\begin{array}{l}\text { Outside biological } \\
\text { shield }\end{array}$ & 290 & 0.04 & $<0.1$ \\
\hline Axial, downward & $\begin{array}{l}\text { Level }+7.1 \\
\text { center line }\end{array}$ & & $1.1 \times 10^{4}$ \\
\hline Not shown in figures & $\begin{array}{c}2 \mathrm{~m} \text { above } \\
\text { plane }+7.1\end{array}$ & & $2.3 \times 10^{4}$ \\
\hline
\end{tabular}

$\dagger$ From Aalto et al. ${ }^{24}$

$\$$ Not comparable to the measured value because in the latter streaming effects dominate.

plant coupled with the limitations on measurements make such a survey an inferior means for studying the accuracy of shielding prediction methods. In such a conservatively designed plant, only a gross design error resulting in excessively high fluxes would draw any attention. Thus the Ågesta survey was a successful design verification that confirmed the original analysis in a highly satisfactory way.

\subsection{BOILING WATER: PATHFINDER}

Designed as a demonstration boiling-water-reactor plant, the Pathfinder plant was built near Sioux Falls. S. D., to supply $66 \mathrm{Mw}(\mathrm{e})$ to the area power network. Start-up was in March 1964, and the reactor was shut down in September 1967 for repair of a condenser-tube break. Bjeldanes ${ }^{26}$ reports that subsequent inspections in the reactor vessel disclosed failures in the steam separators and a baffle in the fuel hold-down structure. After various considerations of required repairs, plant size, and operating costs, the reactor was decommissioned, and the plant was later converted to a fossile-fuel system. 
Table 10.6-

\begin{tabular}{|c|c|c|c|}
\hline \multirow[b]{2}{*}{$\begin{array}{l}\text { Measuring line, def- } \\
\text { inition of point }\end{array}$} & \multirow[b]{2}{*}{$\begin{array}{l}\text { Coordinate, } \\
\mathbf{c m}\end{array}$} & \multicolumn{2}{|c|}{$\begin{array}{c}S(n, p) \\
\operatorname{dis}^{-1} \sec ^{-1}\end{array}$} \\
\hline & & $\begin{array}{c}\text { Predicted by } \\
\text { NRN }\end{array}$ & Measured \\
\hline $\begin{array}{l}\text { Axial, upward } \\
\left(A_{1} \text { and } A_{2}\right) \\
\text { Bottom of the lid } \\
\text { box } \\
\text { Below the concrete } \\
\text { lid: }\end{array}$ & 60 & $1.45 \times 10^{6}$ & $(1.4 \pm 1.3) \times 10^{6}$ \\
\hline $\begin{array}{c}A_{1} \\
A_{2} \\
\text { Radial }(R)\end{array}$ & $\begin{array}{l}610 \text { to } 760 \\
610 \text { to } 760\end{array}$ & $\begin{array}{l}\left(1 \times 10^{-5}\right) \ddagger \\
\left(1 \times 10^{-5}\right) \ddagger\end{array}$ & $\begin{array}{l}5 \times 10^{-2} \\
(5 \text { to } 13) \times 10^{-2}\end{array}$ \\
\hline $\begin{array}{l}\text { Thermal shield } \\
\text { Outside the pressure } \\
\text { vessel }\end{array}$ & $\begin{array}{l}44 \\
54\end{array}$ & $\begin{array}{l}2.85 \times 10^{6} \\
7.27 \times 10^{5}\end{array}$ & $\begin{array}{l}(3.1 \pm 1.3) \times 10^{6} \\
(1.95 \pm 0.10) \times 10^{5}\end{array}$ \\
\hline $\begin{array}{l}\text { Outside the biologi- } \\
\text { cal shield } \\
\text { Axial, downward } \\
\text { Below pressure ves- } \\
\text { sel, center line }\end{array}$ & 290 & $3.08 \times 10^{-5}$ & $(3.1 \pm 0.2) \times 10^{2}$ \\
\hline
\end{tabular}

$\dagger$ From Aalto et al. ${ }^{24}$ Fast-neutron fluxes are given as $S(n, p)$ reaction rates. To obtain $\phi>1 \mathrm{MeV}$ for fission spectrum, multiply by $\left(9 \times 10^{2}\right)$.

$\$$ Not comparable to the measured value in which streaming effects dominate.

The following description of the design is taken from a shield-analysis report by Holl and Stephen. ${ }^{27}$ The plant consists of four connected buildings: the reactor building, the turbine and control building, the fuel-handling and waste-disposal building, and the water-treatment building. A plan view of the operating floor is shown in Fig. 10.29. This plant layout evidently resulted from many iterations and reflects a good deal of planning for management of radiation problems. Each building may be characterized by its principal radiation source. The water-treatment building was to contain unused water and have no radiation sources. The reactor and fuel-handling buildings are controlled by active and spent fuel; however, the fuel-handling building is also affected by activated corrosion products (called crud by the authors) in the used elements and equipment. Radiation levels in the turbine building were to be controlled by gaseous activation products from $(n, p)$ reactions in oxygen of the water; levels were underpredicted here, 
and this created the only unforeseen radiation problem in the plant. We will return to this problem in the discussion of the survey measurements.

Figure 10.30 is an elevation of the plant showing the containment shell of the reactor building with the adjacent structures. The core is located below grade to take advantage of earth fill as shielding. The primary

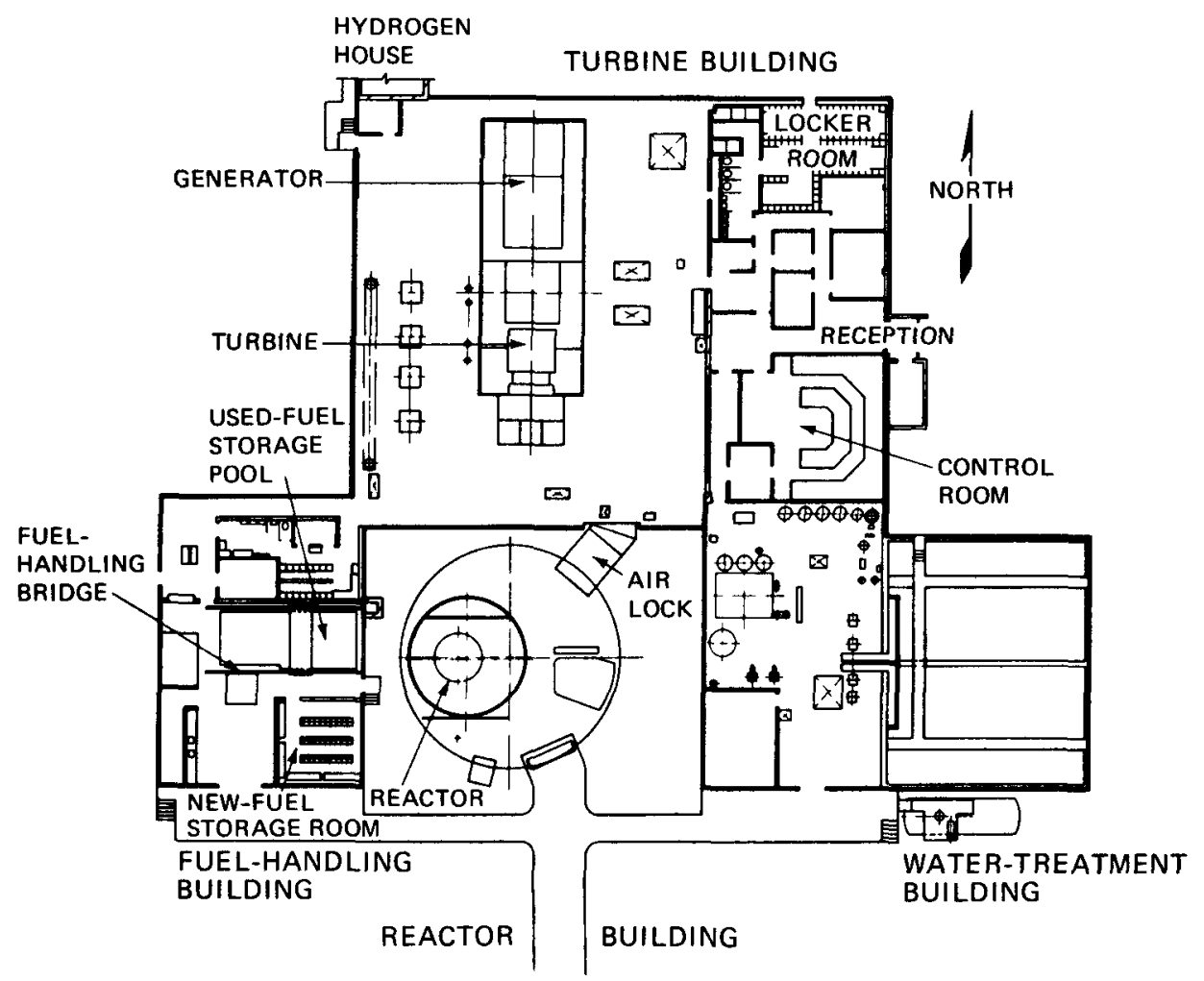

Fig. 10.29_-Pathfinder operating floor. (From Holl and Stephen. ${ }^{27}$ )

biological shield is ordinary concrete about $3 \mathrm{~m}$ thick. A clearance space of about $1 \mathrm{ft}$ between the reactor vessel and the shield provides a water-cooling space. Penetrations for reactor controls and instruments are shadowed by 91-cm-thick concrete plugs. Above the reactor a $5.5-\mathrm{m}$ pool of water attenuates radiation to the operating floor.

The turbine is recessed into the operating floor. Original plans called for restricting access (limiting working times) near the turbine. The control room and administrative offices are adjacent to the turbine room. 


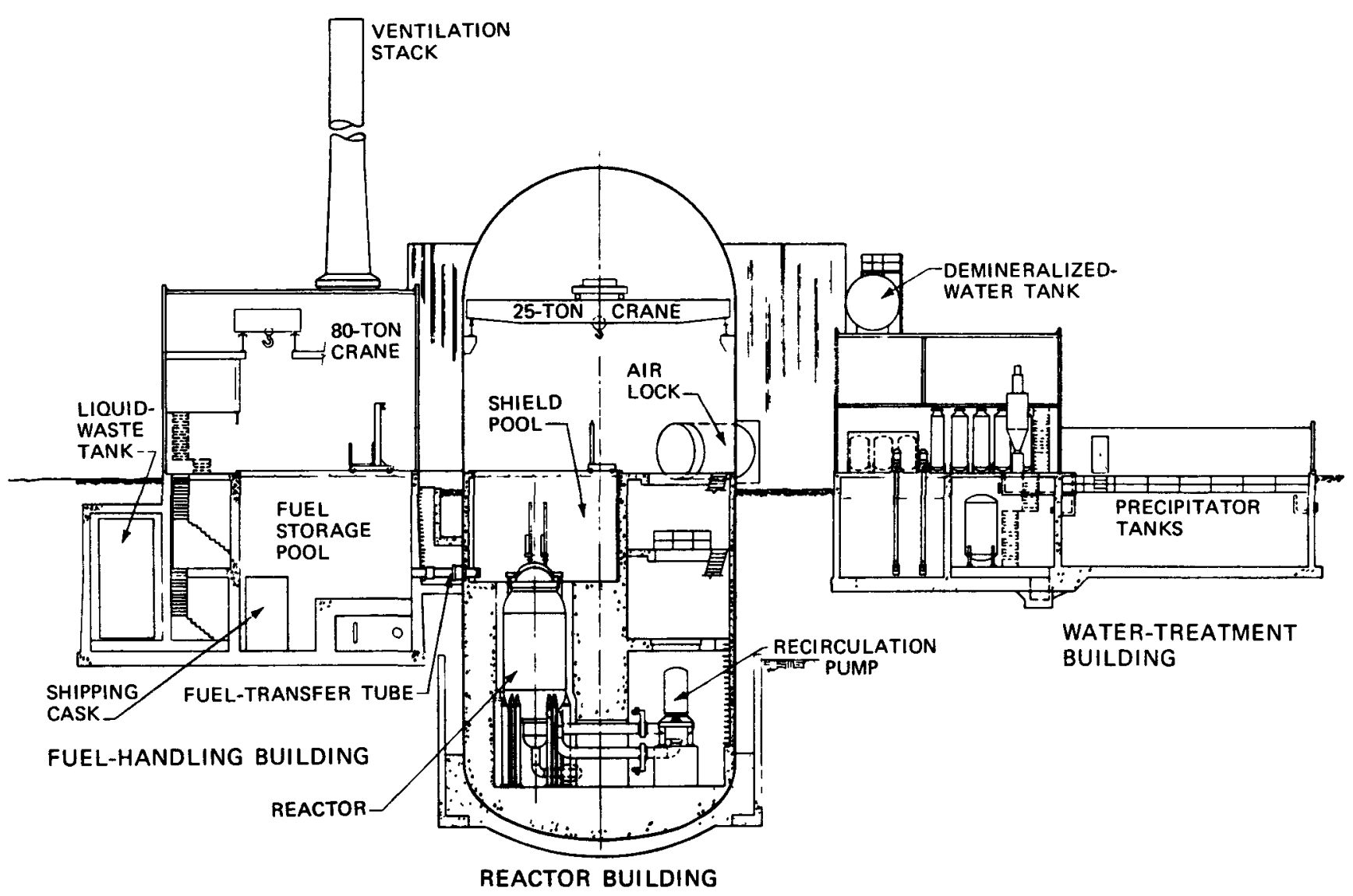

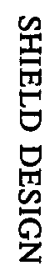

Fig. 10.30-Pathfinder south elevation. (From Holl and Stephen. ${ }^{27}$ ) 


\subsubsection{Calculations}

As with other systems described, the initial shield analysis for Pathfinder was done by hand from attenuation curves. The detailed calculations were done with fast-neutron removal theory and gamma-ray linear-absorption buildup factors. A point-kernel machine program was used that could adequately handle the large number of regions, materials, and compositions encountered in the design. Duct scattering was done with the SimonClifford equations (Sec. 7.10.2).

The list of radiation sources that must be considered in a boiling-water system is impressive. Gamma-ray sources in the core include those from prompt fission; delayed fission; capture $\left({ }^{235} \mathrm{U},{ }^{238} \mathrm{U}\right.$, zirconium in fuel-element cladding, and hydrogen); and inelastic scattering. Decay gamma rays from the shutdown core must be considered as a function of core history and time after shutdown for maintenance planning.

Gamma rays from other materials include captures in iron and various activation products from the crud $\left({ }^{16} \mathrm{~N},{ }^{17} \mathrm{~N}\right.$, and $\left.{ }^{19} \mathrm{O}\right)$, and potential activities from ${ }^{17} \mathrm{~N}$ neutrons.

The crud activity is a result of the high water velocity, which erodes activated species from the core and carries them throughout the system. The activity consists of ${ }^{59} \mathrm{Fe},{ }^{60} \mathrm{Co},{ }^{56} \mathrm{Mn},{ }^{51} \mathrm{Cr},{ }^{95} \mathrm{Zr}$, and ${ }^{65} \mathrm{Ni}$. Various assumptions were made for the buildup and plate-out of the crud in calculating the total gross activity.

Two $(n, p)$ reactions and related captures deserve further mention: ${ }^{16} \mathrm{O}(n, p){ }^{16} \mathrm{~N}$ with a 7.3 -sec half-life,

$$
{ }^{16} \mathrm{~N} \rightarrow{ }^{16} \mathrm{O}+\beta+\gamma(6 \mathrm{MeV})
$$

$80 \%$ include the gamma ray. The other reaction sequence, ${ }^{17} \mathrm{O}(n, p)^{17} \mathrm{~N}$, has an $8-\mathrm{MeV}$ threshold and a very low cross section $(7 \mu \mathrm{b})$, but

$$
{ }^{17} \mathrm{~N} \rightarrow \beta+{ }^{17} \mathrm{O} \rightarrow{ }^{16} \mathrm{O}+n
$$

with a 4-sec half-life; the beta has an energy of $3.7 \mathrm{MeV}$, and the neutron, 1 $\mathrm{MeV}$. The neutron can be troublesome since the nitrogen is carried by the water and steam to all parts of the system.

There is also a capture reaction in ${ }^{18} \mathrm{O}$, producing ${ }^{19} \mathrm{O}$, which has a 29-sec half-life,

$$
{ }^{18} \mathrm{O}+n \rightarrow{ }^{19} \mathrm{O} \rightarrow{ }^{19} \mathrm{~F}+\beta+\gamma(1.4 \mathrm{MeV})
$$


Calculated concentrations of ${ }^{16} \mathrm{~N},{ }^{17} \mathrm{~N}$, and ${ }^{19} \mathrm{O}$ in the water and steam loops at full power were $7.4 \times 10^{9}, 1 \times 10^{7}$, and $4.3 \times 10^{8}$ atoms per pound of water or steam, respectively. Thus the dominant activity is ${ }^{16} \mathrm{~N}$.

Predictions were made for a detailed schedule of dose points and multiplied by a factor of 3 to include a safety margin.

\subsubsection{Survey Measurements}

Lewellen, Lacy, and $\mathrm{Raby}^{28}$ report the results of radiation surveys made at $3,20,40,60,80$, and $90 \%$ of full power. The large number of intermediate power levels was apparently run because some of the radiation levels were not expected to increase linearly with reactor power. Standard portable survey meters were used: a beta-gamma Gieger-tube probe and a $\mathrm{BF}_{3}$ neutron detector.

Results of the radiation survey can be summarized as follows: Although many of the survey measurements differed somewhat from the calculations, no discrepancies related to radiation directly from the core. Normal overdesign (a factor of about 3 in intensity) of the primary shield prevented any radiation problems in the reactor building. The only serious discrepancy occurred in the turbine building, where intensities varied from 3 to 10 times the predicted levels near the turbine itself. Accordingly, a concrete wall $2.4 \mathrm{~m}$ high by $9.1 \mathrm{~m}$ long was erected on the operating floor between the turbine and the administrative section of the building. The addition is shown in Fig. 10.31; the added attenuation in the direction of the administrative side ranged from 2.5 to 5 , which reduced the radiation to desired levels.

\subsection{SHIP PROPULSION: N.S. SAVANNAH}

Important as safety is for fixed plants, it is doubly so for propulsion systems. A fixed-plant location is chosen for remoteness from high population density; a ship must berth at any deep-water harbor, frequently in a city's high-density area.

The reactor plant of the N.S. Savannah was selected as an example of the pressurized-water type because safety considerations, weight, and space limitations on the shield presented a more difficult design problem than land-based power plants. In addition, a detailed set of measurements was made on the finished design under operating conditions. The following discussion of the reactor plant is mostly taken from the Smith and Turner shield design summary reports ${ }^{2 \cdot 30}$ and the shield test report by Blizard, 


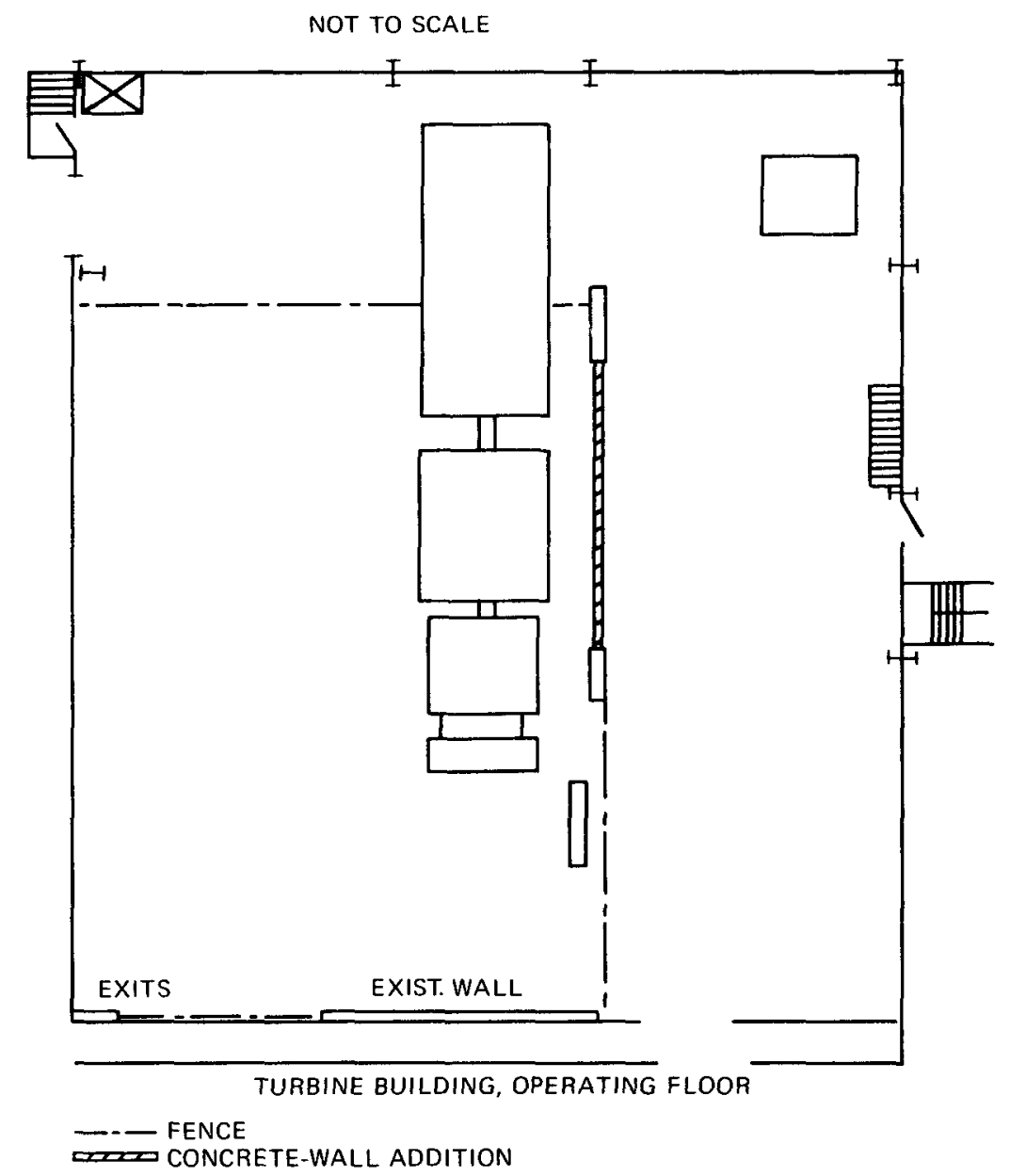

Fig. 10.31-Shield wall and fence additions. (From Lewellen et al. ${ }^{28}$ )

Blosser, and Freestone. ${ }^{31}$ Bear in mind that the shield design dates from the mid-fifties.

\subsubsection{Description of Ship, Reactor, and Main Shielding}

The N.S. Savannah is a single-screw, combination passenger-cargo ship with an overall length of $181 \mathrm{~m}$. She has a molded beam of $24 \mathrm{~m}$ and draws, when fully loaded, $9 \mathrm{~m}$, displacing 20,000 tons. The vessel has three complete decks, A, B, and C (from top down), with platform decks at various levels. A promenade deck extends over A deck for about one-third of the ship's length; the forward end being slightly forward of amidships. The 
promenade deck is devoted exclusively to public rooms and spaces; all passenger staterooms are on A deck. The passenger dining room and some of the crew spaces are on B deck. The remainder of the crew spaces are on $\mathrm{C}$ deck. Below on the smaller D deck various machinery and equipment are located.

The Savannah reactor is the pressurized-water type, fueled with slightly enriched $\left(4.4 \%{ }^{235} \mathrm{U}\right) \mathrm{UO}_{2}$. The fuel is in the form of 1.08 -cm-diameter pellets, clad in $0.089-\mathrm{cm}$-thick stainless steel, contained in bundles of stainless-steel tubes. The core is $229 \mathrm{~cm}$ long overall, with an active fuel length of $168 \mathrm{~cm}$ and an equivalent core diameter of $158 \mathrm{~cm}$. Typically, an initial loading contains $312.4 \mathrm{~kg}$ of ${ }^{235} \mathrm{U}$ and $6787.5 \mathrm{~kg}$ of ${ }^{238} \mathrm{U}$. The predicted lifetime of such a core is $52,200 \mathrm{Mwd}(>750$ days at full power).

The primary shield for the Savannah reactor consists of an annulus of light water, $84 \mathrm{~cm}$ thick, contained in the primary shield tank surrounding the reactor. The water annulus is supplemented by lead in thicknesses from 2.5 to $10 \mathrm{~cm}$ at the primary-shield-tank outer wall. Outside the primary shield tank are boilers, pumps, demineralizers, and other parts of the steam-production system. The core, pressure vessel, primary shield, and steam-production system are enclosed in the containment vessel, a $10.7-\mathrm{m}$ diameter 6.3-cm-thick steel cylinder with hemispherical ends. The total length of the containment vessel is $15.4 \mathrm{~m}$. Figure 10.32 shows a model of the containment vessel and the relative elevations of the various decks.

The secondary shield is outside the containment vessel. Above the midplane of the containment vessel, the secondary shield consists of a layer of lead, varying between 12.7 and $15.2 \mathrm{~cm}$ in thickness, and a minimum of $20 \mathrm{~cm}$ of polyethylene. Below the midplane of the containment vessel, the shield is primarily concrete in thicknesses ranging from 84 to $122 \mathrm{~cm}$. Fore and aft, on the hemispherical ends, ilmenite concrete is used; ordinary concrete is used at the sides. Collision mats consisting of alternate layers of redwood and steel replace some of the concrete on the sides.

Water-storage tanks, approximately $1.5 \mathrm{~m}$ deep, extend the secondary shield below the containment vessel from the tank tops to the hull. A schematic diagram of the shield is shown in Fig. 10.33.

\subsubsection{Shielding for Refueling and Control-Rod Maintenance}

Special provisions for refueling and servicing are worth noting. Before entrance can be gained to the reactor after shutdown for refueling and control-rod maintenance, the insulation covering, pressure-vessel head, control-rod assembly, flow-baffle assembly and upper grid assembly must be removed. The pressure-vessel head is secured by studs and a seal weld. 


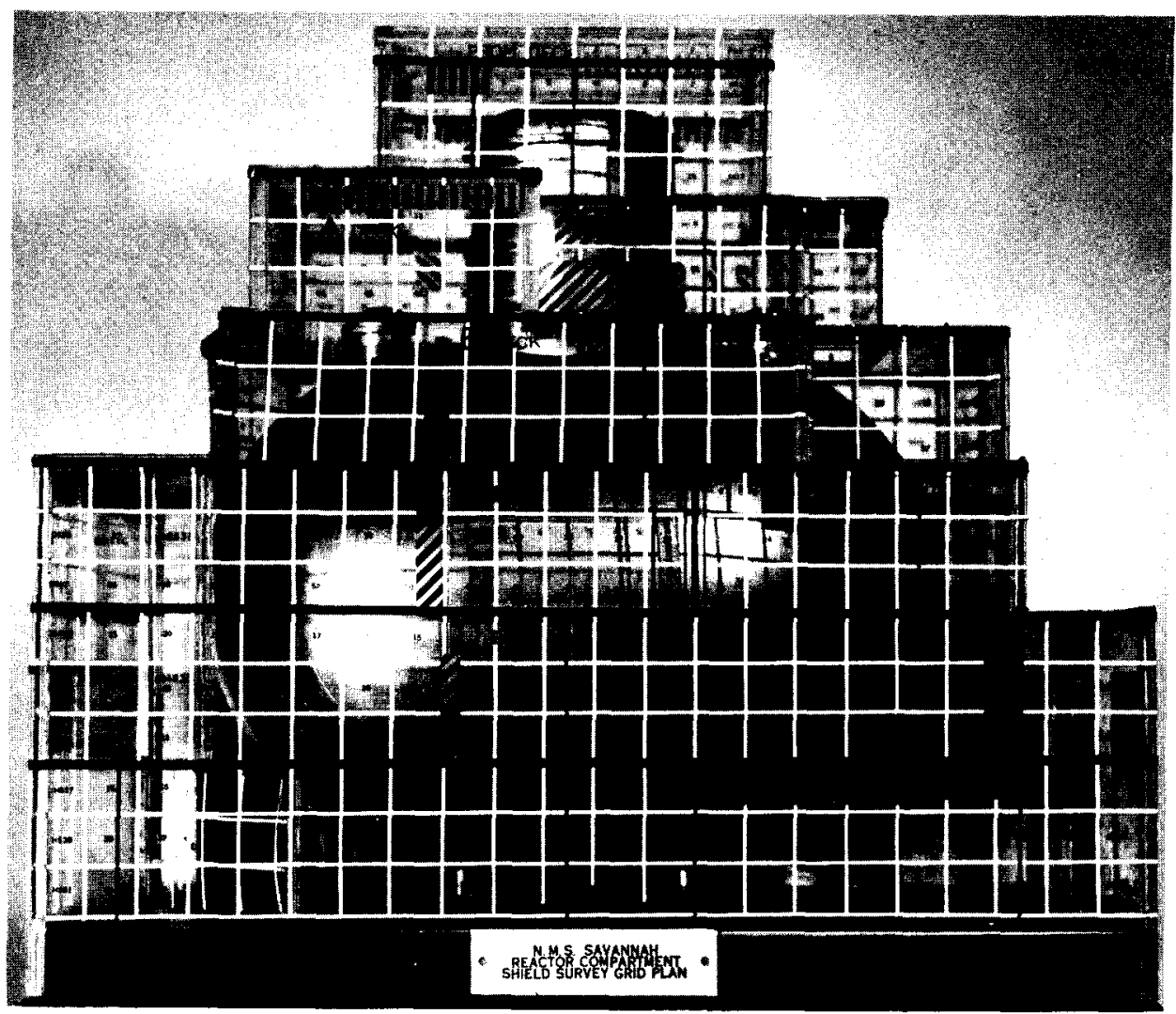

Fig. 10.32 - The New York Shipbuilding Corporation's model of the shield of the N.S. Savannah. (From Blizard et al. ${ }^{31}$ )

The stud-removal operation takes place with the reactor water level up in the head. The water level is lowered before the seal-weld cutting operation begins. Additional water in the upper extension of the shield tank reduces dose rates from core fission products and activated internals to 1 to 2 $\mathrm{mR} / \mathrm{hr}$. Distance attenuation from primary-loop corrosion and fissionproduct activity further reduces these dose-rate contributions.

Before the dismantled pressure-vessel head is removed, a permanent tank above the pressure-vessel flange is filled with transitory shield water to limit dose rates from the reactor internals and spent core. (These are exposed when the head is removed.) The head is then removed, and a telescopic internals cask is lowered onto the pressure-vessel flange. The upper flow-baffle assembly is then withdrawn into the extended cask. Figure 10.34 is a schematic view of the internals cask in place showing the upper 


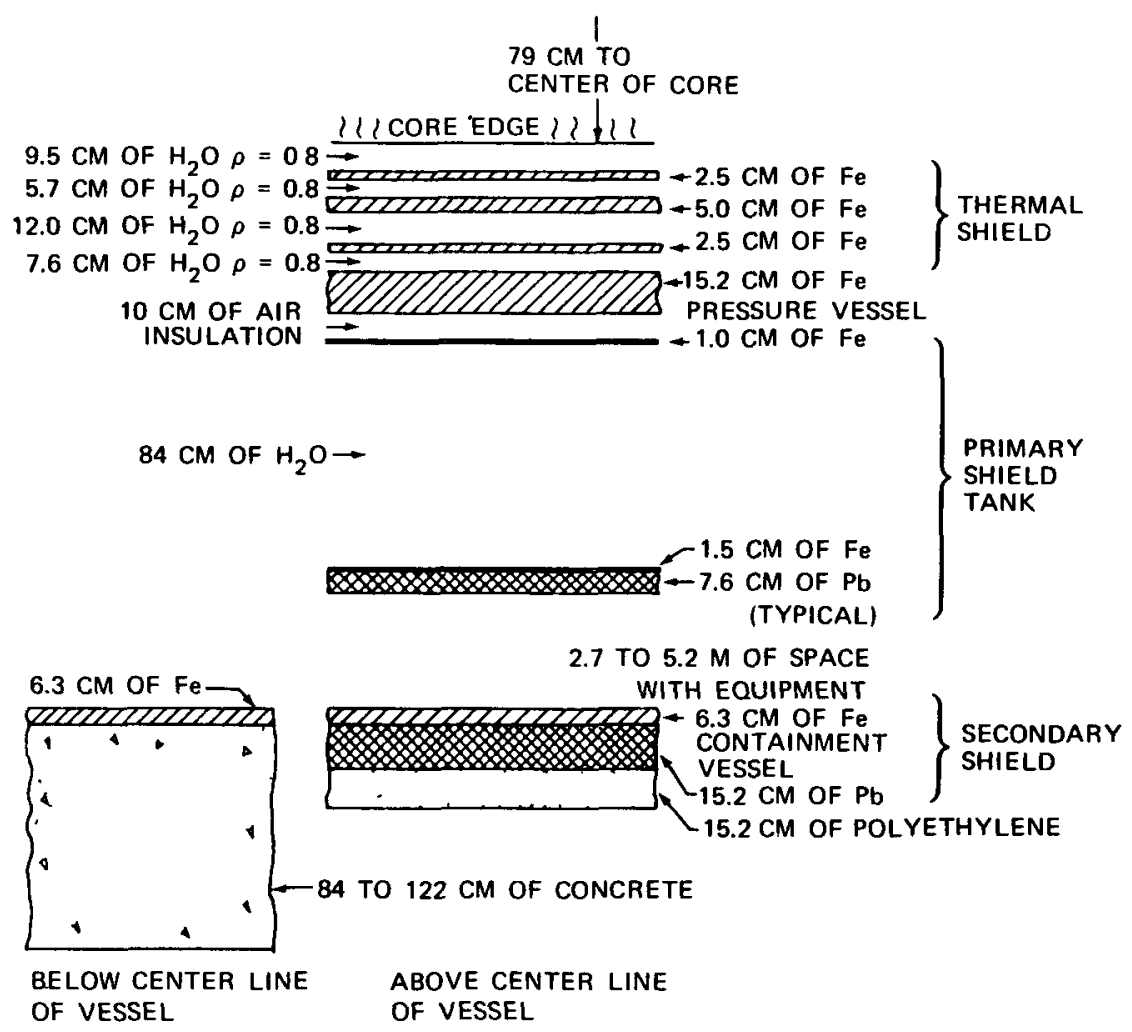

Fig. 10.33 - Schematic cross section at the midplane of the N.S. Savannah reactor shield. (From Blizard et. al. ${ }^{31}$ )

flow-baffle assembly withdrawn. The transitory shield water is also shown. The cask is telescoped to remove the more highly activated grid assembly.

After the flow baffle and grid assembly have been removed, a shield plug, or manipulator (equivalent to about $51 \mathrm{~cm}$ of cast iron, for gamma-ray attenuation), is placed on the pressure-vessel flange, and the transitory water level is lowered. The refueling cask, with its control-rod extension, is positioned on a port in the shield plug, and the port is positioned over the fuel element or control rod to be removed. Figure 10.35 is a schematic diagram of the shield plug and refueling cask in place.

\subsubsection{Shield Design Criteria}

The design requirements for the shielding on the N.S. Savannah were chosen to ensure that the passengers would receive no more radiation than is allowed to the general public, even though they were to live continuously on 


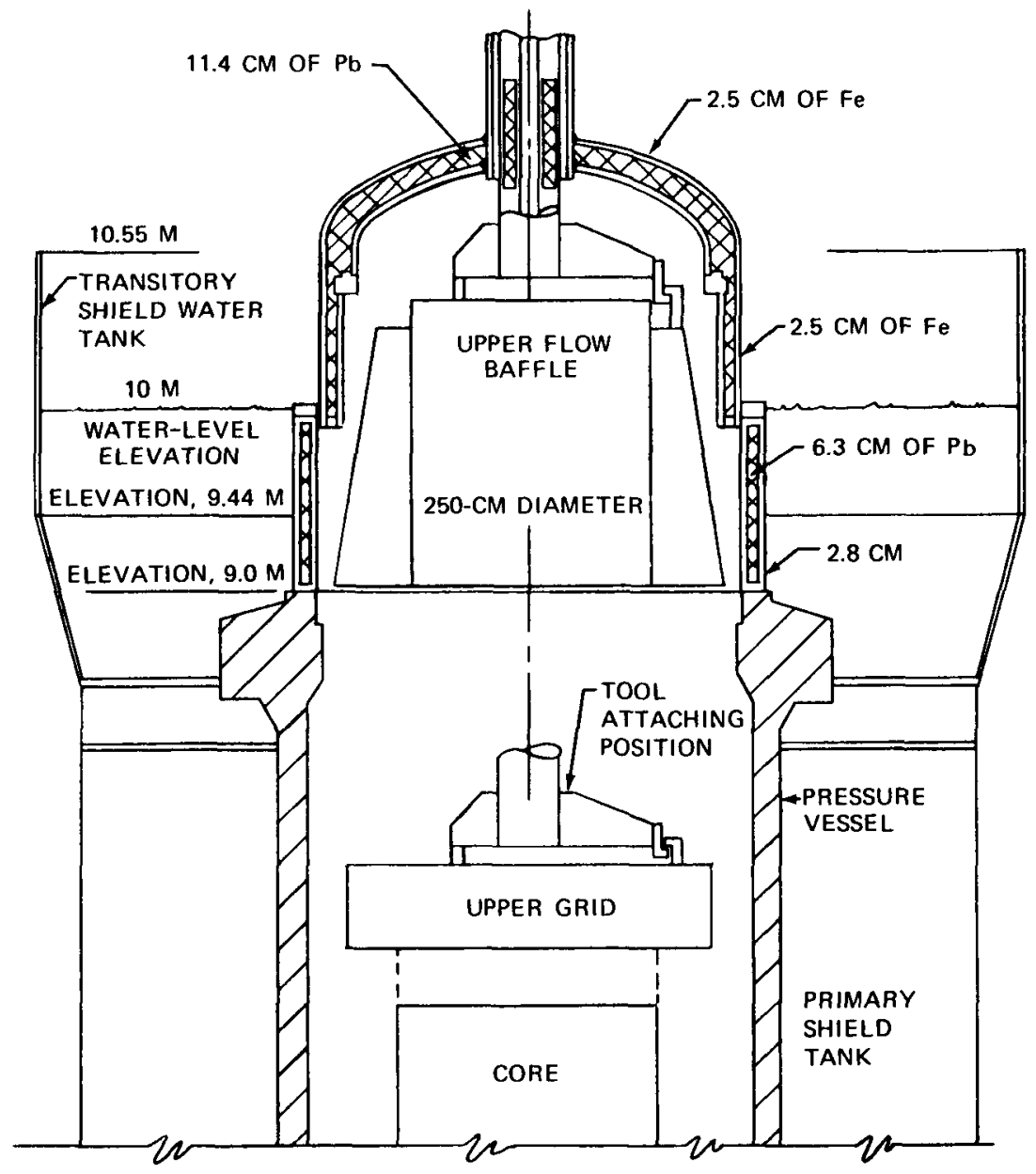

Fig. 10.34-Telescopic internals cask. (From Smith and Turner. ${ }^{29}$ )

board ship with the reactor constantly at full power. The requirements are also that the crew of the vessel receive no more radiation than workers in atomic energy installations, even though they remain on board ship all the time, also with the reactor constantly at full power. The third requirement for the shielding is that stevedores handling cargo will receive no more radiation than that permitted for the general public, even though they continuously handle cargo in the cargo spaces with the reactor at one-fifth full power. These requirements have dictated dose rates that are extremely low in passenger spaces and well below ordinary permissible levels in crew and cargo spaces owing to the presumption that these spaces are occupied continuously with the reactor at full power. 


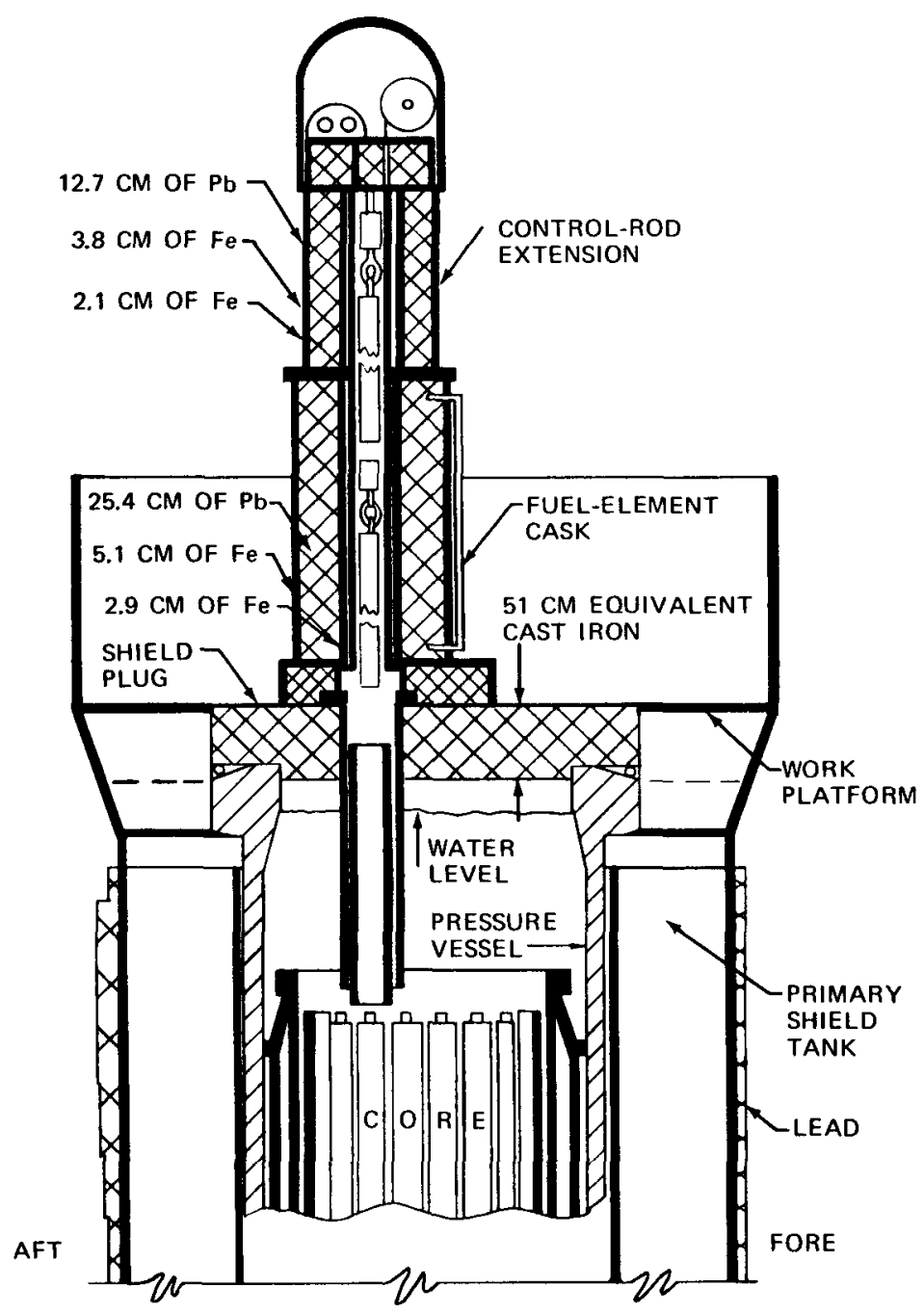

Fig. 10.35-Shield plug and refueling cask. (From Smith and Turner. ${ }^{29}$ )

The design dose rates used to ensure that these requirements were met are summarized in Table 10.7.

\subsubsection{Lead-Polyethylene Shield Construction}

The design of a compact lead-polyethylene shield that can withstand the design stresses is somewhat unusual; consequently some of the details are shown here. 
Table 10.7-DESIGN DOSE RATES FOR N.S. SAVANNAH $\dagger$

\begin{tabular}{|c|c|c|c|}
\hline Location & Operating conditions & $\begin{array}{l}\text { Type } \\
\text { access }\end{array}$ & $\begin{array}{l}\text { Design dose } \\
\text { rate }\end{array}$ \\
\hline $\begin{array}{l}\text { Access spaces outside } \\
\text { secondary shield wall } \\
\text { (passenger spaces) }\end{array}$ & Full-power operation & Unrestricted & $0.5 \mathrm{rem} /$ year \\
\hline $\begin{array}{l}\text { Access spaces outside } \\
\text { secondary shield wall } \\
\text { (crew spaces) }\end{array}$ & Full-power operation & $\begin{array}{l}\text { Unrestricted } \\
\text { (crew) }\end{array}$ & $5.0 \mathrm{rem} /$ year \\
\hline Cargo holds & $\begin{array}{l}\text { Operation at one-fifth } \\
\text { full power }\end{array}$ & Unrestricted & $500 \mathrm{mrem} /$ year \\
\hline Inside containment & Reactor operating & None permitted & \\
\hline Inside containment & $\begin{array}{l}\text { Reactor shut down } \\
1 / 2 \text { hr or longer. } \\
\text { No fission products } \\
\text { in primary loop }\end{array}$ & Limited & $200 \mathrm{mrem} / \mathrm{hr}$ \\
\hline $\begin{array}{l}\text { Locations occupied during } \\
\text { fuel-transfer operations }\end{array}$ & $\begin{array}{l}\text { Reactor shut down } \\
3 \text { days or longer; } \\
\text { refueling operations }\end{array}$ & Limited & $\begin{array}{l}200 \mathrm{mrem} / \mathrm{hr} \\
\text { for transient } \\
\text { conditions, } \\
10 \mathrm{mrem} / \mathrm{hr} \\
\text { for continuing } \\
\text { conditions }\end{array}$ \\
\hline
\end{tabular}

†From Blizard et al. ${ }^{31}$

Polyethylene shielding is made from sheets; differences in shield construction consist mostly in variations in the method of supporting and covering the laminations after they have been placed.

Two properties of polyethylene which are of importance in shield fabrication are its low softening point $\left(180\right.$ to $\left.260^{\circ} \mathrm{F}\right)$ and its relative chemical inertness. The first of these permits the slabs to be shaped with relative ease in the field. The second makes the use of solvent cements generally impractical. Instead of cement, corrugated nails are staggered in alternate layers to fabricate the shield. Sheet size and gauge were chosen for maximum ease of fabrication, and the edges of the polyethylene plates were staggered slightly to prevent streaming.

In the secondary shield the polyethylene was applied over the lead on top of the containment vessel. Rib plate stiffeners and intercostals welded to the shielded surface (containment vessel) were used both to strengthen the shield and to provide a means of attaching the cover plate over the polyethylene. This cover plate was thin steel and served to protect the polyethylene surface from the hazards of combustion and physical damage. 

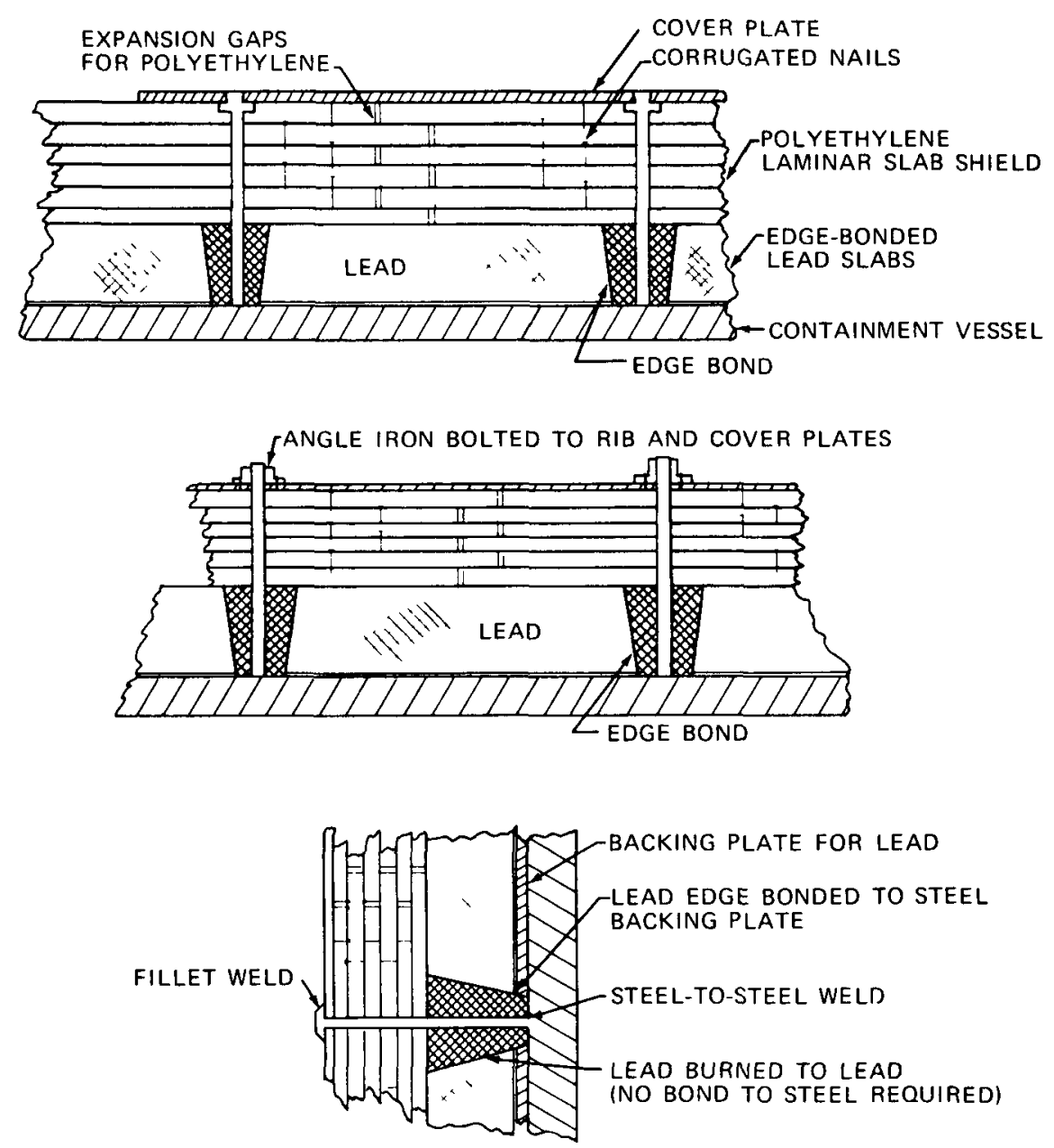

Fig. 10.36-Typical lead-polyethylene shield construction details. (From Smith. ${ }^{30}$ )

It also provided a convenient surface on which to hang pipes, electrical connections, etc. Typical lead-polyethylene shield-arrangement details are sketched in Fig. 10.36. Note the gap spacing shown for polyethylene in these sketches; inadequate allowance for expansion could cause serious structural damage and buckling in the shield.

\subsubsection{Attenuation Calculations}

The attenuation calculations for the primary shield and the secondary shield are discussed separately in the following sections since different techniques were used. 
(a) Primary Shield. Two principal methods of calculating neutron attenuation were used. In all cases conservative assumptions were made since it was recognized that the calculational models were relatively crude and uncertainties existed in the basic cross-section data.

One method involved prediction of the neutron fluxes in the reflector, thermal shields, and pressure vessel with a computer code utilizing two-group diffusion theory. The calculated fast and thermal flux profiles are shown in Fig. 10.37 along with the fast flux distribution through the shield tank, which was calculated with the method described at the end of this section.

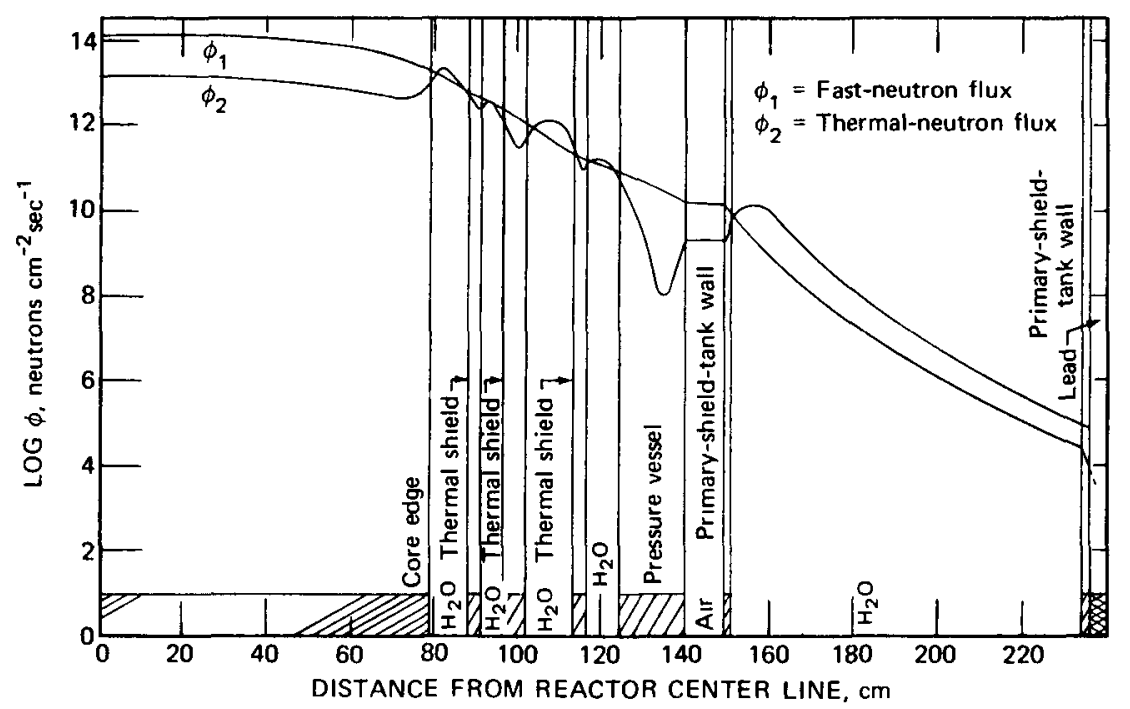

Fig. 10.37-Neutron-flux profile radial center line, $69 \mathrm{Mw}$. (From Smith and Turner. ${ }^{29}$ )

Diffusion theory is inadequate for the deep penetration through the primary shield tank. For this calculation an empirical kernel was used for the attenuation of fast neutrons by water along with the removal-cross-section concept to account for the additional attenuation due to the steel. The kernel was obtained by fitting the fast-flux results of the moments-method calculations for a point fission source in an infinite medium of water by the following equation:

$$
\phi=\frac{1}{4 \pi r^{2}}\left(\mathrm{e}^{-0.123 r}+0.0276 e^{-0.085 r}\right)
$$


where $r$ (in $\mathrm{cm}$ ) is the penetration in water. Basic to the use of Eq. 10.6-1 was the assumption that the energy spectrum approached an equilibrium shape in water for penetrations greater than $40 \mathrm{~cm}$ and that $r$ was the equivalent amount of water (actual water plus equivalent steel).

The attenuation of gamma rays was calculated with the kernel method with infinite-medium buildup factors.

For the radial attenuation of core gamma rays (fission plus capture and inelastics born in the core), the self-shielding factors for uniform cylindrical sources were used. Since self-absorption factors have not been determined in

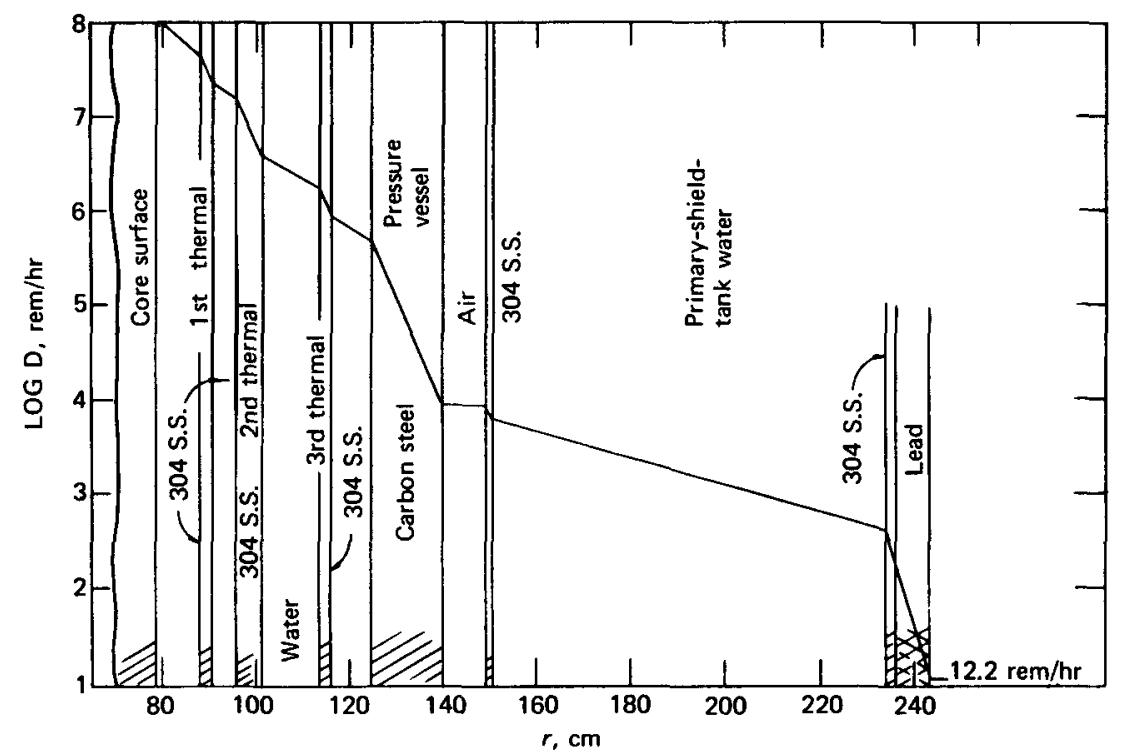

Fig. 10.38-Primary gamma dose rate, radial center line, $69 \mathrm{Mw}$. (From Smith and Turner. ${ }^{29}$ )

general for radiation from the end of a cylinder, this was handled by representing a $1-\mathrm{cm}$-thick disk of core source by an isotropic disk source and interpreting numerically along the core axis. The radial profile of the core gamma rays is shown in Fig. 10.38.

Calculating the secondary gamma flux through a series of water and steel slab shields involved an expression that described the rate of production of gamma rays by capture of thermal neutrons and attenuation in the source slabs (slab assumption was used since the radius of curvature is large compared to the thickness of the steel) and shield materials on either side. An exponential neutron removal was assumed in each source slab, and the 


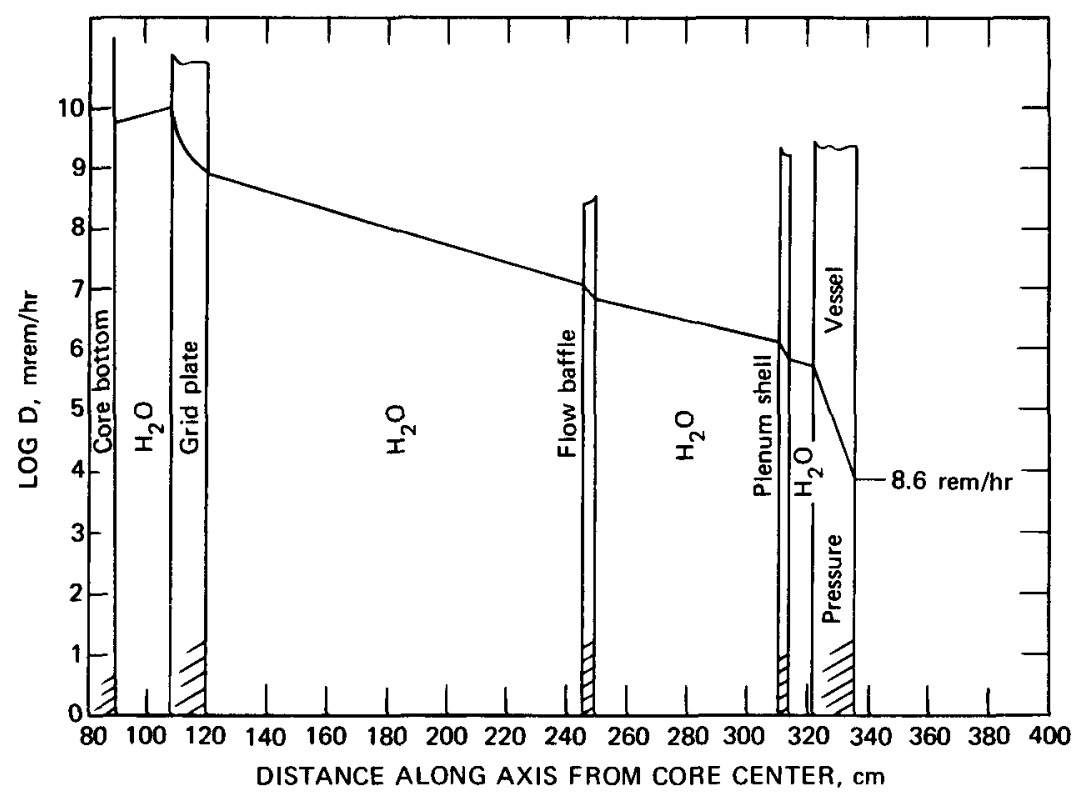

Fig. 10.39-Secondary gamma dose rate through bottom shield axis. (From Smith and Turner. ${ }^{29}$ )

number of source slabs was chosen such that the calculated thermal flux profile through the thermal shield could be matched closely by a simple exponential in each source slab. The results of these calculations for secondary gamma rays are shown in Fig. 10.39.

(b) Secondary Shield. In calculating fluxes or dose rates for points outside the secondary shield, integration over the original isotropic source volume becomes unreliable because of the implications of the combined geometries of the primary and secondary shields. For this situation the pressure-vessel top and the primary shield tank top and outer walls were chosen as surface sources for the subsequent secondary-shield attenuation calculations.

The problem then resolves itself into establishing an angular flux density on these surfaces for both neutrons and gamma rays and then numerically integrating over source surfaces with due regard to the directional orientation of the flux. It was assumed that both neutron- and gamma-ray flux densities could be represented by a cosine distribution, flux density on the surfaces being calculated by point-kernel integration over the volume sources contained within the primary shield tank. With surface source corrected for the intensity in the particular direction, the kernel calculations for 
attenuation through the secondary shield become similar to that for the primary shield calculations with the exception of a correction for the iron removal cross section. The minimum thickness of $15.2 \mathrm{~cm}$ on the secondary shield surface was not sufficient to produce an equilibrium spectrum in hydrogen. Data from iron-water mock-ups at ORNL were analyzed to provide an estimate of the effect of nonequilibrium spectral conditions in hydrogenous material following metal to obtain the necessary corrections to the removal cross sections.

\subsubsection{Measurements}

Accurate measurements of the low dose rates specified in the design were a difficult task. For this purpose a mobile laboratory was developed. ${ }^{31}$ Three types of dosimeters were used in the Savannah survey. All gamma-ray dose-rate measurements were obtained with an anthracene scintillation detector in which the sensitive element is a $2.5-\mathrm{cm}$-diameter $1.3-\mathrm{cm}$-thick anthracene crystal. The choice of anthracene was made because the energy lost within anthracene by a secondary electron resulting from a given-energy gamma-ray photon is very nearly proportional to the (computed) singlecollision dose over the energy range from $100 \mathrm{keV}$ to $10 \mathrm{MeV}$. The instrument has a sensitivity of about 13,300 counts $\min ^{-1} \mathrm{mrem}^{-1} \mathrm{hr}$. Thermal-neutron dose rates (proportional to flux density) were measured with a 1-in.-diameter $\mathrm{BF}_{3}$ proportional counter having an active length of $12 \mathrm{in.}$ In the construction of this counter, high sensitivity was achieved by filling the tube with $96 \%{ }^{10} \mathrm{~B}$-enriched $\mathrm{BF}_{3}$ at the relatively high pressure of $76 \mathrm{~cm} \mathrm{Hg}$. This pressure, essentially $1 \mathrm{~atm}$, results in a sensitivity of approximately 7400 counts $\min ^{-1} \mathrm{mrad}^{-1}$ (tissue) $\mathrm{hr}^{-1}$ and also makes the counter more resistant to accidental damage during use.

Fast-neutron dose rates were measured with a modified long counter that uses the $\mathrm{BF}_{3}$ tube of the thermal-flux detector as the sensitive element within a $20-\mathrm{cm}$-diameter $30-\mathrm{cm}$-long cadmium-covered polyethylene cylinder. The polyethylene thicknesses at the end and side of the cylinder had been adjusted by experiments with monoenergetic neutrons from a Van de Graaff accelerator to give a counter response proportional to the multicollision tissue-dose curve to within $\pm 10 \%$ over the energy range from 0.025 to approximately $10 \mathrm{MeV}$. The sensitivity of this instrument is approximately 7800 counts $\min ^{-1} \mathrm{mrad}^{-1}$ (tissue) $\mathrm{hr}^{-1}$.

The instrumentation described was carefully calibrated against known sources and fluxes and compared to standard instrumentation in a series of experiments at the ORNL Bulk Shielding Facility (BSF). The BSF 
experiments used an idealized mock-up of the Savannah's shield, thus ensuring that the comparisons would be valid for the energy spectrum that would be encountered aboard the Savannah.

The experimental procedures used in the Savannah survey were developed and tested in a series of studies of land-based reactors made during the months preceding this study. By the time the mobile laboratory was lifted aboard the Savannah, methods and procedures were firm, and a group of trained and experienced personnel was available to act as a nucleus for the large group required for the survey. Before the survey started, the secondary shield was completely marked with a rectangular $0.9-\mathrm{m}$ grid (see Fig. 10.32). The general procedure for the survey consisted in obtaining a 1-min or 0.5 -min count, depending on counting statistics, in the center of each grid with both the thermal-neutron and gamma-ray dosimeters. After the count the detector was slowly swept over the area within the grid to detect any small area of unusual leakage. The entire shield was covered with both thermal-neutron and gamma-ray dosimeters. Fast-neutron dose rates were measured at carefully chosen locations over the shield, which permitted a good evaluation of the ratio of fast to thermal neutrons over the shield.

\subsubsection{Comparison of Measurements and Calculations}

The reactor was held at approximately its full power of $69 \mathrm{Mw}$ for the entire duration of the detailed shield survey. The dose rates recorded thus represent the maximum to be expected. A detailed comparison of the measurements and calculations is shown in Table 10.8, which has been abridged from Blizard et al. ${ }^{31}$ The point numbers identifying the data positions were selected by Smith and Turner ${ }^{29}$ for later comparison with measurements. The locations on each deck are shown in Fig. 10.40, with point numbers corresponding to those of Table 10.8.

Note that the measured fast-neutron dose rates in Table 10.8 are reported in absorbed dose units ( $\mathrm{mrad}$ ) and are converted to dose equivalent (mrem) for comparison with calculations by multiplying by a relative biological equivalence factor varying from 8 to 9 depending on energy spectrum (or total shield thickness). These units and their relations are discussed in Sec. 3.3.4.

In general, the actual dose rates appear to be considerably lower than the predicted dose rates, which reflects the intended conservatism in the design. Spot checks of the comparisons show overestimates of the gamma-ray dose rate and underestimates of the neutron dose rate. 
Table 108 -COMPARISON OF MEASURED DOSE RATES WITH CALCULATED DOSE RATES AT SPECIAL POINTS $\dagger$

\begin{tabular}{|c|c|c|c|c|c|c|c|}
\hline \multirow[b]{3}{*}{$\begin{array}{l}\text { Point } \\
\text { number }\end{array}$} & \multicolumn{4}{|c|}{ Observed dose rate } & & & \\
\hline & \multicolumn{2}{|c|}{ Neutron } & \multirow[b]{2}{*}{$\begin{array}{l}\text { Gamma, } \\
\text { mrem/hr }\end{array}$} & \multirow[b]{2}{*}{$\begin{array}{l}\text { Total, } \\
\text { mrem/hr }\end{array}$} & \multicolumn{3}{|c|}{ Babcock \& Wilcox 11441 calculated dose rate } \\
\hline & $\begin{array}{c}\text { Fast } \\
\mathrm{mrad} / \mathrm{hr}\end{array}$ & $\begin{array}{l}\text { Thermal, } \\
\text { mrem/hr }\end{array}$ & & & $\begin{array}{l}\text { Neutron, } \\
\text { mrem/hr }\end{array}$ & $\begin{array}{l}\text { Gamma, } \\
\text { mrem/hr }\end{array}$ & $\begin{array}{l}\text { Total, } \\
\text { mrem/hr }\end{array}$ \\
\hline \multicolumn{8}{|c|}{ Promenade Deck } \\
\hline 44 & $150 \times 10^{3}$ & $131 \times 10^{-4}$ & $0925 \times 10^{-2}$ & $223 \times 10^{-2}$ & $10 \times 10^{-2}$ & $419 \times 10^{-2}$ & $429 \times 10^{1}$ \\
\hline 45 & 0988 & 116 & 142 & 228 & 0 & 95 & 095 \\
\hline 46 & 0976 & 0771 & 0569 & 142 & 07 & 179 & 186 \\
\hline 47 & 165 & 152 & 114 & 258 & & & \\
\hline 48 & 151 & 0412 & 109 & 239 & 10 & 110 & 120 \\
\hline 54 & 140 & 0570 & 153 & 275 & 24 & 84 & 108 \\
\hline 56 & 0897 & 209 & 0624 & 142 & 16 & 110 & 126 \\
\hline 57 & 0845 & 0369 & 0360 & 110 & & & \\
\hline 71 & 109 & 0232 & 0823 & 176 & 43 & 84 & 127 \\
\hline 72 & 143 & 0318 & 068 & 191 & 46 & 84 & 130 \\
\hline \multicolumn{8}{|c|}{ A Deck } \\
\hline 1 & 343 & 358 & 347 & 646 & 14 & 192 & 206 \\
\hline 2 & 332 & 433 & 417 & 707 & 14 & 191 & 205 \\
\hline 3 & 198 & 134 & 226 & 397 & 16 & 127 & 143 \\
\hline 4 & 283 & 345 & 279 & 527 & 14 & 214 & 228 \\
\hline 5 & 417 & 419 & 430 & 793 & 14 & 238 & 252 \\
\hline 6 & 268 & 241 & 384 & 617 & 19 & 181 & 200 \\
\hline 7 & 276 & 126 & 293 & 532 & 29 & 146 & 175 \\
\hline 14 & 226 & 125 & 205 & 401 & 50 & 152 & 202 \\
\hline 17 & 884 & 0803 & 170 & 932 & 47 & 272 & 319 \\
\hline 22 & 1911 & 0792 & 172 & 337 & 19 & 89 & 108 \\
\hline 23 & 155 & 0697 & 112 & 246 & 50 & 90 & 140 \\
\hline 26 & 975 & 0422 & 0694 & 909 & 50 & 170 & 220 \\
\hline 803 & 235 & 117 & 245 & 452 & 90 & 183 & 273 \\
\hline 804 & 186 & 0232 & 126 & 286 & 90 & 124 & 214 \\
\hline \multicolumn{8}{|c|}{ C Deck } \\
\hline 252 & $776 \times 10^{3}$ & $1277 \times 10^{-4}$ & $1378 \times 10^{-2}$ & $2059 \times 10^{-2}$ & $332 \times 10^{-2}$ & $235 \times 10^{-2}$ & $567 \times 10^{-1}$ \\
\hline 253 & 225 & 276 & 219 & 416 & 0 & 95 & 095 \\
\hline 261 & 456 & 166 & 0820 & 576 & 04 & 108 & 112 \\
\hline 324 & 537 & 1090 & 1579 & 2052 & 336 & 249 & 585 \\
\hline 325 & 187 & 249 & 453 & 617 & 0 & 18 & 018 \\
\hline 611 & 294 & 264 & 213 & 469 & 0 & 337 & 337 \\
\hline 612 & 315 & 247 & 346 & 620 & & & \\
\hline 613 & 233 & 225 & 132 & 1525 & 0 & 202 & 202 \\
\hline \multicolumn{8}{|c|}{ D Deck } \\
\hline 276 & 1051 & 0781 & 0644 & 969 & 16 & 67 & 083 \\
\hline 278 & 141 & 0400 & 0706 & 192 & 06 & 59 & 065 \\
\hline 332 & 138 & 0517 & 0777 & 202 & 16 & 85 & 101 \\
\hline 813 & 202 & 101 & 224 & 399 & 23 & 156 & 179 \\
\hline 815 & 113 & 0370 & 163 & 261 & 26 & 694 & 720 \\
\hline \multicolumn{8}{|c|}{ Tank top } \\
\hline 297 & 0390 & 0465 & 0343 & 0684 & 09 & 95 & 104 \\
\hline 307 & 0338 & 0317 & 0338 & 0532 & 19 & 29 & 048 \\
\hline 334 & 0910 & 0190 & 0273 & 106 & 15 & 26 & 041 \\
\hline 640 & 124 & 0486 & 0685 & 176 & 285 & 1214 & 1499 \\
\hline 641 & 0754 & 0317 & 0288 & 0939 & 21 & 62 & 083 \\
\hline
\end{tabular}

tAdapted from Blizard et al ${ }^{31}$ 

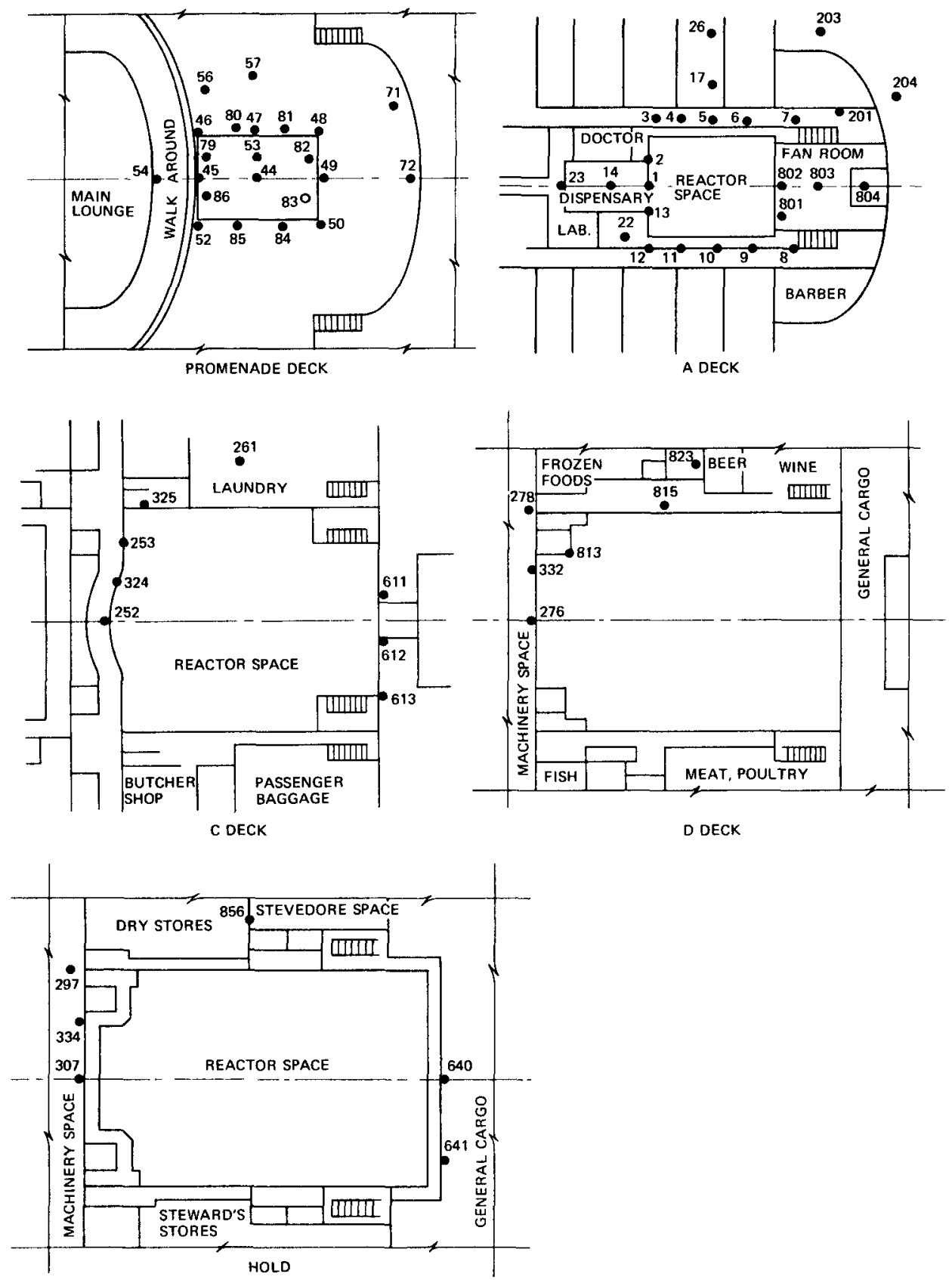

Fig. 10.40-Special point locations aboard the N.S. Savannah. (From Blizard et al. ${ }^{31}$ ) 
The measurements showed, however, that the dose rates did not exceed tolerance limits in areas where passenger access is permitted. The design dose rate was exceeded only in a small region on $D$ deck, to which only the crew is permitted access and which can easily be controlled.

\subsection{SPACE POWER: SNAP-10A FLIGHT TEST}

The Snapshot-1 flight test, which took place in April 1965, is the only known space flight of a reactor. Strictly speaking, this was an experiment, and it represents a test system in development rather than a finished design application. However, the magnitude of the program, the preflight design development effort, and the postflight analysis give us the same pattern of input that we have sought for the previous discussion of reactor-shield applications in this chapter. We should, nevertheless, bear in mind that the Space Nuclear Auxiliary Power (SNAP)-10A is not a finished product but an intermediate development model.

The SNAP-10A reactor is described by Glasstone ${ }^{32}$ as a very small system, being only $35 \mathrm{~cm}$ in diameter and about $46 \mathrm{~cm}$ long. It is cooled with $\mathrm{NaK}$ (sodium-potassium) and controlled by rotating beryllium reflector material in semicylindrical drums toward the core. The core is about $23 \mathrm{~cm}$ in diameter and $39.5 \mathrm{~cm}$ long. Fuel elements are enriched uranium and zirconium hydride clad in Hastelloy with hydrogen serving as a moderator. The choice of materials was based largely on the need for compact design and high-temperature operation. The $\mathrm{NaK}$ coolant leaves the core at $545^{\circ} \mathrm{C}$ and is pumped to a thermoelectric converter for production of electricity. The reactor operated at about $40 \mathrm{kw}(\mathrm{t})$ and produced a little over $0.5 \mathrm{kw}$ of electric power.

The SNAP-10A-Agena spacecraft was launched into a near-polar orbit Apr. 3, 1965. After 6 days of monitoring and manual control of the reactor by ground commands, the reactor was switched to automatic temperaturecoefficient control. Subsequent operation was automatic without further ground command. The reactor was to have operated for at least 6 months, but on May 16 loss of signals from the spacecraft indicated that the reactor had shut down. The failure was apparently not in the reactor itself but in a voltage regulator.

\subsubsection{Shield Analysis}

Belcher, Flynn, and Thomson ${ }^{33}$ summarized an extensive preflight shield analysis to ensure that the shield met its design objectives. These 
objectives were that the 1-year gamma and fast-neutron levels not exceed $10^{7} \mathrm{R}$ or $10^{12}$ neutrons $/ \mathrm{cm}^{2}$, respectively, across a $152-\mathrm{cm}$-diameter plane located $5.3 \mathrm{~m}$ below the reactor core. This plane is called the reference-dose plane, as shown in Fig. 10.41. The weight objective for the shield was $102 \mathrm{~kg}$, including container and structure, which was certainly a stringent restriction.

The gamma-ray- and neutron-level specifications were intended to limit radiation effects to solid-state electronics equipment. Belcher et al. ${ }^{33}$ estimate an approximate gross leakage of $10^{8} \mathrm{R}$ and $10^{16}$ neutrons $/ \mathrm{cm}^{2}$ and a corresponding attenuation requirement of $10^{4}$ for fast neutrons and 10 for gamma rays. They chose $\mathrm{LiH}$ as the shield material for its high neutron-shielding efficiency (light weight) and high-temperature properties, estimating that about $60 \mathrm{~cm}$ would be required.

They also point out, as is evident in Fig. 10.41, that the control drums and sides of the reactor were not shadowed by the shield from the thermoelectric converter and the near part of the Agena stage. Not shown on the sketch is the $\mathrm{NaK}$ piping around the shield. The control drums, converter, and $\mathrm{NaK}$ piping all constitute sources of scattered neutrons in the near section of the Agena structure. Had the shadow shield been designed with an annular bulge and shortened to avoid a weight increase, it might have been more effective. An alternative arrangement within the $102-\mathrm{kg}$ weight limit would be an interesting design exercise. Belcher et al. ${ }^{33}$ state that it was impossible to rectify this condition without exceeding the weight limitations.

Although the specifications refer to the reference-dose plane, most of the measurements and predictions use the SNAP-Agena mating plane (also shown on Fig. 10.41) as a primary reference for radiation intensities. We will use only the SNAP-Agena plane to avoid confusion.

The core region was divided into an array of effective point sources, and a ray-tracing technique using removal cross sections determined attenuation through the core and $\mathrm{LiH}$ shield. Attenuation measurements for $\mathrm{LiH}$ were repeated at the Atomics International (AI) Shield Test Facility in Canoga Park, Calif., and the General Dynamics Aerosystems Test Facility in Fort Worth, Tex. From the measurements a $\mathrm{LiH}$ attenuation coefficient of 0.156 $\mathrm{cm}^{-1}$ for the $0.7 \mathrm{~g} / \mathrm{cm}^{3}$ density was estimated at operating temperature $\left(\sim 370^{\circ} \mathrm{C}\right)$.

As might be suspected from the discussion of the geometry, neutrons scattered around the shield sufficiently exceeded the number penetrating 


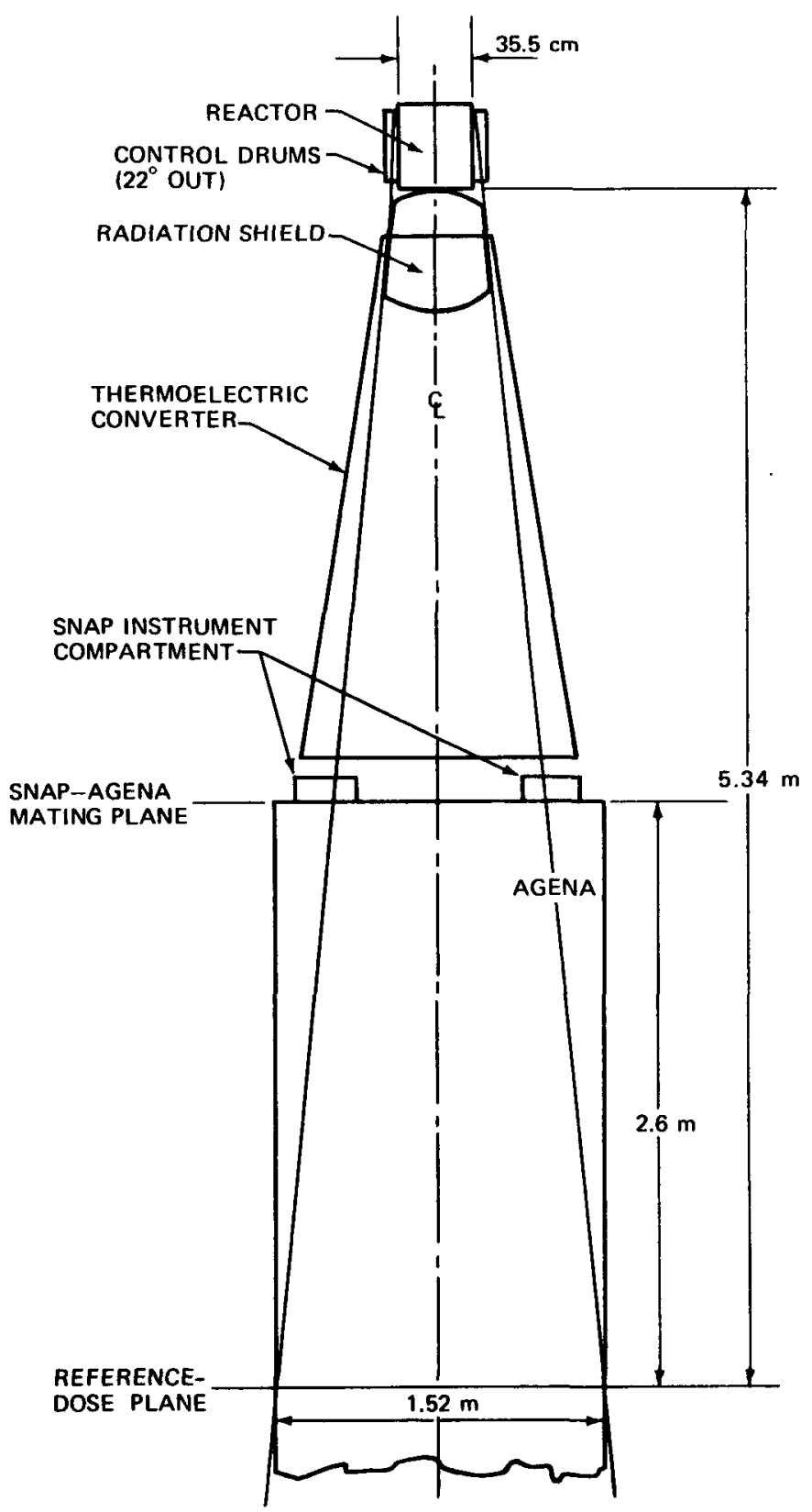

Fig. 10.41 - SNAP-10A radiation shield geometry. (From Belcher et al. ${ }^{33}$ ) 
through the shield that the latter component was unimportant in analyzing the flight-test data.

Gamma-ray exposure through the shield was computed by the same ray-tracing method as the fast neutrons. The sources considered were fission gamma rays, captures in the core, and activation from the $\mathrm{NaK}$ coolant. Neutron capture in ${ }^{23} \mathrm{Na}$ produces ${ }^{24} \mathrm{Na}$, which decays by producing two gamma rays, 2.76 and $1.38 \mathrm{MeV}$. The decay of ${ }^{42} \mathrm{~K}$ gives a $1.51-\mathrm{MeV}$ gamma ray about $25 \%$ of the time. The moments-method buildup factors (Sec. 4.8.1) were used. None of the gamma-ray intensities exceeded the $10^{7}$ $\mathrm{R} /$ year maximum at the SNAP-Agena mating plane. The computed total consisted mostly of gamma rays from the core.

The principal heating rate in the shield was due to neutron capture in ${ }^{6} \mathrm{Li}:$

$$
{ }^{6} \mathrm{Li}+n \rightarrow{ }^{3} \mathrm{H}+\alpha+4.8 \mathrm{MeV}
$$

The alpha has a very short range and may be considered deposited at the capture site. Neutron kinetic energy in elastic collisions with hydrogen produces almost as much heat as the ${ }^{6} \mathrm{Li}$ captures. Captures in ${ }^{7} \mathrm{Li}$ (the principal isotope) produce gamma rays, which give unimportant intensities in this case. At $40 \mathrm{kw}$ the heating in the shield was computed to be about 160 watts with a maximum power density of $0.3 \mathrm{watt} / \mathrm{cm}^{3}, 49 \%$ from ${ }^{6} \mathrm{Li}(n, \alpha)$, $39 \%$ from neutron elastic scattering, and $12 \%$ from gamma-ray absorption.

\subsubsection{Flight-Test Results}

Bennett, Krakar, and Kruse $^{34}$ report the nuclear environment and compare it with the analysis just described. We have not described the method of calculating the important scattering contributions from the control drum, converter, and reactor sides because the estimates of Belcher et al. ${ }^{33}$ were admittedly crude and were revised for inclusion with the flight results of Bennett $e t$ al. ${ }^{34}$ We will discuss these estimates later.

Fast-neutron-flux densities were measured with a ${ }^{237} \mathrm{~Np}$ fission chamber. The fission cross section has a threshold of $0.65 \mathrm{MeV}$, which makes this chamber well-suited for fast-neutron fluxes limited by radiation-effects considerations. Neutrons below the threshold do not significantly contribute to radiation damage. The counter was enclosed in cadmium to eliminate thermal-neutron interaction with impurities in the ${ }^{237} \mathrm{~Np}$.

A similar fission chamber with ${ }^{235} \mathrm{U}$ was used for thermal-neutron measurements; actually two sensors were used, one cadmium-wrapped, to 
obtain the thermal-flux density by subtraction of the two signals. Gammaray exposures were obtained with a $\mathrm{CO}_{2}$-filled ionization chamber.

Additional neutron measurements were made with a radiation-sensitive transistor, which responds to the cumulative fast-neutron-flux density.

Telemetry and data processing need not concern us in this discussion except to note they were necessarily elaborate and contributed to reproducibility within a nominal factor of 1.22 in fast-neutron flux or gamma-ray exposure.

Table 10.9-SNAPSHOT RADIATIONN-DETECTOR

RESULTS AT SNAP-10A-AGENA MATING PLANE

\begin{tabular}{ccc}
\hline & $\begin{array}{c}\text { Fast-neutron- } \\
\text { flux density, } \\
\mathbf{1 0}^{14} \text { neutrons } \\
\mathrm{cm}^{-2} \text { year }^{-1}\end{array}$ & $\begin{array}{c}\text { Gamma-ray } \\
\text { exposure, } \\
\mathbf{1 0}^{6} \text { R year }^{-1}\end{array}$ \\
\hline LMSC detectors & & \\
RAI-1 & 0.48 & \\
RAI-2 & 1.09 & \\
AI detectors & & \\
166 & 0.67 & \\
167 & 0.63 & \\
168 & 0.41 & \\
169 & 0.42 & \\
170 & 0.50 & \\
171 & 0.47 & \\
172 & 0.52 & \\
138 & & 5.6 \\
\hline
\end{tabular}

Table 10.9 gives flux densities for the detectors located on the mating plane we have used for reference. Each value for Lockheed Missiles and Space Company (LMSC) detectors represents a mean of 1500 telemetry samples; for AI detectors each value represents a mean of 10 telemetry samples from each of 20 data acquisitions. The former are fission-chamber measurements and the latter are the solid-state detectors. We have converted the rate data of Belcher $e$ al. to 1-year totals.

The flight data prompted an immediate review of the preflight analysis. The fast-neutron-flux densities were found to be underestimated by a factor of 6.5 to 7.8 ; gamma exposures were within $10 \%$ of prediction in this area. Revised estimates were made of the scattering from the thermoelectric converter-radiator, increasing the scattered component by a factor of 


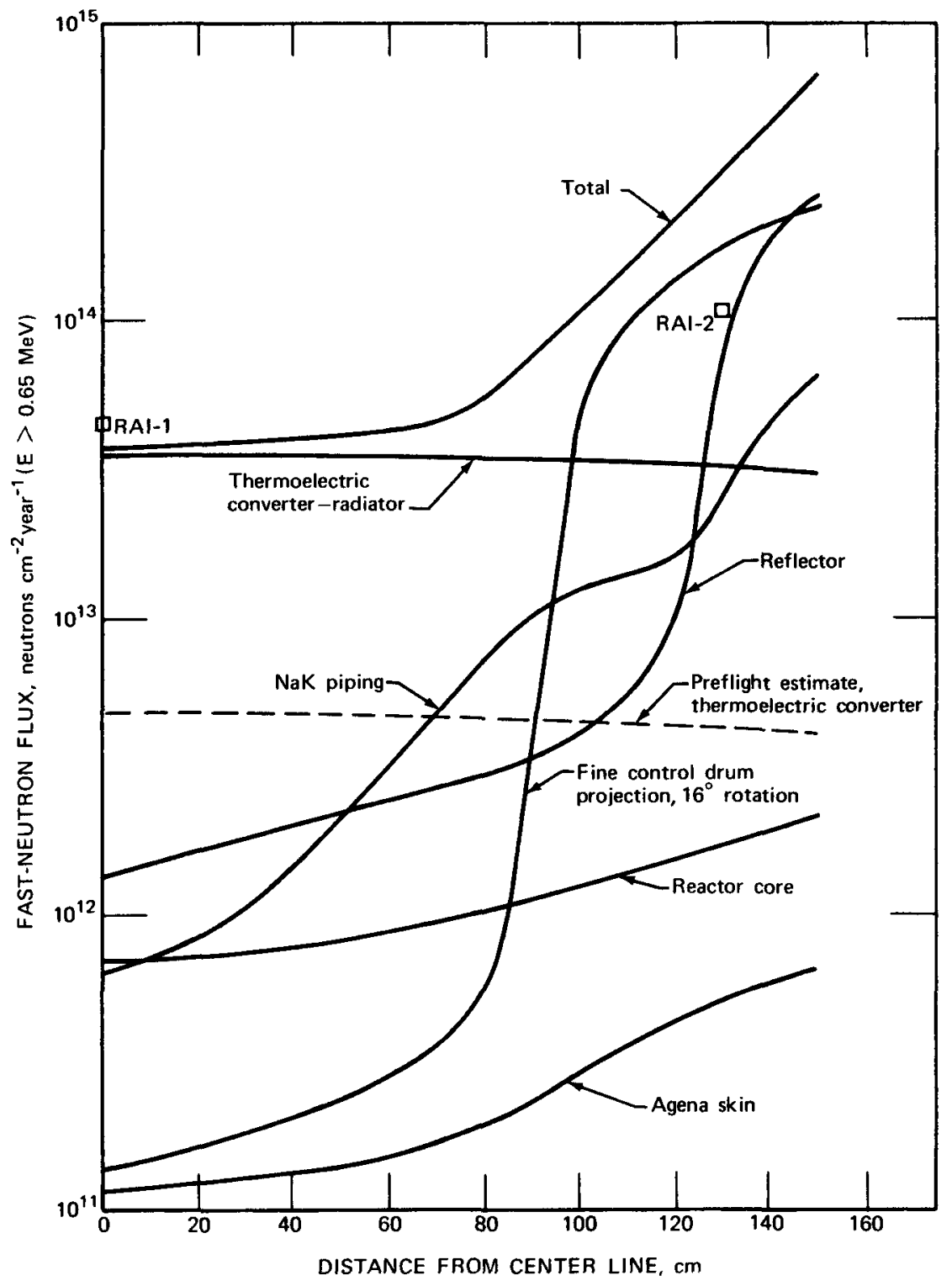

Fig. 10.42-Revised AI fast-neutron flux at SNAP-10A-Agena mating plane below out-rotated control drums. (From Bennett et al. ${ }^{34}$ )

about 8 . The various contributions to the fast-neutron-flux density are shown in Fig. 10.42. The projection of the shadow shield to this plane of the vehicle has a $51-\mathrm{cm}$ radius. Transmission through the shield is contained in the component marked reactor core. The rest of the components do not 


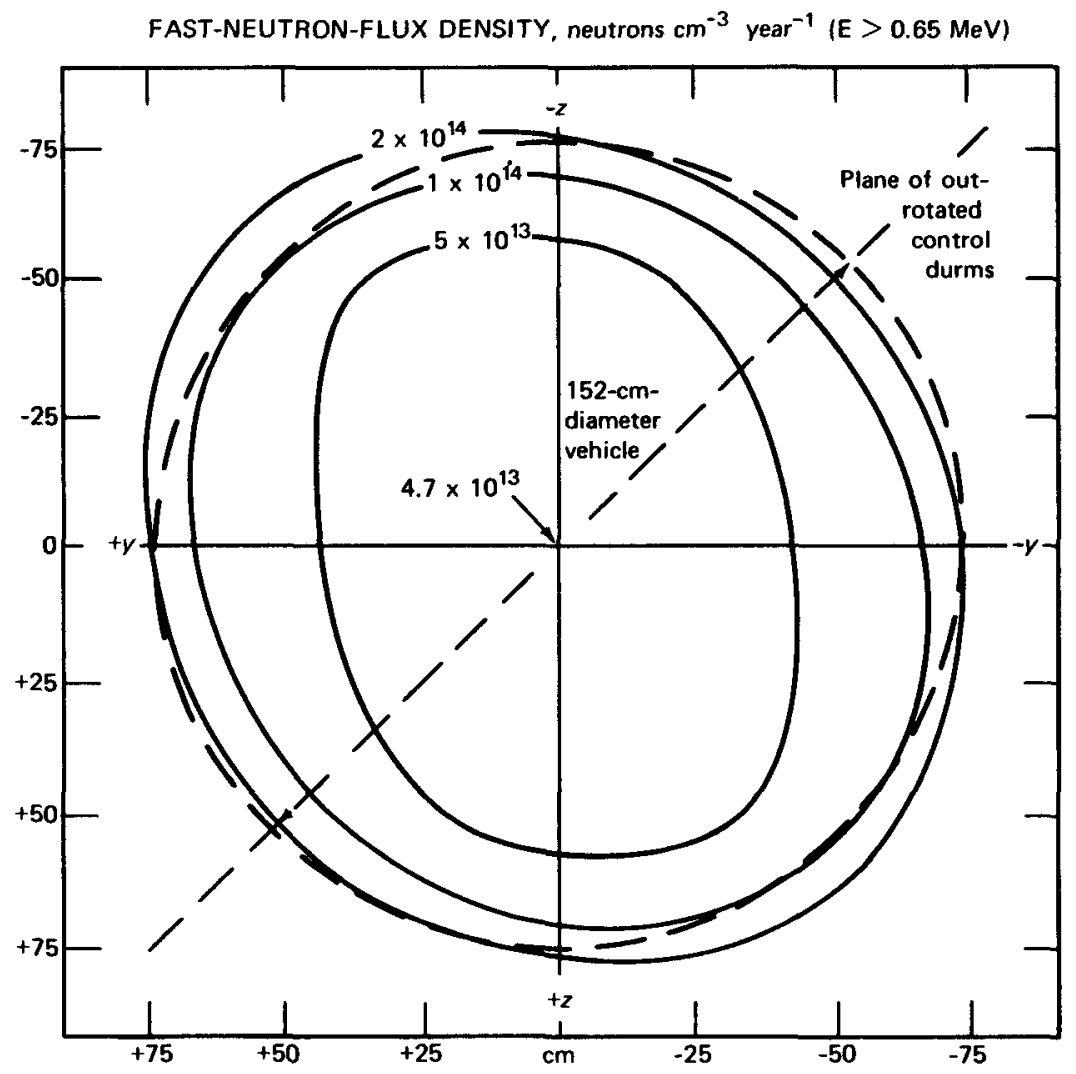

Fig. 10.43-Polar graph of fast-neutron data at SNAP-Agena mating plane. (From Bennett et al. ${ }^{34}$ )

penetrate the shield. The early preflight prediction of scattering from the thermoelectric converter is shown as a dashed line. The revised estimate of this component raised the total at the center line by a factor of 5 ; near the skin of the vehicle $(76 \mathrm{~cm})$, the total was unaffected. Shown in the figure are two measurements from Table 10.9 that are in reasonable agreement.

This was a complex set of scattering sources, and the predictions were indeed within the accuracy of the approximate methods used. This is a clear-cut instance where a Monte Carlo calculation of the scattered contributions is the only method that could be expected to give the desired accuracy (presumably within a factor of 2 ).

The flight-test measurements were compared with the predictions, and a model was constructed that gave a better estimate of the radiation environment throughout the vehicle. The polar plot for our plane of reference is shown in Fig. 10.43. Note that the effect of the control drums is 
to push the isoflux lines inward toward the center. Intensities beyond the mating plane could not be satisfactorily modeled without accounting for the attenuation contributed by some 19 pieces of equipment aboard the Agena stage. Accordingly, attenuation measurements made during preflight testing were used to derive removal cross sections for each piece of equipment. ${ }^{34} \mathrm{~A}$ point-kernel calculation was made from the mating plane to each location to generate the data to model the radiation environment. The resulting model, based on hybrid combination of preflight prediction, in-flight measurement, and empirical adjustment, reproduced miasured data within a factor of 2 .

\section{REFERENCES}

1. H. E. Hungerford, Problems in Design of Fast Reactor Shields, Trans. Amer. Nucl. Soc., 11: 702 (1968).

2. R. J. Beaudry, H. E. Hungerford, R. F. Nantey, and W. F. Chaltron, Design of the Radiation Shield System for the Enrici Fermi Atomic Power Plant, Report APDA-203, Atomic Power Development Associates, Inc., May 1967.

3. R. J. Beaudry, Operational Shield Tests for the Enrico Fermi Atomic Power Plant, Report APDA-221, Atomic Power Developnent Associates, Inc., July 1968.

4. H. E. Hungerford, Shielding, Chap. 8, in Fa.it Reactor Technology, Plant Design, J. G. Yevick (Ed.), M.I.T. Press, Cambridge, Mass, 1966.

5. H. V. Lichtenberger, F. W. Thalgott, 'W. Y. Kato, and M. Novick, Operating Experience and Experimental Results Obtained from a NaK-Cooled Fast Reactor, in Proceedings of the First United Nations International Conference on the Peaceful Uses of Atomic Energy, Geneva, 1955, Vıl. 3, p. 345, United Nations, New York, 1956.

6. J. L. Koch, H. O. Monson, W. R. Simmons, M. Levenson, F. Verber, E. Hutter, R. Jaross, T. R. Spalding, J. R. Simanton, and A. Lovoff, Construction and Design of EBR-II, in Proceedings of the Second United Nations International Conference on the Peaceful Uses of Atomic Energy, Geneva, 1958, Vol. 9, p. 323, United Nations, New York, 1958.

7. C. P. Zaleski and L. Vautrey, Le Réacteur Rapide Surrégénérateur, Rapsodie, Vols. I and II, French Report CEA-2193, 1962.

8. H. Cartwright, J. Tatlock, and R. R. Matthews, Dounreay Fast Reactor-Basic Problems in Design, in Proceedings of tize Second United Nations International Conference on the Peaceful Uses of Atomic Energy, Geneva, 1958, Vol. 9, p. 323, United Nations, New York, 1958.

9. A. I. Leipunsky, V. G. Grabin, N. N. Aristarkhov, I. I. Bondarenko, O. D. Kazachovsky, O.' L. Lubimtsev, S. A. Pashkov, M. S. Pinkhasik, K. K. Renne, U. Y. Stavissky, F. A. Ukraintsev, and L. N. Usachev, Experimental Fast Reactors in the Soviet Union, in Proceedings of the Second United Nations International Conference on the Peaceful Uses of Atomic Energy, Geneva, 1958, Vol. 9, p. 348, United Nations, New York, 1958. 
10. Power Reactor Development Company, Enrico Fermi Atomic Power Plant Technical Information and Hazards Summary Report, Vol. 3, June 1961.

11. Title 10, Code of Federal Regulations, Chapter 1, Atomic Energy Commission, Part 20, Standard for Protection Against Radiation, in Federal Register, Superintendent of Documents, U. S. Government Printing Office, Washington, D. C., Nov. 17, 1960.

12. L. F. Zartman and G. B. Greenough (Eds.), Proceedings of US/UK Graphite Conference, London, Dec. 16-18, 1957, USAEC Report TID-7565, Part I, AEC Division of Reactor Development and United Kingdom Atomic Energy Authority, March 1969.

13. R. F. Mantey, Temperature Distribution and Thermal Stresses in a Concrete Shield Due to Gamma Radiation, Trans. Amer. Nucl. Soc., 1: 66 (1958).

14. H. P. Flatt and D. C. Baller, AIM-5, A Multigroup One-Dimensional Diffusion Equation Code, Report NAA-SR-4694, North American Aviation, Inc., Mar. 1, 1960.

15. S. Preiser, G. Rabinowitz, and E. de Dufour, A Program for the Numerical Integration of the Boltzmann Equation, Report UNUCOR-632, United Nuclear Corporation, 1960.

16. B. G. Carlson, C. Lee, and W. J. Worlton, DSN and TDC Neutron Transport Codes, USAEC Report LAMS-2346, Los Alamos Scientific Laboratory, 1962.

17. W. J. Worlton, $S_{n}$ Reactor Code DTK, a Predecessor to DTF-IV, USAEC Report LA-3373, Los Alamos Scientific Laboratory, 1965.

18. A. Hassitt, A Computer Program to Solve the Multigroup Diffusion Equations, British Report TRG-Report-229, Mar. 8, 1962.

19. C. B. Mills, Neutron Cross Sections for Fast and Intermediate Nuclear Reactors, USAEC Report LAMS-2255, Los Alamos Scientific Laboratory, 1959.

20. E. Fisher, Streaming of Neutrons in Shields, Nucl. Sci. Eng., 1(3): 222-238 (1956).

21. T. Rockwell III (Ed.), Reactor Shielding Design Manual, USAEC Report TID-7004, 1956.

22. J. Adamson, A. M. Judd, and N. I. McNair, Neutron Flux in the Shield of the Dounreay Fast Reactor, J. Brit. Nucl. Energy Soc., 4: 119-126 (1965).

23. D. C. G. Smith, Physics of the Dounreay Fast Reactor, in Physics of Fast and Intermediate Reactors, Symposium Proceedings, Vienna, 1961, International Atomic Energy Agency, Vienna, 1962 (STI/PUB/49).

24. E. Aalto, R. Sandlin, and A. Krell, Some Comparisons of Measured and Predicted Primary Radiation Levels in the Ågesta Power Plant, Swedish Report AE-325, 1968.

25. L. Hjarne and M. Leimdorfer, A Method for Predicting the Penetration and Slowing Down of Neutrons in Reactor Shields, Nucl. Sci. Eng., 24: 165-174 (1966).

26. M. N. Bjeldanes, Pathfinder, Nucl. News, 13(6): 56 (June 1970).

27. R. J. Holl and D. W. Stephen, Pathfinder Atomic Power Plant Shielding Analysis, USAEC Report ACNP-62016, Allis-Chalmers Manufacturing Company, July 15, 1962.

28. J. W. Lewellen, P. S. Lacy, and T. M. Raby, Pathfinder Atomic Power Plant. Shielding and Radiation Survey Measurements, USAEC Report ACNP-67520, Allis-Chalmers Manufacturing Company, June 1967.

29. W. R. Smith and M. A. Turner, Nuclear Merchant Ship Reactor Shield Design Summary Report, USAEC Report BAW-1144-1, Babcock \& Wilcox Company, Aug. 1, 1959. 
30. W. R. Smith, Nuclear Merchant Ship Reactor, Shield Fabrication for the NMSR, USAEC Report BAW-1111, Babcock \& Wilcox Company, May 1958.

31. E. P. Blizard, T. V. Blosser, and R. M. Freestone, Jr., Radiation Leakage Survey of the Shield of the Nuclear Ship Savannah, USAEC Report ORNL-3336, Oak Ridge National Laboratory, Aug. 29, 1962.

32. Samuel Glasstone, Sourcebook on Atomi: Energy, 3rd ed., p. 612, D. Van Nostrand Co., Inc., Princeton, N. J., 1967.

33. J. A. Belcher, W. A. Flynn, and R. J. Thornson, SNAP 10A Radiation Shield Analysis, USAEC Report NAA-SR-9647, Atomics International, Oct. 15, 1964.

34. C. M. Bennett, J. J. Krakar, and W. G. Kruse, SNAP-10A Flight Vehicle Nuclear Environment and Radiation Effects, USAEC Report TID-22418(LMSC-A764793), Lockheed Missiles and Space Company, 1965. 


\section{Gamma Rays from Inelastic Neutron Scattering and Fission}

\section{Appendix A}

Table A.1 gives representative data on important elements in the neutronenergy region of interest. Table A.2 gives gamma-ray energy release vs. time after fission.

Table A.1-GAMMA RAYS FROM INELASTIC NEUTRON SCATTERING $\dagger \ddagger$

\begin{tabular}{|c|c|c|c|c|c|c|c|c|c|c|}
\hline \multirow[b]{2}{*}{$Z$} & \multirow[b]{2}{*}{ Element } & \multirow{2}{*}{$\begin{array}{c}\text { Energy of } \\
\text { prominent } \\
\text { gamma rays, } \\
\text { MeV }\end{array}$} & \multirow{2}{*}{$\begin{array}{c}\text { Average } \\
\text { anisotropy, } \\
\mathbf{W}\left(0^{\circ}\right) / \mathbf{W}\left(90^{\circ}\right)\end{array}$} & \multicolumn{7}{|c|}{$\begin{array}{c}d \sigma / d \Omega\left(90^{\circ}\right), \mathrm{mb} / \mathrm{sr} \\
E_{n}, \mathrm{MeV}\end{array}$} \\
\hline & & & & 1.0 & 1.5 & 2.0 & 3.0 & 3.5 & 4.0 & 5.0 \\
\hline 3 & $\mathbf{L i}$ & 0.478 & 1.0 & 15 & & & & & 22 & \\
\hline \multirow[t]{7}{*}{5} & ${ }^{10} \mathrm{~B}$ & $0.478(n, \alpha)$ & $1.0 \ddagger$ & 21.3 & 8.9 & 13.0 & 9.5 & 8.2 & 10.6 & \\
\hline & & 0.717 & $1.0+$ & & 0.3 & 3.4 & 4.0 & 3.8 & 5.4 & \\
\hline & & 1.02 & & & & & & 1.1 & 1.2 & \\
\hline & & 1.43 & & & & & & & 0.7 & \\
\hline & & 2.15 & & & & & & 0.5 & 0.9 & \\
\hline & & 2.86 & & & & & & & 0.5 & \\
\hline & & $3.37(n, p)$ & & & & & & & 0.3 & \\
\hline 5 & ${ }^{11} \mathrm{~B}$ & 2.13 & 1.0 & & & & 0.5 & 2.3 & 5.3 & \\
\hline 6 & C & 4.4 & 2.0 & & & & & & & 4.0 \\
\hline \multirow[t]{4}{*}{7} & $\mathbf{N}$ & 1.6 & & & & & & & 0.1 & 0.2 \\
\hline & & 2.31 & & & & & $<0.1$ & $<0.1$ & 0.2 & 0.3 \\
\hline & & 4.90 & & & & & & & & \\
\hline & & 5.10 & & & & & & & & \\
\hline \multirow[t]{2}{*}{9} & $\mathbf{F}$ & 0.110 & 1.0 & 25 & & & & & & \\
\hline & & 0.198 & & 66 & & & & & & \\
\hline \multirow[t]{3}{*}{11} & ${ }^{23} \mathrm{Na}$ & 0.44 & 1.0 & 42 & 44 & 45 & 44 & 45 & 50 & \\
\hline & & 0.63 & & & & & & & 2 & \\
\hline & & 1.64 & & & & & 5 & 10 & 15 & \\
\hline \multirow[t]{2}{*}{12} & ${ }^{24} \mathrm{Mg}$ & 1.37 & 1.4 & & & & 39 & 38 & 36 & \\
\hline & ${ }^{26} \mathrm{Mg}$ & 1.81 & 1.4 & & & & 47 & 64 & 70 & \\
\hline \multirow[t]{6}{*}{13} & ${ }^{27} \mathrm{Al}$ & 0.84 & 1.0 & & & & & 9.5 & 9.9 & 8.8 \\
\hline & & 1.01 & 1.0 & & & & & 18.1 & 19.5 & 18.6 \\
\hline & & 1.72 & 1.0 & & & & & 7.0 & 8.0 & 5.1 \\
\hline & & 2.21 & 1.0 & & & & & 17.0 & 16.8 & 14.6 \\
\hline & & 2.73 & 1.0 & & & & & 2.4 & 3.4 & 3.9 \\
\hline & & $\begin{array}{l}3.00 \\
\quad \text { (doublet) }\end{array}$ & 1.2 & & & & & 8.0 & 13.6 & 12.0 \\
\hline & ${ }^{28} \mathrm{Si}$ & 1.77 & 1.45 & & & & 32 & 30 & 39 & \\
\hline \multirow[t]{5}{*}{18} & ${ }^{40} \mathrm{Ar}$ & 0.68 & 1.0 & & & & 6.0 & 9.6 & 7.6 & 10.8 \\
\hline & & 1.08 & 0.9 & & & & 6.4 & 8.0 & 5.2 & 7.2 \\
\hline & & 1.45 & 1.3 & & & & 41 & 54 & 61 & 60 \\
\hline & & 1.77 & 1.8 & & & & & 2.2 & 6.5 & 9.2 \\
\hline & & 2.53 & 1.6 & & & & & 5.0 & 3.8 & 10.4 \\
\hline
\end{tabular}

(Table continues on next page.) 
Table A.1 - (Continued)

\begin{tabular}{|c|c|c|c|c|c|c|c|c|c|c|}
\hline \multirow[b]{2}{*}{$Z$} & \multirow[b]{2}{*}{ Element } & \multirow{2}{*}{$\begin{array}{c}\text { Energy of } \\
\text { prominent } \\
\text { gamma rays, } \\
\text { MeV }\end{array}$} & \multirow{2}{*}{$\begin{array}{c}\text { Average } \\
\text { anisotropy, } \\
\mathrm{W}\left(0^{\circ}\right) / \mathrm{W}\left(90^{\circ}\right)\end{array}$} & \multicolumn{7}{|c|}{$\begin{array}{c}d \sigma / d \Omega\left(90^{\circ}\right), \mathrm{mb} / \mathrm{sr} \\
E_{n}, \mathrm{MeV}\end{array}$} \\
\hline & & & & 1.0 & 1.5 & 2.0 & 3.0 & 3.5 & 4.0 & 5.0 \\
\hline \multirow[t]{3}{*}{19} & $\mathbf{K}$ & 2.52 & & & & & 5 & 7 & 10 & \\
\hline & & 2.81 & & & & & 1.5 & 7 & 10 & \\
\hline & & 3.05 & & & & & & 3 & 7 & \\
\hline \multirow[t]{6}{*}{20} & & 0.51 & & & & & & 0.5 & 6.9 & 15.2 \\
\hline & (Nat.) & & & & & & & & & \\
\hline & & 0.768 & 0.9 & & & & & 4.6 & 5.8 & 10.4 \\
\hline & & 1.158 & & & & & & 3.7 & 2.1 & 3.0 \\
\hline & & 3.730 & 2.4 & & & & & & 2.6 & 7.7 \\
\hline & & 3.900 & 1.7 & & & & & & 0.5 & 7.0 \\
\hline \multirow[t]{6}{*}{24} & ${ }^{52} \mathrm{Cr}$ & 1.43 & 1.4 & & & 40 & 60 & 40 & & \\
\hline & & 2.37 & 1.3 & & & & 3 & 6 & & \\
\hline & & 2.65 & & & & & 4 & 4 & & \\
\hline & & 2.77 & & & & & 1 & 4 & 7 & \\
\hline & & 2.96 & & & & & & 14 & & \\
\hline & & 3.16 & & & & & & 5 & & \\
\hline \multirow[t]{9}{*}{26} & ${ }^{56} \mathrm{Fe}$ & 0.845 & 1.8 & 21.1 & 35.5 & 83.0 & 86.0 & 89.2 & 107.6 & \\
\hline & & 1.24 & 1.6 & & & & 9.7 & 10.4 & 17.2 & \\
\hline & & 1.81 & 1.4 & & & & 12.3 & 11.3 & 14.9 & \\
\hline & & 2.12 & 1.5 & & & & & 8.4 & 12.1 & \\
\hline & & 2.30 & 1.1 & & & & & 4.8 & 6.8 & \\
\hline & & 2.60 & 1.0 & & & & & & 9.2 & \\
\hline & & 3.45 & 0.9 & & & & & & 4.2 & \\
\hline & & 2.76 & 1.08 & & & & & & 3.2 & \\
\hline & & 3.60 & 1.27 & & & & & & 2.8 & \\
\hline \multirow[t]{11}{*}{26} & ${ }^{54} \mathrm{Fe}$ & 1.41 & 1.2 & & & & 65.1 & 61.5 & 69.5 & 80.3 \\
\hline & & 1.13 & 1.25 & & & & 13.0 & 17.3 & 22.8 & 27.2 \\
\hline & & 1.56 & $>1.3$ & & & & & 4.6 & 8.3 & 6.9 \\
\hline & & 2.96 & 1.55 & & & & & 6.1 & 7.7 & 5.6 \\
\hline & & 1.75 & $<1.2$ & & & & & 1.6 & 2.5 & 4.1 \\
\hline & & 3.16 & 1.48 & & & & & 5.2 & 8.1 & 8.3 \\
\hline & & 1.93 & 1.7 & & & & & & 4.6 & 7.1 \\
\hline & & 2.42 & & & & & & & & 4.0 \\
\hline & & 2.66 & & & & & & & & 4.5 \\
\hline & & 2.85 & & & & & & & & 1.6 \\
\hline & & 3.37 & & & & & & & & 1.8 \\
\hline \multirow[t]{10}{*}{27} & ${ }^{59} \mathrm{Co}$ & 0.55 & & & & & 16.9 & 19.2 & 22.4 & 29.4 \\
\hline & & 0.68 & & & & & 15.3 & 15.7 & 15.8 & 18.2 \\
\hline & & 1.10 & 1.0 & & 5.6 & & 14.2 & 13.3 & 11.9 & 14.6 \\
\hline & & 1.19 & 1.0 & & 12.6 & & 37.9 & 33.4 & 34.7 & 35.5 \\
\hline & & 1.29 & 1.0 & & 2.7 & & 11.6 & 11.2 & 9.3 & 10.1 \\
\hline & & 1.48 & 1.0 & & 6.4 & & 32.8 & 32.4 & 31.7 & 33.8 \\
\hline & & 1.74 & & & & & 10.9 & 11.7 & 13.0 & 15.2 \\
\hline & & 2.10 & & & & & 8.1 & 12.6 & 15.3 & 18.7 \\
\hline & & 2.50 & & & & & 7.2 & 6.7 & 8.2 & 10.4 \\
\hline & & 2.80 & & & & & 3.3 & 4.0 & 3.9 & 5.2 \\
\hline \multirow[t]{15}{*}{39} & ${ }^{89} Y$ & 0.71 & 0.75 & & & & 5.6 & 5.7 & 5.5 & 5.4 \\
\hline & & 1.31 & 1.1 & & & & 6.0 & 8.5 & 5.7 & 7.6 \\
\hline & & 1.51 & 0.85 & & & & 31.8 & 35.0 & 27.8 & 24.6 \\
\hline & & 1.62 & 0.85 & & & & 4.7 & 8.2 & 7.5 & 7.9 \\
\hline & & 1.75 & 1.25 & & & & 27.7 & 29.6 & 22.6 & 20.2 \\
\hline & & 1.95 & 0.75 & & & & 2.5 & 5.4 & 5.4 & 4.8 \\
\hline & & 1.98 & & & & & & & 3.9 & 4.5 \\
\hline & & 2.10 & & & & & & & & 4.5 \\
\hline & & 2.36 & & & & & & & & 2.6 \\
\hline & & 2.56 & & & & & & & 7.0 & 7.5 \\
\hline & & 2.70 & & & & & & & & 2.7 \\
\hline & & 2.86 & 0.75 & & & & 3.6 & 11.6 & 10.0 & 9.0 \\
\hline & & 3.05 & & & & & & 14.5 & 16.4 & 12.7 \\
\hline & & 3.49 & & & & & & & 2.7 & 2.9 \\
\hline & & 3.85 & & & & & & & & 3.2 \\
\hline
\end{tabular}


Table A.1-(Continued)

\begin{tabular}{|c|c|c|c|c|c|c|c|c|c|c|}
\hline \multirow[b]{2}{*}{7} & \multirow[b]{2}{*}{ Element } & \multirow{2}{*}{$\begin{array}{c}\text { Energy of } \\
\text { prominent } \\
\text { gamma rays, } \\
\text { MeV }\end{array}$} & \multirow{2}{*}{$\begin{array}{c}\text { Average } \\
\text { anisotropy, } \\
\mathrm{W}\left(0^{\circ}\right) / \mathrm{W}\left(90^{\circ}\right)\end{array}$} & \multicolumn{7}{|c|}{$\begin{array}{c}\mathrm{d \sigma} / d \Omega\left(90^{\circ}\right), \mathrm{mb} / \mathrm{sr} \\
E_{n}, \mathrm{MeV}\end{array}$} \\
\hline & & & & 1.0 & 1.5 & 2.0 & 3.0 & 3.5 & 4.0 & 5.0 \\
\hline \multirow[t]{4}{*}{40} & ${ }^{90} \mathrm{Zr}$ & 0.43 & & & & & 10 & 10 & & \\
\hline & & 0.57 & & & & & 13 & 14 & & \\
\hline & & 0.89 & & & & & & 7 & & \\
\hline & & 2.18 & & & & & 51 & 50 & 48 & 48 \\
\hline \multirow[t]{7}{*}{40} & ${ }^{92} \mathrm{Zr}$ & 0.46 & & & 4 & 18 & 10 & 10 & & \\
\hline & & 0.57 & & & 1 & 20 & 20 & 23 & & \\
\hline & & 0.93 & & 10 & 80 & 100 & 130 & 130 & & \\
\hline & & 1.12 & & & & & 20 & 25 & & \\
\hline & & 1.41 & & & & & 10 & 10 & & \\
\hline & & 1.84 & & & & 2 & 8 & 8 & & \\
\hline & & 2.16 & & & & & 4 & 5 & & \\
\hline \multirow[t]{2}{*}{82} & ${ }^{206} \mathrm{~Pb}$ & 0.54 & $0.7(4 \mathrm{MeV})$ & & & & 65 & 97 & 117 & \\
\hline & & 0.80 & $1.2(4 \mathrm{MeV})$ & 13 & 43 & & 118 & 175 & 182 & \\
\hline \multirow[t]{6}{*}{82} & ${ }^{207} \mathrm{~Pb}$ & 0.57 & $1.0(4 \mathrm{MeV})$ & 18 & 42 & & 74 & 137 & 150 & \\
\hline & & 0.894 & $0.82(4 \mathrm{MeV})$ & & 14 & & 43 & 61 & 63 & \\
\hline & & 1.08 & & & & & 10 & 14 & 25 & \\
\hline & & 1.77 & $1.0(4 \mathrm{MeV})$ & & & & 14 & 30 & 44 & \\
\hline & & 2.12 & $1.0(4 \mathrm{MeV})$ & & & & 8 & 16 & 26 & \\
\hline & & 2.64 & $1.32(4 \mathrm{MeV})$ & & & & 2 & 8 & 22 & \\
\hline \multirow[t]{4}{*}{82} & ${ }^{208} \mathrm{~Pb}$ & 0.58 & & & & & & 7 & 48 & \\
\hline & & 0.76 & & & & & & & 7 & \\
\hline & & 0.86 & & & & & & & 16 & \\
\hline & & 2.61 & 1.75 & & & & 40 & 55 & 87 & \\
\hline
\end{tabular}

†From I. L. Morgan, Gamma Rays from Inelastic Neutron Scattering, in Engineering Compendium on Radiation Shielding, Vol. I, R. G. Jaeger (Ed.), Sec. 2.3.2.2, Springer-Verlag, New York, 1968. $\ddagger d \sigma / d \Omega\left(55^{\circ}\right)$. 
Table A.2-GAMMA-RAY ENERGY RELEASE VS. TIME AFTER FISSION $\nmid$

\begin{tabular}{|c|c|c|c|c|c|c|c|c|c|c|}
\hline Time & $\boldsymbol{D}$ & B & $\Gamma_{T}$ & $\Gamma_{1}$ & $\Gamma_{2}$ & $\Gamma_{3}$ & $\Gamma_{4}$ & $\Gamma_{s}$ & $r_{6}$ & $r_{7}$ \\
\hline \multicolumn{11}{|c|}{ The Values are for $10^{10}$ Instantaneous Fissions } \\
\hline 100 & $239 \cdot 10^{5}$ & $347 \cdot 10^{5}$ & $349 \cdot 10^{5}$ & $634 \cdot 10^{3}$ & $279 \cdot 10^{4}$ & $111 \cdot 10^{5}$ & $474 \cdot 10^{4}$ & $979 \cdot 10^{3}$ & $276 \cdot 10^{4}$ & $119 \cdot 10^{5}$ \\
\hline 200 & $159 \cdot 10^{5}$ & $2 \cdot 10^{5}$ & $230 \cdot 10^{5}$ & & $165 \cdot 10^{4}$ & & $374 \cdot 10^{4}$ & & $201 \cdot 10^{4}$ & $778 \cdot 10^{4}$ \\
\hline 400 & $914 \cdot 10^{4}$ & $988 \cdot 10^{4}$ & $121 \cdot 10^{5}$ & $373 \cdot 10^{3}$ & $946 \cdot 10^{3}$ & $328 \cdot 10^{4}$ & $234 \cdot 10^{4}$ & $544 \cdot 10^{3}$ & $117 \cdot 10^{4}$ & $344 \cdot 10^{4}$ \\
\hline $100 \cdot 10^{1}$ & $421 \cdot 10^{4}$ & $403 \cdot 10^{4}$ & $443 \cdot 10^{4}$ & $249 \cdot 10^{3}$ & $584 \cdot 10^{3}$ & $139 \cdot 10^{4}$ & $901 \cdot 10^{3}$ & $287 \cdot 10^{3}$ & $391 \cdot 10^{3}$ & $618 \cdot 10^{3}$ \\
\hline $200 \cdot 10^{1}$ & $226 \cdot 10^{4}$ & $200 \cdot 10^{4}$ & $224 \cdot 10^{4}$ & $151 \cdot 10^{3}$ & $429 \cdot 10^{3}$ & $656 \cdot 10^{3}$ & $463 \cdot 10^{3}$ & $156 \cdot 10^{3}$ & $180 \cdot 10^{3}$ & $204 \cdot 10^{3}$ \\
\hline $400 \cdot 10^{1}$ & $105 \cdot 10^{4}$ & $841 \cdot 10^{3}$ & $106 \cdot 10^{4}$ & $644 \cdot 10^{2}$ & $301 \cdot 10^{3}$ & $229 \cdot 10^{3}$ & $254 \cdot 10^{3}$ & $654 \cdot 10^{2}$ & $961 \cdot 10^{2}$ & $647 \cdot 10^{2}$ \\
\hline $100 \cdot 10^{2}$ & $361 \cdot 10^{3}$ & $257 \cdot 10^{3}$ & $362 \cdot 10^{3}$ & $156 \cdot 10^{2}$ & $133 \cdot 10^{3}$ & $633 \cdot 10^{2}$ & $863 \cdot 10^{2}$ & $223 \cdot 10^{2}$ & $309 \cdot 10^{2}$ & $969 \cdot 10^{1}$ \\
\hline $200 \cdot 10^{2}$ & $167 \cdot 10^{3}$ & $112 \cdot 10^{3}$ & $128 \cdot 10^{3}$ & $632 \cdot 10^{1}$ & $456 \cdot 10^{2}$ & $\cdot 10^{2}$ & $310 \cdot 10^{2}$ & $996 \cdot 10^{1}$ & $926 \cdot 10^{1}$ & $212 \cdot 10^{1}$ \\
\hline $400 \cdot 10^{2}$ & $821 \cdot 10^{2}$ & $484 \cdot 10^{2}$ & $482 \cdot 10^{2}$ & $341 \cdot 10^{1}$ & $201 \cdot 10^{2}$ & $994 \cdot 10^{1}$ & $984 \cdot 10^{1}$ & $271 \cdot 10^{1}$ & $161 \cdot 10^{1}$ & 301 \\
\hline $700 \cdot 10^{2}$ & $445 \cdot 10^{2}$ & $218 \cdot 10^{2}$ & $235 \cdot 10^{2}$ & & $125 \cdot 10^{2}$ & $440 \cdot 10^{1}$ & $319 \cdot 10^{1}$ & 605 & 232 & $04 \cdot 1 \sigma^{1}$ \\
\hline $100 \cdot 10^{3}$ & $289 \cdot 10^{2}$ & $124 \cdot 10^{2}$ & $144 \cdot 10^{2}$ & $177 \cdot 10^{1}$ & $863 \cdot 10^{1}$ & $219 \cdot 10^{1}$ & $148 \cdot 10^{1}$ & 230 & $781 \cdot 1 \sigma^{1}$ & $412 \cdot 10^{-2}$ \\
\hline $200 \cdot 10^{3}$ & $118 \cdot 10^{2}$ & $406 \cdot 10^{1}$ & $549 \cdot 10^{1}$ & 814 & $343 \cdot 10^{1}$ & 508 & 563 & 772 . & $540 \cdot 1 \sigma^{-1}$ & $97 \cdot 1 \sigma^{3}$ \\
\hline $400 \cdot 10^{3}$ & $525 \cdot 10^{1}$ & $149 \cdot 10^{1}$ & $245 \cdot 10^{1}$ & 376 & $124 \cdot 10^{3}$ & 1 & 51 & $437 \cdot 1 \sigma^{1}$ & $477 \cdot 1 \sigma^{1}$ & $877 \cdot 1 \sigma^{-3}$ \\
\hline 100 & $203 \cdot 10^{1}$ & 574 & $100 \cdot 10^{1}$ & 111 & 401 & $\cdot 10^{-1}$ & 387 & $101 \cdot 10^{-1}$ & $282 \cdot 1 \sigma^{-1}$ & $731 \cdot 1 \sigma^{-3}$ \\
\hline 200 & 955 & 289 & 455 & $371 \cdot 10^{-1}$ & 180 & $163 \cdot 1 \sigma^{-1}$ & 203 & $110 \cdot 1 \sigma^{2}$ & $133 \cdot 1 \sigma^{1}$ & $398 \cdot 10^{3}$ \\
\hline $400 \cdot 10^{4}$ & 447 & 134 & 177 & $5^{-1}$ & $992 \cdot 1 \sigma^{1}$ & & 576 . & $255^{\circ}$ & $369 \cdot 1 \sigma^{2}$ & $\cdot 10^{-3}$ \\
\hline $100 \cdot 10^{5}$ & 154 & $466 \cdot 10^{-1}$ & $498 \cdot 10^{-1}$ & $\sigma^{2}$ & $\cdot 10^{-1}$ & $\sigma^{3}$ & $140 \cdot 10^{-2}$ & $211 \cdot 10^{3}$ & $903 \cdot 1 \sigma^{4}$ & $463 \cdot 10^{-5}$ \\
\hline & $544 \cdot$ & $201 \cdot 10^{-1}$ & 160 & & & & $488 \cdot 10^{-4}$ & $160^{\circ}$ & $393 \cdot 1 \sigma^{-5}$ & $10^{5}$ \\
\hline & $168 \cdot 10^{-1}$ & $840 \cdot 10^{-2}$ & 196 & $645 \cdot 10^{-4}$ & 174. & 114 & $271 \cdot 1 \sigma^{4}$ & $913 \cdot 10^{-4}$ & $243 \cdot 10^{-5}$ & $104 \cdot 10^{-5}$ \\
\hline $100 \cdot 10^{6}$ & $471 \cdot 10^{-2}$ & $200 \cdot 10^{-2}$ & $313 \cdot 10^{-3}$ & $116 \cdot 1 \sigma^{-4}$ & $271 \cdot 1 \sigma^{3}$ & $259 \cdot 1 \sigma^{5}$ & $553 \cdot 10^{-5}$ & $171 \cdot 10^{-4}$ & $649 \cdot 1 \sigma^{6}$ & $278 \cdot 10^{6}$ \\
\hline \multicolumn{11}{|c|}{ The Reactor Operating Time Was 1 hr at 1 watt } \\
\hline 100 & & & & $229 \cdot 10^{7}$ & $658 \cdot 10^{7}$ & $153 \cdot 10^{8}$ & & & & \\
\hline 200 & $390 \cdot 10^{8}$ & $382 \cdot 10^{8}$ & & & & & $888 \cdot 10^{7}$ & 25 & $0^{7}$ & $10^{7}$ \\
\hline 400 & $319 \cdot 10^{8}$ & $296 \cdot 10^{8}$ & $342 \cdot 10^{8}$ & $189 \cdot 10^{7}$ & 538 & $976 \cdot 10^{7}$ & $710 \cdot 10^{7}$ & 212 . & $301 \cdot 10^{7}$ & $\cdot 10^{7}$ \\
\hline $100 \cdot 10^{1}$ & $220 \cdot 10^{8}$ & $192 \cdot 10^{8}$ & $222 \cdot 10^{8}$ & $141 \cdot 10^{7}$ & $455 \cdot 10^{7}$ & $612 \cdot 10^{7}$ & $475 \cdot 10^{7}$ & $148 \cdot 10^{7}$ & $187 \cdot 10^{7}$ & $200 \cdot 10^{7}$ \\
\hline $200 \cdot 10^{1}$ & $145 \cdot 10^{8}$ & $120 \cdot 10^{8}$ & $148 \cdot 10^{8}$ & $918 \cdot 10^{6}$ & $377 \cdot 10^{7}$ & $348 \cdot 10^{7}$ & $336 \cdot 10^{7}$ & $939 \cdot 10^{6}$ & $128 \cdot 10^{7}$ & $102 \cdot 10^{7}$ \\
\hline $400 \cdot 10^{1}$ & $835 \cdot 10^{7}$ & $640 \cdot 10^{7}$ & $870 \cdot 10^{7}$ & $455 \cdot 10^{6}$ & $279 \cdot 10^{7}$ & $165 \cdot 10^{7}$ & $211 \cdot 10^{7}$ & $500 \cdot 10^{6}$ & $783 \cdot 10^{6}$ & $399 \cdot 10^{6}$ \\
\hline $100 \cdot 10^{2}$ & $359 \cdot 10^{7}$ & $251 \cdot 10^{7}$ & $345 \cdot 10^{7}$ & $149 \cdot 10^{6}$ & $127 \cdot 10^{7}$ & $605 \cdot 10^{6}$ & $819 \cdot 10^{6}$ & $225 \cdot 10^{6}$ & $287 \cdot 10^{6}$ & $828 \cdot 10^{5}$ \\
\hline $200 \cdot 10^{2}$ & $181 \cdot 10^{7}$ & $120 \cdot 10^{7}$ & $133 \cdot 10^{7}$ & $680 \cdot 10^{5}$ & $474 \cdot 10^{6}$ & $247 \cdot 10^{6}$ & $322 \cdot 10^{6}$ & $104 \cdot 10^{6}$ & $920 \cdot 10^{5}$ & $206 \cdot 10^{5}$ \\
\hline $400 \cdot 10^{2}$ & $930 \cdot 10^{6}$ & $542 \cdot 10^{6}$ & $540 \cdot 10^{6}$ & $393 \cdot 10^{5}$ & $230 \cdot 10^{6}$ & $112 \cdot 10^{6}$ & $108 \cdot 10^{6}$ & $289 \cdot 10^{5}$ & $167 \cdot 10^{5}$ & $307 \cdot 10^{4}$ \\
\hline $700 \cdot 10^{2}$ & $512 \cdot 10^{6}$ & $248 \cdot 10^{6}$ & $269 \cdot 10^{6}$ & $277 \cdot 10^{5}$ & $145 \cdot 10^{6}$ & $498 \cdot 10^{5}$ & $357 \cdot 10^{5}$ & $667 \cdot 10^{4}$ & $251 \cdot 10^{4}$ & $318 \cdot 10^{3}$ \\
\hline $100 \cdot 10^{3}$ & $335 \cdot 10^{6}$ & $143 \cdot 10^{6}$ & $167 \cdot 10^{6}$ & $206 \cdot 10^{5}$ & $100 \cdot 10^{6}$ & $250 \cdot 10^{5}$ & $169 \cdot 10^{5}$ & $262 \cdot 10^{4}$ & $894 \cdot 10^{3}$ & $439 \cdot 10^{2}$ \\
\hline $200 \cdot 10^{3}$ & $138 \cdot 10^{6}$ & $474 \cdot 10^{5}$ & $642 \cdot 10^{5}$ & $953 \cdot 10^{4}$ & $401 \cdot 10^{5}$ & $592 \cdot 10^{4}$ & $664 \cdot 10^{4}$ & $907 \cdot 10^{3}$ & $639 \cdot 10^{3}$ & $828 \cdot 10^{1}$ \\
\hline $400 \cdot 10^{3}$ & $619 \cdot 10^{5}$ & $175 \cdot 10^{5}$ & $289 \cdot 10^{5}$ & $443 \cdot 10^{4}$ & $147 \cdot 10^{5}$ & $230 \cdot 10^{4}$ & $614 \cdot 10^{4}$ & $515 \cdot 10^{3}$ & $565 \cdot 10^{3}$ & $104 \cdot 10^{2}$ \\
\hline $100 \cdot 10^{4}$ & $240 \cdot 10^{5}$ & $679 \cdot 10^{4}$ & $119 \cdot 10^{5}$ & $131 \cdot 10^{4}$ & $474 \cdot 10^{4}$ & $645 \cdot 10^{3}$ & $458 \cdot 10^{4}$ & $119 \cdot 10^{3}$ & $333 \cdot 10^{3}$ & $865 \cdot 10^{1}$ \\
\hline & $113 \cdot 10^{5}$ & $342 \cdot 10^{4}$ & $538 \cdot 10^{4}$ & $438 \cdot 10^{3}$ & $213 \cdot 10^{4}$ & $193 \cdot 10^{3}$ & $240 \cdot 10^{4}$ & $129 \cdot 10^{2}$ & $157 \cdot 10^{3}$ & $470 \cdot 10^{1}$ \\
\hline $400 \cdot 10^{4}$ & $529 \cdot 10^{4}$ & $159 \cdot 10^{4}$ & $209 \cdot 10^{4}$ & $130 \cdot 10^{3}$ & $117 \cdot 10^{4}$ & $511 \cdot 10^{2}$ & $682 \cdot 10^{3}$ & $302 \cdot 10^{1}$ & $437 \cdot 10^{2}$ & $136 \cdot 10^{1}$ \\
\hline $100 \cdot 10^{5}$ & $182 \cdot 10^{4}$ & $551 \cdot 10^{3}$ & $589 \cdot 10^{3}$ & $178 \cdot 10^{2}$ & $547 \cdot 10^{3}$ & $325 \cdot 10^{1}$ & $165 \cdot 10^{2}$ & $250 \cdot 10^{1}$ & $107 \cdot 10^{1}$ & $548 \cdot 1 \sigma^{-1}$ \\
\hline $200 \cdot 10^{5}$ & $644 \cdot 10^{3}$ & $238 \cdot 10^{3}$ & $190 \cdot 10^{3}$ & $267 \cdot 10^{1}$ & $183 \cdot 10^{3}$ & 535 & 577 & $189 \cdot 10^{1}$ & $466 \cdot 1 \sigma^{1}$ & $192 \cdot 1 \sigma^{1}$ \\
\hline $400 \cdot 10^{5}$ & $199 \cdot 10^{3}$ & $995 \cdot 10^{2}$ & $232 \cdot 10^{2}$ & 764 & $206 \cdot 10^{2}$ & 135 & 321 & $108 \cdot 10^{1}$ & $287 \cdot 1 \sigma^{1}$ & $123 \cdot 10^{-1}$ \\
\hline $100 \cdot 10^{6}$ & $558 \cdot 10^{2}$ & $237 \cdot 10^{2}$ & $370 \cdot 10^{1}$ & 137 & $321 \cdot 10^{1}$ & $307 \cdot 1 \sigma^{1}$ & $654 \cdot 1 \sigma^{1}$ & 203 & $768 \cdot 1 \sigma^{2}$ & $329 \cdot 10^{-2}$ \\
\hline
\end{tabular}


The Reactor Operating Time Was 10 hr at 1 watt

\begin{tabular}{|c|c|c|c|c|c|c|c|c|c|c|}
\hline & & & & & & & & & & \\
\hline 100 & $722 \cdot 10^{8}$ & $659 \cdot 10^{8}$ & $780 \cdot 10^{8}$ & $352 \cdot 10^{7}$ & $151 \cdot 10^{8}$ & $199 \cdot 10^{8}$ & $161 \cdot 10^{8}$ & $436 \cdot 10^{7}$ & $670 \cdot 10^{7}$ & $122 \cdot 10^{8}$ \\
\hline 200 & $658 \cdot 10^{8}$ & $572 \cdot 10^{8}$ & $686 \cdot 10^{8}$ & $334 \cdot 10^{7}$ & $144 \cdot 10^{8}$ & $171 \cdot 10^{8}$ & $147 \cdot 10^{8}$ & $407 \cdot 10^{7}$ & $592 \cdot 10^{7}$ & $904 \cdot 10^{7}$ \\
\hline 400 & $580 \cdot 10^{8}$ & $481 \cdot 10^{8}$ & $577 \cdot 10^{8}$ & $306 \cdot 10^{7}$ & $136 \cdot 10^{8}$ & $141 \cdot 10^{8}$ & $127 \cdot 10^{8}$ & $364 \cdot 10^{7}$ & $492 \cdot 10^{7}$ & $558 \cdot 10^{7}$ \\
\hline $100 \cdot 10^{1}$ & $463 \cdot 10^{8}$ & $362 \cdot 10^{8}$ & $439 \cdot 10^{8}$ & $248 \cdot 10^{7}$ & $122 \cdot 10^{8}$ & $101 \cdot 10^{8}$ & $995 \cdot 10^{7}$ & $289 \cdot 10^{7}$ & $360 \cdot 10^{7}$ & $259 \cdot 10^{7}$ \\
\hline $200 \cdot 10^{1}$ & $366 \cdot 10^{8}$ & $272 \cdot 10^{8}$ & $339 \cdot 10^{8}$ & $185 \cdot 10^{7}$ & $107 \cdot 10^{8}$ & $696 \cdot 10^{7}$ & $793 \cdot 10^{7}$ & $220 \cdot 10^{7}$ & $277 \cdot 10^{7}$ & $148 \cdot 10^{7}$ \\
\hline $400 \cdot 10^{1}$ & $271 \cdot 10^{8}$ & $190 \cdot 10^{8}$ & $242 \cdot 10^{8}$ & $121 \cdot 10^{7}$ & $846 \cdot 10^{7}$ & $448 \cdot 10^{7}$ & $577 \cdot 10^{7}$ & $155 \cdot 10^{7}$ & $192 \cdot 10^{7}$ & $712 \cdot 10^{6}$ \\
\hline $100 \cdot 10^{2}$ & $170 \cdot 10^{8}$ & $111 \cdot 10^{8}$ & $129 \cdot 10^{8}$ & $672 \cdot 10^{6}$ & $481 \cdot 10^{7}$ & $241 \cdot 10^{7}$ & $301 \cdot 10^{7}$ & $898 \cdot 10^{6}$ & $871 \cdot 10^{6}$ & $215 \cdot 10^{6}$ \\
\hline $200 \cdot 10^{2}$ & $111 \cdot 10^{8}$ & $680 \cdot 10^{7}$ & $702 \cdot 10^{7}$ & $451 \cdot 10^{6}$ & $278 \cdot 10^{7}$ & $139 \cdot 10^{7}$ & $153 \cdot 10^{7}$ & $453 \cdot 10^{6}$ & $326 \cdot 10^{6}$ & $672 \cdot 10^{5}$ \\
\hline $400 \cdot 10^{2}$ & $676 \cdot 10^{7}$ & $362 \cdot 10^{7}$ & $373 \cdot 10^{7}$ & $325 \cdot 10^{6}$ & $179 \cdot 10^{7}$ & $744 \cdot 10^{6}$ & $620 \cdot 10^{6}$ & $143 \cdot 10^{6}$ & $706 \cdot 10^{5}$ & $117 \cdot 10^{5}$ \\
\hline $700 \cdot 10^{2}$ & $408 \cdot 10^{7}$ & $185 \cdot 10^{7}$ & $209 \cdot 10^{7}$ & $237 \cdot 10^{6}$ & $119 \cdot 10^{7}$ & $350 \cdot 10^{6}$ & $241 \cdot 10^{6}$ & $410 \cdot 10^{5}$ & $143 \cdot 10^{5}$ & $134 \cdot 10^{4}$ \\
\hline $100 \cdot 10^{3}$ & $281 \cdot 10^{7}$ & $115 \cdot 10^{7}$ & $138 \cdot 10^{7}$ & $179 \cdot 10^{6}$ & $847 \cdot 10^{6}$ & $186 \cdot 10^{6}$ & $130 \cdot 10^{6}$ & $196 \cdot 10^{5}$ & $754 \cdot 10^{4}$ & $220 \cdot 10^{3}$ \\
\hline $200 \cdot 10^{3}$ & $126 \cdot 10^{7}$ & $421 \cdot 10^{6}$ & $582 \cdot 10^{6}$ & $873 \cdot 10^{5}$ & $359 \cdot 10^{6}$ & $522 \cdot 10^{5}$ & $645 \cdot 10^{5}$ & $851 \cdot 10^{4}$ & $635 \cdot 10^{4}$ & $857 \cdot 10^{2}$ \\
\hline $400 \cdot 10^{3}$ & $594 \cdot 10^{6}$ & $167 \cdot 10^{6}$ & $278 \cdot 10^{6}$ & $424 \cdot 10^{5}$ & $139 \cdot 10^{6}$ & $220 \cdot 10^{5}$ & $612 \cdot 10^{5}$ & $495 \cdot 10^{4}$ & $557 \cdot 10^{4}$ & $104 \cdot 10^{3}$ \\
\hline $100 \cdot 10^{4}$ & $236 \cdot 10^{6}$ & $668 \cdot 10^{5}$ & $117 \cdot 10^{6}$ & $128 \cdot 10^{5}$ & $465 \cdot 10^{5}$ & $628 \cdot 10^{4}$ & $453 \cdot 10^{5}$ & $115 \cdot 10^{4}$ & $328 \cdot 10^{4}$ & $857 \cdot 10^{2}$ \\
\hline $200 \cdot 10^{4}$ & $112 \cdot 10^{6}$ & $339 \cdot 10^{5}$ & $533 \cdot 10^{5}$ & $433 \cdot 10^{4}$ & $212 \cdot 10^{5}$ & $190 \cdot 10^{4}$ & $238 \cdot 10^{5}$ & $126 \cdot 10^{3}$ & $155 \cdot 10^{4}$ & $466 \cdot 10^{2}$ \\
\hline $400 \cdot 10^{4}$ & $526 \cdot 10^{5}$ & $158 \cdot 10^{5}$ & $208 \cdot 10^{5}$ & $129 \cdot 10^{4}$ & $117 \cdot 10^{5}$ & $506 \cdot 10^{3}$ & $675 \cdot 10^{4}$ & $302 \cdot 10^{2}$ & $432 \cdot 10^{3}$ & $135 \cdot 10^{2}$ \\
\hline $100 \cdot 10^{5}$ & $182 \cdot 10^{5}$ & $550 \cdot 10^{4}$ & $588 \cdot 10^{4}$ & $177 \cdot 10^{3}$ & $546 \cdot 10^{4}$ & $324 \cdot 10^{2}$ & $164 \cdot 10^{3}$ & $250 \cdot 10^{2}$ & $106 \cdot 10^{2}$ & 545 \\
\hline $200 \cdot 10^{5}$ & $644 \cdot 10^{4}$ & $238 \cdot 10^{4}$ & $189 \cdot 10^{4}$ & $267 \cdot 10^{2}$ & $183 \cdot 10^{4}$ & $534 \cdot 10^{1}$ & $577 \cdot 10^{1}$ & $189 \cdot 10^{2}$ & 465 & 192 \\
\hline $400 \cdot 10^{5}$ & $199 \cdot 10^{4}$ & $995 \cdot 10^{3}$ & $231 \cdot 10^{3}$ & $764 \cdot 10^{1}$ & $205 \cdot 10^{3}$ & $135 \cdot 10^{1}$ & $320 \cdot 10^{1}$ & $108 \cdot 10^{2}$ & 287 & 123 \\
\hline $100 \cdot 10^{6}$ & $558 \cdot 10^{3}$ & 237 & $370 \cdot 10^{2}$ & 137 & 321 & 307 & & $10^{1}$ & $768 \cdot 1 \sigma^{-1}$ & $329 \cdot 1 \sigma^{-1}$ \\
\hline & & & & 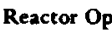 & $\theta$ & & & & & \\
\hline 100 & $930 \cdot 10^{8}$ & $751 \cdot 10^{8}$ & $886 \cdot 10^{8}$ & $475 \cdot 10^{7}$ & $209 \cdot 10^{8}$ & $215 \cdot 10^{8}$ & $175 \cdot 10^{8}$ & $464 \cdot 10^{7}$ & $685 \cdot 10^{7}$ & $123 \cdot 10^{8}$ \\
\hline 000 & $866 \cdot 10^{8}$ & $663 \cdot 10^{8}$ & $792 \cdot 10^{8}$ & $456 \cdot 10^{7}$ & $202 \cdot 10^{8}$ & $187 \cdot 10^{8}$ & $161 \cdot 10^{8}$ & $10^{7}$ & $607 \cdot 10^{7}$ & $906 \cdot 10^{7}$ \\
\hline 400 & $787 \cdot 10^{8}$ & $572 \cdot 10^{8}$ & $683 \cdot 10^{8}$ & $428 \cdot 10^{7}$ & $194 \cdot 10^{8}$ & $157 \cdot 10^{8}$ & 142 & $10^{7}$ & $506 \cdot 10^{7}$ & $559 \cdot 10^{7}$ \\
\hline $100 \cdot 10^{1}$ & $669 \cdot 10^{8}$ & $452 \cdot 10^{8}$ & $544 \cdot 10^{8}$ & $369 \cdot 10^{7}$ & $108 \cdot 10^{8}$ & $117 \cdot 10^{8}$ & $114 \cdot 10^{8}$ & $316 \cdot 10^{7}$ & $374 \cdot 10^{7}$ & $261 \cdot 10^{7}$ \\
\hline $200 \cdot 10^{1}$ & $569 \cdot 10^{\mathrm{z}}$ & $360 \cdot 10^{8}$ & $442 \cdot 10^{8}$ & $305 \cdot 10^{7}$ & $164 \cdot 10^{8}$ & $852 \cdot 10^{7}$ & $933 \cdot 10^{7}$ & $246 \cdot 10^{7}$ & $291 \cdot 10^{7}$ & $150 \cdot 10^{7}$ \\
\hline $400 \cdot 10^{1}$ & $469 \cdot 10^{8}$ & $275 \cdot 10^{8}$ & $341 \cdot 10^{8}$ & $240 \cdot 10^{7}$ & $140 \cdot 10^{8}$ & $596 \cdot 10^{7}$ & $710 \cdot 10^{7}$ & $179 \cdot 10^{7}$ & $205 \cdot 10^{7}$ & $725 \cdot 10^{6}$ \\
\hline $100 \cdot 10^{2}$ & $354 \cdot 10^{8}$ & $188 \cdot 10^{8}$ & $221 \cdot 10^{8}$ & $180 \cdot 10^{\prime}$ & $100 \cdot 10^{8}$ & $372 \cdot 10^{7}$ & $10^{7}$ & $110 \cdot 10^{7}$ & $973 \cdot 10^{6}$ & $224 \cdot 10^{6}$ \\
\hline $200 \cdot 10^{2}$ & $276 \cdot 10^{8}$ & $134 \cdot 10^{8}$ & $151 \cdot 10^{8}$ & $149 \cdot 10^{7}$ & $748 \cdot 10^{7}$ & $247 \cdot 10^{7}$ & $251 \cdot 10^{7}$ & $602 \cdot 10^{6}$ & $405 \cdot 10^{6}$ & $717 \cdot 10^{6}$ \\
\hline $400 \cdot 10^{2}$ & $205 \cdot 10^{8}$ & $870 \cdot 10^{7}$ & $103 \cdot 10^{8}$ & $123 \cdot 10^{7}$ & $571 \cdot 10^{7}$ & $151 \cdot 10^{7}$ & $139 \cdot 10^{7}$ & $247 \cdot 10^{6}$ & $132 \cdot 10^{6}$ & $134 \cdot 10^{5}$ \\
\hline $700 \cdot 10^{2}$ & $150 \cdot 10^{8}$ & $562 \cdot 10^{7}$ & $724 \cdot 10^{7}$ & $986 \cdot 10^{6}$ & $427 \cdot 10^{7}$ & $867 \cdot 10^{6}$ & $879 \cdot 10^{6}$ & $118 \cdot 10^{6}$ & $703 \cdot 10^{5}$ & $226 \cdot 10^{4}$ \\
\hline $100 \cdot 10^{3}$ & $120 \cdot 10^{8}$ & $413 \cdot 10^{7}$ & $564 \cdot 10^{7}$ & $816 \cdot 10^{6}$ & $335 \cdot 10^{7}$ & $575 \cdot 10^{6}$ & $716 \cdot 10^{6}$ & $853 \cdot 10^{5}$ & $620 \cdot 10^{5}$ & $107 \cdot 10^{4}$ \\
\hline $200 \cdot 10^{3}$ & $732 \cdot 10^{7}$ & $220 \cdot 10^{7}$ & $341 \cdot 10^{7}$ & $516 \cdot 10^{6}$ & $185 \cdot 10^{7}$ & $280 \cdot 10^{6}$ & $614 \cdot 10^{6}$ & $567 \cdot 10^{5}$ & $572 \cdot 10^{5}$ & $999 \cdot 10^{3}$ \\
\hline $400 \cdot 10^{3}$ & $440 \cdot 10^{7}$ & $121 \cdot 10^{7}$ & $210 \cdot 10^{7}$ & $297 \cdot 10^{6}$ & $967 \cdot 10^{6}$ & $152 \cdot 10^{6}$ & $579 \cdot 10^{6}$ & $343 \cdot 10^{5}$ & $486 \cdot 10^{5}$ & $103 \cdot 10^{4}$ \\
\hline $100 \cdot 10^{4}$ & $203 \cdot 10^{7}$ & $583 \cdot 10^{6}$ & $100 \cdot 10^{7}$ & $103 \cdot 10^{6}$ & $391 \cdot 10^{6}$ & $498 \cdot 10^{5}$ & $409 \cdot 10^{6}$ & $805 \cdot 10^{4}$ & $288 \cdot 10^{5}$ & $781 \cdot 10^{3}$ \\
\hline $200 \cdot 10^{4}$ & $103 \cdot 10^{7}$ & $314 \cdot 10^{6}$ & $486 \cdot 10^{6}$ & $383 \cdot 10^{5}$ & $197 \cdot 10^{6}$ & $167 \cdot 10^{5}$ & $215 \cdot 10^{6}$ & $965 \cdot 10^{3}$ & $140 \cdot 10^{5}$ & $422 \cdot 10^{3}$ \\
\hline $400 \cdot 10^{4}$ & $504 \cdot 10^{6}$ & $151 \cdot 10^{6}$ & $196 \cdot 10^{6}$ & $119 \cdot 10^{5}$ & $113 \cdot 10^{6}$ & $462 \cdot 10^{4}$ & $611 \cdot 10^{5}$ & $299 \cdot 10^{3}$ & $391 \cdot 10^{4}$ & $122 \cdot 10^{3}$ \\
\hline $100 \cdot 10^{5}$ & $178 \cdot 10^{6}$ & $540 \cdot 10^{5}$ & $576 \cdot 10^{5}$ & $170 \cdot 10^{4}$ & $537 \cdot 10^{5}$ & $308 \cdot 10^{3}$ & $149 \cdot 10^{4}$ & $249 \cdot 10^{3}$ & $963 \cdot 10^{2}$ & $515 \cdot 10^{1}$ \\
\hline & $635 \cdot 10^{5}$ & & $186 \cdot 10^{5}$ & $261 \cdot 10^{3}$ & $180 \cdot 10^{5}$ & $524 \cdot 10^{2}$ & $572 \cdot 10^{2}$ & $188 \cdot 10^{3}$ & $462 \cdot 10^{1}$ & $191 \cdot 10^{1}$ \\
\hline $400 \cdot 10^{5}$ & $197 \cdot 10^{5}$ & $990 \cdot 10^{4}$ & $228 \cdot 10^{4}$ & $760 \cdot 10^{2}$ & $202 \cdot 10^{4}$ & $134 \cdot 10^{2}$ & $319 \cdot 10^{2}$ & $108 \cdot 10^{3}$ & $286 \cdot 10^{1}$ & $123 \cdot 10^{1}$ \\
\hline $100 \cdot 10^{6}$ & $556 \cdot 10^{4}$ & $237 \cdot 10^{4}$ & $370 \cdot 10^{3}$ & $136 \cdot 10^{2}$ & $321 \cdot 10^{3}$ & $306 \cdot 10^{1}$ & $651 \cdot 10^{1}$ & $202 \cdot 10^{2}$ & 765 & 328 \\
\hline
\end{tabular}

(Table continues on next page.) 
Table A.2-(Continued)

\begin{tabular}{|c|c|c|c|c|c|c|c|c|c|c|}
\hline Time & D & $B$ & $\Gamma_{T}$ & $\Gamma_{1}$ & $\Gamma_{2}$ & $\Gamma_{3}$ & $\Gamma_{4}$ & $\Gamma_{5}$ & $\Gamma_{6}$ & $\Gamma_{7}$ \\
\hline \multicolumn{11}{|c|}{ The Reactor Operating Time Was $1000 \mathrm{hr}$ at 1 watt } \\
\hline 100 & $108 \cdot 10^{9}$ & $794 \cdot 10^{8}$ & $958 \cdot 10^{8}$ & $553 \cdot 10^{7}$ & $240 \cdot 10^{8}$ & $219 \cdot 10^{8}$ & $201 \cdot 10^{8}$ & $471 \cdot 10^{7}$ & $704 \cdot 10^{7}$ & $123 \cdot 10^{8}$ \\
\hline 200 & $102 \cdot 10^{9}$ & $707 \cdot 10^{8}$ & $864 \cdot 10^{8}$ & $535 \cdot 10^{7}$ & $233 \cdot 10^{8}$ & $191 \cdot 10^{8}$ & $187 \cdot 10^{8}$ & $442 \cdot 10^{7}$ & $626 \cdot 10^{7}$ & $907 \cdot 10^{\circ}$ \\
\hline 400 & $938 \cdot 10^{8}$ & $615 \cdot 10^{8}$ & $755 \cdot 10^{8}$ & $507 \cdot 10^{7}$ & $225 \cdot 10^{8}$ & $161 \cdot 10^{8}$ & $168 \cdot 10^{8}$ & $398 \cdot 10^{7}$ & $525 \cdot 10^{7}$ & $560 \cdot 10^{\circ}$ \\
\hline $100 \cdot 10^{1}$ & $820 \cdot 10^{8}$ & $496 \cdot 10^{8}$ & $615 \cdot 10^{8}$ & $447 \cdot 10^{7}$ & $211 \cdot 10^{8}$ & $121 \cdot 10^{8}$ & $140 \cdot 10^{8}$ & $322 \cdot 10^{7}$ & $393 \cdot 10^{7}$ & $261 \cdot 10^{?}$ \\
\hline $200 \cdot 10^{\mathrm{l}}$ & $720 \cdot 10^{8}$ & $403 \cdot 10^{8}$ & $514 \cdot 10^{8}$ & $383 \cdot 10^{7}$ & $194 \cdot 10^{8}$ & $889 \cdot 10^{7}$ & $119 \cdot 10^{8}$ & $253 \cdot 10^{7}$ & $309 \cdot 10^{7}$ & $150 \cdot 10^{2}$ \\
\hline $400 \cdot 10^{1}$ & $619 \cdot 10^{8}$ & $319 \cdot 10^{8}$ & $413 \cdot 10^{8}$ & $317 \cdot 10^{7}$ & $171 \cdot 10^{8}$ & $634 \cdot 10^{7}$ & $968 \cdot 10^{7}$ & $186 \cdot 10^{7}$ & $224 \cdot 10^{7}$ & $730 \cdot 10^{6}$ \\
\hline $100 \cdot 10^{2}$ & $503 \cdot 10^{8}$ & $231 \cdot 10^{8}$ & $292 \cdot 10^{8}$ & $257 \cdot 10^{7}$ & $131 \cdot 10^{8}$ & $409 \cdot 10^{7}$ & $676 \cdot 10^{7}$ & $116 \cdot 10^{7}$ & $116 \cdot 10^{7}$ & $229 \cdot 10^{\circ}$ \\
\hline $200 \cdot 10^{2}$ & $424 \cdot 10^{8}$ & $176 \cdot 10^{8}$ & $221 \cdot 10^{8}$ & $225 \cdot 10^{7}$ & $105 \cdot 10^{8}$ & $284 \cdot 10^{7}$ & $507 \cdot 10^{7}$ & $666 \cdot 10^{6}$ & $590 \cdot 10^{6}$ & $765 \cdot 10^{5}$ \\
\hline $400 \cdot 10^{2}$ & $349 \cdot 10^{8}$ & $129 \cdot 10^{8}$ & $171 \cdot 10^{8}$ & $196 \cdot 10^{7}$ & $863 \cdot 10^{7}$ & $187 \cdot 10^{7}$ & $392 \cdot 10^{7}$ & $307 \cdot 10^{6}$ & $314 \cdot 10^{6}$ & $182 \cdot 10^{5}$ \\
\hline $700 \cdot 10^{2}$ & $290 \cdot 10^{8}$ & $966 \cdot 10^{7}$ & $139 \cdot 10^{8}$ & $169 \cdot 10^{7}$ & $709 \cdot 10^{7}$ & $120 \cdot 10^{7}$ & $336 \cdot 10^{7}$ & $175 \cdot 10^{6}$ & $248 \cdot 10^{6}$ & $698 \cdot 10^{\circ}$ \\
\hline $100 \cdot 10^{3}$ & $255 \cdot 10^{8}$ & $806 \cdot 10^{7}$ & $121 \cdot 10^{8}$ & $148 \cdot 10^{7}$ & $607 \cdot 10^{7}$ & $895 \cdot 10^{6}$ & $316 \cdot 10^{7}$ & $138 \cdot 10^{6}$ & $235 \cdot 10^{6}$ & $573 \cdot 10^{\circ}$ \\
\hline $200 \cdot 10^{3}$ & $197 \cdot 10^{8}$ & $580 \cdot 10^{7}$ & $928 \cdot 10^{7}$ & $109 \cdot 10^{7}$ & $431 \cdot 10^{7}$ & $554 \cdot 10^{6}$ & $291 \cdot 10^{7}$ & $984 \cdot 10^{5}$ & $218 \cdot 10^{6}$ & $540 \cdot 10^{2}$ \\
\hline $400 \cdot 10^{3}$ & $150 \cdot 10^{8}$ & $433 \cdot 10^{7}$ & $708 \cdot 10^{7}$ & $748 \cdot 10^{6}$ & $305 \cdot 10^{7}$ & $360 \cdot 10^{6}$ & $260 \cdot 10^{7}$ & $609 \cdot 10^{5}$ & $186 \cdot 10^{6}$ & $495 \cdot 10^{4}$ \\
\hline $100 \cdot 10^{4}$ & $953 \cdot 10^{7}$ & $285 \cdot 10^{7}$ & $438 \cdot 10^{7}$ & $362 \cdot 10^{6}$ & $189 \cdot 10^{7}$ & $162 \cdot 10^{6}$ & $179 \cdot 10^{7}$ & $162 \cdot 10^{5}$ & $119 \cdot 10^{6}$ & $350 \cdot 10^{6}$ \\
\hline $200 \cdot 10^{4}$ & $616 \cdot 10^{7}$ & $186 \cdot 10^{7}$ & $259 \cdot 10^{7}$ & $175 \cdot 10^{6}$ & $131 \cdot 10^{7}$ & $718 \cdot 10^{5}$ & $950 \cdot 10^{6}$ & $408 \cdot 10^{4}$ & $612 \cdot 10^{5}$ & $188 \cdot 10$ \\
\hline $400 \cdot 10^{4}$ & $361 \cdot 10^{7}$ & $107 \cdot 10^{7}$ & $128 \cdot 10^{7}$ & $675 \cdot 10^{5}$ & $897 \cdot 10^{6}$ & $224 \cdot 10^{5}$ & $271 \cdot 10^{6}$ & $282 \cdot 10^{4}$ & $174 \cdot 10^{5}$ & $556 \cdot 10^{2}$ \\
\hline $100 \cdot 10^{5}$ & $148 \cdot 10^{7}$ & $460 \cdot 10^{6}$ & $480 \cdot 10^{6}$ & $121 \cdot 10^{5}$ & $455 \cdot 10^{6}$ & $209 \cdot 10^{4}$ & $696 \cdot 10^{4}$ & $238 \cdot 10^{4}$ & $456 \cdot 10^{3}$ & $352 \cdot 10^{2}$ \\
\hline $200 \cdot 10^{5}$ & $557 \cdot 10^{6}$ & $214 \cdot 10^{6}$ & $156 \cdot 10^{6}$ & $217 \cdot 10^{4}$ & $151 \cdot 10^{6}$ & $443 \cdot 10^{3}$ & $534 \cdot 10^{3}$ & $180 \cdot 10^{4}$ & $437 \cdot 10^{2}$ & $184 \cdot 10^{2}$ \\
\hline $400 \cdot 10^{5}$ & $186 \cdot 10^{6}$ & $943 \cdot 10^{5}$ & $199 \cdot 10^{5}$ & $722 \cdot 10^{3}$ & $175 \cdot 10^{5}$ & $126 \cdot 10^{3}$ & $306 \cdot 10^{3}$ & $103 \cdot 10^{4}$ & $276 \cdot 10^{2}$ & $118 \cdot 10^{2}$ \\
\hline $100 \cdot 10^{6}$ & $544 \cdot 10^{5}$ & $229 \cdot 10^{5}$ & $36 k=10^{4}$ & $130 \cdot 10^{3}$ & $319 \cdot 10^{4}$ & $295 \cdot 10^{2}$ & $624 \cdot 10^{2}$ & $193 \cdot 10^{3}$ & $738 \cdot 10^{1}$ & $316 \cdot 10^{1}$ \\
\hline
\end{tabular}

Definitions of quantities in Table A.2.

Time Time after fission or reactor operation (sec)

D Disintegration rate $\left(\mathrm{sec}^{-1}\right)$

$B$ Beta-ray energy release $\left(\mathrm{MeV} \mathrm{sec}^{-1}\right)$

$\Gamma_{T}$ Gamma-ray energy release ( $\mathrm{MeV} \mathrm{sec}^{-1}$ ) for gamma-ray energies above $0.1 \mathrm{MeV}$

$\Gamma_{1}$ Gamma-ray energy release $\left(\mathrm{MeV} \mathrm{sec}^{-1}\right)$ for gamma-ray energies from 0.1 to $0.4 \mathrm{MeV}$

$\Gamma_{2}$ Gamma-ray energy release ( $\mathrm{MeV} \mathrm{sec}^{-1}$ ) for gamma-ray energies from 0.4 to $0.9 \mathrm{MeV}$

$\Gamma_{3}$ Gamma-ray energy release ( $\mathrm{MeV} \mathrm{sec}^{-1}$ ) for gamma-ray energies from 0.9 to $1.35 \mathrm{MeV}$

$\Gamma_{4}$ Gamma-ray energy release $\left(\mathrm{MeV} \mathrm{sec}^{-1}\right)$ for gamma-ray energies from 1.35 to $1.8 \mathrm{MeV}$

$\Gamma_{5}$ Gamma-ray energy release $\left(\mathrm{MeV} \sec ^{-1}\right)$ for gamma-ray energies from 1.8 to $2.2 \mathrm{MeV}$

$\Gamma_{6} \quad$ Gamma-ray energy release $\left(\mathrm{MeV} \mathrm{sec}^{-1}\right)$ for gamma-ray energies from 2.2 to $2.6 \mathrm{MeV}$

$\Gamma_{7}$ Gamma-ray energy release $\left(\mathrm{MeV} \mathrm{sec}^{-1}\right)$ for gamma-ray energies $>2.6 \mathrm{MeV}$

tData for this table were obtained from J. F. Perkins, Report RR-TR-63-11, U. S. Army Missile Command, July 1963. 


\section{Neutron Fluence-To-Kerma Conversion Factors for Standard-Man Model}

\section{Appendix $B^{\dagger}$}

Table B.1 presents neutron fluence-to-kerma conversion factors calculated by Ritts, Solomito, and Stevens for a standard-man model. The neutron energies range from approximately $0.022 \mathrm{eV}$ to approximately $15 \mathrm{MeV}$, and the compositions of the models are shown in Table B.2. Table B.1 gives the conversion factors for the standard man together with those for the elements making the greatest contributions in the model. Table B. 3 shows the neutron reactions considered for each element.

Table B.1 - NEUTRON FLUENCE-TO-KERMA CONVERSION FACTORS FOR STANDARD MAN $\dagger$

\begin{tabular}{|c|c|c|c|c|c|c|c|c|}
\hline \multirow{2}{*}{$\begin{array}{c}\text { Energy, } \\
\text { MeV }\end{array}$} & \multicolumn{8}{|c|}{ Fluence-to-kerma factor, $\left(\right.$ ergs $\left.^{-1}\right) /\left(\right.$ neutrons $\left.\mathrm{cm}^{-2}\right)$} \\
\hline & Total & Hydrogen & Oxygen & Carbon & Nitrogen & Phosphorus & Sodium & Chlorine \\
\hline $\begin{array}{l}15.14 \\
14.84 \\
14.54 \\
14.24 \\
13.93\end{array}$ & $\begin{array}{l}7.079(-7) \\
6.948(-7) \\
6.811(-7) \\
6.652(-7) \\
6.501(-7)\end{array}$ & $\begin{array}{l}4.633(-7) \\
4.629(-7) \\
4.623(-7) \\
4.617(-7) \\
4.611(-7)\end{array}$ & $\begin{array}{l}1.53(-7) \\
1.46(-7) \\
1.40(-7) \\
1.29(-7) \\
1.18(-7)\end{array}$ & $\begin{array}{l}8.08(-8) \\
7.53(-8) \\
6.89(-8) \\
6.47(-8) \\
6.16(-8)\end{array}$ & $\begin{array}{l}9.35(-9) \\
9.13(-9) \\
8.87(-9) \\
8.67(-9) \\
8.41(-9)\end{array}$ & & & \\
\hline $\begin{array}{l}13.63 \\
13.33 \\
13.03 \\
12.73 \\
12.43\end{array}$ & $\begin{array}{l}6.276(-7) \\
6.119(-7) \\
6.007(-7) \\
5.966(-7) \\
5.949(-7)\end{array}$ & $\begin{array}{l}4.604(-7) \\
4.598(-7) \\
4.591(-7) \\
4.584(-7) \\
4.577(-7)\end{array}$ & $\begin{array}{l}9.90(-8) \\
8.63(-8) \\
7.87(-8) \\
8.03(-8) \\
8.30(-8)\end{array}$ & $\begin{array}{l}5.93(-8) \\
5.72(-8) \\
5.44(-8) \\
4.96(-8) \\
4.61(-8)\end{array}$ & $\begin{array}{l}7.99(-9) \\
7.74(-9) \\
7.63(-9) \\
7.44(-9) \\
7.29(-9)\end{array}$ & & & \\
\hline $\begin{array}{l}12.13 \\
11.83 \\
11.52 \\
11.22 \\
10.92\end{array}$ & $\begin{array}{l}5.936(-7) \\
5.964(-7) \\
5.877(-7) \\
5.818(-7) \\
5.691(-7)\end{array}$ & $\begin{array}{l}4.568(-7) \\
4.559(-7) \\
4.550(-7) \\
4.540(-7) \\
4.531(-7)\end{array}$ & $\begin{array}{l}8.45(-8) \\
9.28(-8) \\
8.90(-8) \\
8.48(-8) \\
7.49(-8)\end{array}$ & $\begin{array}{l}4.45(-8) \\
4.01(-8) \\
3.64(-8) \\
3.59(-8) \\
3.43(-8)\end{array}$ & $\begin{array}{l}7.04(-9) \\
6.77(-9) \\
6.47(-9) \\
6.23(-9) \\
5.95(-9)\end{array}$ & & & \\
\hline $\begin{array}{r}10.62 \\
10.32 \\
10.02 \\
9.72 \\
9.60\end{array}$ & $\begin{array}{l}5.554(-7) \\
5.483(-7) \\
5.421(-7) \\
5.353(-7) \\
5.313(-7)\end{array}$ & $\begin{array}{l}4.522(-7) \\
4.512(-7) \\
4.501(-7) \\
4.478(-7) \\
4.468(-7)\end{array}$ & $\begin{array}{l}6.75(-8) \\
6.81(-8) \\
6.46(-8) \\
5.98(-8) \\
5.70(-8)\end{array}$ & $\begin{array}{l}2.91(-8) \\
2.26(-8) \\
2.12(-8) \\
2.18(-8) \\
2.16(-8)\end{array}$ & $\begin{array}{l}5.85(-9) \\
5.73(-9) \\
5.52(-9) \\
5.28(-9) \\
5.21(-9)\end{array}$ & & & \\
\hline
\end{tabular}

(Table continues on next page.)

† All data in this appendix have been taken from J. J. Ritts, E. Solomito, and P. N. Stevens, Calculations of Neutron Fluence-to-Kerma Factors for the Human Body, USAEC Report ORNL-TM-2079, Oak Ridge National Laboratory, January 1968. 
Table B.1-(Continued)

\begin{tabular}{|c|c|c|c|c|c|c|c|c|}
\hline \multirow{2}{*}{$\begin{array}{c}\text { Energy, } \\
\text { MeV }\end{array}$} & \multicolumn{8}{|c|}{ Fluence-to-kerma factor, $\left(\mathrm{ergs}^{-1}\right) /\left(\right.$ neutrons $\left.\mathrm{cm}^{-2}\right)$} \\
\hline & Total & Hydrogen & Oxygen & Carbon & Nitrogen & Phosphorus & Sodium & Chlorine \\
\hline 9.45 & $5.271(-7)$ & $4.456(-7)$ & $5.40(-8)$ & $2.19(-8)$ & $4.97(-9)$ & & & \\
\hline 9.30 & $5.222(-7)$ & $4.443(-7)$ & $5.00(-8)$ & $2.23(-8)$ & $4.91(-9)$ & & & \\
\hline 9.15 & $5.173(-7)$ & $4.430(-7)$ & $4.74(-8)$ & $2.14(-8)$ & $4.85(-9)$ & & & \\
\hline 9.00 & $5.123(-7)$ & $4.417(-7)$ & $4.78(-8)$ & $1.75(-8)$ & $4.70(-9)$ & & & \\
\hline 8.85 & $5.078(-7)$ & $4.410(-7)$ & $4.54(-8)$ & $1.61(-8)$ & $4.64(-9)$ & & & \\
\hline 8.70 & $5.048(-7)$ & $4.402(-7)$ & $4.37(-8)$ & $1.56(-8)$ & $4.60(-9)$ & & & \\
\hline 8.62 & $5.007(-7)$ & $4.399(-7)$ & $4.01(-8)$ & $1.56(-8)$ & $4.53(-9)$ & & & \\
\hline 8.47 & $4.988(-7)$ & $4.391(-7)$ & $3.90(-8)$ & $1.57(-8)$ & $4.40(-9)$ & & & \\
\hline 8.32 & $5.036(-7)$ & $4.383(-7)$ & $4.38(-8)$ & $1.66(-8)$ & $4.28(-9)$ & & & \\
\hline 8.17 & $5.062(-7)$ & $4.376(-7)$ & $4.24(-8)$ & $2.15(-8)$ & $4.30(-9)$ & & & \\
\hline 8.02 & $4.977(-7)$ & $4.368(-7)$ & $2.94(-8)$ & $2.63(-8)$ & $4.74(-9)$ & & & \\
\hline 7.87 & $5.038(-7)$ & $4.345(-7)$ & $3.81(-8)$ & $2.62(-8)$ & $4.38(-9)$ & & & \\
\hline 7.72 & $5.060(-7)$ & $4.319(-7)$ & $3.89(-8)$ & $3.09(-8)$ & $3.88(-9)$ & & & \\
\hline 7.57 & $4.935(-7)$ & $4.292(-7)$ & $3.65(-8)$ & $2.34(-8)$ & $3.84(-9)$ & & & \\
\hline 7.42 & $4.970(-7)$ & $4.266(-7)$ & $4.11(-8)$ & $2.49(-8)$ & $3.98(-9)$ & & & \\
\hline 7.27 & $4.995(-7)$ & $4.239(-7)$ & $5.32(-8)$ & $1.80(-8)$ & $3.87(-9)$ & & & \\
\hline 7.12 & $4.773(-7)$ & $4.211(-7)$ & $4.13(-8)$ & $1.08(-8)$ & $3.52(-9)$ & & & \\
\hline 6.97 & $4.650(-7)$ & $4.187(-7)$ & $3.13(-8)$ & $1.10(-8)$ & $3.49(-9)$ & & & \\
\hline 6.82 & $4.745(-7)$ & $4.173(-7)$ & $4.14(-8)$ & $1.21(-8)$ & $3.27(-9)$ & & & \\
\hline 6.67 & $4.707(-7)$ & $4.159(-7)$ & $3.94(-8)$ & $1.17(-8)$ & $3.27(-9)$ & & & \\
\hline 6.52 & $4.605(-7)$ & $4.145(-7)$ & $2.97(-8)$ & $1.25(-8)$ & $3.38(-9)$ & & & \\
\hline 6.37 & $4.731(-7)$ & $4.130(-7)$ & $3.89(-8)$ & $1.71(-8)$ & $3.57(-9)$ & & & \\
\hline 6.21 & $4.611(-7)$ & $4.115(-7)$ & $2.46(-8)$ & $2.09(-8)$ & $3.65(-9)$ & & & \\
\hline 6.06 & $4.572(-7)$ & $4.100(-7)$ & $2.77(-8)$ & $1.53(-8)$ & $3.77(-9)$ & & & \\
\hline 5.91 & $4.633(-7)$ & $4.077(-7)$ & $3.73(-8)$ & $1.42(-8)$ & $3.67(-9)$ & & & \\
\hline 5.76 & $4.457(-7)$ & $4.048(-7)$ & $2.32(-8)$ & $1.38(-8)$ & $3.51(-9)$ & & & \\
\hline 5.61 & $4.502(-7)$ & $4.018(-7)$ & $3.08(-8)$ & $1.37(-8)$ & $3.55(-9)$ & & & \\
\hline 5.46 & $4.312(-7)$ & $3.987(-7)$ & $1.47(-8)$ & $1.42(-8)$ & $3.25(-9)$ & & & \\
\hline 5.31 & $4.294(-7)$ & $3.956(-7)$ & $1.70(-8)$ & $1.30(-8)$ & $3.39(-9)$ & & & \\
\hline 5.16 & $4.449(-7)$ & $3.925(-7)$ & $3.46(-8)$ & $1.33(-8)$ & $4.17(-9)$ & & & \\
\hline 5.01 & $4.344(-7)$ & $3.892(-7)$ & $2.76(-8)$ & $1.32(-8)$ & $4.01(-9)$ & & & \\
\hline 4.69 & $4.180(-7)$ & $3.810(-7)$ & $1.79(-8)$ & $1.50(-8)$ & $3.73(-9)$ & & & \\
\hline 4.50 & $4.116(-7)$ & $3.760(-7)$ & $1.66(-8)$ & $1.42(-8)$ & $4.34(-9)$ & & & \\
\hline 4.31 & $4.181(-7)$ & $3.709(-7)$ & $2.91(-8)$ & $1.33(-8)$ & $4.48(-9)$ & & & \\
\hline 4.16 & $4.245(-7)$ & $3.666(-7)$ & $3.37(-8)$ & $1.89(-8)$ & $4.98(-9)$ & & & \\
\hline 4.01 & $4.102(-7)$ & $3.623(-7)$ & $2.24(-8)$ & $2.05(-8)$ & $4.78(-9)$ & & & \\
\hline 3.82 & $4.121(-7)$ & $3.562(-7)$ & $2.91(-8)$ & $2.26(-8)$ & $3.88(-9)$ & & & \\
\hline 3.67 & $4.099(-7)$ & $3.511(-7)$ & $3.05(-8)$ & $2.42(-8)$ & $3.67(-9)$ & & & \\
\hline 3.52 & $4.060(-7)$ & $3.459(-7)$ & $3.09(-8)$ & $2.45(-8)$ & $4.48(-9)$ & & & \\
\hline 3.33 & $3.981(-7)$ & $3.392(-7)$ & $3.46(-8)$ & $2.07(-8)$ & $3.32(-9)$ & & & \\
\hline 3.18 & $3.727(-7)$ & $3.337(-7)$ & $2.06(-8)$ & $1.54(-8)$ & $2.83(-9)$ & & & \\
\hline 3.03 & $3.555(-7)$ & $3.280(-7)$ & $1.53(-8)$ & $9.44(--9)$ & $2.47(-9)$ & & & \\
\hline 2.84 & $3.499(-7)$ & $3.203(-7)$ & $1.14(-8)$ & $1.60(-8)$ & $2.01(-9)$ & & & \\
\hline 2.69 & $3.396(-7)$ & $3.138(-7)$ & $1.10(-8)$ & $1.26(-8)$ & $1.89(-9)$ & & & \\
\hline 2.50 & $3.279(-7)$ & $3.054(-7)$ & $1.01(-8)$ & $1.09(-8)$ & $1.34(-9)$ & & & \\
\hline 2.40 & $3.150(-7)$ & $3.000(-7)$ & $3.40(-9)$ & $1.03(-8)$ & $1.07(-9)$ & & & \\
\hline 2.31 & $3.136(-7)$ & $2.950(-7)$ & $7.36(-9)$ & $9.85(-9)$ & $1.21(-9)$ & & & \\
\hline 2.21 & $3.119(-7)$ & $2.898(-7)$ & $1.08(-8)$ & $9.51(-9)$ & $1.61(-9)$ & & & \\
\hline 2.10 & $3.057(-7)$ & $2.835(-7)$ & $1.14(-8)$ & $9.68(-9)$ & $9.62(-10)$ & & & \\
\hline 2.00 & $2.995(-7)$ & $2.780(-7)$ & $1.13(-8)$ & $9.16(-9)$ & $9.20(-10)$ & & & \\
\hline 1.91 & $2.952(-7)$ & $2.721(-7)$ & $1.31(-8)$ & $8.82(-9)$ & $9.82(-10)$ & & & \\
\hline 1.80 & $2.883(-7)$ & $2.648(-7)$ & $1.31(-8)$ & $8.56(-9)$ & $1.72(-9)$ & & & \\
\hline 1.70 & $2.800(-7)$ & $2.585(-7)$ & $1.21(-8)$ & $8.36(-9)$ & $8.76(-10)$ & & & \\
\hline 1.59 & $2.717(-7)$ & $2.507(-7)$ & $1.18(-8)$ & $8.12(-9)$ & $9.84(-10)$ & & & \\
\hline 1.50 & $2.644(-7)$ & $2.440(-7)$ & $1.15(-8)$ & $7.93(-9)$ & $9.06(-10)$ & & & \\
\hline 1.40 & $2.577(-7)$ & $2.370(-7)$ & $1.13(-8)$ & $7.73(-9)$ & $1.66(-9)$ & & & \\
\hline 1.31 & $2.602(-7)$ & $2.297(-7)$ & $2.23(-8)$ & $7.52(-9)$ & $6.44(-10)$ & & & \\
\hline 1.20 & $2.426(-7)$ & $2.209(-7)$ & $1.39(-8)$ & $7.25(-9)$ & $5.11(-10)$ & & & \\
\hline
\end{tabular}


Table B.1 - (Continued)

\begin{tabular}{|c|c|c|c|c|c|c|c|c|}
\hline \multirow{2}{*}{$\begin{array}{c}\text { Energy, } \\
\text { MeV }\end{array}$} & \multicolumn{8}{|c|}{ Fluence-to-kerma factor, $\left(\mathrm{ergs}^{-1} \mathrm{~g}^{-1}\right) /\left(\right.$ neutrons $\left.\mathrm{cm}^{-2}\right)$} \\
\hline & Total & Hydrogen & Oxygen & Carbon & Nitrogen & Phosphorus & Sodium & Chlorine \\
\hline 1.10 & $2.371(-7)$ & $2.121(-7)$ & $1.63(-8)$ & $7.00(-9)$ & $7.45(-10)$ & & & \\
\hline 1.00 & $2.432(-7)$ & $2.037(-7)$ & $3.21(-8)$ & $6.70(-9)$ & $6.33(-10)$ & & & \\
\hline $9.0(-1)$ & $2.122(-7)$ & $1.939(-7)$ & $1.15(-8)$ & $6.35(-9)$ & $3.39(-10)$ & & & \\
\hline $8.0(-1)$ & $1.980(-7)$ & $1.834(-7)$ & $7.95(-9)$ & $5.97(-9)$ & $3.90(-10)$ & & & \\
\hline $7.0(-1)$ & $1.857(-7)$ & $1.730(-7)$ & $6.61(-9)$ & $5.56(-9)$ & $4.97(-10)$ & & & \\
\hline $6.0(-1)$ & $1.719(-7)$ & $1.609(-7)$ & $5.61(-9)$ & $5.07(-9)$ & $2.80(-10)$ & & & \\
\hline $5.0(-1)$ & $1.592(-7)$ & $1.483(-7)$ & $5.74(-9)$ & $4.52(-9)$ & $5.32(-10)$ & & & \\
\hline $4.5(-1)$ & $1.639(-7)$ & $1.403(-7)$ & $1.90(-8)$ & $4.20(-9)$ & $2.90(-10)$ & & & \\
\hline $4.0(-1)$ & $1.486(-7)$ & $1.318(-7)$ & $1.18(-8)$ & $3.89(-9)$ & $2.96(-10)$ & & & \\
\hline $3.5(-1)$ & $1.339(-7)$ & $1.236(-7)$ & $6.52(-9)$ & $3.51(-9)$ & $2.46(-10)$ & & & \\
\hline $3.0(-1)$ & $1.223(-7)$ & $1.142(-7)$ & $4.73(-9)$ & $3.12(-9)$ & $2.13(-10)$ & & & \\
\hline $2.5(-1)$ & $1.104(-7)$ & $1.037(-7)$ & $3.74(-9)$ & $2.70(-9)$ & $1.93(-10)$ & & & \\
\hline $2.0(-1)$ & $9.649(-8)$ & $9.116(-8)$ & $2.89(-9)$ & $2.23(-9)$ & $1.82(-10)$ & & & \\
\hline $1.5(-1)$ & $8.165(-8)$ & $7.758(-8)$ & $2.16(-9)$ & $1.75(-9)$ & $1.47(-10)$ & & & \\
\hline $1.0(-1)$ & $6.335(-8)$ & $6.056(-8)$ & $1.45(-9)$ & $1.22(-9)$ & $1.08(-10)$ & & & \\
\hline $7.0(-2)$ & $4.973(-8)$ & $4.774(-8)$ & $1.02(-9)$ & $8.76(-10)$ & $9.36(-11)$ & & & \\
\hline $5.0(-2)$ & $3.830(-8)$ & $3.705(-8)$ & $7.30(-9)$ & $6.37(-10)$ & $7.62(-11)$ & & & \\
\hline $3.0(-2)$ & $2.560(-8)$ & $2.472(-8)$ & $4.43(-9)$ & $3.92(-10)$ & $4.97(-11)$ & & & \\
\hline 2.0 & $-8)$ & $-8)$ & 2.98 & $2.66(-10)$ & -11) & & & \\
\hline $1.5(-2)$ & $1.361(-8)$ & $1.316(-8)$ & $2.18(-9)$ & $1.95(-10)$ & $3.14(-11)$ & & & \\
\hline $1.0(-2)$ & $9.550(-9)$ & $9.287(-9)$ & $1.47(-9)$ & $1.34(-10)$ & $2.29(-11)$ & & & \\
\hline $7.0(-3)$ & $6.776(-9)$ & $6.558(-9)$ & $1.03(-9)$ & $9.24(-11)$ & $1.87(-11)$ & & & \\
\hline $5.0(-3)$ & $4.880(-9)$ & $4.832(-9)$ & $7.53(-9)$ & $6.74(-11)$ & $1.56(-11)$ & & & \\
\hline $4.03(-3)$ & $3.951(-9)$ & $3.820(-9)$ & $5.94(-11)$ & $5.32(-11)$ & $1.44(-11)$ & $2.88(-12)$ & & \\
\hline $3.00(-3)$ & $2.964(-9)$ & $2.855(-9)$ & $4.43(-11)$ & $3.96(-11)$ & $1.32(-11)$ & $3.02(-12)$ & & \\
\hline $2.01(-3)$ & $2.002(-9)$ & $1.928(-9)$ & $2.98(-11)$ & $2.67(-11)$ & $1.27(-11)$ & $3.12(-12)$ & & \\
\hline $1.50(-3)$ & $1.495(-9)$ & $1.436(-9)$ & $2.22(-11)$ & $1.99(-11)$ & $1.30(-11)$ & $3.20(-12)$ & & \\
\hline $1.03(-3)$ & $1.030(-9)$ & $9.832(-10)$ & $1.52(-11)$ & $1.36(-11)$ & $1.40(-11)$ & $3.25(-12)$ & & \\
\hline $7.68(-4)$ & $7.774(-10)$ & $7.368(-10)$ & $1.14(-11)$ & $1.02(-11)$ & $1.52(-11)$ & $3.27(-12)$ & & 2.7 \\
\hline $5.13(-4)$ & $5.299(-10)$ & $4.925(-10)$ & $7.60(-12)$ & $6.80(-12)$ & $1.77(-11)$ & $3.30(-12)$ & & $1.84(-12)$ \\
\hline $4.02(-4)$ & $4.244(-10)$ & $3.867(-10)$ & $5.96(-12)$ & $5.34(-12)$ & $1.95(-11)$ & $3.31(-12)$ & & $3.28(-12)$ \\
\hline $3.10(-4)$ & $3.347(-10)$ & $2.986(-10)$ & $4.60(-12)$ & $4.12(-12)$ & $2.19(-11)$ & $3.32(-12)$ & & $1.97(-12)$ \\
\hline $2.01(-4)$ & $2.299(-10)$ & $1.939(-10)$ & $2.98(-12)$ & $2.67(-12)$ & $2.67(-11)$ & $3.33(-12)$ & & $5.91(-14)$ \\
\hline $1.42(-4)$ & $1.764(-10)$ & $1.372(-10)$ & $2.11(-12)$ & $1.88(-12)$ & $3.15(-11)$ & $3.33(-12)$ & & $1.12(-13)$ \\
\hline $1.006(-4)$ & $1.417(-10)$ & $9.753(-11)$ & $1.49(-12)$ & $1.33(-12)$ & $3.73(-11)$ & $3.34(-12)$ & & $3.87(-13)$ \\
\hline $7.1(-5)$ & $1.192(-10)$ & $6.920(-11)$ & $1.05(-12)$ & $9.42(-13)$ & $4.42(-11)$ & $3.34(-12)$ & $3.50(-13)$ & $9.84(-14)$ \\
\hline $5.03(-5)$ & $1.071(-10)$ & $4.94(-11)$ & $7.46(-13)$ & $6.67(-13)$ & $5.237(-11)$ & $3.34(-12)$ & $4.11(-13)$ & $1.10(-13)$ \\
\hline $4.11(-5)$ & $1.036(-10)$ & $4.07(-11)$ & $6.09(-13)$ & $5.45(-13)$ & $5.786(-11)$ & $3.34(-12)$ & $4.54(-13)$ & $1.31(-13)$ \\
\hline $3.43(-5)$ & $1.025(-10)$ & $3.43(-11)$ & $5.09(-13)$ & $4.56(-13)$ & $6.323(-11)$ & $3.34(-12)$ & $4.99(-13)$ & $1.57(-13)$ \\
\hline $2.74(-5)$ & $1.033(-10)$ & $2.78(-11)$ & $4.07(-13)$ & $3.64(-13)$ & $7.065(-11)$ & $3.35(-12)$ & $5.58(-13)$ & $1.95(-13)$ \\
\hline $2.05(-5)$ & $1.077(-10)$ & $2.13(-11)$ & $3.05(-13)$ & $2.73(-13)$ & $8.155(-11)$ & $3.35(-12)$ & $6.44(-13)$ & $2.59(-13)$ \\
\hline $1.60(-5)$ & $1.143(-10)$ & $1.71(-11)$ & $2.38(-13)$ & $2.13(-13)$ & $9.226(-11)$ & $3.35(-12)$ & $7.34(-13)$ & $3.14(-13)$ \\
\hline $1.26(-5)$ & $1.230(-10)$ & $1.40(-11)$ & $1.86(-13)$ & $1.67(-13)$ & $1.041(-10)$ & $3.35(-12)$ & $8.34(-13)$ & $3.79(-13)$ \\
\hline $8.0(-6)$ & $1.454(-10)$ & $1.01(-11)$ & $1.19(-13)$ & $1.06(-13)$ & $1.302(-10)$ & $3.35(-12)$ & $1.04(-12)$ & $5.08(-13)$ \\
\hline & & $7.20(-12)$ & $5.94(-14)$ & $5.32(-14)$ & $1.836(-10)$ & $3.35(-12)$ & $1.46(-12)$ & $7.24(-13)$ \\
\hline $2.0(-6)$ & $2.723(-10)$ & $6.63(-12)$ & $2.97(-14)$ & $2.66(-14)$ & $2.591(-10)$ & $3.35(-12)$ & $2.09(-12)$ & $1.03(-12)$ \\
\hline $1.0(-6)$ & $3.811(-10)$ & $7.59(-12)$ & $1.49(-14)$ & $1.33(-14)$ & $3.656(-10)$ & $3.35(-12)$ & $2.97(-12)$ & $1.45(-12)$ \\
\hline $5.0(-7)$ & $5.354(-10)$ & $9.87(-12)$ & & & & & & \\
\hline $2.5(-7)$ & $7.529(-10)$ & $1.35(-11)$ & & & $7.280(-10)$ & $3.35(-12)$ & $5.89(-12)$ & $2.12(-12)$ \\
\hline $1.7(-7)$ & $1.144(-9)$ & $2.03(-11)$ & & & $1.109(-9)$ & $3.35(-12)$ & $9.00(-12)$ & $2.12(-12)$ \\
\hline $7.2(-8)$ & $1.398(-9)$ & $2.48(-11)$ & & & $1.356(-9)$ & $3.35(-12)$ & $1.10\langle-11)$ & $2.12(-12)$ \\
\hline $4.5(-8)$ & $1.766(-9)$ & $3.14(-11)$ & & & $1.715(-9)$ & $3.35(-12)$ & $1.39(-11)$ & $2.12(-12)$ \\
\hline $2.23(-8)$ & $2.489(-9)$ & $4.18(-11)$ & & & $2.424(-9)$ & $3.35(-12)$ & $1.83(-11)$ & $2.12(-12)$ \\
\hline
\end{tabular}

tThe standard-man model also includes the elements calcium, sulfur, potassium, and magnesium, but their contributions to the kerma are negligible.

$\ddagger$ Read: $7.079 \times 10^{-7}$, etc. 
Table B.2-ELEMENTAL PERCENTAGES AND NUMBER DENSITIES USED IN NEUTRON KERMA CALCULATIONS

\begin{tabular}{|c|c|c|c|c|c|c|c|c|c|c|c|c|}
\hline \multirow[b]{2}{*}{ Element } & \multicolumn{6}{|c|}{ Percent composition } & \multicolumn{6}{|c|}{ Number density, $10^{24}$ atoms $/ \mathrm{g}$} \\
\hline & $\begin{array}{l}\text { Standard } \\
\text { man }\end{array}$ & Lung & Muscle & Bone & Brain & $\begin{array}{c}\text { Red } \\
\text { marrow }\end{array}$ & $\begin{array}{l}\text { Standard } \\
\text { man }\end{array}$ & Lung & Muscle & Bone & Brain & $\begin{array}{c}\text { Red } \\
\text { marrow }\end{array}$ \\
\hline $\mathbf{H}$ & 10 & 10 & 10 & 7.1 & 10 & 10 & $5.977(-2) \ddagger$ & $6.062(-2)$ & $5.977(-2)$ & $4.50(-2)$ & $5.977(-2)$ & $5.977(-2)$ \\
\hline 0 & 60 & 75 & 75 & 39.5 & 71 & $74 \dagger$ & $2.259(-2)$ & $2.864(-2)$ & $2.824(-2)$ & $1.49(-2)$ & $2.674(-2)$ & $2.7866(-2)$ \\
\hline C & 24 & 10 & 11 & 22.4 & 15 & $12.5 \dagger$ & $1.204(-2)$ & $5.09(-3)$ & $5.518(-2)$ & $1.13(-2)$ & $7.52(-3)$ & $6.270(-3)$ \\
\hline $\mathbf{N}$ & 2.9 & 2.6 & 2.6 & 4.7 & 1.9 & 3.2 & $1.25(-3)$ & $1.13(-3)$ & $1.118(-3)$ & $2.05(-3)$ & $8.17(-4)$ & $1.377(-3)$ \\
\hline $\mathrm{Ca}$ & 1.2 & 0.011 & 0.0031 & 12.37 & 0.0086 & & $1.77(-4)$ & $1.68(-6)$ & $4.66(-7)$ & $1.87(-3)$ & $1.29(-6)$ & \\
\hline $\mathbf{P}$ & 1.1 & 0.11 & 0.18 & 13.16 & 0.34 & & $2.14(-4)$ & $2.17(-5)$ & $3.50(-5)$ & $2.57(-3)$ & $6.61(-5)$ & \\
\hline$s$ & 0.24 & 0.24 & 0.24 & 0.41 & 0.17 & & $4.51(-4)$ & $4.57(-5)$ & $4.51(-5)$ & $7.69(-5)$ & $3.19(-5)$ & \\
\hline $\mathbf{K}$ & 0.20 & 0.20 & 0.30 & & 0.30 & & $3.08(-5)$ & $3.13(-5)$ & $4.62(-5)$ & ( & $4.62(-5)$ & \\
\hline $\mathbf{N a}$ & 0.20 & 0.18 & 0.16 & 0.08 & 0.18 & & $524(-5)$ & $4.78(-5)$ & $4.19(-5)$ & $2.08(-5)$ & $4.72(-5)$ & \\
\hline $\mathrm{Cl}$ & 0.20 & 0.25 & 0.18 & & 0.23 & 0.12 & $3.40(-5)$ & $4.31(-5)$ & $3.06(-5)$ & & $3.91(-5)$ & $2.039(-5)$ \\
\hline $\mathbf{M g}$ & 0.03 & 0.0096 & 0.019 & 0.28 & 0.015 & & $7.43(-6)$ & $4.78(-6)$ & $4.71(-6)$ & $6.87(-5)$ & $3.72(-6)$ & \\
\hline
\end{tabular}

tThe values for oxygen and carbon were increased by 1 and $0.5 \%$, respectively.

$\ddagger$ Read: $5.977 \times 10^{-2}$, etc. 
Table B.3-REACTIONS CONSIDERED IN NEUTRON KERMA CALCULATIONS

\begin{tabular}{|c|c|c|c|c|c|c|c|c|c|c|c|}
\hline Type of reaction & $\mathbf{H}$ & $\mathbf{o}$ & C & $\mathbf{N}$ & $\mathrm{Ca}$ & $\mathbf{P}$ & $\mathbf{S}$ & $\mathbf{K}$ & $\mathbf{N a}$ & $\mathrm{Cl}$ & $\mathbf{M g}$ \\
\hline Radiative capture & $\mathbf{x}$ & & & $\mathbf{x}$ & & $\mathbf{x}$ & $\mathbf{x}$ & $\mathbf{x}$ & $x \dagger$ & $\mathbf{x}$ & $\mathbf{x}$ \\
\hline Elastic scattering & $\mathbf{x}$ & $\mathbf{x}$ & $\mathbf{x}$ & $\mathbf{x}$ & $\mathbf{x}$ & $\mathbf{x}$ & $\mathbf{x}$ & $\mathbf{x}$ & $\mathbf{x}$ & $\mathbf{x}$ & $\mathbf{x}$ \\
\hline Inelastic scattering & & $\mathbf{x}$ & $\mathbf{x}$ & $\mathbf{x}$ & $\mathbf{x}$ & $\mathbf{x}$ & $\mathbf{x}$ & $\mathbf{x}$ & $\mathbf{x}$ & & $\mathbf{x}$ \\
\hline$(n, p)$ reaction & & $x \dagger$ & & $\mathbf{x}$ & & $x \dagger$ & & $\mathbf{x}$ & & $\mathbf{x}$ & $\mathbf{x}$ \\
\hline $\begin{array}{l}(n, \alpha) \text { reaction } \\
\left(n, n^{\prime} 3 \alpha\right) \text { reaction }\end{array}$ & & $\mathbf{x}$ & $\begin{array}{l}\mathbf{x} \\
\mathbf{x}\end{array}$ & $\mathbf{x}$ & & $x t$ & & $\mathbf{x}$ & & $x t$ & $\mathbf{x}$ \\
\hline$(n, 2 n)$ reaction & & & & $x \ddagger$ & & $x \neq$ & & $x \neq$ & & & \\
\hline$(n, d)$ reaction & & & & $\mathbf{x}$ & & & & $\mathbf{x}$ & & & \\
\hline$(n, t)$ reaction & & & & $\mathbf{x}$ & & & & & & & \\
\hline$(n, 2 \alpha)$ reaction & & & & $\mathbf{x}$ & & & & & & & \\
\hline$\left(n, n^{\prime} p\right)$ reaction & & & & & & $\mathbf{x}$ & & $\mathbf{x}$ & & & $\mathbf{x}$ \\
\hline$\left(n, n^{\prime} \alpha\right)$ reaction & & & & & & & & $\mathbf{x}$ & & & \\
\hline
\end{tabular}

†Includes beta decay after reaction.

$\$$ Includes positron decay. 


\section{Cylindrical Tissue Phantom}

\section{Appendix C}

Table C.1 - MAXIMUM ABSORBED DOSE AND MAXIMUM DOSE EQUIV ALENT FOR MONOENERGETIC NEUTRONS INCIDENT ON A CYLINDRICAL TISSUE PHANTOM $†$

\begin{tabular}{|c|c|c|c|}
\hline $\begin{array}{c}\text { Neutron } \\
\text { energy, } \\
\text { MeV }\end{array}$ & $\begin{array}{c}\text { Maximum } \\
\text { absorbed } \\
\text { dose, }+ \\
\text { mrad } \\
\text { neutron }{ }^{-1} \mathrm{~cm}^{-2}\end{array}$ & $\begin{array}{c}\text { Maximum } \\
\text { dose } \\
\text { equivalent, } \ddagger \\
\text { mrem } \\
\text { neutron }{ }^{-1} \mathrm{~cm}^{-2}\end{array}$ & $\begin{array}{c}\text { Effective } \\
\text { quality } \\
\text { factor }\end{array}$ \\
\hline Thermal & $4.68(-7) \S$ & $1.15(-6)$ & 2.46 \\
\hline 0.000001 & $5.89(-7)$ & $1.34(-6)$ & 2.27 \\
\hline 0.00001 & $5.18(-7)$ & $1.21(-6)$ & 2.34 \\
\hline 0.0001 & $4.45(-7)$ & $1.01(-6)$ & 2.27 \\
\hline 0.001 & $4.32(-7)$ & $8.85(-6)$ & 2.04 \\
\hline 0.01 & $4.34(-7)$ & $9.92(-6)$ & 2.29 \\
\hline 0.1 & $8.02(-7)$ & $4.86(-6)$ & 6.06 \\
\hline 0.5 & $1.81(-6)$ & $1.89(-5)$ & 10.4 \\
\hline 1 & $3.01(-6)$ & $3.26(-5)$ & 10.8 \\
\hline 2.5 & $3.99(-6)$ & $3.50(-5)$ & 8.77 \\
\hline 5 & $5.72(-6)$ & $4.41(-5)$ & 7.70 \\
\hline 7 & $5.70(-6)$ & $4.03(-5)$ & 7.07 \\
\hline 10 & $7.25(-6)$ & $4.31(-5)$ & 5.94 \\
\hline 14 & $8.31(-6)$ & $6.15(-5)$ & 7.40 \\
\hline
\end{tabular}

†Based on data from J. A. Auxier, W. S. Snyder, and T. D. Jones, Radiation Dosimetry, Vol. I, Chap. 6, F. H. Attix and W. C. Roesch (Eds.), Academic Press, Inc., New York, 1968. $\$$ Multiply by 3600 to convert to (mrad hr-1)/(neutron $\mathrm{cm}^{-2} \mathrm{sec}$ ) or to (mrem hr-1)/(neutron $\mathrm{cm}^{-2} \mathrm{sec}$ ).

$\S$ Read: $4.68 \times 10^{-7}$, etc. 


\section{Coordinate Systems, Vector Operations, and Legendre Polynomials}

\section{Appendix D}

\section{D.1 COORDINATE SYSTEMS}

The three common spatial coordinate systems and the basic unit vectors of each are illustrated in Fig. D.1 and Table D.1.

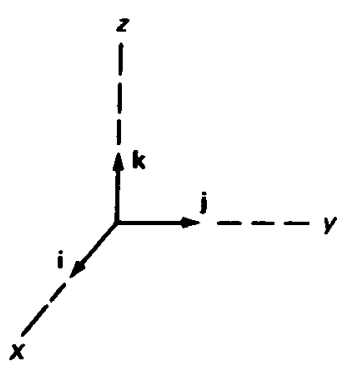

CARTESIAN

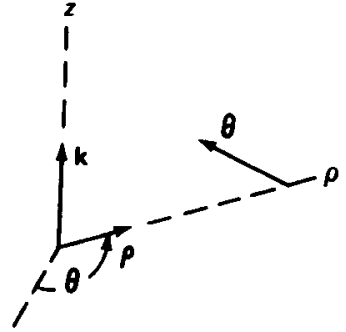

CYLINDRICAL

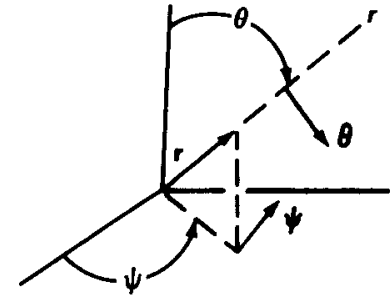

SPHERICAL

Fig. D.1-Principal coordinate systems.

Table D.1 - POSITION VARIABLES

\begin{tabular}{lcc}
\hline System & Independent variables & Unit vectors \\
\hline Cartesian & $x, y, z$ & $i, j, k$ \\
Cylindrical & $\rho, \theta, z$ & $\rho, \theta, k$ \\
Spherical & $r, \theta, \psi$ & $r, \theta, \psi$ \\
\hline
\end{tabular}

\section{D.2 COORDINATE-SYSTEM TRANSFORMATION}

(a) Cartesian-Cylindrical

$$
x=\rho \cos \theta \quad \rho=\left(x^{2}+y^{2}\right)^{\frac{1}{2}}
$$




$$
\begin{aligned}
& y=\rho \sin \theta \quad \cos \theta=x\left(x^{2}+y^{2}\right)^{-1 / 2} \\
& \frac{\partial x}{\partial \rho}=\frac{\partial \rho}{\partial x}=\cos \theta=x\left(x^{2}+y^{2}\right)^{-1 / 2} \\
& \frac{\partial y}{\partial \rho}=\frac{\partial \rho}{\partial y}=\sin \theta=y\left(x^{2}+y^{2}\right)^{-4 / 2} \\
& \frac{\partial x}{\partial \theta}=-\sin \theta=-y ; \frac{\partial \theta}{\partial x}=-y\left(x^{2}+y^{2}\right)^{-1}=-\frac{1}{\rho} \sin \theta \\
& \frac{\partial y}{\partial \theta}=\rho \cos \theta=x ; \frac{\partial \theta}{\partial y}=x\left(x^{2}+y^{2}\right)^{-1}=\frac{1}{\rho} \cos \theta
\end{aligned}
$$

(b) Cartesian-Spherical

$$
\begin{aligned}
& x=r \sin \theta \cos \psi \quad r=\left(x^{2}+y^{2}+z^{2}\right)^{1 / 2} \\
& y=r \sin \theta \sin \psi \quad \cos \theta=z\left(x^{2}+y^{2}+z^{2}\right)^{-1 / 2} \\
& z=r \cos \theta \quad \sin \psi=y\left(x^{2}+y^{2}\right)^{-1 / 2} \\
& \frac{\partial x}{\partial r}=\frac{\partial r}{\partial x}=\sin \theta \cos \psi=x\left(x^{2}+y^{2}+z^{2}\right)^{-1 / 2} \\
& \frac{\partial y}{\partial r}=\frac{\partial r}{\partial y}=\sin \theta \sin \psi=y\left(x^{2}+y^{2}+z^{2}\right)^{-1 / 2} \\
& \frac{\partial z}{\partial r}=\frac{\partial r}{\partial z}=\cos \theta=z\left(x^{2}+y^{2}+z^{2}\right)^{-1 / 2} \\
& \frac{\partial x}{\partial \theta}=r \cos \theta \cos \psi=x z\left(x^{2}+y^{2}\right)^{-1 / 2} \\
& \frac{\partial \theta}{\partial x}=x z\left(x^{2}+y^{2}\right)^{-1 / 2}\left(x^{2}+y^{2}+z^{2}\right)^{-1}=\frac{1}{r} \cos \theta \cos \psi \\
& \frac{\partial y}{\partial \theta}=r \cos \theta \sin \psi=y z\left(x^{2}+y^{2}\right)^{-1 / 2} \\
& \frac{\partial \theta}{\partial y}=y z\left(x^{2}+y^{2}\right)^{-\frac{1}{2}}\left(x^{2}+y^{2}+z^{2}\right)^{-1}=\frac{1}{r} \cos \theta \sin \psi \\
& \frac{\partial z}{\partial \theta}=-r \sin \theta=-\left(x^{2}+y^{2}\right)^{1 / 2}
\end{aligned}
$$




$$
\begin{aligned}
& \frac{\partial \theta}{\partial z}=\frac{z^{2}\left(x^{2}+y^{2}+z^{2}\right)^{-1}-1}{\left(x^{2}+y^{2}\right)^{1 / 2}}=-\frac{1}{r} \sin \theta \\
& \frac{\partial x}{\partial \psi}=-r \sin \theta \sin \psi=-y \\
& \frac{\partial \psi}{\partial x}=-y\left(x^{2}+y^{2}\right)^{-1}=-\frac{1}{r}\left(\frac{\sin \psi}{\sin \theta}\right) \\
& \frac{\partial y}{\partial \psi}=r \sin \theta \cos \psi=x \\
& \frac{\partial \psi}{\partial y}=x\left(x^{2}+y^{2}\right)^{-1}=\frac{1}{r}\left(\frac{\cos \psi}{\sin \theta}\right) \\
& \frac{\partial z}{\partial \psi}=\frac{\partial \psi}{\partial z}=0
\end{aligned}
$$

\section{D.3 VECTOR OPERATORS AND FUNCTIONS}

In the following, $\mathbf{F}(u, v, w)$ is a vector function of the form

$$
\mathbf{F}(u, v, w)=F_{1}(u, v, w) \mathbf{u}+F_{2}(u, v, w) \mathbf{v}+F_{3}(u, v, w) \mathbf{w}
$$

in which $F_{i}(u, v, w)$ is a scalar function of the three variables $u, v$, and $w$, which have the corresponding basis unit vectors $u, v$, and $\mathbf{w}$, respectively.

(a) Gradient Operator $\nabla$

Cartesian: $\nabla F(x, y, z)=\left(\frac{\partial F}{\partial x} \mathrm{i}+\frac{\partial F}{\partial y} \mathrm{j}+\frac{\partial F}{\partial z} \mathrm{k}\right)$

Cylindrical: $\nabla F(\rho, \theta, z)=\left(\frac{\partial F}{\partial \rho} \rho+\frac{1}{\rho} \frac{\partial F}{\partial \theta} \theta+\frac{\partial F}{\partial z} \mathbf{k}\right)$

Spherical: $\nabla F(r, \theta, \psi)=\left(\frac{\partial F}{\partial r} \mathrm{r}+\frac{1}{r}+\frac{\partial F}{\partial \theta} \theta+\frac{1}{r \sin \theta} \frac{\partial F}{\partial \psi} \psi\right)$

(b) Divergence Function $\nabla \cdot \mathrm{F}$

Cartesian: $\nabla \cdot \mathrm{F}(x, y, z)=\frac{\partial F_{1}}{\partial x}+\frac{\partial F_{2}}{\partial y}+\frac{\partial F_{3}}{\partial z}$

Cylindrical: $\nabla \cdot \mathbf{F}(\rho, \theta, z)=\frac{1}{\rho}\left(\frac{\partial F_{1}}{\partial \rho}+\frac{\partial F_{2}}{\partial \theta}+\rho \frac{\partial F_{3}}{\partial z}\right)$ 
Spherical: $\nabla \cdot \mathbf{F}(r, \theta, \psi)=\frac{\partial F_{1}}{\partial r}+\frac{1}{r} \frac{\partial F_{2}}{\partial \theta}+\frac{1}{r \sin \theta} \frac{\partial F_{3}}{\partial \psi}$

(c) Laplacian Operator $\nabla^{2} \quad\left(\nabla^{2} F=\nabla \cdot \nabla F\right)$

Cartesian: $\nabla^{2} F(x, y, z)=\frac{\partial^{2} F}{\partial x^{2}}+\frac{\partial^{2} F}{\partial y^{2}}+\frac{\partial^{2} F}{\partial z^{2}}$

Cylindrical: $\nabla^{2} F(\rho, \theta, z)=\frac{1}{\rho}\left[\frac{\partial}{\partial \rho}\left(\rho \frac{\partial F}{\partial \rho}\right)\right]+\frac{1}{\rho^{2}}\left(\frac{\partial^{2} F}{\partial \theta^{2}}\right)+\frac{\partial^{2} F}{\partial z^{2}}$

Spherical: $\nabla^{2} F(r, \theta, \psi)=\frac{1}{r^{2}}\left[\frac{\partial}{\partial r}\left(r^{2} \frac{\partial F}{\partial r}\right)\right]+\frac{1}{r^{2} \sin \theta}\left[\frac{\partial}{\partial \theta}\left(\sin \theta \frac{\partial F}{\partial \theta}\right)\right]$

$$
+\frac{1}{r^{2} \sin ^{2} \theta}\left(\frac{\partial^{2} F}{\partial \psi^{2}}\right)
$$

(d) Vector Derivative

$$
\frac{\partial \mathbf{F}(x, y, z)}{\partial t}=\frac{\partial F_{1}}{\partial x} \frac{\partial x}{\partial t} \mathbf{i}+\frac{\partial F_{2}}{\partial y} \frac{\partial y}{\partial t} \mathrm{j}+\frac{\partial F_{3}}{\partial z} \frac{\partial z}{\partial t} \mathbf{k}
$$

Similar relations exist for the other coordinate systems.

(e) Divergence Theorem

Let $V$ be a closed and bounded volume as in Fig. D. 2 with $\mathbf{F}$ a vector function everywhere continuous and differentiable in $V$. Then

$$
\int_{V} \nabla \cdot \mathbf{F} d V=\int_{S} \mathbf{F} \cdot \mathbf{n} d A
$$

where $d V=$ differential-volume element of $V$

$$
S=\text { surface of volume } V
$$

$d A=$ differential-area element of $S$

$\mathbf{n}=$ outward-directed unit normal of $d A$

(f) Some Miscellaneous Theorems

(1) $F(\mathbf{u} \cdot \mathbf{v})=(\mathbf{u} F) \cdot \mathbf{v}=\mathbf{u} \cdot \mathbf{v} F$

(2) $\boldsymbol{\nabla} \cdot \mathbf{u} F=F(\nabla \cdot \mathbf{u})+\mathbf{u} \cdot \nabla F$

(3) $\nabla\left(F_{1} F_{2}\right)+F_{1}\left(\nabla F_{2}\right)+F_{2}\left(\nabla F_{1}\right)$

(4) $\nabla^{2}\left(F_{1} F_{2}\right)=F_{1} \nabla^{2} F_{2}+F_{2} \nabla^{2} F_{1}+2\left(\nabla F_{1} \cdot \nabla F_{2}\right)$ 


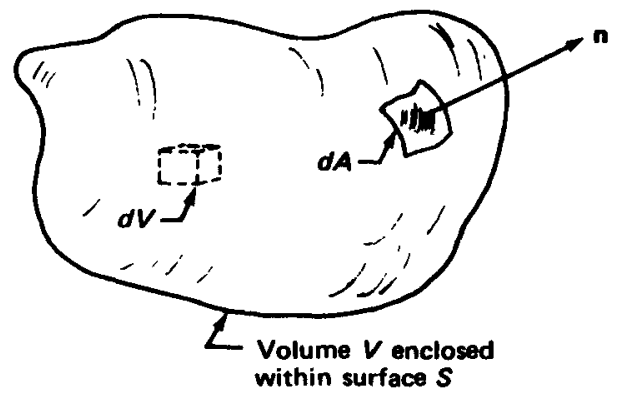

Fig. D.2-Divergence theorem.

(5) $\mathbf{u} \cdot(\mathbf{v}+\mathbf{w})=(\mathbf{u} \cdot \mathbf{v})+(\mathbf{u} \cdot \mathbf{w})$

\section{D.4 DIRAC DELTA FUNCTION}

(a) The Dirac delta function, $\delta\left(x-x_{0}, y-y_{0}, \ldots\right)$, is defined such that

$$
\begin{aligned}
\delta\left(x-x_{0}, y-y_{0}, \ldots\right) & =0 ; x \neq x_{0}, y \neq y_{0}, \ldots \\
& =1 ; x=x_{0}, y=y_{0}, \ldots
\end{aligned}
$$

It has the property that

$\int_{x_{0}-\epsilon_{1}}^{x_{0}+\epsilon_{1}} \int_{y_{0}-\epsilon_{2}}^{y_{0}+\epsilon_{2}} \ldots f(x, y, \ldots) \delta\left(x-x_{0}, y-y_{0}, \ldots\right) d x d y \ldots$

$$
=f\left(x_{0}, y_{0}, \ldots\right)
$$

where $\epsilon_{i}>0$ for $i=1,2, \ldots$

(b) Miscellaneous relations:

(1) $\delta\left(x-x_{0}\right)=\delta\left(-x-x_{0}\right)$

(2) $\delta\left(c x-x_{0}\right)=|c|^{-1} \delta\left(x-x_{0}\right) \quad(c$ is a constant $)$

(3) $\delta\left[\left(x-x_{1}\right),\left(x-x_{2}\right)\right]=\left|x_{1}-x_{2}\right|^{-1}\left[\delta\left(x-x_{1}\right)+\delta\left(x-x_{2}\right)\right]$

(4) $\delta\left(x-x_{1}\right) \cdot \delta\left(x-x_{2}\right)=\delta\left(x-x_{1}\right) \delta\left(x_{1}-x_{2}\right)=\delta\left(x-x_{2}\right) \delta\left(x_{1}-x_{2}\right)$

(5) $\frac{d}{d x} \delta(x)=\frac{1}{\pi} \lim _{k \rightarrow \infty}\left(\frac{k \cos k x}{x}-\frac{\sin k x}{x^{2}}\right)$

(6) $\int_{x_{0}-\epsilon}^{x_{0}+\epsilon} \delta^{\prime}\left(x-x_{0}\right) f(x) d x=-\left.f^{\prime}(x)\right|_{x_{0}}$ 
(7) $\delta^{\prime}\left(x-x_{0}\right)=-\delta^{\prime}\left(x_{0}-x\right)$

(8) $\delta(x)=\frac{1}{\pi} \lim _{k \rightarrow \infty} \int_{0}^{\mathrm{k}} \cos k x d x=\frac{1}{\pi} \lim _{k \rightarrow \infty}\left(\frac{\sin k x}{x}\right)$

(9) $\int_{0}^{x} \delta(y) d y=-\frac{1}{2} \quad(x<0)$

$$
=+\frac{1}{2} \quad(x>0)
$$

(10) $\sum_{n} \psi_{n}(x) \psi_{n}^{*}(y)=\delta(x-y)$, where the set $\psi_{n}(x)$ forms an orthonormal set and $\psi_{n}^{*}(x)$ is the complex conjugate of $\psi_{n}(x)$.

(11) Let the vector $\mathrm{r}=x \mathbf{i}+y \mathbf{j}+z \mathrm{k}$ where $\mathrm{i}, \mathrm{j}$, and $\mathrm{k}$ are unit vectors. Then

$$
\delta(\mathbf{r})=\delta(x) \delta(y) \delta(z)=\frac{1}{(2 \pi)^{3}} \int_{\Omega} e^{i \boldsymbol{\Omega} \cdot \mathbf{r}} d \Omega
$$

and

$$
\begin{aligned}
\int_{a}^{b} \delta(\mathbf{r}) f(\mathbf{r}) d t & =f(0) \quad(a \leqslant 0 \leqslant b) \\
& =0 \text { otherwise }
\end{aligned}
$$

(c) The Kronecker delta function is a discrete analog of the Dirac delta function and is defined for integers $m$ and $n$ by

$$
\begin{aligned}
\delta(m, n,) & =1 \quad(m=n) \\
& =0 \text { otherwise }
\end{aligned}
$$

\section{D.5 LEGENDRE POLYNOMIALS}

(a) The Legendre polynomials $P_{n}(x)$ of degree $n$ are defined by the generating relation

$$
\left(1-2 x t+t^{2}\right)^{-1 / 2}=\sum_{n=0}^{\infty} P_{n}(x) t^{n}
$$

It can be shown that

$$
P_{n}(x)=\sum_{k=0}^{n} a_{k} x^{k}=\sum_{k=0}^{[n / 2]} \frac{(-1)^{k}[2 n-2 k] ! x^{n-2 k}}{2^{n} k !(n-k) !(n-2 k) !}
$$

where $[n / 2]=n / 2$ for even $n$ and $(n-1) / 2$ for odd $n$.

(b) $P_{0}(x)=1 ; P_{1}(x)=x ; P_{2}(x)=\left(3 x^{2}-1\right) / 2 ; P_{3}(x)=\left(5 x^{3}-3 x\right) / 2$ 
(c) Miscellaneous relations:
(1) $\int_{-1}^{1} P_{m}(x) P_{n}(x) d x=\frac{2}{2 n+1} \delta(m, n)$ (orthogonality relation)
(2) $x P_{n}(x)=\frac{1}{2 n+1}\left[(n+1) P_{n+1}(x)+n P_{n+1}(x)\right]$

(recursion relation)

(3) $P_{n}(x)=\frac{1}{\pi} \int_{0}^{\pi}\left[x+\left(x^{2}-1\right)^{1 / 2} \cos \theta\right] d \theta$ (integral form)

(4) $P_{n}(x)=(-1)^{n} P_{n}(-x)$

(5) $P_{n}(x)=\frac{1}{2^{n} n !} \frac{d^{(n)}}{d x^{n}}\left(x^{2}-1\right)^{n}$ (Rodrigues formula)

(6) For $f(x)$ piecewise continuous in $[-1,1]$ and having a first derivative there,

$$
\begin{aligned}
f(x) & =\sum_{n=0}^{\infty} a_{n} P_{n}(x) \text { (expansion theorem) } \\
\text { where } a_{n} & =\left(\frac{2 n+1}{2}\right) \int_{-1}^{1} f(y) P_{n}(y) d y
\end{aligned}
$$

\section{D.6 ASSOCIATED LEGENDRE POL YNOMIALS}

(a) The associated Legendre polynomial of the first kind of degree $n$ and order $m$ is defined by

$$
P_{n}^{m}(\mu)=\frac{\left(1-\mu^{2}\right)^{m / 2}}{2^{n} n !} \frac{d^{(n+m)}}{d \mu^{n+m}}\left(\mu^{2}-1\right)^{n} \quad(0 \leqslant m \leqslant n)
$$

(b) Miscellaneous relations:

(1) $P_{n}^{m}(\mu)=\left(1-\mu^{2}\right)^{m / 2} \frac{d^{(m)}}{d \mu^{m}} P_{n}\left(\mu^{\prime}\right)$

(2) Letting $\mu=\cos \theta$, 


$$
\begin{aligned}
P_{n}^{m}(\cos \theta) & =\sin ^{m} \theta \frac{d(m)}{d \cos ^{m} \theta} P_{n}(\cos \theta) \\
& =\frac{\sin ^{m} \theta}{2^{n} n !} \frac{d^{(n+m)}}{d \cos ^{n+m} \theta}(-\sin \theta)^{n}
\end{aligned}
$$

(3) $P_{n}^{-m}(\mu)=(-1)^{m} \frac{(n-m) !}{(n+m) !} P_{n}^{m}(\mu)$

(4) Two recursion relations are:

$$
\begin{gathered}
\mu P_{n}^{m}(\mu)=\frac{1}{2 n+1}\left[(n+m) P_{n-1}^{m+1}(\mu)+(n-m+1) P_{n+1}^{m}\right] \\
P_{n}^{m}(\mu)=\frac{\left(1-\mu^{2}\right)^{-1 / 2}}{2 n+1}\left[P_{n+1}^{m+1}(\mu)-P_{n-1}^{m}(\mu)\right]
\end{gathered}
$$

(5) The set $P_{n}^{m}(\mu)$ are orthogonal:

$$
\int_{-1}^{1} P_{n}^{m}(\mu) P_{l}^{m}(\mu) d \mu=\frac{2}{2 n+1} \frac{(n+m) !}{(n-m) !} \delta(n, l)
$$

Let $G(\Omega)$ be a function continuous over the unit sphere and having second-order derivatives there. If $\theta$ and $\psi$ are the polar and azimuthal angles, respectively, of $\Omega$, then $G(\Omega)=G(\theta, \psi)$ may be expanded as

(6)

$$
\begin{aligned}
G(\Omega)= & \sum_{n=0}^{\infty}\left[a_{n, 0} P_{n}(\mu)\right. \\
& \left.+\sum_{k=1}^{n}\left(a_{n, k} \cos k \psi+b_{n, k} \sin k \psi\right) P_{n}^{k}(\mu)\right]
\end{aligned}
$$

where $\mu=\cos \theta$

$$
\begin{gathered}
a_{n, 0}=\frac{2 n+1}{4 \pi} \int_{4 \pi} G(\Omega) P_{n}(\mu) d \Omega \\
a_{n, k}=\frac{2 n+1}{2 \pi} m_{4 \pi}^{1} G(\Omega) P_{n}^{k}(\mu) \cos k \psi d \Omega \\
b_{n, k}=\frac{2 n+1}{4 \pi} \int_{4 \pi} G(\Omega) P_{n}^{k}(\mu) \sin k \psi d \Omega \\
(d \Omega=\sin \theta d \theta d \psi=-d \mu d \psi)
\end{gathered}
$$

(7) Let $\omega=\Omega \cdot \Omega^{\prime}$, where $\Omega(\theta, \psi)$ and $\Omega^{\prime}\left(\theta^{\prime}, \psi^{\prime}\right)$ are two unit vectors. The useful addition theorem is an expansion of the Legendre polynomial in terms of the associated functions: 


$$
\begin{aligned}
P_{n}(\omega)=\sum_{m=-n}^{n} F^{2}(n, m) P_{n}^{m}(\mu) P_{n}^{m}\left(\mu^{\prime}\right) e^{i m\left(\psi-\psi^{\prime}\right)}=P_{n}(\mu) P_{n}\left(\mu^{\prime}\right) \\
+2 \sum_{m=1}^{n} F^{2}(n, m) P_{n}^{m}(\mu) P_{n}^{m}\left(\mu^{\prime}\right) \cos m\left(\psi-\psi^{\prime}\right)
\end{aligned}
$$

where $\mu=\cos \theta, \mu^{\prime}=\cos \theta^{\prime}$, and $F(n, m)=\left[\frac{(n-m) !}{(n+m) !}\right]^{1 / 2}$

\section{D.7 ASSOCIATED SPHERICAL-HARMONIC FUNCTIONS}

(a) With the unit vector $\Omega(\theta, \psi)$ defined as above, the associated spheriical harmonics of degree $l$ and order $m$ are defined by

$$
\begin{aligned}
& P_{l, m}(\Omega)=(-1)^{m} F(l, m) e^{i m \psi}\left[\frac{\sin ^{m} \theta}{2^{l l} !} \frac{d^{(l+m)}}{d \mu^{(l+m)}}\left(u^{2}-1\right)^{l}\right] \\
& \text { where } \mu=\cos \theta, F(l, m)=\left[\frac{(l-m) !}{(l+m) !}\right]^{1 / 2}, \text { and }(0 \leqslant m \leqslant l)
\end{aligned}
$$

(b) Miscellaneous relations:

(1) $P_{l, m}(\Omega)=(-1)^{m} F(l, m) P_{l}^{m}(\mu) e^{i m \psi}$

(2) $P_{l, m}(\Omega)=(-1)^{m} F(l, m) P_{l}^{m}(\mu)(\cos m \psi-\mathrm{i} \sin m \psi)$

(3) The complex conjugates are thus given by

$$
\begin{aligned}
& P_{l, m}^{*}(\Omega)=(-1)^{m} F(l, m) P_{l}^{m}(\mu) e^{-i m \psi} \\
& P_{l, m}^{*}(\Omega)=(-1)^{m} F(l, m) P_{l}^{m}(\mu)(\cos m \psi-i \sin m \psi)
\end{aligned}
$$

(4) In the degenerate case where $m=0$

$$
\begin{aligned}
& P_{l, 0}(\Omega)=P_{l, 0}^{*}(\Omega)=P_{l}^{\uparrow}(\mu)=P_{l}(\mu) \\
& P_{l,-m}(\Omega)=(-1)^{m} P_{l, m}^{*}(\Omega)
\end{aligned}
$$

(5) There are two orthogonal properties:

$$
\begin{aligned}
& \int_{4 \pi} P_{l, m}(\Omega) P_{j, k}(\Omega) d \Omega=\frac{4 \pi}{2 l+1} \partial(l, j) \partial(m, k) \partial(m, 0) \\
& \int_{4 \pi} P_{l, m}(\Omega) P_{j, k}^{*}(\Omega) d \Omega=\frac{4 \pi}{2 l+1} \partial(l, j) \partial(m, k)
\end{aligned}
$$

(6) The addition theorem can be written as an expansion of $P_{n}(\omega)$ in spherical harmonics:

$$
P_{n}(\omega)=\sum_{m=-n}^{n} P_{n, m}(\Omega) P_{n, m}^{*}\left(\Omega^{\prime}\right)
$$




\section{Exposure Buildup Factors}

\section{Appendix E}

Table E.1 contains exposure buildup factors. Plots for a given initial energy and penetration depth $\left(\mu_{0} r\right)$ vs. atomic number can be used for interpolation.

Table E.1-EXPOSURE BUILDUP FACTOR $\left(B_{r}\right)$ POINT ISOTROPIC SOURCE $†$

\begin{tabular}{|c|c|c|c|c|c|c|c|c|}
\hline \multirow[b]{2}{*}{ Material } & \multirow{2}{*}{$\begin{array}{c}E_{0} \\
\mathrm{MeV}\end{array}$} & \multicolumn{7}{|c|}{$\mu_{0} r$} \\
\hline & & 1 & 2 & 4 & 7 & 10 & 15 & 20 \\
\hline \multirow[t]{9}{*}{ Water } & 0.255 & 3.09 & 7.14 & 23.0 & 72.9 & 166 & 456 & 982 \\
\hline & 0.5 & 2.52 & 5.14 & 14.3 & 38.8 & 77.6 & 178 & 334 \\
\hline & 1.0 & 2.13 & 3.71 & 7.68 & 16.2 & 27.1 & 50.4 & 82.2 \\
\hline & 2.0 & 1.83 & 2.77 & 4.88 & 8.46 & 12.4 & 19.5 & 27.7 \\
\hline & 3.0 & 1.69 & 2.42 & 3.91 & 6.23 & 8.63 & 12.8 & 17.0 \\
\hline & 4.0 & 1.58 & 2.17 & 3.34 & 5.13 & 6.94 & 9.97 & 12.9 \\
\hline & 6.0 & 1.46 & 1.91 & 2.76 & 3.99 & 5.18 & 7.09 & 8.85 \\
\hline & 8.0 & 1.38 & 1.74 & 2.40 & 3.34 & 4.25 & 5.66 & 6.95 \\
\hline & 10.0 & 1.33 & 1.63 & 2.19 & 2.97 & 3.72 & 4.90 & 5.98 \\
\hline \multirow[t]{8}{*}{ Aluminum } & 0.5 & 2.37 & 4.24 & 9.47 & 21.5 & 38.9 & 80.8 & 141 \\
\hline & 1.0 & 2.02 & 3.31 & 6.57 & 13.1 & 21.2 & 37.9 & 58.5 \\
\hline & 2.0 & 1.75 & 2.61 & 4.62 & 8.05 & 11.9 & 18.7 & 26.3 \\
\hline & 3.0 & 1.64 & 2.32 & 3.78 & 6.14 & 8.65 & 13.0 & 17.7 \\
\hline & 4.0 & 1.53 & 2.08 & 3.22 & 5.01 & 6.88 & 10.1 & 13.4 \\
\hline & 6.0 & 1.42 & 1.85 & 2.70 & 4.06 & 5.49 & 7.97 & 10.4 \\
\hline & 8.0 & 1.34 & 1.68 & 2.37 & 3.45 & 4.58 & 6.56 & 8.52 \\
\hline & 10.0 & 1.28 & 1.55 & 2.12 & 3.01 & 3.96 & 5.63 & 7.32 \\
\hline \multirow[t]{8}{*}{ Iron } & 0.5 & 1.98 & 3.09 & 5.98 & 11.7 & 19.2 & 35.4 & 55.6 \\
\hline & 1.0 & 1.87 & 2.89 & 5.39 & 10.2 & 16.2 & 28.3 & 42.7 \\
\hline & 2.0 & 1.76 & 2.43 & 4.13 & 7.25 & 10.9 & 17.6 & 25.1 \\
\hline & 3.0 & 1.55 & 2.15 & 3.51 & 5.85 & 8.51 & 13.5 & 19.1 \\
\hline & 4.0 & 1.45 & 1.94 & 3.03 & 4.91 & 7.11 & 11.2 & 16.0 \\
\hline & 6.0 & 1.34 & 1.72 & 2.58 & 4.14 & 6.02 & 9.89 & 14.7 \\
\hline & 8.0 & 1.27 & 1.56 & 2.23 & 3.49 & 5.07 & 8.50 & 13.0 \\
\hline & 10.0 & 1.20 & 1.42 & 1.95 & 2.99 & 4.35 & 7.54 & 12.4 \\
\hline
\end{tabular}


Table E.1-(Continued)

\begin{tabular}{|c|c|c|c|c|c|c|c|c|}
\hline \multirow[b]{2}{*}{ Material } & \multirow{2}{*}{$\begin{array}{c}E_{0} \\
\mathrm{MeV}\end{array}$} & \multicolumn{7}{|c|}{$\mu_{0} r$} \\
\hline & & 1 & 2 & 4 & 7 & 10 & 15 & 20 \\
\hline \multirow[t]{8}{*}{ Tin } & 0.5 & 1.56 & 2.08 & 3.09 & 4.57 & 6.04 & 8.64 & \\
\hline & 1.0 & 1.64 & 2.30 & 3.74 & 6.17 & 8.85 & 13.7 & 18.8 \\
\hline & 2.0 & 1.57 & 2.17 & 3.53 & 5.87 & 8.53 & 13.6 & 19.3 \\
\hline & 3.0 & 1.46 & 1.96 & 3.13 & 5.28 & 7.91 & 13.3 & 20.1 \\
\hline & 4.0 & 1.38 & 1.81 & 2.82 & 4.82 & 7.41 & 13.2 & 21.2 \\
\hline & 6.0 & 1.26 & 1.57 & 2.37 & 4.17 & 6.94 & 14.8 & 29.1 \\
\hline & 8.0 & 1.19 & 1.42 & 2.05 & 3.57 & 6.19 & 15.1 & 34.0 \\
\hline & 10.0 & 1.14 & 1.31 & 1.79 & 2.99 & 5.21 & 12.5 & 33.4 \\
\hline \multirow[t]{8}{*}{ Tungsten } & 0.5 & 1.28 & 1.50 & 1.84 & 2.24 & 2.61 & 3.12 & \\
\hline & 1.0 & 1.44 & 1.83 & 2.57 & 3.62 & 4.64 & 6.25 & $(7.35)$ \\
\hline & 2.0 & 1.42 & 1.85 & 2.72 & 4.09 & 5.27 & 8.07 & $(10.6)$ \\
\hline & 3.0 & 1.36 & 1.74 & 2.59 & 4.00 & 5.92 & 9.66 & 14.1 \\
\hline & 4.0 & 1.29 & 1.62 & 2.41 & 4.03 & 6.27 & 12.0 & 20.9 \\
\hline & 6.0 & 1.20 & 1.43 & 2.07 & 3.60 & 6.29 & 15.7 & 36.3 \\
\hline & 8.0 & 1.14 & 1.32 & 1.81 & 3.05 & 5.40 & 15.2 & 41.9 \\
\hline & 10.0 & 1.11 & 1.25 & 1.64 & 2.62 & 4.65 & 14.0 & 39.3 \\
\hline \multirow[t]{9}{*}{ Lead } & 0.5 & 1.24 & 1.42 & 1.69 & 2.00 & 2.27 & 2.65 & $(2.73)$ \\
\hline & 1.0 & 1.37 & 1.69 & 2.26 & 3.02 & 3.74 & 4.81 & 5.86 \\
\hline & 2.0 & 1.39 & 1.76 & 2.51 & 3.66 & 4.84 & 6.87 & 9.00 \\
\hline & 3.0 & 1.34 & 1.68 & 2.43 & 3.75 & 5.30 & 8.44 & 12.3 \\
\hline & 4.0 & 1.27 & 1.56 & 2.25 & 3.61 & 5.44 & 9.80 & 16.3 \\
\hline & 5.1 & 1.21 & 1.46 & 2.08 & 3.44 & 5.55 & 11.7 & 23.6 \\
\hline & 6.0 & 1.18 & 1.40 & 1.97 & 3.34 & 5.69 & 13.8 & 32.7 \\
\hline & 8.0 & 1.14 & 1.30 & 1.74 & 2.89 & 5.07 & 14.1 & 44.6 \\
\hline & 10.0 & 1.11 & 1.23 & 1.58 & 2.52 & 4.34 & 12.5 & 39.2 \\
\hline \multirow[t]{8}{*}{ Uranium } & 0.5 & 1.17 & 1.30 & 1.48 & 1.67 & 1.85 & 2.08 & \\
\hline & 1.0 & 1.31 & 1.56 & 1.98 & 2.50 & 2.97 & 3.67 & \\
\hline & 2.0 & 1.33 & 1.64 & 2.23 & 3.09 & 3.95 & 5.36 & $(6.48)$ \\
\hline & 3.0 & 1.29 & 1.58 & 2.21 & 3.27 & 4.51 & 6.97 & 9.88 \\
\hline & 4.0 & 1.24 & 1.50 & 2.09 & 3.21 & 4.66 & 8.01 & 12.7 \\
\hline & 6.0 & 1.16 & 1.36 & 1.85 & 2.96 & 4.80 & 10.8 & 23.0 \\
\hline & 8.0 & 1.12 & 1.27 & 1.66 & 2.61 & 4.36 & 11.2 & 28.0 \\
\hline & 10.0 & 1.09 & 1.20 & 1.51 & 2.26 & 3.78 & 10.5 & 28.5 \\
\hline
\end{tabular}

†From H. Goldstein and J. E. Wilkins, Jr., Calculations of the Penetrations of Gamma Rays, Final Report, USAEC Report NYO-3075, Nuclear Development Associates, Inc., June 30, 1954. 


\section{Coefficients for Gamma-Ray Buildup Factors}

\section{Appendix F}

Table F.1 -COEFFICIENTS FOR LINEAR, QUADRATIC, AND BERGER FORMS OF EXPOSURE BUILDUP FACTORS FITTED OVER THE RANGE 0 TO 7 MEAN FREE PATHS FROM POINT ISOTROPIC SOURCE †

\begin{tabular}{|c|c|c|c|c|c|c|c|c|c|}
\hline \multirow[b]{2}{*}{ Material } & \multirow[b]{2}{*}{$E, \mathrm{MeV}$} & \multicolumn{2}{|c|}{ Linear } & \multicolumn{3}{|c|}{ Quadratic } & \multicolumn{3}{|c|}{ Berger } \\
\hline & & $A_{1}$ & $\begin{array}{c}\text { Maximum } \\
\text { error, } \neq \\
\%\end{array}$ & $A_{2}$ & $b$ & $\begin{array}{c}\text { Maximum } \\
\text { error, } \\
\%\end{array}$ & $C$ & $D$ & $\begin{array}{c}\text { Maximum } \\
\text { error, } \\
\%\end{array}$ \\
\hline \multirow[t]{9}{*}{ Water } & 0.255 & 8.6524 & F3.1 & -0.2525 & 1.4984 & 30 & 1.7506 & 0.2609 & 10 \\
\hline & 0.5 & 4.6800 & F2.3 & 0.6684 & 0.6750 & 8 & 1.3245 & 0.2078 & 5 \\
\hline & 1 & 1.9953 & 40 & 1.0053 & 0.1666 & 2 & 1.0622 & 0.1052 & 3 \\
\hline & 2 & 1,0301 & 10 & 0.8242 & 0.0346 & 2 & 0.8093 & 0.0408 & 1 \\
\hline & 3 & 0.7397 & 3 & 0.6962 & 0.0073 & 1 & 0.6876 & 0.0125 & 1 \\
\hline & 4 & 0.5884 & 1 & 0.5801 & 0.0014 & 1 & 0.5800 & 0.0024 & 1 \\
\hline & 6 & 0.4321 & 3 & 0.4616 & -0.0050 & 1 & 0.4655 & -0.0126 & 1 \\
\hline & 8 & 0.3406 & 4 & 0.3782 & -0.0063 & 1 & 0.3860 & -0.0214 & 1 \\
\hline & 10 & 0.2877 & 4 & 0.3251 & -0.0063 & 1 & 0.3342 & -0.0257 & 1 \\
\hline \multirow[t]{8}{*}{ Aluminum } & 0.5 & 2.6461 & F1. 5 & 1.0688 & 0.2654 & 2 & 1.2435 & 0.1250 & 3 \\
\hline & 1 & 1.6089 & 30 & 0.9316 & 0.1140 & 2 & 0.9589 & 0.0864 & 3 \\
\hline & 2 & 0.9686 & 13 & 0.7437 & 0.0378 & 2 & 0.7267 & 0.0486 & 2 \\
\hline & 3 & 0.7197 & 5 & 0.6355 & 0.0142 & 1 & 0.6294 & 0.0227 & 1 \\
\hline & 4 & 0.5663 & 3 & 0.5284 & 0.0064 & 1 & 0.5253 & 0.0127 & 1 \\
\hline & 6 & 0.4334 & 2 & 0.4142 & 0.0032 & 1 & 0.4177 & 0.0061 & 1 \\
\hline & 8 & 0.3476 & 1 & 0.3346 & 0.0022 & 1 & 0.3371 & 0.0050 & 1 \\
\hline & 10 & 0.2847 & 2 & 0.2715 & 0.0022 & 1 & 0.2752 & 0.0055 & 1 \\
\hline \multirow[t]{8}{*}{ Iron } & 0.5 & 1.4283 & 25 & 0.8642 & 0.0949 & 1 & 0.9081 & 0.0752 & 2 \\
\hline & 1 & 1.2373 & 20 & 0.8026 & 0.0731 & 1 & 0.8214 & 0.0684 & 2 \\
\hline & 2 & 0.8556 & 12 & 0.6526 & 0.0342 & 5 & 0.7020 & 0.0319 & 3 \\
\hline & 3 & 0.6691 & 9 & 0.5338 & 0.0228 & 1 & 0.5323 & 0.0384 & 1 \\
\hline & 4 & 0.5403 & 7 & 0.4366 & 0.0175 & 1 & 0.4366 & 0.0358 & 1 \\
\hline & 6 & 0.4297 & 8 & 0.3237 & 0.0178 & 1 & 0.3271 & 0.0457 & 1 \\
\hline & 8 & 0.3391 & 8 & 0.2473 & 0.0154 & 1 & 0.2563 & 0.0464 & 1 \\
\hline & 10 & 0.2681 & 8 & 0.1785 & 0.0151 & 1 & 0.1876 & 0.0592 & 1 \\
\hline \multirow[t]{8}{*}{$\operatorname{Tin}$} & 0.5 & 0.5153 & 3 & 0.5479 & -0.0055 & 2 & 0.5608 & -0.0146 & 1 \\
\hline & 1 & 0.7199 & 6 & 0.6153 & 0.0176 & 1 & 0.6219 & 0.0244 & 1 \\
\hline & 2 & 0.6731 & 8 & 0.5455 & 0.0215 & 1 & 0.5498 & 0.0338 & 1 \\
\hline & 3 & 0.5837 & 11 & 0.4284 & 0.0261 & 1 & 0.4379 & 0.0479 & 1 \\
\hline & 4 & 0.5146 & 12 & 0.3420 & 0.0290 & 1 & 0.3583 & 0.0601 & 1 \\
\hline & 6 & 0.4153 & 17 & 0.2082 & 0.0348 & 2 & 0.2369 & 0.0925 & 1 \\
\hline & 8 & 0.3317 & 17 & 0.1371 & 0.0327 & 2 & 0.1692 & 0.1103 & 1 \\
\hline & 10 & 0.2550 & 16 & 0.0945 & 0.0270 & 2 & 0.1232 & 0.1190 & 1 \\
\hline
\end{tabular}


Table F.1 - (Continued $)$

\begin{tabular}{|c|c|c|c|c|c|c|c|c|c|}
\hline \multirow[b]{2}{*}{ Materıal } & \multirow[b]{2}{*}{$E, \mathrm{MeV}$} & \multicolumn{2}{|c|}{ Linear } & \multicolumn{3}{|c|}{ Quadratic } & \multicolumn{3}{|c|}{ Berger } \\
\hline & & $A_{1}$ & $\begin{array}{c}\text { Maximum } \\
\text { error, } \neq \\
\%\end{array}$ & $A_{2}$ & $b$ & $\begin{array}{c}\text { Maximum } \\
\text { error, } \\
\%\end{array}$ & $C$ & $D$ & $\begin{array}{c}\text { Maximum } \\
\text { error, } \\
\%\end{array}$ \\
\hline \multirow[t]{8}{*}{ Tungsten } & 0.5 & 0.1903 & 8 & 0.2692 & -0.0133 & 2 & 0.2938 & -0.0751 & 2 \\
\hline & 1 & 0.3817 & 5 & 0.4269 & -0.0076 & 2 & 0.4425 & -0.0255 & 1 \\
\hline & 2 & 0.4376 & 2 & 0.4164 & 0.0036 & 1 & 0.4172 & 0.0080 & 1 \\
\hline & 3 & 0.4171 & 5 & 0.3515 & 0.0110 & 1 & 0.3501 & 0.0295 & 1 \\
\hline & 4 & 0.4054 & 12 & 0.2540 & 0.0255 & 1 & 0.2710 & 0.0666 & 1 \\
\hline & 6 & 0.3363 & 17 & 0.1435 & 0.0324 & 2 & 0.1771 & 0.1049 & 1 \\
\hline & 8 & 0.2624 & 16 & 0.0957 & 0.0281 & 2 & 0.1245 & 0.1223 & 1 \\
\hline & 10 & 0.2073 & 14 & 0.0748 & 0.0223 & 2 & 0.0974 & 0.1238 & 1 \\
\hline \multirow[t]{9}{*}{ Lead } & 0.5 & 0.1549 & 8 & 0.2273 & -0.0122 & 3 & 0.2526 & -0.0848 & 2 \\
\hline & 1 & 0.2990 & 6 & 0.3613 & -0.0105 & 2 & 0.3779 & -0.0403 & 1 \\
\hline & 2 & 0.3796 & 1 & 0.3787 & 0.0001 & 1 & 0.3862 & 0.0032 & 1 \\
\hline & 3 & 0.3810 & 5 & 0.3164 & 0.0109 & 1 & 0.3267 & 0.0253 & 1 \\
\hline & 4 & 0.3523 & 10 & 0.2389 & 0.0191 & 1 & 0.2530 & 0.0547 & 1 \\
\hline & 5.1 & 0.3219 & 13 & 0.1747 & 0.0248 & 1 & 0.1936 & 0.0839 & 1 \\
\hline & 6 & 0.3034 & 15 & 0.1346 & 0.0284 & 2 & 0.1622 & 0.1027 & 1 \\
\hline & 8 & 0.2419 & 15 & 0.0894 & 0.0257 & 3 & 0.1220 & 0.1112 & 2 \\
\hline & 10 & 0.1933 & 13 & 0.0642 & 0.0217 & 3 & 0.0939 & 0.1167 & 2 \\
\hline \multirow[t]{8}{*}{ Uranium } & 0.5 & 0.1054 & 7 & 0.1637 & -0.0098 & 2 & 0.1825 & -0.0951 & 2 \\
\hline & 1 & 0.2264 & 7 & 0.2990 & -0.0122 & 2 & 0.3204 & -0.0599 & 2 \\
\hline & 2 & 0.3023 & 3 & 0.3250 & -0.0038 & 1 & 0.3321 & -0.0162 & 1 \\
\hline & 3 & 0.3169 & 4 & 0.2760 & 0.0069 & 1 & 0.2814 & 0.0196 & 1 \\
\hline & 4 & 0.3010 & 7 & 0.2199 & 0.0136 & 1 & 0.2283 & 0.0458 & 1 \\
\hline & 6 & 0.2571 & 12 & 0.1314 & 0.0212 & 1 & 0.1476 & 0.0916 & 1 \\
\hline & 8 & 0.2081 & 12 & 0.0885 & 0.0201 & 1 & 0.1081 & 0.1076 & 1 \\
\hline & 10 & 0.1621 & 11 & 0.0638 & 0.0165 & 1 & 0.0798 & 0.1163 & 1 \\
\hline
\end{tabular}

†From D. K. Trubey, A Survey of Empincal Functions Used To Fit Gamma-Ray Buildup Factors, USAEC Report ORNL-RSIC-10, Oak Ridge National Laboratory, February 1966.

$\ddagger$ F3.1 means "factor of 3.1 ," etc.

Table F.2-COEFFICIENTS FOR LINEAR, QUADRATIC, AND BERGER FORMS OF EXPOSURE BUILDUP FACTORS FITTED OVER THE RANGE 0 TO 20 MEAN FREE PATHS FROM POINT ISOTROPIC SOURCES $\uparrow$

\begin{tabular}{|c|c|c|c|c|c|c|c|c|c|}
\hline \multirow[b]{2}{*}{ Material } & \multirow[b]{2}{*}{$E, \mathrm{MeV}$} & \multicolumn{2}{|c|}{ Linear } & \multicolumn{3}{|c|}{ Quadratic } & \multicolumn{3}{|c|}{ Berger } \\
\hline & & $A_{1}$ & $\begin{array}{c}\text { Maximum } \\
\text { error, } \neq \\
\%\end{array}$ & $A_{2}$ & $b$ & $\begin{array}{c}\text { Maximum } \\
\text { error, } \\
\% \neq\end{array}$ & $C$ & $D$ & $\begin{array}{c}\text { Maximum } \\
\text { error, } \\
\%\end{array}$ \\
\hline \multirow[t]{9}{*}{ Water } & 0.255 & 36.1015 & F12 & -12.9947 & 3.0515 & $F \infty$ & 2.5048 & 0.1623 & 30 \\
\hline & 0.5 & 13.0926 & F5.6 & -0.9744 & 0.8743 & F3 & 1.8035 & 0.1224 & 25 \\
\hline & 1 & 3.4788 & F2 & 1.1152 & 0.1469 & 6 & 1.2282 & 0.0649 & 11 \\
\hline & 2 & 1.2549 & 25 & 0.9173 & 0.0210 & 6 & 0.8594 & 0.0240 & 5 \\
\hline & 3 & 0.7863 & 6 & 07218 & 0.0040 & 2 & 0.7004 & 0.0074 & 2 \\
\hline & 4 & 0.5951 & 1 & 0.5907 & 0.0003 & 1 & 0.5826 & 0.0014 & 1 \\
\hline & 6 & 0.4030 & 5 & 0.4471 & -0.0027 & 2 & 0.4853 & -0.0082 & 1 \\
\hline & 8 & 0.3085 & 7 & 0.3561 & -0.0038 & 2 & 0.3741 & -0.0124 & 2 \\
\hline & 10 & 0.2584 & 7 & 0.3002 & -0.0026 & 3 & 0.3206 & -0.0139 & 2 \\
\hline
\end{tabular}

(Table continues on next page.) 
Table F $2-$ (Continued)

\begin{tabular}{|c|c|c|c|c|c|c|c|c|c|}
\hline \multirow[b]{2}{*}{ Material } & \multirow[b]{2}{*}{$E, \mathrm{MeV}$} & \multicolumn{2}{|c|}{ Linear } & \multicolumn{3}{|c|}{ Quadratic } & \multicolumn{3}{|c|}{ Berger } \\
\hline & & $A_{1}$ & $\begin{array}{c}\text { Maximum } \\
\text { error, } \ddagger \\
\%\end{array}$ & $A_{2}$ & $b$ & $\begin{array}{c}\text { Maxımum } \\
\text { error, } \\
\% \ddagger\end{array}$ & $C$ & $D$ & $\begin{array}{c}\text { Maximum } \\
\text { error, } \\
\%\end{array}$ \\
\hline \multirow[t]{8}{*}{ Aluminum } & 05 & 57374 & F3 & 06696 & 03150 & 20 & 14412 & 00850 & 12 \\
\hline & 1 & 25385 & F1 9 & 11185 & 00883 & 10 & 10831 & 00535 & 9 \\
\hline & 2 & 11928 & 30 & 08751 & 00197 & 8 & 07869 & 00266 & 6 \\
\hline & 3 & 08061 & 12 & 06812 & 00078 & 3 & 06504 & 00137 & 3 \\
\hline & 4 & 06075 & 6 & 05503 & 00036 & 2 & 05343 & 00082 & 2 \\
\hline & 6 & 04626 & 6 & 04252 & 00023 & 2 & 04182 & 00063 & 1 \\
\hline & 8 & 03697 & 5 & 03395 & 00019 & 1 & 03366 & 00058 & 1 \\
\hline & 10 & 03087 & 5 & 02750 & 00021 & 1 & 02738 & 00074 & 1 \\
\hline \multirow[t]{8}{*}{ Iron } & 05 & 23773 & F19 & 09019 & 00917 & 3 & 09814 & 00548 & 7 \\
\hline & 1 & 18643 & F1 6 & 09212 & 00586 & 7 & 08932 & 00460 & 7 \\
\hline & 2 & 11194 & 33 & 07423 & 00234 & 6 & 07173 & 00277 & 4 \\
\hline & 3 & 08446 & 25 & 05840 & 00162 & 4 & 05571 & 00261 & 4 \\
\hline & 4 & 06942 & 25 & 04605 & 00145 & 2 & 04518 & 00268 & 3 \\
\hline & 6 & 06134 & 34 & 03201 & 00182 & 1 & 03381 & 00368 & 3 \\
\hline & 8 & 05245 & 40 & 02207 & 00189 & 3 & 02603 & 00428 & 2 \\
\hline & 10 & 04759 & 50 & 01143 & 00225 & 6 & 01902 & 00553 & 1 \\
\hline \multirow[t]{8}{*}{ Tin } & 05 & 05090 & 4 & 05150 & -00005 & 3 & 05457 & -00063 & 3 \\
\hline & 1 & 08495 & 18 & 06666 & 00114 & 3 & 06378 & 00180 & 3 \\
\hline & 2 & 08521 & 25 & 05826 & 00168 & 3 & 05678 & 00254 & 3 \\
\hline & 3 & 08509 & 40 & 04254 & 00264 & 1 & 04533 & 00388 & 3 \\
\hline & 4 & 08643 & F1 58 & 02845 & 00360 & 5 & 03700 & 00518 & 3 \\
\hline & 6 & 10786 & F2 2 & -01374 & 00756 & 40 & 02401 & 00891 & 2 \\
\hline & 8 & 11907 & F2 8 & -04693 & 01032 & F3 & 01669 & 01145 & 1 \\
\hline & 10 & 11075 & F30 & -06523 & 01094 & F 12 & 01190 & 01278 & 5 \\
\hline \multirow[t]{8}{*}{ Tungsten } & 05 & 01550 & 13 & 02206 & -00054 & 5 & 02692 & -00477 & 5 \\
\hline & 1 & 03382 & 9 & 04149 & -00048 & 2 & 04279 & -00150 & 2 \\
\hline & 2 & 04671 & 8 & 04072 & 00037 & 3 & 04163 & 00070 & 4 \\
\hline & 3 & 05919 & 30 & 03255 & 00165 & 2 & 03484 & 00324 & 2 \\
\hline & 4 & 08102 & F1 8 & 00995 & 00442 & 15 & 02727 & 00653 & 1 \\
\hline & 6 & 12616 & F3 & -05462 & 01124 & F4 & 01704 & 01160 & 2 \\
\hline & 8 & 13753 & F3 5 & -09399 & 01439 & $F_{\infty}$ & 01161 & 01405 & 6 \\
\hline & 10 & 12730 & F3 8 & -09502 & 01382 & $\mathbf{F} \infty$ & 00882 & 01510 & 7 \\
\hline \multirow[t]{9}{*}{ Lead } & 05 & 01043 & 15 & 01791 & -00047 & 5 & 02243 & -00500 & 5 \\
\hline & 1 & 02549 & 11 & 03133 & -00036 & 5 & 03530 & -00211 & 4 \\
\hline & 2 & 03947 & 3 & 03695 & 00015 & 2 & 03791 & 00021 & 1 \\
\hline & 3 & 05123 & 30 & 02990 & 00133 & 2 & 03244 & 00279 & 1 \\
\hline & 4 & 06378 & F1 6 & 01449 & 00306 & 10 & 02526 & 00557 & 1 \\
\hline & 51 & 08560 & F2 1 & -01480 & 00624 & 40 & 01904 & 00883 & 2 \\
\hline & 6 & 11247 & F2 8 & -05070 & 01014 & F4 & 01554 & 01143 & 4 \\
\hline & 8 & 14165 & F4 & -11408 & 01589 & $F \infty$ & 01075 & 01440 & 12 \\
\hline & 10 & 12370 & F4 & -10279 & 01408 & $F \infty$ & 00824 & 01513 & 12 \\
\hline \multirow[t]{8}{*}{ Uranıum } & 05 & 00812 & 11 & 01262 & -00037 & 5 & 01635 & -00606 & 5 \\
\hline & 1 & 01914 & 11 & 02556 & -00053 & 5 & 02991 & -00385 & 5 \\
\hline & 2 & 02838 & 5 & 03185 & -00022 & 2 & 03240 & -00084 & 2 \\
\hline & 3 & 04081 & 20 & 02614 & 00091 & 2 & 02781 & 00234 & 1 \\
\hline & 4 & 04991 & 43 & 01621 & 00210 & 6 & 02273 & 00475 & 1 \\
\hline & 6 & 08088 & F2 3 & -02492 & 00658 & F2 & 01426 & 01011 & 2 \\
\hline & 8 & 09323 & F2 9 & -05357 & 00912 & F5 & 01004 & 01274 & 5 \\
\hline & 10 & 09203 & F3 3 & -06560 & 00980 & $F_{\infty}$ & 00721 & 01442 & 7 \\
\hline
\end{tabular}

tFrom D K Trubey 4 Survey of Empirical Functions Used To Fit Gamma Ray Buildup Factors, USAEC Report ORNL RSIC 10 Oak Ridge National Laboratory, February 1966

$\$$ F12 means 'factor of 12 ' etc 
Table F.3 - BERGER FORMULA CONSTANTS FOR ENERGY-DEPOSITION BUILDUP, POINT ISOTROPIC SOURCE, 20-MEAN-FREE-PATH FIT $\dagger$

\begin{tabular}{|c|c|c|c|c|c|c|c|c|c|c|c|c|c|c|c|}
\hline \multirow[b]{2}{*}{$E, \mathrm{MeV}$} & \multicolumn{3}{|c|}{ Water } & \multicolumn{3}{|c|}{ Aluminum } & \multicolumn{3}{|c|}{ Iron } & \multicolumn{3}{|c|}{ Tin } & \multicolumn{3}{|c|}{ Lead } \\
\hline & $C$ & $D$ & $\begin{array}{c}\text { Maximum } \\
\text { error, } \%\end{array}$ & C & $D$ & $\begin{array}{c}\text { Maximum } \\
\text { error, } \%\end{array}$ & $C$ & $D$ & $\begin{array}{c}\text { Maximum } \\
\text { error, \% }\end{array}$ & $C$ & $D$ & $\begin{array}{l}\text { Maximum } \\
\text { error, } \%\end{array}$ & C & $D$ & $\begin{array}{c}\text { Maximum } \\
\text { error, } \%\end{array}$ \\
\hline 0.255 & 2.4039 & 0.1598 & 30 & & & & & & & & & & 0.4551 & -0.0753 & 10 \\
\hline 0.5 & 1.7128 & 0.1201 & 24 & 1.7237 & 0.0902 & 14 & 1.7887 & 0.0550 & 7 & 1.1873 & -0.0069 & 4 & 0.7027 & -0.0278 & 7 \\
\hline 1 & 1.2443 & 0.0660 & 12 & 1.2204 & 0.0553 & 10 & 1.2192 & 0.0466 & 8 & 1.0819 & 0.0134 & 3 & 0.5510 & -0.0014 & 3 \\
\hline 2 & 0.8829 & 0.0243 & 5 & 0.8480 & 0.0268 & 6 & 0.7868 & 0.0280 & 4 & 0.7490 & 0.0245 & 4 & 0.3476 & 0.0251 & 2 \\
\hline 3 & 0.7506 & 0.0065 & 2 & 0.6746 & 0.0134 & 3 & 0.5847 & 0.0255 & 4 & 0.4909 & 0.0380 & 9 & 0.2227 & 0.0532 & 1 \\
\hline 4 & 0.5905 & 0.0012 & 1 & 0.5411 & 0.0079 & 2 & 0.4494 & 0.0281 & 2 & 0.3352 & 0.0534 & 8 & 0.1646 & 0.0818 & 3 \\
\hline 6 & 0.4550 & -0.0079 & 1 & 0.3995 & 0.0060 & 1 & 0.2975 & 0.0353 & 3 & 0.1931 & 0.0820 & 2 & 0.1167 & 0.1060 & 5 \\
\hline 8 & 0.3757 & -0.0121 & 2 & 0.3079 & 0.0060 & 1 & 0.2076 & 0.0420 & 2 & 0.1237 & 0.1030 & 2 & 0.0781 & 0.1269 & 8 \\
\hline 10 & 0.3036 & -0.0133 & 2 & 0.2492 & 0.0074 & 1 & 0.1565 & 0.0528 & 1 & 0.0930 & 0.1148 & 4 & 0.0588 & 0.1327 & 15 \\
\hline
\end{tabular}

tFrom D. K. Trubey, A Survey of Empirical Functions Used To Fit Gamma-Ray Buildup Factors, USAEC Report ORNL-RSIC-10, Oak Ridge National Laboratory, February 1966. 
Table F.4-PARAMETERS $a, b$, AND $c$ FOR ENERGY-DEPOSITION BUILDUP FACTOR IN THE EQUATION $†$

$B(\mu x)=1.0+a(\mu x)+b(\mu x)^{2}+c(\mu x)^{3}$

FOR A POINT ISOTROPIC SOURCE

\begin{tabular}{|c|c|c|c|c|c|c|c|}
\hline \multirow{2}{*}{$\begin{array}{c}Z \text { of } \\
\text { material }\end{array}$} & \multicolumn{7}{|c|}{ Parameters for energy of } \\
\hline & $0.5 \mathrm{MeV}$ & $1 \mathrm{MeV}$ & $2 \mathrm{MeV}$ & $4 \mathrm{MeV}$ & $6 \mathrm{MeV}$ & $8 \mathrm{MeV}$ & $10 \mathrm{MeV}$ \\
\hline \multicolumn{8}{|c|}{ Parameter $a$} \\
\hline $7.5\left(\mathrm{H}_{2} \mathrm{O}\right)$ & 0.840 & 0.970 & 0.830 & 0.589 & 0.460 & 0.381 & 0.312 \\
\hline 2 & 0.22 & 0.97 & 0.76 & 0.65 & 0.54 & 0.45 & 0.39 \\
\hline 4 & 0.44 & 0.98 & 0.76 & 0.63 & 0.51 & 0.42 & 0.36 \\
\hline 6 & 0.64 & 0.99 & 0.76 & 0.60 & 0.48 & 0.40 & 0.34 \\
\hline 8 & 0.83 & 1.00 & 0.76 & 0.58 & 0.46 & 0.37 & 0.31 \\
\hline 10 & 1.01 & 1.01 & 0.76 & 0.56 & 0.43 & 0.34 & 0.29 \\
\hline 12 & 1.19 & 1.02 & 0.76 & 0.54 & 0.41 & 0.32 & 0.26 \\
\hline 14 & 1.32 & 1.03 & 0.76 & 0.52 & 0.38 & 0.30 & 0.24 \\
\hline 16 & 1.42 & 1.04 & 0.76 & 0.51 & 0.36 & 0.28 & 0.22 \\
\hline 18 & 1.50 & 1.05 & 0.76 & 0.49 & 0.34 & 0.26 & 0.20 \\
\hline 20 & 1.55 & 1.06 & 0.75 & 0.47 & 0.33 & 0.24 & 0.18 \\
\hline 22 & 1.59 & 1.07 & 0.75 & 0.46 & 0.31 & 0.22 & 0.17 \\
\hline 24 & 1.62 & 1.08 & 0.75 & 0.44 & 0.30 & 0.21 & 0.16 \\
\hline 26 & 1.64 & 1.09 & 0.75 & 0.43 & 0.28 & 0.20 & 0.15 \\
\hline 28 & 1.64 & 1.10 & 0.74 & 0.42 & 0.27 & 0.19 & 0.14 \\
\hline 30 & 1.64 & 1.11 & 0.74 & 0.41 & 0.26 & 0.18 & 0.14 \\
\hline 32 & 1.63 & 1.11 & 0.74 & 0.39 & 0.25 & 0.17 & 0.13 \\
\hline 34 & 1.61 & 1.12 & 0.73 & 0.38 & 0.24 & 0.17 & 0.13 \\
\hline 36 & 1.59 & 1.12 & 0.73 & 0.37 & 0.23 & 0.16 & 0.13 \\
\hline 38 & 1.56 & 1.13 & 0.72 & 0.36 & 0.22 & 0.16 & 0.12 \\
\hline 40 & 1.53 & 1.13 & 0.72 & 0.36 & 0.22 & 0.15 & 0.12 \\
\hline 42 & 1.49 & 1.13 & 0.71 & 0.35 & 0.21 & 0.15 & 0.12 \\
\hline 44 & 1.44 & 1.13 & 0.71 & 0.34 & 0.21 & 0.15 & 0.12 \\
\hline 46 & 1.39 & 1.13 & 0.70 & 0.33 & 0.20 & 0.14 & 0.11 \\
\hline 48 & 1.34 & 1.12 & 0.70 & 0.32 & 0.20 & 0.14 & 0.11 \\
\hline 50 & 1.29 & 1.11 & 0.69 & 0.32 & 0.19 & 0.14 & 0.11 \\
\hline 52 & 0.24 & 1.10 & 0.68 & 0.31 & 0.19 & 0.13 & 0.11 \\
\hline 54 & 1.19 & 1.09 & 0.67 & 0.30 & 0.18 & 0.13 & 0.10 \\
\hline 56 & 1.15 & 1.08 & 0.67 & 0.30 & 0.18 & 0.13 & 0.10 \\
\hline 58 & 1.10 & 1.06 & 0.66 & 0.29 & 0.17 & 0.12 & 0.10 \\
\hline 60 & 1.05 & 1.04 & 0.65 & 0.29 & 0.17 & 0.12 & 0.10 \\
\hline 62 & 1.01 & 1.02 & 0.65 & 0.28 & 0.16 & 0.12 & 0.10 \\
\hline 64 & 0.96 & 1.00 & 0.64 & 0.27 & 0.16 & 0.11 & 0.10 \\
\hline 66 & 0.91 & 0.98 & 0.64 & 0.27 & 0.16 & 0.11 & 0.09 \\
\hline 68 & 0.87 & 0.96 & 0.63 & 0.26 & 0.15 & 0.11 & 0.09 \\
\hline
\end{tabular}


Table F.4-(Continued)

\begin{tabular}{|c|c|c|c|c|c|c|c|}
\hline \multirow{2}{*}{$\begin{array}{c}Z \text { of } \\
\text { material }\end{array}$} & \multicolumn{7}{|c|}{ Parameters for energy of } \\
\hline & $0.5 \mathrm{MeV}$ & $1 \mathrm{MeV}$ & $2 \mathrm{MeV}$ & $4 \mathrm{MeV}$ & $6 \mathrm{MeV}$ & $8 \mathrm{MeV}$ & $10 \mathrm{MeV}$ \\
\hline 70 & 0.82 & 0.94 & 0.62 & 0.26 & 0.15 & 0.11 & 0.09 \\
\hline 72 & 0.78 & 0.92 & 0.62 & 0.25 & 0.15 & 0.11 & 0.09 \\
\hline 74 & 0.73 & 0.90 & 0.61 & 0.25 & 0.14 & 0.11 & 0.09 \\
\hline 76 & 0.69 & 0.87 & 0.61 & 0.24 & 0.14 & 0.10 & 0.09 \\
\hline 78 & 0.65 & 0.85 & 0.60 & 0.24 & 0.14 & 0.10 & 0.09 \\
\hline \multicolumn{8}{|c|}{ Parameter $b$} \\
\hline $7.5\left(\mathrm{H}_{2} \mathrm{O}\right)$ & 0.600 & 0.175 & 0.037 & 0.0015 & -0.0035 & -0.005 & -0.0055 \\
\hline 2 & 0.53 & 0.126 & 0.055 & 0.05 & 0.05 & 0.05 & 0.05 \\
\hline 4 & 0.50 & 0.125 & 0.053 & 0.04 & 0.04 & 0.04 & 0.04 \\
\hline 6 & 0.464 & 0.125 & 0.051 & 0.03 & 0.03 & 0.03 & 0.03 \\
\hline 8 & 0.436 & 0.124 & 0.049 & 0.015 & 0.015 & 0.015 & 0.015 \\
\hline 10 & 0.402 & 0.123 & 0.047 & 0.010 & 0.010 & 0.010 & 0.010 \\
\hline 12 & 0.367 & 0.121 & 0.045 & 0.005 & 0.003 & 0.002 & 0.001 \\
\hline 14 & 0.336 & 0.120 & 0.043 & 0.007 & 0.005 & 0.003 & 0.002 \\
\hline 16 & 0.305 & 0.118 & 0.041 & 0.008 & 0.006 & 0.004 & 0.003 \\
\hline 18 & 0.275 & 0.116 & 0.039 & 0.011 & 0.008 & 0.006 & 0.005 \\
\hline 20 & 0.244 & 0.113 & 0.038 & 0.013 & 0.010 & 0.008 & 0.007 \\
\hline 22 & 0.215 & 0.109 & 0.037 & 0.015 & 0.012 & 0.010 & 0.009 \\
\hline 24 & 0.188 & 0.105 & 0.036 & 0.017 & 0.014 & 0.010 & 0.009 \\
\hline 26 & 0.160 & 0.100 & 0.036 & 0.019 & 0.015 & 0.011 & 0.009 \\
\hline 28 & 0.142 & 0.094 & 0.036 & 0.021 & 0.017 & 0.011 & 0.009 \\
\hline 30 & 0.103 & 0.088 & 0.036 & 0.022 & 0.018 & 0.011 & 0.009 \\
\hline 32 & 0.091 & 0.082 & 0.036 & 0.023 & 0.019 & 0.010 & 0.008 \\
\hline 34 & 0.070 & 0.079 & 0.036 & 0.023 & 0.019 & 0.010 & 0.008 \\
\hline 36 & 0.050 & 0.067 & 0.036 & 0.023 & 0.019 & 0.009 & 0.007 \\
\hline 38 & 0.031 & 0.058 & 0.036 & 0.022 & 0.018 & 0.009 & 0.007 \\
\hline 40 & 0.014 & 0.050 & 0.036 & 0.022 & 0.018 & 0.008 & 0.006 \\
\hline 42 & 0.0 & 0.041 & 0.036 & 0.021 & 0.017 & 0.007 & 0.005 \\
\hline 44 & -0.013 & 0.032 & 0.036 & 0.021 & 0.017 & 0.006 & 0.004 \\
\hline 46 & -0.025 & 0.022 & 0.036 & 0.020 & 0.016 & 0.005 & 0.003 \\
\hline 48 & -0.035 & 0.013 & 0.036 & 0.020 & 0.016 & 0.004 & 0.002 \\
\hline 50 & -0.043 & 0.004 & 0.036 & 0.019 & 0.015 & 0.004 & 0.002 \\
\hline 52 & -0.048 & -0.005 & 0.035 & 0.018 & 0.015 & 0.003 & 0.001 \\
\hline 54 & -0.053 & -0.013 & 0.032 & 0.018 & 0.014 & 0.003 & 0.001 \\
\hline 56 & -0.056 & -0.020 & 0.028 & 0.017 & 0.013 & 0.002 & 0.001 \\
\hline 58 & -0.058 & -0.027 & 0.025 & 0.017 & 0.012 & 0.002 & 0.0 \\
\hline 60 & -0.060 & -0.033 & 0.022 & 0.016 & 0.011 & 0.001 & -0.001 \\
\hline 62 & -0.062 & -0.037 & 0.018 & 0.015 & 0.010 & 0.001 & -0.001 \\
\hline 64 & -0.063 & -0.040 & 0.015 & 0.015 & 0.009 & 0.001 & -0.002 \\
\hline 66 & -0.064 & -0.043 & 0.012 & 0.014 & 0.008 & 0.0 & -0.003 \\
\hline 68 & -0.065 & -0.045 & 0.008 & 0.014 & 0.007 & -0.001 & -0.003 \\
\hline
\end{tabular}


Table F.4-(Continued)

\begin{tabular}{|c|c|c|c|c|c|c|c|}
\hline \multirow{2}{*}{$\begin{array}{c}Z \text { of } \\
\text { material }\end{array}$} & \multicolumn{7}{|c|}{ Parameters for energy of } \\
\hline & $0.5 \mathrm{MeV}$ & $1 \mathrm{MeV}$ & $2 \mathrm{MeV}$ & $4 \mathrm{MeV}$ & $6 \mathrm{MeV}$ & $8 \mathrm{MeV}$ & $10 \mathrm{MeV}$ \\
\hline 70 & -0.066 & -0.047 & 0.005 & 0.013 & 0.006 & -0.001 & -0.004 \\
\hline 72 & -0.067 & -0.048 & 0.002 & 0.013 & 0.005 & -0.002 & -0.005 \\
\hline 74 & -0.067 & -0.050 & -0.002 & 0.012 & 0.004 & -0.002 & -0.005 \\
\hline 76 & -0.068 & -0.051 & -0.004 & 0.011 & 0.003 & -0.003 & -0.006 \\
\hline 78 & -0.068 & -0.052 & -0.006 & 0.010 & 0.002 & -0.004 & -0.006 \\
\hline \multicolumn{8}{|c|}{ Parameter $c$} \\
\hline $7.5\left(\mathrm{H}_{2} \mathrm{O}\right)$ & 0 & 0 & 0 & 0 & 0 & 0 & 0 \\
\hline 2 & 0 & 0 & 0 & 0 & 0 & 0 & 0 \\
\hline 4 & 0 & 0 & 0 & 0 & 0 & 0 & 0 \\
\hline 6 & 0 & 0 & 0 & 0 & 0 & 0 & 0 \\
\hline 8 & 0 & 0 & 0 & 0 & 0 & 0 & 0 \\
\hline 10 & 0 & 0 & 0 & 0 & $\mathbf{0}$ & 0 & 0 \\
\hline 12 & 0 & 0 & 0 & 0 & 0 & 0 & 0 \\
\hline 14 & 0 & 0 & 0 & 0 & 0 & 0 & 0 \\
\hline 16 & 0 & 0 & 0 & 0 & 0 & 0 & 0 \\
\hline 18 & 0 & 0 & 0 & 0 & 0 & 0 & 0 \\
\hline 20 & 0 & 0 & 0 & 0 & 0 & 0 & 0 \\
\hline 22 & 0 & 0 & 0 & 0 & 0 & 0 & 0 \\
\hline 24 & 0 & 0 & 0 & 0 & 0 & 0 & 0 \\
\hline 26 & 0 & 0 & 0 & 0 & 0 & 0 & 0 \\
\hline 28 & 0.0001 & 0 & -0.0001 & 0.0001 & 0.0001 & 0.0001 & 0.0001 \\
\hline 30 & 0.0002 & 0 & -0.0002 & 0.0002 & 0.0002 & 0.0002 & 0.0002 \\
\hline 32 & 0.0003 & 0 & -0.0002 & 0.0004 & 0.0003 & 0.0003 & 0.0003 \\
\hline 34 & 0.0005 & 0.0001 & -0.0003 & 0.0005 & 0.0004 & 0.0005 & 0.0005 \\
\hline 36 & 0.0008 & 0.0001 & -0.0004 & 0.0006 & 0.0005 & 0.0008 & 0.0008 \\
\hline 38 & 0.0010 & 0.0002 & -0.0005 & 0.0007 & 0.0006 & 0.0010 & 0.0010 \\
\hline 40 & 0.0012 & 0.0002 & -0.0005 & 0.0008 & 0.0006 & 0.0012 & 0.0012 \\
\hline 42 & 0.0014 & 0.0003 & -0.0006 & 0.0008 & 0.0007 & 0.0013 & 0.0014 \\
\hline 44 & 0.0015 & 0.0004 & -0.0006 & 0.0009 & 0.0008 & 0.0014 & 0.0015 \\
\hline 46 & 0.0016 & 0.0005 & -0.0006 & 0.0009 & 0.0009 & 0.0015 & 0.0016 \\
\hline 48 & 0.0017 & 0.0006 & -0.0007 & 0.0010 & 0.0016 & 0.0017 & 0.0017 \\
\hline 50 & 0.0018 & 0.0007 & -0.0007 & 0.0010 & 0.0010 & 0.0017 & 0.0018 \\
\hline 52 & 0.0019 & 0.0008 & -0.0006 & 0.0010 & 0.0011 & 0.0017 & 0.0019 \\
\hline 54 & 0.0020 & 0.0009 & -0.0006 & 0.0010 & 0.0012 & 0.0018 & 0.0020 \\
\hline 56 & 0.0021 & 0.0010 & -0.0006 & 0.0010 & 0.0013 & 0.0018 & 0.0020 \\
\hline 58 & 0.0022 & 0.0010 & -0.0005 & 0.0010 & 0.0013 & 0.0019 & 0.0020 \\
\hline 60 & 0.0023 & 0.0011 & -0.0004 & 0.0010 & 0.0014 & 0.0019 & 0.0020 \\
\hline 62 & 0.0023 & 0.0012 & -0.0004 & 0.0009 & 0.0014 & 0.0020 & 0.0020 \\
\hline 64 & 0.0024 & 0.0013 & -0.0003 & 0.0009 & 0.0015 & 0.0020 & 0.0020 \\
\hline 66 & 0.0025 & 0.0014 & -0.0002 & 0.0009 & 0.0015 & 0.0020 & 0.0020 \\
\hline 68 & 0.0025 & 0.0015 & -0.0001 & 0.0009 & 0.0015 & 0.0020 & 0.0020 \\
\hline
\end{tabular}


Table F.4-(Continued)

\begin{tabular}{cccccccc}
\hline \multirow{2}{*}{$\begin{array}{c}Z \text { of } \\
\text { material }\end{array}$} & $\mathbf{0 . 5 \mathrm { MeV }}$ & $\mathbf{1 ~ M e V}$ & $\mathbf{2 ~ M e V}$ & $4 \mathrm{MeV}$ & $\mathbf{6 ~ M e V}$ & $\mathbf{8 ~ M e V}$ & $\mathbf{1 0 ~ M e V}$ \\
\cline { 2 - 8 } & & & & & & \\
70 & 0.0026 & 0.0016 & 0.0 & 0.0008 & 0.0016 & 0.0020 & 0.0020 \\
72 & 0.0026 & 0.0017 & 0.0001 & 0.0008 & 0.0016 & 0.0021 & 0.0020 \\
74 & 0.0026 & 0.0018 & 0.0002 & 0.0008 & 0.0017 & 0.0021 & 0.0020 \\
76 & 0.0027 & 0.0018 & 0.0003 & 0.0007 & 0.0017 & 0.0021 & 0.0019 \\
78 & 0.0027 & 0.0019 & 0.0004 & 0.0007 & 0.0018 & 0.0021 & 0.0019 \\
\hline
\end{tabular}

†From M. L. Tobias, D. R. Vondy, and M. P. Lietzke, NIGHTMARE-An IBM 7090 Code for the Calculation of Gamma Heating in Cylindrical Geometry, USAEC Report ORNL-3198, Oak Ridge National Laboratory, Feb. 26, 1972.

Table F.5.1 — WATER ENERGY-DEPOSITION BUILDUP DATA

\begin{tabular}{|c|c|c|c|c|}
\hline \multirow[b]{2}{*}{$\mathrm{E}, \mathrm{MeV}$} & \multicolumn{4}{|c|}{ Polynomial coefficients $\beta_{i}$} \\
\hline & $\beta_{0}$ & $\beta_{1}$ & $\beta_{2}$ & $\beta_{3}$ \\
\hline \multicolumn{5}{|c|}{ Operating Gamma Energies } \\
\hline 0.5 & $1.00125(0)$ & $9.26231(-1)$ & $5.06779(-1)$ & $1.05625(-2)$ \\
\hline 1.5 & $9.95465(-1)$ & $9.42191(-1)$ & $8.54431(-2)$ & $-1.40297(-3)$ \\
\hline 2.5 & $9.97165(-1)$ & $7.79558(-1)$ & $2.32124(-2)$ & $-5.09605(-4)$ \\
\hline 3.5 & $9.99216(-1)$ & $6.44960(-1)$ & $5.92178(-3)$ & $-1.60968(-4)$ \\
\hline 4.5 & $1.00080(0)$ & $5.48872(-1)$ & $-6.25612(-4)$ & $-1.31755(-5)$ \\
\hline 5.5 & $1.00198(0)$ & $4.78918(-1)$ & $-3.53293(-3)$ & $5.64411(-5)$ \\
\hline 6.5 & $1.00290(0)$ & $4.26224(-1)$ & $-4.93742(-3)$ & $9.13922(-5)$ \\
\hline 7.5 & $1.00361(0)$ & $4.85274(-1)$ & $-5.63875(-3)$ & $1.09377(-4)$ \\
\hline 8.5 & $1.00419(0)$ & $3.52605(-1)$ & $-5.98227(-3)$ & $1.18446(-4)$ \\
\hline 9.5 & $1.00466(0)$ & $3.25966(-1)$ & $-6.13305(-3)$ & $1.22589(-4)$ \\
\hline \multicolumn{5}{|c|}{ Shutdown Gamma Energies } \\
\hline 0.4 & $9.96370(-1)$ & $1.19891(0)$ & $5.98646(-1)$ & $2.58224(-2)$ \\
\hline 0.7 & $1.00043(0)$ & $8.86758(-1)$ & $3.38048(-1)$ & $5.62186(-4)$ \\
\hline 1.0 & $9.96994(-1)$ & $9.59381(-1)$ & $1.90309(-1)$ & $-1.77445(-3)$ \\
\hline 1.5 & $9.95465(-1)$ & $9.42191(-1)$ & $8.54431(-2)$ & $-1.40297(-3)$ \\
\hline 2.0 & $9.96087(-1)$ & $8.62337(-1)$ & $4.33880(-2)$ & $-8.56510(-4)$ \\
\hline 2.5 & $9.97165(-1)$ & $7.79558(-1)$ & $2.32124(-2)$ & $-5.09605(-4)$ \\
\hline 2.8 & $9.97823(-1)$ & $7.34476(--1)$ & $1.59527(-2)$ & $-3.69642(-4)$ \\
\hline
\end{tabular}

†From M. A. Capo, Polynomial Approximation of Gamma-Ray Buildup Factors for a Point Isotropic Source, USAEC Report APEX-510, General Electric Company, August 1959. 
Table F.5.2 -ALUMINUM ENERGY-DEPOSITION BUILDUP DATA $†$

\begin{tabular}{|c|c|c|c|c|}
\hline \multirow[b]{2}{*}{$\mathrm{E}, \mathrm{MeV}$} & \multicolumn{4}{|c|}{ Polynomial coefficients $\beta_{i}$} \\
\hline & $\beta_{0}$ & $\beta_{1}$ & $\beta_{2}$ & $\beta_{3}$ \\
\hline \multicolumn{5}{|c|}{ Operating Gamma Energies } \\
\hline 0.5 & $9.99375(-1)$ & $1.30777(0)$ & $3.09928(-1)$ & $4.17211(-3)$ \\
\hline 1.5 & $9.89303(-1)$ & $8.80067(-1)$ & $8.17524(-2)$ & $-1.45150(-3)$ \\
\hline 2.5 & $9.96422(-1)$ & $7.10143(-1)$ & $3.04919(-2)$ & $-6.93062(-4)$ \\
\hline 3.5 & $9.99988(-1)$ & $5.84845(-1)$ & $1.31824(-2)$ & $-2.99415(-4)$ \\
\hline 4.5 & $1.00133(0)$ & $4.91214(-1)$ & $6.60650(-3)$ & $-1.34319(-4)$ \\
\hline 5.5 & $1.00177(0)$ & $4.20424(-1)$ & $3.96087(-3)$ & $-6.53646(-5)$ \\
\hline 6.5 & $1.00183(0)$ & $3.65631(-1)$ & $2.95324(-3)$ & $-3.89701(-5)$ \\
\hline 7.5 & $1.00173(0)$ & $3.22193(-1)$ & $2.68803(-3)$ & $-3.25229(-5)$ \\
\hline 8.5 & $1.00156(0)$ & $2.87011(-1)$ & $2.77497(-3)$ & $-3.57358(-5)$ \\
\hline 9.5 & $1.00137(0)$ & $2.57981(-1)$ & $3.03014(-3)$ & $-4.36250(-5)$ \\
\hline \multicolumn{5}{|c|}{ Shutdown Gamma Energies } \\
\hline 0.4 & $9.96852(-1)$ & $1.47519(0)$ & $2.95950(-1)$ & $1.58034(-2)$ \\
\hline 0.7 & $1.02587(0)$ & $1.37596(0)$ & $1.64305(-1)$ & $2.71874(-3)$ \\
\hline 1.0 & $9.95468(-1)$ & $1.05501(0)$ & $1.33496(-1)$ & $-8.37533(-4)$ \\
\hline 1.5 & $9.89303(-1)$ & $8.80067(-1)$ & $8.17524(-2)$ & $-1.45150(-3)$ \\
\hline 2.0 & $9.93089(-1)$ & $7.87367(-1)$ & $4.90708(-2)$ & $-1.04754(-3)$ \\
\hline 2.5 & $9.96422(-1)$ & $7.10143(-1)$ & $3.04919(-2)$ & $-6.93062(-4)$ \\
\hline 2.8 & $9.97858(-1)$ & $6.68707(-1)$ & $2.33484(-2)$ & $-5.37691(-4)$ \\
\hline
\end{tabular}

†From M. A. Capo, Polynomial Approximation of Gamma-Ray Buildup Factors for a Point Isotropic Source, USAEC Report APEX-510, General Electric Company, August 1959. 
Table F.5.3-IRON ENERGY-DEPOSITION BUILDUP DATA $†$

\begin{tabular}{llccr}
\hline & \multicolumn{5}{c}{ Polynomial coefficients $\beta_{i}$} \\
\cline { 2 - 5 } E, MeV & \multicolumn{5}{c}{$\beta_{0}$} & $\beta_{1}$ & $\beta_{2}$ & \multicolumn{1}{c}{$\beta_{3}$} \\
\hline \multicolumn{5}{c}{ Operating Gamma Energies } \\
0.5 & $1.00284(0)$ & $1.59020(0)$ & $1.69181(-1)$ & $1.73772(-4)$ \\
1.5 & $1.00295(0)$ & $8.66308(-1)$ & $6.05394(-2)$ & $-7.72469(-4)$ \\
2.5 & $1.00009(0)$ & $6.37132(-1)$ & $3.03015(-2)$ & $-3.77266(-4)$ \\
3.5 & $9.98723(-1)$ & $4.91866(-1)$ & $2.07774(-2)$ & $-1.64953(-4)$ \\
4.5 & $9.98231(-1)$ & $3.92656(-1)$ & $1.62387(-2)$ & $-3.22198(-5)$ \\
5.5 & $9.98087(-1)$ & $3.21302(-1)$ & $1.35838(-2)$ & $5.86485(-5)$ \\
6.5 & $9.98085(-1)$ & $2.67764(-1)$ & $1.18363(-2)$ & $1.24756(-4)$ \\
7.5 & $9.98142(-1)$ & $2.26206(-1)$ & $1.05957(-2)$ & $1.75005(-4)$ \\
8.5 & $9.98223(-1)$ & $1.93054(-1)$ & $9.66759(-3)$ & $2.14486(-4)$ \\
9.5 & $9.98311(-1)$ & $1.66013(-1)$ & $8.94618(-3)$ & $2.46324(-4)$ \\
\multicolumn{5}{c}{ Shutdown Gamma Energies } \\
0.4 & $1.00480(0)$ & $1.80880(0)$ & $1.90712(-1)$ & $3.23403(-4)$ \\
0.7 & $9.90035(-1)$ & $1.34931(0)$ & $1.81651(-1)$ & $-1.20219(-3)$ \\
1.0 & $1.00093(0)$ & $1.06991(0)$ & $1.12726(-1)$ & $-1.11370(-3)$ \\
1.5 & $1.00295(0)$ & $8.66308(-1)$ & $6.05394(-2)$ & $-7.72469(-4)$ \\
2.0 & $1.00140(0)$ & $7.37006(-1)$ & $4.02749(-2)$ & $-5.39837(-4)$ \\
2.5 & $1.00009(0)$ & $6.37132(-1)$ & $3.03015(-2)$ & $-3.77266(-4)$ \\
2.8 & $9.99533(-1)$ & $5.87104(-1)$ & $2.65084(-2)$ & $-3.01289(-4)$ \\
\hline
\end{tabular}

†From M. A. Capo, Polynomial Approximation of Gamma-Ray Buildup Factors for a Point Isotropic Source, USAEC Report APEX-510, General Electric Company, August 1959. 
Table F.5.4-TIN ENERGY-DEPOSITION BUILDUP DATA $†$

\begin{tabular}{|c|c|c|c|c|}
\hline \multirow[b]{2}{*}{$\mathrm{E}, \mathrm{MeV}$} & \multicolumn{4}{|c|}{ Polynomial coefficients $\beta_{i}$} \\
\hline & $\beta_{0}$ & $\beta_{1}$ & $\beta_{2}$ & $\beta_{3}$ \\
\hline \multicolumn{5}{|c|}{ Operating Gamma Energies } \\
\hline 0.5 & $1.00259(0)$ & $1.25931(0)$ & $-3.63953(-2)$ & $1.57962(-3)$ \\
\hline 1.5 & $1.00156(0)$ & $8.65069(-1)$ & $3.58198(-2)$ & $-6.85867 i-4)$ \\
\hline 2.5 & $9.97988(-1)$ & $5.73675(-1)$ & $2.88829(-2)$ & $3.55381(-5)$ \\
\hline 3.5 & $9.95935(-1)$ & $4.03733(-1)$ & $1.65694(-2)$ & $9.51860(-4)$ \\
\hline 4.5 & $9.96384(-1)$ & $3.00490(-1)$ & $1.29627(-2)$ & $1.20395(-3)$ \\
\hline 5.5 & $9.97424(-1)$ & $2.35258(-1)$ & $1.11403(-2)$ & $1.30735(-3)$ \\
\hline 6.5 & $9.97634(-1)$ & $1.85578(-1)$ & $8.58655(-3)$ & $1.43864(-3)$ \\
\hline 7.5 & $9.97014(-1)$ & $1.51450(-1)$ & $5.30151(-3)$ & $1.59782(-3)$ \\
\hline 8.5 & $9.95564(-1)$ & $1.32875(-1)$ & $1.28515(-3)$ & $1.78488(-3)$ \\
\hline 9.5 & $9.93284(-1)$ & $1.29852(-1)$ & $-3.46252(-3)$ & $1.99984(-3)$ \\
\hline \multicolumn{5}{|c|}{ Shutdown Gamma Energies } \\
\hline 0.4 & $1.00240(0)$ & $1.30553(0)$ & $-4.68267(-2)$ & $1.56464(-3)$ \\
\hline 0.7 & $1.00452(0)$ & $1.16701(0)$ & $-2.65822(-2)$ & $2.33401(-3)$ \\
\hline 1.0 & $1.00398(0)$ & $1.06706(0)$ & $1.52859(-2)$ & $2.68730(-4)$ \\
\hline 1.5 & $1.00156(0)$ & $8.65069(-1)$ & $3.58198(-2)$ & $-6.85867(-4)$ \\
\hline 2.0 & $9.99520(-1)$ & $6.99078(-1)$ & $3.47173(-2)$ & $-4.44094(-4)$ \\
\hline 2.5 & $9.97988(-1)$ & $5.73675(-1)$ & $2.88829(-2)$ & $3.55381(-5)$ \\
\hline 2.8 & $9.97257(-1)$ & $5.13412(-1)$ & $2.50130(-2)$ & $3.30280(-4)$ \\
\hline
\end{tabular}

†From M. A. Capo, Polynomial Approximation of Gamma-Ray Buildup Factors for a Point Isotropic Source, USAEC Report APEX-510, General Electric Company, August 1959. 
Table F.5.5--LEAD ENERGY-DEPOSITION BUILDUP DATA

\begin{tabular}{lcccc}
\hline \multicolumn{5}{c}{ Polynomial coefficients $\beta_{i}$} \\
\cline { 2 - 5 } E, MeV & $\beta_{0}$ & $\beta_{1}$ & $\beta_{2}$ & $\beta_{3}$ \\
\hline \multicolumn{5}{c}{ Operating Gamma Energies } \\
0.5 & $1.02446(0)$ & $4.50964(-1)$ & $-4.10998(-2)$ & $1.48297(-3)$ \\
1.5 & $1.00591(0)$ & $6.85608(-1)$ & $-1.89736(-2)$ & $5.62874(-4)$ \\
2.5 & $1.00341(0)$ & $4.49401(-1)$ & $-2.58366(-4)$ & $3.62998(-4)$ \\
3.5 & $1.00230(0)$ & $2.81788(-1)$ & $8.13507(-3)$ & $4.48166(-4)$ \\
4.5 & $1.00046(0)$ & $2.01870(-1)$ & $7.30625(-3)$ & $9.53997(-4)$ \\
5.5 & $9.96828(-1)$ & $1.71349(-1)$ & $4.27843(-4)$ & $1.64896(-3)$ \\
6.5 & $9.93954(-1)$ & $1.47018(-1)$ & $-4.98319(-3)$ & $2.12918(-3)$ \\
7.5 & $9.91842(-1)$ & $1.28877(-1)$ & $-8.92684(-3)$ & $2.39466(-3)$ \\
8.5 & $9.90492(-1)$ & $1.16925(-1)$ & $-1.14031(-2)$ & $2.44540(-3)$ \\
9.5 & $9.89905(-1)$ & $1.11164(-1)$ & $-1.24120(-2)$ & $2.28140(-3)$ \\
\multicolumn{5}{c}{} \\
& & Shutdown Gamma Energies & \\
0.4 & $1.00415(0)$ & $3.79448(-1)$ & $-3.68912(-2)$ & $1.36614(-3)$ \\
0.7 & $1.01437(0)$ & $6.15504(-1)$ & $-5.27118(-2)$ & $2.13950(-3)$ \\
1.0 & $1.00920(0)$ & $7.47687(-1)$ & $-3.85780(-2)$ & $1.26739(-3)$ \\
1.5 & $1.00591(0)$ & $6.85608(-1)$ & $-1.89736(-2)$ & $5.62874(-4)$ \\
2.0 & $1.00435(0)$ & $5.61596(-1)$ & $-7.47115(-3)$ & $3.80808(-4)$ \\
2.5 & $1.00341(0)$ & $4.49401(-1)$ & $-2.58366(-4)$ & $3.62998(-4)$ \\
2.8 & $1.00300(0)$ & $3.91690(-1)$ & $2.87674(-3)$ & $3.80735(-4)$ \\
\hline
\end{tabular}

†From M. A. Capo, Polynomial Approximation of Gamma-Ray Buildup Factors for a Point Isotropic Source, USAEC Report APEX-510, General Electric Company, August 1959. 
Table F.6-COEFFICIENTS FOR BERGER BUILDUP.FACTOR FUNCTION FOR POINT ISOTROPIC SOURCE TO 20 MEAN FREE PATHS $\dagger$

\begin{tabular}{|c|c|c|c|c|c|c|}
\hline$E, \mathrm{MeV}$ & C & $D$ & $\begin{array}{l}\text { Max. } \\
\text { error, \% }\end{array}$ & $C$ & $D$ & $\begin{array}{l}\text { Max. } \\
\text { error, }\end{array}$ \\
\hline \multicolumn{7}{|c|}{ ORDINARY CONCRETE } \\
\hline \multicolumn{4}{|c|}{ Exposure $\ddagger$} & \multicolumn{3}{|c|}{ Tissue kerma $\S$} \\
\hline 0.5 & 1.3029 & 0.08610 & 18 & 1.3110 & 0.08073 & 12 \\
\hline 1.0 & 1.0914 & 0.04566 & 15 & 1.0221 & 0.05160 & 9 \\
\hline 2.0 & 0.8126 & 0.01980 & 9 & 0.7744 & 0.02456 & 5 \\
\hline 3.0 & 0.6731 & 0.00942 & 4 & 0.6525 & 0.01258 & 3 \\
\hline 4.0 & 0.5953 & 0.00299 & 2 & 0.5672 & 0.00753 & 2 \\
\hline 6.0 & 0.4915 & -0.00159 & 3 & 0.4718 & 0.00198 & 1 \\
\hline 8.0 & 0.4164 & -0.00172 & 6 & 0.4203 & 0.00096 & 1 \\
\hline \multirow[t]{2}{*}{10.0} & 0.3585 & -0.00005 & 9 & 0.3716 & 0.00127 & 1 \\
\hline & \multicolumn{3}{|c|}{ Energy deposition $\ddagger$} & \multicolumn{3}{|c|}{ Energy deposition $\S$} \\
\hline 0.5 & 1.6280 & 0.09272 & 21 & 1.6568 & 0.08538 & 13 \\
\hline 1.0 & 1.2367 & 0.04795 & 17 & 1.1633 & 0.05325 & 10 \\
\hline 2.0 & 0.8850 & 0.01987 & 9 & 0.8203 & 0.02465 & $\mathbf{5}$ \\
\hline 3.0 & 0.7171 & 0.00825 & 3 & 0.6702 & 0.01221 & 3 \\
\hline 4.0 & 0.6154 & 0.00199 & 1 & 0.5672 & 0.00753 & 2 \\
\hline 6.0 & 0.4882 & -0.00205 & 3 & 0.4583 & 0.00168 & 1 \\
\hline 8.0 & 0.4049 & -0.00208 & 6 & 0.3985 & 0.00084 & 1 \\
\hline 10.0 & 0.3435 & -0.00041 & 9 & 0.3609 & 0.00083 & 1 \\
\hline
\end{tabular}

\section{MAGNETITE CONCRETE}

Tissue kerma

$\begin{array}{rr}0.5 & 1.0971 \\ 1.0 & 0.9281 \\ 2.0 & 0.7318 \\ 3.0 & 0.6154 \\ 4.0 & 0.5308 \\ 6.0 & 0.4345 \\ 8.0 & 0.3752 \\ 10.0 & 0.3245\end{array}$

0.5

$\begin{array}{rrr}0.5 & 0.7002 & 0.01624 \\ 1.0 & 0.7237 & 0.03007 \\ 2.0 & 0.6270 & 0.02373 \\ 3.0 & 0.5367 & 0.02158 \\ 4.0 & 0.4645 & 0.02122 \\ 6.0 & 0.3727 & 0.02233 \\ 8.0 & 0.3172 & 0.02588 \\ 10.0 & 0.2667 & 0.02878\end{array}$

Tissue kerma
Energy deposition

0.06458
0.04768
0.02620
0.01836
0.01513
0.01118
0.01153
0.01199

$$
\begin{aligned}
& 8 \\
& 8 \\
& 5 \\
& 4 \\
& 3 \\
& 2 \\
& 2 \\
& 2
\end{aligned}
$$

$\begin{array}{ll}0.06696 & 8 \\ 0.04877 & 8 \\ 0.02591 & 5 \\ 0.01747 & 3 \\ 0.01428 & 3 \\ 0.00705 & 3 \\ 0.01010 & 2 \\ 0.00999 & 2\end{array}$

\section{BARYTES CONCRETE§}

Energy deposition

$\begin{array}{lll}1.3542 & 0.01056 & 4 \\ 1.1102 & 0.02792 & 3 \\ 0.7672 & 0.02229 & 3 \\ 0.5806 & 0.01995 & 3 \\ 0.4689 & 0.01948 & 2 \\ 0.3403 & 0.02045 & 2 \\ 0.2787 & 0.02251 & 2 \\ 0.2420 & 0.02368 & 1\end{array}$


Table F.6-(Continued)

\begin{tabular}{|c|c|c|c|c|c|c|}
\hline$E, \mathrm{MeV}$ & $C$ & $D$ & $\begin{array}{l}\text { Max. } \\
\text { error, \% }\end{array}$ & C & $D$ & $\begin{array}{c}\text { Max. } \\
\text { error, \% }\end{array}$ \\
\hline \multicolumn{7}{|c|}{ AIR \& } \\
\hline & \multicolumn{3}{|c|}{ Tissue kerma } & \multicolumn{3}{|c|}{ Energy deposition } \\
\hline 0.5 & 1.6001 & 0.10094 & 18 & 1.5411 & 0.09920 & 17 \\
\hline 1.0 & 1.1571 & 0.05749 & 11 & 1.1305 & 0.05687 & 11 \\
\hline 2.0 & 0.8363 & 0.02430 & 5 & 0.8257 & 0.02407 & 5 \\
\hline 3.0 & 0.6974 & 0.01017 & 3 & 0.6872 & 0.01002 & 3 \\
\hline 4.0 & 0.6081 & 0.00324 & 1 & 0.6020 & 0.00323 & 1 \\
\hline 6.0 & 0.5146 & -0.00316 & 1 & 0.5080 & -0.00289 & 1 \\
\hline 8.0 & 0.4635 & -0.00362 & 1 & 0.4567 & -0.00349 & 1 \\
\hline 10.0 & 0.4235 & -0.00350 & 1 & 0.4261 & -0.00333 & 1 \\
\hline \multicolumn{7}{|c|}{ SANDq } \\
\hline & \multicolumn{3}{|c|}{ Tissue kerma } & \multicolumn{3}{|c|}{ Energy deposition } \\
\hline 0.5 & 1.4474 & 0.08932 & 14 & 1.6641 & 0.09348 & 16 \\
\hline 1.0 & 1.0876 & 0.05482 & 10 & 1.1729 & 0.05619 & 10 \\
\hline 2.0 & 0.8077 & 0.02503 & 5 & 0.8343 & 0.02532 & 5 \\
\hline 3.0 & 0.6758 & 0.01239 & 3 & 0.6833 & 0.01212 & 3 \\
\hline 4.0 & 0.5875 & 0.00651 & 2 & 0.5844 & 0.00653 & 2 \\
\hline 6.0 & 0.4886 & 0.00091 & 1 & 0.4734 & 0.00100 & 1 \\
\hline 8.0 & 0.4334 & 0.00009 & 1 & 0.4175 & -0.00055 & 1 \\
\hline 10.0 & 0.3883 & -0.00224 & 1 & 0.3800 & -0.00034 & 1 \\
\hline \multicolumn{7}{|c|}{ WOOD } \\
\hline & \multicolumn{3}{|c|}{ Tissue kerma } & \multicolumn{3}{|c|}{ Energy deposition } \\
\hline 0.5 & 1.6187 & 0.10280 & 19 & 1.5079 & 0.09921 & 17 \\
\hline 1.0 & 1.1676 & 0.05802 & 11 & 1.1163 & 0.05688 & 11 \\
\hline 2.0 & 0.8457 & 0.02392 & 5 & 0.8234 & 0.02388 & 5 \\
\hline 3.0 & 0.7051 & 0.00913 & 3 & 0.6949 & 0.00915 & 3 \\
\hline 4.0 & 0.6149 & 0.00253 & 1 & 0.6122 & 0.00276 & 1 \\
\hline 6.0 & 0.5176 & -0.00342 & 1 & 0.5186 & -0.00343 & 1 \\
\hline 8.0 & 0.4727 & -0.00438 & 1 & 0.4729 & -0.00445 & 1 \\
\hline 10.0 & 0.4328 & -0.00390 & 1 & 0.4430 & -0.00403 & 1 \\
\hline \multicolumn{7}{|c|}{ LITHIUM HYDRIDE } \\
\hline \multicolumn{7}{|c|}{ Tissue kerma } \\
\hline 0.5 & 2.0198 & 0.13997 & 34 & & & \\
\hline 1.0 & 1.2652 & 0.07091 & 16 & & & \\
\hline 2.0 & 0.8391 & 0.02272 & 6 & & & \\
\hline 3.0 & 0.6607 & 0.00546 & 2 & & & \\
\hline 4.0 & 0.5778 & -0.00157 & 1 & & & \\
\hline 6.0 & 0.4671 & -0.01017 & 2 & & & \\
\hline 8.0 & 0.4047 & -0.01213 & 3 & & & \\
\hline 10.0 & 0.3676 & -0.01135 & 3 & & & \\
\hline
\end{tabular}

tFrom D. K. Trubey, Gamma-Ray Buildup Factor Coefficients for Concretes and Other Materials, Nuclear Applications and Technology, 9: 441 (September 1970).

$\ddagger$ Constants based on A. B. Chilton, Nuclear Engineering and Design, 6: 205 (1967).

§Constants based on F. H. Clark and D. K. Trubey, Nuclear Applications, 4: 37 (1968).

I Francis H. Clark, Gamma-Ray Buildup Factors for Sand, Air, and Wood (Cellulose), Nuclear Applications, 6: 588 (1969); Francis B. K. Kam and Francis H. S. Clark, Fission Neutron Attenuation and Gamma-Ray Buildup for Lithium Hydride, Nuclear Applications, 3: 433 (1967). 


\section{Graphs and Formulas of Exponential Integral Functions}

\section{Appendix G}

Several exponential functions that are extremely useful in shielding calculations are presented in this appendix. They include the exponential function $e^{-x}$ and the exponential integral functions $E_{n}(x)$, which are expressed in integral form by

$$
\begin{aligned}
& E_{n}(x)=x^{n-1} \int_{x}^{\infty} e^{-y} \frac{d y}{y^{n}} \\
& E_{n}(x)=\int_{1}^{\infty} e^{-x y} \frac{d y}{y^{n}} \\
& E_{n}(x)=\int_{0}^{1} y^{n-2} e^{-x / y} d y
\end{aligned}
$$

and in series form by

$$
E_{n}(x)=\sum_{\substack{m=0 \\ m \neq n-1}}^{\infty} \frac{(-x)^{m}}{(n-1-m) m !}+(-1)^{n} \frac{x^{n-1}}{(n-1) !}\left(\ln \gamma x-A_{n}\right)
$$

where $\ln \gamma=0.577216 \ldots, A_{1}=0, A_{n}=\sum_{m=1}^{n-1} \frac{1}{m}$, and $n=0,1,2,3, \ldots$.

The recursion relation for the exponential integrals is

$$
E_{n}(x)=\frac{1}{n-1}\left[e^{-x}-x E_{n-1}(x)\right]
$$

In particular, the first-order exponential integral is given by

$$
E_{1}(x)=\int_{x}^{\infty} \frac{e^{-y}}{y} d y
$$


and the series representation by

$$
E_{1}(x)=-\ln \gamma x-\sum_{n=0}^{\infty} \frac{(-1)^{n} x^{n}}{n ! n}
$$

In some publications the $E_{1}(x)$ function is denoted by $-E i(-x)$; i.e., $E_{1}(x)=-E i(-x)$. When tables of the exponential function are used, caution should always be exercised not to confuse $-E i(-x)$ with $-E i(x)$.

For negative arguments the $E_{1}(x)$ function is frequently denoted by the symbol $E i(x)$, which is defined by

$$
E i(x) \equiv \int_{-\infty}^{-x} \frac{e^{y}}{y} d y
$$

and related to $E_{1}(-x)$ by

$$
-E i(x)=E_{1}(-x)=\int_{-x}^{\infty} e^{y} \frac{d y}{y}
$$

Graphs of $e^{-x}$ and $E_{n}(x)$ for $n=1,2$, and 3 are presented ${ }^{1}$ in Figs. G.1 to G.6. The function $E i(x)$ is plotted ${ }^{2}$ in Figs. G.7 to G.10.

Some approximations that are frequently used are

$$
\begin{aligned}
\frac{e^{-x}}{x+n}<E_{n}(x) \leqslant \frac{e^{-x}}{x+n-1} \quad(n \geqslant 1) & \\
E_{n}(x)= & \frac{e^{-x}}{x}\left\{1-\frac{n}{x}+\frac{n(n+1)}{x^{2}}\right. \\
& \left.-\left[\frac{n(n+1)(n+2)}{x^{3}}\right]+\ldots\right\} \quad(x \gg 1 ; n=0) \\
E_{n}(x) \cong & \frac{1+x+n}{x+(x+n)^{2}} e^{-x} \quad(x>1) \\
E i(x) \cong & e^{x}\left(\frac{1}{x}+\frac{1}{x^{2}}+\frac{2 !}{x^{3}}+\frac{3 !}{x^{4}}+\ldots\right) \quad(x>10)
\end{aligned}
$$

Reference 3 contains further information about the exponential integrals. 


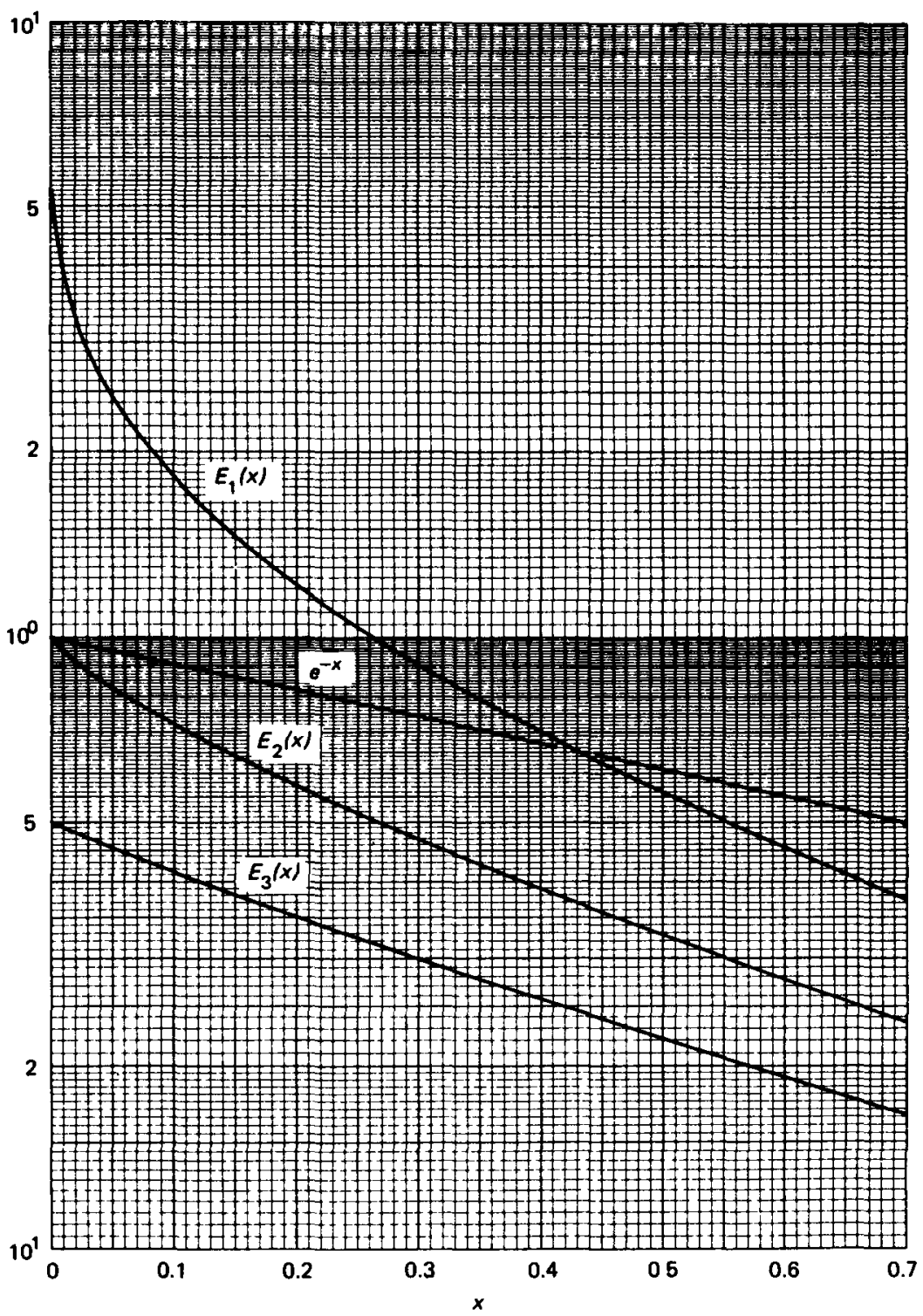

Fig. G.1-The functions $e^{-x}, E_{1}(x), E_{2}(x)$, and $E_{3}(x)$ for $x=0$ to 0.7. (Plotted from data tabulated in Trubey. ${ }^{2}$ ) 


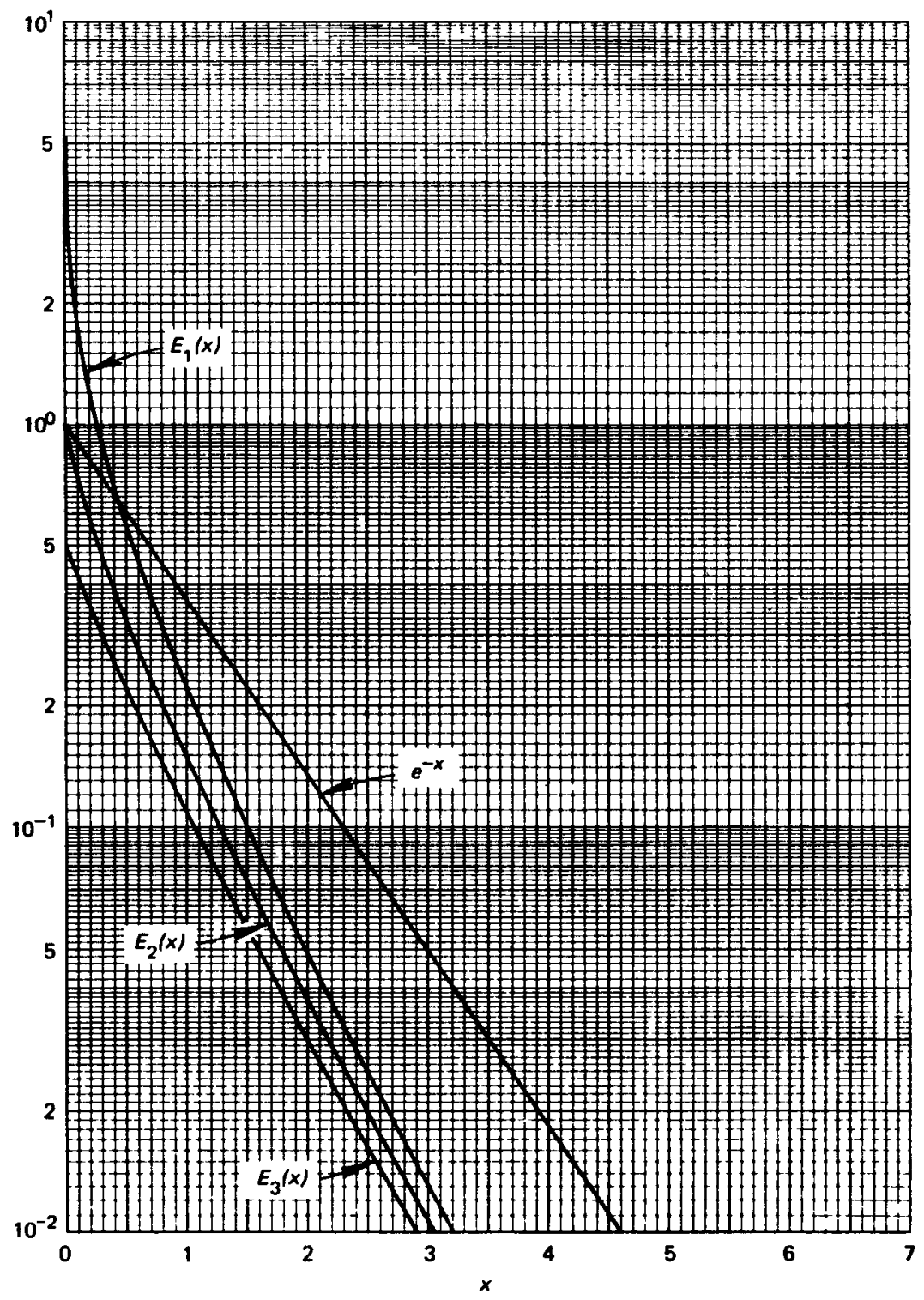

Fig. G.2-The functions $e^{-x}, E_{1}(x), E_{2}(x)$, and $E_{3}(x)$ for $x=0$ to 5. (Plotted from data tabulated in Trubey. ${ }^{2}$ ) 


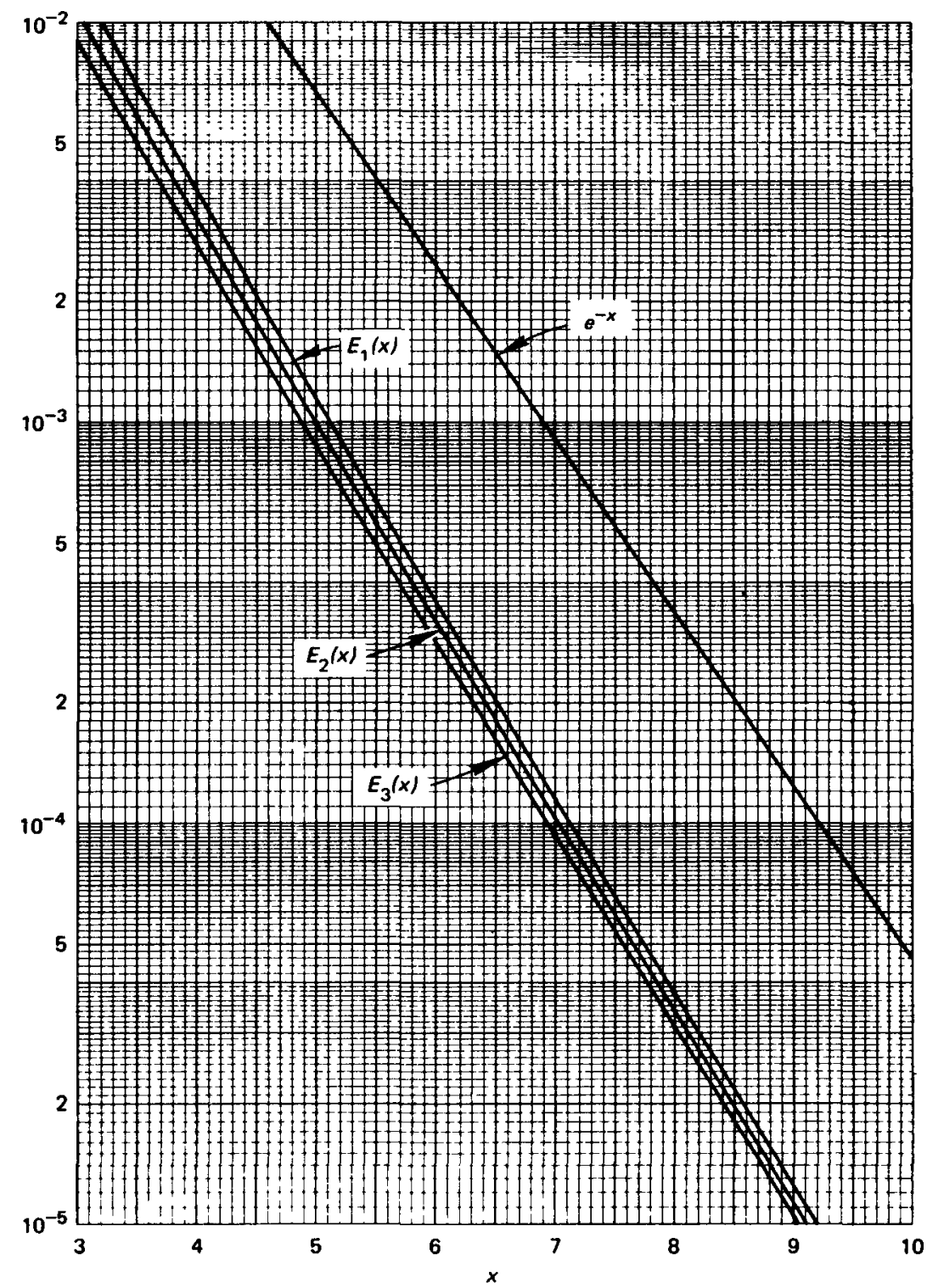

Fig. G.3-The functions $e^{-x}, E_{1}(x), E_{2}(x)$, and $E_{3}(x)$ for $x=3$ to 10 . (Plotted from data tabulated in Trubey. ${ }^{2}$ ) 


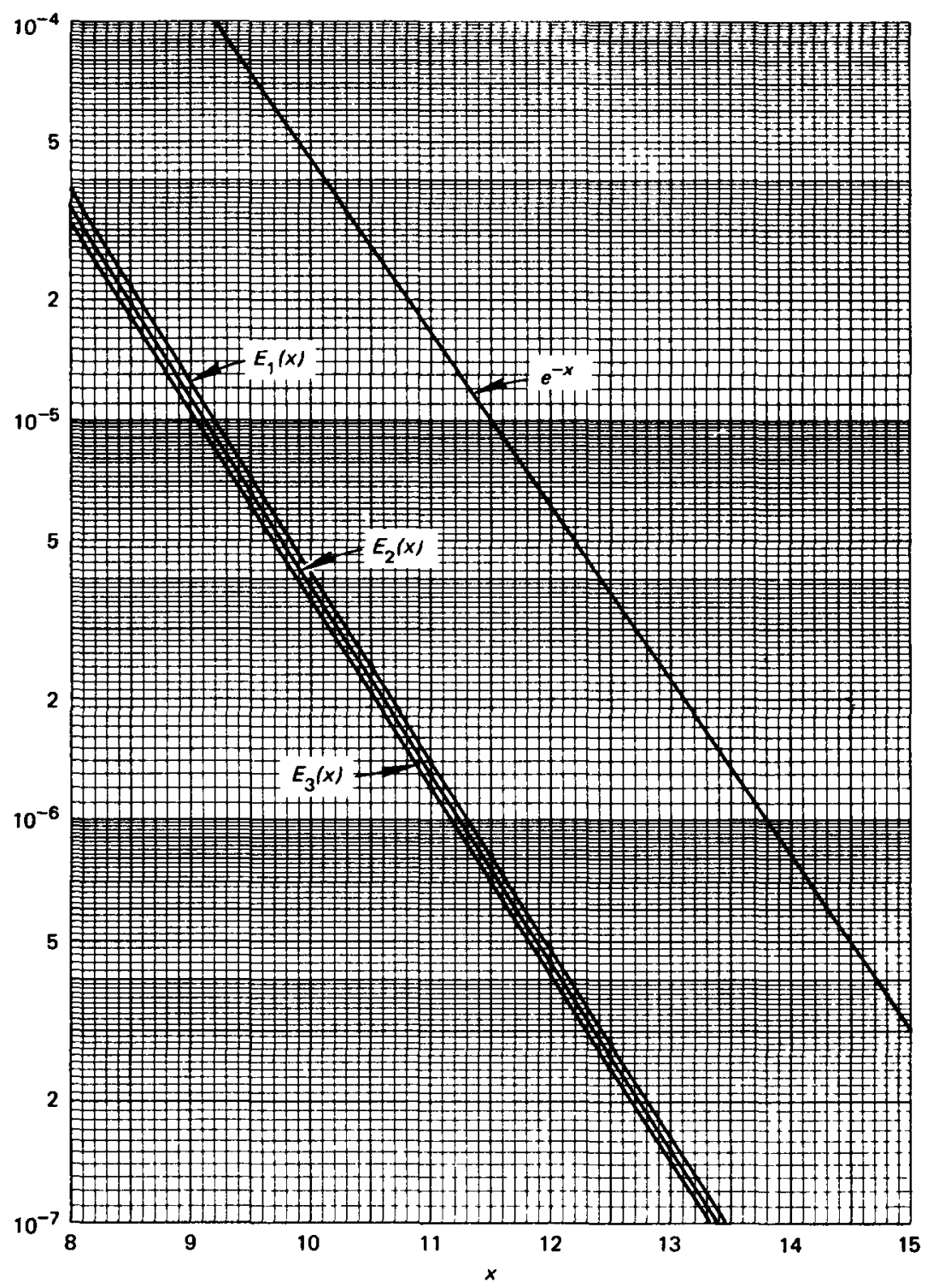

Fig. G.4-The functions $e^{-x}, E_{1}(x), E_{2}(x)$, and $E_{3}(x)$ for $x=8$ to 15. (Plotted from data tabulated in Trubey. ${ }^{2}$ ) 


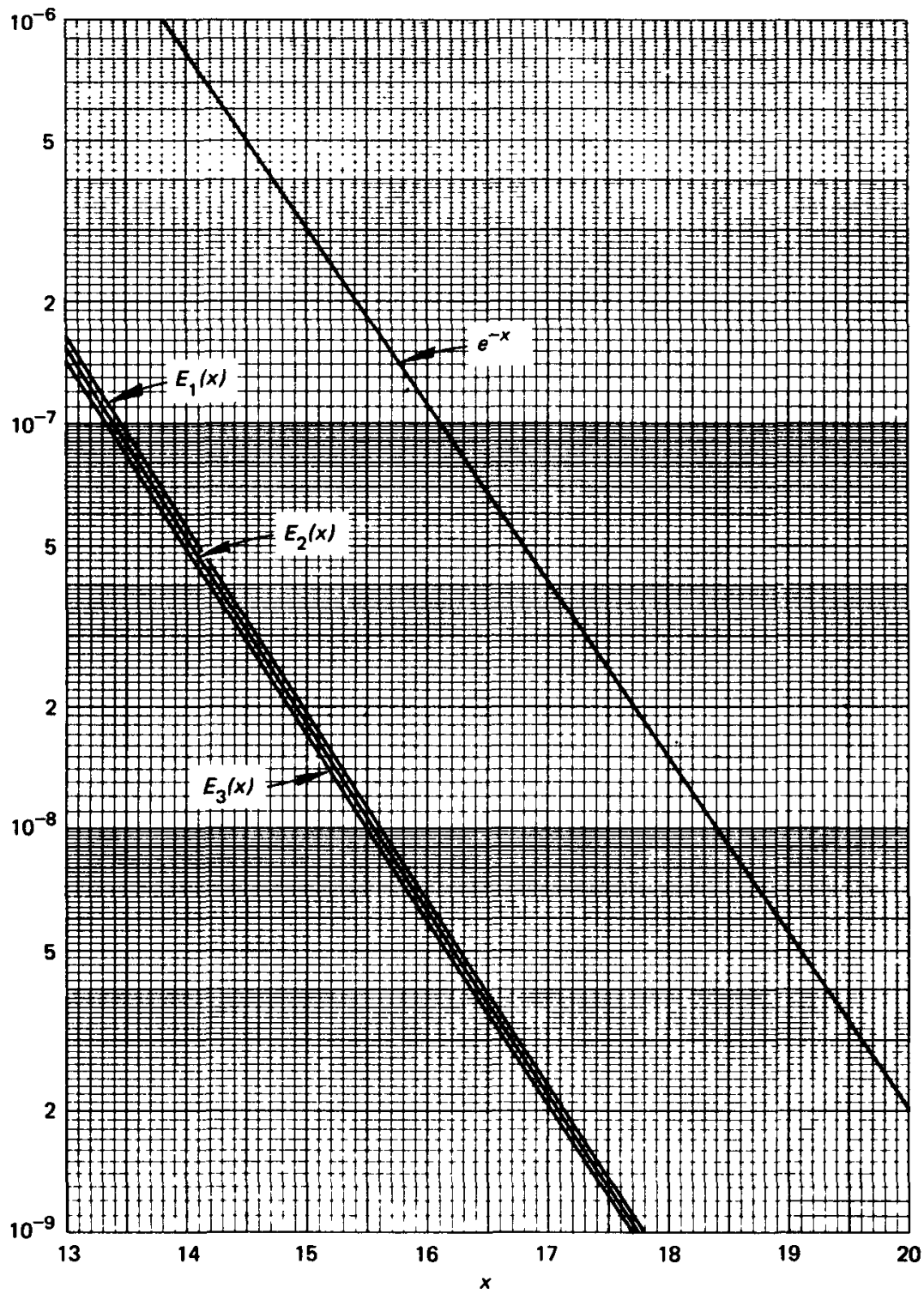

Fig. G.5-The functions $e^{-x}, E_{1}(x), E_{2}(x)$, and $E_{3}(x)$ for $x=13$ to 20 . (Plotted from data tabulated in Trubey. ${ }^{2}$ ) 


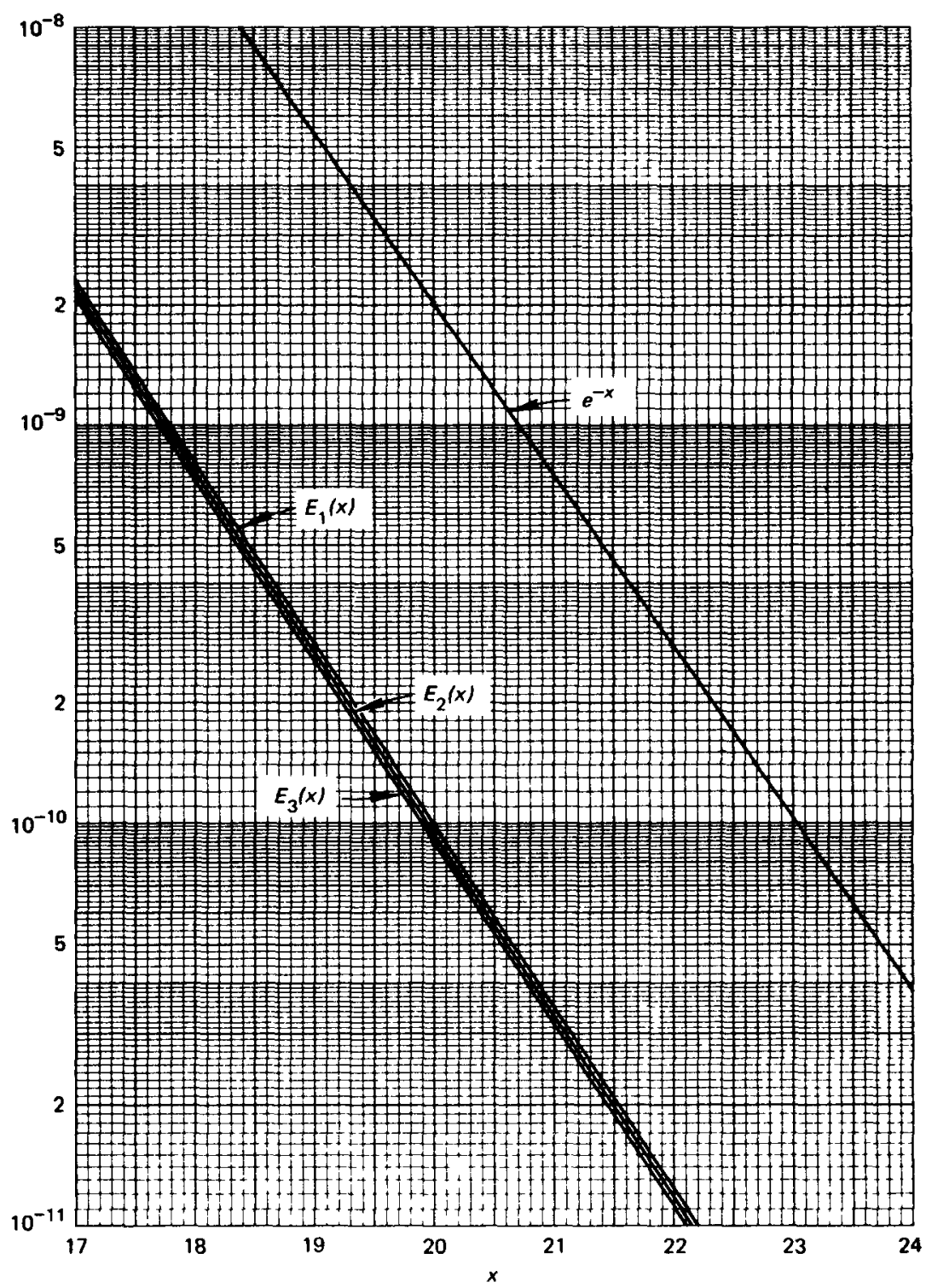

Fig. G.6-The functions $e^{-x}, E_{1}(x), E_{2}(x)$, and $E_{3}(x)$ for $x=17$ to 24. (Plotted from data tabulated in Trubey. ${ }^{2}$ ) 


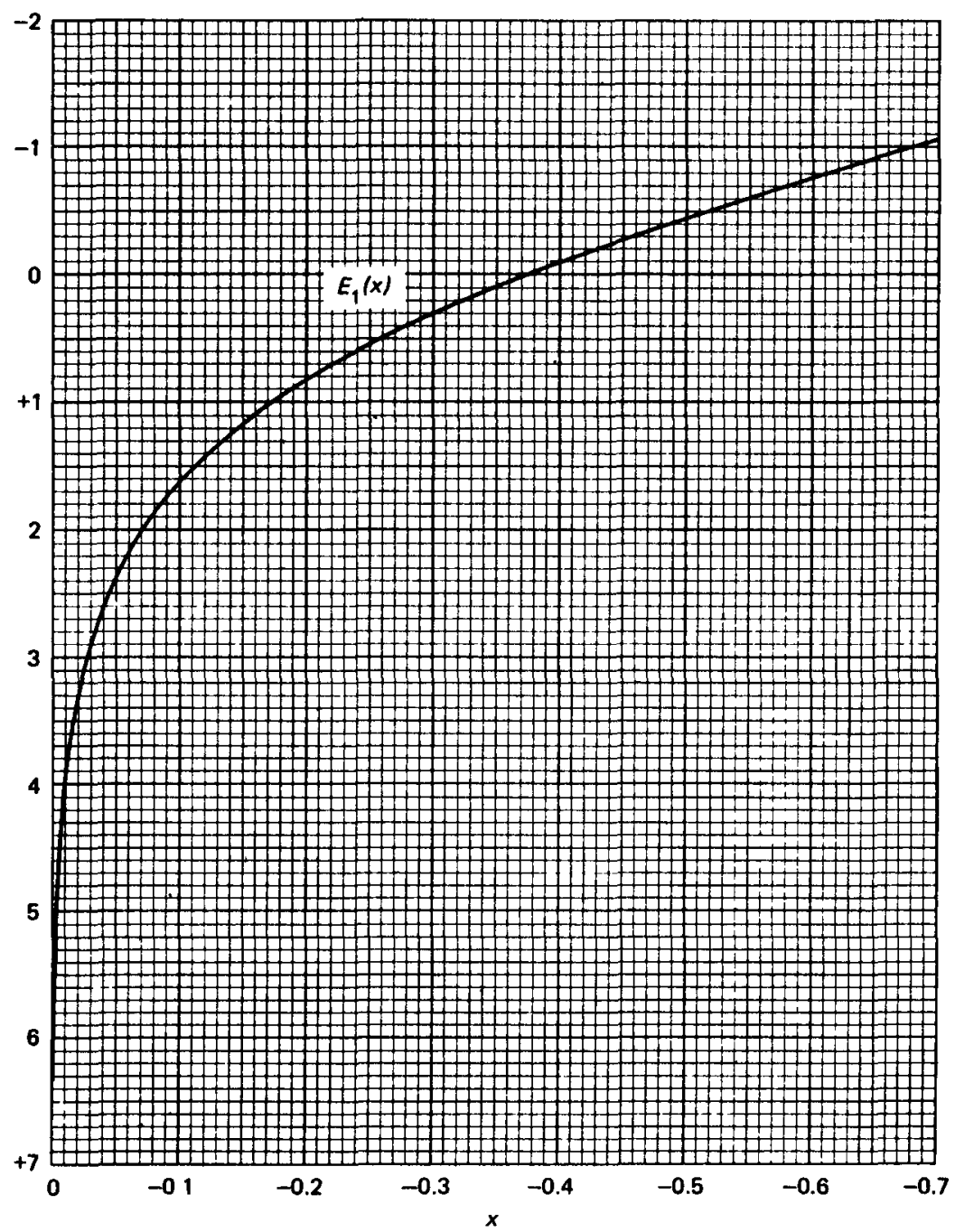

Fig. G.7-The function $E_{1}(x)$ for negative arguments. (Plotted from data in Ref. 1.) 


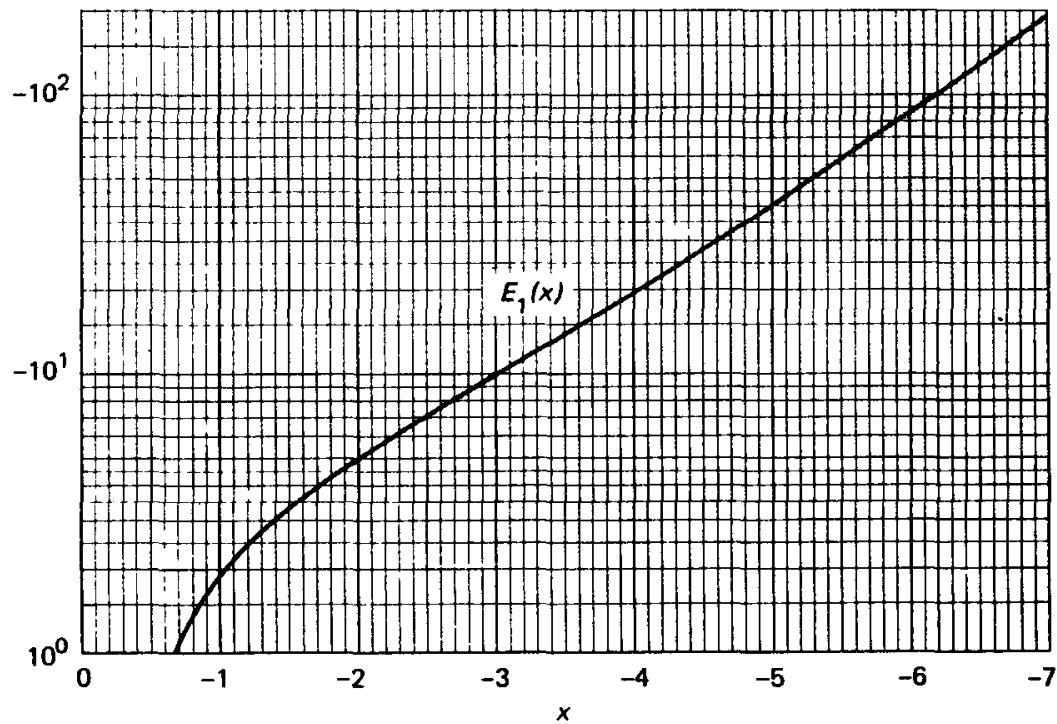

Fig. G.8-The function $E_{1}(x)$ for negative arguments. (Plotted from data in Ref. 1.)

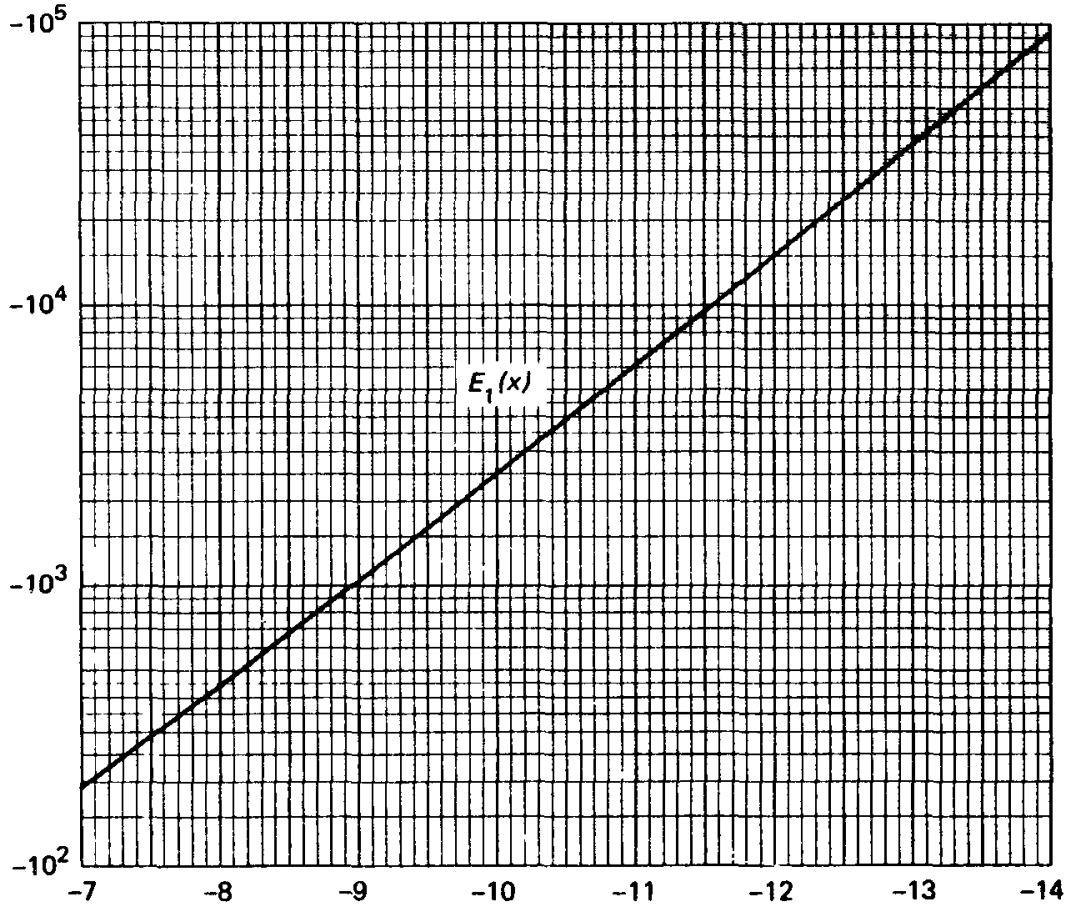

Fig. G.9-The function $E_{1}(x)$ for negative arguments. (Plotted from data in Ref. 1.) 


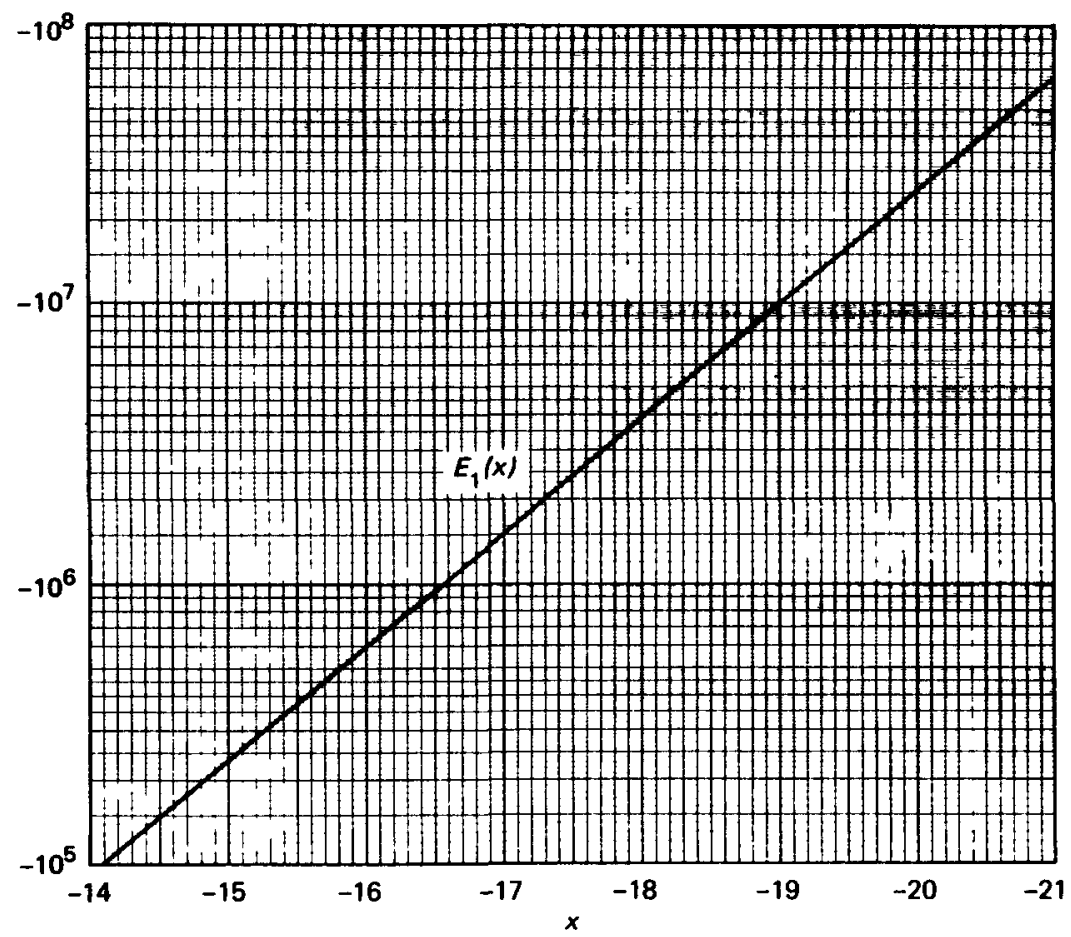

Fig. G.10-The function $E_{1}(x)$ for negative arguments. (Plotted from data tabulated in Ref. 1.)

\section{REFERENCES}

1. Federal Works Agency, Works Project Administration, Tables of Stne, Cosine, and Exponential Integrals, Vols. I and II, prepared under sponsorship of National Bureau of Standards, Superintendent of Documents, U. S. Government Printing Office, Washington, D. C., 1940.

2. D. K. Trubey, A Table of Three Exponential Integrals, USAEC Report ORNL-2750, Oak Ridge National Laboratory, June 18, 1959.

3. M. Abramowitz and I. A. Stegun (Fds.), Handbook of Mathematscal Functions with Formulas, Graphs, and Mathematical Tables, Nationd Bureau of Standards, Applied Mathematics Series 55, Superintendent of Documents, U. S. Government Printing Office, Washington, D. C., 1964. 


\section{Tables of Attenuation Functions for Finite Slab Geometry}

Appendix $\mathrm{H}$

This appendix consists of tabulations of attenuation functions for slab shields and disk or rectangular sources obtained by integrating over a point kernel of the form given for gamma rays in Sec. 4.8.1. The geometry for the disk-source configuration is shown in Figs. 4.7 and 4.8. Data are presented in Tables H.1 and H.2, for sources with both isotropic and cosine angular distributions, respectively, the isotropic-source data being taken from the work of Hubbell, Bach, and Herbold, ${ }^{1}$ and the cosine-source data, from the work of Trubey. ${ }^{2}$ Corresponding data for a rectangular-source configuration are given in Table H.3, from Hubbell, Bach, and Lamkin, ${ }^{3}$ and in Table H.4, from Trubey. ${ }^{2}$ The geometry for the rectangular source is shown in Fig. 4.9.

The use of these attenuation functions is explained in Sec. 4.8.1 and summarized here.

The variables for the disk source (see Tables H.1 and H.2) are

$$
\begin{aligned}
\Gamma\left(\mu t, r_{0} / z, \rho / r_{0}\right) & =\text { dose rate } \\
G(E) & =\text { flux-to-dose-rate conversion factor } \\
S & \left.=\text { source strength (particles } \mathrm{cm}^{-2} \sec ^{-1}\right), 4 \pi \text { solid angle } \\
\mu t & =\text { optical shield thickness (mean free paths) } \\
r_{0} / z & =\text { ratio of source radius to the source-detector distance } \\
\rho / r_{0} & =\text { distance off axis (units of source radius) } \\
y & =\mu t \sec \theta
\end{aligned}
$$

For an isotropic source the unscattered flux attenuation for the dose rate on the disk axis is given by

$$
\frac{4 \pi \Gamma\left(\mu t, r_{0} / z, 0\right)}{S G(E)}=2 \pi\left\{E_{1}(\mu t)-E_{1}\left[\mu t \sqrt{1+\left(\frac{r_{0}}{z}\right)^{2}}\right]\right\}
$$

(Text continues on page 658.) 
Table H.1 - UNSCATTERED FLUX DENSITY FROM A DISK SOURCE

$4 \pi \Gamma /[S G(E)]$

\begin{tabular}{|c|c|c|c|c|c|c|c|c|c|c|c|}
\hline \multirow{2}{*}{$z / r_{0}$} & \multirow[b]{2}{*}{$\mu t$} & \\
\hline & & $\rho / r_{0}=0$ & $\rho / r_{0}=0.2$ & $\rho / r_{0}=0.5$ & $\rho / r_{0}=0.8$ & $p / r_{0}=1.0$ & $\rho / r_{0}=1.2$ & $\rho / r_{0}=1.5$ & $\rho / r_{0}=2.0$ & $\rho / r_{0}=5.0$ & $\rho / r_{0}=10.0$ \\
\hline \multirow[t]{11}{*}{0.1} & 0 & $1.45(1) \ddagger$ & $1.44(1)$ & $1.36(1)$ & $1.15(1)$ & $7.39(0)$ & $3.60(0)$ & $1.83(0)$ & $9.00(-1)$ & $1.28(-1)$ & $3.16(-2)$ \\
\hline & 0.01 & $1.39(1)$ & $1.38(1)$ & $1.31(1)$ & $1.10(1)$ & $7.03(0)$ & $3.33(0)$ & $1.62(0)$ & $7.53(-1)$ & $7.84(-2)$ & $1.17(-2)$ \\
\hline & 0.02 & $1.34(1)$ & $1.33(1)$ & $1.26(1)$ & $1.06(1)$ & $6.71(0)$ & $3.08(0)$ & $1.44(0)$ & $6.31(-1)$ & $4.81(-2)$ & $4.31(-3)$ \\
\hline & 0.05 & $1.20(1)$ & $1.19(1)$ & $1.13(1)$ & $9.54(0)$ & $5.86(0)$ & $2.47(0)$ & $1.02(0)$ & $3.76(-1)$ & $1.13(-2)$ & \\
\hline & 0.1 & $1.01(1)$ & $1.00(1)$ & $9.57(0)$ & $8.11(0)$ & $4.81(0)$ & $1.76(0)$ & $5.99(-1)$ & $1.66(-1)$ & & \\
\hline & 0.2 & $7.38(0)$ & $7.34(0)$ & $7.10(0)$ & $6.12(0)$ & $3.45(0)$ & $9.94(-1)$ & $2.09(-1)$ & $4.56(-2)$ & $1.36(-3)$ & $1.34(-4)$ \\
\hline & 0.5 & $3.50(0)$ & $3.50(0)$ & $3.46(0)$ & $3.17(0)$ & $1.66(0)$ & $2.51(-1)$ & $2.74(-2)$ & $3.45(-3)$ & $2.17(-5)$ & $1.72(-5)$ \\
\hline & 1.0 & $1.38(0)$ & $1.38(0)$ & $1.38(0)$ & $1.32(0)$ & $6.63(-1)$ & $4.25(-2)$ & $1.03(-3)$ & $2.49(-4)$ & & \\
\hline & 2.0 & $3.08(-1)$ & $3.08(-1)$ & $3.08(-1)$ & $3.05(-1)$ & $1.49(-1)$ & $2.11(-3)$ & & & & \\
\hline & 5.0 & $7.22(-3)$ & $7.22(-3)$ & $7.21(-3)$ & $7.21(-3)$ & $3.54(-3)$ & & & & & \\
\hline & 10.0 & $2.61(-5)$ & $2.61(-5)$ & $2.61(-5)$ & $2.61(-5)$ & $1.29(-5)$ & & & & & \\
\hline \multirow[t]{11}{*}{0.2} & 0. & $1.02(1)$ & $1.01(1)$ & $9.42(0)$ & $7.65(0)$ & $5.37(0)$ & $3.25(0)$ & $1.77(0)$ & $8.90(-1)$ & $1.28(-1)$ & $3.16(-2)$ \\
\hline & 0.01 & $9.98(0)$ & $9.87(0)$ & $9.19(0)$ & $7.44(0)$ & $5.20(0)$ & $3.11(0)$ & $1.67(0)$ & $8.13(-1)$ & $1.00(-1)$ & $1.92(-2)$ \\
\hline & 0.02 & $9.74(0)$ & $9.62(0)$ & $8.96(0)$ & $7.25(0)$ & $5.04(0)$ & $2.98(0)$ & $1.57(0)$ & $7.43(-1)$ & $7.83(-2)$ & $1.17(-2)$ \\
\hline & 0.05 & $9.04(0)$ & $8.94(0)$ & $8.32(0)$ & $6.71(0)$ & $4.60(0)$ & $2.63(0)$ & $1.31(0)$ & $5.70(-1)$ & $3.76(-2)$ & $2.63(-3)$ \\
\hline & 0.01 & $8.01(0)$ & $7.92(0)$ & 7.38(0) & $5.93(0)$ & $3.97(0)$ & $2.15(0)$ & $9.78(-1)$ & $3.70(-1)$ & $1.12(-2)$ & $1.01(-3)$ \\
\hline & 0.2 & $6.35(0)$ & $6.28(0)$ & $5.88(0)$ & $4.71(0)$ & $3.17(0)$ & $1.49(0)$ & $5.66(-1)$ & $1.62(-1)$ & $3.48(-3)$ & $3.16(-4)$ \\
\hline & 0.5 & $3.37(0)$ & $3.35(0)$ & $3.19(0)$ & $2.60(0)$ & $1.55(0)$ & $5.79(-1)$ & $1.37(-1)$ & $2.19(-2)$ & $1.62(-4)$ & $4.90(-6)$ \\
\hline & 1.0 & $1.37(0)$ & $1.37(0)$ & $1.34(0)$ & $1.14(0)$ & $6.35(-1)$ & $1.67(-1)$ & $1.73(-2)$ & $1.02(-3)$ & & \\
\hline & 2.0 & $3.08(-1)$ & $3.08(-1)$ & $3.06(-1)$ & $2.80(-1)$ & $1.42(-1)$ & $2.06(-2)$ & $5.93(-4)$ & & & \\
\hline & 5.0 & $7.22(-3)$ & $7.22(-3)$ & $7.21(-3)$ & $7.08(-3)$ & $3.48(-3)$ & $1.04(-4)$ & $7.67(-7)$ & & & \\
\hline & 10.0 & $2.61(-5)$ & $2.61(-5)$ & $2.61(-5)$ & $2.61(-5)$ & $1.27(-5)$ & $2.71(-8)$ & & & & \\
\hline \multirow[t]{11}{*}{0.5} & 0. & $5.06(0)$ & $4.98(0)$ & $4.53(0)$ & $3.69(0)$ & $2.96(0)$ & $2.26(0)$ & $1.49(0)$ & $8.26(-1)$ & $1.27(-1)$ & $3.15(-2)$ \\
\hline & 0.01 & $4.98(0)$ & $4.90(0)$ & $4.46(0)$ & $3.62(0)$ & $2.90(0)$ & $2.21(0)$ & $1.45(0)$ & $7.95(-1)$ & $1.15(-1)$ & $2.58(-2)$ \\
\hline & 0.02 & $4.90(0)$ & $4.82(0)$ & $4.39(0)$ & $3.56(0)$ & $2.84(0)$ & $2.16(0)$ & $1.41(0)$ & $7.66(-1)$ & $1.04(-1)$ & $2.11(-2)$ \\
\hline & 0.05 & $4.68(0)$ & $4.61(0)$ & $4.19(0)$ & $3.38(0)$ & $2.69(0)$ & $2.02(0)$ & $1.30(0)$ & $6.84(-1)$ & $7.74(-2)$ & $1.16(-2)$ \\
\hline & 0.1 & $4.34(0)$ & $4.27(0)$ & $3.87(0)$ & $3.11(0)$ & $2.45(0)$ & $1.81(0)$ & $1,13(0)$ & $5.68(-1)$ & $4.74(-2)$ & $4.30(-3)$ \\
\hline & 0.2 & $3.73(0)$ & $3.66(0)$ & $3.32(0)$ & $2.63(0)$ & $2.03(0)$ & $1.47(0)$ & $8.69(-1)$ & $3.94(-1)$ & $1.79(-2)$ & \\
\hline & 0.5 & $2.38(0)$ & $2.34(0)$ & $2.11(0)$ & $1.63(0)$ & $1.21(0)$ & $8.07(-1)$ & $4.09(-1)$ & $1.38(-1)$ & $1.72(-3)$ & $6.69(-5)$ \\
\hline & 1.0 & $1.16(0)$ & $1.14(0)$ & $1.03(0)$ & $7.78(-1)$ & $5.43(-1)$ & $3.26(-1)$ & $1.30(-1)$ & $2.73(-2)$ & $1.87(-5)$ & \\
\hline & 2.0 & $2.93(-1)$ & $2.90(-1)$ & $2.69(-1)$ & $2.03(-1)$ & $1.32(-1)$ & $6.64(-2)$ & $1.73(-2)$ & $1.62(-3)$ & & \\
\hline & 5.0 & $7.21(-3)$ & $7.20(-3)$ & $7.04(-3)$ & $5.76(-3)$ & $3.29(-3)$ & $1.09(-3)$ & $9.00(-5)$ & $6.03(-7)$ & & \\
\hline & 10.0 & $2.61(-5)$ & $2.61(-5)$ & $2.61(-5)$ & $2.31(-5)$ & $1.22(-5)$ & $2.29(-6)$ & $2.52(-8)$ & & & \\
\hline \multirow[t]{4}{*}{1.0} & 0 & $2.18(0)$ & $2.15(0)$ & $1.99(0)$ & $1.72(0)$ & $1.51(0)$ & $1.30(0)$ & $1.01(0)$ & $6.66(-1)$ & $1.23(-1)$ & $3.13(-2)$ \\
\hline & 0.01 & $2.15(0)$ & $2.12(0)$ & $1.96(0)$ & $1.70(0)$ & $1.49(0)$ & $1.26(0)$ & $9.95(-1)$ & $6.52(-1)$ & $1.17(-1)$ & $2.83(-2)$ \\
\hline & 0.02 & $2.13(0)$ & $2.10(0)$ & $1.94(0)$ & $1.68(0)$ & $1.47(0)$ & $1.20(0)$ & $9.78(-1)$ & $6.38(-1)$ & $1.11(-1)$ & $2.56(-2)$ \\
\hline & 0.05 & $2.05(0)$ & $2.02(0)$ & $1.87(0)$ & $1.61(0)$ & $1.41(0)$ & $1.20(0)$ & $9.29(-1)$ & $5.99(-1)$ & $9.57(-2)$ & $1.90(-2)$ \\
\hline
\end{tabular}




\begin{tabular}{|c|c|c|c|c|c|c|c|c|c|c|c|}
\hline & 0.1 & $1.93(0)$ & $1.90(0)$ & $1.76(0)$ & $1.51(0)$ & $1.31(0)$ & $1.12(0)$ & $8.53(-1)$ & $5.39(-1)$ & $7.45(-2)$ & $1.15(-2)$ \\
\hline & 0.2 & $1.71(0)$ & $1.69(0)$ & $1.55(0)$ & $1.32(0)$ & $1.14(0)$ & $9.62(-1)$ & $7.20(-1)$ & $4.37(-1)$ & $4.52(-2)$ & $4.24(-3)$ \\
\hline & 0.5 & $1.20(0)$ & $1.18(0)$ & $1.07(0)$ & $8.96(-1)$ & $7.56(-1)$ & $6.18(-1)$ & $4.37(-1)$ & $2.35(-1)$ & $1.02(-2)$ & $3.96(-4)$ \\
\hline & 1.0 & $6.64(-1)$ & $6.51(-1)$ & $5.85(-1)$ & $4.73(-1)$ & $3.87(-1)$ & $3.02(-1)$ & $1.95(-1)$ & $8.65(-2)$ & $1.06(-3)$ & $2.27(-6)$ \\
\hline & 2.0 & $2.05(-1)$ & $2.01(-1)$ & $1.77(-1)$ & $1.37(-1)$ & $1.06(-1)$ & $7.67(-2)$ & $4.22(-2)$ & $1.29(-2)$ & $6.65(-6)$ & \\
\hline & 5.0 & $6.54(-3)$ & $6.42(-3)$ & $5.70(-3)$ & $4.21(-3)$ & $2.95(-3)$ & $1.79(-3)$ & $6.51(-4)$ & $7.40(-5)$ & & \\
\hline & 10.0 & $2.58(-5)$ & $2.56(-5)$ & $2.41(-5)$ & $1.78(-5)$ & $1.14(-5)$ & $5.57(-6)$ & $1.13(-6)$ & $2.66(-8)$ & & \\
\hline \multirow[t]{11}{*}{2.0} & 0 & $7.01(-1)$ & $6.96(-1)$ & $6.71(-1)$ & $6.27(-1)$ & $5.91(-1)$ & $5.52(-1)$ & $4.90(-1)$ & $3.92(-1)$ & $1.10(-1)$ & $3.03(-2)$ \\
\hline & 0.01 & $6.94(-1)$ & $6.89(-1)$ & $6.63(-1)$ & $6.20(-1)$ & $5.85(-1)$ & & $4.84(-1)$ & $3.86(-1)$ & $1.07(-1)$ & $2.88(-2)$ \\
\hline & 0.02 & $6.86(-1)$ & $6.81(-1)$ & $6.56(-1)$ & $6.14(-1)$ & $5.78(-1)$ & $5.39(-1)$ & $4.78(-1)$ & $3.81(-1)$ & $1.04(-1)$ & $2.74(-2)$ \\
\hline & 0.05 & $6.65(-1)$ & $6.60(-1)$ & $6.35(-1)$ & $5.93(-1)$ & $5.58(-1)$ & $5.20(-1)$ & $4.60(-1)$ & $3.65(-1)$ & $9.60(-2)$ & $2.35(-2)$ \\
\hline & 0.1 & $6.31(-1)$ & $6.26(-1)$ & $6.02(-1)$ & $5.61(-1)$ & $5.27(-1)$ & $4.90(-1)$ & $4.32(-1)$ & $3.40(-1)$ & $8.40(-2)$ & $1.83(-2)$ \\
\hline & 0.2 & $5.67(-1)$ & $5.63(-1)$ & $5.40(-1)$ & $5.02(-1)$ & $4.70(-1)$ & $4.35(-1)$ & $3.81(-1)$ & $2.96(-1)$ & $6.44(-2)$ & $1.10(-2)$ \\
\hline & 0.5 & $4.13(-1)$ & $4.09(-1)$ & $3.91(-1)$ & $3.60(-1)$ & $3.34(-1)$ & $3.05(-1)$ & $2.62(-1)$ & $1.95(-1)$ & $2.91(-2)$ & $2.41(-3)$ \\
\hline & 1.0 & $2.44(-1)$ & $2.41(-1)$ & $2.28(-1)$ & $2.06(-1)$ & $1.89(-1)$ & $1.70(-1)$ & $1.41(-1)$ & $9.74(-2)$ & $7.83(-3)$ & $2.35(-4)$ \\
\hline & 2.0 & $8.47(-2)$ & $8.36(-2)$ & $7.79(-2)$ & $6.85(-2)$ & $6.09(-2)$ & $5.29(-2)$ & $4.12(-2)$ & $2.50(-2)$ & $6.16(-4)$ & \\
\hline & 5.0 & $3.59(-3)$ & $3.52(-3)$ & $3.17(-3)$ & $2.60(-3)$ & $2.16(-3)$ & $1.72(-3)$ & $1.14(-3)$ & $4.77(-4)$ & $2.28(-7)$ & \\
\hline & 10.0 & $1.89(-5)$ & $1.84(-5)$ & $1.61(-5)$ & $1.24(--5)$ & $9.55(-6)$ & $6.86(-6)$ & $3.45(-6)$ & $9.02(-7)$ & & \\
\hline \multirow[t]{11}{*}{5.0} & 0 & $1.23(-1)$ & $1.23(-1)$ & $1.22(-1)$ & $1.20(-1)$ & $1.19(-1)$ & $1.17(-1)$ & $1.14(-1)$ & $1.07(-1)$ & $6.28(-2)$ & $2.52(-2)$ \\
\hline & 0.01 & $22(-1)$ & $1.22(-1)$ & $1.21(-1)$ & $1.19(-1)$ & $1.18(-1)$ & $1.16(-1)$ & $1.12(-1)$ & $1.06(-1)$ & $6.27(-2)$ & $2.51(-2)$ \\
\hline & 0.02 & $1.21(-1)$ & $1.21(-1)$ & $1.20(-1)$ & $1.18(-1)$ & $1.16(-1)$ & $1.14(-1)$ & $1.11(-1)$ & $1.05(-1)$ & $6.11(-2)$ & $2.41(-2)$ \\
\hline & 0.05 & $1.17(-1)$ & $1.17(-1)$ & $1.16(-1)$ & $1.14(-1)$ & $1.13(-1)$ & $1.11(-1)$ & $1.08(--1)$ & $1.01(-1)$ & $5.85(-2)$ & $2.25(-2)$ \\
\hline & 0.1 & $1.11(-1)$ & $1.11(-1)$ & $1.10(-1)$ & $1.09(-1)$ & $1.07(-1)$ & $1.05(-1)$ & $1.02(-1)$ & $9.60(-2)$ & $5.46(-2)$ & $2.02(-2)$ \\
\hline & 0.2 & $1.01(-1)$ & $1.01(-1)$ & $9.96(-2)$ & $9.81(-2)$ & $9.66(-2)$ & $9.50(-2)$ & $9.20(-2)$ & $8.62(-2)$ & $4.74(-2)$ & $1.61(-2)$ \\
\hline & 0.5 & $7.44(-2)$ & $7.43(-2)$ & $7.35(-2)$ & $7.22(-2)$ & $7.10(-2)$ & $6.96(-2)$ & $6.71(-2)$ & $6.23(-2)$ & $3.10(-2)$ & $8.26(-3)$ \\
\hline & 1.0 & $4.49(-2)$ & $4.48(-2)$ & $4.43(-2)$ & $4.33(-2)$ & $4.25(-2)$ & $4.14(-2)$ & $3.97(-2)$ & $3.62(-2)$ & $1.53(-2)$ & $2.71(-3)$ \\
\hline & 2.0 & $1.64(-2)$ & $1.63(-2)$ & $1.60(-2)$ & $1.56(-2)$ & $1.52(-2)$ & $1.47(-2)$ & $1.39(-2)$ & $1.23(-2)$ & $3.76(-3)$ & $2.94(-4)$ \\
\hline & 5.0 & $7.91(-4)$ & $7.87(-4)$ & $7.66(-4)$ & $7.29(-4)$ & $6.97(-4)$ & $6.60(-4)$ & $5.97(-4)$ & $4.83(-4)$ & $5.71(-5)$ & $3.77(-7)$ \\
\hline & 10.0 & $5.09(-6)$ & $5.05(-6)$ & $4.83(-6)$ & $4.45(-6)$ & $4.13(-6)$ & $3.78(-6)$ & $3.20(-6)$ & $2.27(-6)$ & $5.99(-8)$ & \\
\hline \multirow{11}{*}{10.0} & 0 & $3.13(-2)$ & $3.12(-2)$ & $3.12(-2)$ & $3.11(-2)$ & $3.10(-2)$ & $3.08(-2)$ & $-2)$ & $3.01(-2)$ & $2.51(-2)$ & $1.57(-2)$ \\
\hline & 0.01 & $3.09(-2)$ & & & & & & & & & \\
\hline & 0.02 & $3.06(-2)$ & $3.06(-2)$ & $3.06(-2)$ & $3.04(-2)$ & $3.03(-2)$ & $3.02(-2)$ & $3.00(-2)$ & $2.95(-2)$ & $2.45(-2)$ & $1.53(-2)$ \\
\hline & 0.05 & $2.97(-2)$ & $2.97(-2)$ & $2.97(-2)$ & $2.95(-2)$ & $2.94(-2)$ & $2.93(-2)$ & $2.91(-2)$ & $2.86(-2)$ & $2.37(-2)$ & $1.46(-2)$ \\
\hline & 0.1 & $2.83(-2)$ & $2.83(-2)$ & $2.82(-2)$ & $2.81(-2)$ & $2.80(-2)$ & $2.79(-2)$ & $2.76(-2)$ & $2,72(-2)$ & $2.24(-2)$ & $1.36(-2)$ \\
\hline & 0.2 & $2.56(-2)$ & $2.56(-2)$ & $2.55(-2)$ & $2.54(-2)$ & $2.53(-2)$ & $2.52(-2)$ & $2.50(-2)$ & $2.45(-2)$ & $2.00(-2)$ & $1.18(-2)$ \\
\hline & 0.5 & $1.89(-2)$ & $1.89(-2)$ & $1.89(-2)$ & $1.88(-2)$ & $1.87(-2)$ & $1.86(-2)$ & $1.84(-2)$ & $1.80(-2)$ & $1.43(-2)$ & $7.75(-3)$ \\
\hline & 1.0 & $1.15(-2)$ & $1.15(-2)$ & $1.14(-2)$ & $1.14(-2)$ & $1.13(-2)$ & $1.12(-2)$ & $1.11(-2)$ & $1.08(-2)$ & $8.20(-3)$ & $3.82(-3)$ \\
\hline & 2.0 & $4.21(-3)$ & $4.21(-3)$ & $4.19(-3)$ & $4.16(-3)$ & $4.13(-3)$ & $4.09(-3)$ & $4.03(-3)$ & $3.90(-3)$ & $2.68(-3)$ & $9.31(-4)$ \\
\hline & 5.0 & $2.08(-4)$ & $2.08(-4)$ & $2.06(-4)$ & $2.04(-4)$ & $2.04(-4)$ & $2.01(-4)$ & $1.93(-4)$ & $1.82(-4)$ & $9.37(-5)$ & $1.36(-5)$ \\
\hline & 10.0 & $1.39(-6)$ & $1.38(-6)$ & $1.37(-6)$ & $1.34(-6)$ & $1.31(-6)$ & $1.28(-6)$ & $1.22(-6)$ & $1.10(-6)$ & $3.53(-7)$ & $1.26(-8)$ \\
\hline
\end{tabular}

$\nmid$ From Hubbell et al. ${ }^{1}$

$\ddagger$ Read: $1.45 \times 10^{1}$, etc. 
Table H.2-UNSCATTERED FLUX DENSITY FROM A DISK SOURCE WITH A

\begin{tabular}{|c|c|c|c|c|c|c|c|c|c|c|c|}
\hline \multirow[b]{2}{*}{$z / r_{0}$} & \multirow[b]{2}{*}{$\mu t$} & \multicolumn{10}{|c|}{$2 \Gamma /[\Phi(0) G(E)]$} \\
\hline & & $\rho / r_{0}=0$ & $\rho / r_{0}=0.2$ & $\rho / r_{0}=0.5$ & $\rho / r_{0}=0.8$ & $\rho / r_{0}=1.0$ & $\rho / r_{0}=1.2$ & $\rho / r_{0}=1.5$ & $\rho / r_{0}=2.0$ & $\rho / r_{0}=5.0$ & $\rho / r_{0}=10.0$ \\
\hline 0.1 & $\begin{array}{l}0 \\
0.01 \\
0.02 \\
0.03 \\
0.1 \\
0.2 \\
0.5 \\
1.0 \\
2.0 \\
5.0 \\
10.0\end{array}$ & $\begin{array}{l}9.01(-1) \ddagger \\
8.78(-1) \\
8.56(-1) \\
8.35(-1) \\
7.08(-1) \\
5.70(-1) \\
3.26(-1) \\
1.48(-1) \\
3.75(-2) \\
9.96(-4) \\
3.83(-6)\end{array}$ & $\begin{array}{l}8.97(-1) \\
8.75(-1) \\
8.53(-1) \\
8.33(-1) \\
7.06(-1) \\
5.70(-1) \\
3.26(-1) \\
1.48(-1) \\
3.75(-2) \\
9.96(-4) \\
3.81(-6)\end{array}$ & $\begin{array}{l}8.77(-1) \\
8.55(-1) \\
8.35(-1) \\
8.15(-1) \\
6.95(-1) \\
5.64(-1) \\
3.26(-1) \\
1.48(-1) \\
3.75(-2) \\
9.96(-4) \\
3.83(-6)\end{array}$ & $\begin{array}{l}7.88(-1) \\
7.70(-1) \\
7.53(-1) \\
7.36(-1) \\
6.35(-1) \\
5.23(-1) \\
3.12(-1) \\
1.46(-1) \\
3.74(-2) \\
9.96(-4) \\
3.82(-6)\end{array}$ & $\begin{array}{l}4.30(-1) \\
4.19(-1) \\
4.08(-1) \\
3.97(-1) \\
3.36(-1) \\
2.71(-1) \\
1.57(-1) \\
7.19(-2) \\
1.83(-2) \\
4.91(-4) \\
1.89(-6)\end{array}$ & $\begin{array}{l}9.65(-2) \\
9.10(-2) \\
8.60(-2) \\
8.13(-2) \\
5.65(-2) \\
3.57(-2) \\
1.12(-2) \\
2.16(-3) \\
1.19(-4) \\
5.05(-8) \\
2.85(-13)\end{array}$ & $\begin{array}{c}2.79(-2) \\
2.51(-2) \\
2.27(-2) \\
2.05(-2) \\
1.06(-2) \\
4.55(-3) \\
5.01(-4) \\
1.98(-5) \\
5.43(-8) \\
3.72(-15) \\
\S\end{array}$ & $\begin{array}{c}8.56(-3) \\
7.25(-3) \\
6.15(-3) \\
5.23(-3) \\
1.77(-3) \\
4.24(-4) \\
9.12(-6) \\
2.73(-8) \\
4.86(-13) \\
\S \\
\S\end{array}$ & $\begin{array}{c}4.18(-4) \\
2.57(-4) \\
1.59(-4) \\
9.80(-5) \\
3.59(-6) \\
3.73(-8) \\
8.01(-14) \\
6.52(-23) \\
\S \\
\S \\
\S\end{array}$ & $\begin{array}{c}5.06(-5) \\
1.87(-5) \\
6.96(-6) \\
2.59(-6) \\
2.75(-9) \\
1.84(-13) \\
1.13(-25) \\
\S \\
\S \\
\S \\
\S\end{array}$ \\
\hline 0.2 & $\begin{array}{l}0 \\
0.01 \\
0.02 \\
0.03 \\
0.1 \\
0.2 \\
0.5 \\
1.0 \\
2.0 \\
5.0 \\
10.0\end{array}$ & $\begin{array}{l}8.04(-1) \\
7.88(-1) \\
7.72(-1) \\
7.57(-1) \\
6.60(-1) \\
5.46(-1) \\
3.23(-1) \\
1.48(-1) \\
3.75(-2) \\
9.96(-4) \\
3.83(-6)\end{array}$ & $\begin{array}{l}7.98(-1) \\
7.82(-1) \\
7.67(-1) \\
7.52(-1) \\
6.56(-1) \\
5.43(-1) \\
3.22(-1) \\
1.48(-1) \\
3.75(-2) \\
9.96(-4) \\
3.83(-6)\end{array}$ & $\begin{array}{l}7.61(-1) \\
7.46(-1) \\
7.32(-1) \\
7.18(-1) \\
6.28(-1) \\
5.23(-1) \\
3.15(-1) \\
1.47(-1) \\
3.75(-2) \\
9.96(-4) \\
3.83(-6)\end{array}$ & $\begin{array}{l}6.30(-1) \\
6.18(-1) \\
6.06(-1) \\
5.95(-1) \\
5.23(-1) \\
4.39(-1) \\
2.71(-1) \\
1.31(-1) \\
3.52(-2) \\
9.84(-4) \\
3.83(-6)\end{array}$ & $\begin{array}{l}3.83(-1) \\
3.74(-1) \\
3.66(-1) \\
3.58(-1) \\
3.09(-1) \\
2.54(-1) \\
1.50(-1) \\
6.95(-2) \\
1.79(-2) \\
4.82(-4) \\
1.87(-6)\end{array}$ & $\begin{array}{l}1.54(-1) \\
1.49(-1) \\
1.44(-1) \\
1.40(-1) \\
1.12(-1) \\
8.37(-2) \\
3.84(-2) \\
1.25(-2) \\
1.77(-3) \\
1.01(-5) \\
3.73(-9)\end{array}$ & $\begin{array}{l}5.25(-2) \\
4.98(-2) \\
4.72(-2) \\
4.48(-2) \\
3.14(-2) \\
1.94(-2) \\
5.34(-3) \\
8.10(-4) \\
2.77(-5) \\
2.90(-9) \\
1.64(-15)\end{array}$ & $\begin{array}{c}1.68(-2) \\
1.54(-2) \\
1.42(-2) \\
1.31(-2) \\
7.41(-3) \\
3.42(-3) \\
4.07(-4) \\
1.66(-5) \\
4.61(-8) \\
3.20(-15) \\
\S\end{array}$ & $\begin{array}{c}8.35(-4) \\
6.55(-4) \\
5.13(-4) \\
4.03(-4) \\
7.51(-5) \\
7.13(-6) \\
7.94(-9) \\
1.57(-13) \\
1.25(-22) \\
\S \\
\S\end{array}$ & $\begin{array}{c}1.01(-4) \\
6.15(-5) \\
3.75(-5) \\
2.28(-5) \\
7.24(-7) \\
5.49(-9) \\
3.19(-15) \\
2.24(-25) \\
\S \\
\S \\
\S\end{array}$ \\
\hline 0.5 & $\begin{array}{l}0 \\
0.01 \\
0.02 \\
0.03 \\
0.1 \\
0.2 \\
0.5 \\
1.0 \\
2.0 \\
5.0 \\
10.0\end{array}$ & $\begin{array}{l}5.53(-1) \\
5.45(-1) \\
5.37(-1) \\
5.29(-1) \\
4.78(-1) \\
4.14(-1) \\
2.71(-1) \\
1.36(-1) \\
3.67(-2) \\
9.96(-4) \\
3.83(-6)\end{array}$ & $\begin{array}{l}5.44(-1) \\
5.36(-1) \\
5.28(-1) \\
5.21(-1) \\
4.71(-1) \\
4.08(-1) \\
2.67(-1) \\
1.34(-1) \\
3.64(-2) \\
9.95(-4) \\
3.83(-6)\end{array}$ & $\begin{array}{l}4.94(-1) \\
4.87(-1) \\
4.79(-1) \\
4.73(-1) \\
4.27(-1) \\
3.70(-1) \\
2.43(-1) \\
1.23(-1) \\
3.43(-2) \\
9.79(-4) \\
3.82(-6)\end{array}$ & $\begin{array}{l}3.84(-1) \\
3.78(-1) \\
3.72(-1) \\
3.67(-1) \\
3.30(-1) \\
2.84(-1) \\
1.85(-1) \\
9.37(-2) \\
2.64(-2) \\
8.07(-4) \\
3.42(-6)\end{array}$ & $\begin{array}{l}2.81(-1) \\
2.77(-1) \\
2.72(-1) \\
2.68(-1) \\
2.39(-1) \\
2.03(-1) \\
1.28(-1) \\
5.20(-2) \\
1.65(-2) \\
4.57(-4) \\
1.80(-6)\end{array}$ & $\begin{array}{l}1.86(-1) \\
1.82(-1) \\
1.79(-1) \\
1.75(-1) \\
1.53(-1) \\
1.27(-1) \\
7.48(-2) \\
3.29(-2) \\
7.38(-3) \\
1.37(-4) \\
3.07(-7)\end{array}$ & $\begin{array}{l}9.50(-2) \\
9.27(-2) \\
9.04(-2) \\
8.82(-2) \\
7.43(-2) \\
5.85(-2) \\
2.94(-2) \\
1.02(-2) \\
1.49(-3) \\
8.77(-6) \\
3.29(-9)\end{array}$ & $\begin{array}{l}3.70(-2) \\
3.57(-2) \\
3.47(-2) \\
3.33(-2) \\
2.60(-2) \\
1.85(-2) \\
6.85(-3) \\
1.46(-3) \\
8.58(-5) \\
3.79(-8) \\
2.17(-13)\end{array}$ & $\begin{array}{c}2.06(-3) \\
1.87(-3) \\
1.69(-3) \\
1.54(-3) \\
7.77(-4) \\
2.96(-4) \\
1.72(-5) \\
1.74(-7) \\
2.54(-11) \\
2.39(-22) \\
\S\end{array}$ & $\begin{array}{c}2.52(-4) \\
2.06(-4) \\
1.69(-4) \\
1.39(-4) \\
3.46(-5) \\
4.80(-6) \\
1.35(-8) \\
8.95(-13) \\
5.82(-21) \\
\S \\
\S\end{array}$ \\
\hline 1 & $\begin{array}{l}0 \\
0.01 \\
0.02 \\
0.03 \\
0.1\end{array}$ & $\begin{array}{l}2.93(-1) \\
2.89(-1) \\
2.86(-1) \\
2.83(-1) \\
2.60(-1)\end{array}$ & $\begin{array}{l}2.88(-1) \\
2.84(-1) \\
2.81(-1) \\
2.77(-1) \\
2.55(-1)\end{array}$ & $\begin{array}{l}2.61(-1) \\
2.57(-1) \\
2.54(-1) \\
2.51(-1) \\
2.31(-1)\end{array}$ & $\begin{array}{l}2.15(-1) \\
2.12(-1) \\
2.09(-1) \\
2.07(-1) \\
1.89(-1)\end{array}$ & $\begin{array}{l}1.79(-1) \\
1.76(-1) \\
1.74(-1) \\
1.72(-1) \\
1.56(-1)\end{array}$ & $\begin{array}{l}1.43(-1) \\
1.41(-1) \\
1.39(-1) \\
1.37(-1) \\
1.24(-1)\end{array}$ & $\begin{array}{l}9.85(-2) \\
9.69(-2) \\
9.54(-2) \\
9.38(-2) \\
8.37(-2)\end{array}$ & $\begin{array}{l}5.18(-2) \\
5.08(-2) \\
4.98(-2) \\
4.88(-2) \\
4.23(-2)\end{array}$ & $\begin{array}{l}3.93(-3) \\
3.74(-3) \\
3.56(-3) \\
3.38(-3) \\
2.39(-3)\end{array}$ & $\begin{array}{l}4.98(-4) \\
4.51(-4) \\
4.08(-4) \\
3.69(-4) \\
1.84(-4)\end{array}$ \\
\hline
\end{tabular}




\begin{tabular}{|c|c|c|c|c|c|c|c|c|c|c|c|}
\hline & 0.2 & $2.31(-1)$ & $2.27(-1)$ & $2.04(-1)$ & $1.66(-1)$ & $1.37(-1)$ & $1.08(-1)$ & $7.12(-2)$ & $3.46(-2)$ & $1.46(-3)$ & $6.79(-5)$ \\
\hline & 0.5 & $1.62(-1)$ & $1.59(-1)$ & $1.43(-1)$ & $1.14(-1)$ & $9.20(-2)$ & $7.05(-2)$ & $4.42(-2)$ & $1.91(-2)$ & $3.35(-4)$ & $3.48(-6)$ \\
\hline & 1.0 & $9.03(-2)$ & $8.84(-2)$ & $7.86(-2)$ & $6.16(-2)$ & $4.83(-2)$ & $3.55(-2)$ & $2.04(-2)$ & $7.27(-3)$ & $3.01(-5)$ & $2.57(-8)$ \\
\hline & 2.0 & $2.82(-2)$ & $2.76(-2)$ & $2.44(-2)$ & $1.85(-2)$ & $1.39(-2)$ & $9.51(-3)$ & $4.68(-3)$ & $1.16(-3)$ & $2.78(-7)$ & $1.63(-12)$ \\
\hline & 5.0 & $9.29(-4)$ & $9.13(-4)$ & $8.17(-4)$ & $6.02(-4)$ & $4.14(-4)$ & $2.41(-4)$ & $7.93(-5)$ & $7.24(-6)$ & $4.16(-13)$ & $8.52(-25)$ \\
\hline & 10.0 & $3.80(-6)$ & $3.77(-6)$ & $3.54(-6)$ & $2.65(-6)$ & $1.68(-6)$ & $7.93(-7)$ & $1.45(-7)$ & $2.78(-9)$ & $1.84(-22)$ & $\S$ \\
\hline \multirow[t]{11}{*}{2.0} & 0 & $1.06(-1)$ & $1.04(-1)$ & $9.91(-2)$ & $9.01(-2)$ & $8.27(-2)$ & $7.47(-2)$ & $6.28(-2)$ & $4.50(-2)$ & $6.60(-3)$ & $9.52(-4)$ \\
\hline & 0.01 & $1.04(-1)$ & $1.03(-1)$ & $9.81(-2)$ & $8.91(-2)$ & $8.17(-2)$ & $7.39(-2)$ & $6.20(-2)$ & $4.44(-2)$ & $6.43(-3)$ & $9.05(-4)$ \\
\hline & 0.02 & $1.03(-1)$ & $1.02(-1)$ & $9.70(-2)$ & $8.81(-2)$ & $8.08(-2)$ & $7.30(-2)$ & $6.12(-2)$ & $4.38(-2)$ & $6.26(-3)$ & $8.51(-4)$ \\
\hline & 0.03 & $1.02(-1)$ & $1.01(-1)$ & $9.60(-2)$ & $8.71(-2)$ & $7.99(-2)$ & $7.22(-2)$ & $6.05(-2)$ & $4.32(-2)$ & $6.09(-3)$ & $8.18(-4)$ \\
\hline & 0.1 & $9.50(-2)$ & $9.40(-2)$ & $8.90(-2)$ & $8.06(-2)$ & $7.38(-2)$ & $6.65(-2)$ & $5.54(-2)$ & $3.92(-2)$ & $5.07(-3)$ & $5.74(-4)$ \\
\hline & 0.2 & $8.55(-2)$ & $8.45(-2)$ & $7.99(-2)$ & $7.22(-2)$ & $6.59(-2)$ & $5.91(-2)$ & $4.90(-2)$ & $3.41(-2)$ & $3.89(-3)$ & $3.46(-4)$ \\
\hline & 0.5 & $6.22(-2)$ & $6.15(-2)$ & $5.79(-2)$ & $5.18(-2)$ & $4.69(-2)$ & $4.16(-2)$ & $3.38(-2)$ & $2.26(-2)$ & $1.77(-3)$ & $7.61(-5)$ \\
\hline & 1.0 & $3.67(-2)$ & $3.62(-2)$ & $3.38(-2)$ & $2.98(-2)$ & $2.66(-2)$ & $2.32(-2)$ & $1.83(-2)$ & $1.14(-2)$ & $4.80(-4)$ & $6.16(-6)$ \\
\hline & 2.0 & $1.28(-2)$ & $1.26(-2)$ & $1.16(-2)$ & $9.96(-3)$ & $8.66(-3)$ & $7.33(-3)$ & $5.43(-3)$ & $2.97(-3)$ & $3.67(-5)$ & $4.22(-8)$ \\
\hline & 5.0 & $5.43(-4)$ & $5.32(-4)$ & $4.75(-4)$ & $3.84(-4)$ & $3.14(-4)$ & $2.45(-4)$ & $1.55(-4)$ & $5.95(-5)$ & $2.07(-8)$ & $1.78(-14)$ \\
\hline & 10.0 & $2.87(-6)$ & $2.80(-6)$ & $2.45(-6)$ & $1.86(-6)$ & $1.42(-6)$ & $1.01(-6)$ & $5.16(-7)$ & $1.17(-7)$ & $1.28(-13)$ & $7.29(-25)$ \\
\hline \multirow[t]{11}{*}{5.0} & 0 & $1.94(-2)$ & $1.94(-2)$ & $1.91(-2)$ & $1.87(-2)$ & $1.84(-2)$ & $1.79(-2)$ & $1.72(-2)$ & $1.57(-2)$ & $7.10(-3)$ & $1.80(-3)$ \\
\hline & 0.01 & $1.92(-2)$ & $1.92(-2)$ & $1.90(-2)$ & $1.85(-2)$ & $1.82(-2)$ & $1.78(-2)$ & $1.70(-2)$ & $1.56(-2)$ & 3) & -3) \\
\hline & 0.02 & $1.90(-2)$ & $1.90(-2)$ & $1.88(-2)$ & $1.84(-2)$ & $1.80(-2)$ & $1.76 i-2)$ & $1.68(-2)$ & $1.54(-2)$ & $6.90(-3)$ & $1.72(-3)$ \\
\hline & 0.03 & $1.88(-2)$ & $1.88(-2)$ & $1.86(-2)$ & $1.82(-2)$ & $1.78(-2)$ & $1.74(-2)$ & $1.67(-2)$ & $1.52(-2)$ & $6.80(-3)$ & $1.68(-3)$ \\
\hline & 0.1 & $1.75(-2)$ & $1.75(-2)$ & $1.73(-2)$ & $1.69(-2)$ & $1.66(-2)$ & $1.62(-2)$ & $1.55(-2)$ & $1.41(-2)$ & $6.16(-3)$ & $1.44(-3)$ \\
\hline & 0.2 & $1.59(-2)$ & $1.58(-2)$ & $1.56(-2)$ & $1.53(-2)$ & $1.50(-2)$ & $1.46(-2)$ & $1.39(-2)$ & $1.27(-2)$ & $5.35(-3)$ & $1.15(-3)$ \\
\hline & 0.5 & $1.17(-2)$ & $1.17(-2)$ & $1.15(-3)$ & $1.12(-2)$ & $1.10(-2)$ & $1.07(-2)$ & $1.02(-2)$ & $9.16(-3)$ & $3.51(-3)$ & $5.91(-4)$ \\
\hline & 1.0 & $7.07(-3)$ & $7.05(-3)$ & $6.94(-3)$ & $6.75(-3)$ & $6.57(-3)$ & $6.37(-3)$ & $6.02(-3)$ & $5.34(-3)$ & $1.74(-3)$ & $1.95(-4)$ \\
\hline & 2.0 & $2.58(-3)$ & $2.57(-3)$ & $2.52(-3)$ & $2.43(-3)$ & $2.35(-3)$ & $2.26(-3)$ & $2.11(-3)$ & $1.81(-3)$ & $4.28(-4)$ & $2.12(-5)$ \\
\hline & 5.0 & $1.25(-4)$ & $1.24(-4)$ & $1.20(-4)$ & $1.14(-4)$ & $1.08(-4)$ & $1.02 i-4)$ & $9.08(-5)$ & $7.16(-5)$ & $6.57(-6)$ & $2.88(-8)$ \\
\hline & 10.0 & $8.00(-7)$ & $7.93(-7)$ & $7.57(-7)$ & $6.94(-7)$ & $6.41(-7)$ & $5.82(-7)$ & $4.88(-7)$ & $3.36(-7)$ & $6.77(-9)$ & $5.46(-13)$ \\
\hline \multirow[t]{11}{*}{10.0} & 0 & $4.96(-3)$ & $4.96(-3)$ & $4.94(-3)$ & $4.92(-3)$ & $4.89(-3)$ & $4.86(-3)$ & $4.80(-3)$ & $4.68(-3)$ & $3.57(-3)$ & $1.77(-3)$ \\
\hline & 0.01 & $4.91(-3)$ & $4.91(-3)$ & $4.89(-3)$ & $4.87(-3)$ & $4.84(-3)$ & $4.81(-3)$ & $4.75(-3)$ & $4.64(-3)$ & $3.53(-3)$ & $1.74(-3)$ \\
\hline & 0.02 & $4.86(-3)$ & $4.86 i-3 j$ & $4.85(-3)$ & $4.82(-3)$ & $4.79(-3)$ & $4.76(-3)$ & $4.71(-3)$ & $4.59(-3)$ & $3.49(-3)$ & $1.72(-3)$ \\
\hline & 0.03 & $4.82(-3)$ & $4.81(-3)$ & $4.80(-3)$ & $4.77(-3)$ & $4.74(-3)$ & $4.71(-3)$ & $4.66(-3)$ & $4.54(-3)$ & $3.45(-3)$ & $1.70(-3)$ \\
\hline & 0.1 & $4.49(-3)$ & $4.49(-3)$ & $4.47(-3)$ & $4.45(-3)$ & $4.42(-3)$ & $4.39(-3)$ & $4.34(-3)$ & $4.23(-3)$ & $3.19(-3)$ & $1.54(-3)$ \\
\hline & 0.2 & $4.06(-3)$ & $4.06(-3)$ & $4.04(-3)$ & $4.02(-3)$ & $4.00(-3)$ & $3.97(-3)$ & $3.92(-3)$ & $3.82(-3)$ & $2.85(-3)$ & $1.33(-3)$ \\
\hline & 0.5 & $3.01(-3)$ & $3.00(-3)$ & $2.99(-3)$ & $2.97(-3)$ & $2.95(-3)$ & $2.93(-3)$ & $2.89(-3)$ & $2.81(-3)$ & $2.04(-3)$ & $8.73(-4)$ \\
\hline & 1.0 & $1.82(-3)$ & $1.82(-3)$ & $1.81(-3)$ & $1.80(-3)$ & $1.79(-3)$ & $1.77(-3)$ & $1.74(-3)$ & $1.69(-3)$ & $1.17(-3)$ & $4.31(-4)$ \\
\hline & 2.0 & $6.68(-4)$ & $6.68(-4)$ & $6.64(-4)$ & $6.58(-4)$ & $6.52(-4)$ & $6.45(-4)$ & $6.33(-4)$ & $6.07(-4)$ & $3.81(-4)$ & $1.05(-4)$ \\
\hline & 5.0 & $3.30(-5)$ & $3.30(-5)$ & $3.27(-5)$ & $3.22(-5)$ & $3.18(-5)$ & $3.12(-5)$ & $3.03(-5)$ & $2.83(-5)$ & $1.34(-5)$ & $1.54(-6)$ \\
\hline & 10.0 & $2.20(-7)$ & $2.19(-7)$ & $2.16(-7)$ & $2.11(-7)$ & $2.06(-7)$ & $2.01(-7)$ & $0.91(-7)$ & $1.72(-7)$ & $5.06(-8)$ & $1.37(-9)$ \\
\hline
\end{tabular}

fFrom Trubey. ${ }^{2}$

$¥$ Read: $9.01 \times 10^{-1}$, etc.

\&Effectively zero. 
and, for a cosine current source (isotropic flux), it is given by

$$
\frac{2 \Gamma\left(\mu t, r_{0} / z, 0\right)}{\Phi_{0} G(E)}=\left\{E_{2}(\mu t)-\left[1+\left(\frac{r_{0}}{z}\right)^{2}\right]^{-1 / 2} E_{2}\left[\mu t \sqrt{1+\left(\frac{r_{0}}{z}\right)^{2}}\right]\right\}
$$

where $\Phi_{0}$ is the isotropic flux at the source plane.

The variables for the rectangular source (see Tables H.3 and H.4) are

$\Gamma(\mu t, a, b)=$ dose rate on perpendicular to corner

$H, W=$ height and width of rectangle $(\mathrm{cm})$

$a, b=$ dimensions of rectangle in units of detector distance, $a=H / z, b=$ $W / z$ and $S, G(E)$ and $\mu t$ are as previously defined.

For an isotropic source the unscattered flux attenuation function for the dose rate at a corner position is given by

$$
\begin{aligned}
\frac{4 \pi \Gamma(\mu t, a, b)}{S G(E)}=\int_{0}^{b} \int_{0}^{a} e^{-\mu r} \frac{d x d y}{r^{2}} & \approx \frac{\pi}{2}\left[E_{1}(\mu t)\right. \\
& \left.-E_{1}\left(\mu t \sqrt{1+a^{2}}\right)\right] \quad(\text { for } a \cong b>1)
\end{aligned}
$$

and, for the cosine current source (isotropic flux), it is given by

$$
\begin{aligned}
\frac{2 \Gamma(\mu t, a, b)}{\Phi_{0} G(E)} & =\frac{1}{2 \pi} \int_{0}^{b} \int_{0}^{a} e^{-\mu r} \frac{3}{r} \frac{d x d y}{r^{2}} \\
& \approx \frac{1}{4}\left[E_{2}(\mu t)-\left(1 / \sqrt{1+a^{2}}\right) E_{2}\left(\mu t \sqrt{1+a^{2}}\right)\right] \quad(\text { for } a \cong b>1)
\end{aligned}
$$

where $r^{2}=x^{2}+y^{2}+z^{2}$.

\section{REFERENCES}

1. J. H. Hubbell, R. L. Bach, and R. J. Herbold, Radiation Field from a Circular Disk Source, J. Res. Nat. Bur. Stand., C, 65: 249 (1961).

2. D. K. Trubey, Oak Ridge National Laboratory, unpublished calculations.

3. J. H. Hubbell, R. L. Bach, and J. C. Lamkin, Radiation Field from a Rectangular Source, J. Res. Nat. Bur. Stand., C, 64: 121 (1960). 
Table H.3-UNSCATTERED FLUX AT A CORNER POSITION FROM A RECTANGULAR PLANE SOURCE WITH AN ISOTROPIC ANGULAR DISTRIBUTION $\dagger$

\begin{tabular}{|c|c|c|c|c|c|c|}
\hline \multirow[b]{2}{*}{$\mu t$} & \multicolumn{6}{|c|}{$4 \pi \Gamma /[S G(E)]$} \\
\hline & $\begin{array}{l}b=0.1 \\
a=0.1\end{array}$ & $\begin{array}{l}b=0.2 \\
a=0.1\end{array}$ & $\begin{array}{l}b=0.2 \\
a=0.2\end{array}$ & $\begin{array}{l}b=0.5 \\
a=0.1\end{array}$ & $\begin{array}{l}b=0.5 \\
a=0.2\end{array}$ & $\begin{array}{l}b=0.5 \\
a=0.5\end{array}$ \\
\hline 0 & $9.93(-3) \ddagger$ & $1.97(-2)$ & $3.90(-2)$ & $4.62(-2)$ & $9.16(-2)$ & $2.16(-1)$ \\
\hline 0.01 & $9.83(-3)$ & $1.95(-2)$ & $3.86(-2)$ & $4.57(-2)$ & $9.07(-2)$ & $2.14(-1)$ \\
\hline 0.02 & $9.74(-3)$ & $1.93(-2)$ & $3.82(-2)$ & $4.53(-2)$ & $8.97(-2)$ & $2.11(-1)$ \\
\hline 0.05 & $9.44(-3)$ & $1.87(-2)$ & $3.70(-2)$ & $4.39(-2)$ & $8.69(-2)$ & $2.05(-1)$ \\
\hline 0.1 & $8.99(-3)$ & $1.78(-2)$ & $3.52(-2)$ & $4.17(-2)$ & $8.25(-2)$ & $1.94(-1)$ \\
\hline 0.2 & $8.13(-3)$ & $1.61(-2)$ & $3.18(-2)$ & $3.75(-2)$ & $7.43(-2)$ & $1.74(-1)$ \\
\hline 0.5 & $6.02(-3)$ & $1.19(-2)$ & $2.35(-2)$ & $2.75(-2)$ & $5.44(-2)$ & $1.26(-1)$ \\
\hline 1.0 & $3.64(-3)$ & $7.18(-3)$ & $1.42(-2)$ & $1.64(-2)$ & $3.23(-2)$ & $7.38(-2)$ \\
\hline 2.0 & $1.34(-3)$ & $2.62(-3)$ & $5.14(-3)$ & $5.79(-3)$ & $1.14(-2)$ & $2.53(-2)$ \\
\hline 5.0 & $6.60(-5)$ & $1.28(-4)$ & $2.47(-4)$ & $2.60(-4)$ & $5.04(-4)$ & $1.04(-3)$ \\
\hline \multirow[t]{2}{*}{10.0} & $4.37(-7)$ & $8.26(-7)$ & $1.54(-6)$ & $1.50(-6)$ & $2.83(-6)$ & $5.15(-6)$ \\
\hline & $b=1.0$ & $b=1.0$ & $b=1.0$ & $b=1.0$ & $b=2.0$ & $b=2.0$ \\
\hline$\mu t$ & $a=0.1$ & $a=0.2$ & $a=0.5$ & $a=1.0$ & $a=0.1$ & $a=0.2$ \\
\hline 0 & $7.83(-2)$ & 1.5 & 3.69( & 6.4 & 1) & $-1)$ \\
\hline 0.01 & 7.7 & $-1)$ & $3.65(-1)$ & $6.32(-1)$ & $1.09(-1)$ & $2.17(-1)$ \\
\hline 0.02 & $7.66(-2)$ & $1.52(-1)$ & $3.60(-1)$ & $6.24(-1)$ & $1.08(-1)$ & $2.14(-1)$ \\
\hline 0.05 & $7.40(-2)$ & $1.47(-1)$ & $3.48(-1)$ & $6.01(-1)$ & $1.04(-1)$ & $2.06(-1)$ \\
\hline 0.1 & $7.00(-2)$ & $1.39(-1)$ & $3.29(-1)$ & $5.65(-1)$ & $9.70(-2)$ & $1.93(-1)$ \\
\hline 0.2 & $6.26(-2)$ & $1.24(-1)$ & $2.93(-1)$ & $4.99(-1)$ & $8.53(-2)$ & $1.69(-1)$ \\
\hline 0.5 & $4.47(-2)$ & $8.85(-2)$ & $2.07(-1)$ & $3.45(-1)$ & $5.82(-2)$ & $1.15(-1)$ \\
\hline 1.0 & $2.56(-2)$ & $5.06(-2)$ & $1.17(-1)$ & $1.87(-1)$ & $3.14(-2)$ & $6.20(-2)$ \\
\hline 2.0 & $8.49(-3)$ & $1.67(-2)$ & $3.74(-2)$ & $5.61(-2)$ & $9.57(-3)$ & $1.88(-2)$ \\
\hline 5.0 & $3.85(-4)$ & $7.55(-4)$ & $1.77(-3)$ & $7.99(-3)$ & $3.37(-4)$ & $6.54(-4)$ \\
\hline \multirow[t]{2}{*}{10.0} & $1.67(-6)$ & $3.16(-6)$ & $5.77(-6)$ & $6.49(-6)$ & $1.67(-6)$ & $3.16(-6)$ \\
\hline & $b=2$ & $b=2.0$ & $b=2.0$ & & & \\
\hline$\mu t$ & $a=0.5$ & & $a=2.0$ & $a=0.1$ & $a=0.2$ & $a=0.5$ \\
\hline 0 & & & 41 & 1) & 1) & \\
\hline 0.01 & $5.18(-1)$ & $9.18(-1)$ & $1.38(0)$ & $1.35(-1)$ & $2.68(-1)$ & $6.46(-1)$ \\
\hline 0.02 & $5.11(-1)$ & $9.05(-1)$ & $1.36(0)$ & $1.33(-1)$ & $2.64(-1)$ & $6.35(-1)$ \\
\hline 0.05 & $4.91(-1)$ & $8.67(-1)$ & $1.30(0)$ & $1.26(-1)$ & $2.51(-1)$ & $6.03(-1)$ \\
\hline 0.1 & $4.60(-1)$ & $8.07(-1)$ & $1.20(0)$ & $1.16(-1)$ & $2.31(-1)$ & $5.55(-1)$ \\
\hline 0.2 & $4.03(-1)$ & $7.01(-1)$ & $1.02(0)$ & $9.93(-2)$ & $1.97(-1)$ & $4.72(-1)$ \\
\hline 0.5 & $2.72(-1)$ & $4.62(-1)$ & $6.38(-1)$ & $6.38(-2)$ & $1.27(-1)$ & $3.00(-1)$ \\
\hline 1.0 & $1.44(-1)$ & $2.35(-1)$ & $3.02(-1)$ & $3.27(-2)$ & $6.46(-2)$ & $1.50(-1)$ \\
\hline 2.0 & $4.24(-2)$ & $6.43(-2)$ & $7.46(-2)$ & $9.69(-3)$ & $1.90(-2)$ & $4.28(-2)$ \\
\hline 5.0 & $1.32(-3)$ & $1.75(-3)$ & $1.80(-3)$ & $3.37(-4)$ & $6.54(-4)$ & $1.35(-3)$ \\
\hline 10.0 & $5.79(-6)$ & $6.51(-6)$ & $6.52(-6)$ & $1.67(-6)$ & $3.16(-6)$ & $5.79(-6)$ \\
\hline
\end{tabular}

(Table continues on next page.) 
Table H.3-(Continued)

\begin{tabular}{|c|c|c|c|c|c|c|}
\hline \multirow[b]{3}{*}{$\mu t$} & \multicolumn{6}{|c|}{$4 \pi \Gamma /[S G(E)]$} \\
\hline & $b=5.0$ & $b=5.0$ & $b=5.0$ & $b=10.0$ & $b=10.0$ & $b=10.0$ \\
\hline & $a=1.0$ & $a=2.0$ & $a=5.0$ & $a=0.1$ & $a=0.2$ & $a=0.5$ \\
\hline 0 & $1.19(0)$ & $1.88(0)$ & $2.73(0)$ & $1.47(-1)$ & $2.92(-1)$ & $7.06(-1)$ \\
\hline 0.01 & $1.17(0)$ & $1.84(0)$ & $2.65(0)$ & $1.44(-1)$ & $2.86(-1)$ & $6.92(-1)$ \\
\hline 0.02 & $1.15(0)$ & $1.80(0)$ & $2.58(0)$ & $1.41(-1)$ & $2.81(-1)$ & $6.77(-1)$ \\
\hline 0.05 & $1.08(0)$ & $1.69(0)$ & $2.39(0)$ & $1.33(-1)$ & $2.65(-1)$ & $6.38(-1)$ \\
\hline 0.1 & $9.92(-1)$ & $1.53(0)$ & $2.10(0)$ & $1.21(-1)$ & $2.41(-1)$ & $5.79(-1)$ \\
\hline 0.2 & $8.35(-1)$ & $1.26(0)$ & $1.64(0)$ & $1.02(-1)$ & $2.02(-1)$ & $4.84(-1)$ \\
\hline 0.5 & $5.14(-1)$ & $7.27(-1)$ & $8.53(-1)$ & $6.42(-2)$ & $1.27(-1)$ & $3.01(-1)$ \\
\hline 1.0 & $2.47(-1)$ & $3.20(-1)$ & $3.44(-1)$ & $3.27(-2)$ & $6.47(-2)$ & $1.50(-1)$ \\
\hline 2.0 & $6.51(-2)$ & $7.57(-2)$ & $7.69(-2)$ & $9.69(-3)$ & $1.90(-2)$ & $4.28(-2)$ \\
\hline 5.0 & $1.75(-3)$ & $1.80(-3)$ & $1.80(-3)$ & $3.37(-4)$ & $6.54(-4)$ & $1.35(-3)$ \\
\hline \multirow[t]{2}{*}{10.0} & $6.51(-6)$ & $6.53(-6)$ & $6.54(-6)$ & $1.67(-6)$ & $3.16(-6)$ & $5.79(-6)$ \\
\hline & $b=10.0$ & $b=10.0$ & $b=10.0$ & $b=10.0$ & $b=20.0$ & $b=20.0$ \\
\hline$\mu t$ & $a=1.0$ & $a=2.0$ & $a=\mathbf{5 . 0}$ & $a=10.0$ & $a=0.1$ & $a=0.2$ \\
\hline 0 & $1.28(0)$ & $2.07(0)$ & $3.15(0)$ & $3.80(0)$ & $1.52(-1)$ & $3.02(-1)$ \\
\hline 0.01 & $1.26(0)$ & $2.02(0)$ & $3.04(0)$ & $3.64(0)$ & $1.48(-1)$ & $2.95(-1)$ \\
\hline 0.02 & $1.23(0)$ & $1.97(0)$ & $2.94(0)$ & $3.49(0)$ & $1.45(-1)$ & $2.88(-1)$ \\
\hline 0.05 & $1.15(0)$ & $1.83(0)$ & $2.67(0)$ & $3.10(0)$ & $1.36(-1)$ & $2.70(-1)$ \\
\hline 0.1 & $1.04(0)$ & $1.63(0)$ & $2.30(0)$ & $2.58(0)$ & $1.22(-1)$ & $2.43(-1)$ \\
\hline 0.2 & $8.60(-1)$ & $1.31(0)$ & $1.74(0)$ & $1.86(0)$ & $1.02(-1)$ & $2.03(-1)$ \\
\hline 0.5 & $5.18(-1)$ & $7.34(-1)$ & $8.65(-1)$ & $8.78(-1)$ & $6.39(-2)$ & $1.27(-1)$ \\
\hline 1.0 & $2.47(-1)$ & $3.21(-1)$ & $3.44(-1)$ & $3.45(-1)$ & $3.27(-2)$ & $6.47(-2)$ \\
\hline 2.0 & $6.51(-2)$ & $7.56(-2)$ & $7.69(-2)$ & $7.68(-2)$ & $9.69(-3)$ & $1.90(-2)$ \\
\hline 5.0 & $1.75(-3)$ & $1.80(-3)$ & $1.80(-3)$ & $1.80(-3)$ & $3.37(-4)$ & $6.54(-4)$ \\
\hline \multirow[t]{2}{*}{10.0} & $6.51(-6)$ & $6.53(-6)$ & $6.53(-6)$ & $6.53(-6)$ & $1.67(-6)$ & $3.16(-6)$ \\
\hline & $b=20.0$ & $b=20.0$ & $b=20.0$ & $b=20.0$ & $b=20.0$ & $b=20.0$ \\
\hline$\mu t$ & $a=0.5$ & $a=1.0$ & $a=2.0$ & $a=5.0$ & $a=10.0$ & $a=20.0$ \\
\hline 0 & $7.31(-1)$ & $1.33(0)$ & $2.17(0)$ & $3.38(0)$ & $4.22(0)$ & $4.88(0)$ \\
\hline 0.01 & $7.13(-1)$ & $1.30(0)$ & $2.10(0)$ & $3.25(0)$ & $4.01(0)$ & $4.56(0)$ \\
\hline 0.02 & $6.96(-1)$ & $1.27(0)$ & $2.04(0)$ & $3.12(0)$ & $3.81(0)$ & $4.28(0)$ \\
\hline 0.05 & $6.50(-1)$ & $1.18(0)$ & $1.88(0)$ & $2.79(0)$ & $3.30(0)$ & $3.59(0)$ \\
\hline 0.1 & $5.86(-1)$ & $1.05(0)$ & $1.65(0)$ & $2.36(0)$ & $2.68(0)$ & $2.81(0)$ \\
\hline 0.2 & $4.86(-1)$ & $8.63(-1)$ & $1.31(0)$ & $1.75(0)$ & $1.89(0)$ & $1.92(0)$ \\
\hline 0.5 & $3.01(-1)$ & $5.18(-1)$ & $7.34(0)$ & $8.63(-1)$ & $8.79(-1)$ & $8.82(-1)$ \\
\hline 1.0 & $1.50(-1)$ & $2.47(-1)$ & $3.21(0)$ & $3.44(-1)$ & $3.45(-1)$ & $3.45(-1)$ \\
\hline 2.0 & $4.28(-2)$ & $6.51(-2)$ & $7.56(-2)$ & $7.69(-2)$ & $7.68(-2)$ & $7.68(-2)$ \\
\hline 5.0 & $1.35(-3)$ & $1.75(-3)$ & $1.80(-3)$ & $1.80(-3)$ & $1.80(-3)$ & $1.80(-3)$ \\
\hline 10.0 & $5.79(-6)$ & $6.51(-6)$ & $6.53(-6)$ & $6.53(-6)$ & $6.53(-6)$ & $6.53(-6)$ \\
\hline
\end{tabular}


Table H.4 - UNSCATTERED FLUX AT A CORNER POSITION FROM A RECTANGULAR PLANE SOURCE WITH A COSINE ANGULAR DISTRIBUTION (ISOTROPIC FLUX) $†$

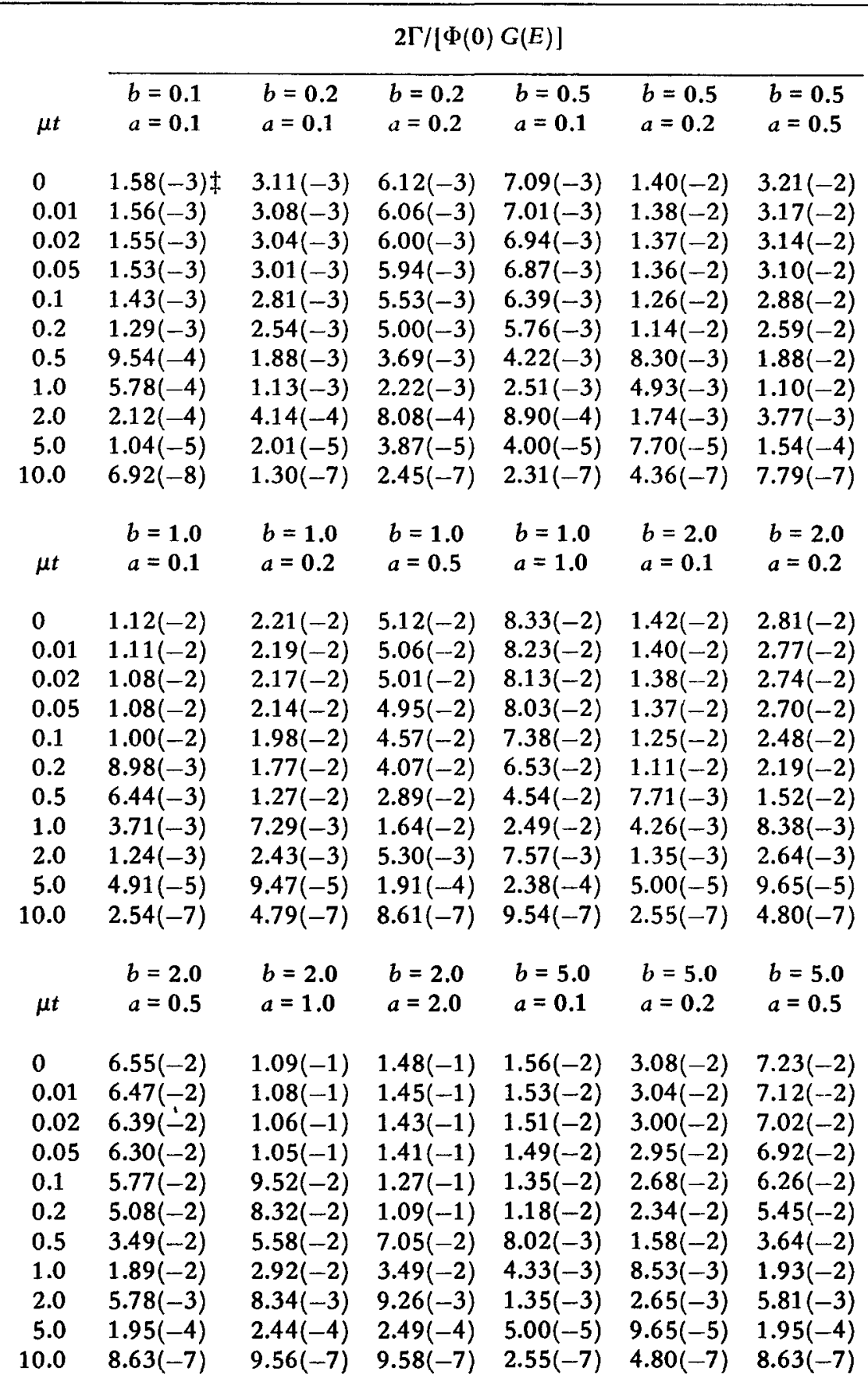

(Table continues on next page.) 
Table H.4-(Continued)

\begin{tabular}{|c|c|c|c|c|c|c|}
\hline \multirow[b]{2}{*}{$\mu t$} & \multicolumn{6}{|c|}{$2 \Gamma /[\Phi(0) G(E)]$} \\
\hline & $\begin{array}{l}b=5.0 \\
a=1.0\end{array}$ & $\begin{array}{l}b=5.0 \\
a=2.0\end{array}$ & $\begin{array}{l}b=5.0 \\
a=5.0\end{array}$ & $\begin{array}{c}b=10.0 \\
a=0.1\end{array}$ & $\begin{array}{c}b=10.0 \\
a=0.2\end{array}$ & $\begin{aligned} b & =10.0 \\
a & =0.5\end{aligned}$ \\
\hline 0 & $1.22(-1)$ & $1.70(-1)$ & $2.06(-1)$ & $1.58(-2)$ & $3.13(-2)$ & $7.34(-2)$ \\
\hline 0.01 & $1.20(-1)$ & $1.67(-1)$ & $2.01(-1)$ & $1.56(-2)$ & $3.08(-2)$ & $7.23(-2)$ \\
\hline 0.02 & $1.18(-1)$ & $1.64(-1)$ & $1.97(-1)$ & $1.58(-2)$ & $3.04(-2)$ & $7.12(-2)$ \\
\hline 0.05 & $1.16(-1)$ & $1.62(-1)$ & $1.93(-1)$ & $1.51(-2)$ & $2.99(-2)$ & $7.01(-2)$ \\
\hline 0.1 & $1.05(-1)$ & $1.43(-1)$ & $1.68(-1)$ & $1.37(-2)$ & $2.70(-2)$ & $6.32(-2)$ \\
\hline 0.2 & $9.02(-2)$ & $1.21(-1)$ & $1.38(-1)$ & $1.19(-2)$ & $2.35(-2)$ & $5.48(-2)$ \\
\hline 0.5 & $5.87(-2)$ & $7.51(-2)$ & $8.10(-2)$ & $8.02(-3)$ & $1.58(-2)$ & $3.65(-2)$ \\
\hline 1.0 & $2.99(-2)$ & $3.59(-2)$ & $3.71(-2)$ & $4.33(-3)$ & $8.53(-3)$ & $1.93(-2)$ \\
\hline 2.0 & $8.38(-3)$ & $9.32(-3)$ & $9.38(-3)$ & $1.35(-3)$ & $2.65(-3)$ & $5.81(-3)$ \\
\hline 5.0 & $2.44(-4)$ & $2.49(-4)$ & $2.49(-4)$ & $5.00(-5)$ & $9.65(-5)$ & $1.95(-4)$ \\
\hline \multirow[t]{2}{*}{10.0} & $9.56(-7)$ & $9.58(-7)$ & $9.58(-7)$ & $2.55(-7)$ & $4.80(-7)$ & $8.63(-7)$ \\
\hline & $\begin{array}{c}b=10.0 \\
a=1.0\end{array}$ & $\begin{array}{c}b=10.0 \\
a=2.0\end{array}$ & $\begin{array}{c}b=10.0 \\
a=5.0\end{array}$ & $\begin{array}{l}b=10.0 \\
a=10.0\end{array}$ & $\begin{array}{c}b=20.0 \\
a=0.1\end{array}$ & $\begin{array}{c}b=20.0 \\
a=0.2\end{array}$ \\
\hline 0 & $1.24(-1)$ & $1.75(-1)$ & $2.15(-1)$ & $2.28(-1)$ & $1.58(-2)$ & $3.14(-2)$ \\
\hline 0.01 & $1.22(-1)$ & $1.71(-1)$ & $2.10(-1)$ & $2.22(-1)$ & $1.56(-2)$ & $3.09(-2)$ \\
\hline 0.02 & $1.20(-1)$ & $1.68(-1)$ & $2.05(-1)$ & $2.16(-1)$ & $1.54(-2)$ & $3.04(-2)$ \\
\hline 0.05 & $1.18(-1)$ & $1.65(-1)$ & $2.01(-1)$ & $2.11(-1)$ & $1.51(-2)$ & $3.00(-2)$ \\
\hline 0.1 & $1.06(-1)$ & $1.46(-1)$ & $1.72(-1)$ & $1.78(-1)$ & $1.37(-2)$ & $2.71(-2)$ \\
\hline 0.2 & $9.08(-2)$ & $1.22(-1)$ & $1.40(-1)$ & $1.43(-1)$ & $1.19(-2)$ & $2.35(-2)$ \\
\hline 0.5 & $5.88(-2)$ & $7.53(-2)$ & $8.13(-2)$ & $8.17(-2)$ & $8.02(-3)$ & $1.58(-2)$ \\
\hline 1.0 & $2.99(-2)$ & $3.59(-2)$ & $3.71(-2)$ & $3.71(-2)$ & $4.33(-3)$ & $8.53(-3)$ \\
\hline 2.0 & $8.38(-3)$ & $9.32(-3)$ & $9.38(-3)$ & $9.38(-3)$ & $1.35(-3)$ & $2.65(-3)$ \\
\hline 5.0 & $2.44(-4)$ & $2.49(-4)$ & $2.49(-4)$ & $2.49(-4)$ & $5.00(-5)$ & $9.65(-5)$ \\
\hline \multirow[t]{2}{*}{10.0} & $9.56(-7)$ & $9.58(-7)$ & $9.58(-7)$ & $9.58(-7)$ & $2.55(-7)$ & $4.81(-7)$ \\
\hline & $b=20.0$ & $b=20.0$ & $b=20.0$ & $b=20.0$ & $b=20.0$ & $b=20.0$ \\
\hline$\mu t$ & $a=0.5$ & $a=1.0$ & $a=2.0$ & $a=5.0$ & $a=10.0$ & $a=20.0$ \\
\hline 0 & $7.37(-2)$ & $1.25(-1)$ & $1.76(-1)$ & $2.18(-1)$ & $2.32(-1)$ & $2.39(-1)$ \\
\hline 0.01 & $7.26(-2)$ & $1.23(-1)$ & $1.72(-1)$ & $2.12(-1)$ & $2.26(-1)$ & $2.31(-1)$ \\
\hline 0.02 & $7.14(-2)$ & $1.21(-1)$ & $1.69(-1)$ & $2.07(-1)$ & $2.20(-1)$ & $2.24(-1)$ \\
\hline 0.05 & $7.03(-2)$ & $1.19(-1)$ & $1.66(-1)$ & $2.02(-1)$ & $2.14(-1)$ & $2.18(-1)$ \\
\hline 0.1 & $6.33(-2)$ & $1.06(-1)$ & $1.46(-1)$ & $1.73(-1)$ & $1.79(-1)$ & $1.80(-1)$ \\
\hline 0.2 & $5.48(-2)$ & $9.08(-2)$ & $1.22(-1)$ & $1.40(-1)$ & $1.43(-1)$ & $1.44(-1)$ \\
\hline 0.5 & $3.65(-2)$ & $5.88(-2)$ & $7.53(-2)$ & $8.13(-2)$ & $8.17(-2)$ & $8.17(-2)$ \\
\hline 1.0 & $1.93(-2)$ & $2.99(-2)$ & $3.59(-2)$ & $3.71(-2)$ & $3.71(-2)$ & $3.71(-2)$ \\
\hline 2.0 & $5.81(-3)$ & $8.38(-3)$ & $9.32(-3)$ & $9.38(-3)$ & $9.38(-3)$ & $9.38(-3)$ \\
\hline 5.0 & $1.95(-4)$ & $2.44(-4)$ & $2.49(-4)$ & $2.49(-4)$ & $2.49(-4)$ & $2.49(-4)$ \\
\hline 10.0 & $8.63(-7)$ & $9.56(-7)$ & $9.58(-7)$ & $9.58(-7)$ & $9.58(-7)$ & $9.58(-7)$ \\
\hline
\end{tabular}

†From Trubey. ${ }^{2}$

$\ddagger$ Read: $1.58 \times 10^{-3}$, etc. 


\section{Random-Number Generators}

\section{Appendix I}

The use of Monte Carlo methods in any calculation depends on a copious supply of random numbers. Many early calculations relied heavily on a table $\dagger$ of a million random digits generated electronically. Whether telephone directories were ever actually tried is not known; however, their use was frequently postulated (too much bias for some digits). The advent of the digital computer made a mathematical algorithm highly desirable since such a function has the property of generating a reproducible sequence provided one starts with the same initial numbers and provided the same computer system is involved. The reproducible sequence is valuable in checking problem results to ascertain that the nonrandom aspects of a program are running properly. The necessity of limiting reproducibility to a given computer system stems from the dependence of the generator on the number of bits per word and on round-off methods. Both these characteristics vary widely with computer type. Computer manufacturers typically supply a random-number-generator routine especially suited to their machines. A characteristic of many of these routines is that they generate the same sequence for the same initial random number. It is thus advantageous if the user is allowed to supply his own initial number so that he can repeat a sequence or generate a new sequence at his option.

Number sequences produced by a mathematical relation are clearly deterministic, not random, and are thus properly called pseudorandom. To avoid semantic difficulties as well as logical contradictions, we acknowledge that the number sequences we seek actually must have special properties that further distinguish them from a random set. In the subsequent discussion, unless otherwise stated, we use random to mean adequate for Monte Carlo application. A classic paper by Hull and Dobell' ${ }^{1}$ serves as an excellent introduction to the problem of devising random-number generators.

†Rand Corporation, A Million Random Digits with 100,000 Normal Deviates, Free Press of Glencoe, New York, N. Y., 1955. 


\section{I.1 PROPERTIES OF RANDOM-NUMBER GENERATORS (RNG's)}

The RNG with which we are concerned is a computer subroutine that generates one random number each time it is called. It thus generates a sequence of $N$ terms

$$
r_{1}, r_{2}, \ldots, r_{n}, \ldots, r_{N}
$$

and it is the sequence of these $N$ terms that must display the properties of randomness. The two basic properties, although conceptually simple, are far from trivial.

1. Equidistribution: The terms in the sequence should be uniformly distributed over the unit interval. Their frequency distribution should be characterized by a horizontal plot as shown in Fig. I.1.

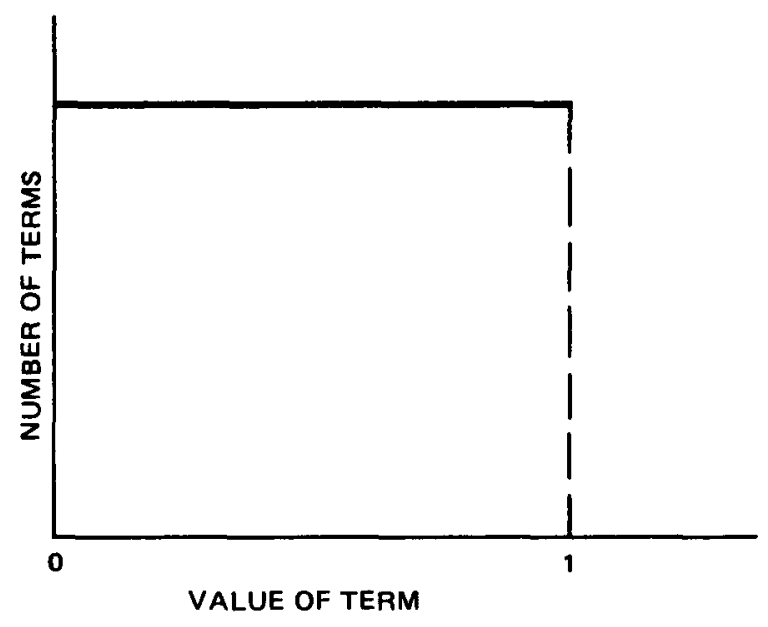

Fig. 1.1-Frequency distribution.

Further, large enough sub-sequences

$$
r_{j}, r_{j+1}, \ldots, r_{j+k}
$$

imbedded in the full sequence should also be equidistributed. For example, if a random-number generator can generate a sequence of a million terms, any sub-sequence of 10,000 terms should also display equidistribution.

2. Independence: It is not enough that the sequence be equidistributed. The sequence $0,0.1,0.2, \ldots, 0.9,1.0,0,0.1,0.2, \ldots, 0.9,1.0,0,0.1, \ldots$ is equidistributed over $[0,1]$ but certainly is not random. Thus each term of 
the sequence should be independent of any other; i.e., each number in $[0,1]$ should have equal probability of being generated each time the RNG is called. Intuitively, patterns in the sequence should not be discernible.

Verifying that an RNG produces sequences satisfying both of these properties is an unsolved problem. Many necessary conditions can be defined, but the key theorems, those involving sufficiency, are yet to be stated and proved.

Testing a generated sequence for equidistribution is simple, but being assured that the next sequence to be generated will also be equidistributed is a far greater challenge. In fact, some workers recommend that the RNG sequence generated in a Monte Carlo calculation be analyzed during the calculation so that if bad luck produced a pathological t sequence, the results could be discarded. This is not a common inclusion in current Monte Carlo codes, however.

Complete testing for independence is impossible, but techniques have been developed which look for the appearance of independence. They usually involve attempts to find a relation (called serial correlation) between the $n$th term and the immediately preceding (e.g., 10) terms for all values of $n$. Testing methods are discussed in the following sections.

\section{I.2 RECURSION EQUATIONS FOR RNG's}

A recursion relation is a function that relates the $n$th term of a sequence to previous terms. Although some RNG's have used algorithms that used more than the immediately preceding term to generate the $n$th term, the common methods compute $r_{n}$ as a function only of $r_{n-1}$ and preselected constants. Since a large quantity (on the order of 10,000 to 100,000 ) of random numbers are used in a typical Monte Carlo calculation, speed of generation is important. Thus the recursion relations used are designed to minimize computing time while retaining sufficient randomness to be of practical use.

Almost all RNG's used in shielding codes are designed for fixed-wordlength machines. The theory and the tests must be modified to account for a binary or a decimal machine as well as for the bit (or digit) length of the computer word. For illustrative purposes we will assume a 36-bit binary machine in this discussion.

tDefined later in this section. 
There are only $2^{36}$ numbers representable in a 36 -bit word computer (actually $2^{35}$ if one bit is reserved for a sign). An RNG in such a machine can thus only generate at most $2^{36}$ rational numbers. Rational numbers requiring more than 36 bits (integers and fractions) as well as the uncountably infinite number of irrational numbers cannot be generated. Thus, although not critical $\left(2^{36}\right.$ fractions would seem to cover the unit interval quite well), the theoretical properties of equidistribution and independence are irrevocably compromised in the computer approximation.

Most RNG's in common use generate sequences of positive random integers, $x_{1}, x_{2}, \ldots, x_{i}$. The desired random fractions $\left(0 \leqslant r_{i}<1\right)$ are obtained by the relation

$$
r_{i}=x_{i} / M
$$

where the integers $x_{i}$ are uniformly distributed over $[0, M]$. In a binary computer, if $k$ bits are allotted to represent the magnitude of the number (i.e., not including the sign bit, if any), $M=2^{k}$ and the $x_{i}$ values can range from 0 to $2^{k}-1$.

Many recursion schemes have been used to generate sequences of pseudorandom integers. Recent work has shown that some of the commonly used generators are inadequate.

Generators that have received the most attention are of the congruential type. Three are discussed in the following paragraphs, the first for historical reasons and the last two because of their widespread use.

1. Middle-digit square. The first RNG put into common use was that of von Neumann ${ }^{2}$ and Metropolis. ${ }^{3}$ In this method an arbitrary first term with a length of $k$ digits is chosen and squared to obtain a $2 k$-digit number. The middle $k$ digits of this intermediate result are extracted to obtain the next random number. The sequence is generated by repeating this process.

This generator appeared adequate and was used for many years. However, when examined more carefully by newer testing methods, it proved to be inadequate and is not in widespread use today.

2. Mixed congruential. This generator requires two constants as well as an initial term of the sequence to be chosen. Its recursion relation is of the form $\dagger$

$$
x_{i} \equiv \lambda x_{i-1}+c(\bmod M) \quad(\text { for } i=1,2, \ldots)
$$

†The expression $A \equiv B(\bmod M)$ is read: $A$ is congruent to $B$ modulo $M$ and means $A$ is the remainder of $B / M$. 
in which $\lambda$ and $c$ are the two constants and $M=2^{k}$ (e.g., $2^{36}$ ), where $k$ is the bit length of the computer word being used.

3. Multiplicative congruential. This is a special case of the mixed generator in which $c=0$. It thus has the advantage of greater speed since an addition step is eliminated. Its recursion relation is

$$
x_{i} \equiv \lambda x_{i-1}(\bmod M) \quad(\text { for } i=1,2, \ldots)
$$

These latter two generators have been extensively studied to determine if one is clearly better than the other. Also, the effect of the constants $\lambda$ and $c$ and the starting value $x_{1}$ on the properties of randomness have been investigated. The complexity of the problem is illustrated by the fact that Hull and Dobell ${ }^{4}$ recommended the mixed method as the best choice in 1964; this was followed by the Fourier analysis by Coveyou and MacPherson, ${ }^{5}$ which resulted in the opposite recommendations in 1967.

The constants $\lambda$ and $c$ are usually fixed when the subroutine is written. The first term, $x_{1}$, is provided as an input variable. Each has an effect on the properties of the RNG. For example, if either $\lambda$ or $x_{1}$ is even, every term in a multiplicative sequence will be even, which will halve the period of the generator. The period (or cycle length), $P$, is the number of terms that will be generated before the sequence repeats itself. In a binary machine with an effective word length of $k$ bits (sign bit not included), $P \leqslant 2^{k}$.

In binary computers $\lambda$ is often chosen to be of the form

$$
\lambda=2^{p}+b(p \text { and } b \text { are small integers })
$$

since the multiplication $\lambda x_{i-1}$ can then be accomplished by the fast shift-and-add operations in the computer. However, for small choices of $p$ (e.g., $p \leqslant 8$ ) where the speed advantage is greatest, the resulting generator was found to be generally inadequate.

Coveyou and MacPherson ${ }^{5}$ examined 32 generators of the mixed and multiplicative type: 7 for 36-bit binary machines; 8 for 48-bit binary machines; 7 for 32-bit binary machines; and 10 for decimal machines. They varied values of $\lambda$ and $c$ and used a Fourier analysis technique to examine the distribution properties of one-to-ten term sub-sequences. Their conclusions are of interest:

1. There is at present no method of generation of pseudo-uniform sequences better than the simple multiplicative congruence method with a carefully chosen multiplier $(\lambda)$. 
2. The multiplier $\lambda$ should not be close to a simple rational multiple of $\mathrm{M}$; if it is, the basic congruence shows that appreciable serial correlation will result.

3. The multiplier $\lambda$ should not be close to a simple rational multiple of the square root of $M$; even though this choice may produce very small correlation between adjacent pairs (adjacent terms in the sequence), serious difficulties result in the triplet distribution.

4. The multiplier $\lambda$ should not be chosen with a small number of ones in the binary representation (e.g., $2^{7}$ ) to facilitate shift-and-add techniques.

5. Above all, the multiplier $\lambda$ must be adequately large (e.g., $5^{15}$ ).

6. The choice of multiplier must be made most carefully for 32-bit word multiplication, ... for (36-bit machines) the choice still needs some care. For (48-bit) and . . 64-bit multiplication ..., a suitable choice should be easy.

These conclusions are reinforced by the work of Van Gelder, ${ }^{6}$ who advises that the bit pattern for the chosen $\lambda$ should not contain long strings of zeros. He further states: “. . . there is no hope for multipliers below $10^{3}$, and it is best to stay above $10^{4}$. For modulus $2^{35}$, the best range (for $\lambda$ ) seems to be about $2^{12}$ to $2^{13}$, with at least one digit to break up the string of zeros."

It is desirable to maximize the period $P$ of the generator. Barnett ${ }^{7}$ gives necessary and sufficient conditions on $\lambda$ and $x_{1}$ to maximize the period of a multiplicative generator on a binary machine. They are, where $x_{i} \equiv \lambda x_{i-1}$ $(\bmod M)$,

$$
\begin{aligned}
x_{1} & = \pm(\bmod 8) \\
1<\lambda & =1(\bmod 2)
\end{aligned}
$$

i.e., $\lambda$ must be greater than 1 and odd. The maximum period obtainable on a computer with a word length of $k$ bits is $2^{k-2}$ numbers where $M=2^{k}$. The period $P=(1 / 4) M$, although the maximum value attainable by $x_{i}$, is still $M-1$. For mixed generators the period can be made to equal $M$. However, this advantage is offset by the reduced computing speed of the mixed method.

\subsection{TESTING RNG's}

Several classes of tests are applied to RNG's. Although not inclusive, the following methods are illustrative of those in use: 


\subsubsection{Global Tests}

Global tests are the simple tests that can be applied to large sub-sequences of the generator. They make use of the following types of relations:

$$
\begin{aligned}
& \lim _{n \rightarrow \infty} \frac{1}{n-k+1} \sum_{i=k}^{n} r_{i}=\frac{1}{2} \quad(k=1,2, \ldots) \\
& \lim _{n \rightarrow \infty} \frac{1}{n-k+1} \sum_{i=k}^{n} r_{i}^{2}=\frac{1}{3} \quad(k=1,2, \ldots) \\
& \lim _{n \rightarrow \infty} \frac{1}{n-k} \sum_{i=k}^{n}\left(r_{i}-\frac{1}{2}\right)^{2}=\frac{1}{12} \quad(k=1,2, \ldots)
\end{aligned}
$$

These three limits state that the average of any $(n-k+1)$ term $t$ of a random sub-sequence in the unit interval should approximate $1 / 2$; the average of the squares, $1 / 3$; and the variance, $1 / 12$. Observe that thesc tests all are necessary conditions for equidistributions. They tell nothing about independence.

However, Coveyou ${ }^{8}$ suggests one such global test for pair-wise correlation:

$$
\lim _{n \rightarrow \infty} \frac{1}{n-k+1} \sum_{i=k}^{n} r_{i} r_{i+1}=\frac{1}{4}+\frac{1}{12 \lambda} \quad(i=1,2, \ldots)
$$

\section{I.3.2 Equidistribution}

The simplest test of equidistribution is to partition the unit interval into small subintervals of equal width (e.g., 0.001) and count the number of terms of any sub-sequence that fall in each subinterval. The frequency function thus obtained should approximate a constant, namely, $N / I$, where $N$ is the number of terms in the sub-sequence and $I$ is the number of subintervals chosen.

More sophisticated methods of analysis are available. Coveyou and MacPherson ${ }^{5}$ start with the necessary and sufficient condition for equidistribution

†Note that the choice of $k(k=1,2, \ldots)$ determines the selection of any sub-sequence within the period of the generator. 


$$
\lim _{n \rightarrow \infty} \frac{k}{n} \sum_{j=1}^{n} e^{2 \pi i k r} j=0
$$

for all $k=1,2, \ldots$, and develop a Fourier analysis that generates figures of merit for the uniformity of distribution of $n$-tuples of consecutive terms in the generated sequence. Their analysis covered $n$-tuples from 1 to 10 terms in length.

\subsubsection{Independence}

Independence is the hardest property to analyze. No necessary conditions have been established. In fact, since each term is deterministically computed from its predecessor, the terms are clearly dependent. One can only hope that no obvious pattern emerges and that relatively short sub-sequences are equidistributed. A standard method of analysis involves the use of $n$-tuples; i.e., sub-sequences of $n$ terms. We will illustrate the technique by use of an example.

Consider an RNG that generates decimal digits (e.g., $2,8,7,3,3, \ldots$ ). If independence holds, each pair of all possible consecutive terms, e.g., $(8,7)$, should be uniformly distributed within the sequence. When a sequence has been generated, each such pair $\left(x_{i}, x_{i+1}\right)$ for $i=1,2, \ldots$, can be tabulated in the matrix shown in Fig. I.2. Each box contains the number of times the pair appeared in the sequence. Hopefully, each pair occurs with the same frequency as any other pair.

When this type of test is applied to RNG's generating fractions in the unit interval, the partition of the unit interval creating the counting bins can be as small as desired. Also, the dimensionality of the matrix can be extended to handle any number of consecutive terms ( $n$-tuples). We should point out that a special type of equidistribution property is being used, that of equidistribution of $n$-tuples, to examine apparent independence. In fact, the Fourier technique of Coveyou discussed is exactly that. When the $n$-tuple is one term, equidistribution over $(0,1)$ in the sense we have used is tested. For $n$-tuples of two or more terms, apparent independence is the basic consideration. Most RNG's are now tested for at least pair and triplet correlation. In his earlier paper, Coveyou ${ }^{8}$ suggests that one should test for correlations of $n$-tuples up to 10 to 20 terms in length.

All the preceding tests admit to standard statistical analysis. A set of sequences can be generated by a given RNG and each test can be applied. The probability that the results represent random sequences can then be estimated by chi-square tests and computation of correlation coefficients. 


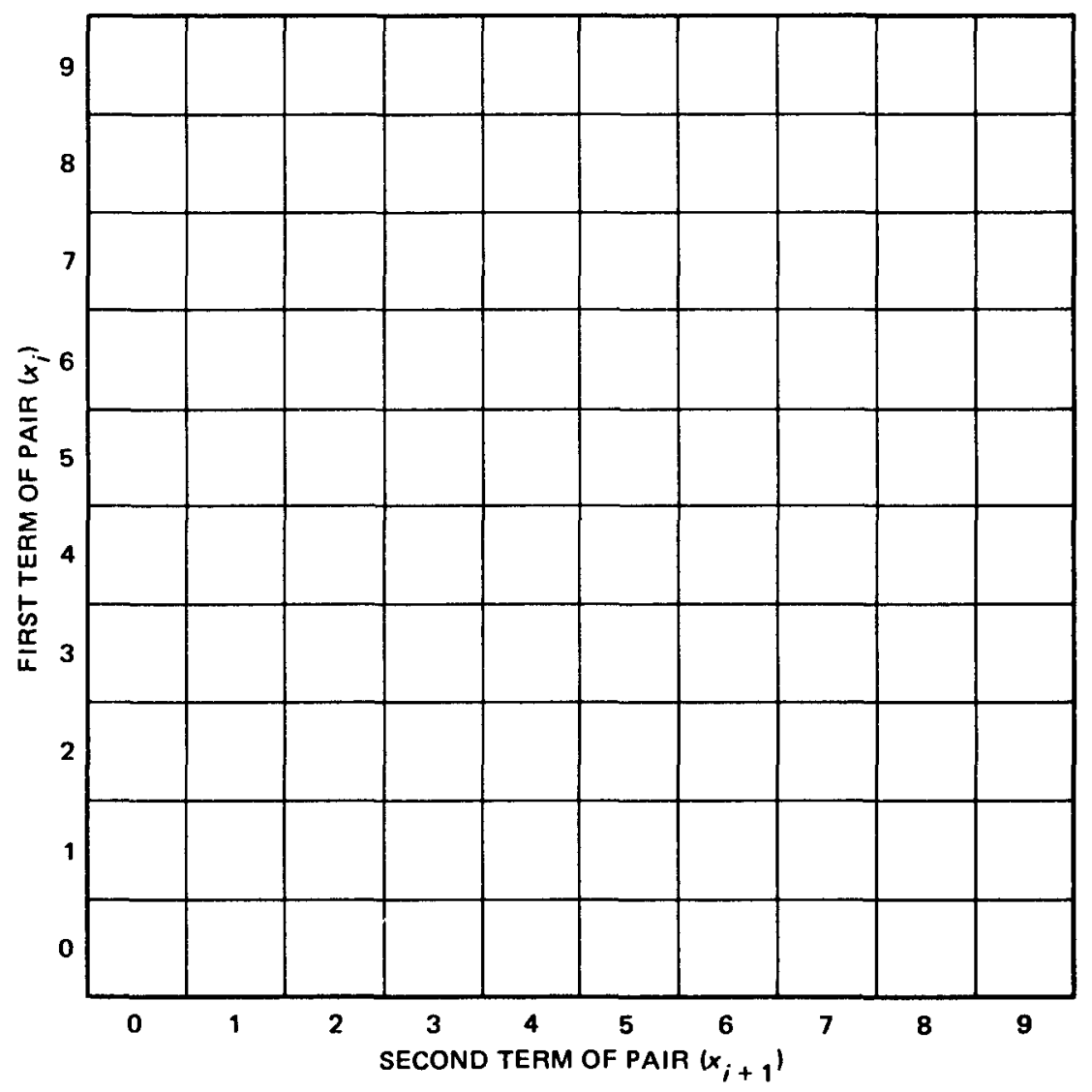

Fig. I.2-Distribution of pairs.

The reader is referred to Refs. 1 and 4 for elementary discussion of such statistical tests.

\section{I.4 PATHOLOGICAL NUMBERS}

In an infinite sequence of random numbers, all the weird sub-sequences that one could imagine should be in evidence. Somewhere the sub-sequence containing 100 consecutive values of 0.1 should appear, long monotonically increasing sub-sequences should be included, etc. The sequence is called pathological when it presents a pattern of this character. Thus, in examining one sequence generated by an RNG, the fact that a clear pattern emerges does not imply necessarily that the RNG is bad. After all, any conceivable sub-sequence has a finite probability. However, in the finite sequences used 
in a Monte Carlo calculation, the appearance of a pathological sub-sequence could spell disaster. Any RNG that exhibits such behavior in any except the rarest of instances should be avoided. In fact, it probably is good that pseudorandom-number generators of the preceding type are sufficiently nonrandom to preclude many of these situations. (The proper choice of $\lambda$ and $x_{1}$ can guarantee that every number in the period appears only once before the period is exhausted, for example.)

\section{I.5 USEFUL RNG's}

The major purpose of this discussion has been to introduce the concepts and hazards inherent in RNG's. Many examples can be found where lengthy and costly Monte Carlo calculations were invalidated because not enough attention was paid to the quality of the RNG. The user of an RNG subroutine should require sufficient evidence of its quality.

With this warning and the material presented here, one should be prepared to examine some cookbook RNG codes that have recently appeared. Marsaglia and $\mathrm{Bray}^{9}$ propose using one- or two-line FORTRAN statements in the body of a program rather than exit to a subroutine. They use the multiplicative recursion relation and give FORTRAN statements for the IBM-7094 and 360 and the UNIVAC 1108 computers. They also discuss the use of two RNG's together, one to generate an ever-changing table of pseudorandom numbers and the other to pick a number from the table at (pseudo) random. Some flaws in their coding were corrected by Grosenbaugh. ${ }^{10}$ Finally, Seraphin ${ }^{1}$ suggests that it is not so bad after all to exit to a subroutine if it is coded in efficient assembly language, and he proposes a multiplicative subroutine for the 32-bit IBM-360.

One point in using the preceding techniques may not be so obvious. The earlier discussion of recursion relations assumed fixed-point (i.e., integer) representations. In fact, most Monte Carlo codes are programmed and implemented with floating-point numbers. Not only are the bits available for integer representation different from the computer word length but also multiplication is handled in a variety of ways. Thus care must be taken when the two-step computation

$$
\begin{aligned}
x_{i} & \equiv \lambda x_{i-1}(\bmod M) \\
r_{i} & =\frac{x_{i}}{M}
\end{aligned}
$$


is coded. The results must be properly floated and normalized for use in the Monte Carlo program.

The last references ${ }^{9-11}$ recommending specific RNG's contain from zero to three short lines discussing the tests to which they have been submitted. It seems that a spirit of caveat emptor exists. Perhaps this is the just due of Monte Carlo practitioners because the father of this subject, John von Neumann, admonished that he who uses arithmetic means to generate random numbers is, of course, in a state of $\sin ^{2}$

\section{REFERENCES}

1. T. E. Hull and A. R. Dobell, SIAM (Soc. Ind. Appl. Math.) Rev., 4: 230-254 (July 1962).

2. J. von Neumann, Various Techniques Used in Connection With Random Digits, National Bureau of Standards, Applied Mathematics Series 12, Superintendent of Documents, U. S. Government Printing Office, Washington D. C., 1951.

3. N. Metropolis, Phase Shifts Middle Squares Wave Equation, in Symposium on Monte Carlo Methods, H. A. Meyer (Ed.), John Wiley \& Sons, Inc., New York, 1956.

4. T. E. Hull and A. R. Dobell, Mixed Congruential Random Number Generators for Binary Machines, J. Assn. Comp. Mach., 11: 31-40 (January 1964).

5. R. R. Coveyou and R. D. MacPherson, Fourier Analysis of Uniform Random Number Generators, J. Assn. Comp. Mach., 14: 100 (January 1967).

6. A. Van Gelder, Some New Results in Pseudo-Random Number Generation, J. Assn. Comp. Mach., 14: 785 (October 1967).

7. V. D. Barnett, The Behavior of Pseudo-Random Sequences Generated on Computers by the Multiplicative Congruential Method, Math. Comput., 16: 63 (1962).

8. R. R. Coveyou, Serial Correlation in the Generation of Pseudo-Random Numbers, $J$. Assn. Comp. Mach., 7: 72 (January 1960).

9. G. Marsaglia and T. A. Bray, One-line Random Number Generators and Their Use in Combinations, Commun. ACM (Assn. Comput. Mach.), 11: 757 (November 1968).

10. L. R. Grosenbaugh, More on Fortran Random Number Generators, Commun. ACM (Assn. Comput. Mach.), 12: 639 (November 1969).

11. D. S. Seraphin, A Fast Random Number Generator for IBM 360, Commun. ACM (Assn. Comput. Mach.), 12: 695 (December 1969). 


\section{Demonstration Monte Carlo Program}

\section{Appendix J}

A slab penetration calculation is described in Sec. 5.9 as a sample Monte Carlo problem to demonstrate some of the techniques presented in this chapter. This appendix contains the input-data requirements, the logic flow chart of the calculation, the FORTRAN listing of the program, the description of a test problem for program check-out, and a specimen listing of the input and output for the test problem.

We emphasize that the random-number-generator subroutine called RAND is not a random-number generator but a table of random numbers with a stepping procedure to make it possible to run the test problem and to obtain the same results given here to ensure that the program is running properly. Application of the program to realistic calculations will require a bona fide random-number generator. Similarly, the subroutine XSEC is a simplified cross-section interpolation routine. Unlike RAND, however, it may be used for realistic calculations for energy ranges over which the cross sections vary in an approximately linear fashion.

A number of explanatory comments ( $C$ statements) have been included in the program listing which can be eliminated in subsequent copies and thereby reduce the key-punch labor considerably.

For each computer system the card reader is referenced by one particular digit and the printer, by another. The user of this program should change $\mathrm{KX}$ to equal his computer's card-reader reference if other than 1 (line 29 in the listing), and $K Y$ to equal his computer's printer reference if other than 3 (line 30 in the listing). If the available FORTRAN compiler does not accept DATA statements, the user should delete the ninth and tenth lines in subroutine RAND and insert 23 assignment statements (each to a card) for $I R$; that is, $I R=22, I R=16, I R=20$, etc., after the line $100 \mathrm{KNT}=23$. 
INPUT-DATA REQUIREMENTS

\begin{tabular}{|c|c|c|c|}
\hline Variable & Format & Definition & Limit \\
\hline NENEG & & Number of print energy groups & $\leqslant 7$ \\
\hline NANG & & $\begin{array}{l}\text { Number of print angle interval (for each } \\
\text { surface) }\end{array}$ & $\leqslant 6$ \\
\hline NHIST & 515 & Number of histories to be run & \\
\hline NDIVT & & $\begin{array}{l}\text { Number of slab increments for } \\
\text { storing absorptions }\end{array}$ & $\leqslant 20$ \\
\hline IB & & Random-number-generator base & Odd \\
\hline EO & & Source energy (MeV) & \\
\hline TT & & Slab thickness $(\mathrm{cm})$ & \\
\hline $\mathrm{COA}$ & $4 \mathrm{E} 10.3$ & $\begin{array}{l}\text { Small angle below which theta is assumed } \\
\text { to be zero; usually } 10^{-5}\end{array}$ & \\
\hline AW & & Atomic weight (amu) & \\
\hline ANG $(J)$ & $6 \mathrm{E} 10.3$ & $\begin{array}{l}\text { Kth bound of the print angle } \\
\text { intervals; ascending order in } \\
\text { degrees }\end{array}$ & $\begin{array}{l}\text { NANG + } 1 \\
\text { values } \\
<90\end{array}$ \\
\hline $\operatorname{FTD}(\mathrm{I})$ & $6 \mathrm{E} 10.0$ & $\begin{array}{l}\text { Flux density-to-dose conversion } \\
\text { factor for the Ith print } \\
\text { energy group }\end{array}$ & $\begin{array}{r}\text { NENEG } \\
\text { values }\end{array}$ \\
\hline $\mathbf{E}(\mathbf{I})$ & $6 \mathrm{E} 10.0$ & $\begin{array}{l}\text { Energy bound of the } I \text { th print } \\
\text { energy interval; ascending order in } \\
\mathrm{MeV}\end{array}$ & $\begin{array}{l}\text { NENEG + } 1 \\
\text { values }\end{array}$ \\
\hline NCSE & I10 & $\begin{array}{l}\text { Number of energies at which cross } \\
\text { sections are input }\end{array}$ & $\leqslant 40$ \\
\hline $\mathrm{EE}(\mathbf{I})$ & $6 \mathrm{E} 10.3$ & $\begin{array}{l}\text { Ith value of the energy at which } \\
\text { cross sections are input; ascending } \\
\text { order in } \mathrm{MeV}\end{array}$ & NCSE values \\
\hline $\operatorname{TOT}(\mathrm{I})$ & $6 \mathrm{E} 10.3$ & $\begin{array}{l}\text { Total macroscopic cross section at } \\
\operatorname{EE}(\mathrm{I})\left(\mathrm{cm}^{-1}\right)\end{array}$ & NCSE values \\
\hline $\operatorname{SCA}(\mathrm{I})$ & $6 \mathrm{E} 10.3$ & $\begin{array}{l}\text { Scattering macroscopic cross section } \\
\text { at } \operatorname{EE}(\mathrm{I})\left(\mathrm{cm}^{-1}\right)\end{array}$ & NCSE values \\
\hline
\end{tabular}


FLOW CHART FOR SAMPLE MONTE CARLO PROGRAM $†$

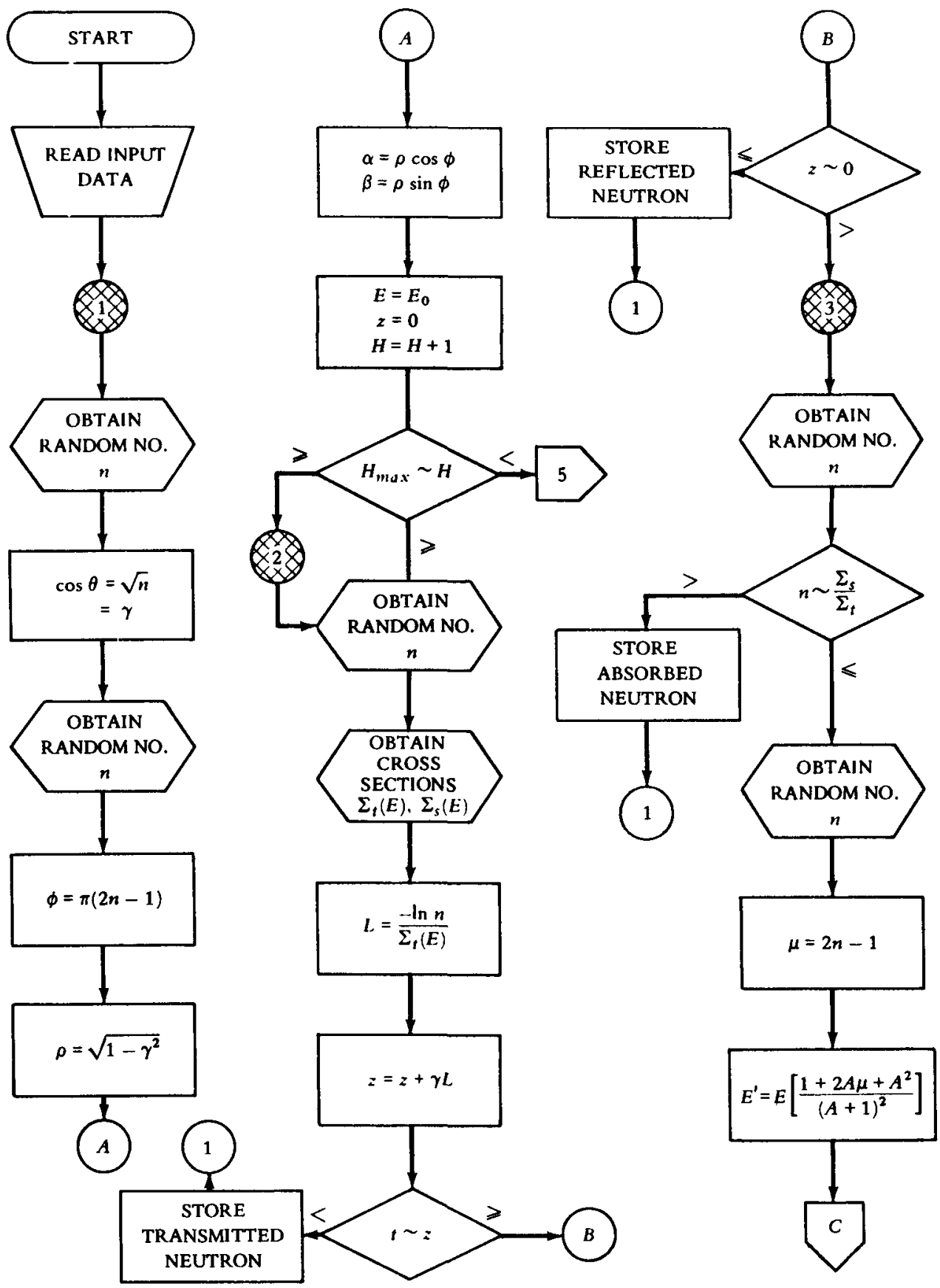

tShaded numbers 1 to 5 refer to the outline of the program logic in Sec. 5.9. 


\section{FLOW CHART (Continued)}
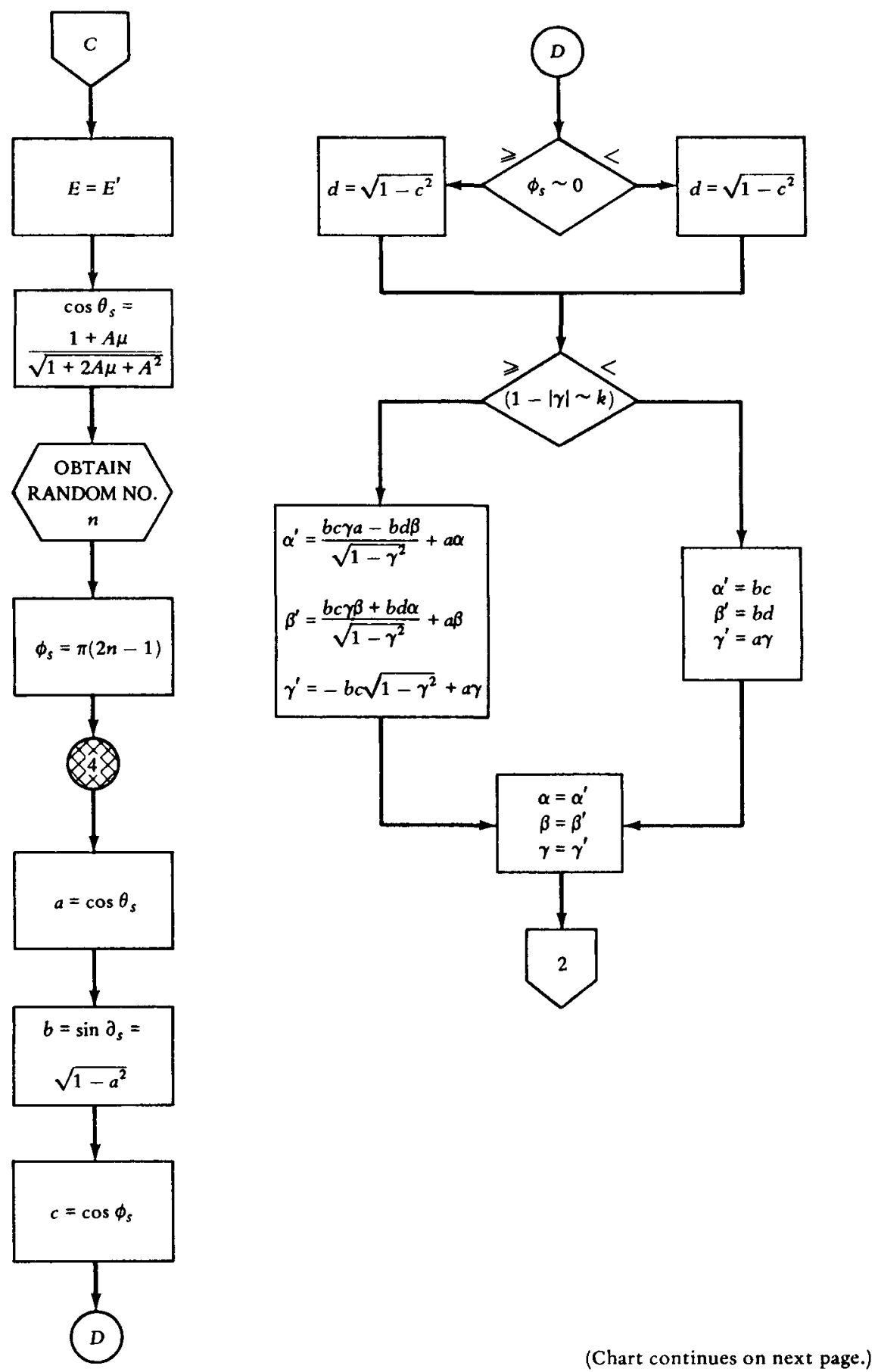
FLOW CHART (Continued)

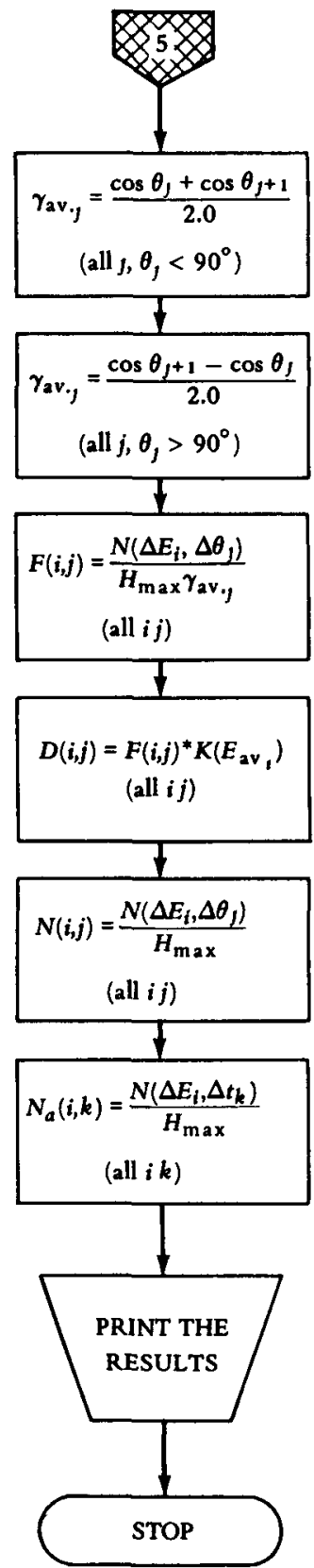




\section{FORTRAN LISTING}

A MONTE CARLO DEMONSTRATICN PROCEDURF

CALCULATES THF TRANSMITTED AND REFLECTFD CURRENT, FLUENCE, AND DOSE AND NUMBER OF ABSORRT IONS FOR A SEMI-INFINUTE SLAB WITH A COSINE CURRFNT lCCATED on A SLAB SURFACE.

DIMENSION XNIT(144)

DIMENSION FTD $(8), F T(8,6)$, DT $(8,6), F R(8,6), D R(8,6), C D A(8), C D B(8)$ COMMON N1, NN , NOT, IOP, EAS, ANG $(6), B N G(6), T 1(21), E(8), T(8,6), R(8,6)$

$1, A(8,20), 2, G$

FOUIVALFNCE (FT(1), XNITI1)), (0TI1), XNIT(49)), (ORI1), XNIT(97)

DO $5001=1,144$

$500 \times N I T I I I=0$.

NH $=0$

READ INPUT PARAMETERS

$$
\begin{array}{ll}
\text { NFNFG } & \text { NO. PRINT ENERGY GRDUPS } \\
\text { NANG } & \text { NC. PRINT ANGLE INTERVALS } \\
\text { NHIST } & \text { NC. HISTORIES } \\
\text { NOIVT } & \text { NO. SLAB ABSORPTION INTERVALS } \\
\text { IB } & \text { RANDOM NUMPER BASE (OODI } \\
\text { EO } & \text { SOURCE ENERGY } \\
\text { IT } & \text { SLAB THICKNESS } \\
\text { COA } & \text { CUT-OFF ANGLE } \\
\text { AH } & \text { ATCMIC WEIGHT } \\
\text { FTO } & \text { FLUX-TO-DOSE CONVERSION FACTORS }
\end{array}
$$

$K X=1$

$K Y=3$

RFAD (KX, 2OINENEG, NANG, NHIST, NDIVI, IB

READ $(K X, 19) E O, T T$, COA, AW

READ THE PRINT ANGLE INTERVAL BCUNDS LESS than 90 DEg. and GENERATE THOSF GREATER THAN 90 DEG.

NN1 = NANG+1

REAO (KX,19) (ANG $(K), K=1, N N I)$

DO $24 M=1, N N 1$

$24 B N G(M)=18 C \cdot 0-A N G(M)$

READ $(K X+19)$ (FTDI 1$), 1=1$, NENEG)

READ THE PRINT ENERGY BCUNCS.

N1 1 NENEG+1

RFAD (KX,19) (E(J), J=1,NI)

20 FCRMAT(10I5)

19 FORMAT (6E 10.3$)$

C

CONVERT PRINT ANGLES FROM DEGRFES TC RADIANS.

QED $=.0174533$

$C C A=C O A * O E D$

DO $25 \mathrm{~N}=1$, NN 1

$\triangle N G(N)=A N G(N) * O E D$

$25 B N G(N)=B N G(N) * Q E D$

C

ZERO DATA STORAGE ARRAYS

DO $23 I=1$, NENEG

DO $21 J=1$, NANG

$T(I, J)=0.0$

$21 R(I, J)=0.0$

.nO $23 K=1$.NOIVT

$23 A(I, K)=0.0$ 


\section{FORTRAN LISTING (Continued)}

$$
\text { c }
$$




\section{FORTRAN LISTING (Continued)}

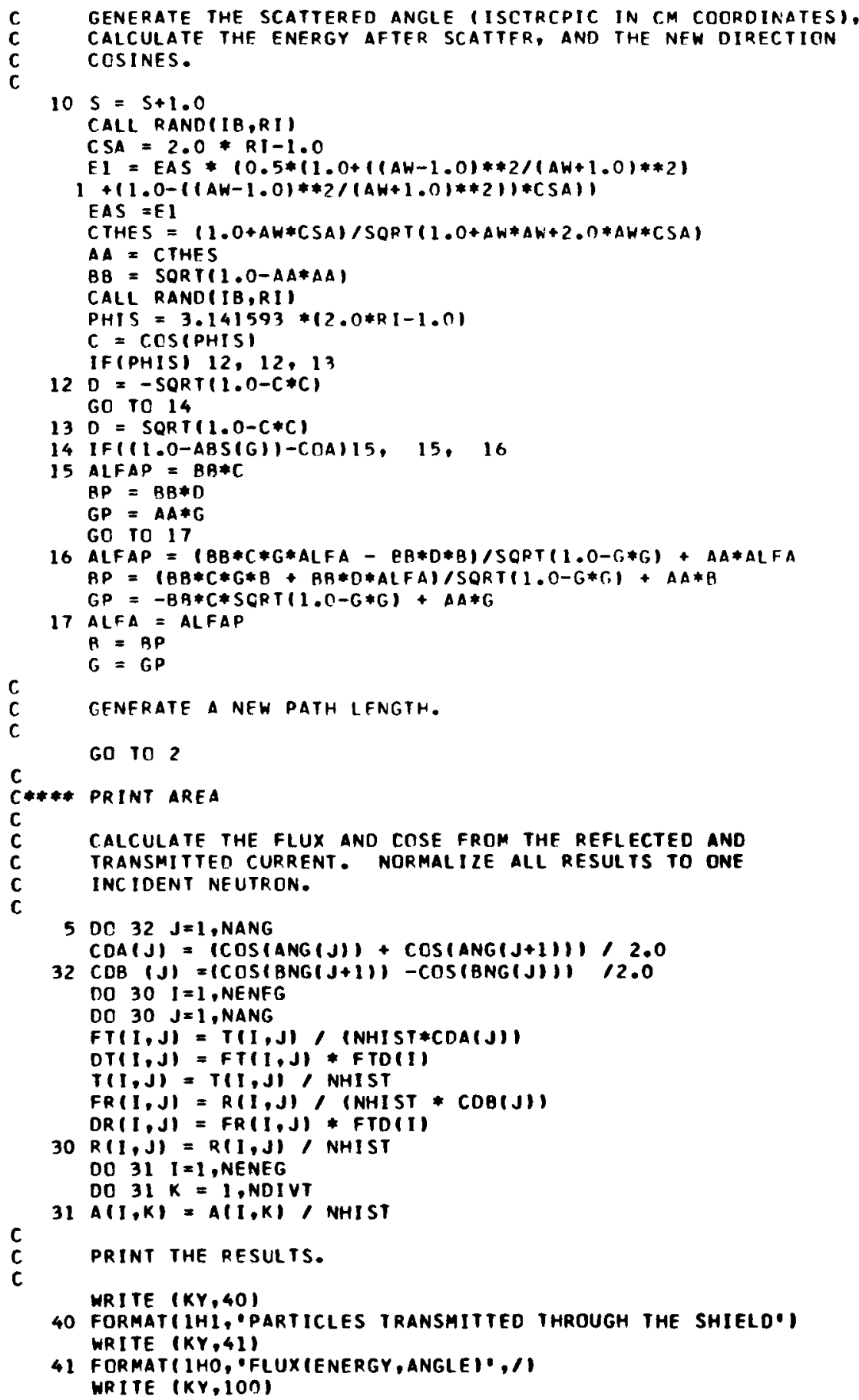




\section{FORTRAN LISTING (Continued)}

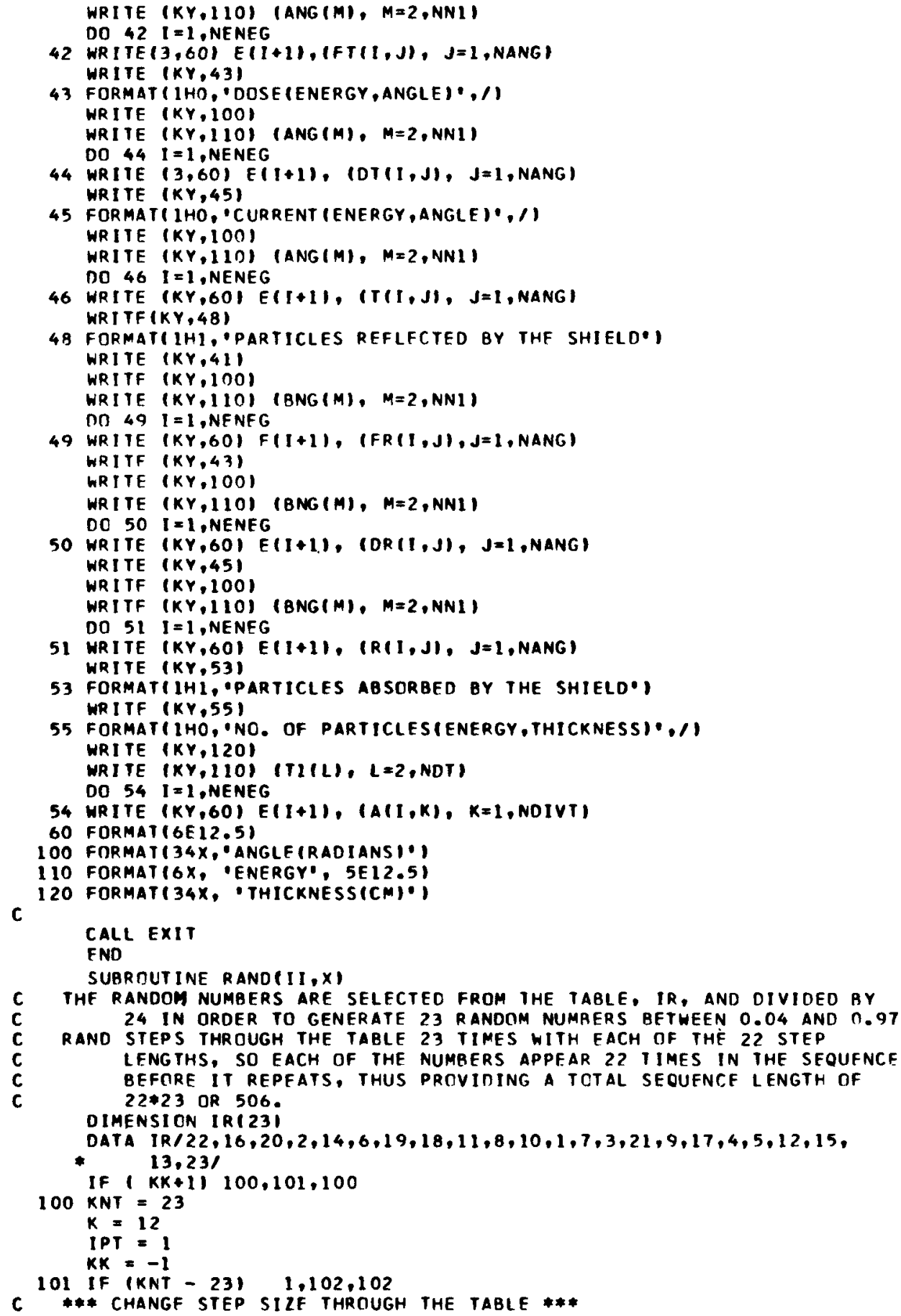


FORTRAN LISTING (Continued)

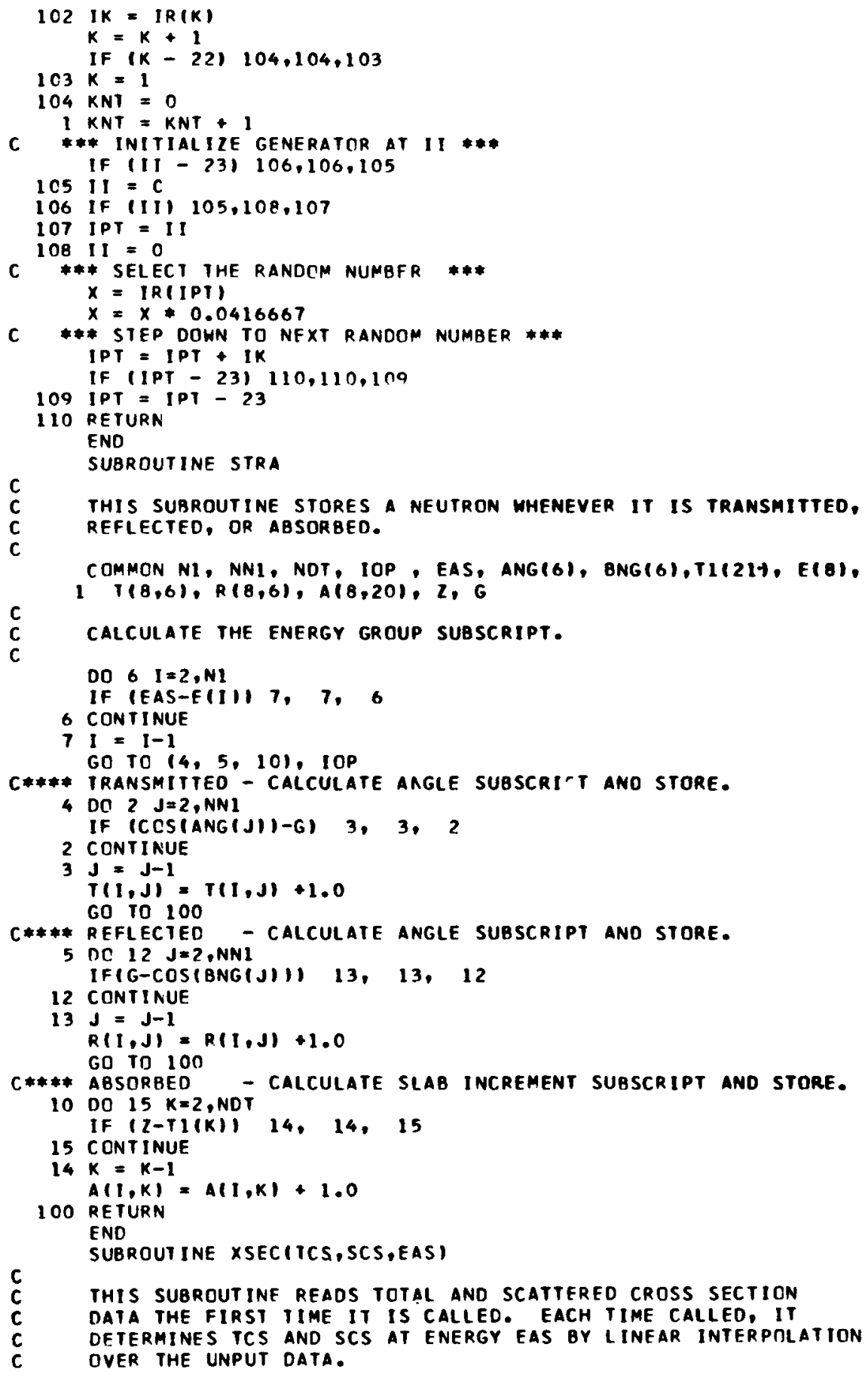


FORTRAN LISTING (Continued)

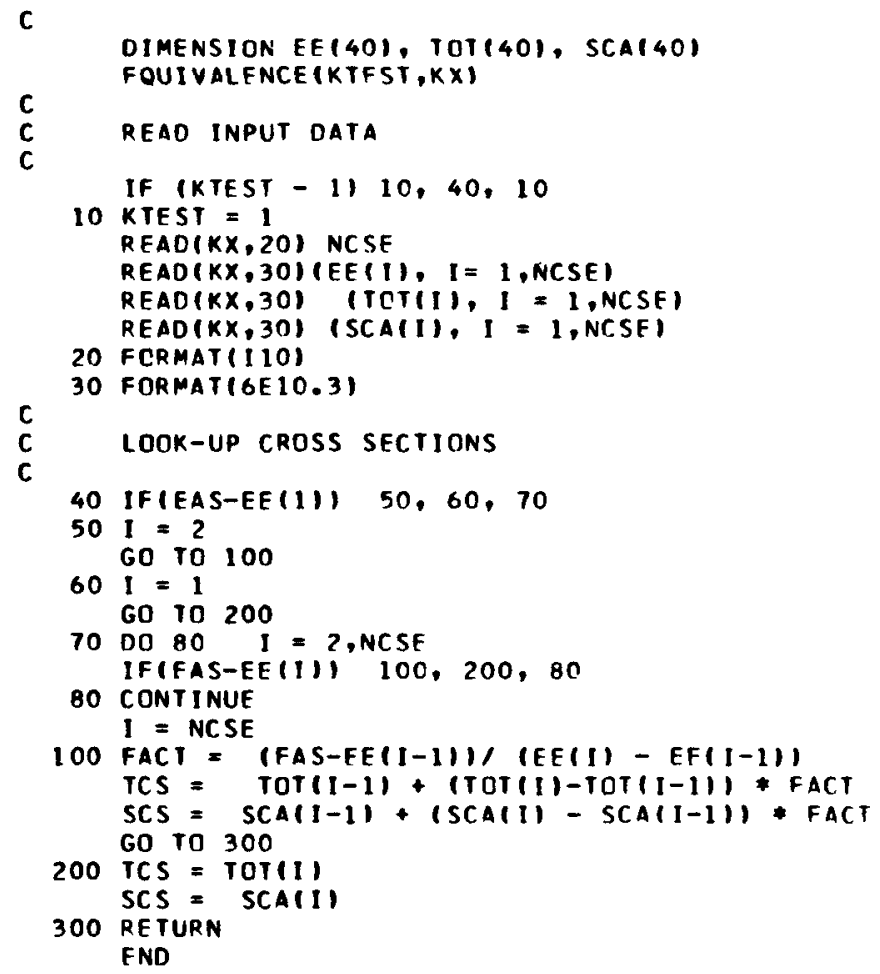

\section{TEST PROBLEM}

A test case has been run with the computer procedure for 3-MeV neutrons incident on a 2 -cm-thick semi-infinite slab of silicon. The reflected and transmitted neutrons arc sorted into six energy intervals and five angle intervals, and the absorbed neutrons are sorted into the same six energy intervals and into ten slab increments. The cross sections that were input are not realistic, but they make the neutron mean-free-path distance approximately 3 to $4 \mathrm{~cm}$. One-hundred histories were run; therefore one should not expect the answers to be nonzero except for a few storage bins. Both the input and output data are shown following the program listing. Summing the output neutron current will show that 19 neutrons were reflected, 42 were transmitted, and the 39 remainder were absorbed. 


\section{TEST PROBLEM}

Input Data

65
$3.00+00$
$0.00+00$
$2.00-09$
$0.00+00$
$3.00+00$
12
0.00
1.50
0.98
0.45
0.10
0.29

$$
\begin{array}{rr}
100 & 5 \\
2.00+00 \\
1.50+01 \\
3.20-09 \\
5.00-01 \\
\\
\\
0.25 \\
1.75 \\
0.80 \\
0.42 \\
0.20 \\
0.28
\end{array}
$$

$$
0.50
$$$$
0.75
$$$$
2.25
$$$$
1.00
$$$$
\begin{aligned}
& 1.00 \\
& 2.50
\end{aligned}
$$$$
0.55
$$$$
0.62
$$$$
0.25
$$$$
0.41
$$$$
0.23
$$$$
0.40
$$$$
0.28
$$

0. 28

0.28

0.27

$$
\begin{aligned}
& 1.25 \\
& 3.00 \\
& 0.49 \\
& 0.37 \\
& 0.31 \\
& 0.26
\end{aligned}
$$

Output Data

PARTICLES TRANSMITTEO THROUGH THE SHIELD

FLUXIENERGY, ANGLE )

ANGLE (RADIANS I

ENERGY $0.26180 E$ OO 0.52360 OE OO 0.7854 OE $000.10472 E$ O $010.15708 E$ OL 0.50000 E OO 0.0

0.10000 OI 0.0

$0.15000 E$ O1 0.0

0.0

0.0

0.0

0.0

0.0

0.0

0.0

0.0

0.0

0.0

0.0

0.0

0.0

0.0

$0.12713 E-010.0$

0.0

0.25000 E 010.0

0.0

$0.30000 E$ O1 $0.30520 E-01$

$0.13101 E 00$

$0.16569 E$ OO $0.40000 E-01$

DOSE (ENERGY, ANGLE)

ANGLE (RADIANS I

ENERGY $0.26180 E$ OO $0.52360 E$ OO $0.78540 E$ O0 $0.10472 E$ O $010.15708 E \quad 01$ $0.50000 E$ OO 0.0

$0.10000 E 010.0$

0.15000 E 010.0

0.0

0.0

0.0

0.0

0.0

0.0

0.0

0.0

0.0

0.0

0.0

0.0

0.0

$0.53397 \mathrm{~F}-100.0$

0.0

0.25000 E 01 1 0.0

0.0

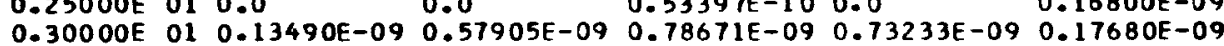

$0.16800 E-09$

CURRENT IENERGY.ANGLEI

ANGLE (RADIANS)

ENERGY $0.26180 E$ OO 0.52360 OD OD 0.7854 OE 00 $0.50000 E$ OO 0.0

$0.10000 E$ O1 0.0

$0.15000 E$ O1 0.0

$0.20000 E$ O1 0.0

0.25000 E 010.0

0.0

0.0

0.0

0.0

0.0

0.0

0.0

$\begin{array}{ll}0.0 & \text { C.0 } \\ 0.10000 E-01 & 0.0\end{array}$

$0.10472 \mathrm{~F}$

0.0

0.0

0.0

0.0

0.0

0.0

0.0

0.0

$0.30000 E$ OL $0.30000 E-01$

0.12000 E OO

0.14000 E $00 \quad 0.10000$ E 00

$0.10000 E-01$

$0.10000 E-01$ 
TEST PROBLEM (Continued)

Output Data (cont1nued)

PARTICLES REFLECTED BY THE SHIELD

FLUXIENERGY,ANGLE!

ANGLE (RADIANS)

ENERGY $0.28798 E$ O $010.26180 E$ O1 $0.23562 E$ O $010.20944 E$ OL $0.15708 E$ OL 0.50000 OO 0.0 0.10000 E O1 0.0 0.15000 E 010.0 $0.20000 E$ OI 0.0 0.25000 E 010.0 0.0 0.0 0.0 0.0 0.0 0.0 0.0 0.0 0.0 0.0

0.0 0.0 0.0 $0.40040 E$ OO $0.12585 E 00 \quad 0.0$ 0.0 $0.30000 E$ O1 0.0 $0.40040 E$ OO $0.62925 E$ DO $0.67598 E$ DO $0.40000 E-01$

DOSEIENERGY, ANGLE)

ANGLE (RADIANS)

ENERGY $0.28798 E$ OL $0.26180 E$ OL $0.23562 E$ OL $0.20944 E$ OL $0.15708 E$ OL 0.50000 OO 0.0 $0.10000 E$ O1 0.0 $0.15000 E$ OI 0.0 $0.20000 E$ OI 0.0 $0.25000 E$ OI 0.0 $0.30000 E$ OL 0.0

0.0

0.0

0.0

0.0

0.0

0.0

0.0

0.0

$0.16817 E-08$ $0.17698 E-08$

$\begin{array}{ll}0.0 & 0.0 \\ 0.52857 E-09 & 0.0\end{array}$

0.0

0.0

0.0

0.0

0.0

0.0

0.0

0.18 eces-0s

CURRE NT IENERGY , ANGLE)

ANGLE (RADIANS)

ENERGY $0.28798 E$ OL $0.26180 E$ OL $0.23562 E$ OL $0.20944 E$ OL $0.15708 E$ OL $0.50000 E 000.0$ $0.10000 E$ OL 0.0 $0.15000 E$ OL 0.0 0.20000 OL 0.0 $0.25000 E$ OI 0.0 0.30000 E 010.0

0.0

0.0

0.0

0.0

$0.20000 E-01$

$0.20000 E-01$

0.0

0.0

0.0

0.0

0.0

0.0

$0.0 \quad 0.0$

0.0

0.0

0.0

0.0

0.0

$0.10000 E-01$

$0.50000 E-010.70000 E-01$ 0.10000E-01

PARTICles absorbed bY the shield

NO. OF PARTICLES (ENERGY, THICKNESS)

THICKMESSICM)

ENERGY 0.40000 E 00 0.80000E OO 0.12000 E 010.16000 E 010.20000 E 01

0.50000 E 00 O.0

0.10000 E 010.0

0.15000 OL 0.0

0.0

0.0

0.0

0.0

0.0

0.0

0.0

0.0

0.0

0.0

0.0

0.0

$\begin{array}{ll}0.0 & 0.0 \\ 0.11000 E \text { OO } & 0.60000 E-01\end{array}$

0.0

0.0

0.0

0.25000 OL 0.10000 -01 0.0

$0.30000 E$ OI $0.11000 E$ OO $0.60000 E-01$ 0.11000E 00 0.60000E-01 0.40000E-01 


\section{Moments-Method Results for Fission-Neutron Penetration in $\mathrm{Be}, \mathrm{C}, \mathrm{CH}$, $\mathrm{CH}_{2}, \mathrm{H}$, and $\mathrm{H}_{2} \mathrm{O}^{\dagger}$}

\section{Appendix K}

Source strengths are 1 neutron/sec for the point isotropic fission cases and 1 neutron $\mathrm{cm}^{-2} \mathrm{sec}^{-1}$ for carbon, a plane isotropic fission source.

Table K 1 -DIFFERENTIAL NUMBER SPECTRA AND DOSE FOR BER YLLIUM, A POINT ISOTROPIC FISSION SOURCE

\begin{tabular}{|c|c|c|c|c|c|c|c|}
\hline \multirow[b]{2}{*}{ E, MeV } & \multicolumn{7}{|c|}{$r, \mathrm{~g} / \mathrm{cm}^{2}$} \\
\hline & $\mathbf{0}$ & 10 & 20 & 30 & 60 & 90 & 120 \\
\hline \multicolumn{8}{|c|}{ Number Spectra, $4 \pi r^{2} N_{0}(r, E)$, neutrons $\sec ^{-1} \mathrm{MeV}^{-1}$} \\
\hline $\begin{array}{ll}0 & 330 \\
0 & 383 \\
0 & 445 \\
0 & 492 \\
0 & 572\end{array}$ & $\begin{array}{l}315(-1) t \\
327(-1) \\
338(-1) \\
344(-1) \\
351(-1)\end{array}$ & $\begin{array}{l}273(-1) \\
291(-1) \\
330(-1) \\
276(-1) \\
277(-1)\end{array}$ & $\begin{array}{l}103(-1) \\
111(-1) \\
128(-1) \\
102(-1) \\
106(-1)\end{array}$ & $\begin{array}{l}357(-2) \\
395(-2) \\
469(-2) \\
388(-2) \\
421(-2)\end{array}$ & $\begin{array}{l}182(-3) \\
221(-3) \\
289(-3) \\
265(-3) \\
323(-3)\end{array}$ & $\begin{array}{l}152(-4) \\
189(-4) \\
252(-4) \\
238(-4) \\
297(-4)\end{array}$ & $\begin{array}{l}135(-5) \\
169(-5) \\
227(-5) \\
215(-5) \\
269(-5)\end{array}$ \\
\hline $\begin{array}{ll}0 & 601 \\
0 & 734 \\
1 & 10 \\
1 & 34 \\
1 & 63\end{array}$ & $\begin{array}{l}353(-1) \\
356(-1) \\
337(-1) \\
314(-1) \\
280(-1)\end{array}$ & $\begin{array}{l}199(-1) \\
312(-1) \\
262(-1) \\
258(-1) \\
225(-1)\end{array}$ & $\begin{array}{l}746(-2) \\
133(-1) \\
129(-1) \\
140(-1) \\
128(-1)\end{array}$ & $\begin{array}{l}313(-2) \\
578(-2) \\
662(-2) \\
776(-2) \\
741(-2)\end{array}$ & $\begin{array}{l}259(-3) \\
512(-3) \\
744(-3) \\
969(-3) \\
100(-2)\end{array}$ & $\begin{array}{l}242(-4) \\
484(-4) \\
730(-4) \\
969(-4) \\
103(-3)\end{array}$ & $\begin{array}{l}219(-5) \\
438(-5) \\
665(-5) \\
884(-5) \\
944(-5)\end{array}$ \\
\hline $\begin{array}{l}200 \\
244 \\
269 \\
298 \\
364\end{array}$ & $\begin{array}{l}238(-1) \\
190(-1) \\
165(-1) \\
140(-1) \\
936(-2)\end{array}$ & $\begin{array}{l}192(-1) \\
142(-1) \\
864(-2) \\
745(-2) \\
610(-2)\end{array}$ & $\begin{array}{l}107(-1) \\
672(-2) \\
270(-2\rangle \\
274(-2) \\
284(-2)\end{array}$ & 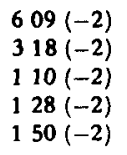 & $\begin{array}{l}782(-3) \\
251(-3) \\
919(-4) \\
129(-3) \\
178(-3)\end{array}$ & $\begin{array}{l}768(-4) \\
183(-4) \\
813(-5) \\
121(-4) \\
181(-4)\end{array}$ & $\begin{array}{l}681(-5) \\
154(-5) \\
831(-6) \\
123(-5) \\
188(-5)\end{array}$ \\
\hline $\begin{array}{l}444 \\
543 \\
663 \\
810 \\
989\end{array}$ & $\begin{array}{l}558(-2) \\
285(-2) \\
122(-2) \\
412(-3) \\
105(-3)\end{array}$ & $\begin{array}{l}442(-2) \\
228(-2) \\
820(-3) \\
312(-3) \\
853(-4)\end{array}$ & $\begin{array}{l}223(-2) \\
118(-2) \\
402(-3) \\
156(-3) \\
455(-4)\end{array}$ & $\begin{array}{l}122(-2) \\
664(-3) \\
229(-3) \\
893(-4) \\
272(-4)\end{array}$ & $\begin{array}{l}162(-3) \\
965(-4) \\
351(-4) \\
150(-4) \\
514(-5)\end{array}$ & $\begin{array}{l}183(-4) \\
1118(-4) \\
450(-5) \\
216(-5) \\
807(-6)\end{array}$ & $\begin{array}{l}205(-5) \\
140(-5) \\
560(-6) \\
297(-6) \\
118(-6)\end{array}$ \\
\hline $\begin{array}{l}121 \\
1475 \\
180\end{array}$ & $\begin{array}{lll}1 & 87 & (-4) \\
2 & 17 & (-5) \\
1 & 47 & (-6)\end{array}$ & 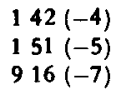 & $\begin{array}{l}712(-5) \\
943(-6) \\
417(-7)\end{array}$ & $\begin{array}{l}413(-5) \\
593(-6) \\
232(-7)\end{array}$ & $\begin{array}{l}765(-6) \\
119(-6) \\
375(-8)\end{array}$ & $\begin{array}{l}122(-6) \\
215(-7) \\
563(-9)\end{array}$ & $\begin{array}{l}184(-7) \\
361(-8) \\
834(-10)\end{array}$ \\
\hline \multicolumn{8}{|c|}{ Dose $4 \pi r^{2} D(r), D$ in mrads $/ h r$} \\
\hline & $101(-2)$ & $785(-3)$ & $369(-3)$ & $191(-3)$ & $233(-4)$ & $238(-5)$ & $238(-6)$ \\
\hline
\end{tabular}

t Read $315 \times 10^{1}$ etc

tAll data in this appendix have been taken from H. Goldstein, Fundamental Aspects of Reactor Shieldıng, Addıson-Wesley Publıshıng Company, Inc, Readıng, Mass, 1959. 
Table K.2-DIFFERENTIAL NUMBER FLUX FOR CARBON, A PLANE ISOTROPIC FISSION SOURCE $N_{0}(x, E)$, neutron $\mathrm{cm}^{-2} \sec ^{-1} \mathrm{MeV}^{-1}$

\begin{tabular}{|c|c|c|c|c|c|c|}
\hline \multirow[b]{2}{*}{$E, \mathrm{MeV}$} & \multicolumn{6}{|c|}{$x, \mathrm{~g} / \mathrm{cm}^{2}$} \\
\hline & 10 & 20 & 30 & 60 & 90 & 120 \\
\hline 18.02 & $0.6453 \times 10^{-6}$ & $0.3134 \times 10^{-6}$ & $0.1585 \times 10^{-6}$ & $0.2995 \times 10^{-7}$ & $0.1020 \times 10^{-7}$ & $0.4588 \times 10^{-8}$ \\
\hline 12.08 & $0.8331 \times 10^{-4}$ & $0.4080 \times 10^{-4}$ & $0.2076 \times 10^{-4}$ & $0.3942 \times 10^{-5}$ & $0.1331 \times 10^{-5}$ & $0.6025 \times 10^{-6}$ \\
\hline 8.10 & $0.1512 \times 10^{-2}$ & $0.6391 \times 10^{-3}$ & $0.2856 \times 10^{-3}$ & $0.4442 \times 10^{-4}$ & $0.1445 \times 10^{-4}$ & $0.5984 \times 10^{-5}$ \\
\hline 5.43 & $0.1580 \times 10^{-1}$ & $0.8383 \times 10^{-2}$ & $0.4576 \times 10^{-2}$ & $0.9661 \times 10^{-3}$ & $0.3227 \times 10^{-3}$ & $0.1444 \times 10^{-3}$ \\
\hline 3.64 & $0.4676 \times 10^{-1}$ & $0.2194 \times 10^{-1}$ & $0.1063 \times 10^{-1}$ & $0.1708 \times 10^{-2}$ & $0.5213 \times 10^{-3}$ & $0.2262 \times 10^{-3}$ \\
\hline 2.44 & $0.1748 \times 10^{0}$ & $0.8833 \times 10^{-1}$ & $0.4592 \times 10^{-1}$ & $0.8427 \times 10^{-2}$ & $0.2502 \times 10^{-2}$ & $0.1010 \times 10^{-2}$ \\
\hline 1.63 & $0.3407 \times 10^{0}$ & $0.1819 \times 10^{0}$ & $0.9959 \times 10^{-1}$ & $0.2031 \times 10^{-1}$ & $0.6070 \times 10^{-2}$ & $0.2370 \times 10^{-2}$ \\
\hline 1.1 & $0.5311 \times 10^{0}$ & $0.2930 \times 10^{0}$ & $0.1653 \times 10^{0}$ & $0.3596 \times 10^{-1}$ & $0.1087 \times 10^{-1}$ & $0.4183 \times 10^{-2}$ \\
\hline 0.73 & $0.7516 \times 10^{0}$ & $0.4234 \times 10^{0}$ & $0.2436 \times 10^{0}$ & $0.5518 \times 10^{-1}$ & $0.1682 \times 10^{-1}$ & $0.6427 \times 10^{-2}$ \\
\hline 0.49 & $0.8507 \times 10^{0}$ & $0.4848 \times 10^{0}$ & $0.2820 \times 10^{0}$ & $0.6543 \times 10^{-1}$ & $0.2008 \times 10^{-1}$ & $0.7652 \times 10^{-2}$ \\
\hline 0.33 & $0.7365 \times 10^{0}$ & $0.4175 \times 10^{0}$ & $0.2419 \times 10^{0}$ & $0.5585 \times 10^{-1}$ & $0.1722 \times 10^{-1}$ & $0.6598 \times 10^{-2}$ \\
\hline 0.221 & $0.9966 \times 10^{0}$ & $0.5694 \times 10^{0}$ & $0.3322 \times 10^{0}$ & $0.7783 \times 10^{-1}$ & $0.2406 \times 10^{-1}$ & $0.9193 \times 10^{-2}$ \\
\hline 0.148 & $0.1442 \times 10^{1}$ & $0.8384 \times 10^{0}$ & $0.4966 \times 10^{0}$ & $0.1197 \times 10^{0}$ & $0.3713 \times 10^{-1}$ & $0.1408 \times 10^{-1}$ \\
\hline 0.099 & $0.2113 \times 10^{1}$ & $0.1252 \times 10^{1}$ & $0.7552 \times 10^{0}$ & $0.1885 \times 10^{0}$ & $0.5881 \times 10^{-1}$ & $0.2214 \times 10^{-1}$ \\
\hline 0.066 & $0.3120 \times 10^{1}$ & $0.1888 \times 10^{1}$ & $0.1159 \times 10^{1}$ & $0.3005 \times 10^{0}$ & $0.9466 \times 10^{-1}$ & $0.3542 \times 10^{-1}$ \\
\hline 0.012 & $0.1401 \times 10^{2}$ & $0.9070 \times 10^{1}$ & $0.5927 \times 10^{1}$ & $0.1765 \times 10^{1}$ & $0.5892 \times 10^{0}$ & $0.2206 \times 10^{0}$ \\
\hline $0.3 \times 10^{-5}$ & $0.4819 \times 10^{5}$ & $0.3630 \times 10^{5}$ & $0.2740 \times 10^{5}$ & $0.1191 \times 10^{5}$ & $0.5277 \times 10^{4}$ & $0.2385 \times 10^{4}$ \\
\hline
\end{tabular}


Table K.3-DIFFERENTIAL NUMBER SPECTRA FOR CH, A POINT ISOTROPIC FISSION SOURCE $4 \pi r^{2} N_{0}(r, E)$, neutron $\sec ^{-1} \mathrm{MeV}^{-1}$

\begin{tabular}{ccccccc}
\hline & \multicolumn{5}{c}{$r, \mathrm{~g} / \mathrm{cm}^{2}$} \\
\cline { 2 - 7 } E, MeV & 10 & 20 & 30 & 60 & 90 & 120 \\
\hline 18.02 & $0.9312 \times 10^{-6}$ & $0.4418 \times 10^{-6}$ & $0.2511 \times 10^{-6}$ & $0.4272 \times 10^{-7}$ & $0.6668 \times 10^{-8}$ & $0.1009 \times 10^{-8}$ \\
12.08 & $0.1100 \times 10^{-3}$ & $0.4664 \times 10^{-4}$ & $0.2421 \times 10^{-4}$ & $0.3167 \times 10^{-5}$ & $0.3760 \times 10^{-6}$ & $0.4431 \times 10^{-7}$ \\
8.10 & $0.1906 \times 10^{-2}$ & $0.6603 \times 10^{-3}$ & $0.2843 \times 10^{-3}$ & $0.2005 \times 10^{-4}$ & $0.1392 \times 10^{-5}$ & $0.1099 \times 10^{-6}$ \\
5.43 & $0.1397 \times 10^{-1}$ & $0.5142 \times 10^{-2}$ & $0.2194 \times 10^{-2}$ & $0.1401 \times 10^{-3}$ & $0.8371 \times 10^{-5}$ & $0.5378 \times 10^{-6}$ \\
3.64 & $0.3850 \times 10^{-1}$ & $0.1099 \times 10^{-1}$ & $0.3872 \times 10^{-2}$ & $0.1620 \times 10^{-3}$ & $0.8243 \times 10^{-5}$ & $0.5170 \times 10^{-6}$ \\
2.44 & $0.9867 \times 10^{-1}$ & $0.2806 \times 10^{-1}$ & $0.8970 \times 10^{-2}$ & $0.2824 \times 10^{-3}$ & $0.1276 \times 10^{-4}$ & $0.7847 \times 10^{-6}$ \\
1.63 & $0.1481 \times 10^{0}$ & $0.4117 \times 10^{-1}$ & $0.1250 \times 10^{-1}$ & $0.3424 \times 10^{-3}$ & $0.1476 \times 10^{-4}$ & $0.9034 \times 10^{-6}$ \\
1.10 & $0.2007 \times 10^{0}$ & $0.5422 \times 10^{-1}$ & $0.1587 \times 10^{-1}$ & $0.4109 \times 10^{-3}$ & $0.1753 \times 10^{-4}$ & $0.1073 \times 10^{-5}$ \\
0.734 & $0.2560 \times 10^{0}$ & $0.6802 \times 10^{-1}$ & $0.1940 \times 10^{-1}$ & $0.4947 \times 10^{-3}$ & $0.2102 \times 10^{-4}$ & $0.1287 \times 10^{-5}$ \\
0.492 & $0.2981 \times 10^{0}$ & $0.7879 \times 10^{-1}$ & $0.2210 \times 10^{-1}$ & $0.5642 \times 10^{-3}$ & $0.2392 \times 10^{-4}$ & $0.1461 \times 10^{-5}$ \\
0.33 & $0.3711 \times 10^{0}$ & $0.9896 \times 10^{-1}$ & $0.2718 \times 10^{-1}$ & $0.6958 \times 10^{-3}$ & $0.2929 \times 10^{-4}$ & $0.1782 \times 10^{-5}$ \\
\hline
\end{tabular}


Table K.4-DIFFERENTIAL NUMBER SPECTRA FOR $\mathrm{CH}_{2.0}$, A POINT ISOTROPIC FISSION SOURCE $4 \pi r^{2} N_{0}(r, E)$, neutron sec ${ }^{-1} \mathrm{MeV}^{-1}$

\begin{tabular}{ccccccc}
\hline & \multicolumn{7}{c}{$r, \mathrm{~g} / \mathrm{cm}^{2}$} \\
\cline { 2 - 6 }$E, \mathrm{MeV}$ & 10 & 20 & 30 & 60 & 90 & 120 \\
\hline 18.02 & $0.7280 \times 10^{-6}$ & $0.3087 \times 10^{-6}$ & $0.1490 \times 10^{-6}$ & $0.1425 \times 10^{-7}$ & $0.1282 \times 10^{-8}$ & $0.1114 \times 10^{-9}$ \\
12.08 & $0.7751 \times 10^{-4}$ & $0.2769 \times 10^{-4}$ & $0.1140 \times 10^{-4}$ & $0.6554 \times 10^{-6}$ & $0.3680 \times 10^{-7}$ & $0.2118 \times 10^{-8}$ \\
8.10 & $0.1204 \times 10^{-2}$ & $0.3352 \times 10^{-3}$ & $0.1041 \times 10^{-3}$ & $0.2627 \times 10^{-5}$ & $0.8284 \times 10^{-7}$ & $0.3221 \times 10^{-8}$ \\
5.43 & $0.7724 \times 10^{-2}$ & $0.2003 \times 10^{-2}$ & $0.5458 \times 10^{-3}$ & $0.9009 \times 10^{-5}$ & $0.1971 \times 10^{-6}$ & $0.5711 \times 10^{-8}$ \\
3.64 & $0.1883 \times 10^{-1}$ & $0.3739 \times 10^{-2}$ & $0.8267 \times 10^{-3}$ & $0.9384 \times 10^{-5}$ & $0.2049 \times 10^{-6}$ & $0.5743 \times 10^{-8}$ \\
2.44 & $0.4193 \times 10^{-1}$ & $0.7368 \times 10^{-2}$ & $0.1424 \times 10^{-2}$ & $0.1294 \times 10^{-4}$ & $0.2847 \times 10^{-6}$ & $0.7728 \times 10^{-8}$ \\
1.63 & $0.6053 \times 10^{-1}$ & $0.9916 \times 10^{-2}$ & $0.1793 \times 10^{-2}$ & $0.1487 \times 10^{-4}$ & $0.3300 \times 10^{-6}$ & $0.8774 \times 10^{-8}$ \\
1.10 & $0.8130 \times 10^{-1}$ & $0.1258 \times 10^{-1}$ & $0.2207 \times 10^{-2}$ & $0.1779 \times 10^{-4}$ & $0.3960 \times 10^{-6}$ & $0.1045 \times 10^{-7}$ \\
0.734 & $0.1039 \times 10^{0}$ & $0.1538 \times 10^{-1}$ & $0.2665 \times 10^{-2}$ & $0.2138 \times 10^{-4}$ & $0.4745 \times 10^{-6}$ & $0.1255 \times 10^{-7}$ \\
0.492 & $0.1259 \times 10^{0}$ & $0.1806 \times 10^{-1}$ & $0.3109 \times 10^{-2}$ & $0.2504 \times 10^{-4}$ & $0.5523 \times 10^{-6}$ & $0.1470 \times 10^{-7}$ \\
0.33 & $0.1612 \times 10^{0}$ & $0.2246 \times 10^{-1}$ & $0.3831 \times 10^{-2}$ & $0.3105 \times 10^{-4}$ & $0.6769 \times 10^{-6}$ & $0.1820 \times 10^{-7}$ \\
\hline
\end{tabular}


Table K.5-DIFFERENTIAL NUMBER SPECTRA FOR $\mathrm{H}_{2}$, A POINT ISOTROPIC FISSION SOURCE $4 \pi r^{2} N_{0}(r, E)$, neutron $\sec ^{-1} \mathrm{MeV}^{-1}$

\begin{tabular}{clcccc}
\hline & \multicolumn{5}{c}{$r, \mathrm{~cm}$} \\
\cline { 2 - 7 }$E, \mathrm{MeV}$ & \multicolumn{1}{c}{10} & \multicolumn{1}{c}{20} & 30 & 60 & 90 \\
\hline 18.017 & $0.1047 \times 10^{-5}$ & $0.6581 \times 10^{-6}$ & $0.4415 \times 10^{-6}$ & $0.1322 \times 10^{-6}$ & $0.42248 \times 10^{-7}$ \\
13.348 & $0.43476 \times 10^{-4}$ & $0.2814 \times 10^{-4}$ & $0.18227 \times 10^{-4}$ & $0.49575 \times 10^{-5}$ & $0.13801 \times 10^{-5}$ \\
8.947 & $0.11671 \times 10^{-2}$ & $0.63120 \times 10^{-3}$ & $0.34302 \times 10^{-3}$ & $0.56543 \times 10^{-4}$ & $0.97823 \times 10^{-5}$ \\
5.997 & $0.87118 \times 10^{-2}$ & $0.39413 \times 10^{-2}$ & $0.17905 \times 10^{-2}$ & $0.18519 \times 10^{-3}$ & $0.21545 \times 10^{-4}$ \\
3.824 & 0.032608 & 0.012542 & $0.4784 \times 10^{-2}$ & $0.33947 \times 10^{-3}$ & $0.32015 \times 10^{-4}$ \\
1.555 & 0.10505 & 0.035939 & 0.011680 & $0.59143 \times 10^{-3}$ & $0.55322 \times 10^{-4}$ \\
0.7344 & 0.16057 & 0.057007 & 0.019296 & $0.81843 \times 10^{-3}$ & $0.69773 \times 10^{-4}$ \\
0.33 & 0.22197 & 0.082713 & 0.029512 & $0.11020 \times 10^{-2}$ & $0.83534 \times 10^{-4}$ \\
\hline
\end{tabular}

Table K.6-DIFFERENTIAL NUMBER SPECTRA FOR WATER, A POINT ISOTROPIC FISSION SOURCE $4 \pi r^{2} N_{0}(r, E)$, neutron $\sec ^{-1} \mathrm{MeV}^{-1}$

\begin{tabular}{cccccccc}
\hline & \multicolumn{7}{c}{$r, \mathrm{~cm}$} \\
\cline { 2 - 7 } E, MeV & 0 & 10 & 20 & 30 & 60 & 90 \\
\hline 10.9 & $4.64 \times 10^{-4}$ & $2.02 \times 10^{-4}$ & $8.77 \times 10^{-5}$ & $3.79 \times 10^{-5}$ & $3.01 \times 10^{-6}$ & $2.35 \times 10^{-7}$ & $1.82 \times 10^{-8}$ \\
6.0 & $1.91 \times 10^{-2}$ & $7.54 \times 10^{-3}$ & $2.90 \times 10^{-3}$ & $1.10 \times 10^{-3}$ & $5.79 \times 10^{-5}$ & $3.07 \times 10^{-6}$ & $1.68 \times 10^{-7}$ \\
2.7 & $1.65 \times 10^{-1}$ & $5.86 \times 10^{-2}$ & $1.62 \times 10^{-2}$ & $4.37 \times 10^{-3}$ & $1.07 \times 10^{-4}$ & $4.14 \times 10^{-6}$ & $2.16 \times 10^{-7}$ \\
1.1 & $3.37 \times 10^{-1}$ & $1.20 \times 10^{-1}$ & $3.14 \times 10^{-2}$ & $6.70 \times 10^{-3}$ & $1.47 \times 10^{-4}$ & $5.62 \times 10^{-6}$ & $2.99 \times 10^{-7}$ \\
0.33 & $3.15 \times 10^{-1}$ & $2.37 \times 10^{-1}$ & $5.06 \times 10^{-2}$ & $1.20 \times 10^{-2}$ & $3.26 \times 10^{-4}$ & $1.01 \times 10^{-5}$ & $5.37 \times 10^{-7}$ \\
\hline
\end{tabular}




\section{Gamma-Ray Differential Energy Spectra for $W$ ater and Lead}

\section{Appendix L}

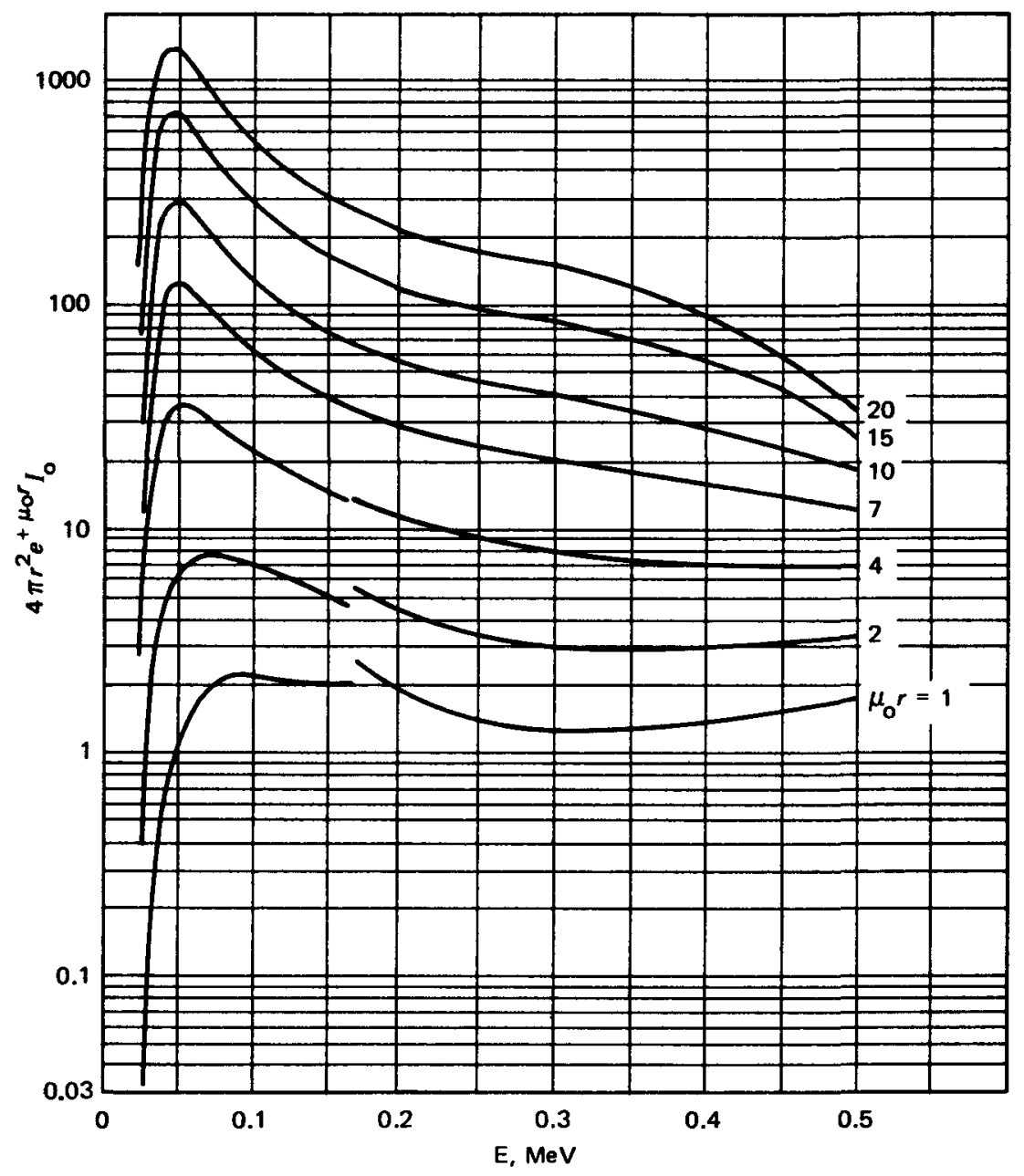

Fig. L.1-Point isotropic 0.5-MeV gamma-ray source in water. 


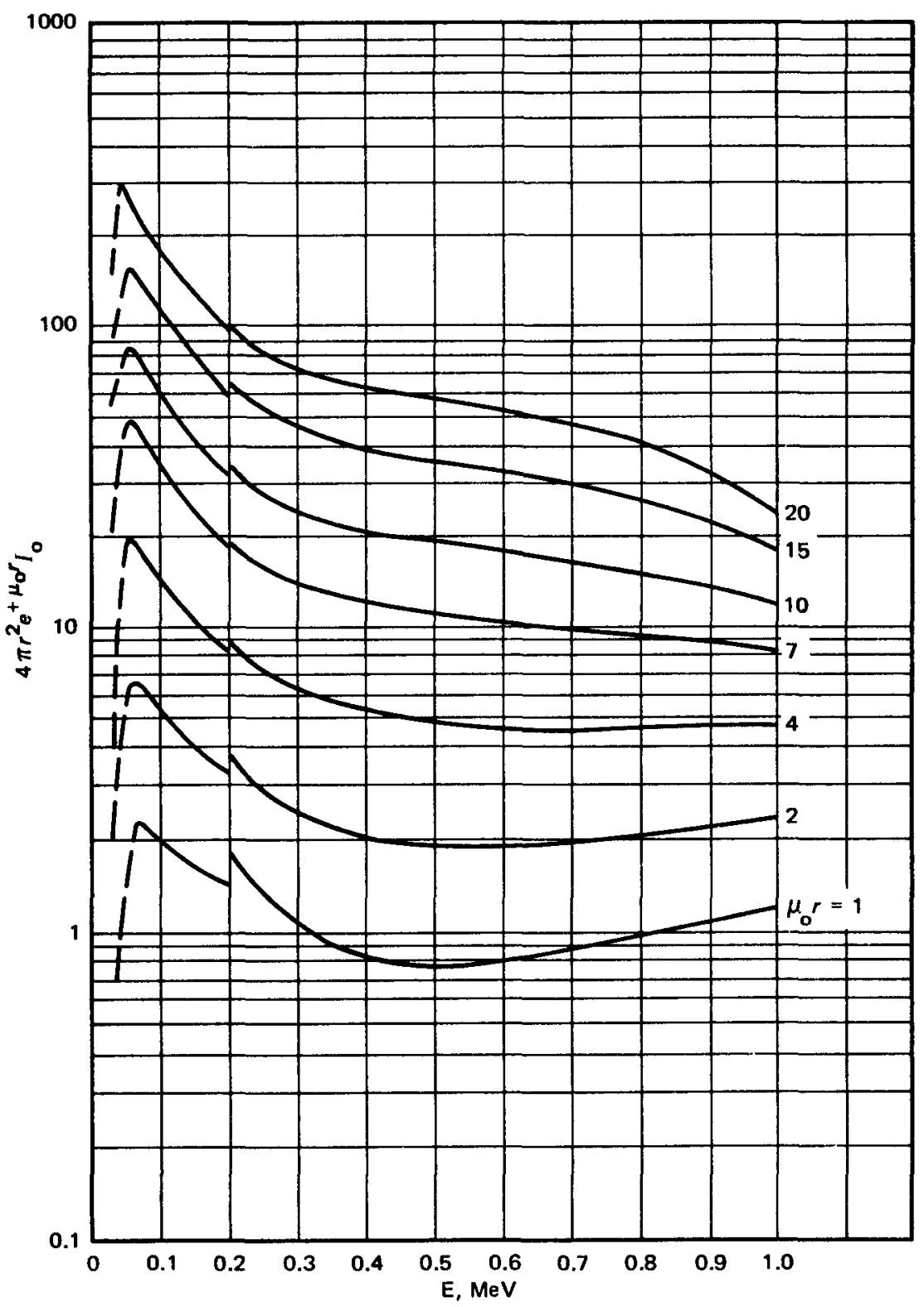

Fig. L.2-Point isotropic 1.0-MeV gamma-ray source in water. 


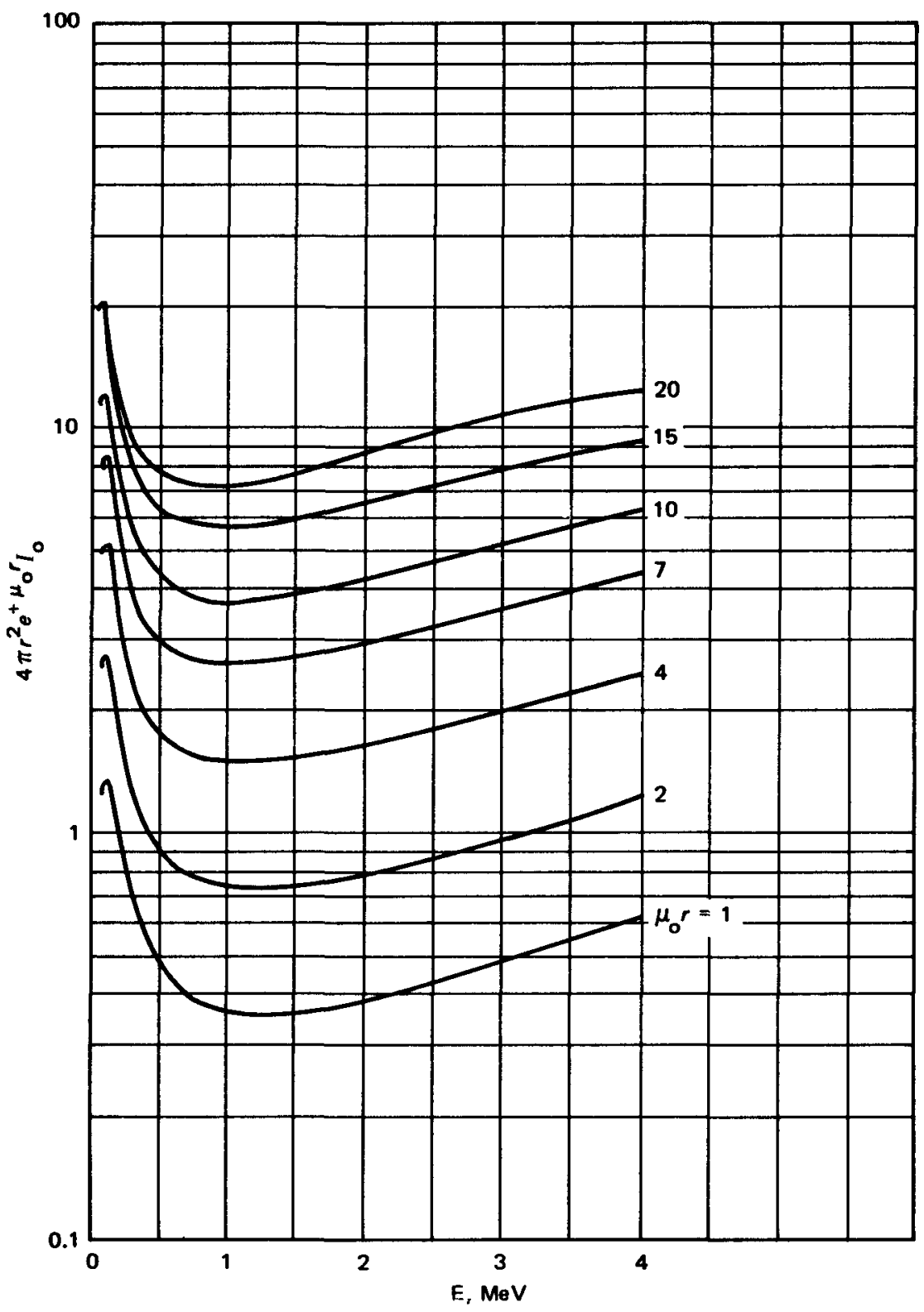

Fig. L.3-Point isotropic 4.0-MeV source in water. 


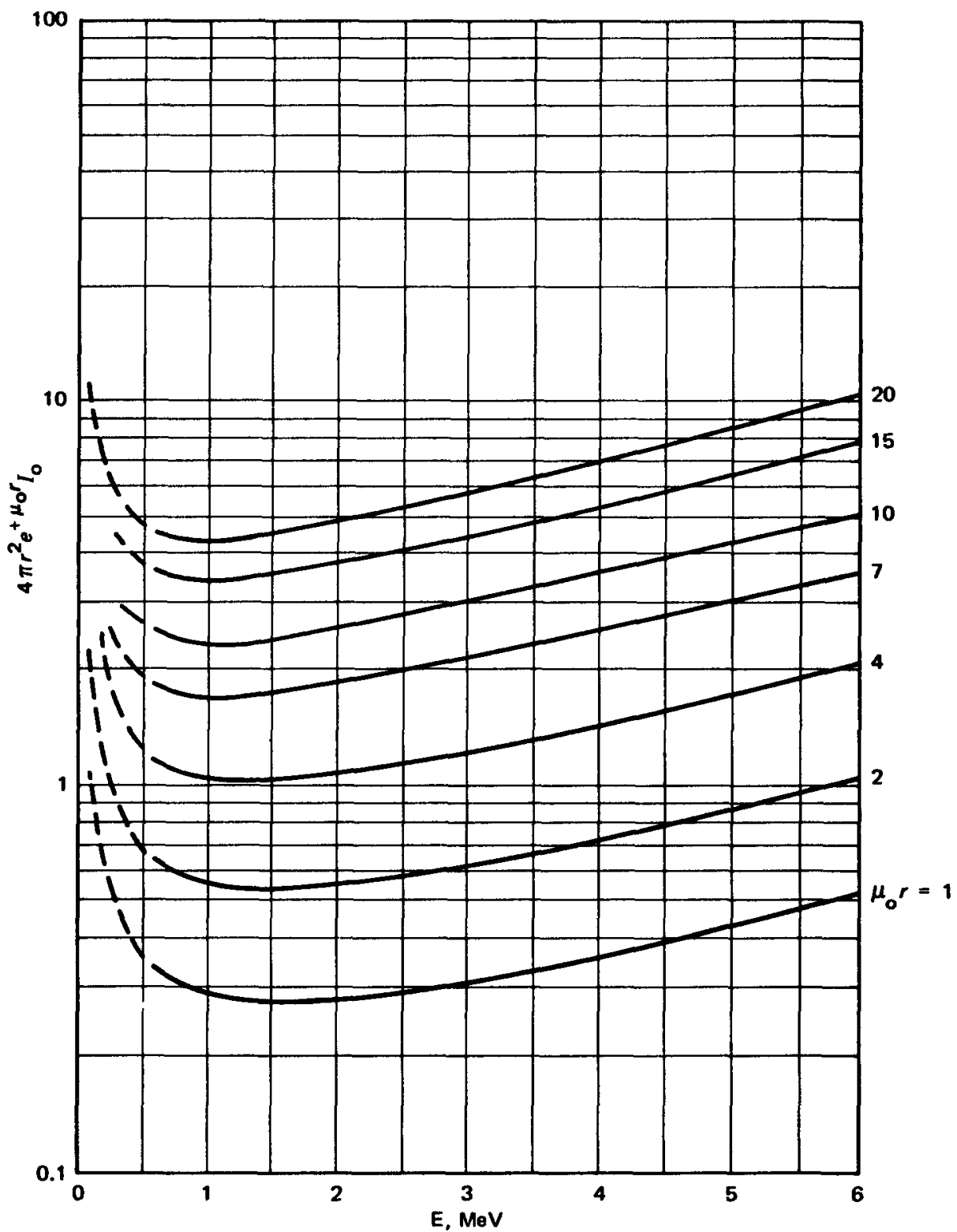

Fig. L.4-Point isotropic 6.0-MeV gamma-ray source in water. 


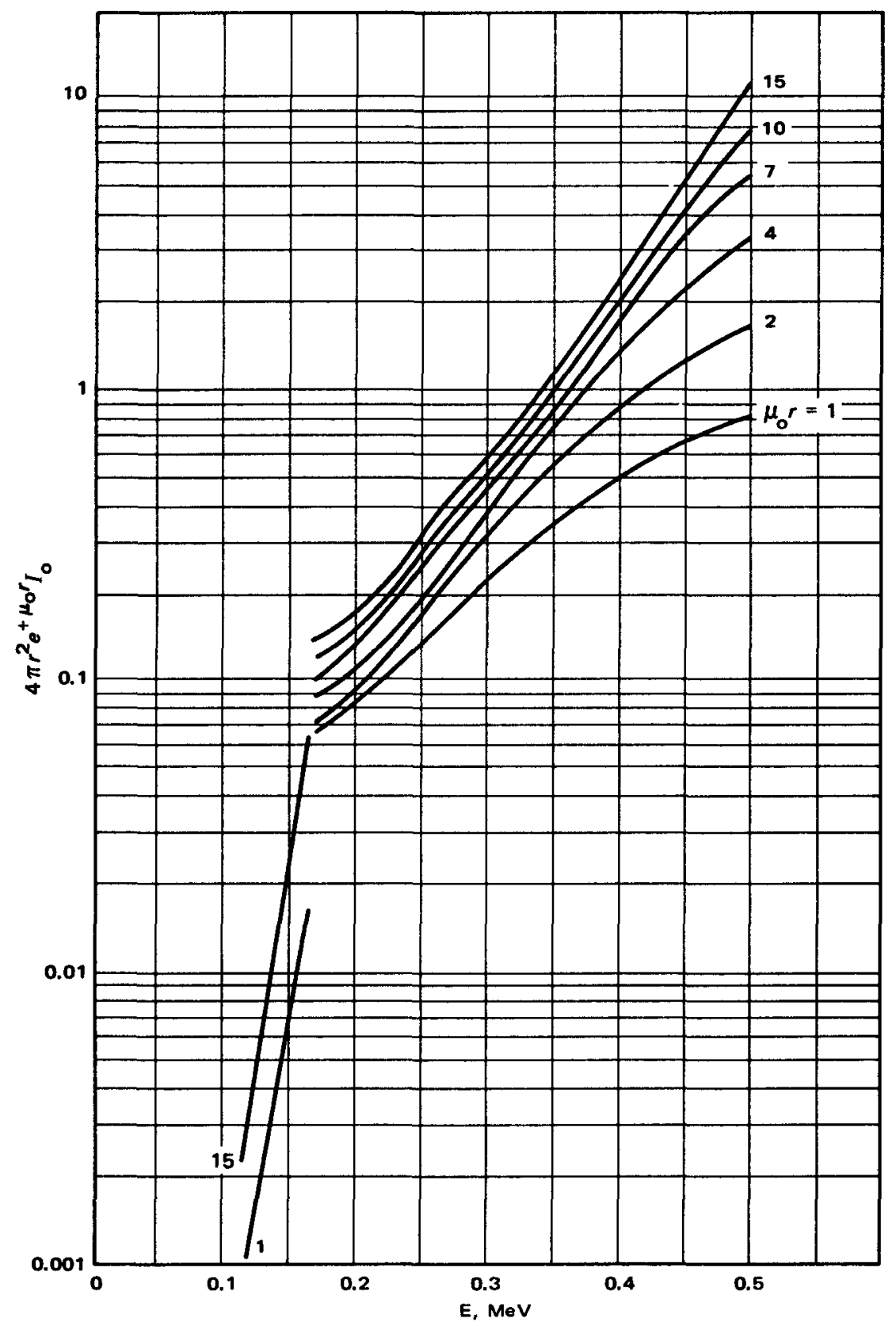

Fig. L.5-Point isotropic 0.5-MeV gamma-ray source in lead. 


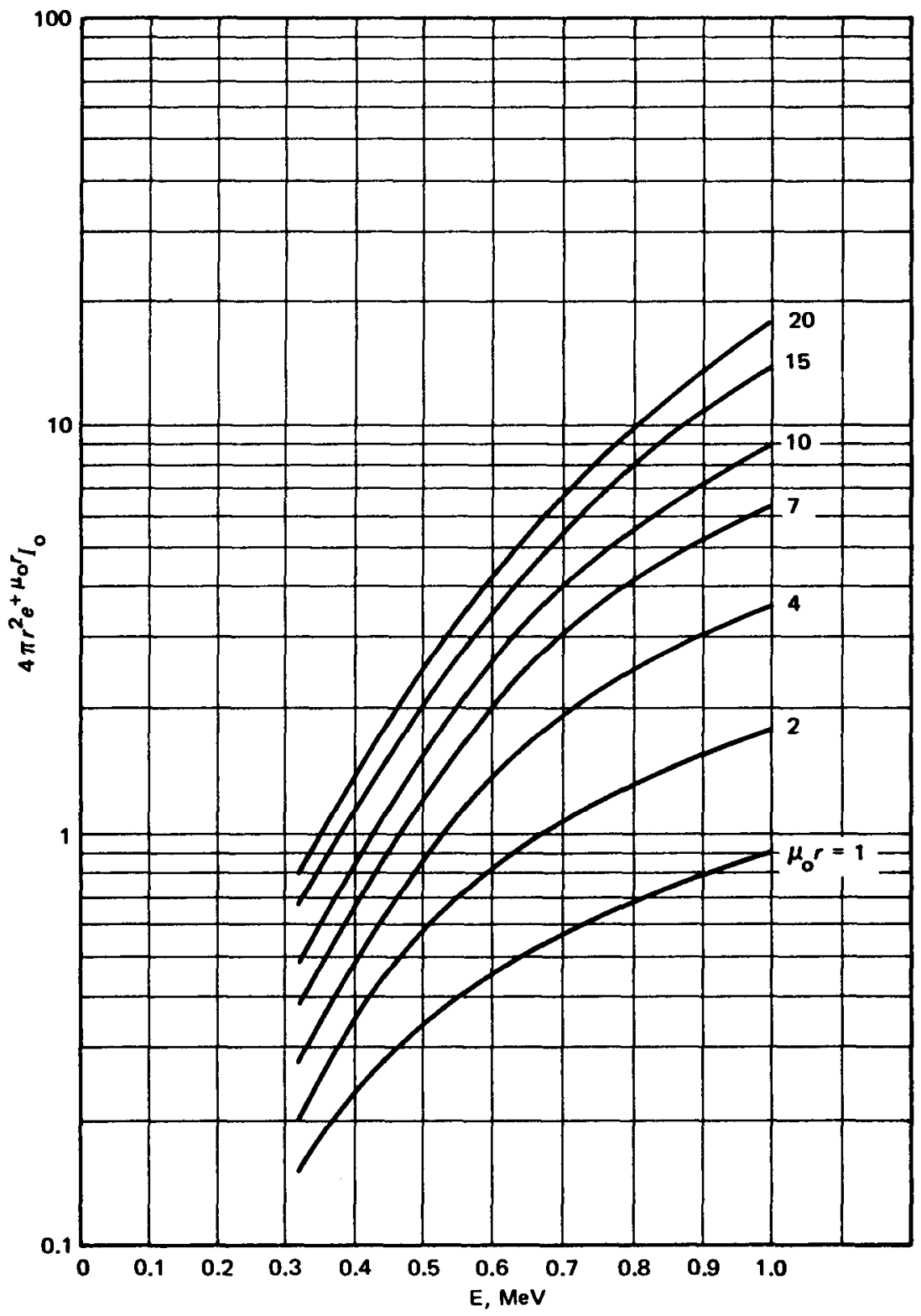

Fig. L.6-Point isotropic 1.0-MeV source in lead. 


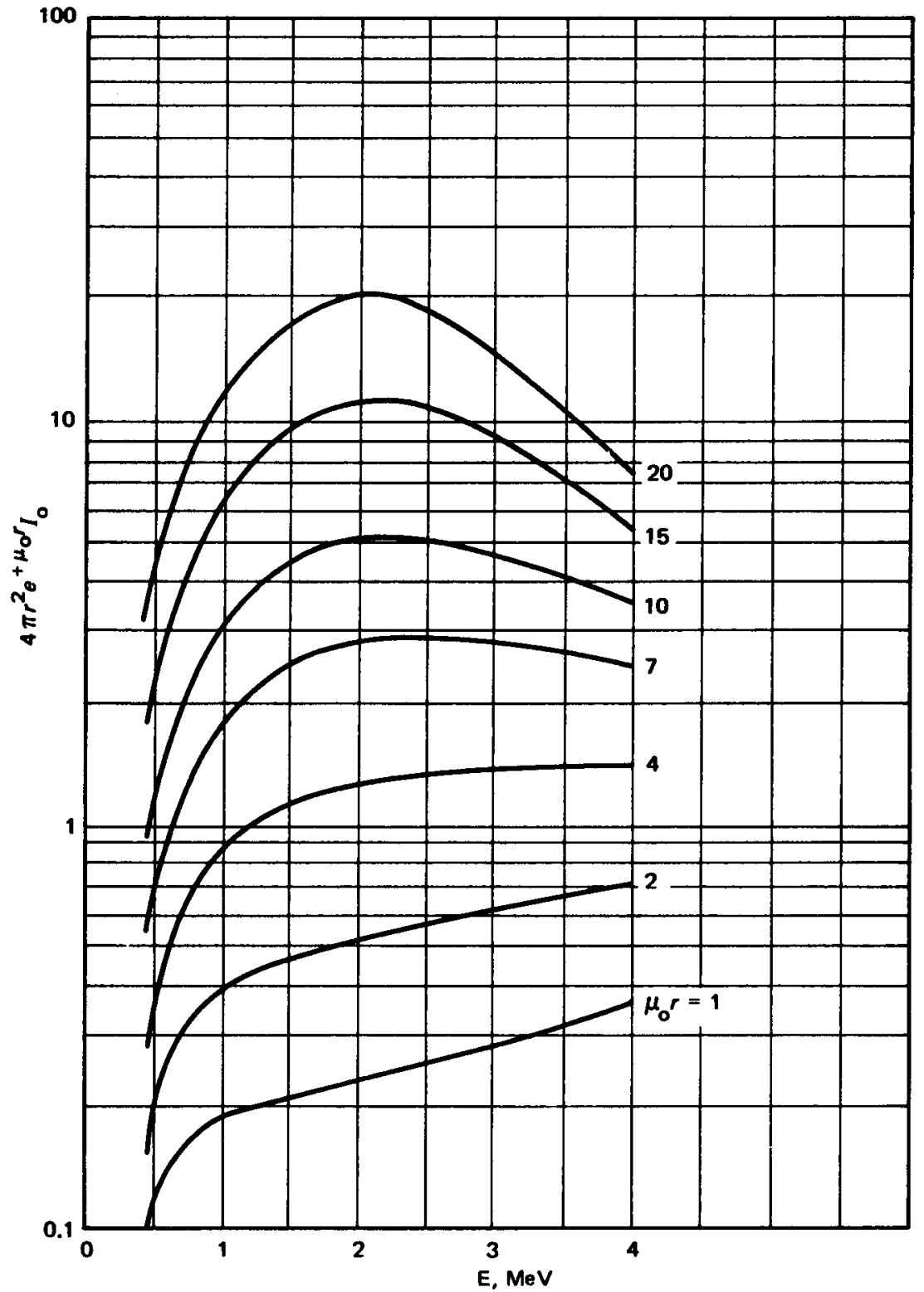

Fig. L.7-Point isotropic 4.0-MeV gamma-ray source in lead. 


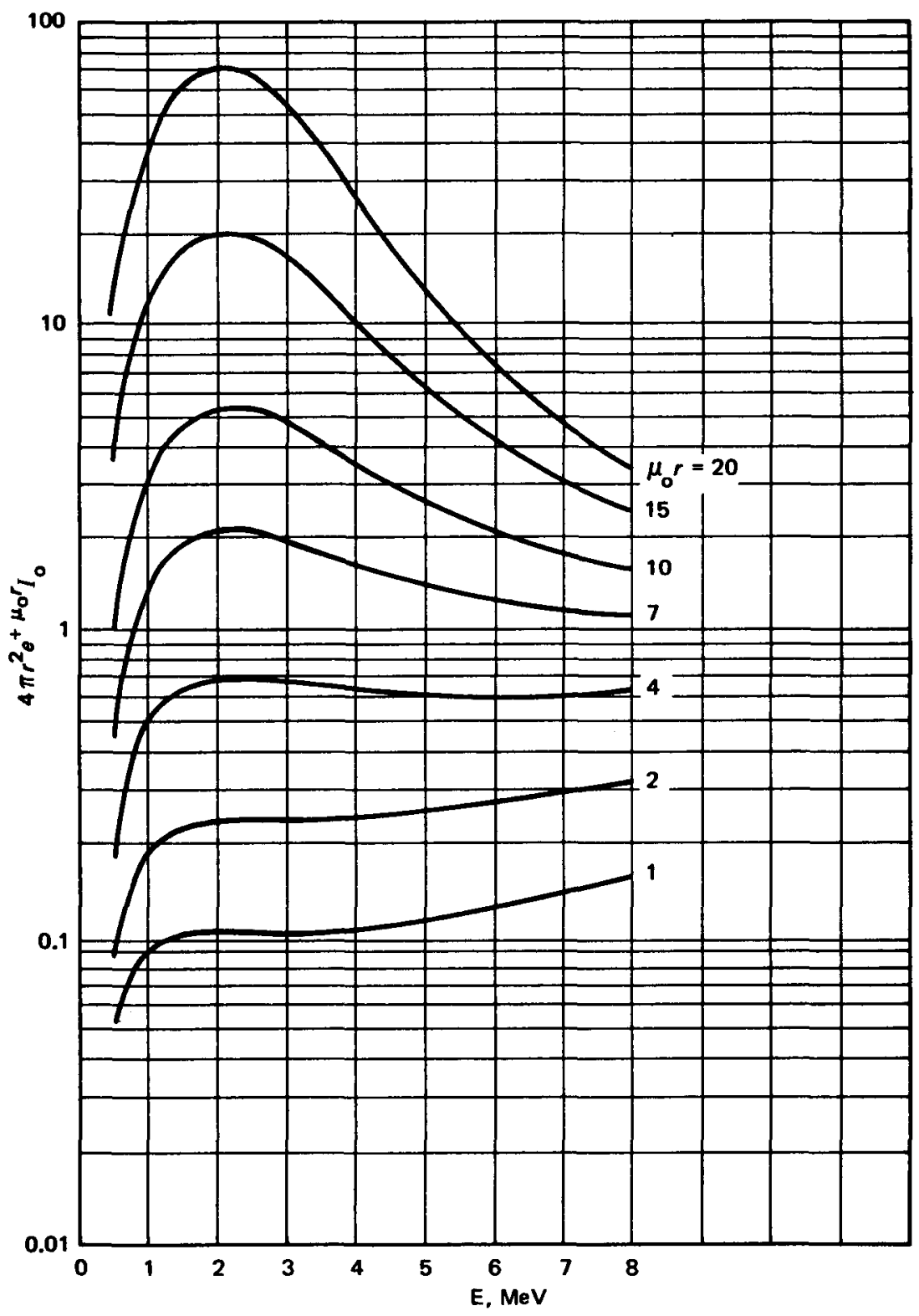

Fig. L.8-Point isotropic 8.0-MeV gamma-ray source in lead. 


\section{Graphs for Neutron Attenuation Calculations}

\section{Appendix M}

This appendix consists of graphs of neutron-penetration results obtained from Monte Carlo calculations by Clark, Betz, and Brown' and by Allen and Futterer. $^{2}$ Table M.1 gives the composition of materials used in the calculations; Figs. M.1 to M.10 show the dose (in ergs/g per incident neutron $/ \mathrm{cm}^{2}$ ) due to monoenergetic beams of neutrons normally incident on ordinary concrete, both on a slab and on a semi-infinite medium (half space). Figures M.11 to M.15 show the neutron-dose transmission factor as a function of polyethylene thickness for monoenergetic neutrons incident at various angles. When the thickness is adjusted according to the key included at the top of each figure, these latter curves apply also to water, to concrete, and to Nevada Test Site soil, both dry and water-saturated.

The use of these curves is explained in Sec. 6.4.3.

\section{REFERENCES}

1. F. H. Clark, N. A. Betz, and J. Brown, Monte Carlo Calculations of the Penetration of Normally Incident Neutron Beams Through Concrete, USAEC Report ORNL-3926, Oak Ridge National Laboratory, January 1967; F. A. R. Schmidt, Revised Neutron Kerma Values at the Boundary of a Semi-Infinite Concrete Medium, USAEC Report ORNL-TM-2284, Oak Ridge National Laboratory, 1968.

2. F. J. Allen and A. T. Futterer, Neutron Transmission Data, Nucleonics, 21(8): 120 (1963). 
Table M.1 - COMPOSITIONS OF MATERIALS USED FOR NEUTRON-TRANSMISSION CALCULATIONS $\dagger$

\begin{tabular}{|c|c|c|c|}
\hline \multirow[b]{2}{*}{ Material } & \multirow{2}{*}{$\begin{array}{l}\text { Density, } \\
\mathrm{g} / \mathrm{cm}^{3}\end{array}$} & \multicolumn{2}{|c|}{ Composition } \\
\hline & & Element & $10^{21}$ atoms $/ \mathrm{cm}^{3}$ \\
\hline $\begin{array}{l}\text { Borated polyethylene } \\
\qquad\left(8 \mathrm{wt} . \% \mathrm{~B}_{\mathbf{4}} \mathrm{C}\right) \ddagger\end{array}$ & 0.97 & $\begin{array}{r}\mathrm{H} \\
\mathrm{C} \\
{ }_{10}{ }^{\mathrm{B}} \mathrm{B} \\
{ }^{11} \mathrm{~B}\end{array}$ & $\begin{array}{c}76.80 \\
39.20 \\
0.658 \\
2.67\end{array}$ \\
\hline Water & 1.00 & $\begin{array}{l}\mathrm{H} \\
\mathrm{O}\end{array}$ & $\begin{array}{l}66.90 \\
33.45\end{array}$ \\
\hline Concrete & 2.26 & $\begin{array}{l}\mathrm{H} \\
\mathrm{O} \\
\mathrm{Al} \\
\mathrm{Si}\end{array}$ & $\begin{array}{c}13.75 \\
45.87 \\
1.743 \\
20.15\end{array}$ \\
\hline $\begin{array}{l}\text { Nevada Test Site } \\
\text { soil (dry) }\end{array}$ & 1.15 & $\begin{array}{l}\mathrm{H} \\
\mathrm{O} \\
\mathrm{Al} \\
\mathrm{Si}\end{array}$ & $\begin{array}{c}8.553 \\
22.68 \\
2.014 \\
9.533\end{array}$ \\
\hline $\begin{array}{l}\text { Nevada Test Site } \\
\quad \text { soil }(100 \% \text { saturated })\end{array}$ & 1.25 & $\begin{array}{l}\mathrm{H} \\
\mathrm{O} \\
\mathrm{Al} \\
\mathrm{Si}\end{array}$ & $\begin{array}{c}16.87 \\
27.00 \\
1.976 \\
8.963\end{array}$ \\
\hline
\end{tabular}

†From F. J. Allen and A. T. Futterer, Nucleonics, 21(8): 120 (1963). \# Several calculations made for pure polyethylene slabs $\left(\rho=0.925 \mathrm{~g} / \mathrm{cm}^{3}\right)$ up to 6 in. thick yielded approximately the same neutron transmission results as those for the borated polyethylene. 


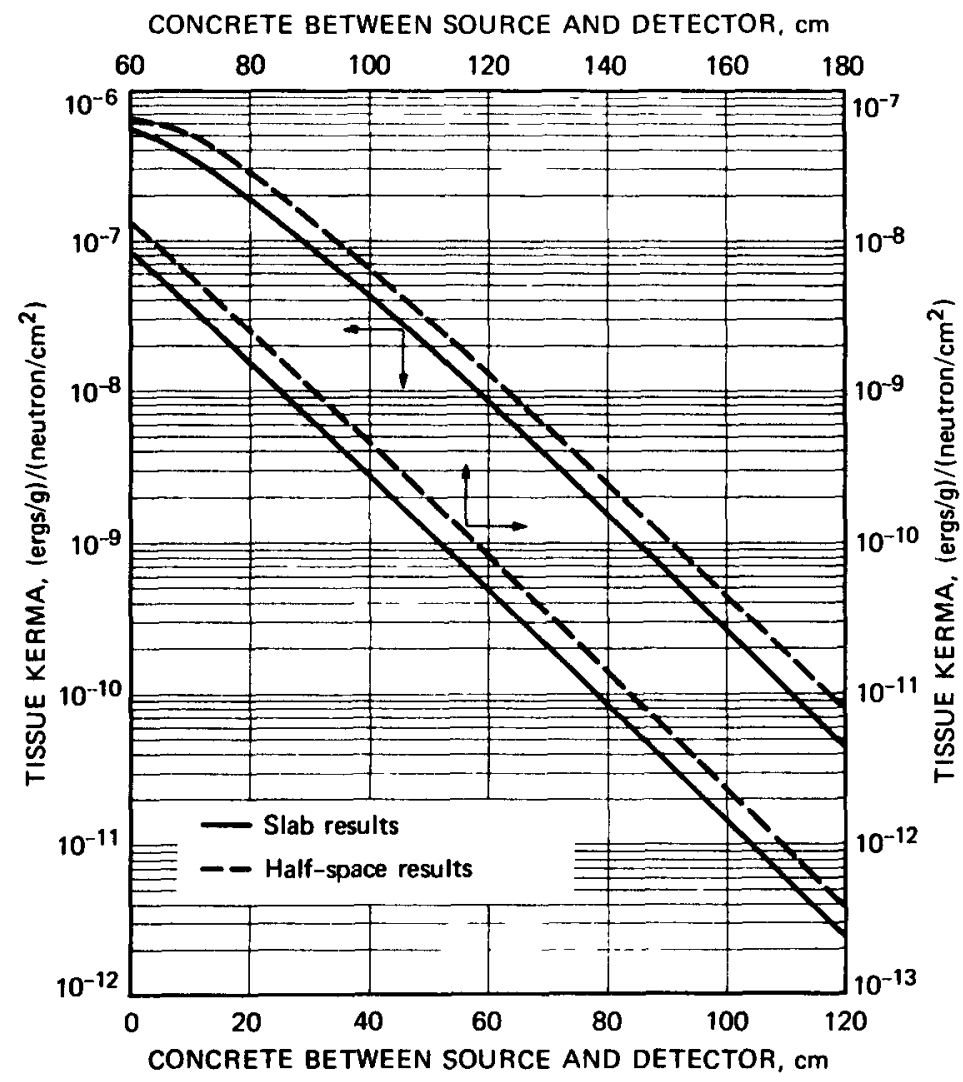

Fig. M.1-Transmission of $14-\mathrm{MeV}$ normally incident neutrons in ordinary concrete. (From Clark et al. ${ }^{1}$ ) 


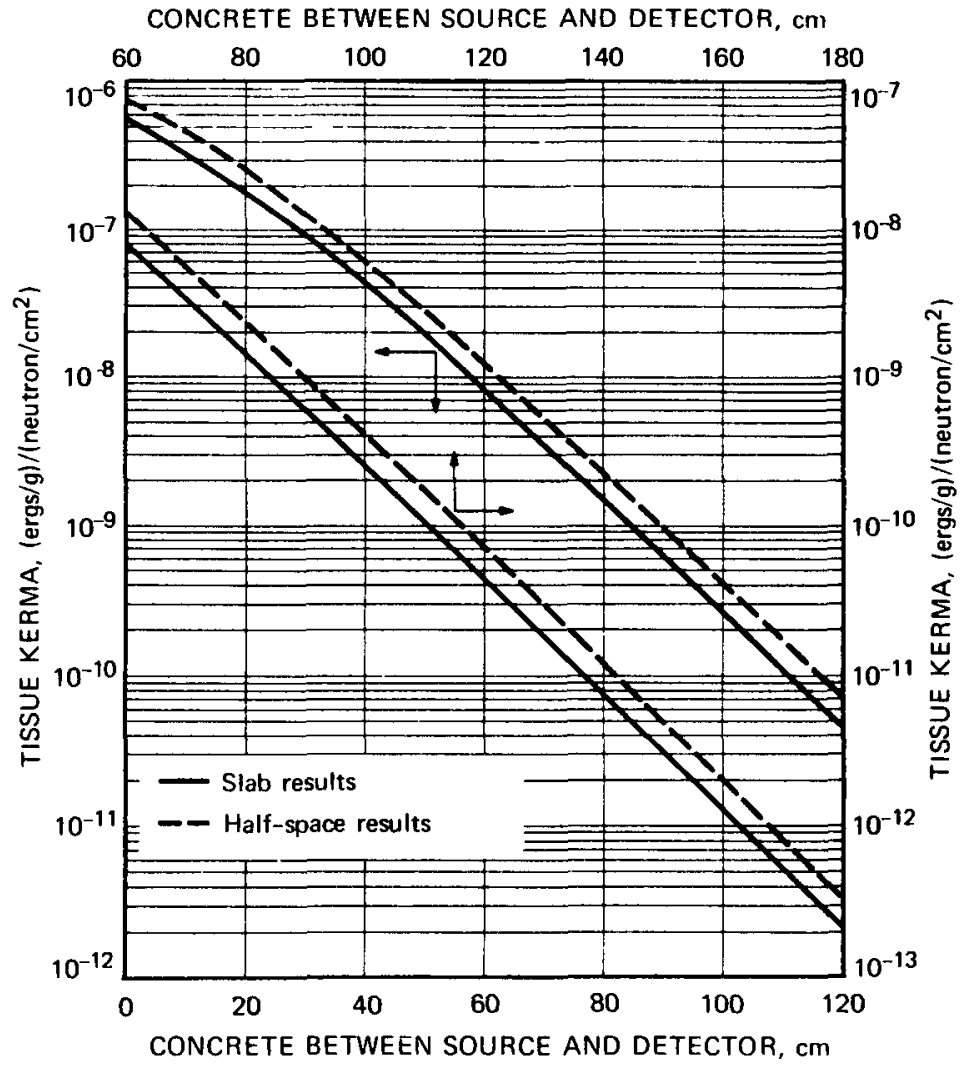

Fig. M.2-Transmission of $12-\mathrm{MeV}$ normally incident neutrons in ordinary concrete. (From Clark et al. ${ }^{1}$ ) 


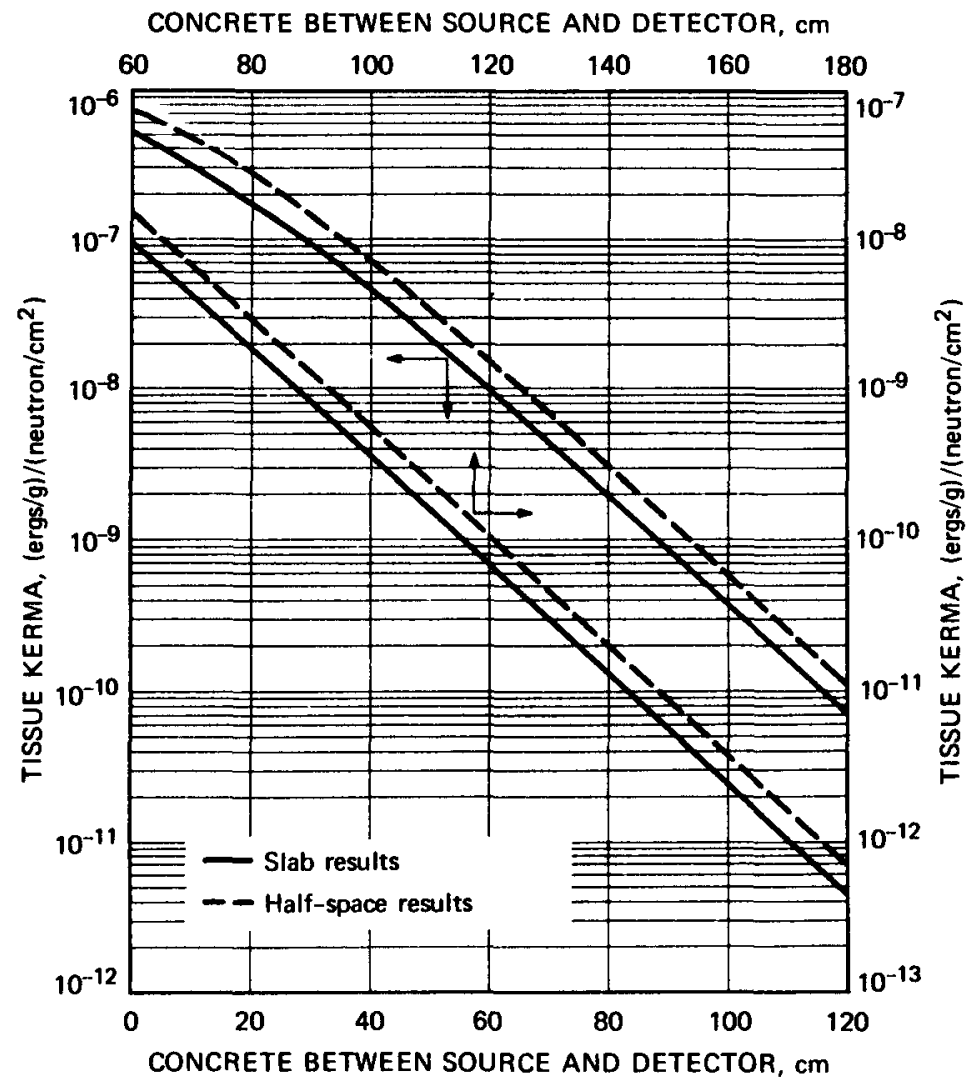

Fig. M.3-Transmission of $10-\mathrm{MeV}$ normally incident neutrons in ordinary concrete. (From Clark et al. ${ }^{1}$ ) 


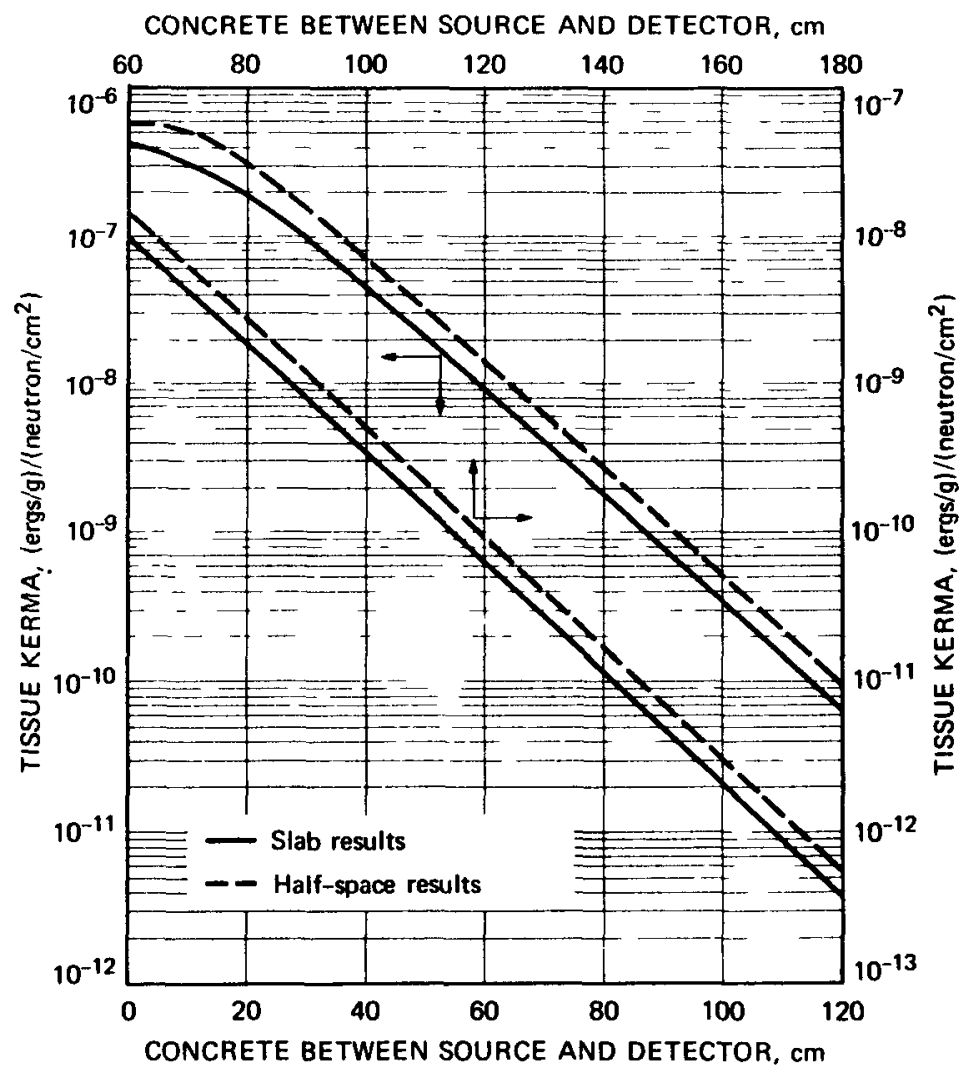

Fig. M.4-Transmission of 8-MeV normally incident neutrons in ordinary concrete. (From Clark et al. ${ }^{1}$ ) 


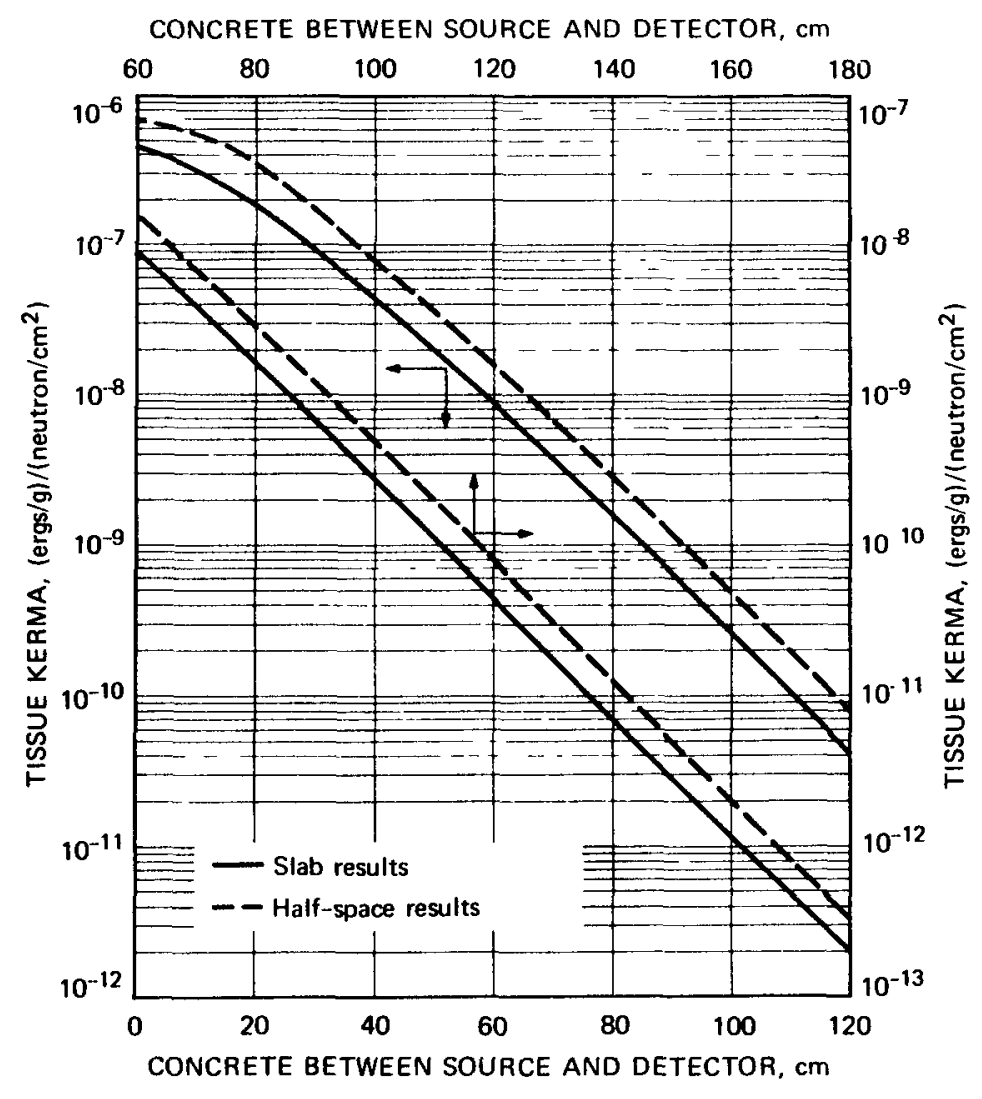

Fig. M.5-Transmission of 6-MeV normally incident neutrons in ordinary concrete. (From Clark et al. ${ }^{1}$ ) 


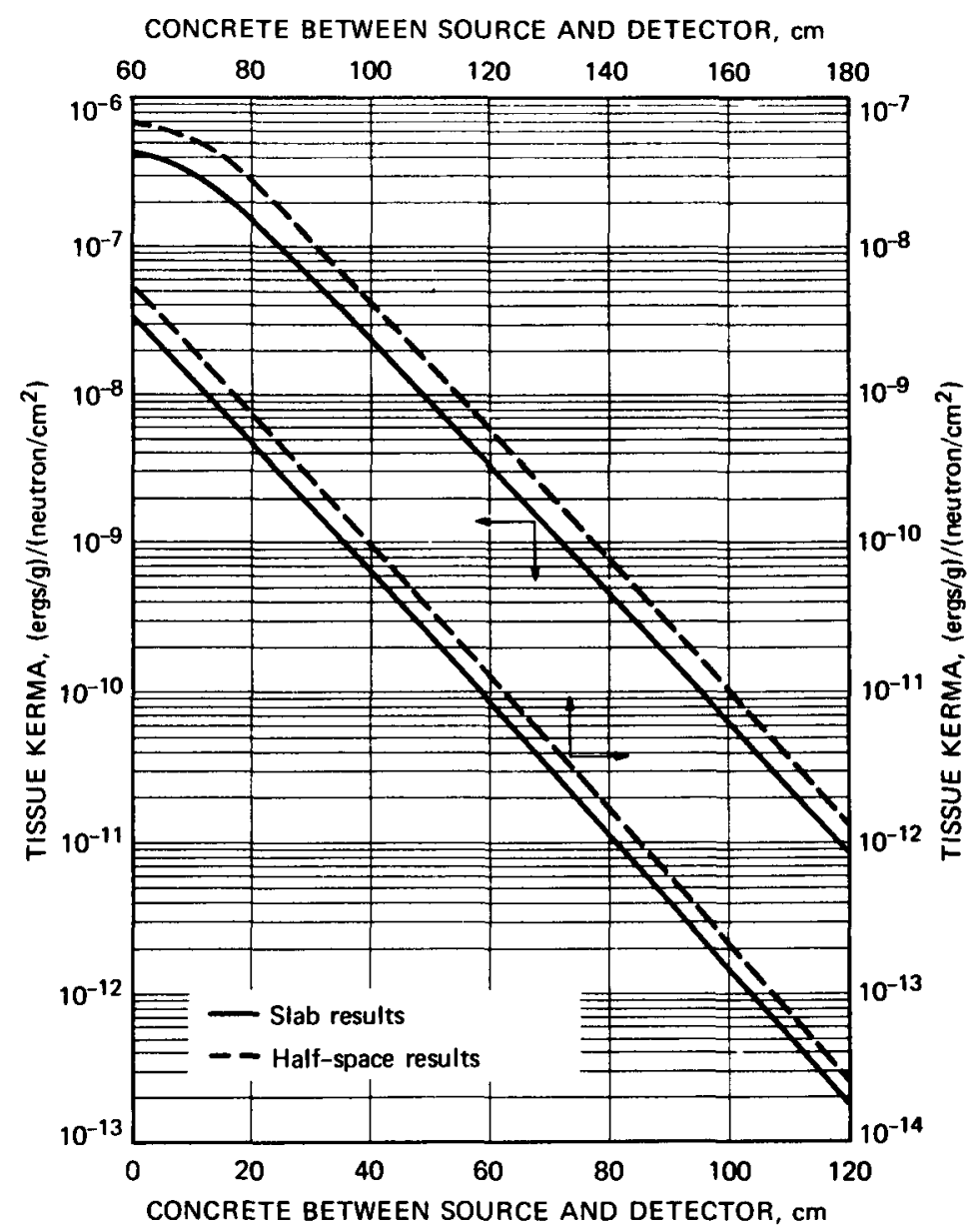

Fig. M.6-Transmission of 4-MeV normally incident neutrons in ordinary concrete. (From Clark et al. ${ }^{1}$ ) 


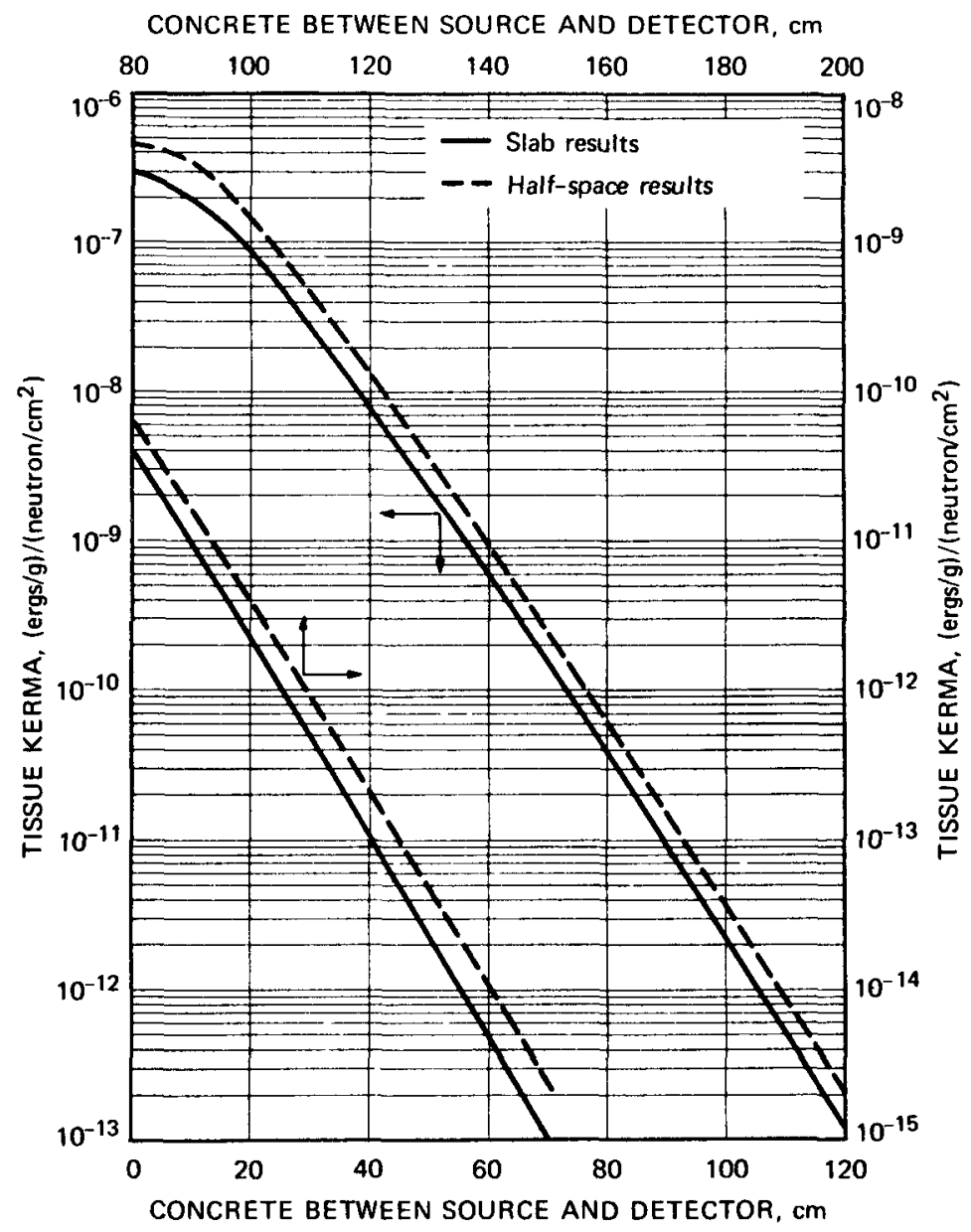

Fig. M.7-Transmission of 3-MeV normally incident neutrons in ordinary concrete. (From Clark et al. ${ }^{1}$ ) 


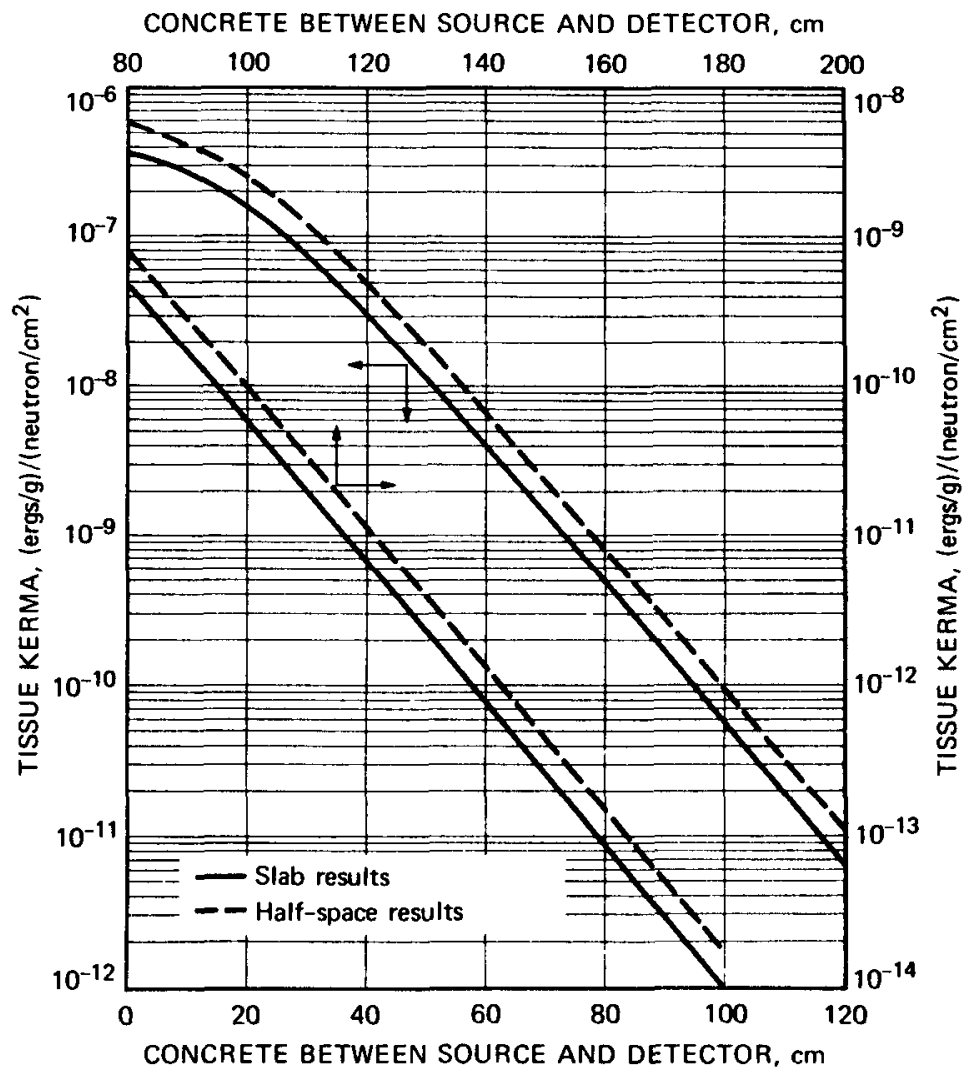

Fig. M.8-Transmission of 2-MeV normally incident neutrons in ordinary concrete. (From Clark et al. ${ }^{1}$ ) 


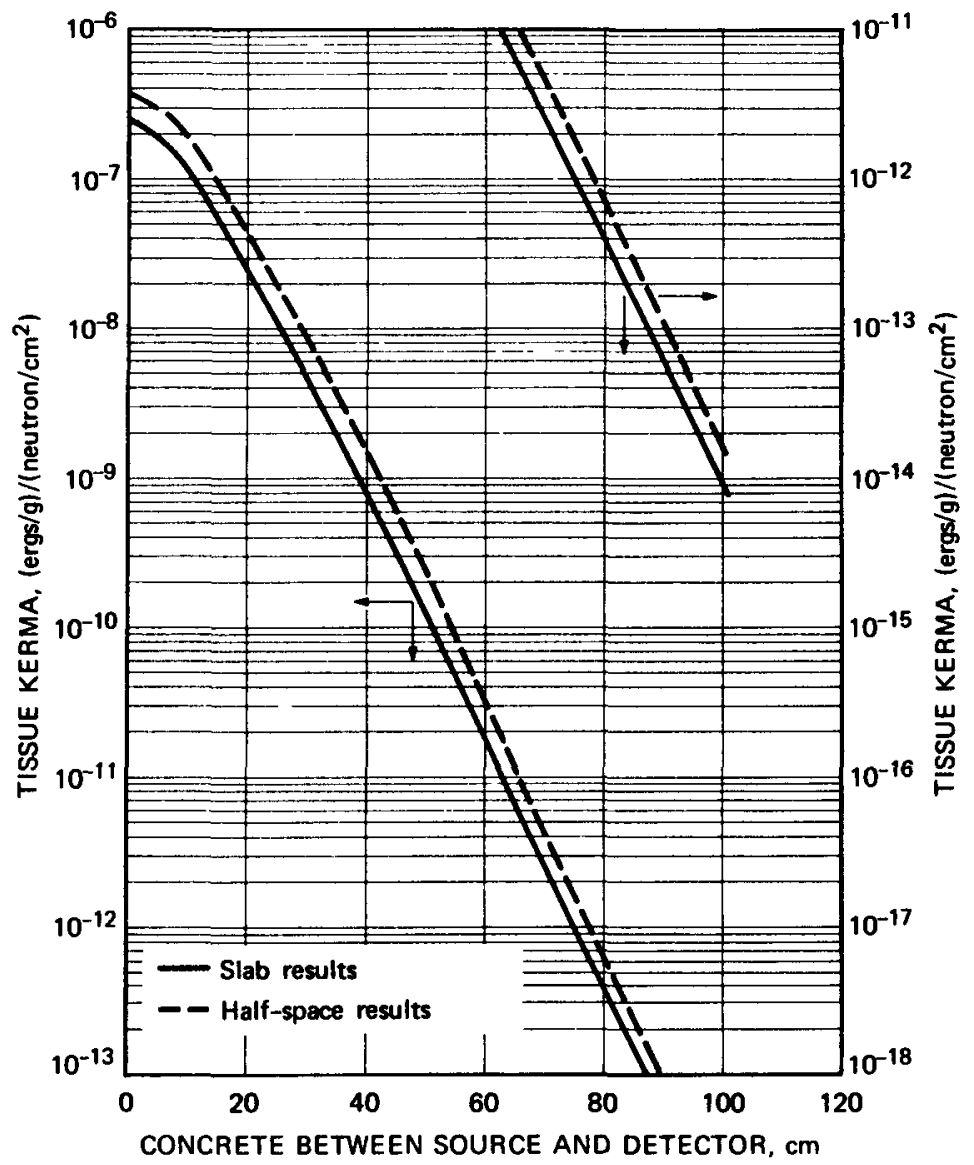

Fig. M.9-Transmission of $1.3-\mathrm{MeV}$ normally incident neutrons in ordinary concrete. (From Clark et al. ${ }^{1}$ ) 


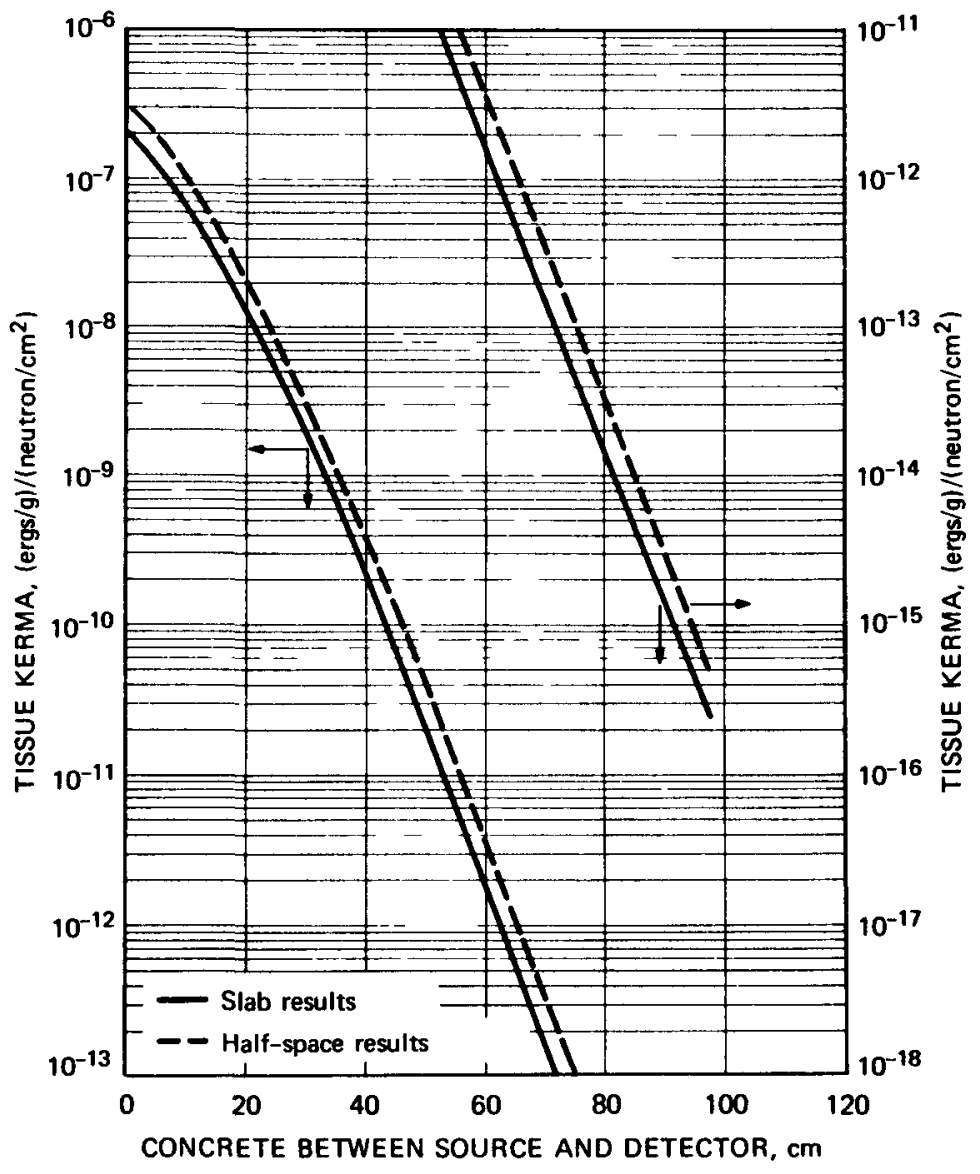

Fig. M.10-Transmission of $0.7-\mathrm{MeV}$ normally incident neutrons in ordinary concrete. (From Clark et al. ${ }^{1}$ ) 


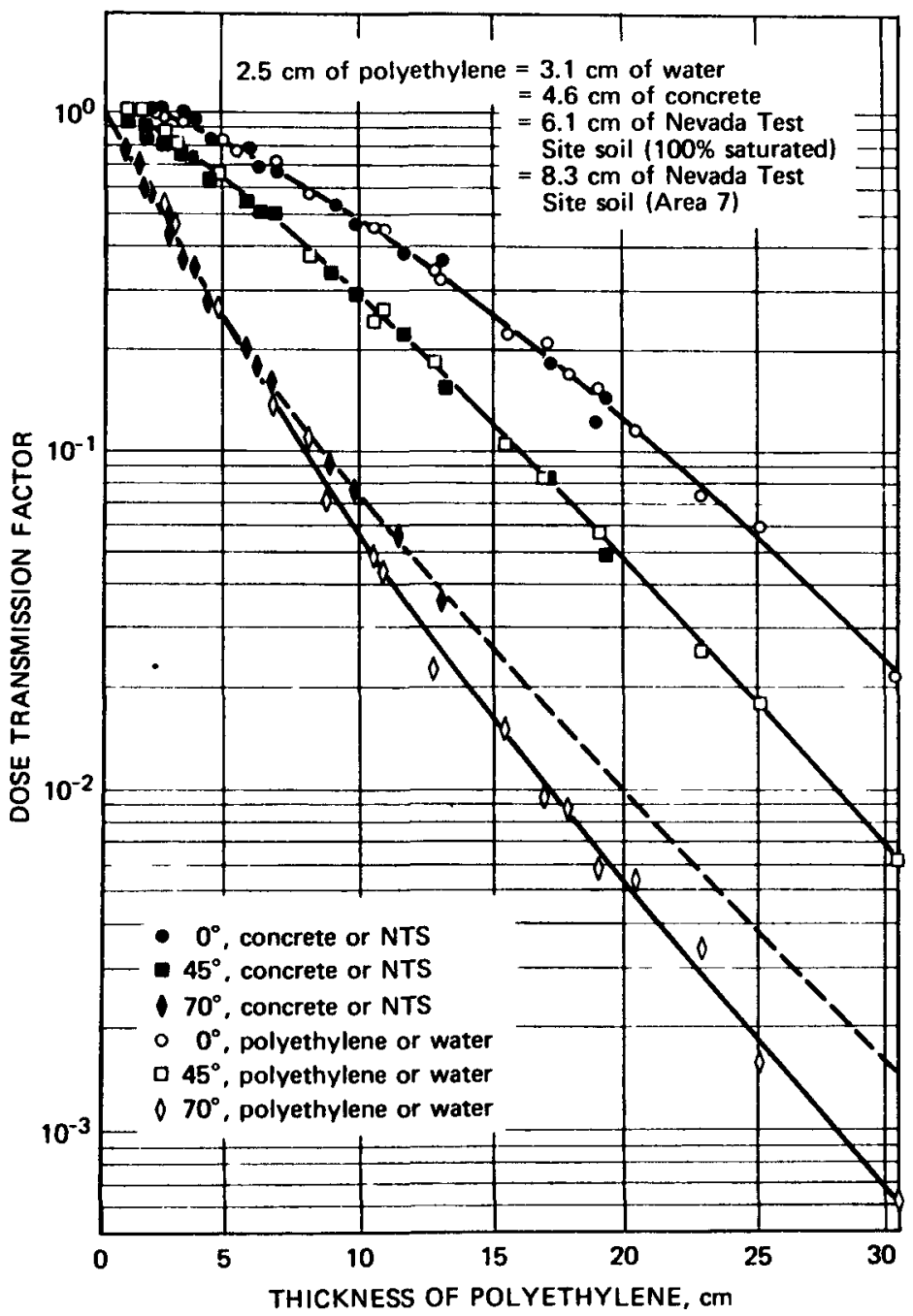

Fig. M.11-Penetration of 5-MeV neutrons incident on several materials at various angles. (Based on data from Ref. 2.) 


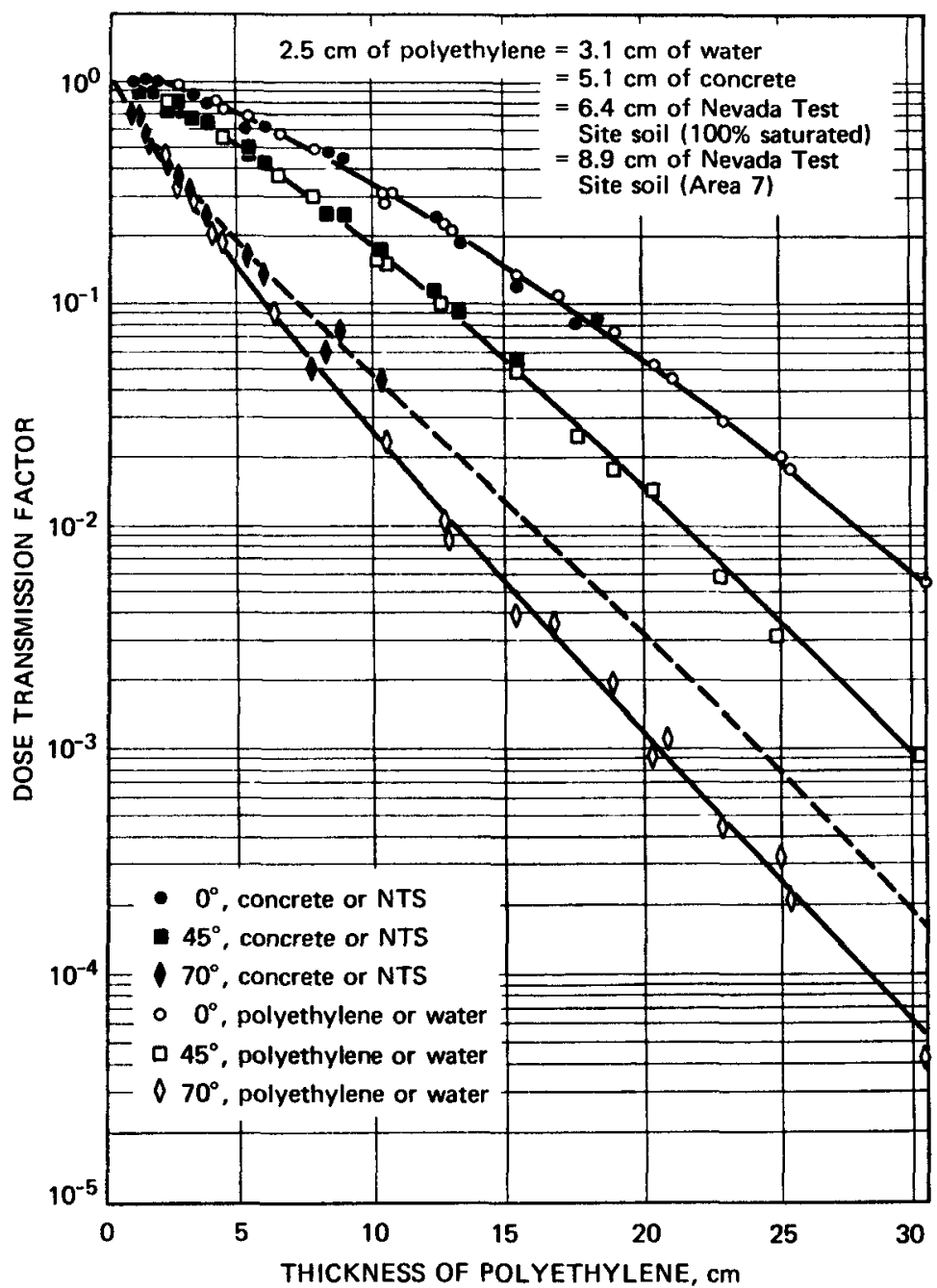

Fig. M.12-Penetration of 3-MeV neutrons incident on several materials at various angles. (Based on data from Ref. 2.) 


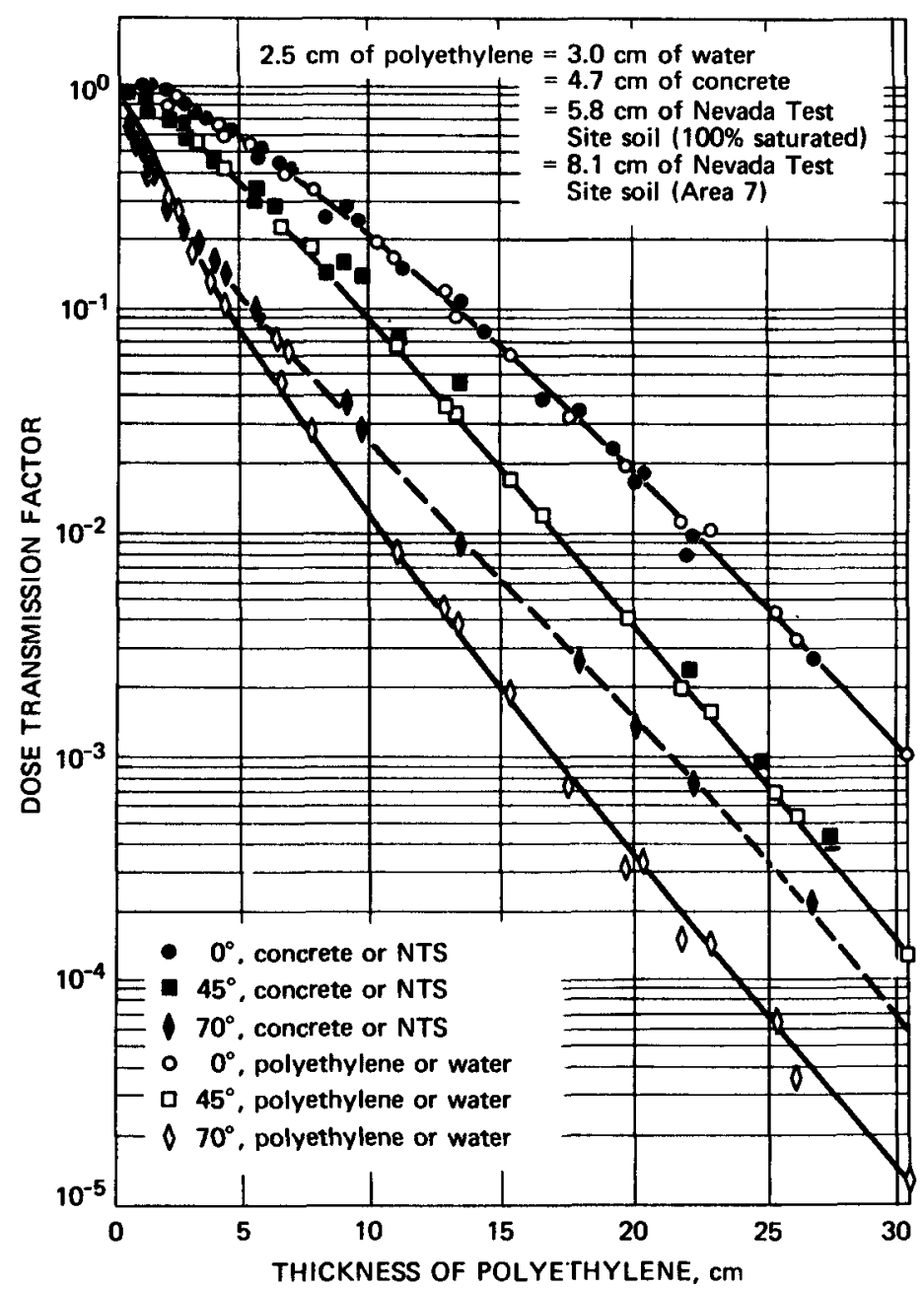

Fig. M.13-Penetration of 2-MeV neutrons incident on several materials at various angles. (Based on data from Ref. 2.) 


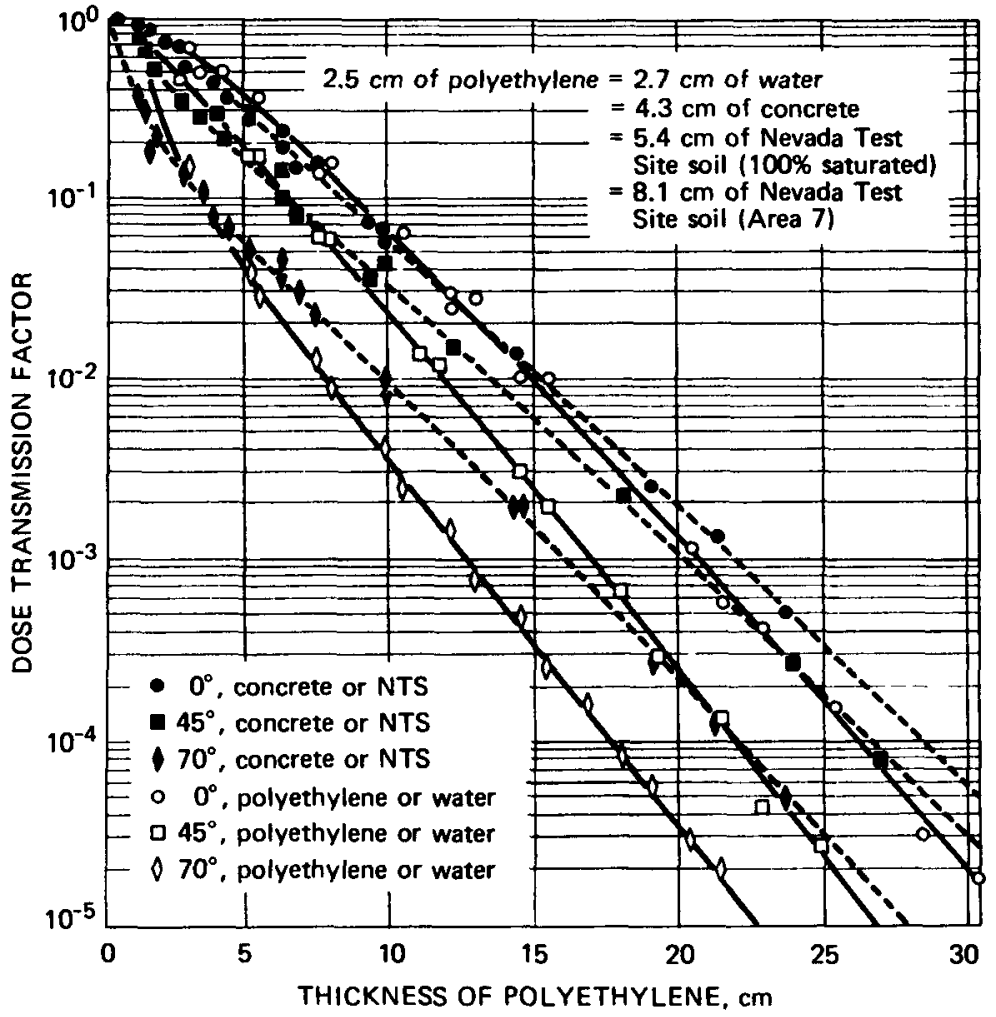

Fig. M.14-Penetration of $1-\mathrm{MeV}$ neutrons incident on several materials at various angles. (Based on data from Ref. 2.) 


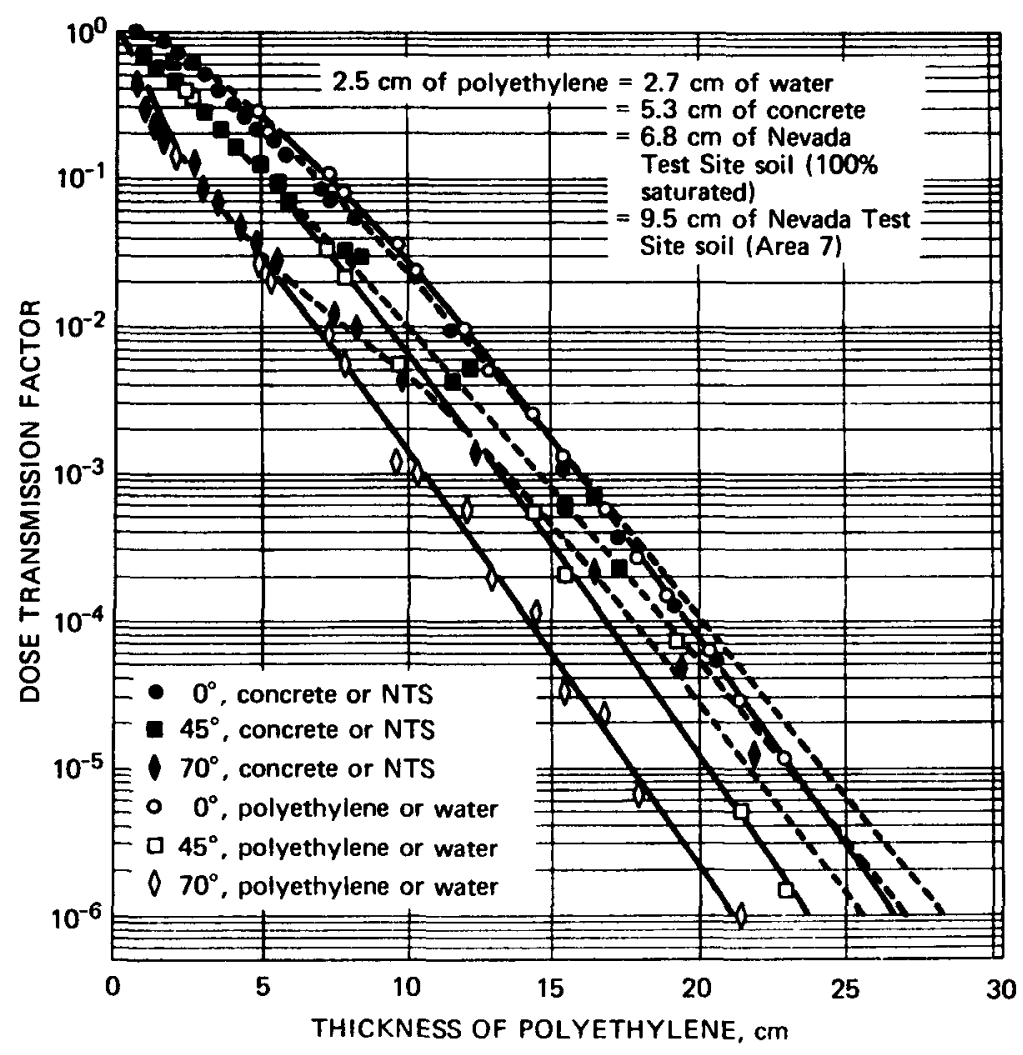

Fig. M.15-Penetration of $0.5-\mathrm{MeV}$ neutrons incident on several materials at various angles. (Based on data from Ref. 2.) 


\section{Graphs of the $\psi$ Function}

Appendix N

A useful function in calculating the shield penetration of the uncollided secondary gamma-ray flux is the $\psi_{0}$ function, defined in Sec. 6.5. The $\psi_{n}$ functions $(n>0)$ can be used to calculate the scattered flux. Values of these functions have been given by Schmidt ${ }^{1}$ for a slab shield and by Claiborne ${ }^{2}$ for a semi-infinite shield. The functions are plotted in Figs. N.1 to N.6, all of which are taken from a compilation of Schmidt. ${ }^{1}$

\section{REFERENCES}

1. Fritz A. R. Schmidt, The Attenuation Properties of Concrete for Shielding of Neutrons of Energy Less than $15 \mathrm{MeV}$, USAEC Report ORNL-RSIC-26, Oak Ridge National Laboratory, August 1970.

2. H. C. Claiborne, Analytical Solutions for Heat Generation Distributions in Regular Geometries, in Engineering Compendium on Radiation Shielding, Article 7.3, Springer-Verlag, New York, 1968. 


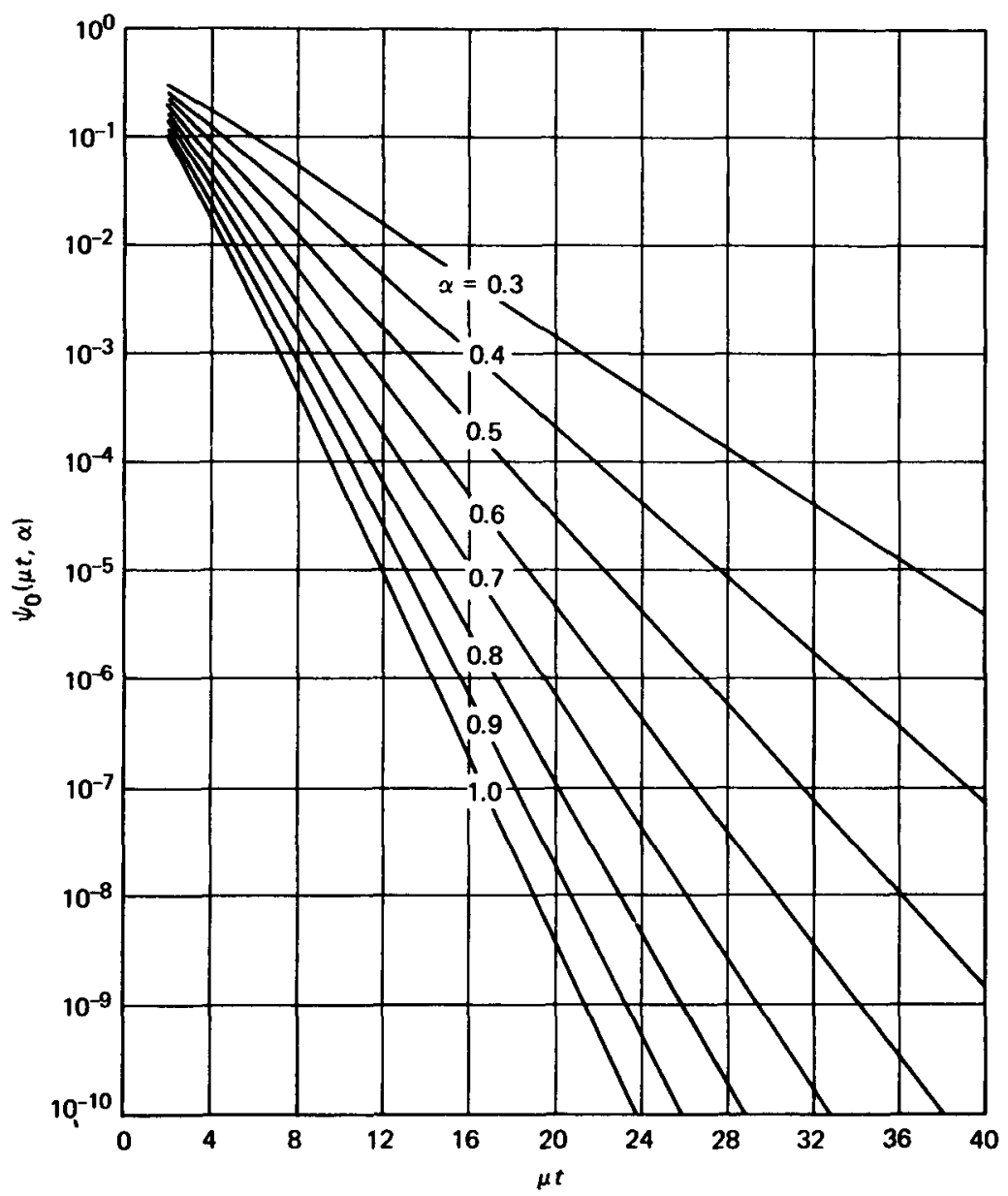

Fig. N.1a-The function $\psi_{0}(\mu t, \alpha)$ for $\alpha \leqslant 1$ for a slab shield. (From Schmidt. ${ }^{1}$ ) 


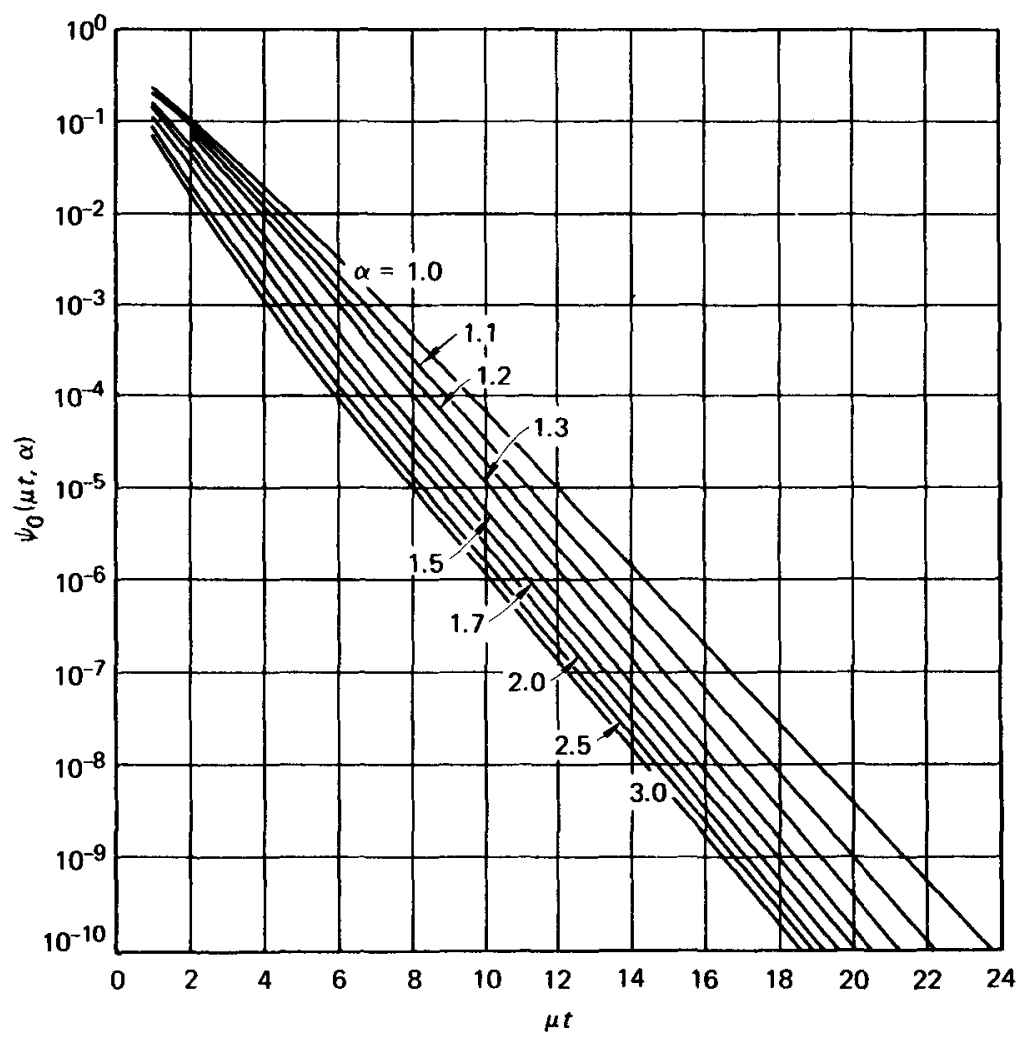

Fig. N.1b-The function $\psi_{0}(\mu t, \alpha)$ for $\alpha \geqslant 1$ for a slab shield. (From Schmidt. ${ }^{1}$ ) 


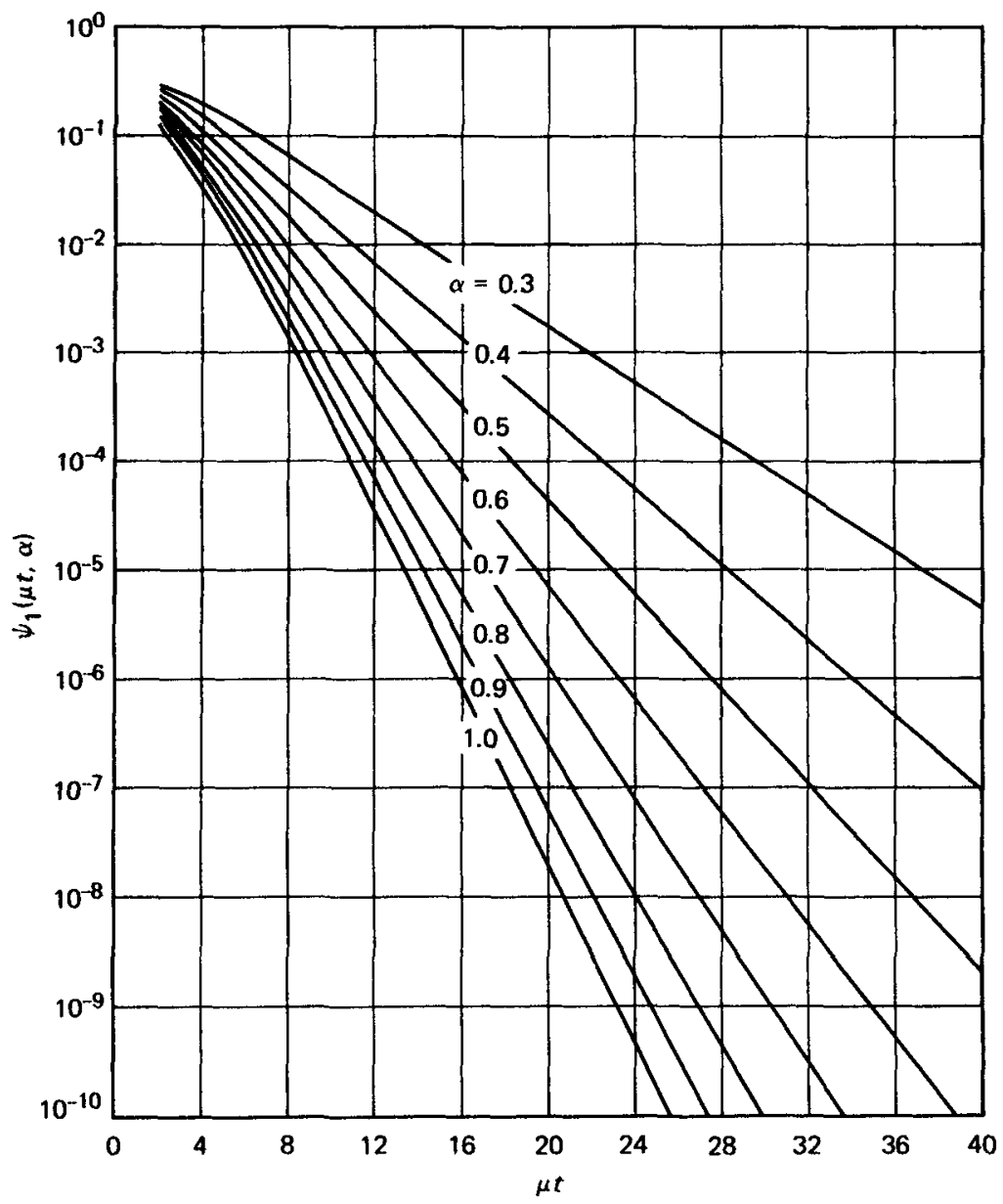

Fig. N.2a-The function $\psi_{1}(\mu t, \alpha)$ for $\alpha \leqslant 1$ for a slab shield. (From Schmidt. ${ }^{\prime}$ ) 


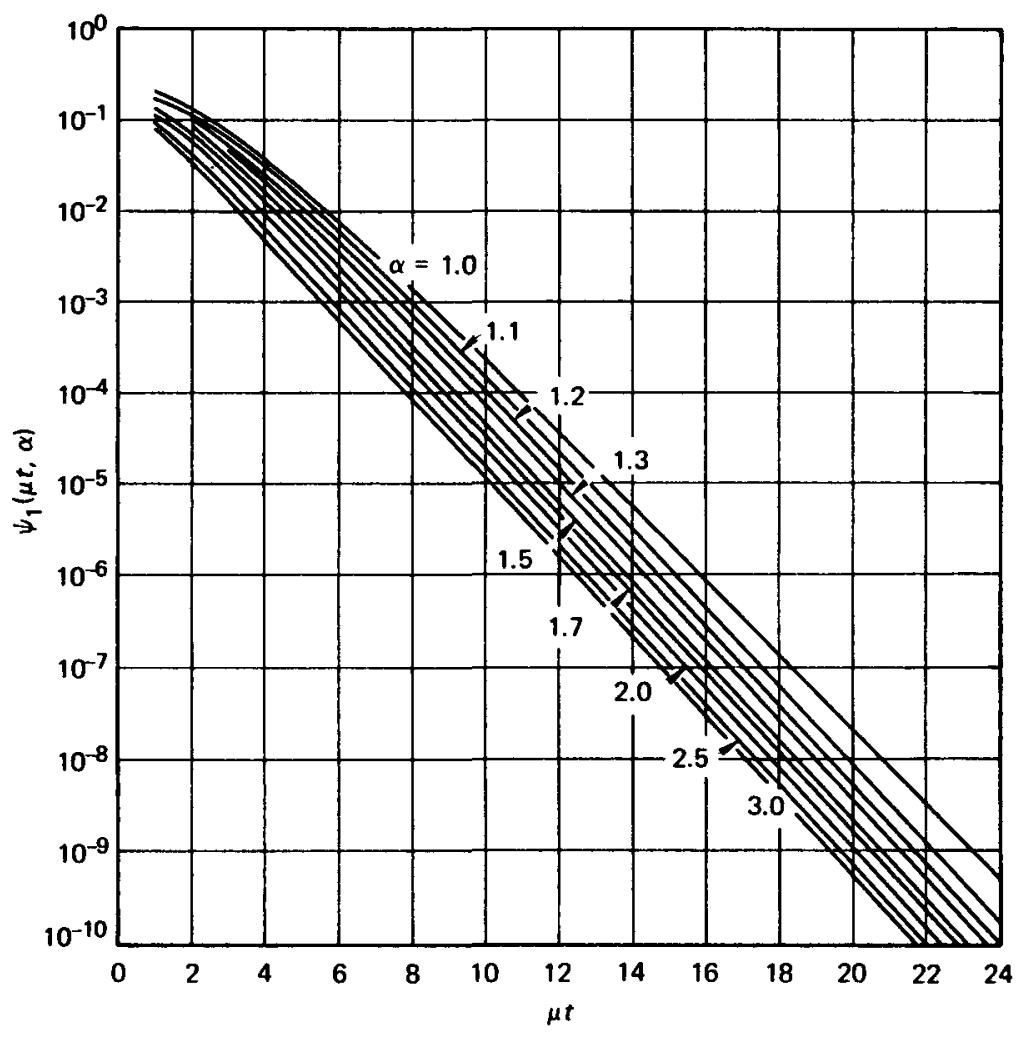

Fig. N.2b-The function $\psi_{1}(\mu t, \alpha)$ for $\alpha \geqslant 1$ for a slab shield. (From Schmidt. ${ }^{1}$ ) 


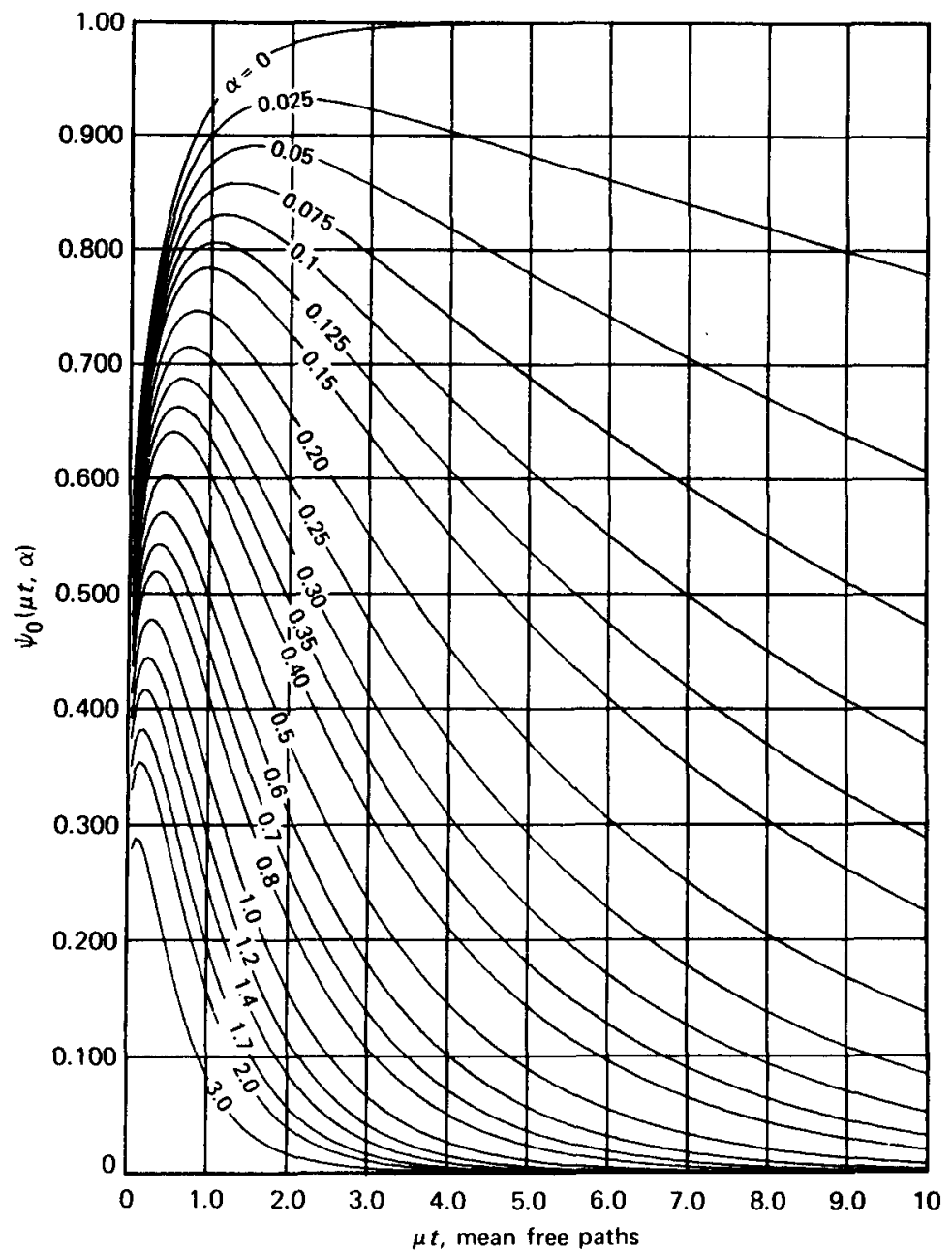

Fig. N.3-The function $\psi_{0}(\mu t, \alpha)$ for a semi-infinite shield. (From H. C. Claiborne, Engineering Compendium on Radiation Shielding, p. 447, Springer-Verlag, New York, 1968.) 


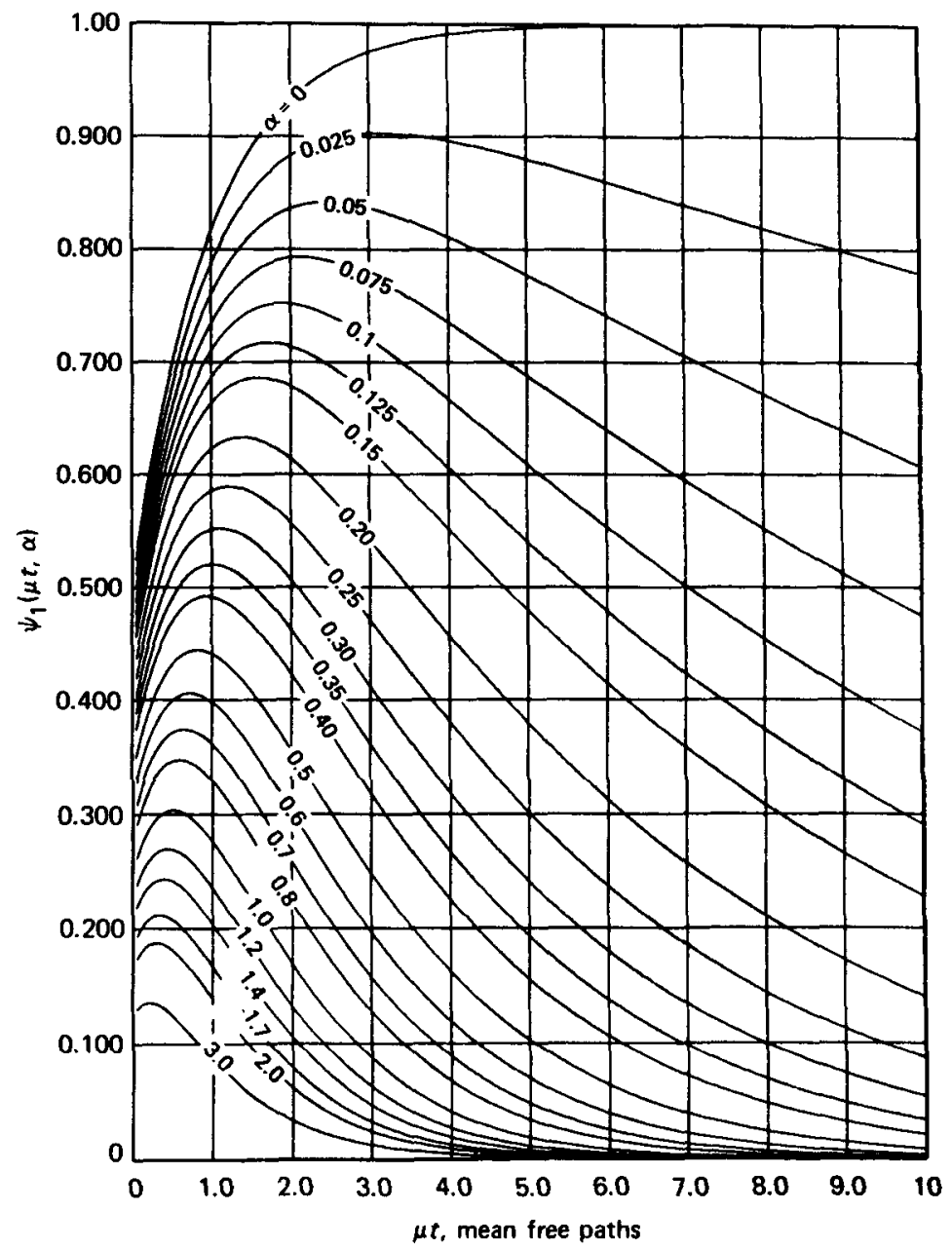

Fig. N.4-The function $\psi_{1}(\mu t, \alpha)$ for a semi-infinite shield. (From H. C. Claiborne, Engineering Compendium on Radiation Shielding, p. 448, Springer-Verlag, New York, 1968.) 


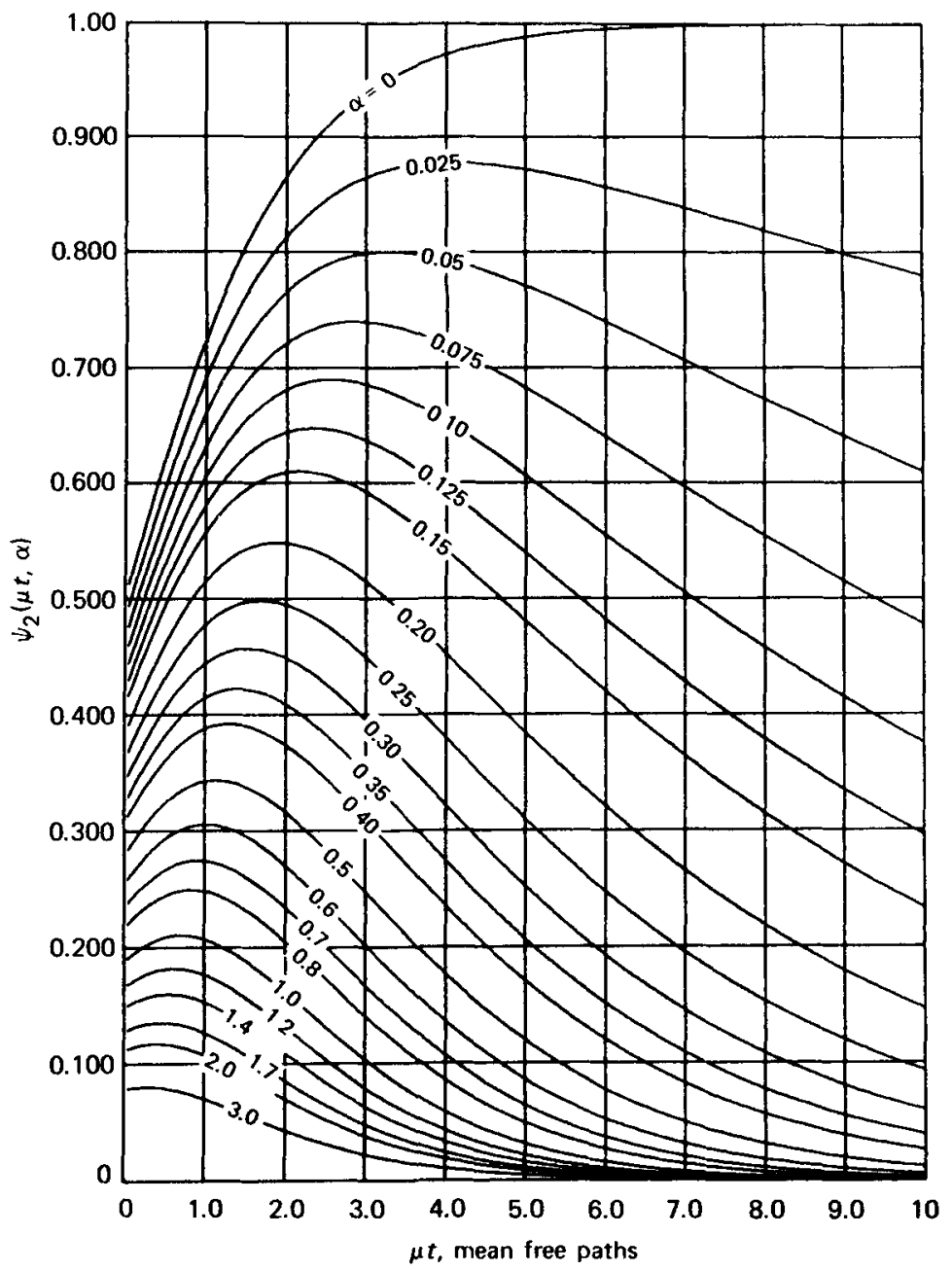

Fig. N.5-The function $\psi_{2}(\mu t, \alpha)$ for a semi-infinite shield. (From H. C. Claiborne, Engineering Compendium on Radiation Shielding, p. 448, Springer-Verlag, New York, 1968.) 


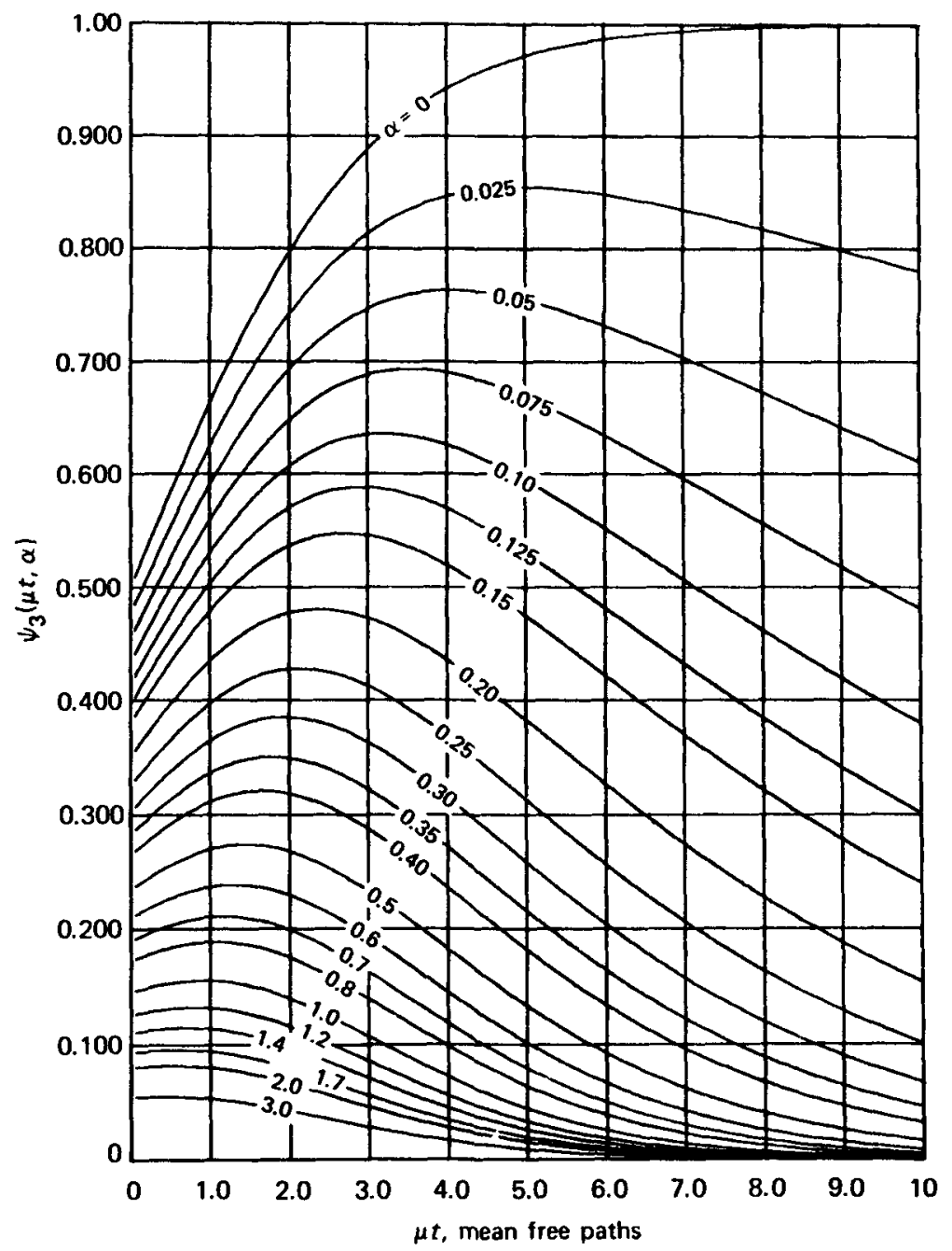

Fig. N.6-The function $\psi_{3}(\mu t, \alpha)$ for a semi-infinite shield. (From H. C. Claiborne, Engineering Compendium on Radiation Shielding, p. 448, Springer-Verlag, New York, 1968.) 


\section{Constants for Empirical Expressions of Albedo Data}

\section{Appendix 0}

Table 0.1-CONSTANTS FOR THE EXPRESSION † FITTING THE MAERKER AND MUCKENTHALER DIFFERENTIAL DOSE ALBEDO DATA FOR FAST NEUTRONS INCIDENT ON CONCRETE

\begin{tabular}{|c|c|c|c|c|c|c|}
\hline \multirow[b]{2}{*}{ Constant } & \multicolumn{6}{|c|}{ Value of constant for $\Delta E_{0}$ of } \\
\hline & $\begin{array}{c}0.2 \text { to } 0.75 \\
\mathrm{MeV}\end{array}$ & $\begin{array}{c}0.75 \text { to } 1.5 \\
\mathrm{MeV}\end{array}$ & $\begin{array}{c}1.5 \text { to } 3 \\
\mathrm{MeV}\end{array}$ & $\begin{array}{l}3 \text { to } 4 \\
\text { MeV }\end{array}$ & $\begin{array}{l}4 \text { to } 6 \\
\mathrm{MeV}\end{array}$ & $\begin{array}{l}6 \text { to } 8 \\
\mathrm{MeV}\end{array}$ \\
\hline $\mathrm{G}_{\mathbf{0}}$ & $6.585(-2) \S$ & $7.045(-2)$ & $7.211(-2)$ & $7.024(-2)$ & $6.856(-2)$ & $5.899(-2)$ \\
\hline$G_{1}$ & $5.048(-2)$ & $4.393(-2)$ & $5.845(-2)$ & $7.452(-2)$ & $8.294(-2)$ & $6.039(-2)$ \\
\hline $\mathbf{G}_{2}$ & $3.710(-2)$ & $7.088(-2)$ & $5.968(-2)$ & $1.000(-1)$ & $9.517(-2)$ & $7.524(-2)$ \\
\hline $\mathrm{G}_{3}$ & $1.544(-2)$ & $1.898(-2)$ & $2.729(-2)$ & $5.591(-2)$ & $7.761(-2)$ & $8.140(-2)$ \\
\hline $\mathrm{G}_{4}$ & $7.837(-3)$ & $2.408(-3)$ & $1.190(-2)$ & $2.646(-2)$ & $4.292(-2)$ & $6.622(-2)$ \\
\hline $\mathrm{G}_{5}$ & 0 & $-3.589(-3)$ & $1.000(-3)$ & $-6.908(-4)$ & $1.824(-2)$ & $3.056(-2)$ \\
\hline $\mathrm{G}_{6}$ & 0 & 0 & $4.637(-3)$ & $-8.087(-4)$ & $5.599(-3)$ & $1.595(-2)$ \\
\hline $\mathrm{G}_{7}$ & 0 & 0 & $6.490(-3)$ & $-1.459(-3)$ & $5.288(-3)$ & $1.277(-2)$ \\
\hline $\mathrm{G}_{\mathbf{8}}$ & 0 & 0 & 0 & $-1.809(-3)$ & $1.046(-2)$ & $9.380(-3)$ \\
\hline $\mathbf{B}_{\mathbf{0}}$ & $6.27(-2)$ & $9.00(-2)$ & $8.80(-2)$ & $9.05(-2)$ & $8.744(-2)$ & $6.374(-2)$ \\
\hline$B_{1}$ & $1.50(-2)$ & $8.5(-3)$ & $1.30(-2)$ & $2.15(-2)$ & $2.817(-2)$ & $1.382(-2)$ \\
\hline $\mathbf{B}_{2}$ & $5.3(-3)$ & $9.7(-3)$ & $6.0(-3)$ & $2.30(-2)$ & $2.344(-2)$ & $1.178(-2)$ \\
\hline $\mathbf{B}_{3}$ & 0 & 0 & 0 & 0 & $1.779(-2)$ & $1.084(-2)$ \\
\hline $\mathbf{B}_{4}$ & 0 & 0 & 0 & 0 & $8.517(-3)$ & $6.801(-3)$ \\
\hline $\mathbf{K}_{1}$ & 1.0 & 1.0 & 1.1 & 0.9 & 1.1 & 1.06 \\
\hline$a_{00}$ & 0.36 & 0.51 & 0.56 & 0.60 & 0.43 & 0.35 \\
\hline$a_{01}$ & 1.29 & 0.32 & 0.18 & 0.15 & 2.02 & 0.95 \\
\hline 202 & 0 & 1.00 & 1.32 & 0.48 & -0.38 & 0 \\
\hline$a_{10}$ & 0.06 & -0.04 & -0.14 & -0.61 & 0.05 & 0.10 \\
\hline$a_{11}$ & -3.06 & -2.46 & -2.76 & -1.08 & -9.13 & -2.28 \\
\hline$a_{12}$ & 0 & 0 & 0 & 0 & 5.93 & 1.11 \\
\hline 220 & -0.20 & 0.05 & 0.05 & 0.32 & 0.04 & 0 \\
\hline$a_{21}$ & 1.68 & 0.95 & 1.14 & 0.30 & 5.97 & 0 \\
\hline$a_{22}$ & 0 & 0 & 0 & 0 & -4.39 & 0 \\
\hline
\end{tabular}

tEquation 7.3-1.

\$From R. E. Maerker and F. J. Muckenthaler, Calculation and Measurement of the Fast-Neutron Differential Dose Albedo for Concrete, Nucl. Sci. Eng., 22: 455 (1965).

§Read: $6.585 \times 10^{-2}$, etc. 
Table 0.2-COMPOSITIONS OF SOIL, IRON, AND POLYETHYLENE USED IN MONTE CARLO CALCULATIONS †

\begin{tabular}{|c|c|c|c|c|c|}
\hline \multirow[b]{3}{*}{ Element } & \multicolumn{5}{|c|}{ Composition (in units of $10^{21}$ atoms $/ \mathrm{cm}^{3}$ ) } \\
\hline & \multicolumn{3}{|c|}{ Nevada Test Site soil } & \multirow[b]{2}{*}{ Iron } & \multirow[b]{2}{*}{$\begin{array}{l}8 \% \text { borated } \\
\text { polyethylene }\end{array}$} \\
\hline & Dry & $\begin{array}{c}50 \% \\
\text { saturated }\end{array}$ & $\begin{array}{c}100 \% \\
\text { saturated }\end{array}$ & & \\
\hline Boron-10 & & & & & 0.658 \\
\hline Boron-11 & & & & & 2.67 \\
\hline Hydrogen & 8.553 & 9.80 & 16.87 & & 76.8 \\
\hline Carbon & & & & & 39.2 \\
\hline Oxygen & 22.68 & 23.30 & 27.00 & & \\
\hline Aluminum & 2.014 & 1.830 & 1.976 & & \\
\hline Silicon & 9.533 & 8.680 & 8.963 & & \\
\hline Iron & & & & 84.9 & \\
\hline Density, & & & & & \\
\hline $\mathrm{g} / \mathrm{cm}^{3}$ & 1.15 & 1.12 & 1.25 & 7.88 & 0.97 \\
\hline
\end{tabular}

†From F. J. Allen, A. Futterer, and W. Wright, Dependence of Neutron Albedos Upon Hydrogen Content of a Shield, Report BRL-1224, Ballistic Research Laboratories, October 1963. 
Table 0.3-VALUES OF THE CONSTANT $k\left(E_{0}\right) \dagger$ FOR THE EXPRESSION FITTING THE DIFFERENTIAL-DOSE-ALBEDO DATA OF ALLEN et al $\ddagger$ FOR FAST NEUTRONS INCIDENT ON VARIOUS MATERIALS $§$

\begin{tabular}{|c|c|c|c|c|c|c|c|c|c|}
\hline \multirow[b]{2}{*}{ Material } & \multicolumn{9}{|c|}{$k\left(E_{0}\right)$ for incident energies of } \\
\hline & $0.1 \mathrm{MeV}$ & $0.25 \mathrm{MeV}$ & $0.5 \mathrm{MeV}$ & $1.0 \mathrm{MeV}$ & $2.0 \mathrm{MeV}$ & $3.0 \mathrm{MeV}$ & $5.0 \mathrm{MeV}$ & $14.0 \mathrm{MeV}$ & Fission \\
\hline Concrete & 0.0948 & 0.1027 & 0.1062 & 0.1323 & 0.1164 & 0.1030 & 0.0834 & 0.0552 & 0.1110 \\
\hline $\begin{array}{l}\text { Dry NTS soil } \\
50 \% \text { saturated }\end{array}$ & 0.0967 & 0.0895 & 0.1002 & 0.1272 & 0.1103 & 0.0979 & 0.0784 & 0.0535 & 0.1050 \\
\hline $\begin{array}{l}\text { NTS soil } \\
100 \% \text { saturated }\end{array}$ & 0.0868 & 0.0957 & 0.0952 & 0.1209 & 0.1074 & 0.0926 & 0.0746 & 0.0533 & 0.1015 \\
\hline NTS soil & 0.0778 & 0.0818 & 0.0839 & 0.1054 & 0.0891 & 0.0791 & 0.0644 & 0.0463 & 0.0868 \\
\hline Iron & 0.1750 & 0.1752 & 0.1801 & 0.1182 & 0.1477 & 0.1508 & 0.1158 & 0.0802 & 0.1366 \\
\hline
\end{tabular}

†Equation 7.3-3.

$\ddagger$ F. J. Allen, A. Futterer, and W. Wright, Dependence of Neutron Albedos Upon Hydrogen Content of a Shield, Report BRL-1224, Ballistic Research Laboratories, October 1963.

$\S$ From R. L. French and M. B. Wells, An Angular Dependent Albedo for Fast-Neutron Reflection Calculations, USAEC Report RRA-M31, Radiation Research Associates, Inc., November 1963. 
Table 0.4-CONSTANTS FOR THE EXPRESSIONS $\nmid$ FITTING THE COLEMAN ET AL., $\ddagger$ DIFFERENTIAL AND TOTAL ALBEDO DATA FOR INTERMEDIATE-ENERGY NEUTRONS INCIDENT ON REINFORCED CONCRETE

\begin{tabular}{|c|c|c|c|c|c|c|c|c|c|c|}
\hline \multirow[b]{2}{*}{ Constant } & \multicolumn{10}{|c|}{ Values of constants for $\Delta E_{0}$ of } \\
\hline & $\begin{array}{c}5.1 \text { to } 200 \\
\text { keV }\end{array}$ & $\begin{array}{c}15.2 \text { to } 55.1 \\
\mathrm{keV}\end{array}$ & $\begin{array}{c}4.2 \text { to } 15.2 \\
\mathrm{keV}\end{array}$ & $\begin{array}{c}1.15 \text { to } 4.2 \\
\text { keV }\end{array}$ & $\begin{array}{c}0.32 \text { to } 1.15 \\
\text { keV }\end{array}$ & $\begin{array}{c}87 \text { to } 320 \\
\mathrm{eV}\end{array}$ & $\begin{array}{c}24 \text { to } 87 \\
\mathrm{eV}\end{array}$ & $\begin{array}{c}6.6 \text { to } 24 \\
\mathrm{eV}\end{array}$ & $\begin{array}{c}1.8 \text { to } 6.6 \\
\mathrm{eV}\end{array}$ & $\begin{array}{c}0.5 \text { to } 1.8 \\
\text { eV }\end{array}$ \\
\hline$E_{1}$ & 0.190 & 0.190 & 0.216 & 0.210 & 0.208 & 0.210 & 0.205 & 0.202 & 0.172 & 0.105 \\
\hline$\epsilon_{2}$ & -0.020 & -0.025 & -0.047 & -0.046 & -0.042 & -0.061 & -0.068 & -0.075 & -0.059 & -0.036 \\
\hline$\beta_{1}$ & 0.020 & 0.025 & -0.004 & -0.005 & -0.005 & -0.003 & -0.003 & -0.002 & 0.021 & 0.115 \\
\hline$\hat{\beta}_{2}$ & 0.300 & 0.295 & 0.307 & 0.310 & 0.305 & 0.296 & 0.283 & 0.270 & 0.218 & 0.125 \\
\hline$\gamma_{1}$ & 0.11 & 0.11 & 0.12 & 0.12 & 0.12 & 0.125 & 0.13 & 0.13 & 0.105 & 0.080 \\
\hline$\gamma_{2}$ & 0.91 & 0.91 & 0.91 & 0.91 & 0.91 & 0.865 & 0.845 & 0.82 & 0.65 & 0.48 \\
\hline$a$ & 0.20 & 0.225 & 0.24 & 0.24 & 0.24 & 0.28 & 0.30 & 0.32 & 0.40 & 0.255 \\
\hline$b$ & 0.56 & 0.69 & 0.70 & 0.70 & 0.70 & 0.72 & 0.73 & 0.74 & 0.77 & -0.072 \\
\hline$c$ & 0 & 0 & 0 & 0 & 0 & 0 & 0 & 0 & 0 & 0.765 \\
\hline$\delta_{1}$ & 0.880 & 0.865 & 0.875 & 0.875 & 0.860 & 0.845 & 0.830 & 0.815 & 0.817 & 0.792 \\
\hline$\delta_{2}$ & -0.208 & -0.177 & -0.200 & -0.232 & -0.205 & -0.210 & -0.228 & -0.230 & -0.244 & -0.232 \\
\hline
\end{tabular}

†Equations 7.3-6 and 7.3-7.

$\ddagger$ W. A. Coleman, R. E. Maerker, F. J. Muckenthaler, and P. N. Stevens, Calculation of Doubly Differential Current Albedos for Epicadmium Neutrons Incident on Concrete and Comparison of the Reflected Subcadmium Component with Experiment, Nucl. Sci. Eng., 27: 411 (1967). 
Table O.5-VALUES OF COEFFICIENTS AND FUNCTIONS FOR THE SEMIEMPIRICAL FORMULA OF MOCKEL † (Eq. 7.3-13)

\begin{tabular}{lcccccc}
\hline Source & $\alpha$ & \multicolumn{1}{c}{$\beta$} & \multicolumn{1}{c}{$\beta$} & \multicolumn{1}{c}{$A$} & \multicolumn{1}{c}{$f(p)$} & \multicolumn{1}{c}{$g(p)$} \\
\hline Norm. & $1.37(1-p)^{0.44}$ & $1.37(1-p)^{0.44}$ & 0.2775 & $(1-p)^{0.388}$ & $0.067 p^{4.48}$ \\
lsotropic & $1.37(1-p)^{0.44}$ & $1.33(1-p)^{0.3675}$ & 0.640 & $(1-p)^{0.5}$ & 0 \\
Cosine & $1.37(1-p)^{0.44}$ & $1.37(1-p)^{0.44}$ & 0.3882 & $(1-p)^{0.41}$ & $0.05 p^{3.33}$ \\
\hline
\end{tabular}

tA. Mockel, Reflection and Transmission by a Strongly Absorbing Slab, Nucl. Sci. Eng., 22: 339 (1965).

$¥$ Norm., normal incidence; isotropic, isotropic current; and cosine, cosine current.

Table O.6-EXPRESSIONS FOR DIFFERENTIAL AND TOTAL THERMALNEUTRON ALBEDOS DUE TO INCIDENT 0.5-eV TO 200-keV NEUTRONS $\dagger$

\begin{tabular}{cll}
\hline \multicolumn{1}{c}{$\Delta E_{0}$} & \multicolumn{1}{c}{$\begin{array}{c}\alpha_{2}\left(\Delta E_{0} \mu_{0} \mu\right), \ddagger \text { thermal neutrons } \\
\text { steradian }^{-1} \text { source neutron }\end{array}$} & $\begin{array}{c}\mathbf{A}_{2}\left(\Delta E_{0} \mu_{0}\right), \\
\text { thermal neutrons/ } \\
\text { source neutron }\end{array}$ \\
\hline $55.1-200 \mathrm{keV}$ & $\mu(1+1.73 \mu)\left(0.0043+0.0058 \mu_{0}\right)$ & $0.029+0.039 \mu_{0}$ \\
$15.2-55.1 \mathrm{keV}$ & $\mu(1+1.73 \mu)\left(0.0052+0.0059 \mu_{0}\right)$ & $0.035+0.040 \mu_{0}$ \\
$4.2-15.2 \mathrm{keV}$ & $\mu(1+1.73 \mu)\left(0.0062+0.0071 \mu_{0}\right)$ & $0.042+0.048 \mu_{0}$ \\
$1.15-4.2 \mathrm{keV}$ & $\mu(1+1.73 \mu)\left(0.0077+0.0073 \mu_{0}\right)$ & $0.052+0.049 \mu_{0}$ \\
$0.32-1.15 \mathrm{keV}$ & $\mu(1+1.73 \mu)\left(0.0090+0.0099 \mu_{0}\right)$ & $0.061+0.067 \mu_{0}$ \\
$87-320 \mathrm{eV}$ & $\mu(1+1.73 \mu)\left(0.011+0.012 \mu_{0}\right)$ & $0.074+0.081 \mu_{0}$ \\
$24-87 \mathrm{eV}$ & $\mu\left[\left(0.0185+0.0150 \mu_{0}\right)+\mu\left(0.0177+0.0235 \mu_{0}\right)\right]$ & $0.095+0.096 \mu_{0}$ \\
$6.6-24 \mathrm{eV}$ & $\mu\left[\left(0.0332+0.0085 \mu_{0}\right)+\mu\left(0.0220+0.0268 \mu_{0}\right)\right]$ & $0.150+0.084 \mu_{0}$ \\
$1.8-6.6 \mathrm{eV}$ & $\mu\left[\left(0.0595+\mu\left(0.0290+0.0305 \mu_{0}\right)\right]\right.$ & $0.248+0.064 \mu_{0}$ \\
$0.5-1.8 \mathrm{eV}$ & $\mu\left[\left(0.124-0.035 \mu_{0}\right)+\mu\left(0.020+0.053 \mu_{0}\right)\right]$ & $0.431+0.001 \mu_{0}$ \\
\hline
\end{tabular}

†From W. A. Coleman, R. E. Maerker, F. J. Muckenthaler, and P. N. Stevens, Nucl. Sci. Eng., 27: 419 (1967).

$\ddagger \mu_{0}=\cos \theta_{0} ; \mu=\cos \theta$. 
Table 0.7-EXPRESSIONS FOR DIFFERENTIAL AND TOTAL THERMALNEUTRON ALBEDOS DUE TO INCIDENT 200-keV TO 9.57-MeV NEUTRONS $\dagger$

\begin{tabular}{ccc}
$\Delta E_{0}$ & $\begin{array}{c}\alpha_{2}\left(\Delta E_{0}, \mu_{0}, \mu\right), \ddagger \text { thermal neutrons } \\
\text { steradian }^{-1} \text { source neutron }\end{array}$ & $\begin{array}{c}\mathrm{A}_{2}\left(\Delta E_{0} \mu_{0}\right), \\
\text { thermal neutrons/source } \\
\text { neutron }\end{array}$ \\
\hline $2.64-9.57 \mathrm{MeV}$ & $\mu(1+1.73 \mu)\left(0.0024+0.0040 \mu_{0}\right)$ & $0.016+0.027 \mu_{0}$ \\
$0.750-2.64 \mathrm{MeV}$ & $\mu(1+1.73 \mu)\left(0.0028+0.0044 \mu_{0}\right)$ & $0.019+0.030 \mu_{0}$ \\
$200-750 \mathrm{keV}$ & $\mu(1+1.73 \mu)\left(0.0036+0.0049 \mu_{0}\right)$ & $0.024+0.033 \mu_{0}$ \\
\hline
\end{tabular}

†From W. A. Coleman, R. E. Maerker, F. J. Muckenthaler, and P. N. Stevens, Nucl. Sci. Eng., 27: 422 (1967).

$\ddagger \mu_{0}=\cos \theta_{0} ; \mu=\cos \theta$.

Table 0.8-VALUES OF PARAMETERS FOR CHILTONHUDDLESTON GAMMA-RAY DIFFERENTIAL DOSE ALBEDO FORMULA (Eq. 7.4-1) $t$

\begin{tabular}{llrr}
\hline Material & $\begin{array}{c}E_{0}, \\
\mathrm{MeV}\end{array}$ & \multicolumn{1}{c}{$C$} & \multicolumn{1}{c}{$C^{\prime}$} \\
\hline Water & 0.2 & $-0.0187 \pm 0.0027$ & $0.1327 \pm 0.0054$ \\
& 0.662 & $0.0309 \pm 0.0047$ & $0.0253 \pm 0.0034$ \\
& 1.00 & $0.0470 \pm 0.0053$ & $0.0151 \pm 0.0025$ \\
& 2.50 & $0.0995 \pm 0.0068$ & $0.0058 \pm 0.0010$ \\
& 6.13 & $0.1861 \pm 0.0107$ & $0.0035 \pm 0.0005$ \\
Concrete & 0.2 & $0.0023 \pm 0.0033$ & $0.0737 \pm 0.0065$ \\
& 0.662 & $0.0347 \pm 0.0050$ & $0.0197 \pm 0.0035$ \\
& 1.00 & $0.0503 \pm 0.0056$ & $0.0118 \pm 0.0025$ \\
& 2.50 & $0.0999 \pm 0.0078$ & $0.0051 \pm 0.0011$ \\
Iron & 6.13 & $0.1717 \pm 0.0103$ & $0.0048 \pm 0.0005$ \\
& 0.2 & $0.0272 \pm 0.0033$ & $-0.0100 \pm 0.0062$ \\
& 0.662 & $0.0430 \pm 0.0045$ & $0.0063 \pm 0.0030$ \\
& 1.00 & $0.0555 \pm 0.0049$ & $0.0045 \pm 0.0021$ \\
& 2.50 & $0.1009 \pm 0.0073$ & $0.0044 \pm 0.0010$ \\
& 6.13 & $0.1447 \pm 0.0101$ & $0.0077 \pm 0.0006$ \\
Lead & 0.2 & $0.0044 \pm 0.0002$ & $-0.0050 \pm 0.0004$ \\
& 0.662 & $0.0308 \pm 0.0015$ & $-0.0100 \pm 0.0007$ \\
& 1.00 & $0.0452 \pm 0.0013$ & $-0.0083 \pm 0.0004$ \\
& 2.50 & $0.0882 \pm 0.0014$ & $0.0001 \pm 0.0002$ \\
& 6.13 & $0.1126 \pm 0.0048$ & $0.0063 \pm 0.0003$ \\
\hline
\end{tabular}

†From A. B. Chilton, C. M. Davisson, and L. A. Beach, Transactions of the American Nuclear Society, 8: 656 (1965). 
Table 0.9-CHILTON'S MODIFIED ALBEDO FOR CONCRE'TE $†$

\begin{tabular}{ccc}
\hline Parameter & ${ }^{137} \mathrm{Cs}(0.662 \mathrm{MeV})$ & ${ }^{60} \mathrm{Co}(1.25 \mathrm{MeV})$ \\
\hline$C$ & 0.0455 & 0.0710 \\
$C^{\prime}$ & 0.0161 & 0.0114 \\
$A_{1}$ & 1.512 & 1.555 \\
$A_{2}$ & -0.606 & -0.629 \\
$A_{3}$ & -0.641 & -0.605 \\
$A_{4}$ & 0.645 & 0.539 \\
$A_{5}$ & -0.157 & -0.168 \\
\hline
\end{tabular}

†From A. B. Chilton, Nucl. Sci. Eng., 27: 482 (1967).

$$
\ddagger \alpha\left(\theta_{0}, \theta, \phi\right)=F\left(\theta_{0}, \theta, \phi\right) \frac{C \cdot 10^{26} \cdot K_{e}\left(E_{0}, \theta_{s}\right)+C^{\prime}}{1+\cos \theta_{0} \sec \theta\left(1+2 E_{0} \text { vers } \theta_{s}\right)^{1 / 2}}
$$

where

$$
\begin{aligned}
F\left(\theta_{0}, \theta, \phi\right)= & A_{1}+A_{2} \text { vers }{ }^{2} \theta_{0}+A_{3} \text { vers }^{2} \theta \\
& +A_{4} \text { vers } \theta_{0} \text { vers }{ }^{2} \theta \\
& +A_{5} \text { vers } \theta_{0} \text { vers } \theta \text { vers } \phi \\
\text { vers } \phi \equiv & 1-\cos \phi .
\end{aligned}
$$

\begin{tabular}{|c|c|c|c|}
\hline Material & Source & $\cos \theta_{0}$ & $b$ \\
\hline \multirow[t]{2}{*}{ Aluminum } & ${ }^{60} \mathrm{Co}$ & $\begin{array}{l}0.50 \\
0.75 \\
1.00\end{array}$ & $\begin{array}{l}0.0070 \\
0.0090 \\
0.0095\end{array}$ \\
\hline & ${ }^{137} \mathrm{Cs}$ & $\begin{array}{l}0.50 \\
0.75 \\
1.00\end{array}$ & $\begin{array}{l}0.0132 \\
0.0167 \\
0.0194\end{array}$ \\
\hline \multirow[t]{2}{*}{ Iron } & ${ }^{60} \mathrm{Co}$ & $\begin{array}{l}0.50 \\
0.75 \\
1.00\end{array}$ & $\begin{array}{l}0.0060 \\
0.0065 \\
0.0065\end{array}$ \\
\hline & ${ }^{137} \mathrm{Cs}$ & $\begin{array}{l}0.50 \\
0.75 \\
1.00\end{array}$ & $\begin{array}{l}0.0091 \\
0.0120 \\
0.0130\end{array}$ \\
\hline \multirow[t]{2}{*}{ Concrete } & ${ }^{60} \mathrm{Co}$ & $\begin{array}{l}0.50 \\
0.75 \\
1.00\end{array}$ & $\begin{array}{l}0.0075 \\
0.0090 \\
0.0100\end{array}$ \\
\hline & ${ }^{137} \mathrm{Cs}$ & $\begin{array}{l}0.50 \\
0.75 \\
1.00\end{array}$ & $\begin{array}{l}0.0133 \\
0.0165 \\
0.0184\end{array}$ \\
\hline
\end{tabular}

Table 0.10 -VALUES OF THE CONSTANT $b$ FITTING THE EXPRESSION OF HAGGMARK et al. $\dagger$ (Eq. 7.4-2) FOR GAMMA-RAY DIFFERENTIAL, DOSE ALBEDOS FOR ALUMINUM, IRON, AND CONCRETE

tFrom L. G. Haggmark, T. H. Jones, N. E. Scofield, and W. J. Gurney, Differential Dose-Rate Measurements of Backscattered Gamma Rays from Concrete, Aluminum and Steel, Nucl. Sci. Eng., 23: 138 (1965). 
Table 0.11—CURVE-FITTED EXPRESSIONS FOR CAPTURE GAMMA-RAY DIFFERENTIAL

AND TOTAL ALBEDOS ARISING FROM THE SLOWING DOWN AND CAPTURE

OF INCIDENT EPICADMIUM $\dagger$

\begin{tabular}{|c|c|c|}
\hline$\Delta E_{0}$ & $\begin{array}{c}\alpha_{D 2}(\mathrm{n}, \gamma)\left(\Delta E_{0}, \mu_{0}, \mu\right), \ddagger \\
\left(\text { rads hr }{ }^{-1} \text { steradian }^{-1}\right) /(\text { incident unit neutron current })\end{array}$ & $\begin{array}{c}A_{D 2(\mathrm{n}, \gamma)}\left(\Delta E_{0}, \mu_{0}\right) \\
(\mathrm{rads} / \mathrm{hr}) /(\text { incident } \\
\text { unit neutron current) }\end{array}$ \\
\hline $55.1-200 \mathrm{keV}$ & $10^{-7} \mu\left(0.43+2.17 \mu-1.67 \mu^{2}\right)\left(0.39+0.51 \mu_{0}\right)(1.10)$ & $10^{-7}\left(1.40+1.83 \mu_{0}\right)$ \\
\hline $15.2-55.1 \mathrm{keV}$ & $10^{-7} \mu\left(0.39+1.78 \mu-1.39 \mu^{2}\right)\left(0.50+0.68 \mu_{0}\right)(1.11)$ & $10^{-7}\left(1.52+2.07 \mu_{0}\right)$ \\
\hline $4.2-15.2 \mathrm{keV}$ & $10^{-7} \mu\left(0.70+2.53 \mu-2.07 \mu^{2}\right)\left(0.37+0.46 \mu_{0}\right)\left(1.12-0.01 \mu_{0}\right)$ & $10^{-7}\left(1.74+2.15 \mu_{0}\right)$ \\
\hline $1.15-4.2 \mathrm{keV}$ & $10^{-7} \mu\left(0.68+2.59 \mu-2.08 \mu^{2}\right)\left(0.40+0.54 \mu_{0}\right)\left(1.12-0.01 \mu_{0}\right)$ & $10^{-7}\left(1.93+2.57 \mu_{0}\right)$ \\
\hline $0.32-1.15 \mathrm{keV}$ & $10^{-7} \mu\left(0.66+3.18 \mu-2.60 \mu^{2}\right)\left(0.46+0.54 \mu_{0}\right)\left(1.14-0.02 \mu_{0}\right)$ & $10^{-7}\left(2.43+2.79 \mu_{0}\right)$ \\
\hline $87-320 \mathrm{keV}$ & $10^{-7} \mu\left(0.89+3.52 \mu-3.09 \mu^{2}\right)\left(0.41+0.59 \mu_{0}\right)\left(1.18-0.06 \mu_{0}\right)$ & $10^{-7}\left(2.56+3.42 \mu_{0}\right)$ \\
\hline $24-87 \mathrm{eV}$ & $10^{-7} \mu\left(1.14+3.98 \mu-3.77 \mu^{2}\right)\left(0.38+0.62 \mu_{0}\right)\left(1.30-0.15 \mu_{0}\right)$ & $10^{-7}\left(2.95+4.20 \mu_{0}\right)$ \\
\hline $6.6-24 \mathrm{eV}$ & $10^{-7} \mu\left(1.40+2.73 \mu-2.50 \mu^{2}\right)\left(0.45+0.65 \mu_{0}\right)\left(1.11-0.04 \mu_{0}\right)$ & $10^{-7}\left(3.08+4.18 \mu_{0}\right)$ \\
\hline $1.8-6.6 \mathrm{eV}$ & $\begin{array}{l}10^{-7} \mu\left[1.34+0.78 \mu_{0}+\mu\left(0.15+2.30 \mu_{0}\right)-\mu^{2}\left(0.60+2.11 \mu_{0}\right)\right] \\
\quad \times\left(1.09-0.03 \mu_{0}\right)\end{array}$ & $10^{-7}\left(3.89+4.14 \mu_{0}\right)$ \\
\hline $0.5-1.8 \mathrm{eV}$ & $\begin{array}{l}10^{-7} \mu\left[2.12+0.72 \mu_{0}+\mu\left(-1.46+2.92 \mu_{0}\right)+\mu^{2}\left(0.40-2.68 \mu_{0}\right)\right] \\
\quad \times\left(1.07-0.02 \mu_{0}\right)\end{array}$ & $10^{-7}\left(4.52+4.33 \mu_{0}\right)$ \\
\hline
\end{tabular}

†From W. A. Coleman, R. E. Maerker, F. J. Muckenthaler, and P. N. Stevens, Nucl. Sci. Eng., 27: 420 (1967). $\ddagger \mu_{0}=\cos \theta_{0} ; \mu=\cos \theta$. 


\section{Radiation Penetration of Cylindrical Ducts ${ }^{\dagger}$}

\section{Appendix P}

Table P.1 - UNCOLLIDED FLUX DENSITY AT EXIT OF CYLINDRICAL DUCT DUE TO RADIATION ARRIVING THROUGH DUCT WALLS (ISOTROPIC SOURCE)

\begin{tabular}{lcccccc}
\hline \multirow{2}{*}{$\left(\frac{a}{Z}\right)$} & \multicolumn{5}{c}{ Flux density per unit surface source intensity for shield thickness of } \\
\cline { 2 - 6 } & $\mu Z=0.1 \dagger$ & $\mu Z=0.2$ & $\mu Z=0.5$ & $\mu Z=1.0$ & $\mu Z=2.0$ & $\mu Z=5.0$ \\
\hline 0.001 & 1.823 & 1.223 & 0.5602 & 0.2198 & 0.04912 & $1.166 \times 1 \sigma^{-3}$ \\
0.002 & 1.823 & 1.223 & 0.5607 & 0.2207 & 0.04935 & $1.184 \times 1 \sigma^{-3}$ \\
0.005 & 1.824 & 1.224 & 0.5620 & 0.2215 & 0.05002 & $1.242 \times 10^{-3}$ \\
0.01 & 1.825 & 1.226 & 0.5643 & 0.2235 & 0.05119 & $1.349 \times 1 \sigma^{-3}$ \\
0.02 & 1.828 & 1.229 & 0.5688 & 0.2278 & 0.05363 & $1.614 \times 1 \sigma^{-3}$ \\
0.05 & 1.834 & 1.239 & 0.5820 & 0.2406 & 0.06171 & $2.977 \times 1 \sigma^{-3}$ \\
0.1 & 1.842 & 1.254 & 0.6024 & 0.2613 & 0.07621 & $7.042 \times 1 \sigma^{-3}$ \\
0.2 & 1.851 & 1.272 & 0.6360 & 0.2982 & 0.1049 & $1.795 \times 1 \sigma^{-2}$ \\
0.5 & 1.825 & 1.277 & 0.6841 & 0.3659 & 0.1687 & $5.223 \times 1 \sigma^{-2}$ \\
0.75 & 1.765 & 1.240 & 0.6804 & 0.3819 & 0.1923 & $6.990 \times 1 \sigma^{-2}$ \\
$1 . \theta$ & 1.689 & 1.185 & 0.6560 & 0.3767 & 0.1980 & $7.753 \times 1 \sigma^{-2}$ \\
\hline
\end{tabular}

tNumber of relaxation lengths.

+All data in this appendix have been taken from D. K. Trubey, A Calculation of Radiation Penetration of Cylindrical Duct Walls, USAEC Report ORNL-CF-63-2-64, Oak Ridge National Laboratory, Feb. 28, 1963. 
Table P.2-UNCOLLIDED FLUX DENSITY AT EXIT OF CYLINDRICAL DUCT DUE TO RADIATION ARRIVING THROUGH DUCT WALLS (COSINE SOURCE)

\begin{tabular}{lcccccc}
\hline & \multicolumn{6}{c}{ Flux density per unit surface source intensity for shield thickness of } \\
\cline { 2 - 6 }$\left(\frac{a}{Z}\right)$ & $\mu Z=\mathbf{0 . 1} t$ & $\mu Z=\mathbf{0 . 2}$ & $\mu Z=\mathbf{0 . 5}$ & $\mu Z=\mathbf{1 . 0}$ & $\mu Z=2.0$ & $\mu Z=\mathbf{5 . 0}$ \\
\hline 0.001 & 1.445 & 1.149 & 0.6539 & $\mathbf{0 . 2 9 7 6}$ & 0.07544 & $\mathbf{0 . 0 0 2 0 2 6}$ \\
0.002 & 1.446 & 1.149 & 0.6545 & $\mathbf{0 . 2 9 8 3}$ & 0.07582 & $\mathbf{0 . 0 0 2 0 5 9}$ \\
$\mathbf{0 . 0 0 5}$ & 1.446 & 1.150 & 0.6564 & 0.3002 & 0.07698 & 0.002166 \\
0.01 & 1.447 & 1.152 & 0.6595 & $\mathbf{0 . 3 0 3 4}$ & 0.07896 & 0.002365 \\
0.02 & 1.450 & 1.156 & 0.6657 & 0.3100 & 0.08314 & 0.002862 \\
0.05 & 1.455 & 1.166 & 0.6836 & 0.3298 & 0.09703 & 0.005458 \\
0.1 & 1.459 & 1.179 & 0.7095 & 0.3610 & 0.1217 & 0.01323 \\
0.2 & 1.453 & 1.188 & 0.7462 & 0.4120 & 0.1682 & 0.03343 \\
0.5 & 1.339 & 1.116 & 0.7510 & 0.4714 & 0.2494 & 0.08728 \\
0.75 & 1.190 & 0.9949 & 0.6827 & 0.4465 & 0.2553 & 0.1036 \\
1.0 & 1.036 & 0.8643 & 0.5953 & 0.3956 & 0.2340 & 0.1014 \\
\hline
\end{tabular}

†Number of relaxation lengths. 
Table P.3-RATIO OF LINE-OF-SIGHT RADIATION TO TOTAL UNCOLLIDED FLUX DENSITY IN DUCT

\begin{tabular}{|c|c|c|c|c|c|c|}
\hline \multirow{2}{*}{$\left(\frac{a}{Z}\right)$} & \multicolumn{6}{|c|}{ Fraction of total flux density for shield thickness of } \\
\hline & $\mu Z=0.1 \dagger$ & $\mu Z=0.2$ & $\mu Z=0.5$ & $\mu Z=1.0$ & $\mu Z=2.0$ & $\mu Z=5.0$ \\
\hline \multicolumn{7}{|c|}{ Isotropic Infinite-Plane Source } \\
\hline 0.001 & $<10^{-6}$ & $<10^{-6}$ & $<10^{6}$ & $<10^{-5}$ & $<1 \sigma^{-4}$ & $<10^{-3}$ \\
\hline 0.002 & $<10^{-5}$ & $<10^{-5}$ & $<10^{-5}$ & $<10^{5}$ & $<1 \sigma^{-4}$ & 0.002 \\
\hline 0.005 & $<10^{-5}$ & $<10^{-4}$ & $<10^{-4}$ & $<10^{4}$ & $<1 \sigma^{3}$ & 0.010 \\
\hline 0.01 & $<10^{-4}$ & $<10^{-4}$ & $<10^{-4}$ & $10^{-3}$ & 0.001 & 0.038 \\
\hline 0.02 & $<10^{-3}$ & $<10^{-3}$ & $10^{-3}$ & $10^{-3}$ & 0.004 & 0.11 \\
\hline 0.05 & $<10^{-3}$ & 0.001 & 0.002 & 0.005 & 0.020 & 0.29 \\
\hline 0.1 & 0.003 & 0.004 & 0.008 & 0.019 & 0.062 & 0.42 \\
\hline 0.2 & 0.011 & 0.015 & 0.031 & 0.062 & 0.16 & 0.52 \\
\hline 0.5 & 0.059 & 0.083 & 0.14 & 0.23 & 0.40 & 0.68 \\
\hline 0.75 & 0.11 & 0.15 & 0.25 & 0.37 & 0.53 & 0.76 \\
\hline 1.0 & 0.17 & 0.23 & 0.34 & 0.48 & 0.64 & 0.82 \\
\hline \multicolumn{7}{|c|}{ Cosine Infinite-Plane Source } \\
\hline 0.001 & $<10^{-6}$ & $<10^{-6}$ & $<10^{-5}$ & $<1 \sigma^{-5}$ & $<1 \sigma^{4}$ & $<1 \sigma^{3}$ \\
\hline 0.002 & $<10^{-5}$ & $<10^{-5}$ & $<10^{-5}$ & $<10^{-4}$ & $<10^{-4}$ & 0.002 \\
\hline 0.005 & $<10^{-4}$ & $<10^{-4}$ & $<10^{-4}$ & $<10^{-4}$ & $<1 \sigma^{3}$ & 0.011 \\
\hline 0.01 & $<10^{-4}$ & $<1 \sigma^{-4}$ & $<10^{-3}$ & $<10^{-3}$ & 0.001 & 0.040 \\
\hline 0.02 & $<10^{-3}$ & $<10^{-3}$ & $<10^{-3}$ & 0.002 & 0.005 & 0.12 \\
\hline 0.05 & 0.002 & 0.002 & 0.004 & 0.008 & 0.025 & 0.27 \\
\hline 0.1 & 0.007 & 0.008 & 0.014 & 0.027 & 0.076 & 0.43 \\
\hline 0.2 & 0.026 & 0.031 & 0.050 & 0.12 & 0.19 & 0.54 \\
\hline 0.5 & 0.14 & 0.16 & 0.22 & 0.31 & 0.45 & 0.71 \\
\hline 0.75 & 0.25 & 0.28 & 0.37 & 0.48 & 0.61 & 0.79 \\
\hline 1.0 & 0.36 & 0.40 & 0.50 & 0.60 & 0.71 & 0.85 \\
\hline
\end{tabular}

tNumber of relaxation lengths. 


\section{Solutions to Exercises}

\section{CHAPTER 2}

2.1 Let $N^{\prime}(t)$ be the number of atoms that have disintegrated at time $t$, where $N(0)=N_{0}$

$$
N^{\prime}(t)=N_{0}-N_{0} e^{-\lambda t}=N_{0}\left(1-e^{-\lambda t}\right)
$$

(a)

$$
p(t)=\frac{d}{d t}\left[N^{\prime}(t)\right]=\lambda N_{0} e^{-\lambda t}
$$

(b)

$$
\begin{aligned}
F_{1}(t) & =\int_{0}^{t} p\left(t^{\prime}\right) d t^{\prime}=\int_{0}^{t} \lambda N_{0} e^{-\lambda t^{\prime}} d t^{\prime} \\
& =N_{0}\left(1-e^{-\lambda t}\right) \\
f_{1}(t) & =\frac{\int_{0}^{t} p\left(t^{\prime}\right) d t^{\prime}}{\int_{0}^{\infty} p\left(t^{\prime}\right) d t^{\prime}}=\frac{N_{0}\left(1-e^{-\lambda t}\right)}{N_{0}}=1-e^{-\lambda t} \\
f_{2}(t) & =\frac{\int_{t}^{\infty} p\left(t^{\prime}\right) d t^{\prime}}{\int_{0}^{\infty} p\left(t^{\prime}\right) d t^{\prime}}=\frac{N_{0} e^{-\lambda t}}{N_{0}}=e^{-\lambda t}
\end{aligned}
$$

(d)

$$
\Delta \mathrm{N}\left(T_{1}, T_{2}\right)=\int_{T_{1}}^{T_{2}} p\left(t^{\prime}\right) d t^{\prime}=N_{0}\left(e^{-\lambda T_{1}}-e^{-\lambda T_{2}}\right)
$$

2.2 (a) See accompanying sketch.

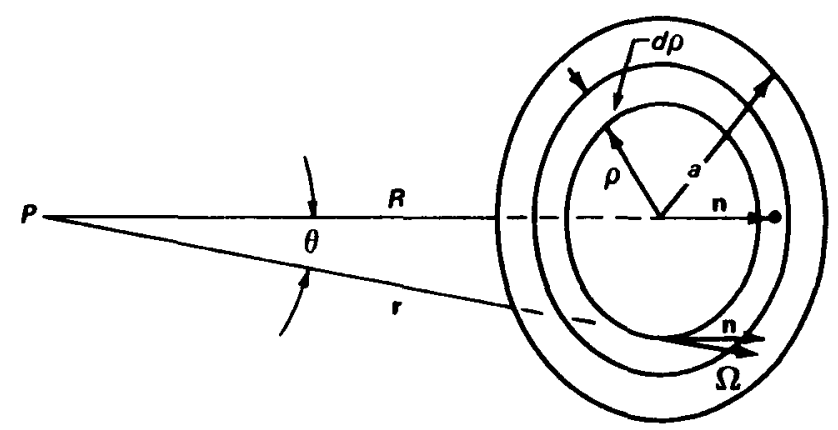

Solution 2.2-Solid-angle calculation. 


$$
\begin{aligned}
d \mathbf{A} & =\Omega d A \\
d \Omega & =\frac{\mathbf{n} \cdot d \mathbf{A}}{|\mathbf{r} \cdot \mathbf{r}|}=\frac{\cos \theta d A}{r^{2}} \\
d A & =2 \pi \rho d \rho, r^{2}=R^{2}+\rho^{2}, \cos \theta=\frac{R}{r}=\frac{R}{\left(R^{2}+\rho^{2}\right)^{1 / 2}} \\
\Omega & \left.=\int_{0}^{\mathrm{a}} d \Omega=\int_{0}^{\mathrm{a}} \frac{2 \pi R \rho d \rho}{\left(R^{2}+\rho^{2}\right)^{3 / 2}}=-\frac{2 \pi R}{\left(R^{2}+\rho^{2}\right)^{1 / 2}}\right]_{0}^{a} \\
& =2 \pi\left[1-\frac{R}{\left(R^{2}+a^{2}\right)^{1 / 2}}\right]
\end{aligned}
$$

(b) As $R \rightarrow 0$ or $a \rightarrow \infty, \Omega \rightarrow 2 \pi$ (half space).

$2.3(a)$

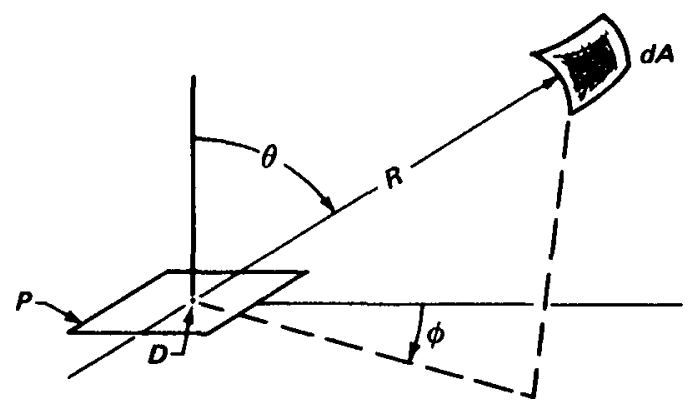

Solution 2.3-Spherical-shell source.

$$
d A=R^{2} \sin \theta d \theta d \phi
$$

The differential source $=S_{a} R^{2} \sin \theta d \theta d \phi$ particles $\mathrm{cm}^{-2} \sec ^{-1}$

$$
\begin{aligned}
= & \frac{1}{4 \pi} S_{a} R^{2} \sin \theta d \theta d \phi \text { particles } \mathrm{cm}^{-2} \\
& \sec ^{-1} \text { steradian }^{-1}
\end{aligned}
$$

The solid angle at $d A$ subtended by a sphere of unit cross-sectional area centered at $D$ is $1 / R^{2}$ steradian (see figure). Thus, for flux density, $\Phi(R)$,

$$
\begin{gathered}
d \Phi(R)=\left(\frac{1}{4 \pi} S_{a} R^{2} \sin \theta d \theta d \phi\right)\left(\frac{1}{R^{2}}\right) \\
\Phi(R)=\int_{\phi=0}^{2 \pi} \int_{\theta=0}^{\pi} \frac{1}{4 \pi} S_{a} \sin \theta d \theta d \phi
\end{gathered}
$$




$$
\left.\Phi=\frac{S_{a}}{2} \cos \theta\right]_{\pi}^{0}=S_{a} \text { particles } \mathrm{cm}^{-2} \sec ^{-1}
$$

(b) From symmetry the net current through the plane $P$ is zero.

2.4 (a) From symmetry the flux density is $(1 / 2) S_{a}$ particles $\mathrm{cm}^{-2} \mathrm{sec}^{-1}$.

(b)

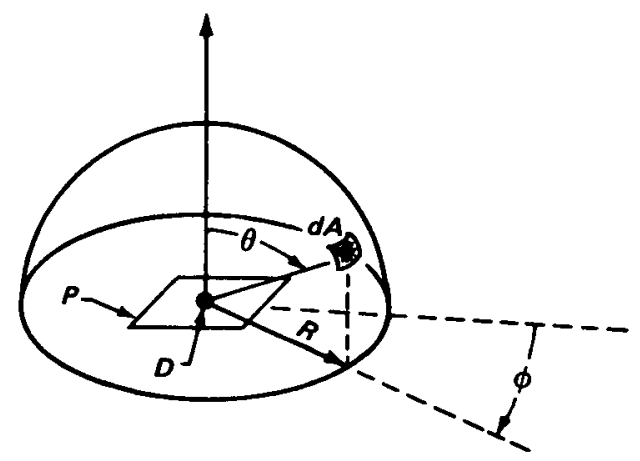

Solution 2.4-Hemispheric shell source.

The differential source strength is given by

$$
d s=\frac{1}{4 \pi} S_{a} R^{2} \sin \theta d \theta d \phi \text { particles } \mathrm{cm}^{-2} \sec ^{-1} \operatorname{steradian}^{-1}
$$

The solid angle subtended at $d A$ from a unit area about point $D$ in plane $P$ (see sketch) is $\cos \theta / R^{2}$. Thus the differential current through $P$ is given by

$$
\begin{aligned}
d \mathrm{~J}(R) & =\left(\frac{1}{4 \pi} S_{a} R^{2} \sin \theta d \theta d \phi\right)\left(\frac{\cos \theta}{R^{2}}\right) \\
\mathrm{J}(R) & =\int_{\phi=0}^{2 \pi} d \phi \int_{\theta=0}^{\pi / 2} \frac{1}{4 \pi} S_{a} \sin \theta \cos \theta d \theta \\
& =\frac{1}{2} S_{a}\left[\frac{1}{2} \sin ^{2} \theta\right]_{0}^{2 \pi}=\frac{1}{4} S_{a} \text { particles } \mathrm{cm}^{-2} \sec ^{-1} \text { directed downward }
\end{aligned}
$$

\section{5 (a)}

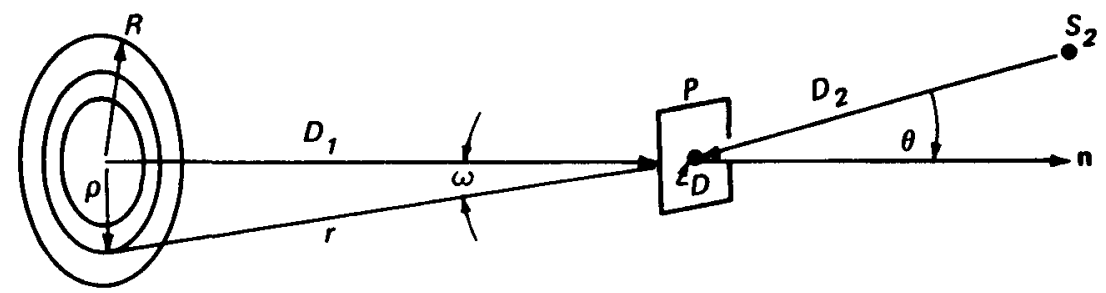

Solution 2.5-Disk and point sources. 
Let $\Phi_{1}\left(D_{1}\right)$ be the flux-density contribution from the disk and $\Phi_{2}\left(D_{2}\right)$ be the flux contribution from the point source. Using the parameter in the sketch,

$$
\begin{aligned}
& \begin{aligned}
d \Phi_{1}\left(D_{1}\right) & =\left(\frac{1}{4 \pi} S_{a} 2 \pi \rho d \rho\right)\left(\frac{1}{r^{2}}\right) \\
\text { where } r^{2} & =\rho^{2}+D_{1}^{2} \text { and } r d r=\rho d \rho \\
\Phi_{1}\left(D_{1}\right) & =\int_{r=D_{1}}^{\left(D_{1}^{2}+R^{2}\right)^{\frac{1}{2}}} \frac{1}{2} S_{a}\left(\frac{1}{r}\right) d r \\
& =\frac{1}{2} S_{a}[\ln r]_{D_{1}}^{\left(D_{1}^{2}+R^{2}\right)^{1 / 2}} \\
\Phi_{2}\left(D_{2}\right) & =\frac{S}{4 \pi}\left(\frac{1}{D_{2}^{2}}\right)=\frac{S}{4 \pi D_{2}^{2}}
\end{aligned}
\end{aligned}
$$

Thus

$$
\Phi(D)=\frac{1}{2} S_{a} \ln \left[\frac{\left(D_{1}^{2}+R^{2}\right)^{1 / 2}}{D_{1}}\right]+\frac{1}{4 \pi}\left(\frac{S}{D_{2}^{2}}\right)
$$

(b) Let $\mathrm{J}_{1}\left(D_{1}\right)$ be the current through $P$ from the disk and $\mathrm{J}_{2}\left(D_{2}\right)$ be the current through $P$ from the point source.

$$
\begin{aligned}
d J_{1}\left(D_{1}\right) & =\frac{1}{4 \pi} S_{a}(2 \pi \rho d \rho)(\cos \omega)\left(\frac{1}{r^{2}}\right) \\
& =\frac{1}{4 \pi} S_{a}(2 \pi r d r)\left(\frac{D_{1}}{r}\right)\left(\frac{1}{r^{2}}\right) \\
J_{1}\left(D_{1}\right) & =\int_{r=D_{1}}^{\left(D_{1}^{2}+R^{2}\right)^{1 / 2}} \frac{1}{2} S_{a} D_{1}\left(\frac{1}{r^{2}}\right) d r \\
& =\frac{1}{2} S_{a} D_{1}\left[\frac{1}{r}\right]_{D_{1}}^{\left(D_{1}^{2}+R^{2}\right)^{1 / 2}} \text { in direction } \mathbf{n} \\
\mathbf{J}_{2}\left(D_{2}\right) & =\frac{S}{4 \pi}\left(\frac{\cos \theta}{D_{2}^{2}}\right) \text { in direction -n }
\end{aligned}
$$

The net current $\mathrm{J}(D)$ at the point $D$ is

$$
\begin{aligned}
\mathrm{J}(D) & =\mathrm{J}\left(D_{1}\right)-\mathrm{J}_{2}\left(D_{2}\right) \\
& =\frac{1}{2} S_{a}\left[1-\frac{D_{1}}{\left(D_{1}^{2}+R^{2}\right)^{\frac{1}{3}}}\right]-\frac{1}{4 \pi}\left(\frac{S \cos \theta}{D_{2}^{2}}\right) \text { in direction n }
\end{aligned}
$$


2.6

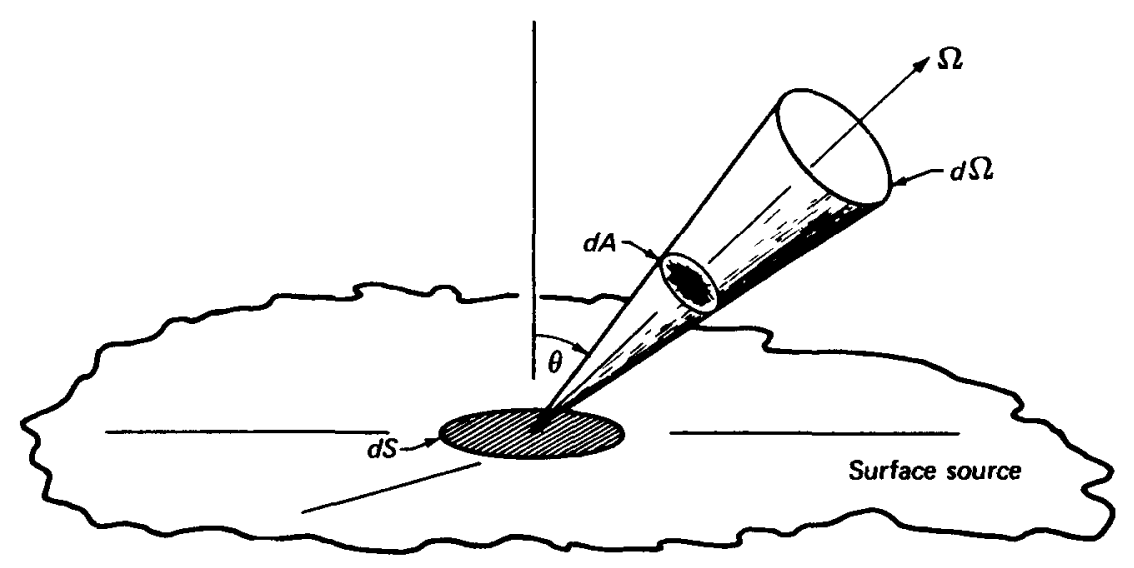

Solution 2.6-Plane array of point source.

Consider in the accompanying sketch the solid angle $d \Omega$ about $\Omega$ passing through the area $d A$, where $d A$ is the projected area on the unit sphere about the differential surface source area $d S . S / c^{2}=$ average surface source (particles $\mathrm{cm}^{-2}$ $\left.\sec ^{-1}\right)$ and $(1 / 4 \pi)\left(S / c^{2}\right) d S=$ particles $\sec ^{-1}$ steradian $^{-1}$ from $d S$ into $d \Omega$ about $\Omega$. But $d S=d A / \cos \theta$. Thus $(1 / 4 \pi)\left(S / c^{2}\right)(d A / \cos \theta)$ particles $\sec ^{-}$steradian $^{-1}$ pass through $d A$ at point $P$, and the flux density at point $P$ is

$$
\left(\frac{1}{4 \pi} \frac{S}{c^{2}} \frac{d A}{\cos \theta}\right)\left(\frac{1}{d A}\right)=\frac{1}{4 \pi} \frac{S}{c^{2} \cos \theta} \text { particles } \mathrm{cm}^{-2} \sec ^{-1} \operatorname{steradian}^{-1}
$$

2.7 (a) Referring to the sketch for part (a) and using polar coordinates about $P$,

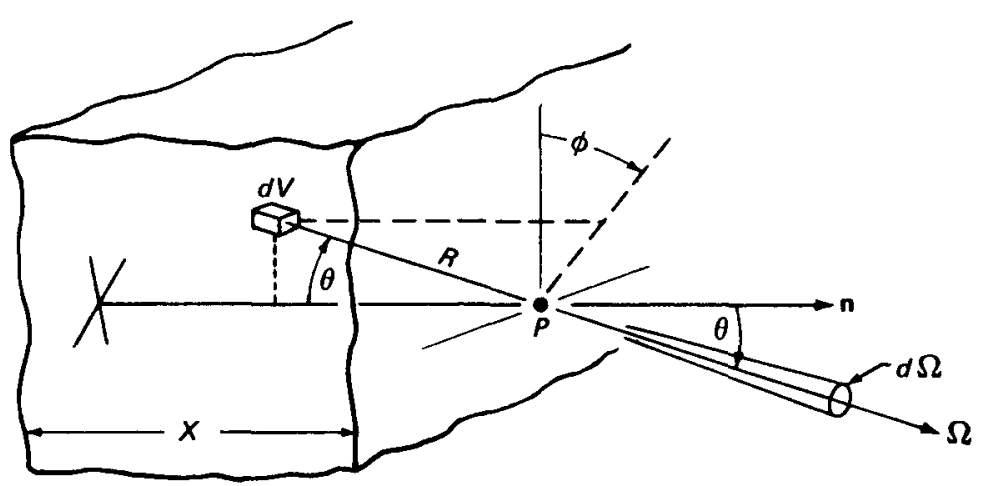

Solution 2.7(a)-Nonabsorbing slab source $G(\theta)$. 


$$
\begin{aligned}
& d V=R^{2} \sin \theta d \theta d \phi d R \\
& d \Omega=\sin \theta d \theta d \phi
\end{aligned}
$$

$\frac{1}{4 \pi} S_{v} d V=$ particles $\sec ^{-1}$ steradian $^{-1}$ into $d \Omega$ about $\Omega$

$$
\frac{1}{R^{2}}=\text { steradians subtended at } d V \text { by a unit area at } P \text { perpendicular to } \Omega
$$

$G(\theta) d \Omega=\int_{R=0}^{X / \cos \theta} \frac{1}{4 \pi} \varsigma_{\nu} R^{2} \sin \theta d \theta d \phi d R \frac{1}{R^{2}}$ particles $\mathrm{cm}^{-2} \sec ^{-1}$ into $d \Omega$

$$
=\frac{1}{4 \pi} S_{v} \frac{X}{\cos \theta} \sin \theta d \theta d \phi
$$

$$
G(\theta)=\frac{1}{4 \pi \cos \theta} S_{\nu} X \text { particles } \mathrm{cm}^{-2} \sec ^{-1} \operatorname{steradian}^{-1}
$$

(b) Referring to the sketch for part (b),

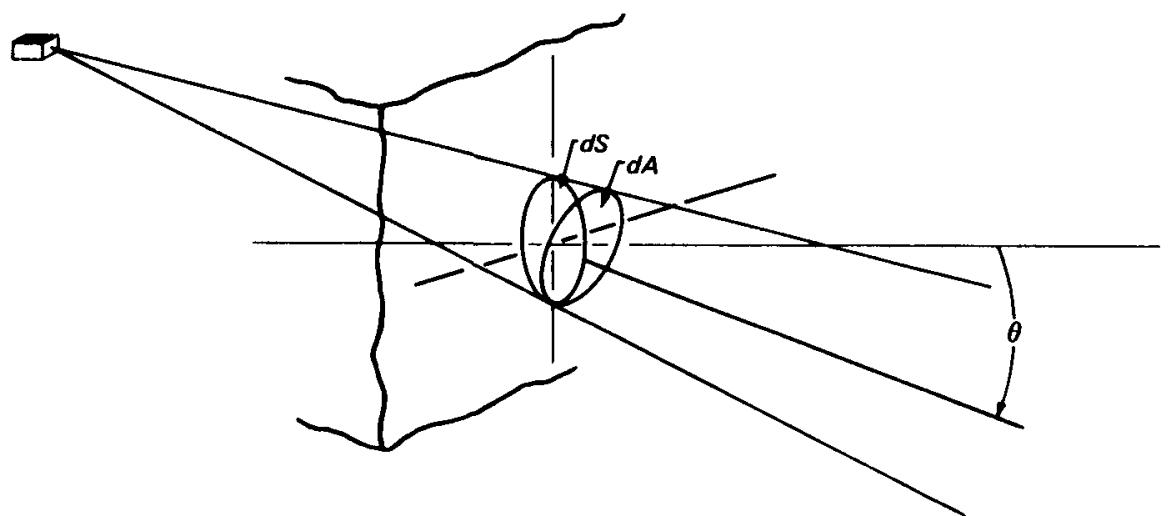

Solution 2.7(b)-I( $\theta)$ from slab source.

$$
\begin{aligned}
\Phi(\theta) d S & =G(\theta) d A \\
d A & =\cos \theta d S \\
\Phi(\theta) d S & =G(\theta) \cos \theta d S \\
\Phi(\theta) & =G(\theta) \cos \theta
\end{aligned}
$$

Thus

$$
\Phi(\theta)=\frac{1}{4 \pi} S_{v} X \text { particles } \mathrm{cm}^{-2} \sec ^{-1} \operatorname{steradian}^{-1}
$$


(c)

$$
\begin{aligned}
\Phi_{a} & =\int_{\Omega} \Phi(\theta) d \Omega=\int_{\phi=0}^{2 \pi} \int_{\theta=0}^{\pi / 2} \frac{1}{4 \pi} S_{\eta} X \sin \theta d \theta d \phi \\
& =\frac{1}{2} S_{v} X
\end{aligned}
$$

(d) The equivalent isotropic surface source emits particles in all directions (into as well as out of the slab). Thus $\Phi_{a}=S_{v} X$.

(e) Referring to the sketch for part (e),

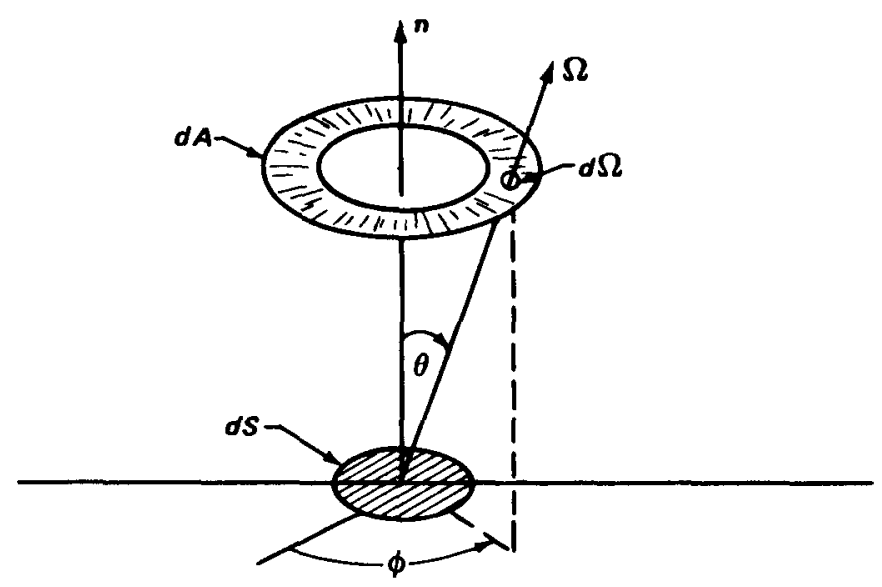

Solution 2.7(e)-H( $\theta)$ function.

$$
\begin{aligned}
H(\theta) d \theta & =\int_{\phi=0}^{2 \pi} G(\theta) d \Omega=\int_{0}^{2 \pi} \frac{1}{4 \pi \cos \theta} S_{v} X \sin \theta d \theta d \phi \\
& =\frac{1}{2} S_{v} X \tan \theta d \theta \\
H(\theta) & =\frac{1}{2} S_{v} X \tan \theta \\
\text { (f) } \Phi\left(\theta_{1} \theta_{2}\right) & =\int_{\theta_{1}}^{\theta_{2}} H(\theta) d \theta=\int_{\theta_{1}}^{\theta_{2}} \frac{1}{2} S_{v} X \tan \theta d \theta \\
& =\frac{1}{2} S_{v} X[\ln \cos \theta]_{\theta_{2}}^{\theta_{1}}=\frac{1}{2} S_{v} X \ln \left(\frac{\cos \theta_{1}}{\cos \theta_{2}}\right)
\end{aligned}
$$

The values for each interval are shown in the table. 
SOLUTION $2.7(f)$

\begin{tabular}{ccccc}
\hline Interval & $\operatorname{Cos} \theta_{1}$ & $\operatorname{Cos} \theta_{2}$ & $\frac{\operatorname{Cos} \theta_{1}}{\operatorname{Cos} \theta_{2}}$ & $\ln \frac{\operatorname{Cos} \theta_{1}}{\operatorname{Cos} \theta_{2}}$ \\
\hline $0^{\circ}$ to $10^{\circ}$ & 1 & 0.985 & 1.016 & 0.0159 \\
$40^{\circ}$ to $50^{\circ}$ & 0.715 & 0.643 & 1.19 & 0.174 \\
$75^{\circ}$ to $85^{\circ}$ & 0.259 & 0.0872 & 2.97 & 1.09 \\
$80^{\circ}$ to $90^{\circ}$ & 0.174 & 0 & $\infty$ & $\infty$ \\
\hline
\end{tabular}

2.8 (a)

$$
\begin{aligned}
N(E) & =0.77 E^{1 / 2} e^{-0.776 E} \\
\frac{d}{d E} N(E) & =\frac{0.77}{2} E^{-1 / 2} e^{-0.776 E}-0.77(0.776) E^{1 / 2} e^{-0.776 E} \\
& =0.77 e^{-0.776 \mathrm{E}}\left|\frac{1}{2 E^{1 / 3}}-0.776 E^{1 / 2}\right|
\end{aligned}
$$

Setting

$$
\begin{gathered}
\frac{d N}{d E}=0 \\
\frac{1}{2 \hat{E}^{1 / 2}}=0.776 \hat{E}^{1 / 2}=0 \\
\frac{1}{2}-0.776 \hat{E}=0 \\
\hat{E}=0.645 \mathrm{MeV} \\
\bar{E}=\frac{\int_{0}^{\infty} N(E) E d E}{\int_{0}^{\infty} N(E) d E}=\frac{\int_{0}^{\infty} 0.77 E^{/ / /} e^{-0.776 E} d E}{\int_{0}^{\infty} 0.77 E^{1 / 3} e^{-0.776 E} d E}
\end{gathered}
$$

(b)

Let $x=0.776 E ; E=x / 0.776$

$$
\begin{aligned}
d E & =\frac{1}{0.776} d x \\
\bar{E} & =\frac{\int_{0}^{\infty}\left(\frac{x}{0.776}\right)^{3 / 2} e^{-x}\left(\frac{1}{0.776}\right) d x}{\int_{0}^{\infty}\left(\frac{x}{0.776}\right)^{1 / 2} e^{-x}\left(\frac{1}{0.776}\right) d x}=\frac{(0.776)^{-5 / 2} \Gamma(5 / 2)}{(0.776)^{-3 / 2} \Gamma(3 / 2)} \\
& =(0.776)^{-1} \frac{(3 / 2) \Gamma(3 / 2)}{\Gamma(3 / 2)}=(0.776)^{-1}(3 / 2)=1.93 \mathrm{MeV} \\
v_{\bar{E}} & =\left(\frac{1.93}{0.525 \times 10^{-18}}\right)^{1 / 2}=1.91 \times 10^{9} \mathrm{~cm} / \mathrm{sec}
\end{aligned}
$$


(c)

$$
\begin{aligned}
& N(E)=0.77 E^{1 / 2} e^{-0.776 E} \\
& E=\frac{m v^{2}}{2} ; \frac{d E}{d r}=m v \\
& N(v)=N(E) \frac{d E}{d r}=0.77\left(\frac{m}{2}\right)^{1 / 2} v e^{-0.776 m v^{2} / 2} m v \\
& =0.77 \frac{m^{* / 2}}{2^{1 / 2}} v^{2} e^{-0.776 m v^{2} / 2} \\
& \bar{v}=\frac{\int_{0}^{\infty} N(v) v d v}{\int_{0}^{\infty} N(v) d v}=\frac{\int_{0}^{\infty} v^{3} \exp \left(-\frac{0.776 m v^{2}}{2}\right) d v}{\int_{0}^{\infty} v^{2} \exp \left(-\frac{0.776 m v^{2}}{2}\right) d v} \\
& \text { Let } y=\frac{0.776 m v^{2}}{2}, v=\left(\frac{2}{0.776 m}\right)^{1 / 2} y^{1 / 2} \\
& d v=\left(\frac{2}{0.776 m}\right)^{1 / 2} y^{-\frac{1}{2}} d y \\
& \bar{v}=\frac{\int_{0}^{\infty}\left(\frac{2}{0.776 m}\right)^{\frac{1}{2}} y^{/ / 2} e^{-y}\left[\frac{1}{2(0.776 m)}\right]^{1 / 2} y^{-1 / 2} d y}{\int_{0}^{\infty}\left(\frac{2}{0.776 m}\right) y e^{-y}\left[\frac{1}{2(0.776 m)}\right]^{1 / 2} y^{-1 / 2} d y}=\frac{2^{1 / 2}}{0.776 m} \frac{\int_{0}^{\infty} y e^{-y} d y}{\int_{0}^{\infty} y^{1 / 2} e^{-y} d y} \\
& =\left(\frac{2}{0.776 m}\right)^{1 / 2} \frac{\Gamma(2)}{\Gamma(3 / 2)}=\left(\frac{2}{0.776 m}\right)^{1 / 2}\left[\frac{1}{(1 / 2)}\right] \frac{\Gamma(1)}{\Gamma(1 / 2)}=\left(\frac{2}{0.776 m}\right)^{3 /}\left(\frac{2}{\sqrt{\pi}}\right) \\
& =2\left(\frac{2}{0.776 m \pi}\right)^{1 / 2} \\
& \bar{E}_{\bar{\nu}}=\frac{m v^{2}}{2}=\frac{m}{2}\left[4\left(\frac{2}{0.776 m \pi}\right)\right] \frac{4}{0.776 \pi}=1.64 \mathrm{MeV} \\
& \bar{v}=\left(0.525 \times 10^{-18}\right)^{-1 / 2}[E(\bar{v})]^{1 / 2}=\left(\frac{1.64}{0.525 \times 10^{-18}}\right)^{1 / 2}=1.77 \times 10^{9} \mathrm{~cm} / \mathrm{sec}
\end{aligned}
$$




\section{CHAPTER 3}

3 .1 (a) Partial densities:

$$
\begin{aligned}
& \rho_{N}=0.7805(0.00125)=0.000976 \mathrm{~g} / \mathrm{cm}^{3} \text { of air } \\
& \rho_{O}=0.2099(0.00143)=0.000300 \mathrm{~g} / \mathrm{cm}^{3} \text { of air } \\
& \rho_{A r}=0.0096(0.00178)=0.0000171 \mathrm{~g} / \mathrm{cm}^{3} \text { of air }
\end{aligned}
$$

(b) Weight percents:

$$
\begin{aligned}
& w_{N}=\frac{0.000976}{0.00129} \times 100 \%=75.5 \% \\
& w_{O}=\frac{0.0003}{0.00129} \times 100 \%=23.2 \% \\
& w_{A r}=\frac{0.0000171}{0.00129} \times 100 \%=1.3 \%
\end{aligned}
$$

(c) Atomic densities: (Avogadro's number is $6.02 \times 10^{23}$ )

$$
\begin{aligned}
& N_{N}=\frac{0.602 \times 10^{24}}{14} \times 0.000976=4.20 \times 10^{19} \text { atoms } / \mathrm{cm}^{3} \text { of air } \\
& N_{O}=\frac{0.602 \times 10^{24}}{16} \times 0.000300=1.13 \times 10^{19} \text { atoms } / \mathrm{cm}^{3} \text { of air } \\
& N_{A r}=\frac{0.602 \times 10^{24}}{39.9} \times 0.0000171=2.58 \times 10^{17} \mathrm{atoms} / \mathrm{cm}^{3} \text { of air }
\end{aligned}
$$

(d) Electron density:

$$
\begin{aligned}
\rho_{e} & =\left(4.2 \times 10^{19}\right) 7+\left(1.13 \times 10^{19}\right) 8+\left(2.58 \times 10^{17}\right) 18 \\
& =3.89 \times 10^{20} \text { electrons } / \mathrm{cm}^{3}
\end{aligned}
$$

(e) Average $Z$

$$
\begin{aligned}
\bar{Z} & =\frac{3.89 \times 10^{20}}{\left(4.2 \times 10^{19}\right)+\left(1.13 \times 10^{19}\right)+\left(2.58 \times 10^{17}\right)} \\
& =\frac{3.89 \times 10^{20}}{5.36 \times 10^{19}}=7.26 \text { electrons/atom }
\end{aligned}
$$

3.2 (a) Atomic density:

$$
\text { Molecules } \mathrm{H}_{2} \mathrm{O}=\frac{0.602 \times 10^{24}}{2+16} \times 1 \mathrm{~g} / \mathrm{cm}^{3}=3.34 \times 10^{22} \mathrm{molecules} / \mathrm{cm}^{3}
$$

There are thus $3.34 \times 10^{22}$ atoms $/ \mathrm{cm}^{3}$ of oxygen and $6.68 \times 10^{22}$ atoms $/ \mathrm{cm}^{3}$ of hydrogen. The atomic density is then:

$$
N\left(\mathrm{H}_{2} \mathrm{O}\right)=\left(3.34 \times 10^{22}\right)+\left(6.68 \times 10^{22}\right)=1.0 \times 10^{23} \text { atoms } / \mathrm{cm}^{3}
$$


(b) Electron density:

$$
\rho_{e}=\left(3.34 \times 10^{22}\right) 8+\left(6.68 \times 10^{22}\right) 1=3.34 \times 10^{23} \text { electrons } / \mathrm{cm}^{3}
$$

(c) Atomic number:

$$
\bar{Z}=\frac{3.34 \times 10^{23}}{1.0 \times 10^{23}}=3.34 \text { electrons } / \text { atom }
$$

3.3 (a) Discussion question.

(b) Total cross section: $\sigma_{t}=3.45+0.041=3.49 \mathrm{~b} / \mathrm{atom}$

Then $\frac{\mu_{\mathrm{t}}}{\rho}=\frac{0.602 \times 10^{24}}{14} 3.49 \times 10^{-.4 \mathrm{~s}}=0.15 \mathrm{~cm}^{2} / \mathrm{g}$

$$
\mu_{t}=0.15 \mathrm{~cm}^{2} / \mathrm{g} \times 0.00125 \mathrm{~g} / \mathrm{cm}^{3}=1.88 \times 10^{-4} \mathrm{~cm}^{-1}
$$

(c)

$$
\begin{aligned}
\frac{\mu_{t}}{\rho}(N) & =\frac{0.602 \times 10^{24}}{14}(0.806+0.025) \times 10^{-24}=0.0357 \mathrm{~cm}^{2} / \mathrm{g} \\
\frac{\mu_{t}}{\rho}(O) & =\frac{0.602 \times 10^{24}}{16}(0.921+0.032) \times 10^{-24}=0.0358 \mathrm{~cm}^{2} / \mathrm{g} \\
\frac{\mu_{t}}{\rho}(A) & =\frac{0.602 \times 10^{24}}{39.9}(2.07+0.17) \times 10^{-24}=0.0338 \mathrm{~cm}^{2} / \mathrm{g} \\
\frac{\mu_{t}}{\rho} \text { air } & =0.0357(0.755)+0.0358(0.232)+0.0338(0.013) \\
& =0.0357 \mathrm{~cm}^{2} / \mathrm{g} \\
\mu_{t}(\text { air }) & =0.0357(0.00129)=4.61 \times 10^{-5} \mathrm{~cm}^{-1}
\end{aligned}
$$

(d)

$$
\lambda=\frac{1}{\mu}=\frac{10^{5}}{4.61}=2.17 \times 10^{2} \mathrm{~m}
$$

(e) At $0.1 \mathrm{MeV}$ :

$$
\begin{aligned}
\sigma_{c o h} & =\frac{4.06\left(3.34 \times 10^{22}\right)+0.493\left(6.68 \times 10^{22}\right)}{3.34 \times 10^{22} \text { molecules } / \mathrm{cm}^{3}}=5.07 \mathrm{~b} / \text { molecule } \\
\sigma_{\text {incoh }} & =\frac{3.94\left(3.34 \times 10^{22}\right)+0.493\left(6.68 \times 10^{22}\right)}{3.34 \times 10^{22}}=4.95 \mathrm{~b} / \mathrm{molecule} \\
\sigma_{p e} & =\frac{0.071\left(3.34 \times 10^{22}\right)+0}{3.34 \times 10^{22}}=0.071 \mathrm{~b} / \text { molecule } \\
\sigma_{p p} & =0
\end{aligned}
$$

At $3.0 \mathrm{MeV}$ :

$\sigma_{\text {incoh }}=\frac{0.921\left(3.34 \times 10^{22}\right)+0.1151\left(6.68 \times 10^{22}\right)}{3.34 \times 10^{22}}=1.16 \mathrm{~b} /$ molecule 


$$
\begin{aligned}
\sigma_{c o h} & =\sigma_{\text {incoh }}=1.16 \mathrm{~b} / \text { molecule } \\
\sigma_{p p} & =\frac{0.032\left(3.34 \times 10^{22}\right)+0.00052\left(6.68 \times 10^{22}\right)}{3.34 \times 10^{22}}=0.033 \mathrm{~b} / \text { molecule } \\
\sigma_{p e} & =0
\end{aligned}
$$

3.4 Disk thickness $=\frac{0.04 \mathrm{~g} / \mathrm{cm}^{2}}{2.7 \mathrm{~g} / \mathrm{cm}^{3}}=0.0148 \mathrm{~cm}$

Disk volume, $V=\pi\left(2^{2}\right) \times 0.0138=0.186 \mathrm{~cm}^{3}$

$$
N_{A l}=\frac{\left(0.602 \times 10^{24}\right)}{27} 2.7=6.02 \times 10^{22} \text { atoms } / \mathrm{cm}^{3}
$$

The total scattering coefficient includes coherent events:

$$
\mu_{c o h}=N_{A l} \sigma_{c o h}=\left(6.02 \times 10^{22}\right)\left(6.79 \times 10^{-24}\right)=0.409 \mathrm{~cm}^{-1}
$$

The scattering coefficient for events that involve energy loss does not include coherent events,

$$
\mu_{\text {incoh }}=N_{A l} \sigma_{\text {incoh }}=\left(6.02 \times 10^{22}\right)\left(6.41 \times 10^{-24}\right)=0.386 \mathrm{~cm}^{-1}
$$

Since $0.1 \mathrm{MeV}$ is below the threshold for pair production, the photon absorption coefficient is due only to photoelectric events:

$$
\mu_{p e}=N_{A l} \sigma_{p e}=\left(6.02 \times 10^{22}\right)\left(0.78 \times 10^{-24}\right)=0.047 \mathrm{~cm}^{-1}
$$

All interactions are given by the sum

$$
\mu_{t}=\mu_{c o h}+\mu_{p e}=0.409+0.047=0.456 \mathrm{~cm}^{-1}
$$

(a) The reaction rate $(\mathrm{RR})=\Phi \mu_{t} V$, where $V$ is the total volume over which interactions are to be considered.

$$
\Phi \mu_{t} V=10^{10}(0.456)(0.186)=8.48 \times 10^{8} \text { photons } / \mathrm{sec} \text { interaction }
$$

(b) Absorption rate $=\Phi \mu_{p c} V=10^{10}(0.047)(0.186)=8.74 \times 10^{7}$ photons $/ \mathrm{sec} / \mathrm{ab}-$ sorbed.

(c) Scattering rate $=\Phi \mu_{c o h} V=10^{10}(0.409)(0.186)=7.61 \times 10^{8}$ photons $/ \mathrm{sec}$

(d) $\quad \Phi_{\text {out }}=\Phi_{\text {in }}-$ total degraded or absorbed

$$
\begin{aligned}
& =\Phi_{\text {in }}-\Phi_{\text {in }}\left(\mu_{\text {incoh }}+\mu_{p e}\right) t \\
& =10^{10}[1-0.433(0.0148)]=0.994 \times 10^{10} \text { photons cm } \mathrm{sec}^{-1}
\end{aligned}
$$

(e)

$$
\begin{aligned}
\text { Fraction } & =\frac{\text { reaction rate }}{\text { total atoms in disk }} \\
& =\frac{8.48 \times 10^{8}}{\left(6.02 \times 10^{22}\right)(0.186)}=7.57 \times 10^{-14}
\end{aligned}
$$


3.5

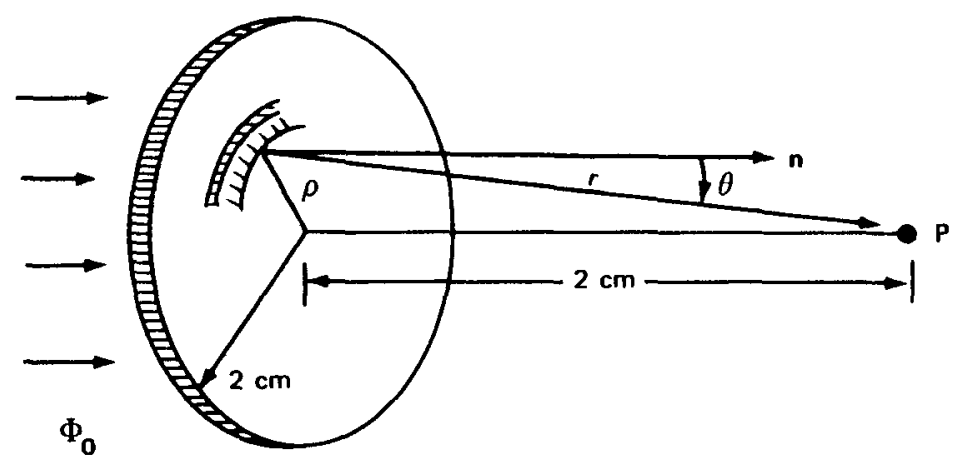

Figure for Solution 3.5 .

$$
\begin{aligned}
& r^{2}= \rho^{2}+2^{2} \\
& \rho d \rho= r d r \\
& \Delta t= 0.0148 \mathrm{~cm} \\
& d V= 2 \pi \rho d \rho \Delta t=2 \pi(0.0148) r d r \\
& \cos \theta= \frac{2}{r} \\
& \rho_{e}=\left(6.02 \times 10^{22} \text { atoms } / \mathrm{cm}^{3}\right)(13 \text { electrons/atom })=7.83 \times 10^{23} \text { electrons } / \mathrm{cm}^{3} \\
& \frac{d \mu_{s}}{d \Omega}= \sigma(\theta) \rho_{e}=\left(7.83 \times 10^{23}\right)\left(10^{-26}\right)\left[29.5-78.3\left(\frac{2}{r}\right)+56.6\left(\frac{4}{r^{2}}\right)\right] \\
& d \Phi_{s}(P)= \frac{\text { source }}{\text { steradian }} \frac{\text { steradians at } \mathrm{P}}{\mathrm{cm}}=\left(\frac{d \mu_{s}}{d \Omega} \Phi_{0} d \nu\right) \frac{1}{r^{2}} \\
& \Phi_{s}(P)= \int_{r=2}^{2 \sqrt{2}} 10^{10}\left(7.83 \times 10^{-3}\right) \\
& \times\left[29.5-\frac{78.3(2)}{r}+\frac{56.6(4)}{r^{2}}\right] \frac{1}{r^{2}}[2 \pi(0.0148) r d r] \\
&=\left(7.27 \times 10^{6}\right)\left[29.5 \ln \sqrt{2}+78.3\left(\frac{1}{\sqrt{2}}-1\right)-56.6(0.25-0.50)\right] \\
&=\left(7.27 \times 10^{6}\right) 1.34=9.75 \times 10^{6} \mathrm{photons} \mathrm{cm}^{-2} \mathrm{sec}-1 \\
&=\left(7.27 \times 10^{6}\right) \int_{2}^{2 \sqrt{2}}\left[\frac{29.5}{r}-\frac{78.3(2)}{r^{2}}+\frac{56.6(4)}{r^{3}}\right] d r \\
& r
\end{aligned}
$$




$$
\begin{aligned}
\vec{\sigma} & =\frac{\int_{0}^{\infty} \sigma(v) \Phi(v) d v}{\int_{0}^{\infty} \Phi(v) d v}=\frac{\int_{0}^{\infty} \sigma(v) n(v) v d v}{\int_{0}^{\infty} n(v) v d v} \\
& =\frac{\int_{0}^{\infty}\left(C v^{-1}\right) A v^{2} e^{-m v^{2} / 2 k T} v d v}{\int_{0}^{\infty} A v^{2} e^{-m v^{2} / 2 k T} v d v} \\
& =\frac{C \int_{0}^{\infty} v^{2} e^{-m v^{2} / 2 k T} d v}{\int_{0}^{\infty} v^{3} e^{-m v^{2} / 2 k T} d v}
\end{aligned}
$$

Let $x=\frac{m v^{2}}{2 k T}, d x=\frac{m}{k T} v d v, v d v=\frac{k T}{m} d x, v=\left(\frac{2 k T}{m}\right)^{1 / 2} x^{1 / 2}$, and $v^{2}=\frac{2 k T}{m} x$

Then $\bar{\sigma}=-C \int_{0}^{\infty}\left(\frac{2 k T}{m}\right)^{1 / 2} x^{1 / 2} e^{-x} \frac{k T}{m} d x$

$$
\int_{0}^{\infty} \frac{2 k T}{m} x e^{-x} \frac{k T}{m} d x
$$

Reference to a table of integrals shows that both numerator and denominator give gamma functions:

$$
\begin{aligned}
& \sigma=\frac{C\left(\frac{2 k T}{m}\right)^{1 / 2} \Gamma(3 / 2)}{\frac{2 k T}{m} \Gamma(2)}=C\left(\frac{2 k T}{m}\right)^{-1 / 2} \frac{[1 / 2 \Gamma(1 / 2)]}{1}=\frac{C}{2}\left(\frac{m \pi}{2 k T}\right)^{1 / 2} \\
& \bar{\sigma}=C\left(\frac{m \pi}{8 k T}\right)^{1 / 2}
\end{aligned}
$$

Since $\sigma=C / v$,

$$
\begin{aligned}
& \frac{1}{\bar{v}}=\left(\frac{m \pi}{8 k T}\right)^{1 / 2} ; \bar{v}^{2}=\frac{8 k T}{m \pi} \\
& E=\frac{m \bar{v}^{2}}{2}=\frac{m}{2}\left(\frac{8 k T}{m \pi}\right) \\
& E=\frac{4 k T}{\pi}
\end{aligned}
$$

$$
\Phi=\frac{2\left(3.7 \times 10^{10}\right)}{4 \pi(100)^{2}}=5.88 \times 10^{5} \text { photons } \mathrm{cm}^{-2} \mathrm{sec}^{-1}
$$

(The factor 2 appears because ${ }^{60} \mathrm{Co}$ emits two photons, 1.33 and $1.17 \mathrm{MeV}$, per disintegration.) 
With an average energy per disintegration of $1.25 \mathrm{MeV}$, the intensity is

$$
I=E \Phi=1.25\left(5.88 \times 10^{5}\right)=7.35 \times 10^{5} \mathrm{MeV} \mathrm{cm}^{-2} \mathrm{sec}^{-1}
$$

(a) Air:

$$
\begin{aligned}
K & =I\left(\frac{\mu_{k}}{\rho}\right)=\left(7.35 \times 10^{5}\right) 0.0268=1.97 \times 10^{4} \mathrm{MeV} \mathrm{g}^{-1} \mathrm{sec}^{-1} \\
K_{\text {air }} & =\left(1.97 \times 10^{4}\right)\left(1.6 \times 10^{-6} \mathrm{ergs} / \mathrm{MeV}\right)=0.0315 \mathrm{erg} \mathrm{g}^{-1} \mathrm{sec}^{-1}
\end{aligned}
$$

(b) Water:

$$
K_{\mathrm{H}_{2} \mathrm{O}}=0.0315\left(\frac{0.0300}{0.0268}\right)=0.0353 \mathrm{erg} \mathrm{g}^{-1} \sec ^{-1}
$$

(c) Iron:

$$
K_{F e}=0.0315\left(\frac{0.0253}{0.0268}\right)=0.0296 \mathrm{erg} \mathrm{g}^{-1} \sec ^{-1}
$$

(d) Lead:

$$
K_{P b}=0.0315\left(\frac{0.0350}{0.0268}\right)=0.0411 \mathrm{erg} \mathrm{g}^{-1} \sec ^{-1}
$$

(e) Tissue:

$$
\begin{aligned}
D & =K=0.0315\left(\frac{0.0290}{0.0268}\right)=0.034 \mathrm{erg} \mathrm{g}^{-1} \sec ^{-1} \\
& =0.034\left(\frac{3600 \mathrm{rads} / \mathrm{hr}}{100 \mathrm{ergs} \mathrm{g}^{-1} \mathrm{sec}^{-1}}\right)=1.23 \mathrm{rads} / \mathrm{hr}
\end{aligned}
$$

3.8

$$
\begin{aligned}
\lambda^{\prime}-\lambda= & 1-\cos \theta ; \cos \theta=1-\lambda^{\prime}+\lambda \\
\cos ^{2} \theta= & \left(1-\lambda^{\prime}+\lambda\right)^{2} ; \cos ^{2} \theta-1=-\sin ^{2} \theta=\left(1-\lambda^{\prime}+\lambda\right)^{2}-1 \\
-\sin ^{2} \theta= & \left(1-\lambda^{\prime}\right)^{2}+2\left(1-\lambda^{\prime}\right)(\lambda)+\lambda^{2}-1 \\
& =1-2 \lambda^{\prime}+\lambda^{\prime 2}+2 \lambda-2 \lambda \lambda^{\prime}+\lambda^{2}-1 \\
& =2\left(\lambda-\lambda^{\prime}\right)+\lambda^{2}-2 \lambda^{\prime} \lambda+\lambda^{\prime 2} \\
& =2\left(\lambda-\lambda^{\prime}\right) \pm\left(\lambda-\lambda^{\prime}\right)^{2}
\end{aligned}
$$

(a) Thus $\sigma(\theta)=\frac{3}{16 \pi}\left(\frac{\lambda}{\lambda^{\prime}}\right)^{2}\left[\frac{\lambda^{\prime}}{\lambda}+\frac{\lambda}{\lambda^{\prime}}+2\left(\lambda-\lambda^{\prime}\right)+\left(\lambda-\lambda^{\prime}\right)^{2}\right]$

The probability of scattering into solid angle $d \Omega=2 \pi \sin \theta d \theta$ (see figures for Solution 3.8) is given by $\sigma(\theta) d \Omega / \sigma_{c}$, where $\sigma_{c}=$ total Compton cross section. Since 


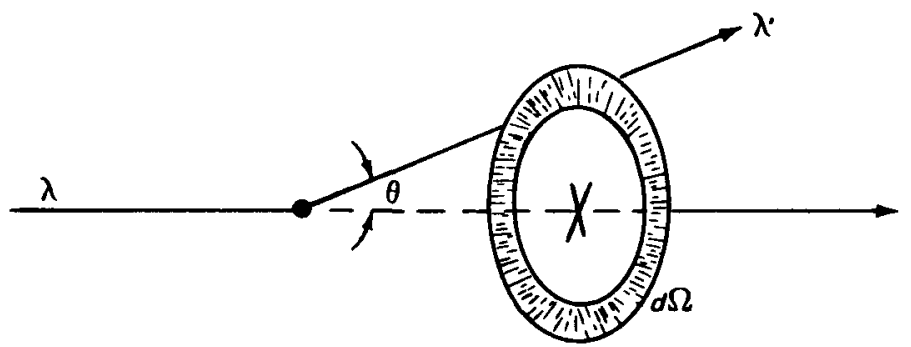

Figure for Solution 3.8 .

$$
\begin{gathered}
\lambda^{\prime}=1+\lambda-\cos \theta, d \lambda^{\prime}=\sin \theta d \theta \\
\sigma(\theta) \equiv \frac{d \sigma}{d \Omega}=\frac{d \sigma}{d \lambda^{\prime}} \frac{d \lambda^{\prime}}{d \theta} \frac{d \theta}{d \Omega}=\sigma\left(\lambda^{\prime}\right) \sin \theta \frac{1}{2 \pi \sin \theta}
\end{gathered}
$$

and

$$
\sigma\left(\lambda^{\prime}\right)=2 \pi \sigma(\theta)
$$

so that

(b)

$$
\sigma\left(\lambda^{\prime}\right)=2 \pi\left\{\frac{3}{16 \pi}\left(\frac{\lambda}{\lambda^{\prime}}\right)^{2}\left[\frac{\lambda^{\prime}}{\lambda}+\frac{\lambda}{\lambda^{\prime}}+2\left(\lambda-\lambda^{\prime}\right)+\left(\lambda-\lambda^{\prime}\right)^{2}\right]\right\}
$$

\section{CHAPTER 4}

4.1 $(\mathrm{n} \cdot \Omega)=\cos \theta$, where $\theta$ is the angle between the planes containing $d A$ and $d S$. When $\theta<\pi / 2, \cos \theta>0$ and the leakage through $d S$ along $\Omega$ is also outward (toward $\mathbf{n}$ ) through $d S$. When $\pi / 2<\theta<\pi$, the leakage along $\Omega$ through $d S$ is opposite in direction to $n$, but then $\cos \theta<0$. Thus the sign of $(n \cdot \Omega)$ properly is positive when the leakage through $d S$ is outward and negative when it is inward.

4.2 We use the Compton wavelength $\dagger \lambda=1 / E$, which implies $d E=-\lambda^{-2} d \lambda$, and first prove two lemmas:

Lemma 1: $I(\lambda)=I(E)$ where $I(\lambda) \equiv \lambda \Phi(\lambda)$ and $I(E) \equiv E \Phi(E)$.

Proof: Between two values of wavelength, $\lambda_{a}>\lambda_{b}$, conservation of particles demands that

$$
\int_{\lambda_{b}}^{\lambda_{a}} \Phi(\lambda) d \lambda=\int_{E\left(\lambda_{a}\right)}^{E\left(\lambda_{b}\right)} \Phi(E) d E
$$


(The limits are reversed since $\lambda$ increases as $E$ decreases.) Thus

$$
\int_{\lambda_{b}}^{\lambda_{a}} \Phi(\lambda) d \lambda=\int_{\lambda_{a}}^{\lambda_{b}} \Phi(E) \frac{d E}{d \lambda} d \lambda=\int_{\lambda_{a}}^{\lambda_{b}} \Phi(E)\left(-\frac{1}{\lambda^{2}}\right) d \lambda=\int_{\lambda_{b}}^{\lambda_{a}} \Phi(E)\left(\frac{1}{\lambda^{2}}\right) d \lambda
$$

The relation must hold for all $\lambda_{a}$ and $\lambda_{b}$; the integrands must be equal, and

$$
\Phi(\lambda)=\frac{1}{\lambda^{2}} \Phi(E) ; \text { thus } \lambda \Phi(\lambda)=E \Phi(E)
$$

Lemma 2: $\lambda^{2} \quad \Sigma_{s}\left(\lambda^{\prime}\right) p\left(\lambda^{\prime} \rightarrow \lambda, \Omega^{\prime} \rightarrow \Omega\right) \Phi\left(\lambda^{\prime}\right)=\Sigma_{s}\left(E^{\prime}\right) p\left(E^{\prime} \rightarrow E, \Omega^{\prime} \rightarrow \Omega\right) \Phi\left(E^{\prime}\right)$

Using conservation of particles scattering from $E^{\prime}\left(\lambda^{\prime}\right)$ into the energy interval $\left(E_{b}, E_{a}\right)$ where $E_{a}>E_{b}$, the following must hold (where $E^{\prime}=1 / \lambda^{\prime}$ ):

$$
\begin{aligned}
& \int_{E=E_{b}}^{E_{a}} \Sigma_{s}\left(E^{\prime}\right) p\left(E^{\prime} \rightarrow E, \Omega^{\prime} \rightarrow \Omega\right) \Phi\left(E^{\prime}\right) d E=\int_{\lambda=\lambda_{a}}^{\lambda_{b}} \Sigma_{s}\left(\lambda^{\prime}\right) p\left(\lambda^{\prime} \rightarrow \lambda, \Omega^{\prime} \rightarrow \Omega\right) \Phi\left(\lambda^{\prime}\right) d \lambda \\
& \int_{\lambda_{b}}^{\lambda_{a}} \Sigma_{s}\left(E^{\prime}\right) p\left(E^{\prime} \rightarrow E, \Omega^{\prime} \rightarrow \Omega\right) \Phi\left(E^{\prime}\right) \frac{d E}{d \lambda} d \lambda=\int_{\lambda_{a}}^{\lambda_{b}} \Sigma_{s}\left(\lambda^{\prime}\right) p\left(\lambda^{\prime} \rightarrow \lambda, \Omega^{\prime} \rightarrow \Omega\right) \Phi\left(\lambda^{\prime}\right) d \lambda \\
& \int_{\lambda_{a}}^{\lambda_{b}} \Sigma_{s}\left(E^{\prime}\right) p\left(E^{\prime} \rightarrow E, \Omega^{\prime} \rightarrow \Omega\right) \Phi\left(E^{\prime}\right)\left(\frac{1}{\lambda^{2}}\right) d \lambda=\int_{\lambda_{a}}^{\lambda_{b}} \Sigma_{s}\left(\lambda^{\prime}\right) p\left(\lambda^{\prime} \rightarrow \lambda, \Omega^{\prime} \rightarrow \Omega\right) \Phi\left(\lambda^{\prime}\right) d \lambda
\end{aligned}
$$

Again the integrands must be equal. Thus

$$
\Sigma_{s}\left(E^{\prime}\right) p\left(E^{\prime} \rightarrow E, \Omega^{\prime} \rightarrow \Omega\right) \Phi\left(E^{\prime}\right)=\lambda^{2} \Sigma_{s}\left(\lambda^{\prime}\right) p\left(\lambda^{\prime} \rightarrow \lambda, \Omega^{\prime} \rightarrow \Omega\right) \Phi\left(\lambda^{\prime}\right)
$$

Now, multiplying the Boltzmann equation (Eq. 4.2-8) by E, we obtain

$$
\nabla \cdot \Omega I(E)+\Sigma_{t}(E) I(E)=\int_{\Omega^{\prime}} \int_{E^{\prime}=E}^{\infty} \Sigma_{s}\left(E^{\prime}\right) p\left(E^{\prime} \rightarrow E, \Omega^{\prime} \rightarrow \Omega\right) \Phi\left(E^{\prime}\right) E d E^{\prime} d \Omega^{\prime}+E S(E)
$$

Making the change of variable to $\lambda$ and using the two lemmas, we obtain

$$
\begin{array}{r}
\nabla \cdot \Omega I(\lambda)+\Sigma_{t}(\lambda) I(\lambda)=\int_{\Omega^{\prime}} \int_{\lambda^{\prime}=\lambda}^{0} \lambda^{2} \Sigma_{s}\left(\lambda^{\prime}\right) p\left(\lambda^{\prime} \rightarrow \lambda, \Omega^{\prime} \rightarrow \Omega\right) \Phi\left(\lambda^{\prime}\right) \\
\quad \times \frac{1}{\lambda} \frac{d E^{\prime}}{d \lambda^{\prime}} d \lambda^{\prime} d \Omega^{\prime}+E S(E)
\end{array}
$$

Now, recalling $\lambda^{\prime} \Phi\left(\lambda^{\prime}\right)=I\left(\lambda^{\prime}\right), d E^{\prime} / d \lambda^{\prime}=-\left(\lambda^{\prime}\right)^{-2}$, and formally defining $S(\lambda)=E S(E)$, we obtain

$$
\nabla \cdot \Omega I(\lambda)+\Sigma_{t}(\lambda) I(\lambda)=\int_{\Omega^{\prime}} \int_{0}^{\lambda} \Sigma_{s}\left(\lambda^{\prime}\right) p\left(\lambda^{\prime} \rightarrow \lambda, \Omega^{\prime} \rightarrow \Omega\right) I\left(\lambda^{\prime}\right) \frac{\lambda}{\lambda^{\prime}} d \lambda^{\prime} d \Omega^{\prime}+S(\lambda)
$$


4.3 The slab geometry is shown in the figure for solution 4.3.

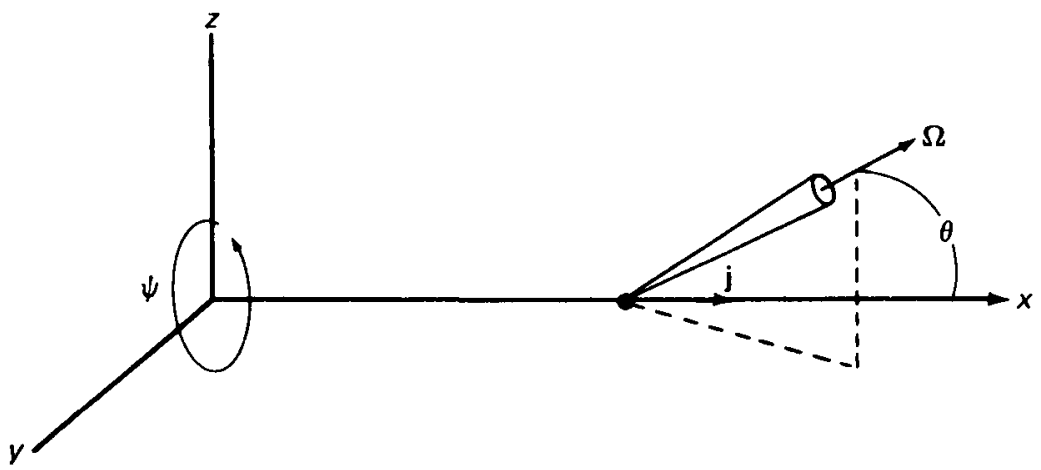

Solution 4.3 , geometry.

We first note that because of the azimuthal symmetry $\partial \Phi / \partial y=\partial \Phi / \partial z=0$ and $\mathrm{i} \cdot \boldsymbol{\Omega}=\cos \theta$. Thus

$$
\nabla \cdot \Omega \Phi(r, \Omega)=\Omega \cdot \nabla \Phi=\Omega \cdot\left(\frac{\partial \Phi}{\partial x} \mathrm{i}\right)=\Omega \cdot \mathrm{i} \frac{\partial \Phi}{\partial x}=\cos \theta \frac{\partial \Phi(\mathrm{r}, \Omega)}{\partial x}
$$

We define $\Sigma_{s} p\left(\Omega^{\prime} \rightarrow \Omega\right)=\Sigma_{s}\left(\Omega, \Omega^{\prime}\right)$; the balance equation becomes

$$
\cos \theta \frac{\partial \Phi(\mathbf{r}, \Omega)}{\partial x}+\Sigma_{t} \Phi(\mathbf{r}, \Omega)=\int_{\Omega^{\prime}} \Sigma_{s}\left(\Omega, \Omega^{\prime}\right) \Phi\left(\mathbf{r}, \Omega^{\prime}\right) d \Omega^{\prime}+S(\mathbf{r}, \Omega)
$$

The differential flux density in the preceding equation has units of particles $\mathrm{cm}^{-2} \sec ^{-1} \operatorname{steradian}^{-1}$. Let $\mu=\cos \theta, d \mu=-\sin \theta d \theta$, and $d \Omega=-d \mu d \psi$. The flux density per unit $\mu$ is given by

$$
\Phi(x, \mu)=\int_{\psi=0}^{2 \pi} \Phi(r, \Omega) d \psi=2 \pi \Phi(r, \Omega)
$$

(Note: $\Phi(\mathbf{r}, \boldsymbol{\Omega})$ is not a function of $\psi$ because of azimuthal symmetry.) Integrating each term over $\psi$, we obtain

$$
\mu \frac{\partial \Phi(x, \mu)}{\partial x}+\Sigma_{t} \Phi(x, \mu)=\int_{\psi=0}^{2 \pi} \int_{\Omega^{\prime}} \Sigma_{s}\left(\Omega, \Omega^{\prime}\right) \Phi\left(x, \Omega^{\prime}\right) d \Omega^{\prime} d \psi+S(x, \mu)
$$

where $\int_{0}^{2 \pi} S(\mathbf{r}, \Omega) d \psi=S(x, \mu)$. Now integrate the inscattering integral over $\psi^{\prime}\left(d \Omega^{\prime}=\right.$ $\left.\sin \theta^{\prime} d \theta^{\prime} d \psi^{\prime}=-d \mu^{\prime} d \psi^{\prime}\right)$ :

$$
\begin{aligned}
& \int_{0}^{2 \pi} \int_{0}^{2 \pi} \int_{0}^{\pi} \Sigma_{s}\left(\Omega, \Omega^{\prime}\right) \Phi\left(x, \Omega^{\prime}\right)\left(\sin \theta^{\prime}\right) d \theta^{\prime} d \psi^{\prime} d \psi \\
& =\int_{\psi=0}^{2 \pi} \int_{\psi^{\prime}=0}^{2 \pi} \int_{\mu^{\prime}=-1}^{1} \Sigma_{s}\left(\Omega, \Omega^{\prime}\right) \Phi\left(x, \Omega^{\prime}\right) d \mu^{\prime} d \psi^{\prime} d \psi
\end{aligned}
$$


Substituting this in the balance equation above completes the derivation. Note that the inscattering integral cannot be integrated over $\psi$ or $\psi^{\prime}$ since, in general, $\Sigma_{s}\left(\Omega, \Omega^{\prime}\right)$ can be a function of $\psi$ and $\psi^{\prime}$. However, see the solution to problem 4.4 for a method of handling this integration.

4.4 The point source geometry is shown in sketch a for solution 4.4.

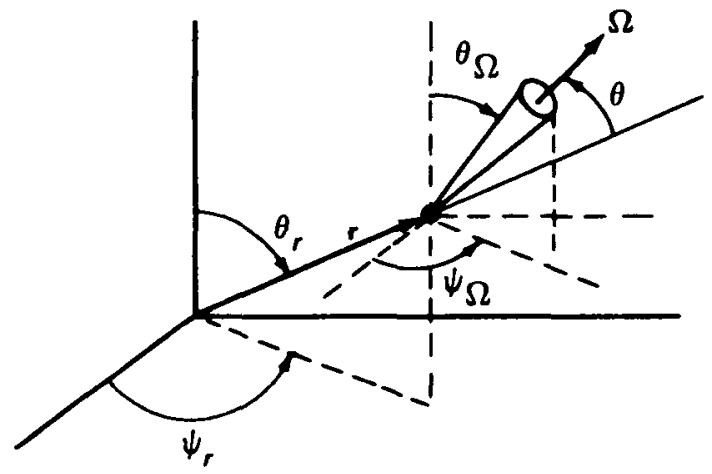

Solution 4.4 , sketch a.

Care must be taken in developing the angle dependencies. The polar angle $\theta$ between $r$ and $\Omega$ has an implicit dependence on the polar angle $\theta_{r}$ of the radius vector $r$. Sketch $b$ for solution 4.4 illustrates the relations:

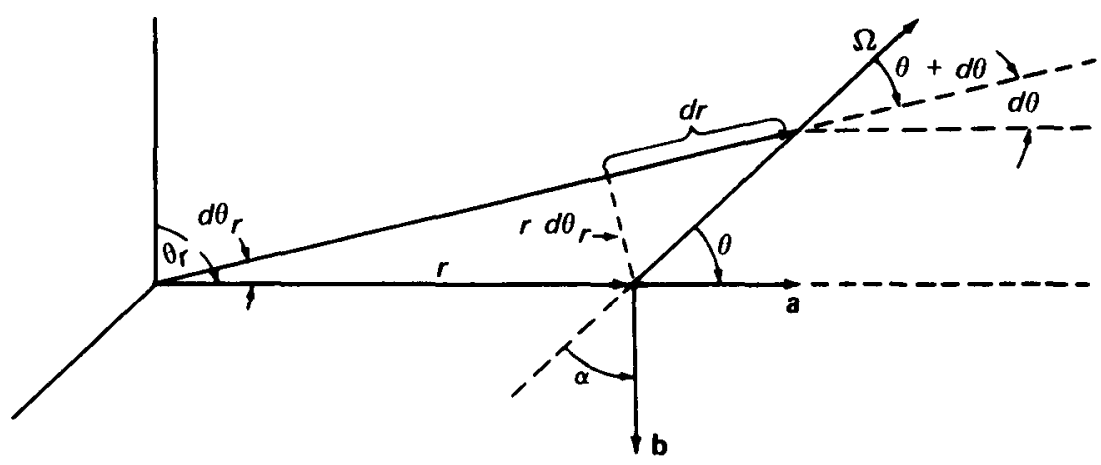

Solution 4.4 , sketch $b$.

where $a$ is the unit vector along $r$ and $b$ is the unit vector associated with $\theta_{r}$ in spherical coordinates. The sketch shows the change $d \theta_{r}$ and $d \theta$ (both negative changes) as $|r|$ goes from $r$ to $r+d r$. Also observe that $\alpha=(\pi / 2)-\theta$ and that $d \theta_{r}=$ $d \theta$. Thus

$\nabla \cdot \Omega \Phi\left(r, \theta_{r} ; \Omega ; E\right)=\Omega \cdot \nabla \Phi\left(r, \theta_{r} ; \Omega ; E\right)=\Omega\left(\frac{\partial \Phi}{\partial r} a+\frac{1}{r} \frac{\partial \Phi}{\partial \theta_{r}} b\right)=\frac{\partial \Phi}{\partial r}(\Omega \cdot a)+\frac{1}{r} \frac{\partial \Phi}{\partial \theta_{r}}(\Omega \cdot b)$ 
But

$$
\begin{aligned}
& (\boldsymbol{\Omega} \cdot \mathbf{a})=\cos \theta \\
& (\boldsymbol{\Omega} \cdot \mathbf{b})=-\cos \alpha=-\sin \left(\frac{\pi}{2}-\alpha\right)=-\sin \theta
\end{aligned}
$$

and

$$
\nabla \cdot \Omega \Phi=\cos \theta \frac{\partial \Phi}{\partial r}+\frac{1}{r}(-\sin \theta) \frac{\partial \Phi}{\partial \theta_{r}}=\cos \theta \frac{\partial \Phi}{\partial r}+\frac{1}{r}(-\sin \theta) \frac{d \Phi}{d \theta}
$$

Let

$\mu=\cos \theta, \frac{d \mu}{d \theta}=-\sin \theta$, and apply the chain rule:

$$
\boldsymbol{\nabla} \cdot \Omega \Phi=\mu \frac{\partial \Phi}{\partial r}+\frac{1}{r}(-\sin \theta) \frac{\partial \Phi}{\partial \mu} \frac{d \mu}{d \theta}=\mu \frac{\partial \Phi}{\partial r}+\frac{1}{r} \sin ^{2} \theta \frac{\partial \Phi}{\partial \mu}
$$

Since $\sin ^{2} \theta=1-\mu^{2}$,

$$
\nabla \cdot \Omega \Phi(r, \Omega, E)=\mu \frac{\partial \Phi}{\partial r}+\frac{1-\mu^{2}}{r} \frac{\partial \Phi}{\partial \mu}
$$

The balance equation becomes

$$
\begin{aligned}
& \mu \frac{\partial \Phi(r, \Omega, E)}{\partial r}+\frac{1-\mu^{2}}{r} \frac{\partial \Phi(r, \Omega, E)}{\partial \mu}+\Sigma_{t}(r, E) \Phi(r, \Omega, E) \\
&=\int_{E^{\prime}} \int_{\Omega^{\prime}} \Sigma_{s}\left(r, E^{\prime}\right) p\left(E^{\prime} \rightarrow E, \Omega^{\prime} \rightarrow \Omega\right) \Phi\left(r, \Omega^{\prime}, E^{\prime}\right) d \Omega^{\prime} d E+S(r, \Omega, E)
\end{aligned}
$$

As in problem 4.3, the differential flux density $\Phi(r, \Omega, E)$ is in units of particles $\mathrm{cm}^{-2} \mathrm{sec}^{-1} \mathrm{MeV}^{-1}$ steradian ${ }^{-1}$. Now

$$
\int_{\psi=0}^{2 \pi} \Phi(r, \Omega, E) d \psi=2 \pi \Phi(r, \Omega, E) \equiv \Phi(r, \mu, E)
$$

Integrate the balance equation over $\psi$ to obtain

$$
\begin{aligned}
\mu \frac{\partial \Phi(r, \mu, E)}{\partial r}+ & \frac{1-\mu^{2}}{r} \frac{\partial \Phi(r, \mu, E)}{\partial \mu}+\Sigma_{t}(r, E) \Phi(r, \mu, E) \\
& =\int_{E^{\prime}} \int_{\psi} \int_{\Omega^{\prime}} \Sigma_{\mathcal{s}}\left(r, E^{\prime}\right) p\left(E^{\prime} \rightarrow E, \Omega^{\prime} \rightarrow \Omega\right) \Phi\left(r, \Omega^{\prime} E^{\prime}\right) d \bar{\Omega}^{\prime} d \psi d E^{\prime}+S(r, \mu, E)
\end{aligned}
$$

where again

$$
\int_{0}^{2 \pi} S(r, \Omega, E) \equiv S(r, \mu, E)
$$


Because of the azimuthal symmetry, the inscattering integral can be written

$$
\int_{E^{\prime}} \int_{\mu^{\prime}=-1}^{1} \frac{1}{2 \pi} \Phi\left(r, \mu^{\prime}, E^{\prime}\right) \int_{\psi} \int_{\psi^{\prime}} \Sigma_{s}\left(r, E^{\prime}\right) p\left(E^{\prime} \rightarrow E, \Omega^{\prime} \rightarrow \Omega\right) d \psi^{\prime} d \psi d \mu^{\prime} d E^{\prime}
$$

We now assume $p\left(E^{\prime} \rightarrow E, \Omega^{\prime} \rightarrow \Omega\right)$ is a function only of $E^{\prime}, E$, and $\mu_{0}$, where $\mu_{0}=$ $\Omega^{\prime} \cdot \Omega=f\left(\mu^{\prime}, \mu\right)$. Define

$$
\Sigma_{s}\left[r, E^{\prime} \rightarrow E, \mu_{0}\left(\mu^{\prime}, \mu\right)\right] \equiv 2 \pi \int_{\psi=0}^{2 \pi} \int_{\psi^{\prime}=0}^{2 \pi} \Sigma_{s}\left(r, E^{\prime}\right) p\left(E^{\prime} \rightarrow E, \Omega^{\prime} \rightarrow \Omega\right) d \psi^{\prime} d \psi
$$

to obtain

$$
\begin{aligned}
\mu \frac{\partial \Phi(r, \mu, E)}{\partial r}+\frac{1-\mu^{2}}{r} & \frac{\partial \Phi(r, \mu, E)}{\partial \mu}+\Sigma_{t}(r, E) \Phi(r, \mu, E) \\
& =\int_{E^{\prime}=0}^{\infty} \int_{\mu^{\prime}=-1}^{1} \Sigma_{s}\left[r, E^{\prime} \rightarrow E, \mu_{0}\left(\mu^{\prime}, \mu\right)\right] \Phi\left(r, \mu^{\prime}, E^{\prime}\right) d \mu^{\prime} d E^{\prime}+S(r, \mu, E)
\end{aligned}
$$

4.5 Using the results of problems 4.2 and 4.3, we can immediately write the Boltzmann equation as

$\mu \frac{\partial I(x, \mu, \lambda)}{\partial x}+\Sigma_{t}(\lambda) I(x, \mu, \lambda)$

$$
=\int_{0}^{\lambda} \int_{0}^{2 \pi} \int_{\Omega^{\prime}} \Sigma_{s}\left(\lambda^{\prime}\right) p\left(\lambda^{\prime} \rightarrow \lambda, \Omega^{\prime} \rightarrow \Omega\right) I\left(x, \Omega^{\prime}, \lambda^{\prime}\right) \frac{\lambda}{\lambda^{\prime}} d \Omega^{\prime} d \psi d \lambda^{\prime}+S(x, \mu, \lambda)
$$

However, for photon scattering by the Compton process (see Sec. 3.2.1), the following relation holds:

$$
\lambda-\lambda^{\prime}=1-\cos \theta_{0}=1-\Omega^{\prime} \cdot \Omega
$$

where $\theta_{0}$ is the scattering angle. Thus

$$
\Sigma_{s}\left(\lambda^{\prime}\right) p\left(\lambda^{\prime} \rightarrow \lambda, \Omega^{\prime} \rightarrow \Omega\right)=\left\{\begin{array}{c}
\Sigma_{s}\left(\lambda^{\prime}, \Omega^{\prime} \cdot \Omega\right) ; 1-\cos \theta_{0}=\lambda-\lambda^{\prime} \\
0 ; 1-\cos \theta_{0} \neq \lambda-\lambda^{\prime}
\end{array}\right.
$$

and we can write $\left(\right.$ since $\left.\cos \theta_{0}=\Omega^{\prime} \cdot \Omega\right)$

$$
\Sigma_{s}\left(\lambda^{\prime}\right) p\left(\lambda^{\prime} \rightarrow \lambda, \boldsymbol{\Omega}^{\prime} \rightarrow \boldsymbol{\Omega}\right)=\Sigma_{s}\left(\lambda^{\prime}, \boldsymbol{\Omega}^{\prime} \cdot \boldsymbol{\Omega}\right) \delta\left(1+\lambda^{\prime}-\lambda-\boldsymbol{\Omega}^{\prime} \cdot \boldsymbol{\Omega}\right)
$$

The inscattering integral can now be integrated over $\psi$ to obtain

$$
\int_{0} \int_{\Omega^{\prime}} \Sigma_{s}\left(\lambda^{\prime}, \Omega^{\prime} \cdot \Omega\right) \delta\left(1+\lambda^{\prime}-\lambda-\Omega^{\prime} \cdot \Omega\right) I\left(x, \Omega^{\prime}, \lambda^{\prime}\right) \frac{\lambda}{\lambda^{\prime}} d \Omega^{\prime} d \lambda^{\prime}
$$


Letting $k=2 \pi \Sigma_{s}\left(\frac{\lambda}{\lambda^{\prime}}\right)$, we obtain

$\mu \frac{\partial I(x, \mu, \lambda)}{\partial x}+\Sigma_{t} I(x, \mu, \lambda)=S(x, \mu, \lambda) \delta(x)$

$$
+\int_{0}^{\lambda} \int_{\Omega^{\prime}} k \frac{\delta\left(1+\lambda^{\prime}-\lambda-\Omega^{\prime} \cdot \Omega\right)}{2 \pi} I\left(x, \Omega^{\prime}, \lambda^{\prime}\right) d \Omega^{\prime} d \lambda^{\prime}
$$

The delta function in the source term forces the source to be zero everywhere except in the $y-z$ plane at $x=0$.

\section{CHAPTER 5}

\subsection{Event space:}

(a) The set $\{1,2,3,4,5,6\}$.

(b) The set of unordered pairs $(21$ members) $\{(1,1) ;(1,2) ;(1,3) ;(1,4)$; $(1,5) ;(1,6) ;(2,2) ;(2,3) ;(2,4) ;(2,5)$; $(2,6) ;(3,3) ;(3,4),(3,5) ;(3,6) ;(4,4)$; $(4,5) ;(4,6) ;(5,5) ;(5,6) ;(6,6)\}$.

(c) The set of ordered pairs (36 members) $\{(1,1) ;(1,2) ; \ldots ;(1,6)$; $(2,1) ;(2,2) ; \ldots ;(2,6) ;(3,1) ; \ldots$; $(3,6) ; \ldots ;(6,6)\}$.

(d) The continuous set of all energies into which fission neutrons can be born.

(e) The set of all energies from $E$ (energy of the incident photon) to $E^{\prime}$ (energy resulting from $180^{\circ}$ scatter),

$$
\text { or }
$$

the set of all photon scattering angles from $0^{\circ}$ to $180^{\circ}$,

or

the set of all electron scattering angles from $0^{\circ}$ to $90^{\circ}$, etc.
Typical event:

A die landing 3.

A pair of dice showing $(2,4)$ (note that the dice are indistinguishable).

A die landing 4 on the first throw and 2 on the second throw.

A neutron emitted from the fission process at $1.0 \mathrm{MeV}$.

A photon leaving the collision point with energy $1.0 \mathrm{MeV}$,

or

a photon scattering through $75^{\circ}$,

or

an electron scattered at an angle of $30^{\circ}$,

etc.

\subsection{For 5.1(a):}

(a) The PDF is the point function having a value of $1 / 6$ at $1,2,3,4,5,6$ and zero elsewhere. 


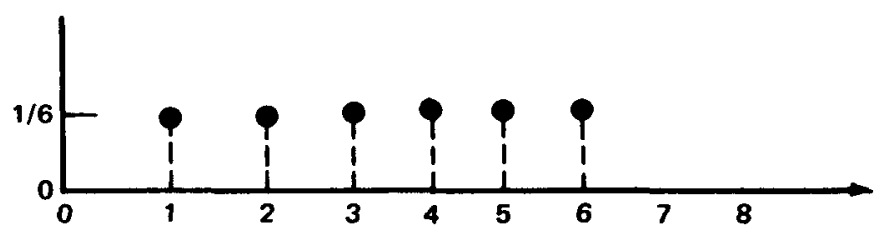

Solution to Exercise 5.2(a). Values of PDF.

(b) The CDF is the function

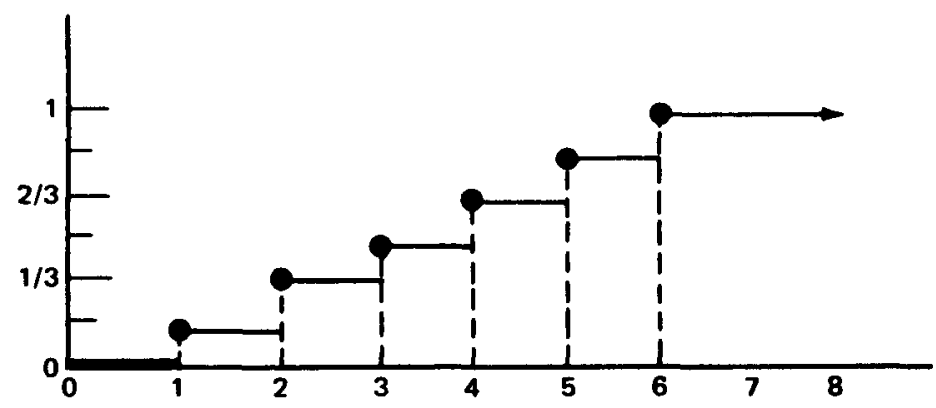

Solution to Exercise $5.2(\mathrm{~b})$. Values of CDF.

(c) A random-variable function is not necessary to define the PDF. At least an ordering of the six events is necessary to plot the CDF.

For 5.1(b):

(d) Values of the PDF are listed in the accompanying matrix. It is zero elsewhere.

\begin{tabular}{|c|c|c|c|c|c|}
\hline $2 / 36$ & $2 / 36$ & $2 / 36$ & $2 / 36$ & $2 / 36$ & $1 / 36$ \\
\hline $2 / 36$ & $2 / 36$ & $2 / 36$ & $2 / 36$ & $1 / 36$ & \\
\hline $2 / 36$ & $2 / 36$ & $2 / 36$ & $1 / 36$ & & \\
\hline $2 / 36$ & $2 / 36$ & $1 / 36$ & & & \\
\hline $2 / 36$ & $1 / 36$ & & & & \\
\hline $1 / 36$ & & & & & \\
\hline
\end{tabular}

Solution to Exercise 5.2(d). Values of PDF. 
(e) To obtain the CDF, we must order the possible outcomes. One way to accomplish this is to assign integers to the outcomes. Let $f(i, j)=k$ be the function defined in the accompanying matrix.

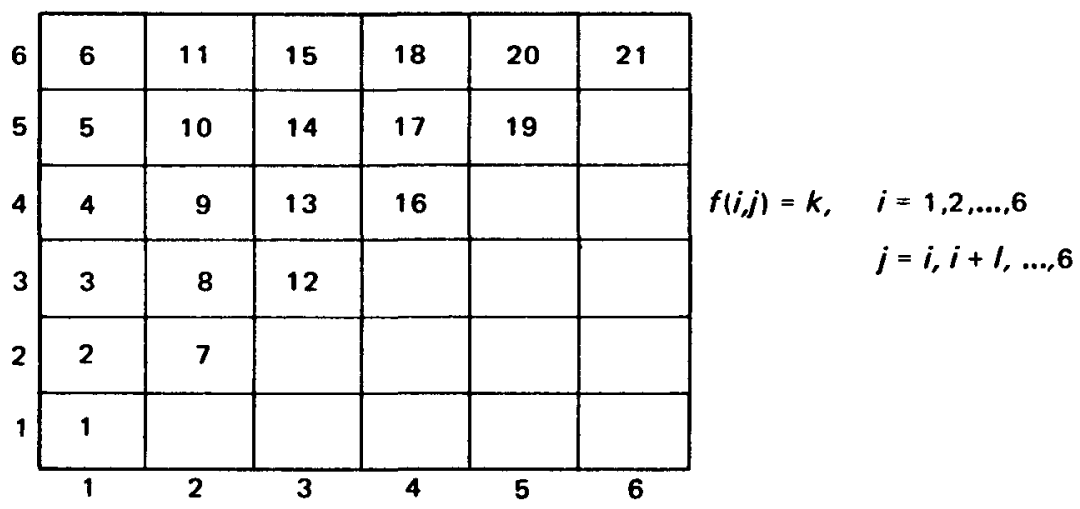

Solution to Exercise 5.2(e). Values of CDF.

The CDF then becomes

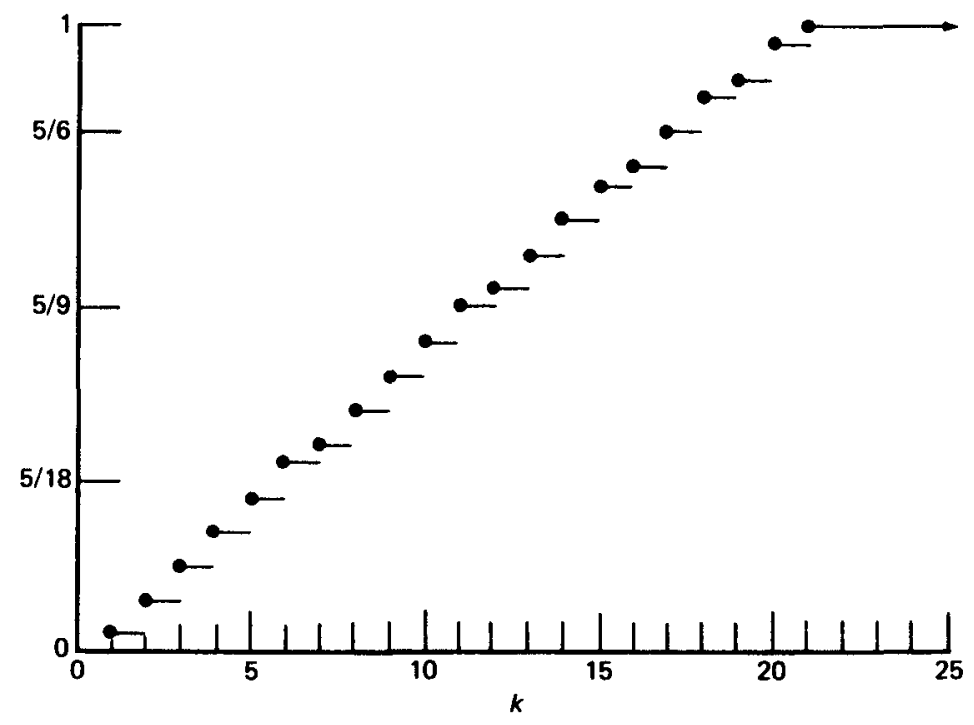

(f) For the PDF, no; for the CDF, yes.

For $5.1(\mathrm{c})$ :

(g) The PDF has a value of $1 / 36$ for each ordered pair and is zero elsewhere. 
(h) The CDF must be constructed as above. It will be a step function increasing $1 / 36$ at each step.

5.3 (a) Figure 2.1 is not a PDF. (It has values greater than 1). Figure 2.4 is a PDF. Its values are in $(0,1)$, and the integral under the curve is 1 . (b) Figure 2.1 can be converted to a PDF by integrating the curve from 0 to infinity and dividing the plotted values by the value of the integral.

5.4 See graph for solution 5.4.

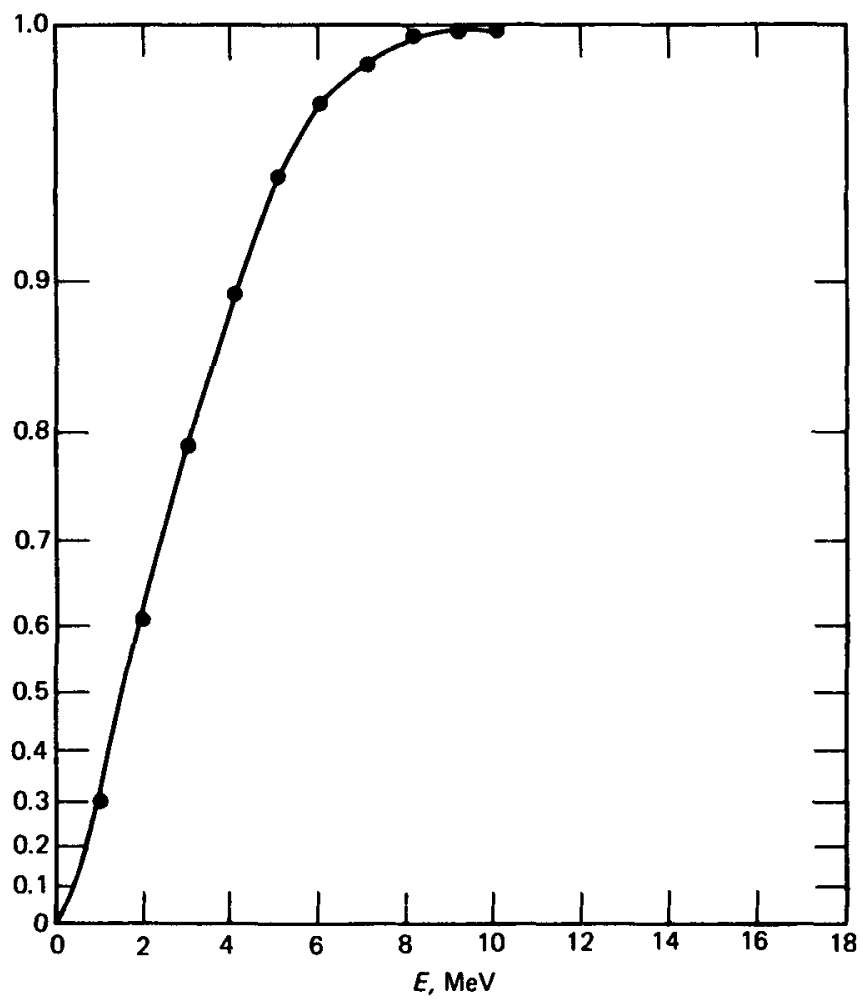

Solution to Exercise 5.4.

5.5 (a) $\int_{0}^{\pi} C \sin x d x=1$ implies $C=1 / 2$. Thus $f(x)=(\sin x) / 2$ and $0<f(x)<1$.

(b) $\mathrm{CDF}=\int_{0}^{x}(\sin y) / 2 d y=(1-\cos x) / 2$.

(c) See graph for solution 5.5 . 


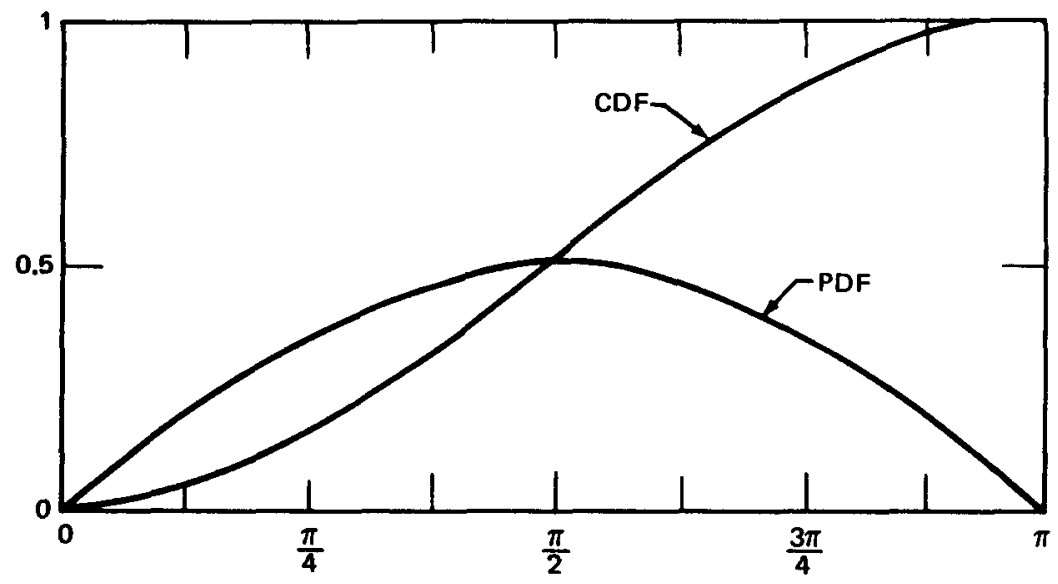

Solution to Exercise $5.5(\mathrm{c})$.

(d) With $0,0.1,0.2, \ldots, 0.9,1.0$, the following choices of $x$ are obtained:

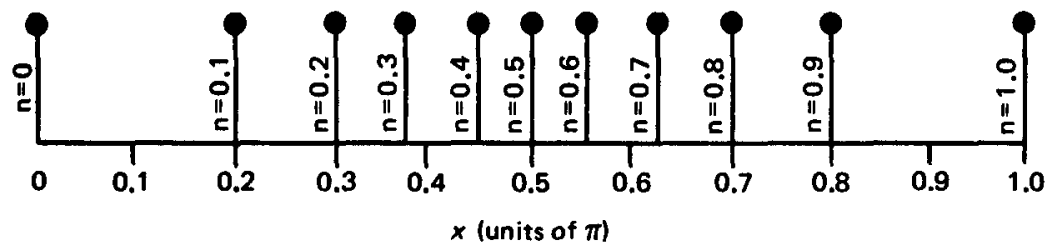

Solution to Exercise 5.5(d).

(e) They are distributed as the PDF. (Notice the bunching toward $x=\pi / 2$ ).

(f) Probably so, if enough random samples, $n$, were chosen with equal probability from $(0,1)$. (But no guarantees for a finite number of choices-you might have a run of bad luck!)

(g) Choosing the samples equally distributed in $(0,1)$ forced the values of $x$ to be distributed exactly as the PDF. Thus we forced more choices to be made in the important region around $\pi / 2$ where the PDF has its maximum value. This was, in fact, systematic sampling (See Sec. 5.3.4). The samples were chosen from the biased distribution

$$
f^{\prime}(x)=f(x) h(x)
$$

where $h(x)=2 / \sin x$ and were adjusted by $1 / h(x)$. 
5.6 (a) The graph is not a PDF. The values are not constrained within $(0,1)$.

(b) The following table for $5.6(\mathrm{~b})$ was the result of 300 trials:

SOLUTION TO EXERCISE 5.6(b)-AUTHOR'S RESULTS

\begin{tabular}{|c|c|c|c|c|c|c|c|}
\hline \multirow{2}{*}{$\begin{array}{l}\text { No. of } \\
\text { rolls }\end{array}$} & \multicolumn{2}{|c|}{$\mathbf{A}$} & \multicolumn{2}{|l|}{ B } & \multicolumn{2}{|l|}{ C } & \multirow{2}{*}{$\begin{array}{l}\text { No. of } \\
\text { rejections }\end{array}$} \\
\hline & Selected & $\%$ & Selected & $\%$ & Selected & $\%$ & \\
\hline 100 & 12 & $20 \%$ & 23 & $39 \%$ & 24 & $41 \%$ & 41 \\
\hline 200 & 20 & $16 \%$ & 44 & $36 \%$ & 58 & $48 \%$ & 78 \\
\hline 300 & 33 & $18 \%$ & 64 & $35 \%$ & 86 & $47 \%$ & 117 \\
\hline Expected & & $17 \%$ & & $33 \%$ & & $50 \%$ & \\
\hline
\end{tabular}

That the distribution is not closer to the expected one is revealed in the smaller table for the distribution of results of tossing the first die (to select the letter to be tested):

$\begin{gathered}\text { SOLUTION TO EXERCISE 5.6(b)-ANALYSIS } \\
\text { OF AUTHORS }\end{gathered}$
\begin{tabular}{cc} 
Letter & $\begin{array}{c}\text { No. of choices } \\
\text { in } 300 \text { rolls }\end{array}$ \\
\hline A & 108 \\
B & 106 \\
C & 86
\end{tabular}

Perhaps it can be explained by the fact that the authors randomly selected the two dice from their well-used set of "liar's dice." Of course, a better explanation is that these results are well within the accuracy to be expected for 300 trials.

\section{CHAPTER 6}

6.1 Use Fig. 6.1 , and interpolate to obtain curves at 85 and $95 \mathrm{~cm}$. Use the $120-\mathrm{cm}$ curve directly.

(a) The spectrum at 1 is given by the $95-\mathrm{cm}$ curve $\times 2 \times 10^{10}$. The spectrum at 2 is the sum of the $85-\mathrm{cm}$ and the $120-\mathrm{cm}$ spectra $\times 1 \times 10^{10}$. Observe that at point 2 the contribution of source $A$ is inconsequential. 
SOLUTION TO EXERCISE 6.1(a)

\begin{tabular}{|c|c|c|c|c|c|c|c|}
\hline & \multicolumn{6}{|c|}{$\mathrm{E}, \mathrm{T} \mathrm{MeV}$} \\
\hline & & 0.1 & 1 & 2 & 3 & 4 & 5 \\
\hline $\begin{array}{c}r=85 \\
\mathrm{~cm} \\
r=95 \\
\mathrm{~cm} \\
r=120 \\
\mathrm{~cm} \\
\text { Spectrum } \\
\text { Spectrum }\end{array}$ & $\begin{array}{l}4 \pi r^{2} N_{0} \\
N_{0} \\
4 \pi r^{2} N_{0} \\
N_{0} \\
4 \pi r^{2} N_{0} \\
N_{0} \\
\text { at } 1 \ddagger \\
\text { at } 2 \ddagger\end{array}$ & $\begin{array}{l}4(-5) \\
4.4(-10) \\
4(-6) \\
3.3(-11) \\
1(-6) \\
5.5(-12) \\
0.66 \\
4.4\end{array}$ & $\begin{array}{l}1.5(-5) \\
1.7(-10) \\
1.5(-6) \\
1.2(-11) \\
3.5(-7) \\
1.9(-12) \\
0.24 \\
1.7\end{array}$ & $\begin{array}{l}9(-6) \\
9.9(-11) \\
1.1(-6) \\
9.1(-12) \\
2.2(-7) \\
1.2(-12) \\
0.18 \\
0.99\end{array}$ & $\begin{array}{l}6(-6) \\
6.6(-11) \\
9(-7) \\
7.4(-12) \\
2.0(-7) \\
1.1(-12) \\
0.15 \\
0.66\end{array}$ & $\begin{array}{l}4(-6) \\
4.4(-11) \\
6(-7) \\
4.9(-12) \\
1.4(-7) \\
7.7(13) \\
0.098 \\
0.44\end{array}$ & $\begin{array}{l}5(-6) \\
5.5(-11) \\
8(-7) \\
6.6(-12) \\
1.6(-7) \\
8.8(-13) \\
0.13 \\
0.55\end{array}$ \\
\hline \multirow{2}{*}{\multicolumn{2}{|c|}{ Spectrum at $2 \ddagger$}} & \multicolumn{6}{|c|}{$E, \uparrow \mathrm{MeV}$} \\
\hline & & 6 & 7 & 8 & 9 & 10 & 11 \\
\hline $\begin{array}{c}r=85 \\
\mathrm{~cm} \\
r=95 \\
\mathrm{~cm} \\
r=120 \\
\mathrm{~cm} \\
\text { Spectrum }\end{array}$ & $\begin{array}{l}4 \pi r^{2} N_{0} \\
N_{0} \\
4 \pi r^{2} N_{0} \\
N_{0} \\
4 \pi r^{2} N_{0} \\
N_{0} \\
\text { at } 1 \neq\end{array}$ & $\begin{array}{l}5(-6) \\
5.5(-11) \\
9(-7) \\
7.4(-12) \\
1.8(-7) \\
9.9(-13) \\
0.15\end{array}$ & $\begin{array}{l}4(-6) \\
4.4(-11) \\
8(-7) \\
6.6(-12) \\
1.5(-7) \\
8.3(-13) \\
0.13\end{array}$ & $\begin{array}{l}3(-6) \\
3.3(-11) \\
6(-7) \\
4.9(-12)\end{array}$ & $\begin{array}{l}2(-6) \\
2.2(-11) \\
3(-7) \\
2.5(-12)\end{array}$ & $\begin{array}{l}1(-6) \\
1.1(-11) \\
1.3(-7) \\
1.1(-12)\end{array}$ & $\begin{array}{l}4(-7) \\
4.4(-12)\end{array}$ \\
\hline Spectrum & at 2 末 & 0.55 & 0.44 & 0.33 & 0.22 & 0.11 & 0.04 \\
\hline
\end{tabular}

$\dagger 4(-5)=4 \times 10^{-5}$, etc.

\$Neutron $\mathrm{cm}^{-2} \mathrm{sec}^{-1} \mathrm{MeV}^{-1}$.

(b) Plot the energy-flux-density spectra $\left(E N_{0}\right)$ and numerically integrate:

SOLUTION TO EXERCISE 6.1(b)

E, $\mathrm{MeV}$

\begin{tabular}{|c|c|c|c|c|c|c|c|c|c|c|}
\hline 0.1 & 1 & 2 & 3 & 4 & 5 & 6 & 7 & 8 & 9 & 10 \\
\hline
\end{tabular}

At 1:

$\begin{array}{llllllllllll}E N_{0} & 0.07 & 0.24 & 0.36 & 0.45 & 0.39 & 0.65 & 0.90 & 0.91 & 0.78 & 0.45 & 0.20\end{array}$

$\begin{array}{ccccccccccccc}\text { At 2: } & & & & & & & & & & & \\ E N_{0} & 0.44 & 1.7 & 2.0 & 2.0 & 1.8 & 2.8 & 3.3 & 3.1 & 2.6 & 2.0 & 1.1 & 0.44\end{array}$

Using $\Delta E=0.4 \mathrm{MeV}$ for numerical integration:

$$
\begin{aligned}
& \int_{0}^{10} E N_{0}(\text { point } 1) d E \cong 5.3 \mathrm{MeV} \mathrm{cm}^{-2} \mathrm{sec}^{-1} \\
& \int_{0}^{11} E N_{0}(\text { point } 2) d E \cong 23 \mathrm{MeV} \mathrm{cm}{ }^{-2} \sec ^{-1}
\end{aligned}
$$




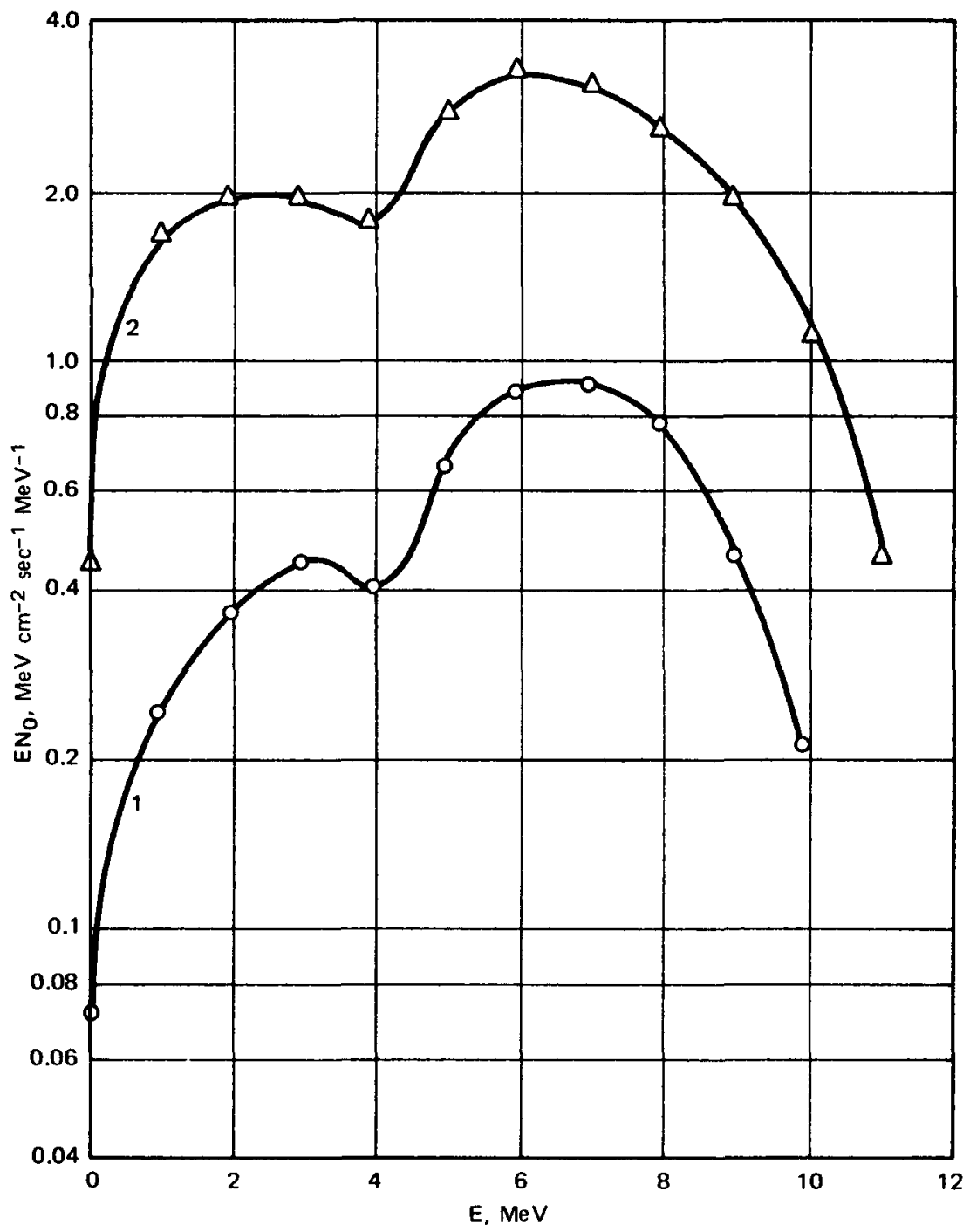

Solution to Exercise 6.1(b). Graphs of energy-flux density at points 1 and 2 .

(c) Use Fig. 6.20:

At point 1

$$
\Phi_{\mathrm{tn}}=\left(2 \times 10^{10}\right)\left(2.5 \times 10^{-10}\right)=5 \text { neutrons } \mathrm{cm}^{-2} \mathrm{sec}^{-1}
$$

At point 2

$$
\Phi_{\mathrm{tn}}=\left(10^{10}\right)\left(1.6 \times 10^{-9}\right)+\left(10^{10}\right)\left(2.2 \times 10^{-11}\right)=16 \text { neutrons } \mathrm{cm}^{-2} \mathrm{sec}^{-1}
$$


6.2 We first observe that $1 \mathrm{mrad} / \mathrm{hr}=2.78 \times 10^{-5} \mathrm{ergs}^{-1} \mathrm{sec}^{-1}$. Next we compute the flux density incident (essentially normally) to the slab.

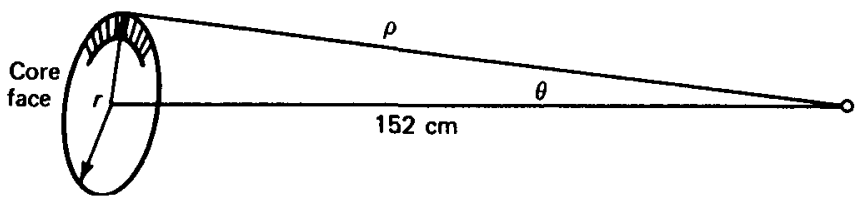

Solution to Exercise 6.2, geometry.

$7.63 \mathrm{~cm}=$ radius of beam tube

$$
\begin{aligned}
d \Phi & =\frac{10^{12} 2 \pi r d r}{4 \pi \rho^{2}} \cos \theta ; r^{2}+152^{2}=\rho^{2}, \cos \theta=\frac{152}{\rho} \\
\Phi & =\int_{r=0}^{7.63} \frac{\left(10^{12}\right) r d r}{2 \rho^{2}} \frac{152}{\rho}=\int_{\rho=152}^{152.19} \frac{152\left(10^{12}\right)}{2} \frac{d \rho}{\rho^{2}} \\
& =\left.5(152) 10^{11}\left(-\frac{1}{\rho}\right)\right|_{152} ^{152.19} \\
& =6.2 \times 10^{8} \text { neutrons } \mathrm{cm}^{-2} \mathrm{sec}^{-1} \text { at point } B
\end{aligned}
$$

To compute the attenuation, we use the curves of Appendix $M$ and the fission spectrum given in Fig. 2.18 to construct Table A. The choices of $\bar{E}$ were dictated by the curves of Appendix $M$. The spectrum $N_{0} / \Delta E$ was obtained from Fig. 2.18 multiplied by $\Delta E$, and the sum over all energy intervals was verified as 1.00 .

SOLUTION TO EXERCISE 6.2 - CONCRETE ATTENUATION DATA

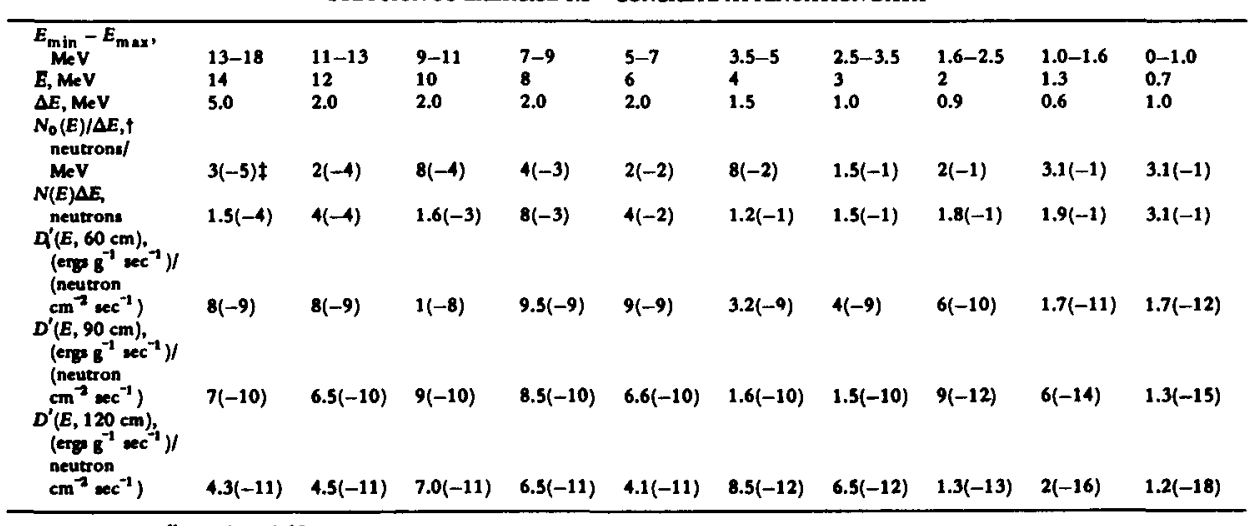


The three normalized dose spectra were obtained from Appendix M; we extrapolated where necessary. The objective is to get a curve of total dose rate vs. slab thickness for the whole spectrum. We now form the product $N(E) \Delta E \Phi D^{\prime}(E, x)=D(E, x)$, using $\Phi=6.2(8)$ neutrons $\mathrm{cm}^{-2} \mathrm{sec}^{-1}$, and construct Table B.

SOLUTION TO EXERCISE 6.2-CONCRETE ATTENUATION DATA

\begin{tabular}{lllll}
\hline $\begin{array}{c}\bar{E}, \\
\mathrm{MeV}\end{array}$ & $\begin{array}{c}N(E) \Delta E, \\
\text { neutrons }\end{array}$ & $\begin{array}{c}D(E, 60), \\
\text { ergs g- } \mathrm{sec}^{-1}\end{array}$ & $\begin{array}{c}D(E, 90), \\
\mathrm{ergs} \mathrm{g}^{-1} \mathrm{sec}^{-1}\end{array}$ & $\begin{array}{c}D(E, 120), \\
\mathrm{ergs} \mathrm{g}^{-1} \mathrm{sec}^{-1}\end{array}$ \\
\hline 14 & $1.5(-4) \dagger$ & $7.5(-4)$ & $7.0(-5)$ & $3.8(-6)$ \\
12 & $4(-4)$ & $2.0(-3)$ & $1.7(-4)$ & $1.6(-5)$ \\
10 & $1.6(-3)$ & $1.0(-2)$ & $0.9(-3)$ & $0.7(-4)$ \\
8 & $8(-3)$ & $4.7(-2)$ & $4.2(-3)$ & $3.2(-4)$ \\
6 & $4(-2)$ & $2.2(-1)$ & $1.6(-2)$ & $1.0(-3)$ \\
4 & $1.2(-1)$ & $2.4(-1)$ & $7.5(-3)$ & $6.5(-4)$ \\
3 & $1.5(-1)$ & $3.8(-1)$ & $1.5(-2)$ & $6.0(-4)$ \\
2 & $1.8(-1)$ & $7.0(-2)$ & $1.0(-3)$ & $1.5(-5)$ \\
1.3 & $1.9(-1)$ & $2.0(-3)$ & $7.0(-6)$ & $2.5(-8)$ \\
0.7 & $3.1(-1)$ & $3.3(-4)$ & $2.5(-7)$ & $2.2(-10)$ \\
Sum & 1.00 & $0.97=D(60)$ & $4.5(-2)=D(90)$ & $2.7(-3)=D(120)$ \\
\hline
\end{tabular}

$\dagger 1.5(-4)=1.5 \times 10^{-4}$.

Plotting $D(60), D(90)$, and $D(120)$ on semilog paper, we find, as expected (the curves in Appendix $M$ suggest it), that they fall on a straight line, and

$$
D(x) \cong 290 e^{-0.096 x}
$$

Thus

$$
\begin{aligned}
2.78 \times 10^{-5} \operatorname{ergs~g}^{-1} \sec ^{-1} & \cong 290 e^{-0.096 x} \\
0.096 x & \cong \ln \frac{290}{2.78 \times 10^{-4}}=\ln 104+\ln 10^{4}=13.9 \\
x & \cong \frac{13.9}{0.09}=145 \mathrm{~cm}
\end{aligned}
$$

6.3 We use Fig. 4.11 and the thickness computed in Problem $6.2(x=145 \mathrm{~cm})$. Extrapolating the $152-\mathrm{cm}$ curve of Fig. 4.11, we find that the thermal-flux density is about $5 \times 10^{-7}$ per incident unit flux density. Thus

$$
\begin{aligned}
\Phi_{\mathrm{th}} & \cong\left(6.2 \times 10^{8}\right) \times\left(5 \times 10^{-7}\right) \\
& \cong 3.1 \times 10^{2} \text { neutrons } \mathrm{cm}^{-2} \mathrm{sec}^{-1}
\end{aligned}
$$


6.4 The source strength of the disk is $5000 \times 3.7 \times 10^{10} \times 2=3.7 \times 10^{14}$ photons $/ \mathrm{sec}$ If we assume that there is no self-shielding, the equivalent surface source is

$$
\frac{3.7 \times 10^{14}}{3.14 \times 10^{2}}=1.18 \times 10^{12} \text { photons } \mathrm{cm}^{-2} \mathrm{sec}^{-1} \text { emitted isotropically }
$$

We plot Berger's coefficients vs. energy (Appendix F) using the 0 to 7 mean-free-path data $(75 \mathrm{~cm} \cong 4.7$ mean free paths) to find $C=0.96$ and $D=0.082$.

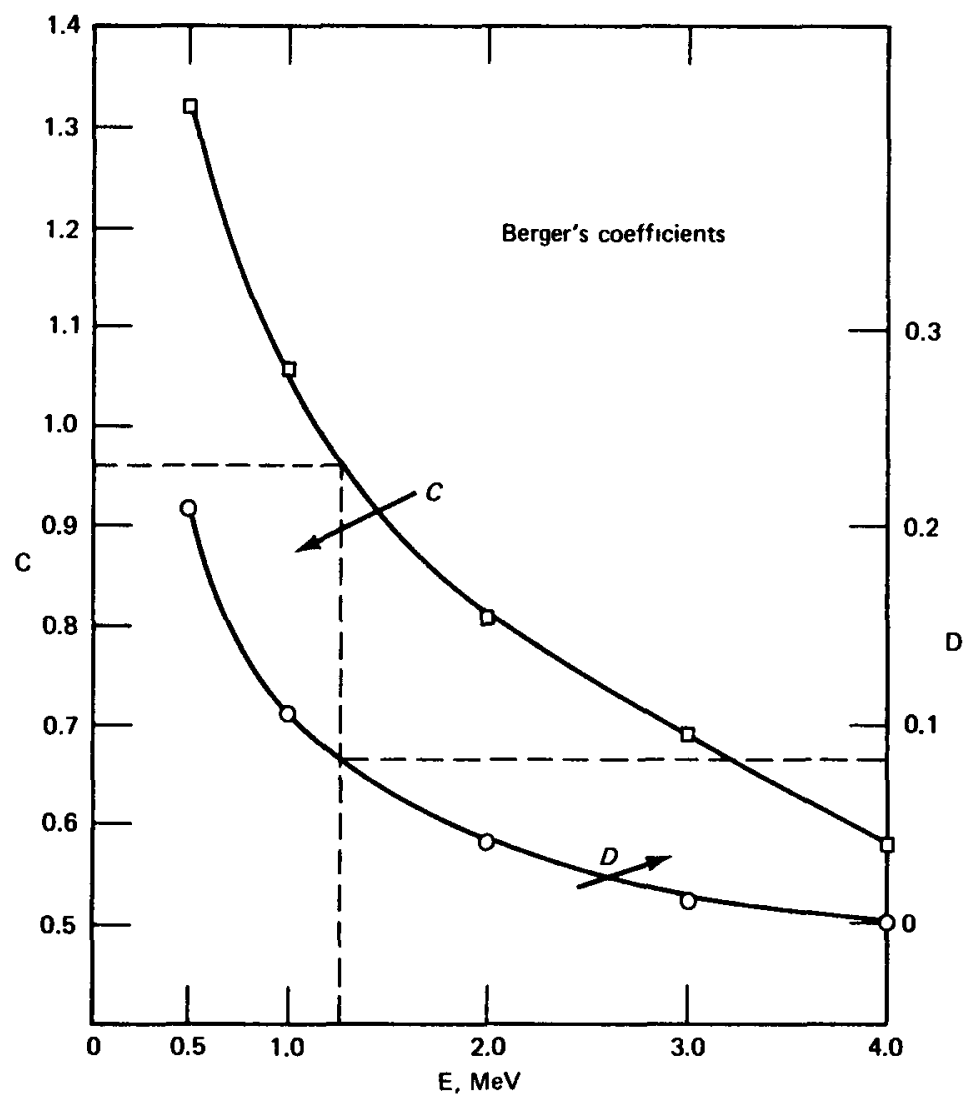

Solution to Exercise 6.4. Interpolation of Berger's coefficients for water.

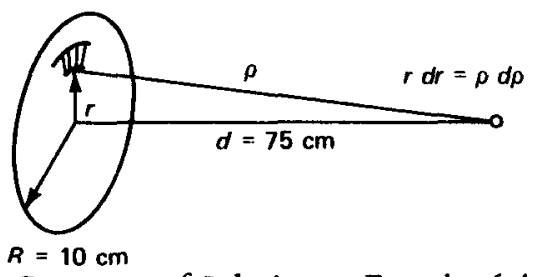

Geometry of Solution to Exercise 6.4. 


$$
D R=\int_{r=0}^{10} \frac{S_{a} r d r}{2 \rho^{2}} e^{-\mu \rho}\left(1+C \mu \rho e^{D \mu \rho}\right)\left(\mu_{a} E\right)
$$

where $S_{a}=1.18 \times 10^{12}$ photons $\mathrm{cm}^{-2} \mathrm{sec}^{-1}$.

Note that the first parentheses enclose the buildup factor and that the coefficient of the first term will be the dose rate without buildup.

$$
D R=\frac{S_{a} \mu_{a} E}{2}\left\{\int_{d}^{b} \frac{e^{-\mu \rho}}{\rho} d \rho+C \mu \int_{d}^{b} e^{-\mu \rho(1 D)} d \rho\right\}\left[b=\left(d^{2}+R^{2}\right)^{1 / 2}\right]
$$

Let $x=\mu \rho ; d \rho=d x / \mu ; \rho=x / \mu$, then

$$
\begin{aligned}
& D R=\frac{S_{a} \mu_{a} E}{2}\left[\int_{\mu d}^{\mu b} \frac{e^{-x}}{x} d x+C \int_{\mu d}^{\mu b} e^{-x(1-D)} d x\right] \\
& =\frac{S_{a} \mu_{a} E}{2}\left\{E_{1}(\mu d)-E_{1}(\mu b)+\frac{C}{1-D}\left[e^{-\mu d(1-D)}-e^{-\mu b(1-D)}\right]\right\} \\
& \mu d=0.064(75)=4.80 ; \mu b=\mu\left(d^{2}+R^{2}\right)^{\frac{1}{2}}=0.064(5625+100)^{1 / 2}=4.84 \\
& \mu d(1-D)=4.80(0.918)=4.406 ; \mu\left(d^{2}+R^{2}\right)^{1 / 2}(1-D)=4.84(0.918)=4.443 \\
& D R=\frac{1.18 \times 10^{12}(0.03)(1.25)}{2}\left[1.45 \times 10^{-3}-1.37 \times 10^{-3}+\frac{0.96}{0.918}\right. \\
& \quad=2.21 \times 10^{10}\left[8.0 \times 10^{-5}+1.05\left(5 \times 10^{-4}\right)\right] \\
& \text { (a) } D R=2.21 \times 10^{10}\left(6.05 \times 10^{-4}\right)=1.34 \times 10^{7} \mathrm{MeV} \mathrm{g}^{-1} \mathrm{sec}^{-1} \\
& \text { (b) The buildup factor is } 6.05 / 0.8=7.6 . \\
& =2.21 \times 10^{10}\left(8 \times 10^{-5}+5.25 \times 10^{-4}\right)
\end{aligned}
$$

†Values of $E_{1}$ are from Appendix G. 
6.5 Since the concrete shield is hydrogenous, we can use neutron removal theory. From Table 4.3 the removal cross section for lead is $\sigma_{R}=3.5$ barns/atom. Thus

$$
\begin{aligned}
\Sigma_{R} & =\frac{A_{v} \mu}{A_{w}} \sigma_{R} \\
& =\frac{0.6 \times 10^{+24}(11.3)}{207} 3.5 \times 10^{-24} \\
& =0.115 \mathrm{~cm}^{-1}
\end{aligned}
$$

The added factor of fast-neutron removal is simply

$$
e^{-\Sigma} R^{t}=\mathrm{e}^{-0.115(5)}=\mathrm{e}^{-0.575}=0.563
$$

The fast-neutron dose rate is reduced by a factor of 0.563 .

6.6 Since the average prompt fission photon energy is in the $\mathrm{MeV}$ region, an approximate, conservative answer can be obtained by using the minimum attenuation coefficient, which occurs at about $3.5 \mathrm{MeV}$ for lead. From Fig. 3.8, $\sigma \cong 15$ barns/atom. Thus

$$
\begin{aligned}
\mu & =\frac{0.6 \times 10^{24}(11.3)}{207}(15)=0.49 \mathrm{~cm}^{-1} \\
e^{-\mu t} & =e^{-0.49(5)}=e^{-2.5}=0.082
\end{aligned}
$$

The gamma dose rate is reduced by a factor of 0.082 . To obtain a more accurate result, we would follow a procedure for the gamma-ray spectrum similar to the treatment of the neutron spectrum in the solution to Problem 6.2. 


\section{Author Index}

Aalton, E., 566-570, 572-575

Abbott, L. S., 1, 8

Adamson, J., 560, 561-565

Alberg, M., 503-504, 506, 508

Albert, R. D., 178, 187-189, 286, 299

Allen, F. J., 272, 291, 319-320, 323-328, 393-394, 700-701, 727-728

Ambarzumian, V. A., 163

Anderson, D. C., 189-190

Aronson, R., 6, 465

Attix, F. H., 616

Auxier, J. A., 101, 109-110, 509, 616

Avery, A. F., 6, 190, 198

Bach, R. L., 175-176, 360, 653

Baksys, I. G., 338

Barrett, M. J., 347

Bayer, C. E., 398

Beach, L. A., 346, 731

Beaudry, R. J., 527, 529-531, 535-536, $538,542-543,545-547,549,551-558$

Beck, H., 503-504, 508

Belcher, J. A., 478-479, 595-597, 599

Bellman, R., 163

Bendall, D. E., 190-192, 194, 375, 377

Benenson, R. E., 375-376

Bennett, C. M., 598, 600-601

Benoit, J. W., 432

Berger, M. J., 6-7, 68, 79, 150, 171-172, $286,304,306,342-343,346-347,425$, 437

Betz, N. A., 291, 700

Bjeldanes, M. N., 574

Blatt, J. M., 240

Blizard, E. P., 6, 8, 55, 68, 92, 180, 291$292,299,430,579,582-583,586,592$, 594

Blosser, T. V., 580

Bokhari, M. S., 57, 482, 485, 487-489, 501, 503-504, 515

Bowman, L. A., 423
Bray, T. A., 672

Broder, D. L., 9

Brown, J., 291, 700

Burrus, W. R., 490

Burton, B. S., Jr., 275, 280

Butkov, E., 463

Butler, J., 6, 190-192

Cain, V. R., 6, 218, 382, 395, 398

Canali, U., 194

Capo, M. A., 7, 171-172, 424, 635-639

Carlson, A. D., 495, 497, 500

Carlson, B. G., 7, 133

Cashwell, E., 6, 228, 239-240

Casper, A. W., 188, 276

Cerbone, R. J., 494-497

Certaine, J., 6, 159

Chaltron, W. F., 527

Chandrasekhar, S., 133, 333-334

Chapman, G. T., 6, 179, 184

Chapman, J. M., 388-393, 400-401, 403

Chase, D. M., 371

Cheka, J. S., 509

Chilton, A. B., 7, 307, 327, 345-347, 381, $383-384,388-391,393-394,641$, $731-732$

Cierjacks, S., 496, 498

Claiborne, H. C., 8, 27, 63, 111, 362, 420, $519,717,722-725$

Clark, F. H. S., 6-7, 232, 291, 456, 458, $641,700,702-711$

Clark, O., 333

Clifford, C. E. (Canada), 401, 405-406

Clifford, C. E. (U.S.A.), 2, 4, 8, 362, 381, 490, 493-495, 498, 514-515

Coleman, W. A., 331, 339-340, 353, 396, 729-731, 733

Collier, W. D., 198

Collins, D. C., 378

Courtney, J. C., 57, 482, 485, 487-489, 501,503 
Coveyou, R. R., 6, 218, 667, 669-670

Cranberg, L., 54

Cranford, W., 465

Curtıs, C. C., 198

Davisson, C. M., 346, 731

Draz, J. A., 334

Dobell, A. R., 663, 667

Doggett, J., 6, 342-343

Doty, D. R., 394

Dresner, L., 8

Dudley, T. E., 13

Dudzıak, D. J., 181, 184-185

Dunn, W. L., 184

Edlund, M. C., 333, 340

Edwards, W., 6

Emmett, M. B., 291

Enginol, T. B., 465

Engle, L. B., 52-53

Engle, W. W., Jr., 7, 425

Eriksson, B., 231

Everett, C., 6, 228, 239-240

Fano, U., 5-6, 149-150, 155

Fasano, A. N., 375-377

Fedorenko, R. P., 464-465

Fenning, F. W., 3

Ferm1, E., 332, 340

Fisher, E., 550

Fisher, P. C., 52-53

Flynn, W. A., 595

Foderaro, A., 68, 84, 91, 291, 426, 428429

Fort1, P., 496

Freestone, R. M., Jr., 490, 580

French, R. L., 7, 298, 324-325, 329, 354, $425,442,444,509,514,728$

Friedman, S. T., 471, 480, 482

Frye, G., 54

Futterer, A. T., 272, 291, 319, 325-328, 700-701, 727-728

Garrett, C. W., 11, 63, 119, 207, 261

Gelbard, E. M., 148, 248

Gilley, L. W., 509

Glasstone, S., 333, 340, 595

Goertzel, G., 6, 218

Goldberg, M. D., 88

Goldstein, H., 1, 5-6, 8, 54-55, 58, 68, 70, $78,80-82,88,150,171-172,265-268$, $422-426,627,687$

Goussev, M. G., 291

Grant, J. S., 401

Greenhow, C. R., 425

Greenspan, H., 338
Gritzner, M. L., 432

Grodstem, G W., 5

Grosenbaugh, L. R., 672

Grotenhu1s, M., 7, 425

Grundl, J. A.,55

Gurney, W. J., 8, 347, 350, 732

Haffner, J. W., 189, 299

Hageman, L. A., 148

Haggmark, L. G., 347-348, 350, 732

Halliday, D. B., 3, 387-388

Halpern, O., 333, 340

Hansen, K. F., 167

Harrison, J. R., 3

Hayward, E., 6

Haywood, F. F., 509-513

Heaton, H. T. Il, 498

Held, K., 465

Henderson, B. J., 101-103

Henry, K. M., 490

Henry, R. L., 327-330

Herbold, R. J., 175, 653

HJarnc, L. H., 196, 567

Holden, N E., 13

Holl, R J., 575-577

Holland, L. B., 278

Hoover, L. J., 426

Horton, C. C., 1, 3, 6, 8, 366, 387-388

Howerton, R. J., 91

Hubbell, J. H., 6-7, 68, 79, 11 5, 172, $175-$ $177,360-361,653,655,660$

Huddleston, C. M., 327, 345, 389, 391, 394

Hull, T. E., 663, 667

Hungerford, H. E., 451, 519-520, 527528,550

Ilseman, H., 194

Ingold, W. C., 391

Irving, D. C., 6

Jacger, R. G., 8, 607

Jaeger, T., 8

Jerri, A. J., 400

Johns, H. E., 505

Johnson, W. P., 432

Jones, A. R., 111

Jones, J. D., 6

Jones, T. D., 109, 616

Jones, T. H., 347, 350, 732

Judd, A. M., 560

Kahn, H., 6, 230, 240

Kalaba, R., 163

Kalos, M. H., 6, 218, 244

Kam, F. B. F., 456, 458, 641

Ke1th, J. R., 432-433

Kimel, W. R., 8 
King, R W , 54

Kirhbride, J , 49

Klahr, C N , 430, 465

Kodras, F D , 300

Komarovsk11, A N , 445

Kopp, H J , 148

Kopsch, D , 496

Kouts H J 411-413

Kovalev, E E, 291

Krakar, J J , 598

Krell, A 566

Kropp, L , 496

Krumbein, A D , 265

Kruse, W G , 598

Kuščer, I , 124

Lacy, P S , 579

Laht1, G P , 460, 462

Lamkın, J C , 176, 360, 653

Lanning, W D , 132

Lathrop, K D , 133, 425-426

Leachman, R B , 56

Le Doux, J C , 381, 383-384 388-391, 393

Lee, C E , 133, 146

Leimdorfer, M 196, 231-232, 341-342, $349-351,567$

Leonard, B P, Jr , 4

Lewellen, J W , 579-580

Lewın, J , 8

Lietzke, M P , 424, 635

Littler, D J , 3

Loy, E T , 509

Lueneburg, R , 333

Lundin, M I , 509

Lyday, R O, Jr , 400

Lynch, R E , 432

MacDonald, J E, 6

MacPherson, R D , 667, 669

McLaughlın, J E , 503-504

McNair, N I , 560

Maerker, R E , 278, 319-320, 324, 330-

$331,337-340,353-354,362,396-400$, $490,726,729-731,733$

Marenschein, F C , 4, 13, 49-52

Mantey, R F , 527

Marable, J H , 426

Marcum, J I , 438-439

Marsaglia, G , 672

Marshall, J D , 378-380, 435, 506-507

Maskewitz, B F , 8

Mason, E A, 167

Mathews, D R, 167

May, V M , 88

Meem, L , 4
Mendelson, M R , 13

Metropolis, N, 666

Miller, J E , 6, 494

Miller, R A 465

Mills, C B 549

Mingle f $O, 167$

Mittelman, P , 6

Mizuta, H 167

Mockel, A , 334-335, 730

Mooney, L G , 327, 329-330, 419, 444, $471,509,514$

Morgan, I L , 607

Morgenau, $\mathrm{H}, 463$

Morris, E E , 347

Moses, E, 334

Moteff, J , 6

Muckenthaler, F I , 7, 278, 319-324, $330-331,337-340,353-354,396-400$, $426,490,726,729-731,733$

Mueller, G O , 425

Murphy, G M , 463

Mynatt, F R, 7, 132 147, 426, 490

Nebe, J , 496

Nelms, A T , 74, 76

Nereson, N , 54

Neuer, J R, 55

Neufeld, J , 108-110, 398

Newcll, J , 398

Newson, $\mathrm{H}, 2$

Nichols, J P , 456, 458-459, 461

Nicks, R , 198

O'Brien, K , 185-186, 503-504, 508

O'Reilly, B D 265

Peelle, R W , 4, 13, 49, 50

Penny, S K , 8, 426

Perkins, J F , 54, 610

Perline, G , 198

Peterson, D M , 282

Peterson, E G , 194-195

Piercey, D C , 375, 377

Plechaty, E F, 91

Pomraning, G C , 335, 340

Ponti, C, 198

Preusch, H , 194, 198

Prevost, R J , 327, 329-330

Price, B T , 3, 8

Price, W J , 184, 516

Profio, E A , 494

Raby, T M , 579

Raso, D J , 342-346, 348, 389

Rickover, $\mathrm{H}, 2$

Ritts, J J , 101-102, 105, 110,611

Rockwell, T III, 8, 550 
Roesch, W. C., 616

Rosen, L., 54

Rothberg, D. M., 185-186

Sabian, G. E., 425

Sanders, F. W., 509

Sandlin, R., 566

Schaeffer, N. M., 1, 4, 354, 419, 519

Schmidt, F. A. R., 717-721

Schmucker, J. E., 181, 184-185

Schrack, R. A., 498

Schreiber, P. W., 300

Schubart, H., 198

Schwartz, R. B., 498

Scofield, N. E., 347, 350, 732

Scoles, J. F., 54

Selph, W. E., 11, 63, 119, 207, 261, 279, 313, 398

Seraphin, D. S., 672

Serduke, J. T., 275, 281

Shamberger, R., 3

Sheffield, R. D., 465

Shelton, F. H., 432-433

Shimizu, A., 167

Shure, K., 6, 132, 185-186, 189-190

Simon, A., 381

Skarsgard, L. D., 505

Slotin, L., 2

Smith, C. V., 275, 281

Smith, D. C. G., 563

Smith, N. M., Jr., 413

Smith, W. R., 579, 584-585, 587, 590, 592

Snyder, W. S., 101, 108-110, 398, 616

Solomito, E., 93, 101, 178, 611

Song, Y. T., 326, 388, 393-394

Spanier, J., 248

Spencer, L. V., 5-6, 8, 149-150, 155, 334

Spinney, K. T., 3, 8, 190, 192, 195, 201, 300,366

Stehn, J. R., 88

Steinberg, H., 465

Stephen, D. W., 575-577

Stevens, P. N., 6, 7, 27, 63, 101, 119, 301, $331,396,426,611,729-731,733$

Stockton, J. R., 178

Storrs, C. L., 184

Straker, E. A., 6, 432-434, 438-441, 490

Suvorov, A. P., 9, 464-465

Taylor, J. J., 7, 171, 306

Terrell, C. W., 391-393, 402
Thompson, R. J., 595

Tipton, C. R., Jr., 451

Tobias, M. L., 424, 635

Todd, H. A., 515

Tonks, L., 409-410

Troubetzkoy, E. S., 89-91, 463, 465

Trubey, D. K., 6, 8, 111, 172, 175, 177, $179,247,291,300,302,304,371,423$, $425,427,432,629-631,641,644-649$, $657,662,734$

Tsypin, S. G., 9

Turner, M. A., 579, 584-585, 588-590, 592

Unseld, H., 496

Verbinski, V. V., 57, 482, 485, 487-490, $498,501,503-504,515$

Veselkin, A., 9

Vogel, R. E., 435

Vondy, D. R., 424, 635

von Neumann, J., 666, 673

Waldman, J., 347

Walker, R. L., 425

Ward, D. R., 509

Watt, B. E., 54

Webster, W. M., 432, 434

Weinberg, A. M., 129, 131

Weisskopf, V. F., 240

Wells, M. B., 6, 324-325, 329, 336-337, $340-342,345-349,352-354,432,435$, $437,506-507,728$

Welton, T. A., 3, 178, 187-189, 286, 299

Western, G. T., 277

Whitesides, G. E., 57, 482, 485, 487-489, 501, 503-504

Wick, G. C., 133

Wigner, E. P., 129, 131, 533

Wilkins, J. E., Jr., 6, 58, 150, 171-172, 422-423, 426

Willoughby, D. F., 495

Wing, G. M., 163

Worlton, J., 133

Wright, W., 272-273, 319, 325-328, 727728

Yegorov, Yu., 9

Yost, K. J., 91, 93, 218

Zerby, C. D., 6, 432, 435

Zinn, W., 2

Zoller, L. K., 179, 182, 478-479 


\section{Subject Index}

Absorbed dose, 95-98, 101, 106, 112, 116, $\mathrm{rad}, 97,104$

Absorbéd dose maximum, 616

Absorbed dose rate, 26, 97

Absorption coefficients, narrow beam, 82-83

Absorption of neutrons, 84

Activation cross sections, 93-94

Activation neutrons, 18

Activation-product gamma rays, 16

Agesta reactor, 566-574

calculational model, 567-570

description of reactor and shield, 566-570

magnetite concrete in, 566

measurements, 570-574

primary shield, 568

streaming in shield, 571

AIM-6 diffusion code, 548-549, 559

Aurcraft Shield Test Reactor (ASTR), 4

Air dose, 99

Arr Force, early nuclear program, 2-5

Air transport, 430-444

alr-over-ground calculations, 437-439

boundary effects, 431

comparison of calculation and measure-

ment, $440-442$

differential data, 435

effect of air density, 435,437

fission-neutron, 432, 434-435, 437

geometry, 431

infinite air medium, 432-437, 439

interface effects, 439-444

neutron (14 MeV), 432-433

single scattering, 432

Albedo, 228, 313-356

application to wall scattering, $355-356$

assumption, 314

differential types, $315-317$

effective, 314,318 fast-neutron, Monte Carlo, 319-322

borated polyethylene, 324-327

concrete, $319-328,726,728$

curve fit, $321-322,325,327$,

$$
\text { 726-728 }
$$

dependence on hydrogen content, 326,329

experimental verification,

$$
322-323,328-330
$$

tron, 324-327, 727-728

sorls, 324-327, 727-728

steel with borated polyethylene

vs thickness, 328-330

water, 324-327

gamma-ray, $341-350$

aluminum, $731-732$

concrete, 343-350, 731-732

contributions by positron

annihilation, 341-342

formulas for concrete, 345 , $348,731-732$

tron, 342-343, 348, 731-732

lead, $731-732$

for spherical walls, 349-351

vs. atomic number, $342-344$

water, $342-343,731-732$

geometry for definitions, 315

intermediate neutron, 331-332, 729

materials data avallable, 314

Mockel's coefficients, 730

neutrons incident at nonthermal

energies, 339-340

less than $200 \mathrm{keV}, 339-340$

up to $957 \mathrm{MeV}, 340$

neutrons incident at thermal energy,

332-339

analytic results, $333-336$

fits to Monte Carlo results, $336-339$, 730-731 
secondary gamma-ray, 350-355

fits from Monte Carlo data, 352-354, 733

thermal-neutron, $319,730-731$

three types, 316

total types, 315, 317-318

Albert-Welton point kernel, 178, 187-188, 286-287, 299

Angular current, 29

Angular current density, 34-39

Angular distribution

monodirectional approximation of, 46-48

plane isotropic source, 45

Angular energy-flux density, 30

Angular flux density, 29, 34

group, 31-32

ANISN code, 7

Annihilation radiation, 16

Anthracene scintillation dosimeter (ASD), 475

Argonne National Laboratory (ANL), 7

graphite pile, 1

Associated spherical-harmonic functions, 625

Associated spherical harmonics, 129

Ato mics International (AI) Shield

Test Facility, 596

Atoms for Peace Conference, 6

Attenuation

exponential, 122

geometric vs. material, 120-123

neutron (see specific transport method,

e.g., Moments method)

shield calculations, $261-309$

See also Shield attenuation calculations

slab, 121-123

spherical shell, 123

Attenuation coefficient, 66

See also Cross sections

linear, 83, 115

photon, 80-83

Attenuation functions for gamma rays, 653

slab geometry, 653-662

cosine source, 656-657, 661-662

disk source, $653-658$

isotropic source, 654-655, 659-660

rectangular source, 658-662

ATTOW removal-diffusion code, 198-199

Average value, 24

Azimuthal angle, 42
Barn book, 92, 94

Barrier factor, 121

BEPO, 3

Beryllium measurements, 478

Beryllium oxide measurements, 478-482

Bettis Atomic Power Laboratory, 2

Biasing, 216, 223-225

collision parameters, 234-236

effect on variance, 250

particle weight, 223-225, 228

path length, 229

plane isotropic source, 224-225

point isotropic source, 223-224

Binding energy of neutrons, 15, 91, 93, 97

Biological damage, 94-112

Biological dose, 94-95

Boiling water reactor, 574-579

calculations, 578

measurements, 579

plant layout, 576

Boltzmann transport equation, 7, 119-120, 123-129, 208, 216-217

applied to discrete ordinates, 134-135

approaches to solving, 128-129

conservative form, 135

integral operator, 136

integro-differential form, 127-128

in moments method, 150-151

second form, 131

transformations, 128

Boral

effect on captures, 481

measurements, $478-482$

Borated graphite

in Dounreay reactor, 560-562

in Fermi reactor, 537-541

Borated materials, thermal absorption in, 93

Boron, $449-450$

in shield fabrication, 525

Boron carbide measurements, 478

Boron steel

in Fermi reactor, 540-541, 545

measurements, 478-482

Boron trifluoride detector, 473

Bremsstrahlung, 17,83

in dose calculations, 101

production of photoneutrons, 497, 501

BREN (Bare Reactor Experiment Nevada), 509

British program, 3-4, 6

Brookhaven National Laboratory (BNL), 3,65 
data files, 492

Broomstıck experıment, 490-492, 494

Buildup factor, effective, 424

Buildup factors for gamma rays

applications, 284-286

Berger form, 171-172, 286, 304, 307, $628,631,640$

coefficients for air, $640-641$

aluminum, 636

concretes, 640

iron, 637

lead, 639

lithium hydride, 640-641

sand, 640-641

tin, 638

water, 635

wood, 640-641

in ducts, 368, 407

energy absorption, 170

energy deposition, 170, 631-632

equivalent $Z$ method, 423

exposure, 170, 626-628,630

finite media, 422

infinite plane source, 285

interpolation method, 424

linear form, 171-172, 628

multiple layers, 423-424

narrow beam geometry, 284

polynomial form, $171-172,305,635$

quadratic form, 171-172, 628

in shield designs, $578,589,598$

in shield heating, 421-425

Taylor form, 171-173, 306, 408

wide beam geometry, 284-285

Bulk Shielding Reactor (BSR), 1, 4, $482-485,591$

\section{17 code, 282}

Cable route openings (see Ducts)

Calcium borate in Fermi reactor, 539-543, 546

Capture gamma rays

energy distribution, 15

sources of, 14-15

spectra, 15

Carbon steel in Fermi reactor, 540-541, 545

Carlso'n's $S_{n}$ (see Discrete ordinates $S_{n}$ method)

Center of mass coordinates, 236

Charged particles

equilibrium, 99-101

heating, $429-430$

sources of, 12

Chicago Ple 3, 2
Coherent scattering of photons, 80-81, 115

Collided radiation, 119-120

Collision kernel, 217

Collision parameters, 234-242

biasing, 235-236

direction cosines, 241-242

gamma rays, 235,240

neutrons, 235-240

Compton effect, 70-74, 97--98, 104, 341

energy deposition, 73

fractional energy loss, 73

in Monte Carlo, 240

Compton wavelength, 71-72, 117

Conceptual design, 520-524

calculations for, 521-522

detaled considerations, 522-524

economic considerations in, 523-524

radiation streaming in, 523

Concrete, 1-3, 447451

barytes, 3, $450-451$

colemanite, $450-451$

construction criteria, 534

in Dounreay reactor, 560562

effect of water content, 456, 459, 461

effective atomic number, 425

ferrophosphorus, $450-451$

heating criteria, 533

iron-portland, 3, 450-451

lumnite, $450-451$

magnetite, 544, 566

monoenergetic neutron transport

data, 291

in Pathfinder reactor, 576

serpentine, 544

in shield fabrication, 526

Convair, Fort Worth, 2-5

Conversion factors (see Neutron conversion factors)

Coordinate systems, 617 center of mass, $86 \quad 87$

laboratory, 86

transformations, 617-619

Cosine distribution, 45-48

Coupled Monte Carlo and discrete ordinate calculational links, 7

CRAM, $548 \quad 550,556$

Cross section, differential, 72, 237

energy absorption, 420

Cross sections, 63-67

See also Kemoval cross section

absorption, 64

activation, 93-94

effect of state of medium, 64

evaluated data, 64-65 
gamma-ray, 68-83

Compton energy absorption, 73

Compton scattering, 73,81

pair production, 74-80

photoelectric effect, 68-70

macroscopic, 65-66

microscopic, 64-65

neutron, 53-94

effect of minima, 490-492, 503

elastic, 84-88

inelastic, 64, 89-91

nonelastic, 93

radiative capture, 92

total, 64

units of, 65

Crud, 575-578

Cumulative distribution function (CDF),

21, 211-215, 258

Cumulative scattering probability, 237

Current, 35

angular, 29

differential net, 35

net, 35

scalar, 36

Current density, 26, 34-39

angular, 34-39

examples, 37-39

net, 60

Curvature coefficient in discrete ordinates, 139

Delbrück scattering, 80

Demonstration Monte Carlo program, $251-254,674-686$

flow chart, 676-678

FORTRAN listing, 679-684

input data requirements, 675

test problem, 684-686

Depleted uranium, 454-455, 457

Detectors

boron trifluoride, 473

concept, 26

gamma-ray (see Gamma-ray detectors)

neutron (see Neutron detectors)

ther moluminescent (TLD), 476

Diamond difference technique, 133. 144-145

breakdown, 145

Differential cross section, 72, 237

Differential distributions, 19-24

first-moment, 24,60

function, 29,42

histogram form, 22

nth-moment, 24

Differential energy-flux density, 31
Differential flux density, 30, 43

Differential net current, 35

Differential particle density, 28

Differential phase-space cell, 124, 134

Differential solid angle, 25

Diffusion theory, $160-162,190$

equation, 161

first-flight correction, 190

group approach, $161-162$

Dirac delta function, 621-622

Direction cosines, $241-242$

Directional distributions, $42-48$

cosinc, 46-48

isotropic, 47

monodirectional approximation, 48

normalization, $46-47$

quadrature schemes in, 146

source term in, 143

Discrete-ordinates $S_{n}$ method, $7,132-149$, 494

advantages and disadvantages,

148-149

anisotropy of gamma-ray scattering, 426

calculation, removal term of, 140-142 curvature coefficient in, 139

derivation of finite-difference equation, $136-144$

group angular flux in, 136

multigroup calculations for, 140

negative flux fix-up, 145

numerical solution, 144-148

ray effect, 148

spherical geometry, 134

Dispersion, 248-250

Distribution functions

cumulative, $21,211-215,258$

sampling from, 209-215

Divergence theorem, 126, 620-621

Domain of function, 210, 214

Dose, 94-112

absorbed, 95-98, 101, 106, 112, 116

maximum, 108-111, 616

rate, 26,97

air, 99

biological, 94-95

first-collision, 99-103

free-air, 99

free-field, 99

maximum permissible (MPD), 112

multicollision, 112

Dose buildup factor, 170

Dose current, 271-272

Dose distribution factor (DF), 108 
Dose equivalent, 105-108, 112 maximum, 108-111, 616

Dosimeters anthracene scintillation (ASD), 475 glass (see Gamma ray detectors) Hurst fast neutron, 472, 511

DOT, 7, 426

Dounreay Fast Reactor, 559-566 borated graphite in, 560-562 calculation model, 562 graphite in, 559 measurements, 563-565 resilon in, 559-560 rotating top shield, 559-566 streaming, 565-566

DTF code, 486-490, 494

DTK code, 486-490, 502-504, 548

Ducts, $356-404$

bent, 378-404

cylindrical, cosine source, $735-736$ isotropic source, 734, 736

line of sight component, 357--367 $\operatorname{cosine}^{\mathrm{n}} \theta$ distribution, 358-359, 362

cylinder annulus, 366-367

cylinder ducts, 365-366

long thin ducts, 363

rectangular ducts, 360-363

rectangular slots, 363-365

types of plane sources, 358-359

relative magnitude of line of sight and wall penetration components, $371-372$

wall penetration component, 367-375

comparison with experiment, 374-375

cylindrical ducts, 370-372

partially penetrating cylindrical

ducts, $372-374$

ray analysis, $368-370$

wall scattered component, 375-404

albedo methods, 380-404

albedo Monte Carlo, 395-404

analog Monte Carlo, 376-380

coplanar vs noncoplanar three legged ducts, 401

corner-lip correction, 383-384

double reflected component, 382

experimental studies, 400-404

first-order reflection, 380

rectangular-duct formula, 391

rectangular ducts, 388-394

Simon-Clifford technique, 385-388

U-shape, 400-402

Z-shape, 400-402
Economic considerations in conceptual design, 523-524

Effect of medium, 57-58

Effective buildup factor, 424

Effective relaxation length, 288, 371

Elastic neutron scattering, 84-88

angular distribution, 87

anisotropy, 88

ENDF/B (evaluated nuclear data file), 65, 492, 494-495

Energy absorption cross section, 420

Energy cutoff in Monte Carlo, 255

Energy deposition

definition, 96-98

methods, 419-430

rate, 67

Energy distribution of fission neutrons, 54-56 of particle reaction neutrons, 18

Energy distributions, 48-50 effect of medium, 57-59 sampling from, 218-219

Energy fluence, 33

Energy imparted by conızing radia tion, 96-98

Energy removal rate, 67

Energy spectra, 20 See also Differential distributions, Energy distributions

Engineering construction follow up, 525 field drawings, 525

in sheld design, 524-525

Enrico Fermi Atomic Power Plant, 519, 527-559

biological dose criteria, 532

borated graphite in, 537-541

boron steel in, 540-541, 545

calcium borate in, $539-543,546$

calculational techniques, 548-552

carbon steel in, 540-541, 545

comparison of measurements and calculations, 553-559

description of plant, 528-532

exotic materials, 548

graphite shield, $537-541$

heat removal system, 532

intermediate heat exchanger, 544

lead in, 541

magnetite concrete in, 544

operating floor shield, 544

primary neutron shield, 535-541

primary shıeld, 528

primary sodium-pıpe shields, 547

radial biological shield, 544 
radiation-damage criteria, 532-533

radiation heating criteria, 533

reactor shield, 534-547

remote maintendnce, 533

rotating plug shield, $539-540$

safety standards, 534

secondary shield wall, $541-543$

serpentine concrete in, 541

shield costs, 548-549

shield criteria, 532-534

shield systems for the auxiliary facilities, 547

shield test program, 527

sodium coolant, 529-559

sodium pump shield plugs, 544

stainless steel in, 540-541

steel shot in, 539, 545

streaming, in shield, 550

streaming paths, $538-547$

thermal shield, 537, 542-543

Entranceways (see Ducts)

Epithermal region, 85

Equation of continuity in diffusion theory, $160-162$

Equidistribution, 664

of random-number generators, 664 , $669-670$

Euler's constant, 306

Evaluated nuclear data file (see ENDF/B)

Evaluation of integrals by Monte Carlo method, 216-218

Event, 209, 258

probability, 207-208

space, 210

Excitation energy, 239

in neutron capture, 91

Expected value, 212-213, 248-250

Experımental shıeldıng, 471-516

importance of, 471

purposes of, 472

Experiments

accelerators, 493-502

air transport and air-ground interface, 507-514

${ }^{6}$ Co gamma-ray, 508-514

cross section evaluation, 490-492

${ }^{137} \mathrm{Cs}$ gamma ray source, 503-507

duct penetration, 514-516

fixed sources, 503-507

LINAC, 498-503

neutron, (14 MeV), 507-514

neutron spectral and angular

distribution, $482-490$

reactor source, $477-493$

various environments, 516
Exponential attenuation, 122

Exponential function, 305

Exponential integral functions, 292 , 642-652

graphs, 644-652

Exponential transform, 229, 232

Exposure, 103-105

Exposure buildup factors, $170,626-628$, 630

See also Buildup factors

Exposure rate, 26, 104

Fallout shielding, 8

Fast breeder reactor, 527-566

Fast neutron energy region, 85

Fast reactor system, special shielding problems of, 527

Federal Register, 109

Fermı age

in moments method, 149

theory, 189

Fermi Nuclear Plant (see Enrico Ferm1 Atomic Power Plant)

Finite difference cell, 132, 134

Finite difference equation, 132

First collision dose, 99-103

First flight estımate, $244-247$

First-last collision model, 442-444, 509

Fission chambers, 473

Fission gamma rays, 13

delayed, 13

energies, 13

intermediate life, 13

prompt, 13

short life, 13

spectra, $49-51$

Fission neutrons, 17

See also Neutron spectra from

fission

a1r transport, 432, 434-435, 437

average energy, 17

delayed emissions, 17

energy distribution of, 54-56

energy range, 17

number, 17

Fission plate, 492

Fission-product gamma rays, 13-14,

$$
\text { 51-54 }
$$

beta-ray energy, 54

disintegration rate, 54

energies, 14

function of reactor operating time, 53

half-lives, 14

long lived, 52

total gamma-ray energy release, 54 
Fitted parameter data application, 277-283

Fluence, 33

Fluorescent radiation, 68

Flux, 28

See also Flux density

removal, 194, 196

Flux density, 26, 28

angular, 29, 34

angular energy, 30

definition, 124

differential, 30,43

differential energy, 31

examples, $37-38,60$

group, 31-32

incremental sphere concept, 31

special limiting process, 26,33

thermal neutron, 298-301

total, 31,32

total energy, 31-32

FORTRAN, 251

Free alr dose, 99

Free field dose, 99

Frequency functions, 210

Gamma ray attenuation (see specific trans port method, e g, Moments method)

Gamma ray attenuation functions (see Attenuation functions for gamma rays)

Gamma ray buldup factors (see Buldup factors for gamma rays)

Gamma ray cross sections (see Cross sections, gamma ray)

Gamma ray detectors, 475

anthracene scintillation dosimeter, 475,591

film, 476

Geiger-Mueller, 571

Geiger tube, 579

glass, 476

glass dosimeters, 571

ion chambers, 571

1onization chamber, 599

$\mathrm{NaI}(\mathrm{Tl})$ spectrometer, 475-503

calibration, 505-506

solid state spectrometers, 475

Gamma ray doses in phantoms, 111

Gamma ray energy release vs time after fission, 608-610

Gamma ray heating, 309, 420-429

comparison of $S_{n}$, Monte Carlo, and

buldup factor methods, 426-427

differential energy spectra, 425

multigroup diffusion code, 425

$S_{n}$ code, 425
Gamma ray sources, 12-17

See also Activation product gamma rays, Capture gamma rays, Fission gamma rays, Fission product gamma rays,

Secondary gamma rays

inelastic neutron scattering, 605-607

relative importance, $12-13$

Gamma rays

activation product, 16

attenuation functions for, 653

See also Attenuation functions for

gamma rays

buildup factors for (see Buldup factors for gamma rays)

capture (see Capture gamma rays)

fission, 13

fission product, $13-14,51-54$

inelastic-scattering, 15-16

kerma rate factors for, 102-103

reaction product, 16

General Dynamics, 478

Aerosystems Test Facility, 596

General Electric Company, 5

Graphite, 449

in Dounredy reactor, 559

in Fermi reactor, 537-541

in shield fabrication, 525

specifications, 534

Green's functions (see Kernel technique)

Ground Test Reactor (GTR), 4

Group angular current, 35

Group angular flux density, 31-32

Group angular flux in discrete ordinates, 136

Group flux density, 31-32

Group net current, 35

Gulf General Atomic, 494, 497

Hanford, 2

Hanford reactor, $1-2$

Haydite, 1

Health Physics Research Reactor

(HPRR), 509

Heavy water, natural uranium, 566-574

Helium 3 filled detectors, 473

History generator, 209

routine for, 218

Hurst fast neutron dosimeter, 472, 511

ICRP (see International Commission on

Radiological Protection)

ICRU (see International Commission on

Radiation Units and Measurements)

Importance functions, 217-218, 230

Independence, in random number sequences, $664-665,670-671$ 
Inelastıc neutron scattering, 84, 88-91

gamma rays from, 90-91

threshold, 88-89

ty pical cross section, 89

Inelast1c-scatterıng gamma rays, 15-16

emission time, 15

energies, 16

Inscatter term

in discrete ordinates, 139-140, 142

in transport equation, 125, 127

Interaction loss term in transport equation, 127

Interaction probability, 29

See also Cross sections

Interaction rates, 63

Interactions of radiation, 63-117

Intermediate neutrons, energy of, 85

International Atomic Energy Agency (I AEA), 8

International Commission on Radiation Units and Measurements (ICRU), 27, 33, 95, 99, 103-104, 106, 112

International Commission on Radiological Protection (ICRP), 107-108

Invariant imbedding, 7, 163-168 advantages and disadvantages, 167-168 application, 167

Ionization chambers gamma ray 475 neutron, 473

Ionizing radiation, energy imparted by, 96-98

Iron, 449

neutron cross section, 494

spectra, 492

Isotropic distributions, $43-45,47,60$

Isotropic point sources, $44,60-61$

Kerma, 99-103

Kerma rate, 26, 100

factors for gamma rays, 102-103

factors for neutrons, 101-102

Kernel technique, 168-188

gamma rays, 169-177

neutrons, 178-188

plane disk source, 172-175

rectangular source, 176-177

KFK data files, 492

Kleın-Nishına formula, 72, 341

Knolls Atomic Power Laboratory

(KAPL), 2, 5

Kronecker delta function, 624

Laplacian operator, 620

Last flight estimate, 244-247

Law of large numbers, 216
Lead, 448, 449

in Fermi reactor, 541

measurements, 478-482

in Savannah reactor, 581,587

Legendre polynomial, $360-362,622-623$ associated, 623-625

in discrete ordinates, 139-144

in moments method, 151-152, 156

in spherical harmonics, 132

Lethargy, 33

Lıd Tank Shieldıng Facılıty, 3, 178-180, 492-493

LIDO reactor, 4

LINAC (linear accelerator), 494

Line source, 40

Linear attenuation coefficient, 83, 115

Linear energy transfer (LET), 105

Lithium hydride, 454-456, 458, 460, 462 measurements, $478-482$

in SNAP-10A shield, 596

LO5 code, 378

Lockheed, 5

Los Alamos Scientific Laboratory

(LASL), 6-7

MAC

difference from Spinney method, $195-196$

removal-diffusion method, 194-196

Macroscopic cross sections (see Cross sections, macroscopic)

Macroscopic transfer coefficient, multigroup, 140

Magic nucle1, 91

Magnetite concrete in Ágesta reactor, 566

in Fermi reactor, 544

Manhattan Project, 1

Masonite, 2

Mass attenuation coefficients, 66, 83, 115

Massachusetts Institute of Technology, 2

Materıals Testıng Reactor (MTR), 4

Mathematical experiment, 20

Maximum absorbed dose, 108-111

Maximum dose equivalent, 108-111

Maxımum permissible dose (MPD), 112

Maxwell-Boltzmann distribution of thermal neutrons, 85

Mean value, 212-213

Mean-value theorem, 137-138

Measured data applications, 274-283

gamma-ray data, 276, 280-281

neutron data, 275-279

Microscopic cross sections (see Cross sections, microscopic) 
Mock up tests, 472,478

MODRIC, 300

Moments method, 5 6, 149-159

application, 265-269

definition of moments, 149

in design cases, 588,598

energy degradation in scattering, 159

Fermı age in, 149

gamma ray differential data, 268-269, 692-695

accuracy, 268

buildup factors, 269

finite media, 268

lead, 696-699

spectra, 269

water, 692-695

neutron differential energy spectra,

$265-268,687-691$

beryllium, 687

carbon, 588

$\mathrm{CH}, 689$

$\mathrm{CH}_{2}, 690$

finite medid, 267268

$\mathrm{H}_{2}, 691$

$\mathrm{H}_{2} \mathrm{O}, 691$

sample problem, 268

spectral hardening with depth, 266

surface source, 268

neutron transport, $158 \quad 159$

polynomial expansion of flux densities, 155-157

sequence of moments calculation, 153 undetermined parameters to construct the flux densities, 158

Monte Carlo codes, debugging of, 255

Monte Carlo data applications, 270-274

comparison with moments method, 270

complexity of, 270

neutron attenuation, in concrete, $702-716$

in soil, polyethylene, and water, $712-716$

principle of reciprocity, $270-271$

sample results, $272-273$

Monte Carlo methods, 6, 207-259

biasing, 216, 223-225, 250

collision parameters, 234-242

cross-section preparation, 235

demonstration program, $251-254$, 674-686

evaluation of integrals by, 216-218

exponential transform, 229-232

first flight estimate, 244-247

importance function, 217-218, 230 last flight estimate, $244-247$

neutron elastic scattering, 236-238

neutron inelastic scattering, 238-240

particle absorptions, 240-241

particle scoring, 242-247

path-length sdmpling, 225-234

probability distribution function,

$210-215,258$

programming suggestions, 254-257

random sampling, 209-215

rejection technique, $214-216,258$

splitting, 234

statistical estimates, $243-244$

statist Ical variance, $247-251$

transport equation, 208, 216-217

MORSE code, 6,256

Most probable value, 24

Multucollision dose, 112

Multigroup calculations for discrete ordinates, 140

Multigroup cross section in Monte Carlo, 256-257

$\mathrm{Nal}(\mathrm{Tl})$ gamma ray spectrometer, 475-503 calibration, 505-506

Narrow beam absorption coefficients, 82-83

National Bureau of Standards (NBS), 5-6

National Neutron Cross Section Center, 65

Nautilus, U S S , 5

Navy nuclear powered submarıne program, 25

Negative flux fix up in discrete ordinates, 145

Net current, 35

Net current density, 60

Net leakage term in transport equation $125 \quad 127$

Neutrinos, 12

Neutron attenuation (see specific transport method, e g , Moments method)

Neutron binding energies, 15, 91, 93, 97

Neutron conversion factors

fluence to kerma, $611-613$

reactions in neutron kerma calculations, 611-615

Neutron cross section (see Cross sections, neutron)

Neutron detectors active, $472-474$

$\mathrm{BF}_{3}$ counters, $473,515,554,579,591$

emulsion, 475

fission counters, 473, $553 \quad 563,598$

fission folls, 553

forls, $474,563,573$ 
${ }^{3} \mathrm{He}$ filled, 473

Hurst fast neutron dosımeter, 472, 511

${ }^{6} \mathrm{~L}, \mathrm{~F}, 486$

long counter, 591

Ne 211 organic scintillators, 494,497

Ne 213, 490-491, 501

Ne 908 lithium gas scintillator 494

passive, 472475

proportional counter, 474

proton recoll counters, 554

solid state, 474

sulfur pellets, 502

track length, 474

Neutron doses in phantoms, 109-110

Neutron elastic scattering

average angle, $428-429$

average energy loss in, 429

in Monte Carlo calculations, 236-238

Neutron emission, 18

Neutron heatıng, $427-429$

Neutron inelastic scattering, $15 \quad 16$

in Monte Carlo, 238-240

Neutron kerma factors, 101-102

Neutron radiative capture, $91-93,98$

Neutron reactions, 83-94

contrasted with gamma ray reactions $83-84$

nonelastic events, $90-91$

Neutron scattering

capture, 86-87

diffraction, 86

elastic, 8488

inelastic, $84,88-91$

potential, 86

resonance, 86--87

Neutron sources, 17-19

polonium-beryllium, 18

Neutron spectra from fission, 54-57

average energy, 56

empirical expression, $54 \quad 55$

${ }^{235} \mathrm{U},{ }^{233} \mathrm{U}$, and ${ }^{239} \mathrm{Pu}, 56$

Neutron spectrometer, 486-491

Neutrons

absorption of, 84

activation, 18

binding energy of, $91,93,97$

fission, 17

intermediate, energy of, 85

kerma rate factors for, $101 \quad 102$

thermal, energy of, 85

Neutrons per fission, 56

Nevada Test Site (NTS), 509

NIOBE, 133, 300, 486-490, 502-504, 548

Nonelastic neutron cross section, 93
Noneldstic redctions, 93

Normalization

of distribution function 211212

of functions, 21

of source strength, $46 \quad 47$

NRN removal-diffusion method,

$196-198,567$

Nuclear Aerospace Resedrch Facility $2-5,478$

Nuclear Data Sheets, 94

Nuclear Development Associates, Inc , 56

Nuclear emulsions, 475

Nuclear powered aircraft, 2-5

Nuclear-powered submarine, 2-5

Nuclear propulsion, 11

Numerical integration techniques 289290

Odk Ridg: National Laboratory (ORNL), $178,256,319,482,492,509,514,591$ early shielding program 2-9

$X 10$ redctor, $1-2$

Odk Ridge Tower Shielding Facility, 4

O5R system, 6, 256, 492

O6R, 256

1DF code, 494- 496

Optimization, $462-465$

conditions for a mınımum, 463

gradient nonlınear programming, 465

linear perturbation theory, 464-465

of shadow shields, 465

shield synthesis, $463 \quad 465$

with discrete ordinates transport, 465

Outsıde Test Tank (OTT), 477-479

$P_{n}$ approximation, 131

Patr production, 74--81, 104

Paraffinized wood, 1

Particle absorptions in Monte Carlo methods, 240241

Particle death, 208

Particle denstty, 26-27

differential, 28

Particle history, 0

Particle reaction neutrons, energy distribution, 18

Particle scoring in Monte Carlo methods, 242-247

Pathfinder Atomic Power Plant, 574-579

calculation, 578

measurements, 579

plant layout, 576

Path length

biasıng, 229

multıregıon geometry, 226-228

sampling of, 225-234 
single infinte region, $226-228$

track length 225-234

Phantom cylindrical, 616

Phantoms for dose calculations, 95, 1.09-111

gamma ray doses $\mathrm{n}, 111$

neutron doses in 109-110

Phase space 2730,124

in diffusion theory 161

invariant imbedding, 163-164

Photoelectric effect, $68-70,80 \quad 104$

Photon attenuation coefficient, $80-83$

Photon interactions 68-73

See also Compton effect, Pair production, Photoelectric effect

relative importance, $78,81-83$

Photoneutrons, 18

threshold photon energies, 18

Photons

coherent scattering of, 80-81, 115

Physical dose, 94-95

Plane source, 44

angular distribution, 45

isotropic, 44

Plutonium-beryllum neutron source,

18

Point isotropic source, 626

Point kernel

Albert-Welton, 178, 187-188, 286-287 299

applications, $6-7,40,288-298,550$, 578,588

See also Kernel technique

first collision approxımation, 294-296

geometric transformations, 291-294

last collision approximation for cylinder, 297-298

last collision approximation for slab, 289, 296-298

from Monte Carlo calculations, 290-291 shield heating, $421-425$

Point source, 39

Polar angle, 42

Polonium-beryllium neutron source, 18

Polyethylene, 453-454, 458, 460

in Savannah reactor, 581,587

Portholes (see Ducts)

Positron emission, 16

Pratt and Whitney, 5

Pressurized water reactor, 579-595

Probability density function (PDF), 210-215, 258

Probability of an event, 207-209, 258

distribution, 210

Proof testing of shield, 526-527
Pseudorandom numbers, 215, 663

Purdue University, 519

QAD, 178

Quadrature schemes in discrete ordinates, 146

Quality factor (QF), 106-108, 110

Rad, 97, 104

Radiation

annihilation, 16

collided, 119-120

fluorescent, 68

interactions of, 63-117

responses to, 94-112

scattered, 119-120

uncollided, 119-123, 292

unscattered, 119-123

Radiation detectors

See also Gamma ray detectors, Neutron detectors

interpretations of output, $476-477$

in a mixed field, 476

types of, 471-498

Radiation heating (see Shield heating)

Radiation heating criteria, 533

Radiation intensity, 26-39

Radiation production, 11-19

See also Gamma ray sources, Neutron sources

Radiation Shielding Information

Center (RSIC), 8, 477, 482

Radiation streaming in conceptual design, 523

Radiation surveys, 527

Radiation transport, 119-201

geometric vs material attenuation, 120-123

slab geometry, 121-122

Rand Corporation, 6

Random number, 214--215, 226, 233-235, 237, 258

Random number generators, 215, 663-673

middle digit square, 666

mixed congruential, 666-667

multuplicative congruential, 667-668

recursion equations, $665-668$

tests of, 668-671

Random number sequences, 664-665, $670-671$

independence in, 664

Random numbers

pathological sequence of, $665,671-672$

Random sampling, 209-215

for cylindrical source, 220 
for disk source, 219

from energy distribution, 218-219

initial direction of source particle,

222

cosine source, 222

isotropic source, 221

of spatial position, 219

for spherical shell source, 220

of trach length, 225-234

Random variable, 210,258

Random walk, 208-209

RASH E removal-diffusion method, 192-194, 562, 567

Ray tracing, 550, 596

Rayletgh scattering, 80

RBE (see Relative biological effectiveness)

Reaction product gamma rays, 16

Reaction rates, 66-67, 83, 115

Reactors (see specific reactors and reactor types)

Recerver concept, 11, 26

Reflection, 46

Rejection technique, 214-216, 258

Relative biological effectiveness (RBE), 94, 105

dose, 105-108

Rem (roentgen equivalent man), 106

Removal cross section, 3, 178-188, 368, 492

additive property, 181

dependence on geometry, 179

for hydrogen deficient shields, 181-187

macroscopic, 179, 183

microscopic, 179-180, 186

minımum thickness of hydrogen, 180

relation to transport cross section, 181

Removal-diffusion methods, 6, 188-201, 560-562, 567

ATTOW, 198-201

differences in current methods, 199-201

MAC, 194-196, 199-201

NRN, 196, 201

RASH E, 192-194, 199-201

SABINE, $198-201$

Spınney method, 190-192

Removal flux, 194, 196

Removal term of discrete ordinates calculation, 140-142

Removal theory, 548, 578, 588, 596

$\mathrm{d}$ sfference between slabs and

homogeneous mixtures, $287-288$

kernel application, 286-288

RENUPAK, 178, 300

Resilon in Dounreay reactor, 559-560
Resonance region energy, 85

Responses to radiation, 94-112

Roeritgen, 104

Roentgen equivalent man (Rem), 106

Russıan roulette technıque, 234

$S_{n}$ method (see Discrete ordinates

$S_{n}$ method)

SABINE removal-diffusion method, 198

Sample space, 258

Samplıng, systematic, 250

Savannah N S , 579-595

attenuation calculations, 587-591

lead in, 581-587

main shielding, 580

maintenance shields, $581-583$

measurements, 591-595

polyethylene in, $453-454,458,460$

secondary gamma flux, 589

shield design criteria, 583-586

Scalar current, 36

Scattered radiation, 119-120

Secondary gamma rays, 84,91

in arr, 5

in dose considerations, 99

graphs for semi infinite shield, 722-725

graphs for slab shields, 718-721

kernel technique, 301-309

medsurements, 481

semı infinite shield, 308, $722-725$

slab shield, 304, $718 \quad 721$

sources of, 14-15

Serpentine concrete in Fermi reactor, 541

Shadow shield in SNAP 10A, 598

Shield attenuation calculations, 261-309 interpolation and curve fitting, 265

types of distributions, 264

use of equilibrium distributions, 265

use of parametric data, 264-283

use of unit source, 264

Shield design, 519-606

1terations, 519-527

principles of, 519-520

Shield heating, 3, 419-430

buldup factor, 421-425

by charged particles, $429-430$

in conceptual design, 523

energy deposition methods, $419-430$

by gamma rays, $420-429$

by neutrons, $427-429$

point kernel, $421-425$

ratio of neutron to gamma ray

heating, 427-429

in SNAP 10A, 598 
Shreld material experiments, 477-500

Shield materials, 443-460

See also specific substance

capture gamma ray dose, 456,460

comparison of gamma ray attenuation,

456-457

comparison of neutron attenuation, 456 , 458

for Lenin, 452

for mobile reactors, $450-462$

for Savannah N S , 452

selection of, 445-447, 521-522

shipping and storage, 452-453

for stationary reactor, $447-450$

testing, 526

thermal problems, 452

used in test reactors, 448

Shıp propulsıon, 579- -595

attenuation calculation, $587-591$

main shrelding, 580

maintenance shields, 581-583

measurements, 591-595

secondary gamma flux, 589

Sievert's integral, 292

Simon-Clifford technique, 578

method for cylindrical ducts, 385-388

Single collision dose (see First collision dose)

Slab attenuation, 121-123

Slab penetration measurements, 477-483

SNAP $10 A$

flight test, 595-602

radiation limits, 596

shadow shield $\mathrm{m}, 598$

shield analysis, 595-598

shield heating, 598

test results, 595-602

Solid angle, 24, 60

differential, 25

Solid state detectors, 474

Solid state gamma ray spectrometers, 475

Source analysis, 261-263

discrete ordinate $S_{n} 263$

fission gamma rays, 262

fission product gamma rays, 262

list of components, 262

low energy neutrons, 262

Monte Carlo solution, 263

reactor codes vs shielding codes, 263

Source term

in discrete ordinates, 143

in transport equation, 127

Space Nuclear Auxiliary Power, 595

See also SNAP 10A

Space power, 595-602

See also SNAP 10A
Spatial distribution, 39-42

line, 39-40

point, 39-40

surface, 39-41

volume, $39,42-43$

Spectra in water

gamma rays, 506-507

neutrons, 498

Spectrum, 49

See also Energy distributions

Spherical harmonic functions, 625

Spherical harmonics method, 7, 129-132

accuracy limitations, 131-132

truncation number, 131

Spherical shell attenuation, 123

Spinney method, 190-192, 300, 562, 567

multigroup diffusion equations, 191

of removal-diffusion calculations, 190-192

slowing down length, 191

variations, 192-201

Spiral duct, 2

Splitting, 234

Stainless steel

See also Iron

in Fermi reactor 540-541

Standard deviation, 213

Standard man composition, 111, 614

Statistical estimation, $217,243-244$

Statistical variance, $247-251$

average or mean, $248-250$

central limit theorem, 247-248

dispersion, 248

expected value, 248-250

Statistical weight, 208

Stochastic processes, 209

Steel shot in Ferm1 reactor, 539, 545

Steradian, 25

Streaming, 3

See also Ducts

in Agesta shield, 571

in Dounreay shield, 565-566

in Fermi shield, 550

Surface source, $40-41$

Survivorship function (distribution), 21, 60

TDC, 548,562

Thermal neutron flux density estimation methods, 298-301

multigroup diffusion, 299-300

Spinney method, 300

transfusion, 300-302

Thermal neutrons

energy of, 85 
Maxwell-Boltzmann distribution of, 85

Thermoluminescent detectors (TLD), 476

Thomson nuclear scattering, 80

Thomson units ( $\mathrm{T} U$ ) , 72

Time of flight measurement, 494-502

Titanium hydride, 454, 456

Total energy flux density, $31-32$

Total flux density, 31-32

Total net current, 35

Tower Shielding Facility, 322, 340, 514

Track length, 29

See also Flux density

detector for, 474

per unit volume, 29, 30

Transfer kernel, 217

Transport equation

Boltzmann (see Boltzmann transport equation)

inscatter term in, 125, 127

interaction loss term in, 127

net leakage term in, 125-127

source term in, 127

Transport kernel, 217

Tungsten, 453-454, 457, 460, 462

Uncollided radiation, 119-123, 292

United Kingdom early shielding program, 3-4, 6

University of Illinois, 7

Unscattered radiation, 119-123

Uranıum, depleted, 454-455, 457
U S Army Ballistic Research Laboratories, 319

Value functions, 218

Variance, 248-249

of function, 213

statistical, $247-251$

Vector derivative, 620

Vector operators, 619-620

Vents (see Ducts)

Voids, 404-414

See also Ducts

disk shaped, 404-409

ray analysis, 409

small random, 412

spherical, in water, 411

Volterra integral equation, 152, 154

Volume source, $41-42$

Water

gamma ray and neutron spectra in, 498, 506-507

measurements in, 448, 482

transport in, 498-503

Wigner effect, 533

Westinghouse Bettis Laboratory, 5

Windscale reactors, 3

$\mathrm{X} 10$ reactor, 2,492

Zirconium hydride measurements, 478

\section{NOTICE}

This book was prepared under the sponsorship of the United States Government Neither the United States nor the United States Atomıc Energy Commıssıon, nor any of their employees, nor any of their contractors, subcontractors, or their employees makes any warranty, express or implied, or assumes any legal liabılity or responsibility for the accuracy, completeness or usefulness of any information, apparatus, product or process disclosed, or represents that its use would not infringe privately owned rights 


\title{
Radioactive Decay Data Tables
}

\author{
A Handbook of Decay Data for Application to \\ Radiation Dosimetry and Radiological Assessments
}

\author{
David C. Kocher \\ Oak Ridge Natıonal Laboratory \\ Oak Ridge, Tennessee \\ Published by \\ Technical Information Center, \\ U S Department of Energy, 1981 \\ 228 pages, $8 \frac{1}{2} \times 11$ inches, paper binding \\ Library of Congress Catalog Card \\ Number 81-607800 (CIP)
}

\section{Contents}

\begin{tabular}{|c|c|}
\hline Chapter 1 & Introduction \\
\hline Chapter 2 & Review of Radioactive \\
\hline & Decay Processes \\
\hline Chapter 3 & $\begin{array}{l}\text { Preparatıon of Radıoactıve } \\
\text { Decay Data Sets }\end{array}$ \\
\hline Chapter 4 & $\begin{array}{l}\text { Computer Code MEDLIST } \\
\text { and Description of Tables } \\
\text { of Radioactıve Decay Data }\end{array}$ \\
\hline Chapter 5 & $\begin{array}{l}\text { Applications of Decay Data } \\
\text { to Radiation Dosimetry and } \\
\text { Radiological Assessments }\end{array}$ \\
\hline Chapter 6 & $\begin{array}{l}\text { Parent-Daughter Activity } \\
\text { Ratios }\end{array}$ \\
\hline Chapter 7 & $\begin{array}{l}\text { Accuracy of the Data and } \\
\text { Uncertain Decay Schemes }\end{array}$ \\
\hline Appendix 1 & Symbols and Definıtıons \\
\hline Appendix 2 & $\begin{array}{l}\text { Index to Tables of } \\
\text { Radıoactive Decay Data }\end{array}$ \\
\hline Appendix 3 & $\begin{array}{l}\text { References for Radioactive } \\
\text { Decay Data Sets }\end{array}$ \\
\hline Appendix 4 & $\begin{array}{l}\text { Dlagrams of Radioactive } \\
\text { Decay Chains }\end{array}$ \\
\hline Appendix 5 & $\begin{array}{l}\text { Tables of Radioactive } \\
\text { Decay Data }\end{array}$ \\
\hline
\end{tabular}

The estimation of radiation dose to man from either external or internal exposure to radionuclides requires a knowledge of the energies and intensities of the atomic and nuclear radiations emitted during the radioactive decay process. The availability of evaluated decay data for the large number of radionuclides of interest is thus of fundamental importance for radiation dosimetry.

Decay data are listed for approximately 500 radionuclides, which include those occurring naturally in the environment, those of potential importance in routine or accidental releases from the nuclear fuel cycle, those of current interest in nuclear medicine and fusion reactor technology, and some of those of interest to Committee 2 of the International Commission on Radiological Protection for the estimation of annual limits on intake via inhalation and ingestion for occupationally exposed individuals. This handbook supersedes Report ORNL/NUREG/TM102 , which was concernedonly with radionuclides from the nuclear fuel cycle. 